\title{
$A 11100985381$
}

NAT'L INST OF STANDARDS \& TECH R.I.C.

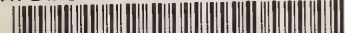

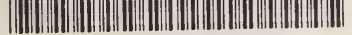

A11100985381

Materials Research S/Accuracy in trace a

QC100 U57 V422;V2;1976 C.1 NBS-PUB-C 19

"थ

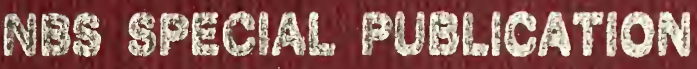

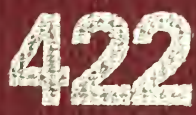

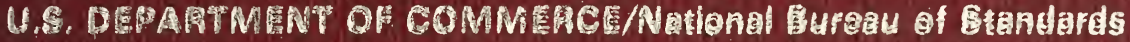

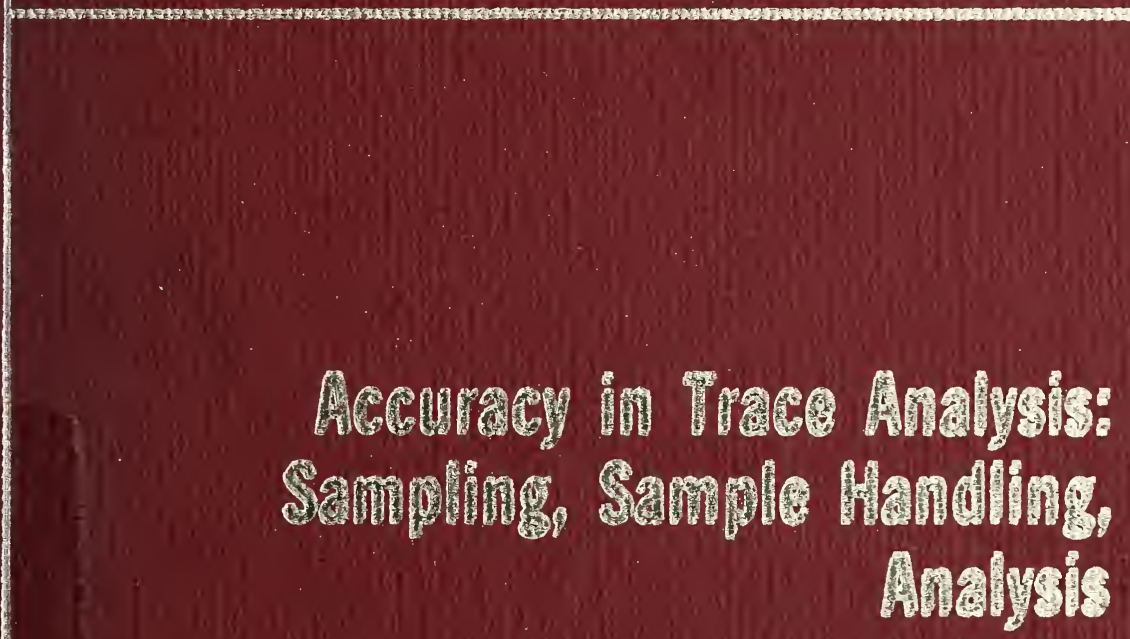

Volume II 



\section{DATE DUE}

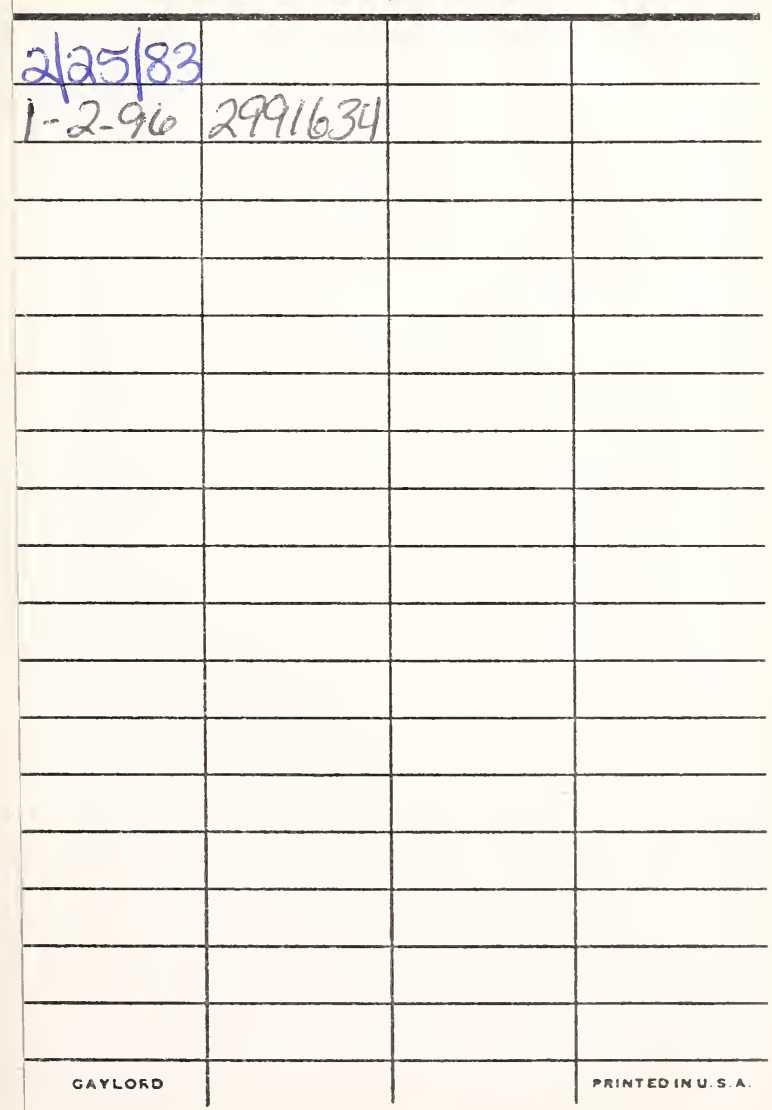





\section{Accuracy in Trace Analysis:

Proceedings of the 7 th Materials

Research Symposium

Held at the

National Bureau of Standards

Gaithersburg, Md.

October 7-11, 1974

Philip D. LaFleur, Editor

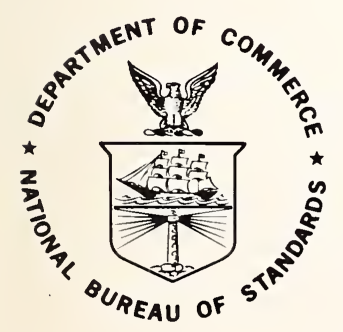

U.S. DEPARTMENT OF COMMERCE, Elliot L. Richardson, Secretary

Dr. Betsy Ancker-Johnson, Assistant Secretary for Science and Technology NATIONAL BUREAU OF STANDARDS, Ernest Ambler, Acting. Director 


\title{
Library of Congress Cataloging in Publication Data
}

Materials Research Symposium, 7th, Gaithersburg, Md., 1974.

Accuracy in Trace Analysis.

(NBS Special Publication; 422)

Supt. of Docs. No.: C 13.10:422

1. Trace Elements-Analysis-Congresses. I. LaFleur, Philip D. II. Title. III. Series: United States. National Bureau of Standards. Special Publication; 422. [DNLM: 1. Chemistry, Analytical-Congresses. 2. Trace Elements-Congresses. W3 MA425 1974a/QD139.T7 M425 1974a]

QC100.U57 No. 422 [QD139.T7] 389'.08s [543]

$75-619250$

\section{National Bureau of Standards Special Publication 422}

Nat. Bur. Stand. (U.S.), Spec. Publ. 422, Vol. II, 636 pages (Aug. 1976)

\author{
CODEN: XNBSAV
}

\section{U.S. GOVERNMENT PRINTING OFFICE WASHINGTON: 1976}

For sale by the Superintendent of Documents,

U.S. Government Printing Office, Washington, D.C. 20402

(Order by SD Catalog No. C13.10:422/vol. II). Price $\$ 20$ per 2-part set.

Stock No. 003-003-01458-3

(Add 25 percent additional for other than U.S. mailing). 


\section{FOREWORD}

The Analytical Chemistry Division of the NBS Institute for Materials Research, a major national laboratory for analytical chemistry is concerned with improving the accuracy and precision of analytical measurements throughout the Nation, and exercises leadership in solving important analytical problems that affect many different segments of the economy as well as the health and safety of our citizens. This laboratory at present has some 100 technical people who are engaged in the development and utilization of more than 60 different analytical techniques ranging from activation analysis to mass spectroscopy and $\mathrm{x}$-ray spectroscopy. The NBS Analytical Chemistry Division is charged with conducting analytical research, preparing and analyzing Standard Reference Materials, and performing service analysis for other divisions within the NBS and the Federal establishment. One important mechanism for the Analytical Chemistry Division in exercising its leadership is in the sponsoring of special conferences and symposia and providing a congenial atmosphere in which scientists from throughout the world may exchange their views and ideas in the field of Analytical Chemistry. Experts are invited to participate in these conferences and an attempt is made to advise all scientists of these meetings and to invite them to contribute papers to these symposia.

The first Materials Research Symposium sponsored by the Institute for Materials Research was held October 3-7, 1966, shortly after NBS moved to their new laboratories in Gaithersburg, Maryland. This first Symposium was also sponsored by the Analytical Chemistry Division and was concerned with trace characterization of materials. Subsequent to that Symposium, more specialized conferences sponsored by the Analytical Chemistry Division have been held on such subjects as quantitative electron probe microanalysis, modern trends in activation analysis, and ion-selective electrodes. "Analytical Chemistry: Key to Progress on National Problems," held in June 1972 was the American Chemical Society's annual summer symposium sponsored by the ACS Division of Analytical Chemistry and the journal Analytical Chemistry, and co-sponsored by the NBS Analytical Chemistry Division. More recently, several workshop-type meetings spontsored or co-sponsored by the Division were held at the Gaithersburg facilities. These include a workshop on oil pollution monitoring, on aerosol measurements, on secondary ion-mass spectrometry and on ozone measurement technology. The proceedings of most of these conferences and most of the workshops are available from 
the Superintendent of Documents, Government Printing Office, Washington, D.C. 20402.

This volume is the proceedings of the 7th Materials Research Symposium also organized by the Analytical Chemistry Division. The theme, Accuracy in Trace Analysis: Sampling, Sample Handling, and Analysis, attracted over 400 scientists, representing a broad spectrum of industrial, governmental, and educational institutions from the United States and from many foreign countries. The format of the Symposium consisted of invited plenary speakers, who discussed specific topics in the areas of sampling, sample handling, data interpretation, analytical methodology, and a number of contributed papers generally corresponding to these topics.

The large attendance at this Symposium as well as the lively and interesting sessions indicate that trace analytical chemistry is one of the key disciplines needed by materials scientists, environmentalists, biologists and engineers to understand and ultimately solve the increasingly complex technological problems now facing the Nation. It is timely that the Analytical Chemistry Division of the Institute for Materials Research sponsor such a symposium at this time.

John D. Hoffman, Director

Institute for Materials Research 


\section{PREFACE}

This volume, the formal report of the proceedings of the 7th Materials Research Symposium held at the National Bureau of Standards in Gaithersburg, Maryland, October 7-11, 1974, is comprised of the invited and contributed papers given at the Symposium. The objective of the Symposium was to define the present status of accuracy and the means of obtaining accuracy in trace analytical chemistry.

Over the past several years there has been an explosion in the number of chemical analyses performed, especially in the areas of environmental quality, plant and animal tissue analyses, biological fluids. high purity materials and geological samples. Because the results of these analyses are used in making important projections and decisions both short- and long-term, it is essential that they be reliable. The various factors which contribute to obtaining accurate analytical results were explored in the Symposium. Particular emphasis was placed on the whole analysis, from taking the sample through the interpretation of the results. rather than concentrating on measurement processes only. The Symposium consisted of four and one-half days of papers. Leading authorities, with broad knowledge of the problems in the areas discussed, were invited to present the keynote lectures. Simultaneous sessions consisting of groups of papers corresponding roughly with the themes of the invited papers were presented in the afternoons.

It is hoped that this volume will provide much of the necessary background information and directions for the future to assist in establishing priority goals and a rationale for solving the difficult problems in accuracy in trace analysis in the next several years.

Identification of commercial materials and/or equipment by the authors in these proceedings in no way implies recommendation or endorsement by the National Bureau of Standards.

An undertaking of the magnitude of this Symposium and of the proceedings. would not have been possible without the cooperation and assistance of a large number of dedicated people. The enthusiastic participation of the invited speakers, the session chairmen and those who contributed papers is deeply appreciated. Many members of the staff of the Analytical Chemistry Division and of the National Bureau of Standards served on numerous committees and assisted during the Symposium in various capacities.

Special thanks are given to Ronald B. Johnson and Robert F. Martin of the Institute for Materials Research. They provided all of the fiscal management of the Symposium and assisted with the other administrative 
matters associated with an operation of this magnitude. Mrs. Sara R. Torrence of the Office of Information Activities, under the direction of W. E. Small, deserves a special note of praise for her work in organizing the accommodations, social program, and coordinating the logistical aspects of the Symposium. Members of the staff of the Office of Technical Publications, under the direction of W. R. Tilley, have given invaluable assistance in the many phases of publishing these proceedings. Special thanks are due to Rebecca J. Morehouse and Miriam K. Oland of the ComputerAssisted Printing Section for producing this book using computerized photocomposition techniques.

Within the Analytical Chemistry Division special thanks are given to Mrs. Barbara Turner for her untiring effort in correspondence with the authors, and especially to Mrs. Rosemary Maddock who has provided the coordination and editorial assistance in the many phases of preparing these proceedings.

Philip D. LaFleur, Chief

Analytical Chemistry Division 


\section{ABSTRACT}

This book is the formal report of the proceedings of the 7th Materials Research Symposium: Accuracy in Trace Analysis. This volume contains the invited and contributed papers presented at the Symposium, and which treat problems of sampling and sample handling as well as the usually-discussed analytical methodology. Many important techniques and methods are described, and extensive references are presented, to give deeper insight into the problems of obtaining accurate results in trace analytical chemistry. Accordingly, this volume should not only stimulate greater interest in research in these areas but should provide a valuable guide for everyday analytical problems.

Keywords: Accuracy; analysis; analytical chemistry; sample handling: sampling; trace analysis. 


\section{CONTENTS}

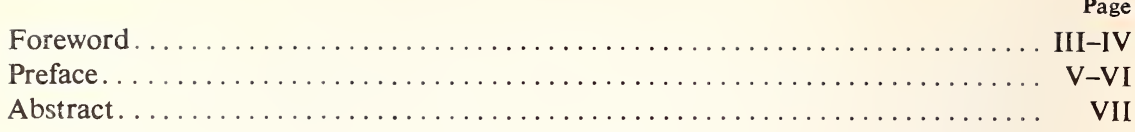

\section{VOLUME I}

\section{Part I. GENERAL CONSIDERATIONS}

The Need for Accuracy in a Regulatory Agency

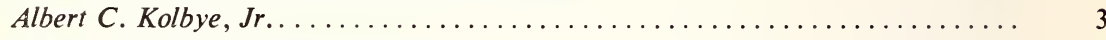

Accuracy and Trace Organic Analyses

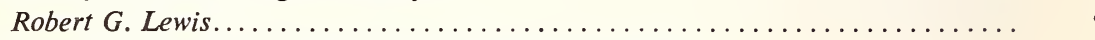

Accuracy and Quality Control in Trace Element Analysis

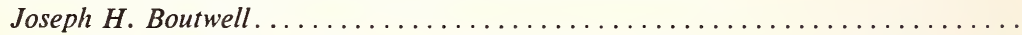

The Role of the National Bureau of Standards Standard Reference Materials in Accurate Trace Analysis

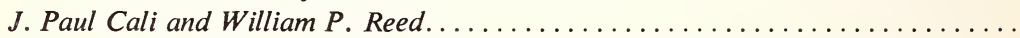

Interpretation of Accuracy of Trace Element Results for Biological Materials

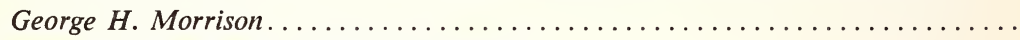

Precision and Accuracy in Silicate Analysis

Samuel S. Goldich...

Use and Interpretation of Water Quality Data

William T. Sayers and Wayne R. Ott.

Interpretation of Clinical Laboratory Data

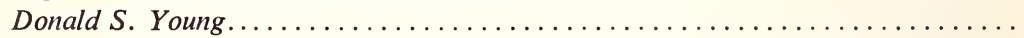

The Analyst and Accuracy

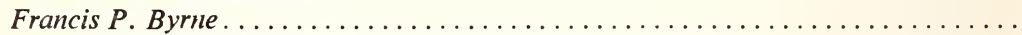

Detection of Systematic Errors by the Analysis of Precision

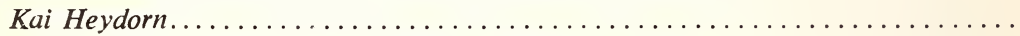

Detection of Systematic Error in Routine Trace Analysis

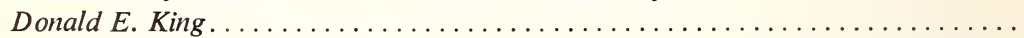

The Application of Cluster Analysis to Trace Elemental Concentrations in Geological and Biological Matrices

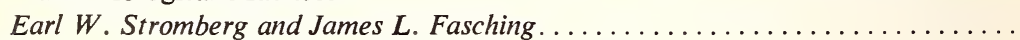

Individual Variation of Trace Metal Content in Fish

John R. Montgomery, Seppo E. Kolehmainen, Mario D. Banus, B. Joan Bendien,

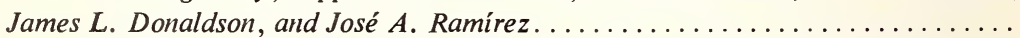

Accuracy of Chemical Analysis of Airborne Particulates. Results of an Intercomparison Exercise

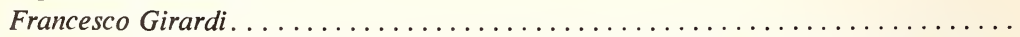

The Estimation of Accuracy in Trace Analysis. Results Obtained from Intercomparisons Organized by the I.A.E.A.

Ludwik Górski, J. Heinonen, and O. Suschny. .

Accuracy Assurance in the Analysis of Environmental Samples

$R$. K. Skogerboe and S. R. Koirtyohann.

Four Laboratory Comparative Instrumental Nuclear Analysis of the NBS Coal and Fly Ash Standard Reference Materials

John M. Ondov, William H. Zoller, I. Olmez, N. K. Aras, Glenn E. Gordon, L. A. Rancitelli, K. H. Abel, R. H. Filby, K. R. Shah, and R. C. Ragaini......

Trace Element Studies of a Selected Portion of the Mahoning River System

I. Mahadeviah, E. Mooney, and R. Munteau. 
Analysis of 11 Elements in Biological Material. Comparison of Neutron Activation Analysis and Atomic Absorption Analysis

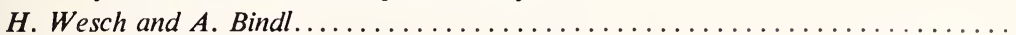

Determination of Zinc in Environmental Matrices: A Comparison of Results Obtained by Independent Methods

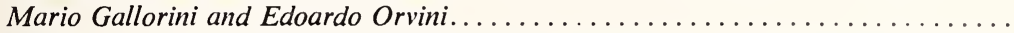

Reliability of Trace Metal Determination in Freshwater by Flameless Atomic Absorption (Graphite Tube Atomization) in Comparison with Other Chemical and Physical Methods

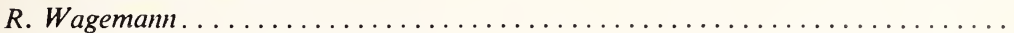

The Preparation and Certification of Trace Mercury in Water Standard Reference Materials

John R. Moody, Paul J. Paulsen, Theodore C. Rains, and Harry L. Rook........

The Influence of Limits of Laboratory Accuracy and Precision on the Interpretation of Blood Lead Analyses for Industrial Lead Hygiene Control

Alfred C. Eckert, Jr.....................................

\section{Part II. SAMPLING}

Sampling and Sample Preparation at the Geological Survey of Canada-The What, Why, and How

John A. Maxwell. ........................................

Sampling of Biological Materials

A. Speecke, J. Hoste, and J. Versieck..........................

Accuracy in Air Sampling

James $P$. Lodge, Jr.

The Reduction of Orders of Magnitude Errors in Lead Analyses of Biological Materials and Natural Waters by Evaluating and Controlling the Extent and Sources of Industrial Lead Contamination Introduced During Sample Collecting and Analysis

Clair C. Patterson and Dorothy M. Settle...........................

Sampling for Clinical Chemistry

Frank A. Ibbott.

High-Purity Reagents for Ultratrace Analysis

Morris Zief and Joseph Horvath.

Contamination of Atmospheric Particulate Matter Collected at Remote Shipboard and Island Locations

Eva J. Hoffman, Gerald L. Hoffman, and Robert A. Duce.................

Sampling and Analysis for Sulfur Compounds in Automobile Exhaust

E. R. Blosser, L. J. Hillenbrand, J. Lathouse, W. R. Pierson, and J. W. Butler. .

Preparation, Analysis, and Sampling Constants for a Biotite

C. O. Ingamells and J. C.

C. Engels. .

An Approximate Method of Computing Errors in Trace Analysis Due to Sampling Heterogeneous Solids

F. J. Flanagan . . .

Sampling and Analysis of Carbon Contained in the Primary Coolant of Pressurized Water Reactors

Sterling A. Meacham.

Sampling and Sample Handling for Activation Analysis of River Water

Sally H. Harrison, Philip D. LaFleur, and William Zoller .

Homogeneity Considerations in Trace Analyses Using the Nuclear Track Technique

B. Stephen Carpenter and G. Michael Reimer. 


\section{Part III. SAMPLE HANDLING}

Problems in Sample Treatment in Trace Analysis

Claude W. Sill.

Dissolution of Organic Materials

T. T. Gorsuch...

The Role of the Analytical Blank in Accurate Trace Analysis

Thomas J. Murphy.

The Analysis of Surfaces and Microstructural Detail

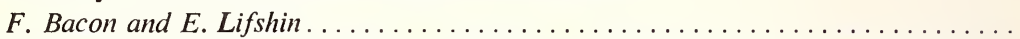

The Effects of Container Composition, Storage Duration, and Temperature on Serum Mineral Levels

Gerald L. Fisher, L. G. Davies, and L. S. Rosenblatt .

Biological Sample Contamination Due to Quartz Container in Neutron Activation Analysis

B. Maziere, A. Gaudry, J. Gros, and D. Comar.

Preparation of Biological Materials for Chromium Analysis

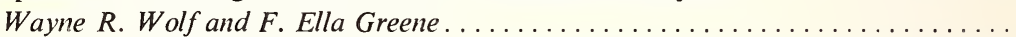

Stability of Chromium Ions at Low Concentrations in Aqueous and Biological

Matrices Stored in Glass, Polyethylene, and Polycarbonate Containers

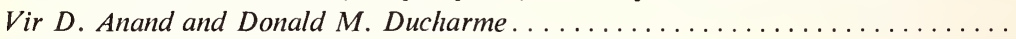

A Possibility of State Analysis by Plasma Spectrometry

Shigeki Hanamura......................................

Preparation and Analysis of Aquatic-Related Samples

N. M. Ferguson, J. R. Lund, R. R. Rickard, and L. T. Corbin............

Stability of Metal Ions in Aqueous Environmental Samples

Anthony Rattonetti.

Resin-Loaded Papers-Sampling and Trace Analysis Using Neutron Activation and X-Ray Spectrography

Stephen L. Law and William J. Campbell . .

\section{VOLUME II}

\section{Part III. SAMPLE HANDLING-Cont.}

Losses, Interferences, and Contamination in Trace Metal Analysis-Some Examples Francis $J$. Amore. . . . . . . . . . . . . . . . . . . . . . . . . . . . . . . . . . .

Sampling Problems and the Determination of Mercury in Surfacewater, Seawater, and Air

H. A. Das and H. A. van der Sloot. . .

Control of Blanks in the Analysis of Phthalates in Air and Ocean Biota Samples

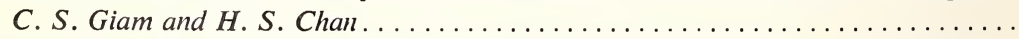

Mass Spectrometric Analysis for Fractional ppb Atomic Content of Boron in Trichlorosilane

John A. Baker.

The Challenge of Getting the Lead Out

Eleanor Berman.

Rapid Homogenization and Drying of Biological Material

Ivan Yordanov Donev. 
Problems in Trace Analysis of Nitrosamines in Foodstuffs

Effects of Storage Conditions on Residues of 2,4-D and 2,4-DCP in Potatoes

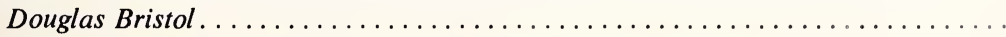

Sampling Problems in the Investigation of High Purity Metals

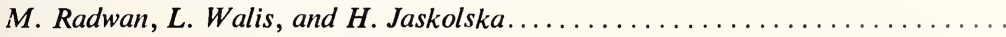

\section{Part IV. ANALYSIS}

Analytical Methodology for Accurate Determination of Trace Constituents in Highly Pure Materials

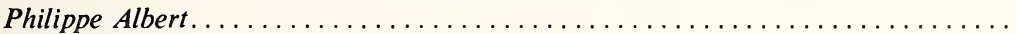

Analytical Methodology-Rocks and Ores

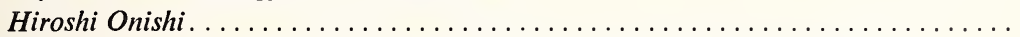

The Past, Present, and Future of the Analysis of Atmospheric Particulates

R. K. Skogerboe.

Analytical Chemistry of Natural Waters

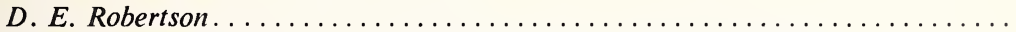

Analytical Methodology for Accurate Analysis in Clinical Chemistry

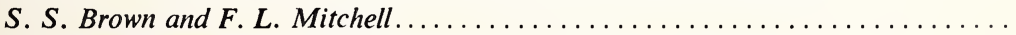

Trace Level Determination of Triclosan by Gas Chromatography

Fritz-Hans Marquardt, Janos Schulze, and Don Smith .

Electrochemical Trace Analyses Based on Faraday's Law

D. J. Curran . . . . . . . . . . . . . . . . . . . . . . . . . . . . . . .

The Non-Faradaic Background in Pulse Polarography

Robert A. Osteryoung and Joseph H. Christie.

Improved Anodic Stripping Voltammetry for Accurate Trace Analysis

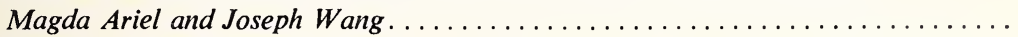

Monitoring Dissolved Copper in Seawater by Means of Ion-Selective Electrodes

Gary $K$. Rice and Raymond J. Jasinski . . . . . . . . . . . . . . . . . .

Electroanalytical Determination and Characterization of Some Heavy Metals in Seawater

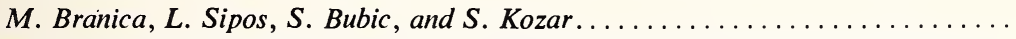

The Accurate Determination of Lead in Biological and Environmental Samples by Isotope Dilution Mass Spectrometry

Lawrence A. Machlan, John W. Gramlich, Thomas J. Murphy, and I. Lynus Barnes.

Rare Earth Element Analysis by Isotope Dilution

Gilbert N. Hanson.

An Accurate Determination of Electrolyte Concentrations in Blood Serum by Isotope Dilution Mass Spectrometry

Ernest L. Garner, Lawrence A. Machlan, John W. Gramlich, Larry J. Moore,

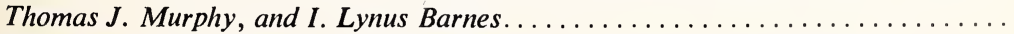

Spiking Isotope Dilution and Its Application to Fluorine Determinations

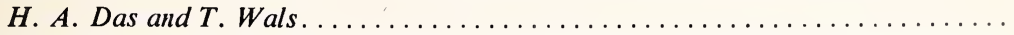

The Determination of Small Amounts of Nitrogen (0 to 20 ppm) in Plain Carbon Steels

H. F. Pronk.

Trace Element Analysis of Nickel and Nickel-Base Alloys-A Review of Current Methods

C. Manning Davis 
Nonuniform Distribution (Both Micro and Macro) of Trace Elements in HighTemperature Alloys

Owen H. Kriege and Jerry Y. Marks.

Monitoring of an Atomic Absorption Spectrophotometer Using Cumulative Sum Statistical Control Charts

John R. Montgomery. ..............................

Signal Characterization and Measurement in Microsample Atomic Absorption Spectrometry

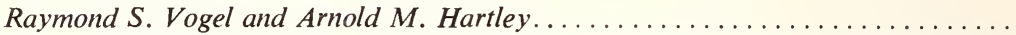

An Intercomparison of Flame and Nonflame Systems in Atomic Absorption Spectrometry

Theodore C. Rains and Oscar Menis.

Atomic Absorption Spectrophotometry of Air Filter Paper Tape Samples

Fausto J. Munoz-Ribadeneira, Maria L. Nazario, and Alice Vega. .............

Analysis of Cadmium in Fish Tissue by Flameless Atomic Absorption with a Tantalum Ribbon

E. R. Blood and G. C. Grant................................

Methodology Considerations in Western Lake Superior Water-Sediment Exchange Studies of Some Trace Elements

John E. Poldoski and G. E. Glass.

Accuracy in Determining Trace Element Concentration in Marine Sediments

Edward D. Wood and Nelson Acosta Cintron.........................

Significant Improvement of Accuracy and Precision in the Determination of Trace

Rare Earths by Fluorescence Analysis

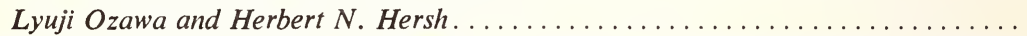

The Advantages of X-Ray Fluorescence Analysis for Trace Elements in Silicate Rocks

Bernard M. Gunn.

Monitoring of Smog Aerosols with Elemental Analysis by Accelerator Beams

T. A. Cahill, R. G. Flocchini, R. A. Eldred, P. J. Feeney, S. Lange, D. Shadoan, and $G$. Wolfe.

Characteristics of Environmental Airborne Fissionable Material

Bruce M. Center, Kurt E. Jackson, and F. H. Ruddy....................

Analytical Design in Activation Analysis: The Role of Accuracy and Precision

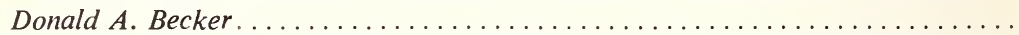

A Search for Accuracy in Activation Analysis of Trace Elements in Different Matrices

Sandro Meloni, Maria T. Ganzerli-Valentini, Vera Caramella-Crespi, Vera Maxia,

Luigino Maggi, Ugo Pisani, Renato Soma, and Pierangelo Borroni.............

Surface Effects on Accuracy in Activation Analysis

G. Revel and M. Fedoroff. . . ..............................

A Study into the Accuracy of a Remote-Controlled System for Multielement Determination in Foodstuffs Using NAA

Rieder Schelenz and Johannes-Friedrich Diehl.

Neutron Activation Analysis of Elements Extracted into Organic Media

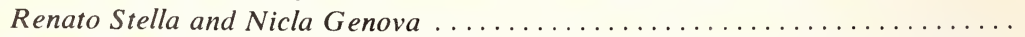

Estimation of Primary Reaction Interferences in Fast Particle Activation Analysis by Calculations Using Cross Section Data

Viliam Krivan ...................................

Activation Analysis with Charged Particles of Intermediate Energy: Performances and Applications

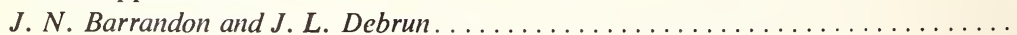


Organomercury and Total Mercury Content of Environmental Matrices as Determined by Neutron Activation Analysis

Edoardo Orvini and Mario Gallorini.

Evaluation of Error in the Instrumental Activation Analysis of Rocks

Rolf J. Rosenberg.

Neutron Activation Analysis for Some Nonstoichiometric Constituents in a Large Number of Natural and Synthetic Beryl Samples

Slobodan M. Ristic.

Control of Sample Configuration as an Aid to Accuracy in Instrumental Neutron Activation Analysis

Robert E. Heft.

Observations on the Quantitative Electron Probe Microanalysis of Particles

C. E. Fiori, K. F. J. Heinrich, R. L. Myklebust, and M. M. Darr

Determination of Elements in Standard Material (Bovine Liver SRM 1577)

I. Y. Donev and L. M. Marichkova. 

Part III. SAMPLE HANDLING - Continued 



\section{LOSSES, INTERFERENCES, AND CONTAMINATION IN TRACE METAL ANALYSIS-SOME EXAMPLES}

\section{Francis Amore}

\author{
Illinois Department of Public Health \\ Division of Laboratories \\ 134 North Ninth Street \\ Springfield, Illinois 62701 U.S.A.
}

The three main problems confronting the analysts in trace metal determinations are losses, interferences, and contamination. These problems occur at all stages of the analysis. Procedures requiring a minimum amount of sample manipulation are, therefore, desirable. Examples such as losses of copper, cadmium, lead and chromium in dairy samples routinely surveyed for these elements in both a dry ashing and wet digestion procedure are discussed. Examples and discussion of interference problems and sources of contamination are also given. The use of blanks and spiking and continuous monitoring practices are emphasized.

Keywords: Analysis; ashing; blood lead; contamination; dairy products; digestion; interferences; losses; mercury; trace metal.

\section{Introduction}

Three main problems confront the analysts in trace metal analysis: (1) losses of the element of interest during sample preparation; (2) interferences in the measurements of the parameter of interest; and (3) contamination of the sample with the element of interest or interfering elements. These problems plague the analysts despite their best efforts because of the many factors over which the analysts have no control. The magnitude of the problems is dependent upon the element of interest, the sample type, and the method of analysis. Because the number of variables are so large and for the most part unknown, it is not possible to obtain a general procedure which works for all elements within a given matrix or 
for the same element in different matrices. It is necessary, therefore, to evaluate the magnitude and extent of the problems for each analysis performed to ensure meaningful results. These problems are becoming increasingly important due to the evermore exacting demands on analytical information as a result of the present concern over the environmental and health aspects of trace metals.

\section{A. Losses}

Losses of the element of interest occur at any stage in the analytical procedure. The overall loss obtained for a given procedure is generally evaluated. If the overall loss is within acceptable limits for a given analysis, it is not necessary to evaluate the losses occurring at each step. If the magnitude of the loss is greater than tolerable, it is necessary to either evaluate the losses at each step and modify or replace the step or steps at which the greatest losses occur, or use a different procedure.

One obvious point at which considerable losses are expected is during the destruction of the organic matrix. Gorsuch $[1,2]$ has discussed the advantages and disadvantages of ashing versus wet digestion. Generalizations are frequently made that the high temperature of dry ashing leads to loss of more volatile elements while with acid digestion there is no element loss. The danger of generalizations such as the above is that most elements do not behave predictably under all circumstances. Recently, Black and Sievers [3] reported on losses of beryllium during wet digestion while Lablanc and Jackson [4] reported on a dry ashing procedure for arsenic. The atypical behavior reported in the above papers emphasizes the need for evaluating each analysis on an individual basis.

A less obvious source of losses is adsorption of the element of interest on the walls of the container during storage prior to analysis. Recently, Issaq and Zielinski [5] reported on losses of lead from aqueous solutions during storage. Rosain and Wai [6] studied the loss of mercury stored in containers of various materials. Others [7-12] studied the adsorption of metals on container walls and concluded that serious losses occur. These losses are dependent upon the container type, metal, and kind and amount of preservative added $[13,16]$. Losses as high as 50 percent occur in 1 hour of storage so that any time there is a delay between preparation of samples and analysis, some consideration must be given to this phenomenon with appropriate precaution made or some means of correction applied for the losses.

In our laboratory, we experience about 5-10 percent losses of $\mathrm{Cu}, \mathrm{Cd}$, $\mathrm{Pb}$, and $\mathrm{Cr}$ at the $0.1 \mu \mathrm{g} / \mathrm{g}$ level from milk samples dry ashed at $450^{\circ} \mathrm{C}$ for 
4 hours in a muffle furnace. The losses are variable from analysis to analysis. The losses are attributed to the physical loss of solid ash during the heating from air currents within the oven, adsorption of metal on the vycor crucibles, and volatilization of some of the metal. Comparable results are obtained by dry ashing and by acid digestion for those elements found to be present in milk at levels above $0.01 \mu \mathrm{g} / \mathrm{g}$; i.e., $\mathrm{Cu}$ and $\mathrm{Cr}$.

Only about 10 percent of the above elements are recovered from cheese samples analyzed by the same procedure as for the milk. This is due to the incomplete dissolution of the ash. The smaller sample sizes, i.e., $5 \mathrm{~g}$ of cheese compared to $10 \mathrm{~g}$ of milk did not improve the results. It is postulated that the metals are tied up as complex phosphates formed during the ashing or possibly as titanates since $\mathrm{TiO}_{2}$ is frequently added to cheeses. It was, therefore, necessary to use a wet acid digestion for the cheese. The high fat content required the use of a sulfuric-perchloric mixture due to the possible formation of unstable nitrates in the presence of nitric acid. With the acid digestion approximately 50 percent of the lead is lost due to its co-precipitation with $\mathrm{CaSO}_{4}$. All of the sulfates are not dissolved in a volume which allows for the measurement of subpart per million levels of lead.

Difficulties were experienced with both an acid digestion and dry ashing of soda pop samples for the determination of tin which is added as a preservative as stannous chloride. Dry ashing produced refractory $\mathrm{SnO}_{2}$ which is not easily dissolved even with aqua regia. Wet digestion yielded the same difficulty unless care was taken to prevent the sample from going to dryness. Even without going to dryness, it was necessary to begin adding some $\mathrm{HCl}$ prior to the completion of digestion with nitric-perchloric; however, this necessitated care to prevent the loss of $\mathrm{SnCl}_{4}$ from solution.

Apparent losses of mercury were encountered in the determination of mercury in dairy products due to "old" stannous chloride reducing agent. The stannous chloride is used to reduce mercury to the elemental form in its analysis by the cold vapor atomic absorption procedure. The $\mathrm{SnCl}_{2}$ hydrolyzed during storage; however, it was adequate to reduce mercury added to a series of aqueous standards. The reagent did not work in digestion of dairy samples even when aqueous mercury was added to the sample. This is thought to be due to the presence of undigested fat in the samples. The exact effect of the undigested material is not known. Preparation of fresh stannous chloride completely overcame the problem.

\section{B. Contamination}

Like losses, contamination of the sample can occur at any point. Such 
things as fingers, dust, dirty glassware, clean glassware sitting on dirty bench tops, containers and reagents all are sources of contamination. Robertson [17] has reviewed some of the sources of contamination of seawater samples. Others have discussed sources of contamination in trace metal analysis $[18,19]$. Several workers $[20,22]$ have reported on the need for high purity acid reagents and methods of preparation while Murau and Gunderson [23] have reviewed methods for the preparation of high purity water.

The danger of contamination of the sample and the precautions necessary to prevent contamination are dependent upon the element involved. For example, lead and copper present far more of a problem than thallium or bismuth. However, some attention must be given to the element of interest in all cases particularly if considerable work is performed with the element in the laboratory. Besides being adsorbed on container surfaces, trace amounts of metals can be leached from the walls [11,24].

We experienced a number of extremely high blood lead values in our early work which were traced to contamination of the sample in the laboratory. We measured blood lead levels of from 120 to $240 \mu \mathrm{g} / 100 \mathrm{ml}$ in about 2 percent of the samples tested. Since randomly high values were also obtained in some standards, contamination of the samples was suspected. Repeat analysis performed on the samples gave values of $<40$ $\mu \mathrm{g} / 100 \mathrm{ml}$ in all cases confirming our suspicion of laboratory contamination. The cause of the contamination was found to be insufficient rinsing of the tubes used after acid cleaning followed by drying the tubes by inversion in metal test tube racks. The problem was eliminated by using care in not contacting the racks with wet tubes.

Blood samples being run for lead by a digestion and anodic stripping procedure were frequently contaminated with both lead and cadmium. The exact source of contamination is not yet determined. It is suspected that several sources are involved including acid reagents, glassware, and air. In one case, the source of contamination was traceable to cigarette smoke which was drawn over the samples in a fume hood during the digestion stage. The smoke was used to illustrate the drawing effect desired during work involving the installation of new fume hoods in the laboratory.

Problems were encountered in the analysis of pop samples for tin by anodic stripping due to contamination of the samples with lead. The lead contamination occurred from the glassware used which was previously used for digesting paint samples for lead and from the reagents. The lead interfered with the measurement of tin because of its similar reduction potential in the supporting electrolyte used. Careful choice and cleaning 
of glassware reduced the problem to a manageable level.

Water samples being analyzed for lead and cadmium were contaminated by cadmium. The source of the contamination appears to be the polarographic cell used, although the reason for the contamination of the cell is not known. At present all attempts to identify the source of cadmium have been unsuccessful. Special precautions are taken prior to the analysis for cadmium. These include careful washing of the cell, cell holder, and electrodes, followed by running deionized water containing some Ultrex hydrochloric acid. The washing and monitoring runs are continued until a negligible amount of cadmium is observed after which the samples are run.

\section{INTERFERENCE}

The nature and type of interferences expected are dependent upon the parameter measured. Sandell [19] discussed interferences in the determination of metals by colorimetric methods. A number of workers have discussed interferences in atomic absorption spectroscopy [25-28]. Pickett and Koirtyohann [29] discussed some interferences in flame emission techniques. Rooney [30] and Barendrecht [31] discussed some interferences in polarographic and anodic stripping methods respectively. References to interferences are found in the biennial reviews in Analytical Chemistry with references to methods in the even year fundamental reviews and references to specific elements in the odd year application reviews.

Although interferences are generally associated with the actual measurement of an analytical parameter, it is possible for interferences to occur at other stages in the analysis. Kaminski [32] recently reported on the interferences of $\mathrm{Al}$ in the extraction of $\mathrm{Cd}$. It is necessary to evaluate the overall procedure for interferences as opposed to merely checking interferences at the measurement stage.

In running dairy products for trace metals by atomic absorption, we encountered nonspecific interferences which gave rise to high results. Levels of cadmium from 0.1 to $0.2 \mu \mathrm{g} / \mathrm{g}$ were obtained without correction. When background correction procedures [33-35] were used, no cadmium was detected corresponding to levels of $<0.01 \mu \mathrm{g} / \mathrm{g}$. The same behavior was observed for $\mathrm{Pb}$ and for $\mathrm{Cu}$ and $\mathrm{Cr}$ to a slightly lesser degree. It should be pointed out that the method of standard addition does not correct for this behavior.

Lead was found to interfere in the determination of thallium in food samples by anodic stripping. This problem was overcome by changing the 
supporting electrolyte. By using a basic media, i.e., $0.01 \mathrm{~N} \mathrm{NaOH}$, the lead peak is shifted about $500 \mathrm{mV}$ negative of the thallium peak. This allowed us to determine thallium, which was the element of interest, and to observe the level of lead in the suspect samples. The addition of EDTA could also be used to achieve the same end, but it would not allow the determination of lead.

Lead also interfered in the determination of $\mathrm{Sn}$ by anodic stripping. In this case, it was necessary to measure the current due to lead and tin, make the solution basic, measure the current due to lead alone at the more negative potential, and calculate the amount of tin by difference. It is also possible to separate the tin by distillation as the bromide prior to analysis. However, for our work, the former procedure was more advantageous.

In doing arsenic analysis using the arsenic-silver diethyldithiocarbamate procedure, a black-colored collection solution was obtained on occasion. The cause of the problem was traced to the use of soap to clean out the tubes after use. The exact species involved in forming the colored compound are not known, nor was any attempt made to identify them. The problem was eliminated by more thorough rinsing of the glassware and by discontinuing a soap wash.

\section{Conclusion}

Although none of the above examples are really new or startling, they serve to point out some of the problems that arise in trace metal analysis. Several important points are evident from these examples which should be of concern to the uninitiated and the experienced. First, it is obvious that problems occur at any point in the analysis so that it is necessary for the analysts to evaluate and check all steps in the procedure. Because losses, contamination, and interferences occur at all stages of the analysis, it is desirable to employ or develop procedures which decrease the number of manipulative steps and the number of reagents involved. Ideally, one would like to make the measurement directly on the sample without any pretreatment, and, in fact, this is possible for a number of cases; however, often times this approach is not possible or else the corrections necessary for matrix effects become awesome. It is necessary to strike a balance so that the number of steps involved is reduced and yet matrix effects are kept to a minimum. Second, it is apparent that problems occur in even the most well-established of procedures so that constant quality control measures are necessary. A number of the problems discussed above were apparent to us only because of the continual 
running of spiked samples and blank solutions. This is done even though aqueous standards are used for quantitation and blank values are normally negligible. Third, it is necessary to reevaluate a method every time the sample type is changed. It is impossible to predict, a priori, whether a given procedure for an element will work in a given matrix simply because it has worked on other types of samples.

Although trace metal analysis has unique problems, it is possible to obtain accurate and precise values by the use of good quality control in the laboratory. The continual check of possible sources of errors greatly decreases the likelihood of obtaining and reporting erroneous results.

\section{References}

[1] Gorsuch, T. T., Analyst 84, 135 (1959).

[2] Gorsuch, T. T., Destruction of Organic Matter, Pergamon Press, New York (1970).

[3] Black, M. S. and Sievers, R. E., Anal. Chem. 45, 1773 (1973).

[4] Lablanc, P. J. and Jackson, A. C., JAOAC 56, 383 (1973).

[5] Issaq, H. J. and Zielinski, W. L., Jr., Anal. Chem. 45, 1328 (1974).

[6] Rosain, R. M. and Wai, C. M., Anal. Chim. Acta 65, 279 (1973).

[7] Robertson, D. E., Anal. Chim. Acta 42,533 (1968).

[8] Benes, P. and Rajman, I., Coll. Czech. Chem. Commun. 34, 1375 (1969).

[9] Benes, P., Coll. Czech. Chem. Commun. 35, 1349 (1970).

[10] Coyne, R. V. and Collins, J. A., Anal. Chem. 44, 1093 (1972).

[11] Struempler, A. W., Anal. Chem. 45, 2251 (1973).

[12] Eickholz, G. C., Nagel, A. E., and Hughes, L. R. B., Anal. Chem. 37, 863 (1965).

[13] Dych, W., Anal. Chem. 40, 454 (1968).

[14] Durst, R. A. and Duhart, B. T., Anal. Chem. 42, 1002 (1970).

[15] West, F. K., West, P. W., and Iddings, F. A., Anal. Chem. 38, 1566 (1966).

[16] West, F. K., West, P. W., and Iddings, F. A., Anal. Chim. Acta 37, 112 (1967).

[17] Robertson, D. E., Anal. Chem. 40, 1067 (1968).

[18] Mizuike, A., Trace Analysis-Physical Methods, Morrison, G. H., Ed., Interscience, New York, pp. 105-115 (1965).

[19] Sandell, E. B., Colorimetric Determination of Trace Metals, Interscience, New York, pp. 14-24 (1958).

[20] Tatsumoto, M., Anal. Chem. 41, 2088 (1969).

[21] Mattinson, J. M., Anal. Chem. 44, 1715 (1972).

[22] Luncet, M. S. and Huey, J. M., Anal. Chem. 46, 1360 (1974).

[23] Murau, P. C. and Gunderson, G., Anal. Chem. 43, 691 (1971).

[24] Woodriff, B. R., Culver, D. S., and Sepper, A. B., Anal. Chem. 45, 230 (1973).

[25] Hwang, J. Y. and Feldman, F. J., Appl. Spectrosc. 24, 371 (1970).

[26] Lewis, L. L., Anal. Chem. 40,28A (1968).

[27] Christian, D. G., Anal. Chem. 41,24A (1969).

[28] Hwang, J. Y., Anal. Chem. 44, 20A (1972).

[29] Pickett, E. E. and Koirtyohann, S. R., Anal. Chem. 41, 28 A (1969).

[30] Rooney, R. C., Chem. Ind., p. 875 (1966). 
[31] Barendrecht, E., in Advances in Electrochemistry and Electrochemical Engineering, Vol. 3, Delahay, P., Ed., pp. 53-109, Interscience, New York (1967).

[32] Kaminski, E. E., Anal. Chem. 46, 1304 (1974).

[33] Dahn, H. L. and Manning, D. C., Amer. Lab. 4, 51 (1972).

[34] Koirtyohann, S. R. and Pickett, E. E., Anal. Chem. 38, 585 (1966).

[35] Hwang, J. Y., Ullucci, P. A., and Mokeler, C. J., Anal. Chem. 44, 2018 (1972). 


\title{
SAMPLING PROBLEMS AND THE DETERMINATION OF MERCURY IN SURFACE WATER, SEAWATER, AND AIR
}

\author{
H. A. Das and H. A. van der Sloot
}

\author{
Reactor Centrum Nederland \\ Westerduinweg 3 \\ Petten (N.H.), The Netherlands
}

\begin{abstract}
Analysis of surface water for mercury comprises the determination of both ionic and organically bound mercury in solution and that of the total mercury content of the suspended matter. Eventually, metallic mercury has to be determined too. These determinations require a sampling procedure which meets the following requirements: a) the concentration of suspended matter is not af fected, b) the separation of the solid fraction from the sample is performed immediately after sampling without losses from the aqueous phase due to adsorption and losses from the solid phase due to desorption at a too low $\mathrm{pH}$, and c) the aqueous phase is processed immediately to prevent losses due to adsorption on the wall of the container. A method for the routine determination of mercury in surface water and seawater was developed and applied to Dutch surface waters. The total sample volume is $2500 \mathrm{ml}$. About $500 \mathrm{ml}$ is used for the determination of the content of suspended matter and the total amount of mercury in the water. The sample is filtered through a bed of previously purified active charcoal at a low flow-rate. The main portion $(\simeq 2000 \mathrm{ml})$ passes a flow-through centrifuge to separate the solid fraction. One liter is used to separate "inorganic" mercury by reduction, volatilization in an airstream and adsorption on active charcoal. The other liter is led through a column of active charcoal to collect all mercury. The procedures were checked with ${ }^{197} \mathrm{Hg}$ radiotracer both as an ion and incorporated in organic compounds. The mercury is determined by thermal neutron activation, followed by volatilization in a tube furnace and adsorption on a fresh carbon bed. The limit of determination is $\simeq 1 \mathrm{ng} 1^{-1}$. The rate of desorption from and adsorption on suspended material has been measured as a function of the $\mathrm{pH}$ of the solution for $\mathrm{Hg}^{+2}$ and various other ions. It can be concluded that only the procedure mentioned above does not disturb the equilibrium. The separation of mercury from air is obtained by suction of $1 \mathrm{~m}^{3}$ through a $0.22 \mu \mathrm{m}$ filter and a charcoal bed. The determination is then performed as in the case of the water samples.
\end{abstract}

Keywords: Activation analysis; adsorption isotherm; environmental samples; mercury; preconcentration; trace elements. 


\section{LIST OF SYMBOLS}

\begin{tabular}{|c|c|c|}
\hline$c$ & concentration in the aqueous sample & $\mu \mathrm{g} / \mathrm{l}$ or $\mathrm{mg} / \mathrm{m}$ \\
\hline$m$ & amount of adsorber & $\mathrm{mg}$ \\
\hline$n$ & $\begin{array}{l}\text { exponent in the Freundlich adsorp- } \\
\text { tion isotherm }\end{array}$ & - \\
\hline V & volume of the aqueous sample & $\mathrm{ml}$ \\
\hline$y$ & adsorbed amount & $\mu \mathrm{g}$ \\
\hline$\alpha$ & $\begin{array}{l}\text { constant of proportionality in the } \\
\text { Henry-isotherm }\end{array}$ & $(\mathrm{mg} / \mathrm{ml})^{-1}$ \\
\hline$\beta$ & $\begin{array}{l}\text { constant of proportionality in the } \\
\text { Freundlich- and Langmuir-isotherm }\end{array}$ & - \\
\hline$\delta$ & constant in the Langmuir-isotherm & $\mathrm{mg} / \mathrm{ml}$ \\
\hline
\end{tabular}

\section{Introduction}

\section{A. General}

The determination of heavy metals in water and air, as distinct from that in sediment and air-dust, is important for the control of pollution. These analyses have to consist of three steps:

1. The elimination of suspended matter, which does not affect the concentration of unbound species in the original water or air sample.

2. The transport to the analyzer system without changing the concentration.

3. The determination proper.

The approach to the problem of water and air analysis, presented in this text, is based on three considerations:

1. In choosing a convenient procedure for the elimination of the suspended material, radiotracers can be used to follow the adsorption and desorption processes and to measure their effects.

2. Adsorption of the trace elements on active charcoal, immediately after the sampling, can be applied to eliminate the deterioration of the sample during transport.

3. Activation analysis with thermal neutrons offers a sufficient sensitivity for the determination of most of the heavy elements to be determined. 


\section{B. Separation of Water and Suspended Matter}

The elimination of the suspended matter can be effected by filtration or centrifugation.

The advantages of filtration are its simplicity and the possibility to use it at any place in the field. Objections which can be raised against filtration are:

a. The filter may adsorb a considerable part of the elements to be determined.

b. Filtration is a slow process. The intimate contact between the water and the particulate matter, already collected on the filter, may cause an exchange between the two phases of the original sample.

The advantage of centrifugation is its rapidity. However, the method cannot be applied everywhere. Moreover, it is necessary to use a flowthrough apparatus in which the particulate matter is separated immediately from the sample.

The change in concentration due to filtration or centrifugation for the various elements should be measured as a function of the $\mathrm{pH}$ and the time needed to perform the separation.

\section{Transport of the Sample}

The adsorption of trace elements on the wall of the sample container is a well-known phenomenon. The percentage and the rate of adsorption depend on a number of variables. The most important are: the molecular species in which the involved element is present; the $\mathrm{pH}$ of the solution: and the container material. Adsorption can generally be eliminated by acidifying the sample solution with double distilled $\mathrm{HNO}_{3}$ to a $\mathrm{pH}$ of 1 or lower. This method is subject to two limitations:

1. The suspended matter has to be removed prior to the acidification. Otherwise the concentration in the solution will increase considerably by desorption from the solid material.

2. Only the total concentration of the element has to be determined. A change in $\mathrm{pH}$ will in general cause a change in the ratio between the "organic" and ionic fractions.

Experiments with radiotracers can serve to determine the adsorption percentage and the rate of adsorption as a function of $\mathrm{pH}$ and time. However, from a practical point of view, it is preferable to process the sample immediately after the removal of the suspended matter. Adsorption on active charcoal can serve this purpose. 


\section{Determination of the Trace Elements}

When the trace elements are adsorbed on active charcoal, the loaded coal can be analyzed for its heavy metal content by thermal neutron activation analysis. The only heavy element which cannot be determined by thermal neutron activation is lead. The (small) charcoal samples can be submitted to intense neutron irradiation without the risk of decomposition. The limit of detection is not governed by the intrinsic sensitivity of the method, but by the blank value of the charcoal. This implies a proper choice and, if necessary, a purification of the material to be used. Up to now, procedures have been developed for the determination of $\mathrm{V}, \mathrm{Mn}$, $\mathrm{Cu}, \mathrm{Zn}, \mathrm{As}, \mathrm{Se}, \mathrm{Mo}, \mathrm{Ag}, \mathrm{Hf}, \mathrm{Au}, \mathrm{Hg}$ and $\mathrm{U}$.

Two practical adsorption techniques can be applied:

1. The sample is filtered through a small column containing $\simeq 2$ grams ( $5 \mathrm{ml})$ of the adsorbent.

2. An amount of $\simeq 2$ to $\simeq 40 \mathrm{mg}$ of active charcoal is added as a $50-1000 \mu \mathrm{l}$ aliquot of a suspension in double distilled water, containing $40 \mathrm{mg}$ charcoal $/ \mathrm{ml}$.

In the first case, the adsorption is quantitative. Use of the charcoal suspension (the second case) results in the adsorption of a considerable fraction, which is fairly constant throughout a series of samples. If necessary, the percentage adsorbed can be determined for each sample separately by using a radioactive spike with a high specific radioactivity. Preferably a carrier-free solution is applied.

The determination of mercury in surface water and in air can be chosen as examples of the procedures which are based on the mentioned principle. The total concentration of mercury in "unpolluted" surface waters is generally well below $100 \mathrm{ng} / \mathrm{l}$. That in air may vary between 1 and 200 $\mathrm{ng} / \mathrm{m}^{3}$.

\section{Investigation of Filtration and Centrifugation with Radiotracers}

\section{A. General}

The following phenomena were measured quantitatively for the principal trace elements, using seawater samples: a. The adsorption on membrane filters of $0.45 \mu \mathrm{m}$ pore diameter; $\mathrm{b}$. The rate of adsorption of freshly added ionic traces. The percentage adsorbed after a long contact time as a function of $\mathrm{pH}$; and $\mathrm{c}$. The rate of desorption from the suspended matter. 
For mercury, the behavior of some organometallic compounds was investigated too.

Apparatus and Chemicals. - The following were used: a glass fiber pressure vessel (type "Amicon") with connections for pressurized air and a filter holder (type "Swinnex 04700"); a "MSE" centrifuge, operating at $\simeq 2000 \mathrm{~g}$; centrifuge tubes of $100 \mathrm{ml}$; membrane filters made of cellulose nitrate, with a pore diameter of $0.45 \mu \mathrm{m}$, Selectron BA 85; concentrated $\mathrm{HNO}_{3}$ of analytical quality.

Radiotracers:

${ }^{59} \mathrm{Fe}$

${ }^{60} \mathrm{Co}$

${ }^{65} \mathrm{Zn}$

${ }^{99} \mathrm{Mo} \rightarrow{ }^{99 \mathrm{~m}} \mathrm{Tc}$

${ }^{115} \mathrm{Cd} \rightarrow{ }^{115 \mathrm{~m}} \mathrm{In}$

${ }^{197} \mathrm{Hg}$

${ }^{212} \mathrm{~Pb}$
$\mathrm{T} 1 / 2$ :

$45 \mathrm{~d}$

5,3 y

$245 \mathrm{~d}$

$66 \mathrm{~h} \rightarrow 6.0 \mathrm{~h}$

$53.5 \mathrm{~h} \rightarrow 4.5 \mathrm{~h}$

$66 \mathrm{~h}$

$10.6 \mathrm{~h}$
(All tracers were present as nitrates in various concentrations.)

\section{B. Adsorption of Trace Elements by Membrane Filters}

Aliquots of a large sample of seawater were filtered through a $0.45 \mu \mathrm{m}$ membrane filter to remove the suspended matter. Radiotracer solution was then added, and the sample was filtered through a fresh membrane filter. This was dissolved in $5 \mathrm{~N} \mathrm{HNO}_{3}$ and counted in the same geometry as an aliquot of the tracer solution. Table 1 gives the results of these experiments. The losses of iron, mercury and lead are serious. The percent loss of mercury increases with decreasing concentration. The specific capacity of the filter for the adsorption of mercury was found to be (1.7 \pm $0.2) \mu \mathrm{g} \mathrm{Hg} / \mathrm{g}$. As the "natural" concentration of mercury in seawater and surface water is $\simeq 0.05 \mu \mathrm{g} / \mathrm{l}$, it is impossible to apply filtration in the determination of mercury in water, unless the adsorption is prevented by lowering the $\mathrm{pH}$ of the solution ( $c f$. section II.C.). The trace element content of the filter material was determined by neutron activation analysis.

Results are:

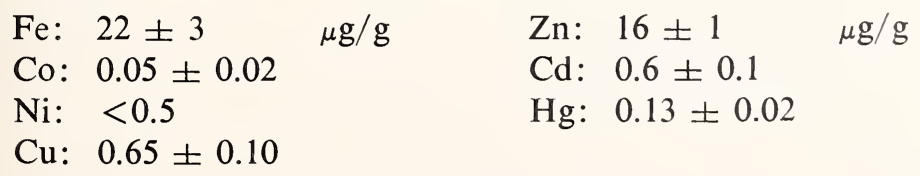


TABLE 1. Adsorption of trace elements on a membrane filter

\begin{tabular}{cccc}
\hline Element & $\begin{array}{c}\text { Concentrations } \\
\text { (ug/l) }\end{array}$ & $\begin{array}{c}\text { Sample } \\
\text { volume }\end{array}$ & $\begin{array}{c}\text { Percentage } \\
\text { adsorbed }\end{array}$ \\
\hline $\mathrm{Hg}$ & 0.2 & 100 & 19 \\
& .2 & 250 & 16 \\
& .2 & 500 & 16 \\
& .2 & 750 & 15 \\
& .4 & 1000 & 14 \\
& .9 & 1000 & 3.5 \\
$\mathrm{~Pb}$ & 0.1 & 1000 & 1.5 \\
& .2 & 1000 & 11 \\
$\mathrm{Zn}$ & 13 & 100 & 5 \\
$\mathrm{Co}$ & 1 & 1000 & 0.5 \\
$\mathrm{Fe}$ & 19 & 1000 & $<.1$ \\
$\mathrm{Mo}$ & 14 & 1000 & 33 \\
\hline
\end{tabular}

a The natural concentration of the seawater has been included.

C. The Rate of Adsorption on Suspended Matter

of Freshly Added Ionic Traces, the Percentage Adsorbed After a Long CONTACT TIME, AND THE INFLUENCE OF THE PH

Experiments were performed with unfiltered seawater $(\mathrm{pH}=7.8)$, which contained $49 \mathrm{mg}$ of suspended matter/liter. The adsorption of zinc, mercury, and lead ions was studied as a function of contact time. The total concentrations of the elements were:

$$
\mathrm{Zn}: 14 \mu \mathrm{g} / \mathrm{l} ; \mathrm{Hg}: 0.5 \mu \mathrm{g} / \mathrm{l} \text {; and } \mathrm{Pb}: 0.2 \mu \mathrm{g} / \mathrm{l} \text {. }
$$

The suspended matter was collected by centrifugation during 10 minutes at $2000 \mathrm{~g}$. Results are given in table 2 and figure 1. The following conclusions can be drawn from these data:

a. The time needed to reach the equilibrium for the elements studies is:

$$
\mathrm{Zn} \geqslant 28 \mathrm{~h} ; \mathrm{Hg} \geqslant 28 \mathrm{~h} \text {; and } \mathrm{Pb} \geqslant 1.5 \mathrm{~h} \text {. }
$$

b. The curves of percentage adsorbed versus contact time cannot be described by a simple exponential curve. 
TABLE 2. Adsorption of $\mathrm{Zn}^{\mathrm{II}_{-}}, \mathrm{Hg}^{\mathrm{II}}$ - and $\mathrm{Pb}^{\mathrm{II}_{-}}$-ions on suspended matter

\begin{tabular}{cccc}
\hline \multirow{2}{*}{$\begin{array}{c}\text { Contact-time } \\
\text { (hours) }\end{array}$} & \multicolumn{3}{c}{ Percentage adsorbed } \\
\cline { 2 - 4 } & $\mathrm{Zn}$ & $\mathrm{Hg}$ & $\mathrm{Pb}$ \\
& & & \\
0.5 & 14 & 24 & 45 \\
1 & 20 & 27 & 56 \\
1.5 & 24 & 41 & 65 \\
4 & 33 & 52 & 76 \\
6 & 31 & 54 & 71 \\
23 & 32 & 56 & 67 \\
28 & 33 & 62 & 68 \\
30 & 37 & 65 & 75 \\
47 & 39 & 66 & 68 \\
& & & \\
Extrapolated & & & $\simeq 70$ \\
value for $t=\infty$ & $\simeq 40$ & & \\
\hline
\end{tabular}

Notes: Concentration of suspended matter, $49 \mathrm{mg} / \mathrm{l}$. Initial concentrations: $\mathrm{Zn} 14 \mu \mathrm{g} / \mathrm{l}$; $\mathrm{Hg} 0.5 \mu \mathrm{g} / \mathrm{l} ; \mathrm{Pb} 0.2 \mu \mathrm{g} / \mathrm{l}$.

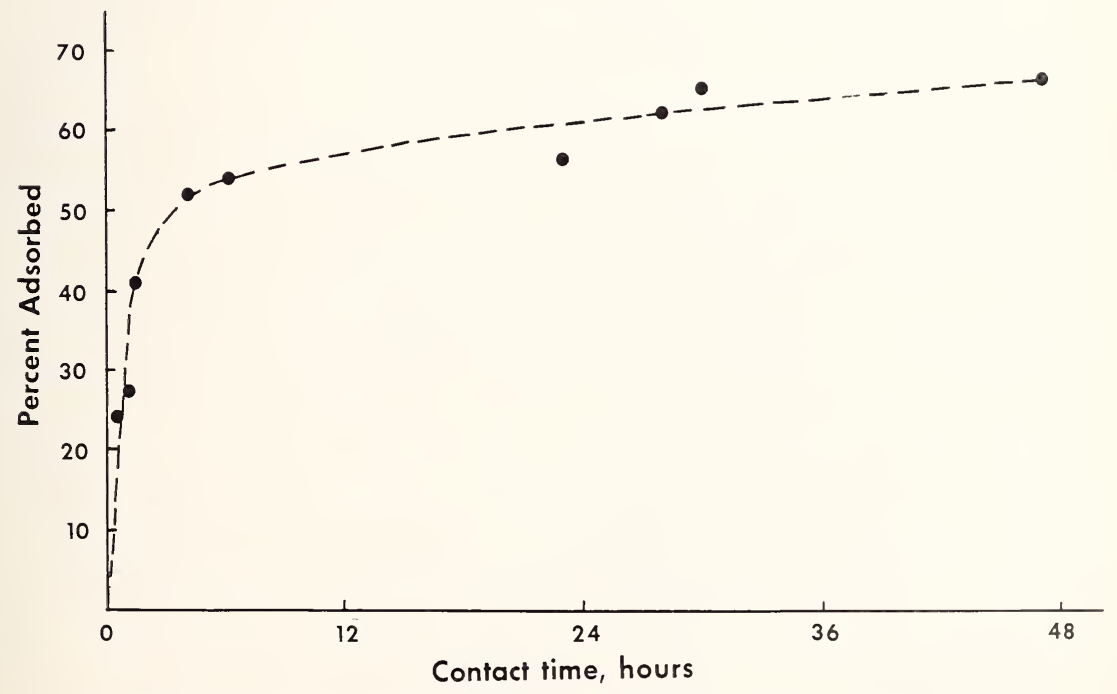

Figure 1. The adsorption of $\mathrm{Hg}^{+2}$ ions on suspended matter. Initial $\mathrm{Hg}^{+2}$ concentration : 0.5 $\mu \mathrm{g} / \mathrm{l}$. 
c. The equilibrium values of the amounts adsorbed are in good agreement with the data quoted in literature $[1,2]$ :

$\mathrm{Zn}: 30$ to 45 percent; $\mathrm{Hg}$ : 60 to 80 percent; and $\mathrm{Pb}: 70$ to 80 percent.

The adsorption behavior of organomercury compounds was studied by using the ${ }^{197} \mathrm{Hg}$-labeled species of $\left(\mathrm{C}_{6} \mathrm{H}_{5}\right) \mathrm{HgCl},\left(\mathrm{C}_{6} \mathrm{H}_{5}\right) \mathrm{HgAc}$, and $\left(\mathrm{C}_{6} \mathrm{H}_{5}\right)_{2} \mathrm{Hg}$. The initial rate of adsorption was very fast for all three compounds. The adsorption of $\left(\mathrm{C}_{6} \mathrm{H}_{5}\right)_{2} \mathrm{Hg}$ was virtually complete within 3 minutes. The percentage adsorbed at equilibrium is $\simeq 75$ for $\left(\mathrm{C}_{6} \mathrm{H}_{5}\right) \mathrm{HgCl}$ and $\left(\mathrm{C}_{6} \mathrm{H}_{5}\right)_{2} \mathrm{Hg}$, and $\simeq 90$ for $\left(\mathrm{C}_{6} \mathrm{H}_{5}\right) \mathrm{HgAc}$.

The behavior of divalent mercury was investigated in more detail. Experiments were performed with various concentrations of suspended matter and various $\mathrm{Hg}$ concentrations. Table 3 summarizes the results obtained for the percentages adsorbed after a long contact time. The following conclusions can be drawn:

a. The adsorption can be described by a Freundlich isotherm, equation (1).

$$
\mathrm{y} / \mathrm{m}=\beta \cdot \mathrm{c}^{n}
$$

b. The average values of the "constants" $\beta$ and $n$ are 12 and 1.2 respectively, if $\mathrm{y} / \mathrm{m}$ is expressed in $\mathrm{ng} \mathrm{Hg}^{+2} / \mathrm{mg}$ suspended matter, and $\mathrm{c}$ in $\mu \mathrm{g} / \mathrm{l}$.

c. The fraction which is adsorbed, $\mathrm{y} / \mathrm{cV}$, is given by equation (2).

$$
\mathrm{y} / \mathrm{cV}=\beta \cdot(\mathrm{m} / \mathrm{V}) \cdot \mathrm{c}^{(n-1)}
$$

The influence of $\mathrm{pH}$ is shown in table 4 . The data in brackets refer to filtration through a membrane filter with $0.45 \mu \mathrm{m}$ pore diameter. The results indicate that adsorption on the filter can be prevented by acidifying the sample solution with double distilled $\mathrm{HNO}_{3}$ to at least $1 N$. However, this is possible only if the desorption from the suspended material is slow.

TABLE 3. The percentage of $\mathrm{Hg}^{\mathrm{II}}$-ions adsorbed by the suspended matter after a long contact

\begin{tabular}{|c|c|c|c|}
\hline $\begin{array}{c}\text { Initial } \mathrm{HgII}^{\mathrm{II}} \text {-concentration } \\
(\mu \mathrm{g} / \mathrm{l})\end{array}$ & $\begin{array}{l}\text { Concentration of } \\
\text { suspended matter } \\
(\mathrm{mg} / \mathrm{l})\end{array}$ & $\begin{array}{l}\text { Percentage } \\
\text { adsorbed }\end{array}$ & $\begin{array}{l}\text { Concentration of the } \\
\text { adsorbed ions } \\
\left.\text { (ng } \mathrm{HgII}^{\mathrm{II}} / \mathrm{mg}\right), \\
\text { suspended matter }\end{array}$ \\
\hline
\end{tabular}
time

$\begin{array}{cccc}0.18 & 38 & 50 & 2.4 \\ .18 & 66 & 82 & 2.2 \\ .22 & 28 & 63 & 5 \\ .50 & 49 & 65 & 6.6 \\ .80 & 43 & 90 & 17\end{array}$


TABLE 4. The influence of the $\mathrm{pH}$ on the adsorption of $\mathrm{Hg}^{\mathrm{II}}$-ions on suspended matter

\begin{tabular}{|c|c|c|c|}
\hline $\begin{array}{l}\text { Initial } \mathrm{HgII}_{-} \\
\text {concentration } \\
(\mu \mathrm{g} / \mathrm{l})\end{array}$ & $\begin{array}{l}\text { Concentration } \\
\text { of suspended } \\
\text { matter }(\mathrm{mg} / \mathrm{l})\end{array}$ & $\begin{array}{l}\text { Percentage } \\
\text { adsorbed }\end{array}$ & $\begin{array}{l}\text { Concentration of the } \\
\text { adsorbed ions } \\
\text { (ng } \mathrm{Hg}^{\mathrm{II}} / \mathrm{mg} \text { ), suspended }\end{array}$ \\
\hline
\end{tabular}

$\begin{array}{lllcc}0.1 & 1.0 & 56 & 4[7]^{\mathrm{a}} & 0.7 \\ .5 & 1.0 & 56 & 5[14] & .9 \\ 1 & 0.5 & 30 & 24 & 4 \\ 1 & 1.0 & 56 & 24 & 4.3 \\ 1.5 & 1.0 & 56 & 42[63] & 7.5 \\ 2 & 1.0 & 56 & 49[71] & 8.8 \\ 3 & 0.5 & 30 & 65[77] & 11 \\ 5 & 0.6 & 30 & 62[87] & 10 \\ 7 & 0.5 & 30 & 63[86] & 11\end{array}$

a Data in brackets refer to filtration through a membrane filter with $0.45 \mu \mathrm{m}$ pore diameter.

\section{The Rate of Desorption from the Suspended Matter}

The desorption from suspended matter was measured as follows:

a. A radioactive tracer was added to a large seawater sample.

b. After 1 day, the solid material was collected by centrifugation and was suspended again in a small volume of fresh seawater. Aliquots of this suspension were added to $250 \mathrm{ml}$ portions of filtered seawater.

c. Immediately after the addition of the sediment, the $\mathrm{pH}$ was adjusted.

d. After a chosen time, the solid material was separated from the sample. The solid material was counted in the same geometry as an aliquot of the suspension originally added.

Figure 2 gives the results obtained. It appears that the rate of desorption is appreciable even if the filtration is completed in 3 minutes. The influence of $\mathrm{pH}$ on the desorption rate can be derived from the percentage desorbed in 3 minutes:

$\begin{array}{lc}\mathrm{pH} & \begin{array}{r}\text { Percent } \\ \text { desorbed }\end{array} \\ 0.1 & 14 \\ 1 & 12 \\ 1.5 & 4 \\ 2 & 2 \\ 3 & 0\end{array}$




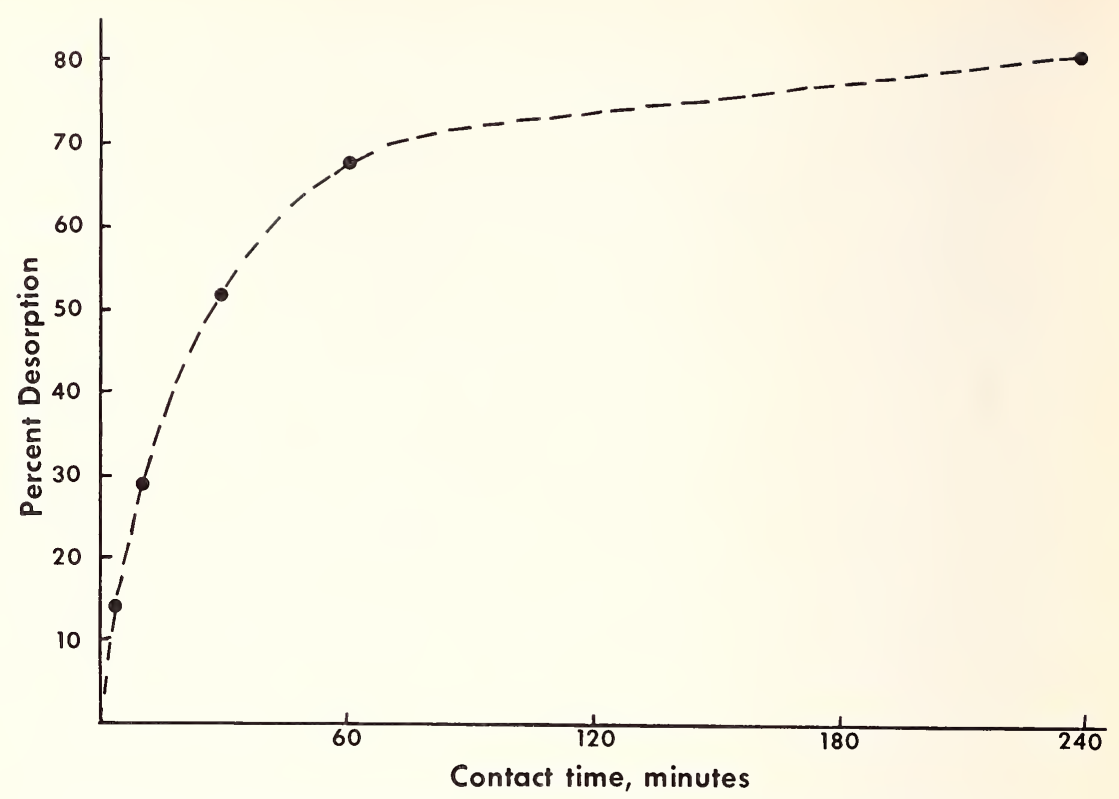

Figure 2. The desorption of $\mathrm{Hg}^{+2}$ ions from suspended matter at a $\mathrm{pH}$ of 0.1 .

\section{E. Conclusion}

Only by rapid centrifugation at the natural $\mathrm{pH}$ can the suspended matter be separated from the samples without disturbing the adsorption and desorption equilibria of the metal ion species investigated. In practice this means that a flow-through centrifuge should be used.

\section{Adsorption of Mercury on the Walls of Containers}

The adsorption of mercury on the wall of the container, both as ions and as organomercury compounds, is well known [3-5]. Table 5 gives the results of a series of experiments with $\mathrm{Hg}$ solutions. The difference between double distilled and tap water is caused by the different $\mathrm{Cl}$ content. The behavior of some organomercury compounds was measured with compounds labelled with ${ }^{197} \mathrm{Hg}(\mathrm{T} 1 / 2=64 \mathrm{~h})$. Results are summarized in table 6. If the samples are not acidified, adsorption on polythene is significant within 24 hours while that on glass can be already appreciable. 


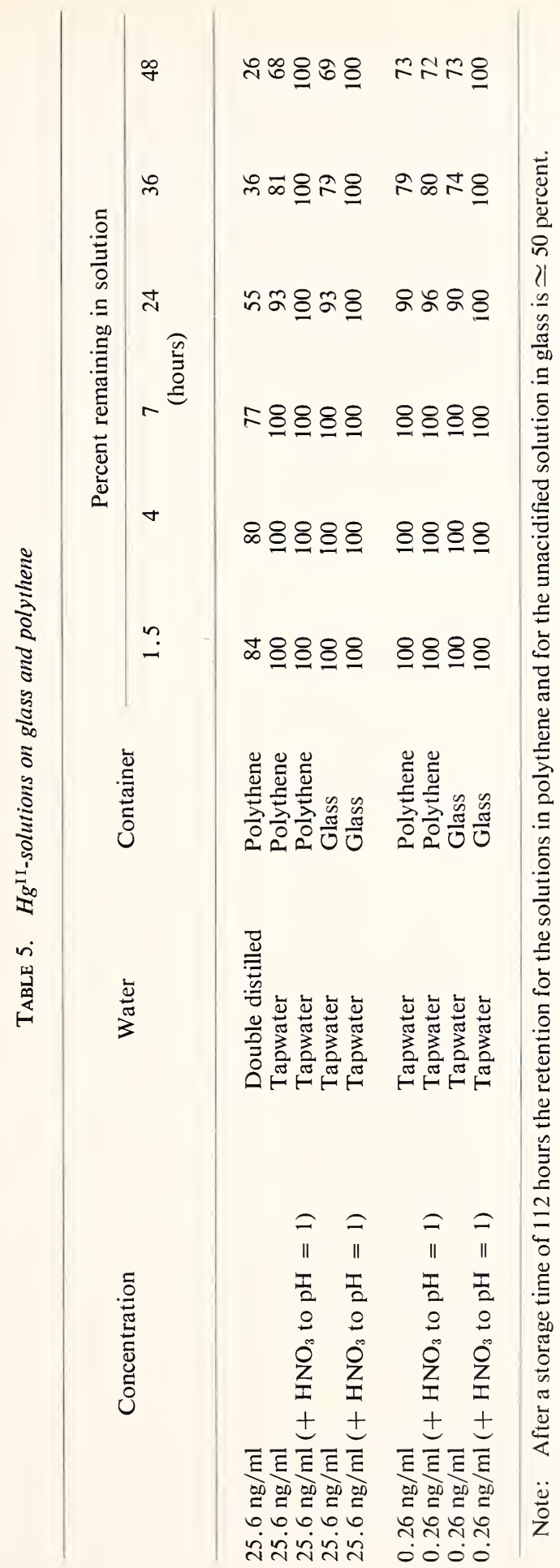


TABLE 6. Adsorption of organomercury compounds on glass and polythene

\begin{tabular}{|c|c|c|c|c|c|c|c|c|}
\hline \multirow{2}{*}{$\begin{array}{l}\text { Compound and } \\
\text { concentration }\end{array}$} & \multirow{2}{*}{$\mathrm{pH}$} & \multirow{2}{*}{ Container } & \multicolumn{6}{|c|}{ Percent remaining in solution } \\
\hline & & & 0 & 1 & $\begin{array}{l}4 \\
\text { (hours) }\end{array}$ & )$^{8}$ & 24 & 48 \\
\hline \multirow{4}{*}{$\begin{array}{l}\left(\mathrm{C}_{6} \mathrm{H}_{5}\right)_{2} \mathrm{Hg} \\
18 \mu \mathrm{g} / \mathrm{l}\end{array}$} & 2 & polythene & 100 & 100 & 100 & 100 & 100 & 97 \\
\hline & 7 & polythene & 100 & 98 & 95 & 86 & 78 & 71 \\
\hline & 7 & glass & 100 & 100 & 100 & 100 & 100 & 97 \\
\hline & 7 & glass & 100 & 100 & 100 & 100 & 100 & 100 \\
\hline \multirow{4}{*}{$\begin{array}{l}\left(\mathrm{C}_{6} \mathrm{H}_{5}\right) \mathrm{Hg}\left(\mathrm{OOCCH}_{3}\right) \\
\quad 2 \mu \mathrm{g} / 1\end{array}$} & 2 & polythene & 100 & 100 & 100 & 100 & 100 & 98 \\
\hline & 7 & polythene & 100 & 98 & 95 & 94 & 90 & 88 \\
\hline & 2 & glass & 100 & 100 & 100 & 100 & 100 & 100 \\
\hline & 7 & glass & 100 & 100 & 100 & 100 & 100 & 100 \\
\hline \multirow{4}{*}{$\begin{array}{l}\mathrm{CH}_{3} \mathrm{HgCl} \\
9 \mu \mathrm{g} / \mathrm{l}\end{array}$} & 1.5 & polythene & 100 & 100 & 100 & 100 & 100 & 100 \\
\hline & 7 & polythene & 100 & 98 & 95 & 93 & 91 & 81 \\
\hline & 1.5 & glass & 100 & 100 & 100 & 100 & 100 & 100 \\
\hline & 7 & glass & 100 & 100 & 99 & 98 & 96 & 95 \\
\hline
\end{tabular}

\section{The Determination of Mercury in Water Samples}

\section{A. INTRODUCTION}

For concentrations smaller than $100 \mathrm{ng} / \mathrm{l}$, activation analysis becomes superior to atomic absorption [6-8] due to the greater sensitivity if a suitable way of concentrating mercury on a solid adsorber can be found. This preconcentration is necessary, as the irradiation of water samples in a nuclear reactor is cumbersome. Olafsson collected inorganic mercury from seawater by amalgamation on a gold foil after reduction with $\mathrm{SnCl}_{2}$ and volatilization [9]. This method is not applicable to organic mercury compounds. Moreover, gold is not a convenient matrix from an activation analysis point of view.

In this investigation, active charcoal is used. A diluted $\mathrm{SnCl}_{2}$ solution, acetone and double distilled nitric acid are the only other reagents used in the method presented here. These reagents can be stripped from mercury easily so that no contamination by the reagents occurs $[10,11]$.

\section{B. Reagents and Apparatus}

- A 1 percent $\mathrm{SnCl}_{2}$ solution, aerated before use to remove any mercury; 
- Acetone of "analytical grade" purity, percolated through a charcoal column before use;

- Double distilled nitric acid. The starting product is of "analytical grade" purity;

- A standard $\mathrm{Hg}^{+2}$ solution $(0.1 \mathrm{mg} \mathrm{Hg} / \mathrm{ml})$;

-Activated charcoal ( 0.5 to $0.75 \mathrm{~mm})$ E. Merck. Chromatographic quality;

- A desiccator with a $48 \% \mathrm{H}_{2} \mathrm{SO}_{4}$ solution ( $52 \%$ relative humidity):

- An aeration vessel ( $c f$. fig. 3);

- A $500 \mathrm{ml}$ receiver, connected to a glass column for an activated charcoal bed ( $c f$. fig. 7);

-Test tubes $(16 \times 160 \mathrm{~mm})$;

-Quartz wool;

-Quartz wool, coated with metallic silver;

-Quartz capsules ( $10 \mathrm{~mm}$ i.d. $\times 70 \mathrm{~mm}$ long);

-A quartz tube with a fritted quartz filter disc for the purification of the activated charcoal;

-Quartz tubes (22 mm i.d. $\times 150 \mathrm{~mm}$ long);

- Glass tubes ( $22 \mathrm{~mm}$ i.d. $\times 150 \mathrm{~mm}$ long);

-A continuous centrifuge, type Heraeus-Christ "Junior-15000";

-Three tube furnaces with the following inner dimensions: $50 \mathrm{~mm} \times$ $250 \mathrm{~mm} ; 35 \mathrm{~mm} \times 200 \mathrm{~mm}$ and $25 \mathrm{~mm} \times 90 \mathrm{~mm}$;

- A well-type $\mathrm{NaI}$ detector, mounted in a sample changer and connected to a 400-channel analyzer with electric typewriter or punchtape read-out.

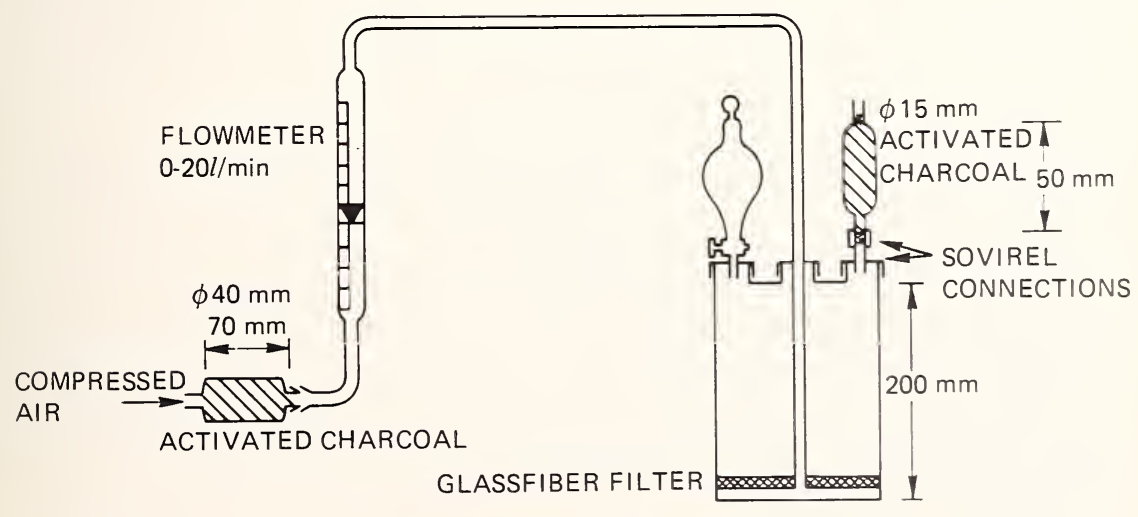

Figure 3. The aeration vessel. 


\section{Separation of Different Forms of Mercury}

The following separations of different species of mercury have been made ( $c f$. fig. 4):

a. The total amount of mercury in the water sample is collected by passing a known volume of the crude sample through a charcoal column. The mercury in solution and that adsorbed on suspended matter is concentrated in this way;

b. An aliquot of the water sample is freed from particulate matter by continuous centrifuging at $10,000 \mathrm{~g}$;

b.1. A known fraction of the clear liquid is passed through a charcoal column to collect the total amount of mercury present in water without suspended solids. The difference between the mercury concentrations obtained for the samples (a. and b.1.) will give the amount of mercury adsorbed on the suspended matter;

b.2. Another fraction (11) of the clear liquid is transferred to an aeration vessel. After reduction with stannous chloride, aeration and subsequent adsorption on activated charcoal, the concentration of inorganic mercury is determined. The amount of dissolved organic mercury can be

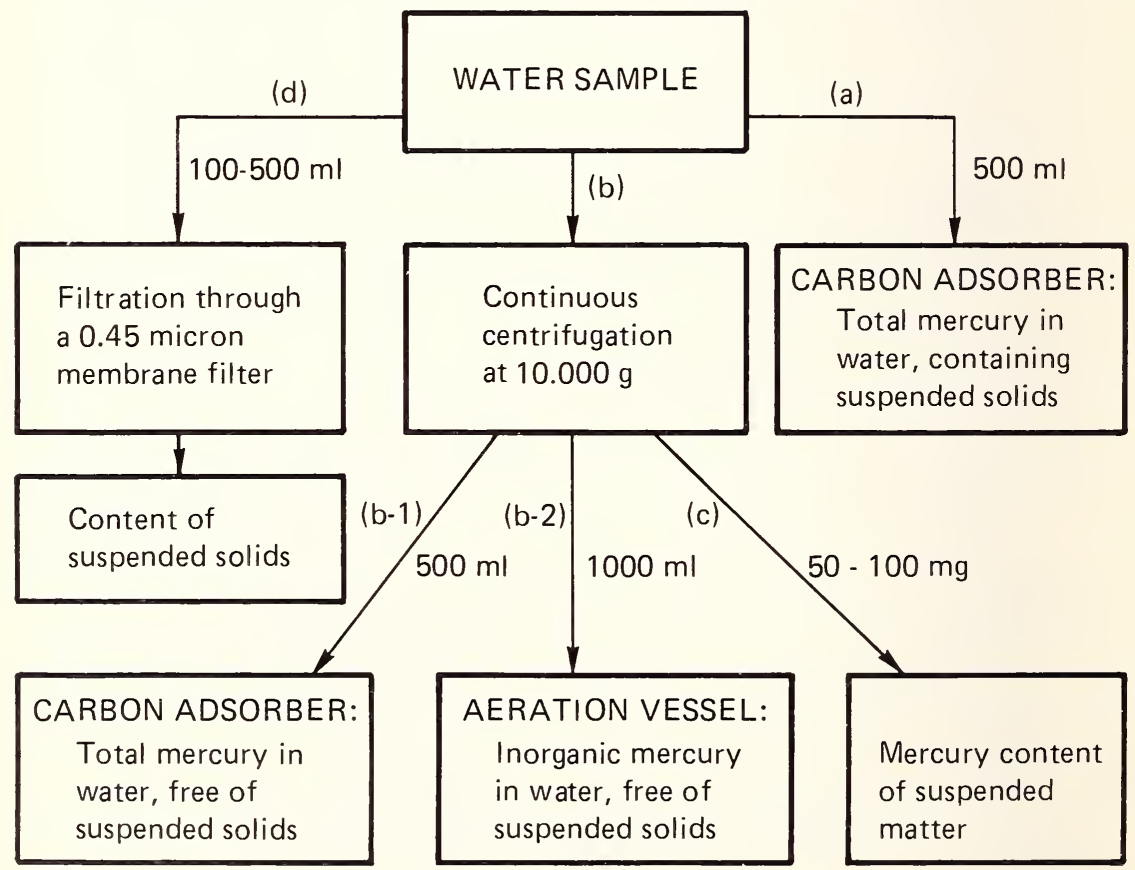

Figure 4. Scheme for the sampling of inorganic, organic, and particulate mercury. 
derived from the difference between the two mercury concentrations determined for the samples (b.1. and b.2.);

c. The amount of mercury on suspended matter can also be determined in another way. Some of the solid concentrated in the centrifuge is taken and weighed after equilibration at constant relative humidity, thus preventing mercury losses caused by other drying procedures. Then the solid is mixed with charcoal and irradiated;

d. Finally the amount of suspended matter is determined by weighing, after filtration of a known volume through a preweighed membrane filter $(0.45 \mu \mathrm{m})$.

\section{The Collection of Inorganic Mercury}

For the determination of inorganic mercury in water the apparatus shown in figure 4 is used. The procedure is based on the reduction to metallic mercury with stannous chloride, and subsequent aeration with a stream of air, which carries the mercury to a charcoal adsorber. The influence of the most important parameters on the recovery of mercury in the charcoal adsorbers has been studied with ${ }^{197} \mathrm{Hg}$ tracers.

\section{Concentration of Stannous Chloride}

The high concentration of stannous chloride $(1.5 \mathrm{~g} / 50 \mathrm{ml})$ used in atomic absorption determinations with an analogous procedure was found to be quite unnecessary, when only reduction of inorganic mercury is preferred. A final concentration of $10 \mathrm{mg} \mathrm{SnCl}{ }_{2}$ per liter proved to be sufficient for complete reduction.

\section{Flow Rate of the Airstream}

The percentage of mercury remaining in the vessel has been determined as a function of the flow rate for solutions of $0.18 \mu \mathrm{g} \mathrm{Hg} / \mathrm{l}$. Complete removal of mercury from the solution is obtained at flow rates higher than $3 \mathrm{l} / \mathrm{min}$. However, to prevent a breakthrough of the charcoal adsorber, the flow rate should not exceed $6 \mathrm{l} / \mathrm{min}$. At flow rates lower than $6 \mathrm{l} / \mathrm{min}$ complete collection of mercury vapor on the charcoal adsorber is observed ( $c f$. figs. 5 and 6). 


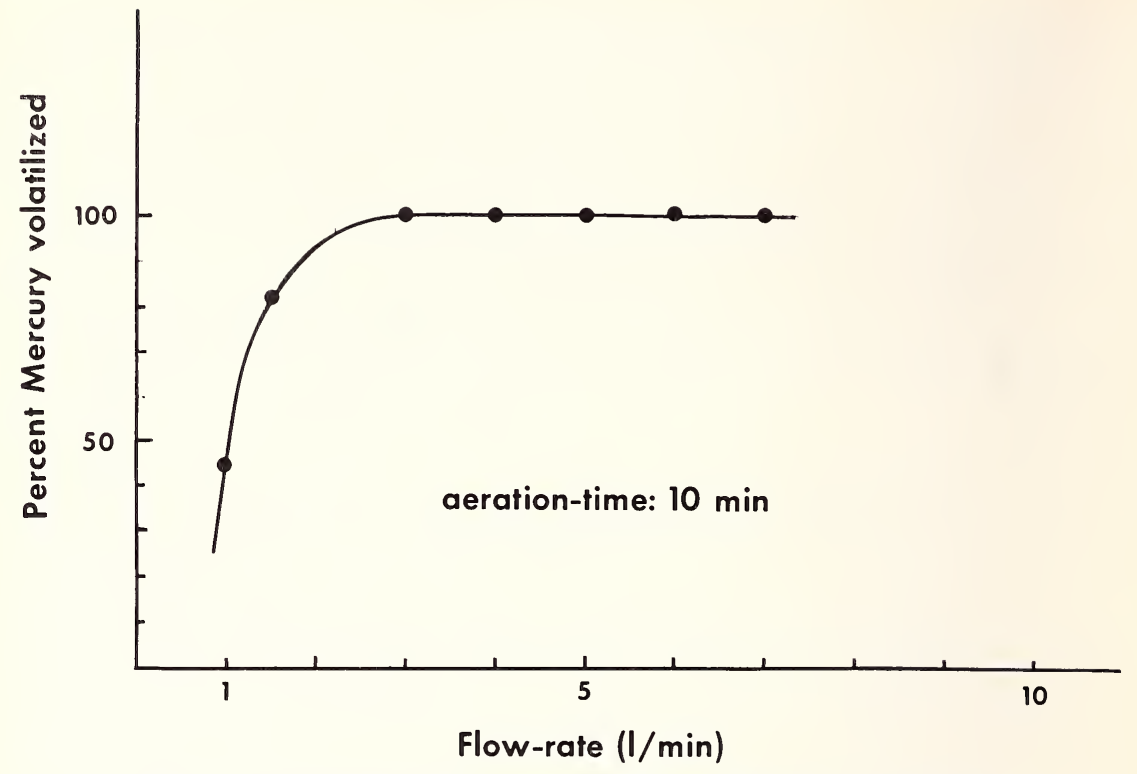

Figure 5. The influence of the flow rate on the volatilization of mercury.

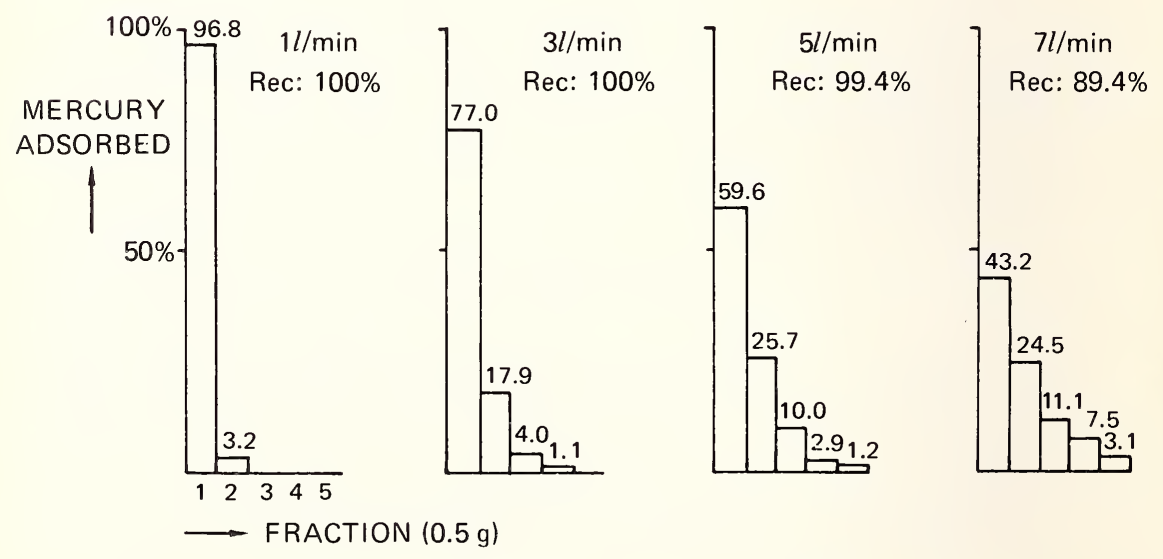

Figure 6. The influence of the flow rate on the adsorption of mercury on activated charcoal. 


\section{Time of Aeration}

It was found that under normal conditions all mercury is volatilized within 5 minutes, so this time was used in further experiments unless otherwise stated. At 4,20 and $40^{\circ} \mathrm{C}$ it takes 5,3 , and $<2$ minutes respectively. This is in accordance with data published in literature $[7,12]$.

\section{Acidity and Salinity of the Sample}

At high acid concentrations a considerable amount of mercury will be retained in solution [12]. Between $\mathrm{pH}=1$ and $\mathrm{pH}=7$ no influence of the acidity on the aeration has been observed. Salt concentrations up to $2 \mathrm{~N}$ $\mathrm{NaCl}$ showed no influence on the yield.

\section{Volume of the Sample}

When small volumes (relative to the volume of the vessel) are processed, the recovery drops to 80 percent in case of a $100 \mathrm{ml}$ sample.

\section{Amount of Charcoal}

The amount of charcoal necessary for complete collection of volatilized mercury has been determined by loading a $5.6 \mathrm{~g}$ charcoal bed with metallic mercury at a flow rate of $51 / \mathrm{min}$ and dividing it in fractions of $0.5 \mathrm{~g}$ each. The first fraction contained 96 percent, the second 4 percent; the others did not contain any mercury. From this result and from the results shown in figure 6 the use of $2.5 \mathrm{~g}$ of charcoal is recommended.

\section{The Reduction of Organic Mercury Compounds}

Only a small fraction ( $\leqslant 0.5$ percent) of organic mercury compounds is reduced under the conditions recommended for the determination of inorganic mercury ( $c f$. section IV.E.). Under more severe circumstances (i.e., stronger reduction, or oxidation followed by reduction, combined with long aeration times) the results given in table 7 were obtained. It can be concluded that a separation between the two species of mercury with the standard procedure is achieved. 
TABLE 7. Volatilization of organic mercury compounds from dilute aqueous solutions

\begin{tabular}{|c|c|c|c|c|}
\hline \multirow{2}{*}{ Compound } & \multirow{2}{*}{$\begin{array}{l}\text { Concentration } \\
\text { in } \mu \mathrm{g} / 1\end{array}$} & \multicolumn{3}{|c|}{ Percentage found on the charcoal adsorber after: } \\
\hline & & Aeration $^{\mathrm{a}}$ & Reduction $^{\mathrm{b}}$ & $\begin{array}{l}\text { Oxidation, re- } \\
\text { duction }^{\mathrm{c}}\end{array}$ \\
\hline
\end{tabular}

$\begin{array}{lrrlr}\left(\mathrm{C}_{6} \mathrm{H}_{5}\right)_{2} \mathrm{Hg} & 2.66 & 0.4 & 8.6 & 25.7 \\ \left(\mathrm{C}_{6} \mathrm{H}_{5}\right) \mathrm{HgCl} & 0.67 & .2 & 3.5 & 13.4 \\ \left(\mathrm{C}_{6} \mathrm{H}_{5}\right) \mathrm{HgAc} & 0.19 & .1 & 8.7 & 17.3 \\ \mathrm{CH}_{3} \mathrm{HgCl} & 1.39 & .2 & 5.8 & 20.7 \\ \left(\mathrm{CH}_{3}\right)_{2} \mathrm{Hg} & 22.6 & .14 & 0.07 & -\end{array}$

\footnotetext{
a Aeration time was 5 minutes at a flow-rate of $51 / \mathrm{min}$.

${ }^{\mathrm{b}}$ Reduction with $\mathrm{SnCl}_{2}$ (final concentration $1 \mathrm{~g} / \mathrm{l}$ ) during $15 \mathrm{~min}$. Aeration time was 20 $\min$.

c Oxidation with $\mathrm{KMnO}_{4}$ (final concentration $0.1 \mathrm{~g} / \mathrm{l}$ ) for 15-20 min, followed by reduction with $\mathrm{SnCl}_{2}$ as described above.
}

\section{E. The Collection of the Total Amount of Mercury}

The apparatus shown in figure 7 is used for the determination of the total amount of mercury in untreated and centrifuged water samples. The mercury, both "inorganic" or "organic," is adsorbed quantitatively on the charcoal column. A small layer of pulverized charcoal is put on top of the column to retain the suspended matter present in the untreated water sample. The influence of the various parameters on the adsorption efficiency was measured with ${ }^{197} \mathrm{Hg}^{+2}$ and ${ }^{197} \mathrm{Hg}$ labeled compounds.

\section{Acidity and Salinity of the Water Sample}

In spite of the high salt content of seawater, no influence on the adsorption of mercury (compounds) at concentrations ranging from $10 \mathrm{ng} / \mathrm{l} \mathrm{up}$ to $10 \mu \mathrm{g} / \mathrm{l}$ was observed. At the normal pH of seawater $(\simeq 8)$ and low mercury concentrations $(50 \mathrm{ng} / \mathrm{l})$, sometimes incomplete adsorption on charcoal occurred. It was found that these losses can be prevented by acidification. After adjusting the $\mathrm{pH}$ to 1 with double distilled nitric acid adsorption is complete.

\section{Flow Rate Through the Column}

At flow rates up to $4 \mathrm{ml} / \mathrm{min}$, the adsorption of mercury is complete, as was checked with mercury tracer. 


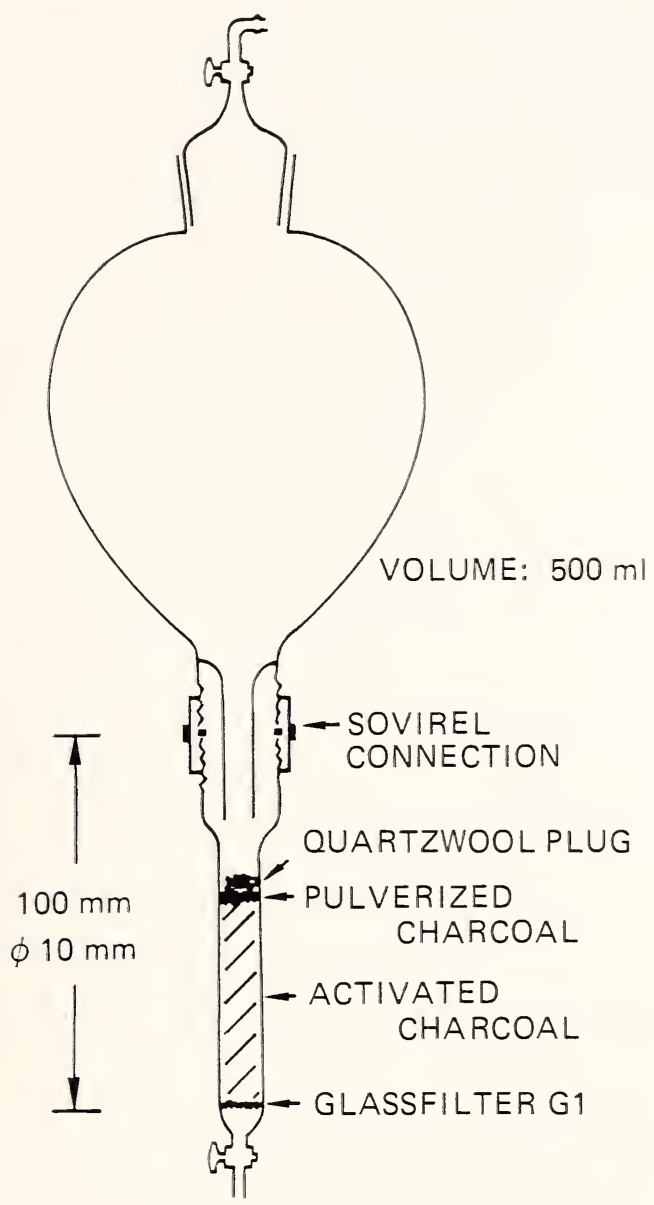

Figure 7. Apparatus for the preconcentration of mercury on charcoal.

\section{Adsorption of Some Mercury Compounds}

The adsorption of metallic mercury, mercury chloride and some organomercury compounds was investigated. The results are summarized in table 8 . It can be said that losses are negligible.

\section{F. The Collection of Particulate Matter}

The mercury content of suspended matter can be determined in a sample collected from the rotor of the centrifuge. Different analytical methods have been used $[13,14]$. The technique applied here is mixing of the sedi- 
TABLE 8. The adsorption of some mercury compounds on activated charcoal

\begin{tabular}{llcc}
\hline Compound & $\begin{array}{c}\text { Dissolved } \\
\text { in }\end{array}$ & $\begin{array}{c}\text { Concentration } \\
\text { in } \mu \mathrm{g} / 1\end{array}$ & $\begin{array}{c}\text { Percentage } \\
\text { adsorbed }\end{array}$ \\
\hline $\mathrm{Hg}^{2}$ & water & 0.1 & 99.8 \\
$\mathrm{Hg}^{2}$ & acetone & .1 & 98.3 \\
$\mathrm{Hg}(\mathrm{O})$ & water & .1 & $93.5^{\mathrm{b}}$ \\
$\left(\mathrm{C}_{6} \mathrm{H}_{5}\right)_{2} \mathrm{Hg}$ & water & 12.7 & 98.9 \\
$\left({ }_{6} \mathrm{H}_{5} \mathrm{HgCl}\right.$ & water & 4.3 & 100.4 \\
$\mathrm{C}_{6} \mathrm{H}_{5} \mathrm{HgAc}$ & water & 1.3 & 100.6 \\
$\mathrm{C}_{6} \mathrm{H}_{5} \mathrm{HgAc}$ & benzene & 1.3 & 99.7 \\
$\mathrm{CH}_{3} \mathrm{HgCl}$ & water & 1.5 & 98.9 \\
$\left(\mathrm{CH}_{3}\right)_{2} \mathrm{Hg}$ & water & 15.4 & 97.1 \\
\end{tabular}

a The labeled organic compounds were prepared by exchange of the unlabeled species with ${ }^{197} \mathrm{Hg}$.

b $\mathrm{The} \mathrm{Hg}(\mathrm{O})$ was obtained by reduction of $\mathrm{Hg}^{2}$ with $\mathrm{SnCl}_{2}$. Losses due to volatilization may have occurred in this case. The mercury content of the effluent was $0.2 \%$ of the original value.

ment or the suspended matter with some charcoal and, after neutron activation transferring the mercury by heating the sediment-charcoal mixture in a tube furnace to $900{ }^{\circ} \mathrm{C}$ with a stream of nitrogen, to a second charcoal adsorber. From experiments with a ${ }^{197} \mathrm{Hg}$ tracer it was found that mercury adsorbed on suspended matter can be recovered quantitatively in this way. Weighing of the sampled suspended matter should be done prior to the heating. Drying of the material for 1 hour at $110^{\circ} \mathrm{C}$ cannot be applied. Experiments with labeled organic mercury compounds revealed that severe losses are suffered in this case.

\section{Compound \\ $\mathrm{CH}_{3} \mathrm{HgCl}$ \\ $\left(\mathrm{CH}_{3}\right)_{2} \mathrm{Hg}$ \\ $\mathrm{Hg}^{+2}$}

Percentage loss after

$1 \mathrm{~h}$ at $110^{\circ} \mathrm{C}$

$$
\begin{array}{r}
8-10 \% \\
80-90 \% \\
0 \%
\end{array}
$$

Therefore, weighing is performed after equilibration at 50 percent relative humidity during at least 16 hours, thus preventing mercury losses due to volatilization. The determination of the content of suspended matter in the water sample $(\mathrm{mg} / \mathrm{l})$ by filtration is performed by weighing under the same conditions. 


\section{G. The Purification of Charcoal and Its Adsorption Capacity}

The mercury content of commercially available "activated" charcoal is 15 to $20 \mathrm{ng} \mathrm{Hg} / \mathrm{g}$. This content can be lowered considerably by heating the charcoal in a quartz tube $(40 \mathrm{~mm}$ o.d. and $300 \mathrm{~mm}$ long), which is placed inside a tube furnace in a vertical position. The temperature of the furnace is maintained at 950 to $1000{ }^{\circ} \mathrm{C}$ and a stream of nitrogen, previously purified by a charcoal scavenger, is passed through the glowing carbon for 30 to 60 minutes. Activated charcoal, purified in this way, contains only 0.1 to $0.2 \mathrm{ng} \mathrm{Hg} / \mathrm{g}$. This residual mercury content determines the lower limit of the determination. A slight increase of the specific adsorption capacity was observed after this treatment. The specific adsorption capacity of charcoal for $\mathrm{Hg}^{+2}$ ions and methylmercury chloride can be calculated from adsorption isotherms determined with the ${ }^{197} \mathrm{Hg}$-labeled compounds. The low solubility in water of the latter compound limits the concentration range which can be studied in this way. The Langmuir plots for mercuric ions and methylmercury chloride are shown in figure 8 . From the expression for the Langmuir isotherm (eq 3), the relation between the fraction adsorbed, $y / c V$, and the concentration, $c$, can be obtained.

$$
\begin{gathered}
\mathrm{y}=\mathrm{m} \cdot \frac{\beta \mathrm{c}}{\delta+\mathrm{c}} \\
\frac{\mathrm{cV}}{\mathrm{y}}=\frac{\mathrm{V}}{\mathrm{m}} \cdot \frac{\delta}{\beta}+\frac{\mathrm{V}}{\mathrm{m} \beta} \cdot \mathrm{c}
\end{gathered}
$$

From the slope and the intercept of the plot, the values of $\beta$ and $\delta$ can be calculated:

$$
\beta \quad \delta \text { in } \mathrm{mg} / \mathrm{ml}
$$

$\begin{array}{lrr}\mathrm{Hg}^{+2} & 0.33 & 0.73 \\ \mathrm{CH}_{3} \mathrm{HgCl} & .23 & .35\end{array}$

At low concentrations, the adsorption can be described by a Henry isotherm:

$$
\mathrm{y}=\mathrm{m} \cdot \alpha \mathrm{c}
$$

This relation was measured for $\mathrm{Hg}^{+2}$ ions in seawater. Known amounts of a $\mathrm{Hg}\left(\mathrm{NO}_{3}\right)_{2}$ standard solution were added to $50 \mathrm{ml}$ aliquots of seawater 


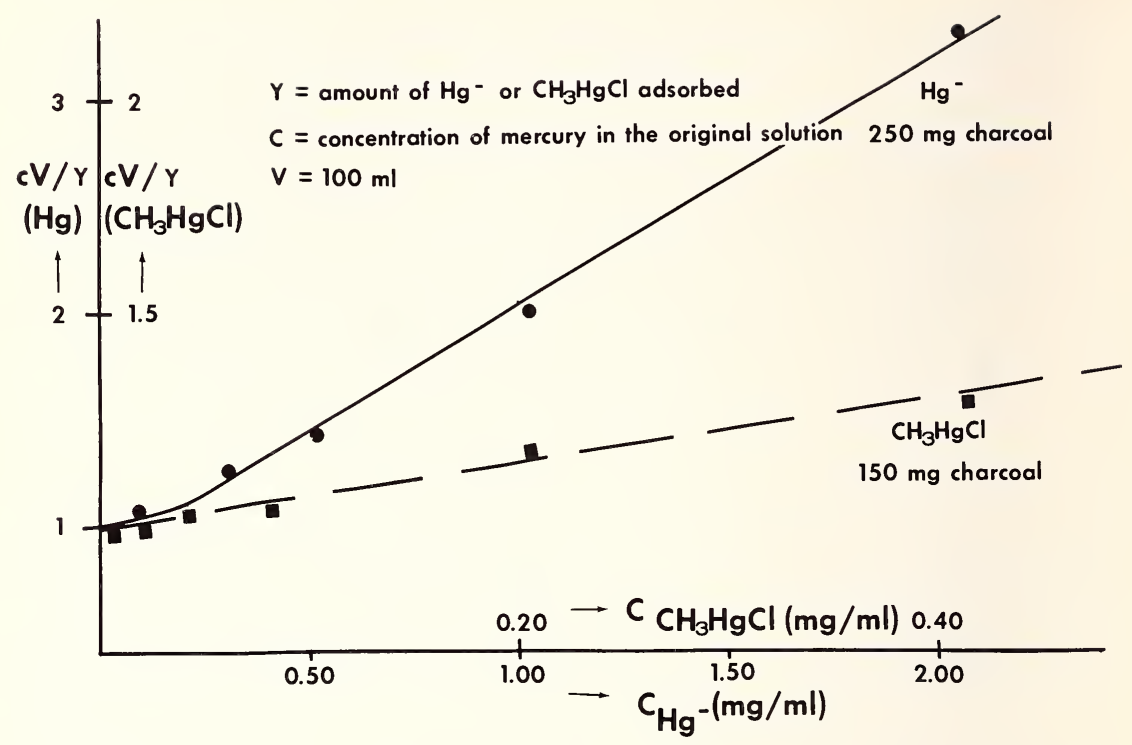

Figure 8. The Langmuir plot for $\mathrm{Hg}^{+2}$ and $\mathrm{CH}_{3} \mathrm{HgCl}$ adsorption on charcoal.

$(\mathrm{pH}=7.4)$. Then $4 \mathrm{mg}$ active charcoal was added as a $100 \mu \mathrm{l}$ portion of a suspension in double distilled water. Results were:

\section{Initial}

$\mathrm{Hg}^{+2}$ concentration

in $\mu \mathrm{g} / \mathrm{l}$

$\mathrm{y} / \mathrm{cV}$

0.80
7.55
15.2
30.3
52

0.84

$.80 \quad$ Average value

$.84 \mathrm{y} / \mathrm{cV}=0.82 \pm 0.02$

152

$.87 \quad \alpha=10.2 \pm 0.2(\mathrm{mg} / \mathrm{ml})^{-1}$

$.74)$

The collection of mercuric ion and methylmercury chloride on $250 \mathrm{mg}$ of charcoal is quantitative for concentrations below $1 \mathrm{mg} \mathrm{Hg}+2 / 100 \mathrm{ml}$ and $2.5 \mathrm{mg} \mathrm{CH} \mathrm{CH}_{3} \mathrm{HgCl} / 100 \mathrm{ml}$ respectively. Methylmercury chloride is probably completely ionized. From the slopes of the Langmuir plots, the adsorption capacities per gram of activated charcoal in case of complete monomolecular coverage are found to be: $\mathrm{Hg}^{+2}: 330 \mathrm{mg} / \mathrm{g}$ and $\mathrm{CH}_{3} \mathrm{Hg}^{+1}$ : $820 \mathrm{mg} / \mathrm{g}$. 


\section{H. IRradiation of the Charcoal Samples}

The charcoal samples, packed in quartz capsules $(10 \mathrm{~mm}$ i.d. $\times 70 \mathrm{~mm}$ long), are irradiated together with a $\mathrm{Hg}$ standard for 8 to 12 hours at a thermal neutron flux of $3 \cdot 10^{12} \mathrm{n} \cdot \mathrm{cm}^{-2} \cdot \mathrm{s}^{-1}$. The standards are prepared by adding $20 \mu \mathrm{l}$ of a solution of $\mathrm{Hg}\left(\mathrm{NO}_{3}\right)_{2} \cdot \mathrm{H}_{2} \mathrm{O}(2.03 \mu \mathrm{g} \mathrm{Hg} / 20 \mu \mathrm{l})$ to activated charcoal in a quartz capsule. The capsule is sealed and the content is homogenized by vigorous shaking. Irradiation takes place in a dry rotating facility, that can hold 19 quartz capsules of the type used in these determinations. To check flux variations, pieces of iron wire are placed at different positions throughout the irradiation flask. After irradiation the samples are cooled for 24 hours to allow for the decay of short-lived nuclides.

\section{The Separation of Mercury from the Radioactive Charcoal}

After irradiation the matrix activity of the charcoal prevents the direct instrumental determination of mercury. For the separation of mercury the apparatus shown in figure 9 is used [14]. Before opening, the quartz capsules are cooled by liquid nitrogen, to avoid the risks of explosion due to pressure buildup during irradiation. The content of a capsule is transferred to the quartz tube that fits in the first tube furnace $(25 \mathrm{~mm}$ i.d. and $200 \mathrm{~mm}$ long). A piece of silver-coated glass wool and a second adsorber are placed in two separate glass tubes. The temperature of the silvercoated plug can be adjusted by means of a small tube furnace. The glass tubes are connected to the quartz tube and the whole array is placed inside the furnaces with temperatures preset respectively at $700{ }^{\circ} \mathrm{C}$ to $800{ }^{\circ} \mathrm{C}$ and $400{ }^{\circ} \mathrm{C}$. The choice of these temperatures becomes apparent

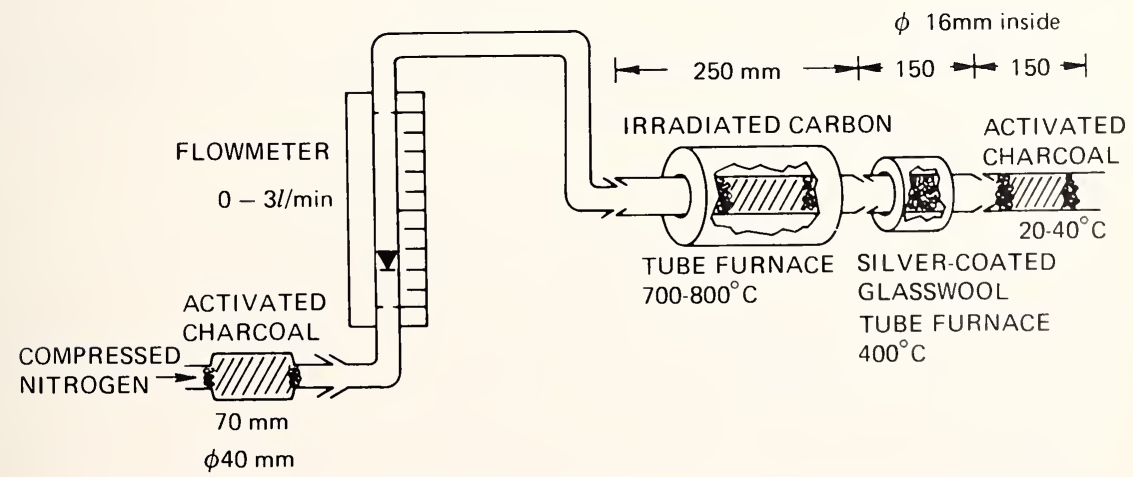

Figure 9. Apparatus for the separation of mercury from a radioactive matrix. 
from figure 10. Two requirements must be fulfilled: The complete removal of mercury from the irradiated charcoal matrix and no adsorption on the silver-coated glass wool plug.

A stream of nitrogen, purified by a charcoal scavenger, is passed through the system at a flow rate of 0.6 to $0.8 \mathrm{l} / \mathrm{min}$. It carries the mercury, volatilized at $700{ }^{\circ} \mathrm{C}$ to $800{ }^{\circ} \mathrm{C}$, to the second adsorber. ${ }^{82} \mathrm{Br}(\mathrm{T} 1 / 2=$ $35.4 \mathrm{~h}$ ), which is partially released at this temperature, is retained in the silver-coated plug. In spite of the fact that only a small percentage of the bromine present in the sample is adsorbed on charcoal and that at $700{ }^{\circ} \mathrm{C}$ to $800{ }^{\circ} \mathrm{C}$ only 10 to 15 percent of the bromine is released, the amount of ${ }^{82} \mathrm{Br}$ adsorbed on the second charcoal adsorber would interfere with the determination of mercury in seawater in case the silver-coated plug is omitted. From experiments with labeled organic mercury compounds it was found that, whatever the chemical form of mercury in the sample was, a quantitative volatilization was obtained.

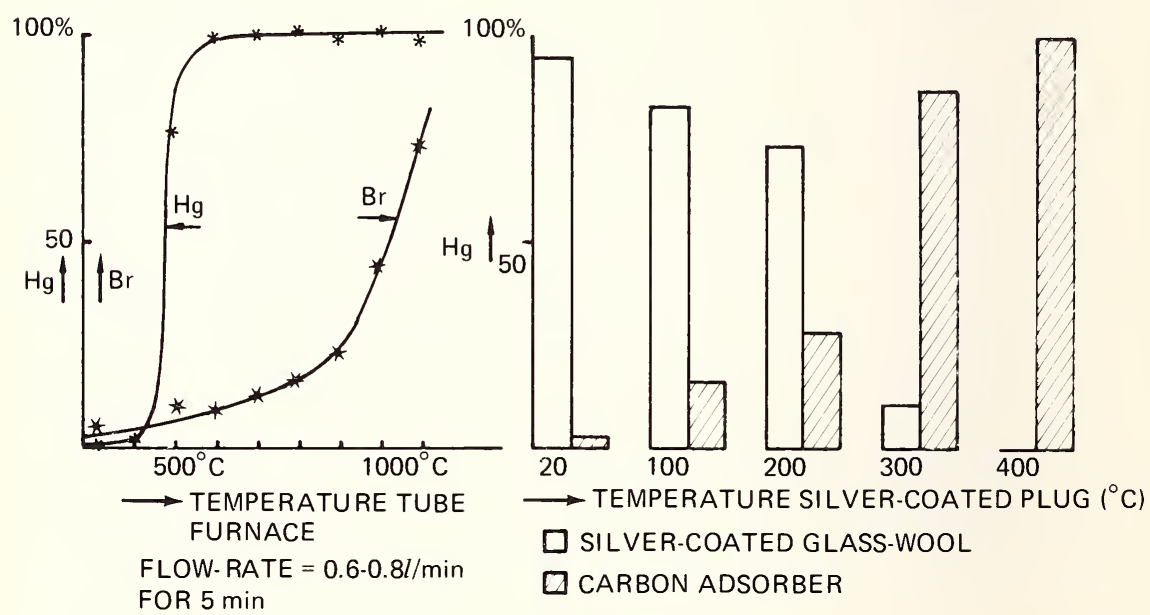

Figure 10. (A) The influence of the temperature on the amount of mercury and bromine released from charcoal. (B) The influence of the temperature on the amount of mercury retained in the silver-coated plug.

\section{J. Procedure for the Determination of InORganic, Total and Particulate Mercury}

\section{The Collection of Inorganic Mercury}

a. One liter of a centrifuged water sample is transferred to the aeration vessel and the adsorber tube containing $2.5 \mathrm{~g}$ charcoal is connected to the vessel; 
b. One milliliter of a 1 percent $\mathrm{SnCl}_{2}$ solution is added and the vessel is closed;

c. The airflow is adjusted to 4 to $6 \mathrm{l} / \mathrm{min}$;

d. After 5 minutes, the aeration is stopped and the charcoal is transferred to a quartz capsule which is then sealed off.

\section{The Collection of Total Mercury}

a. A water sample, either unprocessed or centrifuged, is transferred to a receiver connected with a column containing $1.5 \mathrm{~g}$ of purified charcoal. In case of an unprocessed water sample, $\simeq 100 \mathrm{mg}$ of pulverized charcoal is put on top of the granulated charcoal. The charcoal is wetted with double distilled water before use;

b. The sample is acidified with $\simeq 1 \mathrm{ml}$ of concentrated nitric acid to $\mathrm{pH}$ $=1$;

c. The flow rate through the column is kept below $4 \mathrm{ml} / \mathrm{min}$;

d. Five milliliter of previously purified acetone is fed to the column which is then dried by a gentle stream of air;

e. The charcoal is transferred to a quartz capsule, which is cooled with liquid nitrogen and then sealed off.

\section{The Collection of Particulate Matter}

a. About 50 to $100 \mathrm{mg}$ of suspended matter is taken from the rotor of the centrifuge and transferred to a quartz capsule which has been weighed before;

b. The capsule is placed in a desiccator containing a 48 percent $\mathrm{H}_{2} \mathrm{SO}_{4}$ solution (relative humidity $52 \%$ ) for at least 16 hours;

c. The weight of the suspended matter in the capsule is determined and $0.5 \mathrm{~g}$ of purified charcoal is added;

d. The mixture is homogenized and the capsule is sealed off.

\section{Irradiation of Charcoal Samples}

a. The various charcoal samples are placed in an irradiation can together with mercury standards and flux monitors. The samples are irradiated for 8 to 12 hours at a thermal neutron flux of $3 \cdot 10^{12} \mathrm{n} \cdot \mathrm{cm}^{-2} \cdot \mathrm{s}^{-1}$;

b. After irradiation the samples are cooled for 24 hours. 


\section{The Separation of Mercury from the Radioactive Charcoal}

a. The quartz capsules are opened after cooling with liquid nitrogen and the radioactive charcoal is transferred to a quartz tube connected with two glass tubes containing respectively silver-coated glass wool and a fresh charcoal adsorber;

b. The whole array is placed inside the furnaces of the apparatus shown in figure 9 and a stream of nitrogen is passed through the system at a flow rate of 0.6 to $0.8 \mathrm{l} / \mathrm{min}$. The temperatures of the furnaces are kept at 700 ${ }^{\circ} \mathrm{C}$ to $800{ }^{\circ} \mathrm{C}$ and $400{ }^{\circ} \mathrm{C}$ respectively;

c. After 5 minutes the tubes are removed from the system and the carbon of the second adsorber is transferred to a test tube $(16 \times 160 \mathrm{~mm})$. The mercury standards are processed in the same way as the samples;

d. Samples and standards are counted for at least 5 minutes in a 3 in $\times 3$ in well-type NaI detector, connected to a 400-channel analyzer. The spectra are read out on punchtape or on an electrical typewriter. Flux monitors are counted in a $11 / 2$ in $\times 2$ in $\mathrm{NaI}$ well-type detector connected to a single-channel analyzer.

The observed specific count rate under the $0.077 \mathrm{MeV}$ photopeak of ${ }^{197} \mathrm{Hg}(\mathrm{T} 1 / 2=66 \mathrm{~h})$ is $\simeq 1.6 \times 10^{5}$ counts $/ \mathrm{m} / \mu \mathrm{g} \mathrm{Hg}$ after an irradiation of 12 hours at $3 \cdot 10^{12} \mathrm{n} \cdot \mathrm{cm}^{-2} \cdot \mathrm{s}^{-1}$ and a cooling period of 24 hours. Figure 11 gives the $\gamma$-spectra of the second charcoal adsorber, containing all mercury originally present in the water sample.

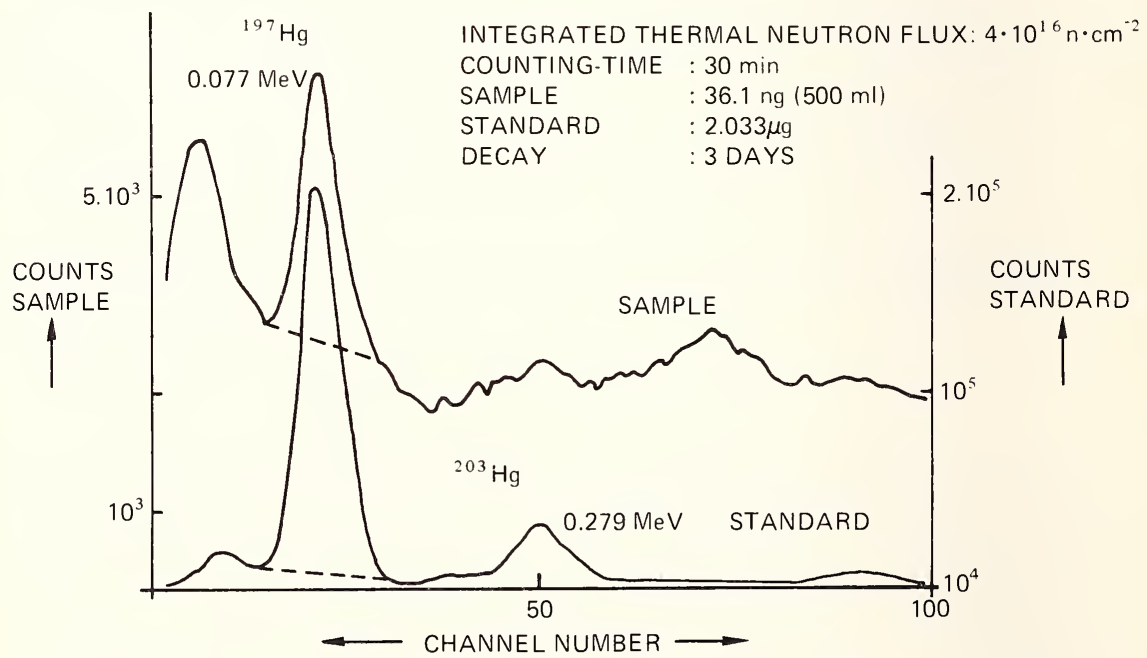

Figure 11. Gamma spectra of a sample and a standard. 


\section{K. Results}

\section{The Mercury Content of Tap Water}

From one large sample of tap water 12 aliquots of $500 \mathrm{ml}$ each were taken and analyzed separately. The following results were obtained:

Total mercury: $31.0 ; 36.2 ; 22.9 ; 35.3 ; 20.7 ; 25.1 ; 27.1 ; 31.8$ and $22.9 \mathrm{ng} / 1$. average: $28( \pm 5) \mathrm{ng} / \mathrm{l}$

Inorganic mercury: $4.2 ; 3.5 ; 4.5$ and $3.9 \mathrm{ng} / 1$.

average: $4.0( \pm 0.4) \mathrm{ng} / \mathrm{l}$.

"Organic" mercury: $24( \pm 5) \mathrm{ng} / \mathrm{l}$.

\section{Double Distilled Water}

Double distilled water was prepared from demineralized water in a quartz apparatus, which has been in continuous operation for a few years. The total mercury content of three aliquots, taken at various days, was: $7.4 ; 7.6$ and $4.6 \mathrm{ng} / \mathrm{l}$.

\section{Seawater}

Three aliquots of a large sample, taken at the beach at Petten, were analyzed. The obtained results are:

Total mercury: $55.1 ; 53.6$ and $46.8 \mathrm{ng} / 1$. average value: $52( \pm 4) \mathrm{ng} / \mathrm{l}$.

Total mercury in the centrifuged sample: $14.4 ; 16.2$ and $22.0 \mathrm{ng} / 1$. average value: $17.5( \pm 3.5) \mathrm{ng} / \mathrm{l}$.

Inorganic mercury: $4.7 ; 4.2$ and $4.3 \mathrm{ng} / 1$. average value: $4.4( \pm 0.4) \mathrm{ng} / \mathrm{l}$.

Mercury content of the particulate matter: $35 \mathrm{ng} / 1$.

Organic mercury in solution: $13 \mathrm{ng} / \mathrm{l}$. 


\section{Surface Waters}

A large sample was taken in a semi-industrial region in the western part of the Netherlands. Three aliquots were analyzed separately. Results are:

Total mercury: $78.2 ; 67.0$ and 74.3 . average value: $73( \pm 6) \mathrm{ng} / \mathrm{l}$.

Total mercury in the centrifuged sample: $39.2 ; 41.1$ and 46.5 . average value: $42( \pm 4) \mathrm{ng} / \mathrm{l}$.

Inorganic mercury: 7.0 and $7.3 \mathrm{ng} / 1$. average value: $7.1( \pm 0.2) \mathrm{ng} / \mathrm{l}$.

Mercury content of the particulate matter: 0.70 and $0.58 \mu \mathrm{g} / \mathrm{g}$. Dissolved organic mercury: $35 \mathrm{ng} / 1$.

\section{The Determination of Mercury in Air}

The determination of mercury in air is analogous to the determination in water. Only the procedure for the determination of the total mercury content is discussed here.

\section{A. Equipment and Reagents}

- An electrical suction pump with variable capacity (0.05 to 2.8 $\mathrm{m}^{3} / \mathrm{h}$ ).

- An integrating flowmeter with decimal reading (to $0.2 \mathrm{l}$ ).

- An adsorption device ( $c f$. fig. 12; type of filter holder: SWINNEX 04700).

-A muffle furnace $(100 \mathrm{~mm}$ i.d. $\times 150 \mathrm{~mm})$.

- A tube furnace $(35 \mathrm{~mm}$ i.d. $\times 150 \mathrm{~mm})$.

-Quartz capsules ( $18 \mathrm{~mm}$ i.d. $\times 40 \mathrm{~mm}$ ).

-Quartz tubes ( $22 \mathrm{~mm}$ i.d. $\times 240 \mathrm{~mm}$ ).

- Membrane filters with $0.22 \mu \mathrm{m}$ pore diameter.

-Activated charcoal (20 to 35 mesh), chromatographic quality, (E. Merck).

- Test tubes $(16 \times 160 \mathrm{~mm})$.

-A 3 in $\times 3$ in NaI well-type crystal connected to a 400-channel analyzer with an electrical typewriter. 


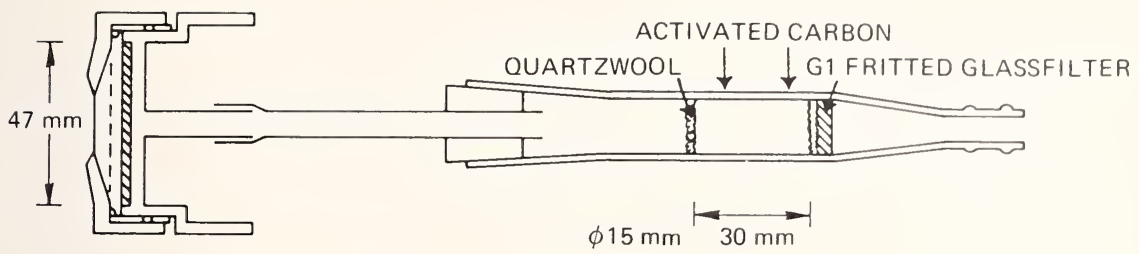

Figure 12. The sampling system for the determination of mercury in air.

\section{B. SAMPLing AND IRRADiATION}

a. The adsorption device is filled with $1.5 \mathrm{~g}$ of purified charcoal ( $c f$. section IV.G.) and a filter is mounted. The apparatus is connected to the suction pump.

b. The flow rate is kept below $5 \mathrm{l} / \mathrm{min}$. For unpolluted air, a sample of 1.5 to $2 \mathrm{~m}^{3}$ is collected.

For higher concentrations, the minimal sample volume is smaller. The distribution of $\mathrm{Hg}, \mathrm{CH}_{3} \mathrm{HgCl}$ and $\left(\mathrm{CH}_{3}\right)_{2} \mathrm{Hg}$ over the carbon adsorber was measured with ${ }^{197} \mathrm{Hg}$ tracers. Figure 13 shows the method and the results for $\left(\mathrm{CH}_{3}\right)_{2} \mathrm{Hg}$. The rest of the procedure is identical to that described in sections IV.J.4. and IV.J.5.
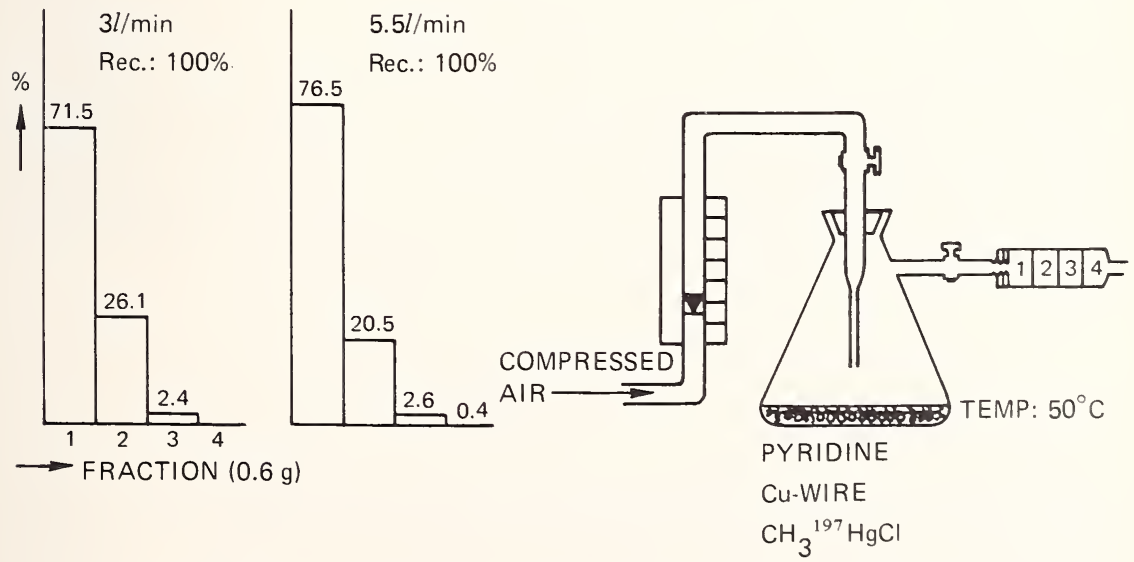

Figure 13. The distribution of dimethylmercury over the carbon adsorber. 


\section{Results}

The specific count rate under the $0.077 \mathrm{MeV}$ photopeak of ${ }^{197} \mathrm{Hg}$ is 1.6 $\times 10^{5}$ count $\min ^{-1} \mu \mathrm{g}^{-1}$ after a cooling period of 2 days. Four groups of samples have been analyzed.

\section{Laboratory Air}

Samples of 0.6 to $3 \mathrm{~m}^{3}$ were taken in a chemical laboratory where mercury is used occasionally. Results obtained in a period of a few months vary between 40 and $68 \mathrm{ng} / \mathrm{m}^{3}$ with a standard deviation of 5 percent per measurement. After removal of the mercury storage bottle, the concentration in the air decreased within a week to $7.4 \mathrm{ng} / \mathrm{m}^{3}$ with the same relative standard deviation. The pressurized air system contained $(5.5 \pm 0.2)$ to $(6.0 \pm 0.2) \mathrm{ng} / \mathrm{m}^{3}$.

\section{Atmospheric Air}

Samples of 2 to $4 \mathrm{~m}^{3}$ of air taken in the vicinity of the Dutch Reactor Center at the North Sea coast on various days were analyzed. The observed range of $\mathrm{Hg}$ concentrations was $(1.8 \pm 0.2)$ to $(2.3 \pm 0.2) \mathrm{ng} / \mathrm{m}^{3}$.

\section{Natural Gas}

Natural gas may contain a considerable $\mathrm{Hg}$ content. Concentrations on the order of $180 \mu \mathrm{g} / \mathrm{m}^{3}$ have been reported [15]. The mercury is partly removed in the purification step. A further decrease may be caused by adsorption on the gastubes during transport. The concentration in the gas, as received in Petten, was found to be $(7.0 \pm 0.5) \mathrm{ng} / \mathrm{m}^{3}$.

\section{Offgas From the Mud Fermentation Reservoir of a Sewage Plant}

The methane gas evolving from a mud-cleaning installation may contain considerable amounts of mercury due to reducing conditions existing in the fermentation tank and the methylation of mercury by bacteria. Values found during a fermentation at $32{ }^{\circ} \mathrm{C}$ were:

$0.060 \mathrm{~m}^{3}$ sample : $158 \mathrm{ng} / \mathrm{m}^{3}$ (S.D.: $12 \%$ ).

$0.330 \mathrm{~m}^{3}$ sample : $139 \mathrm{ng} / \mathrm{m}^{3}$ (S.D.: $4 \%$ ). 


\section{References}

[1] Piro, A., Physicochemical States of Some Trace Elements in Seawater, Report $R T / P R O T$, p. 10 (1972).

[2] Brinkman, F. J. J., Water, 20, 510 (1973).

[3] Coyne, R. V. and Collins, J. A., Anal. Chem. 44, 1093-1096 (1972).

[4] Robertson, D. E., Anal. Chim. Acta 42, 533-536 (1968).

[5] Rosain, R. M. and Wai, C. M., Anal. Chim. Acta 65, 279-284 (1973).

[6] Moffitt, A. E. and Kupel, R. E., Amer. Ind. Hyg. Ass. J., 614-620 (1971).

[7] Gilbert, T. R. and Hume, D. N., Anal. Chim. Acta 65, 461-465 (1973).

[8] Cranston, R. E. and Buckley, D. E., Environ. Sci. Technol. 6, 274-278 (1971).

[9] Olafsson, J., Anal. Chim. Acta 68, 207-211 (1974).

[10] Robertson, D. E., Anal. Chem. 40, 1067-1072 (1968).

[11] Byrne, A. R., Dermelj, M., and Kosta, L., J. Radioanal. Chem. 6, 325-330 (1970).

[12] Kimura, Y. and Miller, V. L., Anal. Chim. Acta 27,325-331 (1962).

[13] Iskander, I. K., Syers, J. K., Jacobs, L. W., Keeney, D. R., and Gilmour, J. I., Analyst 997, 388-393 (1972).

[14] Kosta, L. and Byrne, A. R., N.I.J.S.-Report R-554 (1968).

[15] Achterberg, A. and Zaanen, J. J., Chem. Weekblad 668 (02), 9 (1972). 



\title{
CONTROL OF BLANKS IN THE ANALYSIS OF PHTHALATES IN AIR AND OCEAN BIOTA SAMPLES
}

\author{
C. S. Giam and H. S. Chan \\ Department of Chemistry \\ Texas A\&M University \\ College Station, Texas 77840 U.S.A.
}

\begin{abstract}
For the trace characterization of phthalates, gas chromatographs equipped with electron-capture detectors have been found to be more sensitive than those with flame-ionization detectors in the order of about $10^{2}$. But the response (measured by peak height) of phthalates to electron-capture detectors are in the order of $10^{2}$ less sensitive than that of chlorinated hydrocarbons (DDT, DDE, PCBs, etc.). The response of phthalates to electron-capture detectors is not constant and normally as little as 0.5 nanogram of di-2-ethylhexyl phthalate (DEHP) can be detected.

The following factors limit the detection of phthalates at ultra-low levels (parts per billion to parts per trillion in biota samples) with acceptable accuracy: 1) contamination from solvents, reagents and materials used in the analysis; 2) limitation from the size of the samples; and 3) polychlorinated biphenyls (PCBs) and DEHP contamination in the laboratory and their strong adsorption to glass surfaces.

This paper will describe the problem of background contanination from various sources - reagents, solvents and materials used in the laboratory - the procedure for the removal of these contaminants and the methods for the isolation and separation of phthalates in air and open-ocean biota samples.
\end{abstract}

Keywords: Air analysis; analytical blank; gas chromatography; ocean biota analysis; phthalate trace analysis.

\section{Introduction}

Phthalic acid esters have been used in industry as plasticizers for the past 40 years. The production has increased from approximately $2 \times 10^{8}$ $\mathrm{kg}\left(400\right.$ million lb) in 1961 to $5 \times 10^{8} \mathrm{~kg}$ ( 1 billion lb) in 1971 . The major ester produced is di-2-ethylhexyl phthalate (DEHP) which accounted for about one fourth of the total production. The total production of phtha- 
lates is about 10 times larger than that of the polychiorinated biphenyls (PCBs) which are recognized as worldwide environmental pollutants.

There were laboratory studies on the toxicity of the phthalates [1-3], and evidence that phthalates were present in water [4], food [5], and biological samples [6-8]. Contamination from solvents, reagents, and equipment has been a major problem in phthalate residue analysis [9], especially in the parts per billion ( $\mathrm{ppb}$ ) range. The problem of background contamination is more serious in the trace analyses of phthalates because they are present in almost all equipment and reagents in the laboratory. Even samples of nonplastic materials like cork, glasswool, Teflon sheets and aluminum foil have been found to contain $600,1000,400$ and $300 \mathrm{ppb}$ of DEHP, respectively. Background levels of DEHP and dibutyl phthalate as high as 1500 and $1000 \mathrm{ng}$, respectively, have been reported in spite of rigorous purification [7]. During our investigation of DDT and its metabolites and PCBs in open ocean biota [10-13], we have achieved very low background levels of contaminants. However, even these procedures did not produce a background level satisfactory for the analysis of phthalates. This paper describes the method for the determination of phthalates at ppb levels in marine biota. The method also permits the quantitation of DDTs and PCBs.

A method for detecting specific phthalates in air has not been previously established. Mieure and Dietrich used 5 percent Dexsil 300 on 80100 mesh H.P. Chromosorb W to determine trace organics in air, but the particular phthalate esters present could not be conclusively identified because of GC interference from some of the hydrocarbons present [14]. A simple technique for the sampling, isolation and measurement of PCBs and specific phthalates at low levels $\left(\mathrm{ng} / \mathrm{m}^{3}\right)$ in air has been developed.

\section{Experimental}

\section{A. Gas Chromatography}

Condition 1: A Tracor Model MT-220, nickel-63 (10 mCi) electroncapture detector in a d.c. mode, equipped with a $2 \mathrm{~m}(6 \mathrm{ft}) \times 6 \mathrm{~mm}(1 / 4 \mathrm{in})$ o.d. borosilicate column packed with 3 percent SE-30 on Gas Chrom Q (100-120 mesh). The injector, detector and column temperatures were 250,275 , and $220^{\circ} \mathrm{C}$, respectively. Nitrogen was used as the carrier gas at a flow rate of $60 \mathrm{~cm}^{3} /$ minute. 
Condition 2: A Barber-Colman Model 5360, tritium $(300 \mathrm{mCi})$ electron-capture detector in a pulse mode, equipped with a $2 \mathrm{~m}(6 \mathrm{ft}) \times 6 \mathrm{~mm}$ (1/4 in) o.d. borosilicate column packed with 1.5 percent SP-2250 and 1.95 percent SP-2401 on Supelcon AW-DMCS (100-120 mesh). The injector, detector, and column temperatures were 210,210 , and $195{ }^{\circ} \mathrm{C}$, respectively.

The operating sensitivity for both of the detectors was about $5 \mathrm{ng}$. DEHP giving 50 percent full scale deflection (f.s.d.).

\section{B. REAgents}

Organic solvents are used in the extraction, partition and cleanup processes. High purity solvents are commercially available; solvents used in our laboratory were purchased from Mallinckrodt Chemical Company. Normally, except for diethyl ether, these solvents are sufficiently pure for use without further purification. Only freshly distilled diethyl ether should be used because contamination occurs during storage. Water is purified by liquid-liquid extraction in a 15-liter extractor with petroleum ether as the extracting solvent. All solvents should be checked for contamination prior to use.

Sodium sulfate, sodium chloride and florisil contain about $2 \mathrm{ppb}, 1$ ppb, and $0.05 \mathrm{ppb}$ of DEHP, respectively. They are heated and stored in a $320^{\circ} \mathrm{C}$ oven before use.

\section{EquipMEnT}

The equipment for analysis includes glassware, porcelain ware, filter paper, blender, spatula, forceps, dissecting knife and Teflon stopcocks. Contamination from glassware is one of the major causes of high background levels because the large amount of glassware being used provides a large surface area for adsorption of contaminants. The following procedure describes the decontamination of the equipment used in our analytical procedure.

All equipment is first washed with detergent (Cris-Clene chlorinated, machine dish-washing detergent, Gem Product Company) and laboratory distilled water. Tap water should be checked for its DEHP level before it is used to wash equipment. The tap water in our laboratory was found to contain $1.5 \mathrm{ppb}$ of DEHP. The level of DEHP is less than $0.1 \mathrm{ppb}$ in our laboratory distilled water and it is used to rinse the equipment. After rinsing the equipment with water, it is rinsed with $3 \times 100 \mathrm{~m}$ l distilled acetone (certified A.C.S. grade) and once with $100 \mathrm{ml}$ Nanograde 
acetone. The acetone for rinsing is distilled over potassium permanganate through a $1.6 \mathrm{~m}(5 \mathrm{ft})$ column. Teflon stopcocks which cannot be heated are wrapped with heat-treated aluminum foil after the final rinse. All equipment is heated in the oven for about 10 hours before use. The equipment from the oven is covered with purified aluminum foil while cooling. The covering with aluminum foil prevents the possible contamination from the dust particles in the air. We have found the level of PCBs (Aroclor-1260) in our laboratory is about $40 \mathrm{ng} / \mathrm{m}^{3}$ and DEHP is about 35 $\mathrm{ng} / \mathrm{m}^{3}$. The level is higher in the hood, about $90 \mathrm{ng} / \mathrm{m}^{3}$ for PCBs and 70 $\mathrm{ng} / \mathrm{m}^{3}$ for DEHP. When the equipment has cooled to room temperature, it is rinsed with $2 \times 100 \mathrm{ml}$ Nanograde acetone and once with $150 \mathrm{ml}$ Nanograde petroleum ether. The petroleum ether washing is concentrated to about $0.5 \mathrm{ml}$ and checked with the gas chromatograph for contaminants. If the background level of DEHP is greater than $100 \mathrm{ng}$, the rinsing process is repeated. The number of rinses needed depends on the previous history of the equipment and the length of time in the oven. Aluminum foil and Mason jars are used for storing samples. Aluminum foil is used as the cap liners for the Mason jars and also for wrapping whole fish samples. The level of DEHP in a piece of aluminum foil can be as high as 320 $\mu \mathrm{g} / \mathrm{kg}$. Aluminum foil can be cleaned by heating in a $320^{\circ} \mathrm{C}$ oven. Mason jars are used to store tissue, plankton and sediment samples; they are cleaned as other glassware.

For homogenization or maceration a Virtis blender is preferred because it is more convenient to clean than Waring blenders. The various parts of the Virtis blender - the container, the shaft, and the blades - can be cleaned by heating in a $320^{\circ} \mathrm{C}$ oven. Also cross-contamination between samples can be avoided since Mason jars can be used as the blender jars.

Contamination from filter paper has been a major problem [9]. The complete removal of these contaminants is difficult even by Soxhlet extraction. Whatman GF/A glass fibre filter paper is preferred since it can be cleaned just by heating in the oven. In order to avoid contamination from rubber stoppers, a $5 \mathrm{~cm} \times 5 \mathrm{~cm}$ Teflon sheet $(2 \times 2$ in) with a $2 \mathrm{~cm}$ diameter hole $(3 / 4 \mathrm{in})$ in the center is used for the Buchner funnel in the filtration process. The Teflon sheet can be cleaned by washing with acetone and heated in a $200{ }^{\circ} \mathrm{C}$ oven.

\section{Procedure for Analysis of Biota Samples}

The analytical scheme for the analysis of biota samples is shown in figure 1. Samples are kept at or below $0{ }^{\circ} \mathrm{C}$ until they are ready for analysis. If possible, the skin or shells of biota samples are dissected off and 


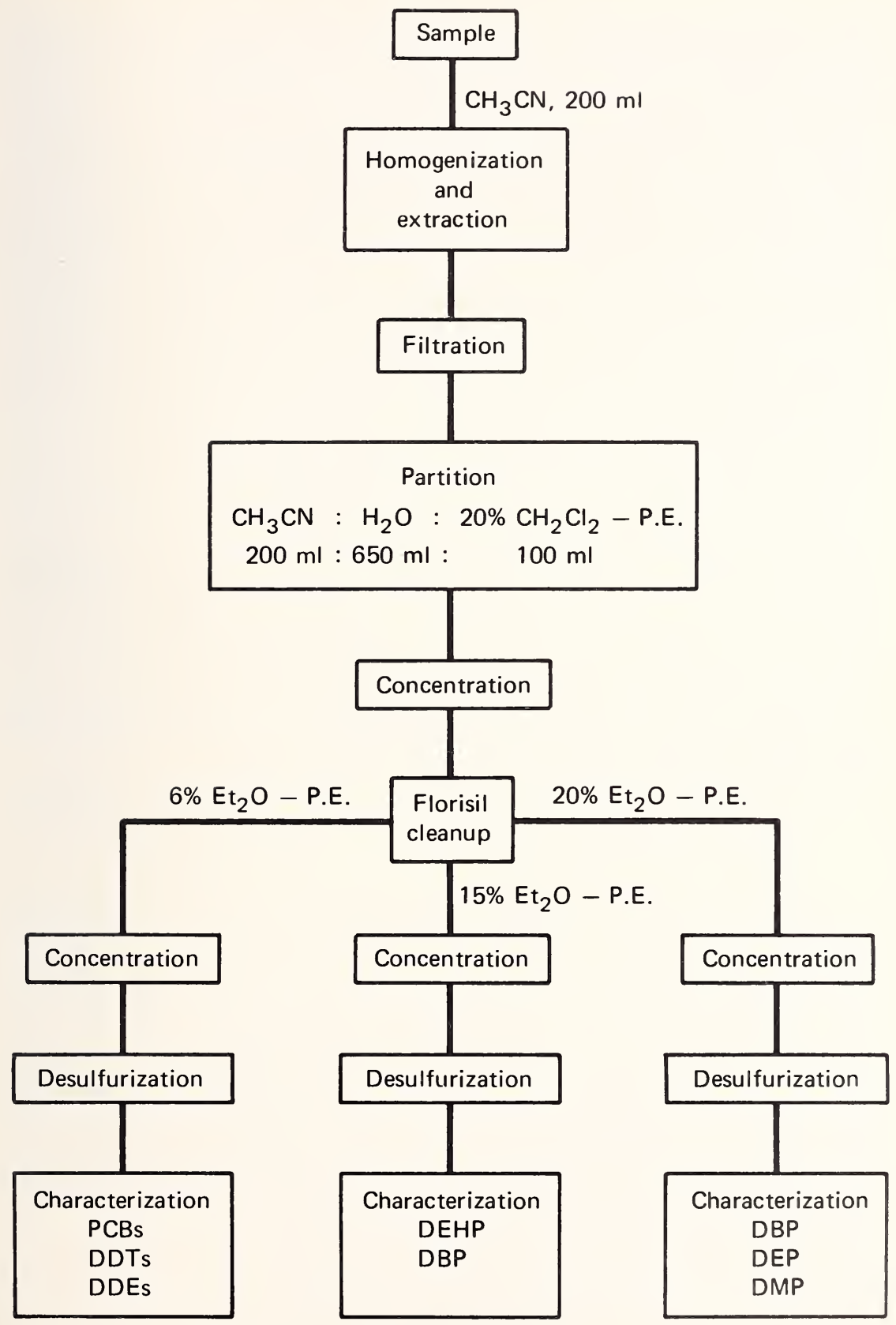

Figure 1. Analytical scheme for the analysis of biota samples. 
only the tissues are used (about 50 to $100 \mathrm{~g}$ for muscle samples and less than $30 \mathrm{~g}$ for liver samples). The tissue sample is put in a weighed blender jar or Mason jar. Nanograde acetonitrile $(100 \mathrm{ml})$ is added to the container and the tissue is macerated for at least 2 minutes. This acetonitrile extract is filtered and another $100 \mathrm{ml}$ of acetonitrile is added to the tissue. It is again blended for 2 minutes, filtered, and then combined with the previous extract in a 1-liter separatory funnel. One hundred $\mathrm{ml}$ methylene chloride-petroleum ether $(1: 5)$ is added to the separatory funnel followed by $650 \mathrm{ml}$ of purified salt water ( 5 percent sodium chloride). After partition, the organic layer is dried by sodium sulfate in a $100 \mathrm{ml}$ stoppered cylinder. Then the extract is transferred to a Kuderna-Danish evaporative concentrator. About $10 \mathrm{ml}$ of isooctane is used to rinse the sodium sulfate and combined with the extract. Then the solution is evaporated to less than $10 \mathrm{ml}$ for the Florisil cleanup. For the cleanup procedure, a $2.2 \mathrm{~cm}$ i.d. glass column is charged with $30 \mathrm{~g}$ of Florisil which was deactivated with 3 percent water (by weight) after heating in a $320^{\circ} \mathrm{C}$ oven for at least 10 hours. About a $2.5 \mathrm{~cm}$ layer ( $1 \mathrm{in}$ ) of sodium sulfate is put on the top of the Florisil. The concentrate is introduced onto the column and eluted with $200 \mathrm{ml}$ of 6,15 , and 20 percent of diethyl ether in petroleum ether, respectively. Each fraction is concentrated to about $5 \mathrm{ml}$ and analysed for chlorinated hydrocarbons and phthalates. Further concentration is done by blowing a gentle stream of nitrogen, purified by passing through a gas drier with molecular sieve pellets $5 \mathrm{~A}$, over the sample.

\section{E. Procedure for Analysis of Air Samples}

A Pasteur disposable capillary pipette or a conventional GC column (6 $\mathrm{mm}$ o.d.) is used as a collection column. Each column is packed with 0.3 $\mathrm{g}$ deactivated Florisil (3\% water $\mathrm{W} / \mathrm{W}$ ) followed by $1 \mathrm{~g}$ granular anhydrous sodium sulfate and a small plug of glass wool. The column is connected to an oil-less vacuum pump by Teflon tubing. The flow rate is about 2 to 3 liters/minute with the free air capacity of the pump about 100 liter/minute. The collected sample is eluted with $4 \mathrm{ml}$ of petroleum ether followed by $4 \mathrm{ml}$ of diethyl ether. The two fractions are collected in graduated centrifuge tubes, concentrated or diluted and analyzed for chlorinated hydrocarbons and phthalates.

\section{Results and Discussions}

Phthalates and peaks that interfere with the identification of phthalates have been found in aluminum foil, glass wool, Florisil, cork, filter papers, 
prepurified nitrogen, sodium chloride, sodium sulfate, tap water, equipment, air in the laboratory and all types of plastic materials; thus, background levels of phthalates in these materials should be checked. The complete removal of phthalates from the background is difficult and time consuming. The type and the level of contaminants depends on the history of the materials and equipment; thus, special sets of equipment should be set aside for trace analysis exclusively. To minimize cleaning effort, use equipment and materials that can be washed with organic solvents and heated in a high temperature oven (over $300{ }^{\circ} \mathrm{C}$ ). All equipment and materials should be kept in the oven until use. Organic solvents and gases should be purified and checked before use. The average background level of the analytical procedure for biota samples is about $10 \mathrm{ng}$ for PCBs, 0.5 $\mathrm{ng}$ for $\mathrm{p}, \mathrm{p}^{\prime}$-DDT, $0.5 \mathrm{ng}$ for $\mathrm{p}, \mathrm{p}^{\prime}$-DDE, $10 \mathrm{ng}$ for DMP, $10 \mathrm{ng}$ for DEP, $100 \mathrm{ng}$ for DBP and $200 \mathrm{ng}$ for DEHP. These background levels for the chlorinated hydrocarbons are about 5 times less and for DEHP and DBP about 10 times less than those reported in the literature [7,9]. The background level of the analytical procedure for air is about $1 \mathrm{ng}$ for DEHP, $0.1 \mathrm{ng}$ for PCBs and $0.1 \mathrm{ng}$ for p, $\mathrm{p}^{\prime}$-DDT.

\section{Acknowledgement}

Financial support for this study was provided by NSF Grant No. GX37349.

\section{References}

[1] Sanders, H. O., Mayer, F. L., Jr., and Walsh, D. F., Toxicity, Residue Dynamics, and Reproductive Effects of Phthalate Esters in Aquatic Invertebrated, Environ. Res. 6, 84-90 (1973).

[2] Mayer, F. L., Jr. and Sanders, H. O., Toxicology of Phthalic Acid Esters in Aquatic Organisms, Environmental Health Perspectives 3, 3-26 (1973).

[3] Autian, J., Toxicity and Health Threats of Phthalate Esters: Review of the Literature, Environmental Health Perspectives 3, 3-26 (1973).

[4] Hites, R. A., Analysis of Trace Organic Compounds in New England Rivers, $J$. Chromatog. Sci. 11, 570-574 (1973).

[5] Cerbulis, J. and Ard, J. S., Methods for Isolation and Detection of Dioctyl Phthalate from Milk Lipids, J. Ass. Off. Anal. Chem. 50, 646-650 (1967).

[6] Mayer, F. L., Jr., Stalling, D. L., and Johnson, J. L., Phthalate Esters as Environmental Contaminants, Nature 238, $411-413$ (1972). 
[7] Williams, D. T., Dibutyl- and Di-(2-ethylhexyl)Phthalate in Fish, J. Agr. Food Chem. 21, 1128-1129 (1973).

[8] Zitko, V., Determination of Phthalates in Biological Samples, J. Environ. Anal. Chem. 2, 241-252 (1973).

[9] Giam, C. S. and Wong, M. K., Problem of Background Contamination in the Analy. sis of Open Ocean Biota for Chlorinated Hydrocarbons, J. Chromatogr. 72, 283-292 (1972).

[10] Giam, C. S., Richardson, R. L., Wong, M. K., and Sackett, W. M., Polychlorinated Biphenyls in Antarctic Biota, Antarctic Journal of the United States 8, 303-305 (1973).

[11] Giam, C. S., Richardson, R. L., Taylor, D., and Wong, M. K., DDT, DDE and PCBs in the Tissues of Reef Dwelling Groupers (Serranidae) in the Gulf of Mexico and the Grand Bahamas, Bull. Environ. Contam. Toxicol. 11, 189-192 (1974).

[12] Giam, C. S., Wong, M. K., Hanks. A. R., Sackett, W. M., and Richardson, R. L., Chlorinated Hydrocarbons in Plankton From the Gulf of Mexico and Northern Caribbean, Bull. Environ. Contam. Toxicol. 9, 376-381 (1973).

[13] Giam, C. S., Hanks, A. R., Richardson, R. L., Sackett, W. M., and Wong, M. K., DDT, DDE, and Polychlorinated Biphenyls in Biota From the Gulf of Mexico and Caribbean Sea-1971, Pestic. Monit. J. 6, 139-143 (1972).

[14] Mieure, J. P. and Dietrich, M. W., Determination of Trace Organics in Air and Water, J. Chromatog. Sci. 11, 559-570 (1973). 


\title{
MASS SPECTROMETRIC ANALYSIS FOR FRACTIONAL PPB ATOMIC CONTENT OF BORON IN TRICHLOROSILANE
}

\author{
John A. Baker \\ Dow Corning Corporation \\ Semiconductor Products Division \\ Solid State Research \& Development \\ Hemlock, Michigan 48626 U.S.A.
}

$\mathrm{HSiCl}_{3}$ is used to make semiconductor grade silicon. This silicon must have a boron content in the low ppb atomic range as measured by resistivity analysis; thus, purity of the raw material $\mathrm{HSiCl}_{3}$ must be known in advance. A mass spectrometric technique was developed to analyze quantitatively the boron content in the $\mathrm{HSiCl}_{3}$ relative to the observed boron deposited in the silicon.

A fixed volume of $\mathrm{HSiCl}_{3}$ was evaporated and hydrolyzed onto a cleaned silicon electrode; the electrode surface area was approximately 20 square $\mathrm{mm}$. A point silicon counter electrode was scanned over the surface of the coated large area electrode to spark the "deposited" residue. To obtain a limit of detection of $3 \mathrm{ppb}$ atomic in the bulk analysis of a solid it is necessary to spark and produce a 300 nanocoulomb exposure on the analyzing photoplate. The evaporation and hydrolysis of $\mathrm{HSiCl}_{3}$ produces a concentration factor as later determined of approximately 1000; thus, a surface exposure of 3 nanocoulomb will give a limit of detection of $0.3 \mathrm{ppb}$ atomic. Experiments were performed on 8 samples covering a wide range of boron content and 16 samples at or near the limit of detection. Mass spectrometric analysis of each $\mathrm{HSiCl}_{3}$ sample was performed and silicon poly rods were grown from each $\mathrm{HSiCl}_{3}$ sample. The poly rods were float zoned 1 and 10 pass to determine by resistivity the boron and donor concentrations in the poly. A calibration curve was then constructed comparing the mass spectrometric boron concentration and the boron as determined by resistivity. The concentration factor of approximately 1000 was thus obtained for the mass spectrometric analysis of boron in $\mathrm{HSiCl}_{3}$. The observed boron content thus refers to the boron deposited in polycrystalline silicon by the $\mathrm{HSiCl}_{3}$ and not the boron content in $\mathrm{HSiCl}_{3}$. This distinction is necessary; to demonstrate that one analytical technique is producing correct results it is necessary to calibrate it against a known standard technique. The measurement of boron in silicon by resistivity is an accurate technique with a low limit of detection and has been well validated. Thus, an analytical technique has been developed to measure impurity concentrations (in this case boron in $\mathrm{HSiCl}_{3}$ ) in the fractional ppb atomic range in liquids with great accuracy. 
Keywords: Boron measurements; mass spectrometry; semiconductor grade silicon; trichlorosilane.

\section{Introduction}

$\mathrm{HSiCl}_{3}$ is used to make semiconductor grade silicon. This silicon must have a boron content in the low part per billion atomic ( $\mathrm{ppb}$ atomic) range as measured by resistivity analysis; thus, purity of the raw material $\mathrm{HSiCl}_{3}$ must be known in advance. A mass spectrometric technique was developed to analyze quantitatively the boron content in the $\mathrm{HSiCl}_{3}$ relative to the observed boron deposited in the silicon. This technique consists of analyzing the residue of $\mathrm{HSiCl}_{3}$ on a silicon electrode caused by evaporation and hydrolysis.

\section{Standard}

A standard must exist for comparison and reference purposes for any new analytical technique. It is normally impossible to obtain reliable standards directly in the ppb atomic region; resistivity measurements on silicon provide one special case in which fractional ppb atomic measurements have been made for many years. The group $3 \mathrm{~A}$ and group $5 \mathrm{~A}$ elements of the periodic table produce electrical carriers in silicon in direct proportion to their concentration. It is possible to remove all the $3 \mathrm{~A}$ and $5 \mathrm{~A}$ elements, with the exception of boron, from the silicon by vacuum float zoning. The distribution coefficient for boron is 0.9 , so after 10 passes in a zoner, 94 percent of the original boron remains; the distribution coefficient for the other elements is sufficiently different from unity, so zero percent of them remain after 10 passes. We, thus, can uniquely identify boron in silicon and measure its concentration accurately by electrical measurement techniques.

\section{Solids Spark Source Mass Spectrometer}

This mass spectrometer normally is used to analyze for impurities in solid materials with a limit of detection of $3 \mathrm{ppb}$ atomic. The host material to be analyzed is prepared in the form of electrodes approximately $1 \mathrm{~mm}$ $\times 2 \mathrm{~mm} \times 7 \mathrm{~mm}$. Two electrodes are placed in a vacuum chamber; a spark 
discharge of $20 \mathrm{kV}$ takes place across the electrode gap at a frequency rate of 3000 pulses per second and a pulse length of 50 microseconds. This spark discharge erodes and ionizes the electrode material; the ionized host atoms with its ionized impurity atoms are swept into the magnetic analyzer and recorded on a photoplate.

When one thinks about the above operation, it is apparent to the observer that bulk analysis need not be the only use made of the instrument. The next logical step is to have one electrode be a large surface area electrode and the second electrode be a point. This second point electrode scans the surface of the flat electrode, thus, producing a surface analysis. This method is employed in the semiconductor industry to perform a layer by layer analysis of a semiconductor device.

Once you are in the frame of mind of thinking about the analysis of thin layers, it is very easy to visualize a thin layer left behind by the evaporation of a liquid. The analysis of impurities left behind in the residue of evaporation of any liquid is as easy to perform as a surface analysis of a solid; an added benefit is a concentration factor which may be obtained by evaporating a large volume of liquid on the surface electrode.

\section{Trichlorosilane Analysis}

A silicon electrode with a surface area of approximately 20 square $\mathrm{mm}$ and the point silicon electrode are placed in a Teflon watch glass and approximately 20 drops of $\mathrm{HSiCl}_{3}$ are poured onto the electrodes, few drops at a time, from a Teflon storage bottle. These drops of $\mathrm{HSiCl}_{\text {; }}$ evaporate (boiling point $31.8^{\circ} \mathrm{C}$ ) and hydrolyze rather rapidly. In the case of $\mathrm{HSiCl}_{3}$, the hydrolysis most likely serves to capture and retain in the thin film some of the more volatile boron containing molecules. The identity of these boron containing molecules can not be determined, thus, we can not discuss boiling points for them. It is assumed that the same percentage of the boron containing molecules will be retained in the thin film from one test to another. This assumption is quite reasonable in that the quality of the $\mathrm{HSiCl}_{3}$ is very consistent from one batch to another; thus, the mix of low boilers to high boilers will be constant. There is no claim that 100 percent of the boron is captured, only a constant amount need be incorporated in the thin film on the silicon electrode. Comparison of the mass spectrometric value with the standard will normalize our data.

The silicon electrodes are chosen from material which contains less than $0.1 \mathrm{ppb}$ atomic boron as determined by resistivity measurements. The normal mode of operation for the mass spectrometer is the analysis 
of bulk samples. A 300 nanocoulomb exposure on a photoplate provides a $3 \mathrm{ppb}$ atomic limit of detection for boron. Thus, we need not be concerned about contribution from the substrate. When we perform a surface analysis we scan the point electrode across the surface of the other electrode. A blue spark is produced by the chlorosilane residue; when this blue color disappears the point electrode is moved to a new position to spark a fresh sample area. This permits us to have uniform sparking conditions with a minimum of the silicon substrate being analyzed. Exposures covering the range $30,10,3,1$ nanocoulombs of charge, are possible before the surface is depleted. This surface analysis is completed with only one electrode surface. It would be possible to recoat the electrode and continue the exposure to obtain maximum exposures in the 100 nanocoulomb range and thereby increase the limit of detection. Fear of contamination resulting from opening the sample chamber repeatedly has discouraged such an approach. The limit of detection for 30 nanocoulomb exposures is $30 \mathrm{ppb}$ atomic for bulk samples. The raw data for the surface analysis is discussed in the same terms in reading the photoplate.

\section{Calibration Curve Construction}

Eight sample lots of trichlorosilane were chosen for growth of polycrystalline silicon. Evaluation of this silicon by 10 pass float zoning and resistivity analysis provided boron values in the single crystal silicon ranging from a high of $200 \mathrm{ppb}$ atomic to a low of $0.07 \mathrm{ppb}$ atomic. These lots of tric nlorosilane were analyzed on the mass spectrometer. A scale factor division by 1040 was necessary to bring the mass spectrometric raw data into correspondence with the resistivity values. This scale factor represents a concentration factor obtained by evaporation of the liquid on the silicon electrode; a comparison of the data for the eight samples is provided in table 1 .

A comment on the meaning of this calibration data is in order. We have produced a calibration curve for determination of boron from a trichlorosilane sample versus the boron content in silicon as determined by resistivity analysis of 10 pass vacuum float zoned silicon. We are not saying that this value quoted for boron is the boron content in the trichlorosilane. We are saying that this value quoted for boron is the boron which will be observed in the silicon made by decomposing trichlorosilane. In actual practice, this is the number which a production department wants to have available, it is a number which relates to their experience and is directly meaningful to them. 
Once this calibration curve was produced, a number (16) of samples were run as unknowns; these samples were chosen to represent trichlorosilane with boron content close to the limit of detection of the mass spectrometric technique. When the calibration curve was produced the limit of detection was $0.13 \mathrm{ppb}$ atomic boron in silicon. A larger surface area for the electrode was used for later samples reducing the limit of detection to $0.03 \mathrm{ppb}$ atomic boron in silicon. The results of this test of the calibration curve are displayed in table 2.

TABLE 1. Mass spectrometric boron determination of trichlorosilane versus 10-pass resistivityanalysis

\begin{tabular}{ccc}
\hline Sample & $\begin{array}{c}\text { Mass spectrometry } \\
\text { (ppb atomic) }\end{array}$ & $\begin{array}{c}\text { Resistivity } \\
\text { (ppb atomic) }\end{array}$ \\
\hline 1 & 21 & 10 \\
2 & 0.6 & 0.39 \\
3 & $<.13$ & .16 \\
4 & .3 & .25 \\
5 & $<.13$ & .17 \\
6 & 24.13 & .18 \\
7 & 238 & 200 \\
8 & & \\
\hline
\end{tabular}

TABLE 2. Comparison of boron content by mass spectrometry and resistivity analysis

\begin{tabular}{ccc}
\hline Sample & $\begin{array}{c}\text { Mass spectrometry } \\
\text { (ppb atomic) }\end{array}$ & $\begin{array}{c}\text { Resistivity } \\
\text { (ppb atomic) }\end{array}$ \\
\hline 1 & 0.11 & 0.23 \\
2 & .12 & .1 \\
3 & .1 & .08 \\
4 & .1 & .18 \\
5 & .1 & .07 \\
6 & .1 & .17 \\
7 & .24 & .25 \\
8 & .1 & .16 \\
9 & .48 & .39 \\
10 & .13 & .05 \\
11 & .10 & .16 \\
12 & .13 & .12 \\
13 & .10 & .12 \\
14 & .128 & .22 \\
15 & .05 & .1 \\
16 & .04 & \\
\hline
\end{tabular}




\section{Conclusion}

A method for analyzing for fractional ppb atomic amounts of boron for liquid samples has been developed. This method consists of evaporating 20 drops of the liquid trichlorosilane on the surface of a silicon area electrode. A solids spark source mass spectrometer is employed to study this thin layer of evaporated material and a calibration curve is produced by comparing the mass spectrometric results with resistivity analysis of silicon grown from the trichlorosilane. Verification of the calibration curve has been performed by measuring a number of unknown samples having boron contents close to the detection limit of the test method. 


\title{
THE CHALLENGE OF GETTING THE LEAD OUT
}

\author{
Eleanor Berman \\ Toxicology Section \\ Division of Biochemistry \\ Cook County Hospital \\ Chicago, Illinois 60612 U.S.A.
}

\begin{abstract}
Developments in spectroscopic instrumentation over the last decade have enabled determinations of the lead content in blood, other biological fluids, and tissues to become routine analyses in clinical laboratories serving metropolitan centers. Though not a technically difficult procedure in itself, maintaining the quality of lead analyses presents a few challenges. One must be cognizant of the pitfalls of a specific method and of the limits of its accuracy and precision. Instrumental limits, the matter of possible extraneous lead contaminants on the subject of analysis, the labware, the in-the-laboratory environment, the reagents, etc. must all be considered.

Factors influencing the integrity of lead determinations in the clinical laboratory, from sample collection to interpretations of analytical results with confidence, will be discussed.
\end{abstract}

Keywords: Atomic absorption spectrometry; blood analyses: contaminations; flameless methods; lead; lead blood analyses; methodology; trace element analyses.

\section{Introduction}

Developments in spectroscopic instrumentation over the last decade have enabled the determinations of trace metal contents in blood, other biological fluids, and tissues to become rather routine clinical laboratory analyses. Judging by the literature of the last 2 years alone, we seem to be victims of a veritable deluge of papers describing various flame emission and atomic absorption spectrophotometric methods.

Because of the long-existing problem of childhood lead poisoning, as well as the need for monitoring industrial exposures, applications of 
atomic absorption instrumentation to lead analyses have been of special interest.

\section{Discussion}

A lead analysis in itself, is not a technically difficult procedure. The ratio between concentration in blood, for example, and detection limits claimed for atomic absorption spectrometry is favorable. A normal blood lead level is about $20 \mu \mathrm{g}$ per $100 \mathrm{ml}(0.2 \mu \mathrm{g}$ per ml), while a level of $0.6 \mu \mathrm{g}$ per $\mathrm{ml}$ is considered diagnostic of lead poisoning. Detection limits for lead at the $283.3 \mathrm{~nm}$ and $217.0 \mathrm{~nm}$ resonance lines are 0.03 and $0.01 \mu \mathrm{g}$ per $\mathrm{ml}$, respectively. The former is the preferred analytical line because of greater instrumental stability at that point.

Supposedly lead is free from spectral interference at these resonance lines. However, the nonspecific molecular absorption generated by the constituents in blood, urine, and tissues affect the absorption signal from lead markedly. Chelation-extraction techniques can be utilized in both eliminating the interferences due to constituents in the biological matrix and in concentrating the lead present. The dithiocarbamate chelate of lead is quantitatively extracted by an organic solvent like methylisobutylketone (MIBK) over a 4 to $11 \mathrm{pH}$ range.

Circulating lead is loosely bound to the red blood cell membrane and can be released for chelation-extraction by either precipitation of blood proteins with trichloracetic acid [1], or more simply hemolyzing the red cells in distilled water containing a surfactant like Triton X-100 [2]. Studies indicate that about 95 percent of the lead can be recovered by chelation-extraction procedures [3]. With good analytical technique, reproducibility of replicates is $\pm 0.03 \mu \mathrm{g}$ per $\mathrm{ml}$. The absorbance signal from a given concentration of lead in MIBK is approximately four times that of a similar concentration in an aqueous solution.

Lead in urine specimens can be chelated directly, and extracted after $\mathrm{pH}$ adjustment. Tissues, hair, nails and other solid materials must be solubilized prior to analysis. Wet or dry ashing at temperatures not exceeding $300{ }^{\circ} \mathrm{C}$ is the preferred method for destroying organic matter, presently. However, alkaline solubilization of tissues in quaternary ammonium hydroxide (TMAH), described recently [4], warrants further investigation.

Flameless sampling devices including the graphite furnace, the carbon rod atomizer and the tantalum strip, extend the capabilities of an atomic absorption instrument. An entire aliquot is atomized as compared to the 
5 or 10 percent atomized by conventional flame aspirators.

Many claims to the contrary, some degree of chemical pretreatment of samples is necessary when utilizing flameless atomization. The trace elements in question are present in microgram amounts and the biological matrix can contain sodium and protein in milligram and gram quantities. These basic relationships are not altered by decreasing the sample size analyzed. Components in the blood matrix, for example, are not eliminated entirely during the drying and charring cycles, and thus produce interference peaks close to, or superimposed on the lead peak. Background correction by a deuterium continuum eliminates but a portion of the nonspecific molecular absorption generated.

Direct analysis in a flameless atomizer of untreated biological materials can at best be considered but a semi-quantitative technique.

Employing the method of standard addition with direct analysis does minimize interferences. However, there is a greater precision error associated with this procedure due in part to uneven drying and spluttering of blood in the graphite tube. The pipetting error is also greater since blood is more viscous than simple aqueous solutions and clings to the pipet tip or tubing.

The severe matrix interferences from the variable solute concentrations in urine preclude direct analysis of these samples even with the method of standard additions.

Combining chelation-extraction methodologies with flameless atomization eliminates the nonspecific molecular absorption due to biological components and also permits the use of smaller aliquots and in addition, analysis time in the instrument is decreased.

Assuming that instrumental performance and analytical technique in general are adequate, maintaining the daily quality of lead and other trace metal analyses does present a few challenges. Providing a sufficiently clean analytical environment in all steps from collecting the actual specimen to attaining the end result is the greatest challenge.

Sample collection vessels, pipettors, etc. can contain hazards for the analyst. One never assumes anything is acceptably free from trace metal contaminants until it has been tested. To cite a few examples - the $7 \mathrm{ml}$ heparinized blood collection tubes were found to be essentially lead free: however, the $1.5 \mathrm{ml}$ size from the identical manufacturer contained sufficient lead to discourage its use. None of the rubber stoppered evacuated tubes tested were found suitable for zinc analysis since sufficient zinc could be leached out through minimal contact of specimen with stopper to produce a spurious elevation of the true zinc level more than fourfold.

Polyethylene or polypropylene pipet tips available for Oxford, Eppen- 
dorf, and other samplers must be acid washed before use. All brands contain generous quantities of lead, cadmium, iron, and zinc many times greater than that present in the aliquot being analyzed. Some of the Teflon tubing used as disposable extensions for the Hamilton type syringes contain these metallic contaminants and require precleaning, also.

It is wise to ban smoking in areas where trace metals are being analyzed.

Water quality and purity of reagents are other factors to be considered.

The analyst should be especially wary of the tissue samples submitted for analysis. Points made concerning cleanliness of collection equipment also apply to tissues, of course. In addition, the composition of various fixative solutions used becomes a major consideration in interpreting findings. A sample of fixative solution should be analyzed along with the tissue in question. Ideally, tissues for trace metal analysis should be submitted in a frozen state free from fixatives.

Because lead is an ubiquitous metal, an environmental contaminant, it is conceivable that the subject presenting himself for a blood lead analysis may carry significant contaminants on his person. An interest in collecting blood samples by finger rather than venipuncture, prompted a demonstration of probable lead deposits on fingers of different subjects. Individual fingers of adults and children were scrubbed with alcohol sponges shown to be lead free. Sponges were then soaked in distilled water containing sodium diethyldithiocarbamate and Triton X-100 for a few hours. Solutions were then extracted into methylisobutyl ketone. Extracts were compared in a Perkin-Elmer Model 403, equipped with a graphite furnace and recorder, against known lead standards similarly treated.

Table 1 shows the relative amounts of soluble lead obtained from fingers of technicians who had washed their hands in the conventional manner prior to scrubbing with the alcohol sponges. Between 0.12 and 0.4 $\mu \mathrm{g}$ of lead were washed off. By comparison, washings taken from the fingers of a visitor, a known chain smoker, contained 0.64 to $1.84 \mu \mathrm{g}$ of lead.

Washings taken at random from the fingers of 20 children waiting to be seen in the Pediatric Clinic at Cook County Hospital were found to contain between 0.34 and $3.76 \mu \mathrm{g}$ of lead. Results are summarized in table 2 .

One need not belabour the fact that blood for lead determinations which was in contact with some of the fingers in question could lead to some rather "interesting" findings. A properly collected venous sample is somewhat less hazardous.

Incidentally, we are not discouraging micro sampling for blood lead analysis. We are merely pointing out another pitfall. 
TABLE 1. Lead $(\mu \mathrm{g})$ washed from technicians' clean fingers

Finger

Technician

\begin{tabular}{|c|c|}
\hline A & \\
\hline
\end{tabular}

Thumb

Index

Second

Third

Fourth

0.22

.16

.16

.12

.22

0.26

0.32

0.18

.36

.2

.16

.22

.3

.4

.24

.22

.2

.16

.22

.16

TABLE 2. Lead washed from fingers of children in pediatric clinic

Lead $(\mu \mathrm{g})$

$$
<0.5
$$

$0.5-0.99$

1.0-1.5

1. 5-2.0

2.0-3.76
Children

(per cent)

\section{Summary}

In summary, factors influencing the integrity of lead analyses performed in a clinical laboratory have been described. Limitations imposed by the analytical procedure and instrumental capabilities were considered. The challenge of maintaining a quality environment for the analysis from specimen collection to reporting of findings with confidence was stressed.

\section{References}

[1] Berman, E., Atom. Abs. Newsletter 3, 111 (1964).

[2] Hessel, D. W., Atom. Abs. Newsletter 7, 55 (1968).

[3] Kopito, L. E., Davis, M. A., and Schwachman, H., Clin. Chem. 20. 205 (1974).

[4] Kaplan, P., Blackstone, M., and Richdale, N., Arch. Environ. Health 27. 387 (1973). 



\title{
RAPID HOMOGENIZATION AND DRYING OF BIOLOGICAL MATERIAL
}

\author{
Ivan Yordanov Donev
}

\author{
Bulgarian Academy of Sciences \\ Institute for Nuclear Research \\ and Nuclear Energy \\ Sofia, Bulgaria
}

The determination of chemical trace elements in biological material presents many methodological difficulties. The most important difficulties of the sample's homogenization and enrichment are connected with the elimination of contamination with other elements or losses of the latter. Another problem is tissue lyophilization.

The paper describes the procedure for a relatively rapid drying and fine homogenization of biological materials for trace element research by means of nondestructive neutron activation analysis. Data are given on a double homogenizer and vacuum drying installation at liquid nitrogen temperatures. Wet homogenization of tissues is carried out in the inner homogenizer. This homogenizer is put into a second one, the lower part of which is utilized for fine mincing.

Each homogenizer has a capacity of up to $15 \mathrm{~cm}^{3}$. The humidity taken away during the drying is approximately 78 to 90 percent, and depends especially on the tissue state.

The procedure was applied with success on serum, blood, plasma and other biological materials.

The total installation allows the simultaneous drying of 13 homogenates. The total time for the drying and homogenization of 13 samples carried out by one trained laboratory assistant, is about 6 to 7 hours.

Keywords: Biological materials; homogenization; lyophilization; noncontaminating; sample preparation.

\section{Introduction}

All specialists well know the difficulties in the exact determination of trace elements in biological materials. A rapid and effective homogeniza- 
tion and drying at lower temperatures are desirable. In connection with the implementation of the IAEA Research Contract 1113/RB on the role of trace elements in the pathogenesis of ischemic heart diseases, and for the work of the laboratory a small installation for homogenization and drying of biological materials at liquid nitrogen temperature was constructed.

\section{Homogenizer}

A scheme of the "combined homogenizer" is given in figure 1. It really represents a combination of two homogenizers. Homogenizer 1 is intended for fine mincing of biological materials. It is fabricated of quartz glass, and the inner side of the narrow part is matted. It is connected -in its upper part - to the general holder 4, made of plexiglas. In the enlarged part of the homogenizer 1 is homogenizer 3 , the lower part of which is perforated and used for rough mechanical mincing of the biological materials. Inside the homogenizer 3 and in the narrower part of homogenizer 1

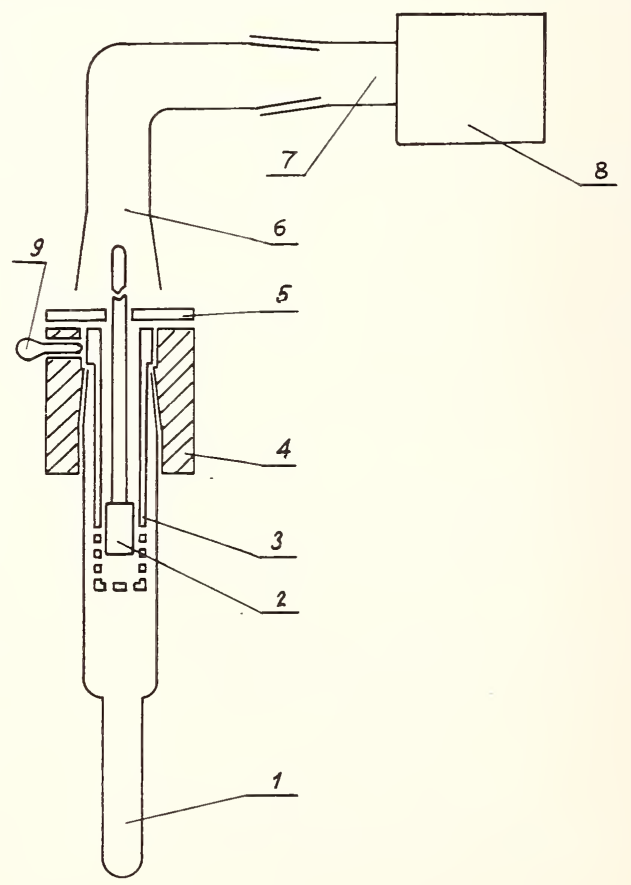

Figure 1. Schematic of "combined homogenizer" used in rapid homogenization and drying of biological materials. 
moves a Teflon piston 2, with plexiglas holder. Homogenizer 3 is tightly connected to homogenizer 1 by means of a conical fitting and fixed to the holder 4 by a screw 9 . Homogenizer 4 is fabricated of plexiglas; quartz could also be used. The combined homogenizer is protected from contamination by a Teflon ring. Tissues and liquids which are too soft, as blood, spleen, etc. are homogenized only in homogenizer 1 .

\section{Procedure}

The biomaterial, roughly cut, is put in the orifice of the homogenizer 3 and then pushed by the piston towards the perforated part. The piston is moved by a motor, the speed of which can be regulated. At its lower part, the piston has a special profile in order to trap the tissues and force them to pass through the holes of the homogenizer. The roughly minced biomaterials gather in the enlarged part of homogenizer 1 and fall into the narrower part. After the mincing of the necessary quantity, homogenizer 3 is removed by loosening the screw 9 . The same piston is used for fine mincing in the narrower matted part of homogenizer 1. After the mincing of the biomaterial to the wanted dimension, the homogenizer is weighed. The homogenate is spread out in a thin film on the inner surface of the quartz homogenizer. Immediately after this operation, the whole homogenizer, up to the conical ring, is cooled in liquid nitrogen.

The vacuum installation represents a glass ring (in fig. 1 the square 8) to which 13 sleeves (7) are soldered horizontally, and which are attached in the form of tubes. The purpose of the shape of the tube is to allow a rotary movement of the homogenizer 1 in two mutually perpendicular directions in case of unexpected thawing of the homogenates. After attachment of all homogenizers the vacuum installation is put in operation gradually. The samples are kept 30 minutes in a completely frozen state. The gradual thawing of homogenates starts soon after the evacuation of the coolers. At liquefaction of the homogenates a quick elimination of water occurs, accompanied by independent freezing, at temperatures higher than those of nitrogen. Some homogenizers, if necessary, can be frozen several times at nitrogen temperatures. After 2 hours at room temperature, the temperature will increase to $30^{\circ} \mathrm{C}$. The homogenates are dry when they come off the homogenizer walls in thin peels.

For complete drying 4 to 6 hours are necessary. The time depends on the thickness of the homogenate spread over the inner surface of the homogenizer. After the drying the ground connector is shut with a glass plug. The dry homogenization is carried out after another weighing with 
the same piston carefully cleaned. The dry homogenate can be minced to a fine powder. A laboratory assistant of average skill can do the work for 13 homogenates in 8 to 9 hours. The capacity of the homogenizer is about $1.5 \times 10^{-5} \mathrm{~m}^{3}$.

\section{Investigation and Results}

Preliminary investigations were carried out with all homogenizers on identical tissue for the determination of differences in drying. The ratio of dry weight to wet weight was chosen as the criterion. Three samples of each dry homogenate were investigated for the quantitative determination of sodium. Table 1 shows the results of water loss in 13 homogenates (in each separate homogenizer) carried out with kidney sections of a patient deceased in an accident, with healthy organs. The differences of mean values are very small, and all statistical quantities show very small values. These data prove that the ring system arrangement of the homogenizers provides equal conditions for extraction of the water, that each homogenizer is mincing the biomaterial to almost the same dimension, and that the spreading of the homogenate is uniform. The loss of water proved to be sufficient; it was established, that at prolonged irradiation with a flux of $5 \times 10^{-13} \mathrm{n} \cdot \mathrm{cm}^{-2} \cdot \mathrm{s}^{-1}$ no ruptured sample containers were found.

TABLE 1. Evaluation of dry to wet weight for thirteen kidney homogenates

\begin{tabular}{ccccc} 
Homogenates & $\begin{array}{c}\text { Dry Wt/Wet Wt } \\
(\%)\end{array}$ & $\begin{array}{c}\text { Standard } \\
\text { deviation }\end{array}$ & $\begin{array}{c}\text { Confidence } \\
\text { interval } \\
\pm\end{array}$ & $\begin{array}{c}\text { Variation } \\
\text { coefficient } \\
(\%)\end{array}$ \\
\hline \multirow{2}{*}{13} & $\begin{array}{l}\text { mean } 20.96 \\
\min 19.89\end{array}$ & 0.89 & 0.54 & 4.26 \\
& $\max 22.01$ & & &
\end{tabular}

The results of determinations of sodium in three samples of each homogenate are given in table 2. Samples (with weights close to the samples) of SRM 1577 (Bovine Liver) were used as standards. The samples of the three groups show very similar values, as seen at the last column of mean values. These data prove the accuracy and precision of the neutron activation method as well as the homogeneity obtained and the uniformity of the drying.

The feeling of exceptional precision, caused by the first two tables, disappears when we examine table 3 . However, it is evident that this mode 
of homogenization and drying allows us to work with relatively low statistical differences of mean values of the ratio dry to wet weight, not only when it concerns the kidneys but other organs also. This is proved also by the small standard deviation, the small confidence interval of the mean, and the small coefficient of variation for six organs of 29 cases of healthy

TABLE 2. Sodium concentrations in thirteen homogenates from the kidneys of a patient dying accidently (in triplicate) ${ }^{\mathrm{a}}$

\begin{tabular}{|c|c|c|c|c|c|}
\hline \multirow{2}{*}{ Homogenizer } & \multirow{2}{*}{$\begin{array}{c}\text { Weight } \\
\text { dry/wet }(\%)\end{array}$} & \multicolumn{3}{|c|}{ Sodium concentration $\mu \mathrm{g} / \mathrm{g}$} & \multirow{2}{*}{$\begin{array}{l}\text { Dry weight } / \text { mea } \\
(I+I I+I I I) /\end{array}$} \\
\hline & & I & II & III & \\
\hline 1 & 20.89 & 2184 & 2170 & 2169 & 2174 \\
\hline 2 & 21.42 & 2204 & 2189 & 2174 & 2189 \\
\hline 3 & 20.33 & 2099 & 2083 & 2173 & 2120 \\
\hline 4 & 20.98 & 2115 & 2149 & 2177 & 2147 \\
\hline 5 & 21.97 & 2190 & 2111 & 2121 & 2141 \\
\hline 6 & 19.89 & 2089 & 2174 & 2115 & 2129 \\
\hline 7 & 22.01 & 2201 & 2144 & 2. 171 & 2173 \\
\hline 8 & 21.73 & 2098 & 2145 & 2177 & 2140 \\
\hline 9 & 20.49 & 2133 & 2103 & 2200 & 2154 \\
\hline 10 & 20.84 & 2169 & 2171 & 2099 & 2146 \\
\hline 11 & 21.08 & 2201 & 2188 & 2102 & 2164 \\
\hline 12 & 21.77 & 2157 & 2117 & 2191 & 2155 \\
\hline 13 & 20.14 & 2139 & 2144 & 2185 & 2156 \\
\hline $\bar{X}$ & 20.96 & 2152 & 2146 & 2158 & 2152 \\
\hline $\mathrm{SD} \pm$ & 0.89 & 70 & 33 & 35 & 19 \\
\hline $\mathrm{SL} \pm$ & 0.54 & 43 & 20 & 21 & 11 \\
\hline $\mathrm{CV} \%$ & 4.26 & 1.5 & 3.3 & 1.6 & 0.9 \\
\hline
\end{tabular}

${ }^{\text {a }} \bar{X}=$ mean values

$\mathrm{SD}=$ standard deviation

$\mathrm{SL}=$ confidence interval

$\mathrm{CV}=$ coefficient of variation

TABLE 3. Mean ratio between dry and wet weight in six organs of 29 control cases

\begin{tabular}{lcccc}
\hline \multicolumn{1}{c}{ Organ } & $\begin{array}{c}\text { dry weight } \\
\text { wet weight } \\
\%\end{array}$ & SD & SL & CV \\
& \multicolumn{1}{c}{ \pm} & \pm & $\%$ \\
\hline Heart & 23.02 & 1.73 & 0.66 & 7.52 \\
Lung & 20.69 & 2.29 & .87 & 11.07 \\
Liver & 27.04 & 1.75 & .68 & 6.47 \\
Kidneys c. & 21.05 & 1.99 & .76 & 9.45 \\
Kidneys m. & 20.74 & 2.01 & .77 & 9.69 \\
Spleen & 23.12 & 2.44 & .93 & 10.55 \\
\hline
\end{tabular}


TABLE 4. Sodium concentrations in six organs of controls and patients dying from ischemic heart diseases

\begin{tabular}{|c|c|c|c|c|c|c|}
\hline Organ & Case & Number & $\begin{array}{l}\text { Sodium, dry } \\
\text { weight } \mu \mathrm{g} / \mathrm{g}\end{array}$ & $\begin{array}{l}\text { SD } \\
\pm\end{array}$ & $\begin{array}{l}\text { SL } \\
\pm\end{array}$ & $\begin{array}{l}\mathrm{CV} \\
\%\end{array}$ \\
\hline Heart & $\begin{array}{l}\text { control } \\
\text { patient }\end{array}$ & $\begin{array}{l}28 \\
37\end{array}$ & $\begin{array}{ll}1 & 024 \\
1 & 229\end{array}$ & $\begin{array}{l}160 \\
468\end{array}$ & $\begin{array}{r}62 \\
157\end{array}$ & $\begin{array}{l}15.6 \\
38.0\end{array}$ \\
\hline Lung & $\begin{array}{l}\text { control } \\
\text { patient }\end{array}$ & $\begin{array}{l}29 \\
31\end{array}$ & $\begin{array}{ll}1 & 936 \\
2 & 068\end{array}$ & $\begin{array}{l}656 \\
535\end{array}$ & $\begin{array}{l}250 \\
196\end{array}$ & $\begin{array}{l}33.9 \\
25.9\end{array}$ \\
\hline Liver & $\begin{array}{l}\text { control } \\
\text { patient }\end{array}$ & $\begin{array}{l}24 \\
37\end{array}$ & $\begin{array}{r}958 \\
1468\end{array}$ & $\begin{array}{l}305 \\
552\end{array}$ & $\begin{array}{l}129 \\
184\end{array}$ & $\begin{array}{l}31.9 \\
37.6\end{array}$ \\
\hline $\begin{array}{l}\text { Kidneys } \\
\text { c. }\end{array}$ & $\begin{array}{l}\text { control } \\
\text { patient }\end{array}$ & $\begin{array}{l}29 \\
37\end{array}$ & $\begin{array}{ll}1 & 990 \\
2 & 255\end{array}$ & $\begin{array}{l}496 \\
725\end{array}$ & $\begin{array}{l}186 \\
241\end{array}$ & $\begin{array}{l}24.9 \\
32.1\end{array}$ \\
\hline $\begin{array}{l}\text { Kidneys } \\
\text { m. }\end{array}$ & $\begin{array}{l}\text { control } \\
\text { patient }\end{array}$ & $\begin{array}{l}29 \\
35\end{array}$ & $\begin{array}{ll}2 & 194 \\
2 & 383\end{array}$ & $\begin{array}{l}580 \\
763\end{array}$ & $\begin{array}{l}221 \\
261\end{array}$ & $\begin{array}{l}26.4 \\
32.0\end{array}$ \\
\hline Spleen & $\begin{array}{l}\text { control } \\
\text { patient }\end{array}$ & $\begin{array}{l}29 \\
37\end{array}$ & $\begin{array}{r}890 \\
11125\end{array}$ & $\begin{array}{l}217 \\
376\end{array}$ & $\begin{array}{r}83 \\
123\end{array}$ & $\begin{array}{l}24.4 \\
33.4\end{array}$ \\
\hline
\end{tabular}

subjects, furthermore, from different sexes and different ages over 40 years. These cases are controls for investigations carried out in connection with the programme WHO/IAEA.

Table 4 shows not yet published differences of sodium concentrations in six organs of controls and patients dying of ischemic heart diseases. It is interesting to note the higher mean values of heart, liver and spleen in patients when compared to those of controls. It is also easy to see the differences in the standard deviations of almost all patient organs. The highest differences in the confidence intervals involve the heart and spleen of patients when compared to those of controls. It is the same with the coefficient of variation.

As a rule, for well known reasons, the standard deviation and the confidence interval of the investigated cases-patients and healthy subjects - are considerably greater than those given for a single organ; sex differences, age differences, and some of the controls were not compared to the corresponding group of patients, but put them into the general list of controls, without taking into consideration the important changes in the water-cell metabolism and the arteriosclerosis. Independently of this, the differences are significant and statistically reliable showing that sodium, parallel with the other elements, has not yet lost its diagnostic significance. 


\title{
PROBLEMS IN TRACE ANALYSIS OF NITROSAMINES IN FOODSTUFFS
}

\section{E. A. Walker and M. Castegnaro}

Unit of Environmental Carcinogens

International Agency for Research on Cancer 150 cours Albert-Thomas, 69008 - Lyon, France

\begin{abstract}
Methods in general use are briefly reviewed. Epidemiological research in the region of the Caspian Littoral has demonstrated certain trends in the distribution of incidence of oesophageal cancer. Samples of typical foodstuffs from both high and low incidence areas have been analyzed for traces of $\mathrm{N}$ nitrosamines. After extraction from the food, the nitrosamines were analyzed by oxidation to nitramines which were then separated by adsorption chromatography and determined by gas chromatography, using an electron capture detector. Initial results suggested a correlation between levels of nitrosodimethylamine and oesophageal cancer incidence. However, the presence of nitrosamines could not be confirmed by mass spectrometry. As a result, the clean-up procedure was modified by inclusion of a further adsorption chromatography stage when some interfering material was eliminated and all levels of nitrosamine were reduced below the $1 \mu \mathrm{g} / \mathrm{kg}$ level. The work demonstrates that even for screening analysis, clean-up procedures must be rigorous when carrying out trace analysis to avoid misleading results.
\end{abstract}

Keywords: Carcinogens; food; nitrosamines; trace analysis.

Since Magee and Barnes in 1956 discovered that nitrosodimethylamine was carcinogenic, $\mathrm{N}$-nitroso compounds have been the subject of considerable attention. Some 100 compounds have now been tested for carcinogenicity, about 75 percent of which are carcinogenic, several in all species of animals tested. As many $\mathrm{N}$-nitroso compounds are also organspecific, this adds considerably to their potential importance.

The ready formation of nitrosamines from secondary amines and nitrite under acidic conditions raises the question of whether such compounds can be found in food or be formed in vivo. In 1969 the International Agency for Research on Cancer called the first of a series of biennial meetings of expert toxicologists, biologists and analytical chemists to collaborate on the question of their analysis. At the 1971 meeting in Heidelberg, a re- 
port of which has been published, a collaborative study was undertaken [1] with results reported at the 1973 meeting held in Lyon [2]. That study was not a true collaboration, but a ring study as the state of the art was still such that workers had confidence only in their own methods and experience. The ring study did, however, demonstrate that several methods were applicable to the analysis of canned cooked meat which had been spiked at the $20 \mu \mathrm{g} / \mathrm{kg}$ level. Table 1 shows a statistical evaluation of the results obtained by 11 different laboratories. The methods used employed a variety of procedures. For example, extraction was carried out either by:

a. digestion in methanolic potash followed by continuous liquid/liquid extraction with methylene chloride,

b. steam distillation from acid and alkali, or

c. cold extraction by macerating with methylene chloride, centrifuging, and washing with acid and alkalies.

In several cases a mixture of these procedures was employed. Further clean-up was then performed by adsorption chromatography or thin-layer chromatography.

TABLE 1. Statistical parameters for analytical results on samples of canned meat

Statistical parameter

True value $(\mu \mathrm{g} / \mathrm{kg})$

Overall mean value found $(\mu \mathrm{g} / \mathrm{kg})$

Average within-laboratory standard deviation

Corresponding coefficient of variation

Standard deviation of means obtained by different laboratories

Corresponding coefficient of variation
NDMA

6.3

4.8

5.2

4.6

NDEA

NDBA

NPy

6.6

6.6

4.7

$\begin{array}{llll}0.942 & 0.650 & 0.898 & 1.801\end{array}$

$19.5 \%$

$14.3 \%$

$18.2 \%$

$38.1 \%$

.801

Notes: NDMA $=$ N-Nitrodimethylamine

NDEA $=\mathrm{N}$-Nitrodiethyllamine

NDBA $=\mathrm{N}$-Nitrodibutylamine

NPy $\quad=$ Ni-Nitropyrrolidine

End determination was made with gas chromatography using a selective nitrogen detector (flame thermionic or Coulson electrometric conductivity) or by conversion to a suitable derivative, i.e., the amine followed by 
conversion to the heptafluorobutylryl derivative, or oxidation of the nitrosamine to the corresponding nitramine. From the results of the study no one method could be singled out as giving the best results. Use of nitrogen selective detectors is more specific but these were more subject to problems than electron capture. High resolution mass spectrometry coupled with gas chromatography was also employed whenever available. It was agreed that this latter method is the only sure one for positive identification of nitrosamines. However, high resolution mass spectrometry/gas chromatography is available only in a limited number of laboratories. In the Agency laboratory we-employed the nitramine approach, having gathered considerable experience in its application to the detection of nitrosamines in alcoholic beverages [3]. In this method, after oxidation the resulting nitrosamines were separated and cleaned-up on a mixed alumina column containing neutral alumina of activity III and basic alumina activity II. Figure 1 shows a chromatogram obtained after oxidation of an extract from apple brandy spiked with eight nitrosamines at the $10 \mu \mathrm{g} / \mathrm{kg}$ level. Figure 2 illustrates the results after clean-up on an alumina column, demonstrating a resulting freedom from background.

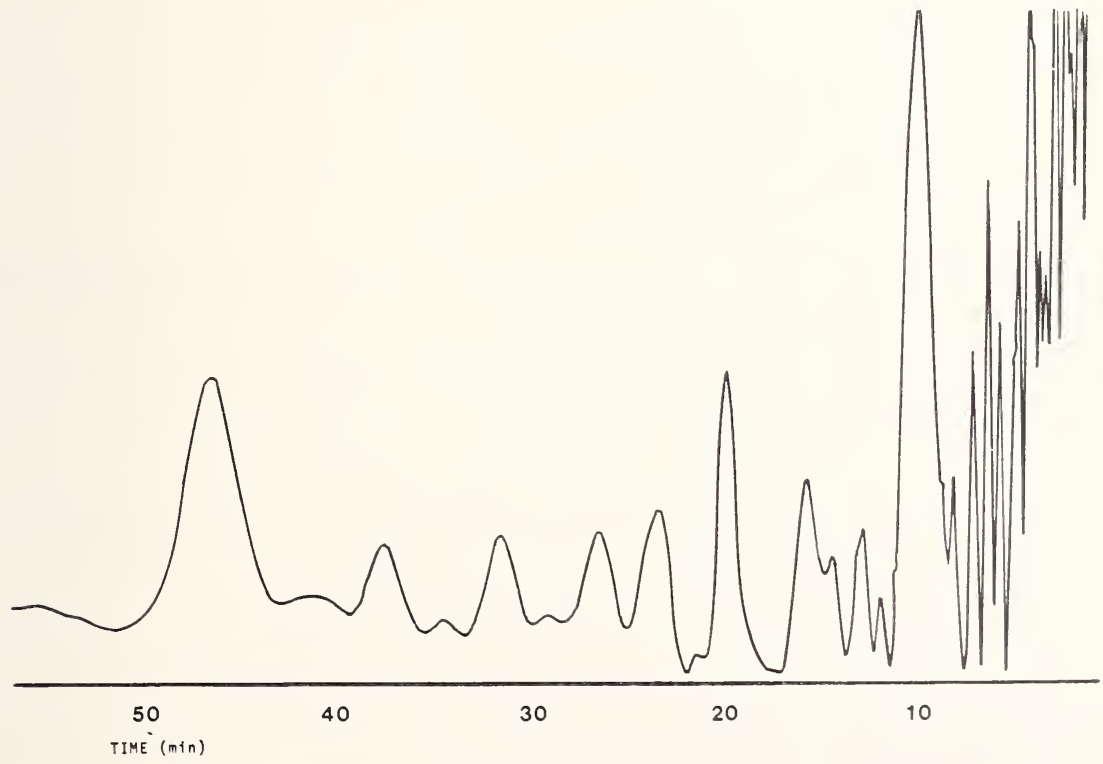

Figure 1. Chromatogram obtained from a calvados to which the following nitrosamines had been added, each at the $10 \mu \mathrm{g} / \mathrm{kg}$ level (before clean up); a) N-Nitrodimethylamine. b) $\mathrm{N}$ Nitromethylethylamine, c) N-Nitrodiethylamine, d) N-Nitrodipropylamine. e) NNitromethylpentylamine, f) N-Nitropiperidine, g) $\mathrm{N}$-Nitrodibutylamine, and h) $\mathrm{N}$ Nitropyrrolidine. 


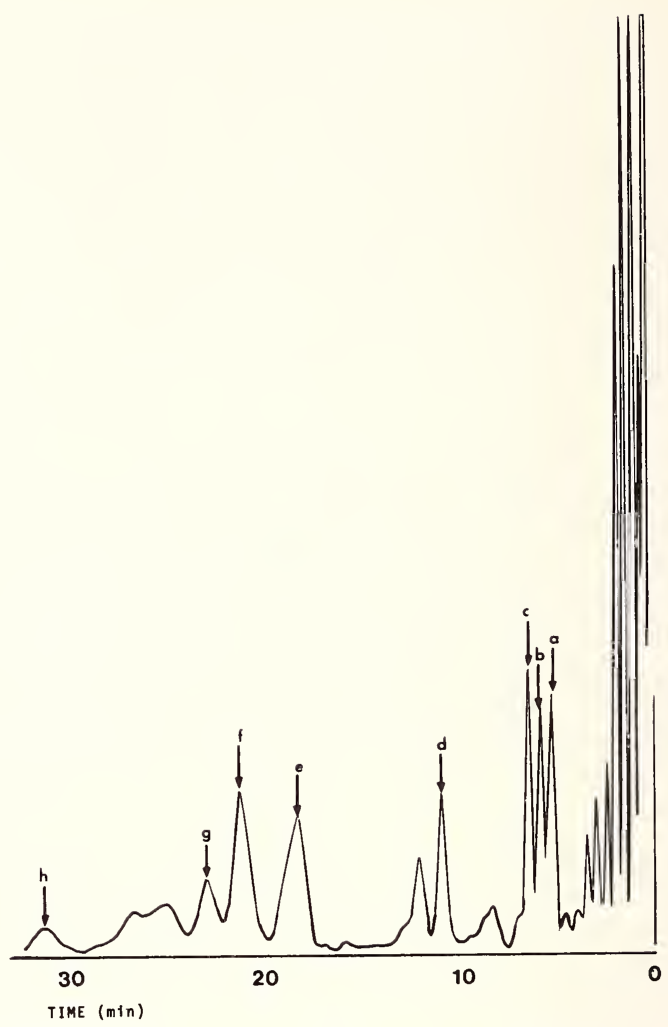

Figure 2. Chromatogram obtained from a calvados to which the following nitrosamines had been added, each at the $10 \mu \mathrm{g} / \mathrm{kg}$ level (after clean up); a) N-Nitrodimethylamine, b) $\mathrm{N}$ Nitromethylethylamine, c) N-Nitrodiethylamine, d) N-Nitrodipropylamine, e) NNitromethylpentylamine, f) N-Nitropiperidine, g) N-Nitrodibutylamine, and h) $\mathrm{N}$ Nitropyrrolidine.

The limit of detection for the method is about $1 \mu \mathrm{g} / \mathrm{kg}$ compared with 25 $\mu \mathrm{g} / \mathrm{kg}$ for an earlier method [4] that used thin-layer chromatography for the clean-up.

As the adsorption chromatography is reproducible for a given batch of alumina, the normal procedure is to analyze separate fraction eluted from the column. Figure 3 shows a nitramine fraction from an apple brandy containing $1 \mu \mathrm{g} / \mathrm{kg}$ of nitrosodimethylamine. The second arrow indicates the position for nitropyrollidine which is eluted in the same fraction. This gives additional support to the gas chromatographic identification of particular nitramines which are eluted in order of increasing polarity [3].

This method was then applied to a number of food samples collected as part of an epidemiological survey in the Caspian Littoral of Iran, the ob- 


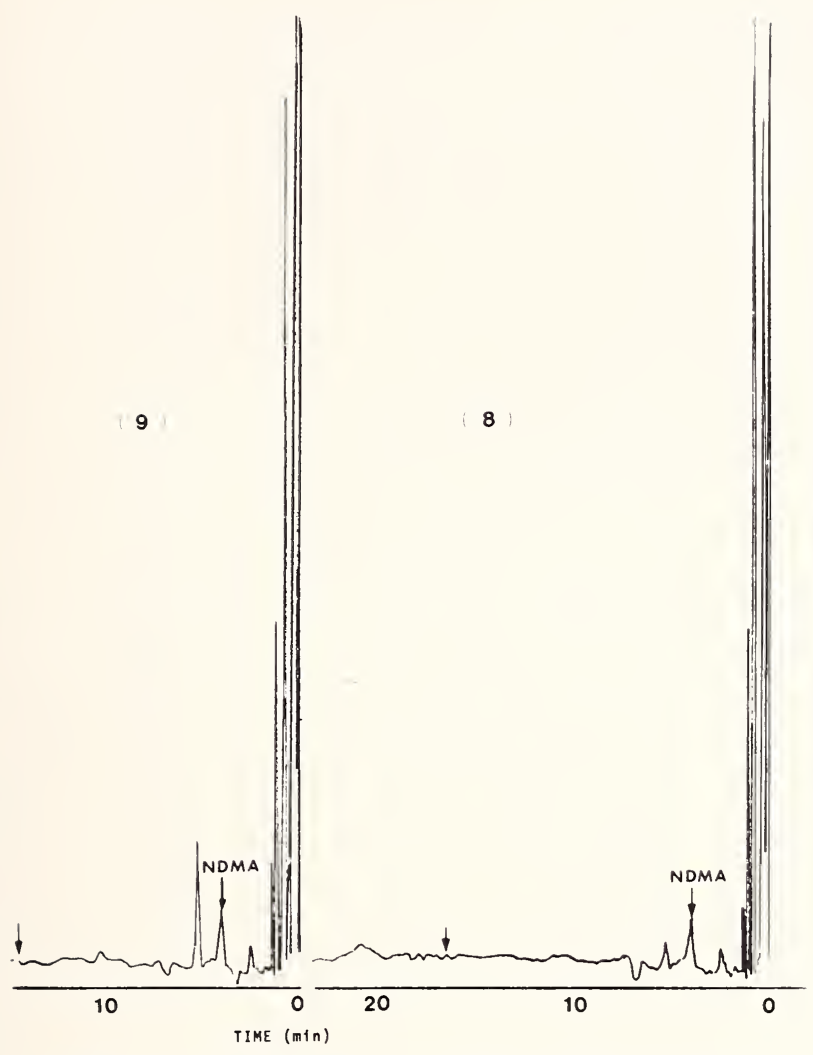

Figure 3. Fractions 8 and 9 of a sample containing NDMA.

jective being comparison of environmental factors between areas of high and low incidence of oesophageal cancer. The preliminary results of this analytical study for nitrosamines were provocative: apparent levels of nitrosodimethylamine in food from the high incidence area, principally in bread which forms the main diet, were in the order of 5 to $20 \mu \mathrm{g} / \mathrm{kg} \mathrm{com-}$ pared with $1 \mu \mathrm{g} / \mathrm{kg}$ or less in food from the low incidence area. Figure 4 shows the typical chromatogram obtained from a dimethylnitramine fraction showing the peak corresponding to the presence of nitrosodimethylamine in the sample. However, when confirmatory analysis was carried out by mass spectrography/gas chromatography (MS/GC) no evidence of nitrosodimethylamine could be found, thus indicating that further clean-up was essential. An additional adsorption chromatography stage was therefore introduced on the nitrosamine extract itself. Although several different column materials were tried, the mixed alumina column 


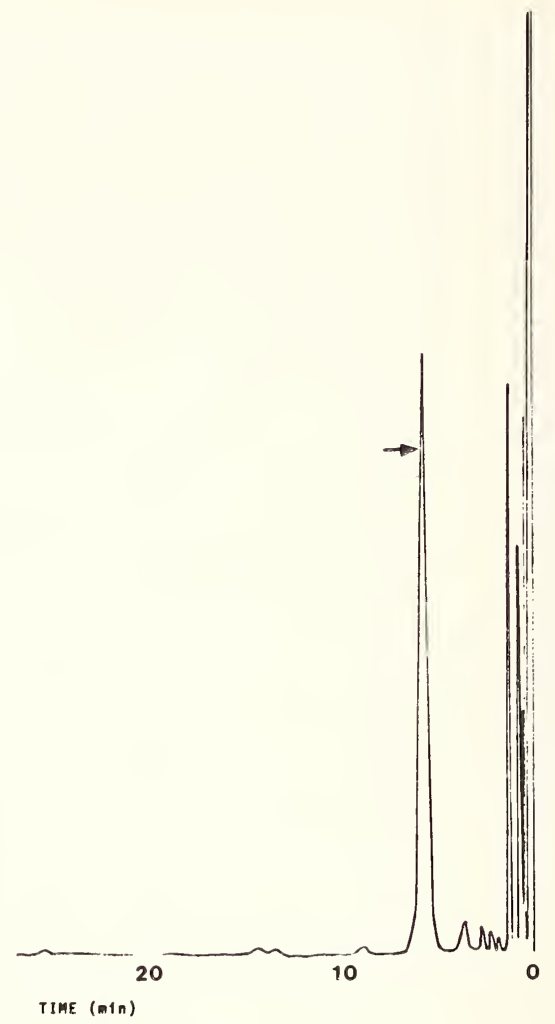

Figure 4. Chromatogram suggesting presence of nitrosodimethylamine using single celumn method.

used in the nitramine clean-up proved the most effective. Figure 5 is a flow diagram outlining the method at present in use. From the first column, two eluate fractions, the first $22 \mathrm{ml}$ and the next $55 \mathrm{ml}$, are collected separately, then oxidized and subsequently rechromatographed. Fractions from the nitramine separation are then analyzed by gas chromatography. In the first fraction, which contains the nitrosodibutylamine and nitrosodipropylamine, a peak was found in the oxidation products corresponding to dimethylnitramine. This peak not only had the same retention time as dimethylnitramine but, on analysis using the Coulson detector, gave a correct response relative to the electron capture detector for the nitramine. It is thus feasible that the samples contain material other than nitrosodimethylamine which in the oxidation treatment gives dimethylnitramine. This will be checked by MS/GC. As may be seen 


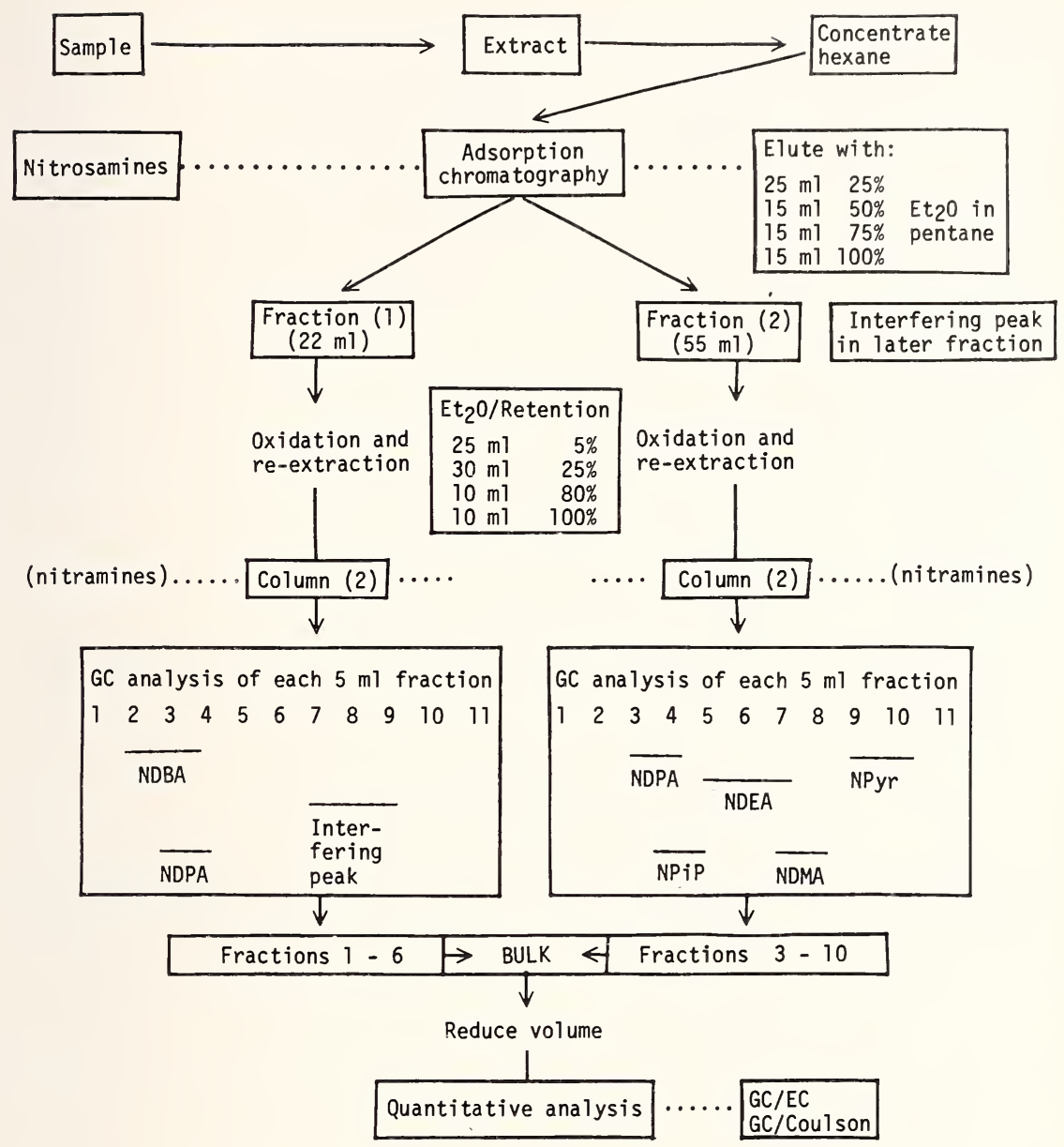

Figure 5. Flow diagram of analytical method.

from figure 6 , the absence of nitramine indicates that this substance is only formed during oxidation. Figure 7 shows the misleading peak in the fraction corresponding to dimethylnitramine and its relative freedom from background interference. A second interfering material was also found in several samples which eluted after nitrosodimethylamine. This also gave an oxidation peak corresponding to dimethylnitramine.

Using this modified method the samples were re-examined. It was found that all apparent nitrosodimethylamine levels were then reduced to $1 \mu \mathrm{g} / \mathrm{kg}$ or less, and the apparent correlation between cancer incidence and occurrence of nitrosamine in the diet no longer existed. It is interesting to note that, using this method, samples taken from the high incidence 


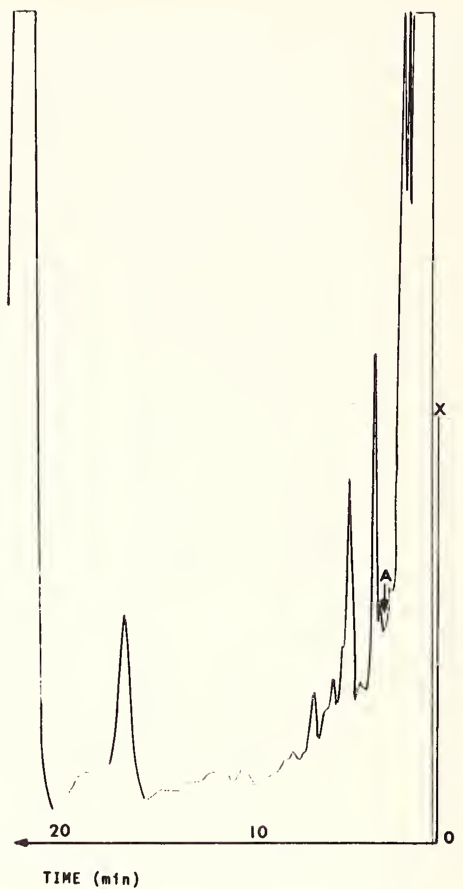

Figure 6. Nitrosodibutylamine fraction before oxidation, where $\mathrm{A}$ indicates position of dimethylnitramine.

area in a different season do show evidence of NDMA in the order of 10 to $20 \mu \mathrm{g} / \mathrm{kg}$. These are now being examined by MS/GC. Figure 8 shows a chromatogram of the assembled fractions using the double column technique. As may be seen from the high recovery of the nitropiperidine this may reintroduce error. In this case the excess is due to an abnormal solvent impurity which came in a different fraction from the nitropiperidine. For this reason we make a practice of analyzing the individual fractions from the column chromatography. This minimizes the risk of misinterpretation and makes correction possible. 


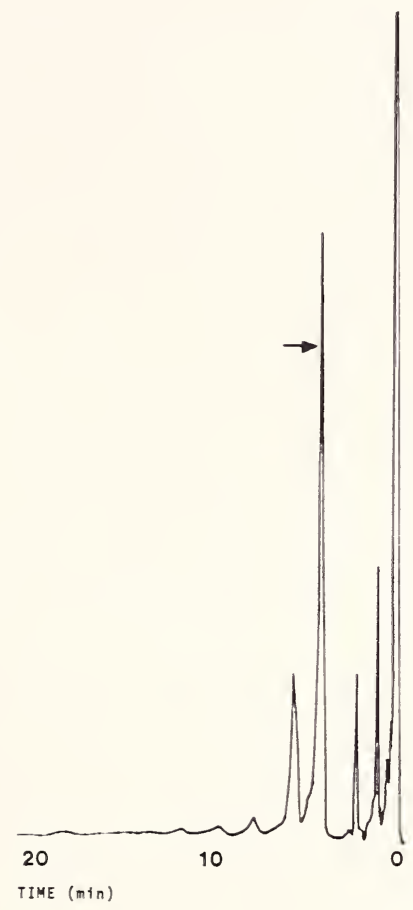

Figure 7. Dimethylnitramine fraction of the oxidation products from the nitrosodibutylamine fraction.

The problems encountered above demonstrate the vital importance and individuality of clean-up procedures appropriate for trace analysis. Methods successfully employed on one food matrix are not necessarily adequate or suitable for another. The problems emphasize the importance of bearing in mind that at the trace level the number of constituents in a natural product is vast and there are thus innumerable possible reactions which can accompany a simple derivatisation of a particular compound. Furthermore all solvents and reagents must be carefully checked and blanks regularly performed. Only by strict attention to detail can useful analyses be obtained. This is particularly true in trace analysis. 


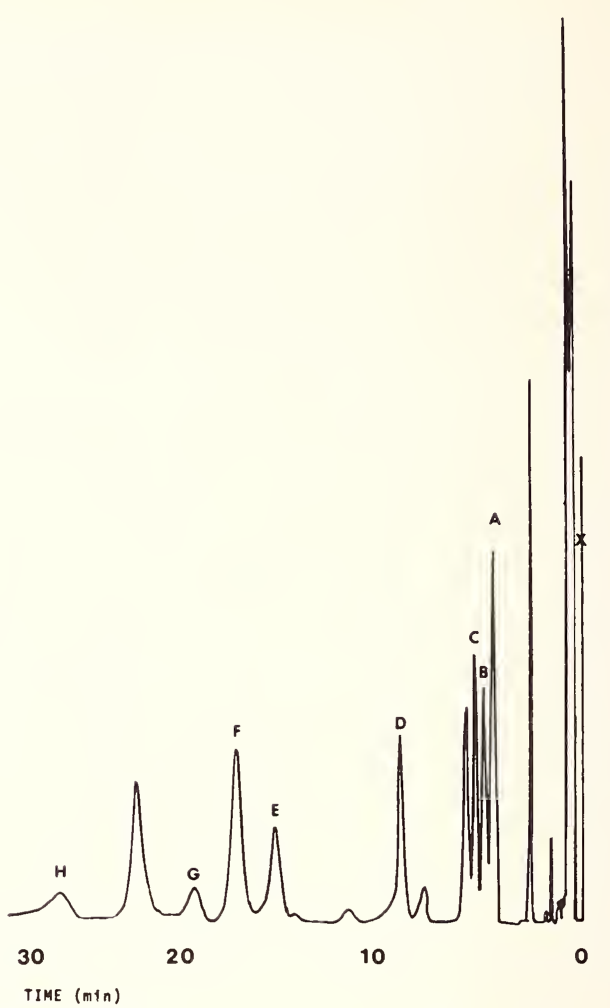

Figure 8. Chromatogram of assembled fraction from sample spiked with $10 \mu \mathrm{g} / \mathrm{kg}$ of $9 \mathrm{~N}$ Nitrosamines under the following chromatographic conditions: column $3 \mathrm{~m} \times 0.6 \mathrm{~cm}$ i.d.; 10 percent Carbowax $20 \mathrm{M}$ on Chromosorb W; column temperature $140{ }^{\circ} \mathrm{C}$; carrier, nitrogen $40 \mathrm{ml} / \mathrm{min}$; and electron capture detector Ni-63. Peaks are identified and recoveries are shown in table 2.

\section{References}

[1] Bogovski, P., Preussman, R., and Walker, E. A., Eds., N-Nitroso Compounds, Analysis and Formation, Proceedings of a Working Conference, October 13-15, 1971, Heidelberg, Federal Republic of Germany, IARC Scientific Publication No. 3, 90-94 (1972).

[2] Walker, E. A. and Castegnaro, M., A Report on the Present Status of a Collaborative Study of Methods for Trace Analysis of Volatile Nitrosamines (in press).

[3] Castegnaro, M., Pignatelli, B., and Walker, E. A., Analyst 99, 156-162 (1974).

[4] Sen, N. P. and Dalpe, C., Analyst 97, 216-220 (1972). 


\title{
EFFECTS OF STORAGE CONDITIONS ON RESIDUES OF 2,4-D AND 2,4-DCP IN POTATOES
}

\author{
Douglas Bristol \\ Residue Research Laboratory \\ Department of Biochemistry \\ North Dakota State University \\ Fargo, North Dakota 58102 U.S.A.
}

Red potatoes grown in North Dakota are treated with low dosage rates of 2,4-D (2,4-dichlorophenoxyacetic acid) at tuber set to improve market quality and yield. Tuber residues of 2,4-D and 2,4-DCP (2,4-dichlorophenol) in potatoes treated at a total rate of 2 ounces of active ingredients per acre were found to range from 0.068 to 0.15 and from less than 0.002 to $0.012 \mathrm{ppm}$, respectively. Samples were analyzed both with and without an acidic tissue hydrolysis step which preceded solvent extraction to determine total and free residue levels, respectively. Conjugated residues of 2,4-D could not be detected in significant quantities.

The stabilities of 2,4-D and 2,4-DCP in stored samples were found to be very dependent upon both the manner in which field samples were processed and the temperatures used for storage. Residue levels of metabolically incorporated 2,4-D in treated potato samples stored whole at $4{ }^{\circ} \mathrm{C}$ decreased over a period of 15 months to less than $0.005 \mathrm{ppm}$ but those of 2,4-DCP remained constant. The observed dissipation rate of 2,4-D was approximately first order with a half-life of about 10-14 weeks. In contrast to these results, obtained under conditions used for commercial storage, the stabilities of 2,4-DCP were reversed in tuber samples which were sliced, fortified and processed immediately after harvest and stored at $-20{ }^{\circ} \mathrm{C}$. Recoveries of 2,4-D from frozen samples were constant over a 15 -month period, but those of 2,4-DCP decreased slowly from 88 to 47 percent. These contrasting results point out the utility and necessity of incorporating dissipation experiments into residue studies conducted on samples stored for significant periods of time.

Keywords: Gas chromatography; potatoes; residues, dissipation of: residues, in potatoes; residues, metabolic; residues, total; residues, volatilization; storage effects; 2,4-dichlorophenol; 2,4-dichlorophenoxyacetic acid. 


\section{Introduction}

One factor that may detract from the reliability and interpretation of results obtained from the analysis of biological and environmental samples for pesticide residues is the effect that storage conditions might have on the pesticide residue in question. A cardinal rule of pesticide residue research, that no analytical result can be better than the original sample assessed [1], certainly applies to storage as well as to sampling considerations.

The best way to avoid complicating storage effects is to analyze samples as soon as possible after they are collected. However, as the number of samples collected for analysis in a residue study is usually large and as residue methods are normally quite time consuming, substantial backlogs of residue samples awaiting analysis are common. Storage of samples for as long as 6 months to 1 year may be unavoidable. Whenever long storage times are foreseen in advance, it is the responsibility of the analyst to prepare fortified samples and to determine experimentally whether or not the particular chemical to be analyzed decomposes, undergoes chemical change, or volatilizes during storage. Protocols for the proper storage of samples and for determining the stability of residues under storage conditions have been outlined $[1,2]$.

The objective of the residue and storage study reported here was to analyze potato tuber samples collected from plants treated at tuber set with low doses of 2,4-dichlorophenoxyacetic acid (2,4-D) as the propylene glycol butyl ether (PGBE) ester. Total residues of 2,4-D and 2,4-dichlorophenol (2,4-DCP), a possible phenolic metabolite, were to be determined by a method that included a step in which the macerated potato tissue was subjected to chemical hydrolysis. Since the required total residue analysis procedure had not yet been developed and verified, it was necessary to store residue samples for an extended period of time. Accordingly, fortified check samples for storage stability determinations were included in the design of the residue study.

\section{Procedures}

Field samples of $4.5 \mathrm{~kg}(10 \mathrm{lb})$ of potato tubers, collected from replicate plots treated with zero, 60 , and $120 \mathrm{~g}(0,2$, and $4 \mathrm{oz})$ active ingredient per acre total rates at 42 and 28 days before harvest, were washed and mixed. Whole and sliced tuber subsamples were prepared and stored as described below. 
Sliced samples were obtained by processing $2.3 \mathrm{~kg}(5 \mathrm{lb})$ field subsamples into French fry type pieces. After thorough mixing, either 200 or 100 $\mathrm{g}$ replicate laboratory samples were weighed out, double packaged in polyethylene freezer bags, labeled, tied close and frozen at $=23{ }^{\circ} \mathrm{C}$. Sets of untreated, control laboratory samples were fortified at different levels with a mixture of 2,4-D and 2,4-DCP to provide for determination of the stability of these chemicals under the conditions of frozen storage. All laboratory samples were stored at below $-20{ }^{\circ} \mathrm{C}$ until analyzed. The total time from harvest through freezing required less than 8 hours.

Whole tubers, consisting of $2.3 \mathrm{~kg}(5 \mathrm{lb})$ subsamples of field samples, were stored in open polyethylene bags at $4{ }^{\circ} \mathrm{C}$ to simulate the conditions employed for commercial storage. These samples were subsampled periodically, processed into laboratory samples and analyzed to check for dissipation of residues.

In addition to the zero, 60 , and $120 \mathrm{~g}$ active ingredients per acre treated samples, sliced and whole samples were obtained from a small field plot treated in the next growing season with a formulation containing uniformly ring-labeled ${ }^{14} \mathrm{C}-2,4-\mathrm{D}$. The analysis of these samples is under way.

Laboratory samples were analyzed for total residues of 2,4-D and 2,4DCP. The procedure developed [3] included a step in which bound and conjugated residues were released from the macerated potato issue by hydrolysis in $1 \mathrm{~mol} / \mathrm{l}$ sulfuric acid at reflux for 1 hour. This step provided for quantitative (98 percent) extraction of metabolically incorporated, ${ }^{14} \mathrm{C}$-labeled residues. After extraction of free 2,4-D and 2,4-DCP residues from aqueous acid into benzene, followed by additional cleanup, the acid and phenol were separated on an acidic alumina column [4,5]. These residues were analyzed separately by gas chromatography utilizing a Coulson conductivity detector operated in the reductive halogen mode.

\section{Results and Discussion}

The overall average recovery of $2,4-\mathrm{D}$, determined in the usual manner, by fortifying control samples at the time of analysis, was 87.9 percent (table 1). This did not differ significantly from the corresponding value ( 87.6 percent) obtained by the analysis of fortified samples which were stored at $-20^{\circ} \mathrm{C}$ and analyzed over a period of 73 weeks (table 2). In addition to the fortified storage samples, a limited number of field treated samples were analyzed after various periods of frozen storage (table 3 ). The metabolic residue levels of 2,4-D in these samples showed no tendency to 
TABLE 1. Recoveries of 2,4-D and 2,4-DCP from freshly fortified potato tuber samples

Fortification level, ppm
No. of samples analyzed
Mean

recovery, $\%^{\mathrm{a}}$

\begin{tabular}{rr}
4 & $89.2 \pm 8.8$ \\
8 & $87.9 \pm 6.8$ \\
4 & $89.5 \pm 6.7$ \\
3 & $84.1 \pm 7.2$ \\
\hline 19 & $87.9 \pm 3.0$
\end{tabular}

$88.6 \pm 27.1$

$78.6 \pm 12.1$

$93.4 \pm 9.4$

$80.5 \pm 13.4$

${ }^{a} \pm 95$ percent confidence limits for the mean.

TABLE 2. Recoveries of 2,4-D and 2,4-DCP from fortified potato tuber samples stored at $-20{ }^{\circ} \mathrm{C}$

\begin{tabular}{|c|c|c|c|c|}
\hline \multicolumn{2}{|c|}{ Fortification level, ppm } & \multirow{2}{*}{$\begin{array}{c}\text { Storage } \\
\text { period, } \\
\text { weeks }\end{array}$} & \multicolumn{2}{|c|}{ Recovery, $\%^{\mathrm{a}}$} \\
\hline $2,4-\mathrm{D}$ & 2,4-DCP & & $2,4-\mathrm{D}$ & 2,4-DCP \\
\hline 0.1 & 0.01 & 12 & 83.8 & - \\
\hline .1 & .01 & 12 & 79.5 & - \\
\hline .2 & .02 & 13 & 81.1 & 87.6 \\
\hline .05 & .005 & 15 & 72.5 & 66.0 \\
\hline .2 & .02 & 15 & 82.8 & 70.3 \\
\hline .05 & .005 & 20 & 88.7 & 67.3 \\
\hline .1 & .01 & 20 & 91.4 & 73.9 \\
\hline .05 & .005 & 25 & 87.7 & 61.6 \\
\hline .2 & .02 & 25 & 85.4 & 65.7 \\
\hline .1 & .05 & 66 & 92.9 & - \\
\hline .1 & .05 & 66 & 91.4 & - \\
\hline .05 & .025 & 67 & $94.8 \pm 2.3$ & $56.4 \pm 1.4$ \\
\hline .1 & .05 & 67 & $91.2 \pm 4.2$ & 41.6 \\
\hline .2 & .1 & 67 & $89.4 \pm 3.8$ & $45.3 \pm 0.8$ \\
\hline .2 & .1 & 68 & 88.0 & 36.2 \\
\hline .05 & .025 & 68 & 96.7 & 50.5 \\
\hline .2 & .1 & 68 & 85.3 & 49.6 \\
\hline .05 & .025 & 68 & 86.7 & 51.7 \\
\hline .1 & .05 & 69 & 85.5 & 37.9 \\
\hline .1 & .05 & 69 & 86.1 & 44.0 \\
\hline .2 & .1 & 73 & 83.1 & 47.7 \\
\hline .05 & .025 & 73 & 94.4 & 47.1 \\
\hline \multirow[t]{2}{*}{.2} & .1 & 73 & 84.2 & 53.8 \\
\hline & & & $\overline{87.6} \pm 2.3^{b}$ & \\
\hline
\end{tabular}

a Mean \pm average deviation of duplicate analysis, where indicated.

b Overall mean \pm 95 percent confidence limits for the mean. 
TABLE 3. Residue levels of metabolically incorporated 2,4-D and 2,4-DCP in field treated samples determined after varivus periods of storage at $-20^{\circ} \mathrm{C}$

\begin{tabular}{|c|c|c|c|}
\hline \multirow{2}{*}{ Sample number } & \multirow{2}{*}{$\begin{array}{l}\text { Storage period, } \\
\text { weeks }\end{array}$} & \multicolumn{2}{|c|}{ Residue level, ppb } \\
\hline & & $2,4-\mathrm{D}$ & 2,4-DC \\
\hline \multirow[t]{2}{*}{ 1973-23-1X } & 5 & 99 & 3.6 \\
\hline & 15 & 103 & 5.6 \\
\hline \multirow[t]{2}{*}{$1973-14-1 X$} & 6 & 116 & 5.1 \\
\hline & 15 & 130 & 4.5 \\
\hline \multirow[t]{2}{*}{$1973-45-1 X$} & 11 & 147 & - \\
\hline & 14 & 124 & 4.8 \\
\hline \multirow[t]{3}{*}{$1972-53-2 X$} & 45 & 108 & - \\
\hline & 46 & 138 & 3.2 \\
\hline & 59 & 104 & 2.7 \\
\hline \multirow[t]{3}{*}{$1972-71-2 X$} & 45 & 117 & 3.1 \\
\hline & 59 & 98 & 3.9 \\
\hline & 60 & 122 & 4.6 \\
\hline \multirow[t]{2}{*}{$1972-21-2 X$} & 45 & 88 & - \\
\hline & 64 & 118 & 1.8 \\
\hline \multirow[t]{2}{*}{$1972-93-1 X$} & 60 & 72 & 2.0 \\
\hline & 65 & 86 & - \\
\hline
\end{tabular}

decrease during frozen storage. Thus, residues of 2,4-D in sliced potato tubers, either incorporated metabolically or by fortification, were stable over a 15 -month period when stored at $-20^{\circ} \mathrm{C}$.

On the other hand, recoveries of 2,4-DCP decreased from an overall average of 84.5 percent in freshly fortified samples (table 1 ) to an average of 46.8 percent in samples stored for $67-73$ weeks at $-20{ }^{\circ} \mathrm{C}$ (table 2 ). In order to confirm the contrasting stabilities of 2,4-D and 2,4-DCP in frozen storage, a set $(n=10)$ of sliced potato samples obtained from a single field sample were fortified in replicate and stored along with control samples at $-20{ }^{\circ} \mathrm{C}$. Recoveries obtained from samples removed from storage after 1 to 25 weeks are presented in figure 1 and indicate clearly that $2,4-\mathrm{D}$ is stable while $2,4-\mathrm{DCP}$ is lost slowly from fortified potato samples stored in this manner.

To determine whether or not the loss of 2,4-DCP involved the presence of potato tissue, aliquots of an aqueous solution containing both 2,4-D and 2,4-DCP were sealed in polyethylene bags, stored at $-20{ }^{\circ} \mathrm{C}$ and analyzed in duplicate, periodically. Concentrations of 2,4-D and 2,4-DCP were determined by measurement of ultraviolet maxima [6] in $0.1 \mathrm{~mol} / \mathrm{l}$ $\mathrm{NaOH}$ solution at $283(\log \mathrm{a}=3.186)$ and $305(\log \mathrm{a}=3.477) \mathrm{nm}$. respectively (fig. 2). After an initial rapid decrease in concentration, the loss of 2,4-DCP from the frozen samples was gradual, whereas the concentration of 2,4-D was unchanged over the 10 -week storage period. Thus, the loss 


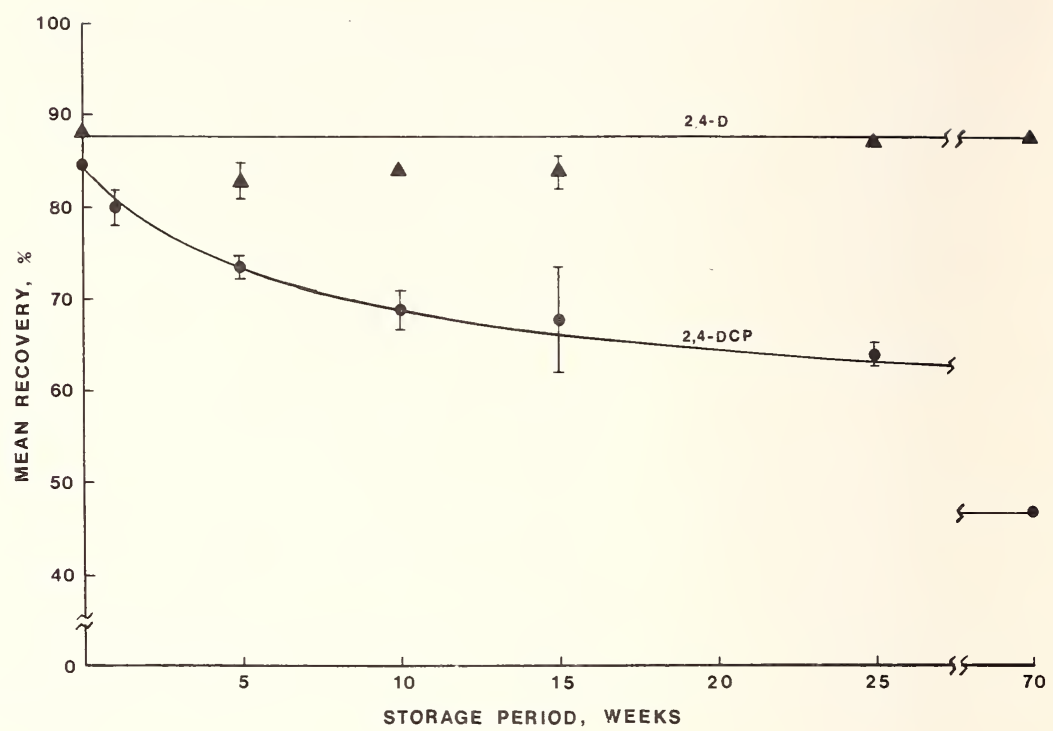

Figure 1. Recoveries of 2,4-D ( $\triangle$ ) and 2,4-DCP (O) from fortified samples stored at -20 ${ }^{\circ} \mathrm{C}$. Values at 1 to 25 weeks are the mean \pm average deviation of duplicate sample analyses. Those at 0 weeks are from table 1 while those at 70 weeks are the mean recoveries from samples in table 2 stored for 66 to 73 weeks.

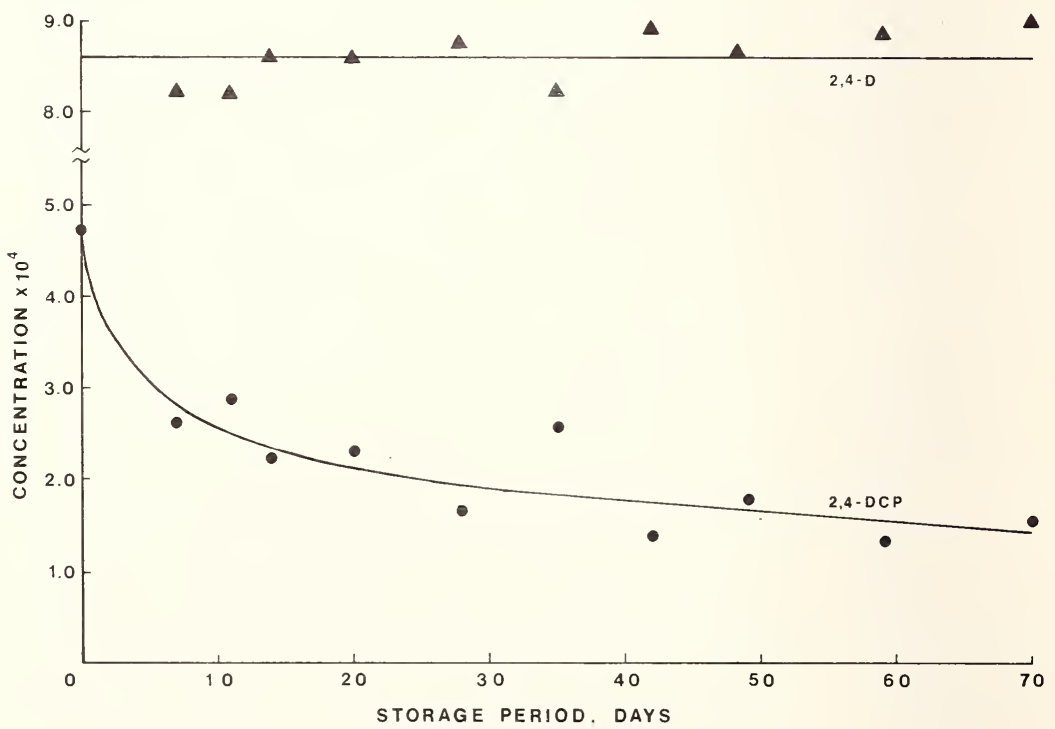

Figure 2. Concentrations of 2,4-D $(\Delta)$ and 2,4-DCP $(\bigcirc)$ in aqueous samples stored at -20 ${ }^{\circ} \mathrm{C}$, duplicate determinations. The mean \pm standard deviation for the concentration of 2,4$\mathrm{D}$ over the entire storage period was $(8.61 \pm 0.30) \times 10^{-4} \mathrm{M}$. 
of 2,4-DCP from fortified tuber samples was not due to metabolism.

A similar experiment in which separate solutions of 2,4-DCP in distilled water and in $0.1 \mathrm{~mol} / 1 \mathrm{NaOH}$ were stored in polyethylene bags at $-20^{\circ} \mathrm{C}$ showed that the loss of 2,4-DCP was prevented by salt formation (fig. 3). These results, plus the observation that plastic bags containing aqueous solutions of 2,4-DCP exuded its characteristic odor, indicate that the observed losses of 2,4-DCP from frozen samples were due to volatilization. The $2,4-\mathrm{DCP}$ residue must be at least partially soluble in polyethylene, through which it diffuses freely and is lost to the surrounding atmosphere of the freezer.

In contrast to the stability of 2,4-D in sliced tuber samples stored at $-20{ }^{\circ} \mathrm{C}$, metabolically incorporated residues of 2,4-D in whole tubers stored at $4{ }^{\circ} \mathrm{C}$ decreased during storage. Assuming first order kinetics, the dissipation curve for 2,4-D in these samples (fig. 4) exhibits a half-life of approximately 12 weeks. Residues of 2,4-D in samples stored for 12 to 15 months at $4{ }^{\circ} \mathrm{C}$ had diminished to less than $0.02 \mathrm{ppm}$, the limit of sensitivity established for the method.

Additional storage and metabolism studies utilizing the samples containing metabolically incorporated, ring-labeled ${ }^{14} \mathrm{C}-2,4-\mathrm{D}$ are in progress. To date, it has been established that the 2,4-D residue which dissipates from whole tubers stored at $4{ }^{\circ} \mathrm{C}$ is converted primarily into water soluble metabolites.

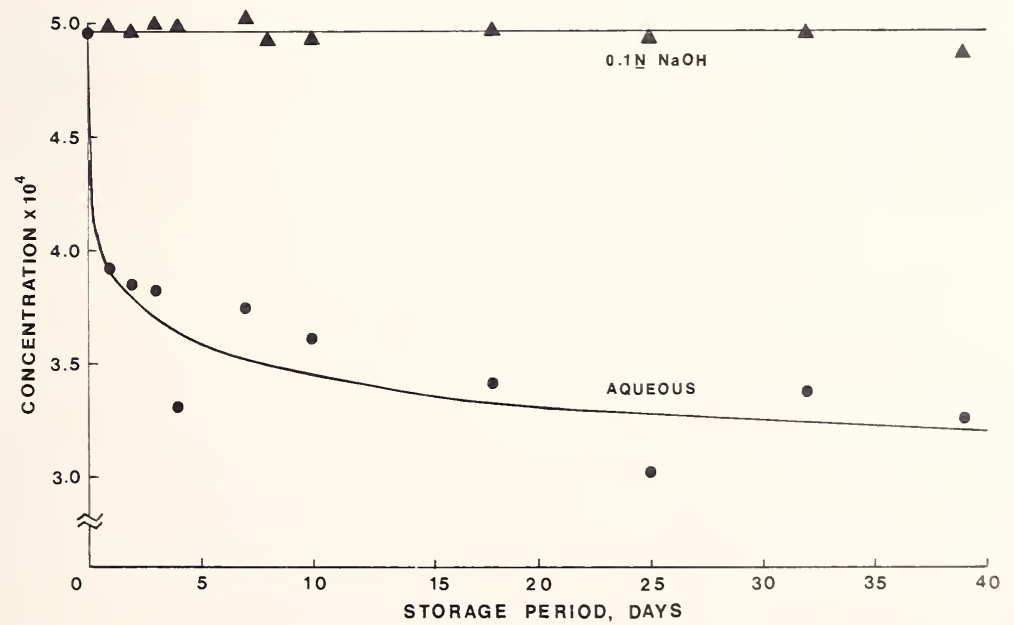

Figure 3. Loss of 2,4-DCP from aqueous $(\mathbf{O})$ and $0.1 \mathrm{~mol} / 1(\mathbf{\Delta}) \mathrm{NaOH}$ solutions stored at $-20^{\circ} \mathrm{C}$, duplicate determinations. The mean \pm standard deviation for the concentration of 2,4-DCP in $0.1 \mathrm{~mol} / 1 \mathrm{NaOH}$ solution over the entire storage period was $(4.96 \pm 0.46)$ $\times 10^{-4} \mathrm{M}$. 


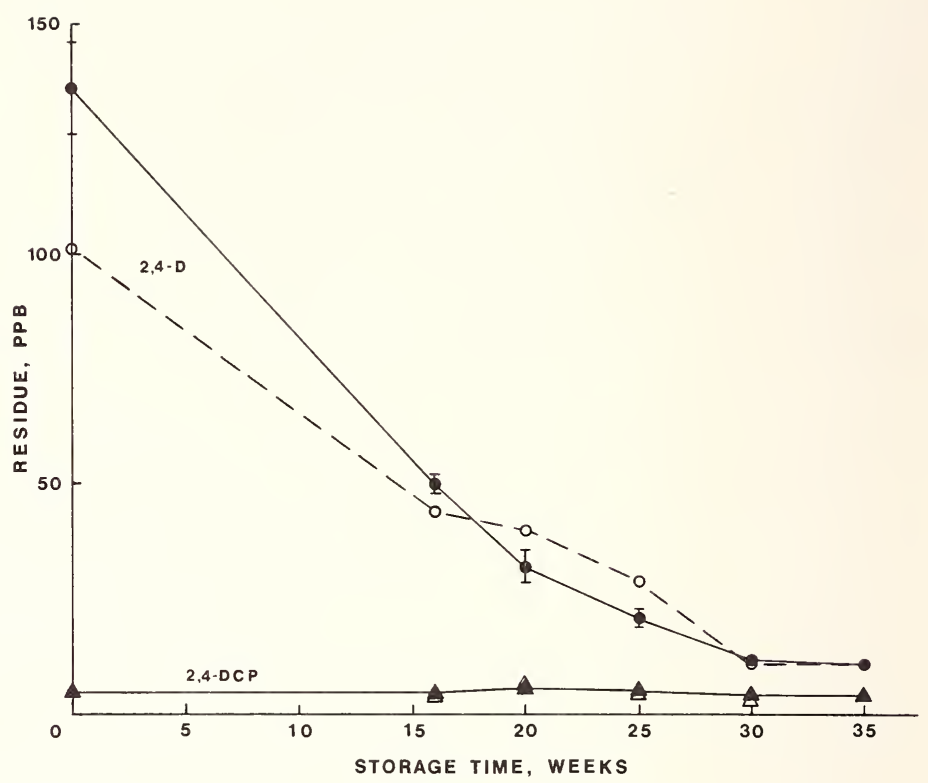

Figure 4. Dissipation of 2,4-D (O) sample 1973-45; (○) sample 1973-23) and 2,4-DCP( $\mathbf{\Delta})$, sample 1973-45; $\Delta$, sample 1973-23) from treated potato tubers stored whole at $4{ }^{\circ} \mathrm{C}$. Sample 1973-45 was analyzed in đuplicate.

"Metabolic" residue levels of 2,4-DCP were very low ( $\sim 0.005 \mathrm{ppm})$ and of questionable significance. However, they did appear to remain constant in samples stored at either $4^{\circ}$ or $-20{ }^{\circ} \mathrm{C}$ (fig. 4 and table 3 , respectively).

\section{Conclusion}

In this study, the stabilities of 2,4-D and 2,4-DCP residues in potato tuber samples were found to be dependent on the temperature used for storage. The results point out the necessity of incorporating storage experiments in residue studies where samples must be stored for significant periods of time, but the stability of the residue being determined is not known. The analyst must exercise good judgment in selecting proper storage conditions (sample form, container, temperature, etc.). To minimize losses, samples containing volatile residues should be stored in all glass containers or in wide mouth glass bottles having Teflon lined screw caps. 


\section{Acknowledgements}

The author expresses sincere thanks to Mr. Leonard Cook for valuable assistance in carrying out the residue analyses and to Dr. Donald C. Nelson, Department of Horticulture, for supplying field samples. Thanks are also due to Dr. H. J. Klosterman of this Department and to Dr. David Jensen of Dow Chemical U.S.A., Ag-Organics Division, Midland, Michigan, for encouragement and helpful suggestions in carrying out this investigation. Gratitude is expressed to Mr. Wes Muelder of Dow Chemical U.S.A. for preparing the formulation containing ${ }^{14} \mathrm{C}$-labeled 2,4-D. Partial financial support for this work from the Red River Valley Potato Growers Association is gratefully acknowledged.

\section{References}

[1] Van Middelem, C. H., Assay Procedures for Pesticide Residues, in Pesticides in the Environment, White-Stevens, R., Ed., Vol. 1, Part II, pp. 317-321, Marcel Dekker, Inc., New York (1971).

[2] Zweig, G.. Ed., Analytical Methods for Pesticides, Plant Growth Regulators and Food Additives, Vol. 1, Academic Press, New York (1963).

[3] Bristol, D. et al., 168th National Meeting of the American Chemical Society, Atlantic City, N.J., Sept. 8-13, 1974, PEST 44.

[4] Jensen, D. et al., Procedure for Residues of 2,4,5-T, 2,4-D, 2,4,5-Trichlorophenol and 2,4-Dichlorophenol in Cattle and Sheep Tissues by Gas Chromatography, Method ACR 71.6, Dow Chemical U.S.A., Ag-Organics Division, Midland. Michigan 48640 (May 1971).

[5] Bjerke, E. et al., Residue Study of Phenoxy Herbicides in Milk and Cream, J. Agr. Food Chem. 20,963-967 (1972).

[6] Aly, O. M. et al., Ultraviolet Spectrophotometry in Residue Analysis, Adv. in Chem. 104, 105 (1971). 



\title{
SAMPLING PROBLEMS IN THE INVESTIGATION OF HIGH PURITY METALS
}

\author{
M. Radwan, L. Walis, and H. Jaskólska \\ Polish Institute of Nuclear Research \\ Warszawa, Poland 03195
}

\begin{abstract}
One of the vital problems in the determination of trace elements in metals is their inhomogeneous distribution in the matrix. Elements which dissolve in the metal matrix at elevated temperatures often concentrate during the crystallization process at the grain boundaries, or inside the grain. Elements which do not dissolve occur in the form of nonmetallic inclusions. The distribution of such inclusions is generally irregular, and obtaining results which are representative for the whole ingot is practically impossible. The error involved can be reduced, if the type and range of segregation of the element to be determined in the alloy is known and taken into account on sampling. Several examples are given.
\end{abstract}

Keywords: High purity metals; inhomogeneity; microsegregation; sampling; surface contamination.

A difficult problem in the analysis of metals and alloys is the selection of sample size for analysis. In practice the choice is made taking into account the sensitivity and the accuracy of the method employed. Such an approach, however, may lead to serious errors due to the inhomogeneity of metals and alloys resulting from segregation under definite conditions of crystallization. This must be taken into account when selecting the size of the sample to be analyzed.

The inhomogeneity of distribution of elements in a matrix is pronounced in the case of limited solubility or lack of solubility. Elements insoluble in a metal (usually present in the form of chemical or intermetallic compounds) tend to accumulate with formation of agglomerates as shown in figure 1 [1]. The results of the determination of such elements will depend on the presence or absence and the size of these inclusions in the sample. As the sample size increases the results become more representative; however, doubts still exist whether they represent the 


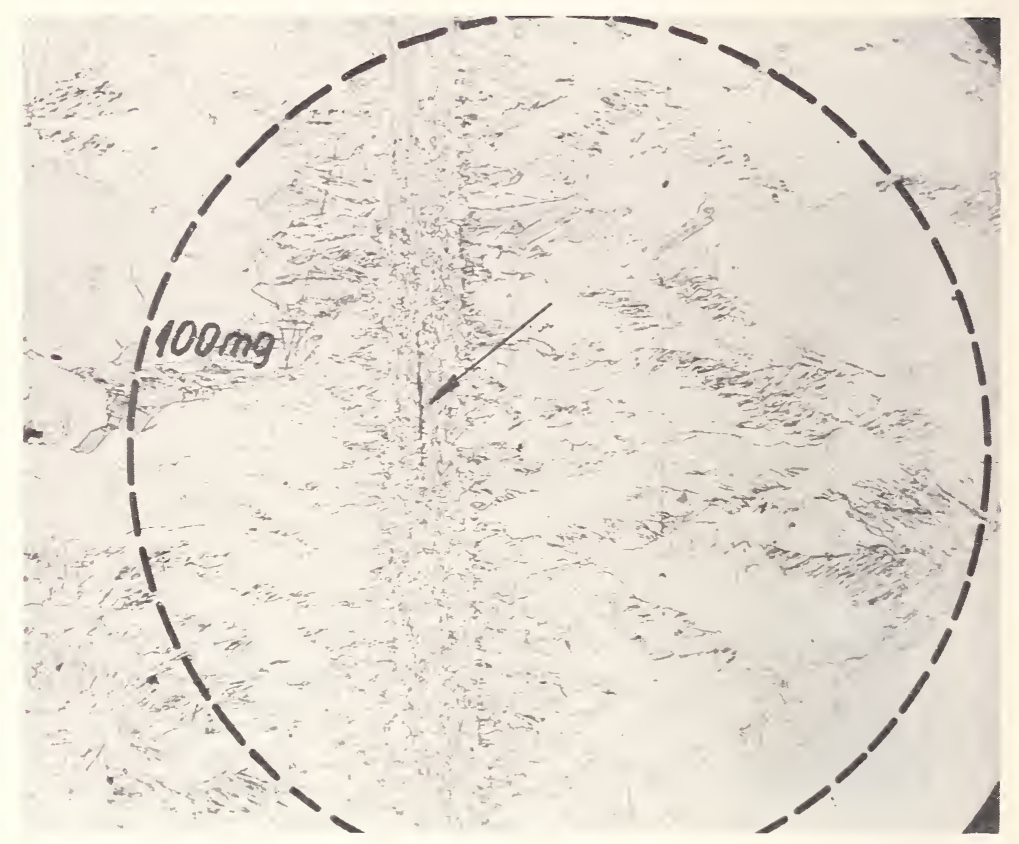

Figure 1. Structure of Armco steel with an inclusion (30X).

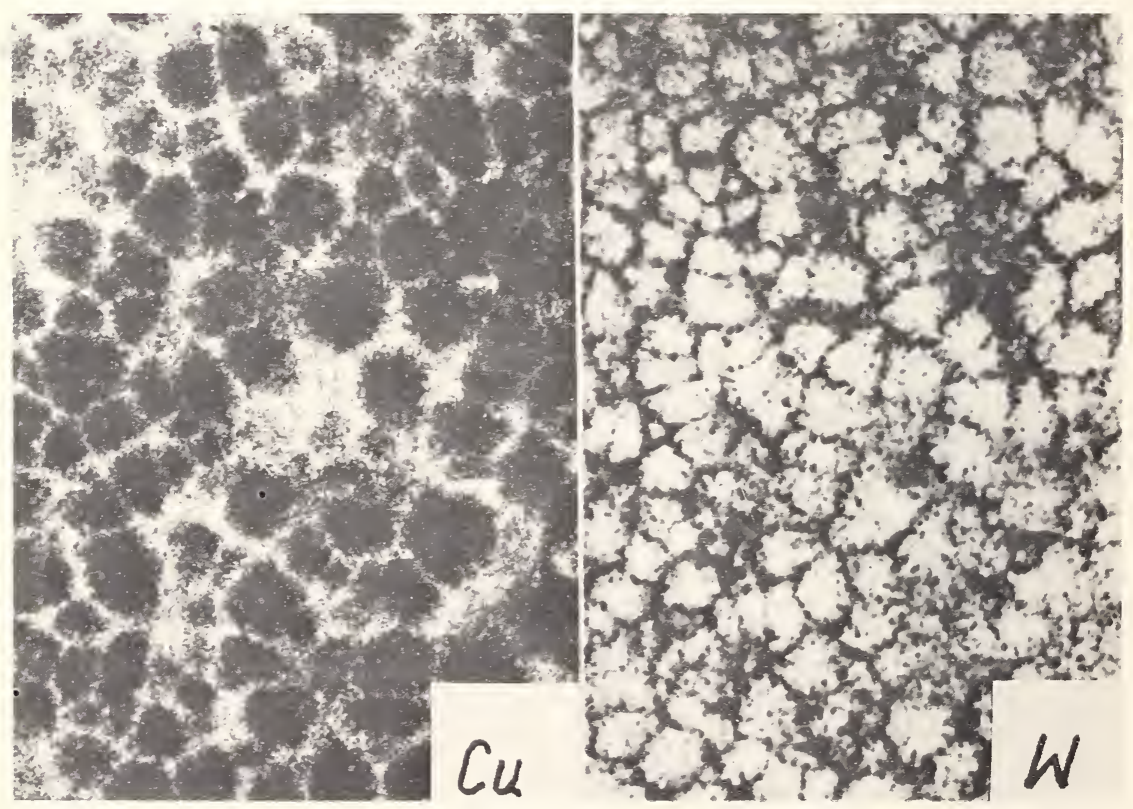

Figure 2. Segregation of copper and tungsten in Fe-C alloy (50X). 
whole material examined (for example, an ingot). This is obvious if one takes into account microsegregation which occurs in addition to macrosegregation. It is then necessary to isolate intermetallic phases or inclusions from the bulk of the material.

Even if the element to be determined dissolves in the liquid metal matrix, its distribution after crystallization becomes inhomogeneous because segregation also takes place during the crystallization process. The element may concentrate at the phase boundary or inside the grain as shown in figure 2 [2] or its concentration may change from the grain boundary (fig. 3 [3]) toward the inside of the sample as a result of hot working and heat treatment. In this case the grain size must be taken into account when choosing the size of the sample to be analyzed. It follows

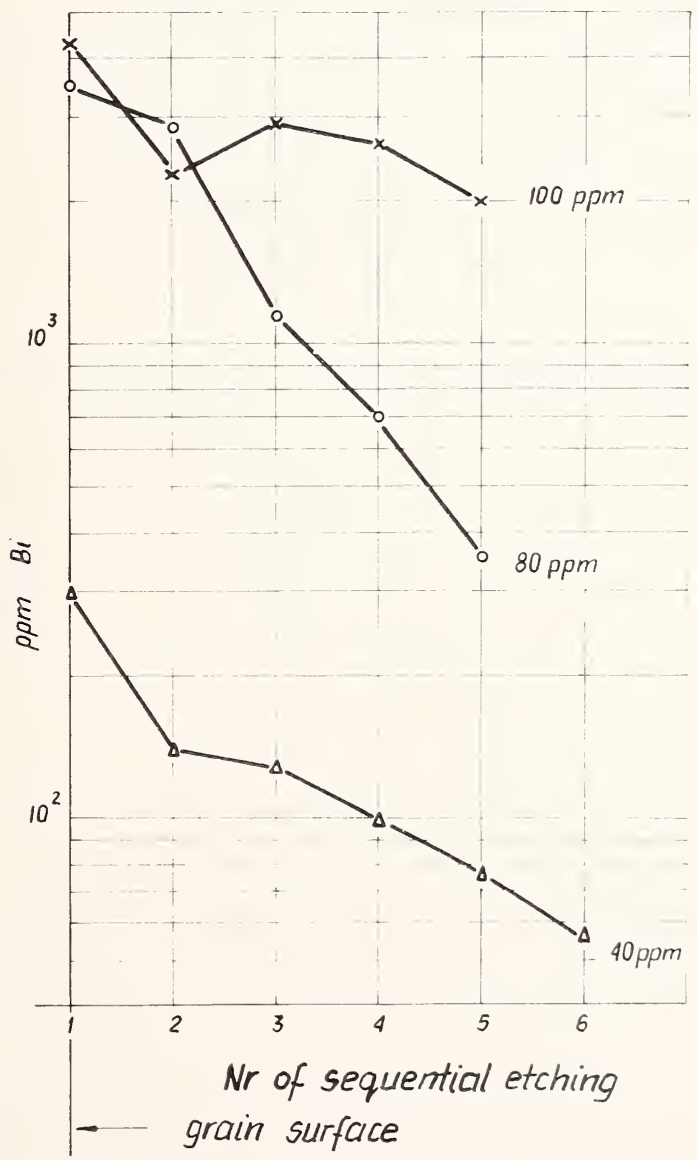

Figure 3. Concentration changes of bismuth in copper grains. 
from figure 4 that in the case of coarse grain structure a sample of a weight of $1 \mathrm{mg}$ may have been contained in a single grain. In this case, elements which segregate at the grain boundary may not be included in the sample. If the sample weight is of about $100 \mathrm{mg}$ this could not occur. In the case of fine grain structure (fig. 5) a $1 \mathrm{mg}$ sample will consist of a few grains and will be more representative.

Even if inhomogeneity resulting from structural segregation (microsegregation) is taken into account the results obtained will not always be representative of a whole ingot. Large errors may occur due to macrosegregation in the ingot, resulting in its inhomogeneity. Such cases, illustrated by autoradiograms, are shown in figures 6 and 7. The accuracy of analysis cannot be improved, in this case, by enlarging the sample but solely by selecting a suitable number of samples and choosing suitable sites for sampling.

The sampling procedure is governed by the purpose of the analysis, i.e., whether the analysis is aimed at the determination of an average concentration of an element or at the determination of concentration differences in individual zones of the ingot. In either case it is necessary to carry out additional examinations in order to evaluate the type and the degree of segregation [1].

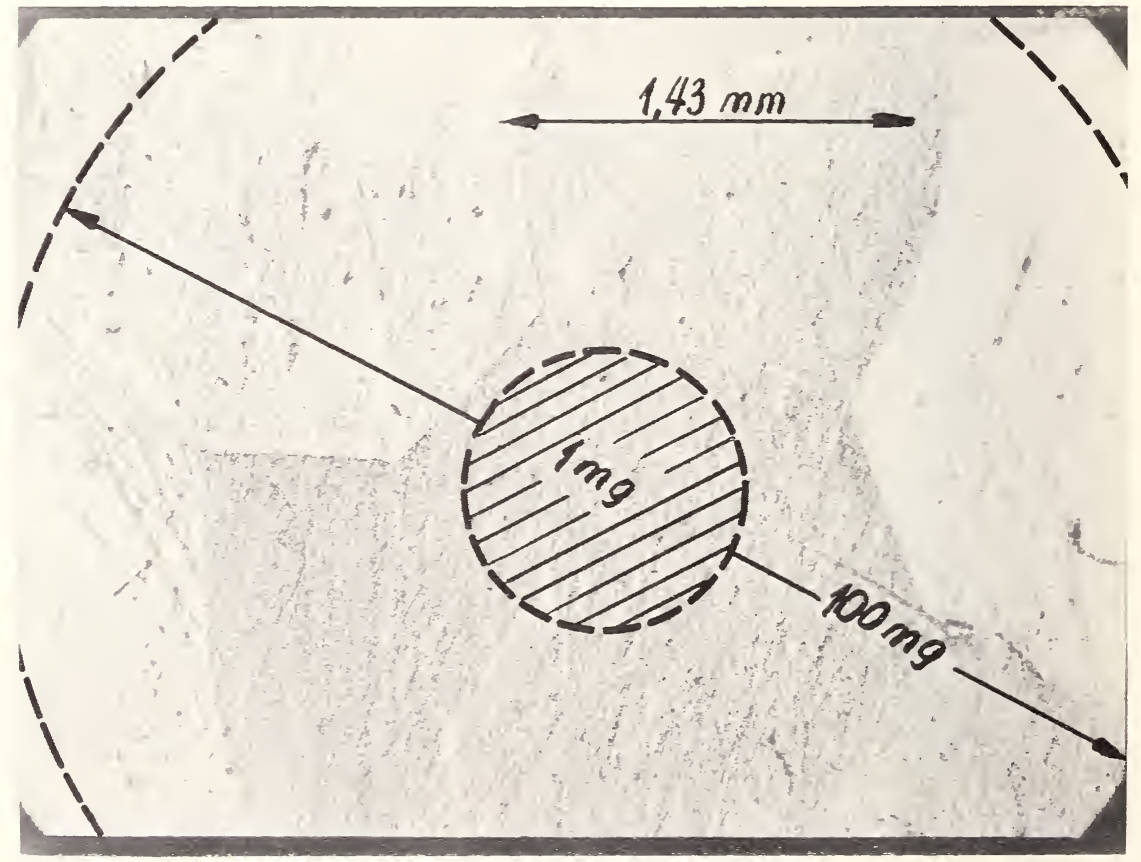

Figure 4. Structure of aluminum (30X) with shown diameters of 1 -mg and 100-mg samples. 


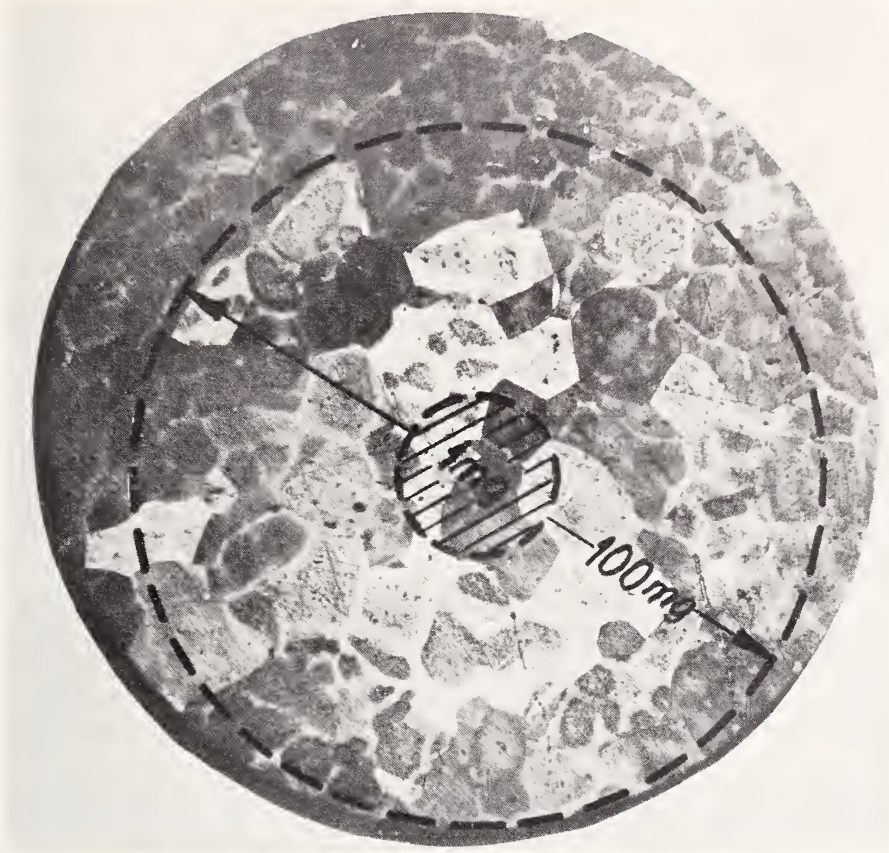

Figure 5. Structure of copper (30X) with shown diameters of 1-mg and 100-mg samples.

When analyzing inhomogeneous materials it is necessary to prepare an average sample. The common procedure is crushing, grinding, and mixing a large sample. However, in the case of metals such treatment is not feasible because:

1. high hardness of many metals can result in serious contaminations with the material of the tools employed,

2. spalling of some impurities, which occur in the form of fragile nonmetallic inclusions, and their separation from the matrix material during mixing (owing to the density differences), and

3 . plasticity of many metals.

In view of these factors an average sample can be prepared only by dissolution. However, this requires that the amount of contamination introduced to the solution from the surrounding atmosphere, vessels, and reagents be accurately determined and corrected for. In trace analysis the blank value may seriously limit the accuracy of the determination.

Preparation of an average sample by dissolution was employed by us in the determination of lanthanum in steel by the neutron activation method [5]. The content of lanthanum in steel was determined in connection with the identification of sources of exogenic inclusions in steel, with the aid of 


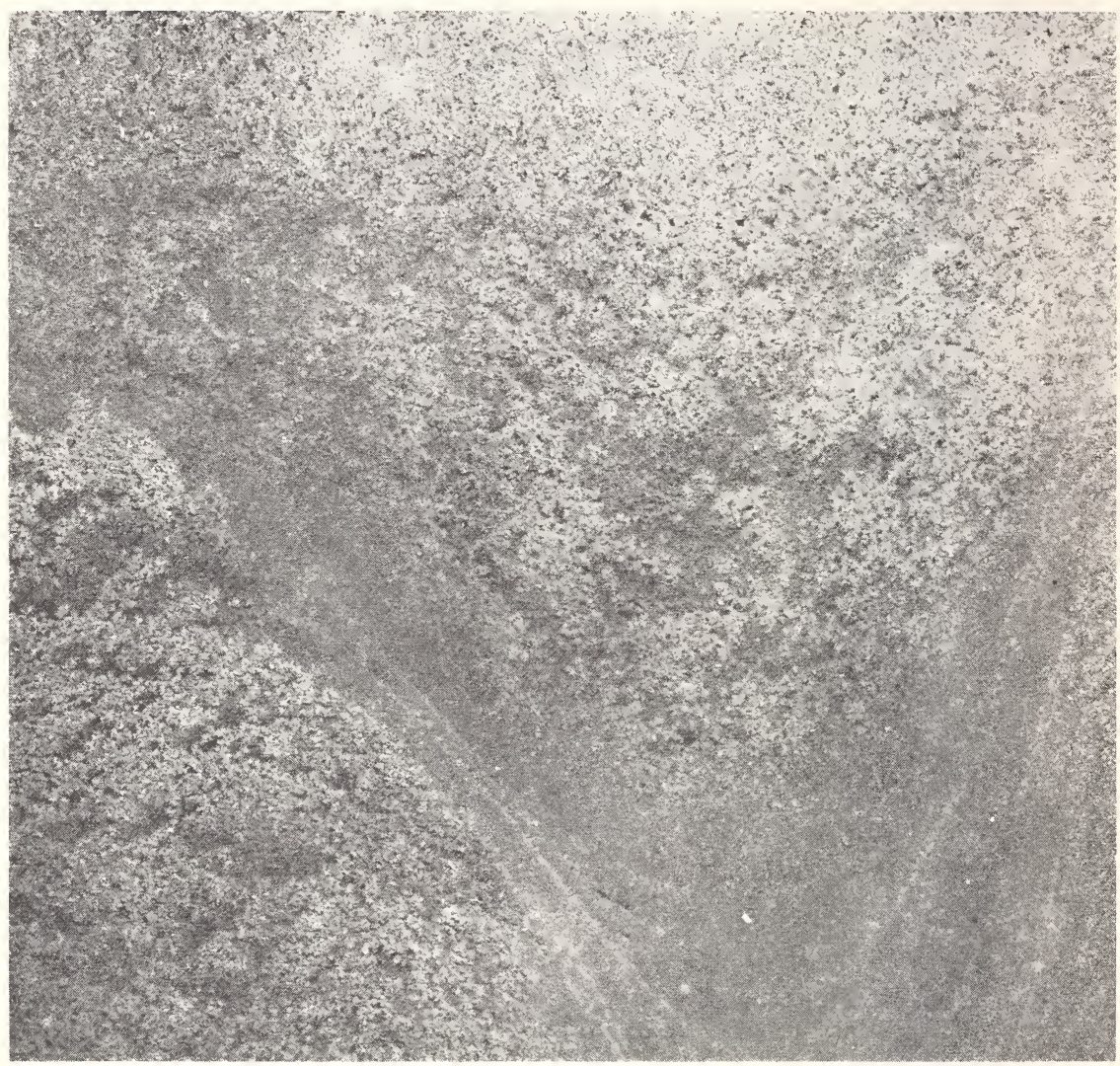

Figure 6. Segregation of tin in aluminum ingot (1X).

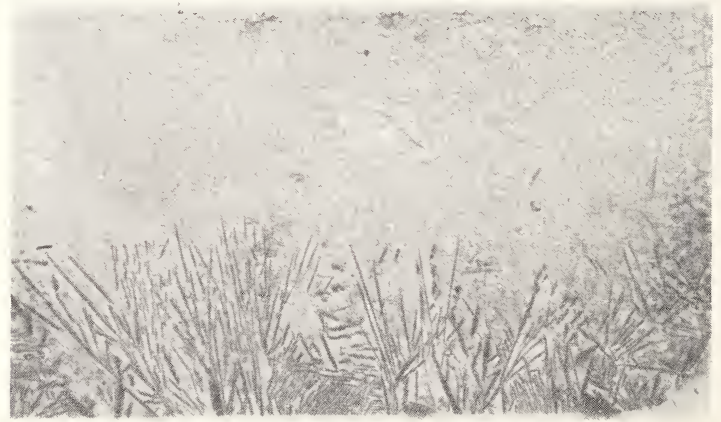

Figure 7. Segregation of copper in a bearing alloy with high tin content (1X). 
an inactive lanthanum tracer. The method applied consisted of the dissolution of a $50 \mathrm{~g}$ steel sample and further treatment of a 1 percent aliquot of the solution. The separation of iron and many other elements from the solution was carried out by electrolysis. The solution was evaporated to dryness and the dry residue, together with a standard, was irradiated in a thermal neutron flux. After a suitable cooling period the irradiated residue was dissolved and sodium was separated by the method of Girardi and Sabbioni [6]. Lanthanum was then determined in the solution by measuring the $1.6 \mathrm{MeV}$ gamma emission of ${ }^{140} \mathrm{La}$ by means of a 512 -channel pulse height analyzer. The lanthanum content determined in the blanks (reagents used for preirradiation treatment, air dust, etc.) was in the range of $10^{-10}$ to $10^{-9} \mathrm{~g}$, while that in the steel samples was commonly in the range of $10^{-8}$ to $10^{-7} \mathrm{~g} / \mathrm{g}$. Hence the amount of lanthanum introduced during the preirradiation treatment did not lead to any significant error.

A similar method was employed in the determination of tin in semiconductor grade indium [7]. Tin was separated from a $1 \mathrm{~g}$ indium sample by dissolution of the sample and iodide extraction of the tin, followed by its precipitation on $\mathrm{Pb}(\mathrm{OH})_{2}$. The $\mathrm{Pb}(\mathrm{OH})_{2}$ precipitate together with the coprecipitated tin was irradiated together with a standard in a nuclear reactor. After irradiaton the tin was substoichiometrically extracted with BPHA. A number of blank determinations were carried out. In most cases the tin content in the blank was lower than the lower detection limit, i.e., $10^{-8} \mathrm{~g} \mathrm{Sn}$. This result was satisfactory because the tin content in the indium sample analyzed was of the order of $10^{-6}$ to $10^{-5} \mathrm{~g} \mathrm{Sn} / \mathrm{g} \mathrm{In}$.

In many cases the impurities concentrate on the surface layers of the metal. About 10 elements were identified in semiconductor silicon plates by means of $\gamma$-spectrometry [8]. By using autoradiography it has been shown that the elements are inhomogeneously distributed in the plane of the plate surface (figs. 8 and 9). Qualitative analysis as well as the distribution of black spots on the autoradiograms suggested that the observed contaminations were concentrated on the surface. This was proved by removing thin layers (of a total thickness of $0.3 \mu \mathrm{m}$ ) from the central region of a plate. Most of the contaminating elements were removed (fig. 10). The example shows that the interpretation of the results of determinations must be done very carefully. In cases similar to that discussed above it is necessary to take off surface layers and to carry out the determinations for each layer and for the rest of the plate separately. If the determinations were carried out on a whole plate, treated as one sample, the result would be misleading.

The weight of the analyzed sample must be accurately known, but it cannot always be directly determined. When determining the concentration profiles of dopants in semiconductor silicon plates, thin surface layers 


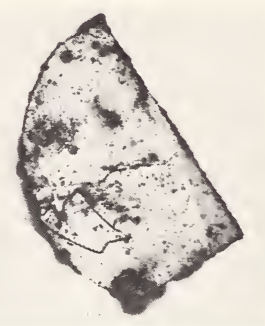

Figure 8 An autoradiogram of an irradiated Si plate (1X).

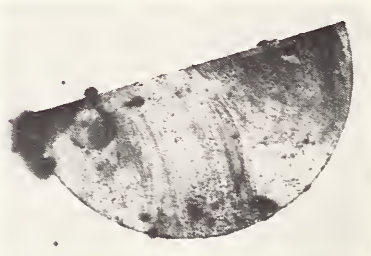

Figure 9. An autoradiogram of an irradiated Si plate (1X).

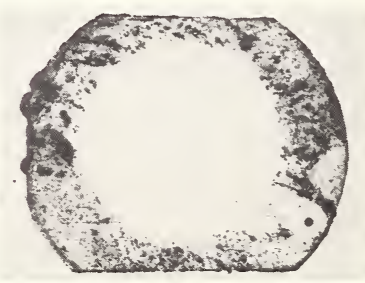

Figure 10. An autoradiogram of an irradiated Si plate after removing thin layers from the central part of the plate (1X).

were removed by anodic oxidation followed by dissolution of the formed oxide [9]. The thickness of the layer removed in this way depends on many parameters. The literature lacks relevant data. The dependence of the thickness of the removed layer on some parameters was examined and it was found for plates not implanted and implanted with phosphorus that the layers removed in the same way differed in thickness by a factor of 2 [10]. Hence the use of a single calibration curve for many types of silicon, which is common practice, can result in serious errors in the determination of concentration profiles. 


\section{References}

[1] Waliś, L., Radwan, M., Štverak, B., and Roušar, I., Radioisotopy 12, 359 (1971).

[2] Waliś, L. and Radwan, M., Neue Hütte 17, 44 (1972).

[3] Grošel, J. and Radwan, M., Isotopenpraxis 7, 449 (1971).

[4] Waliś, L., Postępy Techniki Jądrowej 11, 1285 (1967).

[5] Jaskólska, H., Rowińska, L., and Radwan, M., J. Radioanal. Chem. 20, 419 (1974).

[6] Girardi, F. F. and Sabbioni, E., J. Radioanal. Chem. 1, 169 (1968).

[7] Jaskólska, H., J. Radioanal. Chem. (in print).

[8] Jaskólska, H., Waliś, L., and Rowińska, L., Elektronika (in print).

[9] Duffek, E. F.. Benjamini, E. A., and Mylroie, C., Electrochem. Technol. 3, 75 (1965).

[10] Jaskólska, H., Waliś, L., Golkowska, H., and Wojtowicz-Natanson, B., (prepared for printing). 

Part IV. ANALYSIS 



\title{
ANALYTICAL METHODOLOGY FOR ACCURATE DETERMINATION OF TRACE CONSTITUENTS IN HIGHLY PURE MATERIALS
}

\section{Philippe Albert}

\author{
Directeur des Recherches \\ Service du Cyclotron \\ C.N.R.S. Orleans \\ Orleans Cedex, France
}

High purity materials analysis is a difficult problem and different analytical methods are used. This paper is devoted to the discussion of the principles of analytical methodology in two ways: 1 . the accurate determination of one or a few trace elements in a high purity material, and 2 . the accurate determination of the "complete analysis" in a high purity material with the purpose of defining the true number of "nines," at the level of six or seven "nines," in the conventional definition in percent purity. In the first case the ultimate goal of analytical measurement is a numerical result of known accuracy. The necessary conditions for the accurate and precise analytical determination of an element in a high purity material are discussed and the calibration, the "blank" and the "clean rooms," surface contamination, chemical recovery, and finally the need of certified purity reference materials are debated. In the second case the main problem is to make a selection of some complementary multielement trace analysis methods.

Discussion includes identifying and resolving the problems and identifying what areas require further research. Conclusions are made on general philosophy and attitude concerning the necessity of a general approach to guarantee the accuracy of trace analysis.

Keywords: Contamination; gas analysis; high purity: materials analysis; neutron activation analysis.

\section{Introduction}

When I accepted to speak at this conference, I realized that this was a difficult task. Indeed, if chemical analysis is always a delicate matter, trace analysis is a source of extreme difficulties. 
The first thing to do probably is to attempt to attach a precise meaning to the word "trace" and to some other words that will be much used during this symposium. Next will be to set the limits of this presentation.

However, in this introduction it is necessary to say a few words on the importance of trace analysis in pure materials. This importance can undoubtedly be proven by giving a few examples of the sum of effort and of money that has been devoted to this problem in many countries by numerous private or governmental organizations.

In the United States, industry and universities have created specialized laboratories for trace analysis and, in many instances, have made very expensive equipment available to outside users. The National Bureau of Standards has been reinstated in new buildings and now has considerable means at its disposal. In France, the A.E.C. has developed similar laboratories at Saclay, Grenoble and Fontenay-aux-Roses. One of them, the "laboratoire Pierre Süe" specializes in neutron activation analysis and was built jointly by the A.E.C. and the C.N.R.S.

Recently, the C.N.R.S. has built a cyclotron that will be in large part devoted to trace analysis by activation with charged particles.

Among the numerous conferences dealing with the subject in which we are interested today, several important ones are cited [1-6].

B. Hannay, and more recently Robert A. Laudise, Head of the Crystal Chemistry Research Department of the Bell Telephone Laboratories, have shown that trace analysis is of primary importance in the semiconductor industry at different stages of the production: research, development, and quality control [1-2]. The properties of many metals and alloys also depend upon trace elements; consequently the metallurgical industry needs trace analysis not only for quality control, but also for the study of basic phenomena that are induced by the trace elements [7-9].

At this symposium a number of communications dealing with the determination of the proper experimental conditions to achieve good accuracy have been presented; a few results of intercomparison of methods have been given also.

For this conference some general principles from the results of intercomparisons organized by the O.E.C.D., by the "Bureau Eurisotop" of the European communities, and by the "Bureau National de Métrologie" in France will be discussed.

However, the second part of this presentation will be a discussion of the different methods used for trace analysis.

Now, let us try to define the concept of "trace concentration" and discuss the notion of precision and accuracy in trace analysis. 


\section{Preliminary Definitions}

\section{A. Definition of "Trace"}

In some fields, like geology and geochemistry, the concentration of a "trace element" can range from about 1 to $100 \mathrm{ppm}\left(10^{-6}\right.$ to $\left.10^{-4} \mathrm{~g} / \mathrm{g}\right)$. For specialists in the field of semiconductors, however, this concentration ranges from 0.001 to $1 \mathrm{ppb}\left(10^{-12}\right.$ to $\left.10^{-9} \mathrm{~g} / \mathrm{g}\right)$, while concentrations of 1 to $100 \mathrm{ppm}$ correspond to doped material.

To clarify the definition of trace, H. Kaiser has proposed [10] the terminology "scope of analysis" which is based on three numbers:

$S=$ the weight of the sample.

$q=$ the total weight of the impurity contained in the sample.

$c=$ the concentration of the impurity.

In figure 1 three examples are given:

$S \cdot c(0 ; 6)$ means: Sample $=1 \mathrm{~g} ; c=10^{-6}$; consequently the total weight of the impurity $(q)$ is $1 \mu \mathrm{g}$.

$S \cdot c(1 ; 9)$ means: Sample $=0.1 \mathrm{~g} ; c=10^{-9}$; then $q=10^{-10} \mathrm{~g}$.

For $S \cdot c(3 ; 3) S=10^{-3} \mathrm{~g} ; c=10^{-3}$; and $q=10^{-6} \mathrm{~g}$.

The three numbers $q, c$ and $S$ are related by the following equation:

$$
\log q=\log c+\log S
$$

The scope of analysis is then represented as shown in figure 1 by the two figures in the parentheses and by the two indexes subscripted to these parentheses. For example, $(1 ; 9)_{2,2}$ means $c=10^{-9}$ with a possible variation between $10^{-10}$ and $10^{-8}$ and $S=10^{-1} \mathrm{~g}$ with a variation between $10^{-2}$ $\mathrm{g}$ and $1 \mathrm{~g}$.

\section{B. Definition of Highly Pure Material and of Trace Constituent}

Different studies have lead to two conclusions:

-Some trace constituents modify the properties of the "pure" matrices in which they are present.

- There is interaction between some of these trace elements, the effect of this interaction being either an increase or a decrease of the observed phenomena. 


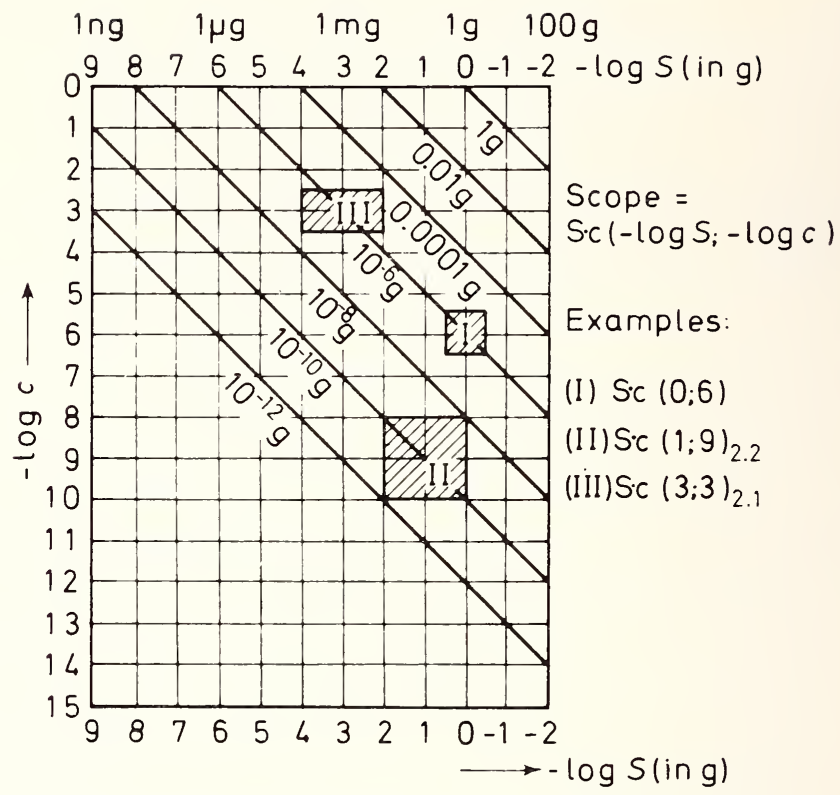

Sample weight: S(ing)

Concentration: c(as decimal fraction)

Quantity of component: $q$ (ing)

$\log q=\log c+\log S$

Figure 1. "Scope" of analysis: Working Scales. $\mathrm{S}=$ sample weight (in grams), c= concentration as the decimal fraction, $\mathrm{q}=$ quantity of component (in grams) and $\log q=$ $\log c+\log S$.

It is then quite clear that to be able to study and explain these phenomena, it is necessary to:

$-\mathrm{Be}$ able to determine all the elements with a sensitivity such that the sum of the detection limits for the undetected elements is at least smaller than the smallest concentration for which the influence of an impurity can be observed.

- Be able to perform the determinations with adequate precision and accuracy in the desired ranges of concentrations.

- Be able to perform a multielemental analysis on a small sample, either because there is not much sample available or because the concentrations vary throughout a sample out of which smaller samples are taken to observe the variation of the concentrations (example: zone refining). 


\section{Definitions of Precision and Accuracy}

An accurate determination is supposed to give the "real concentration." It is well known that it is much safer to say that a "reproducible value" with an associated precision is obtained than to say that the value represents the "true concentration." Indeed, it has been well established that a value can be obtained with a high precision (i.e., reproducibility) and can, at the same time, be very inaccurate.

The reasons for this may be the following:

- Interference from the matrix or from another impurity.

- Blank problem.

- Loss during chemical separation or dissolution.

- Incorrect standardization.

These causes of error are very difficult to deal with when a method is used for trace analysis.

\section{Methods Used for Trace Analysis}

During the last 10 years there has been a considerable improvement in physical and chemical methods of analysis: among these are: polarography, atomic absorption, atomic fluorescence, spectrofluorimetry. flame emission spectrometry. spark source mass spectrometry, isotopic dilution with spark source mass spectrometry, ionic microprobes. infrared spectrometry, etc.

Nuclear methods also have been considerably improved because of the use of high resolution detectors (Ge-Li), the use of computers, and because many new radiochemical separations have been developed. The use of high energy charged particles and photons was found to be particularly suitable to determine low concentrations of $\mathrm{C}$. N, and O. Methods using low energy charged particles were developed for thin film studies. Also, the "nuclear track technique" and isotopic dilution with radioisotopes and substoichiometry have been highly developed. Table 1 gives the "scopes of analysis" of several of these methods.

TABLE 1. Scope of analysis for several highly sensitive methods used for trace analysis

$\begin{array}{lll}\text { Neutron activation analysis } & : & \mathrm{S} \cdot \mathrm{C}(0 ; 9)_{2,3} \\ \text { Charged particle activation analysis } & : & \mathrm{S} \cdot \mathrm{C}(1 ; 8)_{1,3} \\ \text { Spark source mass spectrometry } & : & \mathrm{S} \cdot \mathrm{C}(3 ; 8)_{1,1} \\ \text { Ion probe Slodzian-Castaing } & : & \mathrm{S} \cdot \mathrm{C}(5 ; 6)_{1,2}\end{array}$

s See text or reference [5] for explanation of abbreviations used. 


\section{A. Determination of Trace Elements in AN Ultra-PURE MATERIAL}

This point will be discussed by giving several examples.

Example 1. Determination of oxygen in aluminum.

Fifteen years ago, the results obtained by the method of reducing fusion were of the order of 10 to $20 \mathrm{ppm}$ [15].

Ten years ago in 1964, we could show using ${ }_{2}^{3} \mathrm{He}$ activation with the HILAC of the Lawrence Radiation Laboratory in Berkeley, that the oxygen content of aluminum (quality 99.999\%) was of the order of $0.4 \mathrm{ppm}$. A few years later, we were able to demonstrate using the same technique [12-15] that the oxygen content of aluminum (quality $99.999 \%$ ) is $0.21 \pm$ $0.02 \mathrm{ppm}$ and that the oxygen content of ultra-pure aluminum obtained by zone refining is $\leqslant 0.04 \mathrm{ppm}$.

In 1969, the "Bureau Eurisotop" of the European communities launched a project on the determination of oxygen in nonferrous metals [16]. Many specialized laboratories participated in this project, using all methods available [17]. The conclusions that can already be drawn from this study are the following:

1. For $\mathrm{Al}(99.5 \%)$ and $\mathrm{Al}(99.999 \%)$, the dispersion of the results was high, regardless of the method used.

2. This dispersion could be the result of inexperience of the laboratories, whichever method was used, or improper adaptation of the apparatus and techniques to a new material or to a material whose purity is different from what it was previously.

3. Several causes for the dispersion were clearly pointed out:

- Two laboratories using reducing fusion did not use the correct apparatus.

- There was the influence of surface oxygen; it is well known that aluminum is covered with a thin film of alumina, whose thickness has to be measured exactly in order to make a correction (for most methods). The influence of the surface oxygen is shown in table 2.

- In $14 \mathrm{MeV}$ neutron activation, a new cause of error was discovered $[17,18]$. Because of the reaction ${ }^{16} \mathrm{O}(\mathrm{n}, \mathrm{p})^{16} \mathrm{~N}$ on the oxygen atoms of the rabbit of the pneumatic transfer system, ${ }^{16} \mathrm{~N}$ atoms were implanted in the sample by recoil (see table 3 ).

4. An increase in the quality of the results was observed as more and more comparisons were performed. A relatively important variation of 
TABLE 2. Influence of surface oxygen on the determination of bulk oxygen for aluminum and copper samples using reducing fusion ${ }^{\mathrm{a}}$

\begin{tabular}{|c|c|c|c|c|c|}
\hline Metal & $\begin{array}{l}\text { Sample } \\
\text { weight } \\
\text { (g) }\end{array}$ & $\begin{array}{l}\text { Sample } \\
\text { area } \\
\left(\mathrm{cm}^{2}\right)\end{array}$ & $\begin{array}{l}\text { Surface oxygen } \\
\text { after etching in } \\
\text { the best conditions } \\
\left(\mathrm{mg} / \mathrm{cm}^{2}\right)\end{array}$ & $\begin{array}{r}\text { Bulk } \\
\text { oxygen } \\
\text { content } \\
(\mu \mathrm{g} / \mathrm{g})\end{array}$ & $\begin{array}{c}\text { Error } \\
\text { in the } \\
\text { determination } \\
(\%)\end{array}$ \\
\hline \multicolumn{6}{|l|}{ Aluminum } \\
\hline $99.5 \%$ & 2 & 4.7 & $0.45 \pm 0.15$ & 1 & $105 \pm 35$ \\
\hline $99.99+\%$ & 2 & 4.7 & $.20 \pm .10$ & 0.2 & $235 \pm 117$ \\
\hline Copper & 2 & 2.4 & $0.30 \pm 0.10$ & 1 & $36 \pm 12$ \\
\hline
\end{tabular}

a Eurisotop, report 76.

TABLE 3. Influence of various parameters on the determination of bulk oxygen in aluminum samples, using $14 \mathrm{MeV} \mathrm{NAA^{ \textrm {a } }}$

\begin{tabular}{|c|c|c|}
\hline $\begin{array}{l}\text { Activation and } \\
\text { counting conditions }\end{array}$ & $\begin{array}{c}\text { Apparent } \\
\text { oxygen content } \\
(\mu \mathrm{g} / \mathrm{g})\end{array}$ & $\begin{array}{l}\text { Number of } \\
\text { determinations }\end{array}$ \\
\hline $\begin{array}{l}\text { 1. Transfer system } \\
\text { with air, sample } \\
\text { without container. } \\
\text { 2. Nitrogen as } \\
\text { carrier gas } \\
\text { 3. Etching after } \\
\text { irradiation in } \\
\mathrm{NaOH},(160 \mathrm{~g} / 1) \\
\text { at } 95^{\circ} \mathrm{C} \text { for } 5 \mathrm{~s}, \\
\text { water rinse, counting } \\
12 \mathrm{~s} \text { after irradiation } \\
\text { (air as carrier gas) }\end{array}$ & $\begin{array}{r}17.8 \pm 0.7 \\
9.4 \pm 1.2 \\
1.6 \pm 0.4\end{array}$ & $\begin{array}{l}12 \\
60\end{array}$ \\
\hline
\end{tabular}

${ }^{a}$ From Dugain, F., Speecke, A., Andre, M., and Michout, C. [18].

the results obtained by reducing fusion was noted, as seen in table 4 . Table 5 shows the values that were finally retained.

Example 2. Determination of oxygen in copper.

This study was performed under the same conditions as for aluminum. The same problems were observed but the dispersion was less. Indeed, the difficulties of this analysis are not so severe as in the case of aluminum:

- Less oxygen on the surface of the samples (table 4).

- Easier reduction; possibility to use hydrogen reduction with large $(\sim 20 \mathrm{~g})$ samples. 
TABLE 4. Results of oxygen determination in 99.5 and 99.999 percent aluminum ${ }^{\mathrm{a}}$

\begin{tabular}{|c|c|c|c|c|c|}
\hline \multirow{2}{*}{ Methods } & \multirow{2}{*}{ Laboratories } & \multicolumn{2}{|c|}{$99.5 \%$} & \multicolumn{2}{|c|}{$99.999 \%$} \\
\hline & & Series 1 & Series 2 & Series 1 & Series 2 \\
\hline $\begin{array}{l}\text { Reducing } \\
\text { Fusion }\end{array}$ & $\begin{array}{l}\mathrm{A} \\
\mathrm{B} \\
\mathrm{C}\end{array}$ & $\begin{array}{l}2.8 \pm 0.6 \\
6.4 \pm 1.9 \\
0.8 \pm 0.3\end{array}$ & $\begin{array}{l}1.5 \pm 0.6 \\
4.0 \pm 0.5\end{array}$ & $2.5 \pm 0.7$ & $\begin{array}{l}1.2 \pm 0.6 \\
2 \text { to } 30\end{array}$ \\
\hline $\begin{array}{l}14 \mathrm{MeV} \\
\text { neutron } \\
\text { activation }\end{array}$ & $\begin{array}{l}\text { A } \\
B \\
C \\
D \\
E\end{array}$ & $\begin{array}{l}0.9 \pm 0.5 \\
3.9 \pm .8 \\
2.9 \pm 1.5 \\
2.3 \pm 1.5\end{array}$ & $1.3 \pm 0.5$ & $\begin{array}{l}1.2 \pm 0.6 \\
2.8 \pm 1.5\end{array}$ & 25 \\
\hline $\begin{array}{l}\text { Activation } \\
\text { by helium-3 }\end{array}$ & $\begin{array}{l}\text { A } \\
\text { B }\end{array}$ & $\begin{array}{l}1-57 \\
4-7\end{array}$ & $\begin{array}{r}0.6 \pm 0.1 \\
.5 \pm .1\end{array}$ & $\begin{array}{l}3.7-33 \\
0.16 \pm 0.02\end{array}$ & $\begin{array}{r}0.2 \pm 0.03 \\
.2 \pm .02\end{array}$ \\
\hline$\gamma$ photons & $\begin{array}{l}\mathrm{A} \\
\mathrm{B}\end{array}$ & $\begin{array}{l}\leq 1 \\
\leq 1\end{array}$ & $0.6 \pm 0.1$ & $\leq 0.3$ & \\
\hline
\end{tabular}

${ }^{a}$ From Eurisotop prereport.

TABLE 5. Comparison of results for oxygen determination in aluminum by three different methods ${ }^{\mathrm{a}}$

Aluminum, 99.5\%

Aluminum, $99.99+\%$

Reducing fusion

(1 laboratory)

$1.2 \pm 0.6$

$14 \mathrm{MeV}$ neutron activation

(1 laboratory)

Helium-3 activation

(2 laboratories)

$0.5 \pm 0.2$

$\gamma$ photon activation

$0.55 \pm 0.15$

$0.20 \pm 0.03$

$0.60 \pm 0.10$

a Eurisotop, information note, July 1974.

The results obtained by the different laboratories whose results were finally retained are given in table 6 . It is worth remembering that analysis by charged particle activation was performed on $\sim 10$ to $15 \mathrm{mg}$ samples, while analysis by hydrogen reduction required $\sim 20 \mathrm{~g}$ samples.

The results obtained by reducing fusion and $14 \mathrm{MeV}$ NAA are not shown in this table; they were not retained by the Commission because they were considered to be inaccurate. Indeed, the mean values obtained by these methods are almost consistently higher than those shown in table 
TABLE 6. Oxygen determination in pure industrial copper $(O F H C)^{\mathrm{a}}$

$$
\bar{X} \pm s
$$

12

Helium-3 activation

(1 laboratory)

$\gamma$ photon activation (1 laboratory)

Hydrogen reduction A laboratory B laboratory
$1.3 \pm 0.3$

$1.5 \pm 0.5$

1.5

$1.3 \pm 0.2$

$1.1 \pm .3$
Number of determinations

a From Euristop information note, July 1974, ITE No. 79.

6. There is a considerable scatter of these mean values, and the associated precision is poor. (See fig. 7 and fig. 8 of the communication by J. N. Barrandon and J. L. Debrun of this symposium.)

Example 3. Analysis of several elements in high purity $\mathrm{Al}$ and $\mathrm{Fe}$.

These studies were performed by several laboratories including the C.E.C.M. at Vitry and the "Pierre Süe at Saclay for activation analysis. In the case of iron, the "Bureau National de Métrologie" [19] had a project for the preparation of a standard "pure iron." Problems were encountered for the determination of carbon below the ppm level; this had already been observed [20-22].

For sodium, potassium, manganese, arsenic, copper, chromium, molybdenum, zinc, phosphorus, sulfur, and zirconium, important differences were observed between the results obtained by activation and those obtained by spark source mass spectrometry $[8,9,23]$.

Some examples of these differences are given in tables 7 and 8 .

Consideration of infrared spectrometry, spark source mass spectrometry, ion probe (Slodzian-Castaing) and counting of particles emitted during nuclear reactions:

Infrared absorption spectrometry is used to determine boron, carbon and oxygen in silicon and germanium [6], but must be standardized with the help of other methods like activation analysis [24]. Using optimal experimental conditions it is possible to determine the three elements above at the atomic ppb level and even for boron down to 0.04 atomic ppb [6].

Spark source mass spectrometry is well adapted to multielemental semiquantitative analysis down to 5 to $50 \mathrm{ppb}$ depending upon the elements and the experimental conditions.

It seems even possible [25-26] for the analysis of carbon, nitrogen, and 
TABLE 7. Example of discrepancies between NAA and SSMS for the analysis of sodium, potassium and manganese in aluminum and iron ${ }^{\mathrm{a}}$

\begin{tabular}{lccccr}
\hline & \multicolumn{2}{c}{ Aluminum $(\mu \mathrm{g} / \mathrm{g})$} & & \multicolumn{2}{c}{ Iron $(\mu \mathrm{g} / \mathrm{g})$} \\
\cline { 2 - 3 } \cline { 5 - 6 } Element & SSMS & NAA & & SSMS & NAA \\
\hline NA & $1-5$ & $0.04-0.05$ & & $0.07-0.15$ & $0.006-0.001$ \\
K & $0.6-4$ & $\leq 0.0015$ & & $0.25-1$ & $\leq 0.1$ \\
Mn & $0.1-0.15$ & $0.04-0.013$ & & $0.7-0.6$ & 0.1 \\
& .17 & .007 & & $.3-.25$ & .1 \\
S & & & $7 \pm 2$ & $1.3 \pm 0.6$ \\
\hline
\end{tabular}

a See reference [14] and BNM results.

Notes: $\operatorname{SSMS}=$ spark source mass spectrometry.

NAA $=$ neutron activation analysis.

TABLE 8. Standardization of SSMS by means of NAA in the case of the analysis of sodium in aluminum ${ }^{\mathrm{a}}$

\begin{tabular}{|c|c|c|c|}
\hline \multirow{2}{*}{$\begin{array}{l}\text { Relative } \\
\text { sodium } \\
\text { content of } \\
\text { standard }\end{array}$} & \multicolumn{2}{|c|}{ NAA $(\mu \mathrm{g} / \mathrm{g})$} & \\
\hline & $\begin{array}{c}\text { Vitry } \\
\text { laboratory }\end{array}$ & $\begin{array}{c}\text { Grenoble } \\
\text { AEC } \\
\text { laboratory }\end{array}$ & values only) \\
\hline
\end{tabular}

a See reference [14].

Notes: $\mathrm{NAA}=$ neutron activation analysis.

SSMS = spark source mass spectrometry.

oxygen to lower the blanks to $0.07 \mathrm{ppm}, 0.04 \mathrm{ppm}$ and $0.02 \mathrm{ppm}$ respectively. This is achieved by cryogenic pumping at $4.2 \mathrm{~K}$ (liquid helium), the vacuum being equal to $3 \cdot 10^{-8} \mathrm{~mm} \mathrm{Hg}$ near the spark source.

The ion probe of Slodzian-Castaing is extremely sensitive; indeed it has been reported [6] that concentrations of 1 to $0.01 \mathrm{ppm}$ were measured with a sample equal to $10^{-6}$ to $10^{-7} \mathrm{~cm}^{3}$ in volume. The major problem is in the standardization, since the relative emission coefficients may vary considerably from one type of sample to another. This standardization can be achieved by means of several other methods: ion implantation, ac- 
tivation analysis, and direct measurements involving nuclear reactions. Using the last technique, it is possible to measure the repartition of the light elements in films a few microns thick, the concentrations being of the order of $1 \mathrm{ppm}$ to several hundreds of $\mathrm{ppm}$, and the resolution in depth being about $1000 \AA[6]$.

With this method, the creation of images is particularly interesting because of the high resolution obtained: 1 micron (beam of 250 microns in diameter), $100 \AA$ for the resolution in depth, image X100 or X200.

\section{B. "Global" Analysis of Trace Elements in High Purity Material}

In this type of analysis, most elements, if not all, must be determined.

This can only be achieved by using several methods of multielemental analysis. How should the choice of the methods be made and which method should be trusted more than the others? I will try to give some answers, with the help of a work sponsored by the O.E.C.D. [27] and with the help of some studies that are being made at the B.N.M. [19].

Conclusions of the O.E.C.D. project on the analysis of $C u$ and $G e$ :

Spark source mass spectrometry and neutron activation are the only techniques that gave results for a large number of elements, detected or undetected.

The results obtained by mass spectrometry present a very high dispersion (20 laboratories participated for $\mathrm{Cu}$ and 7 for $\mathrm{Ge}$ ) and it is not possible to ascertain probable mean values for the detected elements, indeed there are 2 and even 3 orders of magnitude between results obtained by different laboratories, especially for $\mathrm{Cu}$. In germanium, mass spectrometry could give results for only 24 elements.

For quite a few elements there is a great discrepancy between activation and mass spectrometry, the results being much higher using the latter technique:

for Oxygen : 2 to 500 times higher

for Chromium : 100 to 2000 times higher

for Nickel : 80 to 180 times higher

for Zinc : 200 to 2000 times higher

for Silver : 100000 to 200000 times higher

The interesting point is that except for oxygen all activation results were obtained by direct $\gamma$-ray spectrometry, without chemistry, and these results may then be really trusted. 
Remarks on the results of the B.N.M. project on high purity iron:

Emission spectrometry gives limits of detection for 13 undetected elements, and these limits range from 1 to $15 \mathrm{ppm}$. Copper, chromium, molybdenum, manganese, and nickel are detected and determined at concentrations ranging from 0.2 to $8 \mathrm{ppm}$.

Twenty elements were detected with spark source mass spectrometry and limits of detection from $50 \mathrm{ppb}$ to $1 \mathrm{ppm}$ were obtained for others.

Nine elements were detected with neutron activation and limits of detection from 1 to $100 \mathrm{ppb}$ were given for others.

Four elements were detected using charged particles and photon activation analysis.

It is worth noting that activation is more sensitive than spark source mass spectrometry for some elements and vice-versa. The following table shows some elements for which neutron activation is much better than SSMS.

\section{General Principles of Methodology for Trace Analysis in High Purity Materials}

1. The most important quality of a method for trace analysis is the accuracy. Good precision may result in very reproducible inaccurate measurements.

To try to obtain an accurate analysis one can compare the results of several independent methods, used by specialists working independently.

2. We must be aware of the fact that it is possible to encounter occasions where the value obtained by a single method may be the "true value," even if other independent methods all obtain an identical result, different from the value found by the single method.

3. If a method is subject to contamination, it must be routinely checked by a reference method.

4. Purity can only be defined when the analysis is complete.

5. A complete analysis necessitates the use of several multielemental methods.

6. The methods for trace multielemental analysis that must be complementary, are in our opinion at the present time, spark source mass spectrometry and the different methods of activation (thermal, epithermal and fast neutrons, charged particles, and photons).

The ion probe analyzer of Slodzian-Castaing will probably be a very good method for multielemental analysis, at trace levels, after a few more years of development. 
In conclusion, we have at our disposal a number of powerful methods and it is now very important to develop programs of intercomparison of these methods. This is the only manner in which we will be able to increase our knowledge of the actual possibilities of these methods. Then it will be possible to place these techniques into the hands of a greater number of laboratories, for the best benefit of science.

\section{References}

[1] Laudise, R. A., Opportunities for Analytical Chemistry in Solid State Research and Electronics, in Analytical Chemistry: Key to Progress on National Problems, Meinke, W. W. and Taylor, J. K., Eds., NBS Spec. Publ. 351, pp. 19-64, U.S. Government Printing Office, Washington, D.C. 20402 (1972).

[2] Meinke, W. W. and Scribner, B. F., Eds., Trace Characterization: Chemical and Physical, Proceedings of 1 st Materials Research Symposium, National Bureau of Standards, Gaithersburg, Maryland, October 3-7, 1966, NBS Monogr. 100, 580 pages, U.S. Government Printing Office, Washington, D.C. 20402 (1967).

[3] DeVoe, J. R. and LaFleur, P. D., Eds., Modern Trends in Activation Analysis, Proceedings of the 1968 International Conference on Modern Trends in Activation Analysis, National Bureau of Standards, Gaithersburg, Maryland, October 7-11, 1968, NBS Spec. Publ. 312. Vols. I and II, 1334 pages, U.S. Government Printing Office, Washington, D.C. 20402 (1969).

[4] The Activation Analysis of Minute Quantities of Elements - International Colloquium of the C.N.R.S., J. Radioanalyt. Chern. 17 (1-2) (1973); 18 (1-2) (1973); and 19 (12) (1974).

[5] Modern Trends in Activation Analysis, Proceedings of the 1972 International Conference on Modern Trends in Activation Analysis, C.E.N. Saclay, France, October 2-6, 1972, reprinted from J. Radioanalyt. Chem. 15 (2) (1973); 16 (1-2) (1973). Elsevier, Amsterdam (1974).

[6] Abstracts of Colloquium on Material and Technical Characteristics of Semiconductors, D.G.R.S.T., Lafeuille. D., Ed., LETI-CEN Grenoble, Cedex, France.

[7] Chaudron, G., Monograph on High Purity Metals, Masson, Paris (1960).

[8] New Properties of Ultra High Purity Metals, International Colloquium of C.N.R.S., C.N.R.S., Paris (1960).

[9] Physical and Chemical Properties of Ultra High Purity Iron, International Colloquium of C.N.R.S., Scientific Memoirs of Metallurgy Review 65.3 (1968).

[10] Ultra High Purity Metals, Seminar of the American Scciety for Metals, October 2122, 1961, American Society for Metals, Metals Park, Ohio. Reinhold (1962).

[11] Kaiser, H., in International Congress on Analytical Chemistry, Proceedings, April 3, 1972, Kyoto, Japan, Pure and Applied Chemistry 34, (1) 35 (1973).

[12] Albert, P., Report of the C.E.C.M. Mission to the U.S.A., C.N.R.S., Vitry (1964).

[13] Deyris, M., Thesis, U. of Paris. Paris, 1966.

[14] Revel, G., Thesis, U. of Paris, Paris, 1965.

[15] Vialatte, B., Thesis, U. of Paris, Paris, 1972; Vialatte. B. and Revel. G.. Anal. Chem. 44. 1077 (1972); and Vialatte, B., J. Radioanalyt. Chern. 17. 301 (1973). 
[16] Private Communication from G. Propstl, Office of Eurisotop, 200 Rue de la Loi, 1040 Brussels, Belgium.

[17] Kraft, G., Office of Eurisotop Information Bulletin No. 71, 1972; Vandecasteele, G., Speecke, A. and Hoste, J., No. 68; Engelmann, C., No. 74; Boisot, P., Cuypers, M., Kraft, G., and Pauwels, J., ITE Report No. 76; and Quaglia, L. and Weber, G., No. 79, Part 1.

[18] Dugain, F., Andre, M., and Speecke, A., Radiochem. Radioanalyt. Letters 4, (2) 35 (1970); Dugain, F. and Michaut, C., Radiochem. Radioanalyt. Letters 9, (2) 119-125 (1972); and Dugain, F. and Michaut, C., J. Radioanalyt. Chem. 15, 585-590 (1973).

[19] Private Communication from G. Denegre and A. Marshal, National Bureau of Metrology, 21 Ruc Casimir Perier, Paris, France.

[20] Revel, G., Chaudron, T., Debrun, J. L., and Albert, P., C.R. Acad. Science 266, 322 (1968) and Revel, G., J. Radioanalyt. Chem. 3, 421 (1969).

[21] Kapitza, S. P., Samosyuk, V. N. et al., Radiochem. Radioanalyt. Letters 5, (4-5) 217 (1970).

[22] Engelmann, C., Thesis, U. of Paris at Orsay, Orsay, France (1970); Isotop. Radiat. Technol. 8, 118 (1970); J. of Radioanalyt. Chem. 7, 89 and 281 (1971); and Radiochem. Radioanal. Letters 5, (6) 319 (1970).

[23] Roth, E., Cornu, A., and Albert, P., Zeit Anal. Chem. 218B, (24) (1966).

[24] Nozaki, T. et al., J. of Electrochem. Soc. 117, 1566 (1970); J. Appl. Rad. Isotopes 22, 607 (1971).

[25] Rook, H. L. and Schweikert, E., Anal. Chem. 41, 958 (1969).

[26] Millett, E. J., Mullard Research Laboratory, Redhill, Surrey, England (personal communication).

[27] O.E.C.D., Organization for Economic Cooperation and Development, 2 rue Andre Pascal, Paris, France. 


\title{
ANALYTICAL METHODOLOGY - ROCKS AND ORES
}

\author{
Hiroshi Onishi \\ Japan Atomic Energy Research Institute \\ Tokai-mura \\ Ibaraki-ken, Japan
}

To Goldschmidt we owe early knowledge of trace element geochemistry. $\mathrm{He}$ analyzed a variety of geomaterials by emission spectrochemical analysis during 1930-37. Colorimetric (spectrophotometric) procedures have been developed beginning with Sandell since 1936. Studies in trace element geochemistry have been greatly advanced by means of neutron activation analysis since approximately 1950. Isotope dilution mass spectrometry is gaining a central position as a reliable trace element analysis method; this has become possible through scrupulous control of blanks.

The blank can be related to the constituent in question and the interfering (positively or negatively) substances. Correction for contamination during sample preparation is usually very difficult. The blank is affected by reagents, vessels, and atmosphere or environment, all of which require the close attention of the analyst. Ways for reducing blank values are briefly mentioned.

Methods recently described for determination of trace elements in geomaterials are: spectrophotometric and fluorimetric, emission spectrochemical, atomic absorption and flame emission, $\mathrm{x}$-ray fluorescence, nuclear (activation, nuclear track, etc.), electrochemical, mass spectrometry, and gas chromatography. Examples of comparison of methods are given. The following are to be considered in choosing the proper analytical technique: objective of analysis, sensitivity and detection limit, accuracy, precision or reproducibility, determination of single element or multielements, time and cost, and analyst.

Keywords: Accuracy and precision; comparison of trace analytical methods; contamination; rocks and ores; sample preparation; trace element analysis.

\section{Introduction}

If we define about $100 \mathrm{ppm}$ as an upper limit of a trace element in a sample, we have a large number of trace elements in igneous rocks (table 1) $[1,2]$. In this table, elements with parentheses are present as traces in 


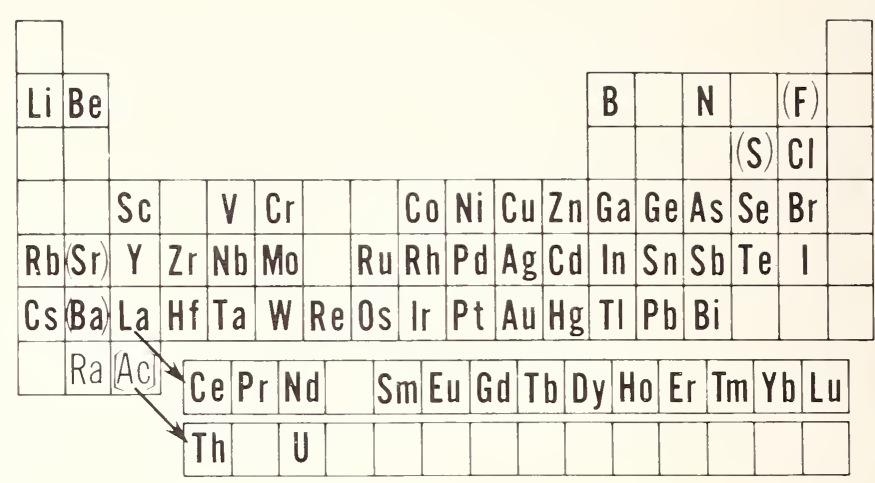

TABLE 1. Trace elements in igneous rocks

some types of igneous rocks. Trace elements in meteorites, e.g., chondrites, are not always the same as those in igneous rocks.

In this paper, methods of trace analysis of rocks and ores are surveyed. An attempt is made to compare the methods so that choosing the proper method is facilitated.

\section{Methods of Trace Analysis of Rocks and Ores}

The following methods, often coupled with separation and concentration, are used for determination of trace elements:

Spectrophotometry and fluorimetry. See, for example, Sandell [3] and Jeffery [4].

Emission spectrochemical methods. See, for example, Ahrens and Taylor [5].

Atomic absorption and flame emission spectrophotometry.

$\mathrm{X}$-ray fluorescence.

Nuclear methods.

Electrochemical methods .

Mass spectrometry. See, for example, Smales and Wager [6].

Gas chromatography.

Fire assay for $\mathrm{Au}$ and $\mathrm{Ag}$.

A review of the past shows that emission spectrochemical analysis was used extensively by Goldschmidt during 1930-37. Colorimetric (spectrophotometric) procedures were being developed by Sandell as early as 1936. Neutron activation analysis made its debut in approximately 1950 . 
Isotope dilution mass spectrometry, which was first used in about 1955 , is gaining a central position as a reliable trace element analysis method. This has become possible through scrupulous control of blanks.

It may not be inappropriate to mention samples which have contributed greatly to trace element analysis and geochemistry. They are:

U.S. Geological Survey G-1 and W-1. Fairbairn et al. [7].

New USGS rocks, G-2, GSP-1, AGV-1, PCC-1, DTS-1, and BCR-1. See, for example, Flanagan [8].

NBS Trace Element Standard Reference Materials:

Trace Elements in Glass, 500 ppm. SRM 610-611

Trace Elements in Glass, 50 ppm. SRM 612-613

Trace Elements in Glass, 1 ppm. SRM 614-615

Trace Elements in Glass, 0.02 ppm. SRM 616-617

Lunar materials:

Apollo 11-17. Proceedings of Lunar Sci. Conferences [9-12]

Luna 16, 20. Luna 20 issue [13].

\section{Blanks, Background, and Noise}

Because accuracy of trace analysis depends on control of blanks and on elimination of effect of other substances (matrix), "blanks" will be discussed briefly. Sometimes the terms blank, background, and noise are used without distinction. Although it may be difficult to draw a line of demarcation among these terms, better definitions of them would be helpful. First we shall see what has been said about blanks. The following selection is chosen somewhat arbitrarily.

According to Hillebrand and Lundell [14], the purpose of a blank analysis is to correct for impurities derived from the reagents or through attack on the vessels used. They caution that the difference in the size of the precipitate between the sample and blank analyses may give an unduly low blank value.

Lundell [15] states that, when a substance is determined, e.g., spectroscopically, after separation and concentration, the sensitivity of such a method is bounded only by the analyst's ability to control his blanks.

Mellor and Thompson [16] remark that the reagents are best tested by blank analyses. However, the blank analysis does not necessarily give absolute certainty, because the reactions without the sample are not necessarily the same as with the sample.

Kolthoff et al. [17] mention that the blank is best carried along simul- 
taneously with the sample. In general, a small blank correction is better than none at all.

Next we shall see what has been said about background. Evaluation of background in x-ray analysis was made by Hevesy [18]. He states that the primary $\mathrm{x}$-ray method suffers from a continuous background, while the secondary $\mathrm{x}$-ray method (x-ray fluorescence) does not.

According to Morrison and Skogerboe [19], when signal reduction (absorption) methods are used, a blank is used to define or set the signal at a particular level. When signal increase (emission) methods are used, the signal for the analyte is compared to that for the background or blank. Inherent in the latter is the noise due to the electronic system.

In spectrochemical analysis, background is due mainly to the emission of continuous radiation from the electrode tips, incandescent particles within the source column, and scattered radiation within the spectrograph [5].

In flame emission spectrophotometry, correction for background is very important [20], and wavelength scanning and nonscanning are used [21]. The use of a synthetic blank is an attempt to eliminate the background effect [20]. In atomic absorption, background absorption occurs as molecular absorption and probably also as scattering by particles in the flame [21].

Background in x-ray fluorescence is defined as the intensity one measures from a sample at the goniometer position for a characteristic line of an element when the particular element is absent from a sample which is otherwise the same in every respect [19]. The background may be due to low resolution, scattering of primary $\mathrm{x}$-rays by the sample, and fluorescent $\mathrm{x}$-rays from analyzing crystal.

Background radiation, which is due to the presence of traces of $U, T h$, $\mathrm{K}$, etc. and to the cosmic radiation, can be reduced by means of proper shielding and anticoincidence circuitry.

In polarography, the diffusion current is measured as the difference between the total current and the small background current ("residual current") observed with the supporting electrolyte alone [22].

In mass spectrometry, background is due to "memory," low resolution, scattering of adjacent ions, etc. [23]. Pretreatment of filaments is often necessary in solid analysis.

In gas chromatography of some metal chelates, how to draw the base line sometimes becomes a problem. This is often related to the resolution of peaks. Thus background correction may often be regarded as a way of drawing a base line on the spectrum.

Although the term "noise" is often used in a broad sense, it should 
preferably be restricted to electrical noise. For electrical noise see, for example, Lewin [24]. In general, corrections for the blank, background, and noise, or corrections for their fluctuations are the limitations to the smallest concentration that can be accurately determined. For discussions on limit of detection, see $[25,26]$. It is suggested that the standard deviation of blank determinations be quoted as the performance characteristic relevant to the power of detection [27].

\section{Reducing Blank Values}

Contamination and losses may occur during sample preparation (crushing, grinding, etc.), decomposition or dissolution of the sample, separation and concentration, and determination or measurement. Correction for contamination during sample preparation is usually very difficult. The blank is affected by the reagents, vessels, and atmosphere or environment, all of which require close attention of the analyst. The element in question (analyte) and interfering substances contribute to the blank. Examples of negatively interfering substances are sulfide ions in a metal analysis and organic substances from ion-exchange resin in color development. The method of decomposing the sample should be selected from the standpoint of effectiveness of the method and availability of pure reagents for decomposition.

A difficulty in blank correction is the effect of matrix on blank analysis. As mentioned above, Hillebrand and Lundell [14] have pointed out that the difference in the size of the precipitate affects the blank value in gravimetric analysis. Moore et al. [28] have described the following experience in isotope dilution analysis. When the blank comprises a significant percentage (i.e., 1 to $10 \%$ ) of the sample size, the separately measured blank is usually smaller by a factor of about 2 than what the actual blank correction should be. It is suspected that the sample itself acts as a carrier during the sample dissolution and separation, whereas the blank is simply the condensate of the various reagents and whatever is leached from the ion exchange resin used for the separation. Ideally, a blank sample, i.e., a matrix that does not contain analyte, is needed.

In determining tin colorimetrically with toluene-3,4-dithiol in the presence of a dispersing agent, Onishi and Sandell [29] experienced that the color was not produced at low tin concentration $(<2.5 \mu \mathrm{g}$ per $10 \mathrm{ml})$. Therefore, to obtain the reagent blank, $5 \mu \mathrm{g}$ of tin was run through the whole procedure and $5 \mu \mathrm{g}$ was deducted from the amount of tin found.

In isotope dilution mass spectrometry, special efforts have been made 
to reduce blank values so that accurate results can be obtained. It is a great advantage of activation analysis that, after irradiation, contamination from reagents and vessels does not affect the result.

As examples of reducing blank values, we may cite studies by Maienthal on iron [30], by Barnes et al. on lead [31,32], and by Arden and Gale on lead [33] and uranium [34]. These workers have lowered the blank coming from the environment by using a clean laboratory and the blank coming from the reagents by purification.

\section{Procedures Recently Described for Determination of Trace Elements}

Tables 2-8 list procedures recently described for determination of trace elements in rocks and ores. The list is not exhaustive; procedures by which standard samples have been analyzed mainly are considered. It may be added that the Proceedings of Lunar Science Conference [9-12] give invaluable information about trace analysis methods. The role of activation analysis in earth sciences has been discussed by Smales [35] and Morrison [36]. Applications of mass spectrometry have been surveyed by Herzog [37]. Usefulness of an ion microprobe mass spectrometer [38] remains to be tested.

Because of limited space, individual methods in tables 2-8 cannot be discussed. Only one paper [31] may be mentioned as a demonstration of high precision (and presumably high accuracy) obtainable with isotope dilution mass spectrometry. Parts per billion to ppm levels of $\mathrm{Pb}, \mathrm{Th}$, and $\mathrm{U}$ in NBS glasses have been determined at NBS and USGS. The precision of the order of 0.5 percent ( $95 \%$ limit of error) is obtained by the method even at the 20-ppb level for these elements.

Separation and concentration methods used for trace element analysis of rocks and ores are not discussed in this paper. 
TABLE 2. Spectrophotometric and fluorimetric methods

Element

Reagent

References

\begin{tabular}{|c|c|}
\hline Co & $\begin{array}{l}\text { 4-(5-Bromo-2-pyridylazo)-1,3- } \\
\text { diaminobenzene } \\
\mathrm{As}_{2} \text { in } \mathrm{CCl}_{4}\end{array}$ \\
\hline Mo, W & Toluene-3,4-dithiol \\
\hline $\begin{array}{l}\mathrm{Mo} \\
\mathrm{Nb}\end{array}$ & $\begin{array}{l}\text { Catalytic } \\
\text { 4-(2-Pyridylazo)-resorcinol }\end{array}$ \\
\hline $\mathrm{Sb}$ & Rhodamine B \\
\hline Sc & 4-(2-Thiazolylazo)- resorcinol \\
\hline Sn & Phenylfluorone \\
\hline Th & Arsenazo III \\
\hline $\mathrm{Tl}$ & Rhodamine $\mathrm{B}^{\mathrm{a}}$ \\
\hline V & Benzoylphenylhydroxylamine \\
\hline V & $\begin{array}{l}\text { As phosphotungstovanadic acid } \\
\text { (visual comparison) }\end{array}$ \\
\hline W & Toluene-3,4-dithiol \\
\hline $\mathrm{Zr}$ & Quercetinª \\
\hline
\end{tabular}

Kiss, E., Anal. Chim. Acta 66 385 (1973).

Grimaldi, F. S., and Schnepfe, M. M., Anal. Chim. Acta 53, 181 (1971).

Kawabuchi K., and Kuroda, R., Talanta 17, 67 (1970).

Fuge, R., Analyst 95, 170 (1970).

Greenland, L. P., and Campbell, E. Y., Anal. Chim. Acta 49, 109 (1970).

Schnepfe, M. M., Talanta 20, 175 (1973).

Shimizu, T., and Momo, E., Anal. Chim. Acta 52, 146 (1970).

Smith, J. D., Anal. Chim. Acta 57, 371 (1971).

Korkisch, J., and Dimitriadis, D., Talanta 20, 1199 (i973).

Matthews, A. D., and Riley, J. P., Anal. Chim. Acta 48, 25 (1969).

Donaldson, E. M., Talanta 17, 583 (1970).

Roberts, J. L., Talanta 18, 1070 (1971).

Quin, B. F., and Brooks, R. R., Anal. Chim. Acta 58, 301 (1972).

Desai, S. R., and Sudhalatha, K., Anal. Chim Acta 55, 395 (1971).

a Fluorimetric. The others are spectrophotometric methods.

TABLE 3. Emission spectrochemical methods

Elements

Remarks

References
$\mathrm{Ba}, \mathrm{Co}, \mathrm{Cr}, \mathrm{Cu}, \mathrm{Ni}$, A $\mathrm{Sr}, \mathrm{V}$ adsorbed on a cation-exchange resin, and the resin is analyzed.
Govindaraju, K., Analysis 2, 367 (1973).

$$
\begin{aligned}
& \text { Pt, Pd, Ag } \\
& \text { Co, Ga, Sc }
\end{aligned}
$$
Concentration by fire assay
Koleva, E. G., and Arpadjian, S. H., Talanta 17, 1018 (1970).
Results of analysis of a lunar soil sample agree with those of NAA.
Taylor, S. R., et al., Geochim. Cosmochim. Acta 37, 2665 (1973).


TABLE 4. Atomic absorption and flame emission methods

$\mathrm{Li},{ }^{\mathrm{a}} \mathrm{Rb},{ }^{\mathrm{a}} \mathrm{Cs},{ }^{\mathrm{a}} \mathrm{Sr},{ }^{\mathrm{b}} \mathrm{Ba}^{\mathrm{b}}$

$\mathrm{Rb},{ }^{\mathrm{a}} \mathrm{Cs}^{\mathrm{a}}$

$\mathrm{Rb},{ }^{\mathrm{b}} \mathrm{Cs}^{\mathrm{b}}$

$\mathrm{Au}, \mathrm{Ru}, \mathrm{Rh}, \mathrm{Pd}, \mathrm{Pt}$

$\mathrm{Bi}, \mathrm{Cd}, \mathrm{Hg}, \mathrm{Pb}, \mathrm{Sr}, \mathrm{Tl}$

$\mathrm{Ba}, \mathrm{Co}, \mathrm{Cr}, \mathrm{Cu}, \mathrm{Ni}, \mathrm{V}$

$\mathrm{Ag}$

$\mathrm{Ag}, \mathrm{Au}$

$\mathrm{Au}$

Be, V

$\mathrm{Hg}$

$\mathrm{Hg}^{\mathrm{c}}$

Ir

$\mathrm{Li}$

$\mathrm{Li}, \mathrm{Zn}$

$\mathrm{Na}, \mathrm{K}$

$\mathrm{Pb}$

$\mathrm{Pd}, \mathrm{Pt}$

$\mathrm{Pt}$

$\mathrm{Rh}$

$\mathrm{Sn}$

$\mathrm{V}$

$\mathrm{V}$, Mo

$\mathrm{Zn}$
Abbey, S., Geol. Surv. Canada Paper 71-50 (1972).

Allen, W. J. F., Anal. Chim. Acta 59, 111 (1972).

Langmyhr, F. J., and Thomassen, Y., Z. Anal. Chem. 264, 122 (1973).

Mallett, R. C., et al., Talanta 19, 181 (1972).

Heinrichs, H., and Lange, J., Z. Anal. Chem. 265, 256 (1973).

Warren, J., and Carter, D., Proc. Soc. Anal. Chem. 11, 53 (1974).

Chao, T. T., Ball, J. W., and Nakagawa, H. M., Anal. Chim. Acta 54, 77 (1971); Moloughney, P. E., and Graham, J. A., Talanta 18, 475 (1971); Walton, G., Analyst 98, 335 (1973); Chowdhury, A. N., Das, A. K., and Das, T. N., Z. anal. Chem. 269, 284 (1974).

Bratzel, M. P., Jr. et al., Anal. Chem. 44, 372 (1972).

Hildon, M. A., and Sully, G. R., Anal. Chim. Acta 54, 245 (1971).

Terashima, S., Bunseki Kagaku 22, 1317 (1973)

Omang, S. H., and Paus, P. E., Anal. Chim. Acta 56, 393 (1971); Aston, S. R., and Riley, J. P., ibid. 59, 349 (1972).

Muscat, V. I., and Vickers, T. J., Anal. Chim. Acta 57, 23 (1971).

Grimaldi, F. S., and Schnepfe, M. M., Talanta 17, 617 (1970).

Stone, M., and Chesher, S. E., Analyst 94, 1063 (1969); (1969); O'Gorman, J. V., and Suhr, N. H., ibid. 96, 335 (1971).

Abbey, S., Geol. Surv. Canada Paper 67-37 (1967).

Strelow, F. W. E., Von S. Toerien, F., and Weinert, C. H. S. W., Anal. Chim. Acta 50, 399 (1970).

Moldan, B., Rubeska, I., and Miksovsky, M., Anal. Chim. Acta 50, 342 (1970).

Schnepfe, M. M., and Grimaldi, F. S., Talanta 16, 591 (1969).

Simonsen, A., Anal. Chim. Acta 49, 368 (1970).

Schnepfe, M. M., and Grimaldi, F. S., Talanta 16, 1461 (1969).

Moldan, B., et al., Anal. Chim. Acta 52, 91 (1970).

Pearton, D. C. G., et al., Anal. Chim. Acta 44, 353 (1969).

Korkisch, J., and Gross, H., Talanta 20, 1153 (1973).

Rosman, K. J., Geochim. Cosmochim. Acta 36, 801 (1972).

a Emission

${ }^{\mathrm{b}}$ Absorption. The elements without mark are also determined by absorption.

c Atomic fluorescence 
TABLE 5. X-ray fluorescence methods

\begin{tabular}{|c|c|c|}
\hline Elements & Remarks & References \\
\hline Many elements & $\begin{array}{l}\text { The use of thin, uniform speci- } \\
\text { mens minimizes matrix effects. }\end{array}$ & $\begin{array}{l}\text { Giauque, R. D., } \\
\text { et al., Anal. Chem. } \\
\text { 45, } 671 \text { (1973). }\end{array}$ \\
\hline Many elements & $\begin{array}{l}\text { Preparation of samples for } \\
\text { analysis is described, and } \\
\text { techniques of correction for } \\
\text { matrix effects are tested. }\end{array}$ & $\begin{array}{l}\text { Murad, E., Anal. } \\
\text { Chim. Acta 67, } 37 \\
\text { (1973). }\end{array}$ \\
\hline Rare earths, Y, Sc & $\begin{array}{l}\text { The elements are collected on } \\
\text { ion-exchange paper prior to } \\
\text { determination. }\end{array}$ & $\begin{array}{l}\text { Eby, G. N., Anal. } \\
\text { Chem. 44, } 2137 \\
\text { (1972). }\end{array}$ \\
\hline $\mathrm{Au}$ & $\begin{array}{l}\text { Au is collected on ion-exchange } \\
\text { paper. The proposed method is } \\
\text { compared with other methods. }\end{array}$ & $\begin{array}{l}\text { Green, T. E., Law, } \\
\text { S. L., and Camp- } \\
\text { bell, W. J., Anal. } \\
\text { Chem. 42, } 1749 \\
\text { (1970). }\end{array}$ \\
\hline $\mathrm{Co}, \mathrm{Ni}$ & $\begin{array}{l}\text { The elements are collected on a } \\
\text { chelating resin, Chelex } 100 \text {. }\end{array}$ & $\begin{array}{l}\text { Blount, C. W., et al., } \\
\text { Anal. Chem. 45, } \\
1045 \text { (1973). }\end{array}$ \\
\hline
\end{tabular}


TABLE 6. Nuclear methods

Elements

Many elements

$\mathrm{Ba}, \mathrm{Co}, \mathrm{Cr}, \mathrm{Cu}, \mathrm{Sc}$

Rare earths, Ta, U

Many elements

Many elements

Many elements

As, $\mathrm{Sb}$

$\mathrm{Au}, \mathrm{Cu}, \mathrm{Ga}, \mathrm{La}, \mathrm{Mn}, \mathrm{Sb}$

$\mathrm{Au}, \mathrm{Ru}, \mathrm{Pd}, \mathrm{Os}, \mathrm{Ir}, \mathrm{Pt}$

$\mathrm{Au}, \mathrm{Ru}, \mathrm{Pd}, \mathrm{Os}, \mathrm{Ir}, \mathrm{Pt}$

$\mathrm{Au}, \mathrm{Sc}$

$\mathrm{Bi}, \mathrm{Cd}, \mathrm{Hg}, \mathrm{Tl}$
INAA

INAA $^{a}$ with epithermal neutrons.

INAA. Ni and Ti are determined by photon activation.

INAA

INAA and NAA using group separations.

INAA and NAA using separations.

NAA using separations.

NAA using hydrated antimony pentoxide for $\mathrm{Na}$ removal.

NAA using radiochemical separation scheme.

NAA using ion-exchange separation.

NAA with an internal-reference method.

NAA using radiochemical separation scheme.
Brunfelt, A. O., and Steinnes, E., Anal. Chim. Acta 48, 13 (1969).

Schmitt, R. A., Linn, T. A., Jr., and Wakita, H., Radiochim. Acta 13, 200 (1970).

Hertogen, J., and Gijbels, R., Anal. Chim. Acta 56, 61 (1971).

Schock, H. H., Z. Anal. Chem. 263, 100 (1973).

Morrison, G. H., et al., Anal. Chem. 41, 1633 (1969); Morrison, G. H., and Nadkarni, R. A., J. Radioanal. Chem. 18, 153 (1973).

Brunfelt, A. O., and Steinnes, E., Talanta 18, 1197 (1971).

Steinnes, E., Analyst 97, 241 (1972).

Gills, T. E., Marlow, W. F., and Thompson, B. A., Anal. Chem. 42, 1831 (1970).

Millard, H. T., Jr., and Bartel, A. J., Activation Analysis in Geochem. and Cosmochem. p. 353 (1971).

Nadkarni, R. A., and Morrison, G. H., Anal. Chem. 46, 232 (1974)

Nomura, K., et al., Anal. Chim. Acta 51, 399 (1970).

Marowsky, G., Activation Anal. in Geochem. and Cosmochem. p. 391 (1971). 
TABLE 6. Nuclear methods-Continued

\section{Elements}

Remarks

References
Cd, In, Y, rare earths

$\mathrm{Co}, \mathrm{Cu}, \mathrm{Ga}, \mathrm{W}, \mathrm{Zn}$

$\mathrm{Rb}, \mathrm{Cs}, \mathrm{Ba}$, rare earths

$\mathrm{Au}$

$\mathrm{Bi}$

$\mathrm{Hg}$

Mo

Pt metals

Pd, Pt

$\mathrm{Pd}$

$\mathrm{Ru}$, Os, Ir
NAA using radiochemical separation scheme.

NAA using anion-exchange separation.

NAA using radiochemical separation scheme.

$\mathrm{Au}$ is collected on ion-exchange paper before neutron activation. The method is compared with other methods.

NAA using separation.

NAA using ion-exchange separation.

Activation with epithermal neutrons followed by separations.

INAA

NAA after fire-assay concentration.

NAA using substoichiometric extraction.

NAA using radiochemical separation scheme.
Rey, P., Wakita, H., and Schmitt, R. A., Anal. Chim. Acta 51, 163 (1970).

Johansen, O., and Steinnes, E., Talanta 17, 407 (1970).

Brunfelt, A. O., Roelandts, I., and Steinnes, E., Analyst 99, 277 (1974).

Green, T. E., Law, S. L., and Campbell, W. J., Anal. Chem. 42, 1749 (1970).

Santoliquido, P. M., and Ehmann, W. D., Geochim. Cosmochim. Acta 36, 897 (1972).

Ishida, K., Kawamura, S., and Izawa, M., Anal. Chim. Acta 50, 351 (1970).

Steinnes, E., Anal. Chim. Acta 57, 249 (1971).

Turkstra, J., Pretorius, P. J., and de Wet, W. J., Anal. Chem. 42, 835 (1970).

Rowe, J. J., and Simon, F. O., Talanta 18, 121 (1971).

Briscoe, G. B., and Humphries, S., Talanta 17, 371 (1970); Nadkarni, R. A., and Haldar, B. C., Radiochem. Radioanal. Lett. 14, 25 (1973).

Gijbels, R., et al., Activation Anal. in Geochem. and Cosmochem. p. 359 (1971). 
TABLE 6. Nuclear methods-Continued

Elements

Remarks

References

$\mathbf{R u}$

Rare earths

$\mathrm{Sb}$

Sn

$\mathrm{Sr}, \mathrm{Ba}$

$\mathrm{Ta}$

Th

$\mathrm{U}$

U

$\mathbf{U}$

$\mathrm{Ce}, \mathrm{Co}, \mathrm{Nb}, \mathrm{Ni}, \mathrm{Rb}$, $\mathrm{Sr}, \mathrm{Y}, \mathrm{Zr}$
INAA

NAA. Correction for $U$ is discussed.

NAA using separation.

NAA using separation.

NAA using separation.

INAA

NAA using separation.

INAA with epithermal neutrons.

INAA

Delayed neutron counting, nondestructive.

Photon activation, nondestructive.
Gijbels, R., Talanta 18, 587 (1971).

Potts, M. J., Early, T. O., and Herrmann, A. G., Z. Anal. Chem. 263, 97 (1973),

Treuil, M., et al., J. Radioanal. Chem. 18, 55 (1973).

Lombard, S. M., Marlow, K. W., and Tanner, J. T., Anal. Chim. Acta 55, 13 (1971).

Johansen, O., and Steinnes, E., Analyst 94, 976 (1969).

Higuchi, H., et al., Anal. Chim. Acta 44, 431 (1969).

Sukhov, G. V., Firsov, V. I., and Lontsikh, S. V., Zh. Anal. Khim. 28, 312 (1973).

Norton, E. F., and Stoenner, R. W., Anal. Chim. Acta 55, 1 (1971); Desai, H. B., Parthasarathy, R., and Das, M. Sankar, Talanta 19, 363 (1972).

Steinnes, E., and Brune, D., Talanta 16, 1326 (1969).

Hofmann, H. P., Z. Anal. Chem. 263, 108 (1973).

Mo, T., Suttle, A. D., and Sackett, W. M., Geochim. Cosmochim. Acta 37, 35 (1973).

Kato, T., Morita, I., and Sato, S., J. Radioanal. Chem. 18, 97 (1973). 
TABLE 6. Nuclear methods-Continued

\begin{tabular}{|c|c|c|}
\hline Elements & Remarks & References \\
\hline $\mathbf{B}, \mathbf{U}$ & Nuclear track technique. & $\begin{array}{l}\text { Carpenter, B. S., } \\
\text { Anal. Chem. 44, } \\
600 \text { (1972). }\end{array}$ \\
\hline Th & $\begin{array}{l}\text { Fission track technique. } \\
\text { Unsuitable when the } \mathrm{Th} / \mathrm{U} \\
\text { ratio is low. }\end{array}$ & $\begin{array}{l}\text { Reimer, G. M., and } \\
\text { Carpenter, B. S., } \\
\text { Nature 247, } 101 \\
\text { (1974). }\end{array}$ \\
\hline $\mathbf{U}$ & Fission track technique. & $\begin{array}{l}\text { Murali, A. V., } \\
\text { Parekh, P. P., and } \\
\text { Das, M. Sankar, } \\
\text { Anal. Chim. Acta } \\
\text { 50, } 71 \text { (1970); } \\
\text { Fisher, D. E., Anal. } \\
\text { Chem. 42, 414 } \\
\text { (1970); Matsuda, } \\
\text { H., et al., Talanta } \\
\text { 19, 851 (1972). }\end{array}$ \\
\hline $\mathrm{Bi}$ & $\begin{array}{l}\text { Substoichiometric radioisotope } \\
\text { dilution. }\end{array}$ & $\begin{array}{l}\text { Greenland, L. P., } \\
\text { and Campbell, } \\
\text { E. Y., Anal. Chim. } \\
\text { Acta 60, } 159 \text { (1972). }\end{array}$ \\
\hline In & $\begin{array}{l}\text { Substoichiometric radioisotope } \\
\text { dilution. }\end{array}$ & $\begin{array}{l}\text { Greenland, L. P., } \\
\text { and Campbell, E. } \\
\text { Y., Anal. Chim. } \\
\text { Acta 67, } 29 \text { (1973). }\end{array}$ \\
\hline Th, U & Gamma ray spectroscopy. & $\begin{array}{l}\text { For example, Eldridge, } \\
\text { J. S., et al., Proc. } \\
\text { Third Lunar Sci. } \\
\text { Conf. 2, } 1651 \\
\text { (1972). }\end{array}$ \\
\hline
\end{tabular}

- INAA = instrumental neutron activation analysis. 
TABLE 7. Mass spectrometry

Elements

Remarks

References

Alkali metals, alkaline earths, rare earths

$\mathrm{Pb}, \mathrm{Th}, \mathrm{Tl}, \mathrm{U}$

$\mathrm{Rb}, \mathrm{Sr}$

$\mathrm{Ga}$

$\mathrm{Pb}$

Rare earths

$\mathrm{U}$

$\mathrm{Zn}$

Many elements

Many elements
Isotope dilution with group separation.

Isotope dilution.

Isotope dilution.

Isotope dilution.

Isotope dilution.

Isotope dilution.

Isotope dilution.

Isotope dilution.

Spark source.
Murugaiyan, P., et al., Talanta 15, 1119 (1968).

Barnes, I. L., et al., Anal. Chem. 45, 880 (1973).

Moore, L. J., et al., Anal. Chem. 45, 2384 (1973).

De Laeter, J. R., Geochim. Cosmochim. Acta 36, 735 (1972).

Arden, J. W., and Gale, N. H., Anal. Chem. 46, 2 (1974).

Schnetzler, C. C., Thomas, H. H., and Philpotts, J. A., Anal. Chem. 39, 1888 (1967).

Arden, J. W., and Gale, N. H., Anal. Chem. 46, 687 (1974).

Rosman, K. J. R., Geochim. Cosmochim. Acta 36, 801 (1972)

Taylor, S. R., Geochim. Cosmochim. Acta 29, 1243 (1965); Nicholls, G. D., et al., Anal. Chem. 39, 584 (1967); Morrison, G. H., and Kashuba, A. T., ibid. 41, 1842 (1969); Chupakhin, M. S. and Polyakov, A. L., Zh. Anal. Khim. 27, 523 (1972); Taylor, S. R. et al., Geochim. Cosmochim. Acta 37, 2665 (1973).

Jackson, P. F. S., and Strasheim, A. Analyst 99, 26 (1974).

TABLE 8. Other methods

Elements

Method

References

$\mathrm{Fe}, \mathrm{Ni}, \mathrm{Ti}$

$\mathrm{Pb}$

$\mathrm{Be}$
Differential cathode ray polarography.

Anodic stripping analysis.

Gas chromatography.
Maienthal, E. J., Anal. Chem. 45, 644 (1973).

Khasgiwale, K. A., Parthasarathy, R., and Das, M. Sankar, Anal. Chim. Acta 59, 485 (1972).

Eisentraut, K. J., Griest, D. J., and Sievers, R. E., Anal. Chem. 43, 2003 (1971). 


\section{Comparison of Methods - Some Examples}

Because accuracy of a method is difficult to assess, comparison of methods through analysis of an identical sample is always encouraged.

\section{A. Gold in Ores [39]}

Fire assay, wet extraction and spectrophotometry, and neutron activation analysis have been evaluated. The fire assay is the fastest and most efficient method for multiple and routine analysis of ores. Wet extraction plus spectrophotometry provides an alternative to the fire assay. The results obtained by the neutron activation analysis for a variety of ores indicate the difficulty in obtaining a representative sample of the small size required. Nadkarni and Morrison [40] state that there may not be "true" values for the noble metals because of sampling problems for these elements in terrestrial rocks. The "true" preferably should be replaced by "representative," because the result may be true only for that particular sample. The situation indicates a manner of occurrence of the noble metals. Performing the separation before neutron activation permits the use of large samples [41], but control of the blank is necessary.

\section{B. Barium and Strontium in Silicates [42]}

Various techniques have been evaluated. Isotope dilution mass spectrometry is considered the reference method for barium and strontium. Strontium can be satisfactorily determined by atomic absorption spectrophotometry. Emission spectrometry, with a spark-solution technique and automated equipment [43], gives satisfactory results for both barium and strontium.

\section{Gallium in Ores [44]}

Gallium can be determined easily by a variety of methods. A comparison of the gallium determination by a spectrophotometric Rhodamine B method and by an atomic absorption method based on preliminary isopropyl ether extraction, indicates similar results. 


\section{Platinum in Glass [45]}

Less than $1 \mathrm{ppm}$ of platinum in glass can be determined, after preliminary separation, by a spectrophotometric p-nitrosodimethylaniline method or by an X-ray fluorescence procedure. Spark-source mass spectrography is too insensitive for the direct determination of platinum in this matrix.

\section{E. RARE EARTHS IN ROCKS}

One of the remarkable developments in trace element analysis in the last 15 years is the determination of individual rare earth elements. According to Haskin and Korotev [46], neutron activation analysis and isotope dilution mass spectrometry are accurate and sensitive. Precision for isotope dilution is a fraction of a percent, that for neutron activation ranges from about 1 to about 10 percent, depending on the element. Low blanks are necessary for isotope dilution. Useful data are also obtained by spark-source mass spectrometry and $\mathrm{x}$-ray fluorescence, with precision ranging upwards from about 10 percent.

\section{F. URANium in Ores [47]}

Four different ores containing 0.01 to 0.7 percent $\mathrm{U}_{3} \mathrm{O}_{8}$ have been analyzed by several techniques, including spectrophotometric, fluorimetric, $x$-ray fluorescence, radiometric, and titrimetric procedures. Statistical analysis shows that there is no significant difference between the results obtained by the spectrophotometric [2-(5-bromo-2-pyridylazo)-5diethylaminophenol], fluorimetric, or x-ray methods.

\section{G. Additional Remarks}

Many methods have been described for determination of rhenium in molybdenites. They include spectrophotometric (Yatirajam and Kakkar, 1969, 1970, 1971), spectrochemical (Nebesar, 1967), x-ray fluorescence (Solt et al., 1969), neutron activation (Terada et al., 1967; Linn et al., 1972), and polarographic (Duca and Calu, 1972) methods. Except the neutron activation method of Linn et al., separations are used. For evaluation of these methods, it seems desirable to have standard or common molybdenite samples.

Various methods of separating and determining thorium in natural materials have been reviewed [48]. 


\section{Choosing the Proper Analytical Technique}

Factors that may be considered in choosing the proper analytical technique are listed below.

\section{Objective of analysis or content of information needed.}

2. Sensitivity and detection limit. These factors are related to sample size. When sample available is limited, sensitive methods should be used. In this case, there is a greater possibility of inhomogeneity.

3. Accuracy. Accuracy may be judged by comparison of results with those obtained by other methods. Blanks, effect of other elements, and necessity of standard samples for calibration or standardization are to be considered.

\section{Precision or reproducibility.}

5. Determination of single element or multielements.

6. Time required for analysis and cost.

7. Availability of instrumentation is also a factor.

8. Analyst. Every method is subject to variability depending upon a number of factors, the most important being the analyst [36]. Choice of the method depends on personal taste and on the competence of the analyst. Therefore, education of the analyst(s) is always necessary.

\section{References}

[1] Taylor, S. R., Geochim. Cosmochim. Acta 28, 1273 (1964).

[2] Wedepohl, K. H., Ed., Handbook of Geochemistry, Vols. II-1, II-2, II-3, SpringerVerlag, Berlin $(1969,1970,1972)$.

[3] Sandell, E. B., Colorimetric Determination of Traces of Metals, 3d Edition, Interscience, New York (1959).

[4] Jeffery, P. G., Chemical Methods of Rock Analysis, Pergamon, Oxford (1970).

[5] Ahrens, L. H. and Taylor, S. R., Spectrochemical Analysis, $2 d$ Edition, AddisonWesley, Reading, Massachusetts (1961).

[6] Smales, A. A. and Wager, L. R., Eds., Methods in Geochemistry, Interscience, New York (1960).

[7] Fairbairn, H. W. et al., U.S. Geol. Surv. Bull. 980 (1951).

[8] Flanagan, F. J., Geochim. Cosmochim. Acta 31, 289 (1967).

[9] Proceedings of the Apollo 11 Lunar Sci. Conf., Pergamon, New York (1970).

[10] Proceedings of the Second Lunar Sci. Conf., The MIT Press, Cambridge, Massachusetts (1971).

[11] Proceedings of the Third Lunar Sci. Conf., The MIT Press, Cambridge. Massachusetts (1972).

[12] Proceedings of the Fourth Lunar Sci. Conf., Pergamon, New York (1973). 
[13] Geochim. Cosmochim. Acta 37, No. 4 (1973).

[14] Hillebrand, W. F. and Lundell, G. E. F., Applied Inorganic Analysis, Wiley, New York (1929).

[15] Lundell, G. E. F., Ind. Eng. Chem., Anal. Ed. 5, 221 (1933).

[16] Mellor, J. W. and Thompson, H. V., A Treatise on Quantitative Inorganic Analysis, $2 d$ Edition, Charles Griffin, London (1938).

[17] Kolthoff, I. M., Sandell, E. B., Meehan, E. J., and Bruckenstein, S., Quantitative Chemical Analysis, 4th Edition, Macmillan, New York (1969).

[18] Hevesy, G. V., Chemical Analysis by X-Rays and Its Applications, McGraw-Hill, New York (1932).

[19] Morrison, G. H., Ed., Trace Analysis: Physical Methods, Wiley-Interscience, New York (1965).

[20] Dean, J. A., Flame Photometry, McGraw-Hill, New York (1960).

[21] Pickett, E. E. and Koirtyohann, S. R., Anal. Chem. 41 (14), 28A (1969).

[22] Lingane, J. J., Electroanalytical Chemistry, $2 d$ Edition, Interscience, New York (1958).

[23] Ogata, K., Kagaku no Ryoiki 27, 383 (1973).

[24] Lewin, S. Z., Anal. Chem. 33 (3), 23 A (1961).

[25] Kaiser, H., Anal. Chem. 42 (2), 24A (1970); 42 (4), 26A (1970).

[26] Ingle, J. D., Jr., J. Chem. Educ. 51,100 (1974).

[27] Wilson, A. L., Talanta 20, 725 (1973).

[28] Moore, L. J., Moody, J. R., Barnes, I. L., Gramlich, J. W., Murphy, T. J., Paulsen, P. J., and Shields, W. R., Anal. Chem. 45, 2384 (1973).

[29] Onishi, H. and Sandell, E. B., Anal. Chim. Acta 14, 153 (1956).

[30] Maienthal, E. J., Anal. Chem. 45, 644 (1973).

[31] Barnes, I. L., Garner, E. L., Gramlich, J. W., Moore, L. J., Murphy, T. J., Machlan, L. A., Shields, W. R., Tatsumoto, M., and Knight, R. J., Anal. Chem. 45, 880 (1973).

[32] Barnes, I. L., Murphy, T. J., Gramlich, J. W., and Shields, W. R., Anal. Chem. 45, 1881 (1973).

[33] Arden, J. W. and Gale, N. H., Anal. Chem. 46, 2 (1974).

[34] Arden, J. W. and Gale, N. H., Anal. Chem. 46, 687 (1974).

[35] Smales, A. A., Activation Analysis in Geochemistry and Cosmochemistry, p. 17 (1971).

[36] Morrison, G. H., J. Radioanal. Chem. 18, 9 (1973).

[37] Herzog, L. F., Int. J. Mass Spectrom. Ion Phys. 4, 253, 337 (1970).

[38] Anderson, C. A. and Hinthorne, J. R., Science 175, 853 (1972).

[39] Chow, A. and Beamish, F. E., Talanta 14, 219 (1967).

[40] Nadkarni, R. A. and Morrison, G. H., Anal. Chem. 46, 232 (1974).

[41] Green, T. E., Law, S. L., and Campbell, W. J., Anal. Chem. 42, 1749 (1970).

[42] Ingamells, C. O., Suhr, N. H., Tan, F. C., and Anderson, D. H., Anal. Chim. Acta 53, 345 (1971).

[43] Suhr, N. H. and Ingamells, C. O., Anal. Chem. 38, 730 (1966).

[44] Lypka, G. N. and Chow, A., Anal. Chim. Acta 60, 65 (1972).

[45] Fuller, C. W., Himsworth, G., and Whitehead, J., Analyst 96, 177 (1971).

[46] Haskin, L. A. and Korotev, R. L., Analysis and Application of Rare Earth Materials, p. 183 (1973).

[47] Florence, T. M., Pakalns, P., and Dale, L. S., AAEC/E-237 (1972).

[48] Karalova, Z. K., Zh. Anal. Khim. 28, 1389 (1973). 


\title{
THE PAST, PRESENT, AND FUTURE OF THE ANALYSIS OF ATMOSPHERIC PARTICULATES
}

\author{
R. K. Skogerboe \\ Department of Chemistry \\ Colorado State University \\ Fort Collins, Colorado 80521 U.S.A.
}

The determination of the composition of airborne particulate matter is a major task of escalating importance. The general requirements imposed on the analytical methods are typical: The multielement composition must be determined accurately and precisely over a wide concentration range. These accurate results are to be generated by the analytical method even though there may be major variations in the general composition of the particulate samples or in their "preparative" histories. Other emerging requirements include the determination of composition on a continuous basis in the field even at exceedingly low concentrations and/or the specification of composition as a function of particle size.

A comparative evaluation of the various analytical methods used for characterization of particulates is presented from the perspective of these requirements. The state of the art for several competitive analytical methods is summarized and their advantages and limitations defined. A synopsis of ongoing research is presented and potentially fruitful directions for analytical development are indicated.

Keywords: Atmospheric particulates; direct analysis techniques; emission spectroscopy; particulate analysis; particulate characterization; sampling methods: $x$-ray fluorescence.

\section{Introduction}

Present knowledge regarding the chemistry of the atmosphere has been developed by researchers working in widely variant disciplines over the past few decades. The chemistry of the atmosphere is very diverse in character. Many of the constituents undergo, or are formed by, heterogeneous reactions and interactions including those involving gasgas, gas-liquid, gas-solid, liquid-solid, and solid-solid phase systems. 
Further, the constituents and/or reactants may originate from anthropogenic sources, man's activities, or both. The characterization of a complex system of this type is truly a challenging analytical problem. In fact, few atmospheric scientists would dispute the statement that the current lack of knowledge in the area of atmospheric chemistry can be attributed directly to the inability to characterize (analyze) accurately many of the constituents of interest. The development of improved analytical measurement techniques-more sensitive, more selective, more accurate -is regarded as essential to the solution of atmospheric chemistry problems. In a previous paper, Dr. Lodge discussed the present status of the problem of characterizing gaseous constituents; the present discussion is concentrated on atmospheric particulate analysis.

Atmospheric measurement techniques may be classed in two general groups [1]. Remote measurement methods are generally carried out in the field but remote from the actual sample being characterized. Such measurements may often be made over relatively large areas from a single location. Certainly such measurements offer very distinct advantages, but the extension of their use and applicability definitely will require the development of numerous analytical methods of greater sensitivity than presently available. The second category includes those measurements requiring physical collection or manipulation of the sample. It may be noted that the primary reason for defining this type of measurement may be attributed directly to the lack of adequate remote measurement methods. Some of these characterizations may involve the direct, in situ, measurement of a property of the sample, e.g., light scattering. The majority, however, require the physical removal of the sample to the lab for analysis. Particulate analyses most generally fall into the latter category. Hence the present discussion deals largely with laboratory based measurements.

\section{The Problem}

The purpose of characterizing atmospheric particulates is generally twofold. In addition to the chemical composition of the particulates, it is usually also necessary to determine their size distributions in order to assess their atmospheric transport characteristics and/or their potential for impact on some segment of the ecosystem, e.g., their potential for retention in lungs. The most rigorous analytical requirement is that of determining the chemical composition as a function of particle size. While differentiation between various compounds present in the particulates is im- 
plied by the term chemical composition, the concentration levels involved, coupled with the general complexity of a particulate matrix, generally precludes the possibility of carrying out definitive speciation measurements. It is almost universally necessary to accept the elemental concentrations as the gross indicator of the chemical composition.

In an attempt to put these general requirements into perspective with regard to the analytical requirements, consider the following. Air pollution regulations often stipulate that a particulate loading of $100 \mu \mathrm{g} / \mathrm{m}^{3}$ should be the maximum allowed for an urban environment. Filters used to collect such particulates usually operate at a flow of approximately 1 $\mathrm{m}^{3} / \mathrm{min}$ so an upper limit of $6 \mathrm{mg}$ of particulate should be collected per hour of sampling $(144 \mathrm{mg} / 24 \mathrm{~h})$. If we assume that the elements to be determined are those classed by EPA as Priority 1 (Ag, As, Be, Cd, Cr, $\mathrm{Cu}, \mathrm{F}, \mathrm{Hg}, \mathrm{Li}, \mathrm{Mn}, \mathrm{Ni}, \mathrm{Pb}, \mathrm{Sb}, \mathrm{Se}, \mathrm{Sn}$, and $\mathrm{Zn}$ ), then the analysis problem can be envisioned. Seventeen elements, each of which typically will be present at a bulk concentration of less than $1000 \mu \mathrm{g}$ per $\mathrm{g}$ of total particulate, must be determined on a relatively small sample. If the particulate size distribution-composition relationship is to be determined, the amount of sample available for analysis in each size classification will be reduced in accordance with the parameters of the size distribution being sampled. In effect, the analysis problem is generally that of multielement trace analysis on microsamples. In this perspective, it is not difficult to understand why measurement techniques capable of real-time, in-field analyses are lacking.

\section{Strategies for Solving the Problem(s)}

The purpose of the following discussion is to examine, on a comparative basis, current methods and those in development which offer means for solving the particulate characterization problem. To put this examination into the proper perspective, it is only necessary to consider the more common approaches used for sample collection.

\section{Sampling Methods}

When the analytical information required is limited to the general composition of the entire atmospheric particulate population, the most common sampling approach used involves pumping a measured volume of air through a filter which removes the particulate. Such filters may be made 
from paper, glass, fiber, cellulose acetates, plastic materials such as polystyrene or Teflon, and graphite (a more recent addition). Filters of various pore sizes may be utilized but the most common are those with mean pore sizes around 0.4 to $0.5 \mu \mathrm{m}$. Particulate size distribution measurements [2-4] have indicated that as much as 50 to 85 percent of the solid aerosol mass may have diameters below $0.5 \mu \mathrm{m}$, so it is reasonable to question how a filter with essentially the same pore size can be expected to collect the total particulate load. Three mechanisms are responsible for the filtration process $[1,5]$. All particles larger than the pore size are collected by interception. The very small particles having diameters approaching the mean free paths of molecules in the atmosphere being sampled undergo extensive diffusion (Brownian motion) on entry into the filter pores and accumulate within the pores by: collision with the pore walls, electrostatic attraction within the pores, or agglomeration with other small particles to develop sizes consistent with interceptive collection [5]. Particles in the middle size range are collected by inertial impaction or impingement as illustrated by figure 1. The streamlines of air and particle flow approaching a filter face may be considered essentially coherent until a point is reached where the direction of flow must change to pass through the pores. The inertial forces tend to make the particles move in a straight line while the drag forces tend to accelerate the particles in the changing direction of airflow. If the inertia of the particle is great enough, it will be collected on the filter by impingement; if not, it will enter the pore and may be collected on the pore wall by impingement or

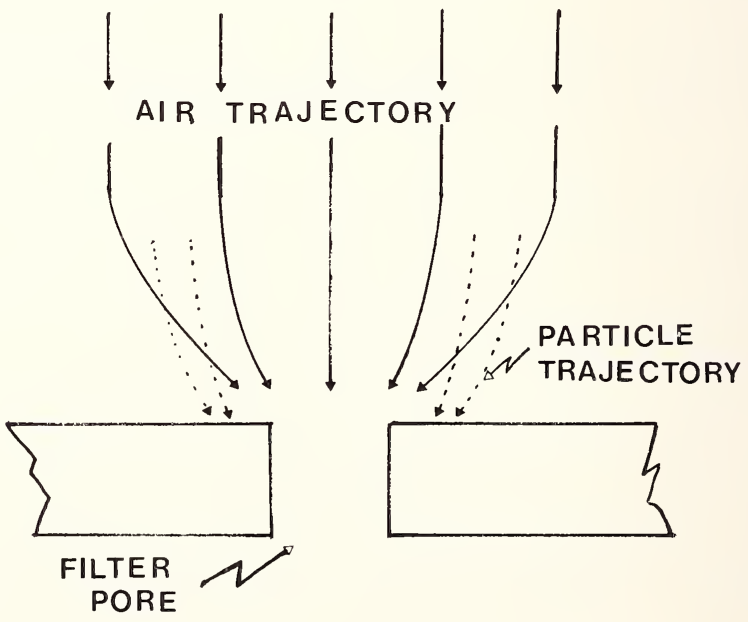

Figure 1. Illustration of the inertial impingement mechanism for the collection of particles smaller than the filter pore size. 
diffusive action. Because these three mechanisms are simultaneously operative, filters are normally considered to be highly efficient for the collection of particulates. This is, of course, subject to certain limiting conditions which will be discussed below.

When sample size distribution information is required, the sampling systems used generally separate the particles collected into size strata by inertial impingement methods. Cascade impactors, for example, consist of a succession of impaction plates (stages) in an airflow arrangement such that the degree of acceleration of the airflow increases at each stage. Heavier particle sizes are collected on the first stage and the lightest (smallest) particles are collected on the last stage where the air velocity is highest. Since this stratification is based on an inertial sorting mechanism, the "sizes" of the particles collected on each stage depend on their densities as well as their physical dimensions. The term used is aerodynamic diameter which is descriptive of the settling velocity of a particle in a fluid medium such as air. The aerodynamic diameter is therefore indicative of the transport characteristics of the particles [1]. A typical cascade impactor size profile for particulates containing lead is given in table 1. By calculating the cumulative percentage of the total mass of lead as shown in table 1 and plotting this versus the maximum aerodynamic particle size for each stage, figure 2 is obtained. The mass median equivalent diameter (MMED) of the particle size distribution is defined as that size coincident with the median of the distribution, i.e., 50 percent of the total mass collected is less than this size. For the figure 2 data, that size is $0.4 \mu \mathrm{m}$. Since unit density is assumed, any actual density difference will be reflected accordingly in the actual physical size.

TABLE 1. Example size distribution of lead collected with a cascade impactor

$\begin{array}{cccc}\begin{array}{c}\text { Stage } \\ \text { number }\end{array} & \begin{array}{c}\text { Aerodynamic diameter } \\ \text { range, } \mu \mathrm{m}\end{array} & \begin{array}{c}\mu \mathrm{g} \mathrm{Pb} \\ \text { collected }\end{array} & \begin{array}{c}\text { Cumulative pe } \\ \text { of total lea }\end{array} \\ 1 & 8-14 & 1.57 & 100 \\ 2 & 5-8 & 1.07 & 93.5 \\ 3 & 4-5 & 1.67 & 89.1 \\ 4 & 2.5-4 & 1.57 & 82.2 \\ 5 & 1.2-2.5 & 1.87 & 75.7 \\ 6 & 0.8-1.2 & 2.87 & 67.9 \\ 7 & 0.4-0.8 & 1.47 & 56.1 \\ 8 & 0.1-0.4 & 1.27 & 50.0 \\ \text { Backing } & <0.1 & 10.81 & 44.7 \\ \text { filter } & & & \end{array}$

Total $=24.17 \mu \mathrm{g}$ 


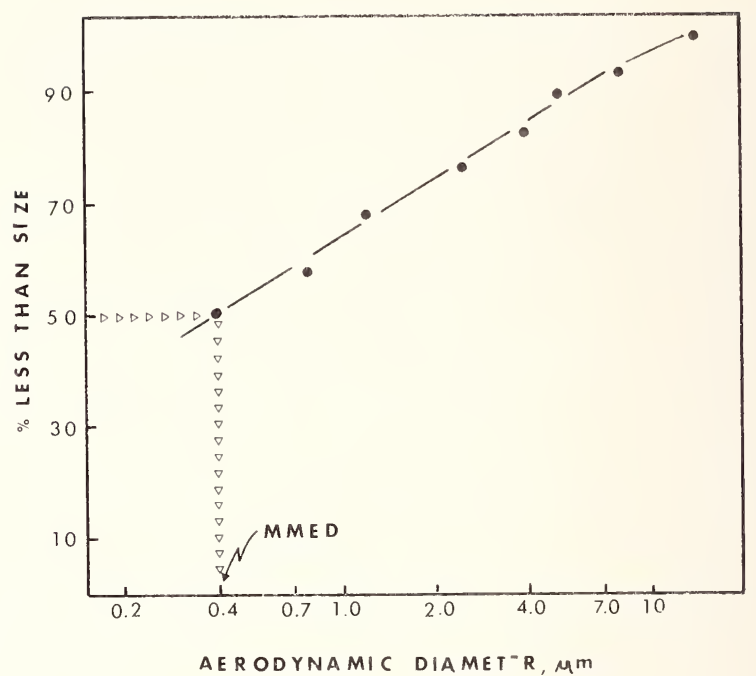

Figure 2. A typical cumulative distribution plot for the determination of the mass median equivalent diameter of lead-bearing particles. (Data are from table 1.)

A dichotomous sampler system which uses the inertial separation phenomenon to segregate the particle size distribution into two discrete ranges and collect them on filters has been described by Dzubay and Stevens [6]. The two ranges selected are 0 to $2 \mu \mathrm{m}$ and 2 to $10 \mu \mathrm{m}$. The former is regarded as the "respirable" size range so information pertinent to the population exposure question may be obtained with this type of sampling system [7]. Data from an atmospheric sample collected in St. Louis [6], for example, has shown that the atmospheric concentrations of the three toxic elements $\mathrm{As}, \mathrm{Pb}$, and $\mathrm{Se}$ are all enriched by factors of 3 or more in the respirable range relative to the larger particle size range. Natusch, Wallace, and Evans [8] have also shown that several toxic elements are concentrated preferentially in the respirable particles. Although filter sampling procedures for the collection of total particulate are most widely used at present, it is becoming increasingly apparent that procedures which provide concentration versus size information will become much more popular. In addition to this development, one may also expect that information based on shorter sampling periods will be required in order to obtain more accurate assessment of population exposures during those hours of peak activity in selected locations, e.g., city shopping areas. These developments will require greater sensitivity of the analytical techniques used. In addition to the analytical techniques used, the sensitivity of any particular determination may be dramatically influenced by the sample preparation methods utilized. 


\section{Sample Preparation}

The filters and/or cascade impactor stages used for collection of atmospheric particulates may serve as a limitation on the analytical sensitivity due to blank contributions. Several reports [9-14] have shown that these blanks may be inordinately high for a variety of different types of filters and quite variable within any particular lot of filters. Such observations led Gandrud and Lazrus. [14] to develop a means for precleaning cellulose filters, but the method works only for elements which form readily soluble salts. Perhaps the best means of correcting for the blank would be by carrying out a nondestructive analysis to determine the blank on each filter prior to use. Even then, high blanks for a particular element would ultimately limit the ability to determine that element at low concentrations. The most reasonable alternative is, consequently, the development of cleaner filter materials. Some examples will be discussed below.

Other sample treatment problems may originate from the methods used for sample preparation. If the sample must be dissolved, reagent blank contributions may add to those from the sample collection substrate material. The reagent blank problem may be serious due to the nature of the particulates collected. A reasonably high percentage of particulates are the products of combustion processes; they often exist in complex, refractory forms. This necessitates the use of rigorous wet oxidation media such as nitric, perchloric, and sulfuric acid mixtures for dissolution. Blank contributions from reagents and container leaching generally increase as the rigor of the wet oxidation system increases. While simplification of this problem can be obtained through the use of dry oxidation techniques, the possibility of loss of analytical species cannot be divorced from such techniques. Such losses are almost certainly matrix dependent $[15,16]$; the wide compositional variability of particulate samples may preclude the possibility of generalizing the dry ashing loss problem. In view of these problems, a general sampling and analysis philosophy which is consistent with the requirements of high analytical accuracy and versatility may be formulated. The sampling approach and medium should be amenable to analysis with a minimum of sample handling and/or pretreatment, i.e., direct analysis, in order to minimize the contamination/loss situation. The analytical method(s) should permit the accurate determination of several elements with a high degree of specificity at low absolute levels. At least a few analytical approaches are capable of satisfying these requirements. The remaining discussion will be devoted to these. 


\section{Direct Analysis Techniques}

Two recent publications from the author's lab $[12,13]$ have described a sampling medium that largely eliminates the filter blank problem and permits sensitive, multielement determinations. This work was based on the method described by Woodriff and Lech [17] who used spectrographic purity graphite cups as air filters coupled with a nonflame atomization technique for the determination of lead by atomic absorption. Porous graphite, by its nature, has been described as ideal for filtration of atmospheric particulates [18]. The availability of highly pure graphite in a variety of geometric forms coupled with its filtration characteristics led to its use in the author's laboratory.

Porous cup graphite electrodes available at low cost on an off-the-shelf basis were used as air filters [12,13]. An emission spectrographic method was developed which permitted the direct analysis of the graphite cups for 14 elements of interest; the list could be expanded considerably. Vaporization of the particulates and excitation of the spectra were carried out with a dc arc in a unique medium using $\mathrm{HCl}$ gas to convert rapidly the majority of sample constituents to volatile halides. In addition to enhancing the sensitivity that could be achieved, the volatile halide generation system may also serve as a means of eliminating interference effects that may originate from compositional variations between samples. While this possibility requires further investigation for proof, present comparisons involving three independent methods of analysis of diverse atmospheric particulate samples have consistently produced results that agree within 10 percent or better.

The graphite cup filter, emission spectrographic analysis approach has been used extensively for a variety of atmospheric modelling programs. One such study involved the characterization of atmospheric transport of particulates in a "city canyon" configuration. The location of the samplers and the measured concentrations of lead which demonstrate the concentration pattern for all elements determined are given in figure 3. The samplers were located at midblock at three heights approximately coincident with the top of each floor of the three-story buildings. Two series were above the sidewalks; a third series was above the four-lane street center and single stations were located atop the buildings on either side. The concentration pattern observed was generally characteristic of cross-street wind conditions. The north/northeast wind in this instance sweeps down the street keeping the particulate concentrations low against the west side while a relatively static zone exists on the windward side. When these conditions existed it was noted that particulate concentrations tended to build up to abnormally high levels through accumulation in this zone. 


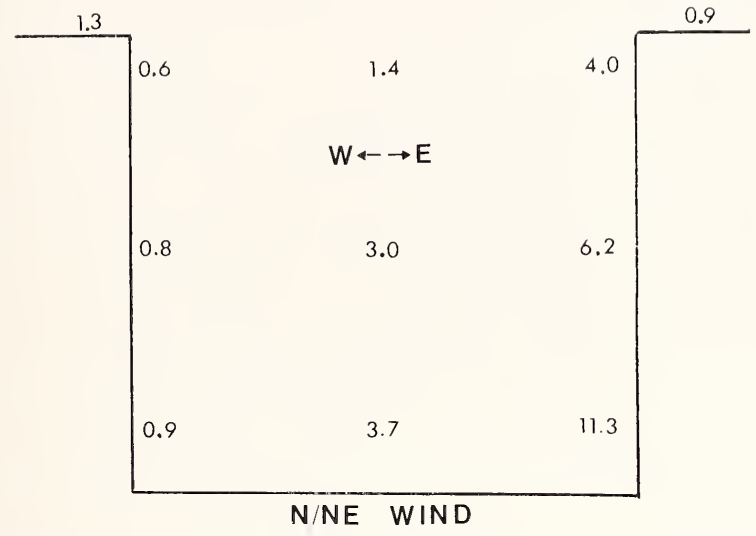

Figure 3. Particulate lead concentration profiles in a city street canyon configuration.

Measurements such as these with the graphite cup system have been used to develop useful atmospheric models for numerous particulate species in the city canyon configuration.

Previous mention was made of the predicted trend toward the use of shorter sampling periods. The data in figure 4 are indicative of the need for such sampling and further demonstrate the utility of the graphite cup sampling system. Analysis of particulate samples collected over half-hour intervals in a city canyon show that the concentrations of lead and other

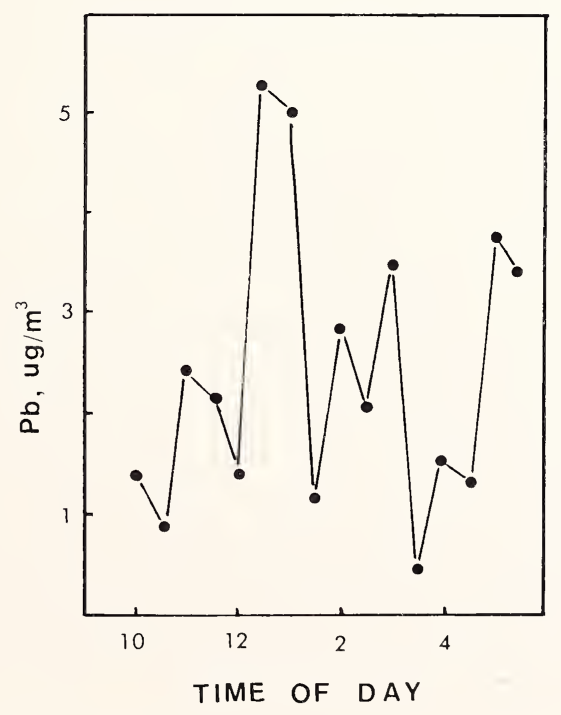

Figure 4. Time variation of the particulate lead concentration in a city street canyon. 
elements undergo rather dramatic short-term variations that may be related to traffic density. A sample collected over a 24-h period at the same location indicated an average lead concentration, for example, of 0.35 $\mu \mathrm{g} / \mathrm{m}^{3}$. This mean value was reduced relative to the average concentration of $2.1 \mu \mathrm{g} / \mathrm{m}^{3}$ determined over the 9 a.m. to $6 \mathrm{p} . \mathrm{m}$. period due to the low traffic density at night. In this case, the 24-h average was six times lower than the daytime average and 10 to 15 times lower than peak concentrations observed during periods of high street population, e.g., the noon hour in figure 4 . In essence, if the exposure of population to toxic trace elements is to be realistic, the concentrations of these trace elements for the periods that people are in a particular exposure location should be determined. Twenty-four hour averages, and particularly those taken atop buildings, do not reflect the true exposure case for individuals spending a few hours at ground level, e.g., traffic policemen. Health agencies are recognizing this, so we may expect more monitoring stations to be dedicated to shorter-term particulate sampling.

While the graphite cup sampler, emission spectrographic method has been shown to be a convenient and reliable means of determining trace element concentrations in particulates, it is limited by the fact that the cups used are not amenable to high sample flow rates. A nominal flow rate of 1 to $2 \mathrm{l} / \mathrm{min}$ or 0.06 to $0.12 \mathrm{~m}^{3} / \mathrm{h}$ is typical. Several elements can be determined at absolute levels of $1 \mathrm{n}$ which permits analyses at concentrations of about $0.01 \mu \mathrm{g} / \mathrm{m}^{3}$ for a 1-h collection. The sensitivity for other elements such as As and Se is 50 to 100 times poorer so their determination at normal atmospheric concentrations is not possible on even longer-term samples. The most obvious solution to this limitation is in using filters offering higher sampling rates. A development in this area is currently in progress in the author's lab. Porous graphite discs, $47 \mathrm{~mm}$ in diameter and spectrographically pure, offering sampling rates of 10 to $20 \mathrm{l} / \mathrm{min}$ have recently become available. These are designed for use in the filter sample holders so commonly used by monitoring agencies and are amenable to direct analysis by several techniques. Dowell and Fagan [19], for example, collected particulates on the graphite discs, ground the entire disc to a powder with the addition of an internal standard, and analyzed the powder by a dc arc emission spectrometric method. The limitation encountered in this method originates from the 100 -fold dilution of the particulate trace element concentrations by the graphite filter substrate. For such filters, more than 90 percent of the particulates collected are concentrated within a few microns of the filter face. Accordingly, two techniques which sample largely the filter surface are being applied to this analysis. A rotating point-to-plane, high voltage spark, emission spectrometric 
technique is being evaluated. Results to date indicate that more than 20 elements can be determined via this technique at atmospheric concentration levels below $0.01 \mu \mathrm{g} / \mathrm{m}^{3}$ based on a half-hour sampling interval. The long-range intent is to use this method preceded by nondestructive $\mathrm{x}$-ray fluorescence determinations carried out on the same filter samples. The use of the $\mathrm{x}$-ray method will extend the general analytical capability to include elements such as $\mathrm{S}, \mathrm{Br}$, and $\mathrm{Cl}$ which cannot be determined readily by emission spectroscopy. It is anticipated that these developments coupled with the use of the low blank graphite disc filters will add new dimensions to the atmospheric particulate analysis capabilities.

In considering the general nature of the particulate analysis problem, it is surprising that $\mathrm{x}$-ray fluorescence techniques have not been used more extensively. The samples collected on filters are generally ideal for x-ray measurements. The sample thickness is relatively thin so any self-absorption corrections required should not be inordinately complex; absorption by light elements may well be the principal problem. Rhodes and Hunter [20] have indicated that particle size effects may be important but have formulated simplified formulas for corrections that may be required. The particulates themselves, and/or the filter substrate, may form a fairly uniform backscatter medium. Dzubay et al. [21] have shown that the backscatter problem can be reduced through the use of polarizing optics. In brief, the direct multielement analysis capabilities of $\mathrm{x}$-ray fluorescence coupled with its fairly uniform sensitivity for a large number of elements surely indicates that a number of the problems previously mentioned can be solved conveniently via the use of this technique. The reports by Birks and Gilfrich $[10,22]$ indicate that multicrystal spectrometers can be used to analyze simultaneously up to 24 elements with good detection capability. The development of high resolution, low background, solid state detection systems [23] has improved the alternative of analysis by energy dispersive $\mathrm{x}$-ray methods. A system of this type has been used in conjunction with a series of secondary fluorescers for the analysis of a large range of elements found in urban aerosols [6]. Studies such as these should lead to the increased use of $\mathrm{x}$-ray fluorescence for particulate analysis.

In the author's view, future developments in atmospheric particulate analysis should be directed at simultaneous multielement analysis systems. The sample collection media used should be selected on the basis of low blank and minimal sample handling/pretreatment criteria. Wherever possible direct analyses should be carried out to avoid the sample contamination and analyte loss problems. The use of independent analysis techniques to provide accuracy cross-check data on any one sample should certainly be considered. The present discussion has 
emphasized the possibilities associated with the use of two such techniques: emission spectroscopy and x-ray fluorescence. These are capable of satisfying the majority of the criteria outlined. While other techniques, such as neutron activation analysis and inorganic mass spectrometry, are also capable of satisfying these criteria, it is not likely that they will be able to compete on the basis of sample throughput rates or other economic considerations. The primary utilization of such techniques will consequently be restricted to a supportive role.

\section{Acknowledgement}

The author's research efforts mentioned herein were supported by NSF Grant number GI 34813X.

\section{References}

[1] Butcher, S. S. and Charlson, R. J., An Introduction to Air Chemistry, Academic Press, New York (1972).

[2] Lee, R. E., Jr., Science 178, 567 (1972).

[3] Whitby, K. T., Husar, R. B., and Lui, B. Y. H., J. Colloid Interface Sci. 39, 117 (1972).

[4] Whitby, K. T., Charlson, R. E., and Wilson, W. E., Science 183, 1098 (1974).

[5] Davies, C. N., Air Filtration, Academic Press, New York (1973).

[6] Dzubay, T. G. and Stevens, R. K., Presented at Second Joint Conf. on Sensing Environmental Pollutants, Washington, D.C. (December 1973).

[7] Amdur, M. O., Proc. Am. Phil. Soc. 14, 3 (1970).

[8] Natusch, D. F. S., Wallace, J. R., and Evans, C. A., Jr., Science 183, 202 (1974).

[9] Bowman, H. R., Conway, J. G., and Asaro, F., Environ. Sci. Technol. 6, 558 (1972).

[10] Birks, L. S. and Gilfrich, J. V., Report No. EPA-R2-72-063, Environmental Protection Agency (November 1972).

[11] Luke, C. L., Kametoni, T. Y., Kessler, J. E., Loomis, T. C., Bove, J. L., and Nathanson, B., Environ. Sci. Technol. 6, 1105 (1972).

[12] Seeley, J. L. and Skogerboe, R. K., Anal. Chem. 46, 415 (1974).

[13] Skogerboe, R. K., Monitoring Trace Metal Particulates: An Evaluation of the Sampling and Analysis Problems, in Instrumentation for Monitoring Air Quality, ASTM STP 555, American Society for Testing and Materials, Philadelphia, Pennsylvania, 125-136 (1974).

[14] Gandrud, B. and Lazrus, A. L., Environ. Sci. Technol. 6, 457 (1972).

[15] Tölg, G. Talanta 19, 1489 (1972).

[16] Gleit, C. E. and Holland, W. D., Anal. Chem. 34, 1454 (1962).

[17] Woodriff, R. A. and Lech, J. F., Anal. Chem. 44, 1323 (1972).

[18] Katz, M., Measurement of Air Pollutants, World Health Organization, Geneva, 1969. 
[19] Dowell. H. J. and Fagan, A. W., Paper presented at the 11 th National Meeting of the Society for Applied Spectroscopy. Dallas, Texas. September 1972.

[20] Rhodes. J. R. and Hunter. C. B., X-Ray Spectrometry 1. 113 (1972).

[21] Dzubay, T. G.. Jarrett, B. V., and Jaklevic, J. M., Nucl. Instrum. and Methods 115. 297 (1974).

[22] Birks. L. S. and Gilfrich. J. V., Report No. EPA-650/2-73-006, Environmental Protection Agency (June 1973).

[23] Goulding. F. S., Jaklevic. J. M., Jarrett. B. V., and Landis, D. A., in Advances in XRay. Analysis, Heinrich et al., Eds., Vol. 15, p. 470, Plenum Press, New York (1972). 



\title{
ANALYTICAL CHEMISTRY OF NATURAL WATERS
}

\author{
D. E. Robertson
}

\author{
Battelle \\ Pacific Northwest Laboratory \\ Richland, Washington 99352 U.S.A.
}

\begin{abstract}
Analysts who have had more than a casual experience in trace element analysis of natural waters will unanimously agree that achieving accurate and precise results is indeed a difficult task. The reasons for these difficulties are numerous and begin with the collection of the sample. Many of the factors which govern the accuracy and precision attainable by present techniques and instrumentation for the measurement of trace element analysis of waters, from the sampling through the actual analysis, are discussed. Several case studies are presented which illustrate the parameters which must be carefully evaluated to ensure sufficient accuracy and precision in water analysis.
\end{abstract}

Keywords: Accuracy; analytical chemistry; contamination; errors; natural water; sample storage; sampling.

\section{Introduction}

Since the beginning of recorded history man has sought to characterize the nature of water, the hydrological cycle, and the role of water in biotic and abiotic processes. Recently, we have become concerned with the quality of our natural waters, because the dispersions of man's industrial wastes are creating deleterious effects upon the aquatic environment. The oceans must be free of devastating pollution to maintain the delicate ecological balance which sustains the abundant marine food chains. Fresh waters must be of sufficient purity to be used for domestic consumption, for agricultural purposes, for recreation and asthetic uses, and for supporting freshwater food chains.

Our understanding and appreciation of the intricate biogeochemistry of natural waters is directly proportional to our ability to accurately measure their composition and their physicochemical properties. Analytical chemistry plays a key role in characterizing natural waters, in assessing 
their quality and in providing information for implementing methods for their wise use and conservation.

The title assigned to this presentation is extremely broad in scope and potentially encompasses many types of chemical measurements often encountered in water analysis. However, for the sake of brevity and to stay within the realm of my own personal expertise, I will concentrate my remarks on the analysis of trace elements in natural waters, and in particular, seawater. This paper describes some of the important factors which govern the accuracy and precision attainable in the analysis of trace elements in waters. Typical means of evaluating these factors are illustrated in the presentation of a case study involving our investigation of the geographical and vertical oceanic distribution of the toxic heavy metal mercury.

\section{The Present State of the Art}

Despite all of the recent sophisticated, highly sensitive analytical techniques and instrumentation which have become available to the water analyst, the accurate and precise measurement of trace elements in natural waters is still a very difficult task. This difficulty can be best illustrated by the results of several interlaboratory calibration exercises in which identical seawater aliquots were analyzed for a variety of trace elements by a number of different investigators. In the 1970 intercalibration study of Brewer and Spencer [1] analysts from 26 laboratories, working independently, and using their preferred methods of analysis, measured the concentrations of various trace elements in carefully prepared aliquots of seawater from two samples of deep Atlantic and Pacific Ocean water. The lack of interlaboratory agreement on the concentrations of many elements often exceeds a factor of 10 . For example, the results of the Co measurements from this exercise are shown in figure 1. The reported Co concentrations ranged from 0.014 to $0.461 \mu \mathrm{g} / \mathrm{l}$. It is readily apparent that the atomic absorption spectrometry (AAS) measurements consistently gave higher Co concentrations compared to the neutron activation analysis (NAA) and X-ray fluorescence (XRF) methods. In this case, the most accurate Co concentration was, in all likelihood, the lowest value $(0.014$ $\mu \mathrm{g} / \mathrm{l})$ determined by instrumental neutron activation analysis. The AAS analyses of Co in seawater were so close to the analytical detection sensitivities that some unknown interference gave apparent Co concentrations which were 7 to 33 times too high. 


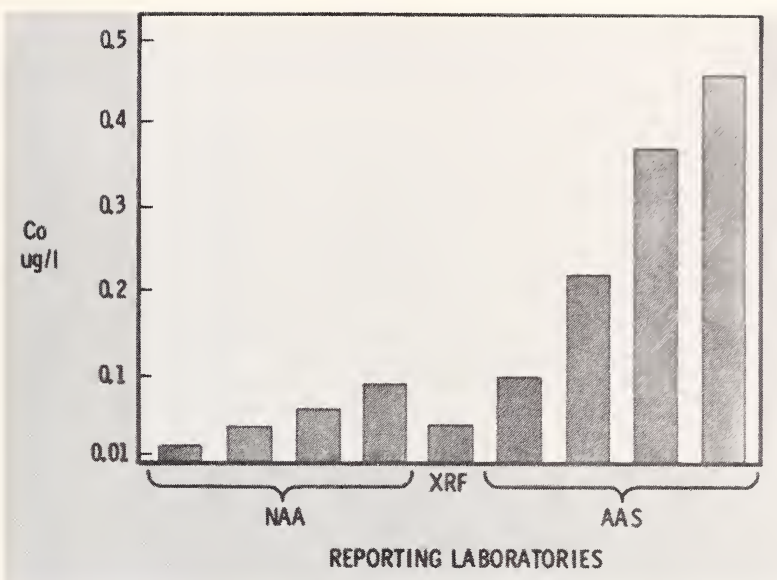

Figure 1. Interlaboratory analysis of cobalt in seawater.

tilaboratory intercalibration exercise to evaluate various accepted methods of measuring $\mathrm{Pb}$ in seawater [2]. The results of one set of measurements are shown in figure 2. The $\mathrm{Pb}$ concentration of the seawater sample was standardized by Patterson using very carefully controlled chemical preconcentration and isotope dilution-mass spectrometry (IDMS), and contained $0.014 \pm 0.003 \mu \mathrm{g} / \mathrm{l}$ of $\mathrm{Pb}$. It is interesting to note that three of the four AAS methods gave $\mathrm{Pb}$ concentrations very close to $0.055 \mu \mathrm{g} / \mathrm{l}$. Although the precision of the three AAS methods was quite good, the accuracy was off by a factor of about 4 , indicating some type of systematic contamination occurred which was peculiar to the AAS method. The anodic stripping voltametry (ASV) results ranged from 0.18 to $1.3 \mu \mathrm{g} / \mathrm{l}$; values 13 to 93 times too high, indicating severe contamination or instrumental interferences.

The above examples are merely representative of the extreme difficulties associated with the measurement of elemental constituents near or below the parts-per-billion $(\mu \mathrm{g} / \mathrm{l})$ concentration range in a complex mixture such as seawater. The difficulties experienced in trace element analysis of natural waters does not apply only to seawater; the problems involved in freshwater analyses are equally as severe. So the present state of the art for measuring trace elements in natural waters leaves something to be desired. Nevertheless, those analysts who very carefully evaluate their procedures and eliminate the inherent sources of error are achieving reasonably accurate and precise results, and are making significant contributions in aquatic and environmental chemistry. 


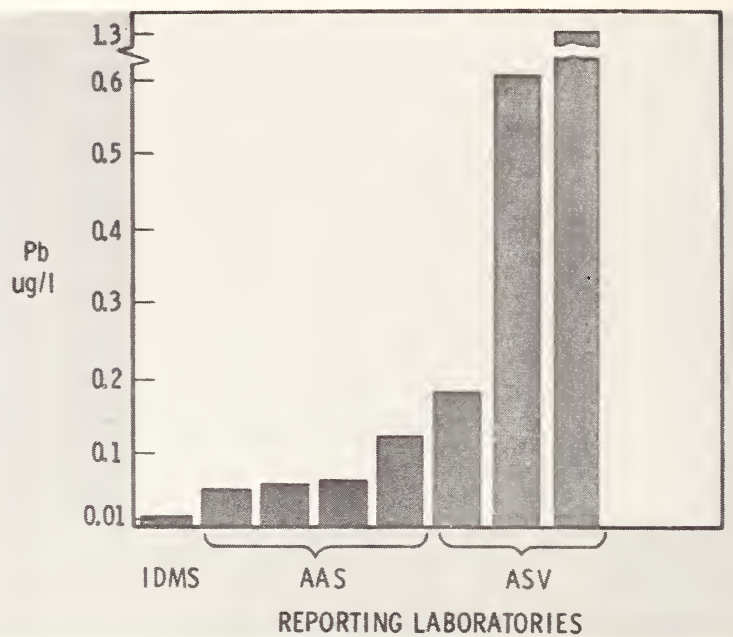

Figure 2. Interlaboratory analysis of lead in seawater.

\section{The Aquatic Environment}

What makes the trace element analysis of natural waters such a difficult analytical task? There are several reasons. First, the concentrations of most trace elements are present in most natural waters near or below the part per billion level, and this level is on the borderline of the detection sensitivities of many of the popular analytical techniques presently in use. Secondly, at these extremely low concentrations contamination problems are often overwhelming; and thirdly, natural waters are so complex in their physicochemical and biological makeup that very subtle partitioning among biotic and abiotic fractions greatly complicates storage and analysis of these waters.

Figure 3 illustrates a model of the major biogeochemical and physical processes occurring in the oceans. Essentially the same processes are active in freshwater environments. Trace elements contained in natural waters are subjected to all of these complex processes, and as a result occur in natural waters in a variety of physicochemical species. Table 1 lists the possible physicochemical forms that can exist for trace elements contained in natural waters. Trace elements may be present in a variety of inorganic and organic dissolved species, colloidal forms and associated with particulate matter. Most natural waters contain all of these forms, and numerous trace elements are simultaneously distributed throughout many of the various fractions. 
ROBERTSON

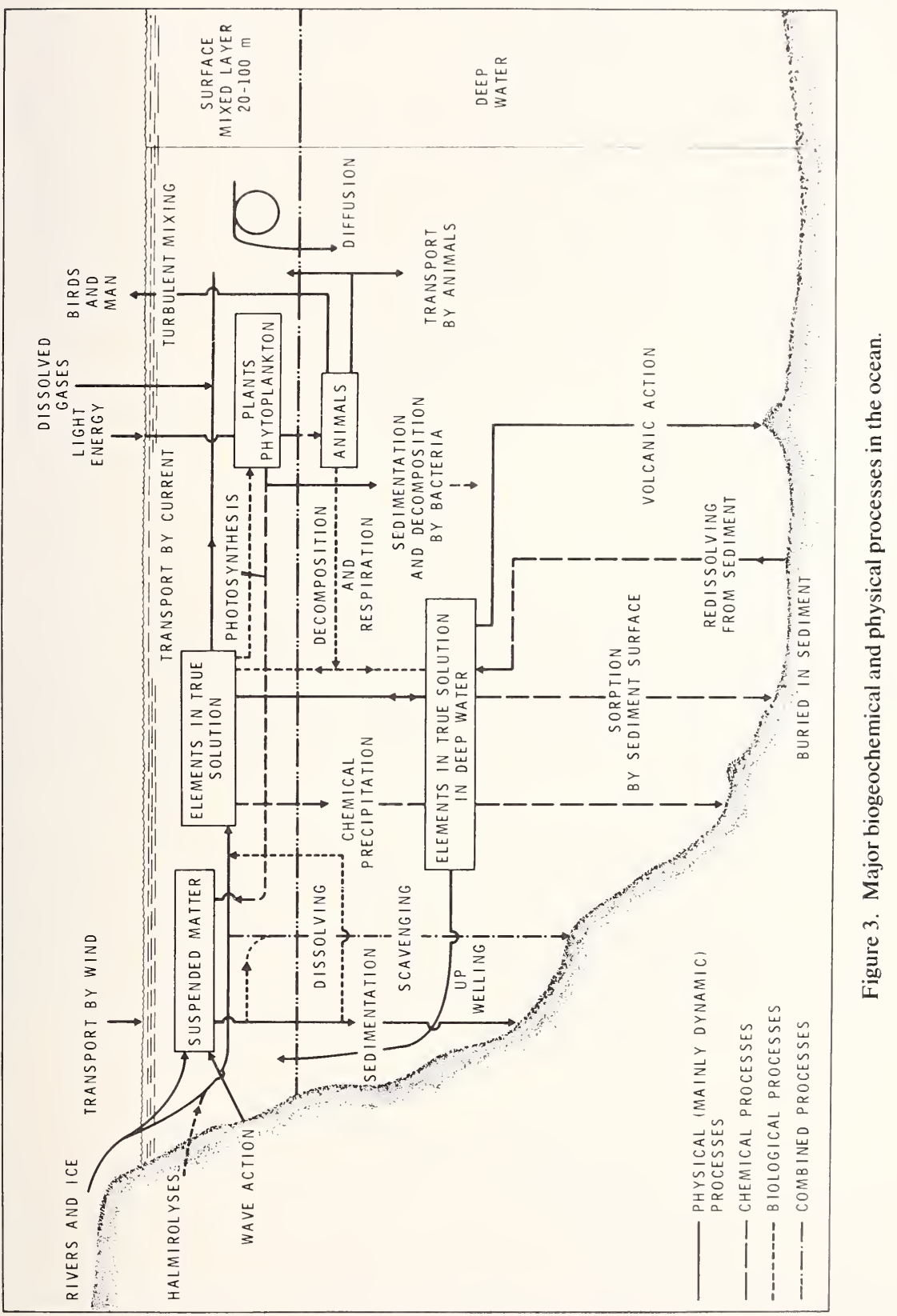


TABLE 1. Physicochemical forms of trace elements in natural waters

True solution
$\quad(<0.001 \mu \mathrm{m})$

Colloidal

$(0.001-0.10 \mu \mathrm{m})$

Particulate

$(0.1-50 \mu \mathrm{m})$
Simple hydrated ions

Inorganic complex ions

Organic chelates

Molecules and polymeric species

Ion pairs

Mineral substances

Hydrolysis and precipitation products

Biopolymers and detritus

Organic particles

(1) Plankton

(2) Detritus

(3) Bacteria and microorganisms

Inorganic particles

(1) Mineral substances

(2) Precipitated and coagulated particles

For example, transition elements such as iron, zinc, and copper have been shown to exist simultaneously in seawater as inorganic complex ions, organic chelates, and in association with mineral substances, hydrolysis and precipitation products, plankton and detritus. Depending upon the analytical method used for measuring these elements in seawater, the total distribution or only one particular physicochemical form may be measured. For example, if instrumental neutron activation analysis, involving no preconcentrations or separations were used, all forms (total) of the trace elements would be measured. However, if anodic stripping voltametry were used, only the simple hydrated or inorganic complexed ions would be measured, since the voltametry technique measures only dissolved, ionic species. Thus, the extreme complexity of natural waters can greatly influence the analyses of their trace element constituents and the interpretation of the results.

\section{Main Sources of Error}

The major sources of error in trace element analyses of natural waters are due to contamination, chemical interferences and instrumental disturbances. Contamination is undoubtedly the most universal problem plaguing trace analysts - and the most difficult to eliminate. Contamination may be classified into three categories:

1. Positive contamination, resulting from the addition of extraneous matter to the constituent being determined. 
2. Negative contamination, which causes erroneously low results due to unexpected losses.

3. Pseudocontamination, resulting from uncontrolled variation of parameters, such as time and method of sampling.

Examples of positive contamination are the leaching of constituents from container materials into the sample during storage or chemical manipulations and the introduction of airborne contaminants during the handling of uncovered samples. Negative contamination is illustrated by losses of volatile elements such as As. Cd. Hg. and Se. during evaporation of acidified waters. or by losses resulting from adsorption of elements from solution onto container walls or filters.

A good example of pseudocontamination occurred at our laboratory when seawater stored in polyethylene bottles was preserved by freezing. During the freezing processes a highly concentrated brine was formed at the bottle cap and partially extruded past the cap as the water continued to freeze and expand. The resulting seawater samples were markedly reduced in salinity and trace element content when thawed and analyzed.

Chemical and instrumental interferences can also be interpreted as kinds of contamination since they result in either positive or negative bias of the data. These disturbances are discussed in the following section which deals with the role of contamination associated with the main parameters which affect the accuracy and precision of trace element analysis of natural waters.

\section{Parameters Affecting Accuracy}

The main parameters affecting the accuracy and precision in trace element analysis of natural waters are:

- Sampling

- Containment

- Handling

- Analysis

- Interpretation

Contamination is an inherent problem in all of the above parameters. and chemical and instrument interferences can be major sources of error in the latter three.

The sampling aspects of trace element analysis are so important that an entire session of this symposium has been devoted to this subject. In the collection of natural waters representative sampling is a most critical initial step in the analysis. Since the sampling of natural waters has been 
discussed by Dr. Patterson, it should suffice here to say that to ensure the collection of representative water samples requires the implementation of "clean lab" techniques. I have only to add that unless the complete history of any sample is known with certainty, the analyst is well advised not to spend his time analyzing it.

\section{A. Containment}

The difference between a representative and a contaminated natural water sample often resides in the quality of the container. Some trace element impurities can be leached from glass surfaces and contaminate natural waters stored therein. Polyethylene, polypropylene or Teflon are the preferred materials for storage containers. The advantages of using plastic containers over glass containers for storing high purity solutions have been discussed in detail elsewhere [3].

A problem of somewhat the opposite nature can also be experienced in storing natural waters, due to losses of trace elements from solution by adsorption onto the container surfaces. The adsorption problem is particularly serious if natural waters are stored over long time periods at their normal, essentially neutral $\mathrm{pH}$. Serious losses from solution of many of the transition and rare earth elements, and the groups III, IV, V, and VI elements by adsorption onto container walls have been reported $[3,4]$. Normally, these adsorption losses can be prevented or minimized by acidifying the natural waters to a $\mathrm{pH}$ of 1 to 2 with high purity acid. In some cases, freezing the water samples immediately after collection retards both leaching of impurities from the container and adsorption losses to the container walls.

Another problem in containing natural waters is the change in partitioning of trace elements between dissolved and particulate phases which can occur during long storage periods. In natural waters equilibrium is not usually established between dissolved and particulate phases because of the dynamic input and removal processes for these constituents. However, when an unfiltered natural water sample is confined within a storage container equilibration of these phases begins and the in situ character of the water sample can be altered. Growth of biological organisms can also have a profound effect upon the partitioning of trace elements in the water. These problems can be minimized if the water samples are filtered and acidified before storage. However, extreme care must be taken to avoid contamination during the filtering process and by the addition of acid to the sample. 


\section{B. Handling}

During the analysis of natural waters the samples can be subjected to a variety of physical and chemical manipulations. Such procedures might include measuring, weighing, filtration, preconcentration, desiccation or freeze drying. If these operations are not carefully controlled, contamination resulting from faulty handling of the samples can occur.

Improperly cleaned labware is a frequent source of contamination. Impurities can be leached from "dirty" labware by natural waters during measuring and weighing procedures. Glass and plastic labware often must be soaked for several days in hot, strong acid solutions and then carefully rinsed with high purity water before they become free from releasing a particular contaminant.

The handling of samples frequently requires exposure to the ambient atmosphere for various periods of time. Unless a work area is designed and used specifically as a clean room for the preparation of samples, it can be

remarkably prolific source of airborne contamination. Air currents

? ted in fume hoods and by air-conditioning and heating fans con-

atiy circulate laboratory dust throughout a room. This dust can be aeposited on labware and other apparatus used during the analysis, or on the actual samples if they are left exposed to the atmosphere. A preferred method of minimizing airborne contamination is through the use of positive pressure, laminar-flow clean hoods. These hoods blow filtered, stream-lined air across an enclosed work area, preventing any airborne room dust from entering the clean area.

Filtration of natural waters is a common practice in removing suspended particulate phases. Contamination of water samples during filtration can be a serious problem if proper precautionary measures are not enforced. Trace metal contaminants may be leached from the filtering apparatus and the filters themselves. Most filter media contain significant concentrations of many elements, and produce high blank values when determining trace element concentrations in the particulate phases. Another contamination hazard during filtering is the sucking of airborne contaminants into the sample by vacuum systems. Several means of minimizing contamination during filtering have been suggested [3].

Natural waters are frequently evaporated as a preconcentration step prior to trace element analysis. Such procedures usually require that the samples remain in containers for extended periods at elevated temperatures. Under these conditions numerous contaminants may be leached from the containers. Many trace elements form volatile compounds when heated in acid solutions, and losses of $\mathrm{Hg}, \mathrm{As}, \mathrm{Se}, \mathrm{Cr}, \mathrm{Sb}, \mathrm{V}, \mathrm{Ge}, \mathrm{Te}, \mathrm{Sn}$, 
$\mathrm{Re}, \mathrm{Si}, \mathrm{P}$ and $\mathrm{Ru}$ can occur if acidified natural waters are evaporated under certain conditions.

Often trace elements occur in natural waters in concentrations so low that preconcentration is necessary before the analysis. A wide variety of preconcentration techniques are commonly employed including solvent extraction, coprecipitation, cocrystallization, ion exchange and adsorption. These techniques all require further handling of the samples, including the addition of various reagents. Manipulations such as centrifuging and filtering are frequently employed for phase separations. All of these processes increase the risk of contamination.

Preconcentration techniques often isolate only one form of the several physicochemical species of a trace element which may exist in solution. Thus, only a specific fraction of the total amount of a trace element may be preconcentrated by a given technique. This, in a sense, could be classified as pseudocontamination, since erroneous conclusions could be drawn from the results.

\section{ANALYSIS}

During the actual quantification or instrumental determination of the concentration of a trace element, errors affecting the accuracy of the measurement can occur due to either chemical interferences or instrumental interferences. These types of interferences are sometimes very subtle and can go undetected. Chemical interferences, or matrix effects, can have both a positive and negative impact upon the measurement. For example, in the atomic absorption spectrophotometry of strontium, small concentrations of either $\mathrm{Al}$ or Si produce a serious depression in the absorbance of strontium, thus leading to erroneously low results. The opposite effect can occur when $\mathrm{Cr}$ absorbance in a $10 \mathrm{ppm} \mathrm{Cr}$ solution can be enhanced up to 60 percent by the presence of $50 \mathrm{ppm}$ of Ti and V.

For essentially every measurement technique there exists some type of chemical interference which can affect the accuracy. It is imperative that analysts be very comprehensive in evaluating their analytical techniques to avoid subtle chemical interferences.

Instrumental interferences are likewise ubiquitous if careful controls are not established. In every method of instrumental analysis there exists a certain background interference, or "noise level," that limits the sensitivity of the method. If the instrumental background is not constant with time, these fluctuations can "contaminate" a series of measurements made near the detection sensitivity of the instrument, since the data would be biased by the instrument's response. Instrumental disturbances can 
result, for example, from fluctuations in power supplies, light sources, emission sources, receivers, amplifiers and recorders. Also, variations in room temperatures, differences in flame and electrode temperatures, differences in sample geometries, etc., may create instrument-response fluctuations that reduce precision and accuracy of an analysis. Each method of instrumental analysis has its own peculiar problems, and an analyst must therefore be familiar with the potential sources of error in his instruments.

\section{INTERPRETATION}

Errors in trace element measurements are sometimes caused by faulty interpretation of data. These errors most frequently occur when one is working near the detection limit of the analytical method. For example, nonlinearity of working curves at very low concentrations may introduce errors if insufficient data points have not adequately defined the working curve. Interpretation errors may also exist when one assumes that he is measuring all forms of a trace element present in the sample, when in fact, his analytical technique is only measuring one specific form of that element. Misinterpretation of curve slopes, optical and emission spectra and signal-to-noise ratios can all lead to serious errors.

\section{Mercury in the Oceans - A Case Study in Evaluating Accuracy}

To illustrate the important parameters which must be considered and evaluated to ensure accurate and precise measurements of trace elements in natural waters, I would like to discuss the results of our $\mathrm{Hg}$ analysis of Atlantic Ocean seawater samples made during our present participation in the Geochemical Ocean Sections Study (GEOSECS). I have singled out $\mathrm{Hg}$ because of the present intense interest in this toxic heavy metal as an environmental contaminant, and because I feel we have an important story to tell concerning its distribution and behavior in the oceans. Also, $\mathrm{Hg}$ is a rather difficult trace element to measure and its analysis exhibits many of the problems encountered during trace element studies of natural waters.

The GEOSECS project is a multi-institutional program designed to provide detailed measurements of ocean constituents along Arctic to Antarctic sections of the Atlantic and Pacific Oceans. The objectives of this study are to better characterize the geochemistry of the oceans, and utilize the oceans' chemical constituents to trace the large-scale mixing and circulation processes in the oceans. The GEOSECS program is the most extensive and sophisticated geochemical study of the oceans that has ever 
been accomplished. Pacific Northwest Laboratory is a participant in this program, providing measurements of trace element and radionuclide constituents in the oceans (see table 2). The GEOSECS Atlantic and Pacific Ocean cruise tracks are shown in figure 4. The Atlantic track was completed in the spring of 1973 and the Pacific track was completed during the summer of 1974. The Atlantic and Pacific tracks occupied 121 and 147 stations, respectively, providing the most complete set of seawater samples for characterization that have ever been collected. Figure 5 illustrates a cutaway view of the Western Basin of the Atlantic Ocean extending from the Greenland Sea in the north to the Southern Ocean in the Antarctic. Seventy-four stations were occupied along this track; 45 of which were major seawater sampling stations. At each major station, shown in figure 5, approximately 44 to 66 seawater samples were collected between the ocean surface and the sea floor. These samples are represented in figure 5 by the dots under each station.

Twenty-two of the Western Atlantic Ocean stations have been analyzed for $\mathrm{Hg}$. Over 1000 seawater samples, blanks and standards have been analyzed to provide the data to characterize the oceanic distribution of $\mathrm{Hg}$ in the western basin of the Atlantic Ocean.

The analytical method is based on the cold vapor, ultraviolet absorptiometric detection of vaporized elemental $\mathrm{Hg}$. Very high sensitivity is achieved by sweeping the generated $\mathrm{Hg}$ vapor through a long, focusing absorption cell in a dual beam Laboratory Data Control Mercury Analyzer $^{R}$. Briefly, the method, which measures total mercury, consists of adding $100 \mathrm{ml}$ of seawater or freshwater to a carefully precleaned 250 $\mathrm{ml}$ Pyrex glass T/S flask and oxidizing the sample with $2.5 \mathrm{ml}$ of concentrated $\mathrm{HNO}_{3}, 2.0 \mathrm{ml}$ of 5 percent $\mathrm{KMnO}_{4}$ and $2.0 \mathrm{ml}$ of 5 percent $\mathrm{K}_{2} \mathrm{~S}_{2} \mathrm{O}_{8}$ at $80{ }^{\circ} \mathrm{C}$ for 2 hours in a hot water bath. The samples are cooled to room temperature and $1 \mathrm{ml}$ of 12 percent $\mathrm{NH}_{2} \mathrm{OH} \cdot \mathrm{HCl}$ is added to reduce the excess $\mathrm{KMnO}_{4}$ and the $\mathrm{MnO}_{2}$ which is formed. The flask is then purged with air to remove any $\mathrm{Cl}_{2}$ gas which interferes with the $\mathrm{Hg}$ measurement. Ten $\mathrm{ml}$ of 10 percent $\mathrm{SnCl}_{2}$ is added, the flask is quickly stoppered, and after a 90 -second waiting period the reduced, vaporized elemental $\mathrm{Hg}$ is swept from the flask in a stream of $\mathrm{N}_{2}$ gas into the absorption cell of the LDC Mercury Analyzer. The absorption of the UV beam by the vaporized $\mathrm{Hg}$ atoms is proportional to the $\mathrm{Hg}$ concentration. Quantification is made by comparing the peak height response of the sample with standard curves prepared by spiking seawater with known quantities of standard $\mathrm{Hg}$ solutions. Details of the sampling technique have been published elsewhere [5]. 
TABLE 2. Trace element and radionuclide constituents measured at Battelle-Northwest during GEOSECS

\section{Trace elements}

INAA NAA and AA

$\begin{array}{lll}\mathrm{Zn} & \mathrm{Hg} & { }^{7} \mathrm{Be} \\ \mathrm{Co} & \mathrm{Cd} & \\ \mathrm{U} & \mathrm{As} & { }^{95} \mathrm{Zr}-{ }^{95} \mathrm{Nb} \\ \mathrm{Sb} & \mathrm{Se} & { }^{106} \mathrm{Ru} \\ \mathrm{Sc} & \mathrm{Ag} & \\ \mathrm{Cs} & \mathrm{Cr} & { }^{144} \mathrm{Ce} \\ \mathrm{Rb} & \mathrm{Mn} & { }^{239} \mathrm{Pu} \\ \mathrm{Fe} & & \end{array}$

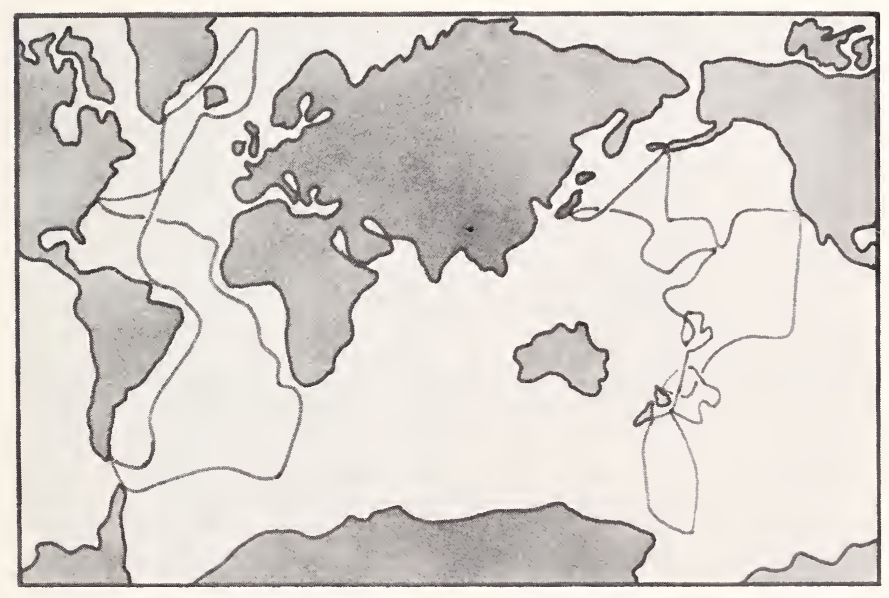

Figure 4. GEOSECS cruise tracks. 


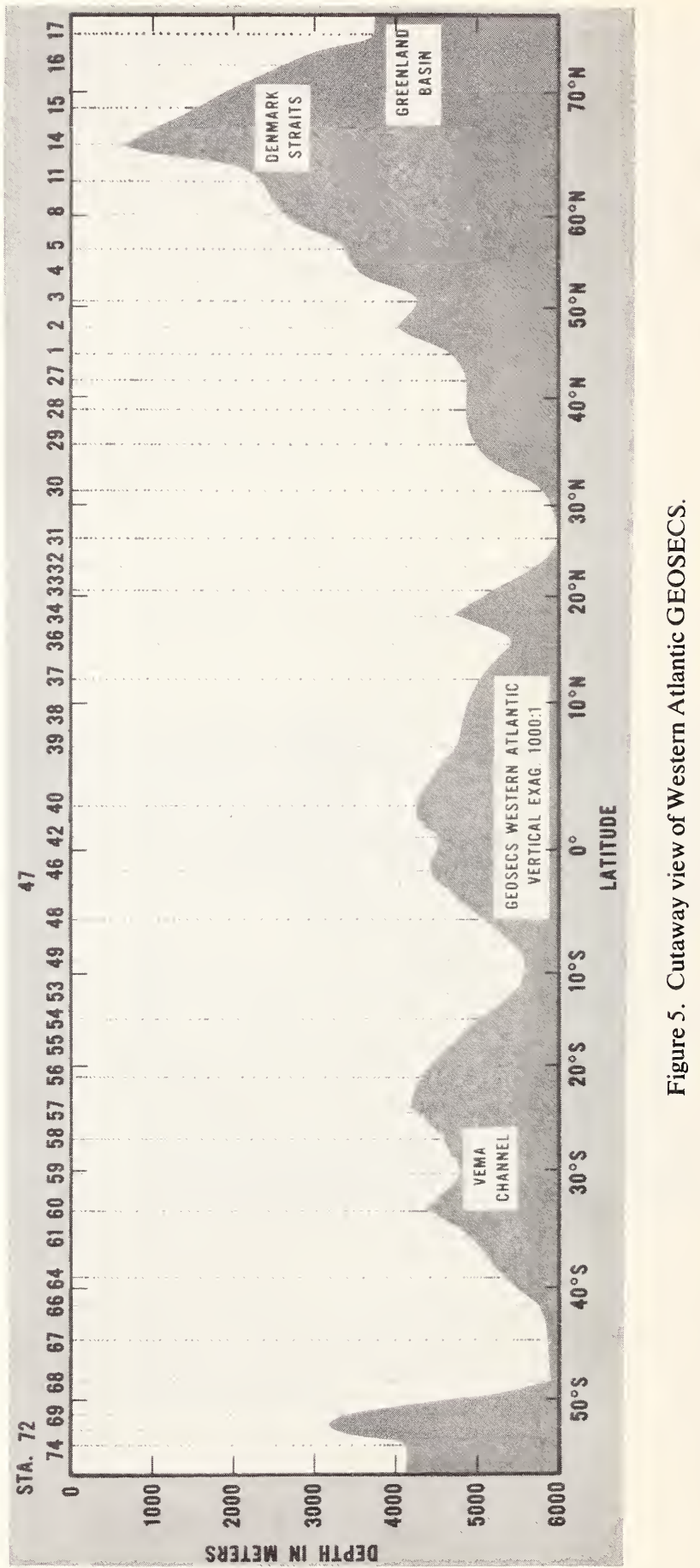


The $\mathrm{Hg}$ concentrations in the Western Atlantic Ocean are highly variable. Table 3, which presents the $\mathrm{Hg}$ analyses of seawater from four widely separated stations, illustrates the great range of $\mathrm{Hg}$ levels in the ocean. The $\mathrm{Hg}$ distribution in the western basin of the Atlantic Ocean is characterized by extremely low concentrations ( 20 to $40 \mathrm{ng} / \mathrm{l}$ ) over most of the ocean between about $60^{\circ} \mathrm{S}$ to $15^{\circ} \mathrm{N}$ latitude. At approximately $15^{\circ} \mathrm{N}$ a very sharp concentration gradient exists. South of $15^{\circ} \mathrm{N}$ the $\mathrm{Hg}$ concentrations range between 2 to $20 \mathrm{ng} / \mathrm{l}$ from the surface to the ocean floor, whereas north of $15^{\circ} \mathrm{N}$ the concentrations range between 20 to $80 \mathrm{ng} / \mathrm{l}$. Between about $20^{\circ} \mathrm{N}$ to $45^{\circ} \mathrm{N}$ a large core of water located at depths between 1500 to 3400 meters was observed to contain highly elevated levels of $\mathrm{Hg}$ (160 to $1370 \mathrm{ng} / \mathrm{l}$; see, for example, Station 27 in table 3). No systematic concentration gradients of $\mathrm{Hg}$ extending from the ocean surface or from the ocean floor were observed at this location suggesting that the core of high $\mathrm{Hg}$ water had not originated from the surface or bottom waters at $20^{\circ} \mathrm{N}$ to $45^{\circ} \mathrm{N}$. The water masses located between 1500 to 3400 meters at this location have been identified from salinity-temperaturedensity data as Labrador Sea water (1500-2000 meters), Iceland-Scotland overflow water (2800-3200 meters) and Denmark Straits overflow water (3700-4000 meters). Mediterranean Sea water flows into the western basin of the Atlantic Ocean at this latitude between a depth of 1000 to 1400 meters. The observed core of high $\mathrm{Hg}$ seawater thus originated in the north polar regions by chilling and sinking of surface waters, followed by their subsequent southward transport at mid-depths. Since the north polar regions have been shown to be relatively pristine and uncontaminated by man's industrial wastes, the high $\mathrm{Hg}$ concentrations in the North Atlantic mid-depth water masses must have resulted from natural, large-scale inputs of $\mathrm{Hg}$ to the oceans in the high northern latitudes. The only mechanism which can be conceived to mobilize such enormous amounts of $\mathrm{Hg}$ into the oceans is degassing of $\mathrm{Hg}$ from the Earth's mantle during submarine volcanism and tectonic activity. The Iceland area is the most volcanic region of the Earth. Iceland is actually a surface manifestation of the great Mid-Atlantic Ridge, the gigantic submarine mountain range which transects the entire Atlantic Ocean floor in a north-south direction. Near Iceland the Mid-Atlantic Ridge is extremely active volcanically, with both surface and submarine eruptions occurring at frequent intervals due to fresh magma being extruded at the ridge crest. South of Iceland the Mid-Atlantic Ridge is split by a huge transform fault known as the Gibbs Fracture Zone. This fault was created when two segments of the ridge were displaced from each other by oppositely spreading tectonic plates. As the plates flow from the spreading center, faulting and 


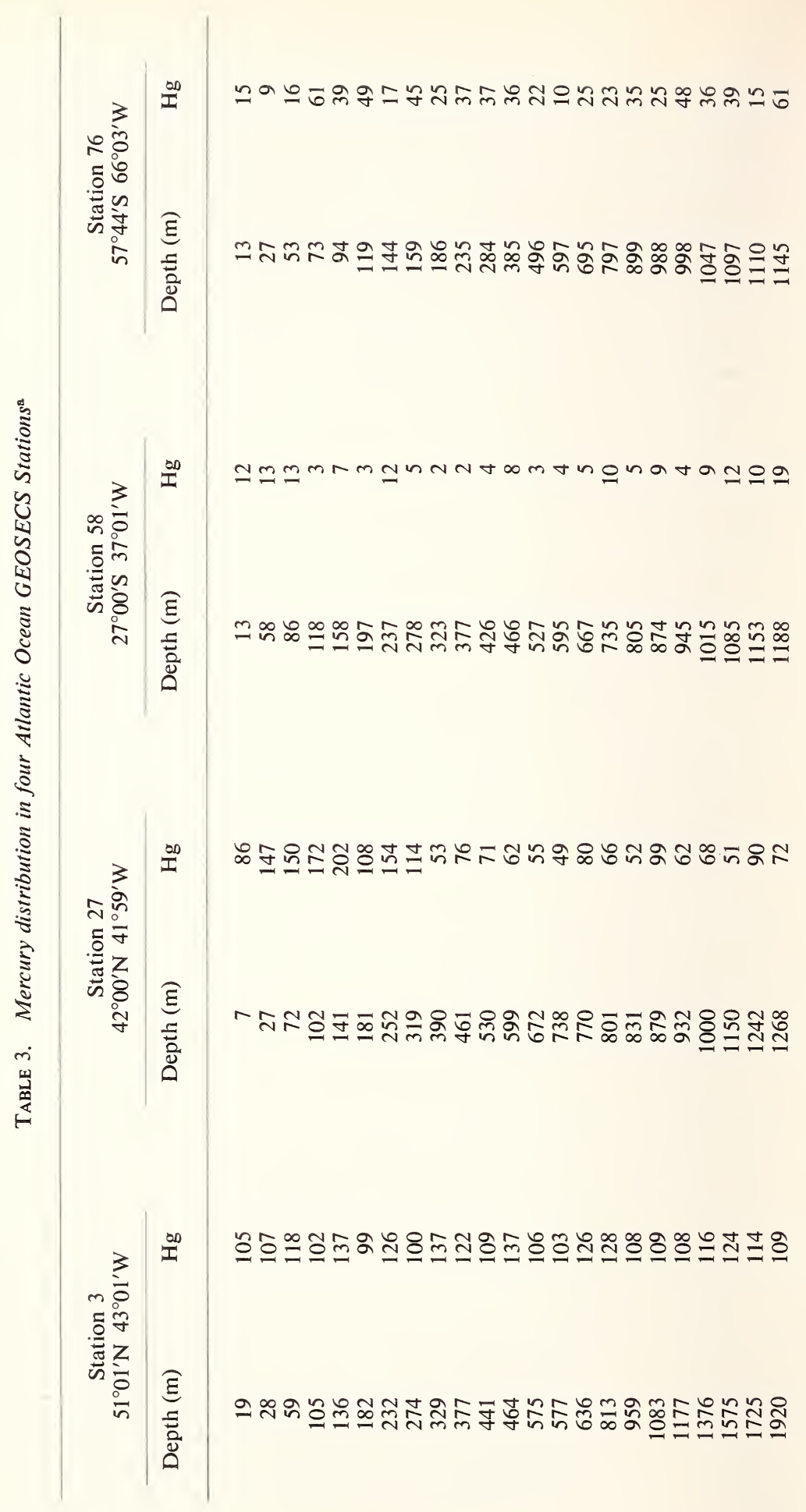




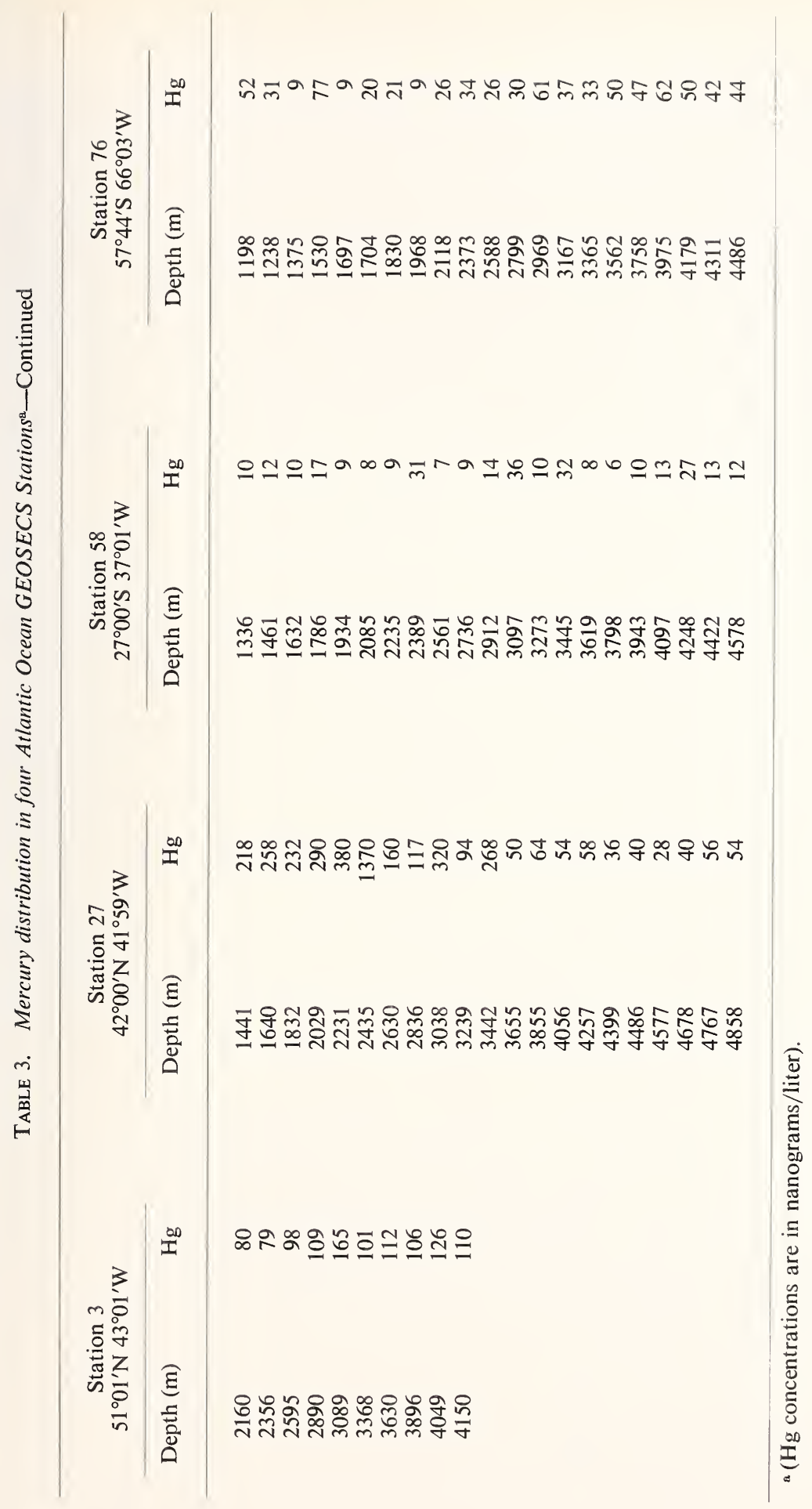


volcanic eruptions occur and lava flows along the length of the ridge, building mountains and escarpments (see ref. 6 for an excellent detailed discussion of these processes).

It appears rather certain that these enormous geophysical volcanic and tectonic processes are the sources of $\mathrm{Hg}$ in the deep ocean. Extremely high concentrations of $\mathrm{Hg}$ are associated with volcanic gases and liquids released to the environment during volcanism and geothermal processes.

Another mechanism by which high levels of $\mathrm{Hg}$ could be transported into the western basin of the Atlantic Ocean between $20^{\circ} \mathrm{N}$ and $45^{\circ} \mathrm{N}$ is by the lateral, east-to-west flow of Mediterranean Sea water across the volcanically active seamounts in the region of the Azores. This water enters the western basin of the Atlantic between depths of about 1000 to 1400 meters.

To obtain an appreciation for the magnitude of these processes, most of which actually go undetected on the ocean floor, we can evaluate the welldocumented submarine eruption of Surtsey which occurred in relatively shallow water off the southern coast of Iceland and eventually built up enough lava and ash to produce a new, permanent island in the North Atlantic Ocean. Between 1963 to 1967 , Surtsey produced over $10^{15} \mathrm{~cm}^{3}$ of lava and ash. If we assume that magma contains $1 \mathrm{ppm}$ of $\mathrm{Hg}$, and that most of it is lost by degassing when the magma is extruded, the Surtsey eruption could have released:

magma $\times$ density $\times \mathrm{Hg}$ content $=$ total $\mathrm{Hg}$ released $\left(10^{15} \mathrm{~cm}\right)\left(3 \mathrm{~g} / \mathrm{cm}^{3}\right)\left(1 \times 10^{-6} \mathrm{~g} \mathrm{Hg} / \mathrm{g}\right)=3 \times 10^{9} \mathrm{~g}$ of $\mathrm{Hg}$

This enormous amount of $\mathrm{Hg}$ is roughly the same order of magnitude estimated to be released to the global environment annually by all of man's industrial activities [7]. Thus, a single volcanic eruption such as Surtsey could mobilize enough $\mathrm{Hg}$ to rival all of man's industrially derived annual releases of mercury to the environment.

This knowledge is extremely important since it places the global mercury pollution problem into a proper perspective. While localized coastal pollution of the oceans with mercury can present serious problems, the open oceans are apparently not significantly increased in $\mathrm{Hg}$ concentrations by industrially mobilized mercury. These findings emphasize the fact that we must understand the natural distributions and behavior of heavy metal pollutants in the environment before we can intelligently assess man-made impacts. 


\title{
A. Evaluation of the Accuracy of Mercury Measurements
}

In any serious attempt to study trace elements in natural waters a comprehensive evaluation of the critical parameters mentioned in the foregoing sections must be done to ensure the accuracy and validity of the results. This is especially true for our GEOSECS $\mathrm{Hg}$ measurements since these data are providing the first real comprehensive baseline measurements for this important heavy metal contaminant. In addition, these data will revolutionize our thinking concerning global pollution of $\mathrm{Hg}$, since this is the first real evidence which illustrates the enormous magnitude of natural mobilization mechanisms compared to anthropogenic $\mathrm{Hg}$ pollution.

We know that we are experiencing some minor uncertainties in the measurement of $\mathrm{Hg}$ in our GEOSECS seawater samples. This is apparent from the analyses of several replicate samples taken at the same depths at nearly all of the GEOSECS stations. Occasionally, replicate seawater samples would differ in $\mathrm{Hg}$ concentrations by 30 to 50 percent and would on rare occasions differ by up to a factor of 2 . However, these deviations are not of a high enough magnitude or frequency to obviate the largescale, systematic variations of mercury that we have observed in the oceans. Nevertheless, it is imperative to know just how close we have come to defining the actual $\mathrm{Hg}$ distribution in the western basin of the Atlantic Ocean. We have therefore carefully evaluated the parameters which we felt were necessary to authenticate our mercury data and provide a measure of the degree of accuracy and precision we think we have attained.

The factors that we have considered are shown in table 4 .

TABLE 4. Parameter evaluation for ensuring accuracy in analysis of natural waters

\author{
Sampling Techniques \\ Storage Methods \\ Consistent and Known Yields of Chemical Manipulations \\ Consistent Procedural Blanks \\ Verification by Another Analytical Method \\ Analysis of Standard Reference Materials \\ Determine Precision at Representative Concentrations \\ Chemical and Instrumental Interferences
}




\section{B. Sampling Technique}

It is always important to determine if the sampling technique is providing representative, uncontaminated water samples. The sampling devices used in the GEOSECS expeditions were 30-liter modified, free-flushing Niskin bottles. The surgical rubber tubing formerly used to snap the end plugs in place were replaced with Teflon coated stainless steel springs to prevent serious contamination which results from various metals leaching from the rubber. The bottles, shown in figure 6 , were arranged on two rosettes, each containing 10 to 12 bottles, respectively. One rosette included a custom-made instrument package which contained a conductivity-temperature-depth sensor, a dissolved oxygen probe, a nephelometer and a bottom proximity pinger. Upon arriving at a sampling station, the

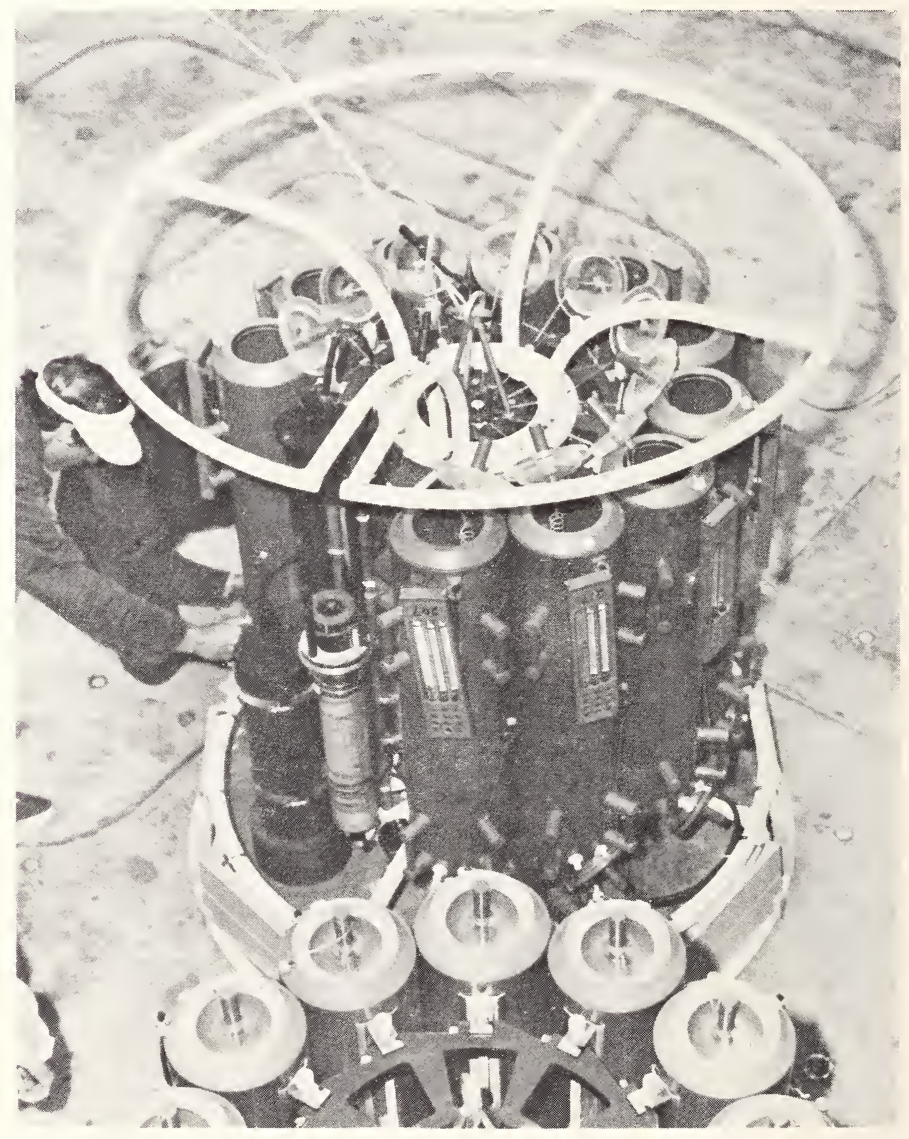

Figure 6. GEOSECS Niskin bottle water sampling rosettes. 
two rosettes were attached to a coaxial lowering cable and lowered to within several meters of the sea floor. During descent the in situ salinity, temperature, oxygen and light scattering were measured as a function of depth and recorded on shipboard displays and in a computer memory. Data from the underwater sensor package were transmitted by frequency shift keying along the coaxial lowering cable. During the ascent the Niskin bottles were tripped shut at any desired depth to collect samples at meaningful intervals. Usually a deep and a shallow cast, and sometimes an additional intermediate cast were taken at each station. Once the rosettes were brought on board ship, the water samples for trace element analysis were drained within 1 hour directly into precleaned 2-liter polyethylene storage bottles.

To determine that no significant positive or negative contamination of the water samples had occurred during containment in the Niskin bottles, two 30-liter Niskin bottles were filled with seawater containing an ultralow level of $\mathrm{Hg}$. Duplicate aliquots of the seawater were then immediately removed from the Niskin bottles, acidified, and stored in specially precleaned glass bottles. This was repeated after intervals of 6 and 18 hours to determine if $\mathrm{Hg}$ concentrations in the water contained in the Niskin bottles had remained constant. Several days later the acidified seawater samples were measured for $\mathrm{Hg}$. The results of this experiment are shown in figure 7. A very slight increase in $\mathrm{Hg}$ concentrations in the seawater occurred during the 18 hours of storage in the Niskin bottles, raising from an initial average value of $2 \mathrm{ng} / \mathrm{l}$ to $4 \mathrm{ng} / \mathrm{l}$ after 6 hours, and to 8

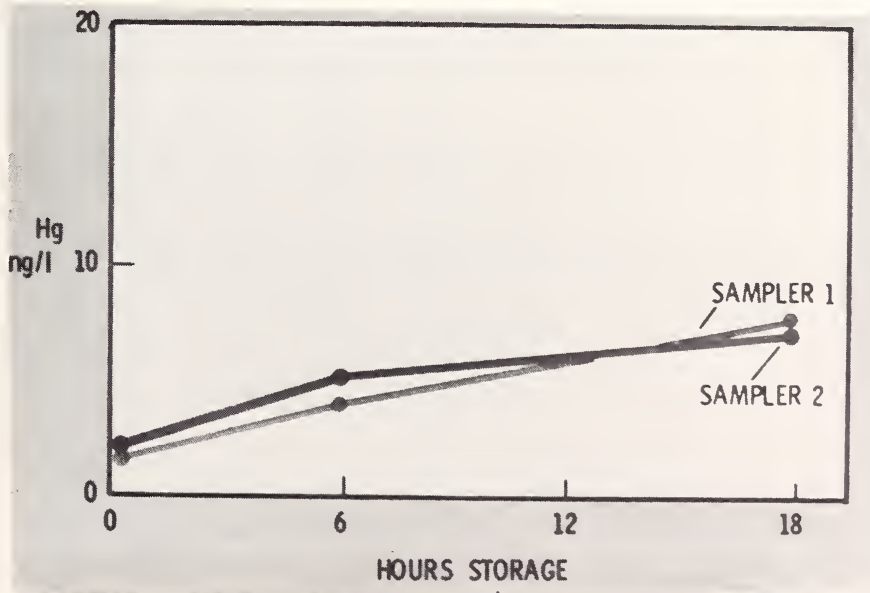

Figure 7. Evaluation of Niskin water samplers for mercury contamination. 
$\mathrm{ng} / \mathrm{l}$ after 18 hours. However, during the actual GEOSECS expedition the Niskin bottles were drained within an hour after bringing them aboard, so any contamination derived from the Niskin bottles would have been essentially negligible.

Attached to each GEOSECS Niskin bottle is a reversing thermometer to provide a secondary check of the temperature of the water at the depth where the sample was taken. Occasionally, a thermometer would break when reversed or the whole thermometer packet would be lost. To determine if thermometer breakage or losses could contribute to $\mathrm{Hg}$ contamination, the sampling log book was searched to record all such instances. Happily, no correlation existed between broken or lost thermometer occurrences and the elevated $\mathrm{Hg}$ levels in the Atlantic Ocean, and the thermometer losses do not appear to influence the data in any way.

\section{Storage}

The GEOSECS seawater samples for trace element analysis are drained directly from the Niskin bottles into precleaned 2-liter Bel-Art ${ }^{R}$ polyethylene bottles with pure polyethylene screw caps. The bottles were precleaned by soaking in $3 \mathrm{~N} \mathrm{HCl}$ and rinsing with high purity water. The seawater is then acidified to $\mathrm{pH} 1.6$ with high purity hydrochloric acid, the bottles are capped tightly and sealed in plywood boxes which are stored in the ship's hold. The seawater was not filtered.

To determine if positive or negative contamination of the GEOSECS seawater samples occurred during the several months of storage before analysis of $\mathrm{Hg}$, a series of experiments were performed.

In the first storage test to determine if various forms of $\mathrm{Hg}$ can be lost by adsorption onto container walls, mercury-203 labeled ionic $\mathrm{Hg}^{++}$and methylmercuric chloride were added to seawater samples contained in polyethylene bottles. One set of seawater samples was adjusted to $\mathrm{pH} 1.6$ with hydrochloric acid and the other set was maintained at a $\mathrm{pH}$ of 8.0, which is the normal $\mathrm{pH}$ of seawater. At scheduled intervals the amounts of ionic $\mathrm{Hg}$ and methyl $\mathrm{Hg}$ remaining in solution were determined. The results are shown in figure 8 . At $\mathrm{pH} 8.0$ both ionic $\mathrm{Hg}$ and methyl $\mathrm{Hg}$ are nearly quantitatively removed from solution by adsorption onto the container walls. However, at $\mathrm{pH} 1.6$ both the ionic and methyl $\mathrm{Hg}$ remained in solution for up to 80 days without any indication that serious losses by adsorption onto the containers would occur. The GEOSECS seawater samples, therefore, appear to be adequately preserved to prevent adsorption losses. 


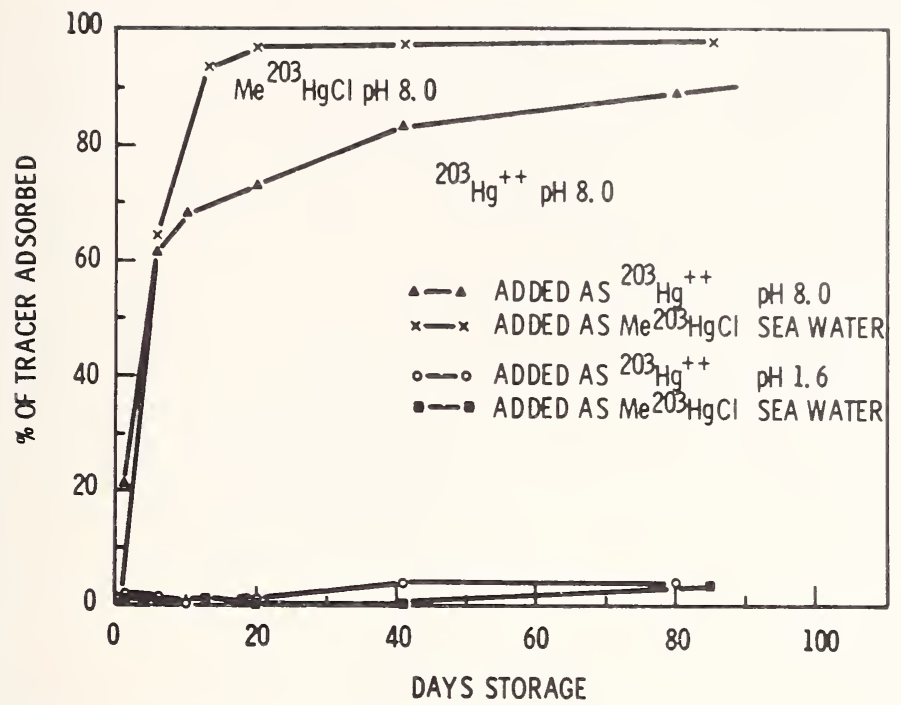

Figure 8. Behavior of ionic $\mathrm{Hg}$ and methyl $\mathrm{Hg}$ in seawater stored in polyethylene bottles.

The next storage test was designed to evaluate possible leaching of $\mathrm{Hg}$ from the polyethylene 2-liter Bel-Art ${ }^{R}$ bottles used for storing GEOSECS seawater samples. An interlaboratory test was conducted with Michael Bothner of the University of Washington. Three replicate Bel-Art ${ }^{R}$ bottles from the same batch were filled with low $\mathrm{Hg}$ seawater at each laboratory, the $\mathrm{pH}$ was adjusted to 1.6 with hydrochloric acid and the $\mathrm{Hg}$ content of the water was measured immediately and then at scheduled intervals extending over 100 days. One set of samples was placed on the bench-top and another set was enclosed in plastic bags and placed on the floor. These samples were compared with seawater which was acidified and stored in carefully precleaned Pyrex glass bottles. The results of this test are shown in figure 9. We were disturbed to find that the $\mathrm{Hg}$ levels in the seawater stored in the polyethylene bottles drastically increased, whereas no change in concentration occurred in the seawater stored in the Pyrex bottles. It was evident that the $\mathrm{Hg}$ levels in the polyethylene bottles placed on the floor increased at a much faster rate compared to the polyethylene bottles placed on the bench top. This seemed peculiar since the bottles were all from the same batch and should be expected to contain roughly the same levels of $\mathrm{Hg}$ impurities. This experiment was repeated with another set of Bel-Art ${ }^{R}$ bottles from the same batch and again the results were the same as before. This led us to believe that perhaps another mechanism besides leaching of $\mathrm{Hg}$ from the containers was contaminating our seawater samples with $\mathrm{Hg}$-namely, diffusion of airborne 


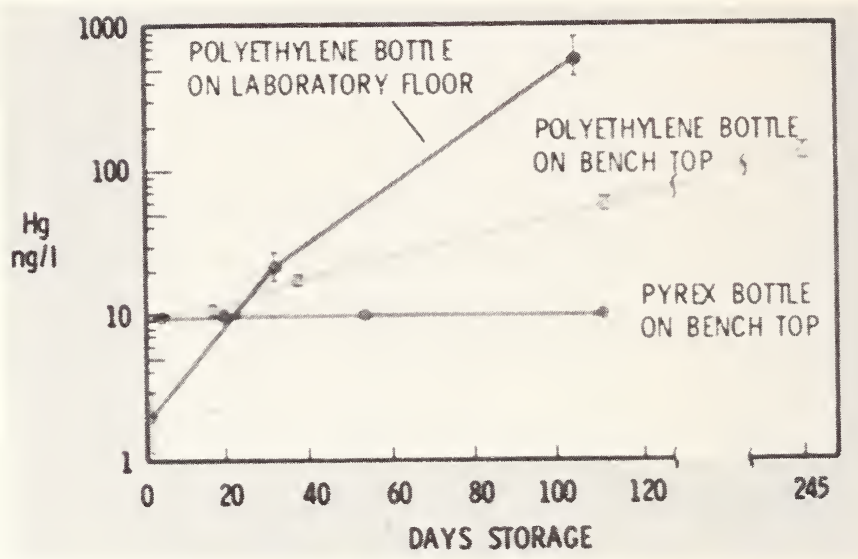

Figure 9. Hg contamination of seawater samples stored in polyethylene bottles.

$\mathrm{Hg}$ vapor through the polyethylene walls of the storage bottles. The diffused $\mathrm{Hg}$ appears to accumulate in the acidified seawater, perhaps becoming dissolved and stabilized as the $\mathrm{HgCl}_{3}{ }^{-}$or $\mathrm{HgCl}_{4}=$ anionic complex. We suspected that perhaps during an earlier occupation of the laboratory, elemental mercury from thermometers, manometers or other apparatus could have spilled on the floor causing tiny droplets of $\mathrm{Hg}$ to fall into cracks of the floor tile or roll behind the baseboards. If this were true relatively high concentrations of airborne $\mathrm{Hg}$ could be emanating from the laboratory floor, with the highest levels being closest to the floor.

The floor was subsequently checked for $\mathrm{Hg}$ contamination using a Bacharach Instrument Company Model MV-2 Mercury Sniffer, which has a sensitivity of $0.01 \mathrm{mg} / \mathrm{m}^{3}$ of air. Floor level air concentrations of $\mathrm{Hg}$ were less than $0.01 \mathrm{mg} / \mathrm{m}^{3}$, but when the floor was smeared near the baseboards with tissue paper, detectable $\mathrm{Hg}$ vapor was found to emanate from the tissue, and a reading of $0.08 \mathrm{mg} / \mathrm{m}^{3}$ was obtained when the tissue was placed near the air intake of the Mercury Sniffer. Mercury vapor from this source, although undetectable by the Sniffer, was thought to account for the rapid rate of $\mathrm{Hg}$ increase in the seawater samples stored on the floor in polyethylene bottles.

To prove that mercury vapor can rapidly diffuse through the walls of polyethylene bottles containing acidified seawater, the following simple experiment was performed. A $70 \mathrm{mg}$ drop of elemental $\mathrm{Hg}$ was placed in an open vial, and the vial was placed on the bottom of a 20 -liter, heavy walled polyethylene bottle. A 2-liter. precleaned Bel-Art ${ }^{R}$ polyethylene bottle was filled with $\mathrm{pH} 1.6$ seawater having a $\mathrm{Hg}$ concentration of $2 \mathrm{ng} / \mathrm{l}$. The Bel-Art ${ }^{R}$ bottle was tightly capped and placed on a small stand at the 
bottom of the 20-liter bottle approximately $15 \mathrm{~cm}$ away from the vial containing the $\mathrm{Hg}$ droplet. The 20-liter bottle was tightly closed to seal in $\mathrm{Hg}$ vapor released from the droplet of elemental $\mathrm{Hg}$. If complete equilibrium was established, the air concentration of $\mathrm{Hg}$ within the 20-liter bottle would be about $20 \mathrm{mg} / \mathrm{m}^{3}$, or about $0.4 \mathrm{mg}$ of $\mathrm{Hg}$ in 20 liters of air. After 5 days, the Bel-Art ${ }^{R}$ bottle containing the acidified seawater was removed from the sealed 20-liter bottle. The outside surface of the Bel-Art ${ }^{R}$ bottle was washed with distilled water and wiped dry to avoid cross-contamination from surface-adsorbed $\mathrm{Hg}$. The $\mathrm{Hg}$ content of the seawater was then measured and found to be greater than $2000 \mathrm{ng} / \mathrm{l}$, an increase of over 1000 -fold. Thus, diffusion of airborne $\mathrm{Hg}$ vapor through polyethylene container walls can create a serious contamination problem if the circulating ambient air contains significant quantities of $\mathrm{Hg}$.

This newly discovered contamination mechanism caused great concern to us, but we felt that somehow our GEOSECS samples were not contaminated by this source of $\mathrm{Hg}$ since hundreds of the seawater samples that we analyzed contained extremely low ( 2 to $20 \mathrm{ng} / \mathrm{l}$ ) concentrations of $\mathrm{Hg}$. These samples had been stored for a period of up to a year before the $\mathrm{Hg}$ analysis. When it became apparent to us that where the samples are stored can be a critical factor, we began an experiment to duplicate the exact method of storage and handling that was followed throughout the GEOSECS work.

As stated earlier, the GEOSECS seawater samples for trace element analysis were drawn directly from the Niskin sampling bottles into precleaned 2-liter Bel-Art ${ }^{R}$ polyethylene bottles and acidified to $\mathrm{pH} 1.6$ with hydrochloric acid. The filled bottles were then placed into plywood boxes which were sealed and stored in the ship's hold. The samples were shipped directly to our laboratory in the plywood boxes and remained in them until they were transferred to new enclosed storage cabinets located in our laboratory basement.

To determine if Bel-Art ${ }^{R}$ bottles could be effectively used for storing pH 1.6 seawater if they were isolated from $\mathrm{Hg}$-contaminated air, the following test was performed. Eight identical acid-rinsed Bel-Art ${ }^{R}$ bottles were filled with $\mathrm{pH} 1.6$ seawater containing $4.0 \mathrm{ng} \mathrm{Hg} /$ liter. Four of the bottles were encased in a sealed plywood box to duplicate the GEOSECS storage procedure, and four were again placed on the contaminated laboratory floor. After storage periods of 18, 40 and 100 days, the $\mathrm{Hg}$ concentrations in the bottles were determined (see fig. 10). For comparison purposes, the results of the storage tests showing increased $\mathrm{Hg}$ levels in acidified seawater in the Bel-Art ${ }^{R}$ bottles stored at benchtop are included in figure 10. The $\mathrm{Hg}$ levels in the seawater stored in the bottles 


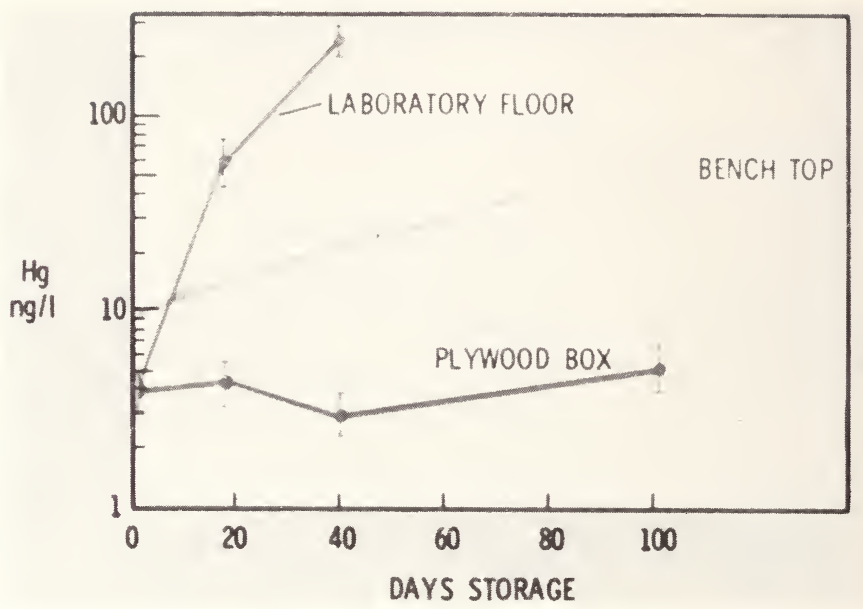

Figure 10. Evaluation of storage conditions of seawater in polyethylene bottles.

sitting on the floor again increased at about the same rate as previously observed, and after 40 days the $\mathrm{Hg}$ concentrations rose to levels between 190 and $240 \mathrm{ng} / \mathrm{l}$ in the four bottles. However, the seawater in the bottles stored in the plywood box did not significantly change from a $\mathrm{Hg}$ concentration of $4 \mathrm{ng} / \mathrm{l}$ after storage for 100 days. Thus, in the case of Bel-Art ${ }^{R}$ bottles, leaching of mercury from the bottle walls does not appear to be a contaminating mechanism, and the bottles appear to be completely suitable containers for storing acidified seawater if the bottles are isolated from large volumes of circulating, $\mathrm{Hg}$-containing air. Such protection was apparently afforded by the sealed plywood boxes and the new enclosed storage cabinets. So, we feel rather confident (and lucky) that the seawater storage methods used in the GEOSECS program did not result in serious contamination of the samples with $\mathrm{Hg}$. However, the storage procedure may have introduced some of the uncertainty observed in the analyses.

\section{Consistent and Known Yields of Chemical and InStRUMENTAL MANipUlations}

Chemical preconcentration and separation manipulations must be free of large random variations if highly accurate and precise results are to be obtained. If added tracers are not used to measure the chemical yield for each sample, the reproducibility of the method must be determined by replicate analyses of a single sample. As many replicate determinations as 
possible should be made to provide a good statist I $_{\text {cal }}$ estimate of the reliability of the method. Standards prepared in the same matrix as the sample should be determined with each set of samples to ensure that chemical yields and instrument responses have not varied with time.

Figure 11 illustrates the standard working curve used in quantifying the $\mathrm{Hg}$ measurements of the GEOSECS seawater samples. The curve was prepared by the method of standard additions using $\mathrm{Hg}$-free seawater as the solution matrix. The consistency of this curve has been checked on numerous occasions and has been found to be very reproducible. During the actual analysis of the GEOSECS samples $\mathrm{Hg}$ standards are always analyzed with the samples to ensure that the chemistry and instrumentation are responding accurately. The instrumentation has been extremely stable for over a year's operation.

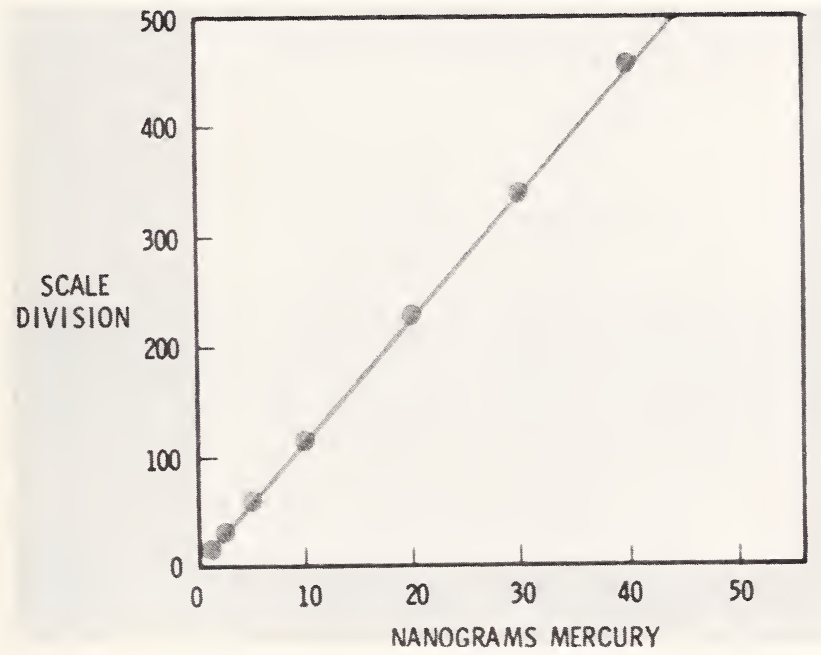

Figure 11. Standard working curve for $\mathrm{Hg}$ analysis.

\section{E. Consistent Procedural Blanks}

One of the biggest problems in trace element analysis of natural waters is the occurrence of large, random increases in the procedural blank. These spurious blanks can be caused by a variety of contamination mechanisms and instrumental errors, and their presence affects the degree of accuracy that can be attained in an analysis.

In developing our $\mathrm{Hg}$ procedure we had to work hard at eliminating contamination sources which caused high random blanks. The labware used in the procedure had to be very carefully precleaned numerous times 
before consistently low procedural blanks could be obtained. The labware had to be frequently used or slight $\mathrm{Hg}$ contamination could reoccur and raise the procedural blank. Surprisingly, the reagents used in the procedure always gave consistently undetectable (less than $0.1 \mathrm{ng} / \mathrm{l}$ ) $\mathrm{Hg}$ concentrations. During the analyses of the GEOSECS seawater samples, numerous blanks were always included with the samples so that a good statistical value of the blank could be obtained. On one occasion we unknowingly had the opportunity to better evaluate the procedural blank during the analyses of 20 GEOSECS seawater samples which had been inadvertently unacidified at the time of collection. During the several months of storage all of the $\mathrm{Hg}$ in the seawater had completely adsorbed to the polyethylene container walls, providing $20 \mathrm{Hg}$-free seawater samples. The analyses of these samples gave an average procedural blank of 0.22 nanograms of $\mathrm{Hg}$ with a standard deviation of 0.08 nanograms. This is typical of the blank values obtained when we are routinely measuring $\mathrm{Hg}$, and we are confident that we have eliminated spuriously high random blanks from our procedure.

\section{F. VerificAtion of Results by ANOther Analytical Method}

All methods of analysis, both chemical and instrumental, have potential sources of error which are peculiar to a given technique. Often these sources of error are very subtle and can go undetected during the analysis. A good way to evaluate the accuracy of a measurement is to measure the constituent by another type of analytical technique.

The accuracy of the cold vapor atomic absorption technique for measuring $\mathrm{Hg}$ in seawater has been checked by a neutron activation analysis (NAA) method [5]. The NAA method involves cocrystallizing $\mathrm{Hg}$ with thionalid, neutron activating the thionalid and then counting the sample directly on a $\mathrm{Ge}(\mathrm{Li})$ gamma-ray spectrometer to measure the ${ }^{203} \mathrm{Hg}$ neutron activation product. The results of this comparison are shown in table 5.

TABLE 5. Analysis of $\mathrm{Hg}$ in seawater by flameless $A A$ and $N A A$

Sample

1

2
Neutron activation

$0.05 \pm 0.004$

$.13 \pm .01$

Flameless AA

$0.048 \pm 0.005$

$.12 \pm .01$ 
The agreement between the two methods was quite satisfactory and indicated that the cold vapor atomic absorption technique was providing accurate results.

\section{G. Analysis of Standard Reference Materials}

Another method of evaluating the accuracy of an analytical method is to analyze standard reference materials of known, well documented composition. Usually standard reference materials have been calibrated by a variety of analytical methods so that the certified values of the measured constituents are considered to be highly accurate. Excellent geological, metallurgical and biological standard reference materials have been developed, but there are few reliable natural water reference standards available. This is due mainly to our lack of knowledge concerning the optimum methods of preparing and preserving such standards without contaminating them in one form or another. Usually standard reference solutions used in evaluating the accuracy of trace element measurements in natural waters are standard solutions, prepared in high purity dilute acids and containing elemental concentrations 1 to 3 orders of magnitude higher than normally found in natural waters. Although this is not desirable, since such solutions are not true standard reference waters, it is probably a necessary requirement to avoid the problems of storing very low levels of trace elements in complex natural water matrices. Standard reference solutions can be diluted to natural water trace element levels just before analyzing them.

A series of standard solutions containing $\mathrm{Hg}$ in various forms were obtained from the EPA. The solutions were diluted to the range of 20 to 100 $\mathrm{ng} \mathrm{Hg} / \mathrm{l}$ and were analyzed in triplicate. The results, shown in table 6, indicate agreement within analytical uncertainty between the measured and suggested values.

TABLE 6. Analysis of $F W Q A$ standard $\mathrm{Hg}$ solutions

\begin{tabular}{cccc}
\hline Sample & Chemical form & FWQA value $\mu \mathrm{g} / \mathrm{l}$ & This study $\mu \mathrm{g} / \mathrm{l}$ \\
\hline 1 & $\mathrm{HgCl}_{2}$ & 0.34 & $0.33 \pm 0.02$ \\
2 & $\mathrm{HgCl}_{2}$ & 4.2 & $4.2 \pm .1$ \\
3 & $\mathrm{HgCl}, \mathrm{CH}_{3} \mathrm{HgCl}$ & 6.3 & $6.5 \pm .1$ \\
4 & $\mathrm{CH}_{3} \mathrm{HgCl}$ & 4.2 & $4.4 \pm .1$ \\
\hline
\end{tabular}




\section{H. Establishing Analytical Precision}

Once the accuracy of an analytical method has been evaluated, it is necessary to establish the degree of analytical precision or reproducibility of the method. This is important, especially if one is trying to detect small natural geographical, vertical or temporal variations in concentrations of trace elements in natural waters. To do this requires a good statistical knowledge of the variance of the experimental uncertainty, so that the natural variations may be measured with confidence. The precision is determined by replicate analysis of a single sample or a set of samples. Replicate analyses should be performed on samples which are typical of the range in concentration observed during a study, since the precision deteriorates as trace element concentrations approach the analytical detection limit of a method.

The precision of our cold vapor atomic absorption method was evaluated by replicate analyses of two seawater samples; one containing $9 \mathrm{ng}$ $\mathrm{Hg} / \mathrm{l}$ and the other containing $130 \mathrm{ng} \mathrm{Hg} / \mathrm{l}$. The results are shown in table 7.

Based on six replicate determinations the average $\mathrm{Hg}$ concentration in the low level sample averaged $9.1 \mathrm{ng} / \mathrm{l}$ with a standard deviation of 1.3 $\mathrm{ng} / \mathrm{l}$, or 14 percent. The intermediate level sample, based on nine replicate determinations, averaged $130 \mathrm{ng} / \mathrm{l}$ with a standard deviation of $2.7 \mathrm{ng} / \mathrm{l}$, or 2.1 percent. This precision is quite good and allows rather small natural variations in the mercury distribution in the oceans to be observed.

TABLE 7. Precision of $\mathrm{Hg}$ analysis by flameless $A A$

Low level seawater

$\mathrm{Hg}$ (ng/l)
Intermediate level seawater

$\mathrm{Hg}(\mathrm{ng} / \mathrm{l})$

\begin{tabular}{crcc}
\hline 1 & 10 & 1 & 125 \\
2 & 11 & 2 & 131 \\
3 & 8 & 3 & 132 \\
4 & 8 & 4 & 132 \\
5 & 10 & 5 & 134 \\
6 & 8 & 6 & 130 \\
Avg. & 9.1 & 7 & 130 \\
$\sigma$ & 1.3 & 8 & 127 \\
$\%$ S.D. & $14 \%$ & 9 & 131 \\
& & Avg. & 130 \\
& & $\sigma$ & 2.7 \\
& & $\%$ S.D. & $2.1 \%$ \\
\hline
\end{tabular}




\section{Chemical and Instrumental Interferences}

The chemical constituents in seawater which are known potential interferences in the UV absorptiometric determination of mercury are $\mathrm{Cl}_{2}$ gas, organic gases or vapors and water vapor.

To give an indication of $\mathrm{Cl}_{2}$ and other interferences a mercury trap consisting of granulated silver in Tygon tubing $(0.6 \mathrm{~cm}$ dia. $\times 5 \mathrm{~cm}$ long) was inserted in the gas line between the sample flask and the optical cell. This trap was found to remove mercury vapor from the gas leaving the sample solution. Since the silver did not remove $\mathrm{Cl}_{2}$, the trap was useful in analysis of test seawater samples to determine if $\mathrm{Cl}_{2}$ was still present in the samples. This trap is also useful for determining the presence of other interfering substances, such as organic vapors and water vapors, provided they are not also removed from the carrier gas by the silver metal. No interferences were identified in this manner in analysis of seawater containing effluent from both a sewage treatment plant and a pulp mill, or samples of interstitial water from sediments.

No special instrumental errors could be identified with the cold vapor atomic absorption procedure.

\section{Summary}

I have demonstrated, using $\mathrm{Hg}$ as an example, the parameters which must be evaluated in the trace element analysis of natural waters if accurate and precise results are to be obtained. If these parameters are carefully investigated and corrective measures employed, an analyst can usually be assured that he is producing reliable results - or, at the worst will know the degree of reliability of his data. In the case of our $\mathrm{Hg}$ analyses of GEOSECS seawater samples, we feel confident that the results we have obtained are a reasonably accurate picture of the actual geographical and vertical distribution of $\mathrm{Hg}$ in the western basin of the Atlantic Ocean. The analytical method certainly has the capability of producing consistently accurate and precise measurements of $\mathrm{Hg}$. The modest amount of uncertainty that we have experienced in our $\mathrm{Hg}$ study is undoubtedly due to minor sampling and storage problems. However, the uncertainty is not of a magnitude which obviates the observed large scale geographical and vertical variations of $\mathrm{Hg}$ in the ocean. 


\section{Acknowledgement}

This paper is based on work performed under United States Atomic Energy Commission Contract AT(45-1)-1830.

\section{References}

[1] Brewer, P. G. and Spencer, D. W., Trace Element Intercalibration Study. Reference No. 70-62, Woods Hole Oceanographic Institution, Woods Hole, Massachusetts, December 1970.

[2] Anonymous, Meeting Report: Interlaboratory Lead Analyses of Standardized Samples of Seawater. Marine Chemistry 2, 69-84 (1974).

[3] Rober:son, D. E., Contamination Problems in Trace-Element Analysis and Ultrapurification, in Ultrapurity-Methods and Techniques, Zief, M. and Speights, R., Eds., Chapter 12, pp. 208-253, Marcel Dekker, Inc., New York (1972).

[4] Tolg, G., Extreme Trace Element Analysis, Talanta 19, 1489-1521 (1972).

[5] Bothner, M. and Robertson, D. E., Mercury Contamination of Seawater Samples Stored in Polyethylene Bottles, In Press.

[6] Menard, H., The Deep-Ocean Floor, Scientific American 221, 126-145 (1969).

[7] Weiss, H. V., A Review of Lead, Sulfur, Selenium and Mercury in Permanent Snowfields, in Marine Electrochemistry, Berkowitz, J. B. et al., Eds., pp. 385-398, The Electrochemical Society, Princeton, New Jersey (1973). 


\title{
ANALYTICAL METHODOLOGY FOR ACCURATE ANALYSIS IN CLINICAL CHEMISTRY
}

\author{
S. S. Brown and F. L. Mitchell \\ Division of Clinical Chemistry \\ Medical Research Council \\ Clinical Research Center \\ Harrow, Middlesex, England
}

Methods of measurement in clinical chemistry need to be continuously assessed to determine the degree of accuracy and precision required to ensure their optimum value for clinical purposes. Constant review is necessary since improvements in technology often show that much of what was once thought to be biological variation within and between individuals can be laboratory error. When biological variation is clearly known, it is pointless to provide measurements which may be costly when done beyond the required degree of accuracy and precision. In many cases however, that degree is not yet attainable in routine work.

Once determined, these levels of efficiency must be maintained and comparability of results ensured between laboratories and nations. Only comparatively recently have the principles which are required to achieve this, been formulated and understood. The first stage was the elimination, as far as possible, of human error by the large-scale introduction of automation and electronic data processing, then when precision was thus brought under control, it became possible to investigate accuracy.

Work in this direction using special reference technology, is proving that many of the well established techniques are far from satisfactory. New substances are required to be measured increasingly, and often, a technique may be accurate and not precise or vice versa, only an ability to monitor and maintain accuracy and precision with confidence in the clinical chemistry laboratory, will ensure continued progress in the use of clinical measurement in medicine. Ways of doing this will be discussed.

Keywords: Accuracy: clinical analysis; clinical chemistry: health: precision. 


\section{Introduction}

Some of those present may know a publication of the British Society for Analytical Chemistry-Official Standardized and Recommended Methods of Analysis. One small section of this book deals with blood and other body fluids, and has as its preface "Nowhere more than in the field of clinical chemistry is the analyst likely to work with more complex material or to encounter a greater variety of potentially interfering substances." This paper may be taken as a brief exposition of this particular text.

The quantitative assay of chemical compounds in body fluids now makes an important contribution to clinical diagnosis, the choice of therapy, and the monitoring of treatment. Indeed, in his opening remarks, Dr. Roberts indicated that several billion such determinations are performed each year in the United States, and it is fair to conclude that so much reliance is placed on the results that a large share of responsibility in medicine and surgery has shifted from the bedside to the laboratory.

Ten or so years ago, it was generally assumed that if a prescribed protocol was correctly carried out in the clinical laboratory, the result obtained would be "correct", i.e., reasonably accurate and precise. It has taken many years to recognize the fallacy of this approach, and Dr. Boutwell and Dr. Mandel pointed out in their earlier presentations some of the elaborate techniques now used in clinical chemistry to ensure the quality of analytical output. So far the emphasis has been on the achievement and maintenance of good precision, but in the last year or two, attention has been directed toward the much more difficult problem of assurance of accuracy. It is not easy to identify why this problem has been neglected so long, but a major factor is probably the belief that if a measurement has an established clinical usefulness it does not matter greatly what is actually measured. In other words, provided that there is some confidence that the same chemical species - whatever it might be -is being measured on each occasion, then high accuracy is not required. This philosophy is illustrated by the so-called flocculation tests which have been used for many years in the investigation of liver function. They undoubtedly had clinical value, but until recently, little was known of the precise chemical nature of the flocculants. To be realistic, the empirical approach is still with us, if we honestly review the chemical processes involved in estimations like serum cholesterol or protein-bound iodine.

Many hundreds of different compounds, often at trace levels of concentration, are now assayed routinely in hospital laboratories, using a wide variety of analytical techniques. These substances range from the sim- 
plest species $-\mathrm{H}^{+}$-through sodium, potassium, calcium, zinc, lead; through organometallic derivatives such as bilirubin and hemoglobin; through well-characterized proteins such ais albumin and transferrin; through less well characterized proteins such as the immunoglobulins and insulin; to enzymes of unknown structure such as creatine kinase and $\gamma$ glutamyltransferase. Historically, analytical methods have evolved by a trial and error, with laboratories in centers of excellence adopting new methodologies fairly rapidly as they become available, and discarding the old. This process takes place more slowly in the less advanced or more remote laboratories, so that in African bush hospitals for example, it is possible to find many of the unsatisfactory techniques of bygone years still flourishing, apparently. The problems facing clinical chemistry in the so-called developing countries of the third world are in many ways different from those in the developed nations, and they require separate investigation and appraisal. Equipment and procedures designed for industrialized temperate countries may have no useful place elsewhere.

Equipment manufacturers and chemical suppliers have made important contributions to improving clinical laboratory performance. Thus in the days when $\mathrm{pH}$ was defined as the negative logarithm of hydrogen ion concentration, the measurement of blood $\mathrm{pH}$ was a highly demanding, and somewhat irreproducible procedure. This has changed, with the definition of $\mathrm{pH}$ as an operational function and with standard buffers, rugged electrodes and highly stable micro-voltmeters readily available. The quality control of $\mathrm{pH}, \mathrm{pCO}_{2}$, and $\mathrm{pO}_{2}$ measurements is still difficult; the state of the art was indicated by Dr. Boutwell when he spoke of the need for drawing a fresh blood specimen from a member of the laboratory staff in order to obtain an adequate sample for quality control purposes. In a somewhat different, but equally important, area of methodology, the last 10 years have witnessed the widespread adoption of atomic absorption spectroscopy in the clinical laboratory. The assays which used to be the prerogative of extraordinarily skilled analysts with mysterious chelating agents are now usable by all. Here again, however, it is too easy to lose sight of the need for vigilant control of both precision and accuracy.

It is also interesting to trace the impact which the advance of technology has had, for example, on the measurement of steroids. In the professional lifetimes of many of today's clinical chemists, such measurements have advanced from bio-assay, using many thousands of mice, to colorimetry or fluorimetry, first on crude extracts, and then on fractions purified by column chromatography, paper or thin-layer chromatography. Gas chromatography later made its impact, and now radio-immunoassay. Mass spectrometry is already in use in some laboratories and rapid ad- 
vances are being made in this particular technique.

The armamentarium of methodology available in clinical chemistry is thus formidable, and the choice of the best technique in any particular case is not necessarily straightforward. Usually the advantages of a new technological approach are recognized quite quickly, but change for change's sake may take place before the real advantage has been proved. The criteria for excellence of methodology are now accepted to concern both accuracy and precision (which involve problems of calibration, reproducibility, and specificity) and also practicability (which involves problems of labor, skill and time required, together with the capital and running costs of the instruments or techniques used).

\section{Accuracy and Precision Required}

In analytical clinical chemistry, possibly more than in other branches of analytical science, the technique giving the most accurate answer is not necessarily the one to be selected for routine application. In Mr. Cali's phraseology, the "end use requirement," in the case of the clinician, may not justify such a high level protocol. In fact the clinician's requirements for speed and sensitivity impose demands upon the analyst which conflict directly with the aim of achieving good precision and accuracy. At the same time, as Dr. Ibbott indicated in his paper, sampling factors are very relevant. Furthermore, if the concentration of a substance in the blood of a population of normal individuals varies over a range of \pm 10 percent there is little point in carrying out measurements to an accuracy of \pm 1 percent to identify an abnormal individual. The level in a given individual however, is usually under much tighter control, so that if one particular person is being studied over a period of time a higher degree of accuracy may be required. In the past it was commonly assumed that the reported "normal" or reference range for a concentration of a substance was entirely due to the biological variation, when a major proportion in fact was due to the unwelcome contribution of imprecision of measurement. On many fronts, the adeptness of nature to maintain homeostasis has proved to be more thorough than was originally thought.

It is highly desirable therefore, to delineate biological variation with the most accurate technique available, thereby determining the degree of latitude allowable if the requirements of routine clinical work are to be met by an economical, sufficiently rapid technique. In practice, valid studies of normal variation are difficult to design, but high speed automated analysers are now available with sufficiently good precision and 
throughput to justify an attack on the problem using an accurately calibrated material for standardization. Such a project is jointly being pursued by the Clinical Research Center and the National Institutes of Health in Bethesda.

This point of view is in no way meant to imply that there is no place left in the clinical laboratory for relatively simple "screening" techniques, such as are used in the study of phenylketonuria in infants, or alcohol ingestion in car drivers. If the only technique suitable for a clinical requirement is not economical or practical for the large numbers which may be required, then it may be proper to institute a two-tier system, i.e., to adopt a rapid screening technique which produces a proportion of false positives, but not false negatives; this is then backed by the more laborious and/or expensive, but more specific and accurate method. Dr. Young will no doubt be covering this aspect in his paper on the interpretation of results.

\section{Reference Technology and its Impact on Accuracy}

When group quality assurance schemes are introduced into a network of clinical laboratories, there is usually an initial improvement in precision. This soon levels out, but often before an acceptable general performance has been attained. Close examination of the findings, either in the organizing center or in the individual laboratories often reveals that the different methods used by different laboratories often give different mean values and coefficients of variation. It is relatively easy to investigate those techniques which show poor precision, and take the necessary action (hence the reason for initial improvement after a scheme commences), but problems with accuracy cannot be revealed without knowledge of the true concentration of an analyte in the material being assayed.

As an example, it is pertinent to consider one particularly important component of blood, calcium. The amount of total calcium is maintained in the body within very close limits, and any marked deviation from these reflects serious disease. Accurate measurement is very important and it was therefore appropriate that NBS and NIH should choose to develop an arcurate serum calcium assay as their first joint venture into clinical chemistry. The history of this venture has been documented elsewhere by Mr. Cali; briefly, a definitive method for the assay of calcium was developed using isotope dilution-mass spectrometry. This was applied to carefully prepared pooled serum samples to establish accurately the calci- 
um concentrations; using these samples, a reference method based on atomic absorption spectroscopy was proposed. Our own laboratory set about to evaluate this Reference Method, and to apply it systematically to samples distributed by the Wellcome Group Quality Control Programme. The intention was to test the applicability of the different routine techniques used for serum calcium measurements in the various laboratories participating in the Programme. To monitor our own accuracy, however, batches of sera were assayed at the Clinical Research Center using the Reference Method, and at the National Bureau of Standards using the Definitive Method. The results were pleasing (fig. 1) even though a small positive bias was evident, which was almost certainly due to a matrix effect in the atomic absorption process itself [1].

When the results of applying the Reference Method were compared with the separate mean values obtained by the 300 or so laboratories in the Programme, it was found that there was a concentration-related bias; that is, the Group mean values tended to be lower than the Reference Method values at high calcium concentrations, but higher at low concentrations. In an effort to trace the origin of this bias, laboratories were

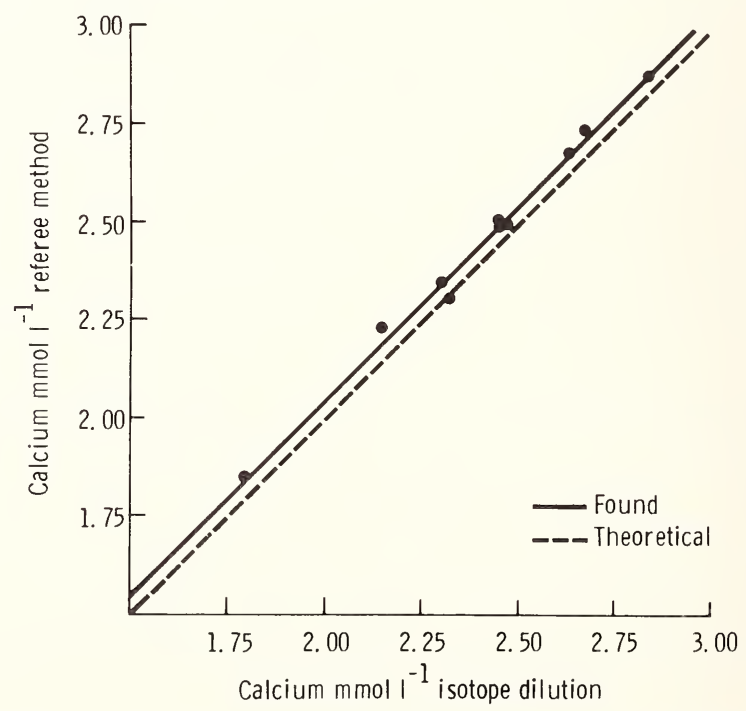

Figure 1. Assay of total calcium in serum samples - relationship between results obtained by the Definitive Method and by the Reference Method. (Figure reproduced, with permission, from ref. 1). 
grouped according to the type of analytical method used for the calcium assay. With appropriate statistical methods, it was then established that the overall performance of laboratories using automated methods was good, with an even scatter about a line showing a small constant bias, or inaccuracy, over the working concentration range. Laboratories using manual methods however, were clearly responsible for the concentrationrelated bias found in the overall results (fig. 2).

This is the first time that a reference method has been available to check accuracy in a proficiency testing survey, and for calcium measurements at least, it is clearly possible to identify analytical methods which are liable to be poorly performed and to look forward to improvement in the measurement of calcium in body fluid. Hopefully, we can also look forward to having reference technology available for many types of assay, even though its development and the organization of its worldwide availability are formidable tasks.

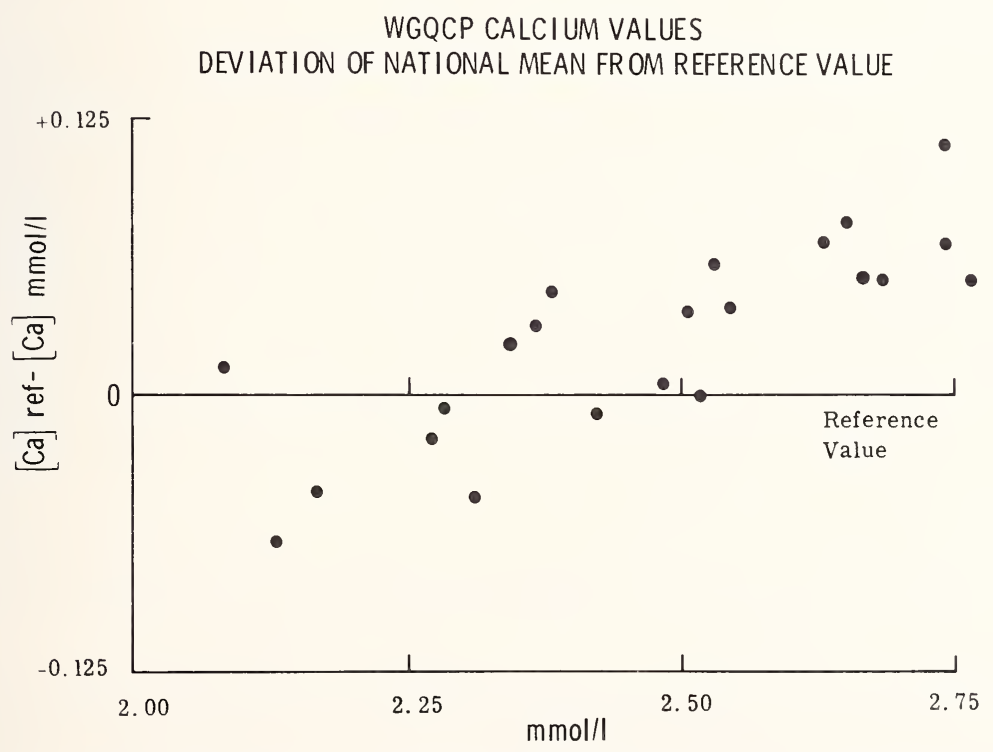

Figure 2. Assay of total calcium in serum samples - difference between results obtained by the Reference Method of analysis and the corresponding mean values obtained by participants in the Wellcome Group Quality Control Programme plotted against the Reference Method Values. 


\section{Special Analytical Problems for the Clinical Chemist}

To recapitulate, the clinical chemist has problems which most other analysts do not have. The substances to be measured range in nature from the simplest elements to the most complex molecules, and are present in a matrix of somewhat variable composition, to which may be added one or another of a wide variety of drugs. In these circumstances it is important to stress the following points:

1. Calibration of a method with an appropriate high purity standard reference material does not necessarily lead to highly accurate measurements, since the physical and chemical properties of the test samples may significantly modify the final measurement.

2. Considerable care must be taken in designing an appropriate "blank" reaction, or in making a decision to dispense with a blank measurement.

3. Enzyme assays are done on a considerable scale, and since in this context no standard reference material is available, reliance for reproducibility has to be placed solely on the rigorous standardization of assay techniques.

The importance of reference technology stems from point number 1 , since it is now realized that accuracy can only be monitored by the comparison of actual results with those obtained by a technique whose accuracy had been rigorously proved. Many methods and instruments can only be satisfactorily calibrated using standards with similar composition to that of the test material. This is particularly evident if high concentrations of protein are present since the physical properties are changed so as to modify the performance of pipettes, peristaltic pumps, atomizers, etc. The problem is particularly acute in the calibration of multichannel machines when, because of mutual interference with each other's concentration, it is impossible to make up a standard "cocktail" of more than a small number of pure components. For this reason, as well as that mentioned previously, such machines almost always have to be calibrated with a previously assayed biological material, and the accuracy of the "primary" measurement is critical.

Many of the techniques in common use lack complete specificity and give blank measurements of varying magnitude. The measurement of such blanks often doubles the work, or with an automated method, it may monopolize a second channel. There is a tendency therefore to advocate the subtraction of a "serum blank" from the analytical result under the assumption that all sera contain the same amount of nonspecific "impurity." Such practice can never be completely satisfactory since a constant serum or other biological fluid blank cannot exist. Even blank measurements 
cannot completely compensate for nonspecificity, since no blank determination can completely mimic blank interference in the test assay. Either a reagent is not included, or the test is modified in some other way. When the nature of the interfering substance is known and is being measured on the same multichannel instrument, the machine's computer can be made to "programme the interference out" of the measurement concerned. This approach is sound, but complicated. Undoubtedly the best answer to the blank problem is to strive toward techniques giving the highest specificity. Efforts are constantly being made to modify the chemistries of assay procedures to this end and to exploit for example, the usefulness of specific enzymes such as hexokinase, uricase, alcohol dehydrogenase and cholesterol oxidase. Since all assays differ considerably in the chemistries used, it is not possible to make general suggestions for improvement. However, most measurements in clinical chemistry are at present made by colorimetry, and it is possible to investigate this to see where improvement is feasible and likely. Two approaches are practical:

1. Most assays rely on a single end-point measurement which is usually taken when a reaction has gone to completion; occasionally, specificity may be improved by measuring absorption at several wavelengths straddling the absorption peak of the chromophore; this is the basis of the socalled Allen correction [2], where a simple calculation using three absorption measurements can compensate for spectral interference.

2. The absorption produced by the analyte may develop at a different rate from that of nonspecific chromogens present; the contribution of these components can then be separated by following the optics of the reaction kinetically. Since the ready availability of facilities to do this would be a major step toward improving specificity, and thus accuracy, it is relevant to consider the capabilities of appropriate instruments.

\section{Choice of Instruments for Spectrophotometric Measurements}

In recent years, several instruments have been developed to measure enzyme activity by following an enzymic reaction kinetically. Ideally such reactions should be followed continuously, but single cuvette instruments can do nothing else during the period of the reaction, and the sample throughput of such instruments is low.

The problem of simultaneous multiple kinetic measurement was solved with the introduction of the GeMSAEC principle [3]. In this, optical cuvettes are mounted at the periphery of a centrifuge rotor, and centrifugal action is used to mix reagents and samples so as to initiate reaction. Opti- 
cal density is monitored by a light beam which passes through the cuvettes as they rotate so that scanning is in effect continuous. A computer monitors and processes the information from the detector so as to calculate the rate of the reaction proceeding in each cuvette. Extraordinarily high precision can be attained with this technique, but a major disadvantage is the need for batch operation since the rotor has to be stopped after each set of measurements, and at least part of it removed for reloading. There is also difficulty in adapting the principle to multichannel working.

The Vickers multichannel analyzer, accepting 300 samples for analysis per hour, is the fastest multichannel machine at present available, but it will not do kinetic measurements. At the Clinical Research Center we are studying the possibility of combining the advantages of the GeMSAEC and Vickers approaches, and have evolved a system which we have termed DACOS (Discrete Analyzer with Continuous Optical Scanning) [4]. In this system a rotating beam of light of suitable wavelength passes through a reaction cuvette and is reflected back to the optical axis, and thence to a detector, by a mirror and prism arrangement (fig. 3). By rotating the prisms, the beam can be made to traverse successive cuvettes arranged in two concentric rings (figs. 4 and 5). The cuvette assembly is it-

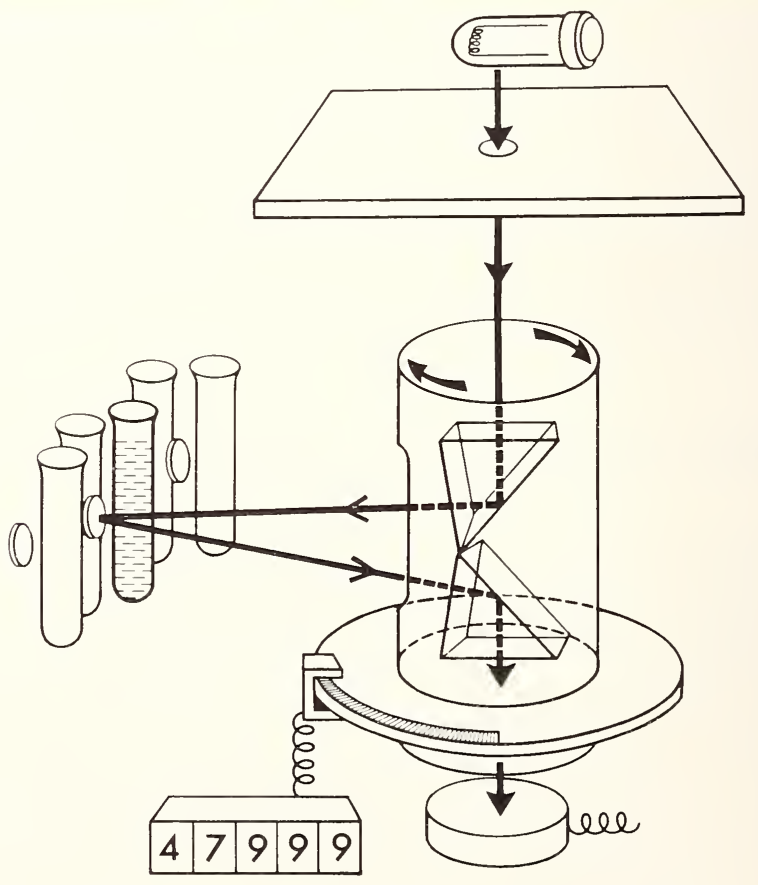

Figure 3. Optical layout of the experimental kinetic analyzer DACOS (Discrete Analyzer with Continuous Optical Scanning). 


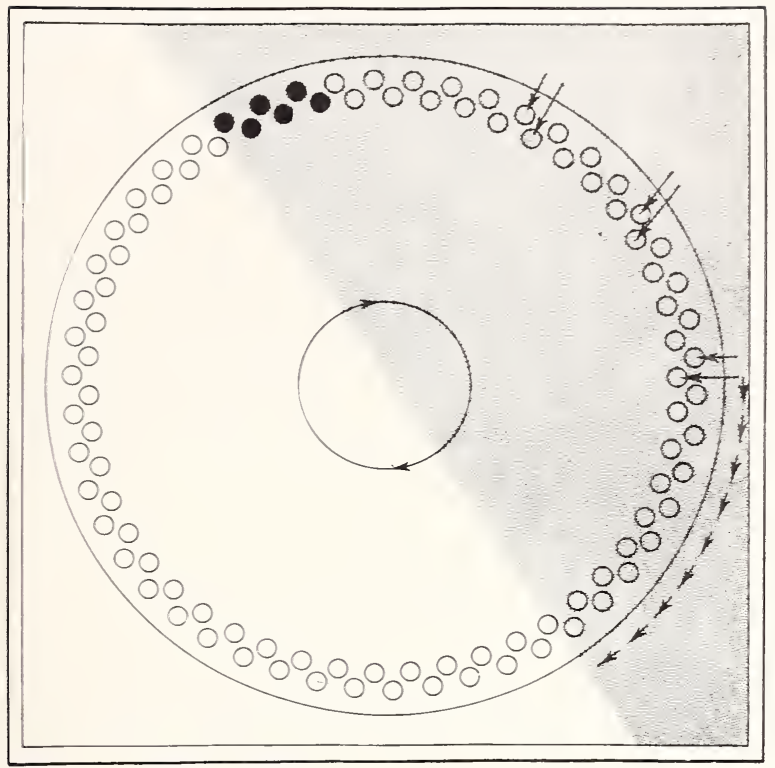

Figure 4. Plan view showing disposition of sample cuvettes in the kinetic analyzer DACOS. Optical measurements are made while cuvettes reside in the unshaded area: cleaning of cuvettes and reagent additions take place in the shaded area.

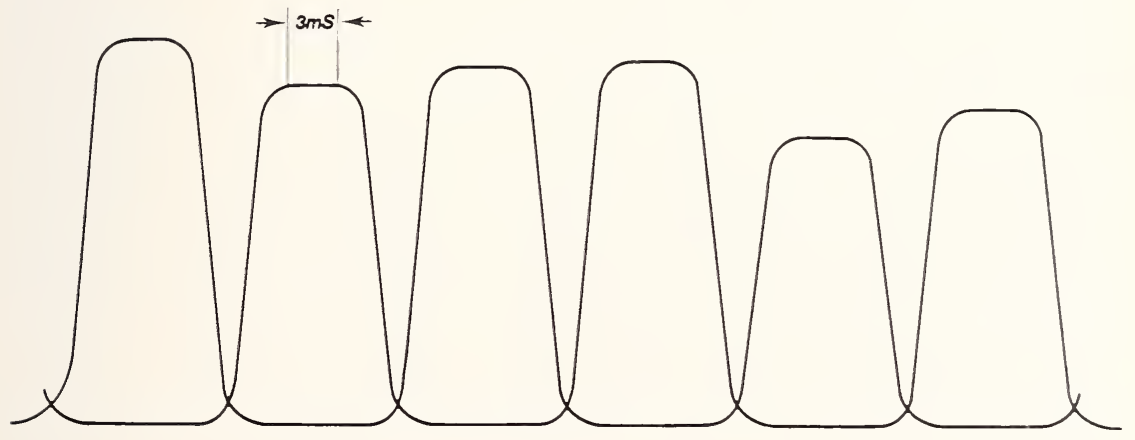

$\leftarrow 10 \mathrm{~ms} \longrightarrow 1$

Figure 5. Pattern of light signals indicating the optical absorbances of solutions in successive cuvettes in the kinetic analyzer DACOS. 
self also rotating in a stepwise manner, so as to utilize the Vickers tube laundry and filling devices, and the net result is that 300 samples can be analyzed every hour, but with a 5-min scanning period for each reaction. By incorporating a fast wavelength switching device in the optical path, successive scans of a given tube can yield the information needed for dual wavelength normalization.

It can be envisaged that a system of 2,3 or 4 such assemblies could be designed, with a common sampling device, so as to afford a 4-, 6- or 8channel instrument. It would therefore be possible to cover in one such instrument, many of the measurements which are routinely required, and at the same time, obtain specificity from the colorimetric reactions involved.

\section{Instruments for the Future}

There is no reason why colorimetric techniques should remain the basis of the majority of analytical methods used in clinical chemistry; in fact the accurate calibration of spectrophotometers over a wide range of wavelengths and absorbances is very difficult.

The popularity of colorimetry has stemmed from its relatively simple chemistry, ease of end-point measurement and ready adaptability to automation. Even reagent preparation is now being done in the factory where the quality control of bulk production can reduce this major source of error considerably. Perhaps the factory should be asked to take over much more of the responsibility for analytical procedures by further exploiting the photographic principle of carrying out reactions in layers of material on film. There is no reason why chemistry done in this way should have less precision and accuracy than that done more conventionally, and it could well be more precise and accurate. Advantages in other directions would be considerable.

Mass spectrometry has recently become available to all chemists and is no longer the sole province of the specialist physicist or physical chemist. Instruments are still expensive and beyond the reach of most hospital laboratories, but small relatively simple quadrupole machines are already in use for specific tasks, and with the rapid advance of technology in this field, the principle of mass spectrometry, especially with regard to ease of operation, will be immunoassay with end-point measurement based either on radioisotope counting or some other highly sensitive and specific system of detection. The prospects for enzyme immunoassay, for example, are very bright. 


\section{Laboratory Management}

Thus far, only errors emanating from the shortcomings of technology itself have been mentioned. Wherever human intervention is necessary, however, human error is to be suspected, which increases with the throughput of work, and with boredom or fatigue. Robinson [5], has shown that there is a linear decrease in the precision of measuring serum potassium by flame photometry as the number of assays completed without a break increases. When a 20 -min break was allowed between batches, precision plateaued at an acceptable level. This effect is referred to in industry as "The 4 o'clock phenomenon."

The relationship between physical and environmental working conditions and the quality of output has been well documented. Effective management can be brought to bear with success in such areas as laboratory design (including decor, spacing, height of benches, stools, shelves, etc.), environment (including heating, humidity, lighting, noise prevention, etc.), ergonomics and equipment maintenance. Good work cannot come out of a laboratory with unhappy, discontented staff.

Undoubtedly the greatest impact on the reduction of errors in clinical chemistry has been through the introduction of automation and electronic data processing. Prior to this, random error in the best laboratories affected at least 5 percent of all results; in fully-automated computerized laboratories, this has now been reduced to less than 1 percent. Improvement beyond this is difficult since the remaining errors are truly random (e.g., the collection of blood from the wrong patient), and are difficult both to detect and to trace.

In his book Zero Defects, J. F. Halpin [6] pointed out that throughout their lives, people are conditioned to accept the fact that they are not perfect and therefore expect to make mistakes: in fact it might be said that a few mistakes a day are required if one is to be accepted as a human being! This state of affairs can largely be corrected by good management if it is realized that the reason behind a lack of perfection can simply be that perfection has not been expected. When it is demanded, it is achieved. Halpin's programme for introducing near perfection into an organization is a management technique aimed at getting the employee to think positively about each and every task, and to realize that both he and the management share a common aim. The series of steps to be followed by management are: to present a challenge; to back the challenge with action of its own; to establish high standards; to check results; to act in accordance with the results to recognize accomplishment. The Zero Defects concept was born in the U.S. missile industry where the smallest imperfection in construction could have the most disastrous consequences. Institution of 
the programme in general industry has achieved dramatic results, e.g., a 54 percent drop in hardware faults in missile part production, a 98 percent improvement in delivery and shipping actions, hundreds of thousands of payroll computations made without error, etc. A Zero Defects programme applied to laboratory work might also produce dramatic results.

Having introduced this paper with a quotation from a British author, and gone on to discuss the work of an American writer, it may be appropriate to close with the words of a French philosopher. Needless to say, these words demonstrate strong negative bias - "London is the city where they purvey the most inaccurate news and produce the worst possible arguments, based on information which is entirely false" (Voltaire).

\section{References}

[1] Pickup, J. F., Jackson, M. J., Price, E. M., and Brown, S. S., Clin. Chem. 20, 1324 (1974).

[2] Allen, W. M., J. Clin. Endocr. Metab. 10, 71 (1950).

[3] Anderson, N. G., Anal. Biochem. 28, 545 (1969).

[4] Snook, M., Renshaw, A. E., and Rideout, J. M., Z. Klin. Chem. Klin. Biochem. 5, 236 (1974).

[5] Robinson, R., Lancet, i, 433 (1967).

[6] Halpin, J. F., Zero Defects, McGraw-Hill, New York (1966). 


\title{
TRACE LEVEL DETERMINATION OF TRICLOSAN BY GAS CHROMATOGRAPHY
}

\author{
Fritz-Hans Marquardt, Janos Schulze, and Don Smith \\ CIBA-GEIG Y Corporation \\ Greensboro, North Carolina 27409 U.S.A.
}

\begin{abstract}
The triclosan (2-hydroxy-2',4',4-trichlorodiphenyl ether) content in various substrates, for example, blood, can be determined by extraction into hexane, formation of the acetyl derivative, and gas chromatography over OV-17 with an electron capture detector, either with or without aldrin as internal standard. The precision and accuracy of this method is discussed.
\end{abstract}

Keywords: Bacteriostats; blood analysis; gas chromatography; trace analysis; triclosan: 2-hydroxy-2' $, 4^{\prime}, 4$-trichlorodiphenyl ether.

\section{Introduction}

Practicing analytical chemists are faced almost routinely now with problems which are related to trace analysis. This emphasis on parts per billion and picograms, instead of the usual percent and milligrams, has refocused the interest on a completely new set of factors which limit the precision and accuracy of analytical techniques.

In this paper we shall discuss, with a practical example, some of the intricacies of trace analysis and show that even a limited statistical analysis may assist in the localization of errors.

The method we use as an example is the determination of triclosan (2hydroxy-2', 4',4-trichlorodiphenyl ether) in blood [1]. Our involvement with this method was based on the need to establish triclosan concentrations in a large number of blood samples, as part of a comprehensive toxicological investigation of bacteriostats. We, therefore, needed a simple, highly sensitive and accurate procedure, which would also be adaptable to automation. 


\section{Method Development}

Gas chromatography with an electron-capture detector was selected as the method of measurement because the triclosan molecule includes three chlorine atoms and this detector system is highly sensitive to chlorine. An additional feature of the method was its suitability for automation. Automatic injectors allow almost continuous operation and consequently, the analysis of a large number of samples with a minimum of work. We have also found that aldrin could be utilized as an internal standard and that acetylation was an excellent derivation technique for triclosan in blood extracts.

The details of the method are shown in figure 1. The procedure consists of three distinct parts: the extraction of triclosan from blood (part " $E$ "), the addition of internal standard and derivatization of triclosan (part "D") and the gas chromatographic analysis (part "G"). Figure 2 shows the conditions for gas chromatography.

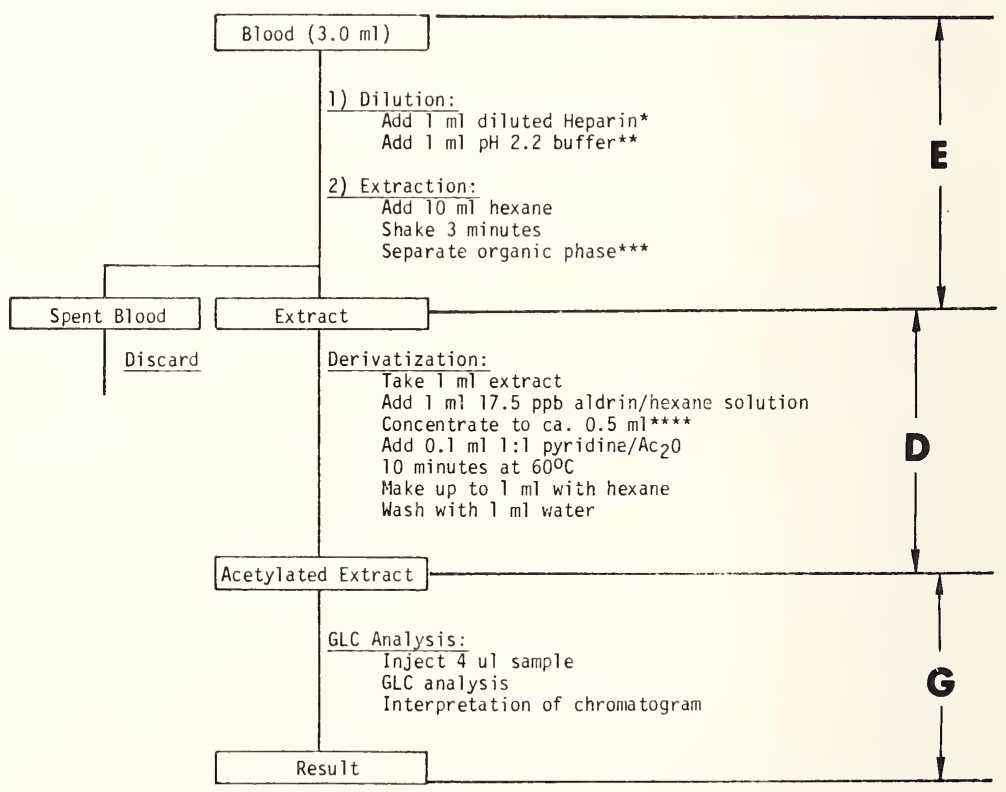

* Sodium Heparin Injection USP diluted tenfold with deionized water.

** $\quad 1.07 \mathrm{~g} \mathrm{Na} 2 \mathrm{HPO}_{4} .7 \mathrm{H}_{2} 0,20.6 \mathrm{~g}$ citric acid in 1 liter deionized water.

$\star * \star$ Freeze at $-200 \mathrm{C}$, Ehaw at room temperature, centrifuge at moderate speed.

$\star \star \star \star \star$ At room temperature under a stream of nitrogen.

Figure 1. Analysis Procedure for the determination of free triclosan in blood by gas chromatography. 
Gas Chromatograph: Hewlett-Packard 5750 equiped with Ni-63 electron capture detector

- GC Column:

4 feet by $1 / 4$ inch packed with 2 percent

OV-17 on $80 / 100$ mesh Chromasorb WHP or

3 percent OV-17 on Supelcoport

- Column temperature:

$230{ }^{\circ} \mathrm{C}$

- Detector temperature:

$290^{\circ} \mathrm{C}$

- Injection port temperature: $240{ }^{\circ} \mathrm{C}$

- Carrier gas: 5 percent methane in argon; carrier gas flow $=45$ to $50 \mathrm{ml} / \mathrm{min}$

Figure 2. Gas chromatography conditions for triclosan analysis.

\section{Qualitative Discussion of Procedure}

This analytical method yields chromatograms (fig. 3), in which aldrin and acetylated triclosan have clearly resolved peaks with retention time of $\sim 1$ and 2 minutes respectively. The quantitative interpretation is therefore easily done, and the sensitivity of the method is such that 5 percent of a full scale deflection can be observed for $100 \mathrm{pg}$ of triclosan. This is equivalent to a lower detection limit of $6 \mathrm{ppb}$ triclosan in blood, which could be further improved by inclusion of an additional concentration step in the method, or by decreasing the amount of hexane used in the extraction. It should be noted that high quality chromatograms were obtained only when the samples were washed (i.e., extracted with water) after acetylation. Without such a washing, considerable tailing of the solvent peak was observed as shown on the right side of figure 3 . Without washing of the sample, aldrin cannot be used as internal standard because solvent tailing interferes with the aldrin peak.

Another problem faced during method development was the limited dynamic range of the electron-capture detectors. Thus, for the triclosan signal, a linear relationship was observed between the amount injected and the signal intensity for injections up to $800 \mathrm{pg}$ of material (fig. 4). This upper limit was increased at will by diluting the eluate of the chromatographic column with purge gas prior to admitting it to the detector. However, dilution with purge gas naturally decreased the sensitivity of the method, consequently utilization of purge gas depended on the triclosan concentration of the sample analyzed. For aldrin, a linear relationship was observed between the amount injected and the signal intensity for up to $130 \mathrm{pg}$ of material. Our method, which uses $65 \mathrm{pg}$ of aldrin as internal standard, can therefore accommodate considerable fluctuations of the in- 


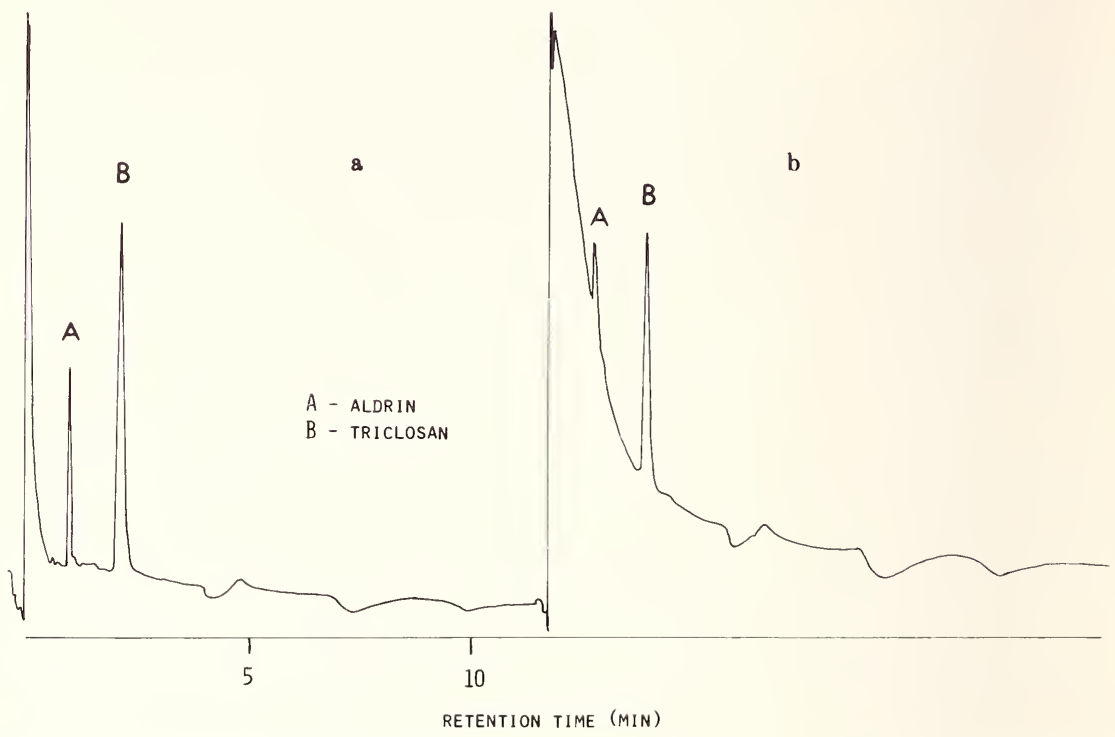

Figure 3. Gas chromatograms for the determination of triclosan in blood. a. Including a water washing in the analytical procedure; $b$. without this water wash.

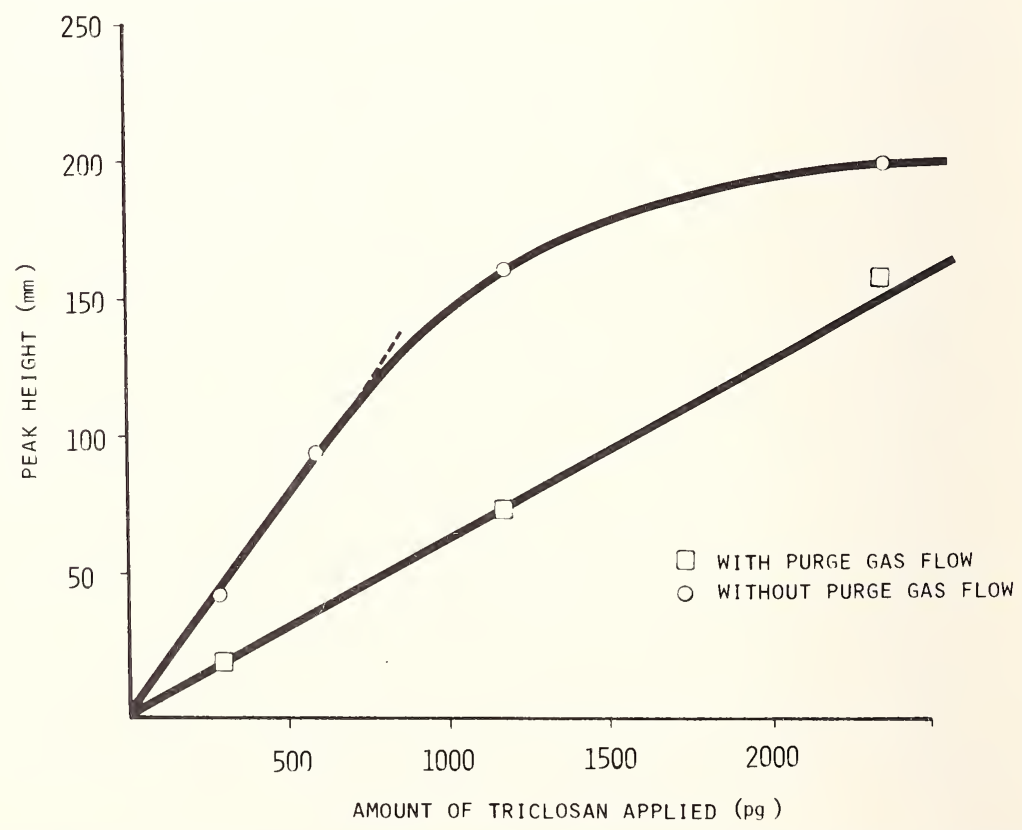

Figure 4. Typical calibration curves for the gas chromatographic determination of triclosan. 
jected volumes. This was important, for automatic injectors have the disadvantage that injected sample volumes may fluctuate (for example due to air bubbles in the syringe).

Utilization of the acetate derivative of triclosan produced a peak which was sharper and more symmetrical than that of the underivatized material. Acetylation was chosen over other commonly used derivatizing techniques, since it has been reported [2] to be the only quantitative reaction of phenols in blood extracts. It was interesting to observe that acetylation increased the area of the triclosan signal by only 13 percent, while it increased the signal intensity of bi-phenolic bacteriostats, such as hexachlorophene, by a factor of $\sim 100$ [3].

In closing the discussion about the chromatogram, we want to mention that sometimes we observed up to three negative signals that followed the triclosan peak. To prevent interference of these negative signals with the peaks of the next chromatogram, we had to set the interval between sample injection at 12 minutes.

Utilizing a 12 minute analysis cycle, and assuming single injections for each sample, one gas chromatograph equipped with an automatic injection system was able to handle 120 samples per day.

\section{Quantitative Discussion of the Procedure}

After the method development work was finished, we wanted to determine how the individual steps influenced the precision of the whole method. For this purpose, the procedure was divided into the three major parts, extraction, derivatization and gas chromatography, and special experiments were designed which made their statistical analysis possible.

The first investigation included only the gas chromatography portion of the method consisting of injection of the sample, the effects of instrumentation, separation on the column, detector sensitivity and quantitative evaluation of the chromatogram. The precision of this portion was established by 9 injections of a hexane solution containing acetylated triclosan (146 ppb) and aldrin (17.5 ppb). This study resulted in a standard deviation of 2.0 percent (table 1 ).

Next, the additions of the internal standard and the acetylation were studied as variables. A simulated blood extract was prepared by dilution of a hexane stock solution. One $\mathrm{ml}$ of this hexane solution containing 146 ng triclosan was submitted to the steps of parts $D$ and $G$ of the method (see fig. 1) including addition of internal standard, acetylation and gas chromatography by single injection. This analysis was repeated 9 times 
independently, and table 2 shows the results of the study. The standard deviation for parts $D$ and $G$ of the method was 2.9 percent. This error was not due to incomplete acetylation since it had been shown prior to this study that the experimental conditions of the method led to complete acetylation of triclosan.

TABLE 1. Gas chromatographic analysis of acetylated triclosan

\begin{tabular}{cccc} 
& \multicolumn{3}{c}{ Peak height } \\
\cline { 2 - 4 } $\begin{array}{c}\text { Sample } \\
\text { No. }\end{array}$ & Aldrin & Triclosan & Ratio \\
\hline & & & \\
\hline & 59 & 156 & 2.64 \\
2 & 61 & 159 & 2.61 \\
4 & 60 & 159 & 2.65 \\
5 & 64 & 160 & 2.50 \\
6 & 62 & 157 & 2.53 \\
7 & 62 & 158 & 2.55 \\
8 & 61 & 158 & 2.59 \\
9 & 62 & 158 & 2.55 \\
$\bar{X}$ & 60 & 157 & 2.62 \\
$s$ & & & 2.58 \\
& & & $2.0 \%$ \\
\hline
\end{tabular}

TABLE 2. Triclosan determination by gas chromatography

\begin{tabular}{lll} 
& \multicolumn{3}{c}{ Peak height } \\
\cline { 2 - 3 } Sample & Triclosan & Ratio
\end{tabular}

$\begin{array}{llll}1 & 79 & 136 & 1.72 \\ 2 & 83 & 137 & 1.65 \\ 3 & 73 & 119 & 1.63 \\ 4 & 72 & 120 & 1.67 \\ 5 & 93 & 147 & 1.58 \\ 6 & 72 & 122 & 1.69 \\ 7 & 90 & 143 & 1.59 \\ 8 & 91 & 146 & 1.60 \\ 9 & 84 & 138 & 1.64 \\ \bar{X} & & & 1.64 \\ s & & & 2.9 \%\end{array}$


Finally, the complete method, including the sampling of the blood and its extraction with hexane, was carried out nine times, analyzing dog blood containing $190 \mathrm{ppb}$ triclosan (received from an oral toxicity study). This test established that the standard deviation for the complete method was 6.0 percent (table 3 ).

TABLE 3. Triclosan determination in blood by gas chromatography

\begin{tabular}{llll}
\hline & \multicolumn{3}{c}{ Peak height } \\
\cline { 2 - 4 } Sample & \multicolumn{3}{c}{ Ratio } \\
\cline { 2 - 4 } & Aldrin & Triclosan & \\
\hline & & 57 & 1.02 \\
2 & 56 & 16 & 1.00 \\
3 & 51 & 54 & 1.04 \\
4 & 56 & 60 & 1.07 \\
5 & 61 & 56 & 0.92 \\
6 & 60 & 56 & .93 \\
7 & 60 & 61 & 1.02 \\
8 & 53 & 59 & 1.11 \\
9 & 55 & 54 & 0.98 \\
$\bar{X}$ & & & 1.01 \\
$s$ & & & $6.0 \%$ \\
\hline
\end{tabular}

Application of the principle of additivity of variances to these results shows that, while step G (gas chromatography) and step D (addition of aldrin and derivatization) each introduces a standard deviation of only about 2 percent to the overall error, more than twice this deviation is introduced by sampling of blood and its extraction (fig. 5).

Our present efforts are concentrated on investigating these parts of the method. We hope to improve the overall precision of the method by improving sampling and extraction.

The accuracy of the method was determined in two different ways. In one study blood was spiked at different levels in the range of 10 to 100 $\mathrm{ppb}$ with triclosan and the recovery checked at each level. It was found that the recoveries were all above 93 percent (table 4), with a standard deviation of 2 percent between these three recovery values. Another study compared the results obtained from radioactive measurements and from gas chromatographic analysis of blood of dogs which had been fed appropriate doses of radio-labelled triclosan. For this study the method had to be altered by treating of the blood with $1 \mathrm{ml}$ of concentrated hydrochloric acid at $100{ }^{\circ} \mathrm{C}$ for 1 hour to hydrolyze the conjugates of triclosan and by repeating the extraction with hexane. Also here the recoveries were better than 93 percent (table 5). 


\section{Experimental PART Conclusion values METHOD}

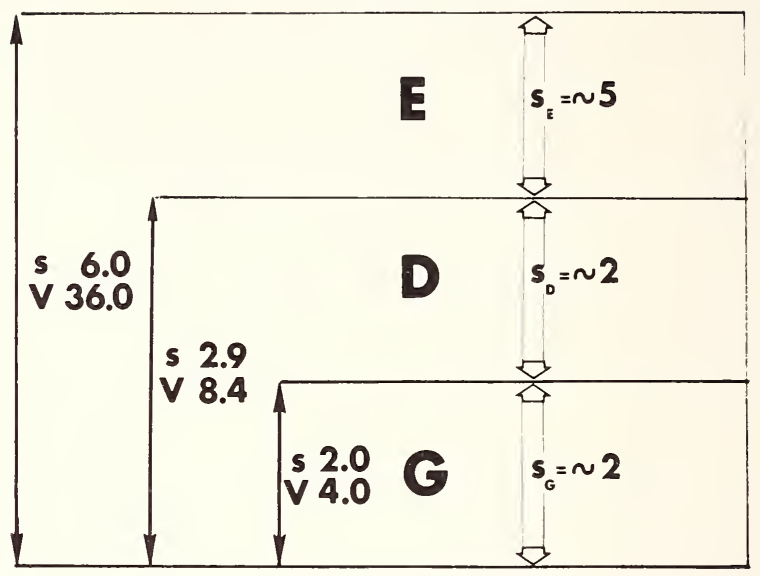

Figure 5. Analysis of the precision of the determination of triclosan in whole blood by the principle of additivity of variances. $s=$ relative standard deviations; $\mathrm{V}=$ variances.

TABLE 4. Recovery of triclosan from fortified whole blood

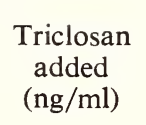

\begin{tabular}{ccc}
\multicolumn{3}{c}{ Recovery (\%) } \\
\hline $\begin{array}{cc}1 \mathrm{st} \\
\text { determination }\end{array}$ & determination & Average
\end{tabular}

$\begin{array}{rrrr}100 & 92.4 & 95.2 & 93.8 \\ 50 & 98.6 & 97.5 & 98.1 \\ 12.5 & 96.0 & 96.0 & 96.0\end{array}$

TABLE 5. Recovery of total triclosan from whole blood

\begin{tabular}{ccccc}
$\begin{array}{c}\text { Concentration } \\
\text { by LSC } \\
(\mu \mathrm{g} / \mathrm{ml})\end{array}$ & $\begin{array}{c}1 \mathrm{c} \\
\text { determination }\end{array}$ & $\begin{array}{c}2 \mathrm{~d} \\
\text { determination }\end{array}$ & $\begin{array}{c}3 \mathrm{~d} \\
\text { determination }\end{array}$ & Average \\
\cline { 2 - 5 } & & & 98.6 & 97.5 \\
7.28 & 96.3 & 97.7 & 96.9 & 93.4 \\
\hline 113.93 & 95.0 & 88.4 & & \\
\hline
\end{tabular}


In summary, we have described a typical biochemical analytical method which can determine trace level triclosan for which we show recoveries of at least 93 percent and a standard deviation of 6 percent for analyses by single injections. Statistical analysis of the precision of this method led to the conclusion that the largest error was introduced in the method by the sampling and extraction steps and that further improvements of the method may be realized by changing these steps.

\section{Acknowledgement}

We wish to acknowledge the careful and conscientious method development work of Mrs. Carol Spitzer.

\section{References}

[1] Schulze, J., Marquardt, F-H., Lyman, F., and Spitzer, C. JAOC̄S, (submitted for publication).

[2] Ulsamer, A. G., JAOAC 55, 1294 (1972).

[3] Terry, D. G. and McQueen, E. C., J. Chrom. 76, 233 (1973); Porcaro, P. J. and Schubiak, P., Anal. Chem. 40, 1232 (1968). 



\title{
ELECTROCHEMICAL TRACE ANALYSES BASED ON FARADAY'S LAW
}

\author{
D. J. Curran \\ Department of Chemistry \\ University of Massachusetts \\ Amherst, Massachusetts 01002 U.S.A.
}

Thin layer electrochemical measurements and constant current coulometry can provide useful absolute methods for trace analysis.

The difficulty in distinguishing the coulombs consumed by the Faradaic process from the total coulombs consumed in the experiment can be minimized by the use of a null concept with constant current coulometry. After adjustment of the system to the null point, for example potentiometric, the sample is added and the system returned to the null by coulometric titration. Since the electrochemical potential of the system is the same before and after passage of the electrolysis current, no charge can be lost or gained from charging or discharging of the double layer. Several examples of determinations based on this concept are presented.

The coulometric method involves the use of an electrogenerated titrant in almost all cases. A more direct approach to the use of Faraday's Law is provided by thin layer methods, where direct electrochemical conversion of the species of interest is carried out stoichiometrically. Corrections for background coulombs must be made with these techniques. Very small volumes of solutions as dilute as $10^{-5} \mathrm{M}$ can be handled and several examples are presented. In addition, it is possible to combine thin layer methods with electrochemical preconcentration techniques to yield a versatile tool for trace analysis.

Keywords: Automatic titrations; coulometric titrations; current integration; digital timing; epinephrine: linear sweep voltammetry; manganese: micrometer cell; thin layer electrochemistry: vanadium.

\section{Introduction}

Faraday's Law of Electrolysis is the basis for a number of methods of electrochemical analysis. The advantages and disadvantages involved in 
its application are well known but among the former, freedom from the need for chemical standards or standard solutions is very appealing in terms of trace analysis. The purpose here is to discuss two such methods, coulometric titrations and thin layer linear sweep voltammetry, in an effort to point out their potentialities.

\section{Coulometric Titrations}

Coulometric titration is a well established method for the determination of a number of substances but work at the sub-microgram level is not common [1-11]. Roughly, 10 ng of material require about ten microcoulombs of electricity for complete electrolysis. The typical solution volume is $50 \mathrm{ml}$ so the corresponding concentration would be about 0.2 parts per billion (ppb). The measured variables in the experiment are the cell current, which is usually held constant, and the time of electrolysis. The cell current is regarded as the sum of the current due to the Faradaic process of interest plus current due to all other processes such as charging current, adsorption current, or current due to Faradaic impurities. It is necessary that the latter processes constitute a negligible fraction of the total cell current to obtain accurate results. Further, the magnitude of the total background current establishes a lower limit on the cell current which can be used with a given error. The background current (residual current) depends on a number of instrumental and chemical variables and is difficult to predict but a reasonable rule of thumb might be a current density of 1 $\mu \mathrm{A} / \mathrm{cm}^{2}$. Thus a cell current density of $1 \mathrm{~mA} / \mathrm{cm}^{2}$ could be used with an error of -0.1 percent relative. Such a current passed at a $1 \mathrm{~cm}^{2}$ electrode would produce 10 microcoulombs of electricity in only 10 milliseconds. Two problems arise from this conclusion: the times of electrolyses usually encountered in the literature of coulometric titrations are much longer than this (several hundred seconds), and the time required to charge the double layer is longer than this (perhaps 50 milliseconds). Neither of these difficulties is fatal.

The first problem has resulted from the desire to make the time measurement with a precision of a few ppt and the common practice of using electric stopclocks for this purpose. A remedy is to use solid state devices to generate and measure timing signals. Digital readout of time to the nearest microsecond is readily achieved in this fashion. Figure 1 shows a block diagram of the timing section of a coulometric titrator we have developed [12]. Four timing ranges of 0 to $0.999999,0$ to $9.99999,0$ to 99.9999, and 0 to 999.999 seconds are provided. The electrolysis may 


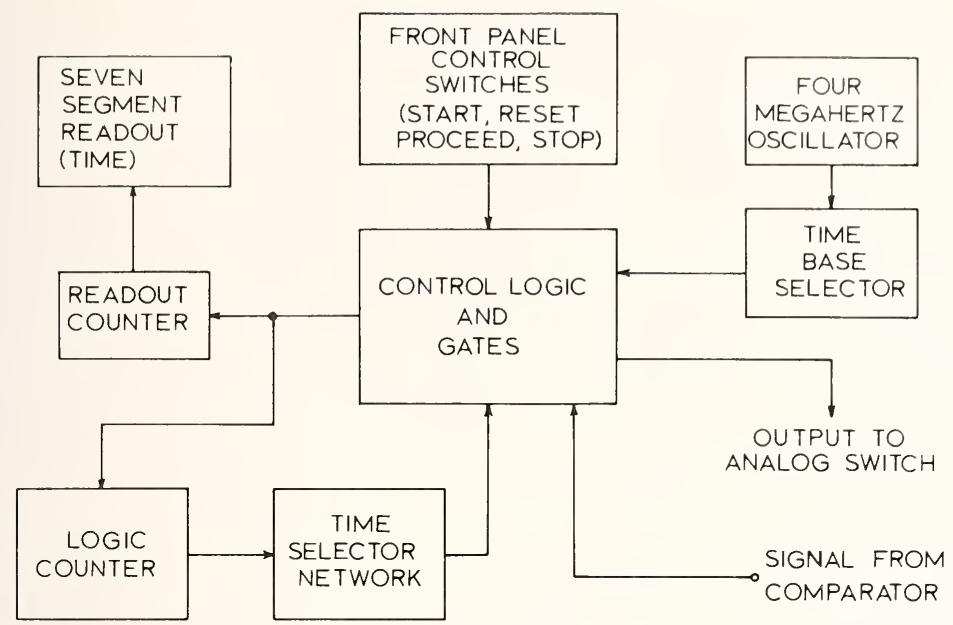

Figure 1. Digital timing section.

either run continuously or until a preset time is reached when a logic pulse is generated to shut off the current. Switching times of less than a microsecond have been achieved.

Before discussing the performance of the titrator it is necessary to return to the double layer charging problem. While not a true capacitor, the electrode-solution interface has capacitive properties and it requires a change in charge to change the electrode potential. These coulombs are normally lost or gained as far as the Faradaic process is concerned and, of course, it is necessary to change the electrode potential to generate a titrant. However, if the generator electrode potential is the same at both the start and finish of the experiment no loss or gain of coulombs can occur. The price which must be paid for this is to wait long enough for the double layer to charge or discharge. With this stipulation, the procedure of pretitration which is commonly employed in coulometric titrations achieves this result. It is most easily seen in terms of potentiometric end point detection. The solution to which the sample is added is brought to some preselected end point potential by the generation of titrant. The sample is added and titrant generated until the indicator electrode returns to the preselected potential. At equilibrium, the generator and indicator electrodes must be at the same potential. At the generator electrode, the double layer charges or discharges through the Faradaic reaction to return to equilibrium. Clearly, the elapsed electrolysis time can be less than the time it takes to change the charge on the double layer provided the elapsed experiment time is longer.

Table 1 shows some data and results obtained by L. B. Jaycox [12] for 
TABLE 1. Data and results for the coulometric titration of vanadium $(V)$ with iron (II)

\begin{tabular}{ccccc}
\hline $\begin{array}{c}\text { Amount } \\
\text { taken } \\
(\mu \mathrm{g})\end{array}$ & $\begin{array}{c}\text { Amount } \\
\text { found } \\
(\mu \mathrm{g})\end{array}$ & $\begin{array}{c}\text { Average end } \\
\text { point time } \\
(\mathrm{s})\end{array}$ & $\begin{array}{c}\text { Relative } \\
\text { precision } \\
\%\end{array}$ & $\begin{array}{c}\text { Relative } \\
\text { error } \\
\%\end{array}$ \\
\hline 225.8 & 226.0 & 44.38 & \pm 0.1 & +0.1 \\
22.58 & 22.58 & 4.432 & \pm .1 & \pm .0 \\
4.516 & 4.510 & 0.8852 & \pm .1 & -.1 \\
2.258 & 2.255 & .4425 & \pm .1 & -.1 \\
0.2200 & 0.2116 & .0415 & \pm 2.5 & -3.9 \\
.1100 & .1046 & .0205 & \pm 1.5 & -4.9 \\
\hline
\end{tabular}

${ }^{a}$ Average of six determinations.

the determination of vanadium (V) in sulfuric acid solution by electrogenerated $\mathrm{Fe}(\mathrm{II})$ with potentiometric end point detection using a platinum indicator electrode and a platinum generator electrode. The reactions are:

$$
\mathrm{Fe}^{+3}+\mathrm{le}^{-} \rightleftharpoons \mathrm{Fe}^{+2}
$$

and

$$
\mathrm{VO}_{2}^{+}+2 \mathrm{H}^{+}+\mathrm{Fe}^{+2} \rightleftharpoons \mathrm{VO}^{++}+\mathrm{Fe}^{+3}+\mathrm{H}_{2} \mathrm{O}
$$

All of these titrations were done manually in a solution volume of about $50 \mathrm{ml}$. Successive sample of $\mathrm{V}(\mathrm{V})$ were added using either a micrometer driven microburet or calibrated pipets. Samples containing more than 2 $\mu \mathrm{g}$ of $\mathrm{V}(\mathrm{V})$ were determined with a precision and accuracy of $0.1 \mathrm{ppt}$. Elapsed electrolysis times to reach the end point were as little as 442.4 milliseconds. Samples containing $110.0 \mathrm{ng}$ of the metal ion were determined with a precision of \pm 1.5 percent and a relative error of -4.9 percent. The average end point time was 20.5 milliseconds and around 2.5 minutes were required for each determination after addition of sample. The solution concentration is about $2 \mathrm{ppb}$ in $\mathrm{V}(\mathrm{V})$.

The titrator will also run in an automatic mode and table 2 has some results for the coulometric titration of permanganate ion with electrogenerated ferrous ion in sulfuric acid solution [12]. Supporting electrolyte volumes in this case were $10 \mathrm{ml}$. A platinum-iridium alloy was used as the indicator electrode and its potential was measured against a lead amalgam-lead sulfate reference electrode using a very high input impedance differential amplifier circuit. The output of this electrode pair was sent to a comparator circuit which developed a logic pulse to turn off the current when the preset end point potential was reached. Timing circuits were also used to provide end point anticipation. The generator elec- 
Curran

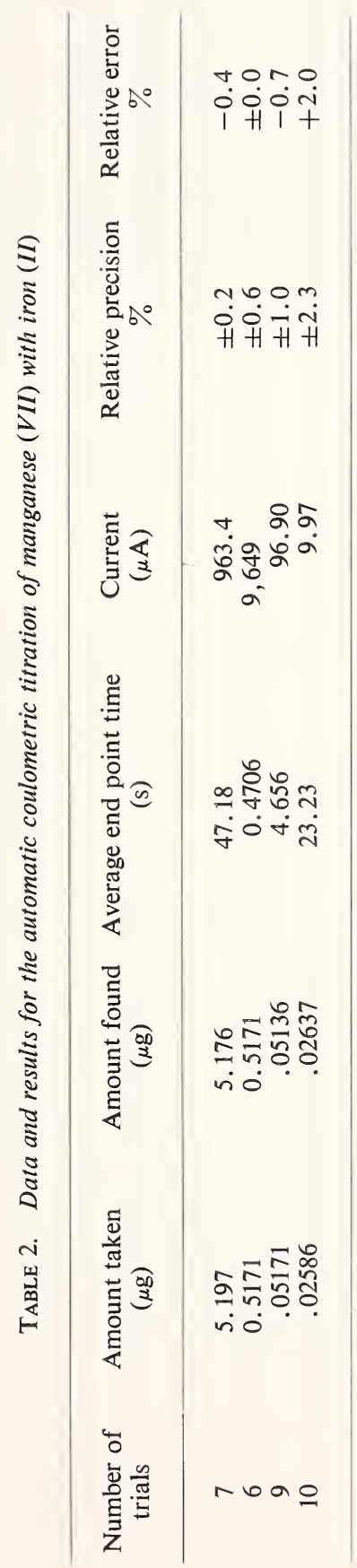


trode was platinum. A hydrazine solution was used in the counter electrode compartment to prevent the generation of oxygen. Two and one-half ppb solutions were determined with a precision and accuracy of 2 percent and much higher accuracy and precision were found for more concentrated samples.

The data in tables 1 and 2 serve to show the potential of coulometric titrations for determinations at the ppb level. They are fast, accurate, precise and readily automated. The latter three are well known advantages of coulometric titrations in general. The former has not been considered adequately in the past. To take full advantage of these attributes, improvements in end point detection are needed. Existing methods should be examined to see if their full capabilities are being utilized in terms of sensitivity and new, highly sensitive methods are required. Some of our work using fluorescent indicator dyes in this regard has been reported [13].

\section{Thin Layer Electrochemistry}

Electrochemical thin layers of solution are those which are dimensionally thinner than the thickness of the diffusion layer which arises from diffusional mass transport of material from the bulk of solution to the electrode surface. Typically they are $5 \times 10^{-3} \mathrm{~cm}$ or less. Since mass transport is not a consideration, such a process cannot be rate controlling and an array of normally steady-state techniques become stoichiometric when carried out in thin layers. In other words, complete conversion of the electroactive species takes place. Three reviews of the subject are available [14-16]. In many instances, the area under the response curve is either the current-time integral or proportional to it. Faraday's Law is therefore readily applied. Background currents must again be considered and a separate experiment with only the supporting electrolyte will yield the number of coulombs which must be subtracted from the sample result.

The above comments apply to the technique of linear sweep voltammetry, and to cyclic voltammetry, in thin layers. The shape of the currentvoltage curve in the former case is a normal distribution curve with the peak current occurring at the polarographic half-wave potential for a reversible system. In a cyclic experiment, the first half of the voltage sweep corresponds identically to the linear sweep experiment and the second half of the sweep returns the electrode to its initial potential. If the system is reversible, all of the material which was reduced or oxidized in the forward sweep must be oxidized or reduced during the backward 
sweep and the current-voltage curve for the reverse process is the mirror image on the voltage axis of that for the forward process. The method is nondestructive in this instance. If the electrode reaction has irreversible character, the curve shifts on the potential axis and a skew in the shape appears for the linear sweep case. The same skew will appear in the cyclic response curve and the peak potentials will shift apart.

Work in our laboratories was performed by R. R. Fike [17]. Currentvoltage curves were studied using a PAR Model 170 Electrochemistry System (Princeton Applied Research Corp., Princeton, N.J.). Although an integration mode exists in this instrument, it was desired to record the response curve and obtain the current-time integration simultaneously. The output of the current-to-voltage conversion amplifier is available on the instrument. Its maximum value is $\pm 10.00 \mathrm{~V}$ for each full scale setting of the current selector control. This signal was processed to introduce the numerical value of the Faraday and presented to a voltage-to-frequency converter (Model 2210, Hewlett Packard Co., Dymac Division, Palo Alto, Calif.). The output of this unit was processed to produce one positive going pulse for every cycle of output and then made TTL compatible to drive events counters. This integration system proved to be electronically accurate to a few ppt and provided a readout directly proportional to equivalents. The proportionality constant, $\mathrm{k}$, depended only on the current setting of the PAR and had values ranging from $10^{-12}$ to $10^{-16}$ equivalents per count for full scale currents of $1 \mathrm{~mA}$ to $0.1 \mu \mathrm{A}$ respectively.

The design of a thin layer cell is critical. The chief difficulty is to produce a cavity to confine the solution to thin layer conditions which will not allow material to enter or leave and which will provide reasonable means for introducing electrodes having the proper geometrical relationships. The latter point refers to the fact that the working and counter electrodes are optimally plane parallel and facing each other and that the working and reference electrodes have as uniform and as small an IR loss between them as possible. All of these features are necessary to prevent diffusional and IR drop distortion of the response curve and are particularly important when working with very dilute solutions. A number of designs are based on standard micrometers [16]. A report of an early version of our cell has appeared elsewhere [18]. The essential feature is a thin layer cavity defined by the face of the working electrode which is mounted on the detachable anvil of the micrometer and a porous boundary of Vycor glass which has been heat treated to reduce its pore size. The cylindrical wall of the cavity is sealed by a paint or a mechanical shield surrounding the anvil. A unique feature is placement of both the counter 
and reference electrodes below the porous boundary. This arrangement, in conjunction with positive feedback to minimize uncompensated IR drop, provided good performance and permitted study of solutions as dilute as $10^{-5} M$.

Catecholamines are readily oxidized at a platinum electrode. The reaction for epinephrine has been reported as a quasi-reversible two electron oxidation to the quinone in $1 M$ sulfuric acid [19]. The oxidation is more complex than this at lower acidities and the rates of coupled chemical reactions differ for different catecholamines at the same acidity but this need not concern us here. Table 3 shows some results for the determination of epinephrine using thin layer linear sweep voltammetry with current integration [17]. The sweep rate was $5 \mathrm{mV} / \mathrm{s}$ and the potential span covered was +0.490 to $+0.690 \mathrm{~V}$ versus a saturated calomel electrode constructed with $\mathrm{NaCl}$ instead of $\mathrm{KCl}$. Very good accuracy was obtained as revealed in column five of the table. Results correct to within a few ppt were found for samples containing as little as 36 picomoles of epinephrine. The error increased to +10 percent for the 8 picomole samples. The precision is not as good as the accuracy. This was a severe test of the cell in that the micrometer was reset for every determination. It would appear that the cell volume cannot be reproduced to better than 1 or 2 percent relative. This is reasonable for cavity volumes of less than a microliter and cavity thicknesses of 2 to $4 \times 10^{-3} \mathrm{~cm}$. A cell having fixed dimensions would undoubtedly improve the precision. The time required for each potential sweep was 40 seconds.

TABLE 3. Thin layer electrochemical determination of epinephrine by linear sweep voltammetry and current integration

\begin{tabular}{ccccr}
\hline $\begin{array}{c}\text { Sample } \\
\text { concentration } \\
(\mathrm{mM})\end{array}$ & $\begin{array}{c}\text { Picomoles }^{\mathrm{b}} \\
\text { found }\end{array}$ & $\begin{array}{c}\text { Relative } \\
\text { precision } \\
\%\end{array}$ & $\begin{array}{c}\text { Concentration } \\
\text { found } \\
(\mathrm{mM})\end{array}$ & $\begin{array}{c}\text { Recovery } \\
\%\end{array}$ \\
\hline & & & & \\
\hline $.59_{6}$ & 3604 & \pm 1.2 & $4.95_{1}$ & 99.9 \\
0.991 & 721.7 & \pm 0.3 & 0.991 & 100.0 \\
$.495_{6}$ & 357.9 & \pm 2.6 & $.491_{6}$ & 99.2 \\
.0991 & 72.78 & \pm 1.6 & .1000 & 100.9 \\
$.0495_{6}$ & 36.21 & \pm 5.9 & $.0497_{4}$ & 100.4 \\
.00991 & $7.96_{5}$ & \pm 16.5 & .01096 & 110.4 \\
& & & & \\
\hline
\end{tabular}

a Based on independent assay of the epinephrine.

b Average of five determinations.

c Based on an average volume of $0.728 \pm 0.004 \mu$ l calculated from the information in columns 1 and 2 using the first five entries. 
These data demonstrated that thin layer electrochemical techniques are applicable to quantitative determinations of solutions with concentrations in the part per million range. Very little sample is needed and high precision and accuracy is possible. Further development appears warranted for applications in trace analyses.

\section{References}

[1] Lingane, J. J., Electroanalytical Chemistry, 2d edition, pp. 484-616, Interscience, New York (1958).

[2] DeFord, D. D., Anal. Chem. 28, 660 (1956).

[3] DeFord, D. D. and Bowers, R. C., Anal. Chem. 30, 613 (1958).

[4] DeFord, D. D., Anal. Chem. 32,31R (1960).

[5] Bard, A. J., Anal. Chem. 34, 57R (1962).

[6] Bard, A. J., Anal. Chem. 36, 70R (1964).

[7] Bard, A. J., Anal. Chem. 38, 88R (1966).

[8] Bard, A. J., Anal. Chem. 40,64R (1968).

[9] Bard, A. J., Anal. Chem. 42, 22R (1970).

[10 Í Davis, D. G.,Anal. Chem. 44,79R (1972).

[11] Davis, D. G., Anal. Chem. 46, 21 R (1974).

[12] Jaycox, L. B., Ph. D. Thesis, University of Massachusetts, Amherst, Mass.

[13] Jaycox, L. B., Cadwgan, G. E., and Curran, D. J., Anal. Letters 6, 1061 (1973).

[14] Reilley, C. N., Pure Appl. Chem. 18, 137 (1968).

[15] Hubbard, A. T. and Anson, F. C., The Theory and Practice of Electrochemistry With Thin Layer Cells, in Electroanalytical Chemistry, Bard, A. J., Ed., Vol. 4, p. 129, Marcel Dekker, New York (1971).

[16] Hubbard, A. T., Crit. Rev. in Anal. Chem. 3, 201 (1973).

[17] Fike, R. R., Ph. D. Thesis, University of Massachusetts, Amherst, Mass.

[18 [ Fike, R. R., J. Electrochem. Soc. 117, (2), 56C (1972).

[19] Hawley, M. D., Tatawandi, S. V., Piekanski, S., and Adams, R. N., J. Am. Chem. Soc. 89, 447 (1967). 



\title{
THE NON-FARADAIC BACKGROUND IN PULSE POLAROGRAPHY
}

\author{
Robert A. Osteryoung and Joseph H. Christie
}

\author{
Department of Chemistry \\ Colorado State University \\ Fort Collins, Colorado 80521 U.S.A.
}

The dropping mercury electrode, with its continually renewed fresh surface, offers advantages for real sample analysis. However, continuous expansion of the electrode drop requires a continuous non-faradaic current to maintain the charge density on the electrode surface. The techniques of pulse polarography attempt to minimize this non-faradaic component by differencing currents flowing before and after application of a potential step to the drop. Nevertheless, since these currents are measured neither at the same potential nor at the same time in drop life, the difference current will contain a non-faradaic contribution, which is a nonlinear function of a potential.

Extensive computer simulations of the effect of the non-faradaic background on the analytical utility of pulse polarography at trace concentrations indicate that inaccurate results may be obtained if the true baseline is not known (as is likely for a real matrix). The magnitude of the background limits the amount of analyte that may be detected and the nonlinearity of the background determines the accuracy with which the superimposed faradaic signal may be measured.

Recognizing that the double layer charging current is dependent only on the potential and the time in drop life at which the current is measured while the faradaic current depends on the history of the entire drop, we have devised three variants of pulse polarography which compensate for the non-faradaic background current. These variants of pulse polarography are: 1. Alternate drop pulse polarography. The output current is the difference in current for a drop whose potential is changed late in drop life and a drop maintained at the step-to potential throughout its life. 2. Twin electrode pulse polarography. The current output is the difference in current between two matched drops, both in the test solution, one of which is pulsed while the other is maintained at the step-to potential.

Keywords: Background correction; differential pulse polarography: dropping mercury electrode; electroanalysis: pulse polarography; trace analysis. 


\section{New Pulse Polarographic Techniques}

At high sensitivities, pulse polarography at the dropping mercury electrode is ultimately limited by the dc double layer charging current due to expansion of the drop. This limitation is most severe in the analysis of real samples for which the true blank current in the absence of the material of interest cannot be determined.

Three procedures to eliminate the problem of the dc capacity current have been devised [1].

Recognizing that the measured current, $i_{m}$, in dc polarography is the sum of the faradaic plus charging currents, we may write, assuming that the faradaic current is given by the Ilkovic equation

$$
\frac{i_{m}}{\mathrm{~km}^{2 / 3}}=\sqrt{\frac{7}{3}} \frac{n \mathrm{Ft}^{1 / 6} \mathrm{D}^{1 / 2} \mathrm{C}}{\sqrt{\pi(1+\epsilon)}}-\frac{2}{3} q^{m} \mathrm{t}^{-1 / 3}
$$

where $\epsilon=\exp \left[(n \mathrm{~F} / \mathrm{RT})\left(\mathrm{E}-\mathrm{E}_{1 / 2}\right)\right]$ and where $k$ is $0.8515 \mathrm{~cm}^{2} / \mathrm{g}^{2 / 3}, q^{m}$ is the surface charge density, $t$ is the time in drop life at which the current is measured.

For normal pulse polarography, the equivalent equation for the current is

$$
\frac{i_{m}}{\mathrm{~km}^{2 / 3}}=\frac{i_{n p}}{\mathrm{~km}^{2 / 3}}-\frac{2}{3} \frac{q^{m}\left(\mathrm{E}_{2}\right)}{(\tau+\delta)^{1 / 3}}
$$

where

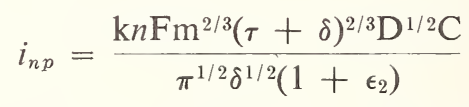

where $\tau$ is the delay time and $\delta$ is the pulse width. The potential is held at $\mathrm{E}_{1}$ during the delay time; the pulse potential is $\mathrm{E}_{2}$.

If we subtract an ordinary polarogram for $\mathrm{t}=\delta+\tau$ (eq 1) from a normal pulse polarogram for the same system, the resultant difference contains no double layer charging contribution and will be purely faradaic.

This faradaic current will be the difference between the faradaic pulse current (eq 3) and the faradaic dc polarographic current (the first term of the r.h.s. of eq 1):

$$
\frac{\Delta i_{f}}{\mathrm{~km}^{2 / 3}}=\frac{n \mathrm{~F}(\tau+\delta)^{2 / 3} \mathrm{D}^{1 / 2} \mathrm{C}}{\sqrt{\pi\left(1+\epsilon_{2}\right)}}\left[\frac{1}{\sqrt{\delta}}-\sqrt{\frac{7}{3}} \frac{1}{(\tau+\delta)^{1 / 2}}\right]
$$

The price paid for elimination of the capacitative term is that this current difference is smaller than the pulse faradaic current by the factor $[1-\sqrt{7 \delta / 3(\tau+\delta)}]$. For all reasonable values of $\delta / \tau$, this factor will be 
greater than 0.5 . (For a $4 \mathrm{~s}$ delay time, and a $40 \mathrm{~ms}$ pulse width, the factor is $0.848-\mathrm{a} 15 \%$ diminution in normal pulse wave height.)

\section{A. Alternate Drop Pulse Polarography}

This new technique derives its name from the characteristic that only every other drop is pulsed from $E_{1}$ to $E_{2}$; the intervening drop remains at potential $\mathrm{E}_{2}$ throughout its entire life. The output is the difference in current flowing at time $(\tau+\delta)$ for the pulsed and the nonpulsed drops. Since these currents are measured at the same potential and at the same time in the life of the drops, the double layer charging component is absent from the difference. In the normal pulse alternate drop mode, $\mathrm{E}_{1}$ is constant and the output current difference is given by eq (4). The current difference has the same potential dependence as the faradaic normal pulse current, but is diminished by the diminution factor $[1-\sqrt{7 \delta / 3(\tau+\delta)}]$.

In the differential alternate drop mode, the potential $\mathrm{E}_{1}$ varies along with $\mathrm{E}_{2}$ such that $\mathrm{E}_{2}-\mathrm{E}_{1}=\Delta \mathrm{E}$ is a constant. The difference in current flowing at $\tau+\delta$ for the pulsed drop and the current for the nonpulsed drop is then given by

$$
\Delta i_{f}=i_{d p}-\left[i_{p o}\left(\mathrm{E}_{2}, \tau+\delta\right)-i_{p o}\left(\mathrm{E}_{1}, \tau+\delta\right)\right]
$$

where

$$
i_{d p}=\mathrm{km}^{2 / 3} \frac{n \mathrm{~F}(\tau+\delta)^{2 / 3} \mathrm{D}^{1 / 2} \mathrm{C}}{\pi^{1 / 2} \delta^{1 / 2}}\left[\frac{\epsilon_{1}\left(1-\sigma^{2}\right)}{\left(1+\epsilon_{1}\right)\left(1+\sigma^{2} \epsilon_{1}\right)}\right]
$$

with

$$
\sigma^{2}=\exp \left(\frac{n \mathrm{~F}}{\mathrm{RT}} \Delta \mathrm{E}\right)
$$

and $i_{p_{0}}$, the polarographic current

$$
i_{p o}=\sqrt{\frac{7}{3}} \frac{\mathrm{knFm}^{2 / 3} \mathrm{t}^{1 / 6} \mathrm{D}^{1 / 2} \mathrm{C}}{\sqrt{\pi(1+\epsilon)}}
$$

Since the term in brackets in equation (5) has exactly the same potential dependence as $i_{d p}$, we can write

$$
\Delta i_{f}=i_{d p}\left[1-\sqrt{\frac{7 \delta}{3(\tau+\delta)}}\right]
$$

As before, the differential alternate drop pulse current will be of the same form as the ordinary differential pulse current, but will be 
diminished in magnitude by the diminution factor $[1-\sqrt{7 \delta / 3(\tau+\delta)}]$. The double layer charging current is again absent.

The potential wave-forms and timing for alternate drop pulse polarography are shown in figure 1.

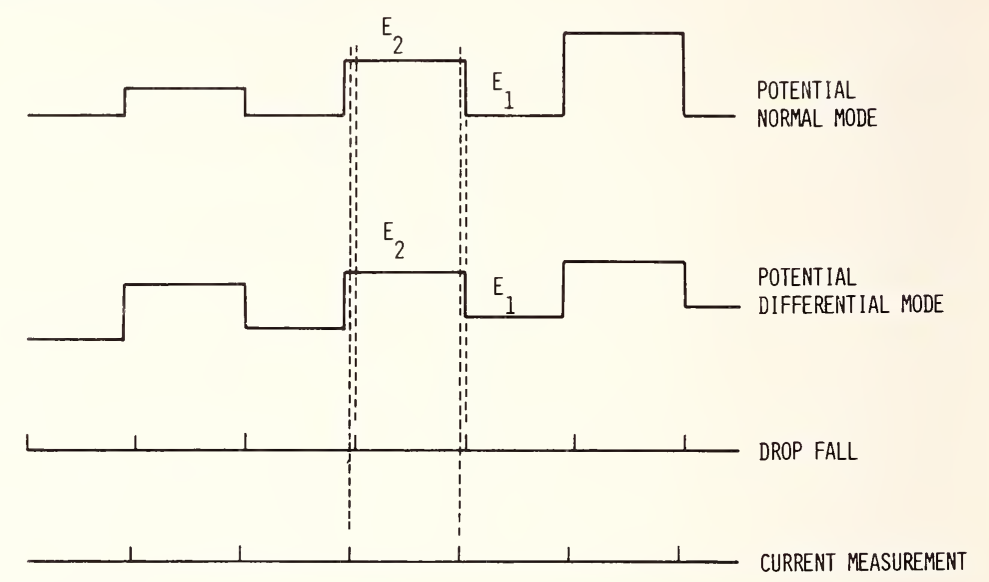

Figure 1. Schematic wave-forms and timing for alternate drop pulse polarography.

\section{B. Twin Electrode Pulse Polarography}

Rather than using one electrode and applying the pulses only to alternate drops, an equivalent result may be obtained by using two matched, synchronized dropping electrodes and applying the pulse to only one electrode. This use of twin electrodes differs from the more normal usage in which the two electrodes are in different solutions, e.g., one electrode is in a "blank" solution, and the same potential wave-form is applied to both electrodes. In twin electrode pulse polarography, both electrodes are in the same test solution, but the potential wave-forms applied to the two electrodes are different. Specifically, a pulse polarographic wave-form is applied to one electrode, while an ordinary polarographic wave-form is applied to the other. The wave-forms for differential twin electrode pulse polarography are shown in figure 2 . If the capillaries are exactly matched, the measured current difference will be the same as for alternate drop pulse polarography.

Since it is impractical to exactly match the flow rates of two different capillaries, we must consider the effect of capillary mismatch. Figure 3 shows calculated twin electrode differential pulse polarograms for 5 percent mismatch; also shown for comparison is an ordinary differential 
pulse polarogram. It should be noted that the capacitative background arising from capillary mismatch in twin electrode differential pulse polarography has the form of an integral capacitance curve, rather than a differential capacitance curve as in ordinary differential pulse polarography. This combination of a peaked faradaic response and a diminished and flattened baseline is an attractive feature of the twin electrode technique.

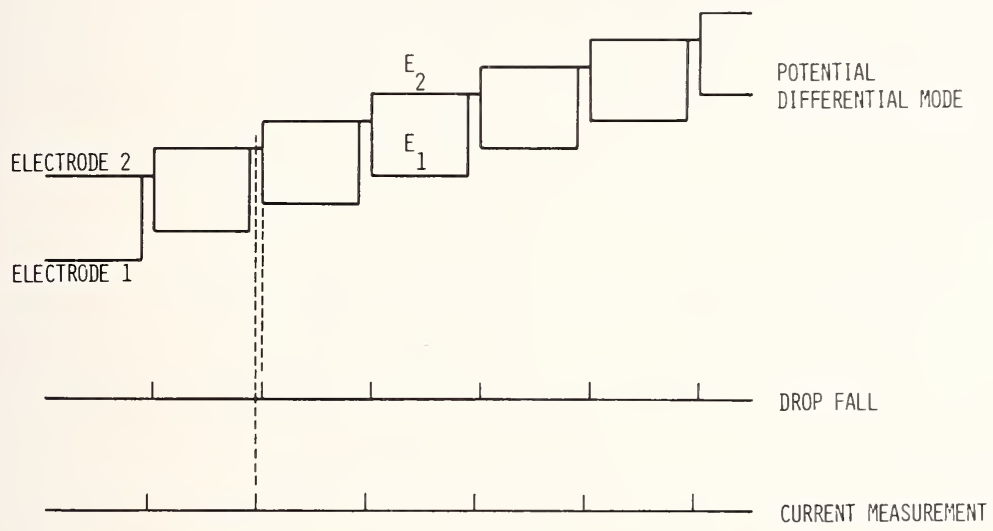

Figure 2. Schematic wave-form and time for twin electrode differential pulse polarography.

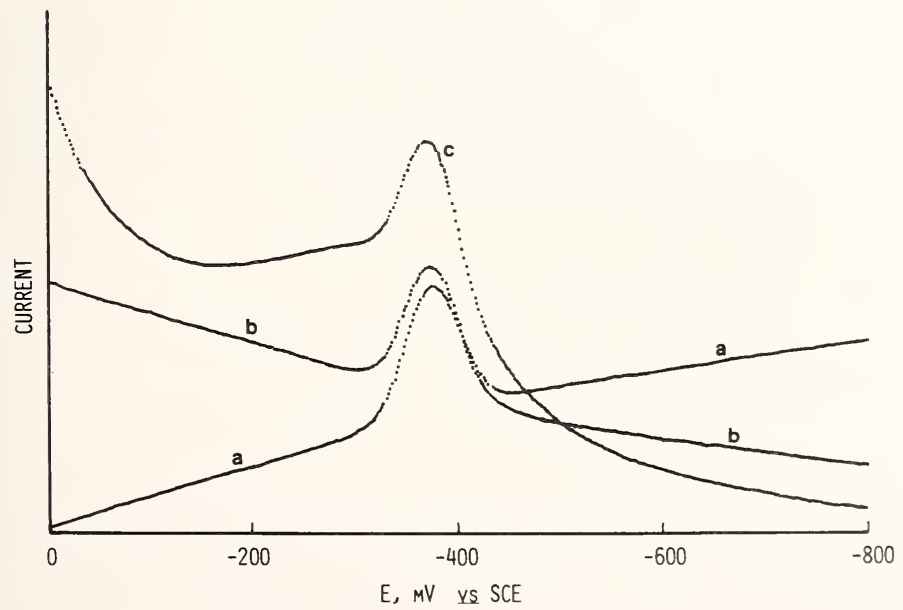

Figure 3. Simulated twin electrode differential pulse polarograms for $\mathrm{Pb}(\mathrm{II})$ in $0.1 \mathrm{~F} \mathrm{KCl}$. Curves $\mathrm{a}$ and $\mathrm{b}$ are for \pm 5 percent mismatch of flow rate. Curve $\mathrm{c}$ is a simulated ordinary differential pulse polarogram on the same scale. $\mathrm{Pb}(\mathrm{II})=0.1 \mu \mathrm{F}: \delta=10 \mathrm{~ms} ;(\tau+\delta)=2.0$ $\mathrm{s} ; \Delta \mathrm{E}=50 \mathrm{mV}$. 


\section{Constant Potential Pulse Polarography}

The simple recognition that the non-faradaic current depends only on the potential and the time in drop life while the faradaic current depends on the history of the drop leads to the third and simplest new variant of pulse polarography. If all measurements are made at the same time in drop life and at the same potential, the capacitative contribution will be constant. A new pulse polarographic wave-form which allows such measurement is shown in figure 4 . The name-constant potential pulse polarography-derives from the fact that all pulses are to the same potential $\mathrm{E}_{2}$ while the potential $\mathrm{E}_{1}$ is variable. This wave-form might be considered the inverse of the ordinary normal pulse polarographic waveform.

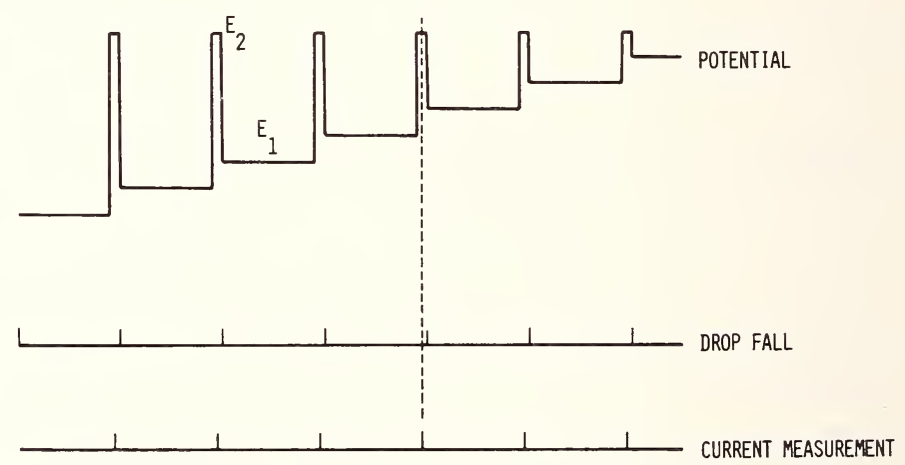

Figure 4. Schematic wave-form and timing for constant potential pulse polarography.

This new variant will give a wave-shaped output superimposed on a constant capacitative component. The faradaic current for any values of $E_{1}$ and $E_{2}$ will be given by

$$
\begin{aligned}
\Delta i_{f}=\frac{n \mathrm{Fkm}^{2 / 3}(\tau+\delta)^{2 / 3} \mathrm{D}^{1 / 2} \mathrm{C}}{\sqrt{\pi}}\left\{\frac{1}{\sqrt{\delta}}\left(\frac{1}{1+\epsilon_{2}}-\frac{1}{1+\epsilon_{1}}\right)\right. & \\
& \left.+\sqrt{\frac{7}{3}} \cdot \frac{1}{\sqrt{\tau+\delta}} \cdot \frac{1}{1+\epsilon_{1}}\right\}
\end{aligned}
$$

We can assess the height of the wave as follows: Let $E_{2}$ be very cathodic of the half-wave potential so that $1 /\left(1+\epsilon_{2}\right)=1$. If $E_{1}$ is very anodic of the half-wave potential, the current will be the normal pulse polarographic current (let $1 /\left(1+\epsilon_{1}\right)=0$ in eq 9 ). If $E_{1}$ is very cathodic of the half-wave potential, the drop is on the diffusion plateau during its entire life and the 
current is simply the polarographic current (let $1 /\left(1+\epsilon_{1}\right)=1 /\left(1+\epsilon_{2}\right)=1$ in eq 9). The wave height is therefore the difference:

$$
\text { wave height }=\frac{n \mathrm{Fkm}^{2 / 3}(\tau+\delta)^{2 / 3} \mathrm{D}^{1 / 2} \mathrm{C}}{\sqrt{\pi \delta}}\left[1-\sqrt{\frac{7 \delta}{3(\tau+\delta)}}\right]
$$

Similar arguments will give the same value for the wave height independent of the value of $E_{2}$ relative to the half-wave potential.

\section{Experimental Results}

\section{A. Alternate Drop Pulse Polarography}

In figure 5 are shown normal mode ordinary and alternate drop pulse polarograms for $\sim 150 \mathrm{ppb} \mathrm{Pb}(\mathrm{II})$ in $1 \mathrm{~F} \mathrm{HCl}$. We see immediately that " e baseline for the alternate drop pulse polarogram is not flat, as theory $w$ suld lead us to expect, but rather that the baseline has a definite upward viope. We attribute this sloping baseline to the phenomenon which Barker called "capillary response" and which he has discussed in some detail [2]. This capillary response results from the slow relaxation of the potential of the solution layer between the mercury thread and the capillary wall and in the cracks in the end of the capillary. The current due to this capillary response decays as $\mathrm{t}^{-x}$ where $\mathrm{x}>1 / 2$ and increases with pulse amplitude (with a power dependence somewhat greater than unity). This capillary response current thus results in an upward sloping baseline and leads to an erroneous pulse width dependence of the pulse current. Some expedients have been suggested for capillary treatment $[3,4]$ which might alleviate this problem. Some of these expedients are under investigation in our laboratory [5].

For a constant pulse amplitude, the capillary response might be expected to be constant and therefore should have less effect on differential pulse polarograms. In figure 6 are shown ordinary and alternate drop differential pulse polarograms for $\sim 60 \mathrm{ppb} \mathrm{Pb}(\mathrm{II})$ in $1 \mathrm{~F} \mathrm{HCl}$. The short drop time of $0.5 \mathrm{~s}$ was chosen as a worst case. The superiority of the alternate drop variant is seen most clearly in this figure. The baseline is quite flat, in marked contrast to the ordinary differential pulse curve; the noise levels are about the same. The diminution in the peak height seems to be about that predicted by theory although it is impossible to determine the true height of the peak in the upper curve. 


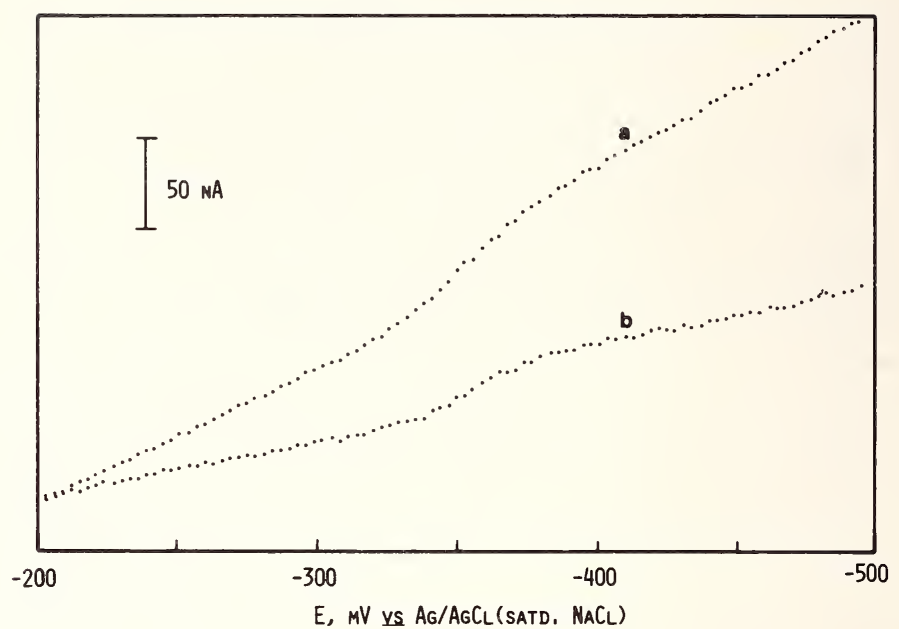

Figure 5. Normal pulse polarograms for $\sim 150 \mathrm{ppb} \mathrm{Pb}(\mathrm{II})$ in $1 \mathrm{~F} \mathrm{HCl}$. Pulse width $=10 \mathrm{~ms}$; drop time $=1$ s. (a) Ordinary; (b) alternate drop.

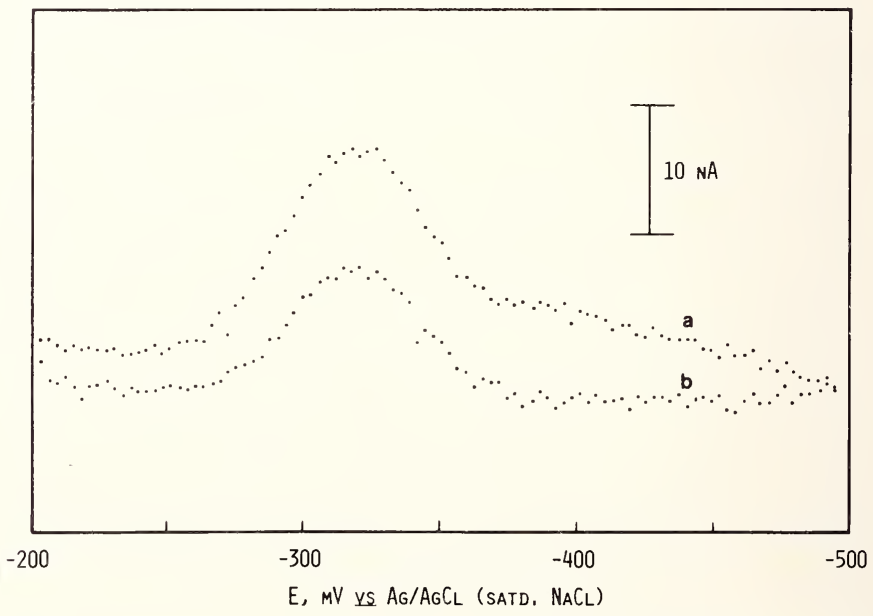

Figure 6. Differential pulse polarograms for $\sim 60 \mathrm{ppb} \mathrm{Pb}(\mathrm{II})$ in $1 \mathrm{~F} \mathrm{HCl}$. Pulse width $=10$ $\mathrm{ms}$; drop time $=0.5 \mathrm{~s} ; \Delta \mathrm{E}=50 \mathrm{mV}$. (a) Ordinary; (b) alternate drop. 


\section{B. Constant Potential Pulse Polarography}

Figure 7 shows constant potential pulse polarograms for $\mathrm{Pb}(\mathrm{II})$ in $1 \mathrm{~F}$ $\mathrm{HCl}$ for $\mathrm{E}_{2}$ values after the wave, in the wave, and before the wave; also shown for comparison is an ordinary normal pulse polarogram for the same system on the same scale. In these curves we see the capillary response effect quite clearly, but we also observe a marked decrease in the slope of the baseline due to the elimination of the effect of drop growth.

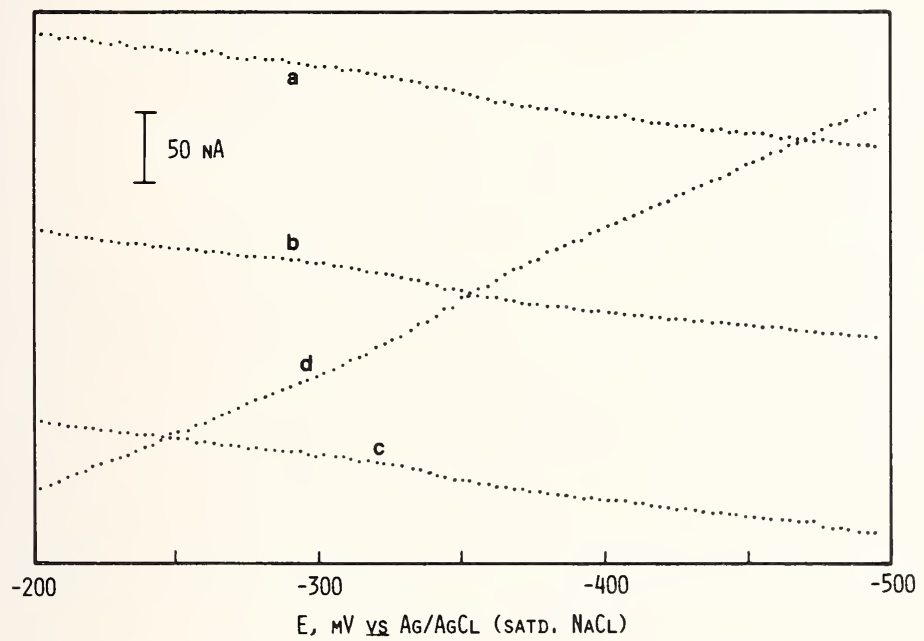

Figure 7. Constant potential pulse polarograms for $\sim 100 \mathrm{ppb} \mathrm{Pb}(\mathrm{II})$ in $1 \mathrm{~F} \mathrm{HCl}$. Pulse width $=10 \mathrm{~ms}$; drop time $=0.5 \mathrm{~s}$. (a) $\mathrm{E}_{2}=-500$; (b) $\mathrm{E}_{2}=-350$; (c) $\mathrm{E}_{2}=-200$; (d) ordinary normal mode.

\section{Acknowledgement}

This work was supported by the National Science Foundation under Grant No. GP 31491 X.

\section{References}

[1] Christie, J. H., Innovations in Pulse Polarography, Ph. D. Thesis, Colorado State University, Fort Collins (1974).

[2] Barker, G. C. and Gardner, A. W., Pulse Polarography, A.E.R.E. C/R 2297. Atomic Energy Research Establishment, Harwell, Berkshire (1958).

[3] Cooke, W. D., Kelley, M. T., and Fisher, D. J., Anal. Chem. 33, 1209 (1961).

[4] Smoler, I., Coll. Czech. Chem. Communs. 19,238 (1954).

[5] Osteryoung, R. A. and Jackson, L. L., unpublished work, Colorado State University, Fort Collins (1974). 



\title{
IMPROVED ANODIC STRIPPING VOLTAMMETRY FOR ACCURATE TRACE ANALYSIS
}

\author{
Magda Ariel and Joseph Wang \\ Department of Chemistry \\ Technion-Israel Institute of Technology \\ Haifa, Israel
}

The unfavorable "signal to noise" ratio obtained during measurements carried out in very dilute metal ion solutions sets the lower concentration limit in anodic stripping voltammetry (ASV) determinations. The noise, deriving largely from the background current during the stripping step, is composed of a capacity current and a faradaic current contribution. DASV (differential ASV) and ASWC (anodic stripping with collection), carried out with in situ mercury film formation at rotating glassy carbon disc or ring-disc electrodes respectively, efficiently reduce residual current interference and significantly improve the reliability of ASV methods, as applied to real sample analyses.

DASV is carried out at identical twin working electrodes balanced against each other and immersed in the sample solution, to which $\mathrm{Hg}^{+2}$ ions have been added. Preelectrolysis, carried out at both electrodes at the same potential but for different deposition periods, is followed by a stripping step during which the oxidation currents are substracted.

In ASWC, a rotating ring disc electrode is employed; conventional ASV at the disc is supplemented, during the stripping step, by simultaneous redeposition at the ring, kept at a suitably constant cathodic potential throughout the stripping scan, allowing unequivocal quantitative evaluation of collection current peaks. Very rapid scan rates may be employed during the disc stripping steps, resulting in improved sensitivity without any loss in accuracy.

The method, combined with thin film ASV, with the mercury film codeposited in situ with the trace metals present on a glassy carbon ring disc electrode, yields excellent results in the analysis of trace metal solutions in the ppb (and lower) range.

Keywords: Anodic stripping voltammetry; background current compensation; differential anodic stripping voltammetry; electrochemical analysis; twin electrode balancing. 


\section{Introduction}

The everwidening significance attached to the presence of minute concentrations of metal ions in a variety of complex materials, as related to environmental, clinical or technological considerations, has led to continued efforts aimed at enhancing the sensitivity of methods used for their analytical determination. Ideally this should be achieved without unduly sacrificing either the precision, accuracy or ease associated with routine operation.

The extension of unmodified anodic stripping voltammetry (ASV) to very low metal ion concentration ranges is largely limited by the unfavourable signal to noise ratio, arising from the compounded contributions of charging current $\left(i_{c}\right)$ and faradaic currents $\left(i_{f, r}\right)$ due to electrolysis of the medium or of other electroactive species present (residual or background current, $\left.i_{r}=i_{c}+i_{f, r}\right)[1,2]$. Methods suggested for decreasing this background current (e.g., [1,3]) have, in general, been only partly effective.

Differential measurement, well established for the compensation of background signals in a variety of techniques, has also been early proposed for voltammetry (e.g., [4,5]); problems incurred in matching the two working electrodes involved limited its analytical exploitation.

Differential anodic stripping voltammetry (DASV), exploiting identical twin working electrodes immersed in the cell containing the sample solution but subjected to different deposition times was proposed by Kemula [6] and used, among others, for the determination of zinc ions in sea water [7] and the overcoming of dissolved oxygen interference [8].

In this work, experimental parameters involved in DASV were studied; their appropriate control turns DASV into a remarkably effective, interference free electroanalytical tool.

Two approaches, both exploiting glassy carbon based, twin thin film mercury (TMF) electrodes were explored: DASV $(t)$, using different deposition times $(t)$ and DASV $(\omega)$, hitherto unreported, using different rates of electrode rotation $(\omega)$ during the deposition step. In both, all residual currents arising from factors other than deposition time $(\mathrm{DASV}(t))$ or rotation rate (DASV $(\omega))$ are compensated by recording the difference of the currents obtained during the deposition step. Since the charging current is completely compensated, very rapid potential scans may be used, enhancing sensitivity and shortening the duration of the determination.

In situ mercury film deposition [9], ensuring the continuous renewal of the electrode surface and permitting the use of extremely thin films, 
further enhances sensitivity and reproducibility. Solutions of $10^{-9} M$ may be analyzed using short ( 1 to $3 \mathrm{~min}$ ) deposition periods; linear standard addition curves are obtained, with a 10 percent relative standard deviation.

In contrast to unmodified ASV, undistorted, easily evaluable stripping current peaks are recorded even in the vicinity of the limits set by the useful potential range of the system; in addition, oxidation or reduction currents due to the presence, in the sample. of significant concentrations of nonamalgam forming electroactive components, which would have swamped the sought for stripping current, are effectively compensated in DASV (fig. 1). These advantages, together with the extreme operational simplicity of the technique, make DASV an attractive analytical tool.

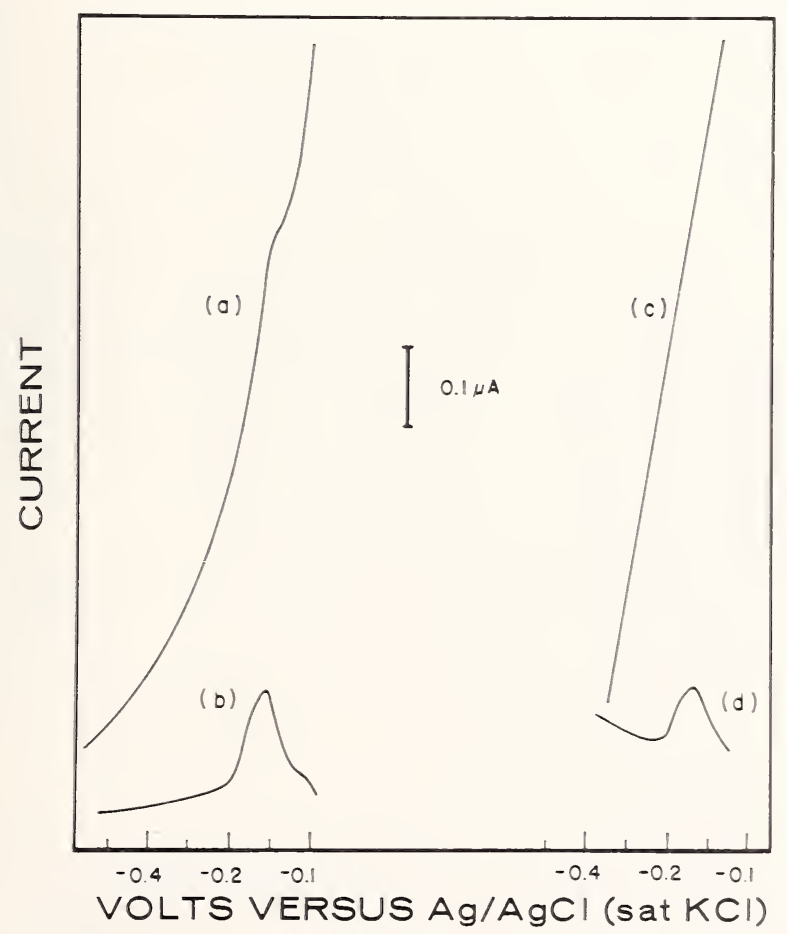

Figure 1. DASV - background current compensation. Curves (a) and (b), ASV and DASV $(t)$ voltammograms recorded in deaerated $4.9 \times 10^{-8} M$ cupric ion solution. in acetic acid/ammonium acetate $(0.02 \mathrm{M} / 0.02 \mathrm{M})$ supporting electrolyte buffer: curves (c) and (d). as for (a) and (b) respectively but without deaeration. Deposition at $-1.0 \mathrm{~V}$ for 1 minute: potential scan rate $6 \mathrm{~V} \mathrm{~min}^{-1}$. 


\section{Experimental}

\section{A. Apparatus}

A $300 \mathrm{ml}$ pyrex glass cell and a 5 hole Teflon cover were employed, with twin glassy carbon (GC 305 Tokai Electrode Mfg. Co., Ltd., Tokyo) electrodes [9] symmetrically flanking an $\mathrm{Ag} ; \mathrm{AgCl}$ ( $\mathrm{KCl}$ (sat) reference electrode (to which all potentials reported in this work are referred) (fig. $2)$ ). In $\operatorname{DASV}(\omega)$ the mutual effect of electrode rotation on each other's diffusion layer was minimized by placing a glass partition between them.

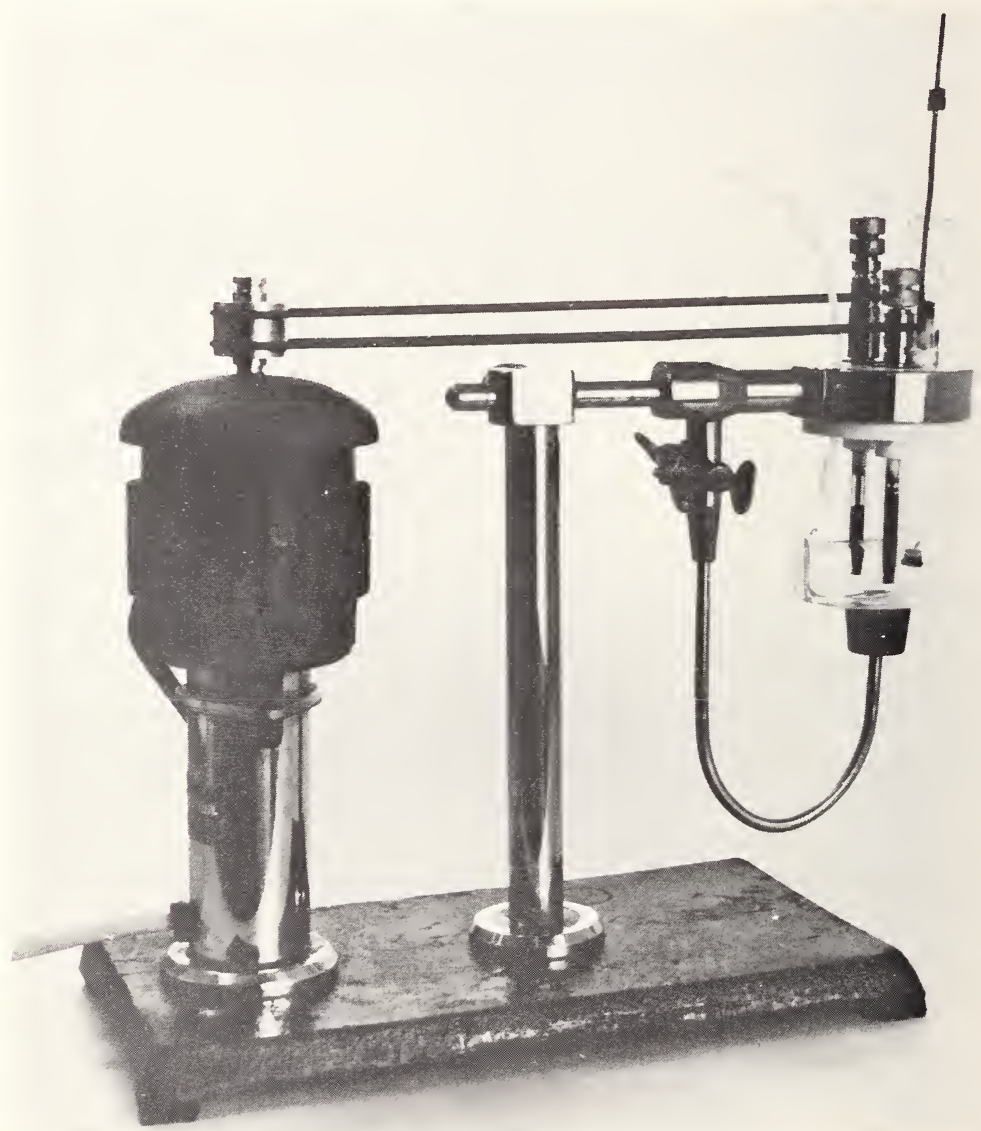

Figure 2. Synchronous motor and electrochemical cell. 
A synchronous motor, capable of independently rotating each electrode at 0,1410 or $2675 \mathrm{rpm}$ was used. A locally built potential alternator [10] imposed a chosen controlled potential on the "background" electrode during the deposition step in DASV $(t)$. A PAR Model 174 Polarographic Analyzer, equipped with the 174/51 Accessory, for differential work [11] and a recorder (either a Leeds and Northrup X/L 680 or a Servogor RE 511) completed the instrumental setup. The cell and stock solutions were protected from airborne contaminants by placement in a laminar airflow cabinet.

\section{B. Reagents And Solutions}

Suprapur (Merck) nitric and acetic acid, ammonium hydroxide and triple distilled water [12] were used. Metal ion stock solutions, $10^{-3} M$, prepared by dissolving the metal in nitric acid and diluting as required, were stored in polyethylene containers. Solutions $10^{-5} \mathrm{M}$ were freshly prepared daily. A $5.7 \times 10^{-4} M$ mercuric ion solution was used for the in situ mercury film deposition. Stock buffer solutions were: acetic acid/ammonium acetate $(0.4 M / 0.4 M)(\mathrm{pH}-4.8)$ and ammonium hydroxide/ammonium chloride $(0.5 \mathrm{M} / 0.5 \mathrm{M})$ ( $\mathrm{pH}-8)$ solutions.

\section{Procedure}

The sample, $5 \mathrm{ml}$ of the mercuric ion solution and $4 \mathrm{ml}$ buffer stock solution (total approximate volume, $100 \mathrm{ml}$ ) are introduced into the cell and purged (when required) with purified nitrogen. During deaeration the working electrodes are kept at $+0.4 \mathrm{~V}$ and rotated. Balancing is next effected by carrying out simultaneously on both electrodes a deposition step, using identical deposition potential, period and rate of electrode rotation. This is followed by concerted rotation stoppage, a 15 -second rest period and a linear potential scan, to $-0.05 \mathrm{~V}$, during which the difference of the currents passing through the electrode is recorded. Perfect balance should result in a straight horizontal line: imperfect balance may be corrected using the electrode and potential compensators [11]. The success of this operation is checked by repeating the above deposition-stripping cycle and inspecting the resulting voltammogram. (Further problems encountered in balancing are discussed below.)

The procedure, up to this point is common to both DASV $(t)$ and $\operatorname{DASV}(\omega)$; from now on it differs, according to the method chosen.

In DASV $(t)$ one of the working electrodes is kept at a suitably cathodic 
potential, during deposition, while the other, "background" electrode, is kept by the potential alternator, at $-0.05 \mathrm{~V}$; both are rotated at the same rate. At the end of the deposition period, rotation is stopped, the deposition potential imposed on both electrodes during a 15 -second rest period, followed by a rapid, anodic potential scan to $-0.05 \mathrm{~V}$ and accompanied by the recording of the differential voltammogram. Deposition periods and rates of potential scan, adjusted according to sample concentration, range from 5 minutes for a $10^{-9} M$ to 0.5 minutes for a $10^{-7} M$ sample and up to $12 \mathrm{~V} \cdot \mathrm{min}^{-1}$.

In DASV $(\omega)$ the balanced background current is recorded after deposition on both electrodes, both rotated at the higher rate of the two rates to be used in the subsequent differential step, at the same deposition potential. Maximum sensitivity is achieved with one of the working electrodes rotated at the highest rate available $(\omega=2675 \mathrm{rpm})$ and the "background" electrode at rest $(\omega=0 \mathrm{rpm})$ (the deposition current at a RDE is proportional to $\left.\omega^{1 / 2}[13]\right)$.

After deposition, electrode rotation is stopped; a 15-second rest period is followed by the stripping step and the differential current recorded as in $\operatorname{DASV}(t)$.

Between determinations, the electrodes are kept at $-0.05 \mathrm{~V}$, to remove possible contaminants.

The required standard additions are introduced through an opening in the cell cover, using microliter pipets $(0.2 \mathrm{ml}$ maximum volume).

At the end of a day's work the mercury film which has accumulated is wiped off the electrode face with a soft tissue wet with $2 M$ nitric acid and the electrode is rinsed with distilled water.

\section{Results and Discussion}

\section{A. Electrode Balance-Evaluation of Results}

The linear correlation obtained between the peak stripping current (measured by taking the distance between the differential voltammogram peak and the background current at the peak potential) and the concentration of the metal ion determined (fig. 3) justifies the use of the method of standard additions for quantitative evaluation. A linear correlation is also obtained between peak areas (charge) and ion concentration; this proved useful whenever split or distorted peaks had to be evaluated [10].

Electrode balancing, a required operation before the differential deposi- 


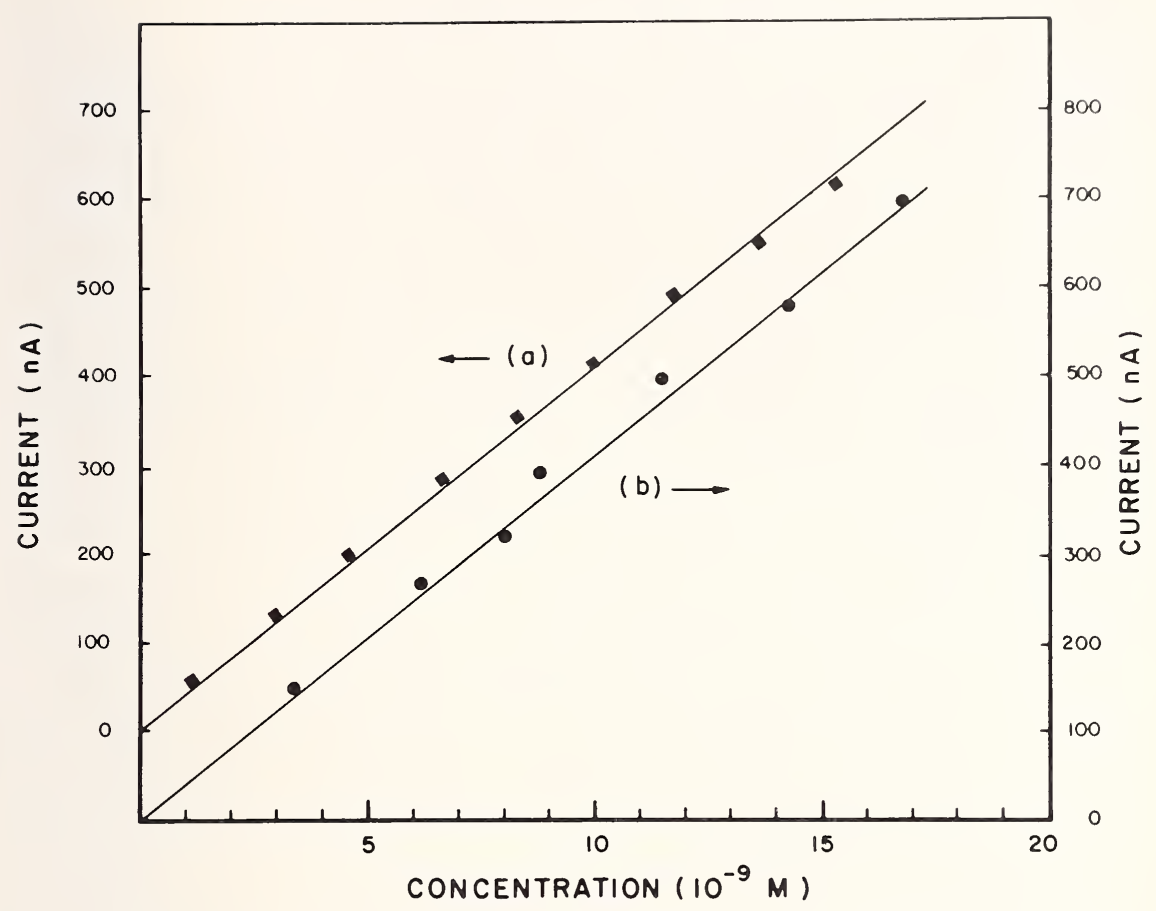

Figure 3. Cadmium (DASV $(t))$ and lead $(\operatorname{DASV}(\omega))$ standard addition curves. (a) cadmium ion in ammonium hydroxide/ammonium chloride $(0.02 \mathrm{M} / 0.02 \mathrm{M})$ and (b) lead ion in acetic acid/ammonium acetate $(0.02 \mathrm{M} / 0.02 \mathrm{M})$ supporting electrolyte buffer. Deposition (a and b) at $-1.1 \mathrm{~V}$ for 3 minutes. Rates of electrode rotation in DASV $(\omega)(b),(2765-0) \mathrm{rpm}$.

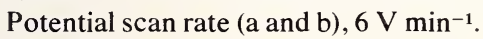

tion step, is often tedious and especially so in low concentration ranges. Perfect compensation of the charging current is not accompanied by the simultaneously complete compensation of the faradaic current of the deposited metal ion (e.g., fig. 4); the residual, unbalanced faradaic current may be up to 20 percent of the peak current recorded in the subsequent differential step. This effect, not yet completely understood, may be caused by differences in morphology of the twin TMF electrode surfaces, resulting from experimental parameters $[3,14]$. Since the charging current is determined by the real and the faradaic current largely by the geometric area of the electrode, their complete compensation cannot be expected to occur at the same compensator setting.

In the case of DASV employing hanging mercury drop electrodes balancing is easy [10]; this further supports the "morphology" assumption (obviously, in this case, the real and geometrical areas are the same). 
Fortunately, the residual (unbalanced) faradaic background current and the differential peak current are additive (fig. 4). Linear calibration curves, extending over a wide concentration range, are obtained whenever the differential peak current, corrected for this residual current, is plotted against metal ion sample concentration. Considerable time and effort may therefore be saved by limiting the balancing step to complete compensation of the charging current and correcting the subsequently obtained differential stripping peak current by adding (or subtracting, as the case may be) the residual, unbalanced faradaic background current. This approach requires the same deposition time to be used in the final balancing and the differential stripping step.

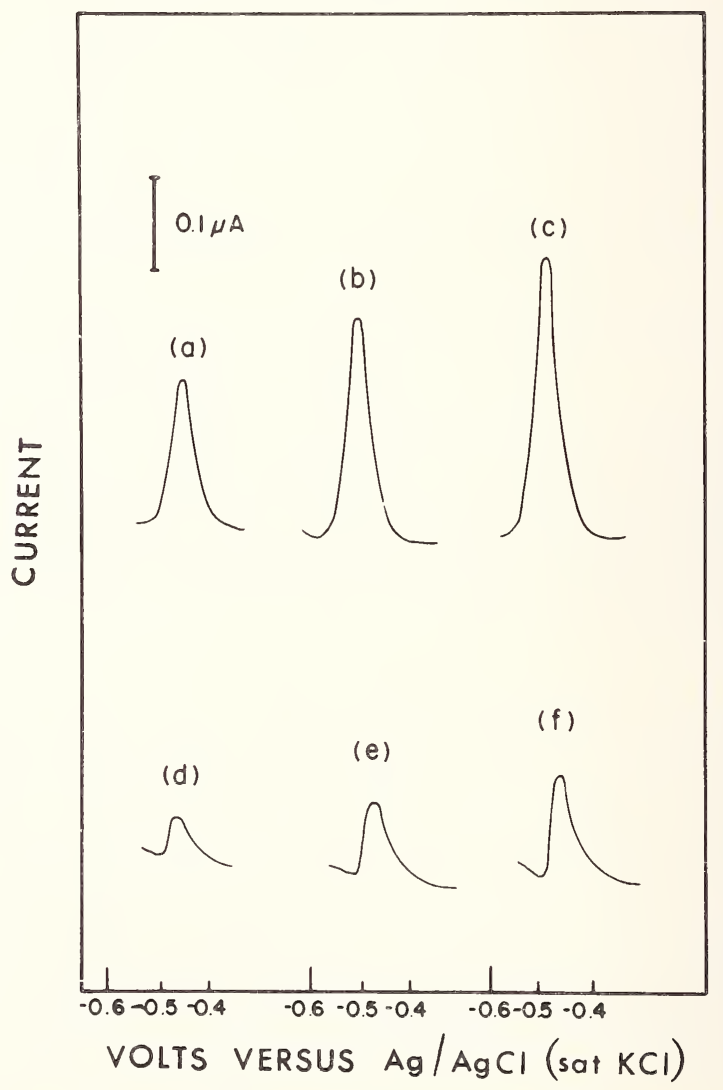

Figure 4. Differential stripping current voltammograms and corresponding residual (unbalanced) faradaic background currents. (a) and (d), (b) and (e), (c) and (f), $2.7 \times 10^{-9} \mathrm{M}$, $4.0 \times 10^{-9} M$ and $5.3 \times 10^{-9} M$, lead ion in acetic acid/ammonium acetate $(0.02 M / 0.02 M)$ supporting electrolyte buffer. Deposition at $-1.0 \mathrm{~V}$ (d), (e) and (f) 4 minutes, both working electrodes; (a), (b) and (c) 4 minutes, one electrode only, with the "background" electrode at $-0.05 \mathrm{~V}$. Potential scan rate $6 \mathrm{~V} \mathrm{~min}^{-1}$. 


\section{B. Advantages of the Method}

Unmodified ASV and DASV (both at TMF electrodes) were applied to systems in which the first does not seem fully satisfactory; the results obtained were unequivocally in favor of DASV.

\section{Shortened Analysis-Increased Sensitivity}

ASV sensitivity at TMF electrodes as compared to ASV at the HMDE is enhanced by the favorable surface to volume ratio. Further attempts to increase sensitivity by using rapid potential scans are defeated by the considerable charging current $\left(i_{c}\right)$ obtained (e.g., fig. 5, right side; $i_{c}=C_{d} \mathrm{Av}$; A-electrode surface area, v-rate of potential scan). The nonlinear relationship between the differential capacity, $C_{d}$, and electrode potential, presents difficulties in electronic charging current compensation.

In contrast in DASV the complete compensation of the charging current permits rapid scans to be used in the stripping step; in view of the significantly increased sensitivity (the stripping peak current at a TMF electrode is proportional to scan rate [15]), deposition periods may be shortened.

Figures 6 and 7, comparing ASV and DASV voltammograms obtained with a solution $\left(10^{-9} M\right)$ of a number of metal ions, under identical experimental conditions, illustrate this fact.

The shorter deposition periods employed in DASV (i.e., the lower metal concentrations attained in the mercury film amalgam) lessen the danger of intermetallic compound formation; time dependent changes in film morphology (e.g., slow adsorption) are also minimized.

Calibration curves for cadmium and lead ions, obtained with 3-minute deposition periods are shown in figure 3. Relative standard deviations for $3 \times 10^{-7} M-2 \times 10^{-9} M$ solutions were 2 to 7 percent respectively. Response rate of available recorders, instrument noise and reagent purity set the detection limit of the method. 


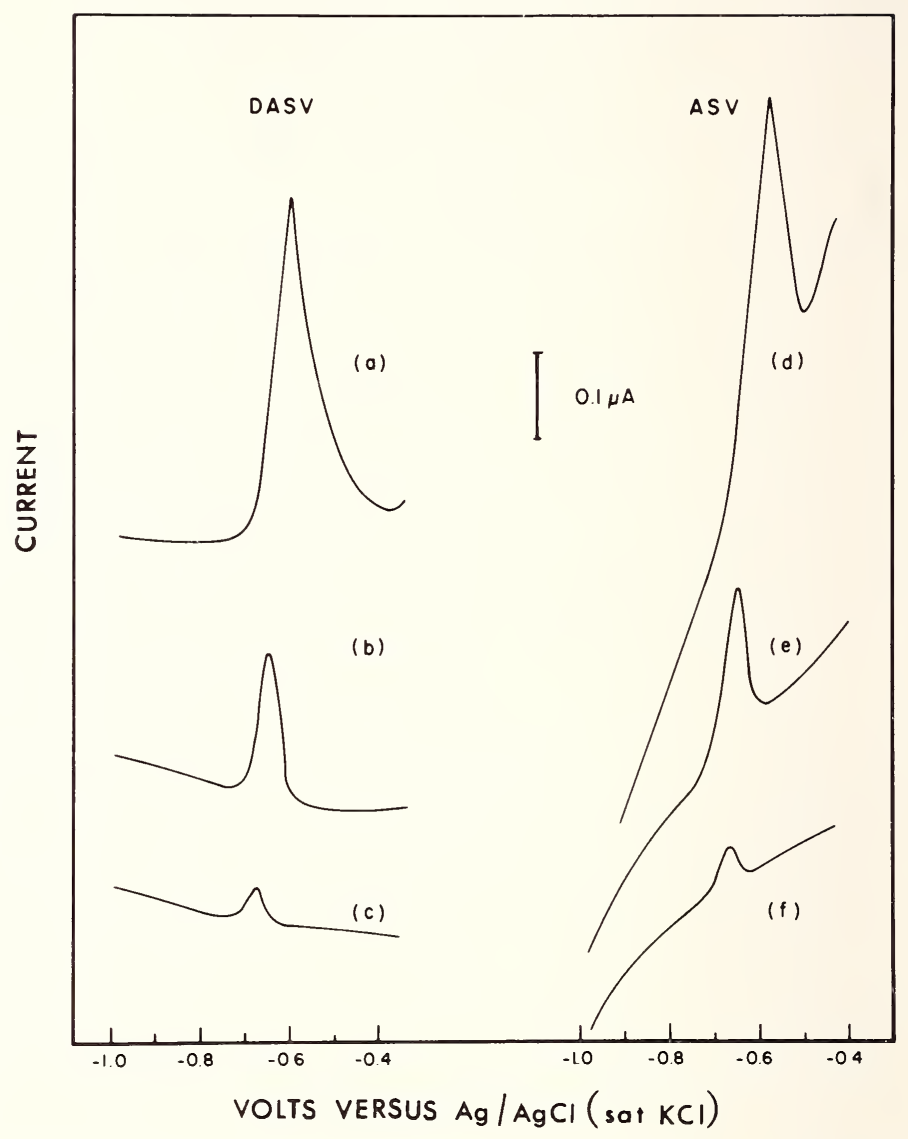

Figure 5. Influence of potential scan rate. (a), (b) and (c) DASV(t) voltammograms recorded in deaerated $2.3 \times 10^{-8} \mathrm{M}$ cadmium ion solution, in acetic acid/ammonium acetate $(0.02 \mathrm{M} / 0.02 \mathrm{M})$ supporting electrolyte buffer, at different potential scan rates, (a) $12 \mathrm{~V} \mathrm{~min}$ min $^{-1}$, (b) $3 \mathrm{~V} \mathrm{~min}-1$ and (c) $0.6 \mathrm{~V} \mathrm{~min}-1$; (d), (e) and (f) ASV voltammograms recorded under conditions identical to (a), (b) and (c), respectively. Deposition at $-1.1 \mathrm{~V}$ for 1 minute. 


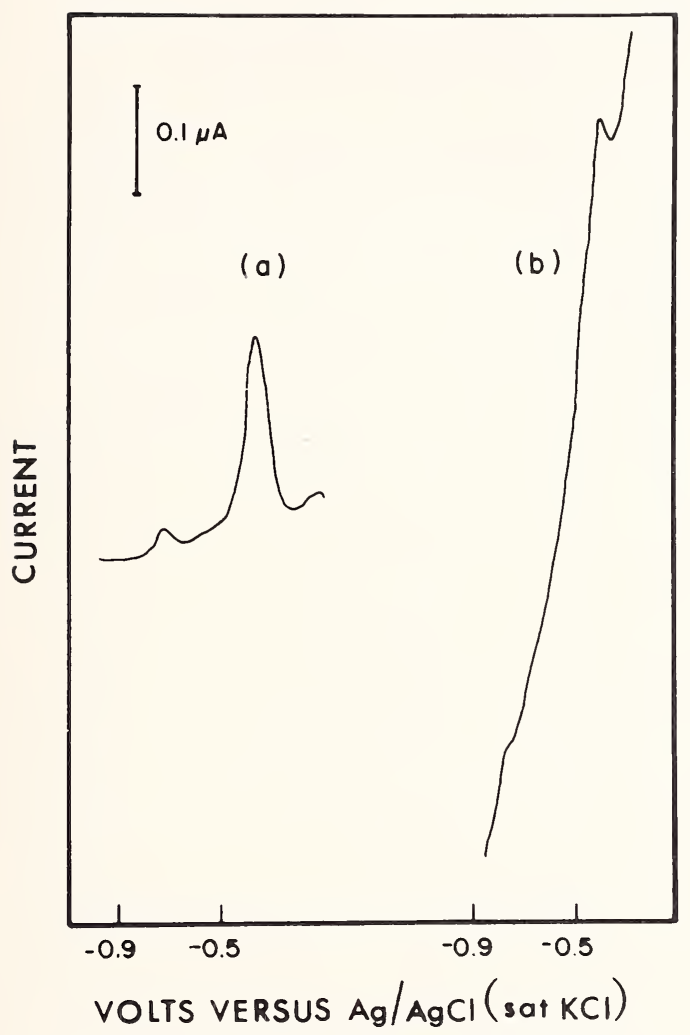

Figure 6. Comparison of DASV( $(t)$ and ASV. (a) and (b) DASV(t) and ASV voltammograms recorded in deareated $2.5 \times 10^{-9} \mathrm{M}$ cupric ion solution, in ammonium hydroxide/ammonium chloride $(0.02 M / 0.02 M)$ supporting electrolyte buffer. Deposition at $1.1 \mathrm{~V}$ for 1 minute. Potential scan rate $12 \mathrm{~V} \mathrm{~min}-1$. The smaller peak at $-0.7 \mathrm{~V}$, is due to cadmium ions present in the blank solution. 


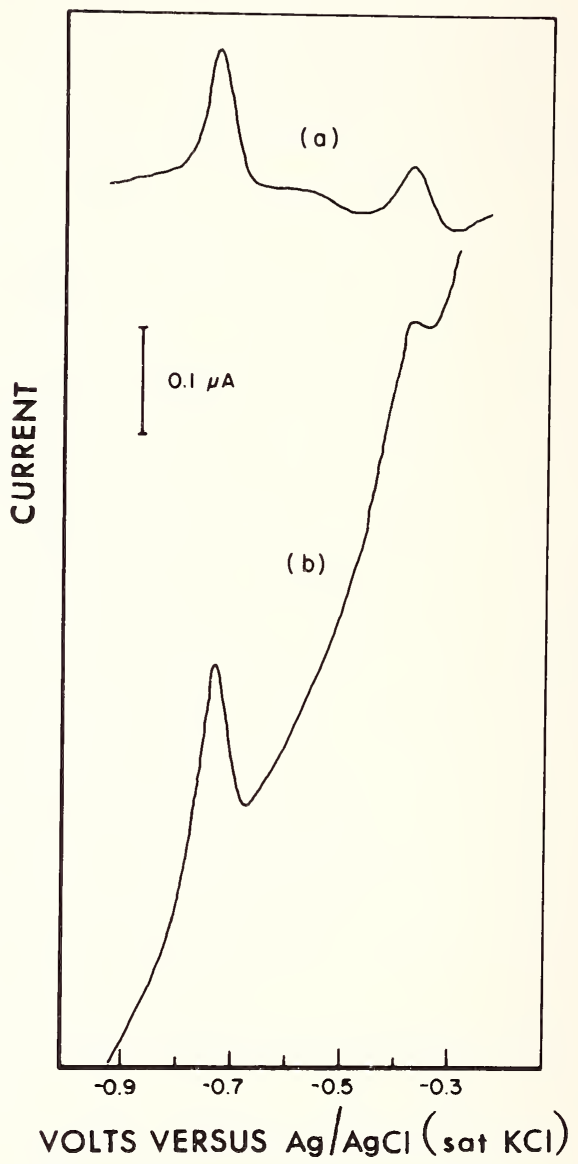

Figure 7. Comparison of DASV( $\omega)$ and ASV. (a) and (b) DASV( $(\omega)$ and ASV voltammograms recorded in deaerated $2.3 \times 10^{-9} \mathrm{M}$ cadmium ion solution, in ammonium hydroxide/ammonium chloride $(0.02 \mathrm{M} / 0.02 \mathrm{M})$ supporting electrolyte buffer. Deposition at $1.0 \mathrm{~V}$ for 5 minutes; rate of electrode rotation, ASV $2765 \mathrm{rpm}, \operatorname{DASV}(\omega)(2765-0) \mathrm{rpm}$. Potential scan rate $12 \mathrm{~V} \mathrm{~min}{ }^{-1}$. The smaller peak, at $\sim-0.4 \mathrm{~V}$, is due to cupric ions present in the blank solution. 


\section{BACKGROUND CURRENT INTERFERENCE}

Electroactive interferents present in the sample solution and causing background currents which distort or even swamp ASV stripping current peaks may be subdivided as follows:

\section{Medium Decomposition}

The ASV determination of a number of metal ions is hampered by the overlap of their stripping current peaks and currents resulting from electrolysis of the medium. Methods proposed to overcome this problem e.g., [16] usually complicate the determination by introducing additional steps. In DASV, medium electrolysis currents (independent of the deposition period) are fully compensated during the balancing step (fig. 8); metal ions such as copper (near the mercury oxidation limit) and zinc (near the hydrogen evolution limit) are easily determined.

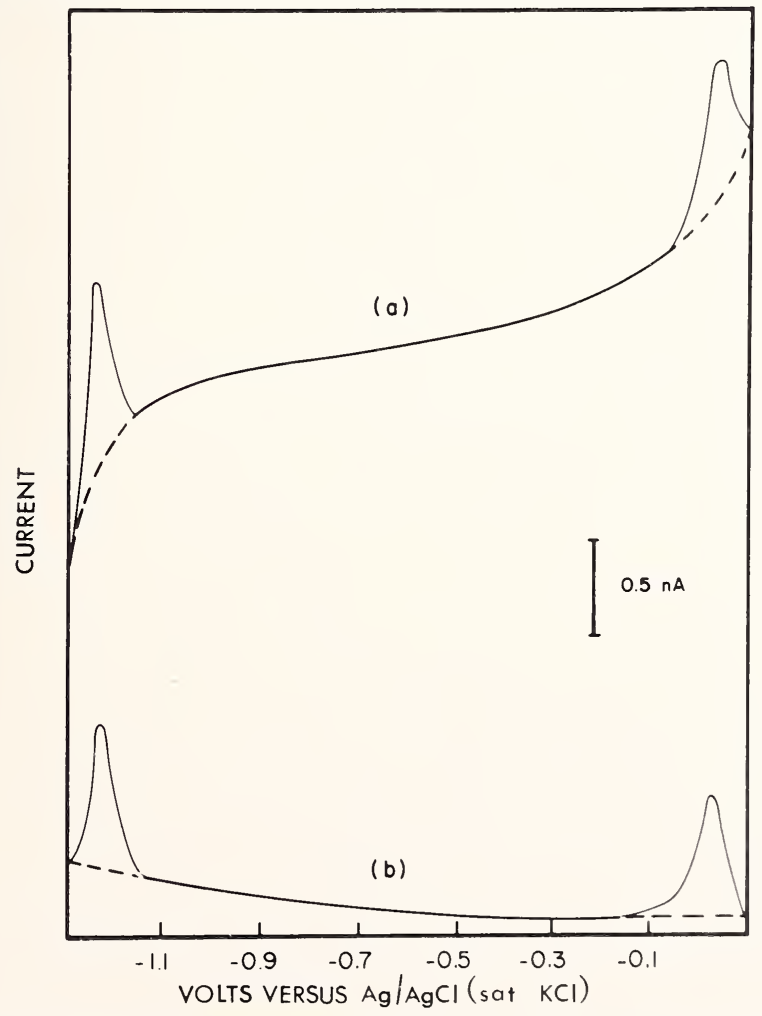

Figure 8. DASV background current compensation. (a) and (b) ASV and DASV voltammograms, respectively, showing improved peak shapes obtained with the latter for zinc $(\mathrm{Ep} \sim-1.2 \mathrm{~V})$ and copper $(\mathrm{Ep} \sim \mathrm{OV})$. 


\section{Electroactive Macro Components}

a. Dissolved Oxygen. This is an interferent prevalent in many electroanalytical determinations; its removal, aside from being time consuming, is sometimes impossible or inadvisable (e.g., in situ determinations in large sample reservoirs or in the case of environmental water samples, where deaeration also removes carbon dioxide, affecting carbonate - bicarbonate equilibria). In the present work DASV without prior removal of dissolved oxygen was thoroughly studied.

Figures 1 and 9 illustrate the effect of dissolved oxygen for a sample containing cadmium, lead and copper ions. Dissolved oxygen affects

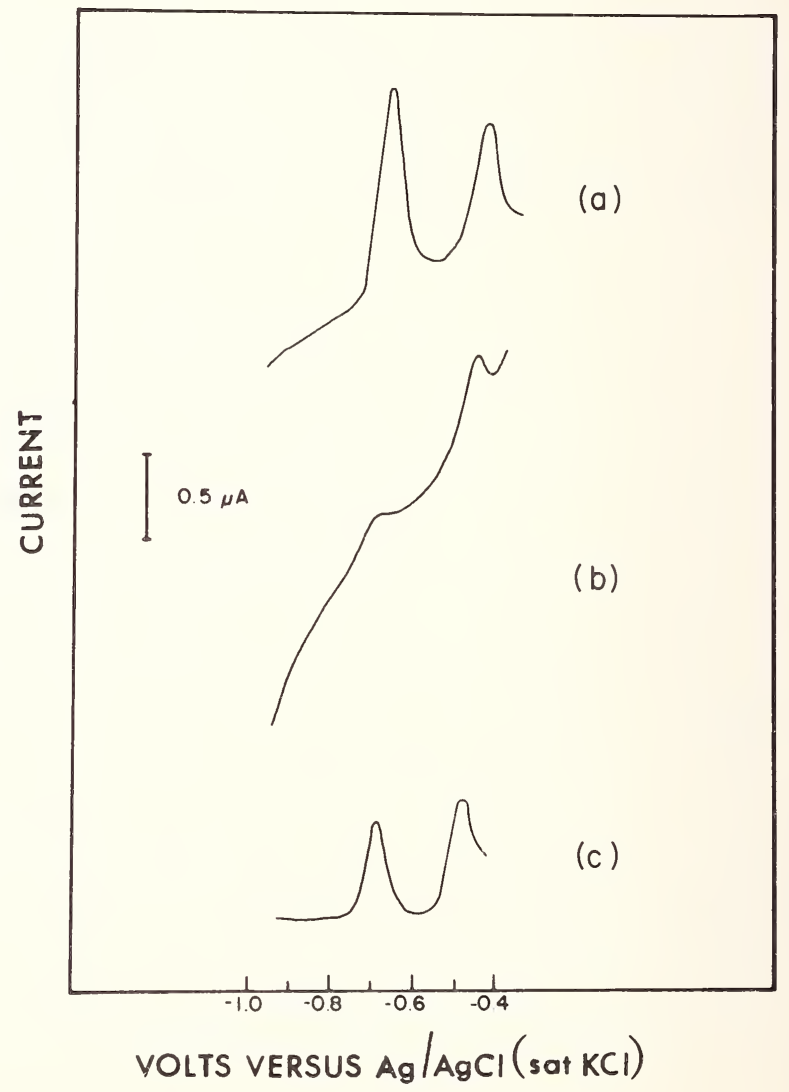

Figure 9. The effect of dissolved oxygen on voltammogram shape. (a), (b) and (c), $4.6 \times$ $10^{-8} \mathrm{M}$ cadmium ion, $2.7 \times 10^{-8} \mathrm{M}$ lead ion solution, in acetic acid/ammonium acetate $(0.02 M / 0.02 M)$ supporting electrolyte buffer. (a) ASV, oxygen removed prior to deposition; (b) ASV, dissolved oxygen present; (c) DASV $(t)$, dissolved oxygen present. Deposition at $-1.0 \mathrm{~V}$ for 3 minutes. Potential scan rate $6.0 \mathrm{~V} \mathrm{~min}{ }^{-1}$. 
ASV through 1) high background currents due to its reduction; 2) precipitation of metal hydroxides by hydroxyl ions formed during the reduction of oxygen in neutral media [8]; and 3) chemical oxidation of metals in the electrode amalgam. The rate of the latter depends on the nature of the metal, its concentration in the amalgam (which in turn, depends on factors such as the concentration of the metal ion in the sample, the length of the deposition period and the volume of the TMF). In contrast to stripping current peaks which, when obtained for copper, cadmium and lead in DASV at HMDEs in the presence and absence of dissolved oxygen, were of similar height [10], DASV at TMF electrodes, in the presence of oxygen, resulted, for some metals and concentration ranges, in lowered peaks (figs. 1 and 9). In spite of this, the linear correlation between peak height and metal ion concentration which continues to hold in most cases, permits quantitative determinations to be carried out without prior oxygen removal.

b. Non amalgam forming electroactive metal ions. Their presence in significant concentrations, frequently incurred in complex samples, constitutes an interference in unmodified ASV; methods for overcoming it (for methods for the elimination of ferric ion interference see [16-18]) often complicate routine procedures. DASV provides an elegant solution; figure 10 illustrates this for the case of copper determination in the presence of a thousandfold concentration of ferric ions.

To sum up DASV, as compared to ASV, offers increased ease, rapidity and sensitivity. The use of an in situ deposited TMF is but one instance of a number of possible variations, such as thicker films whenever the extremely thin film constitutes a disadvantage (e.g., in the presence of dissolved $\mathrm{O}_{2}$ ) or the use of predeposited films for in situ determinations. Detection limits may be further lowered by employing more sophisticated potential functions in the stripping step [1], by prolonging the deposition period or further increasing the potential scan rate.

Recently published modifications of ASV [2,3] solve some of the problems discussed: ASWC [3] offers similar sensitivity and ease of determination near the limits of the useful potential range to those achieved by DASV and is free of charging current background problems: it does not, however solve the problem of dissolved oxygen and electroactive macro components. Rapid ASV [2] shortens the duration of analysis but does not compensate any background currents. DASV does it all; its operative simplicity makes it a fitting method for semiautomatic or automatic operation and especially for determinations to be carried out in situ by remote control. Work towards this goal is currently in progress. 


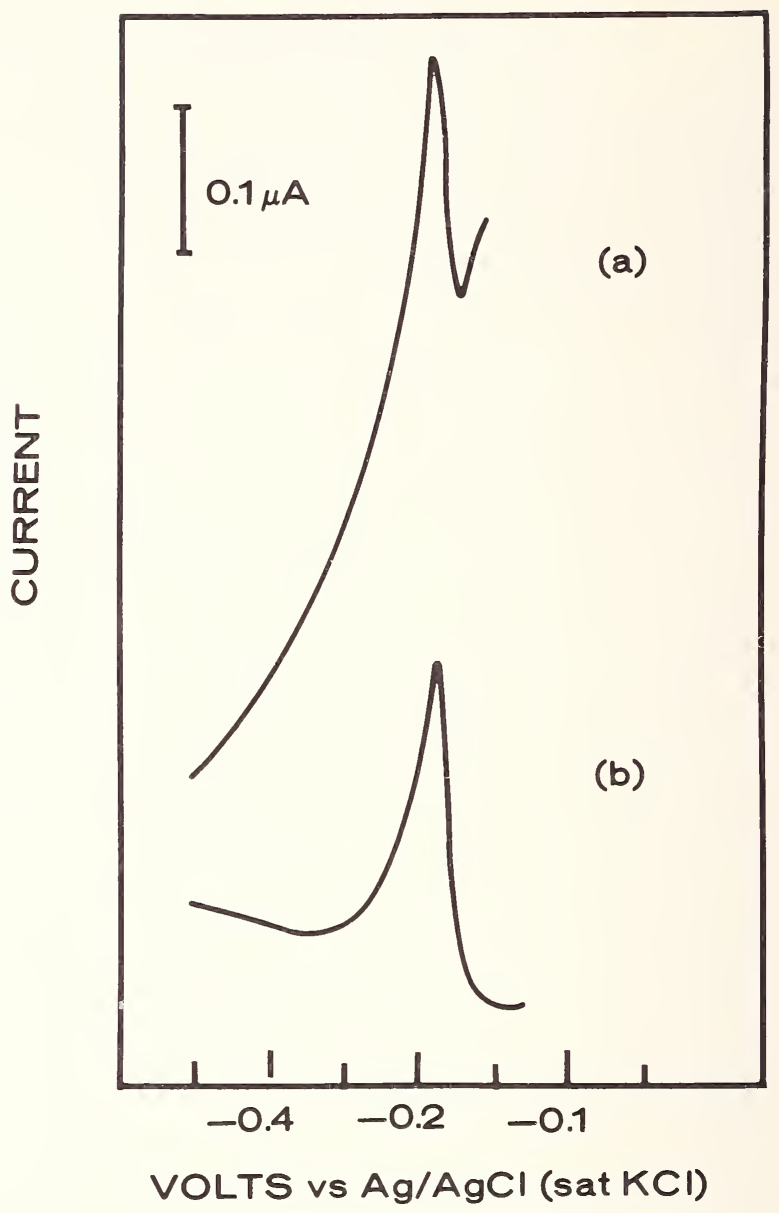

Figure 10. DASV in the presence of a major electroactive solution component. (a) and (b), ASV and DASV $(t)$ voltammograms recorded in deaerated $2.4 \times 10^{-7} M$ cupric ion, $2.5 \times$ $10^{-4} M$ ferric ion solution, in acetic acid/ammonium acetate $(0.02 M / 0.02 M)$ supporting electrolyte buffer. Deposition at $-1.0 \mathrm{~V}$ for 0.5 minute. Potential scan rate $1.2 \mathrm{~V} \mathrm{~min}{ }^{-1}$.

\section{Acknowledgements}

The authors gratefully acknowledge partial support of this research by the United States - Israel Binational Science Foundation. 


\section{References}

[1] Neeb, R., Inverse Polarographie und Voltammetrie, Verlag Chemie GmbH, Weinheim, Bergstr. (1969).

[2] Clem, R. G., Litton, G., and Ornelas, L. D., New Cell for Rapid Anodic Stripping Analysis, Anal. Chem. 45, 1306-1317 (1973).

[3] Laser, D. and Ariel, M., Anodic Stripping with Collection Using Thin Mercury Films, J. Electroanal. Chem.49, 123-132 (1974).

[4] Davis, H. M. and Seaborn, J. E., A Differential Cathode-Ray Polarograph, in Advances in Polarography, Longmuir, I. S., Ed., Vol. 1, pp. 239-250, Pergamon Press, Oxford (1969).

[5] Martin, K. J. and Shain, I., Differential Voltammetry Using the Hanging Mercury Drop Electrode, Anal. Chem. 30, 1808-1810 (1958).

[6] Kemula, W., Polarographic Methods of Analysis, Pure Appl. Chem. 15, 283-296 (1967).

[7] Zirino, A. and Healy, M. L., pH-Controlled Differential Voltammetry of Certain Trace Transition Elements in Natural Waters, Environ. Sci. Technol. 6, 243-249 (1972).

[8] Shimpff, W. K., Advances in Anodic Stripping Voltammetry for the in situ Analysis of Trace Metals in the Presence of Oxygen, Ph. D. Thesis, Univ. of Michigan (1971).

[9] Florence, T. M., Anodic Stripping Voltammetry with a Glassy Carbon Electrode Mercury-Plated in situ, J. Electroanal. Chem. 27, 273-281 (1970).

[10] Wang, J., Differential Stripping Voltammetry, M.Sc. Thesis, to be presented to the Senate of the Technion - I.I.T., Haifa (1974).

[11] Princeton Applied Research Corp., Instruction Manual Linear Sweep Module Accessory Model 174/51, Princeton, N.J. (1971).

[12] Taylor, J. K., Ed., NBS Technical Note 455, Michrochemical Analysis Section: Summary of Activities, July 1967 to June 1968, p. 119, U.S. Government Printing Office, Washington, D.C., October 1968.

[13] Levich, V. G., Physico-Chemical Hydrodynamics, Prentice-Hall, Englewood Cliffs. N.J. (1962).

[14] Hume, D. N. and Carter, J. N., Characteristics of the Mercury Coated Graphite Electrode in Anodic Stripping Voltammetry: Application to the Study of Trace Metals in Environmental Water Systems, Chem. Anal. (Warsaw) 17, 747-759 (1972).

[15] Roe, D. K. and Toni, J. E. A., An Equation for Anodic Stripping Curves of Thin Mercury Film Electrodes, Anal. Chem. 37, 1503-1506 (1965).

[16] Ariel M., Eisner, U., and Gottesfeld, S., Trace Analysis by Anodic Stripping Voltammetry, II. The Method of Medium Exchange, J. Electroanal. Chem. 7, 307-314 (1964).

[17] Phillips, S. L. and Shain, I., Application of Stripping Analysis to Trace Determination of Tin, Anal. Chem. 34, 262-265 (1962).

[18] Koster, G. and Ariel, M., Electrochemical Flow Cell, J. Electroanal. Chem. 33, 339349 (1971). 



\title{
MONITORING DISSOLVED COPPER IN SEAWATER BY MEANS OF ION-SELECTIVE ELECTRODES
}

\author{
Gary K. Rice and Raymond J. Jasinski \\ Central Research Laboratories \\ Texas Instruments Incorporated \\ P.O. Box 5936, M.S. 147 \\ Dallas, Texas 75222 U.S.A.
}

The application of an ion-selective electrode method for continuously monitoring $\mathrm{Cu}^{++}$in seawater, without sample pretreatment, is described.

The necessary sensitivity of the $\mathrm{Cu}^{++}$selective electrodes was achieved by preconditioning the electrodes and by use of a flow cell configuration. Response times, even at trace concentrations, were found to be adequate for monitoring small changes in the $\mathrm{Cu}^{++}$concentration of seawater.

Evaluating the effects of solid materials on the chemistry of $\mathrm{Cu}^{++}$in seawater was simplified by the speed and convenience of the ion-selective electrode technique. In addition to the adsorption of metal ions on solid surfaces, $\mathrm{Cu}^{++}$electrode analysis indicated that contaminants from some solid materials form complexes with $\mathrm{Cu}^{++}$in solution. Other materials apparently introduce cations such as $\mathrm{Fe}^{+++}$which can liberate $\mathrm{Cu}^{++}$from some complexes. It was also found that changes in chemical properties such as $\mathrm{pH}$ measurably affect the distribution of $\mathrm{Cu}^{++}$in seawater.

Operation of a trace sampling and analysis system aboard a ship is complicated by chemical and electrical effects of the ship's hull on the continuous seawater flow stream. A method was found for minimizing electrical "noise" in the flow stream and to distinguish the $\mathrm{Cu}^{++}$potential of chemical contamination due to the ship from the ambient background of natural seawater.

The results indicate that it should be practical to develop trace analytical methods which require little or no sample pretreatment for true in situ analysis thus avoiding many of the problems of sampling and sample handling.

Keywords: Adsorption; copper; cupric ion; flow cell; ion-selective electrode; seawater analysis. 


\section{Introduction}

Ion-selective electrodes based on insoluble metal sulfides have been applied to determination of trace cations in aqueous solutions. The first of these, $\mathrm{Ag}_{2} \mathrm{~S}$ electrodes, respond to as little as $1 \mu \mathrm{g} \mathrm{Ag}^{+} / 1$; even lower concentrations of free $\mathrm{Ag}^{+}$can be measured when $\mathrm{Ag}^{+}$complexing agents are present [1]. Mixed metal sulfide/silver sulfide electrodes were then developed to extend the response to other cations, i.e., those which form sulfide precipitates more soluble than $\mathrm{Ag}_{2} \mathrm{~S}$; electrodes sensitive to $\mathrm{Cu}^{++}$are made from a mixture of $\mathrm{CuS}$ and $\mathrm{Ag}_{2} \mathrm{~S}$ [1]. $\mathrm{Cu}^{++}$ electrodes of this type have been applied to the determination of $\mathrm{Cu}^{++}$ to $9 \mu \mathrm{g} / \mathrm{l}$ in simple aqueous solutions [2]. The response of such electrodes to $\mathrm{Cu}^{++}$in sea-water has recently been accomplished and the detection limit extended to less than $2 \mu \mathrm{g} / \mathrm{l}[3]$.

This development has made possible a technique for the continuous in situ monitoring of $\mathrm{Cu}^{++}$at sea which is the subject of this paper. The specific factors to be discussed are the following:

1. Steady-state electrode response to $\mathrm{Cu}^{++}$concentrations in natural seawater.

2. Extraneous factors which might offset electrode potentials such as temperature response and chemical interference.

3. Chemical effects of materials which might contact seawater samples, specifically the sampling system which supplies a continuous flow of sea-water for analysis.

4. Problems with obtaining significant electrode data in flow streams at sea.

5. The performance of the analytical method and sampling procedures at sea.

\section{Experimental}

Orion $\mathrm{Cu}^{++}$electrode potentials were measured with a precision of \pm $0.1 \mathrm{mV} v s$. Orion double-junction reference electrodes (referred to here as DJ) by a Corning Model 101 Digital Electrometer as previously described [3]. Steady-state electrode potentials were defined for this study as potentials changing by less than $0.1 \mathrm{mV} / \mathrm{min}$. A Corning Model 103 three-channel amplifier was added to the instrumentation to allow simultaneous measurement of as many as four electrodes $v s$. a single reference electrode in the same solution. The multi-channel amplifier also provided electrode potentials on analog outputs which were recorded $v s$. 
time by a Texas Instruments Servo-Riter II dual channel strip chart recorder.

All laboratory ware was acid washed, and the general laboratory procedures to minimize contamination were adapted as previously described [3]. Samples collected at sea were shipped and stored in acidwashed polyethylene bottles. $\mathrm{Cu}$ concentrations in seawater samples returned to the laboratory were determined by atomic adsorption spectrometric analysis of $\mathrm{Cu}$ extracted into a solution of 2 percent oxine in ethyl propionate [3].

\section{Electrode System}

The potential, E, of an electrochemical cell selective to $\mathrm{Cu}^{++}$follows the form of the Nernst equation:

$$
\mathrm{E}=\mathrm{E}_{0}+\mathrm{S} \log \left[\mathrm{Cu}^{++}\right] \text {. }
$$

The constant term, $\mathrm{E}_{0}$, contains thermodynamic constants, reference electrode potential, liquid junction potentials, and activity coefficients. Absolute accuracy (reproducibility) of the electrode measurement depends on the constancy of $E_{0}$ which is experimentally assured only by standards and samples having identical chemical composition and temperature (at normal pressure). "Electrode slope," $\mathrm{S}$, which is equal to $\Delta E / \Delta \log \left[\mathrm{Cu}^{++}\right]$, determines the sensitivity of the measurement.

Electrodes once preconditioned (see below) in seawater typically exhibit reproducible linear slopes of at least $50 \mathrm{mV} / \log \left[\mathrm{Cu}^{++}\right]$which is larger than the thermodynamically predicted $30 \mathrm{mV} / \log \left[\mathrm{Cu}^{++}\right]$. A slope value, once obtained, is stable (for a given electrode) and can be treated as an empirical constant which remains invariant as long as the electrode is exposed to a limited $\mathrm{Cu}^{++}$concentration range (approximately 2 orders of magnitude).

For a constant slope of $50 \mathrm{mV} / \log \left[\mathrm{Cu}^{++}\right]$a measurable potential change of $0.1 \mathrm{mV}$ corresponds to a change in [ $\mathrm{Cu}^{++}$] of only 0.5 percent. An especially attractive feature of potentiometry in trace analysis is that this sensitivity is maintained throughout the concentration range over which the slope remains constant. The slope of $\mathrm{Cu}^{++}$electrode response in seawater decreases at low $\mathrm{Cu}^{++}$concentration (fig. 1), but it will be shown that under certain conditions the region of constant slope may be extended to at least the $1 \mu \mathrm{g} / \mathrm{l}$ level.

The initial pretreatment of $\mathrm{Cu}^{++}$electrodes by long-term immersion in seawater was neccssary before adequate sensitivity to trace $\mathrm{Cu}^{++}$could 
be obtained [3]. This preconditioning, in the laboratory, was typically carried out with $500 \mathrm{ml}$ of stirred seawater in a Teflon beaker. Recorded potentials of new electrodes or electrodes previously exposed to relatively high $\mathrm{Cu}^{++}$concentrations (greater than $1 \mathrm{~g} / \mathrm{l}$ ) exhibited a decaying approach to a steady-state potential when placed in new seawater. When steady potentials were achieved, the electrode solution was replaced with more new seawater. This process was repeated until the potential change on transfer to new seawater became small $(<1 \mathrm{mV})$.

The response of such preconditioned electrodes to additions of $\mathrm{Cu}^{++}$to seawater collected 10 miles off Port Isabel, Texas, is graphed in figure 1 where the $\mathrm{Cu}$ concentration for the first data point was established by atomic adsorption analysis (AA). Responses of three different Orion $\mathrm{Cu}^{++}$ electrodes and one Orion $\mathrm{S}=$ electrode $\left(\mathrm{Ag}_{2} \mathrm{~S}\right)$ illustrate potentials of electrodes with different response characteristics. These changes in electrode response due to preconditioning and the stirring rates dependencies have produced data from which some electrode-solution interactions can be elucidated.

Preconditioning processes have also been noted by others to increase the sensitivity of $\mathrm{Cu}^{++}$electrode response to low free $\mathrm{Cu}^{++}$ concentrations in laboratory solutions. A preconditioning procedure of

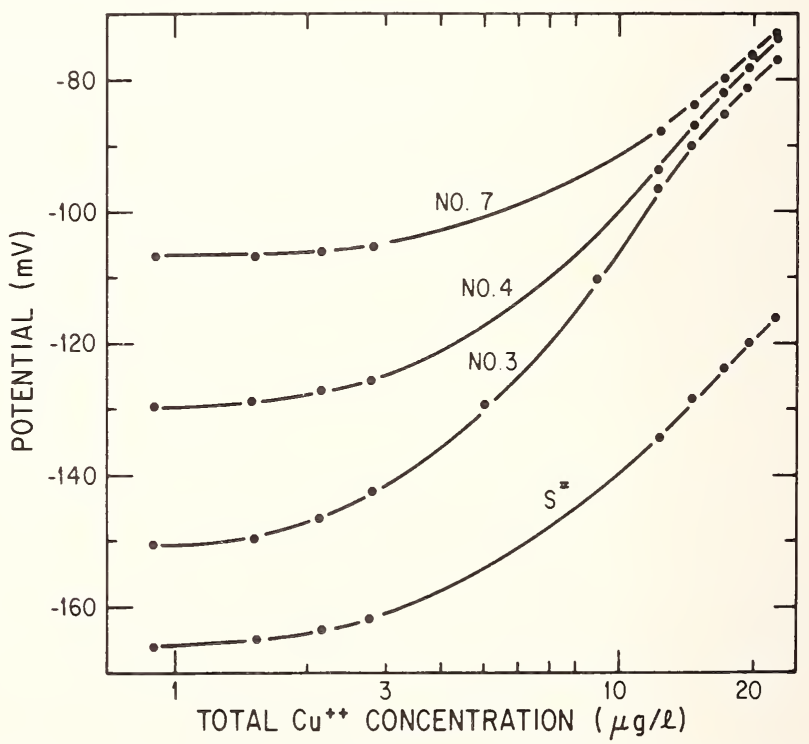

Figure 1. Response of electrodes to additions of $\mathrm{Cu}^{++}$to seawater. First data point at 0.9 $\mu \mathrm{g} / \mathrm{l}$ from atomic absorption analysis. 
soaking $\mathrm{Ag}_{2} \mathrm{~S} / \mathrm{CuS}$ on graphite electrodes in $\mathrm{CN}$ - or EDT A solution [4] has been attributed to a decrease in free $\mathrm{Cu}^{++}$concentration at the electrode surface; the sensitivity of these electrodes was increased at very low free $\mathrm{Cu}^{++}$concentrations in metal-ion buffered solutions. An acid cleaning procedure has been recently described for increasing Orion $\mathrm{Cu}^{++}$ electrode sensitivity in solutions with low concentrations of $\mathrm{Cu}\left(\mathrm{NO}_{3}\right)_{2}$ [5].

This cleaning procedure and the data in this paper can be explained by a depletion of CuS from the electrode surface. In figure $1, \mathrm{Cu}^{++}$ electrodes with greater sensitivity near $1 \mu \mathrm{g} \mathrm{Cu} u^{++} / 1$ also had potentials approaching that of $\mathrm{Ag}_{2} \mathrm{~S}$ in the same solution indicating that preconditioning indeed decreased the $\mathrm{Cu}^{++}$concentration (activity) at, or in, the electrode-solution interface.

Stirring rate also has an effect on electrode potential. The fact that lower flow rates across the electrode surface result in more positive potentials has been attributed to dissolution of $\mathrm{Cu}^{++}$or $\mathrm{Ag}^{+}$from the electrode surface [3]. Further data is shown in figure 2 which compares the flow rate dependency of a sensitive (No. 2) electrode with one that was

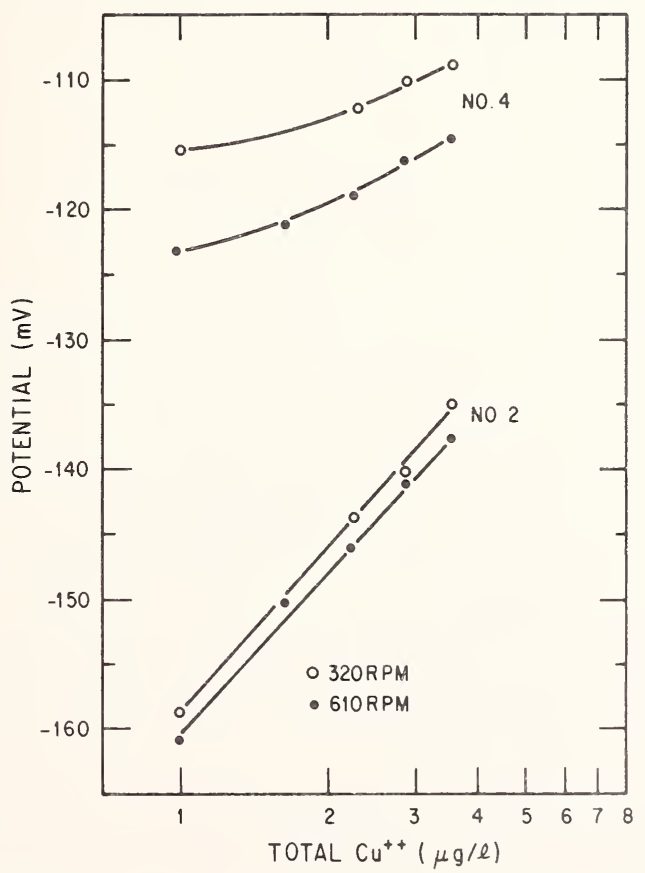

Figure 2. Dependence of potentials on flow rates across ion-sensitive surfaces of two $\mathrm{Cu}^{++}$ electrodes with different sensitivities. Flow rates were varied by changing stirring speed. 
less sensitive (No. 4). Besides more negative potentials, the more sensitive electrode shows less effect from changing flow rate. Also, the sensitivity of electrode No. 4 was increased slightly at low $\mathrm{Cu}^{++}$ concentrations by increased flow across the electrode surface.

That both the pretreatment and flow rate effects imply a dissolution process was verified by the observation that formerly polished ion sensitive surfaces had an etched appearance after prolonged exposure to seawater. Further evidence of dissolution was provided by previously preconditioned electrodes which detected an increase in $\mathrm{Cu}^{++}$(or $\mathrm{Ag}^{+}$) concentration in the seawater sample used to precondition insensitive electrodes.

Even in the "preconditioned" low dissolution rate state it is likely that the potentials of $\mathrm{Cu}^{++}$electrodes were still, at least partially, determined by a slow dissolution process. A slow dissolution rate proportional to $\mathrm{Cu}^{++}$concentration in seawater, through a $\mathrm{S}=$ equilibrium shift or another process, would produce the expected potential change from increased $\mathrm{Cu}^{++}$concentration as well as an additional increase in potential from dissolution products at the electrode-solution interface, i.e., a mixed potential. Such a process could help explain the observed electrode responses larger than $30 \mathrm{mV} / \log \left[\mathrm{Cu}^{++}\right]$.

\section{Temperature Response}

Since an electrode system in a seawater flow stream would be subjected to some variation in sample temperature, the effects of temperature on electrode potential were determined over a small temperature range. Variation of electrode potentials due to temperature can be described by the temperature differential of the Nernst equation [6], but uncertainty in the evaluation of some of the resulting terms usually requires that temperature coefficients be determined experimentally. This is especially true for applications in seawater where the distribution of complexed $\mathrm{Cu}^{++}$is shifted by changing temperature.

Temperature effects were determined from $\mathrm{Cu}^{++}$and $\mathrm{DJ}$ electrodes stirred and thermostated in natural seawater in an electrode cell which was partially sealed to retard evaporation. The electrode potentials, plotted in figure 3, varied nearly linearly over the small temperature range $\left(8^{\circ}\right)$. The slope in figure 3 represents the temperature response, $\mathrm{dE} / \mathrm{dT}$, of $-1.5 \mathrm{mV} / \mathrm{deg}$ for this electrode system. 


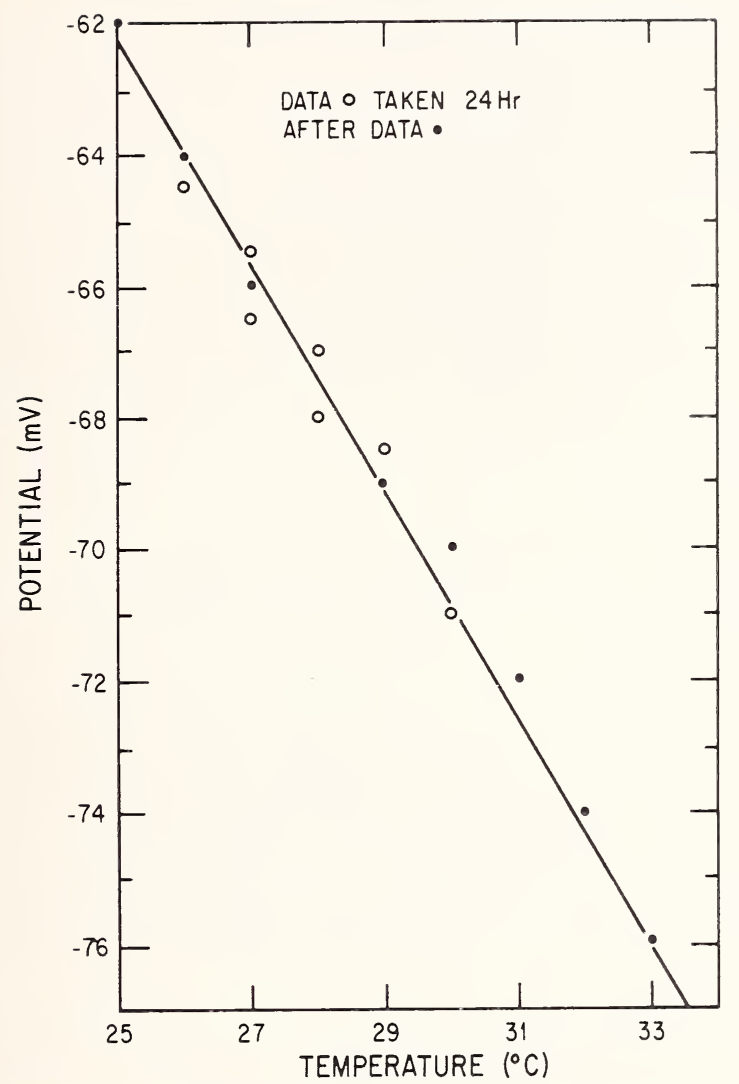

Figure 3. Temperature response of $\mathrm{Cu}^{++} v s$. double-junction electrode pair in natural seawater.

\section{Chemical Interference}

A positive interference from $\mathrm{Fe}^{3+}$ has been reported with Orion $\mathrm{Cu}^{++}$ electrodes [2]. Since monitoring was to be carried out at sea aboard a steel-hulled ship, the effects of $\mathrm{Fe}^{3+}$ on the $\mathrm{Cu}^{++}$electrode potential in natural seawater were determined. $\mathrm{Fe}^{3+}$ did not affect the $\mathrm{Cu}^{++}$electrode potential of Port Isabel seawater until more than $100 \mu \mathrm{g} / 1$ of $\mathrm{Fe}^{3+}$ had been added. Since the potential decreased rather than increased it is possible that ferric hydroxide coprecipitated $\mathrm{Cu}^{++}$. Further addition of larger amounts of $\mathrm{Fe}^{3+}$, up to $1.2 \mathrm{mg} / \mathrm{l}$, had only minor effects on the $\mathrm{Cu}^{++}$ electrode potential.

It was determined, however, that seawater which had been exposed to latex tubing gave electrode potentials more negative than usual, and when 
$\mathrm{Fe}^{3+}$ was added to this water, the electrode potential increased. These results are interpreted as complexation of $\mathrm{Cu}^{++}$by some material leached from latex and subsequent displacement of $\mathrm{Cu}^{++}$from the complex by $\mathrm{Fe}^{3+}$. Equilibrium displacement of $\mathrm{Cu}^{++}$by $\mathrm{Fe}^{3+}$ is reasonable since most organic ligands form $\mathrm{Fe}^{3+}$ complexes which are more stable than the corresponding complex with $\mathrm{Cu}^{++}[7]$. There were some seawater samples which gave varying degrees of positive potential increase on addition of $\mathrm{Fe}^{3+}$. But throughout this study all responses of $\mathrm{Cu}^{++}$electrodes due to the addition of $\mathrm{Fe}^{3+}$ to seawater could be attributed to displacement of complexed $\mathrm{Cu}^{++}$.

\section{Sampling Equipment Construction Materials}

The material of choice for containing seawater to be analyzed by $\mathrm{Cu}^{++}$ electrodes is Teflon; polyethylene is a good second choice [3]. In either continuous or discrete seawater sampling systems mechanical requirements or the commercial use of available components may result in other materials containing the seawater sample. For this reason, a brief evaluation was carried out to determine what effect, if any, seawater sample contact with various materials would have on the results of analysis by $\mathrm{Cu}^{++}$ electrodes.

The simplicity and speed of the ion-selective electrode for determining changes in trace $\mathrm{Cu}^{++}$concentration makes the method well suited for surveying candidate materials for use in a sampling system. The change in electrode potential was determined on immersion of a test material into a stirred seawater solution. The potential change, $\Delta \mathrm{E}$, due to contact of seawater with selected materials are listed in table 1 where $\Delta E=(E$ after contact $)-(\mathrm{E}$ for natural seawater). Negative $\Delta \mathrm{E}$ values were interpreted as adsorption of $\mathrm{Cu}^{++}$or introduction of complexing agents which decreased the concentration of free $\mathrm{Cu}^{++}$. Positive $\Delta \mathrm{E}$ values indicated that free $\mathrm{Cu}^{++}$(or $\mathrm{Ag}^{+}$or $\mathrm{Hg}^{++}$) concentration was increased by seawater contact with the test material. Positive $\Delta \mathrm{E}$ 's can be translated into equivalent $\mathrm{Cu}^{++}$concentrations by use of the response curve for electrode No. 3 in figure 1 .

Some additional information was obtained by measuring $\Delta \mathrm{E}$ for the addition of $\mathrm{Cu}^{++}$to the seawater in contact with the test material. Examples of this data in table 1 represent the effect of material contact on seawater as $\mathrm{Cu}^{++}$concentration varies.

The first four materials in table 1 are flexible polymers commonly used for seals in pumps and valves. Obviously, use of those materials which 
TABLE 1. $\mathrm{Cu}^{++}$electrode potential shift due to contact of seawater with solid materials

\section{Material}

\section{$\Delta E(\mathrm{mV})$}

(Natural seawater $+1.3 \mu \mathrm{g} / 1 \mathrm{Cu}^{++}$ or natural seawater*)

Viton A
Kel F
Hapalon
Nordel
Stainless steel tubing
316 Stainless steel fitting
Latex tubing
Latex tubing
PVC pipe
Nylon

Viton A

Kel F

Stainless steel tubing

316 Stainless steel fitting

Latex tubing

PVC pipe

Nylon

$$
\begin{aligned}
& -3.1^{*} \\
& +5.9 \\
& -27.1 \\
& -187.4 \\
& +7.8 \uparrow(3 \mathrm{~h}) \\
& +109 \uparrow \text { a }(10 \mathrm{~min}) \\
& -41.3 \\
& -23.4 \downarrow(18 \mathrm{~min}) \\
& +22.0 \\
& +6.2
\end{aligned}
$$

analyzed by emission spectrograph; contained approximately $500 \mathrm{ppm} \mathrm{Cu}$.

produced the largest changes in potential should be avoided. The potential increase from one piece of 316 stainless steel was unexpected since $\mathrm{Cu}$ is not specified in the composition of this material [8], and the seawater used for this study did not exhibit a significant response due to addition of $\mathrm{Fe}^{3+}$. Analysis of this piece of stainless steel revealed that it did, in fact, contain $\mathrm{Cu}$.

\section{Shipboard System}

The actual monitoring of dissolved $\mathrm{Cu}$ in seawater was carried out at sea onboard the USNS Mizar, a Naval oceanographic research ship. The sampling system consisted of Teflon tubing extended through an amidships well to a depth of 1 meter below the ship's hull. (Sampling depth was approximately 6 meters below the surface.) A submersible pump (nylon impeller and housing) forced seawater to an on-board analytical system where the seawater in a flow manifold was maintained at a constant level by a continuous overflow to waste. Taps on the manifold provided continuous sources of seawater to two $\mathrm{Cu}^{++}$electrode flow cells.

The body of the $\mathrm{Cu}^{++}$flow cell, which is detailed in figure 4, was machined from a block of solid Teflon. The $\mathrm{Cu}^{++}$electrode was locked into place by a compressed O-ring with its ion-sensitive surface facing upward into the flow channel. An Orion double-junction reference electrode, with its annular junction surface machined flat, was locked in place opposite the $\mathrm{Cu}^{++}$electrode. A special machined plastic gage inserted 


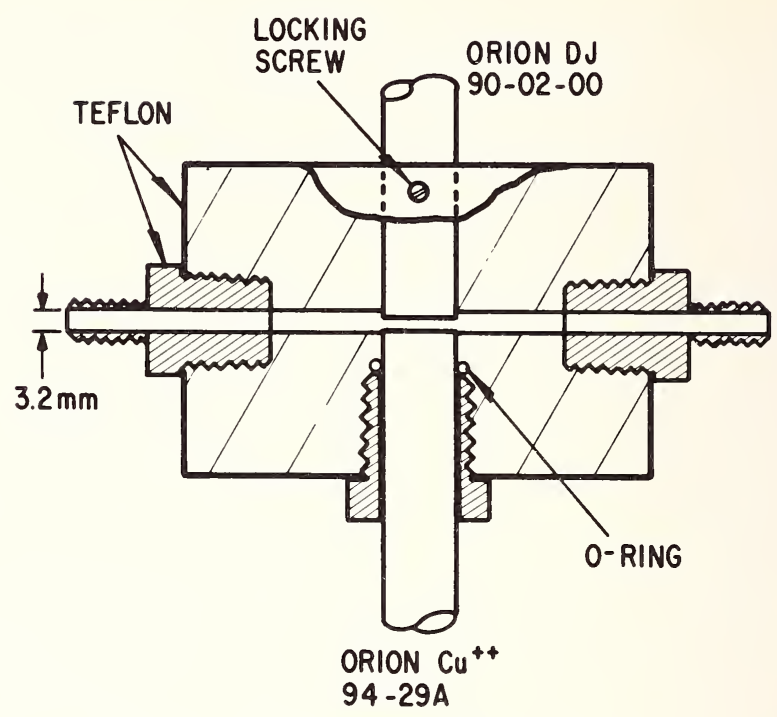

Figure 4. $\mathrm{Cu}^{++}$electrode continuous flow cell.

through one end of the flow passage assured proper placement and spacing of the two electrodes. An interelectrode spacing of $0.8 \mathrm{~mm}$ gave a cross-sectional area between the electrodes equal to that of the flow passage, but the spacing was found to be not critical as long as flow was not restricted.

Seawater from the manifold flowed by gravity to the $\mathrm{Cu}^{++}$cells so that flow rates were easily controlled at 400 to $600 \mathrm{ml} / \mathrm{min}$ by adjusting cell height with respect to the water level in the flow manifold. With the reference electrode removed, high velocity flow could be observed across the entire $\mathrm{Cu}^{++}$sensitive surface which, according to the data in figure 2 should have contributed to maximizing sensitivity at lowest concentrations.

Usually two $\mathrm{Cu}^{++}$flow cells were operated simultaneously and both cell potentials were recorded on strip chart. When the $\mathrm{Cu}^{++}$flow cells were first operated in the continuous flow stream at sea, the recorded potentials exhibited rapid variations which would normally be associated with electrical interference. The problem was traced to a variable potential between the ship's hull and seawater in the flow stream. This potential signal was recorded in figure $5(\mathrm{~A}) v s$. the DJ reference electrode in one of the $\mathrm{Cu}^{++}$flow cells. The signal probably represents variation in corrosion potentials caused by the ship's motion (one frequency component seemed to be correlated with ship's roll); power leakage from on-board electrical equipment to the sea may have contributed to the problem. 


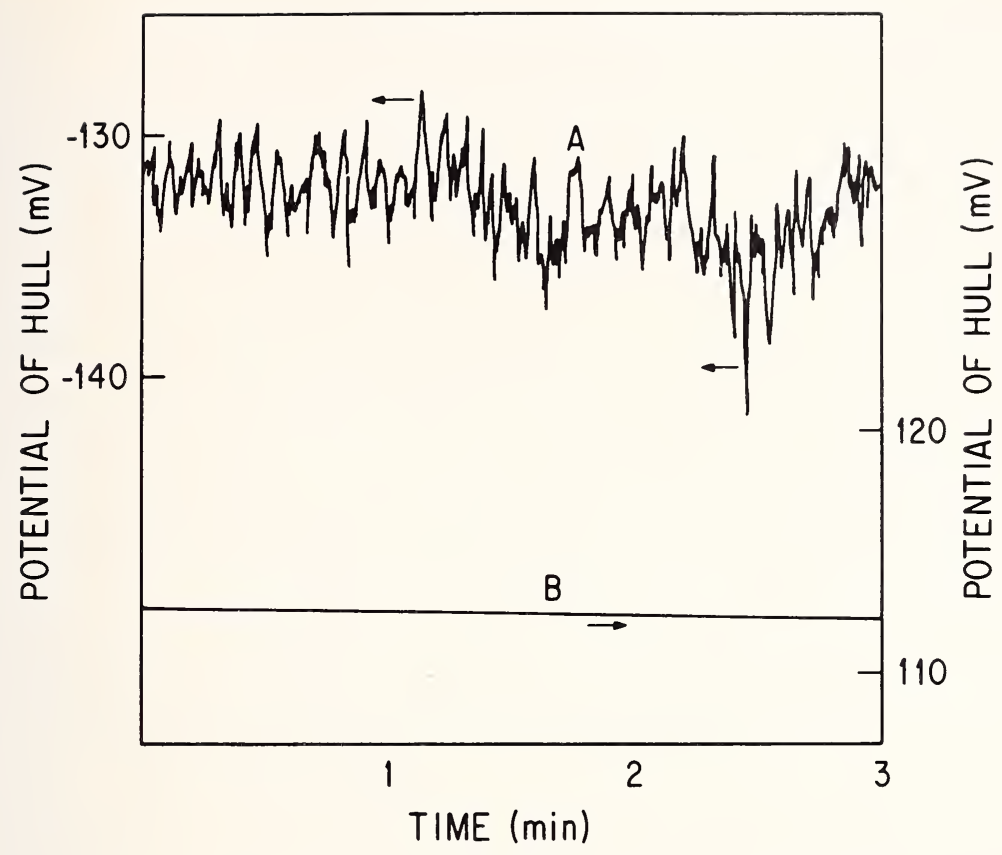

Figure 5. Potentials of ship's hull vs. DJ reference electrode in flow cell. Trace A was before and B after grounded Pt screen was placed in the flow system.

The potential variations were minimized, as shown in figure 5(B), by a cylindrical Pt screen electrode (2.5-cm-diameter by $5-\mathrm{cm}$-long) placed in the flow manifold and connected to ship's ground (hull). This Pt electrode was evidently sufficient to capacitively couple the seawater flow stream to the ship's hull through an electrode double layer established by a $14 \mu \mathrm{A}$ current passed through this electrode. After the flow stream "noise" was drained to ground, stable potentials could be recorded with a resolution limited only by a $5 \mathrm{~Hz} 0.06 \mathrm{mV}$ peak-to-peak signal from a chopper circuit in the electrometer.

\section{Cell Response}

In situ continuous $\mathrm{Cu}^{++}$cell potentials were recorded for seawater through an area of the Caribbean Sea south of Cuba and in the Atlantic Ocean off the coast of Florida. The cell potentials indicated that free $\mathrm{Cu}^{++}$ concentrations in these waters were considerably less than that of the seawater used for the laboratory development of the electrode system. AA 
analysis of samples returned to the laboratory revealed ambient total $\mathrm{Cu}^{++}$background concentrations of 0.15 to $0.50( \pm 0.12) \mu \mathrm{g} / 1\left(\sim 10^{-8} M\right)$. Since no more than about 1 percent of the total $\mathrm{Cu}^{++}$in seawater is not complexed [9], the electrode has to be responding to free $\mathrm{Cu}^{++}$on the order of $10^{-10} \mathrm{M}$ in order to measure total $\mathrm{Cu}^{++}$concentrations at the 0.5 $\mu \mathrm{g} / \mathrm{l}$ level, i.e., that copper in equilibrium.

That response at this level was indeed achieved with the free $\mathrm{Cu}^{++}$ions was demonstrated by removing one electrode from a flow cell after 2 weeks of daily operation and measuring its response to $\mathrm{Cu}^{++}$additions. This addition data, plotted in figure 6 , was obtained at sea, and the $\mathrm{Cu}$ concentration for the first data point was determined by AA analysis of a sample returned to the laboratory. The results in figure 6 show response to at least ambient $\mathrm{Cu}^{++}$concentrations $(0.48 \mu \mathrm{g} / \mathrm{l}$ for this sample).

On comparing figure 6 with figure 1 (data taken before electrode No. 3 had been exposed to flow cell operation), the increase in sensitivity (slope) at lower concentrations is evident. This increase in electrode sensitivity and limit of detection is attributed to a continuation in the flow cell of the previously described electrode conditioning process. The slope increase in the region of $\mathrm{Cu}^{++}$concentrations greater than $1 \mu \mathrm{g} / \mathrm{l}$ approached a value of $70 \mathrm{mV} / \log \left[\mathrm{Cu}^{++}\right]$which, in figure 1 , was established only at concentrations at least five times higher. This increase in sensitivi-

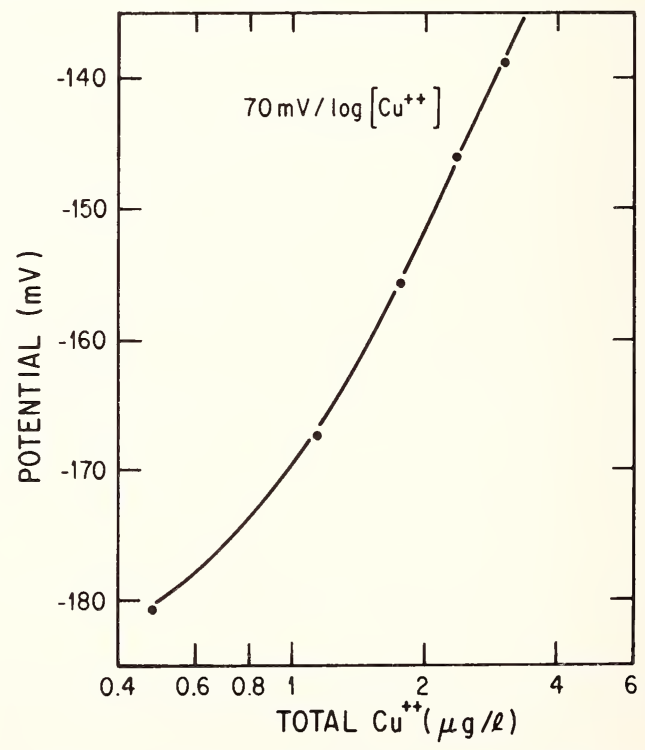

Figure 6. Response of $\mathrm{Cu}^{++}$electrode No. 3 after extended operation in a seawater flow cell. 
ty linearized the slope to $1 \mu \mathrm{g} / 1$ in figure 6 , and has the important consequence that absolute concentrations can now be accurately determined by the known-addition method [10].

This increased sensitivity of the flow cell electrodes combined with the stability of electrode potentials thus made possible the detection of $\mathrm{Cu}^{++}$ concentration changes of less than 1 percent at the ambient $\mu \mathrm{g} / 1$ level.

\section{IX. $\mathrm{Cu}^{++}$Concentration Anomalies}

Since the flow system intake was mounted only 1 meter below the ship, chemical contamination of the sample by the ship's hull could be expected. That this was indeed the case was determined by observing changes in $\mathrm{Cu}^{++}$cell potential as a function of the ship's speed. Slower speeds resulted in more positive cell potentials which fluctuated as the ship rolled about while dead in the water. As the ship started moving again cell potentials returned to their normal, more negative values. This is illustrated for a "worst case" in figure 7.

Usually, however, the effects illustrated in figure 7 were much smaller, and, in some seawater, barely detectable which leads to the conclusion that something more than simple $\mathrm{Cu}^{++}$contamination of seawater by the ship was involved. In any case, with the sample intake only 1 meter below the ship, seawater contaminated by the hull was available at the inlet. The potential data indicate that with the ship underway most seawater con-

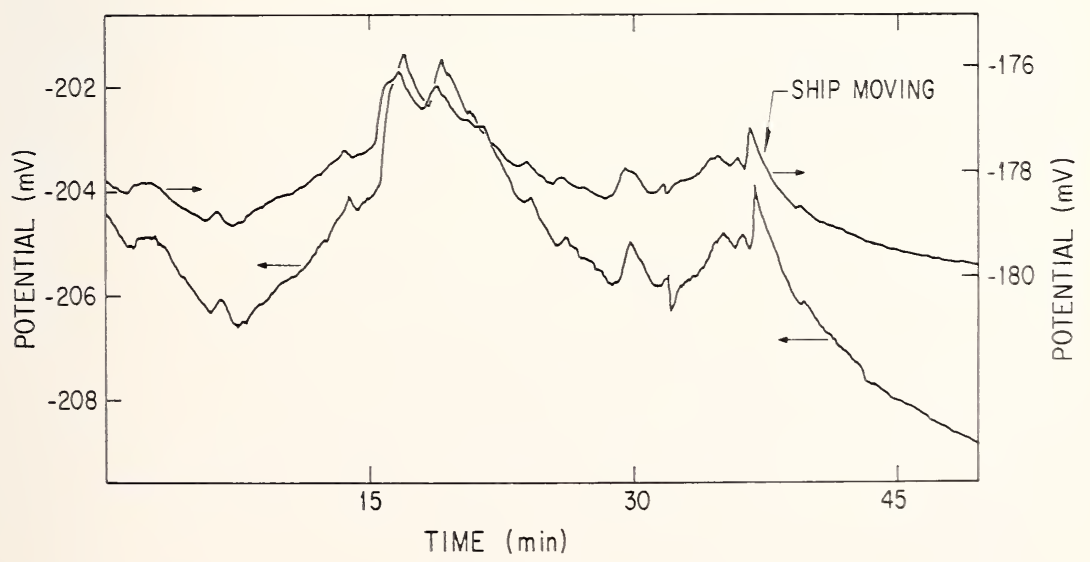

Figure 7. Recorded potentials of two $\mathrm{Cu}^{++}$flow cells with the ship drifting and potential change with ship underway. The two $\mathrm{Cu}^{++}$cells had slightly different sensitivities. and the time offset of corresponding peaks was due to a recorder pen offset. 
taminated by the hull was swept away and was not accessible to the sample intake. These results imply that for monitoring aboard a moving ship sample intakes far removed from the ship's hull (where at 15 knots, mechanical requirements become formidable) may not be necessary. However, the minimum speed for contamination-free sampling would have to be determined for each application.

Another example of anomalous $\mathrm{Cu}^{++}$cell potentials is shown in figure 8 , while the ship was drifting shoreward near Cat Island $\left(24.5^{\circ} \mathrm{N}, 75.5^{\circ}\right.$ $\mathrm{W})$. These results were repeated several times in the same area. It seems unlikely that the dissolution rate of any $\mathrm{Cu}^{++}$source on the surface of the ship would vary so much from one seawater to another; therefore, the results in figures 7 and 8 are ascribed not to changes in total $\mathrm{Cu}^{++}$ concentration (supported by AA analysis of samples returned to the laboratory) but rather to differences in the distribution of complexed $\mathrm{Cu}^{++}$, and subsequent displacement by $\mathrm{Fe}^{3+}$ as discussed above. During a later laboratory investigation seawater collected at approximately the 25 -min mark on figure 8 , indeed, did produce a positive potential change when a pure $\mathrm{Fe}$ wire was immersed in the stirred sample. These results are consistent with $\mathrm{Cu}^{++}$behavior in near-shore waters previously reported [3].

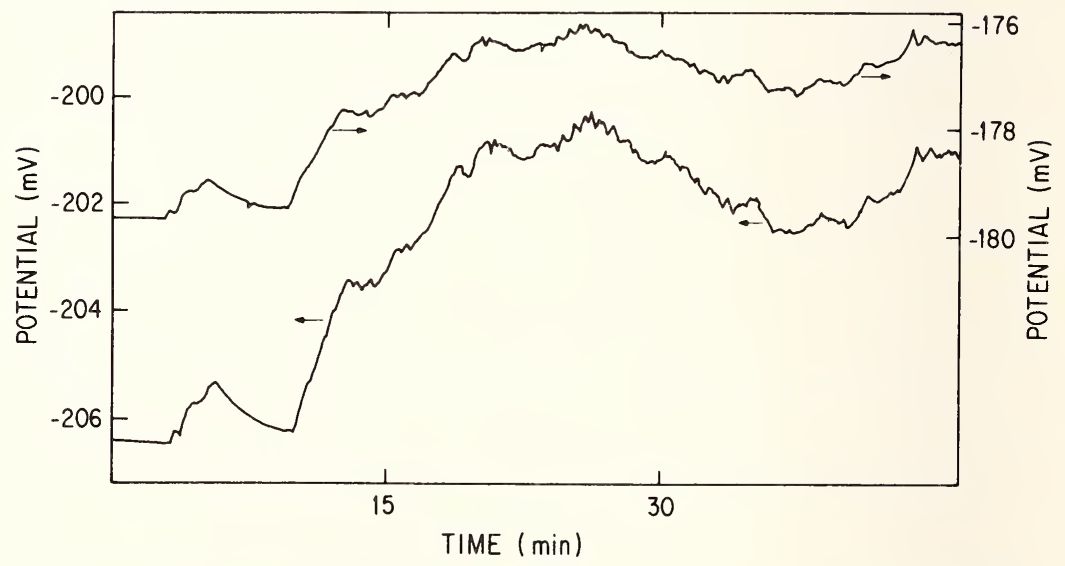

Figure 8. $\mathrm{Cu}^{++}$cell potentials while drifting toward the shore of Cat Island. 


\section{Sampling Devices}

A satisfactory grab sampler for use in trace determinations was devised by modifying a sterile bag sampler manufactured by General Oceanics for biological sampling. The principle of the device entails a sealed polyethylene bag with an inlet which is cut open on messenger activation at depth. Spring loaded flaps then unfold the bag filling it with seawater after which a pinch clamp securely closes the inlet while the sample is retrieved. Contamination of sample by exposed metal parts is minimized by the capability of the sampler to rotate about the sample line and direct the intake tube into ambient ocean currents. As the data in table 1 indicate, it was necessary to modify this system by replacing latex tubes used in the necks of the bags with similar tubes of thin-wall Teflon. The inlets to the bags were not resealed; therefore, the sampler was lowered rapidly and used only for depths of less than 20 meters. Results of atomic absorption analysis of organic extractions of some samples obtained from the bag sampler are listed in table 2 .

Seawater samples from multiple depths were obtained throughout the cruise in Niskin bottles which are commonly used for oceanographic sampling. These bottles were made of PVC and had end closures operated by latex tubes which were extended longitudinally inside the bottle. The previous laboratory study of the effects of PVC and latex on trace $\mathrm{Cu}^{++}$in seawater (summarized in table 1) had indicated that these bottles would be unsuitable for obtaining samples for electrode measurement.

The effect of these materials on seawater to be analyzed for $\mathrm{Cu}^{++}$by AA was not known. Therefore, a sample of seawater from $15 \mathrm{~m}$ was taken in two 8-liter Niskin bottles. AA analysis was made on composite samples collected from the two bottles a few minutes after retrieval and after 4 and 24 hours. The results are reported in table 2.

TABLE 2. Atomic absorption anaylsis of $\mathrm{Cu}^{++}$in seawater 
The $\mathrm{Cu}^{++}$concentration was abnormally high at $\mathrm{t}=0$, but analysis of samples which had longer contact times with the Niskin bottles indicated that after only a few hours most of the $\mathrm{Cu}^{++}$originally present was lost. In order to separate effects of the PVC bottle from those of the latex tubing, a Niskin bottle, with the latex tube removed, was filled with seawater from the flow stream and allowed to stand for 15 hours before a sample was taken for AA analysis. Simultaneously, the latex tube was soaked in approximately 4 liters of seawater during the same 15 hours. The results of AA analysis in table 2 indicate that the latex tube was the main cause for the loss of extractable $\mathrm{Cu}^{++}$in the Niskin bottle.

\section{Conclusions}

The real-time response of $\mathrm{Cu}^{++}$electrodes to trace $\mathrm{Cu}^{++}$in flow cells at sea has been demonstrated at total $\mathrm{Cu}^{++}$concentrations of less than 0.5 $\mu \mathrm{g} / \mathrm{l}$. Advantages of the ion-selective electrode method of trace analysis are excellent sensitivity at trace concentrations, simplicity of procedure, and low cost. The small space requirements of flow cells and supportive instrumentation makes the electrode system well suited for submersed in situ applications which would allow profiling $\mathrm{Cu}^{++}$concentration as a function of depth as well as minimize some of the sample contamination problems.

There are identifiable applications in the fields of geochemistry and oceanography for a technique which can detect $\mathrm{Cu}^{++}$concentration anomalies at the trace level. $\mathrm{Cu}^{++}$sources and sinks in the oceans can be located and defined which has direct applications to mineral exploration and pollution detection and mapping. The continuous, real-time analysis capability of the electrode method makes it a viable survey technique for determining where to sample for trace elements. Biological investigations of $\mathrm{Cu}$ toxicity and the role of $\mathrm{Cu}^{++}$concentrations in primary production will be facilitated by the electrode technique. Since ion-selective electrodes actually measure ion activities, electrode data is directly applicable to developing and testing thermodynamic models of chemical systems.

\section{Acknowledgements}

The authors wish to thank Mr. Roy DeViney for his technical assistance and Mr. Stephen Meyer for his participation during the cruise. Shipboard tests were made possible by Naval Research Laboratory 
Cruise 73-11-07. The assistance of NRL personnel and the cooperation of the captain and the crew of the USNS Mizar are gratefully acknowledged.

This project was sponsored by Advanced Research Projects Agency on Order No. 2199 and administered by the Office of Naval Research under Contract No. 0014-72-C-0368.

\section{References}

[1] Ross, J. W., Solid-State and Liquid-Membrane Electrodes, in Ion-Selective Electrodes, Durst, R. A., Ed., pp. 57-88, NBS Spec. Pub. 314, U.S. Government Printing Office, Washington, D.C. (1969).

[2] Smith, M. and Manahan, S., Anal. Chem. 45, 836-9 (1973).

[3] Jasinski, R., Trachtenberg, I., and Andruchuk, D., Anal. Chem. 46. 364-9 (1974).

[4] Hansen, E. H., Lamm, C. G., and Ruzicka, J., Anal. Chim. Acta 59, 403-26 (1972).

[5] Blaedel, W. J. and Dinewiddle, D. E.; Anal. Chem. 46, 873-7 (1974).

[6] Light, T. S., Industrial Analysis and Control with Ion-Selective Electrodes, in IonSelective Electrodes, Durst, R. A., Ed., pp. 349-74, NBS Spec. Pub. 314, U.S. Government Printing Office, Washington, D.C. (1969).

[7] Sillen, L. G. and Martell, A. E., Stability Constants of Metal-Ion Complexes, Spec. Pub. No. 17, The Chemical Society, London (1964) and Stability Constants Supplement No. 1, Spec. Pub. No. 25 (1971).

[8] Handbook of Chemistry and Physics, 54th edition, p. F-141, Chemical Rubber Co. Press, Cleveland, Ohio (1973).

[9] Zirino, A. and Yamamoto, S., Limnol. Oceanogr. 17, 661-71 (1972).

[10] Durst, R. A., Analytical Techniques and Applications of Ion Selective Electrodes, in Ion-Selective Electrodes, Durst, R. A., Ed., pp. 375-414, NBS Spec. Pub. 314 , U.S. Government Printing Office, Washington, D.C. (1969). 



\title{
ELECTROANALYTICAL DETERMINATION AND CHARACTERIZATION OF SOME HEAVY METALS IN SEAWATER
}

\author{
M. Branica, L. Sipos, S. Bubic, and S. Kozar \\ Center for Marine Research \\ Rudjer Bošković Institute \\ 41000 Zagreb \\ Croatia, Yugoslavia
}

\begin{abstract}
Anodic stripping methods have been widely applied during the last 10 years for determination of some heavy metals in natural water systems. However, relatively little has been done on the physicochemical characterization and determination of species actually present in aquatic systems, which is of special importance for elucidation of respective biogeochemical cycle and fate of radioactive or industrial waste.
\end{abstract}

The experience on application of electroanalytical techniques is presented and discussed with regard to: 1) direct determination of concentrations of some metal ions; 2) characterization of the species actually present; 3 ) study of chelation, hydrolyzation and other interactions between metallic ions and organic ligands in natural aquatic system.

Anodic stripping voltammetry (ASV) with rotating glassy carbon electrode was used for determination of $\mathrm{Cd}, \mathrm{Pb}$, and $\mathrm{Cu}$ and slowly dropping mercury electrode was used for determination of $\mathrm{Zn}$ in the North Adriatic. The reproducibility of measurements, behavior of electrodes, the role of cell and storage bottle materials and sampling procedures are discussed.

A new method for characterization of the ionic state of metals at very low concentrations $\left(10^{-9}\right.$ to $\left.10^{-8} M\right)$, based on voltammetric measurements with accumulation at various constant potentials, is proposed.

Keywords: Adriatic Sea; cadmium; copper; electrochemical analysis; heavy metals; lead; seawater analysis; speciation; stability of water samples; zinc. 


\section{Introduction}

Until now the knowledge on physicochemical characteristics (the species distribution, chemical behavior, interactions and kinetics of equilibration) of trace metals in natural waters has mostly been based on theoretical predictions [1-5]. Only a limited number of papers are supported by experimental studies taken on model systems. Because of lack of sufficient sensitivity the concentrations in the method studied may be several orders of magnitude higher than their occurrence under natural conditions [6-10].

Recently, a method suitable for direct characterization of trace metals in natural concentration levels [11] has been proposed based on ASV with accumulation at various potentials.

One of the greatest problems in trace analysis (at ppb level) is how to keep the concentration unchanged during the sampling, sample storage and determination procedure. Several papers are published describing losses of trace metals from samples due to adsorption and/or contamination because of container materials [12-15].

Anodic stripping voltammetry (ASV) has been successfully applied for the determination of certain soluble fractions of some trace metals in seawater due to its high sensitivity and simplicity. Several reviews on ASV [16-19] and its application to the analysis of seawater [20,21] have been published.

In recent years the most frequently used electrodes were the composite mercury graphite electrode $[22,24]$ or the rotating mercury film glassy carbon electrode $[25,26]$. Their application to the analysis of seawater has also been published [27-29].

Generally, the anodic peak current in the ASV is proportional to the concentration of the electroactive metal ions in the solution. The proportionality depends on several parameters including temperature, rate of stirring, cell geometry, electrode surface and others, and is very difficult to be predicted theoretically. The standard addition method overcomes the problem of reproducibility because it is not very difficult to keep experiments at constant conditions during the analytical procedure. Besides the problems of adsorption or contamination processes, the quantity of added metal ions must satisfy the same species distribution as the metal ions present in the sample. Sometimes the equilibria in natural waters are reached very slowly $[30,31]$.

The rotating glassy carbon electrode (RGCE) with mercury deposition in situ [25] has very reproducible hydrodynamic conditions. At the same time the thickness of the mercury layer is changed due to continuous elec- 
trolysis and deposition of some impurities, which may disturb the determination [32].

The aim of the present paper is to present additional information on applicability, characteristics and reproducibility of the ASV with RGCE employed for determination of trace metals in seawater, connected to the problems of sample storage. The rotation speed of $1500 \mathrm{rpm}$ was kept constant.

\section{A. Reagents and Solutions}

Standard solutions of metal salts of $10^{-4} M$ to $10^{-6} M$ were prepared by diluting $0.1 \mathrm{M}$ reagent grade solutions standardized by means of complexometric titration [35]. The diluted solutions were stored in acid rinsed polyethylene bottles and were acidified with diluted double distilled hydrochloric acid to $\mathrm{pH}=3$.

The seawater samples were stored in 2-liter polyethylene containers at low temperature. The samples for determination of trace metals were collected in polyethylene bottles of $250 \mathrm{ml}$ and stored at $0{ }^{\circ} \mathrm{C}$.

\section{B. Procedures}

The published procedures for determination of $\mathrm{Zn}$ with slowly dropping mercury electrode SDME (Macchi [36]) and of $\mathrm{Cd}, \mathrm{Pb}$, and $\mathrm{Cu}$ with RGCE (Florence [29]) were modified as described in a previous paper [21].

The adsorption/contamination processes in polyethylene storage bottles of traces of some heavy metals were studied as follows.

Thirty polyethylene bottles of $500 \mathrm{ml}$ were filled with $1 \mathrm{~N}$ hydrochloric acid, for at least 2 weeks, and then carefully washed with tetradistilled water. Then they were filled with the same seawater (previously analyzed for $\mathrm{Cd}, \mathrm{Pb}$, and $\mathrm{Cu}$ by $\mathrm{ASV}$ ). Seawater in 10 bottles was acidified to $\mathrm{pH}=$ 2 by the addition of hydrochloric acid. The trace metals were determined every day during the first week, taking the samples always from a different bottle. Three weeks later only three measurements were done. Storage temperature was $0{ }^{\circ} \mathrm{C}$ and $20^{\circ} \mathrm{C}$, respectively.

\section{Results and Discussions}

The anodic peak heights of $\mathrm{Cd}, \mathrm{Pb}$ and $\mathrm{Cu}$ depend on the thickness of 
the mercury layer at the RGCE. It has been measured as a function of a number of repetitive current-voltage cycles and different experimental conditions. In figure 1 this dependence is shown at concentrations of 2 . $10^{-8} M \mathrm{Cd}$ and $\mathrm{Pb}$, and $4 \cdot 10^{-8} M \mathrm{Cu}$.

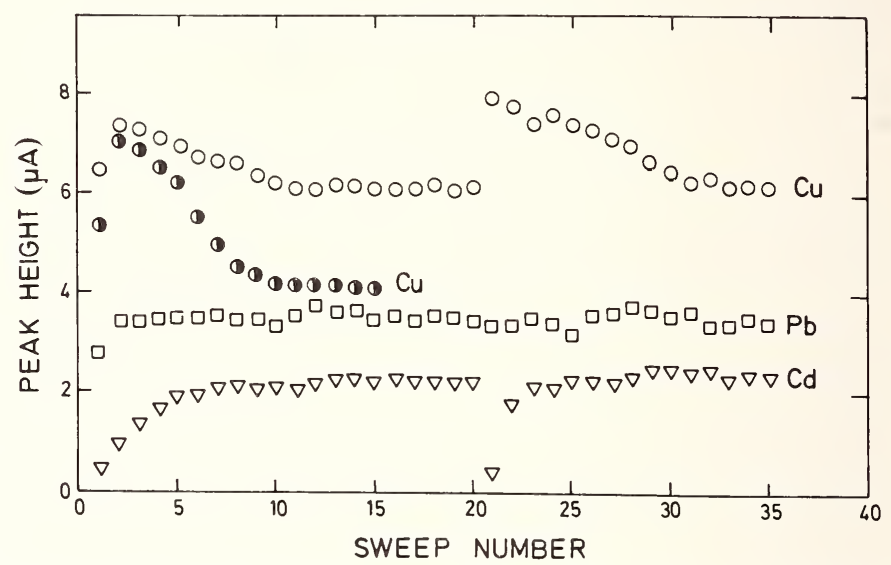

Figure 1. Dependence of peak heights of cadmium, lead and copper in a seawater sample on sweep numbers. The electrode surface was wiped after the twentieth cycle. Deposition time, $5 \mathrm{~min}$ at $-0.9 \mathrm{~V}$ (vs. $\mathrm{Ag} / \mathrm{AgCl}$ ). ( $\mathrm{Cu}$ naturally present, (O) $2 \cdot 10^{-8} \mathrm{M} \mathrm{Cu}$ added, ( $\square) 2 \cdot 10^{-8} M \mathrm{~Pb}$ added, $(\nabla) 2 \cdot 10^{-8} M \mathrm{Cd}$ added.

In a single seawater sample the peak height of lead remains constant even after 30 repeated cycles. The cadmium peak increases in the first 4 to 5 cycles, but after that, remains also constant in the next 25 cycles. The peak height of lead and cadmium is constant between $10^{-5} M$ and 5 . $10^{-5} \mathrm{M}$ of mercuric chloride. The heights of peak of copper are more complicated. At the beginning the peak height decreases toward a constant height after 10 to 15 cycles. The stabilization occurs earlier by increasing the concentration of mercury ions and decreasing the concentration of copper. The peak heights at the first cycle are not proportional to the concentration of $\mathrm{Cu}$, as they are after stabilization. The effects mentioned for $\mathrm{Pb}, \mathrm{Cd}$ and $\mathrm{Cu}$ peaks are observed again after wiping the mercury film from electrode surface.

The continuous decrease of the peak height of $\mathrm{Cu}$ is probably due to the increase of the dissolved amount of $\mathrm{Cu}$ in the mercury layer, which is limited by the low solubility $(0.002 \% \mathrm{Cu} / \mathrm{Hg})$ [37] supposing the same rate of electrolysis. At the given experimental conditions saturation can be reached easily, i.e., at $2 \cdot 10^{-5} \mathrm{M}$ of $\mathrm{Hg}^{2+}$ and $10^{-8} \mathrm{M}$ to $10^{-7} \mathrm{M}$ of $\mathrm{Cu}$ in seawater. However, higher concentrations of $\mathrm{Hg}^{2+}$ are not recommended, 
because of very high residual currents. Because of high solubility of $\mathrm{Cd}$ and $\mathrm{Pb}$ in mercury (5.9\% and $1.3 \%$, respectively) [37] similar effects for these metals have not been observed.

The increasing peak height of cadmium during the first few cycles can be ascribed to the shift of accumulation potential of $\mathrm{Cd}$ to more positive values by increasing of thickness of the mercury film [33].

The shift of the accumulation potential to more positive values with increasing the thickness of the mercury layer is presented in figure 2 , wherefrom it is evident that during the first few cycles the deposition potential of $-0.9 \mathrm{~V}$ lays on the rising part of the curve representing the $\mathrm{Cd}$ anodic peak current vs. deposition potential. On increasing the thickness of the mercury layer, the curve is shifted to more positive values and the deposition potentials of $-0.9 \mathrm{~V}$ reach the plateau. This is the reason why the curve constructed on the basis of peak current vs. deposition potential shows a hysteresis when the potential is increased and decreased (without wiping the electrode surface). One can conclude that the deposition of cadmium must be done at more negative potentials than $-0.9 \mathrm{~V}$ (at about $-1.0 \mathrm{~V} v s$. $\mathrm{Ag} / \mathrm{AgCl}$ ), or by using a thicker mercury layer with the RGCE.

For solution with $\mathrm{pH}=2$, some difficulties arise. The slope of the curve representing the peak height $v s$. deposition potential is suppressed

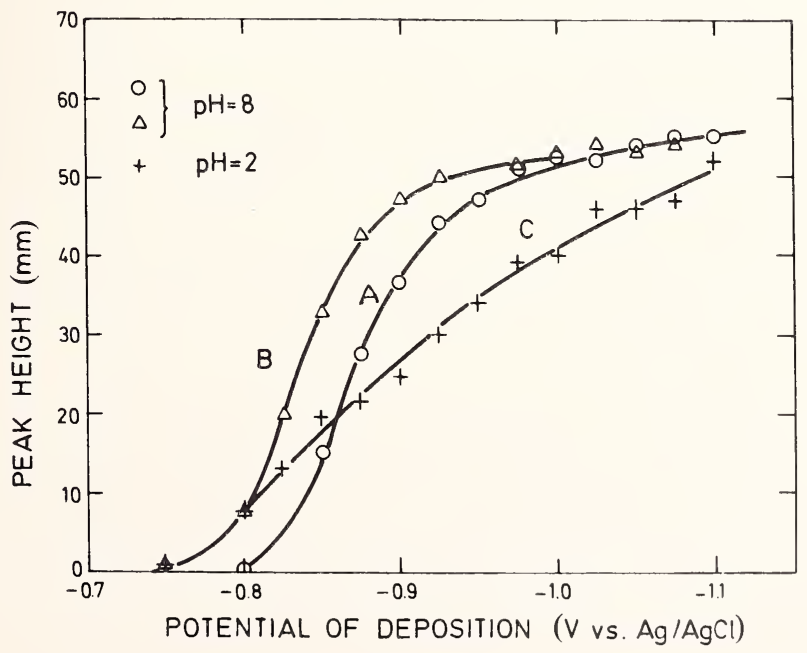

Figure 2. Dependence of peak current of cadmium $v s$. deposition potential, A, stepwise increasing negative deposition potential (forward), B, stepwise decreasing negative deposition potential (backwards after curve $\mathrm{A}$ ) at $\mathrm{pH}=8$, and $\mathrm{C}$, stepwise increasing negative deposition potential at $\mathrm{pH}=2$. Deposition time $3 \mathrm{~min}$. 
because of the interference of hydrogen ions of the reduction and the plateau cannot be reached in the working potential range of the glassy carbon electrode. To avoid the erroneous determination of $\mathrm{Cd}$ (at $\mathrm{pH}=2$ by the standard addition method) long mercury depositions (prior to determination) and precise potential control are recommended.

The measurements show that a procedure consisting of 20 min intensive deaeration and simultaneous mercury deposition at $-0.9 \mathrm{~V}$ ( 2 to 3 . $10^{-5} \mathrm{M} \mathrm{HgCl}_{2}$ ) from a seawater sample is enough to obtain a suitable mercury film for determination of $\mathrm{Cd}, \mathrm{Pb}$ and $\mathrm{Cu}$ by the standard addition method. After application of this procedure, followed by one or two cycles of 5-min depositions and strippings, reproducible peak heights of $\mathrm{Cd}$, $\mathrm{Pb}$, and $\mathrm{Cu}$ can be obtained. A calibration curve for $\mathrm{Cd}$ at the concentration level $10^{-10} \mathrm{M}$ is shown in figure 3 .

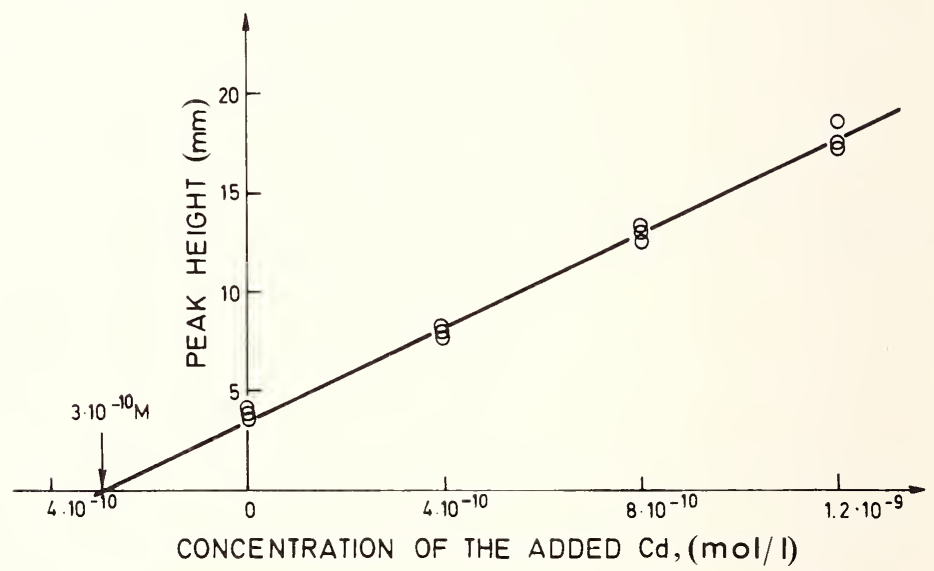

Figure 3. Calibration curve of cadmium (three successive determinations) in seawater by the standard addition method. Deposition time $10 \mathrm{~min}$ at $-1.0 \mathrm{~V}$ (vs. $\mathrm{Ag} / \mathrm{AgCl}$ ).

The reproducibility of determination of cadmium, lead and copper in seawater samples by the standard addition method has been measured in polyethylene cells with 50 and $300 \mathrm{ml}$ of samples (at pH 8 and 2). The results are summarized in table 1 . The reproducibilities of the values obtained are more uniform with $300 \mathrm{ml}$ sample solutions. In $50 \mathrm{ml}$ samples, especially at $\mathrm{pH}=8$, high dissipations may occur due to a relatively large surface to volume ratio $\left(1.4 \mathrm{~cm}^{2} / \mathrm{ml}\right)$. A general conclusion is that using ASV with $\mathrm{RGCE}, \mathrm{Pb}$ and $\mathrm{Cu}$ can be determined with a relative standard deviation of about 10 percent and $\mathrm{Cd}$ of about 20 percent at their natural concentration range in seawater. 


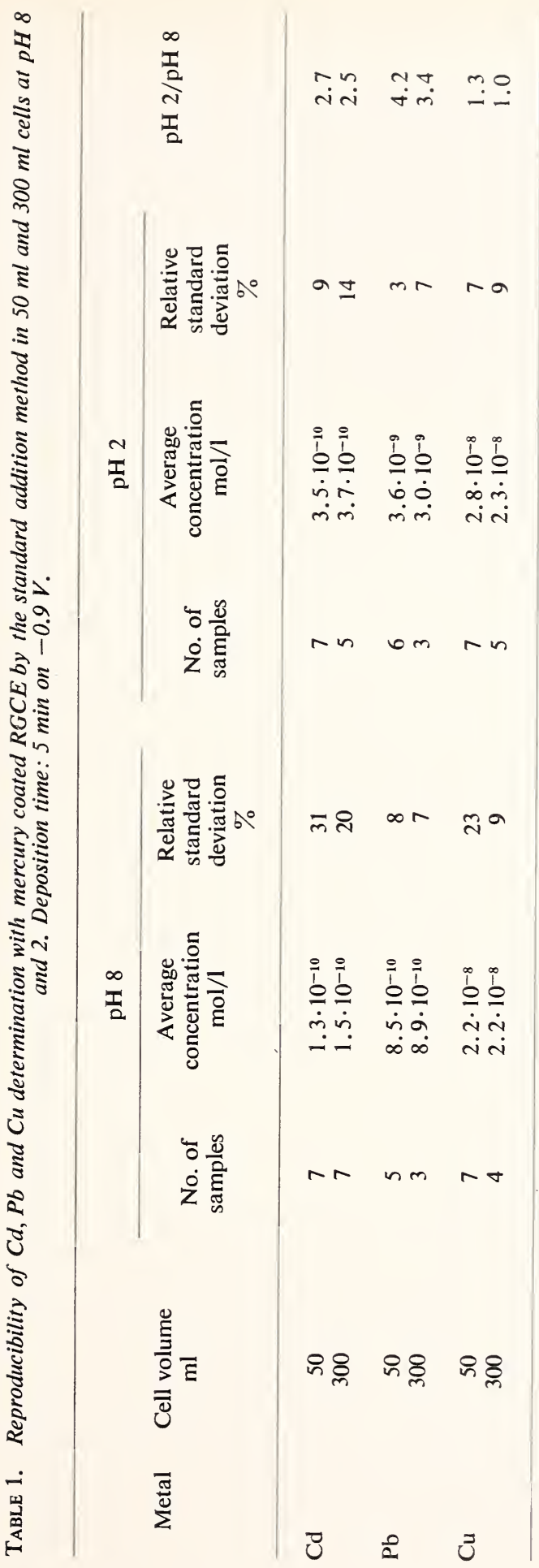


The effect of cell and container walls on the trace metal concentrations has been examined in several experiments. From the results (table 1) it is evident that there is no significant difference between values determined in a volume of $50 \mathrm{ml}$ and volume of $300 \mathrm{ml}$ (i.e., at cell surface to volume ratios from 1.4 to 0.7$)$. One can conclude that during the determination procedure (which lasts 30 to $50 \mathrm{~min}$ ) no significant concentration change occurs due to adsorption/contamination processes in the cell. However, for seawater samples containing $2 \cdot 10^{-8} M \mathrm{Cd}$ and $\mathrm{Pb}$ and $2 \cdot 10^{-7} M \mathrm{Cu}$ (at $\mathrm{pH}=8$ ) in a polyethylene cell with a volume of $50 \mathrm{ml}$ (stored overnight), the peak heights for $\mathrm{Cd}$ and $\mathrm{Pb}$ decrease about 20 percent and those for $\mathrm{Cu}$ about 50 percent.

The change of the concentration of $\mathrm{Cd}, \mathrm{Pb}$ and $\mathrm{Cu}$ in a seawater sample, stored in $500 \mathrm{ml}$ polyethylene bottles at $0{ }^{\circ} \mathrm{C}$ and $20^{\circ} \mathrm{C}$ temperature (at $\mathrm{pH}=8$ and 2 ) is presented in table 2 .

The concentration of $\mathrm{Cd}, \mathrm{Pb}$ and $\mathrm{Cu}$ during the first 7 days was constant with relative standard deviations similar to those presented in table 1. After 3 weeks storage the concentration of cadmium was considerably increased in all samples investigated; which indicates that there was a contamination process, probably from the container walls.

The concentration of $\mathrm{Pb}$ and $\mathrm{Cu}$ was slightly, but not significantly changed after 3 weeks of storage.

Similar results were obtained with seawater samples stored in polyethylene bottles of $250 \mathrm{ml}$ at $0{ }^{\circ} \mathrm{C}$ for 14 days (table 3). According to the results one can conclude that the relatively high concentration decrease (in $50 \mathrm{ml}$ cell) is probably due to the adsorption of metal ions on working and counter electrode compartments. It can also be concluded that the storage procedure may change the composition of a seawater sample in respect to the trace metal content due to contamination or adsorption. The most significant errors are found for the determination of $\mathrm{Cd}$, which naturally appears in seawater in lowest concentrations ( $10^{-10} M$ ). Therefore, samples should be analyzed as soon as possible, avoiding long storage.

The metal ion concentrations in seawater at $\mathrm{pH}=8$ and 2 are different, except for copper (table 1). It is probably due to transformation of electroinactive into electroactive species by decreasing the $\mathrm{pH}$ from 8 to 2 . The ratio of concentrations obtained at the mentioned $\mathrm{pH}$ is not constant, i.e., changing from one to another seawater sample.

The concentrations of $\mathrm{Zn}, \mathrm{Cd}, \mathrm{Pb}$ and $\mathrm{Cu}$ have been determined in more than 400 seawater samples $($ at $\mathrm{pH}=8$ ) collected in the North Adriatic sea in the period from May 1973 to April 1974, using the described analytical procedure. The samples were collected in $250 \mathrm{ml}$ 


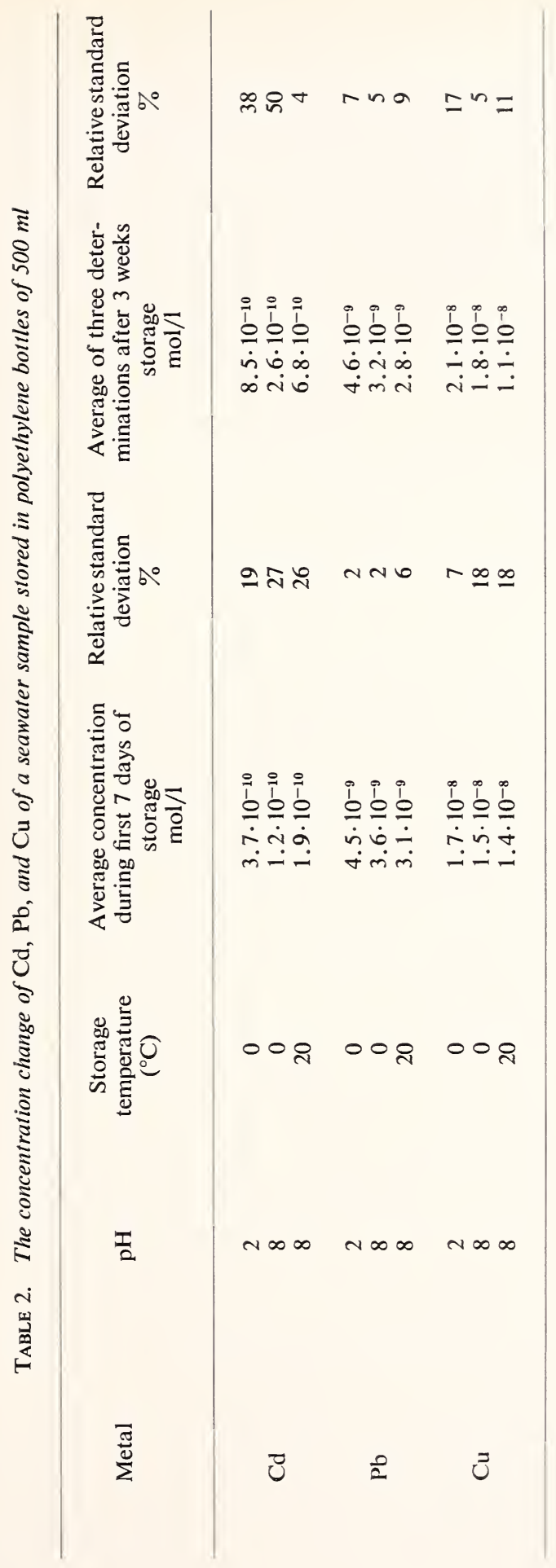




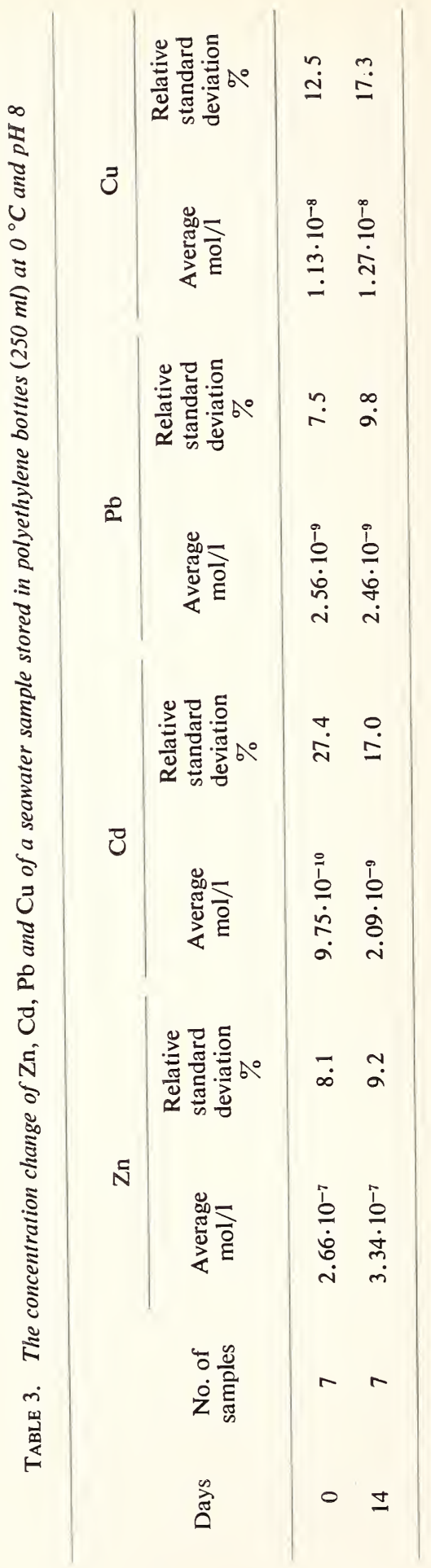


polyethylene bottles and stored at low temperature not longer than 1 week. The results of measurement are summarized in table 4.

TABLE 4. The concentrations of $\mathrm{Zn}, \mathrm{Cd}, \mathrm{Pb}$, and $\mathrm{Cu}$ in the North Adriatic

\begin{tabular}{|c|c|c|c|c|c|}
\hline Metal & $\begin{array}{c}\text { No. of } \\
\text { samples }\end{array}$ & $\begin{array}{c}\text { Average } \\
\mathrm{mol} / \mathrm{l}\end{array}$ & $\begin{array}{c}\text { Relative } \\
\text { standard } \\
\text { deviation } \\
\%\end{array}$ & $\begin{array}{c}\text { Median } \\
\text { mol/1 }\end{array}$ & Concentration range \\
\hline $\mathrm{Zn}$ & 461 & $9.2 \cdot 10^{-8}$ & 50 & $6.9 \cdot 10^{-8}$ & $1.7 \cdot 10^{-8}-4.8 \cdot 10^{-6}$ \\
\hline $\mathrm{Cd}$ & 454 & $8.0 \cdot 10^{-10}$ & 56 & $8.9 \cdot 10^{-10}$ & $4.5 \cdot 10^{-10}-6.6 \cdot 10^{-9}$ \\
\hline $\mathrm{Pb}$ & 463 & $2.9 \cdot 10^{-9}$ & 44 & $2.7 \cdot 10^{-9}$ & $4.8 \cdot 10^{-10}-5.6 \cdot 10^{-8}$ \\
\hline $\mathrm{Cu}$ & 456 & $1.4 \cdot 10^{-8}$ & 56 & $7.9 \cdot 10^{-9}$ & $1.6 \cdot 10^{-9}-2.0 \cdot 10^{-6}$ \\
\hline
\end{tabular}

The question is in which forms these metals are actually present in the sea. Calculations (using stability constants and data on the chemical composition of the seawater) may be used to indicate the ionic forms of trace metals, but direct measurements in natural conditions and actual concentration level have not been available so far.

However, ASV may be a very powerful tool for solving these problems $[11,33]$. A correlation (experimental or theoretical) can be made between the polarographic half-wave potential and the position of curves representing anodic peak current $v s$. deposition potential. On the basis of such curves (the potential changes with the composition of solution) the distribution of ionic species can be determined at concentration levels as low as the detection limit of the ASV.

Using this method the predominant ionic species of $\mathrm{Cd}$ naturally present in seawater were determined as mono and bichloro complexes. The ASV is therefore a useful technique not only for analytical purposes, but also for the study of complexation, hydrolyzation and chelation of trace metals at concentration level about $10^{-10} \mathrm{M}$.

\section{Acknowledgements}

Grateful acknowledgement is made to the National Bureau of Standards, Gaithersburg, Md., U.S.A., and the Republic Council for Scientific Researches of S.R. Croatia, Yugoslavia, for support of this work on Grant No. NBS/IG/-191/JF. 


\section{References}

[1] Sillen, L. G., The Physical Chemistry of Sea Water, in Oceanography, Sears, M., Ed., pp. 549-582, Amer. Assoc. Advan. Sci., Washington, D.C. (1961).

[2] Stumm, W. and Morgan, J. J., An Introduction Emphasizing Chemical Equilibria in Natural Waters, in Aquatic Chemistry.

[3] Sillen, L. G. and Martell, A. E., Stability Constants of Metal Ion Complexes, Spec. Publ. 17, 2d edition, Chem. Soc., London (1964).

[4] Zirino, A. and Yamamoto, S., Limnol. Oceanogr. 17, 661-671 (1972).

[5] Stumm, W. and Bilinski, H., Trace Metals in Natural Waters; Difficulties of Interpretation Arising from our Ignorance of their Speciation, in Advances in Water Pollution Research, Sixth International Conference, June 8-23, 1972, Jerusalem, Pergamon Press, Oxford and New York (1973).

[6] Branica, M., Petek, M., Barić, A., and Jeftić, Lj., Rapp. Comm. int. Mer Médit. 19, 102-106 (1968).

[7] Barić, A. and Branica, M., J. Polarog. Soc. 13, 4-8 (1967).

[8] Zirino, A. and Healy, M. L., Limnol. Oceanogr. 15, 956-958 (1970).

[9] Maljković, D. and Branica, M., Limnol. Oceanogr. 16, 779-785 (1971).

[10] Allen, H. E., Matson, W. R., and Mancy, K. H., J. Water Pollut. Contr. Fed. 42, 573-581 (1970).

[11] Bubić, S. and Branica, M., Thalassia Jugosl. 9, (1973) in press. Proceedings, 23d International Congress, C.I.E.S.M., November 1972, Athens, Greece.

[12] Robertson, D. E., Anal. Chim. Acta 42, 533-536 (1968).

[13] Tolg, G., Extreme Trace Analysis of the Elements, Talanta 19, 1489-1521 (1972).

[14] Struempler, A. W., Anal. Chem. 45, 2251-2254 (1973).

[15] King, W. G., Rodriguez, J. M., and Wai, C. M., Anal. Chem. 46, 771-773 (1974).

[16] Kemula, W. and Kublik, Z., Application of Hanging Mercury Drop Electrodes in Analytical Chemistry, in Advances in Analytical Chemistry and Instrumentation, Reilley, C. N., Ed., Vol. 2, pp. 123-177, Interscience Publishers, New York (1963).

[17] Barendrecht, E., Stripping Voltammetry, in Electroanalytical Chemistry, Bard, A. J., Ed., Vol. 2, pp. 53-109, Marcel Dekker, Inc., New York (1967).

[18] Ellis, W. D., Anal. Chem. 50, A131-A147 (1973).

[19] Brainina, Kh. Z, Talanta 18,513-539 (1971).

[20] Zirino, A., Lieberman, S. H., and Healy, M. L., Anodic Stripping Voltammetry of Trace Metals in Seawater, Proceedings of the Symposium on Marine Electrochemistry, October 9-12, 1972, Miami Beach, Fla.

[21] Bubić, S., Sipos, L., and Branica, M., Thalassia Jugosl. 9, (1973) in press. Proceedings, 23d International Congress, C.I.E.S.M., November 1972, Athens, Greece.

[22] Matson, W. R., Roe, D. K., and Carrit, D. E., Anal. Chem. 37, 1594-1595 (1965).

[23] Matson, W. R., Ph. D. Thesis, Massachusetts Institute of Technology, Cambridge, Mass. (1968).

[24] Hume, D. N. and Carter, J. N., Chemia Analityczna 17, 747-759 (1972).

[25] Florence, T. M., J. Electroanal. Chem. 27, 273-281 (1970).

[26] Batley, G. E. and Florence, T. M., J. Electroanal. Chem. 55, 23-43 (1974).

[27] Barić, A. and Branica, M., Thalassia Jugosl., in press. Proceedings, 22d International Congress, C.I.E.S.M., December 1970, Rome, Italy.

[28] Gilbert, T. R. and Hume, D. N., Anal. Chim. Acta 65, 451-459 (1973). 


\title{
THE ACCURATE DETERMINATION OF LEAD IN BIOLOGICAL AND ENVIRONMENTAL SAMPLES BY ISOTOPE DILUTION MASS SPECTROMETRY
}

\author{
L. A. Machlan, J. W. Gramlich, T. J. Murphy, and I. L. Barnes
}

\author{
Analytical Chemistry Division \\ Institute for Materials Research \\ National Bureau of Standards \\ Washington, D.C. 20234 U.S.A.
}

The accurate determination of lead in biological and environmental samples has become of great interest due to health considerations. Isotope dilution mass spectrometry (IDMS) has proven to be precise and accurate to within 0.15 percent. The IDMS method has permitted the accurate determination of trace quantities of lead in samples of biological and environmental interest as well as determining the inhomogeneity of the lead content.

IDMS has been utilized to provide certified lead values in Standard Reference Materials used by the fuel industry (coal, fuel oil and fly ash). These materials are to be used for comparison standards, quality assurance and developing or verifying analytical methods. The lead content of a series of blood samples has also been determined by IDMS to provide accurate values in the development of a clinical reference method.

The method consists of taking a weighed aliquot of the sample, adding a known amount of separated isotope, decomposing the material with suitable acids, separating the lead by anion exchange, purifying the lead by electrodeposition, determining the lead isotope ratios by thermal ionization mass spectrometry and calculating the lead content of the sample.

\footnotetext{
Keywords: Accurate and precise analysis; biological and environmental samples; blanks; homogeneity of lead content; isotope dilution mass spectrometry; lead analysis.
}

\section{Introduction}

The accurate determination of lead in biological and environmental 
samples has become of great concern due to health considerations. Samples of known lead content are needed for comparison standards, quality assurance, accuracy verification of present analytical methods and use in new method development. Isotope dilution mass spectrometry (IDMS) has proven to be an accurate analytical method [1-3]. The IDMS method is best used for providing accurate values for reference materials and not for routine analysis because of the time and expense involved. The major advantages of the IDMS technique are the high accuracy that can be attained $(0.1 \%$ for some elements, including lead) and the fact that chemical separation and recoveries do not have to be quantitative after the separated isotope and the sample have equilibrated. The major requirements for this method are to provide uniform, purified samples for the mass spectrometric measurement and to keep the blank both low and constant. A uniform, purified lead fraction can be separated from most samples by using anion exchange and electrodeposition. The blank has been reduced to a few nanograms by using a Class 100 [4] clean air environment for all chemical operations and by using specially purified reagents.

\section{Facilities}

Lead contamination from airborne particulates has been a major problem for low level accurate lead analysis for all methods. A clean room facility was a necessity for lead determination, especially at or below the $\mu \mathrm{g} / \mathrm{g}$ level. Particulate contamination was held to a minimum throughout the procedure by using a Class 100 work area for the chemical preparation of the sample and for the mass spectrometer filament loading.

\section{Reagents}

Reagents were another potential source of high lead blanks and high purity reagents were necessary to keep the reagent contribution to the blank at a minimum. The acids and water used were prepared at NBS by subboiling distillation [5]. Typical lead concentration in these reagents were $0.2 \mathrm{ng} / \mathrm{g}$ in $\mathrm{HClO}_{4}, 0.02 \mathrm{ng} / \mathrm{g}$ in $\mathrm{HNO}_{3}, 0.07 \mathrm{ng} / \mathrm{g}$ in $\mathrm{HCl}, 0.05 \mathrm{ng} / \mathrm{g}$ in $\mathrm{HF}$ and $0.008 \mathrm{ng} / \mathrm{g}$ in $\mathrm{H}_{2} \mathrm{O}$. 


\section{Mass Spectrometers}

Isotopic measurements were made on NBS constructed solid sample, single focusing thermal ionization mass spectrometers. Detailed descriptions of these spectrometers have been discussed in other publications [6$8]$.

\section{Procedure}

An appropriate sample size was weighed, usually $1 \mathrm{~g}$, and transferred to a Teflon beaker. A weighed aliquot of separated ${ }^{206} \mathrm{~Pb}$ isotope solution was added to each sample using a syringe [9]. The samples were then decomposed by heating with suitable acids, $\mathrm{HNO}_{3}$ and $\mathrm{HClO}_{4}$ and in addition, HF for some samples. The samples were evaporated to dryness, the residue was dissolved in $1.5 \mathrm{~N} \mathrm{HCl}$ and the lead was separated from most elements using a small anion exchange column. After evaporating to dryness with a few drops of $\mathrm{HClO}_{4}$ and $\mathrm{HNO}_{3}$ the residue was dissolved in dilute $\mathrm{HClO}_{4}$ and the lead was electrodeposited onto high purity platinum wire as a final purification step. The $\mathrm{PbO}_{2}$ was dissolved off the platinum electrode and the solution was evaporated to dryness. The ${ }^{206} \mathrm{~Pb} /{ }^{208} \mathrm{~Pb}$ ratio was then determined by mass spectrometry using silica gel-phosphoric acid as an ionization enhancing agent [10]. Each step of the mass spectrometric analysis was rigorously controlled in order to obtain the highest accuracy. Samples of Standard Reference Material (SRM) 981 (Common Lead) were used to determine the mass spectrometer bias and to ensure that the mass spectrometer was operating correctly. The lead concentration in the sample was then calculated.

\section{Results and Discussion}

\section{A. Isotopic COMPosition}

Separate samples of each material, without any ${ }^{206} \mathrm{~Pb}$ solution being added, were carried through the procedure and the isotopic composition of the lead in each material was determined. The isotopic composition of lead can vary from one material to another and must be determined for each material. Table 1 shows the variations in isotopic composition that were found on lead from four different sources. An error of several per- 
cent could be introduced if these variations were not taken into consideration.

TABLE 1. Isotopic composition of lead in four samples

\begin{tabular}{lrrrr}
\hline \multicolumn{1}{c}{ Isotope } & Coal & Fuel oil & Fly ash & Tuna \\
\hline${ }^{208} \mathrm{~Pb}$ atom \% & 52.217 & 51.975 & 52.025 & 52.134 \\
${ }^{207} \mathrm{~Pb}$ atom \% & 21.276 & 21.268 & 21.034 & 21.515 \\
${ }^{206} \mathrm{~Pb}$ atom \% & 25.146 & 25.340 & 25.598 & 24.966 \\
${ }^{204} \mathrm{~Pb}$ atom \% & 1.361 & 1.417 & 1.343 & 1.386 \\
${ }^{208} \mathrm{~Pb} /{ }^{206} \mathrm{~Pb}$ & 2.077 & 2.051 & 2.032 & 2.088
\end{tabular}

\section{B. BLANKS}

Three blanks for each set of samples were carried through the same procedure used for the samples. The lead blanks for these materials ranged from 2 to $8 \mathrm{ng}$ and represented from 0.02 to 10 percent of the lead in the sample. The precision of an analysis can be no better than the uncertainty in the blank determination. The largest range for any set of three blanks in this study was $2.5 \mathrm{ng}$. Great care must be taken to achieve a low lead blank. Some of the precautions used in this study are: using a Class 100 environment to reduce particulate contamination; using high purity platinum wire (SRM 680) for electrodes in the electrodeposition; keeping a separate set of beakers, ion exchange columns, and platinum wires for blank, isotopic composition and isotope dilution samples; rigorously cleaning beakers, platinum wires and ion exchange columns before use; and using only high purity reagents. These precautions have resulted in reducing the lead blanks from greater than one microgram to a few nanograms.

\section{Lead Concentration}

Table 2 shows that IDMS can be quite precise as well as accurate. These aqueous lead nitrate solutions were prepared for use in developing a clinical reference method for lead analysis in blood. The 95 percent confidence limit for the three solutions is 0.12 percent and the average values checked the known concentration to within 0.10 percent. The second step in this clinical program was to distribute three samples of porcine blood in order to evaluate various methods by using IDMS values as the benchmark values. Table 3 shows the values obtained on these samples 
TABLE 2. Analysis of known aqueous lead solutions

\begin{tabular}{lrrr}
\hline \multicolumn{1}{c}{ Solution } & $\begin{array}{c}1 \\
\mu \mathrm{g} / \mathrm{g}\end{array}$ & $\begin{array}{c}2 \\
\mu \mathrm{g} / \mathrm{g}\end{array}$ & $\begin{array}{c}3 \\
\mu \mathrm{g} / \mathrm{g}\end{array}$ \\
& & & \\
& 0.13923 & 0.49164 & 0.89766 \\
& .13915 & .49194 & .89699 \\
& .13928 & .49159 & .89795 \\
& .13910 & .49151 & .89774 \\
& .13918 & .49210 & .89764 \\
& .13917 & .49184 & .89705 \\
Average & 0.13918 & 0.49177 & 0.89750 \\
Standard deviation & .00006 & .00023 & .00039 \\
$95 \%$ C.L. & .00016 & .00059 & .00100 \\
Known value & .13910 & .49162 & .89666
\end{tabular}

a High purity lead metal was used to prepare these solutions.

Table 3. Lead concentration in Porcine's blood

\begin{tabular}{|c|c|c|c|}
\hline Bottle & Aliquot & $\begin{array}{l}\text { Lead } \\
\mu \mathrm{g} / \mathrm{g}\end{array}$ & $\begin{array}{c}\text { Average } \\
\mu \mathrm{g} / \mathrm{g}\end{array}$ \\
\hline 7 & $\begin{array}{l}1 \\
2\end{array}$ & $\begin{array}{r}0.7847 \\
.7840\end{array}$ & \\
\hline 9 & $\begin{array}{l}1 \\
2\end{array}$ & $\begin{array}{l}.7748 \\
.7752\end{array}$ & 0.7797 \\
\hline 23 & $\begin{array}{l}1 \\
2\end{array}$ & $\begin{array}{l}.3244 \\
.3215\end{array}$ & \\
\hline 27 & $\begin{array}{l}1 \\
2\end{array}$ & $\begin{array}{l}.3191 \\
.3228\end{array}$ & .3220 \\
\hline 12 & $\begin{array}{l}1 \\
2\end{array}$ & $\begin{array}{l}.0320 \\
.0328\end{array}$ & \\
\hline 20 & $\begin{array}{l}1 \\
2\end{array}$ & $\begin{array}{l}.0293 \\
.0266\end{array}$ & .0302 \\
\hline
\end{tabular}

using two aliquots from each of two bottles for each lead concentration level. The highest level shows good agreement between aliquots but some difference between bottles. The results for the other two levels show what may be a few nanograms variation in the lead blank or some inhomogeneity of the blood. 
Table 4 shows the results on four different types of samples. The coal and tuna samples show inhomogeneity for lead. The results for the fly ash samples show a slight inhomogeneity and for the fuel oil the lead concentration is at a level where blank variation could cause most of this spread in results.

TABLE 4. Lead concentration in four materials

\begin{tabular}{|c|c|c|c|c|}
\hline Material & $\begin{array}{l}\text { Coal } \\
\mu \mathrm{g} / \mathrm{g}\end{array}$ & $\begin{array}{c}\text { Fuel oil } \\
\mu \mathrm{g} / \mathrm{g}\end{array}$ & $\begin{array}{c}\text { Fly ash } \\
\mu \mathrm{g} / \mathrm{g}\end{array}$ & $\begin{array}{l}\text { Tuna } \\
\mu \mathrm{g} / \mathrm{g}\end{array}$ \\
\hline & $\begin{array}{l}32.90 \\
25.99 \\
30.22 \\
24.22 \\
27.23 \\
35.60 \\
26.13 \\
32.94 \\
31.34\end{array}$ & $\begin{array}{r}0.0403 \\
.0442 \\
.0396\end{array}$ & $\begin{array}{l}70.95 \\
71.01 \\
71.30 \\
71.11\end{array}$ & $\begin{array}{r}0.490 \\
.743 \\
.457 \\
.456 \\
.494 \\
.498 \\
.402 \\
.424 \\
.447 \\
.492\end{array}$ \\
\hline $\begin{array}{l}\text { Average } \\
\text { Range }\end{array}$ & $\begin{array}{l}29.6 \\
11.4\end{array}$ & $\begin{array}{r}0.0414 \\
.0046\end{array}$ & $\begin{array}{r}71.09 \\
0.35\end{array}$ & $\begin{array}{r}0.49 \\
.34\end{array}$ \\
\hline
\end{tabular}

Lead analysis by IDMS can be accurate and precise to within 0.15 percent. This method is quite useful for homogeneity studies and in providing a lead value for SRM's or benchmark values in method development.

\section{References}

[1] Barnes, I. L., Garner, E. L., Gramlich, J. W., Moore, L. J., Murphy, T. J., Machlan, L. A., Shields, W. R., Tatsumoto, M., and Knight, R. J., The Determination of Lead, Uranium, Thorium and Thallium in Silicate Glass Standard Materials by Isotope Dilution Mass Spectrometry, Anal. Chem. 45, 880-85 (1975).

[2] Moore, L. J. and Machlan, L. A., High Accuracy Determination of Calcium in Blood Serum by Isotope Dilution Mass Spectrometry, Anal. Chem. 44, 2291-96 (1972).

[3] Moore, L. J., Machlan, L. A., Shields, W. R., and Garner, E. L., Internal Normalization Techniques for High Accuracy Isotope Dilution Analyses-Application to Molybdenum and Nickel in Standard Reference Materials, Anal. Chem. 46, 1082-89 (1974).

[4] "Federal Standards," Circular 209d, GSA Business Service Center, Boston, Mass. (1966). 
[5] Kuehner, E. C., Alvarez, R., Paulsen, P. J., and Murphy, T. J., The Production and Analysis of Acids Purified by Sub-Boiling Distillation, Anal. Chem. 44, 2050-56 (1972).

[6] Shields, W. R., Ed., Analytical Mass Spectrometry Section: Instrumentation and Procedures for Isotopic Analysis, U.S. National Bureau of Standards Technical Note 277, 1-99, U.S. Government Printing Office, Washington, D.C. 20402 (1966).

[7] Shields, W. R., Ed., Analytical Mass Spectrometry Section: Summary of Activities July 1966 to June 1967, U.S. National Bureau of Standards Technical Note 426, 1 53, U.S. Government Printing Office, Washington, D.C. 20402 (1967).

[8] Shields, W. R., Ed., Analytical Mass Spectrometry Section: Summary of Activities July 1967 to June 1968, U.S. National Bureau of Standards Technical Note 456, 147, U.S. Government Printing Office, Washington, D.C. 20402 (1968).

[9] Shields, W. R., Ed., Analytical Mass Spectrometry Section: Summary of Activities July 1969 to June 1970, U.S. National Bureau of Standards Technical Note 546, 1110, U.S. Government Printing Office, Washington, D.C. 20402 (1970).

[10] Barnes, I. L., Murphy, T. J., Gramlich, J. W., and Shields, W. R., Lead Separation by Anodic Deposition and Isotope Ratio Mass Spectrometry of Microgram and Smaller Samples, Anal. Chem. 45, 1881-84 (1973). 



\title{
RARE EARTH ELEMENT ANALYSIS BY ISOTOPE DILUTION
}

\author{
Gilbert N. Hanson
}

\author{
Department of Earth and Space Sciences \\ State University of New York \\ Stony Brook, New York 11794 U.S.A.
}

At Stony Brook we have been analyzing igneous rocks, granites through gabbros, basalts, and ultramafic rocks for rare earth elements (REE) as well as the other major, minor, and trace elements in order to determine the origin of these rocks utilizing quantitative trace element models. For rare earths we are using the analytical procedure of J. G. Arth, USGS, Reston, Virginia, which utilizes two Dowex 50Wx8 cation columns, one for nitric acid to get a group separation of the REE and the other for hydrochloric acid to separate the specific REE. The rare earth elements La, Ce, Nd, Sm, Eu, Gd, Dy, Er, Yb, and Lu can usually be analyzed in three to four mass spectrometric runs with a total time for analysis of about 8 hours. The number of REE analyzed should not be reduced or the great advantage of the REE, the regular but not consistent variation from element to element, cannot be fully utilized in an interpretation. Precision is about 1 percent for all elements except for $\mathrm{Lu}$ and $\mathrm{Gd}$. The uncertainty in the accuracy is somewhat greater.

Much of the REE data in the literature has uncertainties of about 5 percent or greater, and for gross comparison of rock types this data is adequate. REE data, however, with uncertainties much greater than 10 percent are no longer acceptable, as they are not useful even for gross comparisons of rock types.

In a detailed study of a magmatic sequence in order to distinguish differentiated fractions versus different original parents and to determine which residual or fractionating minerals are involved, then high precision on the order of 1 percent is absolutely necessary. In such a sequence, since it is only necessary to compare samples with each other, high precision is adequate as one can normalize all samples to a sample in the sequence. In order to compare data with other laboratories, however, high accuracy is also necessary. The uncertainty in the accuracy is mainly in not having adequate rare earth standards.

Keywords: Analytical techniques; fractional crystallization; geochemistry; isotope dilution; partial melting; rare earth elements: trace element models; volcanic rocks. 


\section{Introduction}

The derivation of quantitative trace element models using mineral/melt distribution coefficients for partial melting and for fractional crystallization processes has changed rare earth element (REE) geochemical investigations from comparative studies of rock types and minerals to quantitative studies relating to the genesis of igneous rocks $[1,5,7,20,23]$. The REE are particularly good for these trace element studies, because they are geochemically similar, but different enough so that the various residual minerals in partial melting or fractional crystallization processes affect the REE patterns of the resulting melts in different, but interpretable ways. Thus, since the late 1960's there has been intensive analysis of $\mathrm{REE}$ for genetic interpretations of both lunar and terrestrial rocks as well as a strong emphasis on determinations of mineral/melt distribution coefficients $\left(\mathrm{K}_{d}\right)$ for REE in rocks and by laboratory experiments. Articles reviewing REE geochemistry include Haskin et al. [8], Haskin and Schmitt [9], and Haskin et al. [10].

In order to compare REE in different rocks on a simple graph and to eliminate the Oddo-Harkins effect resulting in high concentrations for the even and low concentrations for the odd atomic numbered REE, concentrations for REE in rocks are generally normalized to chondrites. Four values used for normalizing are shown in table 1. The first three sets of REE values for chondrites in table 1 are similar and variations are a result of variability within the chondrites and interlaboratory precision and calibration. Masuda, Nakamura and Tanaka [14] review the REE analyses of chondrites in the literature and present new high precision, high accuracy REE analyses for chondrites. They recommend that the Leedey chondrite be used for normalization. As can be seen in table 1 the Leedey chondrite has a 20 to 25 percent higher REE content than the three other values used, except for La and Lu which are only about 15 and 10 percent higher respectively.

\section{A. Analytical Techniques}

All but four of the naturally occurring REE can be analyzed by isotope dilution (fig. 1). Pm has no stable isotopes and does not occur in nature. $\mathrm{Pr}, \mathrm{Tb}, \mathrm{Ho}$, and $\mathrm{Tm}$ have only one stable isotope each and cannot be analyzed by isotope dilution. Sm and $\mathrm{Nd}$ have neither interfering elements nor oxides. If $\mathrm{Ba}$ is separated from the sample, $\mathrm{Eu}$ is also easily analyzed. All of the other REE, however, have rare earth element or rare earth oxide interferences and good chemical separation is necessary for their 
TABLE 1. Rare-earth element concentration values for chondrites used for normalizing

\begin{tabular}{lcccc}
\hline & \multicolumn{4}{c}{ Concentration, $\mu \mathrm{g} / \mathrm{g}$} \\
Element & Lab 1 & Lab 2 & Lab 3 & Lab 4 \\
\cline { 2 - 5 } & & & & \\
\hline La & 0.325 & 0.330 & - & 0.378 \\
$\mathrm{Ce}$ & .798 & .880 & 0.787 & .976 \\
$\mathrm{Nd}$ & .567 & .60 & .580 & .716 \\
$\mathrm{Sm}$ & .186 & .181 & .185 & .230 \\
$\mathrm{Eu}$ & .0692 & .069 & .071 & .0866 \\
$\mathrm{Gd}$ & .255 & .249 & .256 & .311 \\
$\mathrm{Dy}$ & .305 & .200 & .303 & .390 \\
$\mathrm{Er}$ & .209 & .200 & .182 & .255 \\
$\mathrm{Yb}$ & $.210^{\mathrm{a}}$ & .034 & .038 & .249 \\
$\mathrm{Lu}$ & .0349 & & .0387
\end{tabular}

${ }^{a}$ Recalibration of tracer concentration shows that the previous value of $0.231 \mu \mathrm{g} / \mathrm{g}$ for $\mathrm{Yb}$ should be reduced 9 percent to $0.210 \mu \mathrm{g} / \mathrm{g}$ (N. Hubbard, oral communication, 1974). Notes:

1. Chondrite composite CCS-3 from R. Schmitt, analyzed by Hubbard and Gast, 1971, precision and accuracy is 3 percent or less [11].

2. Composite of nine chondrites, Haskin et al., 1968, precision and accuracy is 5 to 15 percent [10].

3. Chondrite normalizing values of Philpotts, Martin and Schnetzler, 1971, precision and accuracy 5 percent or less except for Lu which is 10 to 15 percent [17].

4. Leedy chondrite, Masuda, Nakamura, and Tanaka, 1973, precision and accuracy is 2 percent or less [14].

analysis. It is sufficient to separate the REE into a heavy REE fraction, light REE fraction, a $\mathrm{Ce}$ fraction free of $\mathrm{Nd}$, and a $\mathrm{La}$ fraction free of $\mathrm{Ce}$ and $\mathrm{Ba}$. After the rock or silicate mineral is dissolved in $\mathrm{HF}$ and $\mathrm{HClO}_{4}$, the REE are eluted on a column employing Dowex $50 \mathrm{Wx} 8$ cation resin with $\mathrm{HNO}_{3}$ or $\mathrm{HCl}$ in one or two steps [ see for example 12,13,22]. At Stony Brook we use a chemical technique modified by J. G. Arth, U.S. Geological Survey (1973, written communication) using two separate columns. The sample is first loaded on a $1 \times 20 \mathrm{~cm}$ column prepared with $2 \mathrm{~N} \mathrm{HNO}_{3}$ acid to separate the major elements, $\mathrm{Ba}$ and $\mathrm{Sr}$ from the REE, which are separated as a group with $6 \mathrm{~N} \mathrm{HNO}_{3}$. The REE are then separated into fractions on a $1 \times 27 \mathrm{~cm}$ column using $3 N \mathrm{HCl}$.

We run the REE except La using rhenium triple filaments with the center filament temperature at $2000^{\circ} \mathrm{C}$ for the light REE and $2100{ }^{\circ} \mathrm{C}$ for the heavy REE. Because of the small enrichment of ${ }^{138} \mathrm{La}$ tracer and because of the possible interference of ${ }^{138} \mathrm{Ce}$ and ${ }^{138} \mathrm{Ba}$, La is analyzed as $\mathrm{LaO}^{+}$on a single filament of rhenium. The heavy and light REE mass spectrometer runs require monitoring of possible interfering elements or oxides. This is not as difficult as it may seem because the differing work 


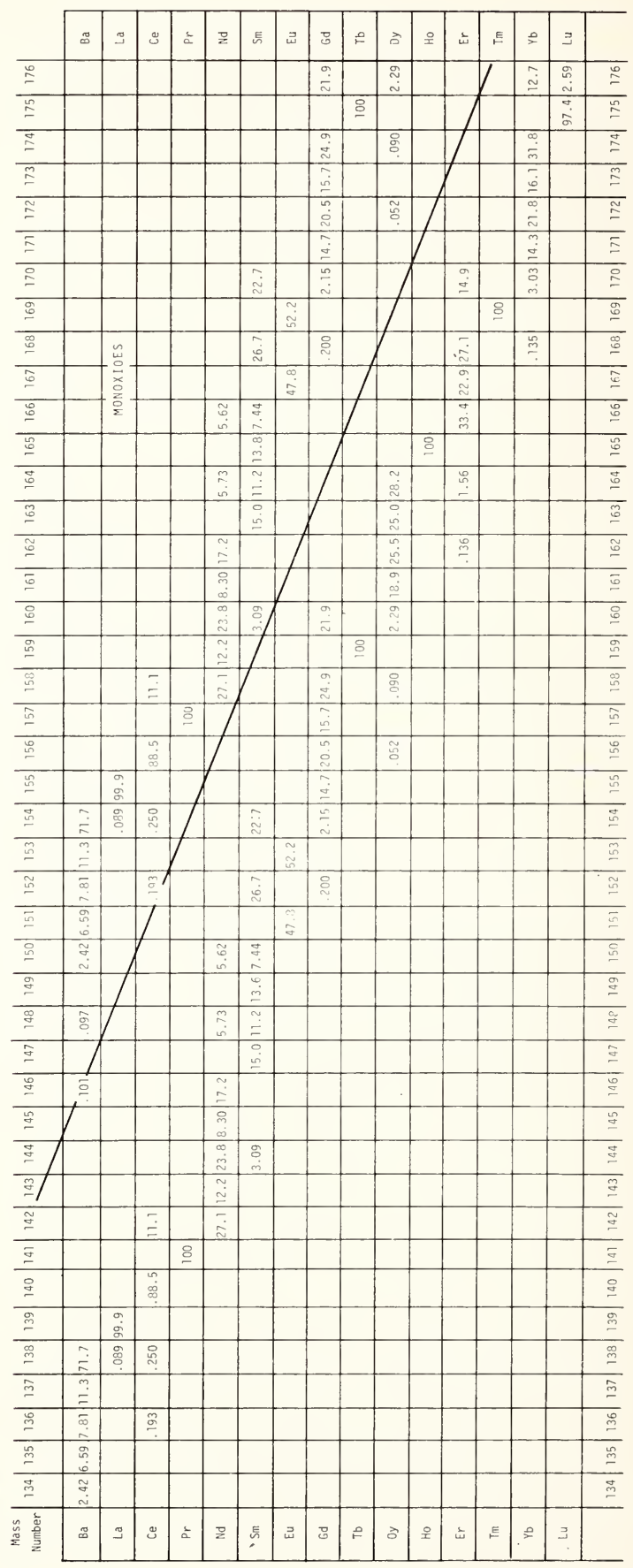

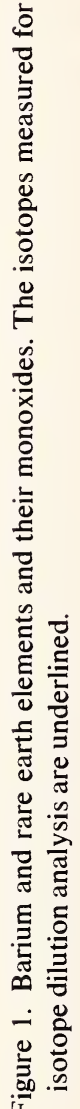


functions allow an element either to be analyzed before it is interfered with by another element or oxide; or to be burned off before the element with which it may interfere is analyzed. Usually the heavy REE fraction, including $\mathrm{Eu}$ and the heavier elements, can be made in a run of less than 5 hours - it is important to have a source pressure of less than $4 \times 10^{-7}$ torr to reduce oxide interferences. A gauss meter is extremely useful for locating the various isotopes and makes it unnecessary to memorize all of the REE isotopes and the effects of adding isotopically enriched tracers.

$\mathrm{Gd}$ and Lu may present problems during analysis. Gd along with $\mathrm{Sm}$, is very important in determining the extent of the Eu anomaly. However, Gd may be difficult to measure because of oxide interferences from La and $\mathrm{Pr}$ and the relatively poor ionization of Gd. Oxide interferences can be reduced using the procedure recommended by $\mathrm{N}$. Hubbard (personal communication NASA, JSC Houston). In this procedure after the more easily ionized REE have been analyzed and the source pressure has reached about $1 \times 10^{-7}$ torr, the liquid nitrogen is removed from the cold finger. After 5 minutes liquid nitrogen is added to the cold finger and $\mathrm{Gd}$ is analyzed immediately. This procedure is repeated until Gd gives the same ratio before and after outgassing. For Lu analysis it is generally very difficult to eliminate the interference of $\mathrm{Yb}$, which is easily ionized. Because of this interference, possible interferences of oxides of Dy, Tb, and Gd, and particularly because of its relatively low abundance, some laboratories do not analyze for $\mathrm{Lu}$.

\section{B. Application of Trace Element Models to ReE}

In applying partial melting or differentiation models in trace element studies it is necessary to know mineral/melt distribution coefficients which are determined either experimentally or on volcanic rocks with a finegrained or glassy matrix and coarser grained minerals (phenocrysts). A relatively large number of distribution coefficients $\left(\mathrm{K}_{d}\right)$ for REE for various minerals are available in the literature and typical examples are shown in figure 2. Although the effects on the distribution coefficients of temperature, pressure or the major element composition of either the minerals and melt are poorly understood, it is still possible to use the quantitative models to great advantage because the patterns for the distribution coefficients are similar for a given mineral even though the values for the distribution coefficients for the individual REE may vary greatly. Thus, although it may not be possible to determine the exact percentage of a mineral left in the residue, it is possible to determine whether that mineral played a significant role in the genesis of the igneous rock studied. If a 


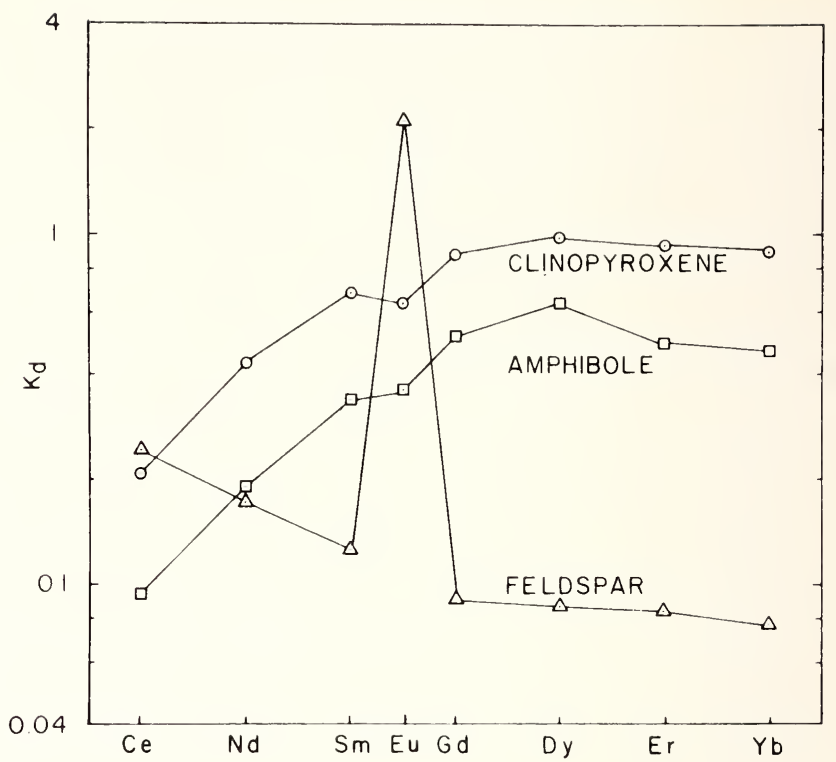

Figure 2. Representative distribution coefficients $\left(\mathbf{K}_{d}\right)$ for clinopyroxene, amphibole and feldspar (plagioclase) from Schnetzler and Philpotts [21].

suite of differentiated volcanic rocks is studied, analysis of phenocrysts and matrix in the volcanics make it possible to determine the distribution coefficients for the minerals involved in fractional crystallization.

The effects on the trace element concentration of melts derived by different extents of a simple partial melting and fractional crystallization (assuming an approach to equilibrium) for various bulk distribution coefficients, D, are shown in figures 3 and 4 . An important consideration for partial melting processes (assuming the melt is in near equilibrium with the residuum) is that when a mineral is no longer present in the residuum due to reaction or melting, the trace element concentration of the melt reflects only the minerals remaining in the residuum and not those that are no longer present. Thus, the trace element concentration of a melt reflects only the mineralogy of the residuum at the time the residuum and melt separate. This is not true, however, of fractional crystallization processes.

For either model if the minerals in the residue have no affinity for the element under consideration, i.e., $\mathrm{D}$ approaches $\mathrm{O}$, then the concentration of the trace element in the melt relative to the concentration in the parent rock or melt, $\mathrm{C}_{l} / \mathrm{C}_{o}$ approaches $1 / \mathrm{F}$. The element goes completely into the melt, therefore the more melt the more dilution of the element. Thus the enrichment of a trace element with a very small $\mathrm{D}$ gives the extent of par- 


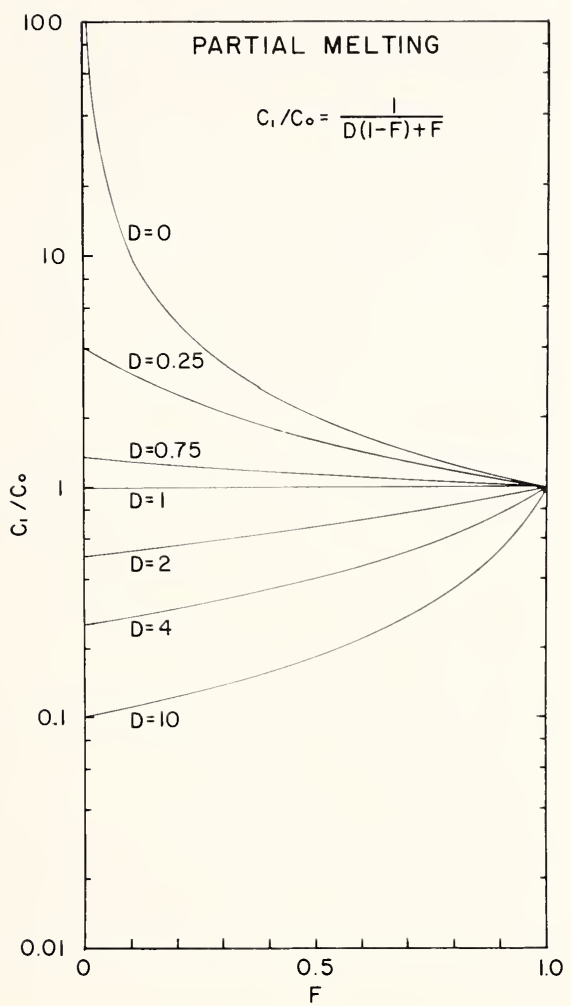

Figure 3. The concentration of a trace element in a melt relative to its concentration in the parent rock, $\mathrm{C}_{l} / \mathrm{C}_{o}$, versus the fraction of partial melting, $\mathrm{F}$. The calculation assumes the melt stays in equilibrium with the residual minerals until the melt separates from the residue. $\mathrm{D}$ is the bulk distribution coefficient for the minerals in the residue, $\mathrm{D}=\Sigma \mathrm{X}^{i} \mathrm{~K}_{d^{i / l}}$. $\mathrm{X}^{i}$ is the weight fraction of each mineral, $\mathrm{K}_{d}{ }^{i / l}$ is the mineral/melt distribution coefficient (weight), $i$ is the mineral, $l$ the melt. This calculation is based on a modal melting model for partial melting, in which D remains constant during melting, presented by Shaw [23]. 


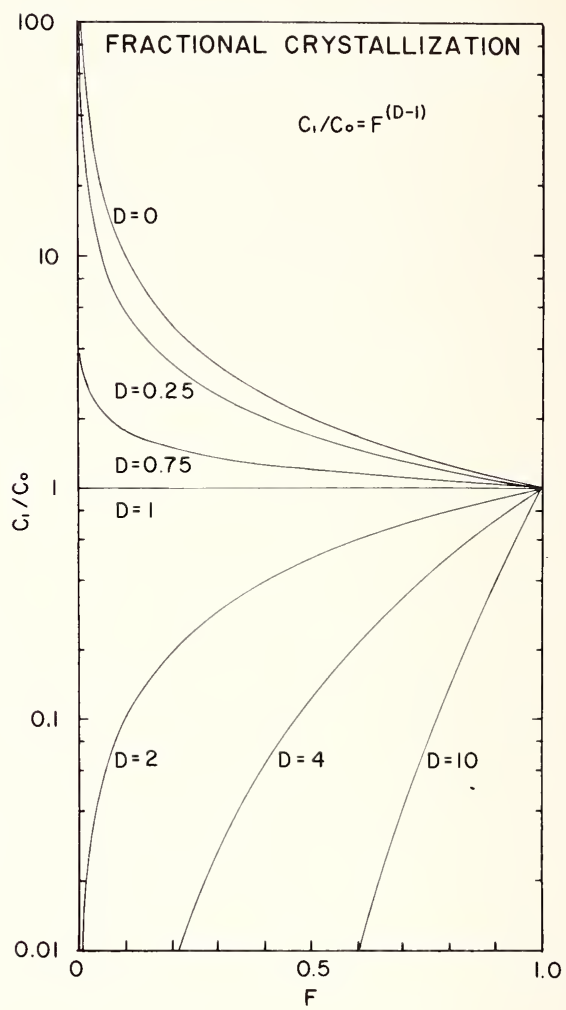

Figure 4. The concentration of a trace element in a melt relative to its concentration in the parent melt, $\mathrm{C}_{l} / \mathrm{C}_{o}$, versus the weight fraction of melt remaining during fractional crystallization, $\mathrm{F}$ for fixed bulk distribution coefficients, D. This model is based on the Rayleigh [19] fractionation law.

tial melting or fractional crystallization, if the concentration of the parent is known.

If $\mathrm{D}$ for a given trace element is about 1 or greater the concentration of that element varies by less than 30 percent for up to 25 percent melting. If only one mineral has a large $K_{d}$ for the given element, and its $K_{d}$ is known, the percent of that mineral in the residue can be calculated. For a large $\mathrm{D}$, $\mathrm{C}_{l} / \mathrm{C}_{0}$ approaches $1 / \mathrm{D}$ and $\mathrm{D} \simeq \mathrm{X}^{a} \mathrm{~K}_{d}{ }^{a / l}$. Thus $\mathrm{C}_{l} / \mathrm{C}_{0} \simeq 1 / \mathrm{X}^{a} \mathrm{~K}_{d}^{a / l}$. Whereas in fractional crystallization, because the minerals are continually being removed from the melt, trace elements with large D's will be strongly depleted with a small percent of fractional crystallization. Thus, if in a series of related igneous rocks the concentration of a trace element presumed to have a large D stays nearly constant the process is probably partial melting. If the trace element's concentration varies strongly with 
a good correlation with the major element composition the process is probably fractional crystallization.

To show actual REE data and to show the effect of uncertainties on the interpretation of the data, REE concentrations normalized to chondrites of selected samples of a differentiated volcanic sequence from Ross Island, Antarctica are presented in figure 5 [24]. There is a factor of 55 difference between the smallest heavy REE concentration and the largest light REE concentration whereas the maximum range for any one REE is a factor of 4 . In order to appreciate the details and to take advantage of our 1 percent precision, figure 6 shows the same samples normalized to the basanitoid with the lowest REE concentration. Figure 6 also allows an easier comparison of the effects of minerals such as are shown in figure 2 on the REE concentration of volcanics derived by fractional crystallization from a parent basanitoid. To a first approximation the effect of the removal of a mineral on the REE pattern is the reciprocal of the mineral/melt $K_{d}$ pattern shown in figure 2 which is enhanced roughly proportional to the percent of the mineral in the residue and to the extent of fractional crystallization.

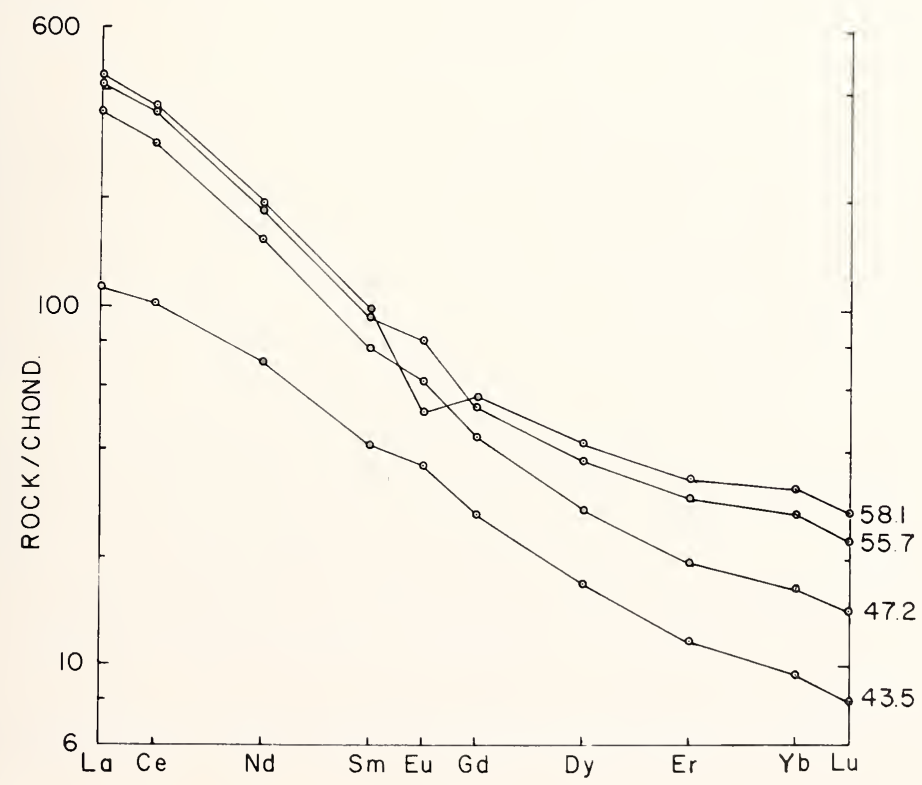

Figure 5. Rare earth elements normalized to chondrites (Hubbard and Gast [11]) for selected volcanic rocks from Ross Island, Antarctica [24]. The numbers are the weight percent silica for each sample. 


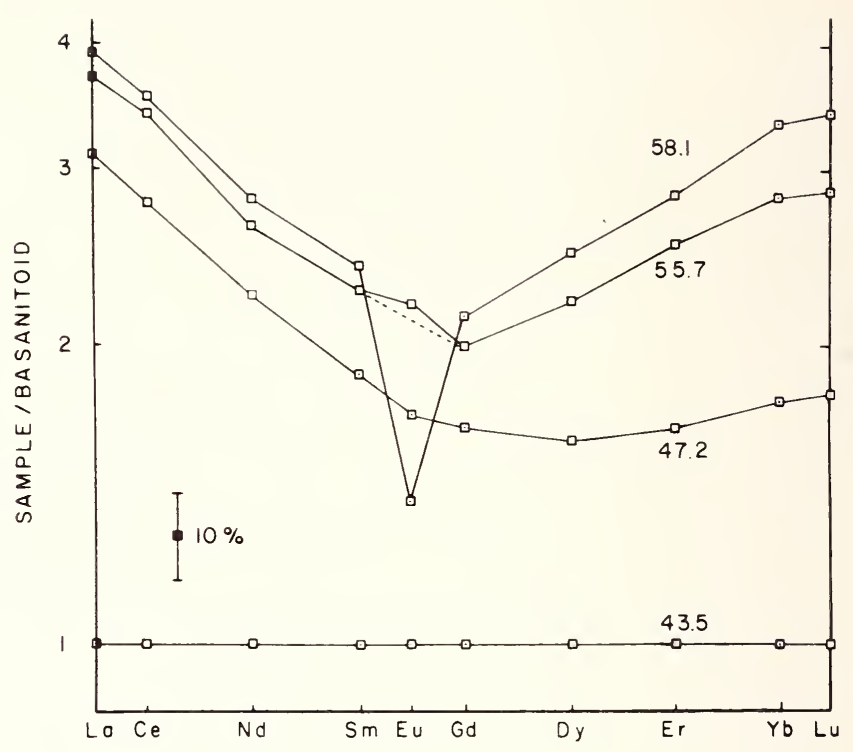

Figure 6. Rare earth elements of rocks in fig. 5 from Ross Island, Antarctica normalized to basanitoid with lowest rare earth element concentration. The precision of 1 percent is represented by the size of the symbols. For comparison of the effect of a 10 percent uncertainty in the analysis, a 10 percent error bar is also shown.

Sun and Hanson [24] have made preliminary model calculations using distribution coefficients from the literature as well as those determined on phenocrysts from the Ross Island volcanics taking into account the variations in the major element concentrations [6]. The calculations show that the REE concentrations can be derived by fractionally crystallizing clinopyroxene, amphibole, olivine, feldspar, apatite, and spinel. Except for the sample with 58.1 percent $\mathrm{SiO}_{2}$, Eu anomalies are less than 5 percent which indicates that plagioclase and anorthoclase feldspar are not major differentiating minerals. If the same data were looked at with a 10 percent rather than the actual 1 percent precision much of the details of the patterns would be lost and some gross features would be less obvious. The small positive Eu anomaly suggesting feldspar (anorthoclase) accumulation rather than precipitation for the sample with 55.7 percent, $\mathrm{SiO}_{2}$ would be indistinguishable. For all samples feldspar precipitation or accumulation of up to 10 percent of the parent liquid would be indiscernable with a 10 percent uncertainty. 


\section{REE REFEREnCE StANDARD}

Although many analysts using isotope dilution claim precision of 2 percent or less, isotope dilution analysis of the same U.S. Geological Survey standard BCR-1 by a number of selected laboratories shows maximum deviations of up to 16 percent (table 2). Gd with a maximum deviation of only 2 percent is difficult to analyze by isotope dilution because of oxide interferences (fig. 1), whereas, Sm with 11 percent maximum deviation is much easier to analyze. Lu with the 16 percent maximum deviation is difficult to analyze because of oxide and $\mathrm{Yb}$ interferences and its relatively low abundances. It would appear then that the major source of uncertainty for absolute abundances is not necessarily the analytical techniques but the lack of adequate reference standards such as are available for $\mathrm{U}, \mathrm{Pb}$, $\mathrm{Rb}, \mathrm{Sr}$ and $\mathrm{K}$ analysis by isotope dilution from the National Bureau of Standards.

TABLE 2. Comparative concentrations of rare earth elements in $B C R-1$ in $\mu g / g$ from selected laboratories

\begin{tabular}{|c|c|c|c|c|c|c|c|c|}
\hline & 1 & 2 & 3 & 4 & 5 & 6 & 7 & 8 \\
\hline $\mathrm{La}$ & - & 26.1 & - & - & 24.4 & 25.5 & - & - \\
\hline $\mathrm{Ce}$ & 53.6 & 54.9 & 54.3 & 54.3 & 54.3 & 54.4 & 52.3 & 53.9 \\
\hline $\mathrm{Nd}$ & 28.2 & 28.8 & 27.7 & 28.3 & 28.9 & 29.1 & 28.9 & 29.0 \\
\hline $\mathrm{Sm}$ & 6.60 & 6.74 & 6.38 & 6.57 & 6.73 & 6.59 & 7.11 & - \\
\hline $\mathrm{Eu}$ & 1.94 & 1.96 & 1.91 & 1.94 & 1.98 & 2.00 & 1.92 & 1.94 \\
\hline $\mathrm{Gd}$ & 6.64 & - & 6.56 & 6.60 & 6.67 & 6.79 & 6.54 & 6.6 \\
\hline Dy & 6.36 & 6.20 & 6.20 & 6.25 & 6.36 & 6.46 & 6.12 & 6.3 \\
\hline $\mathrm{Er}$ & 3.55 & 3.71 & 3.52 & 3.59 & 3.69 & 3.71 & 3.48 & 3.59 \\
\hline $\mathrm{Yb}$ & 3.40 & $3.35^{\mathrm{a}}$ & 3.35 & 3.47 & 3.39 & 3.38 & 3.42 & - \\
\hline $\mathrm{Lu}$ & 0.540 & 0.590 & 0.546 & 0.552 & 0.501 & 0.499 & - & 0.55 \\
\hline \multicolumn{9}{|c|}{$\begin{array}{l}\text { 1. Philpotts et al., 1972, I.D. Ave. of 2, NASA Goddard [18]. } \\
\text { 2. Gast et al., 1970, I.D. Ave. of 2, NASA, Houston [5]. } \\
\text { 3. Arth and Hanson, in Press, I.D. Ave. of 2, SUNY, Stony Brook [2]. } \\
\text { 4. Arth, Oral Communication, I.D. Ave. of 3, U.S.G.S. Denver. } \\
\text { 5. Nakamura and Masuda, 1973, I.D. Ave. of 2, University of Tokyo [15]. } \\
\text { 6. Sun, Simmons, Walker-I.D. Ave. of 3, SUNY, Stony Brook. } \\
\text { 7. O'Nions and Clarke, 1972, I.D., Oxford [16]. } \\
\text { 8. Recommended values of Flanagan, } 1973 \text { [3]. }\end{array}$} \\
\hline
\end{tabular}

a Recalibration of tracer concentration shows that the previous value for $\mathrm{Yb}$ should be reduced 9 percent to $3.35 \mu \mathrm{g} / \mathrm{g}$. (N. Hubbard, Oral Communication 1974). 
Rock reference samples such as BCR-1 are valuable for comparing results among laboratories, but the absolute abundances and the heterogeneity for the REE are not known. With our present understanding of REE geochemistry, the absolute concentrations of REE for a sample would not be necessary if a known homogeneous reference sample were available. Investigators of REE concentrations in rocks could then analyze the reference sample, normalize their sample concentration data to their reference sample analysis and report their concentration values for the standard, making interlaboratory comparisons at the level of laboratory precision possible. If, however, standards for spike calibration were available as well as the absolute values for a homogeneous rock standard, this would allow individual laboratories to judge the reliability of their analytical techniques.

\section{Acknowledgements}

I am gratefui to S. S. Sun, J. G. Arth, and E. C. Simmons for discussions and review. This study was supported by NSF Grants GA 3047 (Geology) and V040762 (Office of Polar Programs).

\section{References}

[1] Albarede, F. and Bottinga, Y., Kinetic Disequilibrium in Trace Element Partitioning between Phenocrysts and Host Lava, Geochim. Cosmochim. Acta 36, 141-56 (1972).

[2] Arth, J. G. and Hanson, G. N., Geochemistry and Origin of the Early Precambrian Crust of Northeastern Minnesota, Geochim. Cosmochim. Acta 39, 325-362 (1975).

[3] Flanagan, F. J., 1972 Values for International Geochemical Reference Samples, Geochim. Cosmochim. Acta 37, 1189-1200 (1973).

[4] Gast, P. W., Trace Element Fractionation and the Origin of Tholeiitic and Alkaline Magma Types, Geochim. Cosmochim. Acta 32, 1057-86 (1968).

[5] Gast, P. W., Hubbard, N. J., and Wiesman, H., Chemical Composition and Petrogenesis of Basalts from Tranquility Base, Geochim. Cosmochim. Acta, supplement 1, 2, 1143-63 (1970).

[6] Goldich, S. S., Stuckless, J. S., Suhr, N. H., and Treves, S. B., Geochemistry of Volcanic Rocks of the Ross Island Area, Antarctica, Abst. Geol. Soc. Am. 5, 638-39 (1973).

[7] Greenland, L. P., An Equation for Trace Element Distribution During Magmatic Crystallization, Am. Mineral. 55, 455-65 (1970).

[8] Haskin, L. A., Frey, F. A., Schmitt, R. A., and Smith, R. H., Meteoritic, Solar, and Terrestrial Rare-Earth Distributions in Physics and Chemistry of the Earth 7, Ahrens, L. H., Press, F., Runcorn, S. K., and Urey, H. C., Eds., pp. 169-321, Pergamon Press, Oxford (1966). 
[9] Haskin, L. A. and Schmitt, R. A., Rare-Earth Distributions, Researches in Geochemistry 2, Abelson, P. A., Ed., pp. 234-58, John Wiley and Sons, Inc., New York (1967).

[10] Haskin, L. A., Haskin, M. A., Frey, F. A., and Wildeman, T. R., Relative and Absolute Terrestrial Abundances of the Rare Earths in Origin and Distribution of the Elements, Ahrens, L. H., Ed., pp. 889-912, Pergamon Press, Oxford (1968).

[11] Hubbard, N. J. and Gast, P. W., Chemical Composition and Origin of Nonmare Lunar Basalts, Proc. Second Lunar Sci. Conf., Geochim. Cosmochim. Acta, supplement 2, 2,999-1020 (1971).

[12] Kay, R. W., The Rare Earth Geochemistry of Alkaline Basaltic Volcanics, Ph. D. thesis, Columbia Univ., 177 pages (1970).

[13] Masuda, A., Lanthanides in the Norton County Achondrite, Geochem. J. 2, 111-35 (1967).

[14] Masuda, A., Nakamura, N., and Tanaka, T., Fine Structures of Mutually Normalized Rare-Earth Patterns of Chondrites, Geochim. Cosmochim. Acta 37, 239-48 (1973).

[15] Nakamura, N. and Masuda, N., Chondrites with Peculiar Rare-Earth Patterns, Earth and Planet. Sci. Letters 19, 429-37 (1973).

[16] O'Nions, R. K. and Clarke, D. B., Comparative Trace Element Geochemistry of Tertiary Basalts from Baffin Bay, Earth and Planet. Sci. Letters 15, 436-46 (1972).

[17] Philpotts, J. A., Martin, W., and Schnetzler, C. C., Geochemical Aspects of Some Japanese Lavas, Earth and Planet. Sci. Letters 12, 89-96 (1971).

[18] Philpotts, J. A., Schnetzler, C. C., Bottino, M. L., Schuman, S., and Thomas, H. H., Luna 16: Some Li, K, Rb, Sr, Ba, Rare Earth, $\mathrm{Zr}$, and $\mathrm{Hf}$ Concentrations, Earth and Planet. Sci. Letters 13, 429-35 (1972).

[19] Rayleigh, J. W. S., Theoretical Considerations Respecting the Separation of Gases by Diffusion and Similar Processes, Phil. Mag. 42, 77-107 (1896).

[20] Schilling, J. G. and Winchester, J. W., Rare-Earth Fractionation and Magmatic Processes in Mantles of the Earth and Terrestrial Planets, Runcorn, S. K., Ed., pp. 267-83, Interscience Publishers, John Wiley and Sons, London (1967).

[21] Schnetzler, C. C. and Philpotts, J. A., Partition Coefficients of Some Rare-Earth Elements between Igneous Matrix Material and Rock-Forming-MineralPhenocrysts-II, Geochim. Cosmochim. Acta 34, 331-40 (1970).

[22] Schnetzler, C. C., Thomas, H. H., and Philpotts, J. A., Determinations of Rare Earth Elements in Rocks and Minerals by Mass Spectrometric, Stable Isotope Dilution Technique, Anal. Chem.39, 1888-90 (1967).

[23] Shaw, D. M., Trace Element Fractionation During Anatexis, Geochim. Cosmochim. Acta 34, 237-43 (1970).

[24] Sun, S. S. and Hanson, G. N., Genesis of McMurdo Volcanics on Ross Island based on Rare Earth Elements and Lead Isotopes, Antarctic J. of the United States 9, 234236 (1974). 



\title{
AN ACCURATE DETERMINATION OF ELECTROLYTE CONCENTRATIONS IN BLOOD SERUM BY ISOTOPE DILUTION MASS SPECTROMETRY
}

\author{
E. L. Garner, L. A. Machlan, J. W. Gramlich, \\ L. J. Moore, T. J. Murphy, and I. L. Barnes \\ Analytical Chemistry Division \\ Institute for Materials Research \\ National Bureau of Standards \\ Washington, D.C. 20234 U.S.A.
}

\begin{abstract}
Concentrations of the electrolytes $\mathrm{Li}, \mathrm{Mg}, \mathrm{Cl}, \mathrm{K}$ and $\mathrm{Ca}$ were determined in three different lots of blood sera by isotope dilution mass spectrometry. Descriptions of the chemistry, mass spectrometry, and some of the precautions necessary to obtain an accurate measurement are given. An internal normalization technique which eliminates a systematic bias component due to variable isotopic fractionation between different analyses, was used to significantly improve the accuracy of the magnesium measurement. The experimental values for the three lots of sera followed a pattern of low, intermediate and high concentration levels for each electrolyte. The magnesium values in $\mu \mathrm{g} / \mathrm{g}$, for example were $8.540 \pm 0.064,24.52 \pm 0.08$, and $41.27 \pm 0.10$. The estimated limit of error for a single analysis, based on the 95 percent confidence limit and the effect of sample impurities, ranged from 0.75 percent for magnesium in one lot of serum to 0.2 percent for the potassium concentrations.
\end{abstract}

Keywords: Accurate; blood; concentrations; determination; dilution; electrolyte; isotope; mass spectrometry; serum.

\section{Introduction}

The basic principles of isotope dilution mass spectrometry have been described [1] and also well established by extensive research in the geochronology of terrestrial and extra-terrestrial materials. The most attractive feature of this analytical technique is applicability to a variety of sample matrices with high accuracy from the nanogram/gram to the per- 
cent concentration range. At the nanogram level the limiting factor on accuracy is the blank, while in the macro range variability of the isotopic fractionation is the limiting factor.

Applicability of the technique is generally restricted to the ability to dissolve the sample and to thermally ionize the analyte during mass spectrometric analysis. While sample dissolution and thermal ionization of the analyte are necessary conditions, they are not sufficient for high accuracy measurements. Other critical factors are loss of sample or separated isotope (spike) before mixing and equilibration, loss of analyte after equilibration, separation of the analyte free of matrix interferences, calibration of the concentration of the spike solution and precise measurement of the isotopic composition of the spike, analyte, and isotope dilution (ID) samples. Because different sample matrices frequently present different problems at various steps in the procedure, research is frequently required to locate and eliminate these problems in both the chemistry and mass spectrometry. Preliminary investigations, especially for a new or different sample matrix, are a first step in an accurate concentration determination.

\section{Experimental}

\section{A. Chemical Facility}

Since environmental particulate matter is a major source of contamination, a clean room facility is a prerequisite for accurate isotope dilution, especially at or below the microgram level. Efforts to minimize particulate contamination must start in the chemical preparation and continue through the mass spectrometric analysis. All chemistry was performed in a specially designed vertical flow clean room.

\section{B. INSTRUMENTATION}

Isotopic measurements were made on four NBS constructed, solid sample, single focusing, thermal ionization mass spectrometers. Essential detailed descriptions of these instruments have been published $[2,3]$. 


\section{ReAgents}

High purity reagents are necessary to keep the reagent contribution to the blank at a minimum. The concentration of most elements in high purity reagents prepared at NBS by sub-boiling distillation in quartz or Teflon stills is less than $1 \mathrm{ng} / \mathrm{g}$ [4]. The entire distillation apparatus is located in a Class 100 clean air environment.

\section{Chemical Procedure}

\section{Multiple Spike}

The number of samples and the random selection of ampoules to be sampled were determined by a statistical method. A $5 \mathrm{~g}$ sample was taken from each of six ampoules for each of the three lots of serum. The method of adding the spike to the sample was weight aliquoting [5,7]. This method of aliquoting is accurate to within 0.01 percent and the effect on the concentration calculations is negligible. The serum samples were spiked with separate weighed aliquots of ${ }^{6} \mathrm{Li},{ }^{41} \mathrm{~K},{ }^{26} \mathrm{Mg}$ and ${ }^{44} \mathrm{Ca}$ solutions. Five $\mathrm{g}$ of $\mathrm{HNO}_{3}$ and $5 \mathrm{~g}$ of $\mathrm{HClO}_{4}$ were added to the sample containers which were covered and heated at a low temperature until the serum was digested. The containers were uncovered, the solutions evaporated to dryness, and the residues, taken up in $\mathrm{H}_{2} \mathrm{O}$, were added to a cation exchange column, $10 \times 0.7 \mathrm{~cm}$, filled with Bio Rad AG 50W-X8 resin. Prior to loading the column, precleaning was accomplished using $55 \mathrm{ml}$ of $6 \mathrm{~mol} / 1 \mathrm{HCl}$ and rinsing with water. The $\mathrm{Li}$ and $\mathrm{K}$ were eluted in separate fractions using $0.3 \mathrm{~mol} / 1 \mathrm{HCl}$, the $\mathrm{Mg}$ was eluted with $0.5 \mathrm{~mol} / \mathrm{l} \mathrm{HCl}$ and the $\mathrm{Ca}$ was eluted with $6 \mathrm{~mol} / \mathrm{l} \mathrm{HCl}$. The fractions were evaporated to dryness, heated with a small volume of $\mathrm{HNO}_{3}$ to destroy organic matter, and the $\mathrm{Li}$ and $\mathrm{K}$ were converted to chlorides by adding a small volume of $\mathrm{HCl}$.

\section{Chlorine Chemistry}

A $1 \mathrm{~g}$ sample was taken from six randomly selected ampoules from each of the three lots of serum and a weighed aliquot of ${ }^{37} \mathrm{Cl}$ spike solution was added. After equilibration the protein was precipitated with ammonium molybdate solution. The chloride in the "protein-free" filtrate was precipitated as $\mathrm{AgCl}$, removed by centrifuging the solution, and dissolved in concentrated ammonium hydroxide solution. The concentration of chlorine was adjusted to $3 \mathrm{mg} / \mathrm{ml}$. 


\section{E. Mass Spectrometric Procedures}

Irrespective of the element or the extent of previous analytical experience, a major concern in all isotopic ratio measurements by thermal ionization is variable fractionation between analyses. For ID samples with less than the optimum amount of spike, this bias is enhanced when propagated through the concentration calculation. In NBS thermalionization mass spectrometry research the traditional and well established method of controlling isotopic fractionation is to identify all critical parameters and determine optimum conditions for each parameter. The end product is an analytical procedure in which all parameters must be reproduced for each analysis and strict adherence to analytical rules observed [3]. Under ideal conditions this method is capable of reproducing the fractionation bias to less than 0.05 percent. However, ID samples are something other than ideal because two critical parameters, sample size and chemical purity, are not always uniform or well known. Under these nonideal conditions a shift from the normal plot of the fractionation curve is obtained and the concentration data will be biased.

Details of the mass spectrometric procedures for $\mathrm{Mg}$ [6], $\mathrm{Cl}$ [7], $\mathrm{K}$ [8] and $\mathrm{Ca}$ [9] have been published. The lithium procedure for electrolytes in blood serum was similar in most aspects to the published potassium analytical method.

A recently developed internal normalization technique [10] accurately corrects each analysis for fractionation bias. This procedure is applicable to polynuclidic elements, with three isotopes being the minimum necessary for a solution to the algebraic equations for fractionation corrections. The general approach of the method is to utilize one isotopic ratio for ID measurements and another isotopic ratio for normalizing purposes.

\section{Results and Discussion}

\section{A. Isotopic Composition}

For most elements it can be safely assumed that variations in natural isotopic composition are small (less than $0.25 \%$ ). This assumption is not necessarily applicable to certain matrices, such as biological materials or to a limited number of high purity commercially processed elements. In our work the isotopic composition of the analyte element is always determined unless the amount of material available is too small. The elec- 
trolytes in each lot of serum were therefore compared to NBS natural isotopic standards (Standard Reference Materials). Each was found to be experimentally identical with its respective standard.

Although the isotopic composition of $\mathrm{Li}$ in the serum samples is experimentally identical to the $\mathrm{Li}$ isotopic standard, a statement of caution about commercially processed samples is necessary. A significant amount of the high purity, commercially available $\mathrm{Li}$ is depleted in ${ }^{6} \mathrm{Li}$. An observed depletion of approximately 100 percent ( 7.4 to $3.5 \%$ relative abundance) has been found for a limited number of shelf samples. Thus, the only reliable method of having precise knowledge of the isotopic composition of this element is an isotopic analysis or use of a standard of known composition.

\section{B. BLANK AND MATRiX INTERFERENCE}

The maximum blank correction for any of the electrolytes was 0.1 percent for lot I Mg samples. The isobaric background contribution of ${ }^{40} \mathrm{~K}$ to the total ${ }^{40} \mathrm{Ca}$ signal was much less than 0.01 percent. Sodium, which was the only other interferential element, has a general deleterious effect on many elements, but was not a significant problem because of its low concentration. For the magnesium analyses the effect of $\mathrm{Na}^{+}$was minimized by using a baffle to reduce the $\mathrm{x}$-axis aperture into the collector. This restriction effectively removed $\mathrm{Na}^{+}$from the collector when $\mathrm{Mg}^{+}$was focused on the faraday cage.

\section{Concentration of Electrolytes}

\section{Lithium}

Concentrations of the electrolytes from each of three lots of serum are shown in table 1. Variability in isotopic fractionation was the major source of error in the $\mathrm{Li}$ measurements. The ${ }^{6} \mathrm{Li} /{ }^{7} \mathrm{Li}$ ratio was observed to behave anomalously in several respects when using a tantalum triple filament mode of ionization. An allowance was made for these effects by using the maximum observed range in the isotopic standard as the limit of error for $\mathrm{Li}$ analyses. 
TABLE 1. Concentration of electrolytes in serum

\begin{tabular}{ccccc}
\hline & & \multicolumn{3}{c}{ Concentration, mmol/1 } \\
& $\begin{array}{c}\text { Blank correction, } \\
\%\end{array}$ & Lot 1 & Lot III & Lot V \\
\cline { 4 - 5 } & & & & \\
\hline $\mathrm{Li}$ & $<0.01$ & 1.004 & 1.968 & 2.954 \\
& $\leq 0.1$ & \pm .005 & \pm .010 & \pm .015 \\
$\mathrm{Mg}$ & 0.3593 & 1.032 & 1.738 \\
& $<0.05$ & \pm .0028 & \pm .005 & \pm .009 \\
$\mathrm{Cl}$ & $<0.01$ & \pm .56 & 102.4 & 114.0 \\
$\mathrm{~K}$ & $\leq 0.3$ & 2.794 & \pm .5 & \pm .6 \\
$\mathrm{Ca}$ & & \pm .006 & 4.765 & 6.867 \\
& & 1.282 & \pm .010 & \pm .014 \\
& & \pm .004 & \pm .253 & 3.264 \\
& & & \pm .007 & \pm .007 \\
\hline
\end{tabular}

\section{Magnesium}

Original plans for $\mathrm{Mg}$ measurements were to use an empirically determined correction factor by analyzing the ${ }^{24} \mathrm{Mg} /{ }^{26} \mathrm{Mg}$ of the isotopic standard, SRM 980. The internal normalization approach was adopted after chemical preparation of the samples was completed. This precluded some essential planning necessary for best results. Matters were further complicated when the samples were overspiked for $\mathrm{Mg}$, because an incorrect preliminary value for the concentration was provided. Finally it was discovered, several months after completing the serum analyses, that the voltage-to-frequency converter was slightly nonlinear $(0.1 \%)$ in the scale range where the ${ }^{24} \mathrm{Mg}$ signal was measured for the ID samples. This condition probably existed at the time of the serum measurements.

The burden of these handicaps is reflected in the data shown in table 1. The ratio ${ }^{24} \mathrm{Mg} /{ }^{26} \mathrm{Mg}$ was used for ID purposes and normalization was accomplished with the ${ }^{24} \mathrm{Mg} /{ }^{25} \mathrm{Mg}$ ratio. The importance of internal normalization was immediately demonstrated, when for some inexplicable reasons samples of lot I responded quite differently than samples of the natural standard and lots III and V. A comparison of concentrations, empirical versus internal normalization, revealed an average systematic bias of approximately 1 percent in the empirical values of lot I samples and less than 0.5 percent for the other lots.

\section{Chlorine}

To the best of our knowledge, this work represents the first determination of chlorine by isotope dilution mass spectrometry. It was anticipated 
that blank contributions and memory (the latter is due to cross-contamination between the sample on the filament and residual chlorine in the mass spectrometer) would be major obstacles. Preliminary investigations demonstrated that the blank would not be a significant factor. Although not anticipated, gross fractionation did occur under certain conditions and rigid adherence to an analytical procedure was necessary.

Memory problems were minimized by reserving one mass spectrometer for blank and separated isotope analysis and a different instrument for ID samples and the natural standard. All ID samples were analyzed in order of increasing ${ }^{35} \mathrm{Cl} /{ }^{37} \mathrm{Cl}$ ratio. A maximum range of 3 percent in the isotopic ratio was used as a predetermined condition for removing and thoroughly cleaning the source. Immediately before each source cleaning, SRM 975, Chlorine Isotopic Standard, was analyzed to evaluate memory effects (which proved to be undetectable). The indicated uncertainty in table 1 is an estimated limit of error and is slightly larger than the t $\sigma$ value calculated from the concentration data. The additional allowance is made for the effects of trace impurities and possible loss of chlorine during the sample mounting procedure.

\section{Potassium}

The vital factor in potassium isotope dilution analysis is purity of the sample matrix on the filament surface. The isotopic fractionation is affected by the relative amount of potassium to impurity, whether organic or inorganic. A plot of the fractionation curve (fig. 1), ${ }^{39} \mathrm{~K} /{ }^{41} \mathrm{~K}$ versus time, for an impure sample is anomalous with the ratio slowly increasing for long periods of time, while the same plot for a high purity standard reflects a decreasing ${ }^{39} \mathrm{~K} /{ }^{41} \mathrm{~K}$ ratio. In both cases the effect appears to be strictly a change in the ratio due to fractionation. (This problem is being studied as part of a potassium mineral survey and atomic weight determination which will be reported elsewhere.)

Although the range of potassium values in table 2 for the six ampoules of each lot was approximately 0.1 percent and the 95 percent confidence limit for the mean is about 0.1 percent, an additional allowance was made for the effects of impurities. The estimated limit of error is 0.2 percent and is based upon observed variation of SRM 985 under variable sample purity conditions.

\section{Calcium}

Precise isotopic measurement of calcium isotopes is dependent upon preheating the samples and producing a transition characterized by a 


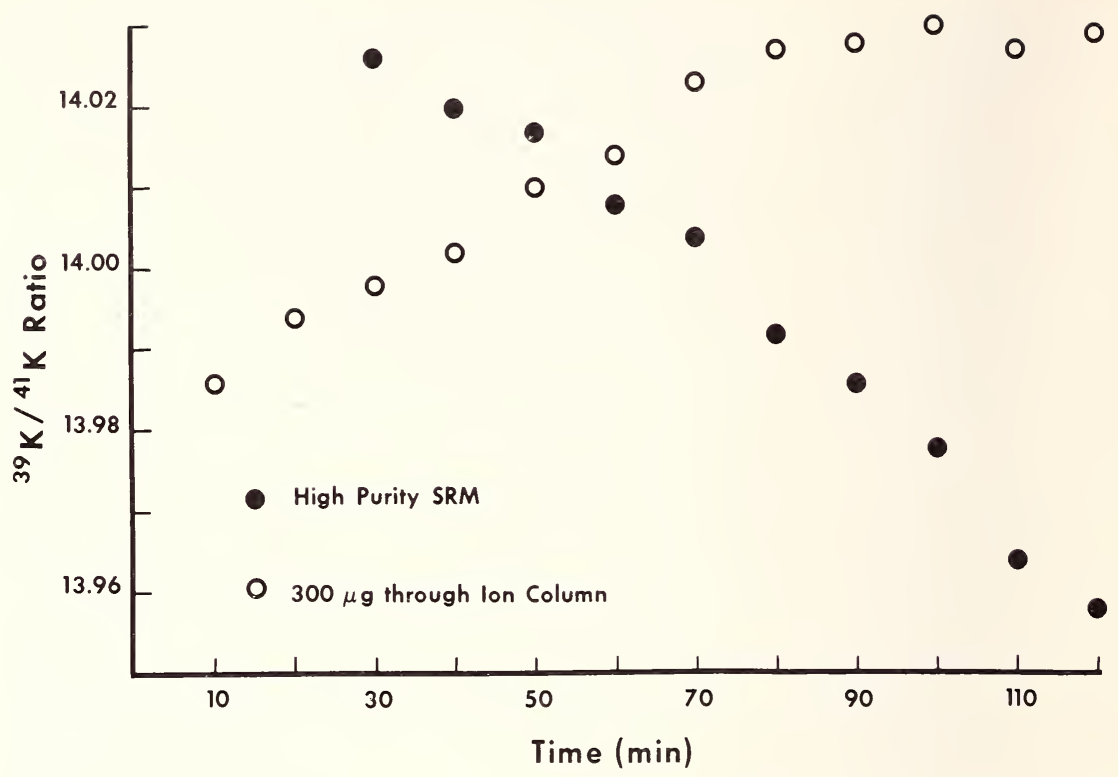

Figure 1. Potassium fractionation.

TABLE 2. Concentration of potassium in serum

\begin{tabular}{crrr} 
& \multicolumn{3}{c}{ Concentration, $\mu \mathrm{g} / \mathrm{g}$} \\
\cline { 2 - 4 } & Lot I & Lot III & Lot $\mathrm{V}$ \\
1 & 106.86 & 182.07 & 262.07 \\
2 & 106.90 & 182.20 & 262.35 \\
3 & 106.93 & 182.13 & 262.28 \\
4 & 106.92 & 182.18 & 262.34 \\
5 & 106.83 & 182.20 & 262.28 \\
6 & 106.90 & 182.15 & 262.17 \\
& & & \\
Average & 106.89 & 182.16 & 262.25 \\
& \pm .21 & \pm .36 & \pm .52
\end{tabular}

rapidly growing signal and a significant change in ${ }^{40} \mathrm{Ca} /{ }^{44} \mathrm{Ca}[11]$. After the transition has occurred, the ${ }^{40} \mathrm{Ca} /{ }^{44} \mathrm{Ca}$ ratios are reproducible to less than 0.2 percent ( $95 \%$ confidence limit). The amount of calcium on the filament appeared to affect the relative ease of producing the transition. Failure to produce the transition for each element and/or the same degree of fractionation would systematically bias the concentration. 
The 95 percent limit of error for lots I and III were expanded to reflect greater difficulty in reproducing the prescribed analytical conditions for $\mathrm{Ca}$ analysis. This type of expansion of the error is avoidable with the internal normalization technique. Ca satisfies the requirements for this procedure but the spike $\left({ }^{42} \mathrm{Ca}\right)$ necessary to use internal normalization was not available at the time of the serum analyses.

\section{Conclusion}

The role of sample impurities in isotope dilution analyses is not usually well understood, but the effect on the ionization process and the isotopic composition is measurable and may be profound. The difficulties with impurities generally translate into a systematic bias which is propagated directly through the concentration calculation. Thus, it is rarely possible to determine the concentration of a trace element with the same degree of accuracy as a high-purity solution or standard.

In mass spectrometric analysis it must be realized that isotopic fractionation is inherent in the thermal ionization process. Evaporation or volatilization of a solid sample will produce some degree of mass dependent fractionation of the light isotopes. This effect has been observed to some degree at NBS in the relative and absolute isotopic abundance studies of nearly 30 elements. A necessary and sufficient fractionation requirement for accurate isotope dilution analyses is relative precision in the isotopic ratio measurement. This precision cannot be achieved without taking steps to minimize the variability of the fractionation between analyses.

Although accurate measurements of trace concentrations by isotope dilution require careful attention to procedures and are time consuming, they can be made in a routine and cost competitive manner. The lack of speed in the technique is more than offset by the benefits which accrue with high accuracy measurements.

\section{Acknowledgement}

The authors wish to thank W. R. Shields, former Section Chief and colleague, for his advice and efforts. We are indebted to: John Mandel for statistical selection of vials analyzed; Mrs. Joy Shoemaker for outstanding performance in preparation of the manuscript. 


\section{References}

[1] Smales, A. A. and Wagner, L. R., Eds., Methods in Geochemistry, 464 pages, Interscience, New York (1960).

[2] Shields, W. R., Ed., Analytical Mass Spectrometry Section: Summary of Activities July 1966 to June 1967, U.S. National Bureau of Standards Technical Note 426, 153, U.S. Government Printing Office, Washington, D.C. 20402 (1967).

[3] Garner, E. L., Machlan, L. A., and Shields, W. R., Standard Reference Materials: Uranium Isotopic Standard Reference Materials, U.S. National Bureau of Standards Special Publication 260-27, 1-150, U.S. Government Printing Office, Washington, D.C. 20402 (1971).

[4] Kuehner, E. C., Alvarez, R., Paulsen, P. J., and Murphy, T. J., The Production and Analysis of Acids Purified by Sub-Boiling Distillation, Anal. Chem. 44, 2050-56 (1972).

[5] Shields, W. R., Ed., Analytical Mass Spectrometry Section: Summary of Activities July 1969 to June 1970, U.S. National Bureau of Standards Technical Note 546, 1110, U.S. Government Printing Office, Washington, D.C. 20402 (1970).

[6] Catanzaro, E. J., Murphy, T. J., Garner, E. L., and Shields, W. R., Absolute Isotopic Abundance Ratios and the Atomic Weight of Magnesium, J. Res. NBS (U.S.), 70A (Phys. and Chem.), 6, 453-8 (1966).

[7] Shields, W. R., Murphy, T. J., Garner, E. L., and Dibeler, V. H., Absolute Isotopic Abundance Ratio and the Atomic Weight of Chlorine, J. Amer. Chem. Soc.84, 1519 22 (1962).

[8] Barnes, I. L., Garner, E. L., Gramlich, J. W., Machlan, L. A., Moody, J. R., Moore, L. J., Murphy, T. J., and Shields, W. R., Isotopic Abundance Ratios and Concentrations of Selected Elements in Some Apollo 15 and Apollo 16 Samples, Proceedings of the Fourth Lunar Science Conference, Geochim. Cosmochim. Acta Suppl. 4, 2, 1197-1207, Pergamon Press (1973).

[9] Moore, L. J. and Machlan, L. A., High Accuracy Determination of Calcium in Blood Serum by Isotope Dilution Mass Spectrometry, Anal. Chem. 44, 2291-96 (1972).

[10] Moore, L. J., Machlan, L. A., Shields, W. R., and Garner, E. L., Internal Normalization Techniques for High Accuracy Isotope Dilution Analyses-Application to Molybdenum and Nickel in Standard Reference Materials, Anal. Chem. 46, 1082-89 (1974). 


\title{
SPIKING ISOTOPE DILUTION AND ITS APPLICATION TO FLUORINE DETERMINATIONS
}

\author{
H. A. Das and T. Wals \\ Reactor Centrum Nederland \\ Westerduinweg 3 \\ Petten (N.H.), The Netherlands
}

Isotope dilution is based on the isolation of a constant substoichiometric amount. This method can be completed by a group of techniques in which this amount is a function of the concentration to be determined. This approach enables the routine analysis of aqueous samples. The practical difference with normal isotope dilution is twofold: a) the activity is added as a spike to both the standard and the sample solution and b) the count rates obtained for the fractions isolated from the standard solutions, are plotted against the concentration in such a way that a linear calibration curve is obtained. Some methods which can be used in the application of this principle are discussed and compared to "classical" isotope dilution. Expressions are derived for the relative statistical error and the limit of detection. As a practical example of these techniques, the determination of fluorine is discussed. The radionuclide used is ${ }^{18} \mathrm{~F}(\mathrm{~T} 1 / 2=110 \mathrm{~min})$, which is prepared carrier free by irradiation of $\mathrm{Li}_{2} \mathrm{CO}_{3}$. The substoichiometric amount is taken by either absorption on glass beads or by imperfect liquid-liquid extraction with $\left(\mathrm{CH}_{3}\right)_{3} \mathrm{SiCl}$ dissolved in benzene. The glass beads or the organic extract are counted integrally. The reciprocal of the count-rate is a linear function of the concentration to be determined. The limit of detection is usually $0.01 \mu \mathrm{g} / \mathrm{ml}$, while the limit of determination is $\simeq 0.05 \mu \mathrm{g} / \mathrm{ml}$. The method is applicable to both $\mathrm{F}^{-}$and $\mathrm{SiF}_{6}=$ and is not sensitive to appreciable $\mathrm{NaCl}$ concentrations. The error is depending on counting statistics and on the ratio reagent amount to be determined. The method can be extended to dried plant material by ashing and dissolving the sample, using ${ }^{18} \mathrm{~F}$ tracer for chemical yield determination.

Keywords: Absorption isotherm; environmental samples; fluorine; isotope dilution; radionuclides; substoichiometry. 


\section{Definitions}

The variables which figure in a quantitative treatment of isotope dilution are:

The concentration of the substance to be determined

The minimal concentration which can be detected above the statistical fluctuation

Sample-weight

The volume of the sample solution

The amount of reagent

The (substoichiometric) amount, isolated by the reagent

The ratio amount of reagent/amount of substance

The fraction of the total amount of the substance to be determined, that is isolated

The number of counts obtained under certain standard conditions for an isolated fraction

The number of counts, added to the solution

The ratio substance (reagent in the compound formed by chemical reaction) $c$

c.

$c_{\min \text { st }}$

$[\mathrm{M} / 1]$

$[\mathrm{M} / 1]$

$[\mathrm{M} / 1]$

[g]

$\alpha$

\section{Introduction}

Radioanalytical methods are based on the general equation

$$
T=\gamma \cdot \frac{c^{*}}{c+c^{*}} \cdot y
$$

Equation (1) indicates the existence of three analytical techniques.

1. The ratio $c^{*} / c$ is constant and much smaller than unity. This holds for activation analysis and radiometry. Equation (1) reduces to

$$
T=\gamma \cdot \frac{c^{*}}{c} \cdot y
$$

In instrumental activation analysis one has $y=c G$ or $y=c V$. Thus $T$ is directly proportional to $c$. This proportionality is preserved when a constant fraction is taken. Calibration is performed by identical processing of standards. 
2. The concentration $c^{*}$ is constant and of the same order as $c$. This situation is met in "classical" isotope dilution. The active standard has a concentration of $c^{*}$. The sample is made active by addition of an equal volume of the standard solution [1]. Experimental conditions are chosen in such a way that $y$ is constant. This is usually achieved by liquid-liquid extraction with a substoichiometric amount of reagent. Application of equation (1) results in

$$
\left.\begin{array}{rl}
T_{\text {sample }} & =\gamma \cdot \frac{c^{*}}{c+c^{*}} \cdot y \\
T_{\text {standard }} & =\gamma \cdot y
\end{array}\right\} c=c^{*}\left[\frac{T_{\text {standard }}}{T_{\text {sample }}}-1\right]
$$

3. The concentration $c^{*}$ is constant and much smaller than $c$. This condition holds when a spike with a high specific activity is added to the sample. It is obvious that $y$ should not be proportional to $c$, in order to have a relation between $T$ and $c$. Consequently, separation methods which take a constant fraction are not applicable. A calibration curve can be constructed by processing a series of standard solutions. This technique is called "spiking isotope technique." The difference with classical isotope dilution is twofold: a) the activity is added as a spike, if possible carrierfree, to both standard and sample solutions and b) the count-rates, obtained for the fractions isolated from the standard solutions, are plotted against the concentration in such a way that a linear calibration curve is obtained. This method enables the application of all systems in which the substoichiometric amount taken is not directly proportional to the concentration to be determined $[2,3]$.

\section{Principle}

\section{A. The Relation $y=f(c)$}

In spiking isotope dilution analysis, a constant amount of reagent is added to a constant volume of a solution, which contains a variable amount of the substance to be determined. Thus, the ratio $p=$ amount of reagent (amount of substance) $=m / c V$ will vary from sample to sample. This causes a corresponding variation of the extracted fraction $q$. It is obvious that equation (4) gives the relation between $q, y$ and $c$.

$$
q=y / c V
$$

The variation of $q$ with $p$ can be measured by means of radiotracers. Apart from classical isotope dilution, at least three variations of spiking 
isotope dilution can be distinguished:

Partial liquid-liquid extraction.

Absorption according to a Langmuir isotherm.

Absorption according to a Freundlich isotherm.

Table 1 gives the corresponding $q=f(p)$ functions.

\section{B. The Relation $T=f(c)$}

The calibration curve gives $T$, the number of counts obtained for the isolated fraction, as a function of $c$, the concentration to be determined. The relation with the $q=f(p)$ curve is given by equation (5).

$$
T=q \cdot T_{0}
$$

Insertion of the experimentally determined relation $q=f(p)$ will give $T$ as a function of $p$. Substitution of $p=m / c V$ gives the $T=f(c)$ curve. Table 2 gives the equations which will lead to linear calibration curves in the four different cases (classical isotope dilution and the three variations of spiking isotope dilution).

\section{The Relative Statistical Error}

It is useful to calculate the value of $p$ at which the relative statistical error, due to counting statistics, is minimal. If the average concentration in the samples is known, the optimal amount of reagent can be calculated from this average and the optimal $p$-value. Table 3 and figure 1 give the relations between relative error, $|\Delta c / c|$, and $p$ for the four different cases. Table 4 gives the relation with the empirical constants $K_{1}$ and $K_{2}$.

\section{The Limit of Detection}

If counting statistics are the only source of error, the limit of detection can be calculated easily. It depends on the smallest significant difference between the obtained result $T$ and the number of counts when only the spike is present, $T_{0}$. If this difference is to be at least three times the standard deviation in $T_{0}$, the value of the minimal detectable concentration, $\left(c_{\min }\right)_{\mathrm{st}}$, can be calculated from the equations, given in table 5 .

\section{Determination of Fluorine in Environmental Samples}

Spiking isotope dilution can be applied profitably to the problem of fluorine determinations in environmental samples. The radionuclide ${ }^{18} \mathrm{~F}$ 


\section{DAS AND Wals}

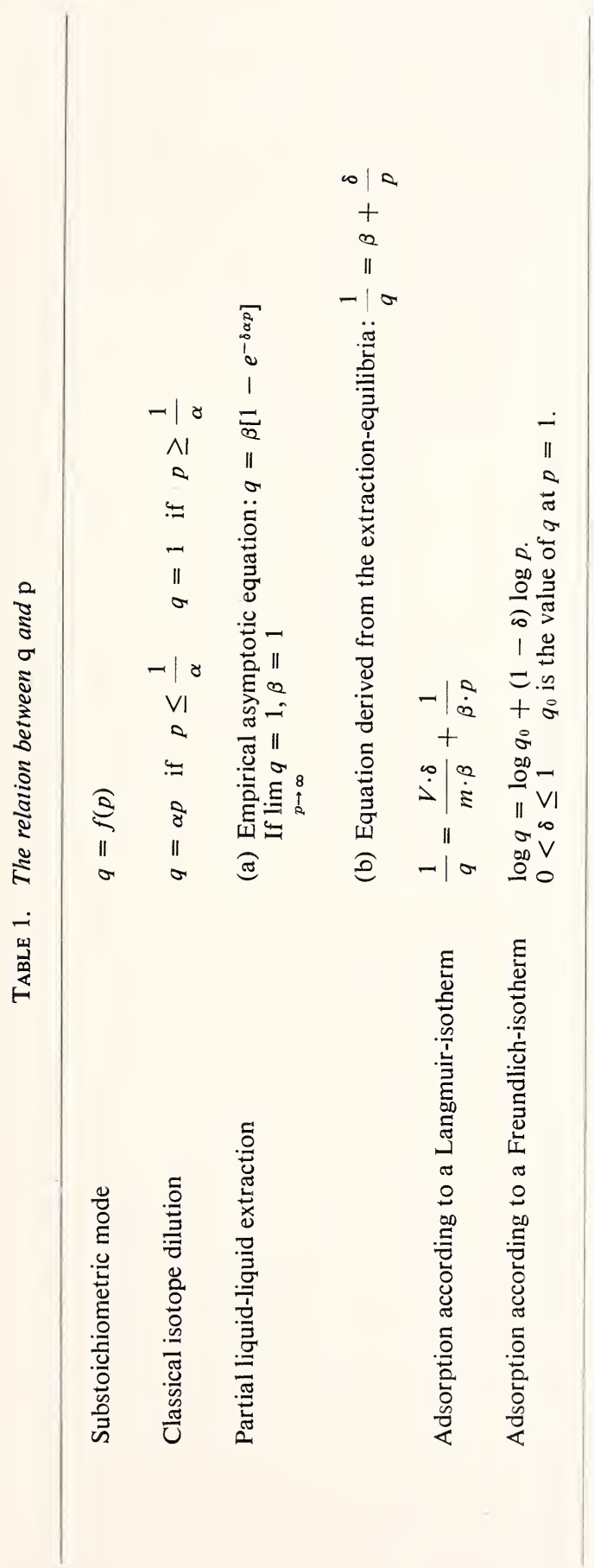




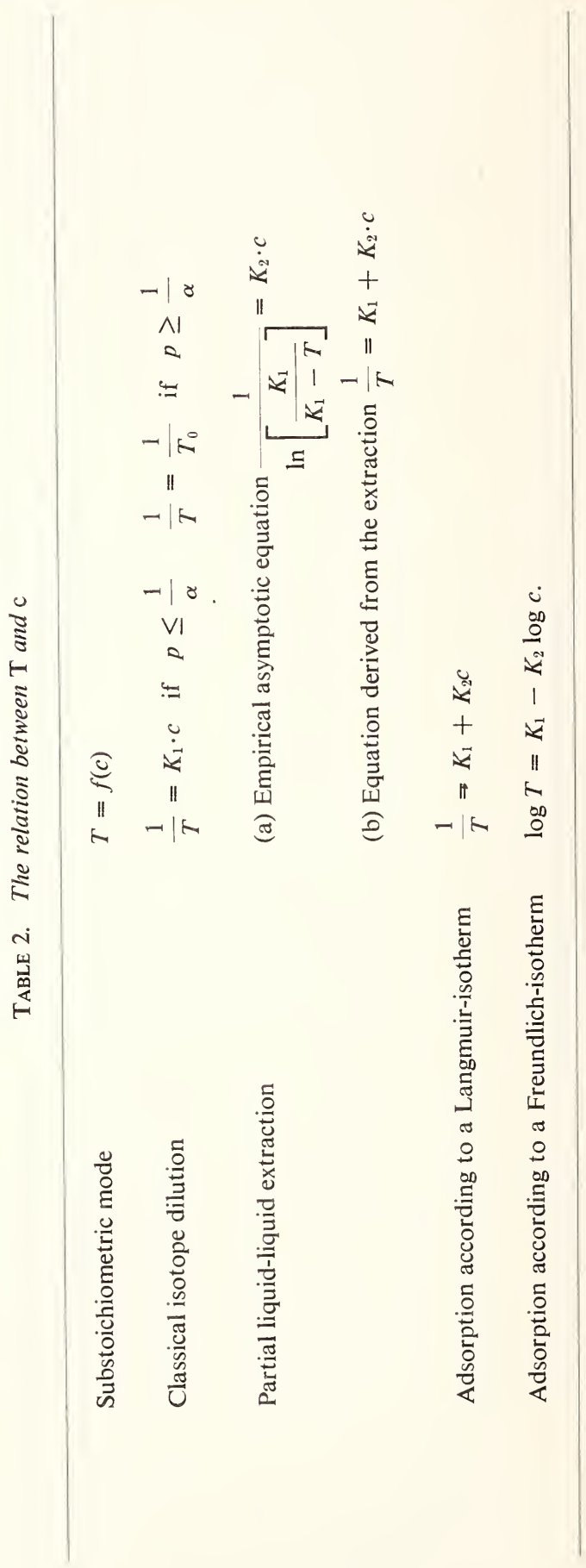


Das AND Wals

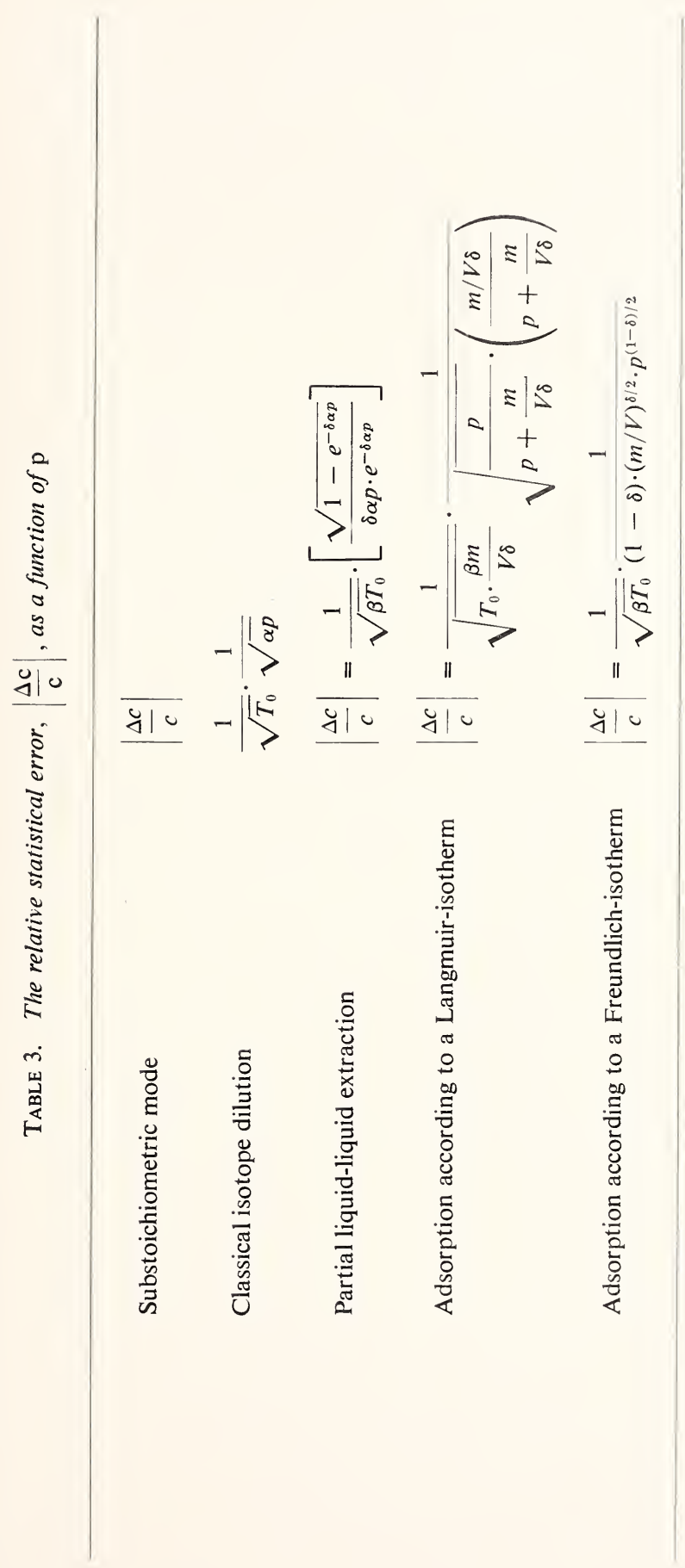




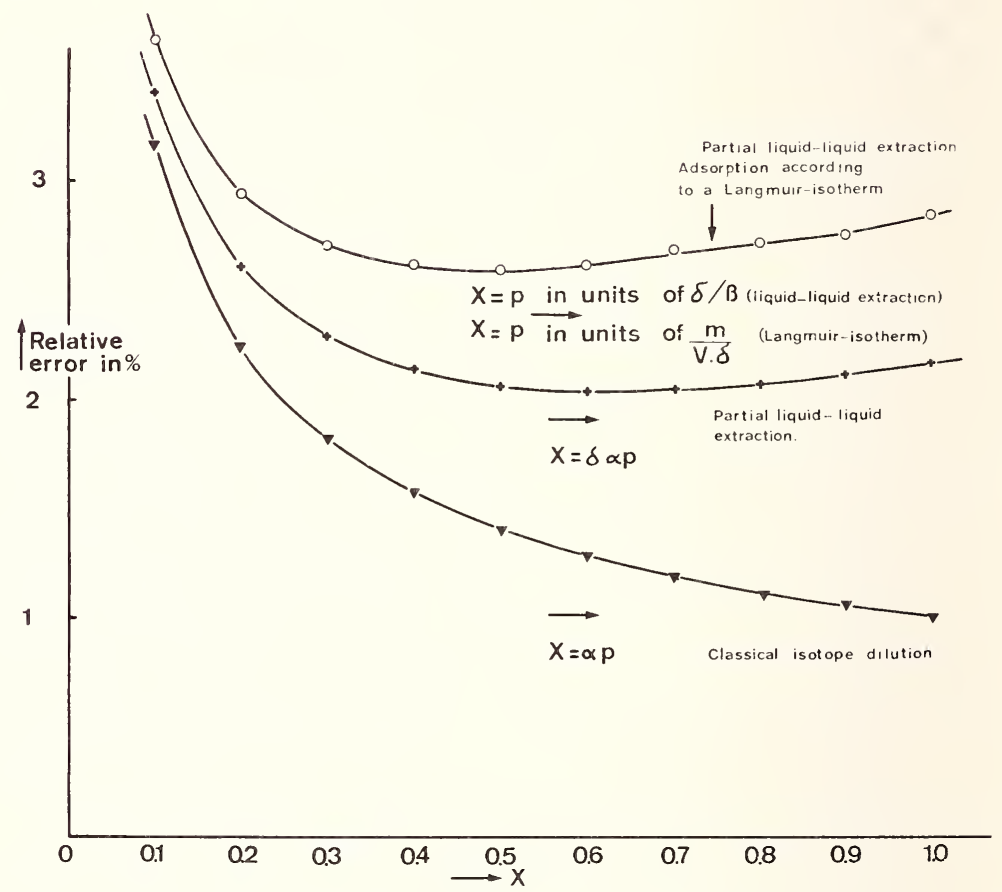

Figure 1. Relative error, $|\Delta c / c|$ in percent. The square root factor which contains $T_{0}$ has been put equal to $10^{2}$ in all cases. 
DAS AND WALS

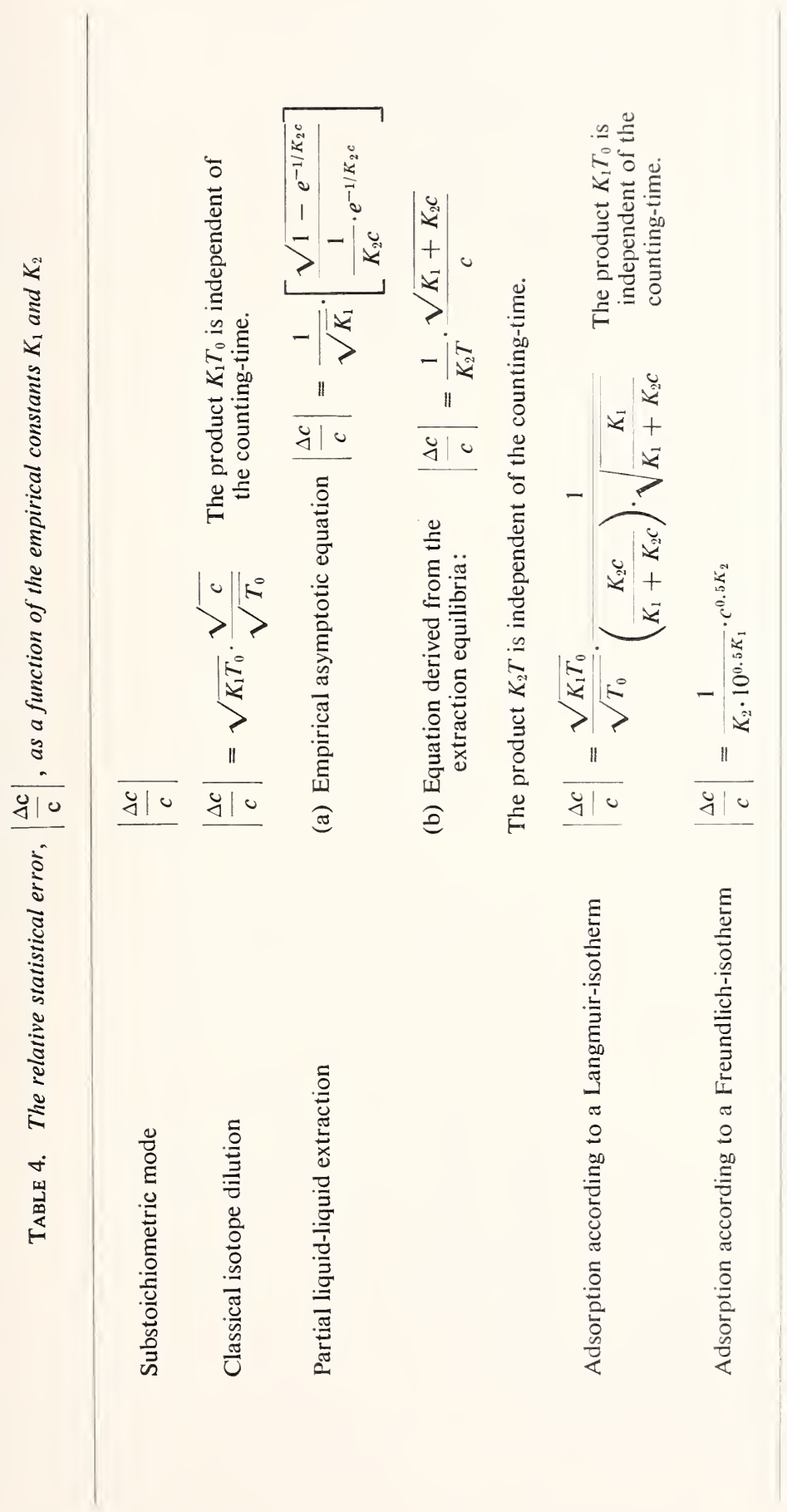




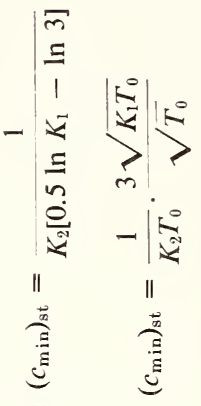

壱

总

है

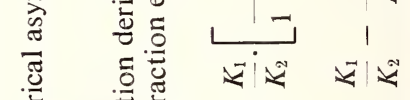

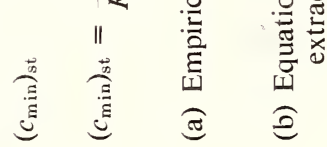

(્) 
$\left(T 1 / 2=110 \mathrm{~min}\right.$ ) can be prepared carrier-free by irradiation of $\mathrm{Li}_{2} \mathrm{CO}_{3}$ with thermal neutrons, using the reactions ${ }^{6} \mathrm{Li}(n, \alpha){ }^{3} \mathrm{H}$ and ${ }^{16} \mathrm{O}(t, n)^{18} \mathrm{~F}$. The following types of samples are to be analyzed:

Type of sample

1) Bone

2) Teeth

3) Dried plant material

4) Air dust on filter paper

5) Tap- and surface-water

6) Urine

7) Air

\section{Sample weight \\ or volume}

50 to $500 \mathrm{mg}$

50 to $1000 \mathrm{mg}$

50 to $5000 \mathrm{mg}$

$\leq 10 \mathrm{mg}$

$\leq 11$

$\leq 500 \mathrm{ml}$

100 to $1500 \mathrm{~m}^{3}$

Sampling by

impinger
Fluorine content

modern 1 to $6000 \mu \mathrm{g} / \mathrm{g}$ fossile $10^{3}$ to $3.10^{4}$

100 to $400 \mu \mathrm{g} / \mathrm{g}$

0.1 to $100 \mu \mathrm{g} / \mathrm{g}$

$\leq 500 \mu \mathrm{g} / \mathrm{g}$

0.05 to $2 \mu \mathrm{g} / \mathrm{ml}$

0.01 to $10 \mu \mathrm{g} / \mathrm{ml}$

$\simeq 0.01 \mu \mathrm{g} / \mathrm{m}^{3}$

The determination in bone can be performed by instrumental neutron activation analysis, using either thermal or fast neutrons. The analysis of teeth can be done by instrumental photon activation analysis, or, for concentrations higher than $\simeq 100 \mu \mathrm{g} / \mathrm{g}$, by instrumental thermal neutron activation analysis. More accurate data can be obtained by dissolving the sample in $3 \mathrm{~N} \mathrm{HNO}_{3}$ and applying the spiking isotope dilution technique, discussed in section 5 .

Instrumental activation analysis cannot be applied to dried plant material. However, it is possible to use the following procedure:

a. Dry ashing at $\simeq 600{ }^{\circ} \mathrm{C}$, and dissolution of the ash in dilute $\mathrm{HNO}_{3}$. The recovery of this step is about 70 percent, depending on the temperature and the time needed to mineralize the sample. If necessary, the solution is filtered. A carrier-free ${ }^{18} \mathrm{~F}$ spike can be used to measure the final recovery after ashing and filtration.

b. Determination of fluoride in the resulting solution, using the technique discussed in section 5 .

Air dust on filter paper can be dissolved by combustion in a Schöninger flask, containing $0.1 \mathrm{~N} \mathrm{NaOH}$ as an absorber. The resulting solution can be analyzed by spiking isotope dilution, using the method discussed in section 4. It is also possible to apply fast neutron activation, followed by steam distillation as $\mathrm{H}_{2} \mathrm{SiF}_{6}$ from concentrated $\mathrm{H}_{2} \mathrm{SO}_{4}$, and anionexchange [4]. Tap water and urine can be analyzed rapidly by spiking isotope dilution. The same holds for air, if sampled with an impinger. 


\section{Determination of Fluoride in Aqueous \\ Samples by Adsorption on Glass Beads}

\section{A. Principle \\ 1. Calibration Curve}

The technique is suited for the analysis of large $(\geqslant 50 \mathrm{ml})$ samples. The solution to be analyzed is spiked with ${ }^{18} \mathrm{~F}^{-}$and a concentration-dependent fraction of the fluoride is taken by adsorption of HF on glass-beads [5]. After adsorption, the beads are washed with demineralized water and counted. The reaction between the glass surface and the HF can be described by a Langmuir isotherm. According to table 2, this situation can be described by the empirical relation

$$
1 / T=\mathrm{K}_{1}+\mathrm{K}_{2} c
$$

The constants $\mathrm{K}_{1}$, in (counts) ${ }^{-1}$, and $\mathrm{K}_{2}$, in [counts $\left.(\mathrm{g} / \mathrm{ml})^{-1}\right]^{-1}$ have to be determined experimentally. The adsorption is in all probability due to chemisorption of $\mathrm{HF}$. The $\mathrm{pH}$-dependence of the adsorption can be explained in terms of the mechanism for the adsorption of anions and their conjugated acids, as discussed by Hingston et al. [6]. However, the agreement between theory and reality is only qualitative (cf. fig. 2).

\section{Influence of Contact Time}

The kinetics of the adsorption process can be described by the empirical formula

$$
T_{t}=T_{\infty}\left(1-e^{-\mu t}\right)
$$

where $T_{t}$ and $T_{\infty}$ stand for the counting results of the glass absorber after time $t$ and after a very long contact time respectively.

\section{Statistical Errors}

The value of $\left(c_{\min }\right)_{\mathrm{st}}$ can be calculated by application of the formula given in table 5. The relative statistical error due to variations of the contact time can be found by differentiation of equation (7). The relative error due to irreproducible losses of HF during the handling of the glass beads after the adsorption is independent of the concentration. Thus:

$$
|\Delta y / y|=|\Delta T / T|=\sigma_{y}
$$


The relative statistical error in the final result, is given by

$$
|\Delta c / c|_{y}=1 / c \mathrm{~K}_{2} \cdot \Delta(1 / T)=\sigma_{y} / c \mathrm{~K}_{2} T
$$

Combination of (6) and (9) results in

$$
|\Delta c / c|_{y}=\sigma_{y}\left(1+\left(\mathrm{K}_{1} / \mathrm{K}_{2}\right) \cdot(1 / c)\right)
$$

The value of $\sigma_{y}$ can be derived from measurements on standard solutions.

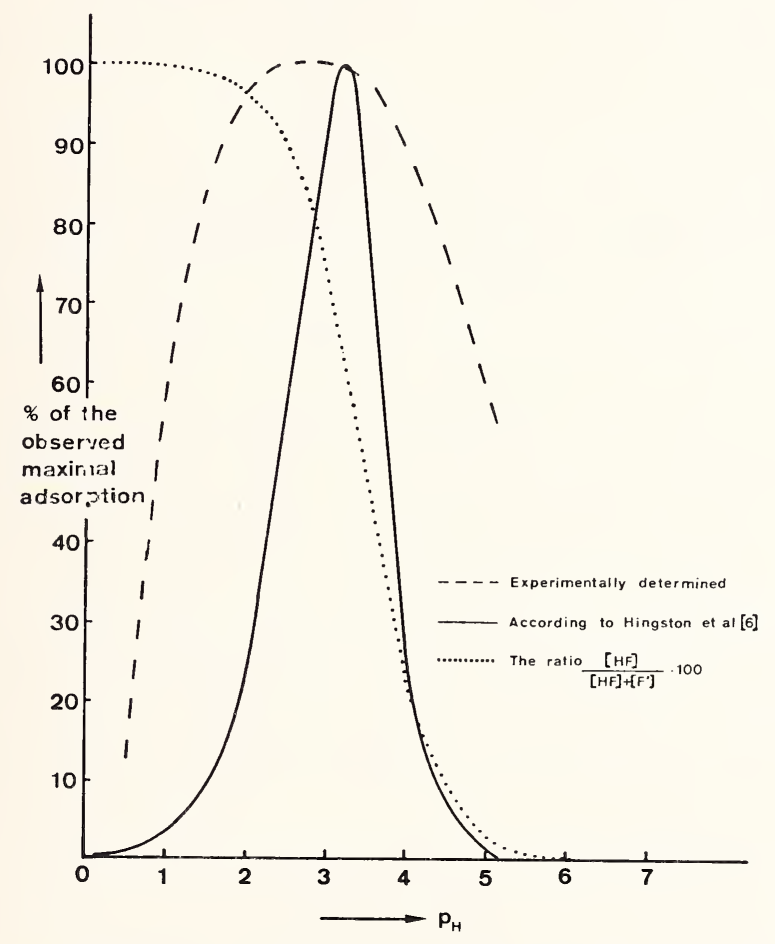

Figure 2. Influence of the $\mathrm{pH}$ on the adsorption behavior of $\mathrm{SiO}_{2}$.

\section{B. EXPERIMENTAL}

\section{Chemicals and Apparatus}

Double distilled water

Demineralized water

Double distilled $0.01 \mathrm{~N}$ and $2 \mathrm{~N} \mathrm{HNO}_{3}$

"Analytical grade" $\mathrm{Li}_{2} \mathrm{CO}_{3}$

"Analytical grade" $\mathrm{NaF}$ 
Glass beads of $3.1 \mathrm{~mm}$ diameter; Kugelfabrik Gebauer, Fulda, Germany

$50 \mathrm{ml}$ polyethene weighing flasks

$200 \mathrm{ml}$ polythene beakers

Quartz capsules of $50 \times 5 \mathrm{~mm}$

A micropipette with disposable polythene $1000 \mu \mathrm{l}$ tips

Plastic sieves

Magnetic stirrers with polythene-covered propellors

$15 \mathrm{ml}$ test tubes.

\section{Procedure}

1) Glass beads are cleaned in portions of $\simeq 10^{4}$ by repeated washings with distilled $0.01 \mathrm{~N} \mathrm{HNO}_{3}$ and, finally, with double distilled water. They are then dried.

2) Portions of 100 beads are weighed out in $50 \mathrm{ml}$ polythene weighing flasks.

3) About $25 \mathrm{mg}$ of $\mathrm{Li}_{2} \mathrm{CO}_{3}$, packed in a quartz capsule, is irradiated for 20 minutes in a thermal neutron flux of $5 \times 10^{13} \cdot \mathrm{cm}^{-2} \cdot \mathrm{s}^{-1}$. After dissolu-

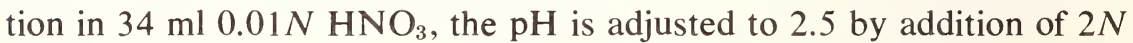
$\mathrm{HNO}_{3}$.

4) The solution to be analyzed is transferred to a $200 \mathrm{ml}$ polythene

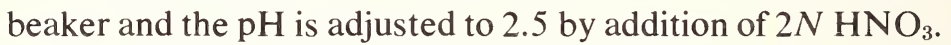

5) A spike of $1000 \mu \mathrm{l}$ of the ${ }^{18} \mathrm{~F}^{-}$solution is added and the solution is stirred for a few seconds to ensure good mixing.

6) A batch of 100 glass beads is added and the solution is stirred for 10 minutes.

7) The beads are filtered off in a plastic sieve and washed for 1 minute in a stream of demineralized water.

8) The beads are transferred to a $15 \mathrm{ml}$ test tube and counted for at least 1 minute with a $3^{\prime \prime} \times 3^{\prime \prime} \mathrm{NaI}$ well-type crystal, connected to a onechannel analyzer. A correction is applied for dead-time. All data are then corrected for decay, using an arbitrary reference time.

9) Standards are made by dissolving "analytical grade" $\mathrm{NaF}$ in double distilled water, diluting to the appropriate concentration range adjusting the $\mathrm{pH}$ to 2.5. The calibration curve is obtained by applying the method of least squares to the results. The calibration curve is valid only for the combination of the involved batches of beads and $\mathrm{Li}_{2} \mathrm{CO}_{3}$.

10) An aliquot of the spiking solution is measured the same geometry, to determine $T_{0}$. 


\section{Results}

\section{Calibration Curve and Statistical Error}

At the working conditions of $\mathrm{pH}=2.5$ and $\mathrm{V}=100 \mathrm{ml}$, the following average values are observed.

$$
\begin{aligned}
& K_{1}=0.75 \times 10^{-5}(c / \mathrm{min})^{-1} \\
& K_{2}=1.5 \times 10^{-5}\left(c / \mathrm{min}(\mu \mathrm{g} / \mathrm{ml})^{-1}\right)^{-1} \\
& \mu=\left(4.0 \pm 0.3 \cdot 10^{-3} \cdot \mathrm{s}^{-1}, \text { at } \mathrm{pH}=2.5\right. \\
& \sigma_{y}=0.03-0.05
\end{aligned}
$$

For a counting time of 1 minute, $\left(c_{\mathrm{min}}\right)_{\mathrm{st}}=5 \cdot 10^{-3} \mu \mathrm{g} / \mathrm{ml}$. It follows from equation (10) that the error due to the handling of the glass beads limits the method to solutions with concentrations above $0.02 \mu \mathrm{g} / \mathrm{ml}$. The uncertainty in the contact time is $\leqslant 10$ seconds. The resulting statistical error is $\leqslant 0.8$ percent.

\section{Synthetic Samples}

Three synthetic samples were prepared and analyzed repeatedly.

Calculated F content observed F content

1) $15.85 \mathrm{mg} \mathrm{NaF}$, dissolved in 10 liter double distilled water

2) $2.0 \mathrm{mg} \mathrm{Na}_{2} \mathrm{SiF}_{6}$ in 1 liter double distilled water

3) $2.0 \mathrm{mg} \mathrm{Na} \mathrm{SiF}_{6}$ in 1 liter double distilled water, containing $310 \mathrm{~m} \times \mathrm{Cl}^{-}$.
$0.72 \mu \mathrm{g} / \mathrm{ml}$

$$
\begin{aligned}
& 0.79 \pm 0.07 \mu \mathrm{g} / \mathrm{ml} \\
& \sigma=9 \% \\
& 1.44 \pm 0.10 \mu \mathrm{g} / \mathrm{ml} \\
& \sigma=7 \% \\
& 1.40 \pm 0.10 \mu \mathrm{g} / \mathrm{ml} \\
& \sigma=7 \%
\end{aligned}
$$

The observed differences are partly due to the contribution of the doubled distilled water $(\simeq 0.05 \mu \mathrm{g} / \mathrm{ml})$ and partly to the nonstoichiometry of the $\mathrm{Na}_{2} \mathrm{SiF}_{6}$. The addition of $\mathrm{Cl}^{-}$to the solution does not affect the result.

\section{Surface Waters}

Two samples of surface water were analyzed, both by direct analysis and standard addition.

$$
\text { Direct analysis }
$$

$$
0.57 \pm 0.05(\sigma) \mu \mathrm{g} / \mathrm{ml}
$$$$
0.32 \pm 0.03
$$

Standard addition

$$
0.55 \pm 0.05(\sigma) \mu \mathrm{g} / \mathrm{ml}
$$

$0.34 \pm 0.03$ 


\section{Urine}

Aliquots of one great urine sample were analyzed directly and by standard addition with $\mathrm{Na}_{2} \mathrm{SiF}_{6}$ results are

Direct analysis: $0.54 \pm 0.05 \mu \mathrm{g} / \mathrm{ml} \quad \sigma=9 \%$

Standard addition: $0.52 \pm 0.13 \mu \mathrm{g} / \mathrm{ml} \quad \sigma=25 \%$.

The direct method was then applied to a few hundreds of urine samples. The obtained data lie between 0.5 and $3 \mu \mathrm{g} / \mathrm{ml}$. The relative standard deviation per measurement is again 7 to 9 percent.

\section{Conclusion}

The radiometric determination of fluoride in aqueous samples is possible down to concentrations of $\simeq 0.02 \mu \mathrm{g} / \mathrm{ml}$. The method is rapid and cheap. Small changes in the ionic strength do not result in systematical errors. The statistical standard deviation per measurement is 9 percent.

\section{Determination of Fluoride in Aqueous \\ Samples by Substoichiometric Extraction with Trimethylchlorosilane}

\section{A. Principle}

\section{Calibration Curve}

This method is suited for the analysis of small $(0.5$ to $10 \mathrm{ml})$ samples. It is based on the partial extraction of $\mathrm{HF}$ from a dilute $\mathrm{HNO}_{3}$ solution by $\left(\mathrm{CH}_{3}\right)_{3} \mathrm{SiCl}$ (TMCS), dissolved in benzene. The reagent reacts in the aqueous phase with $\mathrm{HF}$ forming $\left(\mathrm{CH}_{3}\right)_{3} \mathrm{SiF}$. Again, equation (6) holds. The value of $\left(c_{\min }\right)_{\mathrm{st}}$ can be calculated from the expression given in table 5 .

\section{Influence of Nondissolved Fluoride}

If the sample contains an appreciable amount of nondissolved fluoride, which can exchange with the added ${ }^{18} \mathrm{~F}^{-}$, the result of the determination will be too high. It can be proved easily that the following expression 
holds for the concentration $c^{\prime}$, derived from the measurement:

$$
c^{\prime}=c+(\mathrm{g} / V)\left(1+\left(\mathrm{K}_{1} / \mathrm{K}_{2} c\right)\right)
$$

Here, $g$ stands for the amount of nondissolved but exchangeable fluoride. It is possible to measure the ratio $\mathrm{g} / \mathrm{cV}$ by a separate spiking experiment and thus to correct the obtained value $c^{\prime}$.

\section{Influence of $\mathrm{pH}$}

The recovery of a ${ }^{18} \mathrm{~F}$ spike by extraction from dilute nitric acid was measured as a function of the acidity. The extraction was performed with an equal volume of a $4 \times 10^{-5} \mathrm{M}$ solution of $\left(\mathrm{CH}_{3}\right)_{3} \mathrm{SiCl}$ in benzene. As the fluoride concentration due to the spike is virtually zero, the variation of $1 / K_{1}$ with the acidity is measured in this way. Figure 3 gives the result. It

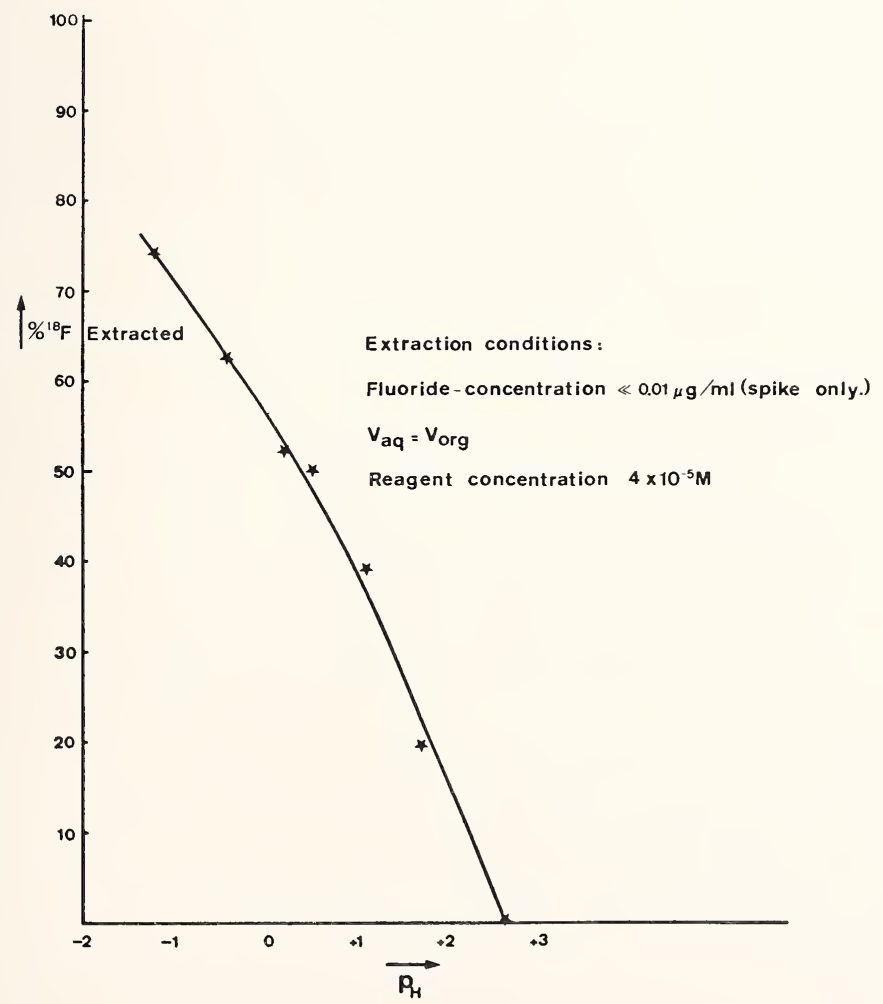

Figure 3. The percentage extracted ${ }^{18} \mathrm{~F}$ as a function of the $\mathrm{pH}$. 
should be kept in mind that below $\mathrm{pH}=2$ all fluoride is present as HF. It can be concluded that in the analysis the solution should have an acidity of at least $0.1 N$. The variation of the recovery $1 / \mathrm{K}_{1}$ with the $\mathrm{pH}$ in the region below $\mathrm{pH}=0$ can be described by

$$
1 / \mathrm{K}_{1}=0.56-0.15 \mathrm{pH}
$$

Thus

$$
\frac{d\left(1 / K_{1}\right)}{d\left[H^{+1}\right]} \cdot \Delta\left[H^{+1}\right]=\frac{0.15}{\left[H^{+1}\right]} \cdot \Delta\left[H^{+1}\right]
$$

An error of 5 percent in the acidity will result in less than 1 percent deviation in the recovery.

\section{Influence of Stirring Time}

The extraction of fluoride by an equal volume of a $4 \times 10^{-5} M$ solution of $\left(\mathrm{CH}_{3}\right)_{3} \mathrm{SiCl}$ in benzene from $5 \cdot 10^{-6}$ to $5 \cdot 10^{-4} \mathrm{M}$ solutions of $\mathrm{NaF}$ in $3 \mathrm{~N} \mathrm{HNO}_{3}$ was measured as a function of time. The rate of extraction was found to be independent of the fluoride concentration. Figure 4 gives the result obtained using a laboratory flask shaker. Equilibrium is reached after $\simeq 5$ minutes.

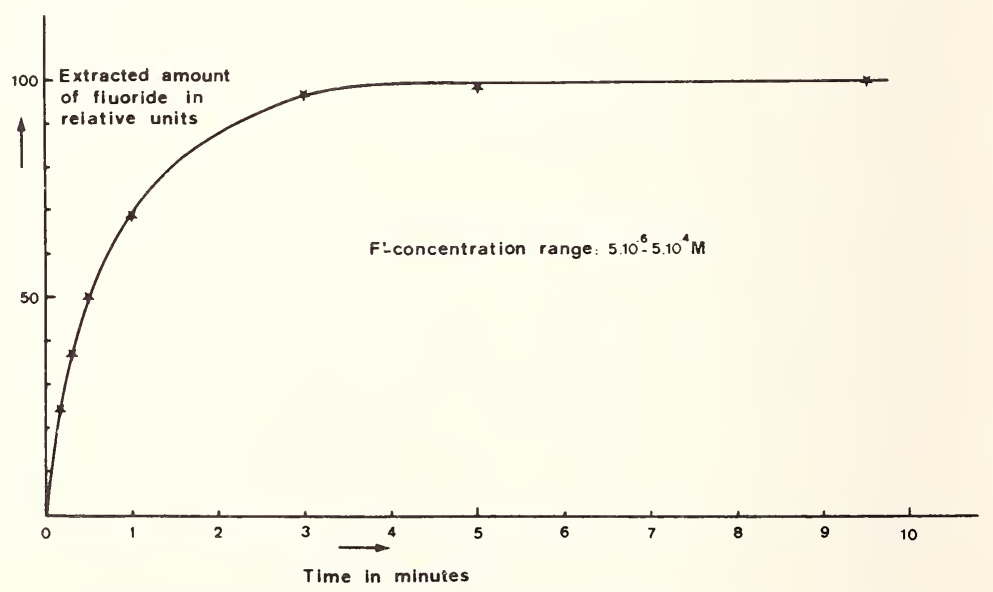

Figure 4. The extracted amount of fluoride as a function of the stirring-time. 


\section{Influence of Indifferent Electrolytes}

Experiments as described in the previous section were performed with solutions of $\mathrm{NaCl}, \mathrm{Mg}\left(\mathrm{NO}_{3}\right)_{2}$ and $\mathrm{Ca}\left(\mathrm{NO}_{3}\right)_{2}$ in $\mathrm{HNO}_{3}$ of various acidities. The concentration of the salts varied from 0.1 percent to 5 percent. No influence of the added electrolyte was observed.

\section{B. EXPERIMENTAL}

\section{Chemicals and Apparatus}

Double distilled water

Double distilled water $\mathrm{HNO}_{3}(15 \mathrm{~N})$

$3 \mathrm{~N} \mathrm{HNO}_{3}$, prepared from double distilled conc. $\mathrm{HNO}_{3}$ and double distilled water.

$\mathrm{NaF}$

$\mathrm{Li}_{2} \mathrm{CO}_{3}$

Benzene

$\left(\mathrm{CH}_{3}\right)_{3} \mathrm{SiCl}$

Quartz capsules of $50 \times 5 \mathrm{~mm}$.

$10 \mathrm{ml}$ polythene vials with snap cap.

$20 \mathrm{ml}$ polythene bottles with screw cap.

A $50 \mathrm{ml}$ polythene bottle with screw cap.

$100 \mathrm{ml}$ polythene bottles with screw cap.

A 1-litre polythene bottle.

Two 1-litre brown glass bottles.

A $5 \mathrm{ml}$ glass pipette with suction balloon.

A micropipette with disposable polythene $100 \mu$ l tips.

A micropipette with a steel plunger in a ground glass barrel.

A flask shaker.

A $3^{\prime \prime} \times 3^{\prime \prime}$ well-type NaI crystal, connected to a one channel analyzer and mounted in a sample changer for test tubes.

A "Griffin" flask shaker.

\section{Procedure}

Preparation of the Reagents

1) About $25 \mathrm{mg} \mathrm{Li}_{2} \mathrm{CO}_{3}$ is put into a polythene vial with a snap cap and irradiated for 4 minutes in a thermal neutron flux of $5 \cdot 10^{13} \cdot \mathrm{cm}^{-2} \cdot \mathrm{s}^{-1}$. 
The $\mathrm{Li}_{2} \mathrm{CO}_{3}$ is then dissolved in $25 \mathrm{ml} 3 \mathrm{~N} \mathrm{HNO}_{3}$ in a $50 \mathrm{ml}$ polythene bottle with screw cap.

2) A $4.3 \times 10^{-5} \mathrm{M}$ solution of TMCS in benzene is prepared by adding $1 \mathrm{ml} \mathrm{TMCS}$ to $200 \mathrm{ml}$ benzene in a brown glass bottle. One millilitre of this stock solution is pipetted to 1 litre benzene in a brown glass bottle.

3) A stock solution of dilute HF, containing $5.3 \times 19^{-4} M$ hydrofluoric acid $(10 \mu \mathrm{g} / \mathrm{ml})$ is prepared by dissolving $23 \mathrm{mg} \mathrm{NaF}$ in 1 litre $3 \mathrm{~N} \mathrm{HNO}_{3}$ in a 1-litre polythene bottle. From this stock solution, standards with concentrations between $\simeq 5.3 \times 10^{-6} M(0.1 \mu \mathrm{g} / \mathrm{ml})$ to $\simeq 5.3 \times 10^{-4} M(10 \mu \mathrm{g} / \mathrm{ml})$ are prepared in $50 \mathrm{ml}$ polythene bottles.

\section{Extraction Procedure and Counting}

1) Four ml of the (approximately neutral) sample is transferred to a 20 $\mathrm{ml}$ polythene bottle with screw cap. One $\mathrm{ml}$ of double distilled $\mathrm{HNO}_{3}(\simeq$ $15 N$ ) is added.

2) Four $\mathrm{ml}$ aliquots of the standards are pipetted in identical $20 \mathrm{ml}$ polythene bottles, and $1 \mathrm{ml}$ of $15 \mathrm{~N} \mathrm{HNO}_{3}$ is added.

3) An aliquot of $1 \mathrm{ml}$ of the $\mathrm{H}^{18} \mathrm{~F}$ solution and $5 \mathrm{ml}$ of the diluted TMCS solution are added. The bottles are closed immediately. They are shaken for at least 5 minutes.

4) The phases are allowed to separate; this takes 2 to 3 minutes. Aliquots of $3 \mathrm{ml}$ are taken with a micropipette with a steel plunger, and transferred to $10 \mathrm{ml}$ polythene tubes, which are closed immediately.

5) The aliquots are counted integrally for 1 minute in the energy region of 0.40 to $0.65 \mathrm{MeV}$. The observed count rates vary between $5 \cdot 10^{3}$ and $5 \cdot 10^{4} \mathrm{c} / \mathrm{min}$. The loss due to dead time $(\tau=3$ to $4 \mu \mathrm{s})$ is negligible, as is the influence of the background.

\section{Results}

\section{Calibration Curve and Statistical Error}

Figure 5 gives a calibration curve obtained for the extraction of fluoride from $5 \cdot 10^{-6}$ to $5 \cdot 10^{-4} M$ solution in $0.6 \mathrm{~N} \mathrm{HNO}_{3}$. The organic phase was a $4 \cdot 10^{-5} \mathrm{M}$ solution of $\left(\mathrm{CH}_{3}\right)_{3} \mathrm{SiCl}$ in benzene. The volume of both the aqueous and the organic phase was $5 \mathrm{ml}$. The straight line can be described by the equation

$$
1 / T=1.8 \times 10^{-5}+1.6 \times 10^{-5} c \cdot(\text { counts } / \mathrm{min})^{-1}
$$

Here, $c$ is expressed in $\mu \mathrm{g} / \mathrm{ml}$. The value of $T_{0}$ was $1.05 \times 10^{-5} \mathrm{c} / \mathrm{min}$. In- 
sertion of the values of $K_{1}$ and $K_{2}$ in the appropriate equation (table 3) yields $\Delta c / c=6 \cdot 10^{-2}$ for a concentration of $1 \mu \mathrm{g} / \mathrm{ml}$. The limit of detection according to table 5 is $\simeq 0.02 \mu \mathrm{g} / \mathrm{ml}$.

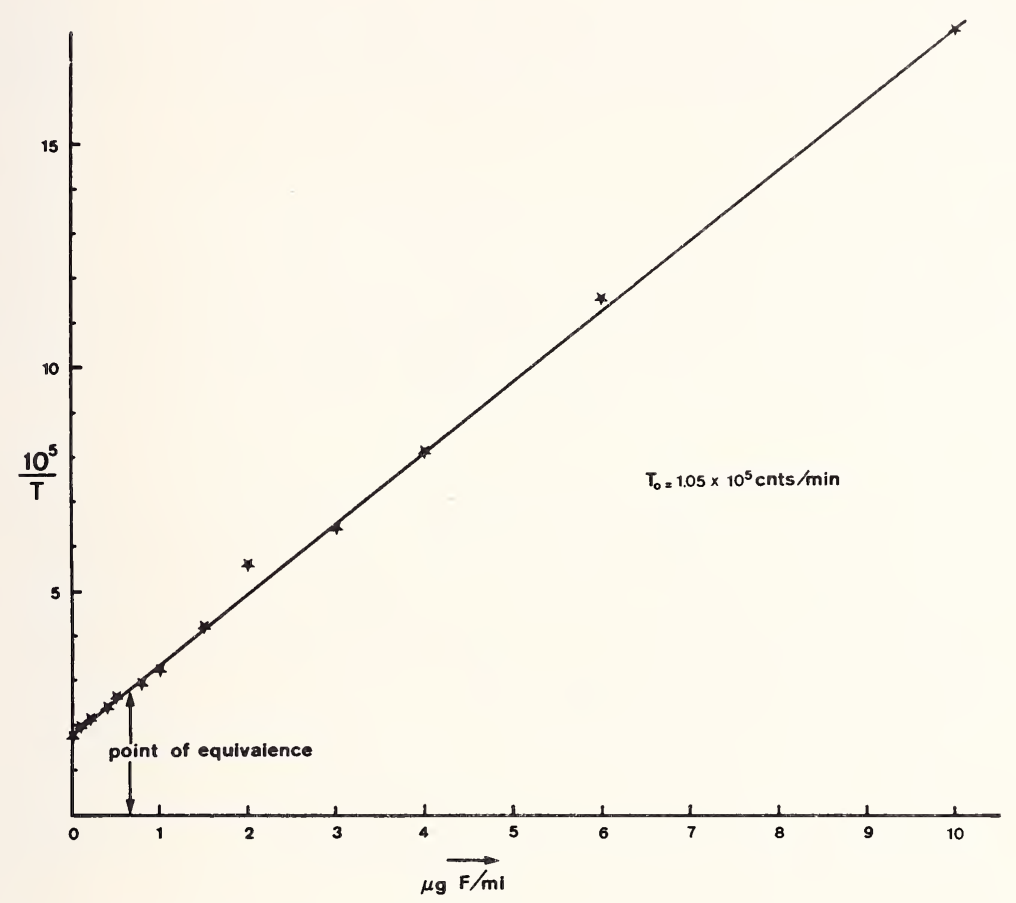

Figure 5. Calibration curve for the extraction from $0.6 \mathrm{~N} \mathrm{HNO}_{3}$.

\section{Fluoride Standard}

A neutral aqueous solution, containing $(1.89 \pm 0.01) \mu \mathrm{g} / \mathrm{ml}$ fluoride was analyzed. The result was $(1.85 \pm 0.03) \mu \mathrm{g} / \mathrm{ml}$, with a standard deviation of $0.05 \mathrm{~g} / \mathrm{ml}$.

\section{Tap Water}

Unfluoridized and fluoridized tap water was analyzed. The values obtained are:

$$
\begin{array}{ll}
\text { Unfluoridized } & (0.61 \pm 0.02) \mu \mathrm{g} / \mathrm{ml}(\mathrm{n}=5) \text { (Petten) } \\
& (0.46 \pm 0.005) \mu \mathrm{g} / \mathrm{ml}(\mathrm{n}=5) \text { (Amsterdam) } \\
\text { Fluoridized } & (1.03 \pm 0.04) \mu \mathrm{g} / \mathrm{ml}(\mathrm{n}=5)
\end{array}
$$


These values are in good agreement with the official data.

\section{Urine}

Urine of a healthy person was filtered and analyzed. The result was $(0.82 \pm 0.02) \mu \mathrm{g} / \mathrm{ml}(\mathrm{n}=5)$. The method was then applied to a few hundred urine samples.

\section{Conclusion}

The determination of fluoride by partial liquid-liquid extraction is rapid and accurate. The method is not sensitive to appreciable variations in the ionic-strength. The lower limit of determination is $\simeq 0.05 \mu \mathrm{g} / \mathrm{ml}$.

\section{References}

[1] Ruzicka, J. and Stary, J., Substoichiometry in Radiochemical Analysis, Pergamon Press, Oxford (1968).

[2] Kyrs, M., Anal. Chim. Acta 33, 245 (1966).

[3] Das, H. A. et al., Spiking Isotope Dilution, J. Radioanal. Chem. (in press).

[4] Van der Mark, W. and Das, H. A., J. Radioanal. Chem. 13, 107 (1973).

[5] Van der Mark, W. and Das, H. A., Determination of Fluoride in Water Samples by Spiking Isotope Dilution, and Absorption on Glass Beads, J. Radioanal. Chem. (in press).

[6] Hingston, F. J. et al., J. Soil Science 23, 177 (1972). 


\title{
THE DETERMINATION OF SMALL AMOUNTS OF NITROGEN (0 to $20 \mathrm{ppm}$ ) IN PLAIN CARBON STEELS
}

\author{
H. F. Pronk, J. de Boer, and N. Butter-Bijwaard
}

Analytical Laboratories

Hoogovens IJmuiden BV

The Netherlands

\begin{abstract}
The paper describes the results of a thorough experimental investigation into the origin and reduction of the blank during sample handling and sample analysis in the determination of the total nitrogen content in plain carbon steels at levels of 0.002 percent and less. Attention is paid to atmospheric contributions, to the blank and to their prevention. The possible harmful effect of nitrite or nitrate compounds on the analytical result is pointed out. Finally a method is recommended which is especially adapted for the determination of very low levels of nitrogen.
\end{abstract}

Keywords: Atmospheric contamination; blank value; indophenol blue reaction; Kjeldahl digestion technique; limit of detection; nitrogen in steel.

\section{Introduction}

With the trend in recent years towards new steelmaking and annealing processes with lower nitrogen levels, the need to determine small amounts of nitrogen in steel has increased. It is now often necessary to determine nitrogen levels of less than $20 \mu \mathrm{g}$ with great accuracy. In determining such small amounts of nitrogen the analytical chemist has to deal with the following general aspects:

A. Sampling, with attention to homogeneity, representativeness and contamination during sample preparation.

B. Sample handling, with attention to contamination from the outside, i.e., atmospheric or environmental contamination. 
C. Analysis, with attention to the choice of the most appropriate method of analysis as well as to the control and to the meaningful measurement of the blank.

Although by this time quite a few physical techniques are available for determining small amounts of nitrogen in steel, all these physical techniques suffer from the fact that they require calibration. In Europe it is a well-established opinion that the best approach to a meaningful calibration is the use of standard reference steel samples with a similar composition as the sample to be analyzed and with known nitrogen contents, preferably analyzed by an independent technique. Therefore, in Europe the Kjeldahl dissolution and distillation technique has been officially accepted in the European standard reference method (Euronorm) for the determination of nitrogen in steel [1]. Since this European standard method does not cover the lower nitrogen contents, the analytical laboratories of Hoogovens started some years ago an investigation into the problems especially related to the determination of low levels of nitrogen in steel. According to Kaiser [2] the limit of detection $(\Delta)$ of a determination depends on the standard deviation of the blank value and can be estimated with 95 percent confidence limit from the relationship:

$$
\Delta=X-X_{\mathrm{B}}=1.96 \sigma_{\mathrm{B}} \sqrt{2}
$$

where: $x=$ the total amount of nitrogen in $\mu \mathrm{g}$ found in the determination; $x_{\mathrm{B}}=$ the amount of nitrogen in $\mu \mathrm{g}$ found in the blank determination; and $\sigma_{\mathrm{B}}$ $=$ the "within days" standard deviation of the blank value.

This relationship emphasizes the special need for tracing and controlling the various factors which may contribute to the overall standard deviation. Generally speaking, the value of the standard deviation increases with the level of the blank itself. Therefore the blank should be as low as possible. This explains the special attention paid in this paper to the origin and reduction of the blank and the special concern with the dissolution and distillation equipment used in the analysis. A further requirement for the blank is that it should be a meaningful representative of the determination itself. Since the harmful effect of nitrate or nitrite compounds on the determination is often underestimated, special attention has been paid to this problem too. The paper finally ends with a description of a recommended method taking into account all factors which were found to have an effect on the limit of detection and on the accuracy of the determination. 


\section{Sampling, Sample Handling, and Analysis}

\section{A. Sampling}

The analytical procedure is based on the use of $2 \mathrm{~g}$ of sample. If this quantity is obtained by milling or drilling of the sample material, it may be considered sufficiently representative and homogeneous. During sampling the usual precautions must be taken such as a clean sample preparation equipment, a dust-proof environment, no smoking, no manual contact, etc. Before analysis the sample should be washed several times with acetone and stored in a weighing bottle placed in a desiccator.

\section{B. Sample Handling}

\section{Environmental-Atmospheric Contamination}

For the determination of nitrogen at the levels being considered it is essential that the analysis should be carried out in a room specially reserved for this purpose. This room should be as far as possible removed from chemical laboratories where ammonia, nitric acid or other nitrogen-bearing compounds are used. Smoking in this room should be forbidden. All reagents used should be stored under impeccable environmental conditions.

\section{Dissolution and Distillation Equipment}

Contrary to the European method, micro-scale equipment was developed in such a way that both the dissolution-decomposition step and the distillation step are carried out protected from atmospheric contact (figs. 1-4). The figures illustrate that arrangements have been made to minimize the atmospheric contact between the inside of the dissolutiondistillation equipment and the environment. The sample is dissolved in the reaction vessel $A$ (fig. 1), the hydrogen evolved escapes through the trap $\mathrm{G}$. The water, condensed from vapor liberated during the dissolution and the subsequent fuming step is collected in vessel F. After fuming and cooling, the vessel $\mathrm{A}$ is quickly transferred to the distillation equipment (figs. 2 and 4). The sodium hydroxide solution is added by means of the vessel $\mathbf{J}$; any ammonia produced on contact with the sample solution is then liberated inside the distillation vessel. The condenser outlet is sealed from the atmosphere as shown in figure 2. 


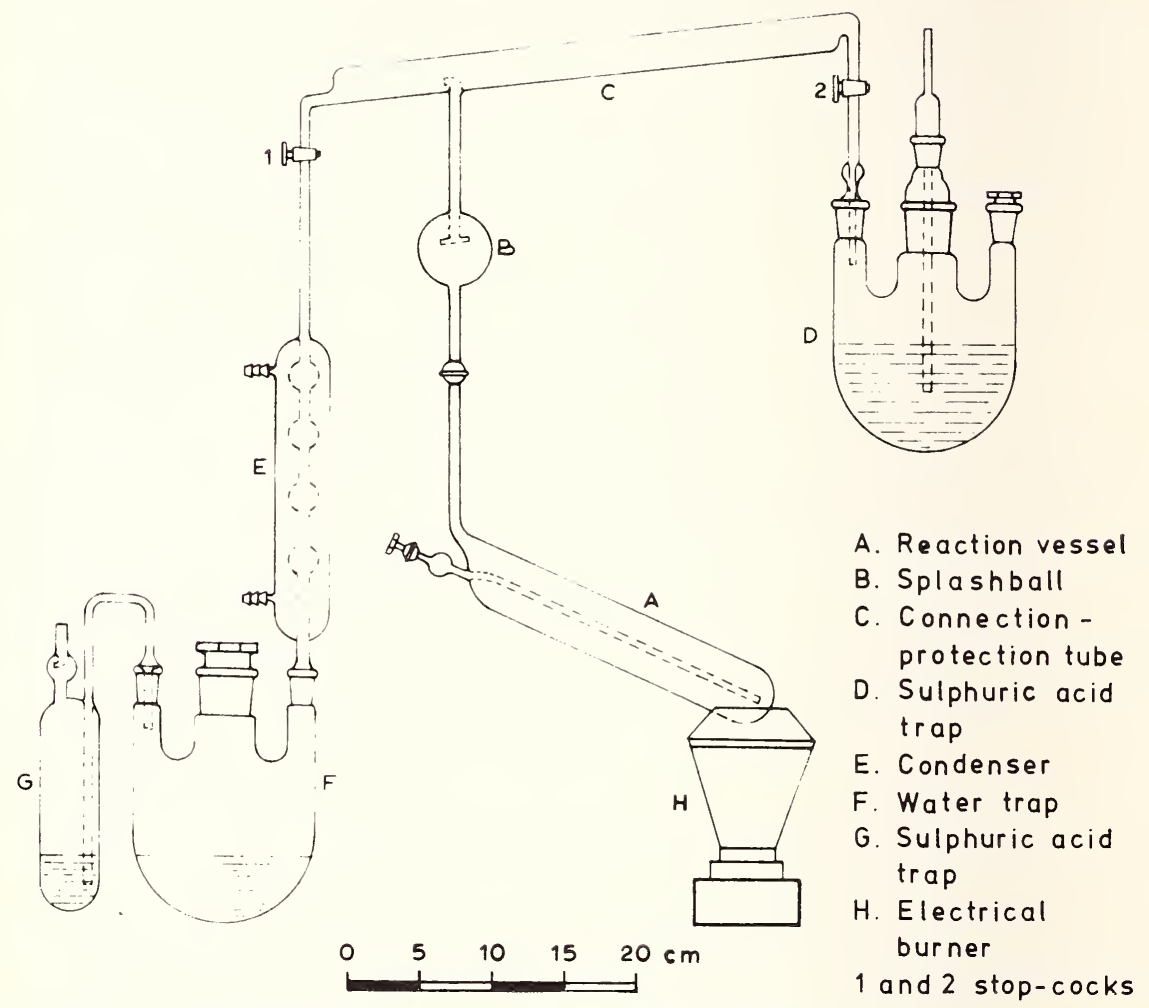

Figure 1. Equipment for the determination of nitrogen in steel (dissolution-decomposition step). 


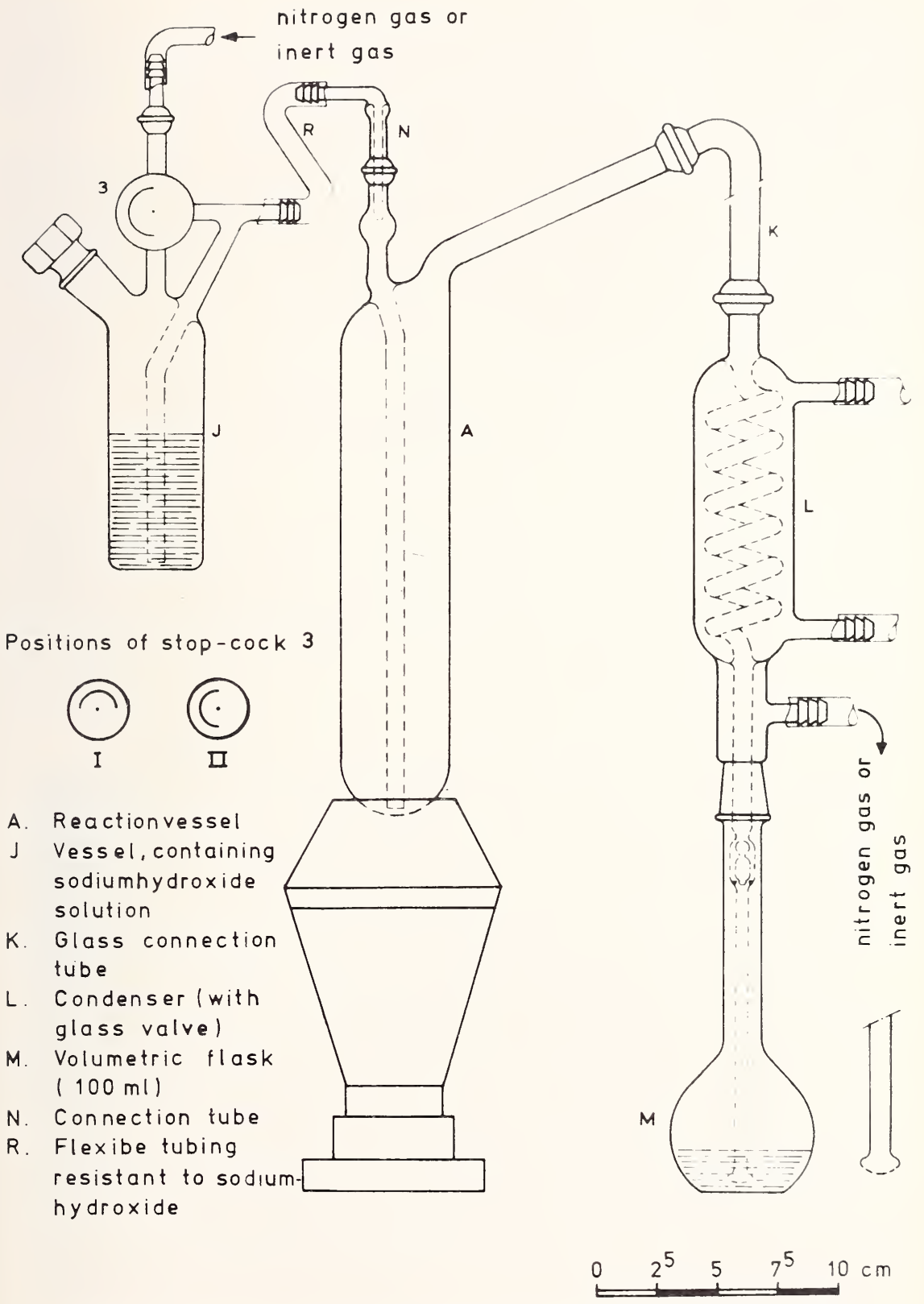

Figure 2. Equipment for the determination of nitrogen in steel (distillation step). 
During the whole operation nitrogen gas (or any other inert gas) is passed through the distillation equipment to avoid the introduction of contaminants from the atmosphere and bumping. The nitrogen flow also serves as the carrier for the ammonia during distillation.

Greatest care should be taken to ensure that all glassware used is kept scrupulously clean and washed well with nitrogenfree water before use. This water should be reserved specially for this work. Figures 3 and 4 illustrate how the individual dissolution and distillation devices can be easily installed in a batch position arrangement.

\section{Analysis}

\section{Choice of Analytical Method}

Among the many chemical and physical methods available, considerable attention has been paid by many workers to the specific and sensitive determination of ammonia based on the reaction between the ammonium ion, sodium phenolate and sodium hypochlorite with sodium nitroprusside as a catalyst [3]. This indophenol blue reaction has been accepted in the Euronorm and has been the subject of an investigation in the Analytical

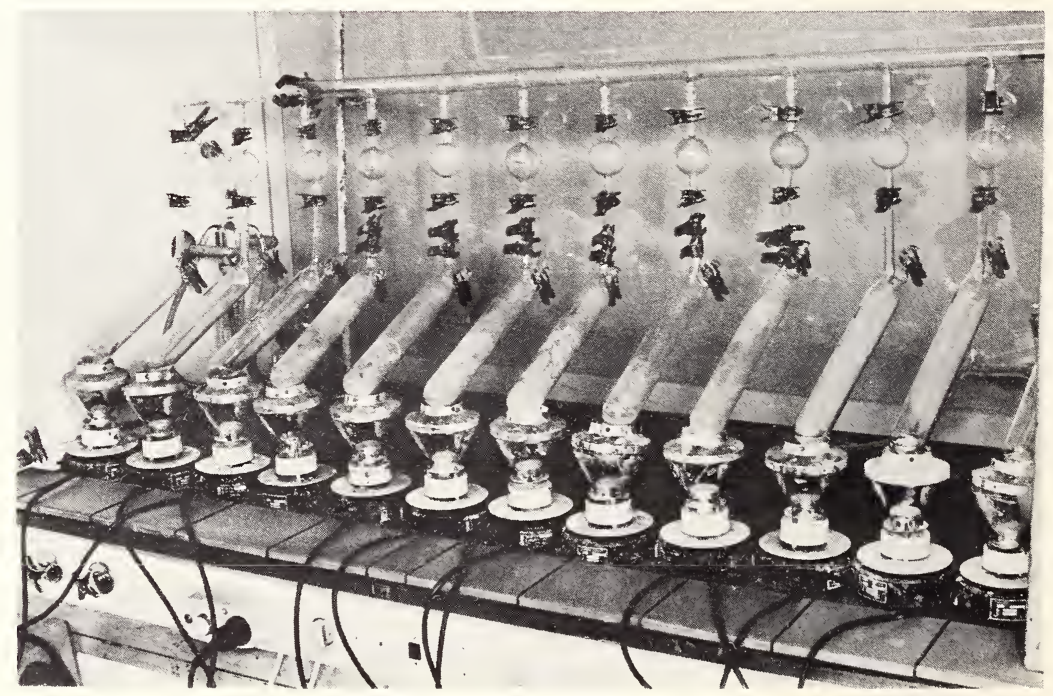

Figure 3. Dissolution-decomposition arrangement. 


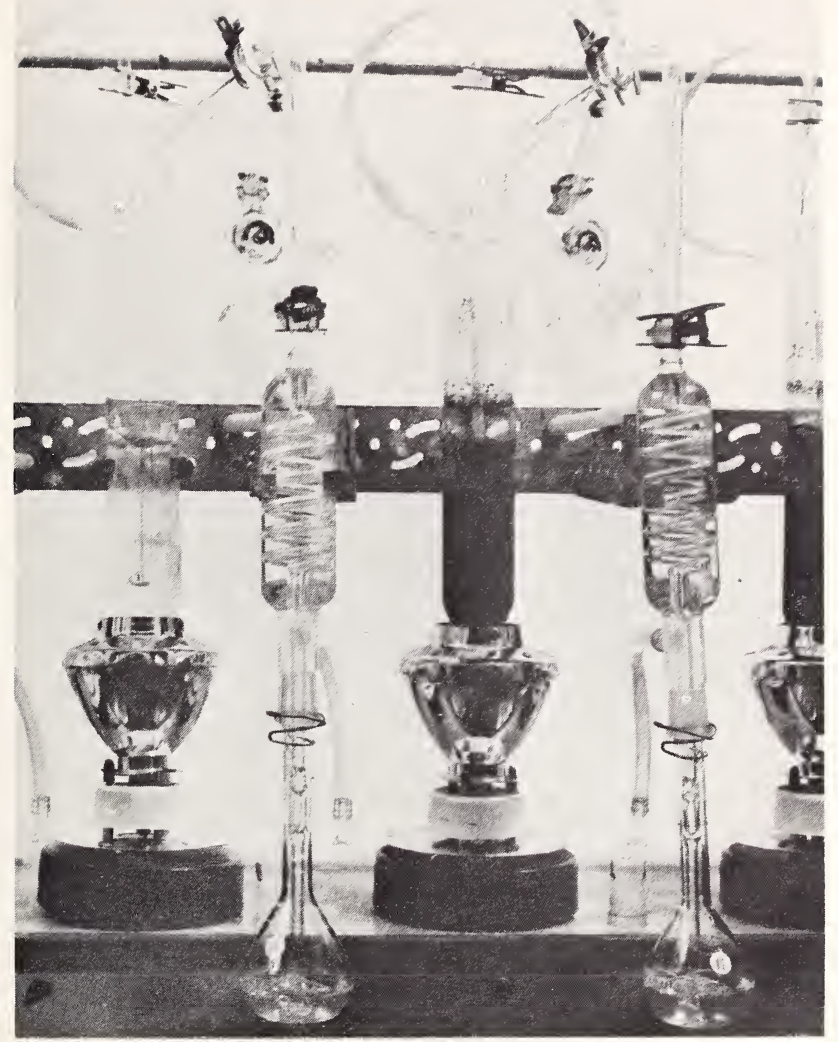

Figure 4. Distillation arrangement.

Laboratories of Hoogovens. Figure 5 shows the absorption spectrum of a test solution and the reagent blank solution against water. Figure 6 shows a calibration graph covering the range 0 to $50 \mu \mathrm{g}$ of nitrogen in 100 $\mathrm{ml}$, which indicates the extreme sensitivity of the reaction.

Due to its sensitivity the method proved to be extremely suitable for the determination of low nitrogen contents. However, the whole procedure must be carried out under carefully controlled analytical conditions; i.e., special attention must be paid to the $\mathrm{pH}$ during the formation of the intensive blue color complex. Following carefully the procedure and the instructions as described in Appendix I, reproducible results can be obtained.

In further agreement with the Euronorm the Kjeldahl dissolution and distillation technique was chosen for the conversion of the nitrogen from the sample into ammonia and for the subsequent separation of the am- 


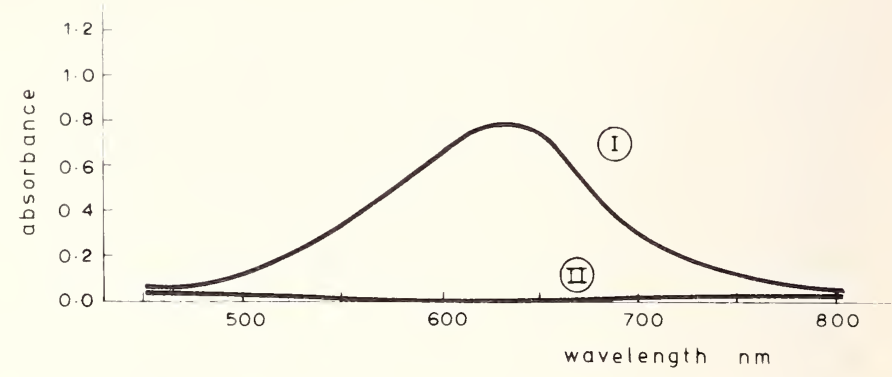

Figure 5. Absorption spectrum of a test solution $(50 \mu \mathrm{g} \mathrm{N} / 100 \mathrm{ml}) \mathrm{I}$ and a reagent blank solution II both measured against water.

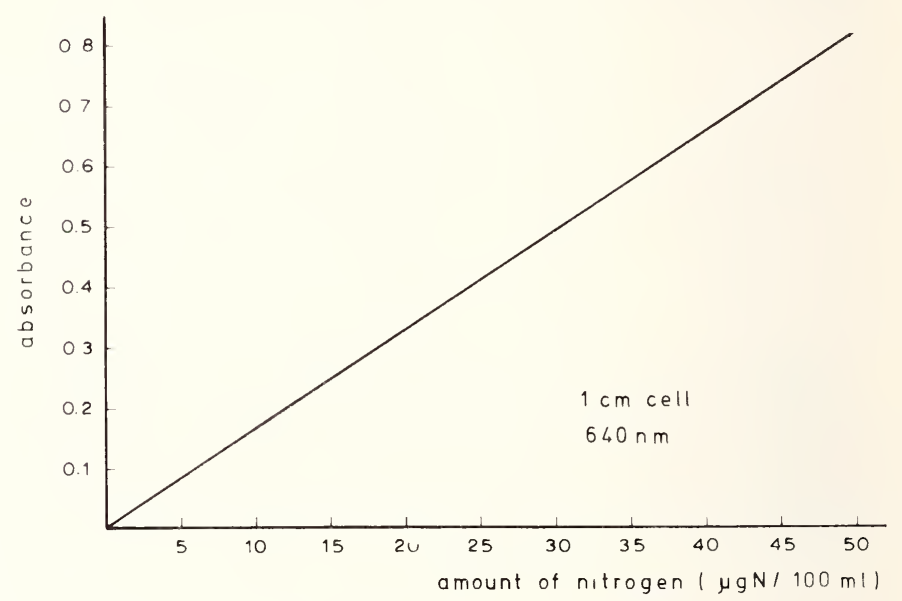

Figure 6. Absorbance versus concentration of nitrogen.

monia by distillation. At variance with the traditional Kjeldahl equipment and the Euronorm, a dissolution-distillation equipment on microscale was developed in view of the low level of detection (fig. 1.)

\section{Blank: Origin and Reduction}

Once the method of analysis, that is semi-micro Kjeldahl technique in combination with indophenol blue reaction under optimum experimental conditions, has been chosen, it remains a well-recognized fact that the determination of small amounts of ammonia is only possible with a blank value as low and as reproducible as possible. In many laboratories this requirement has been the most exasperating obstacle. 
As mentioned in the introduction, the effect of the blank value is demonstrated in the well-known relationship of Kaiser [2]:

$$
\Delta=1.96 \sigma_{\mathrm{B}} \sqrt{2}
$$

Therefore, a good understanding of the origin of the blank is most important. The possibility of the environmental contributions to the blank and their prevention have already been pointed out. Other sources are found in the reagents employed in the method. The water used throughout the procedure must be free from amonia and may easily be prepared from a mixed bed deionizer. Distilled water may have a residual ammonia content. This can be eliminated by passing the water through a cation exchange resin. It is essential that the resin column should be acid-washed before use to ensure its conversion into the hydrogen form. With the exception of the sulphuric acid all the other reagents in practice offer little or no problems. The sulphuric acid reagent generally offers some problems. Various chemical firms market sulphuric acid which is specially reserved for determining low levels of nitrogen. According to the specifications given by these firms, this acid contains a maximum of 0.0002 percent $\mathrm{NH}_{3}$. If this maximum concentration is really present, then the contribution to the blank will be $24 \mu \mathrm{g} \mathrm{N}$ per analysis. This value is inadmissible. When analyzing various lots of sulphuric acid from three suppliers, we found that the amount of ammonia actually present was much lower.

Under the optimum laboratory conditions described above, total blanks of 4 to $5 \mu \mathrm{g} \mathrm{N}$ per analysis can be obtained with sulphuric acid from two of these suppliers. The highest values found with acid from other suppliers were, under the same conditions, approximately $12 \mu \mathrm{g} \mathrm{N}$, which is rather high for the determination of very low amounts of nitrogen.

\section{Blank: Meaningful and Representative Measurement}

It stands to reason that the blank determination should be representative of the actual determination. In case the diluted sulphuric acid, obtained by diluting sulphuric acid $18 \mathrm{M}$ with water, contains nitrate and/or nitrite, this may cause too high analytical results. When the sample material is being dissolved in the diluted sulphuric acid, these nitrogen compounds are reduced to ammonium by hydrogen; this in contrast with the blank procedure where no iron is present. The extent to which this happens, depends on the type of the steel, the amount of the sample, the size of the sample particles and the amount of the nitrogen compound. The Euronorm claims to eliminate the blank problem by taking two dif- 
ferent sample weights: $1 \mathrm{~g}$ and $2 \mathrm{~g}$. The difference between the two results represents the amount of nitrogen in $1 \mathrm{~g}$ of sample.

Upon further investigation of this procedure, especially the effect of $\mathrm{NO}_{3}{ }^{-}$and $\mathrm{NO}_{2}{ }^{-}$on the blank, we found that an incomplete recovery of $\mathrm{NO}_{3}{ }^{-} / \mathrm{NO}_{2}-$ nitrogen may be obtained. In figure 7 the amount of nitrogen recovered from $\mathrm{NO}_{3}$ - reduction is plotted against the weight of the sample using three different types of steel. Each point in the figure has been calculated from the difference between two individual analyses: (sample + $20 \mu \mathrm{g} \mathrm{N}_{\mathrm{NO}_{3}}$ ) - sample. From this figure it can be seen that with an addition of $20 \mu \mathrm{g}$ of $\mathrm{N}$ as $\mathrm{NO}_{3}{ }^{-}$to $1 \mathrm{~g}$ of $\mathrm{BCS} 260 / 2$ only 60 percent is recovered. Furthermore this recovery depends upon the weight of the sample and the steel concerned. Consequently the Euronorm procedure of using 1 and $2 \mathrm{~g}$ will fail under certain conditions. A British suggestion [4] to remove the blank by adding $0.250 \mathrm{~g}$ of reduced iron to the sample and referring this to a second analysis of only $0.250 \mathrm{~g}$ of reduced iron, will fail too unless a special kind of reduced iron exists which is capable of

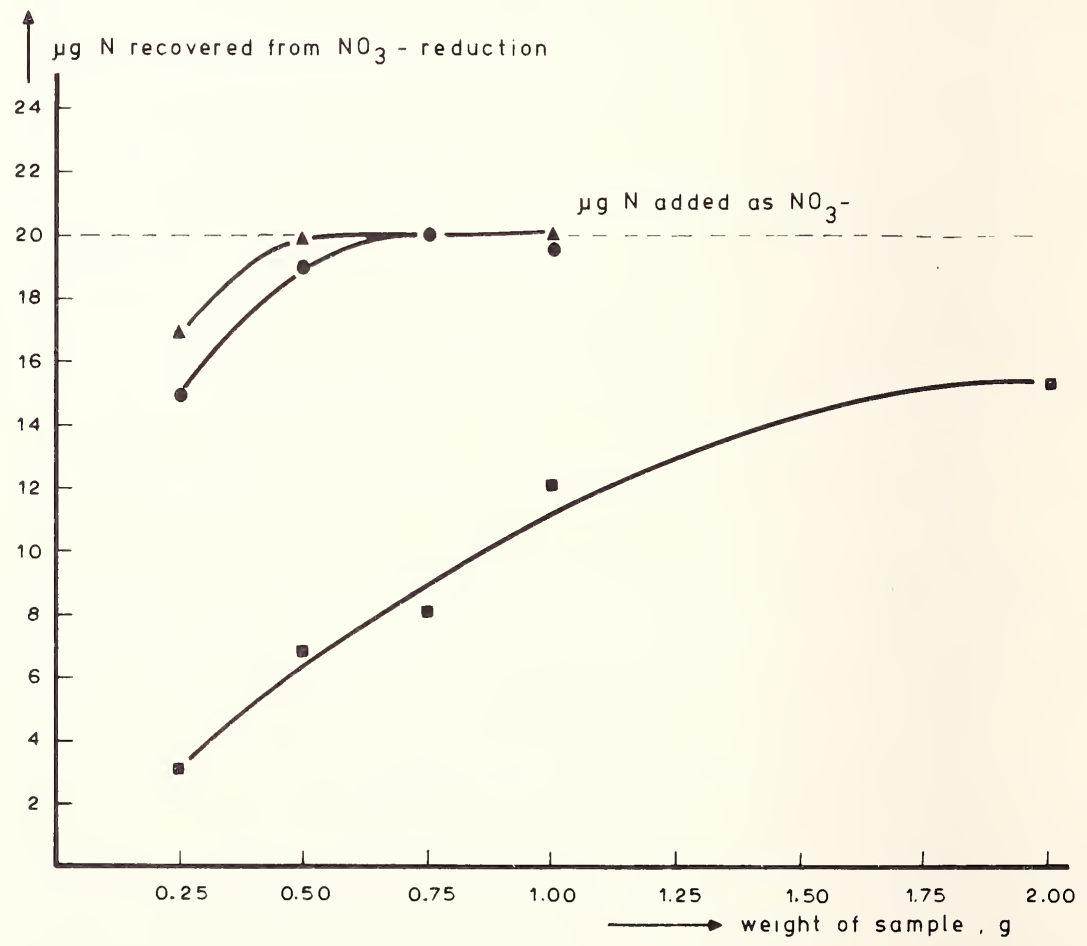

Figure 7. Effect of sample type and sample weight on recovery of nitrate reduction ( $\Delta$ Iron reduced G. R. Merck cat. no. 3819; • Iron sponge Johnson Matthey cat. no. 847; BCS 260/2). 
reducing nitrate and/or nitrite readily and quantitatively. Bearing in mind that the aim of the investigation was the accurate determination of very low levels of nitrogen, all this means that it is necessary to confirm the near absence of nitrogenous oxides in the sulphuric acid used. A procedure for doing this is described in the Appendix II. In case that the amount of $\mu \mathrm{g} \mathrm{N}$ originating from nitrogenous oxides exceeds $1 \mu \mathrm{g} \mathrm{N}$ per $50 \mathrm{ml}$ sulphuric acid, a higher grade and/or better water should be taken. The sulphuric acid brought upon the market by various chemical firms, especially that recommended for the use of Kjeldahl determinations, contains, according to their specifications, a maximum of 0.00002 percent $\mathrm{NO}_{3}{ }^{-}$. This equals $0.6 \mu \mathrm{g} \mathrm{N}$ per analysis. In our current experiences this maximum concentration was never found.

\section{Experimental Results}

\section{A. Total Blank Value-Limit of Detection}

Typical blank values obtained by applying the full blank procedure described in Appendix I are shown in table 1. The table shows the results of five blank determinations being carried out on each of 6 days, covering a period of 2 weeks. A statistical examination of these results gives an overall mean value for the blank of $4.4 \mu \mathrm{g} \mathrm{N}$ with a standard deviation of $0.60 \mu \mathrm{g} \mathrm{N}$. Applying to this reproducibility figure the relationship of Kaiser (see before) a limit of detection of approximately $1.6 \mu \mathrm{g} \mathrm{N}$ is calculated. This equals to 0.00008 percent of nitrogen in steel when using a 2 gram sample. Table 2 shows the results obtained on Johnson Matthey iron

TABLE 1. Blank values for nitrogen on six separate days over a period of two weeks

Nitrogen, Day

Nitrogen, $\mu \mathrm{g} \longrightarrow \begin{array}{llllll}1 & 2 & 3 & 4 & 5\end{array}$

$\begin{array}{llllll}4.0 & 4.2 & 5.3 & 4.8 & 3.3 & 4.8 \\ 3.8 & 4.9 & 4.7 & 4.0 & 2.9 & 5.6 \\ 5.2 & 4.6 & 5.0 & 5.4 & 3.3 & 6.0 \\ 4.0 & 4.0 & 4.5 & 4.2 & 4.2 & 5.3 \\ 4.0 & 3.6 & 4.8 & 3.9 & 3.0 & 4.0\end{array}$

Mean value

4.2

4.3

4.9

4.5

3.3

5.1 


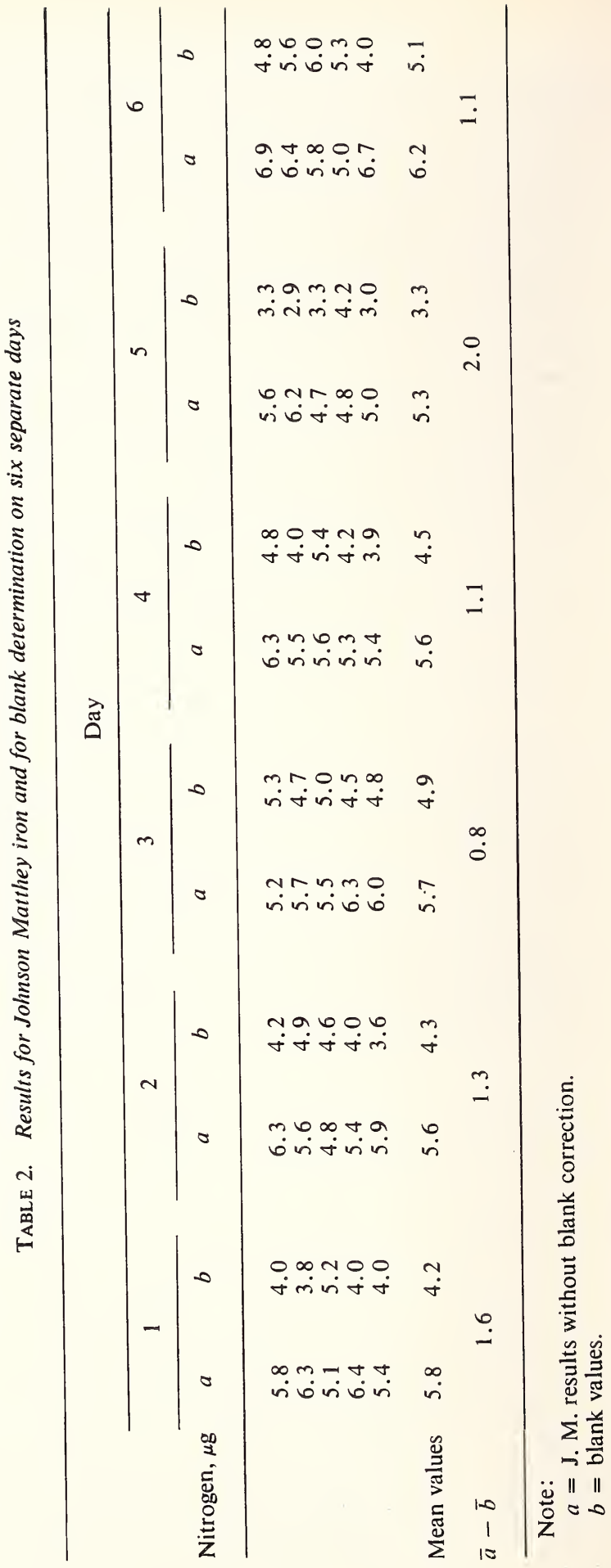


sponge covering a period of 6 days having five test and five blank determinations each day. Referring to only one single test determination in combination with one single blank determination, the nitrogen content of the Johnson Matthey sample cannot be distinguished from the limit of detection, i.e., $1.6 \mu \mathrm{g} \mathrm{N}$ or $0.8 \mathrm{ppm} \mathrm{N}$ when taking a 2 gram sample. If however the mean value of the five test determinations after correction for the mean of the five blank determinations is taken, a limit of the detection of $1.6 / \sqrt{5} \approx 0.7 \mu \mathrm{g} \mathrm{N}$ or $0.3_{5} \mathrm{ppm} \mathrm{N}$ is found. The values in table $2(\mathrm{a}-\mathrm{b}$ ) just exceed this limit of detection, consequently estimating a nitrogen content of the Johnson Matthey iron sponge of 0.00006 percent.

\section{B. Results on Standard Samples}

In the concentration range being considered $(<0.002 \%)$ no officially recognized standard sample is available. Some years ago a sample from the French Institute IRSID was distributed among the members of the European study group for nitrogen in steel. Although the results between the various laboratories showed a very large spread, a mean value of 12.9 parts per million was calculated upon statistical evaluation. In the framework of this investigation this sample IRSID 021-1 was analyzed 24 times over 8 separate days, performing three determinations each day. The results shown in table 3 have all been corrected for the corresponding blank values. From the results an overall mean value of 12.8 parts per million in the sample is calculated with a standard deviation of 0.58 for a single determination and of $0.58 / \sqrt{24} \approx 0.12$ in the mean value.

TABLE 3. Results after blank correction for the sample IRSID 021-1 on eight separate days

Nitrogen, ppm

Day

\begin{tabular}{ccccccccc}
1 & 2 & 3 & 4 & 5 & 6 & 7 & 8 \\
\hline 12.2 & 13.4 & 13.7 & 11.9 & 12.3 & 12.7 & 12.8 & 13.2 \\
13.2 & 13.1 & 13.9 & 11.9 & 12.5 & 13.7 & 12.8 & 13.4 \\
12.7 & 13.1 & 13.3 & 12.3 & 12.1 & 12.7 & 13.4 & 12.4
\end{tabular}




\section{Conclusions}

Starting from the information available from the European standard method for nitrogen in steel which uses the Kjeldahl dissolution and distillation technique in combination with the indophenol blue reaction as the method of analysis, a further expansion towards the lower concentration range appears to be possible. The limiting factor seems to be brought about by the level and the reproducibility of the blank rather than by a lag in sensitivity of the indophenol blue reaction. Reduction of the blank requires special experimental conditions such as a remote laboratory, specially selected reagents and equipment protected from atmospheric contact. The absence of nitrate or nitrite compounds in the reagents will lead to a more meaningful assessment of the nitrogen content in the sample. It is found that under optimum laboratory conditions total blank values of the order of $4 \mu \mathrm{g} \mathrm{N}$ per determination can be obtained in combination with a standard deviation of approximately $0.6 \mu \mathrm{g} \mathrm{N}$. For a single determination this will correspond to a limit of detection of $\sim 0.00008$ percent $\mathrm{N}$ in steel based on 2 gram of sample.

\section{References}

[1] Communauté Européenne du Charbon et de l'Acier, Analyse Chimique des Materiaux Sidérurgiques, Dosage de l'Azote dans les Aciers, Méthode Spectrophotométrique; EURONORM 50-72.

[2] Kaiser, H. and Specker, H., Z. Anal Chem. 149, 46-66 (1956).

[3] Harwood, J. E. and Huyser, D. J., Some Aspects of the Phenol-Hypochlorite Reaction as Applied to Ammonia Analysis, Water Research 4, 501-515 (1970).

[4] Jenkins, R. H., The Determination of Small Amounts of Total Nitrogen in Steel, Proceedings of the Twenty-Fourth Chemists' Conference, May 11-13, 1971, Scarborough, British Steel Corporation, England. 


\section{APPENDIX I}

\section{PHOTOMETRIC DETERMINATION OF SMALL AMOUNTS OF NITROGEN (0-20 ppm) \\ IN PLAIN CARBON STEELS}

\section{Contents}

1. Scope

2. Field of Application

3. Principle of Method

4. Reagents

5. Apparatus

6. Sampling and Samples

7. Procedure

1. Test Portion

2. Determination of the Blank

3. Determination

1. Dissolution

2. Distillation

3. Photometric Determination

4. Determination of the Calibration Factor

8. Expression of Results

\section{Scope}

This method describes a photometric determination of the nitrogen content of plain carbon steel.

\section{Field of Application}

It is possible with this method to determine photometrically the nitrogen, which is transformable in ammonia, to a level of 0.003 percent.

\section{Principle of Method}

The sample is dissolved in dilute sulphuric acid. After the water has been evaporated, the mixture is fumed for 2 hours at 300 to $340^{\circ} \mathrm{C}$, during 
which nitrogen is converted into ammonium. The ammonium formed can be liberated by distillation from an alkaline medium and carried into a distillate receiver by nitrogen or air as a carrier gas. The amount of ammonia-nitrogen is determined photometrically by.measuring the absorbance of the blue complex, produced when sodium phenolate, sodium nitroprusside and sodium hypochlorite are added in succession to the distillate.

\section{Reagents}

1. Distilled or deionized water, specially reserved for determining very low levels of nitrogen.

2. Sulphuric acid $18 M(\rho=1.84 \mathrm{~g} / \mathrm{ml})$, specially reserved for determining very low levels of nitrogen.

3. Sulphuric acid $3 M(1+5)$, specially reserved for determining very low levels of nitrogen; $167 \mathrm{ml}$ of sulphuric acid (4.2.) is added to about $800 \mathrm{ml}$ of water (4.1.) in small amounts with cooling; cool, dilute to 1000 $\mathrm{ml}$ and mix.

4. Sulphuric acid $0.018 M$ - add $10 \mathrm{ml}$ of sulphuric acid (4.2.) cautiously to about $900 \mathrm{ml}$ of water (4.1.) and dilute with water (4.1.) to 1 1; take from this solution $100 \mathrm{ml}$ and dilute again with water (4.1.) to 11 .

5. Sodium hydroxide solution $500 \mathrm{~g} / \mathrm{l}$ - dissolve $500 \mathrm{~g}$ of sodium hydroxide in pellet form in about $700 \mathrm{ml}$ of water (4.1.); heat the solution and boil for 10 minutes; cool and dilute with water (4.1.) to 11 .

6. Sodium phenolate solution - dissolve $8 \mathrm{~g}$ of sodium hydroxide in pellet form in $200 \mathrm{ml}$ of water (4.1.); dissolve in this solution $15 \mathrm{~g}$ of distilled phenol under stirring and dilute to $250 \mathrm{ml}$ in a volumetric flask with water (4.1.); prepare this solution shortly before it is required.

7. Sodium nitroprusside solution - dissolve $0.5 \mathrm{~g}$ of $\mathrm{Na}_{2} \mathrm{Fe}(\mathrm{CN})_{5} \mathrm{NO}$. $2 \mathrm{H}_{2} \mathrm{O}$ in water (4.1.) and dilute with water (4.1.) to 11 in a volumetric flask.

8. Sodium hypochlorite solution $4.5 \mathrm{~g} / 1$ - store the solution in a refrigerator at a temperature of about $2{ }^{\circ} \mathrm{C}$. Note: a) At room temperature 
the hypochlorite solutions are unstable. When stored in a refrigerator (temperature between 0 and $10{ }^{\circ} \mathrm{C}$ ) the stability is better; at a temperature of about $2{ }^{\circ} \mathrm{C}$ the solution can be kept for months. b) The concentration of the sodium hypochlorite solution must be checked after preparation with an iodometric titration. Pipette for this check $10.0 \mathrm{ml}$ of the solution into a conical flask which contains already $25 \mathrm{ml}$ of water, $30 \mathrm{ml}$ of hydrochloric acid $(3 M)$ and $30 \mathrm{ml}$ of a potassium iodide solution $(100 \mathrm{~g} / \mathrm{l})$. Titrate the liberated iodine with a sodium thiosulphate solution $(0.05 \mathrm{M})$, using a starch solution as indicator. The use of sodium thiosulphate solution must be 11 to $13 \mathrm{ml}$.

9. Nitrogen or inert gas, free from ammonia - instead of an inert gas compressed air can also be used; the used gas can be purified from ammonia by passing it through sulphuric acid (4.13).

10. Standard nitrogen solution, $250 \mu \mathrm{g} \mathrm{N} / \mathrm{ml}$ - weigh out accurately $1.179 \pm 0.001 \mathrm{~g}$ of dry ammonium sulphate and dissolve this in water (4.1.) in a volumetric flask of $1 \mathrm{l}$; dilute with water (4.1.) to the mark and mix.

11. Standard nitrogen solution, $5 \mu \mathrm{g} \mathrm{N} / \mathrm{ml}$ - pipette $10.0 \mathrm{ml}$ of the standard nitrogen solution (4.10.) into a volumetric flask of $500 \mathrm{ml}$; dilute with water and mix.

12. Devarda's alloy

13. Sulphuric acid $18 M$

5. Apparatus

Normal laboratory appliances

Glassware for the dissolution and decomposition of the sample (fig. 1).

Glassware for the ammonia distillation (fig. 2). Both the glassware for the dissolution and for the distillation should be present in manifold.

Spectrophotometer or filterphotometer equipped with a filter with sufficiently small band width for measurements at $640 \mathrm{~nm}$ in cells or .5, 1 and $2 \mathrm{~cm}$. 


\section{Sampling and Samples}

Sampling and sample preparation are carried out according to Euronorm 18.

\section{Procedure}

Note: Perform the experimental work in a specially reserved laboratory free from nitrogenous compounds.

1. Test portion - weigh to the nearest $0.001 \mathrm{~g}$, exactly $2 \mathrm{~g}$ of the test sample.

2. Determination of the blank - determine the blank as described in paragraph (7.3.) by analyzing $50.0 \mathrm{ml}$ of sulphuric acid (4.3.) at the same time as the sample, ensuring that the whole procedure is followed under identical conditions; measure the absorbance $\mathrm{A}$ of this blank solution against water at $640 \mathrm{~nm}$ in a $1 \mathrm{~cm}$ cell.

\section{Determination}

1. Dissolution - the sample is dissolved and decomposed, protected from the laboratory atmosphere, in a destruction apparatus as shown in figure 1. Transfer the sample into the reaction vessel (A) and add $50.0 \mathrm{ml}$ of sulphuric acid (4.3.). Next the vessel (A) is put immediately into the position shown in figure 1 , after which the dissolution can start. Stop-cock 1 must be opened and stop-cock 2 closed. Heat with an electrical heater $(\mathrm{H})$ till the hydrogen development has finished, during which the solution is not allowed to boil. Raise the temperature and evaporate the solution to fuming. Continue fuming at a minimum temperature of $320{ }^{\circ} \mathrm{C}$ for two hours avoiding dryness. Next cool while stop-cock 1 is closed and stopcock 2 is opened.

2. Distillation - use for the distillation an apparatus as shown in figure 2. Transfer before starting the distillation $40 \mathrm{ml}$ of sodium hydroxide solution (4.5.) into vessel (J) and switch the three-way stop-cock (3) of this vessel to position I. Connect the volumetric flask (M) which contains already $12 \mathrm{ml}$ of sulphuric acid (4.4.) to the condenser (L). Disconnect the reaction vessel (A) from the destruction apparatus, add as quickly as possible $60 \mathrm{ml}$ of water (4.1.) to the reaction vessel (A) and connect it immediately to the distillation apparatus in the position shown in figure 2 . 
Introduce a gas stream (4.9.) with a gas flow rate of approximately $20 \mathrm{l} / \mathrm{h}$. Transfer then the $40 \mathrm{ml}$ sodium hydroxide solution from the vessel $(\mathrm{J})$ into reaction vessel (A) by switching the three-way stop-cock (3) to position II. Heat the solution in reaction vessel (A) till boiling and finish the distillation when about $50 \mathrm{ml}$ has been distilled. Rinse the end of the condenser (L) with water (4.1.) when removing the volumetric flask (M) containing the distillate.

3. Photometric determination - transfer to the volumetric flask (M) the following reagents in succession, using pipettes:

$5.00 \mathrm{ml}$ of sodium phenolate solution (4.6.)

$5.00 \mathrm{ml}$ of sodium nitroprusside solution (4.7.)

$5.00 \mathrm{ml}$ of sodium hypochlorite solution (4.8.)

Mix well after each addition. Dilute to the mark with water (4.1.) and mix again. The solution is allowed to stand for colour development during one hour at room temperature in a dark place.

Note: The $\mathrm{pH}$ of the final solution must be 11.6 after addition of all the reagents. If all the reagents have been prepared with sufficient care and if all the volumes of reagent solutions have been measured accurately, this condition will be fulfilled.

Measure the absorbance (A) of the solution at $640 \mathrm{~nm}$ in a $1 \mathrm{~cm}$ cell with reference to water. When the absorbance is high or low a $.5 \mathrm{~cm}$ cell or a 2 $\mathrm{cm}$ cell can be used after which the absorbance is recalculated on the basis of a $1 \mathrm{~cm}$ cell.

\section{Determination of the Calibration Factor}

Transfer to each of seven $100 \mathrm{ml}$ volumetric flasks the following portions of the standard nitrogen solution (4.11.): 0.00, 1.00, 2.00, 4.00, 5.00, $8.00,10.00 \mathrm{ml}$. Add $12.0 \mathrm{ml}$ of sulphuric acid (4.4.) to each and dilute with water (4.1.) to about $60 \mathrm{ml}$. Proceed with the solutions as described in paragraph (7.3.3.).

Prepare a graph in which the measured absorbances are plotted against the concentration of nitrogen in $(\mu \mathrm{g})$. Calculate the calibration factor $f$. based on a straight line characteristic of the calibration curve, in terms of $\mu \mathrm{g} \mathrm{N}$ per absorbance unit, measured in a $1 \mathrm{~cm}$ cell.

\section{Expression of Results}

The blank $m_{o}$ paragraph (7.2.) is given in $\mu \mathrm{g}$ by the following equation:

$$
m_{o}=A_{o} \cdot f
$$


in which:

$$
\begin{aligned}
& A_{0}=\text { the absorbance of the blank solution based on a } 1 \mathrm{~cm} \text { cell } \\
& f=\text { calibration factor, determined as described in paragraph (7.4.). }
\end{aligned}
$$

The nitrogen content of the sample is given in percent $(\mathrm{m} / \mathrm{m})$ by the equation:

$$
\text { percent }(m / m) N=\left(A \cdot f-m_{o}\right) /\left(10^{4} \cdot m\right)
$$

in which:

$m_{0}=$ the blank value in $\mu \mathrm{g}$

$m=$ the weight of sample in $\mathrm{g}$

$A=$ the absorbance of the test solution at $640 \mathrm{~nm}$ against water, based on a $1 \mathrm{~cm}$ cell

$f=$ the calibration factor, determined as described in paragraph (7.4.). 


\section{APPENDIX II}

\section{TEST TO $\mathrm{NO}_{3}-\mathrm{NO}_{2}-$ CONTAMINATION OF DILUTED SULPHURIC ACID}

In this test Devarda's alloy is used, which reduces in alkaline medium all the nitrate/nitrite into ammonia. Because the Devarda's alloy itself may contain nitrogen and the used diluted sulphuric acid can be contaminated with ammonia, the test is rather laborious. For this test the distillation apparatus, as shown in figure 2 , is used.

The following sequence of determination is carried out:

1. the direct distillation of $40 \mathrm{ml}$ of sodium hydroxide (4.5.) added through vessel $(\mathrm{J})$

2. the distillation of $50 \mathrm{ml}$ of sulphuric acid (4.3.) to which $40 \mathrm{ml}$ of sodium hydroxide (4.5.) is added through vessel $(\mathrm{J})$

3. the distillation of $0.500 \mathrm{~g}$ of Devarda's alloy (4.13.) to which also $40 \mathrm{ml}$ of sodium hydroxide (4.5.) is added through vessel $(\mathrm{J})$

4. the distillation of $0.500 \mathrm{~g}$ of Devarda's alloy (4.13.) plus $50.0 \mathrm{ml}$ of sulphuric acid (4.3.) to which again $40 \mathrm{ml}$ of sodium hydroxide (4.5.) is added through vessel $(\mathrm{J})$.

For each determination the procedure as described in paragraph (7.3.3.) is followed. Measure the respective absorbances $A_{1}, A_{2}, A_{3}$ and $A_{4}$ against water at $640 \mathrm{~nm}$ in a $2 \mathrm{~cm}$ cell. The absorbance as a result of the converted nitrate/nitrite in the dilute sulphuric acid can be calculated as follows:

$$
A=\left(A_{4}-A_{3}\right)-\left(A_{2}-A_{1}\right)
$$

in which: $A_{4}-A_{3}=$ the absorbance as a result of nitrate/nitrite and ammonia in the sulphuric acid (4.3.); and $A_{2}-A_{1}=$ the absorbance as a result of ammonia in the sulphuric acid (4.3.).

The number of $\mu \mathrm{g}$ of nitrogen that has come from the nitrate/nitrite in $50.0 \mathrm{ml}$ of sulphuric acid (4.3.), is calculated by the following equation:

$$
\mu \mathrm{g} \mathrm{N}_{\mathrm{NO}_{3}-/ \mathrm{NO}_{2}-}=A^{\prime} \cdot f
$$

in which: $A^{\prime}=$ the absorbance $A$ recalculated on a $1 \mathrm{~cm}$ cell; and $f=$ the calibration factor, determined as described in paragraph (7.4.). 



\title{
TRACE ELEMENT ANALYSIS OF NICKEL AND NICKEL-BASE ALLOYS - A REVIEW OF CURRENT METHODS
}

\author{
C. M. Davis \\ The International Nickel Company, Inc. \\ Paul D. Merica Research Laboratory \\ Sterling Forest \\ Suffern, New York 10901 U.S.A.
}

\begin{abstract}
Trace element analysis has been a frontier in the field of analytical chemistry for many years. Twenty years ago 0.01 percent was considered a "trace." Today, specifications require certain nickel-base alloys to have no more than 0.00003 percent $(0.3 \mathrm{ppm})$ of some elements. The everchanging definition of the word "trace" has made trace element analysis a continuing challenge to the analytical chemist. This paper reviews the various approaches to this elusive problem and outlines potential solutions. The emphasis is on elements categorized as being detrimental to the mechanical properties of nickel-base alloys since these are of the greatest concern.

Among the methods reviewed are spark source mass spectrometry, emission spectroscopy, x-ray fluorescence spectroscopy. spectrophotometry, polarography, and atomic absorption spectrophotometry.
\end{abstract}

Keywords: Atomic absorption spectrophotometry; emission spectroscopy: nickel; nickel-base alloys; polarography; spectrophotometry; $\mathrm{x}$ ray fluorescence spectroscopy.

\section{Introduction}

The detrimental effect of certain trace elements on the mechanical properties of nickel-base alloys has been well established. Wood and Cook [1] demonstrated that not only do certain trace elements have detrimental effects on the creep rupture properties of nickel-base superalloys, but that the effect is additive. In 1972 Morykwas [2] published the rigid specification for the acceptable amounts of various trace elements in nickel- and cobalt-base alloy investment castings set by the General Elec- 
tric Company in 1970. In these specifications the maximum allowable limits for bismuth, thallium, and tellurium were: 1, 3, and 5 parts per million, respectively. For lead and silver, 10 ppm was allowed. Since 1972, new specifications have been announced. German specifications for primary nickel are as follows: bismuth, $0.2 \mathrm{ppm}$ max; thallium, $1 \mathrm{ppm}$ max; tellurium, $0.5 \mathrm{ppm}$ max and lead, $1 \mathrm{ppm}$ max.

These stringent specifications are met by a high purity nickel pellet produced by the Inco carbonyl nickel process. With the increasing demand for purer nickel, followed by the commercial production of extremely pure nickel, the analytical chemist was faced with the challenge of analyzing this material.

The purpose of this paper is to highlight some of the responses to this challenge and to review some of the various approaches and techniques used to solve this elusive problem.

\section{Symposia and Reviews}

Growing out of this everincreasing interest in the need for analytical methods for trace element analysis, an ASTM Symposium on Spectrochemical Analysis for Trace Elements was held in 1957 [3]. The paper by Norris [4] on trace elements in metals presents a variety of approaches to this problem, and includes 39 references. Although this paper deals with spectrographic methods, an outline of various "indirect" methods is presented. These involve some type of chemical preconcentration of the trace elements. Obviously not as desirable as direct methods, the "indirect" or preconcentration techniques allow tremendous flexibility in lowering the limit of detection or operating range of concentration. In discussing various separation or concentration techniques, Norris states: "The usefulness of the precipitation method is limited only by the broad range of precipitating agents available and the ingenuity of the person developing the method." The determination of quantities below $1 \mathrm{ppm}$ is cited.

In 1966, a "Symposium on Trace Characterization-Chemical and Physical" was sponsored by the National Bureau of Standards and was held in Gaithersburg, Maryland [5]. This was international in scope and covered a wide variety of techniques in many materials, largely other than alloys. Pertinent to traces in alloys were the papers by Cheng (Paper No. 36, Ref. 5) and by Maienthal and Taylor (Paper No. 5, Ref. 5). The latter paper compared results for trace amounts of tellurium in brass obtained by polarographic, neutron activation and spectrochemical analysis. 
Trace elements affect temper embrittlement in steels. An ASTM task force was formed to study this effect and establish appropriate methods of analysis for the detrimental trace elements. In 1967 a "Symposium on Temper Embrittlement in Steel" was sponsored by the ASTM task force on large turbine and generator rotors (Subcommittee VI of Committee A1 on Steel). This symposium of course focused on steels, rather than on nickel-base alloys, but it, and the review paper by Nadalin, Penkrot, Rudolf, Wolfe and Byrne [6] presented at this symposium are cited here since many of the analytical techniques and approaches are common to both iron- and nickel-base alloys.

In 1970 at the National Meeting of the ASTM, a task group was formed to establish wet chemical methods for the analysis of trace elements in nickel which would finally appear in the ASTM book of standards. This task group is under Subcommittee E-3.05 of Committee E-3 on Chemical Analysis of Metals. Prior to the formation of this task group a similar group was formed for spectrochemical methods for the analysis of trace elements in nickel-base alloys. This group is under E-2 on Spectrochemical Analysis of Metals. In conjunction with the Annual Meeting of the Electrochemical Society in Miami Beach, Florida in October 1972, a symposium on "Trace Elements in High-Temperature Alloys" was chaired by Golden [7]. A variety of papers were presented at this symposium, some on the role of certain trace elements in nickel-base alloys and some on methods of analysis of certain trace elements. Among the techniques discussed were polarography, spark source mass spectroscopy, preconcentration followed by $x$-ray fluorescence spectroscopy, direct and solution methods using $\mathrm{x}$-ray fluorescence spectroscopy, solvent extraction followed by atomic absorption spectrophotometry (a report on the progress of the ASTM task group on chemical methods for traces in nickel) and others.

From the above it is evident that considerable work has been done in the area of trace element analysis. The remainder of this paper will deal with specific methods, instruments and techniques from which one may choose the most suitable approach to this problem in his own laboratory.

\section{Direct Analysis Techniques}

In the search for the ideal method of analysis for sub-ppm quantities, the criteria of accuracy and precision are sometimes compromised. Sensitivity (or detectability), the nature and precision of the blank, and the potential for contamination and control of the blank assume much more 
importance than when higher quantities are analyzed for. In any event, controlling the blank and avoiding contamination can be improved by selecting methods requiring a minimum of sample preparation and chemical treatment prior to making a measurement of concentration. These methods are often called "direct" methods, as compared with the indirect methods which involve the use of chemicals and usually chemical separation techniques; e.g., ion-exchange, solvent extraction, and coprecipitation.

Outstanding among the direct methods are: spark source mass spectrometry, optical emission spectrography, and the $\mathrm{x}$-ray spectrograph. On the horizon is another promising direct technique: flameless atomic absorption spectrophotometry, where fine chips of metal are placed directly into the sample compartment of the instrument rather than oxides or a solution of the sample. This technique is currently being investigated by several laboratories.

Of the techniques just mentioned, the one quite capable of measuring quantities well below the parts per million level is spark source mass spectrometry. Brown [7] reported that many elements could be measured down to $0.05 \mathrm{ppm}$ or lower using a pair of pins machined from the metal sample. Brown et al. [8] reviewed the applications of the spark source mass spectrometer to "high-duty alloys" as well as many other materials. A high degree of accuracy is not required when $0.1 \mathrm{ppm}$ is being measured. The blank is not a serious problem since no chemical treatment is required, and contamination can be controlled by meticulous sample handling. Contamination from a previous sample, "memory effects," can be controlled by proper cleaning and care. One outstanding drawback to this technique is the cost of the equipment. Most laboratories cannot afford the capital outlay for the spark source mass spectrometer.

The optical emission spectrograph, now past its 100th birthday, may be classified as a "direct technique" since the point-to-plane method does not involve a chemical treatment of the sample. The ignited dc arc source on the spectrograph has been used when sensitivity was required. However, the sensitivity was increased by conversion of the sample to the oxide and packing it into a cratered electrode after being mixed with graphite [9]. Later, it was found that by mixing it with a carrier further increases in sensitivity were obtained [10]. In the latter work, a silver chloride-lithium fluoride carrier dc arc technique was used and the stated measurable concentration range for bismuth, lead, and tin in nickel-base alloys was found to be 0.5 to $15 \mathrm{ppm}$.

A more sensitive method using the optical emission spectrograph was published by K. Thorton [11] of the Henry Wiggin and Company, Ltd. in 1969. In this method, a demountable hollow cathode lamp is attached 
to the emission spectrograph. The sample, in the form of small metal chips, is placed directly into a graphite electrode, the hollow cathode, which is then placed in the housing. Excellent sensitivity is obtained for bismuth, lead, tin, antimony, cadmium, zinc, thallium, silver, and tellurium. This technique has been used by International Nickel for over a year for the analysis of the high-purity nickel pellets produced by the Inco carbonyl process. Most of these elements can be measured down to $0.1 \mathrm{ppm}$, some below 0.1 .

Other uses of the optical emission spectrograph in conjunction with separation techniques will be discussed later.

$\mathrm{X}$-ray spectroscopy (fluorescence) is a completely nondestructive and usually direct method of analysis. It has been found to be quite sensitive with the detectors now available. However, the sensitivity is insufficient to measure elements below $1 \mathrm{ppm}$ in a nickel matrix. Because of the excellent precision of this technique it has become useful for trace element analysis after preconcentration. This will be discussed under "Indirect Methods."

\section{Indirect Methods}

Common to the experienced analytical chemist is the concept that when lower quantities of an element are sought, a larger sample size is used. This assumes that a separation technique is employed to separate the matrix from the element sought. It also assumes that the blank is controllable and can be measured. Of course, there is a limit to the size of the sample and amounts of reagents used in this approach. Nevertheless, indirect methods covering a wide range of separation techniques and measuring instruments have been published. A few of these will be cited to assist the chemist faced with selection of the best method for his laboratory. A brief review of the popular chemical separation techniques follows.

\section{A. Solvent Extraction}

Probably the widest used method for the preconcentration of trace metals is solvent extraction. This technique involves the partition of two immiscible liquids, the element sought being separated by being selectively soluble in one liquid and not in the other. The technique involves the formation of a metallo-organic compound containing either the elements sought or the matrix element. In the latter case the matrix is removed, thereby concentrating the remaining elements. Solvent extraction is wide- 
ly used because of its simplicity and the speed of the separation. An ASTM symposium [12] was devoted to the subject. Morrison and Freiser [13] presented an outstanding treatise which not only discusses the theory, but cites many references to practical applications of this powerful technique. Another more recent book by De et al. [14] states that over 5,000 papers have been published on this useful and versatile technique.

\section{B. Co-Precipitation}

This technique, referred to earlier [4], requires the presence of a second element, usually in larger quantities than the element sought, which precipitates under similar conditions as those of the element sought. This second element is often referred to as the "collector" or "carrier." The availability of the micropore filter has contributed to the use of this technique. Applications using precipitation and co-precipitation in conjunction with the optical emission spectrograph and $\mathrm{x}$-ray spectrograph will be cited later.

\section{Ion Exchange}

This valuable method serves to remove the matrix metals or the elements sought, depending on the nature of the resin, the $\mathrm{pH}$, the stability of the compounds present and other factors. Samuelson [15] gives an excellent introduction to this technique.

These methods of separation have been reviewed in the April issue of Analytical Chemistry for many years. In addition, there is an outstanding paper by Kraus and Nelson [16] with 47 references.

These techniques, as well as others, will be found in the references which follow. It should be emphasized that they may be used in conjunction with almost any of the instrumental techniques which are described below.

\section{Spectrophotometric Methods}

Sandell [17] also discusses the above separation techniques, and describes how the final measurements are done using the spectrophotometer or filter photometer. An excellent treatment is also presented on the problems of determining microgram quantities of lead 
using dithizone. This reagent, soluble in carbon tetrachloride and chloroform, has been used for the solvent extraction and color measurement of lead for many years, and it is still used in many laboratories. In our laboratory we have abandoned the use of dithizone primarily because of inconsistent blanks and the need to analyze smaller quantities. Bismuth also forms a colored dithizonate and may be measured spectrophotometrically. In our laboratory arsenic is measured after reduction to the molybdenum blue color spectrophotometrically after preconcentration by solvent extraction.

Color-forming reagents are available for almost all elements of interest in nickel and nickel-base alloys. In the annual reviews of Analytical Chemistry and in Sandell's treatise [17], thousands of references to published procedures demonstrate the broad applicability of the spectrophotometer to trace element analysis.

In 1959, Luke [18] published a colorimetric procedure for trace amounts of tellurium in copper and lead. This method has been adapted by Lewis, Ott and Sine [19] to traces of tellurium in nickel. One part per million of tellurium can be determined spectrophotometrically after complexing the nickel with potassium cyanide.

\section{Polarographic Methods}

Since the printing of the authoritative treatise, "Polarography", by Kolthoff and Lingane [20] in 1941, many papers and books have been published showing the usefulness of this technique in trace element analysis. The cathode ray polarograph has extended downward the limits of detection and thereby has made this technique very practical. One of the major advantages is that no separation is required to measure in the parts per million range. Lead and bismuth can be measured with very good precision. At the symposium on trace elements at the Electrochemical Society Meeting in 1972 [7], Maienthal of the National Bureau of Standards presented data for the determination of traces of antimony, cadmium, lead, bismuth and tellurium in matrices such as steels, nickel-base alloys, cast irons, brasses and bronzes. The results compared favorably with data obtained by other techniques. Though simple in operation, the equipment requires a skilled and experienced analyst to keep it in good operating condition. 


\section{Optical Emission Spectrographic Methods}

The classic method for direct analysis of trace elements is optical emission spectroscopy employing the dc arc as the excitation source. When emission spectroscopy is coupled with separation procedures, many elements may be measured below $1 \mathrm{ppm}$.

In 1966, Balfour, Jukes and Thorton [21] described a preconcentration spectrographic procedure for determining down to $0.5 \mathrm{ppm}$ of $\mathrm{Ag}$, As, $\mathrm{Au}, \mathrm{Gi}, \mathrm{Cd}, \mathrm{Ge}, \mathrm{In}, \mathrm{Mo}, \mathrm{Pb}, \mathrm{Sb}, \mathrm{Sn}$ and $\mathrm{Tl}$ in nickel-base alloys. These elements were co-precipitated with copper as sulfides. The separated precipitate was excited using the dc arc using copper as the internal standard.

Later, in 1970, Golden and Atwell [22] modified the above procedure by using molybdenum as the carrier in the precipitation of the sulfides, as well as the internal standard for the measurements. Multiple (triple) exposures on the same photographic plate, as well as the preconcentration, allowed concentration of traces to be determined well below the $1 \mathrm{ppm}$ level.

Another preconcentration technique used prior to spectrographic analysis is solvent extraction. Using essentially the extraction technique published by Burke [23], the trioctylphosphine oxide-methyl isobutyl ketone (TOPO-MIBK) method for isolating lead and bismuth, Harper [24] extracted lead and bismuth then back-extracted these elements into dilute $(1: 1)$ nitric acid. The acid solution containing the lead and bismuth was evaporated to dryness after addition of indium as an internal standard. The residue was then analyzed by emission spectroscopy. Down to $0.2 \mathrm{ppm}$ of lead and bismuth is determined in this manner.

\section{X-Ray Emission Spectroscopy}

Though not as inherently sensitive as optical emission spectroscopy (dc arc) the $\mathrm{x}$-ray spectrograph is a practical, useful instrument. It presents a simple spectrum which is readily interpreted. Primarily used for the determination of major and minor elements in alloys, it has taken its place as a very popular method for trace analysis. Coupled with preconcentration techniques, particularly co-precipitation, it has proven to be a very versatile and valuable instrument.

In 1967, Davis, Burke and Yanak [25] reviewed the published work including methods for tellurium and selenium. Using tin (II) as a reductant, tellurium, in trace amounts, is reduced to its elemental state. Using 
a microprobe filter the tellurium is separated and the elemental tellurium measured by $x$-ray spectroscopy without removal from the filter pad. Using this method we are able to analyze nickel and nickel-base alloys down to $0.1 \mathrm{ppm}$ tellurium. This work was published by Burke et al. [26]. Selenium has also been reduced to its elemental state, filtered and measured with the x-ray spectrograph. Albright et al. [27] reported very good precision for the analysis of 1 to $100 \mathrm{ppm}$ of selenium by the $\mathrm{x}$-ray spectrometer after reducing selenium to the elemental state and filtering on a millipore filter.

Later, Burke and Yanak [28] found that trace amounts of arsenic could be separated from nickel-base alloys by co-precipitation with tellurium using sodium hypophosphite as a reductant. Excellent recovery of the arsenic was found when tellurium was used as a collector. Efforts to separate the arsenic without the tellurium were unsuccessful.

\section{Atomic Absorption Spectrophotometry}

Atomic absorption spectrophotometry is the newest method for trace element analysis. This technique has become well-established in most metal analytical laboratories because of the relatively simple sample preparation procedure, and ease and rapidity of measurement. The sample preparation for many analyses is simply to weigh the sample, dissolve and dilute to a fixed volume.

Lewis, Sine, and Ott [19] reported that lead and zinc can each be determined in electrolytic nickel down to $2.5 \mathrm{ppm}$, without separation or preconcentration. In the search in our laboratory for a method to analyze nickel for elements down to 1 ppm, Burke [23] concentrated bismuth, lead, antimony, and tin by solvent extraction (trioctylphosphine oxide in methyl isobutyl ketone) and then aspirated the organic phase directly into the flame of the atomic absorption spectrophotometer. This method, adopted by the ASTM task group on analysis of nickel for traces yielded very good precision at the $1 \mathrm{ppm}$ level when the method was submitted to round-robin testing of the procedure [7].

Burke also found that other elements are also extracted by modifications of the same procedure and can be readily measured in the nonaqueous phase. The procedures for traces of silver [29] and thallium [30] have been published.

Using a larger sample weight; e.g., 10 grams, and smaller volumes, this technique has now been extended to 0.1 to $0.2 \mathrm{ppm}$ of these elements (and others) and is now used in our laboratory to confirm results obtained by 
the hollow cathode source on the optical emission spectrograph. This confirmation by using two procedures is especially important for the analysis of our high-purity carbonyl nickel products.

Flameless atomic absorption, a still more recent development, shows great promise in the analysis of sub-ppm quantities of certain critical elements in nickel and nickel-base alloys. The tremendous sensitivity of the nonflame technique eliminates the need for prior concentration techniques, and thereby reduces contamination or high blanks. Welcher, Kriege, and Marks [31] found that this new technique has a detection limit for bismuth, lead, selenium and thallium of $0.1 \mathrm{ppm}$, and for Te, 0.2 $\mathrm{ppm}$. The background due to the nickel matrix was reduced by using a deuterium background correction system carefully aligned with the hollow cathode beam. Sample preparation consisted of acid dissolution in a Teflon beaker, reducing the volume by evaporation, cooling and diluting to volume in a plastic volumetric flask.

In a later paper, Welcher et al. [32] extended the nonflame atomic absorption technique to sub-ppm quantities of tin, indium, antimony, arsenic, germanium, and gallium in nickel-base alloys. Interelement interferences and instrumental variables are discussed.

\section{Conclusion}

The commercial production of an extremely pure nickel, produced by the carbonyl process, resulted from the need for such material. Simultaneously, the rigid specifications for purity have challenged the chemical analyst to devise methods and techniques to analyze nickel and nickelbase alloys at the sub-ppm level. It is hoped that this brief review of the state of the art will be helpful to those presented with this elusive problem.

\section{References}

[1] Wood, D. R. and Cook, R. M., Effects of Trace Contents of Impurity Elements on the Creep-Rupture Properties of Nickel-Base Alloys, Metallurgia 67, 109-117 (1963).

[2] Morykwas, S., Limits on Trace Elements in Investment Casting Alloys, Foundry 100, 106-108 (1972).

[3] Symposium on Spectrochemical Analysis for Trace Elements, ASTM STP 221, Am. Soc. Testing and Materials (1957).

[4] Norris, J. A., Spectrographic Determination of Trace Elements in Metals, Symposium on Spectrochemical Analysis for Trace Elements, ASTM STP 221, 2338, Am. Soc. Testing and Materials (1957). 
[5] Meinke, W. W. and Scribner, B. F., Eds., Trace Characterization, Chemical and Physical, NBS Monograph 100, U.S. Government Printing Office, Washington, D.C., 580 pages (1967).

[6] Byrne, F. P., Nadalin, R. J., Penkrot, J., Rudolf, J. S., and Wolfe, C. R., The Analysis of Rotor Steels for Residual Elements, Symposium on Temper Embrittlement in Steel, ASTM STP 407, pp. 237-250, Am. Soc. Testing and Materials (1967).

[7] Program and Abstracts, Symposium on Trace Elements in High-Temperature Alloys, The Electrochemical Society, 142d Annual Meeting, Miami Beach Florida, Oct. 8-9, 1972 (Paper Nos. 298-305).

[8] Brown, R., Jacobs, M. L., and Taylor, H. E., American Laboratory, Nov.29-30 (1972).

[9] Standard Method for Spectrochemical Analysis of Thermionic Nickel Alloys by the Powder-D-C Arc Technique, ASTM Standards 1973, Part 32 E 129-61, 1915 Race Street, Philadelphia, Pennsylvania (1973).

[10] Atwell, M. G. and Golden, G. S., Appl. Spectrosc. 24, 362-64 (1970).

[11] Thornton, K., Analyst 94, 958-67 (1969).

[12] Symposium on Solvent Extraction in the Analysis of Metals, ASTM STP 238, Am. Soc. Testing and Materials (1958).

[13] Morrison, G. H. and Freiser, H., Solvent Extraction in Analytical Chemistry, John Wiley and Sons, Inc., New York (1957).

[14] De, A. K., Khopkar, S. M., and Chalmers, R. A., Solvent Extraction of Metals, Van Nostrant Reinhold Co., London (1970).

[15] Samuelson, O., Ion Exchangers in Analytical Chemistry, John Wiley and Sons, Inc., New York (1953).

[16] Kraus, K. A. and Nelson, F., Metal Separation by Anion Exchange and Chromatography in Analytical Chemistry. ASTM STP 195, 27-57, Am. Soc. Testing and Materials (1958).

[17] Sandell, E. B., Colorimetric Determination of Traces of Metals, $3 d$ edition, Interscience Publishers, Inc., New York (1959).

[18] Luke, C. L., Anal. Chem. 31, 572-74 (1959).

[19] Lewis, C. L., Ott, W. L., and Sine, N. M., The Analysis of Nickel, Pergamon Press, New York (1966).

[20] Kolthoff, I. M. and Lingane, J. J., Polarography, $2 d$ edition, Interscience Publishers, New York (1952).

[21] Balfour, B. E.. Jukes, D., and Thornton, K., Appl. Spectrosc. 20, 168-171 (1966).

[22] Golden, G. S. and Atwell, M. G., Appl. Spectrosc. 24, 514-18 (1970).

[23] Burke, K.:E., Analyst 97, 19-28 (1972).

[24] Harper, L. E., Solvent Extraction Spectrochemical Method for Trace Amounts of $\mathrm{Pb}, \mathrm{Bi}$, and $\mathrm{Sn}$ in High Temperature Alloys and Raw Materials Used in Their Production, TRW Metals Division Report T10009A (1972).

[25] Davis, C. M., Burke, K. E., and Yanak, M. M., Advances in X-Ray Analysis, Proceedings of the 16th Conference on Applications of X-Ray Analysis, August 9-11, 1967, Denver, Colo., 495 pages, Plenum Press, New York (1968).

[26] Burke, K. E., Yanak, M. M., and Albright, C. H., Anal. Chern. 39, 14-18 (1967).

[27] Albright, C. H., Burke, K. E., and Yanak, M. M., Talanta 16, 309-13 (1969).

[28] Burke, K. E. and Yanak, M. M., Anal. Chem. 41, 963-65 (1969).

[29] Burke, K. E., presented at Joint Eastern Analytical Symposium and National Meeting of the Society for Applied Spectroscopy, New York, N.Y., November 14, 1973.

[30] Burke, K. E., Appl. Spectrosc. 28, 234-37 (1974).

[31] Welcher, G. G., Kriege, O. H., and Marks, J. Y., Anal. Chem. 46, 1227-1231 (1974). 
[32] Welcher, G. G., Kriege, O. H., and Marks, J. Y., presented at the Pittsburgh Conference on Analytical Chemistry and Applied Spectroscopy, Cleveland, Ohio, March $4-8,1974$. 


\title{
NONUNIFORM DISTRIBUTION \\ (BOTH MICRO AND MACRO) OF TRACE ELEMENTS IN HIGH-TEMPERATURE ALLOYS
}

\author{
Owen H. Kriege and Jerry Y. Marks
}

\author{
Pratt \& Whitney Aircraft \\ Materials Engineering \& Research Laboratory \\ East Hartford, Connecticut 06108 U.S.A.
}

Because low melting metals such as bismuth, lead, selenium, tellurium and thallium can have a harmful influence on the mechanical properties of hightemperature alloys, the accurate determination of 0.3 to $10 \mathrm{ppm}$ of these metals is of considerable importance. In developing reliable methods for the determination of trace elements in complex, high-temperature alloys, major sampling problems were encountered. Significant variations in trace metal concentrations were frequently noted in different parts of the same casting. For example, marked variations were sometimes observed between the top and bottom of small cast parts, other specimens had significantly different amounts of trace metals at the surface than at the center of the coating, while the concentration of certain trace metals was a function of wall thickness in some hollow parts. The common practice of sample selection by removal of material from noncritical regions of a casting to preserve the part can evidently lead to serious errors in cases when macro-segregation of trace elements exists. Various production practices which have produced analytically important macro-segregation are summarized in detail.

In addition to macro-segregation, significant intergranular segregation has been observed in high-temperature alloys. There is a pronounced tendency for certain trace elements to concentrate at grain boundaries rather than at the center of grains. With the increased commercial importance of directionally solidified and other large grain cast materials, the importance of this microsegregation is increased, particularly when methods are used which involve the analysis of very small amounts of sample. Techniques for proper sampling of nonuniform, high-temperature alloys are reviewed.

In addition, those methods which are currently used to determine low concentrations of trace metals in high-temperature alloys are reviewed.

Keywords: High-temperature alloys; intergranular segregation: macrosegregation; sampling problems; trace elements. 
Trace amounts of several low melting metals such as bismuth, lead, selenium, tellurium and thallium have a deleterious influence on the mechanical properties of high-temperature alloys. Consequently, it has been necessary to develop reliable procedures for the determination of 0.3 to $10 \mathrm{ppm}$ of these metals in complex alloy systems. In the process of developing these methods, significant segregation of trace elements was noted in many conventionally cast alloy parts. Precise analysis for trace elements in these castings necessitates the utilization of specialized sampling procedures to permit the measurement of both the amount and the location of the contaminant. The various types of segregation which have been observed in castings will be detailed and the effect on sampling noted.

Perhaps the most familiar type of alloy segregation, and one of the easiest to detect, is tramp contamination of the master melt. With the increased use of reclaimed materials, it is possible for an unsuspected trace element to be present in concentrations sufficient to affect high-temperature alloys. Another source of contaminants in the master melt is the ore body from which metals have been extracted. Provided the concentration of the tramp element is no less than the detection limit of the analytical method used, analysis should not be difficult because the element will normally be quite uniformly distributed throughout the melt. No special sampling techniques are required since any portion of the melt will probably be representative of the whole.

Specimens prepared from an uncontaminated master melt may be contaminated in several ways during the casting operation. Depending upon the mode of contamination, the concentration of impurity may be uniform in the casting or appreciably segregated. In either case, considerable problems for the analyst can result. If the specimen is homogeneous with respect to contamination, but there is no technique for rapid nondestructive identification of those castings of a particular master melt which are contaminated, then all castings from that melt are in a questionable status. In some cases, it is possible to remove a small amount of material from noncritical regions of a casting without damaging the part; however, it is essential that the concentration of trace element in the portion analyzed is representative of the entire specimen.

Often tramp metal contamination results in a nonhomogeneous casting. Low melting alloys such as solder or "Cerro alloys" can fall into molds and melt when molten metal is added. In this case, the impurity is usually nonuniformly distributed in the casting. A second source of contamination is from metal-containing binders used in the fabrication of molds. Introduction of hot metal causes the transfer of low melting metals to the 
casting, the contamination being present both on the surface of the casting and in discrete deposits in subsurface grain boundaries. The nonuniformity of contaminant concentration makes effective sampling of these cast specimens extremely difficult. Trace metals can also be introduced into high-temperature alloys through contamination of the wax mold prior to utilization for ceramic mold fabrication. Again, the location of the impurity may be significantly nonhomogeneous. Complete analysis of nonuniform castings necessitates not only measurement of the concentration of the trace metal but also a detailed indication of location as well. In this way, the analyst may aid significantly in the determination of the method of contamination.

The increased utilization of directionally solidified and other slowly cooled casting techniques can present additional problems to the analytical chemist. In some cases, a type of zone refining takes place such that there is a significant difference in the concentrations of impurities (and sometimes minor alloying elements as well) in different parts of the casting. When molten metal is in contact with a ceramic mold or shell for extended periods, there is an increased possibility for interaction. A highly active metal can diffuse from the melt and form an oxide or silicate at the casting/ceramic interface resulting in a region as much as $10 \mathrm{mils}$ in depth which is depleted in the active element. Diffusion of elements from the ceramic to the melt can also be significant when slow casting methods are utilized. Certain sampling techniques such as polishing of solid specimens prior to x-ray fluorescence, or removal of sample turnings for dissolution, can result in serious errors if the region selected for analysis is not representative of the casting.

Castings with large grain size can have important metallurgical advantages: however, accurate analysis necessitates understanding certain types of segregation which can be present. Some constituents (major, minor or trace) in complex alloys tend to be concentrated in grain boundaries while others are enriched in the central portions of each grain. For alloys having fine grain structure, enough material is usually removed for analysis that a representative sample is obtained; however, for coarsegrained materials, significant errors can result if the sample utilized for analysis is enriched or depleted in intergranular areas. This is particularly true for those analytical techniques which utilize small amounts of sample such as spark source mass spectrometry or solid $\mathrm{x}$-ray fluorescence, since the average grain size in a directionally solidified casting may average $1 / 4^{\prime \prime}$ to $1 / 2^{\prime \prime}$ in diameter. Recent studies of concentration gradients within grains have been made using solid x-ray fluorescence or diffused beam microprobe and have confirmed that this type of segregation is quite pronounced for certain elements. It is evident that proper sampling is a 
critical part of the analysis of complex alloy systems and an elaborate analytical procedure may be invalidated if the sample is not representative, or if the exact location of the sample is not specified.

Once a proper sample has been obtained, various methods for the determination of trace metals may be considered. Techniques most often utilized for the determination of trace elements in complex alloys include atomic absorption spectrometry, optical emission spectroscopy, x-ray fluorescence and mass spectrometry. The most versatile of these methods for the determination of trace elements in alloys is atomic absorption analysis using both flame and nonflame atomization. When sensitivity permits, the flame offers a precise and rapid technique for trace amounts of lead, manganese, copper, zinc, silver and cadmium in high-temperature alloys. Relatively large samples $(\sim 1 \mathrm{~g})$ are dissolved, thus eliminating many errors due to segregation in these alloys. Carefully matched solutions are utilized for easy standardization. Nonspecific background absorbance should always be measured, and significant corrections made when trace elements are determined without prior separation. In many cases, this correction is the limiting factor in determining precision and detection limits; consequently, proper alignment of analyte beam and correcting beam (either continuum or nonabsorbing line) are essential. Extraction techniques [1] are sometimes used to concentrate trace elements and to remove matrix elements prior to measurement by the flame atomic absorption method; however, these methods are to be avoided when possible because of lengthy procedures and additional possibilities of sample contamination.

The most important recent advance in the determination of trace elements in complex alloys has been the application of the nonflame atomizer. The excellent sensitivity of the technique makes possible the determination of most trace elements of interest without concentration or separation. Proper choice of acid media, heating programs and simultaneous background correction are essential for best results. This method can give precisions of 5 percent in the determination of ppm of trace elements in complex alloys. Solution standards may be utilized. Methods have been published for the determination of lead, bismuth, selenium, thallium and tellurium [2], while procedures were described at the 1974 Pittsburgh Conference for gallium, indium, tin, antimony and germanium [3] in high-temperature alloys.

Recent work in our laboratory has been concentrated on the development of methods for the direct atomization of important trace elements from solid alloy turnings or chips. Adequate sensitivity has been demonstrated for several elements (lead, bismuth and selenium) and the new technique offers significant time savings. Trace element segregation can 
be a problem with direct atomization of solids since samples exceeding 5 $\mathrm{mg}$ are not effectively atomized. Multiple analysis can somewhat reduce segregation effects on accuracy of analysis. Proper solid standards are required.

Emission spectroscopy incorporating the carrier distillation technique was first used in the determination of traces of lead, bismuth and tin in high-temperature alloys [4]. Moderately large samples $(0.2 \mathrm{~g})$ are dissolved in acid, thus reducing segregation effects on analysis, and then converted to oxides by ignition. The oxides are mixed with silver chloridelithium fluoride carrier and ignited in the $\mathrm{dc}$ arc. The procedure is capable of good precision but is time consuming and requires an experienced spectrographer for proper results. Aqueous solution standards are utilized. More recently a procedure for the determination of lead and bismuth was described in which metal chips are arced directly after mixing with lithium carbonate [5]. Sensitivity is better than for the carrier procedure; however segregation effects are more pronounced because of the smaller sample size. Other emission spectrographic procedures have been described for the determination of several trace elements in complex matrices after first separating the trace elements by precipitation [6,7]. Extraction of tri-n-octylphosphine oxide has also been used to concentrate a number of trace elements before emission spectrographic analysis [8]. Large samples reduce segregation errors to a minimum in both concentration methods.

$\mathrm{X}$-ray fluorescence has been utilized for the measurement of selenium [9], arsenic [10] and tellurium [10] after separating the trace elements by precipitation as metals and collecting on a Millipore pad. Standards preparation is easy and large samples can be used; however, sensitivity for selenium and tellurium is poor. Spark source mass spectrometry has been used for the characterization of complex alloys for trace elements. The method has the advantage of good sensitivity for most elements in the periodic chart and is an adequate survey technique; however, segregation effects and lack of proper standards are problems that must be overcome before the method can be seriously considered for the quantitative measurement of trace elements in alloys. At present, the best overall technique for the quantitative determination of a broad spectrum of trace elements in metallurgical samples is nonflame atomic absorption, because of advantages of simplicity and cost of equipment, availability of standards, ease of sample preparation, sensitivity and accuracy of results. 


\section{References}

[1] Burke, K. E., Analyst (London) 97, 19 (1972).

[2] Welcher, G. G., Kriege, O. H., and Marks, J. Y., Anal. Chem. 46, 1227 (1974).

[3] Welcher, G. G., Kriege, O. H., and Marks, J. Y., Pittsburgh Conference on Analytical Chemistry and Applied Spectroscopy, March 1974, Cleveland, Ohio.

[4] Atwell, M. G. and Golden, G. S., Appl. Spectrosc. 24, 362 (1970).

[5] Atwell, M. G. and Golden, G. S., Appl. Spectrosc. 27, 464 (1973).

[6] Marks, J. Y., Cone, R., and Leao, E., Appl. Spectrosc. 25, 493 (1971).

[7] Atwell, M. G. and Golden, G. S., Appl. Spectrosc. 24, 514 (1970).

[8] Harper, L. E., Solvent Extraction Spectrochemical Method for Trace Amounts of $\mathrm{Pb}, \mathrm{Bi}$, and $\mathrm{Sn}$ in High-Temperature Alloys and Raw Materials Used in Their Production, TRW Metals Division Report T10008A, Cleveland, Ohio (1972).

[9] Albright, C. M., Burke, K. E., and Yanak, M. M., Talanta 16, 309 (1969).

[10] Burke, K. E. and Yanak, M. M., Anal. Chem. 41, 963 (1969). 


\title{
MONITORING OF AN ATOMIC ABSORPTION SPECTROPHOTOMETER USING CUMULATIVE SUM STATISTICAL CONTROL CHARTS
}

\author{
John R. Montgomery \\ Puerto Rico Nuclear Center \\ Mayaguez, Puerto Rico 00708 U.S.A.
}

\begin{abstract}
A method has been devised to monitor the reproducibility of an atomic absorption spectrophotometer utilizing statistical control charts. Standard curves for five elements, $\mathrm{Cu}, \mathrm{Pb}, \mathrm{Zn}, \mathrm{Mn}$. and $\mathrm{Cd}$ have been monitored to determine if the cumulative sum type control charts with "V" mask can detect incipient changes in sensitivity of the atomic absorption spectrophotometer caused by alteration in lamp output, fuel, air mixtures and electronic gear. The cumulative sum chart is easily computed and allows the operator to quickly detect significant changes in the sensitivity of the machine.
\end{abstract}

Keywords: Atomic absorption; cadmium; copper; lead; manganese; sensitivity; spectrophotometry; statistical methods; zinc.

\section{Introduction}

Accuracy in trace metal determination will depend on the sensitivity of the detection instrument. If the sensitivity decreases, thereby changing the lower limit of detection, then the accuracy will decline when determining low levels of trace metals.

There was no system of continuous quality control for the output from our atomic absorption spectrophotometer (AAS) except that of the subjective viewpoint of the technician. This viewpoint is a function of the experience and skill of the technician and can vary greatly. The need for an objective method to continuously monitor the output of the AAS was made apparent during a random sampling of the trace metal standard curve regression equations, specifically the slopes. An alarming change was noticed in the sensitivity levels obtained as compared to the manufacturer's specification [10]. A method was needed to detect and allow cor- 
rection for significant changes in sensitivity. The quality control method, to be effective, must be simple and continuously and easily operated by a relatively unskilled technician. The use of quality control charts was pioneered by Page $[8,9]$ and was augmented by the use of " $V$ " masks as proposed by Barnard [1]. A nongraphical method was proposed by Evan and Kemp [3]. The method used in this study was taken from the procedure in Guttman et al. [6], and includes a combination cumulative sum (Cum Sum) chart and "V" mask.

The use of (Cum Sum) quality control charts, [2,5] is based on the assumption that when a cumulative sum for a normal population, as defined in Eq. (1) is computed,

$$
T m=\sum_{i=1}^{m}\left(X_{1}-\mu_{0}\right)
$$

where $m$ is the sample number, $X_{1}$ is the parameter being sampled and $\mu_{0}$ is the theoretical reference value for the parameter, the resulting Cum Sum should fluctuate randomly around zero [6] when $T m$ is plotted on the ordinate versus $m$ on the abscissa. However, if the average value of $X$ increases, then the slope of the Cum Sum (Eq. 1) would increase rapidly. Similarly, if the reverse occurs, the general slope would decrease rapidly [6]. One of the main values of a Cum Sum chart is that relatively small changes in the mean value, $X$, produce quite different slopes. However, sometimes it is difficult to make an objective decision as to when the process is out of control. The " $\mathrm{V}$ " mask technique furnishes a method to rapidly note an unacceptable change in the process mean. The level of unacceptable change is determined by the analyst. In effect a "V", as shown in figure 1 , with angle theta was located a distance, $d$, from the last plotted Cum Sum. Theta was computed as in Eq. 2 [6]:

$$
\text { Tan theta }=\frac{\delta}{2}
$$

The distance, $d$, was computed as in Eq. 3 [6]:

$$
d=\frac{2}{\delta}\left[\frac{(S)^{2}}{\delta} \text { In } \frac{1-\beta}{\alpha / 2}\right]
$$

where $\delta$ is effectively the upper and lower limits for the theoretical reference values, $S$ is the standard deviation of the theoretical reference value, $\mu_{0}, \beta$ is the probability of a type II error and $\alpha$ is the probability of a type I error. The Cum Sum $(\mathrm{Tm})$ on the ordinate was altered by a constant, $k$, to make the plot more compact [6]. When this is done then Tan theta must be altered in the same manner. An equivalent unit on the ordinate should be two times the standard deviation of the process mean 


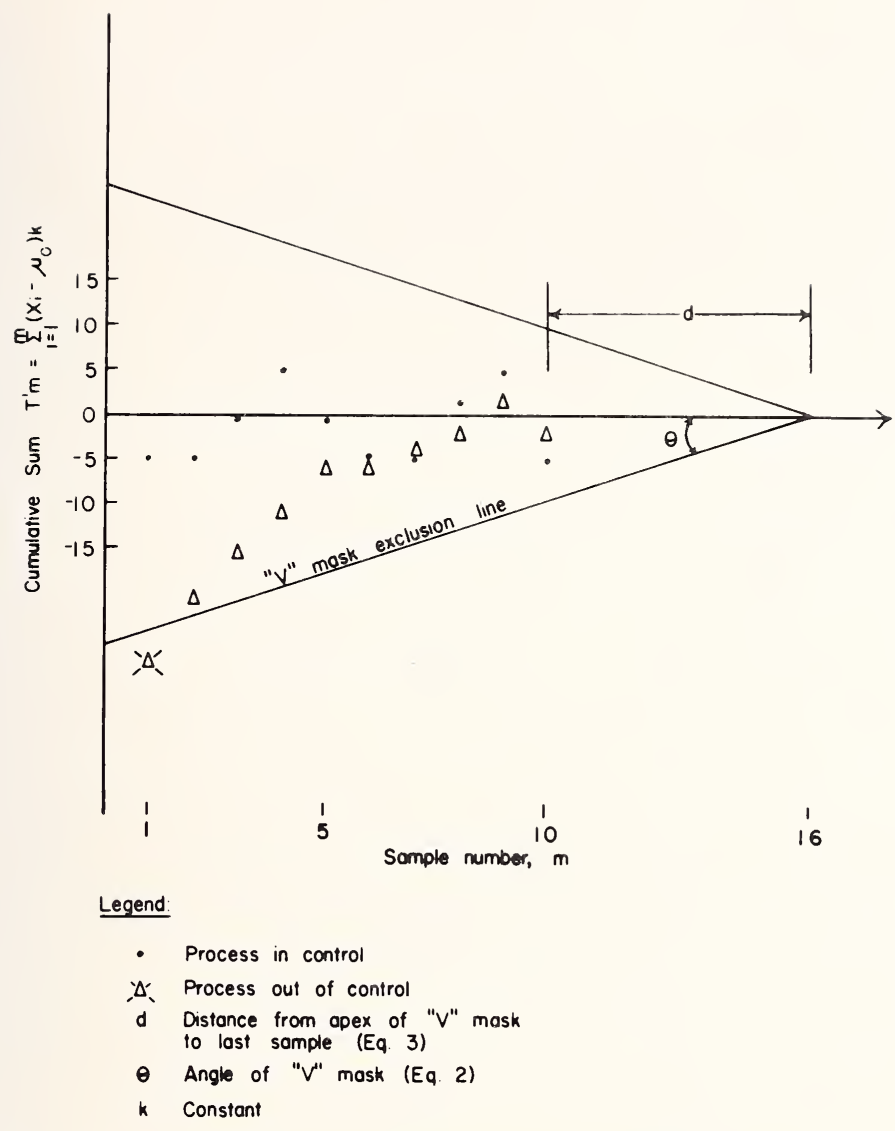

Figure 1. Typical cumulative sum chart with "V" mask.

[11]. The use of a Cum Sum chart is preferable over other quality control charts as it controls both Type I and II errors [6].

\section{Method}

The elements $\mathrm{Zn}, \mathrm{Cu}, \mathrm{Cd}, \mathrm{Pb}$, and $\mathrm{Mn}$ were determined by using a Perkin-Elmer Model 303 atomic absorption spectrophotometer (AAS) with a Perkin-Elmer Model 165 recorder, automatic null recorder readout (303-10103), burner control (040-0139), air/acetylene flame and a 4 inch single slot burner. The wavelength, slit, current, gas flow rates, and standard concentrations used for each metal are shown in table 1 . The theoretical slope for the standard curve was obtained from the manufacturer's 


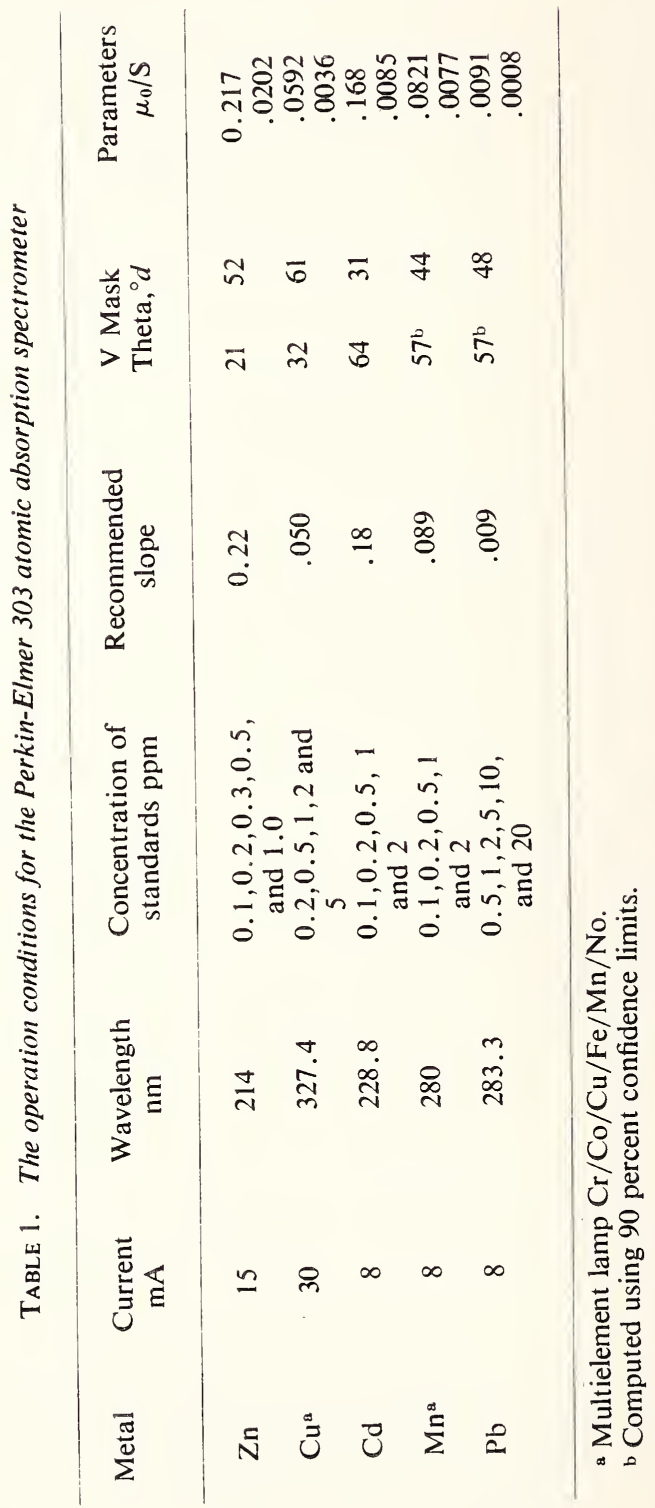


manual [10]. The atomic absorption spectrophotometer model was adjusted until that sensitivity was obtained. The aqueous trace metal standards were prepared weekly and stored in $50 \mathrm{ml}$ linear polyethylene bottles. To compute the cumulative sum (Eq. 1) the mean slope of the trace metal standard curve was used as the theoretical reference value, $\mu_{0}$. The $\mu_{0}$ was computed from the linear least squares equation:

$$
\gamma_{\text {Absorption }}=\mu_{0} \times \mathrm{ppm} \text { metal }
$$

To obtain the natural variation(s) of this slope, for $\mathrm{Zn}^{+}$and $\mathrm{Cu}^{+}$, a set of 5 standards, all within the linear part of the curve (table 1), was run every half hour for 8 hours. The slopes obtained over this 8 hour period were supplemented by slopes obtained from standard curves run over a 3 month period using the same machine settings (table 1). The air flow to the burner or the wavelength setting was altered slightly and the same standards rerun every half hour for 8 hours. The Cum Sum chart was then plotted. The "V" mask was produced using the 95 percent confidence limits of $\mu_{0}$ for $\delta$ in Eq. (2) and (3), for $\mathrm{Zn}, \mathrm{Cu}$, and $\mathrm{Cd}$. The 90 percent confidence limits were used to compute $\delta$ for $\mathrm{Pb}$ and $\mathrm{Mn}$. The standard deviation of $\mu_{0}$ and probabilities of 0.05 and 0.10 for Type I and Type II errors were used to compute $d$ (Eq. 3). The Cum Sum, Tm, was multiplied by a constant $k$ to shrink the ordinate value. Further $\mu_{0}$ values of $\mathrm{Cd}, \mathrm{Pb}$ and $\mathrm{Mn}$ were obtained using the ideal conditions in table 1 over a 3 month period. The values for $\mu_{0}$ under rich flame conditions were obtained from past records. The computed theta and $d$ values for the "V" mask are shown in table 1. The "V" mask was drawn on a plastic overlay. The apex of the "V" was placed $d$ units ahead of the last computed cumulative sum. If all the previous cumulative sums are within the "V" mask arms then the process was considered under control. When a cumulative sum was outside the "V" mask the process was considered out of control.

\section{Results}

The cumulative sum chart for $\mathrm{Zn}$ is shown in figure 2 . The ideal operating conditions produce a slope of $0.217, S=0.0202$. The slope, as taken from the manufacturer's manual, is 0.215 [10]. The cumulative sum chart indicates that the process was in control between point $\mathrm{A}$ to $\mathrm{B}$ (sample number 1 through 30). A definite change in slope was noted from point $B$ to $\mathrm{C}$ when the wavelength was decreased by $0.2 \mathrm{~nm}$ from the ideal process. However, the process was not considered out of control until 14 more standard curves were produced. The Cum Sum chart for $\mathrm{Cu}$ is 

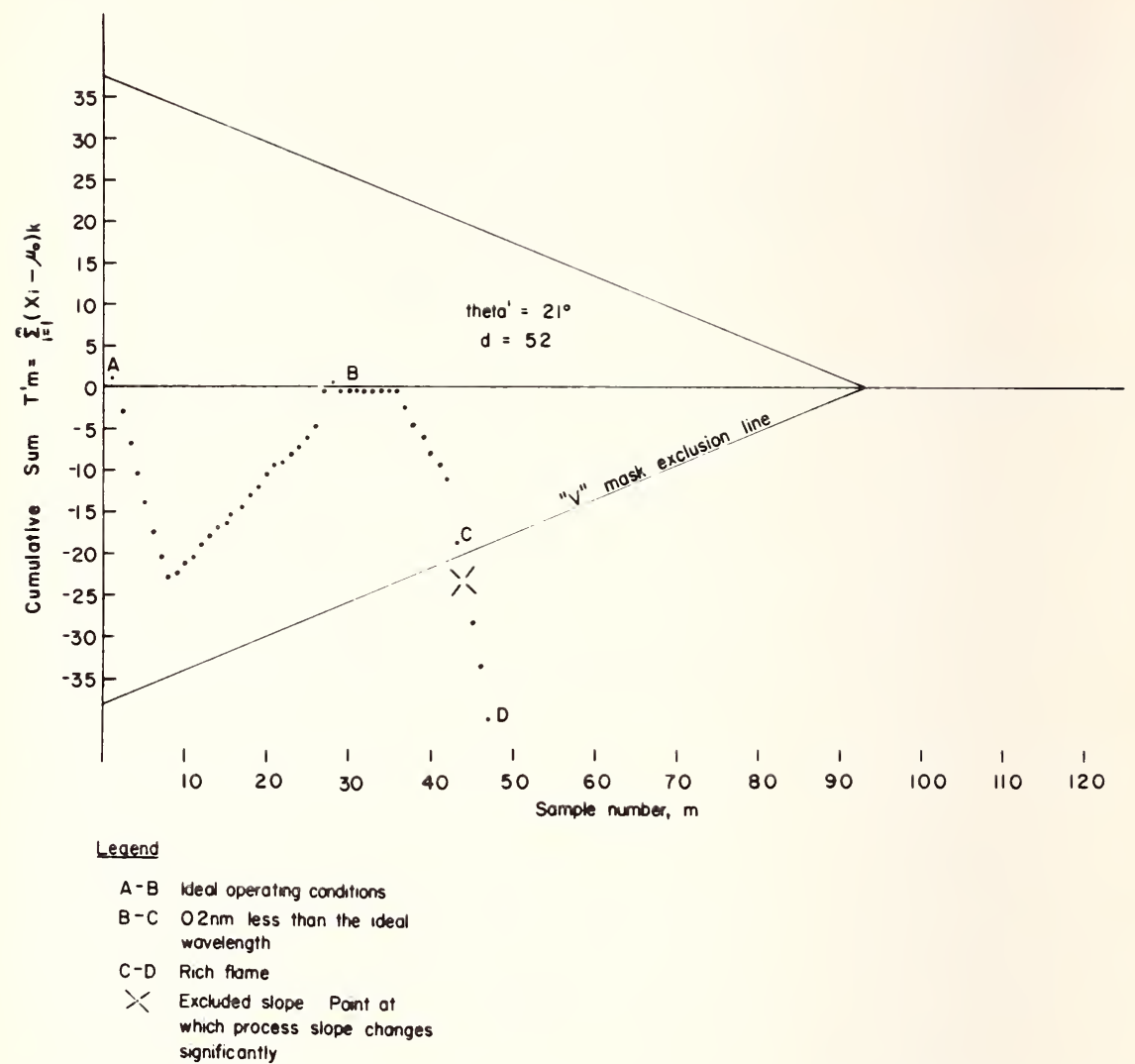

Figure 2. Cumulative sum chart for zinc using a mean slope, $\mu_{0}$ of $0.217, \mathrm{SD} 0.0202$. The ordinate and Tan $\theta$ for the "V" mask are multiplied by 100 .

shown in figure 3 . The ideal operating conditions produced a slope of $0.0592, S=0.0036$. The slope according to the manufacturer should be $0.050[10]$. The Cum Sum chart indicates that the process was in control from point A to B (sample number 1 through 35) (fig. 3). When the flame was made richer (point B to C) (sample number 30 through 41) (fig. 3) the Cum Sum decreases somewhat but the process was still considered "in control." From point C to D (sample number 42 through 47 ) (fig. 3 ) the flame was made leaner, as compared to the conditions used for samples B to C, and the Cum Sum appears to level off. However, as the flame was changed to a rich mixture (point D through E) (fig. 3) the accumulative slopes of less than 0.0592 produced a definite and swift decline in the Cum Sum, and the process was out of control within three samples. 

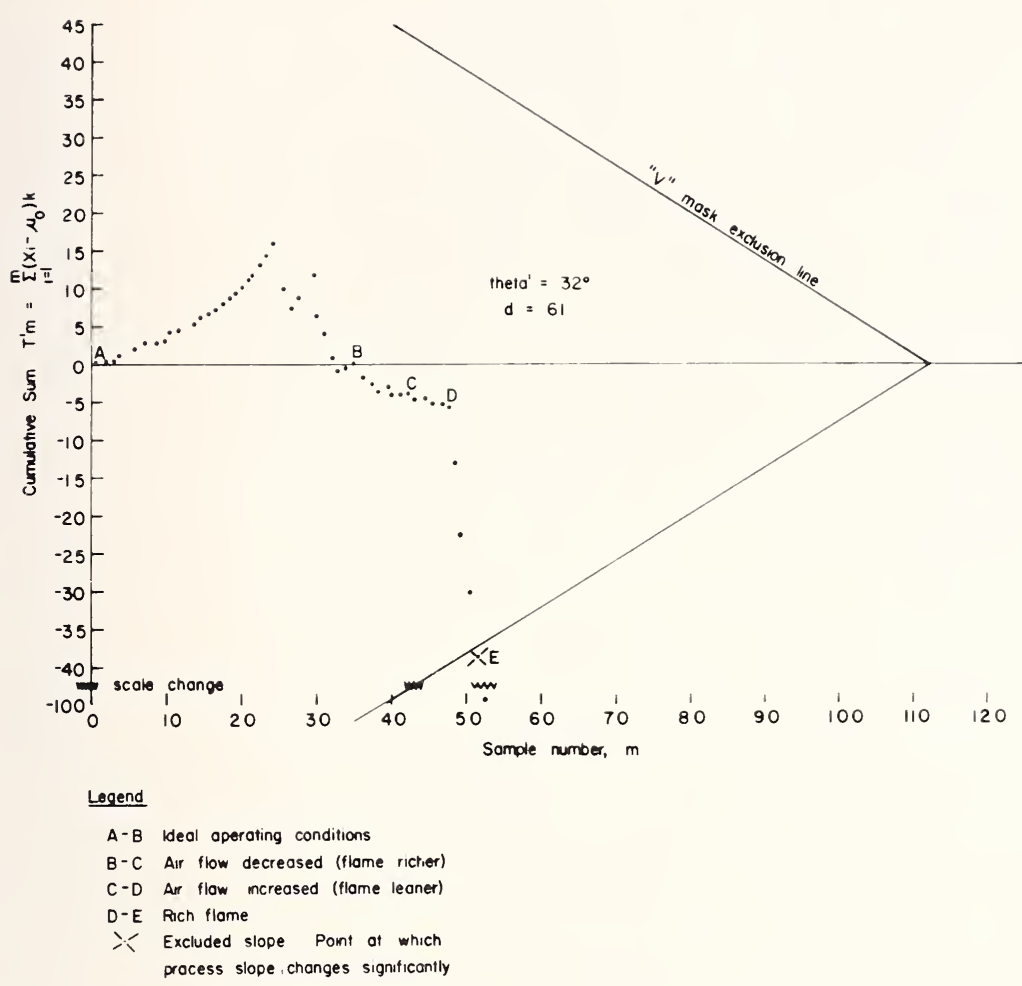

Figure 3. Cumulative sum chart for copper using a mean slope, $\mu_{0}$ of 0.0592 , SD 0.0036 . The ordinate and Tan $\theta$ for the " $V$ " mask are multiplied by 1000 .

The Cum Sum charts for $\mathrm{Cd}, \mathrm{Pb}$ and $\mathrm{Mn}$ (figs. 4, 5, and 6) are not as sensitive as the $\mathrm{Zn}$ and $\mathrm{Cu}$ Cum Sum charts (figs. 2 and 3). This was probably due to the smaller number of standard curve slopes used to arrive at the ideal reference value $\mu_{0}$. The sample numbers for $\mathrm{Zn}$ and $\mathrm{Cu}$, to obtain $\mu_{0}$, were 30 and 35 respectively. The sample numbers for $\mathrm{Pb}$, $\mathrm{Cd}$, and Mn were 20,19, and 12 respectively. Although the calculated $\mu_{0}$ values for $\mathrm{Pb}, \mathrm{Cd}$ and $\mathrm{Mn}$ were very close to the suggested values from the manufacturer (table 1), the $\delta$ value in Eqs. (2) and (3) was derived from the 95 or 90 percent confidence limits of the calculated $\mu_{0}$. Therefore, the $\delta$ value was a function of the sample size used in computing $\mu_{0}$. The larger the sample size, the smaller the confidence limits and hence the value $\delta$ (Eq. 2 and 3) would decrease. The smaller the value $\delta$ the smaller the angle theta (Eq. 2) for the " $V$ " mask. A small change in $\delta$ causes a very large change in theta. The ability of the " $\mathrm{V}$ " mask to quickly detect an unacceptable change in the process slope, was based on both the angle theta and $d$. It is possible to obtain values for theta and $d$ where the unac- 


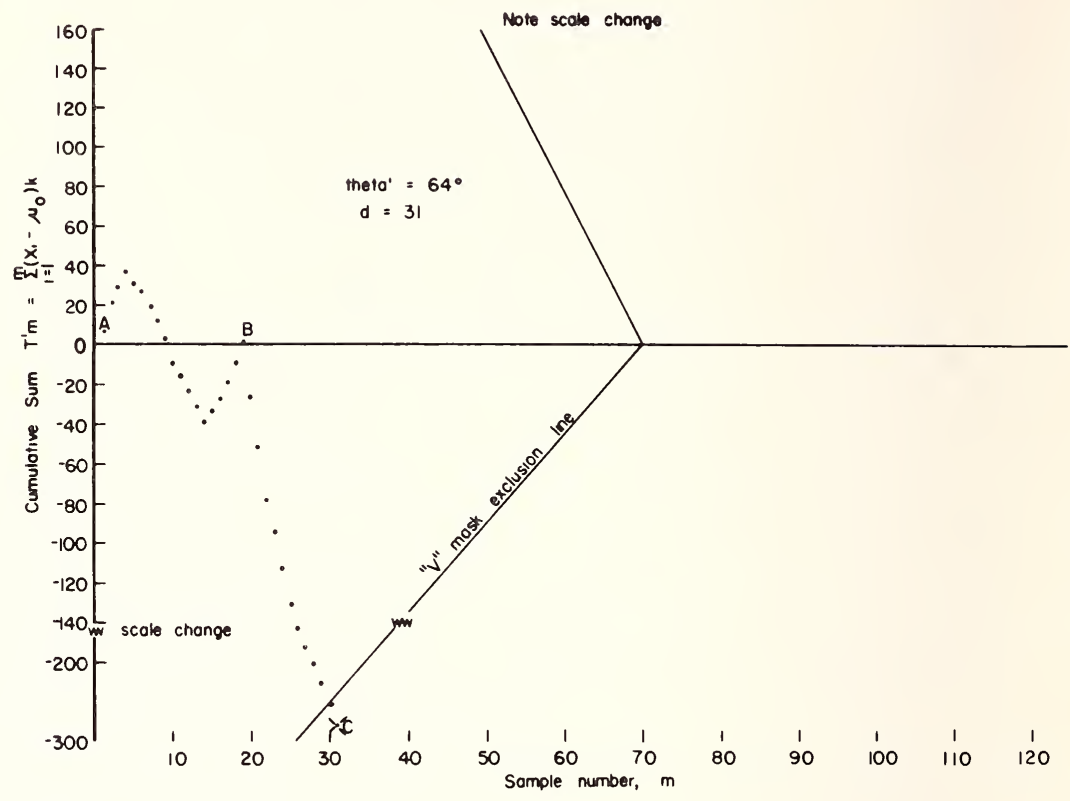

\footnotetext{
Legend

A-B Ideal operaing conditions

$B-C$ Rich flame

․ Excluded slope Point at which

process slope changes significantly
}

Figure 4. Cumulative sum chart for cadmium using a mean slope, $\mu_{0}$, of 0.168, SD 0.0085 .

The ordinate and Tan $\theta$ for the "V" mask are multiplied by 1000 .

ceptable change in standard curve slope would be immediately detected. However, the normal variation in the slopes of the process would also cause all the slopes, even the correct slopes, to be unacceptable. The selection of $d$ and theta (Eq. 2 and 3) must therefore necessarily rest on the experience of the analyst and the past history of the instrument $[4,7]$.

\section{Discussion and Conclusion}

The Cum Sum chart with "V" mask is an easily used and computed quality control chart for the monitoring of an atomic absorption spectrophotometer. The slope of the standard curves for the five metals examined was affected the most by a change in the acetylene/air gas mixture (fig. 2). However, a definite decrease in the Cum Sum was noted (B to C, fig. 2) when the wavelength was decreased $0.2 \mathrm{~nm}$ for $\mathrm{Zn}$. This decrease would probably have caused a definite out of control indication if the 

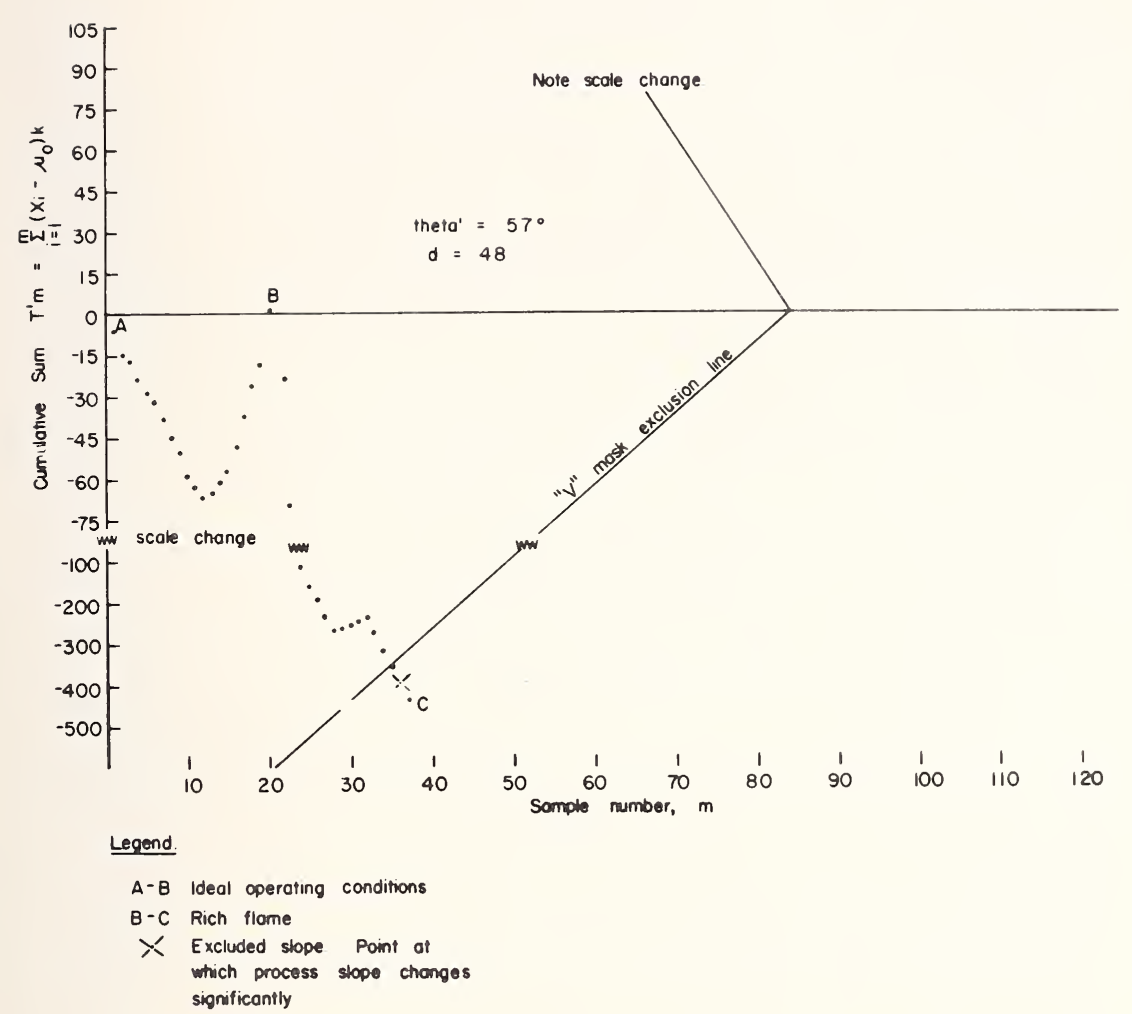

Legend.

Figure 5. Cumulative sum chart for lead using a mean slope, $\mu_{0}$, of $0.009, \mathrm{SD} 0.0008$. The ordinate and Tan $\theta$ for the "V" mask are multiplied by 10,000 .

system was run for a longer length of time. The Cum Sum chart for $\mathrm{Cu}$ was manipulated differently from the chart for $\mathrm{Zn}$. The flame was made richer from samples B to C (fig. 3) and the Cum Sum immediately decreased. This decrease was arrested by making the gas mixture leaner (C to D) and the Cum Sum leveled off. When the gas mixture was made very rich (sample D to E, fig. 3), the Cum Sum responded rapidly and within four samples the process was out of control.

The Cum Sum charts for $\mathrm{Cd}, \mathrm{Pb}$, and Mn (figs. 4, 5, and 6) were not as sensitive as the Cum Sum charts of $\mathrm{Zn}$, and $\mathrm{Cu}$ and needed at least 11 to 14 sample slopes before the system was considered out of control. This insensitivity, as denoted by the large theta and $d$ values, can be remedied by computing the $\mu_{0}$ from a larger sample size. If $\mathrm{Zn}$ and $\mathrm{Cu} \mathrm{Cum}$ Sum charts can be used as an example, it would appear that a minimum sample size of 30 would be necessary to ensure a $\delta$ value of sufficient magnitude to obtain the requisite theta and $d$ values. 


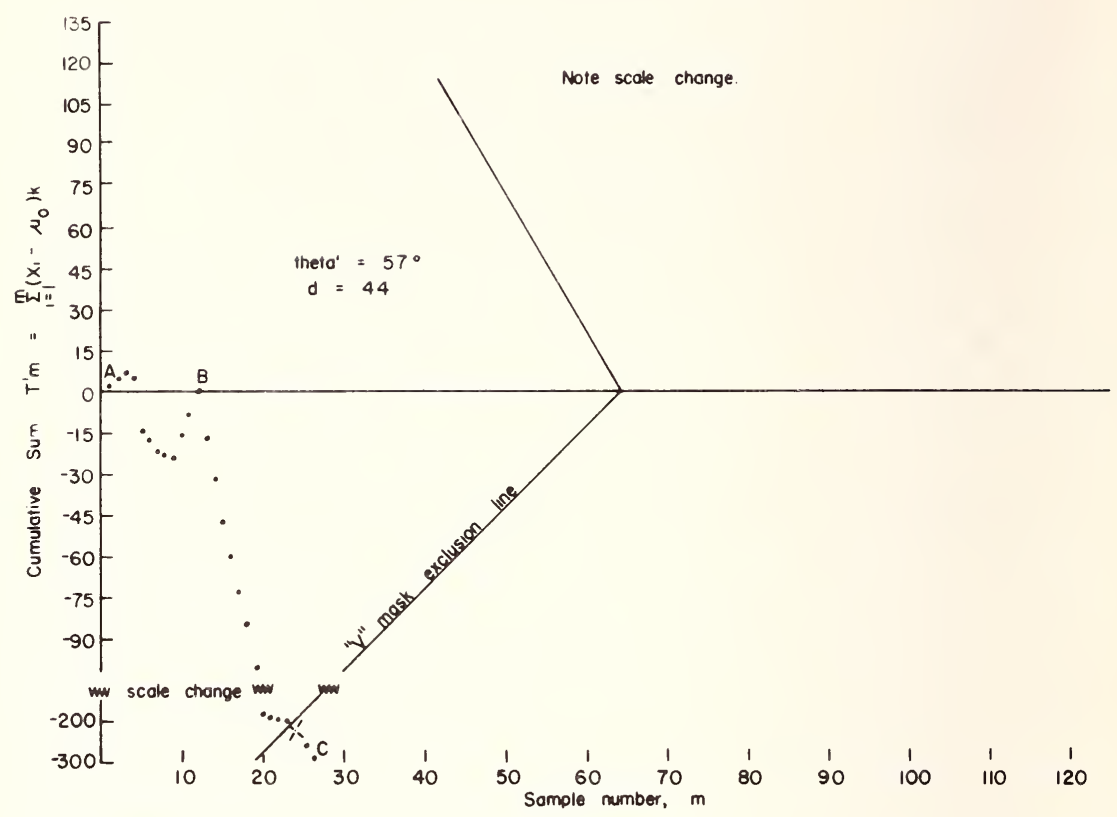

Legend

$$
\begin{aligned}
& \text { A-B Ideal operating conditions } \\
& \text { B-C Rich flame } \\
& \text { Excluded slope Point at which } \\
& \text { process slope changes significantly }
\end{aligned}
$$

Figure 6. Cumulative sum chart for manganese using a mean slope, $\mu_{0}$, of 0.0821 , SD 0.0077 . The ordinate and Tan $\theta$ for the "V" mask are multiplied by 1000 .

This method, although very seldom used in an analytical laboratory, has been found to be very useful in monitoring the output from an atomic absorption spectrophotometer. The technique will be extended to include the monitoring of a DU spectrophotometer and $\mathrm{CHN}$ analyzer.

\section{Acknowledgements}

This research was supported by the U.S. Atomic Energy Commission under contract AT-(40-1)-1833.

I am grateful for the help of Sr. Jose Ramirez, Sra. A. Ramirez, Sr. S. De La Rosa, Dr. J. Echevaria for aid in analysis. Drafting was done by Ms. Mary Price and Sr. D. Carillo and reproduction by Mr. Tom Smoyer. 


\section{References}

[1] Bernard, G. A., Control Charts and Stochastic Processes, J. Roy. Stat. Soc. Series B, 21:239-271.

[2] Evan, W. D., When and How to Use Cum Sum Charts, Technometrics 5:1-22 (1963).

[3] Evan, W. D. and Kemp, K. W., Sampling Inspection of Continuous Processes with No Autocorrelation Between Successive Results, Biometriko 47:363-380 (1960).

[4] Goldsmith, P. L. and Whitfield, H., Average Sum Lengths in Cumulative Chart Quality Control Schemes, Technometrics 3:11-20 (1961).

[5] Johnson, N. L., A Simple Theoretical Approach to Cumulative Sum Charts, $J$. Amer. Stat. Assoc. 56:835-840 (1961).

[6] Guttman, I., Wilks, S. S., and Hunter, J. S., Introductory Engineering Statistics, 2d Edition, John Wiley and Sons, Inc., New York (1965).

[7] Kemp, K. W., The Average Run Length of the Cumulative Sum Charts When a VMask is Used,J. Roy. Stat. Soc. Series B, 23:149-153 (1961).

[8] Page, E. S., Cumulative Sum Charts, Technometrics 3:1-9 (1961).

[9] Page, E. S., Cumulative Sum Schemes Using Gauging, Technometrics 4:97-109 (1962).

[10] Perkin Elmer Corporation, Analytical Methods for Atomic Absorption Spectrophotometry (1973).

[11] Woodward, R. H. and Goldsmith, P. L., Cumulative Sum Techniques, Oliver and Boyd, London (1964). 



\title{
SIGNAL CHARACTERIZATION AND MEASUREMENT IN MICROSAMPLE ATOMIC ABSORPTION SPECTROMETRY
}

\author{
Raymond S. Vogel and Arnold M. Hartley \\ Environmental Research Laboratory \\ Institute for Environmental Studies \\ University of Illinois \\ Urbana, Illinois 61801 U.S.A.
}

\begin{abstract}
Some of the errors encountered in atomic absorption spectrometry are broadband absorption due to light scattering by particles and absorption by molecular species originating from the sample matrix. Instrumentation for microsample techniques have been developed for typical volumes of $50 \mu \mathrm{l}$ or less. The resultant transient absorbance signals are from one to several orders of magnitude greater than the same concentration in the 1-ml or more sample volume typically required by conventional solution aspiration methods. Background signals associated with the microsample methods are sometimes a major fraction of the total absorption signal. Literature dealing with these methods has emphasized the ubiquitous nature of background absorption and so-called "smoke" peaks. Specific absorption by atomic species might be mistaken for nonspecific background absorption resulting in significant errors. An example is given of this potential problem and how mistakes can be avoided.

Absorption signals characteristic of those from nebulizer-burner systems, as well as those from microsample systems are discussed. The conclusion drawn on the basis of the experimental evidence is not that the "smoke" peak attributed by others to nonspecific absorption is actually due to atomic absorption, but rather, when in the use of the microsample atomic absorption techniques, sequential absorption peaks are observed. Each of these peaks must be studied objectively or serious analytical errors may occur.
\end{abstract}

Keywords: Atomic absorption; background correction; Delves Cup; lead; spectrophotometry. 


\section{Introduction}

Atomic absorption spectrometry has become one of the most extensively used techniques for elemental trace analysis applicable to a wide variety of materials. The first commercial instruments that appeared in the early 1960's were simple adaptations of flame photometers or spectrophotometers, but even at that early state of the art, practitioners quickly became aware of the relative ease and rapidity with which results could be obtained by this new technique as compared to the previously used methods. The simplicity of sample preparation and instrumental operation made atomic absorption spectrometry especially appealing to many who had little training in chemistry and none in spectroscopy. As a consequence, problems affecting analytical results often went undetected by the analyst who lacked adequate background and training [1]. The situation was made more hazardous for the uninformed by repeated statements in the early AA literature and in advertising media that the method was entirely free of spectral interferences [2].

One of the most serious of spectral interferences is broadband absorption due to light scattering by particles, and absorption by molecular species originating from the sample matrix. The effect on analytical results is a positive error which can be several hundred percent at trace levels, especially in biological materials and alkali halides [3]. The analytical error goes undetected in the traditional method of standard additions because the background contribution to the absorbance values for the sample and to each standard addition is a constant. Identification of the background effect and means for compensation were first reported by Koirtyohann and Pickett in 1965 [3], and in 1967 an instrumental accessory was commercially made available for automatic background correction [4]. Yet, as recently as 1972, official procedures have been published [5] in which the authors ignored or were not aware of the need for background correction. Currently published work, however, is generally consistent in the recognition of the effect of this factor, and almost all commercial instruments include as standard or optional equipment means for automatic correction for background absorption. Simultaneous background correction of absorption signals in single-beam instruments can be made by instrumental means devised by Koirtyohann and Pickett [3], and by Dick et al. [6].

Instrumentation for microsample techniques of $\mathrm{AA}$ has been developed, such as the L'Vov [7] and Massmann furnace [8], Delves Cup [9], carbon rod [10], and tantalum strip [11], by which samplesolution volumes typically of $50 \mu \mathrm{l}$ or less produce transient absorbance signals one to several orders of magnitude greater than are obtained for 
the same analyte concentration in the 1-ml or more sample volume typically required by conventional solution-aspiration methods.

Background signals associated with these microsample methods are in some cases a major fraction of the total absorption signal, especially for trace metals in biological materials; and the literature dealing with microsample atomic absorption methods has made a strong point of the ubiquitous nature of background absorption and so-called "smoke" peaks. This greatly heightened awareness of workers to background absorption paradoxically can result in oversights similar to the earlier years of atomic absorption spectrometry before background effects were recognized. Objective and systematic investigation is required to correctly characterize the transient signals of the microsample AA methods, for there are again pitfalls along the way in which the unwary analyst might incorrectly mistake specific absorption by atomic species for nonspecific background absorption, and thus incur significant negative errors in the analytical results.

An example will be presented of this potential problem affecting analytical accuracy and how mistakes can be avoided.

\section{Characteristics of Absorption Signals from Nebulizer-Burner Systems}

Absorption signals from conventional solution-aspiration atomic absorption systems are essentially constant with respect to time, since the processes of nebulization, desolvation and dissociation are maintained at steady-state conditions by the continuous a : controlled uptake of sample solution to the nebulizer. For a given sample, the absorption signal at the analytical wavelength is the sum of specific absorbance by the analyte atom population plus broadband absorption and light scatterir.g by the particulate matter and molecular species originating from the sample. Correction for this nonspecific background absorption is made through the use of a nearby "nonabsorbing" line of the analyte element, or a spectral continuum over the instrumental bandwidth at the analytical wavelength [3].

In the steady-state absorption signals of solution-aspiration methods, the analyte and background signals occur simultaneously with respect to time; and the signals remain essentially unvarying over the time interval that sample solution is aspirated. Absorbance specific to the analyte element is obtained by subtracting the time averaged background absorbance from total absorbance over a time interval appropriate to precision requirements. 


\section{Characterization of Absorption Signals}

\section{from Microsample Systems}

The character of absorption signals produced by microsampling atomization techniques are markedly different from the steady-state signals of solution-aspiration methods in two principal ways. First, in microsampling techniques a finite very small volume of sample solution, placed in a metal or graphite cuvette or tube, is vaporized and transferred through an absorption cell by atomization processes not in equilibrium. The resultant absorption signal is a transient which describes the build-up of analyte atom concentration in the cell, a momentary equilibrium when the rate of entry and loss of atoms in the cell is equal, followed by an exponential decay to zero. For a given concentration of analyte, the peak absorbance and time-related half width of the transient absorption signal are functions of atomization rate and residence time in the absorption cell; and factors affecting the rate of the atomization and transfer process proportionally affect peak absorbance [12]. Absorption measurement methods involving peak height rather than the area of the absorption profile must take these factors into consideration. Second, the transient absorption signals describe time-related thermal decomposition and dissociation processes of the sample in sequential peaks of nonspecific absorption in combination with atomic absorption specific of the analyte element. A given absorption profile may have both nonspecific and atomic absorption components, and so correct characterization of the peaks as well as measurement of atomic and nonspecific absorbance of a given absorption profile is necessary for accurate analytical results.

An example of sequential absorption peaks is shown in figure 1 from the original report by Delves [9] on the determination of lead in blood by the now familiar method bearing his name. Here a blood sample partially oxidized by hydrogen peroxide in a nickel cup undergoes pyrolysis and so-called "smoke" absorption signals precede the lead absorption signal. Delves attributed the first peak (B) to background absorption by excess hydrogen peroxide, the second, $(\mathrm{C})$, to combustion products of the sample, and the third, (D), to specific absorption by lead atoms. The analytical measurement for lead was made on the basis of the height of the third peak. No mention was made of background correction.

Another example (fig. 2) taken from a report by another author [13] shows the effect of ashing time of a dried blood sample on the sequential peaks by the Delves Cup method. The author in this case attributed the first peak, (A), to molecular absorption. No mention was made of background correction. 


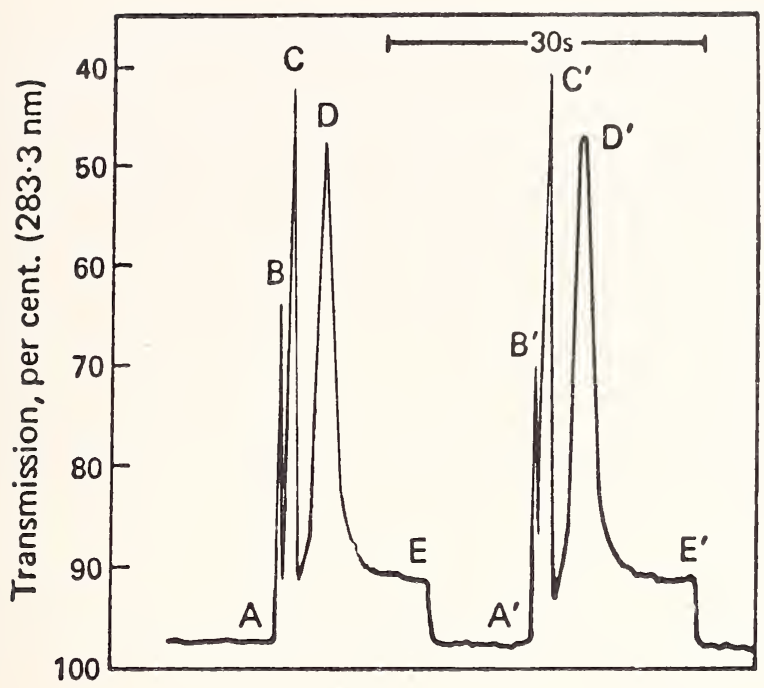

Figure 1. Absorption signals at $283.3 \mathrm{~nm}$ obtained with duplicate $10-\mu$ l samples of blood by the Delves Cup technique. A, $A^{\prime}$ cup inserted into flame; $B, B^{\prime}$ excess $\mathrm{H}_{2} \mathrm{O}_{2} ; C, C^{\prime}$ combustion products; D, $\mathrm{D}^{\prime}$ lead absorption; E, $\mathrm{E}^{\prime}$ cup removed. Courtesy of Delves, N.' T., Analyst 94, 431 (1970).
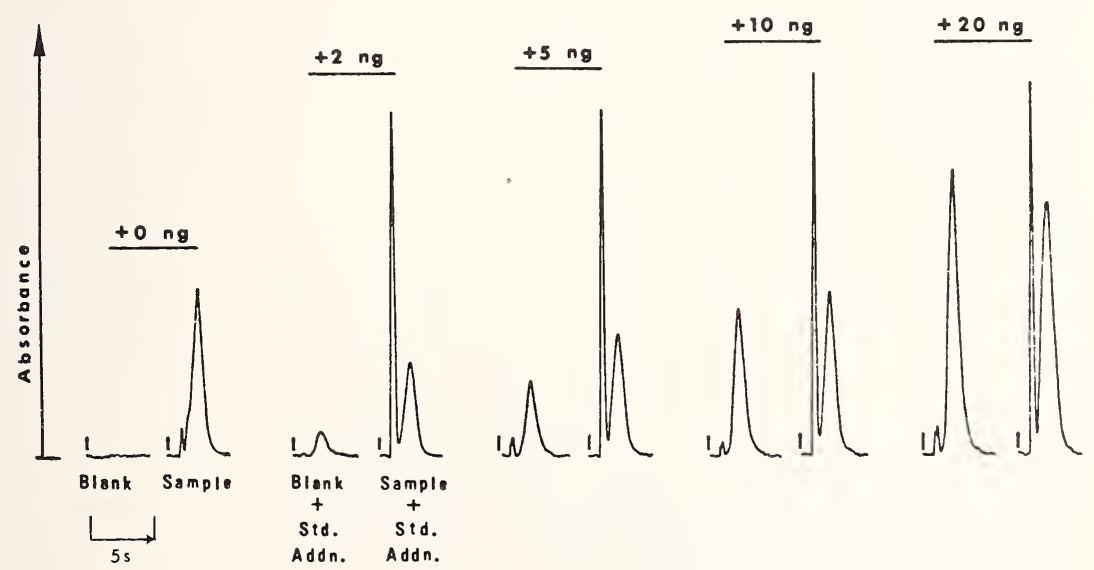

Figure 2. Absorbance vs. time profiles of lead in dry-ashed animal tissue by the Delves Cup technique. 
In current investigations in the authors' laboratory concerned with anomalies of the Delves technique with respect to the determination of lead in animal and plant tissue, the strip-chart recordings frequently showed sequential absorption peaks similar to those of the previous illustrations. In figure 3, absorption peaks of standards and standard additions

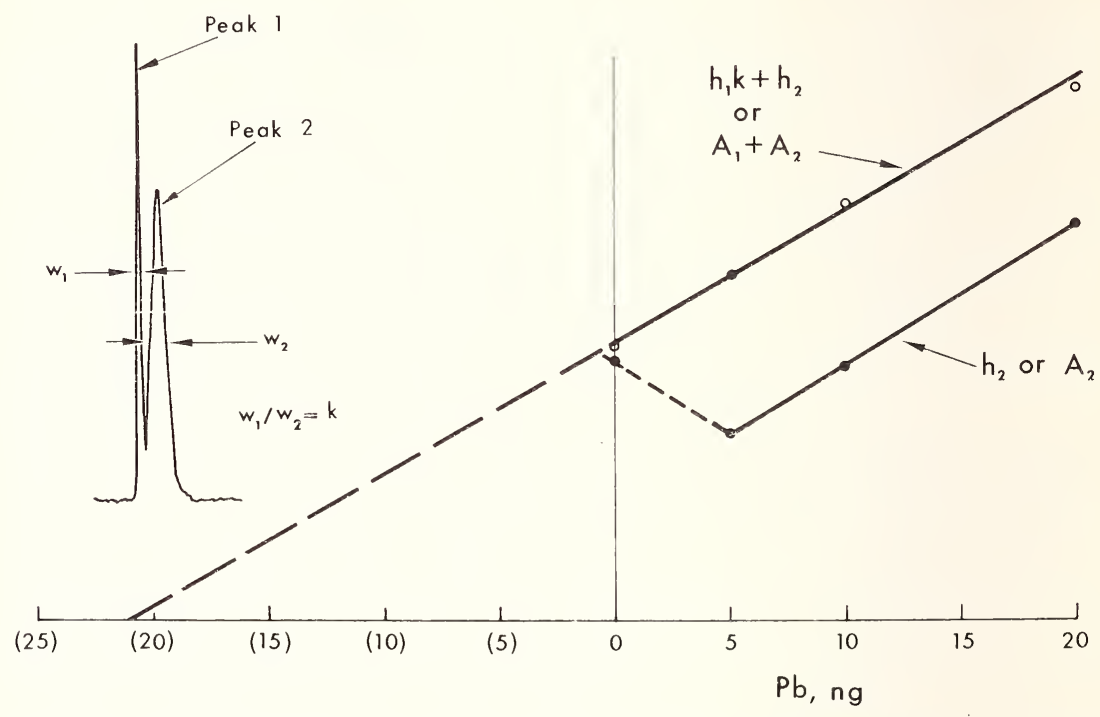

Figure 3. Standard addition plots of lead in dry-ashed animal tissue. $\left(h_{1}\right.$ and $h_{2}$ are peak heights of peaks 1 and 2. $A_{1}$ and $A_{2}$ are the integrated areas of peaks 1 and 2.)

to dry-ashed animal tissue samples dissolved in $3 \mathrm{~N} \mathrm{HCl}$ show a consistent pattern of multiple peaks. The first (and predominant) peak appears in the standard additions to the sample, but is essentially absent in the "unspiked" sample as well as the standards. All absorption profiles in figure 3 were corrected for nonspecific absorption. Thus, it was found that an absorption peak similar to the "smoke" absorption peak reported by others also appears from samples containing no combustible material; and more significantly, when the absorption signals are corrected for nonspecific absorption. To test the hypothesis that the first peak might be due to molecular lead species [13] (a $\mathrm{PbCl}_{2}$ band system in the UV has a principal $(0,0)$ band head at $283.3 \mathrm{~nm})$, strip-chart recordings of background-corrected lead absorption signals from Delves cup preparations were obtained at $\mathrm{Pb} 283.3 \mathrm{~nm}$ and also at $\mathrm{Pb} 217.0 \mathrm{~nm}$-the latter wavelength being free of molecular lead spectral bands. The resultant 
strip-charts showed the same double peaks at both $283.3 \mathrm{~nm}$ and 217.0 $\mathrm{nm}$, thereby not supporting the hypothesis that molecular absorption was responsible for the first peak.

The analytical evidence indicates that, in this example, both of the sequential absorption peaks are due to atomic lead and consequently absorbance measurements must include both peaks. Typical tissue samples which displayed the multiple peaks had been ashed at $490{ }^{\circ} \mathrm{C}$ and the residue then dissolved in $3 N$ hydrochloric acid. Twenty-microliter aliquots were placed in the Delves cups and dried at approximately 140 ${ }^{\circ} \mathrm{C}$ before insertion into the flame cell. Standard additions were made from standard lead solutions also in $3 N$ hydrochloric acid (table 1).

TABLE 1. Digital analysis of the data for the standard addition plots of lead in dry-ashed animal tissue ${ }^{\mathrm{s}}$

Measurement method

Correlation Coefficient

\begin{tabular}{clc}
\hline Sample + & $\begin{array}{l}\text { Standard } \\
\text { addition }\end{array}$ & Analysis result \\
standard addition & $\mathrm{Pb}, \mathrm{ng}$
\end{tabular}

Peak height $1+2^{\mathrm{b}}$

0.9953

0.9990

21. $\pm 2 \%$

Peak height 2

.8170

.9990

19. $\pm 22 \%$

Peak area $1+2$

.9841

.9988

20. $\pm 5 \%$

Peak area 2

.8607

.9988

16. $\pm 20 \%$

${ }^{8}$ See plots in figure 3 .

b Measurement of peak height 1 is normalized by relative half-widths to peak 2 as follows: $h_{1 \mathrm{n}}=h_{1} k$, where $k=w_{1} / w_{2}$.

The sequential lead absorption signals were evolved in a consistent and reproducible pattern. In a given series, the two peaks had remarkably reproducible relative half-widths approximating a ratio of $1: 3$. This was useful when, in the occasional cases where two peaks were not evolved, the identity of the peak could readily be made from the half-width. Information on half-width is of primary importance because analytical use of peak height as the concentration parameter must take into account differences in half-widths, especially if the sum of two peak heights is used as a measure of absorbance. A practical method found for summing peak heights is to treat the areas under the peaks as triangles, and to normalize 
peak heights to the half-width of the second peak. Through exercise of reasonable care in procedural manipulations and reproducing of analytical conditions, the use of normalized peak height measurements gives approximately the same precision as integrated absorption measurements. Since the transient absorption signals from Delves Cup and other microsample AA techniques are similar to those of gas chromatography; techniques used in processing of chromatographic data in terms of peak and baseline detection, peak area integrations, and baseline correction [14] also are directly applicable to the evaluation of the transient absorption signals in atomic absorption spectroscopy.

\section{Conclusions}

The conclusion to be drawn on the basis of the experimental evidence presented is not that the so-called "smoke peak" attributed by others to nonspecific absorption is actually due to atomic absorption; but rather, when in the use of microsample AA techniques sequential absorption peaks are observed, an objective study of the absorption components of each peak must be made, or serious analytical errors may occur.

\section{Acknowledgements}

The assistance of Paul Amberg, Mary Clevenger and John Healy in phases of the experimental work is gratefully acknowledged. This work was supported by the National Science Foundation grant GI-31605 under the RANN Program.

\section{References}

[1] Koirtyohann, S. R., in Monitoring for Lead in the Environment, to be published jointly by Colorado State University, University of Illinois at Urbana-Champaign, and University of Missouri at Columbia.

[2] Kahn, H. L., J. Chem. Educ. 43, A7 and A103 (1966).

[3] Koirtyohann, S. R. and Pickett, E. E., Anal. Chem. 37, 60 (1965).

[4] Kahn, H. L., At. Absorption Newslett.7, 2, (1968).

[5] Hoover, W. L., J. Assoc. Offic. Anal. Chemists, 55, 737 (1972).

[6] Dick, D., Urtamo, S. J., Lichte, F. T., and Skogerboe, R. D.,Appl. Spectros. 27, 467 (1973).

[7] L'Vov, B. V., Spectrochim. Acta 17, 761 (1961). 
[8] Massmann, H., Spectrochim. Acta 23B, 215 (1968).

[9] Delves, H. T., Analyst 95, 431 (1970).

[10] Amos, M. D., Bennett, P. A., Bradie, K. G., Lung, P. W. Y., and Matousek, J. P., Anal. Chem. 43, 211 (1971).

[11] Hwang, J. Y., Ulucci, P. A., Smith, S. B., and Malenfant, S. L., Anal. Chem. 43, 1319 (1971).

[12] Aldous, K. M., Mitchell, D. G., and Ryan, F. J., Anal. Chem.43, 12 (1973).

[13] Cernik, A. A., At. Absorption Newslett. 12, 6 (1973).

[14] Hettinger, J. and Hubbard, J., Amer. Lab. (Feb. 1974). 



\title{
AN INTERCOMPARISON OF FLAME AND NONFLAME SYSTEMS IN ATOMIC ABSORPTION SPECTROMETRY
}

\author{
T. C. Rains and O. Menis \\ Analytical Chemistry Division \\ National Bureau of Standards \\ Washington, D.C. 20234 U.S.A.
}

\begin{abstract}
In atomic absorption spectrometry (AAS) the nonflame devices for producing atomic vapor have recently received wide attention. In comparison to the traditional flame technique they offer increased sensitivity for many elements. On the other hand they seemed to suffer from poorer precision. In this study several of the parameters affecting the precision of the carbon furnace were evaluated and data were compared with NBS Standard Reference Materials (Fly Ash, Coal, and Orchard Leaves). Operating conditions, such as the sample volume, and the temperatures of the drying, ashing and atomization controlled the precision and accuracy of the measurement. The main advantage of the graphite furnace was the elimination of the preconcentration step and its inherent problem of larger blanks.
\end{abstract}

Keywords: Atomic absorption spectrometry; nonflame graphite furnace: sensitivity vs. precision and accuracy; Standard Reference Materials.

\section{Introduction}

The current study was undertaken to evaluate the precision and accuracy of nonflame atomic absorption spectrometry. Historically, a flame was the original mode of producing atomic vapor in AAS [1]. The relative merits of this technique have been reviewed [2]. Recently, greater attention has been given to nonflame devices. These devices can be grouped into categories such as (a) cathodic sputtering, (b) graphite furnace or carbon rod devices, (c) tantalum ribbon, and (d) cold vapor. These methods differ from flame methods in that a more conducive environment is provided in which the ground state atoms are produced. Also, for the graphite 
furnace or carbon rod, a relatively small quantity of sample ( 1 to $50 \mu \mathrm{l}$ ) is used for an analysis, thus providing improved sensitivity over flame methods. However, the nonflame method has an inherent disadvantage in that high precision is difficult to attain.

A major cause of imprecision arises from the interferences encountered in nonflame atomization. These interferences are classified as physical, chemical and interelemental [3]. The physical or nonspecific interferences by light scattering from the incomplete volatization of matrices are of major importance. To minimize this type of interference, the operating parameters (drying, ashing and atomization) of the nonflame cell must be carefully selected and controlled. Even then, background correction may be required.

Early methods of background correction involved the measurement of the unknown solution at a nonabsorbing line which is close to the analytical line and then subtracting this value from the absorbance obtained at the analytical line. This technique suffers from the following disadvantages for nonflame atomic absorption spectrometry, e.g., (1) since two measurements must be made and then subtracted, the two-line sequential method introduces a loss of precision, (2) a nonabsorbing line may not be found closely adjacent to the analytical line thus making background correction difficult for some elements, and (3) the two measurements require additional time for the analysis.

The most recent method for background correction uses a continuous source, either a hydrogen or a deuterium lamp which allows the correction to be made at the same time as the analyte is measured [4]. However, the radiant intensity of these sources is limited to a narrow spectral range in the near ultraviolet region.

To extend the range a xenon arc lamp was used in place of a deuterium lamp. The optical arrangement of the xenon arc has been described previously [5]. This modification increased our capability for simultaneous background correction from a range of 200-350 nm to $200-600 \mathrm{~nm}$.

Again, since chemical interferences in nonflame atomization are different in nature and magnitude than those encountered in flame systems, several operational parameters were investigated to alleviate these interferences. The variables which were studied included the sample size; drying, charring and atomization temperatures; and the effect of single or multiple standard addition. 


\section{Experimental}

\section{A. Apparatus}

A double-beam atomic absorption spectrometer with a premixed laminar flow burner with 5 or $10-\mathrm{cm}$ slot burner heads was used for all flame measurements. Depending upon the analyte, the oxidant-fuel consisted of air-acetylene or nitrous oxide-acetylene.

For the nonflame system, a graphite furnace (HGA-2000), 0.5-m monochromator, hollow cathode lamp, continuum source (xenon or deuterium lamp) and rotating sector mirror were arranged on an optical bench for single or double beam applications [6]. The associated components consisted of power supplies for the graphite furnace, the hollow cathode lamp, the xenon lamp, phototube with a lock-in amplifier, and a chart recorder.

The instrumentation used for the cold vapor technique has been described previously [7].

\section{B. Sample Preparation}

Fly Ash - A 1-gram test portion of SRM-1633 (Fly Ash) is transferred to a $100-\mathrm{ml}$ Teflon beaker. A mixture of $5 \mathrm{ml}$ of $\mathrm{HCl}, 5 \mathrm{ml}$ of $\mathrm{HNO}_{3}$ and $5 \mathrm{ml}$ of $\mathrm{HF}$ is added. The beaker is covered with a Teflon lid and the solution is digested for 1 hour. After cooling, $10 \mathrm{ml}$ of $\mathrm{HClO}_{4}$ is added and the digestion is continued until all the carbonaceous material has been destroyed. The cover is then removed, and the sample is evaporated to dryness and baked until the solids turn brown around the edges. A mixture of $4 \mathrm{ml}$ of $\mathrm{HCl}$ and $75 \mathrm{ml}$ of water is added and the solution is heated at low temperature until all the solids dissolve. The solution is then transferred to a $100-\mathrm{ml}$ volumetric flask and diluted to a calibrated volume.

Coal-A 1-gram test portion of SRM-1632 (Coal) is transferred to a Teflon beaker. The sample is slowly digested for several hours in $25 \mathrm{ml}$ of $\mathrm{HNO}_{3}$ and cooled. A mixture of $5 \mathrm{ml}$ of $\mathrm{HF}$ and $10 \mathrm{ml}$ of $\mathrm{HClO}_{4}$ is added and the digestion is continued at low heat. An excessively high hotplate temperature may cause decomposition and explosion. Digestion is continued until all the carbonaceous material has been destroyed and fumes of perchloric acid appear. The solution is then transferred to a $50-\mathrm{ml}$ volumetric flask and diluted to calibrated volume.

Fuel Oil - A 2-gram test portion of SRM-1634 is transferred to a Teflon beaker. The sample is slowly digested for 2 hours in $20 \mathrm{ml}$ of $\mathrm{HNO}_{3}$. After 
cooling, $10 \mathrm{ml}$ of $\mathrm{HClO}_{4}$ is added and digestion is continued until all carbonaceous material has been destroyed. The solution is then transferred to a $25-\mathrm{ml}$ volumetric flask and diluted to a calibrated volume.

\section{Results and Discussion}

\section{A. Selecting the Drying, Charring And Atomization TEMPERATURES}

The temperatures selected for drying, charring and atomization can be expected to greatly affect the analytical results in nonflame techniques. The operating parameters provided by the instrument manufacturers are to be used only as a guide and failure to carefully check when dealing with a specific matrix will lead to erroneous results. In this respect, the drying, charring and atomization temperatures for lead were investigated. Initial studies showed if the drying temperature of the furnace was above $105^{\circ} \mathrm{C}$, splattering occurred which gave rise to low recoveries.

The charring step is intended to remove the chemical matrix. This process involves fractional distillation of volatile organic and inorganic compounds. The selection of the proper charring temperature is a critical variable in the operation of nonflame AAS. If the temperature is too low, an extra "nonatomic" absorption peak will occur during the atomization step. This "nonatomic" or scatter peak is difficult to separate from the analytical absorption peak. On the other hand, if the temperature is too high, some of the analyte will be lost and the subsequent atomization signal will be reduced. With two nanograms of lead in a nitrate or chloride medium, no significant loss of lead was observed at temperatures of up to $700{ }^{\circ} \mathrm{C}$. Welcher et al. [8] reported no significant loss of lead in a nickel matrix at temperatures as high as $1000{ }^{\circ} \mathrm{C}$. However, in the presence of organic materials (whole blood or methyl isobutyl ketone) losses of lead were observed to occur at temperatures above $400{ }^{\circ} \mathrm{C}$. Under these conditions, when lead is being determined, some of the inorganic components will not be removed from the organic materials and a "nonatomic" contribution will be observed during the atomization step. To correct for this type of interferent, a continuum source (xenon arc) was used. Since the radiant intensity of the xenon arc from 200 to $600 \mathrm{~nm}$ is too high, a neutral density filter was installed in the optical system to facilitate the balancing of the intensities of the xenon arc against the lead hollow cathode lamp. The stability of the xenon arc lamp was better than 0.5 percent. 
TABLE 1. Effect of atomization temperature on absorption of lead ${ }^{\mathrm{a}}$

Atomization temperature Percent absorption \pm standard deviation

$\begin{array}{ll}1600 & 24.6 \pm 1.7 \\ 1800 & 31.1 \pm 0.56 \\ 2000 & 43.9 \pm 0.86 \\ 2200 & 42.7 \pm 0.44 \\ 2400 & 43.6 \pm 1.1\end{array}$

a Two nanograms of lead, wavelength-283.3 nm.

The data on the effect of atomization temperature are shown in table 1. While a temperature of $2400{ }^{\circ} \mathrm{C}$ produced the highest percent absorption, the best precision was attained with an atomization temperature of 2000 ${ }^{\circ} \mathrm{C}$. However, this is dependent in part on the matrices and for some the $1800{ }^{\circ} \mathrm{C}$ temperature produced the highest precision although with some loss of sensitivity. In the case of lead this is due to its very high volatility and rapid evolution, in many instances the response of the recorder (0.2 s full scale) was insufficient to follow the signal.

\section{B. Effect of Sample Volume}

Since the sample in nonflame AAS must be dried, charred and then atomized, the sample volume would be expected to drastically affect the signal and also influence the degree of encountered interferences. In our study 2 nanograms of lead were transferred with an Eppendorf pipet in a sample of 2 to $100 \mu \mathrm{l}$. The best precision was attained with sample volumes from 10 to $50 \mu$ l (table 2). However, with the higher sample volume, a corresponding loss of sensitivity was observed. The extent of spreading of sample over a larger area may contribute to the subsequent loss of sensitivity.

TABLE 2. Effect of sample volume on absorption of two nanograms of lead ${ }^{\mathrm{a}}$

$\begin{array}{cccc}\begin{array}{c}\text { Sample volume } \\ \mu \mathrm{l}\end{array} & \begin{array}{c}\text { Percent } \\ \text { absorption }\end{array} & \begin{array}{c}\text { Standard } \\ \text { deviation }\end{array} & \begin{array}{c}\text { Relative standard } \\ \text { deviation, percent }\end{array}\end{array}$

$\begin{array}{rrrr}2 & 40.2 & 2.2 & 5.4 \\ 5 & 44.3 & 0.97 & 2.2 \\ 10 & 44.2 & .66 & 1.5 \\ 20 & 43.9 & .86 & 2.0 \\ 50 & 39.4 & .63 & 1.6 \\ 100 & 28.1 & .88 & 3.1\end{array}$

a Atomization temperature of $2000{ }^{\circ} \mathrm{C}$.

b Eight consecutive samples. 


\section{Standard Reference Materials}

In tables 3,4 , and 5 are presented the values obtained by AAS (flame and nonflame) for NBS-SRM's Fly Ash, Coal, and Fuel Oil. All the AAS values are in agreement with the certified values. Cadmium in SRM Fly Ash could not be directly determined by the flame method and required a separation and a preconcentration step. It was extracted with ammonium pyrrolidine dithiocarbamate-methyl isobutyl ketone (APDC-MIBK) and then the cadmium was stripped from the organic phase with $1 \mathrm{~N} \mathrm{HCl}$. The backstripping step yielded quantitative recovery of cadmium and improved the precision of the flame method. With the graphite furnace preconcentration was not necessary; however, a 50 percent suppression of the cadmium signal was observed. To overcome this difficulty, the standard addition method was used [8]. Cadmium in SRM's Coal and Fuel Oil could not be determined by the extraction procedure. The high reagent blank was the limiting factor. Since AAS with the graphite furnace or by atomic fluorescence has greater sensitivity, separations were not required and cadmium could be determined directly.

In general, the analytical values by AAS with flame and nonflame methods for Standard Reference Materials were in good agreement. The precision of all flame methods was better than the precision obtained with the graphite furnace. Based on our current experience, the graphite furnace offers a unique advantage for elements present at the subtrace levels $\left(10^{-9}\right.$ to $\left.10^{-6} \mathrm{~g}\right)$. On the other hand, whenever higher concentrations of the analyte are encountered, the flame method is preferred.

TABLE 3. Trace elements in fly ash, NBS-SRM 1633 by atomic absorption spectrometry

\begin{tabular}{|c|c|c|c|c|}
\hline Element & $\begin{array}{c}\text { AAS value } \\
\mu \mathrm{g} / \mathrm{g}\end{array}$ & $\begin{array}{l}\text { Standard } \\
\text { deviation }\end{array}$ & $\begin{array}{l}\text { Relative standard } \\
\text { deviation, percent }\end{array}$ & $\begin{array}{c}\text { Certified } \\
\text { value, } \mu \mathrm{g} / \mathrm{g}\end{array}$ \\
\hline $\mathrm{Be}$ & 12.4 & 0.31 & 2.5 & $(12)^{\mathrm{a}}$ \\
\hline $\mathrm{Cd}$ & $\begin{array}{l}1.46^{\mathrm{b}} \\
1.50^{\mathrm{c}}\end{array}$ & $\begin{array}{l}.05 \\
.15\end{array}$ & 10.4 & $1.45 \pm 0.06$ \\
\hline $\mathrm{Cr}$ & 132 & 3.3 & 2.5 & $121 \pm 2$ \\
\hline $\mathrm{Cu}$ & 130 & 2.2 & 1.7 & $128 \pm 5$ \\
\hline $\mathrm{Hg}$ & $0.134^{\mathrm{d}}$ & 0.004 & 2.9 & $0.14 \pm 2$ \\
\hline $\mathrm{Mn}$ & 493 & 4.1 & 0.8 & $493 \pm 7$ \\
\hline $\mathrm{Ni}$ & $101^{e}$ & 3.3 & 3.2 & \pm 3 \\
\hline $\mathrm{Zn}$ & 216 & 2.4 & 1.1 & \pm 20 \\
\hline
\end{tabular}

${ }^{\text {a }}$ Not certified, for information only.

b Preconcentration with APDC.

c Graphite furnace.

d Cold vapor method.

e Preconcentration with dimethylgloxime. 
TABLE 4. Trace elements in coal, NBS-SRM 1632 by atomic absorption spectrometry

\begin{tabular}{|c|c|c|c|c|}
\hline Element & $\begin{array}{l}\text { ASS value } \\
\mu \mathrm{g} / \mathrm{g}\end{array}$ & $\begin{array}{l}\text { Standard } \\
\text { deviation }\end{array}$ & $\begin{array}{l}\text { Relative standard } \\
\text { deviation, percent }\end{array}$ & $\begin{array}{c}\text { Certified } \\
\text { value, } \mu \mathrm{g} / \mathrm{g}\end{array}$ \\
\hline $\mathrm{Cd}$ & $\begin{array}{r}0.18^{\mathrm{a}} \\
.21^{\mathrm{b}}\end{array}$ & $\begin{array}{l}0.014 \\
.02\end{array}$ & $\begin{array}{l}8.1 \\
9\end{array}$ & $0.19 \pm 0.03$ \\
\hline $\mathrm{Cr}$ & 20.2 & .4 & 2.1 & $20.2 \pm 0.5$ \\
\hline $\mathrm{Cu}$ & $\begin{array}{l}17.9 \\
19.4\end{array}$ & $\begin{array}{r}.2 \\
1.9\end{array}$ & 10 & $18 \pm 2$ \\
\hline $\begin{array}{l}\mathrm{Hg} \\
\mathrm{Zn}\end{array}$ & $\begin{array}{l}0.126^{\mathrm{c}} \\
38.4\end{array}$ & $\begin{array}{c}0.006 \\
.9\end{array}$ & $\begin{array}{l}4.5 \\
2.5\end{array}$ & $\begin{aligned} 0.12 & \pm 0.02 \\
37 & \pm 4\end{aligned}$ \\
\hline
\end{tabular}

a Atomic fluorescence.

b Graphite furnace.

c Cold vapor method.

TABLE 5. Trace elements in fuel oil, NBS-SRM 1634 by atomic absorption spectrometry

\begin{tabular}{cccc}
\hline Element & $\begin{array}{c}\text { AAS value } \\
\mu \mathrm{g} / \mathrm{g}\end{array}$ & $\begin{array}{c}\text { Standard } \\
\text { deviation }\end{array}$ & $\begin{array}{c}\text { Relative standard } \\
\text { deviation, percent }\end{array}$ \\
\hline $\mathrm{Cd}$ & $0.005^{\mathrm{a}}$ & - & - \\
$\mathrm{Fe}$ & 14.1 & 0.6 & 4.2 \\
$\mathrm{Ni}$ & 31.1 & 2.1 & 6.8 \\
$\mathrm{~Pb}$ & $0.05^{\mathrm{a}}$ & $-\overline{8}$ & $-\overline{1}$ \\
$\mathrm{~V}$ & 326 & 6.8 & 2.1 \\
\hline
\end{tabular}

a Graphite furnace.

\section{References}

[1] Walsh, A., Spectrochem. Acta 7, 108-117 (1955).

[2] Herrmann, R., Nebulizers and Burners, chapter in Flame Emission and Atomic Absorption Spectrometry, Deane, J. A. and Rains, T. C., Eds., Vol. 2, pp. 57-93, Marcel Dekker, New York (1971).

[3] Rains, T. C., Atomic Absorption Spectrometry, General Considerations for the Application of Experimental Techniques, chapter in Some Fundamentals of Analytical Chemistry, ASTM STP 564, pp. 50-67, American Society for Testing and Materials (1974).

[4] Kahn, H. L., At. Absorption Newsletter 7, 40-43 (1968).

[5] Rains, T. C., Epstein, M. S., and Menis, O., Anal. Chem. 46, 207-210 (1974).

[6] Rains, T. C. and Menis, O.,J. Assoc. Offi. Anal. Chem. 55, 1339-1344 (1972).

[7] Welcher, G. G., Kriege, O. H., and Marks, J. Y., Anal. Chem. 46. 1227-1231 (1974).

[8] Rains, T. C., Chemical Aspects of Atomic Absorption, chapter in Atomic Absorption Spectroscopy, ASTM STP 443, pp. 19-36, American Society for Testing and Materials (1969). 



\title{
ATOMIC ABSORPTION SPECTROPHOTOMETRY OF AIR FILTER PAPER TAPE SAMPLES
}

\author{
F. J. Muñoz-Ribadeneira, Maria L. Nazario \\ and Alice Vega \\ Puerto Rico Nuclear Center \\ Jobos Bay Project, Radioecology Division \\ College Station \\ Mayaguez, Puerto Rico 00708 U.S.A.
}

\begin{abstract}
Several techniques for the preparation of air paper tape samples for trace metal analysis by Atomic Absorption Spectrophotometry (AAS) have been investigated. Known amounts of $\mathrm{Cr}, \mathrm{Mn}, \mathrm{Co}, \mathrm{Ni}, \mathrm{Cu}, \mathrm{Zn}, \mathrm{Cd}$, and $\mathrm{Pb}$ were used to study the recovery of each under the effects of several preparative and digestion procedures. Conclusions obtained by means of a statistical analysis of the results are given. Statistical analysis of data obtained from the application of the most successful techniques when applied to natural air particulate samples are also presented.
\end{abstract}

Keywords: Air sampling; atomic absorption spectrophotometry; high temperature ashing; trace elements.

\section{Introduction}

A review of the literature indicated that several chemical elements in chloride, nitrate, and fluoride forms can be volatilized at high ashing temperatures (HAT) [1,2]. Sulfatation of the samples can reduce this volatilization, thus increasing the recovery of metals [3]. It has also been found that salts of these metals, under the influence of HAT, can form some more insoluble compounds with glass mats [3].

\section{Experimental}

\section{A. Preliminary Experiments}

In order to test the results from these investigations in our facilities, 20 replications of air tape samples $(10 \times 2.54 \mathrm{~cm})$ were cut from a paper tape 
roll (Type W-41 Bendix Environmental Division) and placed in $100 \mathrm{ml}$ Pyrex beakers which had been washed three times with hot $\mathrm{HCl}(2 \mathrm{~N})$, rinsed with deionized distilled water, and dried. Standards of the metals $\mathrm{Cr}, \mathrm{Mn}, \mathrm{Co}, \mathrm{Ni}, \mathrm{Cu}, \mathrm{Zn}, \mathrm{Cd}$, and $\mathrm{Pb}$ (Instrumentation Laboratory, Inc.) were added with a certified micropipette over the paper tapes. The concentration of the standards was selected to obtain an easily readable percent of absorption ( $>5$ percent). The standards were chlorides except for lead which was used as a nitrate. The samples were then dried under an infrared lamp and later put into a muffle oven, first for 30 minutes at 300 ${ }^{\circ} \mathrm{C}$ and then for 1 hour at 430 to $450{ }^{\circ} \mathrm{C}$. Residues were leached with hot $\mathrm{HCl}(2 \mathrm{~N})$. Hydrochloric acid was used in order to reduce the acid interferences [4]. The atomic absorption (AA) analysis was carried out using a Perkin Elmer Model No. 303, and for each element the manufacturer's recommendations were followed [5].

\section{B. Preliminary Results}

Table 1 shows the low recoveries of the chlorides of $\mathrm{Zn}, \mathrm{Cd}, \mathrm{Cu}, \mathrm{Ni}$, and lead nitrate and indicates the tendency of these compounds to volatilize at the muffle temperatures used in these investigations. Results coming from the sulphuric acid treatment show the effect of the $\mathrm{H}_{2} \mathrm{SO}_{4}$ as an ashing aid to suppress the volatilization of the metals.

It may also be indicated that under the influence of the heat treatment in the muffle, some kinds of more insoluble compounds are formed between the traces of these metals and Pyrex glass of the beakers. In most cases, an increase in the yield of metal recuperation is found in samples submitted to several washing-extractions with hot $\mathrm{HCl}(2 N)$. A statistical analysis of this data points out the convenience of repeating the $\mathrm{HCl}$ treatment on the calcination residue of the paper tape samples.

\section{Other ExPERIMENTS}

Other experiments were conducted with the following conditions:

a) a 95 percent recovery of the metals would be acceptable;

b) a 5 percent standard deviation from the mean was considered reasonable;

c) perchloric acid should not be used, and Pyrex beakers should be used instead of Teflon beakers and/or platinum crucibles;

d) the acid inventory should be kept as low as possible; 


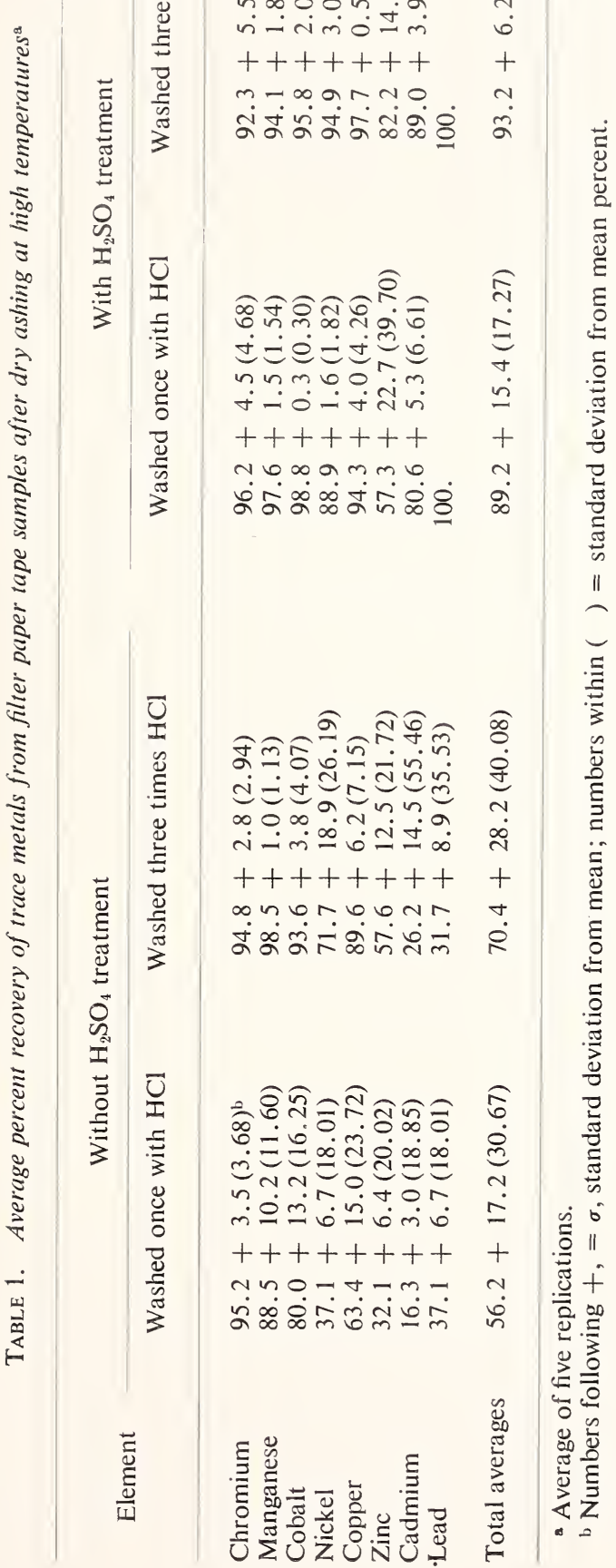


e) organic matter should not be present in the metal solutions in order to avoid enhancement of the signal in the spectrophotometer detector.

In order to satisfy these requirements the following was agreed:

a) perchloric acid could be replaced by hydrogen peroxide;

b) the acid inventory could be reduced if the air tape samples were burned with alcohol prior to their acid dissolution.

Table 2 shows the variables taken into account in the design of experiments for this new phase of the investigation. Additional notes for table 2:

a) Alcohol burning: after the addition of the standards, the samples were dried as in previous experiments, wetted with $2 \mathrm{ml}$ of alcohol of spectroscopic quality, and burned.

b) Chemical treatment (1): to each of the samples, $6 \mathrm{ml}$ of inverse aqua regia $\left(3 \mathrm{HNO}_{3}+1 \mathrm{HCl}\right)$ were added. The beakers were covered with watch glasses $7 \mathrm{~cm}$ in diameter and put on a hot plate at a slow boil. The watch glass permits some reflux of the acids, and the reaction may last up to the time that the paper tape should dissolve. The samples were then brought to a rapid boil, and the evaporation was continued almost to dryness. Four $\mathrm{ml}$ of concentrated $\mathrm{HNO}_{3}$ and $1 \mathrm{ml}$ of $\mathrm{H}_{2} \mathrm{O}_{2}$ ( $30 \%$ by volume) were added. The samples were again boiled, first slowly and then at higher temperatures. When the samples were almost dried, three drops of concentrated $\mathrm{H}_{2} \mathrm{SO}_{4}$ were added to test for the presence of organic matter (found to be negligible or nonexistent). Samples were then evaporated until the $\mathrm{SO}_{3}$ fumes had completely disappeared. Samples Code P and $\mathrm{T}$ were submitted to the heat treatment in the muffle oven following the experimental procedures previously described.

c) Chemical treatment (2): the residues of the calcination, as well as those coming from the other chemical treatments, were extracted three times with hot $(2 \mathrm{~N}) \mathrm{HCl}$. The results obtained in these experiments are presented in table 3 .

\section{Discussion}

Table 3 indicates that samples submitted to treatments $\mathrm{P}$ and $\mathrm{Q}$ show the lowest metal recovery. Table 4, column one, indicates no statistical difference in the results. These two sets of samples were submitted to a common procedure, i.e., alcohol burning of samples prior to other treatments. Table 4, column three, also indicates that the statistical comparison between $\mathrm{P}$ and $\mathrm{T}$ is significantly favorable for $\mathrm{T}$ for $\mathrm{Ni}, \mathrm{Cu}, \mathrm{Zn}$, $\mathrm{Cd}$, and $\mathrm{Pb}$. These two sets of samples were submitted to the $\mathrm{H}_{2} \mathrm{SO}_{4}$ treatment and later to the effects of heating at high temperatures in the 
TABLE 2. Experimental design to test the effects of different variables used in the chemical preparation of air tape samples ${ }^{\mathrm{a}}$

\begin{tabular}{ccccc} 
& \multicolumn{4}{c}{ Variables $^{\text {b }}$} \\
\cline { 2 - 5 } $\begin{array}{c}\text { Experiment } \\
\text { code }\end{array}$ & $\begin{array}{c}\text { Alcohol } \\
\text { burning }\end{array}$ & $\begin{array}{c}\text { Chemical } \\
\text { treatment (1) }\end{array}$ & $\begin{array}{l}\text { Muffle } \\
\text { heating }\end{array}$ & $\begin{array}{c}\text { Chemical } \\
\text { treatment (2) }\end{array}$
\end{tabular}

$\begin{array}{ll}\mathrm{P} & * \\ \mathrm{Q} & * \\ \mathrm{R} & - \\ \mathrm{T} & -\end{array}$

a Five replications for each one of the code set of samples.

$\mathrm{b} *=$ Presence of the variable.

- = Variable absent.

TABLE 3. Average percent of metal recovery from filter paper tape samples submitted to different chemical treatment

\begin{tabular}{|c|c|c|c|c|}
\hline \multirow{2}{*}{ Elements } & \multicolumn{4}{|c|}{ Variables involved as indicated in Table 2} \\
\hline & $P$ & $Q$ & $R$ & $T$ \\
\hline Chromium & 87.5 & 94.0 & 99.1 & 90.0 \\
\hline$\sigma$ & 10.3 & 5.5 & 0.6 & 7.8 \\
\hline$\sigma \%$ & 11.43 & 5.85 & 0.60 & 8.67 \\
\hline Manganese & 90.2 & 91.9 & 97.5 & 96.2 \\
\hline$\sigma$ & 6.4 & 4.6 & 2.3 & 4.1 \\
\hline$\sigma \%$ & 7.09 & 5.04 & 2.35 & 4.26 \\
\hline Cobalt & 91.5 & 98.7 & 98.0 & 94.5 \\
\hline$\sigma$ & 6.0 & 1.0 & 1.6 & 4.3 \\
\hline$\sigma \%$ & 6.55 & 1.01 & 1.63 & 4.55 \\
\hline Nickel & 81.7 & 87.5 & 98.0 & 92.4 \\
\hline$\sigma$ & 7.9 & 6.7 & 0.8 & 4.7 \\
\hline$\sigma \%$ & 9.67 & 7.66 & 0.82 & 5.08 \\
\hline Copper & 82.9 & 90.0 & 95.5 & 97.6 \\
\hline$\sigma$ & 10.5 & 6.0 & 2.3 & 1.8 \\
\hline$\sigma \%$ & 12.66 & 6.67 & 2.41 & 1.84 \\
\hline Zinc & 46.0 & 57.0 & 96.5 & 90.4 \\
\hline$\sigma$ & 8.4 & 8.7 & 2.0 & 5.9 \\
\hline$\sigma \%$ & 18.26 & 16.26 & 2.07 & 6.52 \\
\hline Cadmium & 36.5 & 35.0 & 94.5 & 88.6 \\
\hline$\sigma$ & 3.0 & 5.7 & 3.8 & 6.0 \\
\hline$\sigma \%$ & 8.21 & 16.28 & 4.02 & 6.77 \\
\hline Lead & 55.0 & 63.0 & 100.0 & 100.0 \\
\hline$\sigma$ & 10.1 & 8.5 & - & - \\
\hline$\sigma \%$ & 18.15 & 13.49 & - & - \\
\hline Total average & 71.5 & 77.0 & 97.1 & 93.7 \\
\hline$\sigma$ & 22.8 & 22.7 & 1.8 & 4.0 \\
\hline$\sigma \%$ & 32.09 & 29.48 & 1.90 & 4.26 \\
\hline
\end{tabular}

Notes: $\sigma=$ standard deviation from mean

$\sigma \%=$ standard deviation from mean percent 
TABLE 4. Statistical analysis on data obtained by atomic absorption spectrophotometry from samples submitted to different chemical treatments

\begin{tabular}{lcccccc}
\hline $\begin{array}{c}\text { Ele- } \\
\text { ments }\end{array}$ & $P Q$ & $P R$ & $P T$ & $Q R$ & $Q T$ & $R T$ \\
\hline & & & & & \\
$\mathrm{Cr}$ & $1.244(\mathrm{~N})$ & $2.517(\mathrm{Y})$ & $0.432(\mathrm{~N})$ & $2.061(\mathrm{~N})$ & $0.937(\mathrm{~N})$ & $2.601(\mathrm{Y})$ \\
$\mathrm{Mn}$ & $0.255(\mathrm{~N})$ & $2.326(\mathrm{Y})$ & $1.765(\mathrm{~N})$ & $3.044(\mathrm{Y})$ & $1.850(\mathrm{~N})$ & $0.703(\mathrm{~N})$ \\
$\mathrm{Co}$ & $2.647(\mathrm{Y})$ & $2.701(\mathrm{Y})$ & $0.908(\mathrm{~N})$ & $0.829(\mathrm{~N})$ & $2.127(\mathrm{~N})$ & $1.705(\mathrm{~N})$ \\
$\mathrm{Ni}$ & $1.252(\mathrm{~N})$ & $4.590(\mathrm{Y})$ & $2.603(\mathrm{Y})$ & $1.822(\mathrm{~N})$ & $1.338(\mathrm{~N})$ & $2.626(\mathrm{Y})$ \\
$\mathrm{Cu}$ & $1.312(\mathrm{~N})$ & $2.621(\mathrm{Y})$ & $3.086(\mathrm{Y})$ & $1.914(\mathrm{~N})$ & $2.713(\mathrm{Y})$ & $1.608(\mathrm{~N})$ \\
$\mathrm{Zn}$ & $2.034(\mathrm{~N})$ & $13.078(\mathrm{Y})$ & $9.672(\mathrm{Y})$ & $9.895(\mathrm{Y})$ & $7.105(\mathrm{Y})$ & $2.189(\mathrm{~N})(90 \%)$ \\
$\mathrm{Cd}$ & $0.411(\mathrm{~N})$ & $26.789(\mathrm{Y})$ & $17.434(\mathrm{Y})$ & $19.423(\mathrm{Y})$ & $14.402(\mathrm{Y})$ & $2.613(\mathrm{Y})$ \\
$\mathrm{Pb}$ & $1.363(\mathrm{~N})$ & $(\mathrm{Y}) *$ & $(\mathrm{Y})$ & $(\mathrm{Y}) *$ & $(\mathrm{Y})^{*}$ & $(\mathrm{~N})$ \\
& & & & & &
\end{tabular}

Notes:

" $t$ " Student distribution function at $P(0.05)$ and 8 degrees of freedom $=2.306$.

$(\mathrm{N})=$ no statistical significance.

$(Y)=$ statistical significance at $P(0.05)$ confidence level.

$*=$ the difference is so large that it is accepted as significant.

muffle, but $\mathrm{T}$ was not submitted to alcohol burning. As observed in table 4 , there is a difference in the results, and these differences are statistically significant and are especially shown for the more easily volatilized elements; their low recovery could be attributed to the high temperatures associated with flaming that had been produced by the alcohol burning of the air paper tapes.

For ail the chemical elements studied in this investigation there are significant favorable statistics on data obtained from the set of samples $\mathbf{R}$ compared to samples $P$. Samples R received the aqua regia- $\mathrm{HNO}_{3}-\mathrm{H}_{2} \mathrm{O}_{2}$ treatment $(\mathrm{ANH})$ and were not submitted to any alcohol burning or muffle treatments. This is clearly seen in column two of table 4.

The statistical comparison of data between $Q$ and $R$ and $Q$ and $T$ also indicates significant difference in favor of sets $\mathrm{R}$ and $\mathrm{T}$ in chemical elements that could be more volatilized at higher temperatures. Samples called $\mathrm{R}$ and $\mathrm{T}$, as indicated before, were not submitted to alcohol burning, and the negative effect of paper flaming can be observed in columns four and five of table 4.

Table 3 shows that the yield of metal recovery from samples submitted to the ANH treatment (R) are higher than those submitted to the $\mathrm{H}_{2} \mathrm{SO}_{4}$ muffle heating $(\mathrm{T})$; it also indicates that the overall percent of metal recovery is higher and the variations in the data are smaller. If the same statistical analysis already mentioned is applied for the overall data between samples $\mathrm{R}$ and $\mathrm{T}$, it is found that the differences are statistically significant at $\mathbf{P}(0.05) ;\left(t_{c}=2.953\right)$. In data from table 4 , column six, these differences are statistically significant for $\mathrm{Ni}, \mathrm{Cd}$, and $\mathrm{Cr}$. This can be attributed to the fact that $\mathrm{Ni}$ and $\mathrm{Cd}$ could be volatilized by muffle treat- 
ment, and $\mathrm{Cr}$ under heat could be retained in the Pyrex beaker and extracted with more difficulty by the $\mathrm{HCl}(2 N)$. All of this is an indication that improved recovery is obtained when samples are submitted to wet ashing compared to ashing at high temperatures, even when samples first receive the $\mathrm{H}_{2} \mathrm{SO}_{4}$ treatment.

\section{Application to Natural Aerosol Samples}

The investigated chemical treatments (sulfatation of samples prior to HAT, alcohol burning, and wet digestion of the samples using the inverse aqua regia-nitric acid-hydrogen peroxide method $(\mathrm{ANH})$ ) were used to determine the yield of metal recovery from natural aerosols obtained from Mayaguez, Puerto Rico, during December 1973. For this purpose, three air tape Bendix Model 23,000 machines were operated at the same time for 5 days. The air particulate material obtained during one day was made up into samples, and the paper tapes obtained from each machine were analyzed for their metal content. Results are presented in table 5 .

Data from table 5 indicate that the blanks of the filter paper tapes did not have any metal contaminant except zinc. For all the analyses that were carried out, the best yield of metal was obtained from samples submitted to wet ashing with the HAT method. A statistical analysis of data also indicates that the results obtained by this method are highly significant compared to data obtained from samples submitted to the other methods, alcohol burning and sulfatation coupled with HAT. Data for $\mathrm{Zn}$ and $\mathrm{Pb}$ also indicate the convenience of not using the alcohol burning procedure for sulfatation prior to the HAT. 
TABle 5. Analysis of Mayaguez, Puerto Rico, air particulate matter on paper tape filters as a function of different chemical treatment of samples $\left(\mu \mathrm{g} / \mathrm{m}^{3}\right)$

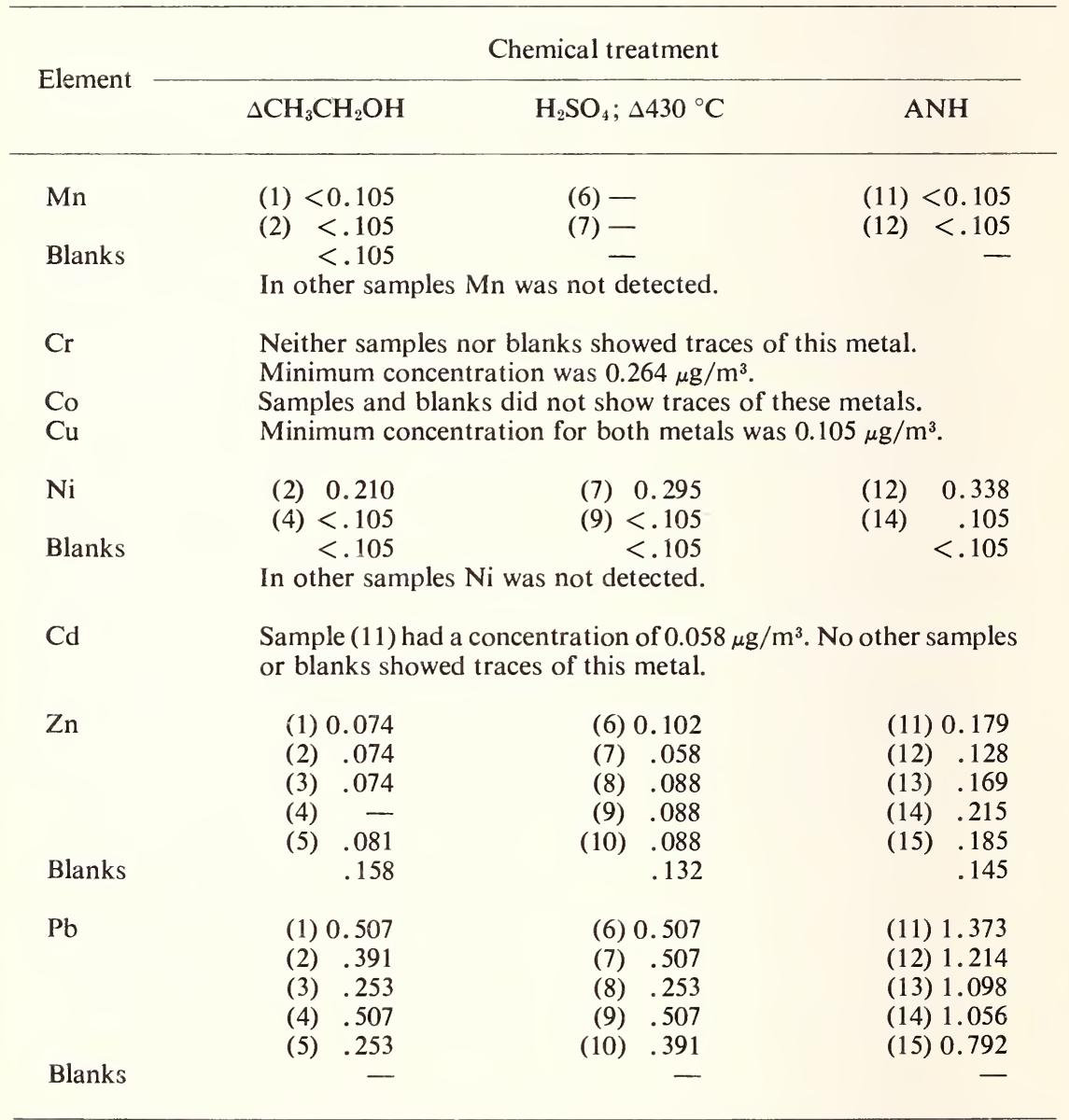




\section{Acknowledgement}

The Puerto Rico Nuclear Center is operated by the University of Puerto Rico for the U.S. Atomic Energy Commission, contract No. AT-(401)-1833.

\section{References}

[1] Kometani, T. Y., Effect of Temperature on Volatilization of Salts During Dry Ashing of Tetrafluoroethylene Fluorocarbon Resin, Anal. Chem. 38, 1596 (1966).

[2] Thompson, R. J., Morgan, G. B., Purdue, L. J., Analysis of Selected Elements in Atmospheric Particulate Matter by Atomic Absorption, Atomics Absorption Newsletter 3.53 (1970).

[3] Kometani, T. Y., Bove, J. L., Nathason, B., Siemberg, S., and Magyar, M., Dry Ashing of Airborne Particulate Matter on Paper and Glass Fiber Filters for Trace Metal Analysis by Atomic Absorption Spectrophotometry, Environ. Sci. Technol. 7, 617 (1972).

[4] Barnett, W. D., Acid Interferences in Atomic Absorption Spectrophotometry, Anal. Chem. 4, 695 (1972).

[5] Analytical Methods for Atomic Absorption Spectrophotometry, Perkin Elmer Corporation, Supplement March 1973, Norwalk, Connecticut (1973).

[6] Romo, L. A., Principios de Investigación Cientifica, Editorial Tip Cat Casals, Barcelona, Spain, 167 (1964). 



\title{
ANALYSIS OF CADMIUM IN FISH TISSUE BY FLAMELESS ATOMIC ABSORPTION WITH A TANTALUM RIBBON
}

\author{
E. R. Blood and G. C. Grant \\ Department of Chemistry \\ Virginia Commonwealth University \\ Richmond, Virginia 23284 U.S.A
}

\begin{abstract}
Bluegills (Lepomis machrochirus) of the same age and approximately the same size after acclimation were exposed to known amounts of cadmium (ppm level), sacrificed at selected time intervals and frozen whole in plastic bags until dissection and analysis. The heart, liver and kidney were removed entirely and portions of skin, muscle, gill, gut and bone were taken at the same time. Analyses were made by atomic absorption, following wet digestion in concentrated acid. All results are expressed as micrograms cadmium per gram of wet tissue. Typical values reported are in the range of 0.2 to $20 \mu \mathrm{g} \mathrm{Cd}$ per gram of wet tissue with a typical precision of $10-15$ percent rsd for repetitive aliquots from the same organ. Corresponding organs from different fish, however, gave mean values differing from one another by as much as a factor of five.
\end{abstract}

Keywords: Atomic absorption; bluegills; cadmium; fish; heavy metal; tissue; trace element analysis; water pollution.

\section{Introduction}

Cadmium is increasingly recognized as an important environmental pollutant with toxic effects on human and animal life at relatively low levels [1-3]. Continuing and increasing usage of cadmium by battery, alloy, pigment, plastics, electroplating and semiconductor manufacturers has resulted in the release of large quantities to the atmosphere and into waterways [4-6]. Environmental concentrations of cadmium are of serious concern because cadmium accumulates in the human body throughout life-from approximately $1 \mu \mathrm{g}$ at birth to about $30 \mathrm{mg}$ with about one-third to be found in the kidneys [7]. Based on animal studies, cadmium is preferentially retained by the kidney and liver [2]. 
In view of the known accumulation of cadmium in biological tissues a detailed study was begun to determine the rate of uptake in the common bluegill (Lepomis macrochirus Raf.) exposed to known amounts of cadmium in a carefully controlled aquatic environment. An important objective was the relative rates of uptake in vital organs including: heart, skin, muscle, gut, gill, kidney, liver and bone. A recent study of the toxicity of $\mathrm{ZnSO}_{4}$ to rainbow trout dealt with only acute toxicity as measured by fish mortality [8]. Another study examined the chronic toxicity of copper, cadmium and zinc mixtures at sub-lethal concentrations to the fathead minnow using mortality, physical characteristics and reproduction as bioassay methods [9]. While these studies increase our understanding of biological effects for relatively concentrated heavy metal pollutants in aquatic systems, they provide no evidence for the actual rate of accumulation of toxic metals nor the distribution of these in vital organs. Moreover, only rarely in natural waters do the concentrations of toxic metals attain the levels used in most acute and chronic bioassay studies. Thus, experimental evidence for heavy metal accumulation and distribution in organisms exposed to environmentally realistic levels of heavy metal pollutants in natural waters should make possible more reliable and general predictions for the long term effects of such pollutants.

The present work is a study of analytical methods for the analysis of cadmium in fish tissue by atomic absorption spectrophotometry using the higher sensitivity afforded by flameless atomization of the sample.

\section{Experimental}

All analyses were performed on an Instrumentation Laboratory (IL) Model 153 Double Beam Atomic Absorption Spectrophotometer, modified electronically to allow subtraction of nonspecific absorption from a hydrogen continuum placed in channel B as previously described by Hwang [10]. Samples were dried and vaporized on the tantalum ribbon of an IL Model 355 Flameless Sampler. Instrumental parameters were optimized according to procedures previously described [10,11] and are given in table 1.

All solid reagents and concentrated acids were A.C.S. reagent grade and were used without further purification. Distilled water, which had been passed through a mixed bed ion exchange column, was used throughout this work. Glassware, polypropylene test tubes and polyethylene storage bottles were standard laboratory equipment. Glassware was cleaned with concentrated $\mathrm{H}_{2} \mathrm{SO}_{4}$ containing $\mathrm{KMnO}_{4}$ followed 
TABLE 1. Instrumental parameters

Model 153 A. A. Spectrophotometer

Mode

Hollow cathode A A-B (background correction) Cadmium

B $\quad \mathrm{H}_{2}$ continuum

Lamp current A

$3.5 \mathrm{~mA}$

B $\quad 20 \mathrm{~mA}$

P.M. voltage

$700 \mathrm{~V}$

Slit width

$320 \mu \mathrm{m}$

Wavelength

$228.8 \mathrm{~nm}$

Model 355 Flameless Sampler

Mode

Purge gas

Gas flow rate

Automatic

Argon

Dry setting

10 S.C.F.H.

$170 \mathrm{C}(2.5$ turns $)$

Dry time

140 seconds

Analyze setting

$1600 \mathrm{C}$ (7.0 turns)

by repeated water rinses. Polypropylene test tubes were soaked at least 24 hours in $5 \mathrm{w} / \mathrm{v}$ percent Triton X-100 solution followed by water rinses. Hatchery obtained fish were approximately the same size and weight (5 to $10 \mathrm{~cm}, 3$ to $5 \mathrm{~g}$ ). Twenty-eight fish were placed in all glass aquaria containing reconstituted water $\left(30 \mathrm{mg} \mathrm{MgSO}_{4} / 1,30 \mathrm{mg} \mathrm{CaSO} / 1,48 \mathrm{mg}\right.$ $\mathrm{NaHCO}_{3} / \mathrm{l}$ and $2 \mathrm{mg} \mathrm{KCl} / \mathrm{l}$ ). After 2 weeks of acclimation, sufficient $\mathrm{CdCl}_{2}$ was added to obtain $3 \mathrm{ppm} \mathrm{CdCl}_{2}$ in each tank. Fish were removed at set intervals throughout the 2-week experiment and frozen in plastic bags until analysis.

For analysis, the digested sample was diluted to $25 \mathrm{ml}$ in a volumetric flask and $20 \mu \mathrm{l}$ aliquots were removed immediately for analysis with an Eppendorf pipet to prevent adsorption of cadmium onto the glass surfaces. Since peak heights observed for cadmium standards decreased approximately 20 percent during the lifetime of a tantalum ribbon (50 to 100 aliquots) and varied from ribbon to ribbon, no attempt was made to use a standard curve. Instead, the unknown concentration was calculated from the ratio of peak heights of a cadmium standard chosen to have a similar peak height. The normal analysis procedure was to analyze two aliquots of standard, four of the sample followed by two of the standard. Any observed irregularities in the standards resulted in the analysis of additional aliquots. 


\section{Results and Discussion}

Figure 1 shows the typical effects of using the background correction made on a $5.0 \mathrm{ppb}$ cadmium standard. Each peak is from a $20 \mu \mathrm{l}$ aliquot from an Eppendorf pipet. The background correction made for standards typically gave 1) absence or sharply reduced matrix forepeak, 2) increased noise level, 3) reduced precision (higher rsd) and 4) approximately a 5 percent change in mean peak height.

In an effort to improve the relative precision of the analyses, the use of a micrometer syringe was investigated. Analysis of successive aliquots of a $5.0 \mathrm{ppb}$ solution gave 1.5 and 3.0 percent rsd for the micrometer and Eppendorf pipets respectively (10 aliquots). However, significantly, such precision with the micrometer syringe was attainable only with very careful technique because significant loss of peak height was observed whenever the solution remained inside the syringe for even a few minutes, indicating rapid adsorption of cadmium onto either the glass or needle which is consistent with other recent work [12]. The increased convenience of use, the freedom from adsorption and the freedom from crosscontamination (using disposable tips) were judged to be well worth the loss in relative precision. Also, in agreement with these adsorption studies [12], peak heights for freshly diluted $1 \mathrm{ppb}$ or $5 \mathrm{ppb}$ standard solutions were (within day to day reproducibility) the same as for solutions aged for periods of several months in polyethylene bottles. Nevertheless, standards were usually freshly diluted every few days from more concentrated stock solutions and contact time with glass vessels was minimized.

Typical results using background correction for the analyses of the various fish organs are shown in figure 2. Cadmium peaks were typically 10 to 20 percent lower using background correction indicating a significant nonspecific absorption (or light scattering) during the analyze cycle. Background correction was therefore routinely used.

During the initial phases of this work, $10 \mathrm{ml}$ glass vials with snap caps were used as containers for the wet acid digestions. In agreement with other recent work [3], reproducibility was found to be very poor with recoveries of standards as low as 74 percent; therefore, all further acid digestions were done in polypropylene test tubes.

Two wet acid digestion mixtures were compared for the analysis of fish tissue. In the first, 1 milliliter of the acid mixture ( 3 parts conc $\mathrm{HNO}_{3}$ by volume: 1 part conc $\mathrm{H}_{2} \mathrm{SO}_{4}$ : 1 part conc $\mathrm{HClO}_{4}$ ) was placed together with a weighed sample in a covered $3.5 \mathrm{ml}$ polypropylene test tube for 2 hours at $74{ }^{\circ} \mathrm{C}$ in a water bath and finally, diluted to $25 \mathrm{ml}$. The second procedure, reported recently [13], was initiated by addition of $1 \mathrm{ml}$ of 


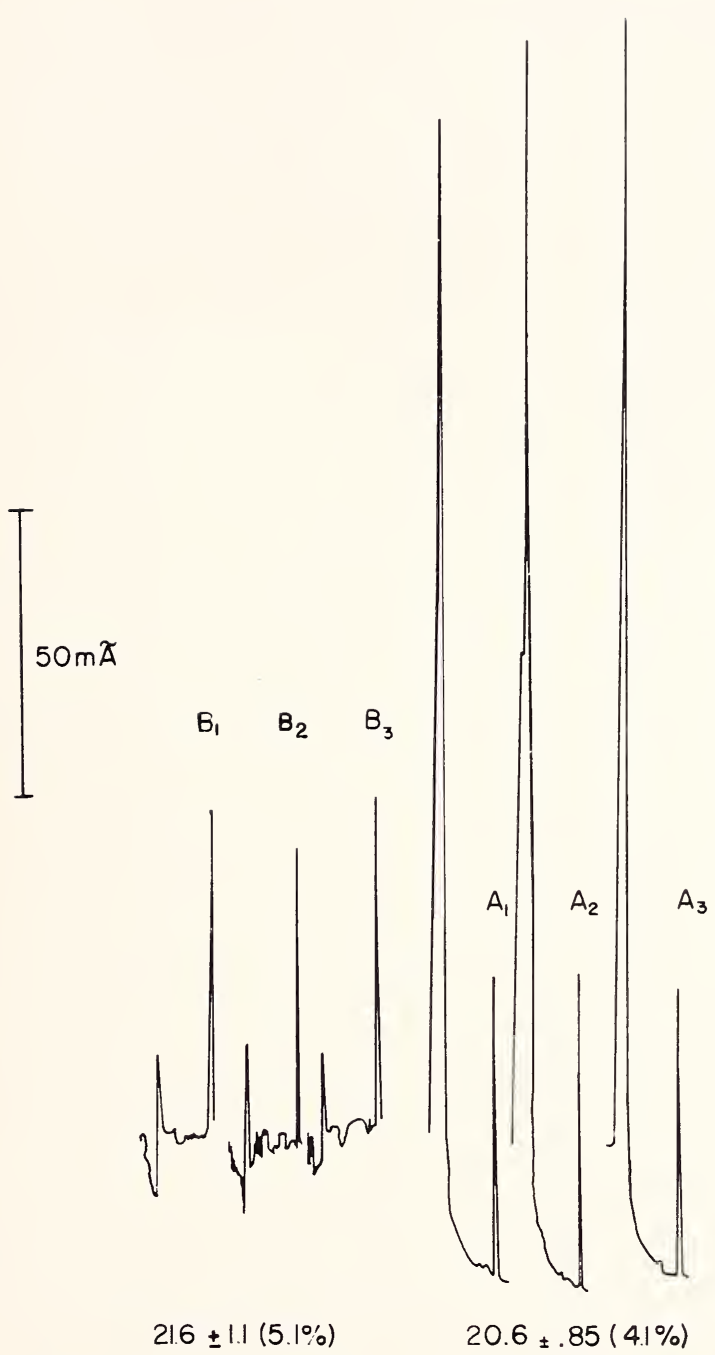

Figure 1. Effect of background correction on $5.0 \mathrm{ppb}$ cadmium standard. Repetitive vaporization of $20 \mu \mathrm{l}$ aliquots of $5.0 \mathrm{ppb}$ aqueous $\mathrm{CdCl}_{2}$ solution using a tantalum ribbon heated electrically to $1600{ }^{\circ} \mathrm{C}$. Peaks $A_{1}, A_{2}$ and $A_{3}$ were obtained in normal mode using a Cd lamp at $228.8 \mathrm{~nm}$. Peaks $\mathrm{B}_{1}, \mathrm{~B}_{2}$ and $\mathrm{B}_{3}$ were obtained in A-B mode (background correction).

concentrated $\mathrm{HNO}_{3}$ to the sample, heated for 15 minutes at 80 to $90{ }^{\circ} \mathrm{C}$ followed by addition of $1 \mathrm{ml}$ of 10 percent $\mathrm{H}_{2} \mathrm{O}_{2}$ and heating for an additional 15 minutes. 


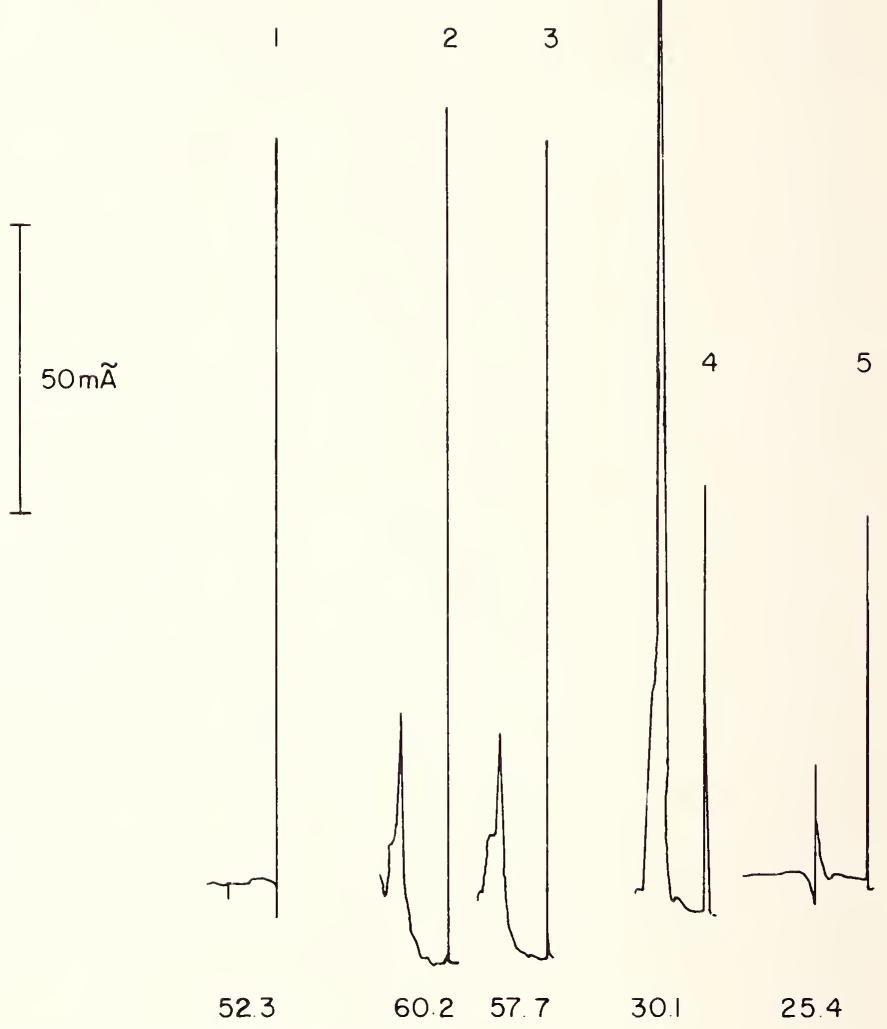

Figure 2. Effect of background correction on cadmium analysis in bluegill skin. Twentymicroliter aliquots were dried at $170{ }^{\circ} \mathrm{C}$ for 140 seconds and analyzed at $1600{ }^{\circ} \mathrm{C}$ in the flameless sampler. The samples were: (1)Bluegill skin with hydrogen lamp background correction, (2,3) same as 1 but with no background correction, (4) $5.0 \mathrm{ppb} \mathrm{CdCl}_{2}$ without background correction, and (5) $5.0 \mathrm{ppb} \mathrm{CdCl}_{2}$ with background correction.

Results for samples digested by both procedures are given in table 2 . Analysis of National Bureau of Standards SRM 1577 by both digestion procedures gave results within the error tolerance given but the $\mathrm{HNO}_{3} / \mathrm{H}_{2} \mathrm{O}_{2}$ method gave only 85 percent recovery as compared with 95 percent for the $\mathrm{HNO}_{3} / \mathrm{H}_{2} \mathrm{SO}_{4} / \mathrm{HClO}_{4}$ method in good agreement with the recovery of a $0.500 \mathrm{ppm}$ standard run through the identical digestion procedures. 
TABLE 2. Recovery of cadmium after acid digestion of standards

Sample

Percent recovery

$\begin{array}{cc}\mathrm{HNO}_{3} / \mathrm{H}_{2} \mathrm{O}_{2} & \mathrm{HNO}_{3} / \mathrm{H}_{2} \mathrm{SO}_{4} / \mathrm{HClO}_{4} \\ \text { digestion } & \text { digestion }\end{array}$

Bovine Liver

(NBS SRM 1577)

85

(0.23 ppm)

88.6

79.2

83.5

87.9

mean 84.8 mean
93

$(0.25 \mathrm{ppm})$

99.9

95.7

93.1

96.1

96.2

TABLE 3. Comparison of cadmium found in Bluegill tissue after acid digestion

\begin{tabular}{lcccr} 
Sample & $\begin{array}{c}\text { ppm Cdb } \\
\left(\mathrm{HNO}_{3} / \mathrm{H}_{2} \mathrm{O}_{2}\right)\end{array}$ & $\begin{array}{c}\text { r.s.d. } \\
(\%)\end{array}$ & $\begin{array}{c}\text { ppm Cd } \\
\left(\mathrm{HNO}_{3} / \mathrm{H}_{2} \mathrm{SO}_{4} / \mathrm{HClO}_{4}\right)\end{array}$ & $\begin{array}{c}\text { r.s.d. } \\
(\%)\end{array}$ \\
\hline $\begin{array}{l}\text { Muscle } \\
\text { Muscle }\end{array}$ & 0.91 & 9.9 & 1.25 & 15.9 \\
Skin & 1.41 & 7.6 & 2.87 & 14.5 \\
& 8.6 & 3.2 & 10.9 & 7.0
\end{tabular}

a A portion of the wet homogenate from a ground glass homogenizer.

${ }^{\mathrm{b}} \mathrm{ppm} \mathrm{Cd}$ is $\mu \mathrm{g} \mathrm{Cd}$ per gram of wet tissue.

Additional comparison of the two digestions is possible using the data in table 3 for muscle and skin. The muscle sample was carefully homogenized before weighing portions for the digestions so that the differences in the ppm Cd found reflect real differences in the digestion step or matrix interferences in the subsequent analysis. Although the relative precision was better for the $\mathrm{HNO}_{3} / \mathrm{H}_{2} \mathrm{O}_{2}$ method, the percent recovery was always smaller indicating systematic errors. Additionally, although the $\mathrm{HNO}_{3} / \mathrm{H}_{2} \mathrm{O}_{2}$ digestion appears to require less operator time, it actually requires more time when multiple samples are run because it must be watched constantly whereas the $\mathrm{HNO}_{3} / \mathrm{H}_{2} \mathrm{SO}_{4} / \mathrm{HClO}_{4}$ digestion can be left unattended. Furthermore, several samples foamed over the test tube upon addition of $\mathrm{H}_{2} \mathrm{O}_{2}$ necessitating repetition of the experiments. For these reasons, the $\mathrm{HNO}_{3} / \mathrm{H}_{2} \mathrm{SO}_{4} / \mathrm{HClO}_{4}$ digestion method is recommended for fish tissue. The concentrated acid mixtures were found to have 7.5 and $10.3 \mathrm{ppb} \mathrm{Cd}$ for the $\mathrm{HNO}_{3} / \mathrm{H}_{2} \mathrm{O}_{2}$ and $\mathrm{HNO}_{3} / \mathrm{H}_{2} \mathrm{SO}_{4} / \mathrm{HClO}_{4}$ 
mixtures, respectively, which was low enough for most of the samples analyzed so that the use of expensive ultrapure acid reagents could be avoided. All sample and standard peak heights were corrected for the contribution of the acid blank, which was $0.41 \mathrm{ppb}$ after dilution to $25 \mathrm{ml}$.

Typical results for eight organs of three bluegills digested with the $\mathrm{HNO}_{3} / \mathrm{H}_{2} \mathrm{SO}_{4} / \mathrm{HClO}_{4}$ acid mixture are given in table 4 . In the case of the heart, kidney and liver the sample weight reflects the entire organ which was excised without connective tissue. Samples of the other organs were chosen from representative portions. The analyte concentrations after correction for the acid mixture blank $(0.41 \mathrm{ppb})$ ranged from 0.1 to $9 \mathrm{ppb}$ and were comparable to blank levels only for the heart and muscle. Since

TABLE 4. Cadmium in fish exposed to $3 \mathrm{ppm} \mathrm{CdCl}_{2}$ for one week

\begin{tabular}{|c|c|c|c|c|c|c|}
\hline Organ & $\begin{array}{l}\text { Sample weight } \\
\text { (mg) }\end{array}$ & $\begin{array}{c}\text { ppm Cda } \\
\text { (each fish) }\end{array}$ & $\begin{array}{l}\text { Absolute } \\
\text { standard } \\
\text { deviation }\end{array}$ & $\begin{array}{l}\text { r.s.d. } \\
(\%)\end{array}$ & $\begin{array}{l}\text { Average } \\
\text { ppm Cd }\end{array}$ & $\begin{array}{l}\text { r.s.d. } \\
(\%)\end{array}$ \\
\hline Heart & $\begin{array}{l}1.8 \\
2.1 \\
2.4\end{array}$ & $\begin{array}{l}1.52 \\
6.34 \\
5.62\end{array}$ & $\begin{array}{r}0.18 \\
.57 \\
1.01\end{array}$ & $\begin{array}{r}12 \\
9 \\
18\end{array}$ & 4.5 & 58 \\
\hline Skin & $\begin{array}{l}22.3 \\
48.1 \\
17.8\end{array}$ & $\begin{array}{l}1.61 \\
1.07 \\
1.26\end{array}$ & $\begin{array}{r}0.24 \\
.13 \\
.38\end{array}$ & $\begin{array}{l}15 \\
12 \\
30\end{array}$ & 1.3 & 21 \\
\hline Muscle & $\begin{array}{l}45.1 \\
85.0 \\
29.0\end{array}$ & $\frac{0.20}{-075}$ & $\frac{.02}{.02}$ & $\frac{10}{27}$ & .14 & 51 \\
\hline Gut & $\begin{array}{l}34.3 \\
26.6 \\
20.6\end{array}$ & $\begin{array}{r}5.25 \\
.61 \\
5.02\end{array}$ & $\begin{array}{l}.11 \\
.02 \\
.10\end{array}$ & $\begin{array}{l}2 \\
3 \\
2\end{array}$ & 3.6 & 72 \\
\hline Gill & $\begin{array}{r}10.6 \\
15.1 \\
9.6\end{array}$ & $\begin{array}{r}6.67 \\
6.49 \\
.94\end{array}$ & $\begin{array}{l}.33 \\
.45 \\
.56\end{array}$ & $\begin{array}{r}5 \\
7 \\
60\end{array}$ & 4.7 & 69 \\
\hline Kidney & $\begin{array}{r}17.8 \\
9.7 \\
10.8\end{array}$ & $\begin{array}{l}4.13 \\
2.26 \\
3.04\end{array}$ & $\begin{array}{l}.37 \\
.79 \\
.33\end{array}$ & $\begin{array}{r}9 \\
35 \\
11\end{array}$ & 3.1 & 30 \\
\hline Liver & $\begin{array}{l}19.9 \\
38.0 \\
17.7\end{array}$ & $\begin{array}{l}4.91 \\
5.18 \\
6.71\end{array}$ & $\begin{array}{l}.20 \\
.21 \\
.81\end{array}$ & $\begin{array}{r}4 \\
4 \\
12\end{array}$ & 5.6 & 17 \\
\hline Bone & $\begin{array}{l}8.9 \\
4.9 \\
4.8\end{array}$ & $\begin{array}{r}2.28 \\
2.59 \\
.32\end{array}$ & $\begin{array}{l}.34 \\
.60 \\
.04\end{array}$ & $\begin{array}{l}15 \\
23 \\
12\end{array}$ & 1.7 & 71 \\
\hline
\end{tabular}


20 microliter aliquots of a $1 \mathrm{ppb}$ solution exceed the detection limit for cadmium by this technique $\left(2 \times 10^{-12} \mathrm{~g}\right)$ by a factor of 10 and since the amount of cadmium per aliquot could be further increased by increasing the size of the aliquot to $50 \mu \mathrm{l}$, dilution of the digested sample to a smaller volume or increasing the sample size, instrumental sensitivity is more than adequate for analysis of all organs studied here. For analysis of still lower levels than detected here the use of specially purified reagent acids will result in an acid blank concentration of cadmium reduced by a factor of $\sim 10$.

Comparison of the relative standard deviations in table 4 for each organ with those for aqueous standards at comparable concentrations indicates a loss in precision due to the matrix remaining after acid digestion only for the skin, kidney and bone. The loss in precision for the heart and muscle is attributable to nearness of the sample concentration to the acid blank for the reagent acid used. Examination of the last column in table 4, comparing organs from three fish exposed to the same amount of cadmium, reveals that reproducibility is far worse between fish than could be accounted for by the precision of the method or the accuracy of analysis (from the Bovine Liver data). Thus the variation in cadmium levels is attributed primarily to physiological differences between fish. Similar variations in the cadmium content of human liver from different portions of the same liver have been attributed to the heterogeneous distribution within the organ [13].

In conclusion, flameless atomic absorption with background correction has been shown to have adequate sensitivity and reproducibility for the analysis of eight fish organs to permit detailed studies of uptake and distribution of cadmium in fish exposed to elevated levels in aquatic systems.

\section{References}

[1] Lee, D., Ed., Metallic Contaminants and Human Health, Academic Press, New York (1972).

[2] Friberg, L., Piscator, M., and Norberg, G., Cadmium in the Environment, CRC Press, Cleveland (1971).

[3] Mc Caull, J., Environment 13, 3 (1971).

[4] Parker, C., Rowe, J., and Sandoz, D., American Laboratory 5, 53 (1973).

[5] Hem, J., Water Resources Res. 8, 661 (1972).

[6] Flick, D., Kraybill, H., and Dimitroff, J., Envir. Res. 4, 71 (1971).

[7] Louria, D. B. et al., Annals of Internal Medicine 76, 307 (1972).

[8] Solbe, J. F. de L. G., Water Res. 8, 389-391 (1974).

[9] Eaton, J. G., Water Res. 7, 1723-1736 (1973).

[10] Hwang, J. Y. et al., Anal. Chem. 44, 795 (1972).

[11] Hwang, J. Y. et al., Anal. Chem. 44, 2018 (1972). 
[12] Struempler, A. W., Anal. Chem. 45, 2251 (1973).

[13] Ullucci, P. A. and Hwang, J. Y., Determination of Cadmium in Biological Materials by Flame and Non-Flame Atomic Absorption Techniques. Presented at the 1973 Pittsburgh Conference (March 1973). 


\title{
METHODOLOGICAL CONSIDERATIONS IN WESTERN LAKE SUPERIOR WATER-SEDIMENT EXCHANGE STUDIES OF SOME TRACE ELEMENTS
}

\author{
J. E. Poldoski and G. E. Glass \\ U.S. Environmental Protection Agency \\ National Water Quality Laboratory \\ 6201 Congdon Boulevard \\ Duluth, Minnesota 55804 U.S.A.
}

\begin{abstract}
Trace element measurements on western Lake Superior water were taken as a function of geographical area and distance above and below the watersediment interface. The objective of detecting gradients of certain constituents in the system, however, required an extensive examination of required methodology. Investigations of this methodology indicate that the values obtained from a particular sample type are constrained by a number of uncertainties and operational definitions. Data were taken using different methods and experiments were conducted at various points in the analytical scheme to determine the influence of sample collection, handling and analysis steps. Results were of aid in determining where emphasis should be placed to improve the end result.
\end{abstract}

Keywords: Anodic stripping voltammetry; flameless atomic absorption; interstitial water; Lake Superior chemical analysis; trace element water analysis; water filtration; water sampling apparatus.

\section{Introduction}

During the summer of 1972 , a new program of studies was initiated by the National Water Quality Laboratory to determine effects from iron ore taconite tailings being discharged into western Lake Superior. Laboratory studies [1,2] characterizing the tailings indicated that at least portions could dissolve and release certain elements such as manganese and silica. Providing realistic values for parameters describing the lake conditions and detecting any significant differences between the appropriate tailings and nontailings areas of the lake were goals of the study $[3,4]$. 
Previous works [5-8] on the chemistry of natural aquatic systems have suggested that sediments play a significant role in lake dynamics. Since sediments vary considerably, they can possibly act as reservoirs or sinks for substances in the water column, particularly trace elements. The basic approach applied in this work consisted of obtaining chemical analyses on water samples which corresponded closely to total dissolved concentrations. Chemical compositions were obtained on both water and interstitial water as a function of geographical area and distance above and below the water-sediment interface and this helped to provide some needed information describing the chemical dynamics of the lake's western arm.

The object of this paper is to briefly convey some considerations in the methodology which includes sampling methods, comparisons of sample preparation steps, comparisons of analytical methods, effects of analytical working parameters, blanks, and reproducibility. The results are largely viewed as routine considerations for establishment of appropriate methods for the particular monitoring requirements.

\section{Sampling}

Western Lake Superior has a typical water temperature of about $4{ }^{\circ} \mathrm{C}$ at depths which reach down near 300 meters and trace element concentrations tend to be extremely low. Sampling and field experiments were performed aboard a 98 foot vessel utilizing Van Dorn and Niskin water samplers (1 to 30 liter capacities), an epoxy encapsulated submersible pump (Model 1 P683A Teel') a Shipek dredge, a Phleger gravity corer $(3.8 \mathrm{~cm} \times$ $61 \mathrm{~cm}$ cellulose acetate butyrate liner) and a Benthos type 217 gravity corer $(6.3 \mathrm{~cm} \times 122 \mathrm{~cm}$ cellulose acetate butyrate liner). Mid-depth samples were taken with 30 liter Niskins at 31 and 180 meters above the sediment surface and at 30 meters below the lake surface with the submersible pump and the 30 liter Niskin samplers. The pump also provided water for core storage baths at lake temperature and for washing equipment. Figure 1 conceptually indicates some simple modifications that were made with the commercially available apparatus to facilitate sampling possible concentration gradients in the top 10 centimeters of interstitial water and in the water column from approximately 0.03 to 3 meters above the sediment surface. For bottom water, the arrangement of sampling bottles (c) was simultaneously triggered to the closed position by the action of the foot contacting the sediment surface as the assembly was slowly lowered. The Benthos corer check valve (b) assembly was modified to allow more

IThe U.S. Environmental Protection Agency neither recommends nor endorses any commercial product; trade names are used only for identification. 


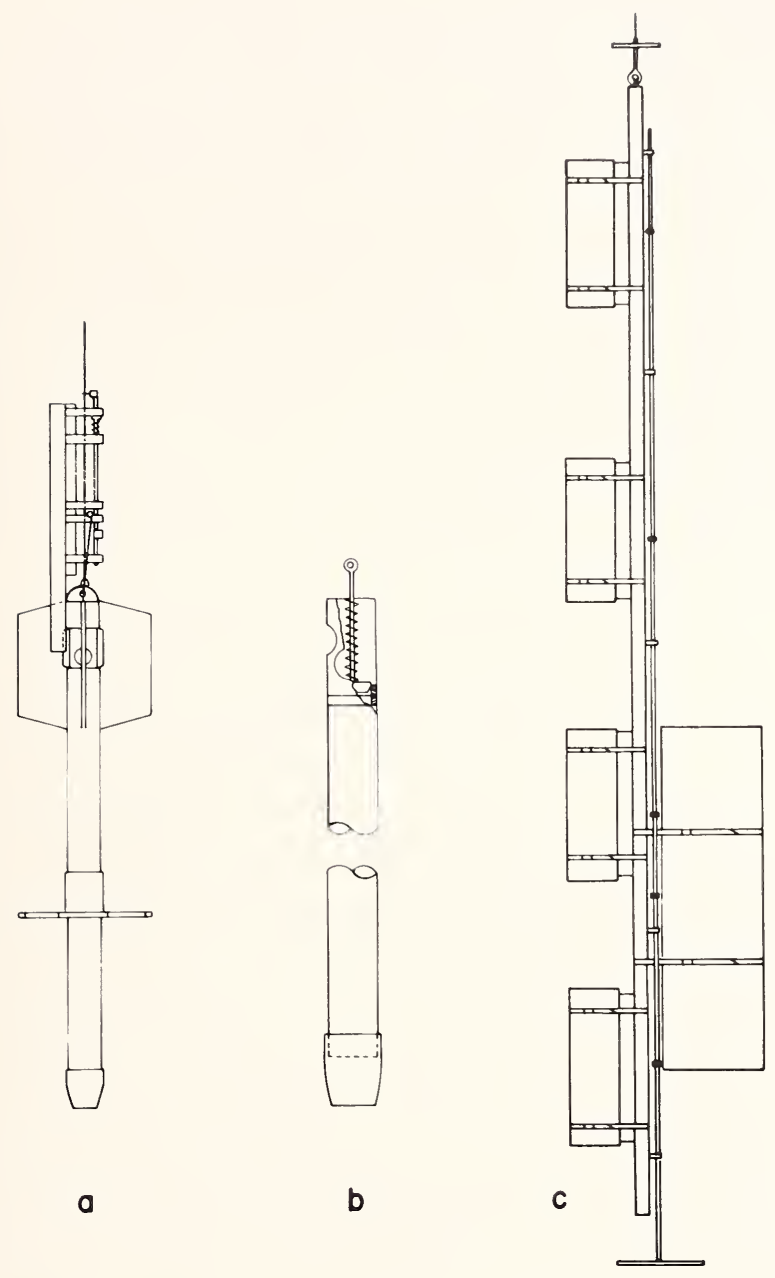

Figure 1. Modified samplers for sediment and bottom water, a) Benthos corer showing attached Niskin tripping mechanism, b) Check valve assembly showing enlarged vent holes. spring, and valve stem, c) Arrangement of Van Dorn and Niskin water samplers with the foot-bottom contact tripping mechanism. 
efficient water flow through the core tube as it was dropped. This was accomplished by boring larger vent holes and compressing the spring-loaded valve completely open with a cord attachment from the valve stem to a tripping mechanism of a Niskin sampler. The mechanism (a) was simply bolted to a length of angle iron mounted on the core body and the valve was closed via messenger immediately after the corer was dropped. The flushing efficiency was checked by filling the corer with a known concentration of dye solution, dropping it from 0.7 meters above the sediment surface and obtaining a core. The dye concentration remaining in the water above the core was measured to be about 10 percent of the original concentration. This experiment did not take into account any velocity differences during the actual core sampling. In further sampling, water above the core sample was defined to be that immediately above the sediment surface.

\section{Sample Handling}

Water samples were routinely collected in polyethylene containers which had been initially washed with 25 percent perchloric acid followed by storage of a $0.01 M$ solution for at least 1 week prior to use. For core samples, one group was sectioned into polyethylene whirlpak ${ }^{2}$ bags (Matheson) on board and generally treated in an aerobic fashion. Another group was kept intact in stoppered core tubes containing overlying water. Precautions were taken to minimize disturbance, temperature changes, and exposure to light and air until after processing under anaerobic conditions. As soon as possible after collection, sample filtration was routinely performed utilizing $0.1 \mu \mathrm{m}$ membrane filters (Millipore). Each sample had its own filter blank and the procedure with observed typical blanks and lake sample analyses are reported in table 1. The deionized (Millipore) distilled water employed for the blank was of high quality and generally had little or no detectable concentrations of the elements under study. Although this blank could not be considered a strict sample correction blank, it was a good approximation and it did provide for individual checks on sample contamination. A new membrane was used for each sample and the apparatus was routinely acid and deionized water rinsed to reduce contamination. To further minimize the contamination potential, all filtrates were collected directly in the decontaminated storage bottles. Both unacidified samples and those acidified to be $0.01 \mathrm{M}$ in perchloric acid (G. F. Smith, double vacuum distilled) were routinely stored at $5{ }^{\circ} \mathrm{C}$ in the absence of light and analyzed as soon as possible. 
TABLE 1. Routine water filtration procedure with typical blank and Lake Superior sample concentrations

Element Blank concentration $(\mu \mathrm{g} / \mathrm{l})$
Lake water range $(\mu \mathrm{g} / \mathrm{l})$
Interstitial water range

$(\mu \mathrm{g} / \mathrm{l})$

$\begin{array}{lc}\mathrm{Mn} & \leq 0.2 \\ \mathrm{Cu} & \leq 0.5 \\ \mathrm{Fe} & <1 \\ \mathrm{Cd} & 0.07 \\ \mathrm{P} & <1 \\ \mathrm{SiO} & <50 \\ \mathrm{Ca} & \leq 200 \\ \mathrm{Mg} & \leq 40 \\ \mathrm{Na} & <0.05 \\ \mathrm{~K} & <0.05\end{array}$

$<0.2-2$

$1.0-1.6$

$<1-2$

$0.01-0.02^{\mathrm{a}}$

2,51

$12,300-13,000$

$2,700-2,800$

$1,000-1,200$

$330-400$
4-8, 100

$1-17$

$17-10,000$

$0.2-2$

$<60-2,100$

$21,000-64,500$

$14,000-66,000$

$4,000-13,000$

$2,700-9,000$

$1,200-2,600$

a Unfiltered samples from laboratory lake water intake.

Notes: lake water filtration procedure $(0.1 \mu \mathrm{m})$ :

1. $100 \mathrm{ml}$ Lake Superior water wash.

2. $100 \mathrm{ml}$ deionized water wash.

3. $50 \mathrm{ml}$ deionized water collected (blank).

4. $50 \mathrm{ml}$ sample collected.

Table 2 shows the combined analytical and sample processing reproducibility for both Lake Superior water containing suspended taconite tailings and interstitial water of sediment core samples which were processed utilizing the aerobic and anaerobic sample processing methods, subsequently discussed. Selected samples were homogenized, split, and taken through the preparation and analytical procedures in a duplicate fashion. For water samples, elevated standard deviations are observed for manganese and iron; however, this is at least partially due to the fact that about half the samples were near the analytical detection limit. In the case of sediments, interstitial water standard deviations of 10 percent or greater are attributed not only to analysis variations but also to variations associated with the sample preparation step. This was observed for manganese, copper, cadmium, phosphorous, and particularly iron. In the case of manganese, the precision appears to be better for the anaerobic method. For the major constituents and copper, overall variations given for sampling, handling, and analysis in reference [4] were generally found to be similar to or only slightly greater than the variations due to just the sample preparation and analysis steps presented here.

To investigate any possible absorption or leaching effects by the filter and apparatus, a selection of lake water samples was (in triplicate) passed through $0.45 \mu \mathrm{m}$ membrane filters. Half the volume for each of the filtrates was again passed through $0.1 \mu \mathrm{m}$ filters. Measured constituent 
TABLE 2. Reproducibility of duplicate sample processing and analysis

\begin{tabular}{|c|c|c|c|c|}
\hline Element & Sample type & $\begin{array}{l}\text { Sample } \\
\text { concentration } \\
\text { range } \\
(\mu \mathrm{g} / \mathrm{l})\end{array}$ & $\begin{array}{l}\text { Relative standard } \\
\text { deviation of } \\
\text { duplicate } \\
\text { samples }\end{array}$ & $\begin{array}{l}\text { Number of } \\
\text { duplicate } \\
\text { samples }\end{array}$ \\
\hline $\mathrm{Mn}$ & $\begin{array}{l}\mathrm{a} \\
\mathrm{b} \\
\mathrm{c}\end{array}$ & $\begin{array}{l}0.3-31 \\
1-3,200 \\
860-3,800\end{array}$ & $\begin{array}{l}30 \\
12 \\
6.6\end{array}$ & $\begin{array}{r}14 \\
6 \\
4\end{array}$ \\
\hline $\mathrm{Cu}$ & $\begin{array}{l}\mathrm{a} \\
\mathrm{b} \\
\mathrm{c}\end{array}$ & $\begin{array}{l}1-3 \\
2-14 \\
1-6\end{array}$ & $\begin{array}{l}12 \\
19 \\
25\end{array}$ & $\begin{array}{l}9 \\
5 \\
4\end{array}$ \\
\hline $\mathrm{Fe}$ & $\begin{array}{l}\mathrm{a} \\
\mathrm{b} \\
\mathrm{c}\end{array}$ & $\frac{1-5}{17-60}$ & $\frac{18}{66}$ & $\frac{9}{4}$ \\
\hline $\mathrm{Cd}$ & $\begin{array}{l}\mathrm{a} \\
\mathrm{b} \\
\mathrm{c}\end{array}$ & $\overline{\overline{-}}$ & $\overline{\bar{z}}$ & $\overline{-}$ \\
\hline$P$ & $\begin{array}{l}\mathrm{a} \\
\mathrm{b} \\
\mathrm{c}\end{array}$ & $\begin{array}{r}8-24 \\
<25-60 \\
-\end{array}$ & $\begin{array}{l}5.8 \\
30 \\
-\end{array}$ & $\begin{array}{r}4 \\
2 \\
-\end{array}$ \\
\hline $\mathrm{SiO}_{2}$ & $\begin{array}{l}\mathrm{a} \\
\mathrm{b} \\
\mathrm{c}\end{array}$ & $\begin{array}{r}3,000-12,000 \\
20,000-53,000 \\
21,000-30,000\end{array}$ & $\begin{array}{l}4.3 \\
3.1 \\
3.2\end{array}$ & $\begin{array}{r}17 \\
6 \\
4\end{array}$ \\
\hline $\mathrm{Ca}$ & $\begin{array}{l}\mathrm{a} \\
\mathrm{b} \\
\mathrm{c}\end{array}$ & $\begin{array}{r}10,000-14,000 \\
3,000-24,000 \\
25,000-34,000\end{array}$ & $\begin{array}{l}1.4 \\
2.4 \\
4.7\end{array}$ & $\begin{array}{r}17 \\
6 \\
4\end{array}$ \\
\hline $\mathrm{Mg}$ & $\begin{array}{l}\mathrm{a} \\
\mathrm{b} \\
\mathrm{c}\end{array}$ & $\begin{array}{r}2,000-3,000 \\
700-6,000 \\
5,000-7,000\end{array}$ & $\begin{array}{l}2.5 \\
2.8 \\
5.9\end{array}$ & $\begin{array}{r}17 \\
6 \\
4\end{array}$ \\
\hline $\mathrm{Na}$ & $\begin{array}{l}\mathrm{a} \\
\mathrm{b} \\
\mathrm{c}\end{array}$ & $\begin{array}{c}1,000-8,000 \\
1,000-3,000 \\
-\end{array}$ & $\begin{array}{l}4.6 \\
7.9 \\
-\end{array}$ & $\begin{array}{r}17 \\
6 \\
-\end{array}$ \\
\hline K & $\begin{array}{l}\mathrm{a} \\
\mathrm{b} \\
\mathrm{c}\end{array}$ & $\begin{array}{l}400-15,000 \\
1-2 \\
-\end{array}$ & $\begin{array}{l}3.4 \\
16 \\
-\end{array}$ & $\begin{array}{r}17 \\
6 \\
-\end{array}$ \\
\hline
\end{tabular}

$\mathrm{a}=$ Lake Superior water.

$\mathrm{b}=$ Sediment interstitial water, aerobic sample processing, method A.

c $=$ Sediment interstitial water, anaerobic sample processing, method C. 
concentrations of the single filtration were divided by those of the double filtration and the calculated mean ratios for copper, manganese, iron, silica, calcium, magnesium, sodium, and potassium were $0.90,1.05,0.69$, $1.02,1.03,1.03,1.04$, and 1.02 , respectively. This did not indicate significant uptake or release of constituents during filtration steps, except for iron. In addition, filtrate concentrations were measured as a function of volume filtered and little or no dependence was observed.

The selection of a filter membrane pore size was a particularly significant consideration since the lake contained high concentrations of particulates in certain locations [9] and the data of most relevance was that corresponding closely to the dissolved fraction. Figure 2 depicts results for at least duplicate experiments where membrane filter pore sizes of $0.45,0.1,0.05$, and $0.01 \mu \mathrm{m}$ were employed to filter a Lake Superior water sample containing particulates due to taconite tailings. It is seen that manganese concentrations significantly decrease with decreasing pore size down to $0.1 \mu \mathrm{m}$ which then shows no further dependence. Copper concentrations remain relatively constant throughout the entire range; however, iron shows a continual decrease possibly due to the characteristic colloidal forms of iron normally present in natural waters.

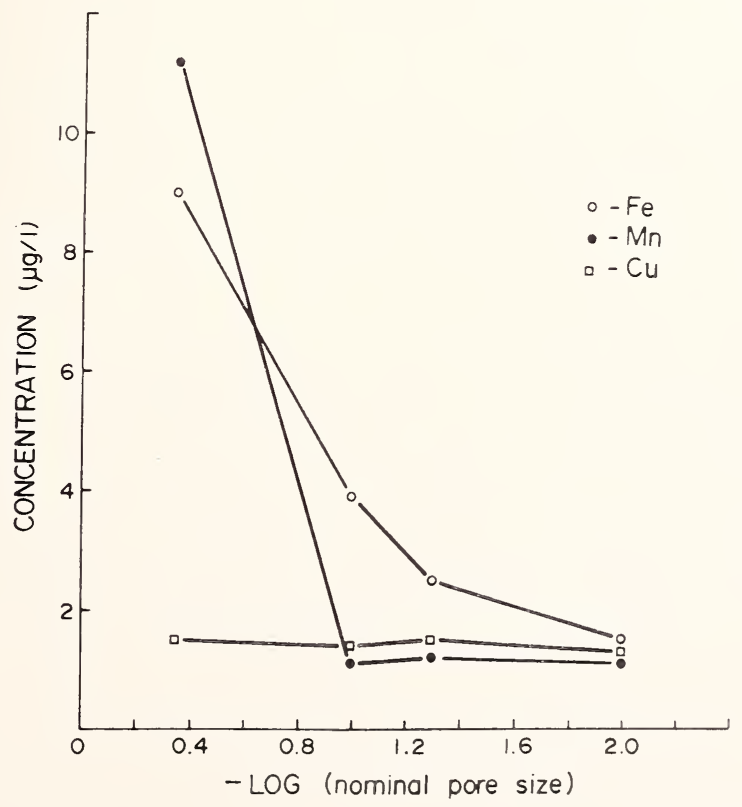

Figure 2. Effect of filter pore size on constituent concentrations of Lake Superior water containing particulates due to taconite tailings. 
Silicon dioxide, calcium, magnesium, sodium, and potassium concentrations also did not show any measurable pore size dependence within this range. These data and similar filtration experiments on additional samples indicated that except for iron, $0.1 \mu \mathrm{m}$ membrane filtration was a better choice for these samples than the commonly employed $0.45 \mu \mathrm{m} \mathrm{mem-}$ brane filtration.

Different handling and sample preparation steps for removing water from sediments can give rise to large analytical differences. Introduction of oxygen into these reduced systems can obviously oxidize elements, and thus affect the chemistry. Such processes as absorption, complexation, precipitation or coprecipitation reactions of course can play major roles in affecting the fraction of an element in the dissolved state [10-13]. It has also been suggested that feldspar particulates can be a factor in oxidation processes [14]. In addition to these considerations, effects from changes in hydrostatic pressure, light, biomass, and temperature during the sampling and handling processes may be additional variables. It is emphasized at this point that the terms "oxidized" and "reduced" used here are meant only in a relative sense for differentiation purposes.

With these thoughts in mind, essentially two classes of interstitial water sample handling were employed-aerobic - and anaerobic methods. The results from each method were used to establish a range of concentrations corresponding to dissolved components in oxidized versus reduced sediment types. Several methods have been employed for sediment-water exchange studies [15-19]. In this work, three specific routes were employed for separating water from the sediments (34 to $85 \%$ moisture content) and they involved the following methods: A) aerobic-refrigerated centrifugation of approximately 25 grams of homogenized sediment sample in filtering centrifuge tubes (Millipore) resulting in about $10 \mathrm{ml}$ of sample; B) anaerobic-mixing approximately 70 grams of sediment with about $50 \mathrm{ml}$ of nitrogen purged deionized distilled water (2- to 3-fold dilution) and immediately filtering the homogenized mixture under a watersaturated blanket of nitrogen utilizing an aseptic membrane filtration apparatus (Millipore) producing about $30 \mathrm{ml}$ of sample; C) anaerobicutilizing the simple interstitial water preparation cell, illustrated in figure 3 , which was constructed from commonly available PVC pipe fittings and epoxy glue. The design provided for use of an inert atmosphere during the steps of centrifugation and subsequent filtration of an undiluted sediment sample. Filtration subsequent to the centrifugation step avoided plugging of the filter, especially by glacial clays. Polyethylene glove bags, filled with water-saturated prepurified nitrogen, were employed to maintain an inert atmosphere during sample handling. After adding about 50 grams of sediment to the cell, it was pressurized through the check valve with 


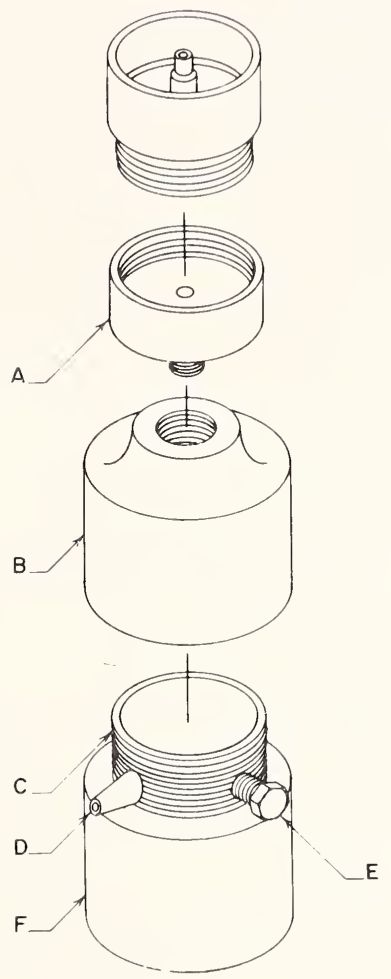

Figure 3. Interstitial water preparation cell A) plastic in-line $47 \mathrm{~mm}$ diameter Millipore filter holder employed with 0.1 micron filter, B) $2 \times 1 / 2$ in reducer coupling (not shown is $1 / 2 \times$ $1 / 4$ in reducer bushing between $A$ and $B$ ). C) polypropylene pressurizing check valve (Belart), D) $1 / 4$ in plug (pressure release valve), E) 2 in end cap (permanently attached to $\mathrm{C}$ with epoxy glue).

water saturated nitrogen and centrifuged at $2400 \mathrm{rpm}$ for 1 hour. The resultant supernatant $(\sim 20 \mathrm{ml})$ was filtered by carefully inverting the cell with the attached filter and applying additional nitrogen pressure.

For several elements, the results of about 40 direct comparisons of method A with method B on split homogenous samples are given in table 3. One half of a split sample was treated anaerobically and the other half aerobically. Contrast between methods is quite obvious, particularly in the cases of manganese, iron, and phosphate where method A gives mean values which are more than an order of magnitude below those of method B. Except for copper, all other parameters tend to show higher values for method B. This may be due in part to the dissolution of sediment when water was added in the preparation step for method B. However, measured concentrations for several samples processed by method $\mathrm{C}$ agreed 
TABLE 3. Comparison of anaerobic and aerobic methods for interstitial water preparation.

Element $\begin{array}{lll}\text { Mean } & \begin{array}{l}\text { Standard } \\ \text { deviation } \\ \text { of ratio }\end{array} & \begin{array}{c}\text { Number of } \\ \text { samples }\end{array}\end{array}$

$\begin{array}{lccr}\mathrm{Mn} & 51 & 117 & 43 \\ \mathrm{Cu} & 1.1 & 1.0 & 37 \\ \mathrm{Fe} & 453 & 467 & 7 \\ \mathrm{P} & 25 & 30 & 37 \\ \mathrm{SiO}_{2} & 1.7 & 0.5 & 43 \\ \mathrm{Ca} & 2.1 & 1.2 & 43 \\ \mathrm{Mg} & 2.4 & 1.4 & 43 \\ \mathrm{Na} & 1.4 & 0.4 & 43 \\ \mathrm{~K} & 1.2 & 0.4 & 38\end{array}$

${ }^{\mathrm{a}}$ Ratio $=$ method $\mathrm{B}$ concentration/method $\mathrm{A}$ concentration.

with those obtained by method B on similar sediment samples which suggested that increased dissolutions was not a major reason for the observed differences. In the case of copper, there seems to be a 1:1 correlation between methods $\mathrm{A}$ and $\mathrm{B}$, however, the variability is quite high. Calcium, magnesium, and silica show consistently lower values by method A. It is interesting to note that a remarkably consistent 1:1 linear correlation between calcium and magnesium is obtained if the ratio of the concentration by method A divided by that of method B for calcium is plotted versus the same ratio for magnesium. This may be indicative of related mixed phase reactions occurring for calcium and magnesium.

These considerations indicate that the amount of dissolved element in sediments can change markedly depending on the oxidizing conditions during sample handling. It is felt that anaerobic sample handling is the procedure that best approximates the actual interstitial water conditions existing in Lake Superior. In addition, these thoughts likely have implications with regard to proper sampling procedures for anoxic bottom waters in stratified lakes and harbors.

\section{Analytical Considerations}

Flameless atomic absorption was employed for the analysis of manganese, copper, iron, and cadmium utilizing either Perkin Elmer Models 403 or 305 B spectrophotometers coupled to the HGA-2000 graphite furnace and a model 56 recorder. Nitrogen purge gas was employed in the interrupt mode. The flameless technique was particularly advantageous 
because of its capability to handle the low concentrations and limited sample volumes encountered, but, still avoiding potentially contaminating steps associated with other methods. Standard flame atomic absorption methods were employed for calcium, magnesium, sodium and potassium using the model 403 and spectral settings were those suggested by Perkin Elmer [20,21]. The heteropoly blue method [22a] for reactive silicon dioxide and the ascorbic acid method for reactive orthophosphate [22b] were routinely employed colorimetric methods using a Cary model 14 uvvisible spectrophotometer. As alternative analytical methods, flameless atomic absorption for silica, anodic stripping voltammetry with a mercury film electrode $[23,24]$ for copper and the standard colorimetric procedures for iron, as iron (II) orthophenanthroline [22c], and manganese, by persulfate oxidation to the permanganate [22d], were employed. Considering all the procedures, estimates of standard curve reproducibility ranged about \pm 2 to 10 percent relative, depending on the method.

Flameless atomic absorption tended to show drift in response of standards due to gradual deterioration of the graphite tube. Injection volumes from 10 to $100 \mu$ l were routinely employed; however, only one size was generally used for the analysis of some samples because of a nonlinear volume-response relationship, especially for cadmium. Figure 4 illustrates typical instrumental responses for the flameless technique.

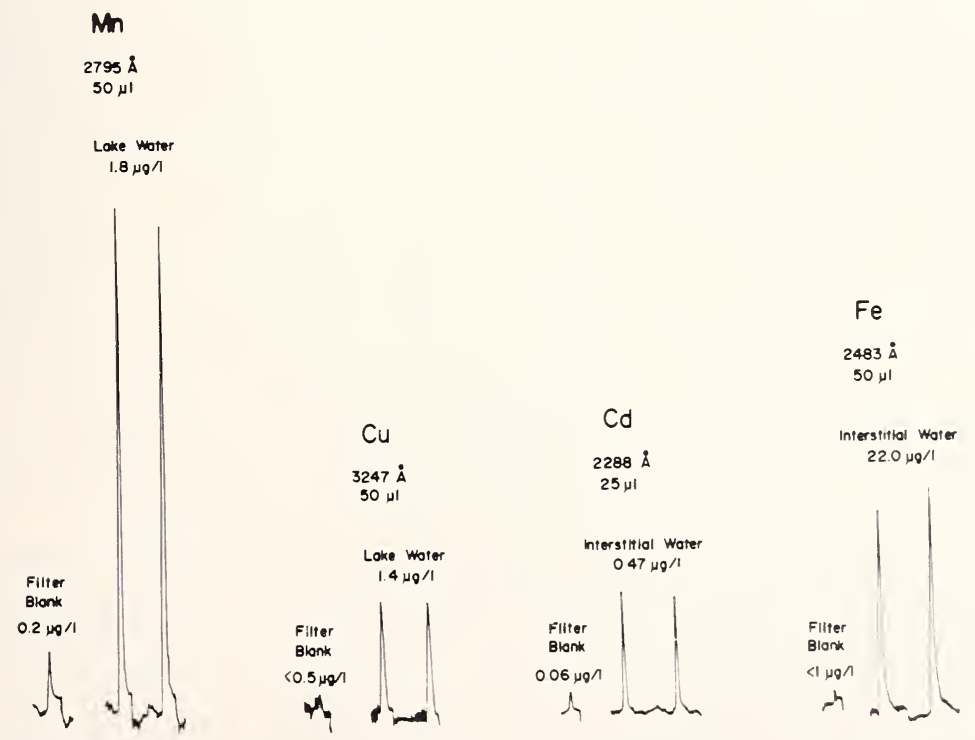

Figure 4. Flameless atomic absorption analyses of Lake Superior samples. 
Working standards were regularly prepared by appropriately diluting $1000 \mathrm{mg} / 1$ stock solutions with deionized distilled water which was routinely checked for impurities. Storage was in either decontaminated polyethylene or FEP Teflon bottles and heavy metal solutions were usually made $0.01 \mathrm{M}$ in perchloric acid, which was also checked for impurities. Stock solutions were prepared from reagent grade sources by direct weighing or a standardization procedure.

In addition to using deionized water standard curves, the method of standard addition was also routinely applied to selected samples and a standard sample of Lake Superior water. The slope of the standard addition curve divided by the slope of the deionized water standard curve was defined as the recovery and this was generally within 10 percent of 1.00 for all the elements except for cadmium which tended to be low for interstitial water $(0.78)$.

To determine the extent of background absorption for flameless AA analyses, several interstitial water samples were analyzed with and without deuterium arc background correction. With about 18 samples analyzed for manganese, copper, and iron, the average quotients of the nonbackground corrected values divided by the corresponding background corrected values were $1.03,1.12$, and 1.15 , respectively. For cadmium, reproducible results could be obtained only with background correction. Data indicated that an abnormally large background absorption was not present; however, the background correction was generally beneficial.

Effects of graphite furnace temperature settings on analytical results for interstitial water samples are illustrated in figures 5 and 6 . The ratio of the average peak height for the sample divided by that of the deionized water standard is plotted versus temperature setting. For charring, the peak heights and peak ratios remained essentially constant for copper, manganese, and iron in a temperature and time range where the metals are normally nonvolatile. Cadmium shows considerable variation within the studied temperature range, which can neither be attributed to a volatilization loss at or below $800^{\circ} \mathrm{C}$ nor to nonspecific background absorption. Increase of atomization temperature shows pronounced changes in the plotted ratios for copper and cadmium. The peak at $2000{ }^{\circ} \mathrm{C}$ for copper is likely due to background absorption. The uses of higher atomization temperatures $\left(2600{ }^{\circ} \mathrm{C}\right)$ and $0.01 \mathrm{M}$ perchloric acid in the samples tended to minimize matrix effects and produce well-formed and reproducible atomization peaks. For the case of cadmium, the ratios changed from about 1.2 at $1500{ }^{\circ} \mathrm{C}$ to 1.8 at $2000{ }^{\circ} \mathrm{C}$. At $1500{ }^{\circ} \mathrm{C}$, atomization peaks were irreproducible and irregularly shaped. The presence of the $0.01 \mathrm{M}$ perchloric acid in the samples was observed to improve reproducibility 


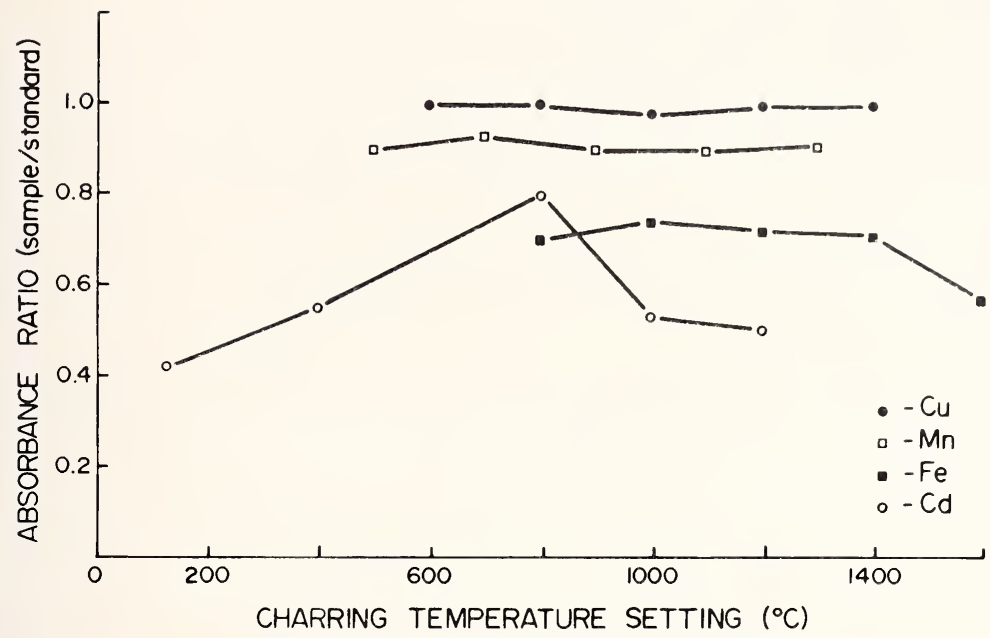

Figure 5. Effect of charring temperature on the ratio of sample to standard response (average of duplicate experiments on interstitial water).

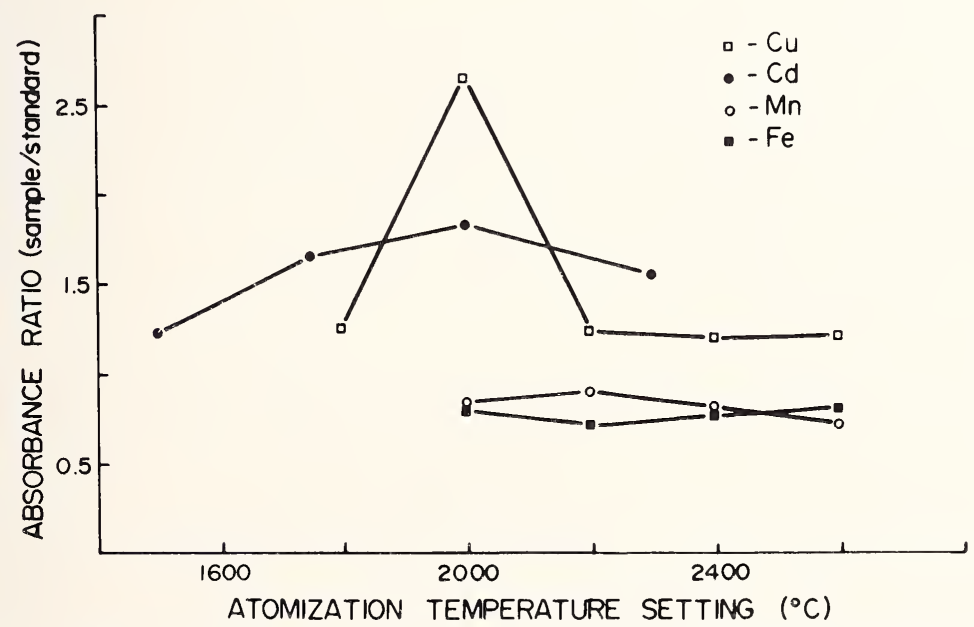

Figure 6. Effect of atomization temperature on the ratio of sample to standard response (average of duplicate experiments on interstitial water).

and decrease the volatility of cadmium such that $800{ }^{\circ} \mathrm{C}$ charring temperatures could be used without significant volatilization loss. The absorbance ratio for manganese and iron show essentially no changes throughout the studied temperature range. It is apparent from this data on both charring and atomization that significant variation can particularly occur for cadmium and this further suggests that the method of additions should be em- 
ployed for its analysis in addition to the use of alternate analytical methods for checking analytical accuracy and calibration. Based on optimum reproducibility, sensitivity and the rate of atomization the following temperature ranges were chosen for the samples: Atomization-copper, iron, and manganese 2400 to $2600{ }^{\circ} \mathrm{C}$, cadmium $2000{ }^{\circ} \mathrm{C}$, silica - maximum temperature; Charring-(30 seconds maximum) copper, manganese, and iron 900 to $1300{ }^{\circ} \mathrm{C}$, cadmium $400{ }^{\circ} \mathrm{C}, 800{ }^{\circ} \mathrm{C}$, silica $1500{ }^{\circ} \mathrm{C}$.

The accuracy of analytical results was investigated by analyzing interstitial water samples for copper, manganese, iron, and silica employing flameless atomic absorption and alternate analytical methods. Results summarized in table 4 show reasonable agreement between the methods for manganese, silica, and iron. In the case of copper, the anodic stripping voltametric determinations are strikingly lower than the flameless atomic absorption values by a factor of two or three. Copper has been studied in natural waters and reported to contain essentially nonelectroactive copper complexes which can be decomposed by a persulfate oxidation [25]. Anodic stripping analyses of oxidized and unoxidized samples were correlated with the total and "nonlabile" copper concentrations, respectively. The anodic stripping analyses performed in this work were run at pH 2 with no prior oxidations. The observed differences in analytical results are probably due to the presence of highly stable and irreversible copper complexes which associate and dissociate slowly and/or copper associated with nonfilterable colloidal forms. It is therefore felt that the flameless atomic absorption values more truly represent the total copper content of the samples.

TABLE 4. Comparison of flameless atomic absorption to alternate analytical methods for the analysis of Lake Superior samples.

\begin{tabular}{lclrrr}
\hline Element & $\begin{array}{l}\text { Concentration } \\
\text { range } \mu \mathrm{g} / \mathrm{l}\end{array}$ & $\begin{array}{l}\text { Analytical } \\
\text { methods }\end{array}$ & $\begin{array}{c}\text { Mean } \\
\text { ratiof }\end{array}$ & $\begin{array}{c}\text { Standard } \\
\text { deviation } \\
\text { of ratio }\end{array}$ & $\begin{array}{c}\text { Number } \\
\text { of } \\
\text { samples }\end{array}$ \\
\hline $\mathrm{Mn}$ & $1,600-10,000$ & a,b & 0.96 & 0.18 & 16 \\
$\mathrm{Cu}$ & $0.5-13$ & a,c & .43 & .11 & 9 \\
$\mathrm{Fe}$ & $2,400-9,800$ & a,d & 1.20 & .10 & 6 \\
$\mathrm{SiO}_{2}$ & $20,000-44,000$ & a,e & 0.89 & .06 & 8 \\
\hline
\end{tabular}

a Flameless atomic absorption.

b Colorimetric manganese.

- Anodic stripping voltammetry.

d Colorimetric iron.

- Colorimetric silica.

${ }^{\mathrm{f}}$ Ratio = alternate method concentration/flameless atomic absorption concentration. 


\section{Acknowledgement}

The conscientious work of Mr. Richard Sinn and Ms. Barbara Halligan is gratefully acknowledged.

\section{References}

[1] Effects of Taconite on Lake Superior 1970, Proceedings of the Conference in the Matter of Pollution of Lake Superior and Its Tributary Basin-Minnesota-WisconsinMichigan, U.S.D.I., F.W.P.C.A., 2nd session Vol. 1, Document 0-401-869, pp. 309324, U.S. Government Printing Office, Washington, D.C. (April 1970).

[2] Studies Regarding the Effect of the Reserve Mining Company Discharge on Lake Superior, U.S. EPA, Washington, D.C., May 2, 1973, May 18, (Supplement 1 and 2) 1973.

[3] Glass, G. E., A Study of Western Lake Superior: Surface Sediments, Interstitial Water, and Exchange of Dissolved Components Across the Water-Sediment Interface, in Studies Regarding the Effect of the Reserve Mining Company Discharge in Lake Superior, pp. 1028-1121, U.S. EPA, Office of Enforcement and General Counsel, Washington, D.C. (1973).

[4] Glass, G. E. and Poldoski, J. E., Proceedings XIX Congress International Association of Limnology (SIL) Winnepeg, Canada (August 1974).

[5] Brooks, R. R., Presley, B. J., and Kaplan, I. R., Trace Elements in the Interstitial Waters of Marine Sediments, Geochim. Cosmochim. Acta. 29.259-313 (1965).

[6] Duchart, P., Calvert, S. E., and Price, N. B., Distribution of Trace Metals in the Pore Waters of Shallow Water Marine Sediments, Limnol. \& Oceanog. 18, 605-10 (1973).

[7] Lerman, A. and Brunskill, G. J., Migration of Major Constituents from Lake Sediments into Lake Water and Its Bearing on Lake Water Composition, Limnol. \& Oceanog. 16, 880-90 (1971).

[8] Mortimer, C. H., Chemical Exchanges Between Sediments and Water in the Great Lakes, Speculations on Probable Regulatory Mechanisms, Limnol. \& Oceanog. 16, 387-404 (1971).

[9] Baumgartner, D. J., Water Clarity in Relation to Fine Particulate Matter in Lake Superior, 1972, U.S. EPA Pacific Northwest Environmental Research Laboratory, Corvallis, Oregon.

[10] Stumm, J. and Morgan, J. J., Aquatic Chemistry, Wiley-Interscience, N.Y. (1970).

[11] Troup, B. M., Bricker, O. P., and Bray, J. T., Oxidation Effect on Analysis of Iron in Interstitial Water of Recent Anoxic Sediments, Nature 249. 237-39 (1974).

[12] Singer, P. C., Complexation of Iron (II) by Organic Matter and Its Effect on Iron (II) Oxygenation, Environ. Sci.\& Technol. 8, 569-73 (1974).

[13] Gardiner, J., Chemistry of Cadmium In Natural Water II. The Adsorption of Cadmium on River Muds and Naturally Occurring Solids, Water Res. 8, 157-64 (1974).

[14] Hem, J. D., Increased Oxidation Rate of Manganese Ions in Contact with Feldspar Grains, U.S. Geol. Survey Prof. Paper, No. 475-C, pp. 216-217, Denver, Colorado (1963).

[15] Reeburgh, W. S., An Improved Interstitial Water Sampler, Limnol. \& Oceanog. 12. (1967). 
[16] Bischoff, J. L., Greer, R. L., and Luistro, A. O., Composition of Interstitial Waters of Marine Sediments: Temperature of Squeezing Effect, Science 167, 1245-46 (1970).

[17] Keeley, J. and Engler, R., Discussion of Regulatory Criteria for Ocean Disposal of Dredged Material: Elutriate Test Rationale and Implementation Guidelines, Miscellaneous Paper D-74-12 (1974), U.S. Army Engineer Waterways Experiment Station, Vicksburg, Mississippi.

[18] Toth, S. J. and Ott, A. N., Characterization of Bottom Sediments: Cation Exchange Capacity and Exchangeable Cation Status, Environ Sci. \& Technol. 4, 935-39 (1970).

[19] Manheim, F. T., A Hydraulic Squeezer for Obtaining Interstitial Water from Consolidated and Unconsolidated Sediment, U.S. Geol. Survey Prof. Paper 550-C, pp. C256-C261 (1966).

[20] Analytical Methods for Atomic Absorption Spectrophotometry, 1971, Perkin Elmer Corp., Norwalk, Connecticut 06856 (1971).

[21] Slavin, W., Atomic Absorption Spectroscopy, Wiley-Interscience, N.Y. (1968).

[22] Standard Methods for the Examination of Water and Wastewater, 13 ed., (a) pp. 306-08, (b) pp. 532-34, (c) pp. 433-36, and (d) pp. 439-42, Am. Public Health Assoc., Washington, D.C. (1971).

[23] Poldoski, J. E. and Glass, G. E., Unpublished Work, National Water Quality Laboratory, Duluth, Minnesota 55804.

[24] Matson, W. R., Roe, D. K., and Carritt, D. E., Composite Graphite-Mercury Electrode for Anodic Stripping Voltametry, Anal. Chem. 37, 1594-5 (1965).

[25] Chau, Y. K. and Lum-Shue-Chan, K., Determination of Labile and Strongly Bound Metals In Lake Water, Water Res. 8, 383-88 (1974). 


\title{
ACCURACY IN DETERMINING TRACE ELEMENT CONCENTRATIONS IN MARINE SEDIMENTS
}

\author{
E. D. Wood* and Nelson Acosta Cintron \\ Puerto Rico Nuclear Center \\ College Station \\ Mayaguez, Puerto Rico 00708 U.S.A.
}

\begin{abstract}
When sampling the environment, one desires not only an accurate analysis of a particular sample, but also an analysis which is representative of the region sampled and one that can be repeated within reasonable limits. Replicate sediment samples were collected in the course of mapping a portion of the sea floor on the west coast of Puerto Rico. The location is in the discharge region of the Guanajibo River. Ten replicate grab samples were taken by independent positioning of the ship. The samples were stripped with hydrochloric acid and the resulting solutions analyzed by atomic absorption spectrometry (AAS) for $\mathrm{Ca}, \mathrm{Cd}, \mathrm{Co}, \mathrm{Cr}, \mathrm{Cu}, \mathrm{Fe}, \mathrm{Mg}, \mathrm{Ni}, \mathrm{Pb}, \mathrm{Sr}$ and $\mathrm{Zn}$. The standard deviations of replicate analysis of individual samples were generally less than for the individual samples themselves. Chemical standards prepared in our laboratory were used to determine the value of the samples. Particle size and homogeneity are factors considered with respect to the number of replicates required and sample size. The average concentrations for several elements in a set of 10 samples with confidence limits for 95 percent level as determined by AAS are as follows: $\mathrm{Cd}, 0.840 \pm 0.011 ; \mathrm{Co}, 56.5 \pm 0.2 ; \mathrm{Cr}, 696 \pm 8 ; \mathrm{Cu}, 30.7 \pm 0.4 ; \mathrm{Fe}$, $60000 \pm 1010 ; \mathrm{Mn}, 827 \pm 5 ; \mathrm{Ni}, 487 \pm 5 ; \mathrm{Pb}, 17.2 \pm 0.2 ;$ and $\mathrm{Zn}, 70.3 \pm 0.8$ $\mu \mathrm{g} / \mathrm{g}$ dry sediment. A composite sample from this location was prepared for use as a standard in future sediment analysis.
\end{abstract}

Keywords: Accuracy; Atomic Absorption Spectrometry; Caribbean; precision; Puerto Rico; replicates; sediments; standard; trace elements.

\section{Introduction}

Over the past few years, attention has been focused on those activities of man which tend to worsen the quality of the aquatic environment. We

*Present address: Bureau of Land Management, U.S. Department of Interior, New Orleans, La. 70113. 
have discharged our wastes into the nearest body of water without concern. This practice continued at an increasing rate until it became quite apparent that major efforts would be necessary to avert mankind's selfdestruction. Knowledge of the present state of a system is a major step toward determining its ability to support life, its immediate threat to man, and what steps are needed to rectify an adverse situation. Obviously polluted regions need to be sampled to establish reclamation programs and base lines for regions where new contamination sources are anticipated.

One could, of course, rush out and collect a large number and variety of samples and analyze for everything under the sun but this would be wasteful, expensive and time consuming. Rather, initially it should be decided what knowledge of the system is needed and to what accuracy because effort tends to increase exponentially with accuracy.

Trace elements leached during the weathering process, in addition to those added by industrial and domestic sources, are carried via streams and sewers to lakes and bays and usually on to the ocean. However, there may be "traps" along the way caused by changes in the physicochemical parameters. Estuaries are prime locations for trace elements to accumulate due to multiple inputs and high gradients of $\mathrm{pH}, \mathrm{E}_{H}$, ionic conductivity, currents and suspended particle loads. Trace elements are often incorporated into the sediments under such conditions.

While investigating the behavior of trace elements during transport, deposition and biological uptake, it was decided that an initial survey of the river-ocean system was necessary to determine the distribution and level of trace elements in sediments. This study was expanded to include three river discharge regions on the west coast of Puerto Rico (fig. 1).

Variations in sample processing, laboratory standards and conditions of analysis soon indicated a need for a natural sediment standard. One station in the Guanajibo River (GUB-443) discharge region was chosen as a source of a standard sediment (fig. 1). Sample locations are determined by using the first number, 4 , as east-west coordinate in figure 1 and the second number, 43, as the north-south coordinate. This sediment is receiving extensive analysis and a portion of it will be included in future analyses of other sediment samples to serve as a check on the analytical method. The results of replicate analyses of sediment from Guanajibo Bay station GUB-443 are discussed below. 


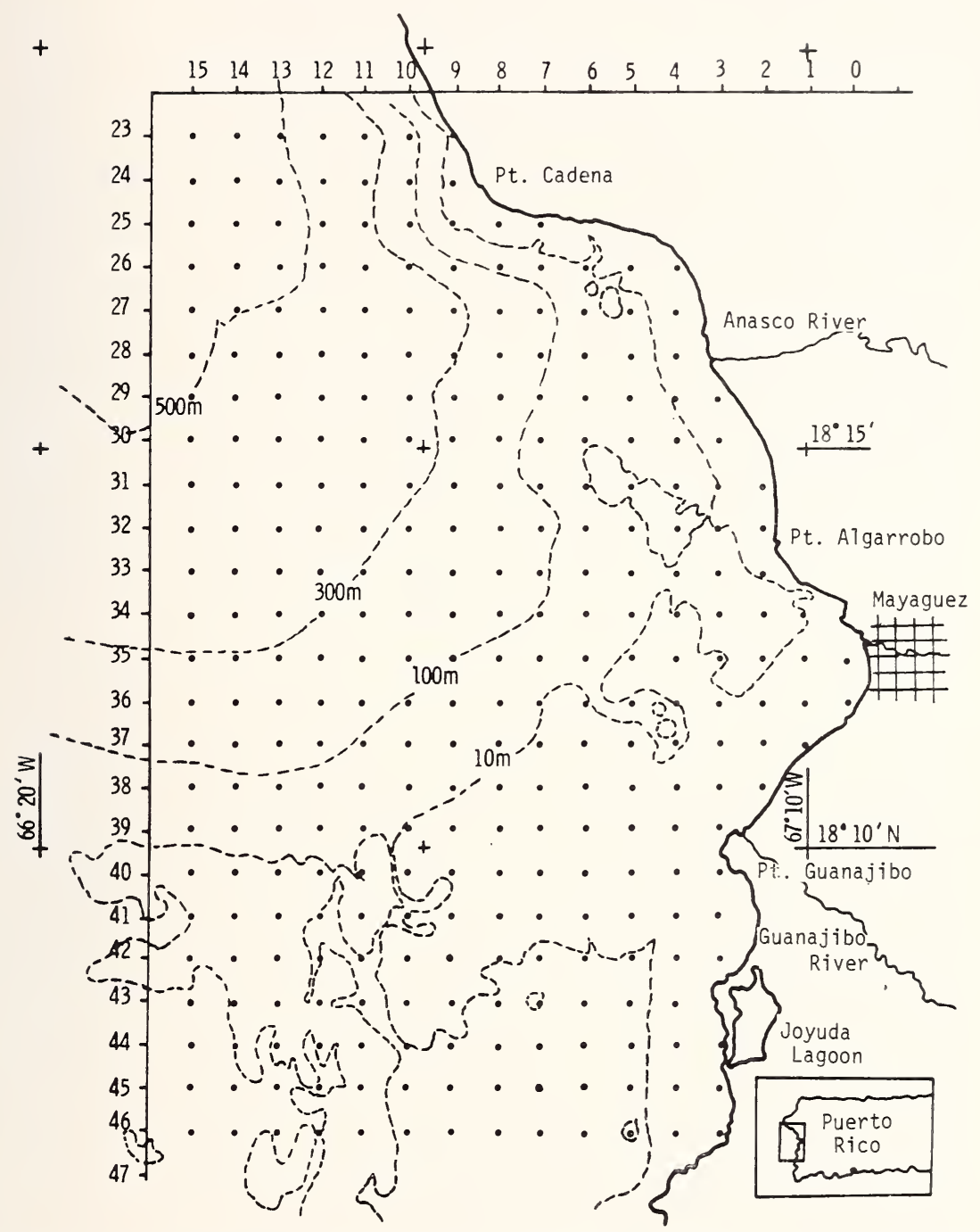

Figure 1. Study region for trace elements in sediments on the west coast of Puerto Rico. Sample numbers are determined from the horizontal numbers followed by the vertical reference numbers. 


\section{Methods and Materials}

\section{A. SAmpling}

The nearshore waters of western Puerto Rico were sampled from the RV R.F. Palumbo using a Shipek grab. The grab has a spring loaded bucket which is triggered by the impact with the sea bed. The bucket then turns through $180^{\circ}$ enclosing the nearly undisturbed sample for its trip to the deck of the ship. Typically, the bucket is removed from the grab and taken to a wet laboratory where the inner portion of the sample is removed with a plastic scoop and placed in an acid cleaned plastic sediment container. The samples are then frozen until ready for analysis. Sample locations were determined by using a combination of radar, channel marker buoys, land marks and depth soundings. A total of 10 samples was taken at station GUB-443, each by independent positioning of the ship. Each sample contained an excess of $500 \mathrm{~g}$ of sediment. The error on positioning was approximately a radius of $50 \mathrm{~m}$.

\section{B. Sample Preparation}

This general survey of trace elements in sediments required only a simple reproducible method. Prior to analysis the samples were thawed and mixed. They were then subjected to hydrochloric acid which dissolved most trace elements not occluded in silicious material. Sufficient wet sediment to give $\geqslant 50 \mathrm{~g}$ of dry sediment was placed in a $250 \mathrm{ml}$ beaker, covered with a ribbed watch glass, and dried to a constant weight at 120 ${ }^{\circ} \mathrm{C}$ in a drying oven. After drying and weighing, the samples were treated with $175 \mathrm{ml}$ of $6 \mathrm{~N} \mathrm{HCl}$ and stirred periodically. (Some sediments contain a high percentage of carbonates and therefore require more acid added slowly in a larger beaker.) About 24 hours were allowed for the initial stripping. The supernate was then decanted and a second addition of 125 $\mathrm{ml}$ of $6 \mathrm{~N} \mathrm{HCl}$ was added to ensure complete removal of all readily accessible trace elements. The remainder of the sediment was then treated with several $50 \mathrm{ml}$ distilled water rinses, and the latter were added to the acid strippings. The final volume of strippings was measured, well mixed and a $50 \mathrm{ml}$ aliquot removed for analysis. This portion was centrifuged at $3000 \pm 200 \mathrm{rpm}$ for 5 minutes to separate suspended particulate matter.

The concentration of many of the elements determined was too high to analyze directly by atomic absorption. Therefore, dilutions were made in the ratios: $1: 50,1: 100,1: 250,1: 500,1: 2500,1: 5000$, and $1: 10,000$. The original solutions and their diluents were placed in dry acid cleaned 
linear polyethylene bottles for analysis using a Perkin-Elmer Model 303 atomic absorption spectrophotometer.

Standard solutions of each element analyzed were run before and after each set of analyses. Standards were also run between every 10 samples when a large number was analyzed. The data were recorded on chart paper as percent absorption for each sample.

\section{Calculations}

Percent absorption was converted to absorbance using a conversion table. The standards were recorded on linear graph paper as absorbance against concentration $(\mathrm{ppm})$. Final concentrations were reported as micrograms of element per gram of dry sediment after the proper dilution, blank and conversion factors were accounted for. The elements determined and the corresponding AAS settings are given in table 1.

TABLE 1. Atomic absorption spectrometry settings for the elements analyzed

\begin{tabular}{lcccccc}
\hline Element & $\begin{array}{c}\text { Wave } \\
\text { length }\end{array}$ & $\begin{array}{c}\text { Slit } \\
\text { width }\end{array}$ & $\begin{array}{c}\text { Source } \\
\text { ma }\end{array}$ & Absorptivity & Scale & $\begin{array}{c}\text { Noise } \\
\text { suppression }\end{array}$ \\
\hline $\mathrm{Ca}$ & 211 & 4 & 15 & 0.047 & 1 & 1 \\
$\mathrm{Cd}$ & 229 & 4 & 8 & .10 & 1 & 1 \\
$\mathrm{Co}$ & 241 & 3 & 30 & .073 & 1 & 2 \\
$\mathrm{Cr}$ & 358 & 4 & 30 & .012 & 1 & 1 \\
$\mathrm{Cu}$ & 325 & 3 & 30 & .038 & 1 & 1 \\
$\mathrm{Fe}$ & 248 & 3 & 30 & .019 & 1 & 2 \\
$\mathrm{Mg}$ & 285 & 4 & 15 & .52 & 1 & 1 \\
$\mathrm{Mn}$ & 279 & 3 & 30 & .056 & 1 & 1 \\
$\mathrm{Ni}$ & 232 & 3 & 30 & .024 & 2 & 2 \\
$\mathrm{~Pb}$ & 283 & 4 & 8 & .008 & 2 & 2 \\
$\mathrm{Sr}$ & 230 & 3 & 20 & .026 & 1 & 2 \\
$\mathrm{Zn}$ & 214 & 4 & 15 & .17 & 1 & \\
\hline
\end{tabular}

\section{Sediment Sizes}

A sample of the sediment was dried and sieved. Almost 51 percent of the sample was collected on the +40 screen $(62.5 \mu \mathrm{m})$. Sixteen percent was collected on the +30 screen and 32 percent was finer than +40 . A few shell fragments were collected on the -10 screen but accounted for less than 0.1 percent of the total. 


\section{Results}

The corresponding mean, $\vec{x}$, estimate of the standard deviation, $s$, and confidence limits for the mean at the 95 percent level for the elements analyzed are given in table 2 . These means are for 10 independent samples and include errors in sampling, analysis and inhomogeneity of the area sampled. Only strontium has a percent relative standard deviation greater than 3 percent (3.2\%). Thus, based on this set of samples, replicate analysis will be within 10 percent of the mean 95 percent of the time. This is acceptable for most environmental work.

Several of the samples were selected for random repeat runs on the AAS to check the precision of the instrument for each element analyzed. The number of runs, $n$, means and standard deviations are given in table 2. The percent relative standard deviations for the replicate samples and the repeated AAS runs are compared in figure 2. The results are quite acceptable for most of the elements analyzed, especially for Co and $\mathrm{Mn}$. However, it is apparent that problems exist for $\mathrm{Fe}, \mathrm{Sr}$ and $\mathrm{Zn}$.

The elemental values determined in GUB-443 sediment were compared to values in shale [1] because of similar metal concentration levels and formation (fig. 3). Incomplete dissolution may have accounted for lower values of $\mathrm{Cu}, \mathrm{Sr}$ and $\mathrm{Zn}$ in GUB-443 than in shale, while $\mathrm{Co}, \mathrm{Cr}$, $\mathrm{Mg}$ and $\mathrm{Ni}$ values were higher in the sediment probably because of major serpentinite deposits in the Guanajibo River drainage area (fig. 1). Cadmium was also higher in the sediment than in shale.

The original intent of the sampling in this region was to delineate the trace element distributions and to correlate this distribution to river discharges [2]. The distribution of copper which correlates well with river plume patterns is shown in figure 4 . Copper had a correlation coefficient of minus $0.64 \pm 0.12$ at the 95 percent level compared with the nearness-to-source parameter. The sampling was done on a $1000 \mathrm{~m}$ grid.

A comparison of the values for copper at GUB-443 and the four nearest samples is given in table 3 . The expected range on the single sample value is plus or minus about 10 percent. The samples to the north (GUB-442) and southeast (GUB-344) are not significantly different from GUB-443. However, the sample to the south (GUB-444) appears to be significantly larger and the sample to the west (GUB-543) is definitely smaller.

Anomalies, such as the increase in copper south of GUB-443, i.e., downstream from the apparent source, may require further sampling. This may be accomplished by replicates at GUB-444, sampling for local lateral distribution, and/or measuring other parameters such as associated trace 


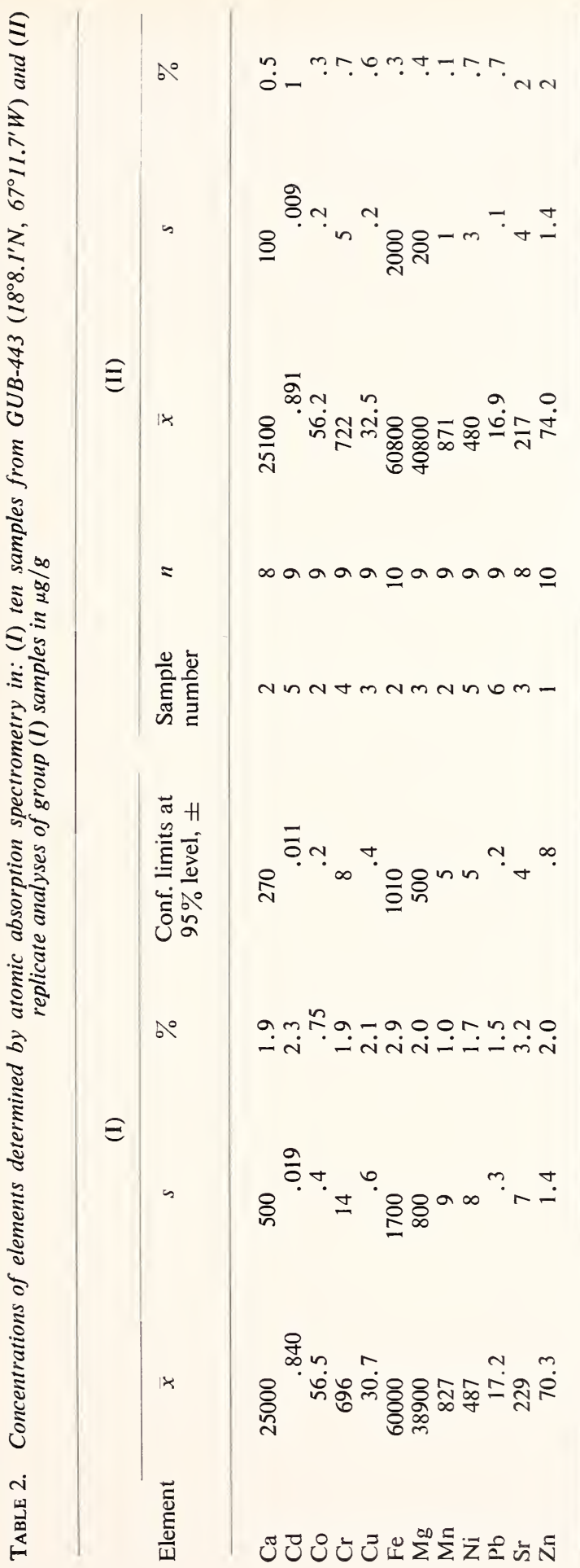




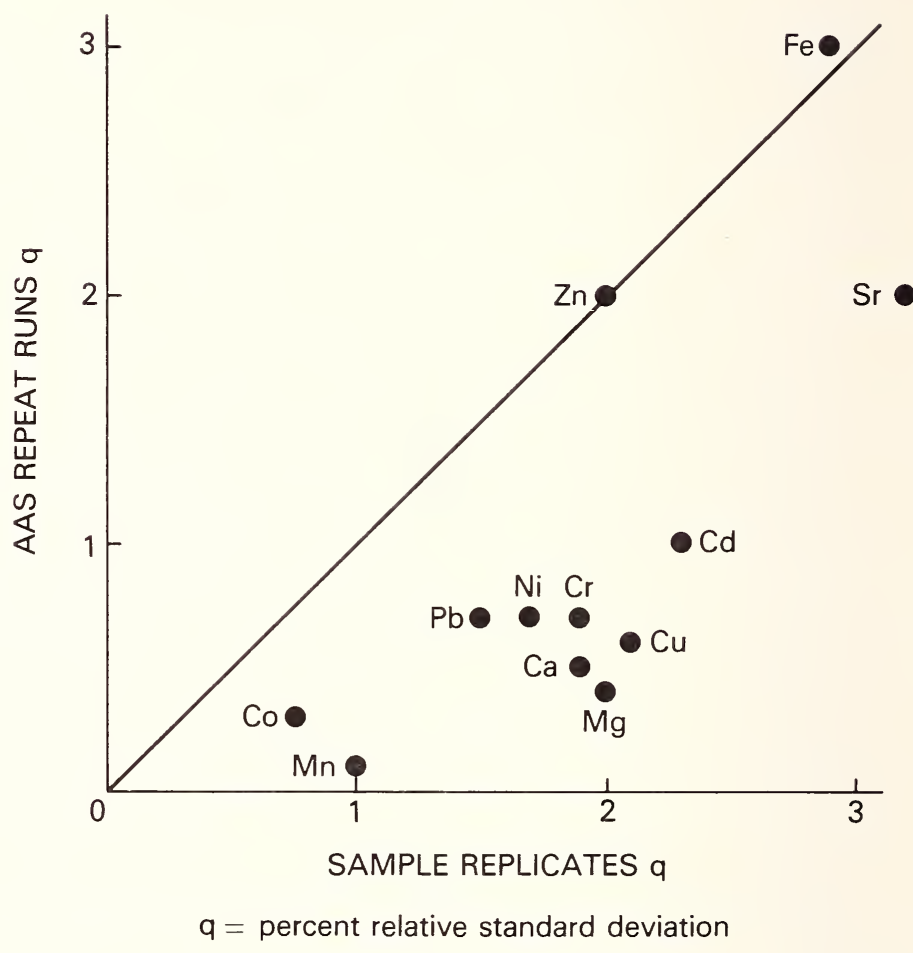

Figure 2. A comparison of the percent relative standard deviations, $q$, for metal concentrations in 10 replicate sediment samples and repeated runs on the AAS. 


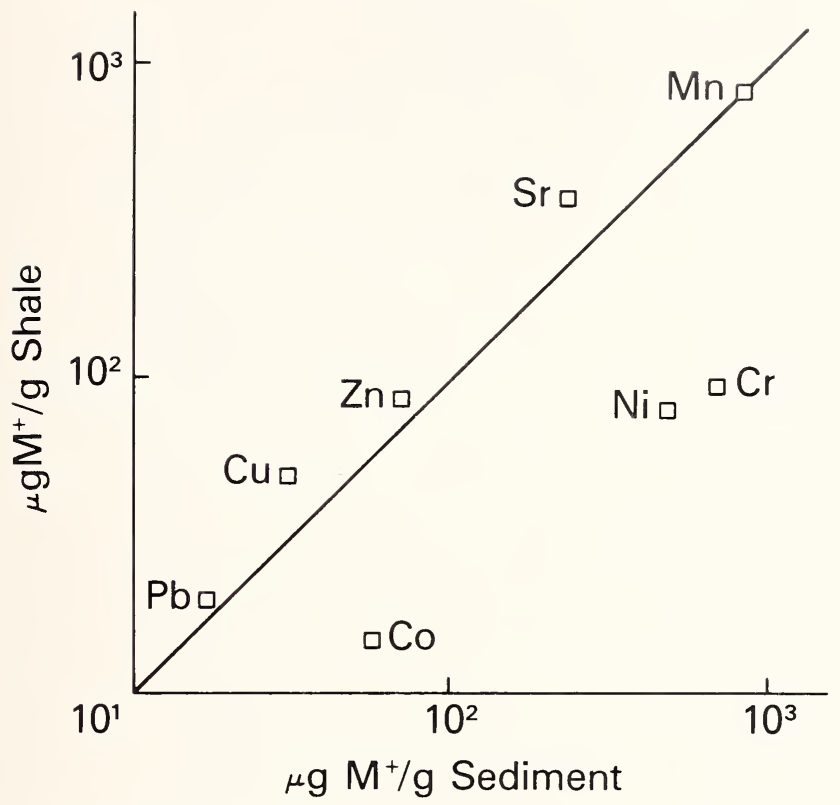

Figure 3. A comparison of metal concentrations in GUB-443 sediment and shale. 


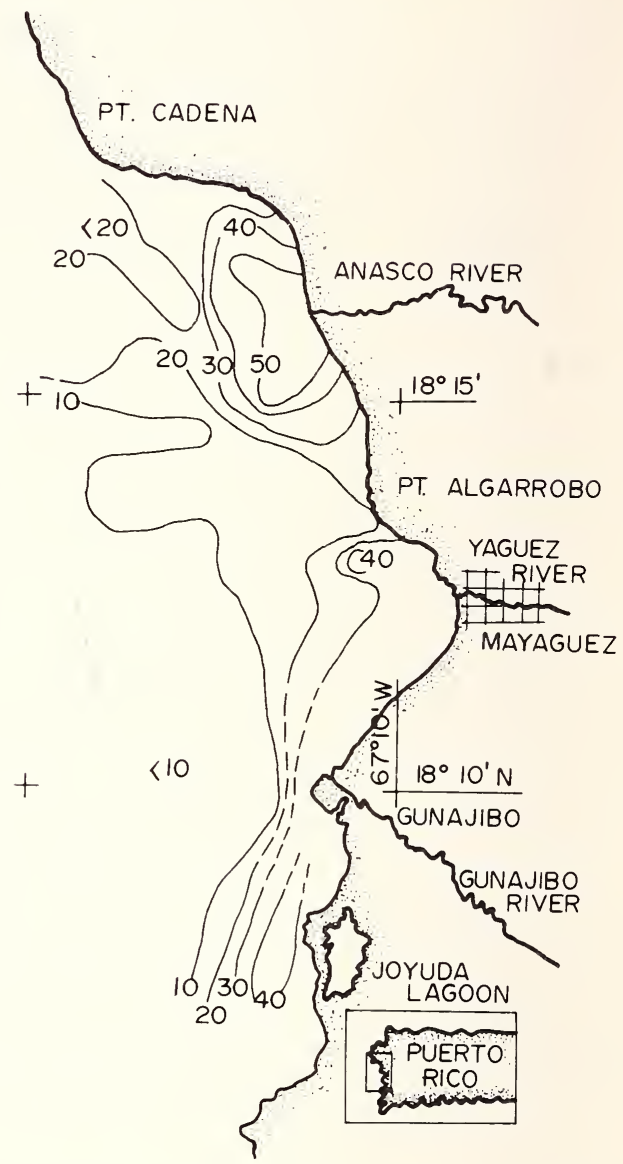

Figure 4. The distribution of copper $(\mu \mathrm{g} / \mathrm{g})$ in marine sediments is closely related to river plume patterns.

TABLE 3. Comparison of copper values determined at GUB-443 and the four nearest samples

$\underset{\text { Sample }}{\text { number }} \quad$ Direction $\quad \mu \mathrm{g} \mathrm{Cu} / \mathrm{g}$ sediment $\quad s \quad n$
number

$\begin{array}{rll}\text { GUB-443 } & - & 30.7 \\ 442 & \mathrm{~N} & 35.7 \\ 344 & \mathrm{SE} & 29.4 \\ 444 & \mathrm{~S} & 43.7 \\ 543 & \mathrm{~W} & 17.5\end{array}$

$0.6 \quad 10$
1
1
1
1


element concentrations, $\mathrm{E}_{H}$, major element concentrations, or bathymetric anomalies.

These detailed studies may require a change in the approach outlined above. For example, the complete dissolution of the sample and a number of replicates and/or larger sample sizes may be required. The sample size is usually increased as the grain size and inhomogeneity increase.

Several methods are available to determine the number of replicate samples that are necessary for the desired confidence limits $[3,4]$. However, these methods are purely statistical and do not take into account the variability of parameters found in natural systems. Usually, the number of replicates is kept to a minimum to reduce the overall effort.

A practical method is to analyze a number of replicates (e.g., 10) at a "typical" spot in the region of interest. Then a statistical analysis of the results is used to determine the number of replicates to be taken in the future based upon the desired confidence limits.

A computer program was devised to do multiple mean and standard deviation calculations on the trace element values obtained from the analyses of the $10 \mathrm{GUB}-443$ replicates to obtain a measure of the range of these values. The calculations were made on the first 2 values, then the first 3 and so on through 10. Then the order was changed by placing the last values first and moving the others up one. The mean and standard deviations were again determined in the same manner as before. This pattern was repeated for a total of 10 runs. Ten runs cannot be compared to the 10 factorial possible combinations of these values, but they do give a good estimate of the range of $x$ and $s$ as $n$ increases from 2 to 9.

Plots of $\bar{x}, s$ and $q$ are shown for two trace elements $(\mathrm{Cr}$ and $\mathrm{Cu})$ in figures 5 and 6 . The range was used for $n=1$ in the plots of $x$ against $n$. The other values were the high and low means as determined in the program above. In the plots of $s$ against $n$, the high and low values were plotted as well as their averages, $s$. The top plot in figures 5 and 6 is the percent relative standard deviation, $q$, against $n$ where

$$
q=\frac{\bar{s}}{\bar{x}}(100)
$$

and $\bar{x}$ is the mean determined for all 10 samples. This should result in a smooth curve, a function of $1 / \mathrm{n}^{1 / 2}$; however, in practice the plots of $q$ against $n$ can usually be reduced to two straight lines. The $n$ at or to the right of the intersection of these two straight lines then represents an optimum number of replicates for this element at this location. An inspection of the elements $\mathrm{Cd}, \mathrm{Co}, \mathrm{Cr}, \mathrm{Cu}, \mathrm{Mn}, \mathrm{Ni}, \mathrm{Pb}$, and $\mathrm{Zn}$ show a spread of $n=$ 3 to $n=5$ as being the optimum number of replicates. 


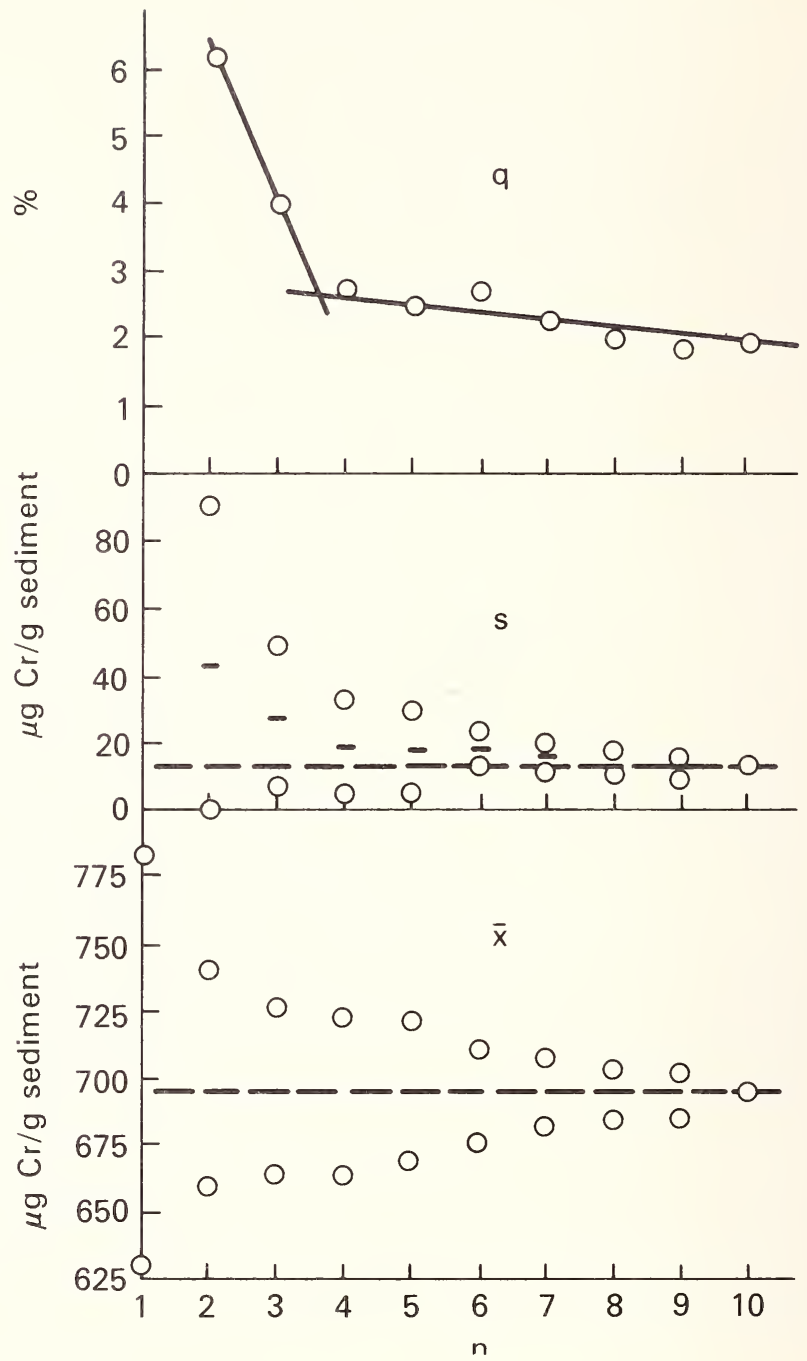

Figure 5. Plots used to determine optimum number of replicates for chromium in sediment from GUB-443.

\section{Discussion}

If there is a need for replicate samples, at least three should be taken and usually little is gained by taking more than five replicates. An exception to this may be when small differences are being sought between two similar values. Precision and accuracy can be improved by increasing the 


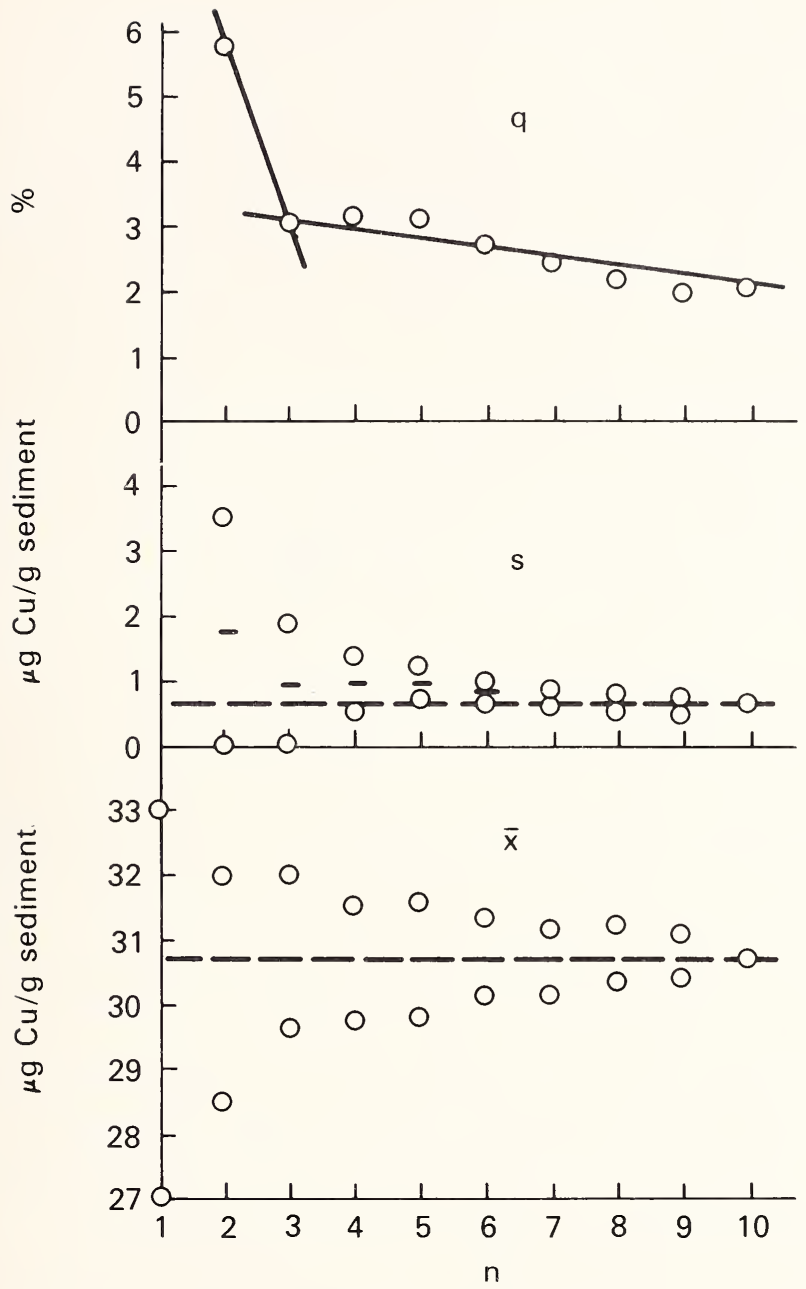

Figure 6. Plots used to determine optimum number of replicates for copper in sediment from GUB-443.

sample size and/or the number of samples. There is a limit to this if nondestructive neutron activation is used for the analyses. For large grained sediment samples or inhomogeneous samples it is better to dissolve a large sample and irradiate a portion of the solution [5]. 


\section{Summary}

The degree of accuracy required in a particular environmental investigation should be determined initially, realizing that the error in one step of the process may be several orders of magnitude greater than in another.

The chemical analysis should be as accurate as practical. However, extreme accuracy in the analysis of a sample may not be warranted if natural variability is high and only orders of magnitude are required. Preliminary sampling may be necessary if little is known of a region, to determine the level of trace element concentrations, as well as sample size and the number of replicates necessary to accurately describe the system. The use of natural standards in addition to laboratory standards is desirable as a check on the analytical procedure and the degree of accuracy.

\section{Acknowledgement}

This work was carried out under Atomic Energy Commission contract AT(40-1) 1833.

\section{References}

[1] Turekian, K. K. and Wedepoh!, K. H., Distribution of the Elements in Some Major Units of the Earth's Crust, Geol. Soc. America Bull. 72:175-192 (1961).

[2] Wood, E. D. and Acosta Cintron, N., The Distribution of Trace Elements in Sediments of Several Tropical River-Ocean Systems. Submitted to Mineral Cycling in Southeastern Ecosystems Symposium, Augusta, Ga. May 1974.

[3] Natrella, M. G., Experimental Statistics, Nat. Bur. Stand. (U.S.), Handb. 91, 505 pages, U.S. Government Printing Office, Washington, D.C. (1963).

[4] Perrin, C. L., Mathematics for Chemists, Wiley-Interscience, New York, 453 pages (1970).

[5] Wood, E. D. and Hood, D. W., Determination of Gold in Geological Materials by Neutron Activation Analysis, Activation Analysis in Geochemistry and Cosmochemistry, Brunfelt, A. O. and Steinnes, E., Eds., Universitetsforlaget, Oslo, 468 pages (1971). 


\title{
SIGNIFICANT IMPROVEMENT OF ACCURACY AND PRECISION IN THE DETERMINATION OF TRACE RARE EARTHS BY FLUORESCENCE ANALYSIS
}

\section{Lyuji Ozawa and Herbert N. Hersh}

\author{
Zenith Radio Corporation \\ Chicago, Illinois 60639 U.S.A.
}

\begin{abstract}
Most of the rare earths in yttrium, gadolinium and lanthanum oxides emit characteristic fluorescent line spectra under irradiation with photons, electrons and $x$ rays. The sensitivity and selectivity of the rare earth fluorescences are high enough to determine the trace amounts $(0.01$ to $100 \mathrm{ppm})$ of rare earths. The absolute fluorescent intensities of solids, however, are markedly affected by the synthesis procedure, level of contamination and crystal perfection, resulting in poor accuracy and low precision for the method (larger than $50 \%$ error). Special care in preparation of the samples is required to obtain good accuracy and precision.

It is found that the accuracy and precision for the determination of trace (less than $10 \mathrm{ppm}$ ) rare earths by fluorescence analysis is improved significantly, while still maintaining the sensitivity, when the determination is made by comparing the ratio of the fluorescent intensities of the trace rare earths to that of a deliberately added rare earth as reference. The variation in the absolute fluorescent intensity remains, but is compensated for by measuring the fluorescent line intensity ratio. Consequently, the determination of trace rare earths (with less than $3 \%$ error) is easily made by a photoluminescence technique in which the rare earths are excited directly by photons. Accuracy is still maintained when the absolute fluorescent intensity is reduced by 50 percent through contamination by $\mathrm{Ni}, \mathrm{Fe}, \mathrm{Mn}$ or $\mathrm{Pb}$ (about $100 \mathrm{ppm}$ ). Determination accuracy is also improved for fluorescence analysis by electron excitation and x-ray excitation. For some rare earths, however, accuracy by these techniques is reduced because indirect excitation mechanisms are involved. The excitation mechanisms and the interferences between rare earths are also reported.
\end{abstract}

Keywords: Fluorescence method analysis; rare earths, as an internal reference; rare earths, determination of trace amounts. 
Most of the naturally occurring trace rare earths in purified yttrium, gadolinium and lanthanum oxides emit characteristic fluorescent lines under irradiation with photons, electrons and $\mathrm{x}$ rays, and the sensitivity and selectivity of these rare earth fluorescences are high enough to determine trace amounts $(0.001$ to $100 \mathrm{ppm})$ [1-3]. The luminescence of these traces in the oxides, however, is markedly affected by the synthesis procedure, the level of non-rare earth contamination and the crystal perfection, resulting in poor accuracy and low precision for the method. Differences and other uncertainties in the geometry of the experimental arrangement also give rise to considerable variation in the results.

We describe here a significant improvement in accuracy, precision and sensitivity of the fluorescence method of determining trace rare earths less than $10 \mathrm{ppm}$. In this method we compare the ratio of the luminescence intensities of the rare earths to that of a deliberately added rare earth acting as an internal reference. The variation in the luminescence intensity measurements remains, but is compensated for by measuring the fluorescence line intensity ratio. Consequently, a determination of trace rare earths can easily be made with less than 3 percent error.

The reference rare earth should not interfere with the rare earth to be detected, either by quenching or enhancing its luminescence. Figure 1 shows the onset of such interferences as the concentration of each possible reference rare earth is increased. It can be seen that $\mathrm{Ce}$ and Printerfere at concentrations greater than $5 \mathrm{ppm}$ more strongly than other RE's (except in $\mathrm{Y}_{2} \mathrm{O}_{3}$ containing $\mathrm{Tb}$ and $\mathrm{Ho}$ ). From the viewpoint of least interference and high fluorescence efficiency, terbium is the best internal reference.

Figure 2 shows the results of the cathodoluminescence analysis of dysprosium using terbium. The measured fluorescent intensities of terbium and dysprosium show much scatter from sample to sample and as a function of concentration, but the fluorescent intensity ratio ( $\mathrm{Dy} / \mathrm{Tb})$ falls smoothly on a straight line having a slope of 0.8 . The explanation for the slope of 0.8 is as follows: the concentration dependence curve of the terbium fluorescence has unit slope under electron and photon irradiation but the dysprosium curve has 0.8 slope under electron irradiation and has unit slope under photon irradiation; consequently, the slope of the Dy/Tb fluorescence ratio curve is 0.8 for cathodoluminescence and 1.0 for photoluminescence.

Figure 3 shows results using photon excitation for $\mathrm{Y}_{2} \mathrm{O}_{3}$ containing equivalent amounts of $\mathrm{Eu}, \mathrm{Dy}$ and $\mathrm{Pr}$ as a function of concentration. At greater than 3 ppm, Pr interferes with Eu and Dy. Each rare earth intensity ratio curve extrapolates smoothly to the zero-point, and the straight line for $\operatorname{Pr}$ (no interference with Eu and Dy) and the smooth curves for $\mathrm{Eu}$ 


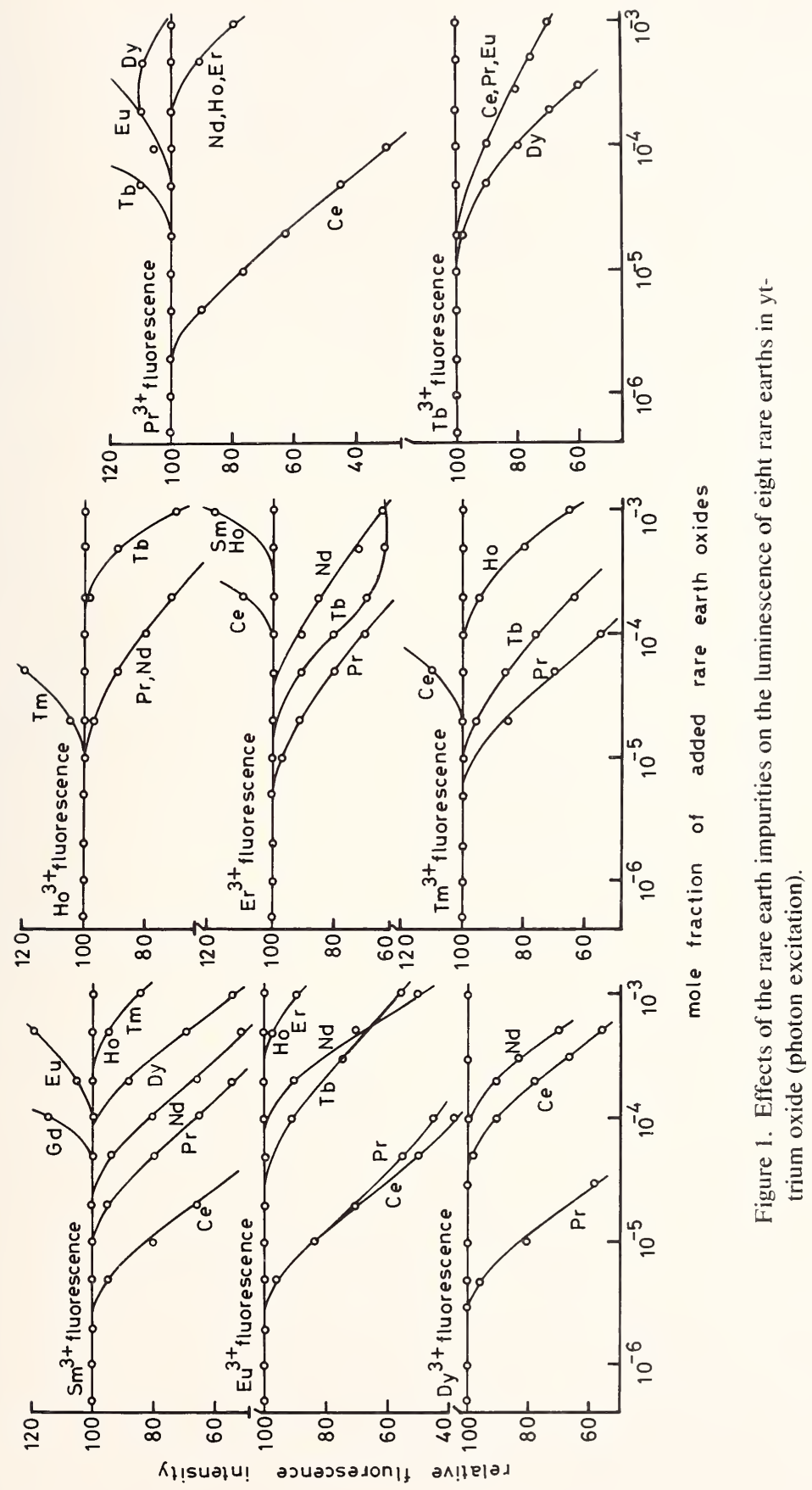




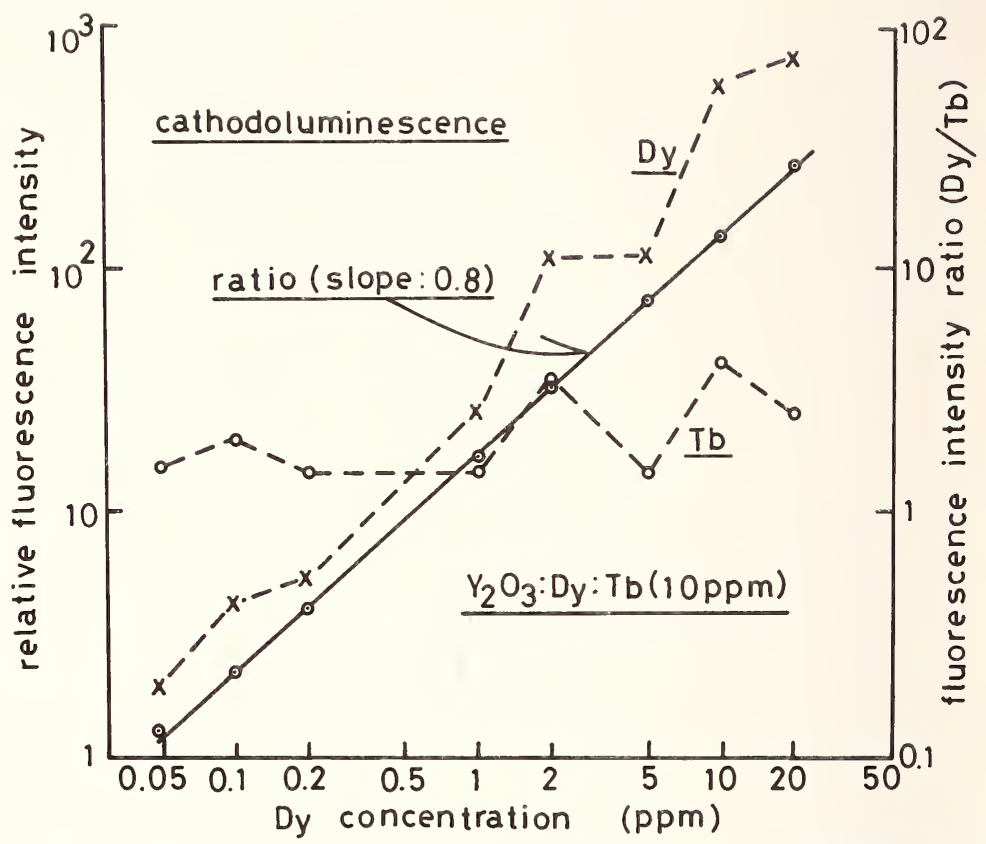

Figure 2. Cathodoluminescence intensity of $\mathrm{Tb}(10 \mathrm{ppm})$ and of Dy as a function of concertration compared to the intensity ratio $(\mathrm{Dy} / \mathrm{Tb})$.

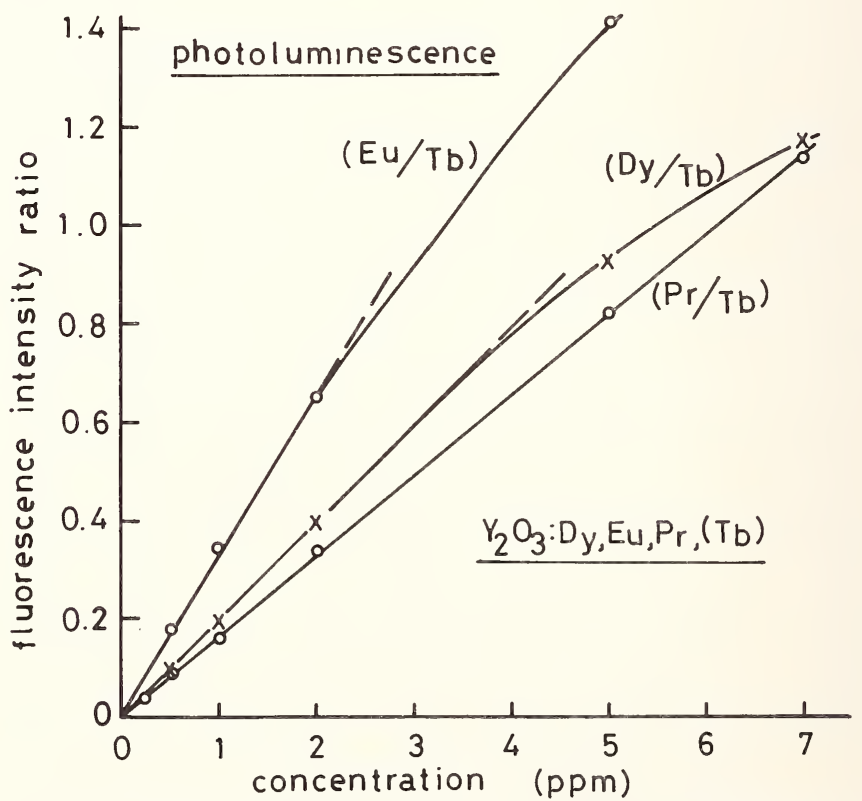

Figure 3. Photoluminescence intensity ratios $(\mathrm{Eu} / \mathrm{Tb}),(\mathrm{Dy} / \mathrm{Tb})$ and $(\mathrm{Pr} / \mathrm{Tb})$ as a function of concentration $([\mathrm{Dy}]=[\mathrm{Eu}]=[\mathrm{Pr}])$. Tb concentration is $5 \mathrm{ppm}$. 
and Dy (interference with $\operatorname{Pr}$ at $[\mathrm{Pr}]>3 \mathrm{ppm}$ ) are consistent with figure 1. Figure 3 thus indicates the high accuracy obtainable. High accuracy is still maintained in the presence of $100 \mathrm{ppm} \mathrm{Ni}, \mathrm{Fe}$, $\mathrm{Mn}$ or $\mathrm{Pb}$, even though the luminescence is decreased by 50 percent.

\section{References}

[1] Ozawa, L. and Toryu, T.,Anal. Chem. 40, 187-190 (1968).

[2] Wickersheim, K. A., Buchanan, R. A., and Sobon, L. E., Anal. Chem. 40, 807-809 (1968).

[3] Jaworowski, R. J., Cosgrove, J. F., Bracco, D. J., and Walters, R. M., Spectrochimica Acta 23 B, $751-763$ (1968). 



\title{
THE ADVANTAGES OF X-RAY FLUORESCENCE ANALYSIS FOR TRACE ELEMENTS IN SILICATE ROCKS
}

\author{
Bernard M. Gunn
}

Département de Géologie

Université de Montreal

Montreal, Canada

\begin{abstract}
$\mathrm{X}$-ray fluorescence (XRF) analysis has been successfully used to analyze over 1000 rock, cement and ore samples per year for elements including $\mathrm{F}$, $\mathrm{Na}, \mathrm{Mg}, \mathrm{Al}, \mathrm{Si}, \mathrm{P}, \mathrm{S}, \mathrm{Cl}, \mathrm{V}, \mathrm{K}, \mathrm{Ca}, \mathrm{Ti}, \mathrm{Mn}, \mathrm{Fe}, \mathrm{Cr}, \mathrm{Co}, \mathrm{Ni}, \mathrm{Cu}, \mathrm{Zn}, \mathrm{Ga}, \mathrm{Rb}, \mathrm{Sr}$, $\mathrm{Y}, \mathrm{Zr}, \mathrm{Ba}, \mathrm{Cs}, \mathrm{Ce}, \mathrm{La}, \mathrm{Pb}$, Th, and $\mathrm{U}$. Detection limits are never greater than $10 \mathrm{ppm}$ and optimally are as low as $1 \mathrm{ppm}$ for 60 second counting times. Accuracy is mainly limited by the accuracy of available standards which for major and minor elements are not known to better than 0.1 to 0.01 percent. Data reduction and matrix corrections are made by computer and programs have been developed in both FORTRAN IV and FOCAL-11 language.
\end{abstract}

Keywords: Mineral analysis; trace analysis; $x$-ray fluorescence.

\section{Introduction}

When excited by an incident beam of electrons, gamma rays or high energy $\mathrm{x}$ rays, elements in a sample fluoresce secondary $\mathrm{x}$ rays of wavelength which are characteristic to each element, and which increase in energy regularly with atomic number of the emitting element. As the spectra are simple, complete resolution of the different wavelengths can be achieved with a crystal monochromator. Satisfactory resolution can also be attained by an energy dispersive system for elements heavier than calcium, but energy differences between the $\mathrm{K} \alpha$ lines of elements lighter than calcium are too small for effective resolution by the energy dispersive system, the resolution being never better than $130 \mathrm{eV}$ compared with about $5 \mathrm{eV}$ for a good crystal.

The output from a spectrometer of wavelength dispersive type is digital 
and computer processable. The intensity of the emitted x-ray beam is, unfortunately, not only proportional to the concentration of the element in a sample, but is also inversely related to the average mass absorption characteristics of the sample. The bulk mass absorption coefficients of two silicate samples may differ by 100 percent, and the method relies on first establishing the slope of the emitted intensity per percent of element concentration, by means of a standard of known concentration. Obviously, unless the difference in mass absorption can be allowed for, the method has little use, and the method can be no better than the accuracy to which the concentration of the element in the standard has been determined.

The following is a summary of procedures which may be used in XRF analysis, and of possible sources of error.

\section{Summary of Procedures}

\section{A. Preparation for Analysis}

(a) For elements lighter than iron it is necessary to fuse the powdered sample in a 2:1 mixture with lithium tetraborate. The glass bead produced must have an optically flat surface, preferably polished, for analysis. Greater dilution or the use of heavy absorbers is highly undesirable because of reduced countrate and homogeneity problems unless only a partial analysis is required.

(b) For elements heavier than iron a pressed powder briquette, cemented together with a few drops of polyvinyl alcohol cement, is all that is necessary.

\section{B. Replication ERrors}

If the glass bead in (a) above is thoroughly stirred both as powder and in the melt, replication will be within the statistical counting uncertainty, i.e., $\pm 0.2 / \mathrm{SiO}_{2}$. In a test of sample homogeneity we recently made up 42 replicate powder pellets. These gave maximum and minimum figures for strontium of 141 and $143 \mathrm{ppm}$, and 22.7 and $21.1 \mathrm{ppm}$ rubidium. These were all within the counting uncertainty of $\pm 1.8 \mathrm{ppm}$. The lighter the element the greater is the error in sample reproducibility due to the shallower depth of penetration of the $\mathrm{x}$ rays. For nickel, replication may be as great as twice the statistical error, and for chromium up to three times. Chromi- 
um should be determined on a glass bead but we usually sacrifice accuracy for speed in this case.

\section{Volatiles}

Providing the total amount of volatiles in a rock does not exceed 5 percent, no serious error ensues when results are summed to 100 percent free of volatiles, though the flux/rock ratio is in error by this amount. When the total volatile content is 5 percent or greater a loss on ignition determination should be done for each sample before major element analysis. The loss on ignition for each sample and for the flux has to be entered into the computer program.

\section{BACKGROUNDS}

For the major elements, the background is low and constant, the peak/background ratio for 1 percent $\mathrm{TiO}_{2}$ being 745:1. The computer program may either subtract a fixed background count, or the actual, if provided. Trace element procedures differ according to the amount of element, e.g., for nickel in the 5 to $200 \mathrm{ppm}$ range a background is measured on either side of the peak. At 200 to $2000 \mathrm{ppm}$ only one background is necessary and over $2000 \mathrm{ppm}$ a predetermined constant background correction is all that is required.

Background slope or curvature corrections are needed for any concentration under $2000 \mathrm{ppm}$. A spec-pure glass plate is always used for standardization, and the computer program calculates curvature factors based on this.

\section{E. Counting Errors}

The computer program will calculate only intensity data bracketed between standards which differ by less than three times the possible statistical error. The possible error is the square root of the sum of the squares of the errors in the standard, unknowns and glass plate both for peaks and background. This possible error at the 95 percent confidence level is printed out after every determination.

\section{F. Counting Efficiency}

Obviously, as the counting error is inversely proportional to $\mathrm{N}$, the 
number of counts accumulated, an x-ray fluorescence spectrometer with an efficient optical system will give better results, or require shorter operating time. Typical intensity yields are given in table 1 . The spectrometer used is now 8 years old; newer spectrometers may attain up to 30 percent more counts per percent element. The use of new crystals such as graphite and thorium acid phthalate, together with a $2 \mu \mathrm{m}$ polycarbonate flowcounter window, greatly improves the analysis of light elements. One micrometer stretched-polypropylene windows have been found to be too unreliable. A thorium acid phthalate crystal has $21 / 2$ times the refractive efficiency of rubidium acid phthalate which is in turn twice as efficient as potassium acid phthlate.

\section{G. Matrix Errors}

The difference in fluorescent yield of an element in the same concentration in samples of different matrix may be as great as 20 percent in geological materials fused in tetraborate, and in excess of 100 percent for trace elements in powder pellets. The element being analyzed may either be absorbed or enhanced by a second element present. In figure 1 the effect of absorption by $\mathrm{MgO}$ in $\mathrm{Al}_{2} \mathrm{O}_{3}$ and the enhancement of magnesium by aluminum is shown. The relationship between every element pair in a sample is somewhat similar. The characteristics of each parabolic curve can be described by an equation of the type $\mathrm{C}_{A}=\mathrm{R}_{A}(1+\alpha \mathrm{B})[1]$. The expression for the enhancement curve is more complex and preliminary work shows that the condition of a heavy self-absorbing element in a light matrix is again described by a symmetric, positive curve. Unfortunately, little information is as yet available for very few elements or oxide pairs.

\section{H. Computer Routine MATRIX}

For major elements in silicate samples this FORTRAN IV or FOCAL-1 1 program accepts an array of nominal conceritrations obtained by ratioing the $\mathrm{x}$-ray intensity of an unknown against that of a standard. Absorption coefficients [3] are read in or stored permanently in the computer. Two mass absorption coefficients have to be calculated for each sample, one for the incident primary beam for which a value of average effective exciting wavelength is assumed, and one for the monochromatic emitted beam. For the major elements the procedure is iterative, about five iterations being necessary for each analysis. The basic calculation follows: 


$$
C_{A}=C_{s} \frac{R_{A}}{R_{s}} \frac{\left(A_{1}+A_{2}\right)_{A}}{\left(A_{1}+A_{2}\right)_{s}}
$$

where $C_{A}=$ concentration of element $A$

$C_{s}=$ known concentration in standard

$R_{A}=$ intensity rate of element $\mathrm{A}$ in sample

$R_{s}=$ intensity of the same element in standard

$A_{1}=$ absorption coefficient for the average effective exciting wavelengths $A_{2}=$ absorption coefficient for emitted radiation $A_{1}$ and $A_{2}$ are calculated for both sample and standard.

It will be seen that no correction for enhancement is made. We have never been able to show that rocks higher in $\mathrm{Al}_{2} \mathrm{O}_{3}$, for example, give anomalously high $\mathrm{MgO}$ results. The $\mathrm{W}-1$ diabase standard with 14.8 percent $\mathrm{Al}_{2} \mathrm{O}_{3}$ and 6.6 percent $\mathrm{MgO}$ gives correct results for the DTS dunite (50\% $\mathrm{MgO}, 0.2 \% \mathrm{Al}_{2} \mathrm{O}_{3}$ ).

\section{Program X-TraCE}

For the trace elements (below 1\%), a single calculation of absorption coefficients for both standard and unknown reduces the error. If the major element composition is not known, the absorption coefficient may be measured and calculated directly (program NORRISH), or approximated by taking the inverse of the Compton-scattered peak intensities (program $\mathrm{X}$-COMP).

\section{J. Limitation of Matrix Programs}

We have not been able to detect any consistent residual matrix error when analyzing geological materials. However, if it is attempted to use pure $\mathrm{Fe}_{2} \mathrm{O}_{3}$ as a standard for iron in a basalt, or pure $\mathrm{SiO}_{2}$ as a standard for silica in a rock, it is necessary to assume different average effective exciting wavelengths [4]. As we do not normally determine iron ores and similar materials, using silicate rock standards, the present system has so far proved adequate (table 2).

\section{K. Computer Output}

Several alternative forms of handling intensity data have been developed at the Université de Montréal.

1. Central Computer: Intensity data are output onto data cards which 


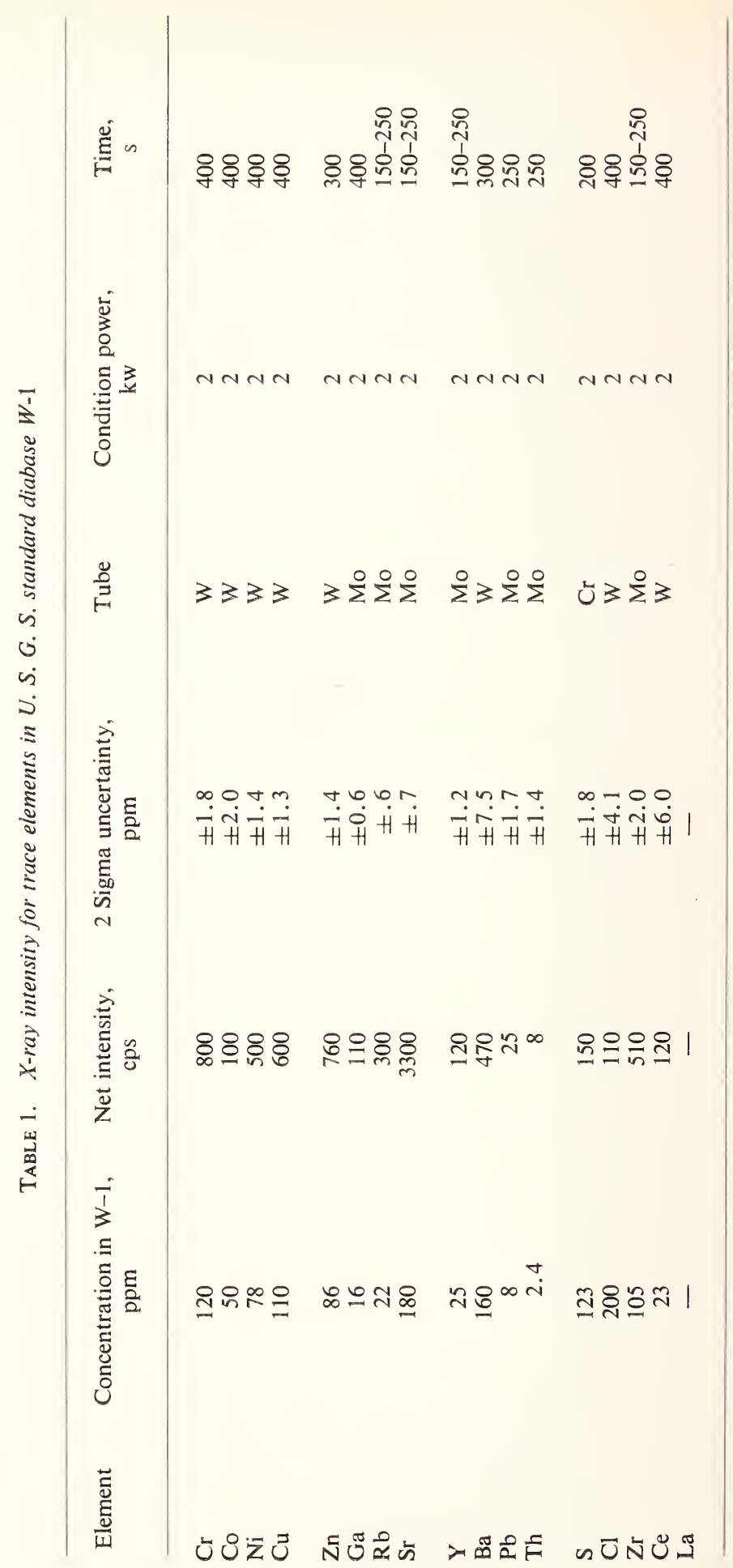




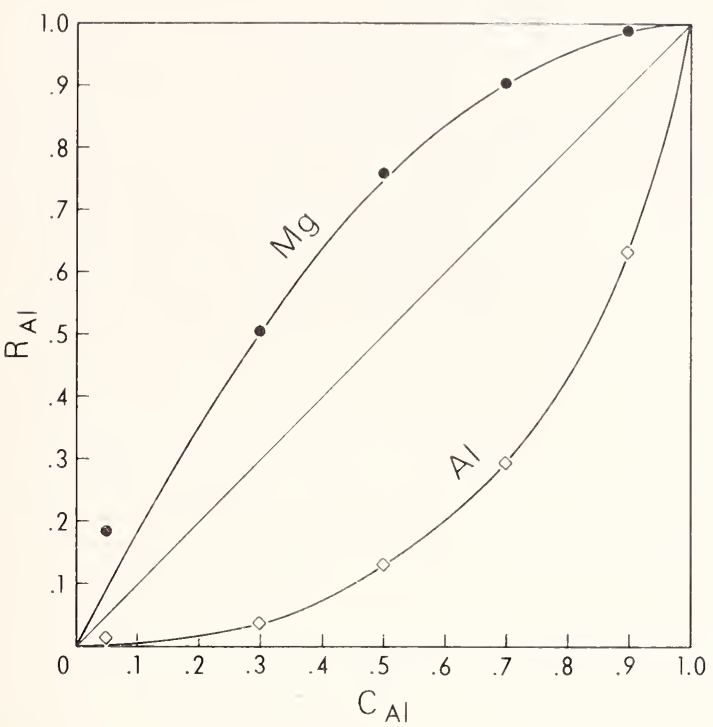

Figure 1. Preliminary diagram showing the absorption and enhancement effects in $\mathrm{Al}_{2} \mathrm{O}_{3}-\mathrm{MgO}$ mixtures, the $\mathrm{Al}$ radiation being strongly absorbed by the $\mathrm{Mg}$ which is in turn secondarily enhanced by the $\mathrm{Al} \mathrm{K} \alpha$ radiation originating within the sample. Note the asymmetry of the absorption curve, possibly due to the presence of varying amounts of oxygen; more likely, this is due to the selective absorption of primary wavelengths near the $\mathrm{Al}$ absorption line.

are read through a CDC 200 series terminal to the Central Cyber 74 computer. Results are both printed and punched onto a card deck for filing. The time for calculation of 120 analyses for 10 elements is 27 seconds and costs $\$ 7.05$. The rental of the on-line key punch is $\$ 75$ per month.

2. Real-time Computer: An 8K PDP-11 is interfaced to the spectrometer. Data reduction and matrix correction routines in FOCAL-11 language are stored on paper tape. Nominal results are printed immediately together with comments on precision, duplication error, and machine conditions. The time required to make the matrix corrections for a single analysis is 21 seconds for 5 iterations. The cost was $\$ 9000$ for the PDP11 and teletype, $\$ 400$ for the interface, and about $\$ 2000$ for software.

3. Terminal to Central Computer: A T.I. Silent 700 terminal costing $\$ 3000$ was linked by acoustic coupler to the computer center and data from the spectrometer read in at intervals in the form of paper tape. This was found to be the least reliable approach and was abandoned. 


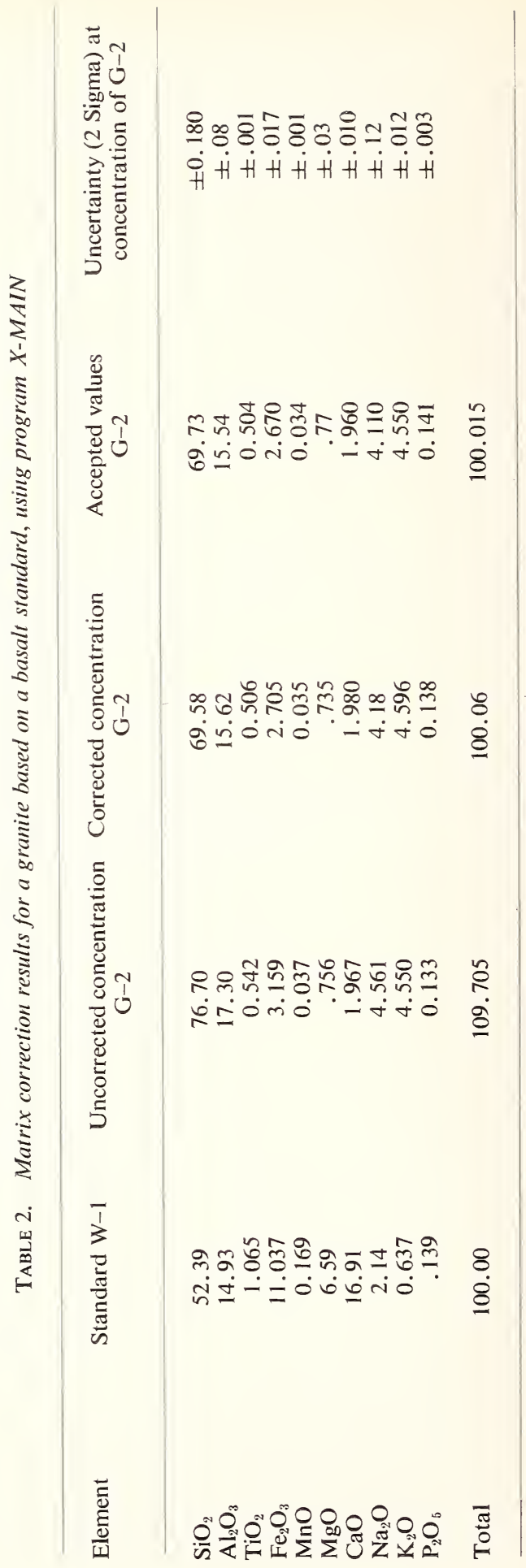




\section{Data Handling}

As an XRF spectrometer may produce 1000 or more reliable analyses for up to 30 elements per year, these must be stored on computer file. We maintain a file of about 12,000 rock analyses (GEOKEM) and a series of data retrieval, plotting, statistical and presentation routines all of which are stored on disc at the main computer center.

\section{Accuracy}

The precision of XRF consistently exceeds the accuracy of the available standards for almost all elements. Thus, while the elements $\mathrm{K}, \mathrm{Ti}, \mathrm{Mn}$ in rocks are reproducible to 0.002 percent, no standard is available with accuracy better than 0.01 percent.

Detectable differences between XRF and the methods used at the U.S. Geological Survey were found only for $\mathrm{Al}_{2} \mathrm{O}_{3}$ of the 10 major elements [5]. Isotope dilution results agreed within $\pm 0.2 \mathrm{ppm}$ in a study of rubidium in Icelandic basalt, well within the calculated uncertainty of $\pm 0.4 \mathrm{ppm}$ [6]. The agreement between a routine set of XRF results and ID is given in table 3. An unpublished comparison of 20 Archaean metabasalts determined for $\mathrm{K}$ in the 0.002 to 0.5 percent range showed the XRF to be consistently $20 \mathrm{ppm}$ high. The graphite crucibles used for fusion had been previously used for granites. Refusion of fresh powder in Pt crucibles gave results without systematic bias.

TABLE 3. Comparison of analyses of Rb and Sr standard rocks by isotope dilution methods (Fairbairn and Hurley, 1971) and routine x-ray fluorescence methods used in this laboratory

\begin{tabular}{cccccc}
\hline Standard & \multicolumn{2}{c}{ Isotope dilution results } & & \multicolumn{2}{c}{ X-ray fluorescence results } \\
Rock & $\mathrm{Sr}$ & $\mathrm{Sr}$ & & $\mathrm{Rb}$ & $\mathrm{Sr}$
\end{tabular}

\begin{tabular}{lllrl}
\hline W-1 & 22.0 & 180.0 & $22.6 \pm 0.2$ & $185.0 \pm 1.0$ \\
BCR & $48.3 \pm 0.1$ & $334 \pm 2.0$ & $49.0 \pm 0.3$ & $333.0 \pm 0.8$ \\
AGV & $68.0 \pm 1.0$ & $661.0 \pm 6.0$ & $70.0 \pm 0.6$ & $665.0 \pm 0.4$ \\
GSP & $251.0 \pm 1.0$ & $236 \pm \pm 1.0$ & $264.0 \pm 1.4$ & $231.0 \pm 0.6$ \\
G-2 & $171 \pm \pm 5.0$ & $479 \pm 6.0$ & $177.0 \pm 0.5$ & $477.2 \pm 1.0$
\end{tabular}

Spiking procedures may be used for trace elements only when a salt of the element has absorption characteristics similar to those of the sample being spiked. A granite spiked with 200 ppm of strontium as $\mathrm{SrNO}_{3}$ when 
used as a standard gave $251 \mathrm{ppm}$ in G-1 compared with the accepted value of $250 \mathrm{ppm}$ [4]. If pure fluxes were available this restriction would not apply, but even spec-pure $\mathrm{Li}_{2} \mathrm{~B}_{4} \mathrm{O}_{7}$ contains at least $10 \mathrm{ppm}$ each of the alkali elements. Were it possible to use uncontaminated flux material, pure element, oxide or a salt of any kind could be used for spiking.

\section{Conclusions}

$\mathrm{XRF}$ analysis is probably the most rapid and precise method of analysis currently available. No other method allows an inexperienced operator to produce trace element data accurate to 1 to $2 \mathrm{ppm}$ in times of as little as 60 seconds. The ready computerization of output eliminates computational error.

When better interelement data are available it should be possible to analyze any solid or liquid material to 0.5 percent accuracy. At present the accuracy possible in geological material is limited by the standards available and is especially poor for $\mathrm{F}, \mathrm{P}, \mathrm{S}, \mathrm{Ca}, \mathrm{K}, \mathrm{V}, \mathrm{Ti}, \mathrm{Mn}, \mathrm{Ga}$, and $\mathrm{Zr}$. Isotope dilution results are generally more precise than XRF at levels below $10 \mathrm{ppm}$ for $\mathrm{Sr}, \mathrm{Rb}$ and $\mathrm{K}$.

\section{References}

[1] Lachance, G. R. and Traill, R. G., A Practical Solution to the Matrix Problem in XRay Analysis, Can. Spectr. 11, 43-48 (1966).

[2] Rasberry, S. D. and Heinrich, K., Calibration for Interelement Effects in X-Ray Fluorescence Analysis, Anal. Chem. 46,81-89 (1974).

[3] Heinrich, K., The Electron Microprobe, John Wiley and Sons, New York, 1035 pages (1964).

[4] Gunn, B. M., The Incident Attenuation Factor and the Determination of Some Heavy Elements in Standard Rocks, Can. Spectr. 12, 3-8 (1967).

[5] Gunn, B. M., Trace Element Partition During Olivine Fractionation of Hawaiian Basalts, Chem. Geol. 8, 1-13 (1971).

[6] Hart, S. R., Gunn, B. M., and Watkins, N. D., Interlava Variation of Alkali Elements in Icelandic Basalt, Amer. Jour. Sci. 270,315-318 (1971).

[7] Fairbairn, H. W. and Hurley, P. M., Evaluation of X-Ray Fluorescence and Mass Spectrometric Analyses of $\mathrm{Rb}$ and $\mathrm{Sr}$ in Some Silicate Standards, Geochim. Cosmochim. Acta. 35, 149-156 (1971). 


\title{
MONITORING OF SMOG AEROSOLS WITH ELEMENTAL ANALYSIS BY ACCELERATOR BEAMS
}

\author{
T. A. Cahill, R. G. Flocchini, R. A. Eldred, P. J. Feeney, \\ S. Lange, D. Shadoan, and G. Wolfe
}

\author{
Crocker Nuclear Laboratory and \\ The Department of Physics \\ University of California \\ Davis, California 95616 U.S.A.
}

The California Air Resources Board has operated an aerosol monitoring network of up to 15 stations since January 1973, using impactors to collect size segregated samples and ion-excited $\mathrm{x}$-ray emission to perform the subsequent elemental analyses. Two-stage Lundgren-type rotary drum impactors with afterfilters collect particles in the 0.1 to $0.6 \mu \mathrm{m}, 0.6$ to $5 \mu \mathrm{m}$, and 5 to 20 $\mu \mathrm{m}$ size ranges. Alpha beams from an isochronous cyclotron, possessing energies of $18 \mathrm{MeV}$, are used to excite $\mathrm{x}$-rays in the aerosol samples, which are then detected by a $\mathrm{Si}(\mathrm{Li}) \mathrm{x}$-ray detector. On-line data collection and reduction codes generate aereal densities for elements between sodium and uranium, with sensitivities in the nanogram per cubic meter range for most elements. During the first year of operation, about 12,000 analyses were performed as part of this program. Each analysis normally included between 15 and 25 elements. In order to insure that all results were accurate, extensive programs were undertaken in quality assurance. The first concern involved proper operation of the aerosol collection systems. Uranene dye studies were able to generate information on the particle size cuts induced by the impactor slits, as well as indicate the importance of nonoptimum collection effects such as bounce-off from the drum surfaces. However, extensive studies with real aerosols were required before the problem of bounce-off for dry, silicious aerosols was solved by coating the drums with $530 \mu \mathrm{g} / \mathrm{cm}^{2}$ mylar upon which was deposited about $50 \mu \mathrm{g} / \mathrm{cm}^{2}$ paraffin. Penetration of the Nuclepore final filter contributed important corrections to the smallest size range, based upon the work of Spurney and Lodge. Operation of the collection system was verified through extensive comparisons on an element by element basis with high-volume filter samplers, and a nominal collection error of \pm 15 percent was assigned from these causes. Absolute accuracy of the analysis system was established through the use of 28 gravimetric thin film standards, and confirmed by numerous interlaboratory and inter-method comparisons, all of 
which showed that the nominal analytical accuracy of \pm 10 percent was quite conservative. Major corrections for light elements, loading effects, which limited accuracy to \pm 30 percent for sodium in the worst case.

Keywords: Aerosol; California aerosol monitoring program; elemental analysis; ion beam analysis; monitoring; particle sizing; time resolution.

\section{Introduction}

The importance of the solid and liquid components of the atmosphere, the atmospheric aerosol, to air quality monitoring programs has long been recognized. Compared to gaseous pollutants, the aerosol is usually the dominant factor in visibility reduction, transport of medium and heavy toxic elements, and soiling. It serves as the final step in many of the processes occurring in the atmosphere that remove gaseous pollutants. Thus, knowledge of aerosol sources, transport, transformations, and sinks is of paramount importance in many situations.

Attempts to include the atmospheric aerosol in monitoring programs have, however, been frustrated by the complexity of the task. First and most important, the aerosol consists of an ensemble of particles with an unlimited variety of sizes and shapes. This information is essential for evaluation of transport properties, health effects, and visibility reduction, while being very useful in source identification.

A second point of great importance involves the elemental and chemical complexity of these particles. While relatively few chemical compounds dominate gaseous pollutants (although hydrocarbons pose interesting problems), no attempt has been seriously made to enumerate important chemical states found in aerosols. A listing of elements found in an average urban aerosol to a level of 0.1 percent of the most abundant component commonly reaches 20 to 30 elements. Such a listing for gaseous pollutants is usually limited to five elements.

Considering these difficulties, it is not surprising that normally only the total mass of aerosol collected on a filter is included in aerosol monitoring efforts, although some estimate of soiling and occasional analyses for a few key chemicals or elements are included. A major barrier to better aerosol monitoring has been the difficulty of obtaining enough material in a particle sizing device for subsequent analyses. Common use of destructive techniques such as atomic absorption spectrophotometry also plays a role in limiting the number of analyses that can be made once a sample has been collected.

Advances in energy dispersive $x$-ray spectroscopy, based on use of the 
$\mathrm{Si}(\mathrm{Li})$ or $\mathrm{Ge}(\mathrm{Li})$ solid state high resolution x-ray detectors, have allowed some of these difficulties to be surmounted. Here one has a nondestructive technique with a very broad elemental range and relatively uniform minimum detectable limits that is suited to (and in fact, requires) limited amounts of sample material. This in turn allows one to use commonly available particle sizing devices. A final point is that, in a multielement mode, the cost of each elemental determination in an average urban aerosol is less than $\$ 1.00$, thus allowing extensive determinations of elemental levels at reasonable cost.

The program run by the University of California at Davis for the California Air Resources Board has attempted to capitalize upon these advances. A 15 stations network has been established in California, based upon continuous sampling of aerosols in three particle size ranges (20 to $3.6 \mu \mathrm{m} ; 3.6$ to $0.65 \mu \mathrm{m} ; 0.65$ to $0.1 \mu \mathrm{m}$ ), followed by analysis for sodium and heavier elements by ion-excited x-ray emission (IEXE) and other methods. New problems in quality control and verification of results have arisen, however, due not only to the enormous quantity of positive identifications ( $>200,000$ in the first year) but also to the details of sample collection, transport, and analysis. The methods used to perform the verifications necessary to establish validity of the data form the remainder of this paper.

\section{Validation of Systems Operation}

\section{A. Description of Sample Collection}

Aerosol samples were collected by means of Lundgren type rotating drum impactors [1] which operated for 7 days unattended at up to 15 sites in California. Samples were collected in three size ranges by two rotating drums and an after filter. Although increasing the number of stages increases information about the particle size distribution of the collected aerosol, the cost of analysis becomes prohibitive. Initially, it was decided to design a unit with two stages. The cut-point would be set at the minimum of the "bimodal distribution" [2] which occurs at approximately $4 \mu \mathrm{m}$. However, two important reasons dictated a third stage.

1. The size distribution of the atmospheric aerosol between 0.1 and 4 $\mu \mathrm{m}$ was known to be very important in terms of health effects, visibility degradation, source identification, and transport properties. To aid in 
elucidating these phenomena, a cut-point in this size range would be extremely important.

2. By establishing a cut-point near the peak in the mass distribution, as much mass as possible could be collected on the impaction stages, thus minimizing filter loading problems.

A cut-point of $0.65 \mu \mathrm{m}$ was chosen. This proved to be a fortunate choice since the data revealed that it separated automotive aerosols (less than $0.65 \mu \mathrm{m}$ ) from stationary fossile source, sulfur-dominated aerosols (between 0.65 and $3.6 \mu \mathrm{m}$ ). Thus, in the final analysis, the size ranges were:

Stage 1 (rotating impaction surface)-about $20 \mu \mathrm{m}$ (set by intake manifold design) to $3.6 \mu \mathrm{m}$.

Stage 2 (rotating impaction surfaces) -3.6 to $0.65 \mu \mathrm{m}$.

Stage 3 (after filter) -0.65 to $0.1 \mu \mathrm{m}$.

The aerodynamic properties for stages 1 and 2 are similar to stages 2 and 4 of the Lundgren impactor. The pump supplied with the unit is a $1 / 10 \mathrm{hp}$ Gast carbon vane rated at $1.25 \mathrm{cfm}$ at $10^{\prime \prime} \mathrm{Hg}$ (see fig. 1 for diagram of system).

The rotary drum stages were covered with $530 \mu \mathrm{g} / \mathrm{cm}^{2}$ of type S mylar upon which about $50 \mu \mathrm{g} / \mathrm{cm}^{2}$ of paraffin was deposited. This was obtained from a 3 percent paraffin in toluene solution. Nuclepore filters with a 0.8 $\mu \mathrm{m}$ pore size were used. Nuclepore was chosen because its surface deposition properties allowed accurate $\mathrm{x}$-ray analysis of low $\mathrm{Z}$ elements ( $\mathrm{Na}$ to Si). Using the mass distribution of Whitby et al. [3] and the theory of Spurny and Lodge [4] a collection efficiency of 48 percent was determined for particles between 0.1 to $0.65 \mu \mathrm{m}$. High efficiency filters could have been used but quantitative information on low $\mathrm{Z}$ elements ( $\mathrm{Na}$ to $\mathrm{Si}$ ) would have been lost. In the higher efficiency filters, particles are entrapped through the media in an unknown distribution. Therefore, although nuclepore was inefficient, it was chosen as the filter medium because the necessary correction factors were better understood and documented.

\section{B. Validation of Sample Collection Techniques}

\section{Particle Loss in Impaction}

Particle loss occurs when particles adhere to any unanalyzable structural component of the impactor. The importance of this process was established through the following studies.

a. Uranene dye studies performed by Environmental Research Cor- 


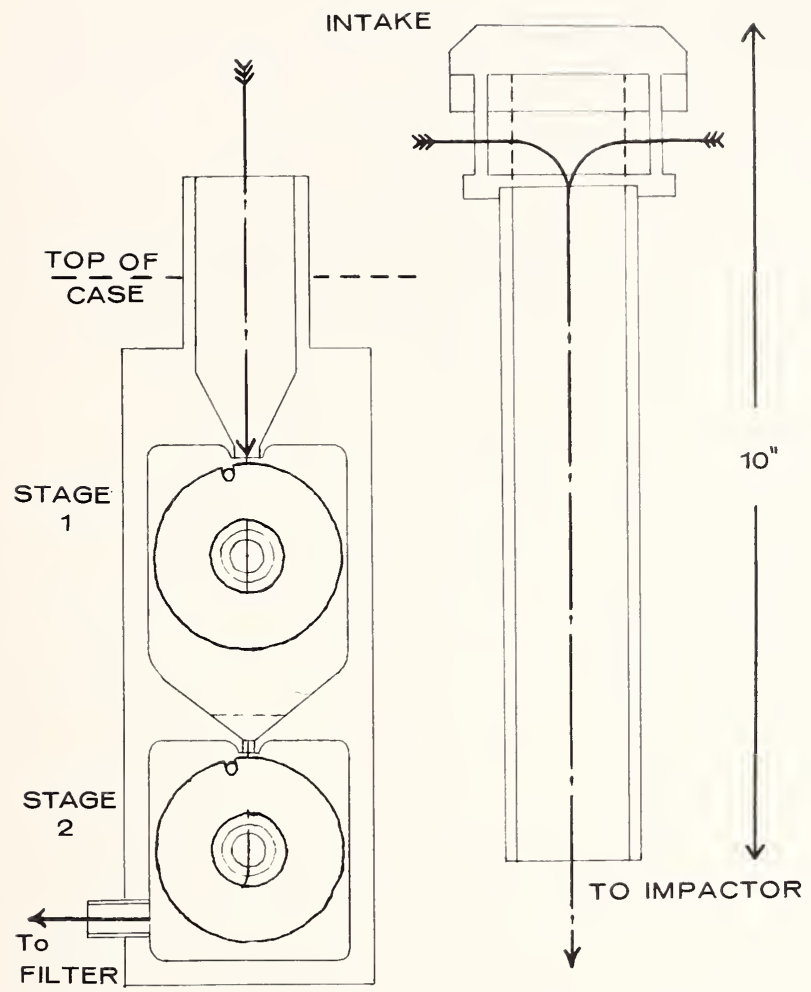

Figure 1. Diagram of aerosol sample collection system.

poration supplied values for particle loss that were less than 5 percent, for all particle sizes from 0.65 to $20 \mu \mathrm{m}$. This was done by collecting uranene dye particles from all nonanalyzable surfaces in the unit and comparing the deposition to that seen on the drum surface.

b. Paraffin studies were performed when the units first arrived in order to verify collection efficiency for realistic dry aerosols [5]. Nine identical units were operated for 48 hours in a random array on the roof of the Physics/Geology building at Davis. Foils were prepared with paraffin loadings from $0 \mu \mathrm{g} / \mathrm{cm}^{2}$ to $50 \mu \mathrm{g} / \mathrm{cm}^{2}$, with two impactors used at each coating thickness. The results, shown in figure 2 , indicated that saturation of the paraffin capture improvement at $\sim 150$ percent of the uncoated value occurred at $\sim 12 \mu \mathrm{g} / \mathrm{cm}^{2}$. Thus, the $50 \mu \mathrm{g} / \mathrm{cm}^{2}$ used appeared adequate.

c. Numerous comparisons were made with samples collected on filters, again using naturally occurring aerosols. Filter penetration effects limited the accuracy of these tests, but they were adequate to establish losses at 


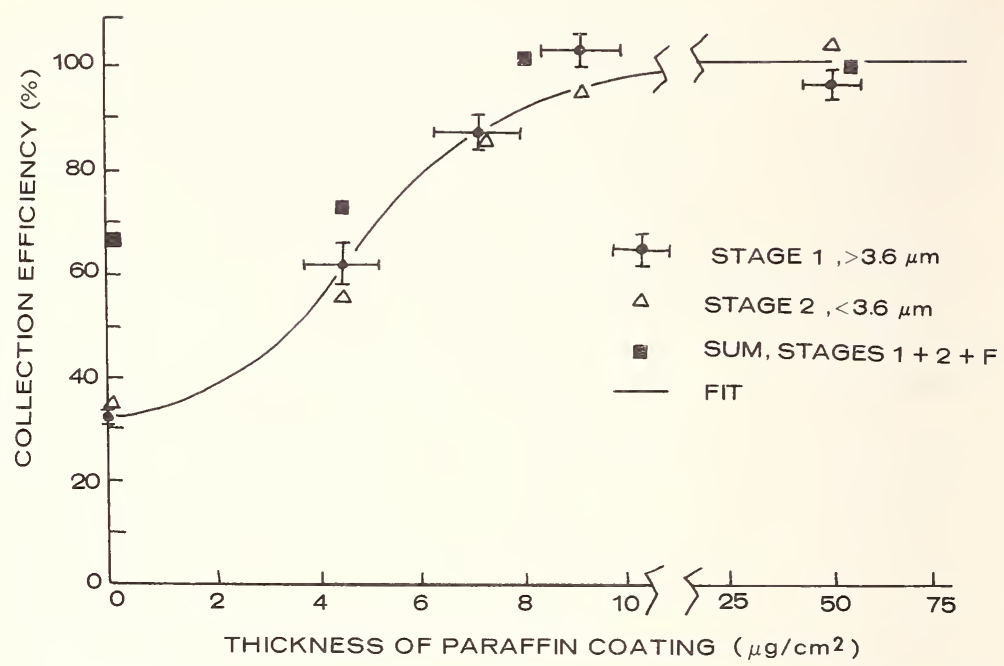

Figure 2. Results of studies to determine adequate paraffin coating thickness.

less than 10 percent for aerosols below $20 \mu \mathrm{m}$ effective diameter. Loss of soil derived aerosols larger than this diameter, however, were a major effect.

d. A limited number of samples were analyzed for total elemental content, hydrogen and above, by elastic alpha scattering, for both stages and the filters. These tests gave results totalling between 75 and $115 \mu \mathrm{g} / \mathrm{m}^{3}$, in reasonable agreement with average mass values obtained by gravimetric methods from high volume filter samplers at the same sites. These comparisons will be greatly expanded in the coming year.

\section{Particle Loss in Filtration}

As was mentioned in section II.A., a surface deposition filter was chosen in order to minimize matrix effects associated with the absorption of low $\mathrm{Z}$ x-rays such as those from sodium in the filter medium. However, a major amount of filter penetration occurred, and corrections had to be applied.

a. The importance of these effects was calculated by folding the penetration values established by Spurny and Lodge [4] at each size range into the mass distribution established by Whitby et al. [3] in the 0.1 to $0.8 \mu \mathrm{m}$ size range. This resulted in a calculated capture efficiency of 48 percent in the 0.1 to $0.65 \mu \mathrm{m}$ interval. 
b. Lead aerosols in California have been shown by this and other studies to have a size distribution typical of the anthropogenic peak of Whitby et al. [6]. In addition, lead aerosols are monitored by standard high volume samplers at many locations. A comparison of $38 \mathrm{Hi}-\mathrm{Vol}$ lead values with the results of the impactor were used to evaluate filter penetration, giving a value of $(54 \pm 5 \%)$ for the capture efficiency of the nuclepore filter. This value was used for the correction factor for all elements occurring in the less than $0.65 \mu \mathrm{m}$ size range.

\section{Particle Sizing Effects}

a. Uranene dye studies performed by Environmental Research Corporation provided the primary standard by which the size cuts were set (fig. 3). Note also that these cuts were very similar to well documented values for the aerodynamically similar stages 2 and 4 of the standard Lundgren impactor $(\rho=1)$.

$\begin{array}{ll}\text { Stage 1-Multiday }-3.6 \mu \mathrm{m} & (1 \mathrm{cfm})^{*} \\ \text { Stage 2-Lundgren-5.0 } \mu \mathrm{m} & (4 \mathrm{cfm}) \\ \text { Stage 2-Multiday }-0.65 \mu \mathrm{m} & (1 \mathrm{cfm}) \\ \text { Stage 4-Lundgren-0.5 } \mu \mathrm{m} & (4 \mathrm{cfm})\end{array}$

* Airflow rates are given in cubic feet per minute.

b. Confirmation of the particle sizing capabilities of the unit were obtained through scanning electron microscopy (SEM) pictures of each stage and the filter. Several elemental profiles were also taken on the SEM. Several dozen scans were taken, and while loss of volatiles and density variations did not allow direct comparison with uranene dye studies, semi-quantitative agreement was obtained. In particular, particles seen on the after filter were properly sized, with the few larger particles seen being "fluffy" substances of low melting point, mainly light element composition, and low density. This was important in evaluating results obtained showing major amounts of soil derived elements in the less than $0.65 \mu \mathrm{m}$ particle size range. The upper size range was also determined by SEM scans to occur at around $14 \mu \mathrm{m}$ diameter for real particles. Since elemental data and SEM morphology indicated soil derivation for most of the mass, a density of $\sim 2 \mathrm{~g} / \mathrm{cm}^{3}$ was assumed for these particles, resulting in an effective aerodynamic diameter of $\sim 20 \mu \mathrm{m}$. This agreed with rough calculations for intake capture at 3 knot winds.

c. Evaluations of nonoptimum collection effects as they affected particle sizing were made as part of the paraffin tests (II.B.1). As shown in table 1, particle sizing errors for uncoated stages were major, in fact, twice as serious as particle loss effects. Thus, the improvement shown in 


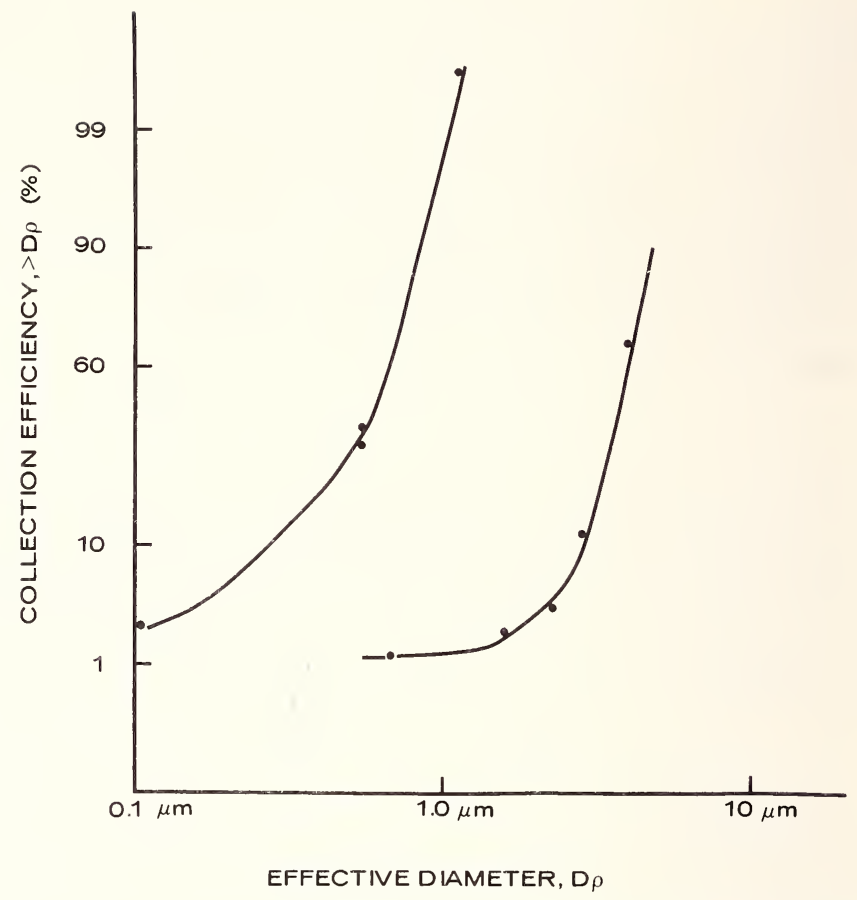

Figure 3. Uranene dye studies provided the primary standard for determining size cuts.

TABLE 1. Summary of sample collection corrections

1. Large particle cut-off

2. First stage cut point

3. Second stage cut point

4. Filter cut point

5. Impaction capture efficiency

6. Filter capture efficiency

7. Sizing errors due to nonoptimum collection
$D \rho=20 \mu \mathrm{m}, \rho=1$, variable $\mathrm{w} /$ wind velocity

$D \rho=3.6 \mu \mathrm{m}, \rho=1$

$D \rho=0.65 \mu \mathrm{m}, \rho=1$

$D \rho=0.1 \mu \mathrm{m}, \rho=1$, effective

$(94 \pm 4) \%$

$(54 \pm 5) \%$

$<5 \%$ 
sizing efficiency as the paraffin coating increased not only set limits on sizing errors but also particle loss effects.

d. Important indirect evidence of the proper operation of the unit with regards to sizing was given by the variations seen in elemental values from stage to stage during this program. On many occasions, values on the first stages were 100 times the values on the filter, or conversely, values on the second stage plus filter were 30 times the values on the first stage. This probably represents the strongest quantitative evidence of correct sizing in many instances.

\section{Description of Sample Transport AND HANDLING}

In order to establish a fast, inexpensive and reliable method for transporting samples and clean replacement materials between sampling sites and the analytical laboratories at Davis, incoming and outgoing samples were mailed weekly in a specially designed container which housed the two rotary drums on which were placed the mylar coated strips and seven petri dishes containing the $47 \mathrm{~mm}$ nuclepore filters. The package was designed to minimize damage in the mails and contamination that could occur in transport. The samples were received in house, logged in, checked against the accompanying flow record, marked, and prepared for analysis. After being cut, the mylar samples were mounted into glassless plastic slide frames and placed into appropriately coded slide trays. The nuclepore was mounted as received in slide frames.

\section{Validation of Sample Transport AND HANDLING TECHNIQUES}

\section{Contamination During Transport and Handling}

It was necessary to evaluate contamination in transport and handling. This was accomplished in the following manner. Mylar was cut to size and wrapped around the drums. The drums then were dipped into a paraffin toluene solution and allowed to dry, the standard procedure for all drums. The drums were then placed in the specially designed mailer and sent to a remote site. The drums were placed in an operating unit. The intake to the two-stage section was then disconnected. The drums were allowed to go through the normal week-rotation. At this point the drums were sent back to Davis where they were handled exactly as any other sample to be analyzed. It should be emphasized that the drums were left in a running 
impactor and the possibility existed for some aerosol to settle out. The following table lists the ratio, (handling amount)/(average actual amount). The average actual amount was determined from the results of site operational during the same time period throughout the area, and represents an average aerosol. The results were as follows:

$\begin{array}{cc}\text { Element } & \text { Percent } \\ \mathrm{Al} & 5.2 \\ \mathrm{Si} & 9.0 \\ \mathrm{~S} & 1.5 \\ \mathrm{Cl} & 2.0 \\ \mathrm{~K} & 11.4 \\ \mathrm{Ca} & 7.9 \\ \mathrm{Fe} & 8.3 \\ \text { All others } & <.5\end{array}$

The experiment was repeated at a later date in exactly the same manner with the following exception: the drums were not placed in an operating unit. The following ratios were obtained:

$\begin{array}{cc}\text { Element } & \text { Percent } \\ \mathrm{Al} & 1.0 \\ \mathrm{Si} & 2.0 \\ \mathrm{~S} & <1.5 \\ \mathrm{Cl} & <1.5 \\ \mathrm{~K} & 2.0 \\ \mathrm{Ca} & <1.5 \\ \mathrm{Fe} & <1.5 \\ \text { All others } & <1.5\end{array}$

This represents a more reasonable estimate of the contamination problem. This is well within the stated uncertainties of the system and no corrections are applied.

\section{Particle Losses in Transport and Handling}

The effect of particles being shaken or removed from the coated drums were found to be negligible. One method of showing this is to divide a sample immediately after collection, preparing one immediately for analysis and allowing the other to proceed through the mailing process. The ratio (mailed)/(unmailed) will then give the loss due to "shake-off."

This ratio was found to be essentially one and no correction is applied $(<2$ percent). Although one cannot say that this will always be the case 
with all types of aerosols, the upper size limit of $20 \mu \mathrm{m}$ and the paraffin coating appear to combine to prevent major losses, even for dry soilderived aerosols. (See table 2.)

\section{TABLE 2. Summary of sample transport and handling corrections}

1. Contamination in handling

2. Loss of particles during transport

3. Loss of material during storage
$\$ 15 \mathrm{ng} / \mathrm{m}^{3} \mathrm{Si},<10 \mathrm{ng} / \mathrm{m}^{3} \mathrm{Al}, \mathrm{K} ;<6 \mathrm{ng} / \mathrm{m}^{3}$ others $<2 \%$ of Si value at most locations; less for other elements

$<2 \%$

$\ll 10 \%$

\section{Loss of Material During Storage}

Since the analytical method was nondestructive, all samples were stored for further analysis if necessary. Losses of materials can in principle occur during extended storage, but no evidence of this effect for any element has been detected after one year $(<10$ percent). This study is in an early stage. but better evidence should become available during the coming year.

\section{E. Description of the Analytical System}

A diagram of the system is given in figure 4 . An $18 \mathrm{MeV}$ alpha beam from the cyclotron passed through remotely readable graphite collimators and impinged on the thin target which was mounted at an angle of $45^{\circ}$ to the incoming beam. The target slide changer operated under realtime computer control. The beam spot was made uniform by use of a diffusion foil, $0.25 \mathrm{mil} \mathrm{Al}$. The beam was then collected by the Faraday cup and integrated to a precision of about 2 percent to give the total charge Q that passed through each sample. X-rays passed through an active filter and a $25 \mu \mathrm{m} \mathrm{Be}$ window and were converted into electrical pulses by a $10 \mathrm{~mm}^{2}$ $\times 3 \mathrm{~mm} \mathrm{LN}$ cooled $\mathrm{Si}(\mathrm{Li})$ detector and associated pulsed optical feedback circuitry. ${ }^{1}$ Data were accumulated in a PDP $15 / 40$ computer with ND 2200 ADC's integral to the system, giving a spectrum of characteristic $x$ rays and a smooth background.

The number of characteristic X-rays, $\mathrm{N}_{z}$, corresponding to some transition of element $Z$ was correlated with the areal density $(\rho t)_{z}$ of the element present in the sample by $\mathrm{N}_{z}=\mathrm{A}_{z}(\rho \mathrm{t})_{z} \mathrm{Q}$. where $\mathrm{A}_{z}$ is a constant determined through use of gravimetric standards. Major effort was expended

${ }^{1}$ Supplied by Kevex. Inc., and Modified at the Crocker Nuclear Laboratory 


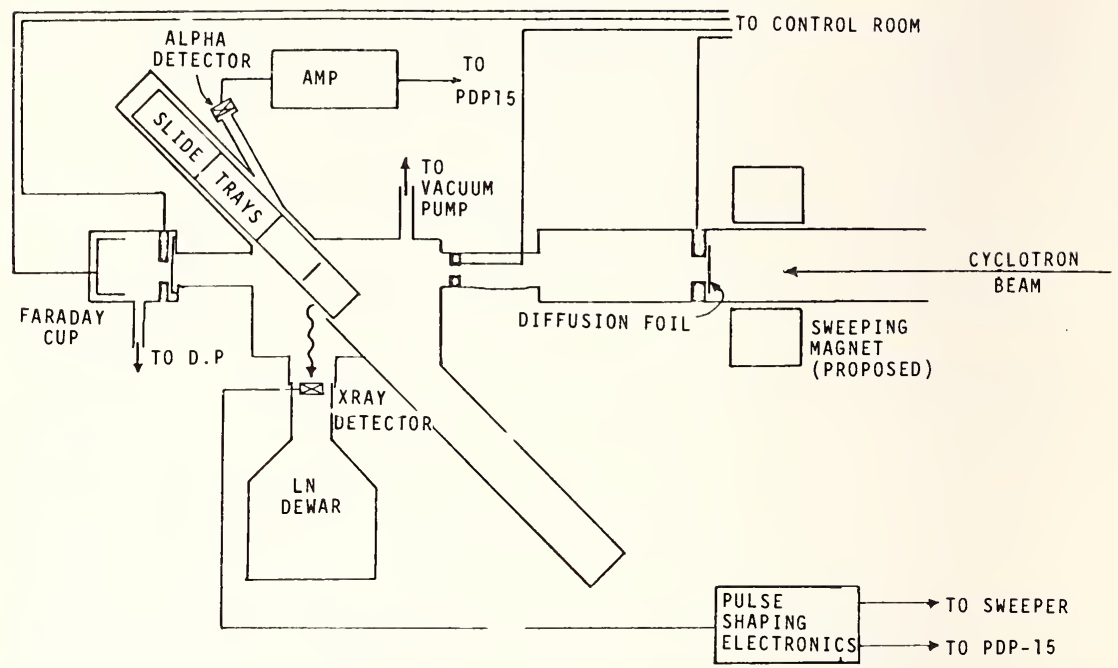

Figure 4. Diagram of the aerosol analysis system.

in design of the electronics and beam handling in order to insure accurately in the acquisition of the events comprising $\mathbf{N}_{z}$. Dead time corrections were automatically applied [7].

All relevant acquisition parameters were entered on the system teletype using a priority interrupt switch in the CAMAC system. These were stored along with the data. After the pre-set charge was accumulated, the spectrum and parameters were recorded on magnetic tape for permanent storage and on an internal disk for immediate analysis by the analysis subroutines. These subroutines, which run concurrently with the acquisition are described elsewhere [8].

An average analysis normally required about 100 seconds of accelerator time and about 25 seconds of computer time, run concurrently. Elements sodium and heavier were routinely detected with minimum detectable limits ranging from a few nanograms per centimeter squared to, when interferences were present, several hundred nanograms per centimeter squared.

\section{F. Validation of the Analytical System}

Validation of the analytical system presented a number of problems, since the amount of given element recorded in an aerosol sample varied from a few nanograms to a few micrograms for many elements from sodium to uranium. The following techniques were used to insure accuracy. 
1. In order to determine the constant $\mathrm{A}_{z}, 28$ gravimetric standards for elements between sodium and uranium were purchased. ${ }^{2}$ The values for each standard were quoted in $\mu \mathrm{g} / \mathrm{cm}^{2}$ to an uncertainty of \pm 5 percent by the manufacturer. These foils were run in the same configuration as the aerosol samples, and provided primary calibration for the system. Total error in $\mathrm{A}_{z}$ in operational conditions was about \pm 7 percent.

2. In order to check the standards, as well as to provide calibration for elements for which no standard was purchased, atomic cross sections for ion excitation at a number of ion energies were extracted. These were plotted, and fit with a polynomial expansion (fig. 5). This fit resulted in values superior to those provided by individual foils, as shown by the need for a 15 percent adjustment for the zinc value as shown in figure 5 .

${ }^{2}$ Micromatter Corporation, Seattle, Washington.

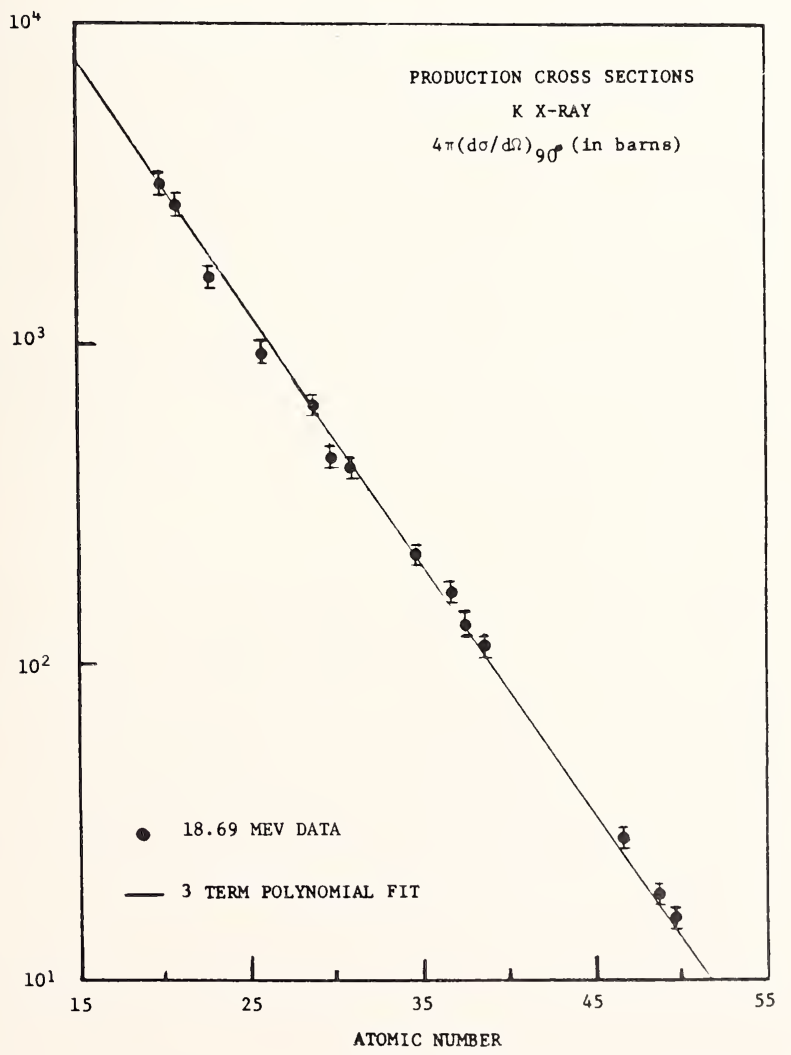

Figure 5. Plot of atomic cross section values were used to check standards or to provide calibration for elements not covered by standards. 
3. Calibration of the system was verified through reanalysis of samples on the calibrated $\mathrm{x}$-ray fluorescence analysis system also present at the laboratory. This also allowed studies to be made of losses of volatiles, since no vacuum was required.

4. Formal and informal interlaboratory comparisons have been routinely utilized to verify operation of the system. A list of such studies made during the past year is shown in table 3. These comparisons confirmed that the standard error of \pm 10 percent quoted was routinely achieved.

5. Although considerable effort was expended at the commencement of every analytical run to verify system operation through reperformance of system checks and analysis of six to eight gravimetric standards, a program of regular reanalysis of aerosol samples has been instituted that results in about 5 percent of all samples being checked during each year. Results to date indicate reproducibility to better than \pm 5 percent for elements present in amounts that allow adequate statistical precision.

6. Tests have been run to detect changes that occur in samples during exposure to vacuum and beam irradiations. In samples run to very high excitations (64 times normal integrated beam flux), some loss of halogens has been detected $(\sim 25 \%)$. This effect appeared to be linear with excitation, and thus was negligible for normal analyses. Likewise, in exposure to vacuum for 24 hours, 8 times normal exposure, no loss of any element on an aerosol sample was detected $(<5 \%)$.

7. Beam energy loss through the aerosol samples was calculated to be $500 \mathrm{keV}$. The change in cross section is negligible $(<5 \%)$. Beam scattering by the aerosol sample was also calculated. At $18 \mathrm{MeV}$ and the thickness of the aerosol negligible scattering was expected. This was verified by experiment.

8. Matrix effects for aerosol samples have two major sources. The first is particle size effects due to self-absorption of low energy x-rays in a single particle. Using information on particle size gained from SEM photographs of actual aerosol samples, it was possible to establish correction factors for average particle sizes on the first and second stages using the model described by Rhodes and Hunter [15]. Relative numbers of particles within each size range were derived from the bimodal mass distribution [2]. The results of these calculations are given in the summary for each stage.

Particle size correction factors were verified through comparison with sized dust samples of kncwn elemental composition. ${ }^{3}$ In addition, a convincing $\mathrm{Na}$ to $\mathrm{Cl}$ ratio of $0.65 \pm 0.06$ was found when these corrections 


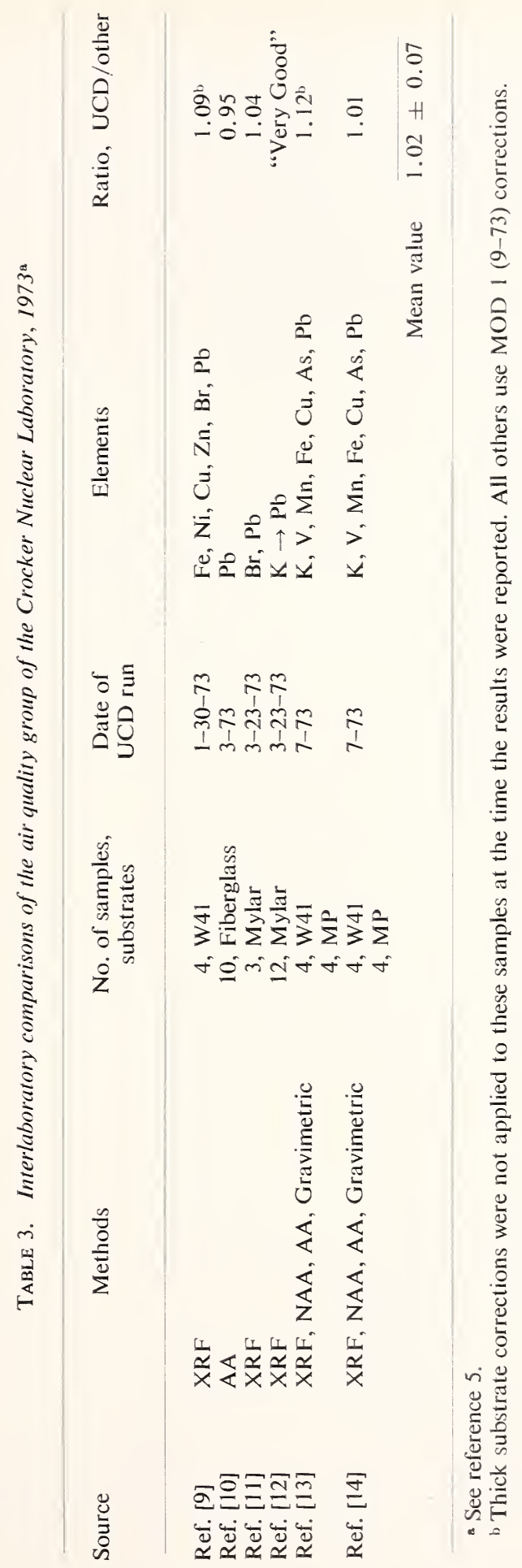


were applied to aerosol samples dominated by sea salt, giving a molar equivalence for $\mathrm{NaCl}$ to \pm 10 percent.

Secondly, self-absorption manifests itself as a target loading problem as well as a particle size problem. This has proven to be a small effect for low $Z(Z<10)$ matrices and higher $x$-ray energy regions $\left(E_{x}>6.5 \mathrm{keV}\right)$. Selfabsorption corrections lend themselves nicely to computer analysis providing certain assumptions are valid. The prime assumption is that monoenergetic $\mathrm{x}$-rays passing through the specimen are attenuated exponentially with depth at a rate characterized by a self-absorption coefficient $(\mu)$ for that material [16].

For aerosol matrices where carbon, oxygen, hydrogen and nitrogen comprise the majority of the matrix, it is further assumed that the $\mu$ values for the target-composite are continuous over the range of analysis. Experimentally it has been found that aerosol targets falling within the con-

\section{TABLE 4. Summary of analytical corrections and uncertainties}

1. Statistical uncertainty in peak counts - variable

2. Uncertainty in gravimetric standards - $-5 \%$

3. Integration of ion beam - $\quad \pm 2 \%$

4. Ion beam attenuation $-<5 \%$

5. Electronic corrections, accuracy $\quad-\quad \pm 5 \%$

6. Peak integration and background $- \pm 7 \%$, (variable) uncertainties

Thus, reproducibility system uncertainty

$-\quad \pm 5 \%$

$-\quad \pm 10 \%$

7. Loss of volatile elements (beam $+\quad-<5 \%$ vacuum)

8. Particle size corrections:

$\begin{array}{lccc} & \text { Stage } 1 & \text { Stage } 2 & \text { Filter Stage } \\ \mathrm{Na} & 2.49 & 1.32 & <10 \% \\ \mathrm{Mg} & 1.96 & 1.22 & <10 \% \\ \mathrm{Al} & 1.68 & 1.16 & <10 \% \\ \mathrm{Si} & 1.49 & 1.12 & <10 \% \\ \mathrm{P} & 1.37 & 1.09 & <10 \% \\ \mathrm{~S} & 1.26 & 1.07 & <10 \% \\ \mathrm{Cl} & 1.31 & <10 \% & <10 \% \\ \mathrm{~K} & 1.12 & <10 \% & <10 \% \\ \text { others } & <10 \% & <10 \% & <10 \%\end{array}$

9. Loading Corrections (for $120 \mu \mathrm{g} / \mathrm{m}^{3}$ aerosol)

$\begin{array}{lccc} & \text { Stage } 1 & \text { Stage } 2 & \text { Filter Stage } \\ \mathrm{Na} & <10 \% & 1.70 & 1.22 \\ \mathrm{Mg} & <10 \% & 1.39 & 1.11 \\ \mathrm{Al} & <10 \% & 1.23 & 1.07 \\ \mathrm{Si} & <10 \% & 1.14 & 1.07 \\ \text { others } & <10 \% & <10 \% & <10 \%\end{array}$

Note: Uncertainty in particle size and loading corrections are estimated at \pm 20 percent for most ambient aerosols, although, especially for the particle size corrections, worse cases are common. These corrections thus increase the total error for the four lightest elements. 
straints of the above assumptions absorb $x$-radiation effectively as if they were composed of a single low- $\mathrm{Z}$ element. This effective $\mathrm{Z}$ can easily be determined by summing elemental amounts present in the target. Once this value is determined for a particular matrix, it is a simple task for the computer to linearly interpolate between theoretical curves of integral $\mathrm{z}$ values to obtain self-absorption corrections to within 10 percent. Thus, these corrections were applied individually to each stage and each sample. Typical values for an average $\left(120 \mu \mathrm{g} / \mathrm{m}^{3}\right)$ aerosol are given in the summary (table 4).

\section{Conclusions}

Experience gained during the first year of the California aerosol monitoring program has shown that, with a continuing systematic program of quality control, one can generate the enormous number of values possible with energy dispersive $\mathrm{x}$-ray systems with acceptable total uncertainties. To accomplish this end, however, the program was required to expend almost 20 percent of its total resources in this one area.

\section{References}

[1] Lundgren, D. A., J. Air Poll. Control Assoc. 20, 603 (1970); Lundgren, D. A., Atmospheric Aerosol Composition and Concentration as a Function of Particle Size and Time, presented at 62d Annual APCA meeting, New York, 1969; Lundgren, D. A., An Aerosol Sampler for Determination of Particle Concentration as a Function of Size and Time, J. Air Poll. Control Assoc. 17, (4), (April 1967).

[2] Butcher, S. S. and Charlson, R. J., An Introduction to Air Chemistry, Academic Press, New York, (1972).

[3] Whitby, K. T., Husar, R. B., and Liu, B. Y. H., J. Colloid Interface Sci. 39, 177 (1972).

[4] Spurny K. R. and Lodge, J. P., Jr., Collection Efficiency Tables for Membrane Filters, National Center for Atmospheric Research Publications, NCAR-TN/STR-77. Boulder, Colorado, (Aug. 1972).

[5] Cahill, T. A. and Feeney, P. J., Final Report to the California Air Resources Board on Contract ARB-502, Contribution of Freeway Traffic to Airborne Particulate Matter, UCD-CNL, 169 (1973).

[6] Hidy, G. M., et al., Characterization of Aerosols in California, Report on ARB Contract No. 358; see also ref. 5.

[7] Cahill, T. A., Final Report to the California Air Resources Board and Project Clean Air, University of California, Riverside on Contract PCA-038, Cyclotron Analysis of Atmospheric Contaminants, UCD-CNL 162 (1972); Flocchini, R. G., Feeney, P. J., Sommerville, R. J., and Cahill, T. A., Sensitivity versus Target Backings for Ele- 
mental Analysis by Alpha Excited X-Ray Emission, Nuclear Instruments and Methods 100,397-402 (1972).

[8] Harrison, J. F. and Eldred, R. A., Automatic Data Acquisition and Reduction for Elemental Analysis of Aerosol Samples in Advances in X-Ray Analysis, J. B. Newkirk and C. O. Ruud, Eds., Vol. 17, pp. 560-570, Plenum Press (1973).

[9] Bonner, N. and Camp, D., Final Report to the California Air Resources Board, Elemental Analysis of Air Filter Samples Using X-Ray Fluorescence, Contract ARB037.

[10] Ranzieri, A., California Division of Highways, Sacramento, California.

[11] Private Communication

[12] Reported at meeting on aerosol analysis organized by: J. Cooper, Battelle Northwest, Seattle, Washington (March 1973). For estimating mean value, used factor 0.98 from notes taken at meeting.

[13] Special section, Denver X-Ray Meeting, organized by J. Rhodes (CSI), D. Camp (LLL) and J. Cooper (BNW).

[14] Camp, D. C., Intercomparison of Trace Element Determinations from Five Analytical Techniques, ANS Transactions 17, 106 (1973). Numerical value from notes taken at meeting.

[15] Rhodes, J. R. and Hunter, C. B., Particle Size Effects in X-Ray Emission Analysis, Journal of X-Ray Spectrometry 1, 113-117 (1972).

[16] Brady, F. P. and Cahill, T. A., Report to the National Science Foundation on Grant GI-32932, Development of X-Ray Fluorescence Analysis and Application, UCDCNL 166 (1973). 


\title{
CHARACTERISTICS OF ENVIRONMENTAL AIRBORNE FISSIONABLE MATERIAL
}

\author{
Bruce M. Center, Kurt E. Jackson, and F. H. Ruddy \\ Nuclear Radiation Center \\ and \\ Department of Chemistry \\ Washington State University \\ Pullman, Washington 99163 U.S.A.
}

\begin{abstract}
Methods are reported for performing a quantitative assay of environmental air samples for fissionable material. Determination of size characteristics using the nuclear track technique is given and a comparison is made with size characteristics obtained from an aerodynamic (cascade impactor) separation. Special emphasis is placed on the important problem of detection of plutonium in the environment.
\end{abstract}

Keywords: Alpha particle tracks; fission radiography; solid dielectric track recorders; ${ }^{239} \mathrm{Pu}$ track.

\section{Introduction}

In the past three decades, the worldwide inventory of the man-made element plutonium has been steadily increasing. The advent of breeder reactors is expected to greatly increase the amount of plutonium produced and reprocessed. The increasing risk of environmental insult due to the accidental release of plutonium is one of the problems which must be dealt with in the next decade. Plutonium is one of the most toxic substances known to man, being lethal in microgram amounts. A recent report by Bair and Thompson [1] has focused on the hazards of all forms of plutonium including airborne insoluble particulate plutonium. The toxicity of particulate forms of alpha emitters in the lung is presently a subject of debate [2]; however, it is clear that methods of environmental assay that can provide information on identity, particle size, and dose rate to the surroundings from airborne particulate alpha radioactivity are needed at this time. 


\section{Methods}

The methods reported herein employ solid dielectric track recorders [3] as the principal means of autoradiographic detection of $\alpha$-particles and neutron induced fission fragments. Air samples may be taken by conventional methods and then subjected to autoradiography using dielectric track detectors. Particles containing alpha emitting isotopes will give rise to clusters of alpha particle tracks in a cellulose nitrate track detector. Similarly, particles containing fissionable material may be identified by neutron irradiation followed by observation of clusters of fission tracks in mica track detectors. For each individual particle the alpha decay rate, mean fission cross section, and ratio of decay rate to fission cross section is obtained. From these measurements one may infer particle size, isotopic composition of the particles, and dose rate to the surrounding material.

The methods described above have been applied to an air filter sample taken from a contaminated lab inside a plutonium reprocessing facility. The diameter distribution (assuming ${ }^{239} \mathrm{PuO}_{2}$ spheres as the source) is shown in figure 1. This type of distribution is typical of particles which penetrate an air filter and is consistent with the measurements of Hayden [4] using similar techniques. Several particles with diameters close to 1 $\mu \mathrm{m}$ were observed. These correspond to "hot particles" as discussed by Tamplin and Cochran [2]. A $1-\mu \mathrm{m}{ }^{239} \mathrm{PuO}_{2}$ particle, if inhaled, would deliver a dose of $180 \mathrm{rad} / \mathrm{h}$ to a volume element of the lung tissue defined by the range of a ${ }^{239} \mathrm{Pu}$ alpha particle (assuming tissue with an average density of $1.0 \mathrm{~g} / \mathrm{cm}^{3}$ ).

The ratio of decay rate to fission cross section was measured for the particles on this filter and was found to be $(10 \pm 2) \times 10^{8} \mathrm{~cm}^{-2} \mathrm{~s}^{-1}$ in agreement with the ratio for plutonium. The ratio was also measured for ${ }^{241} \mathrm{Am}$ and the values for both isotopes are shown in table 1 along with values calculated from the known half-lives and fission cross sections [5].

The problem of detecting smaller amounts of plutonium in the presence of an environmental background of fissionable isotopes $\left({ }^{235} \mathrm{U},{ }^{238} \mathrm{U},{ }^{232} \mathrm{Th}\right)$ and naturally occurring alpha emitters (mainly the daughters of ${ }^{222} \mathrm{Rn}$ ) is more difficult. Samples of the local ambient air have been taken using a BGI cascade impactor which partitions airborne particulates according to aerodynamic size. The impactor used in this study has greater than 50 percent efficiency on each stage for particles greater than the following diameters: stage 1, $9.4 \mu \mathrm{m}$; stage 2, $3.8 \mu \mathrm{m}$; stage $3,2.5 \mu \mathrm{m}$; stage $4,1.8$ $\mu \mathrm{m}$; stage 5, $0.01 \mu \mathrm{m}[6]$. Fission radiography has been applied to samples from each stage in order to determine the relationship between the size of the fissionable particle and the aerodynamic characteristics of this particle or particles to which it may be attached. Such information is criti- 


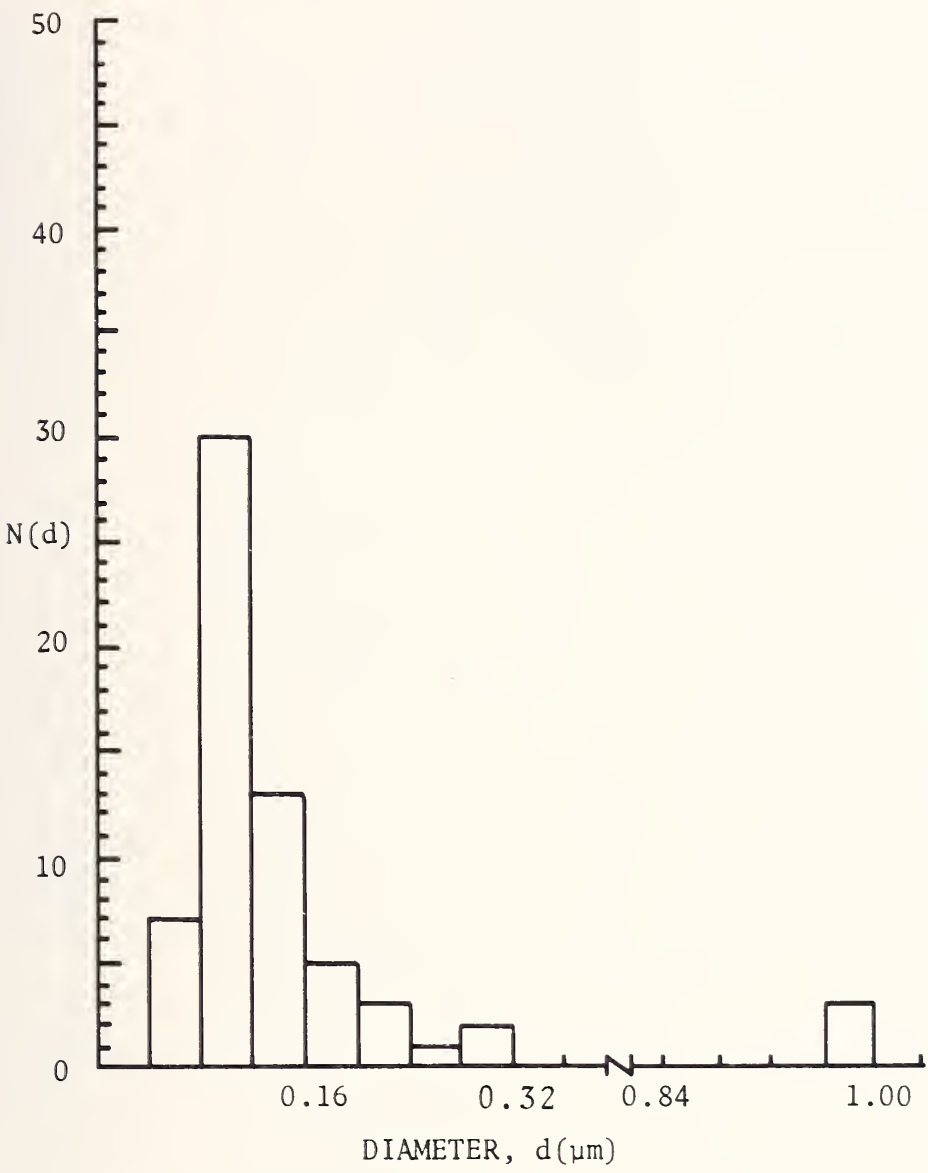

Figure 1. Diameter distribution of plutonium particles on a reprocessing area air filter. The diameters were calculated based on the assumption of spheres of $\mathrm{PuO}_{2}$.

TABLE 1. Experimental and calculated half-life to fission cross section ratios for ${ }^{239} \mathrm{Pu}$ and ${ }^{241} \mathrm{Am}$

$$
\text { Experiment }\left(\mathrm{cm}^{-2}-\mathrm{s}^{-1}\right) \quad t_{1 / 2} / \sigma_{f}\left(\mathrm{~cm}^{-2}-\mathrm{s}^{-1}\right)
$$

$\begin{array}{lll}{ }^{241} \mathrm{Am} & (1.64 \pm 0.13) \times 10^{13} & (1.54 \pm 0.03) \times 10^{13} \\ { }^{239} \mathrm{Pu} & (9.56 \pm 2.16) \times 10^{5} & (12.2 \pm 0.1) \times 10^{5}\end{array}$


cal to the evaluation of inhalation risks. The diameter distribution (assuming natural isotopic abundance $\mathrm{U}_{3} \mathrm{O}_{8}$ spheres) is shown for each stage in figure 2. Qualitatively, the shapes of the distributions are very similar. Most of the fissionable material is concentrated on stages 2 to 4 with much less material associating itself with particles greater than $9.4 \mu \mathrm{m}$ diameter or less than $1.8 \mu \mathrm{m}$ diameter. Plutonium particles from a reprocessing plant would exist mainly as particles with diameters less than $1 \mu \mathrm{m}$, but would probably adhere to larger particles. Several larger particles were found on stages 2 to 4 with $\mathrm{U}_{3} \mathrm{O}_{8}$ diameters that indicated pure particles of $\mathrm{U}_{3} \mathrm{O}_{8}$ but the presence of enriched uranium or other fissionable material cannot be excluded.

An investigation of the natural alpha radioactivity of local ambient air samples has also been undertaken. Conditions which favor the detection of the short-lived $\left({ }^{218} \mathrm{Po},{ }^{218} \mathrm{At}\right)$ members of the radon decay chain as well as conditions which favor detection of the entire chain were employed. In both cases no large clusters of $\alpha$ particles were found which would indicate a concentration of radioactive material on one particle. Clusters of up to three tracks were found, probably corresponding to the sequential decay of one atom in the radon decay series. It is thus thought that natural radioactivity is not an important background problem in $\alpha$ radiography if a sufficiently long delay time ( 1 day) is employed and only clusters with $\geqslant$ 3 tracks are considered. In very long $\alpha$ radiographs, interference from particles of uranium or thorium will undoubtedly be a factor.

Our proposed procedure for characterization of air samples taken from an area of suspected contamination by plutonium (or other toxic alpha emitters) is as follows: The sample is covered with a thin $\left(0.72 \mathrm{mg} / \mathrm{cm}^{2}\right)$ layer of makrofol in order to keep the dust particles in place so that they might be found in each of a series of radiographs. In addition, the makrofol cover acts as a spacer and improves the visual characteristics of the track cluster as well as enhancing the registration of alpha particles with low angles of incidence. The sample is subjected to alpha radiography to identify track clusters corresponding to concentrations of alpha radioactivity. The same particles are then subjected to fission track radiography in order to determine which of these particles correspond to isotopes of uranium or heavier elements. The $\alpha$ decay rate is used as a measure of dose from the particles, and the $\alpha$ and fission rate data are used in conjunction to identify the isotopes present in the sample. These characteristics may be measured on ${ }^{239} \mathrm{Pu}$ particles containing as little as $10^{7}$ atoms or $2 \times 10^{-4} \mathrm{pCi}\left(3.7 \times 10^{-2} \mathrm{~d} / \mathrm{s}\right)$. 

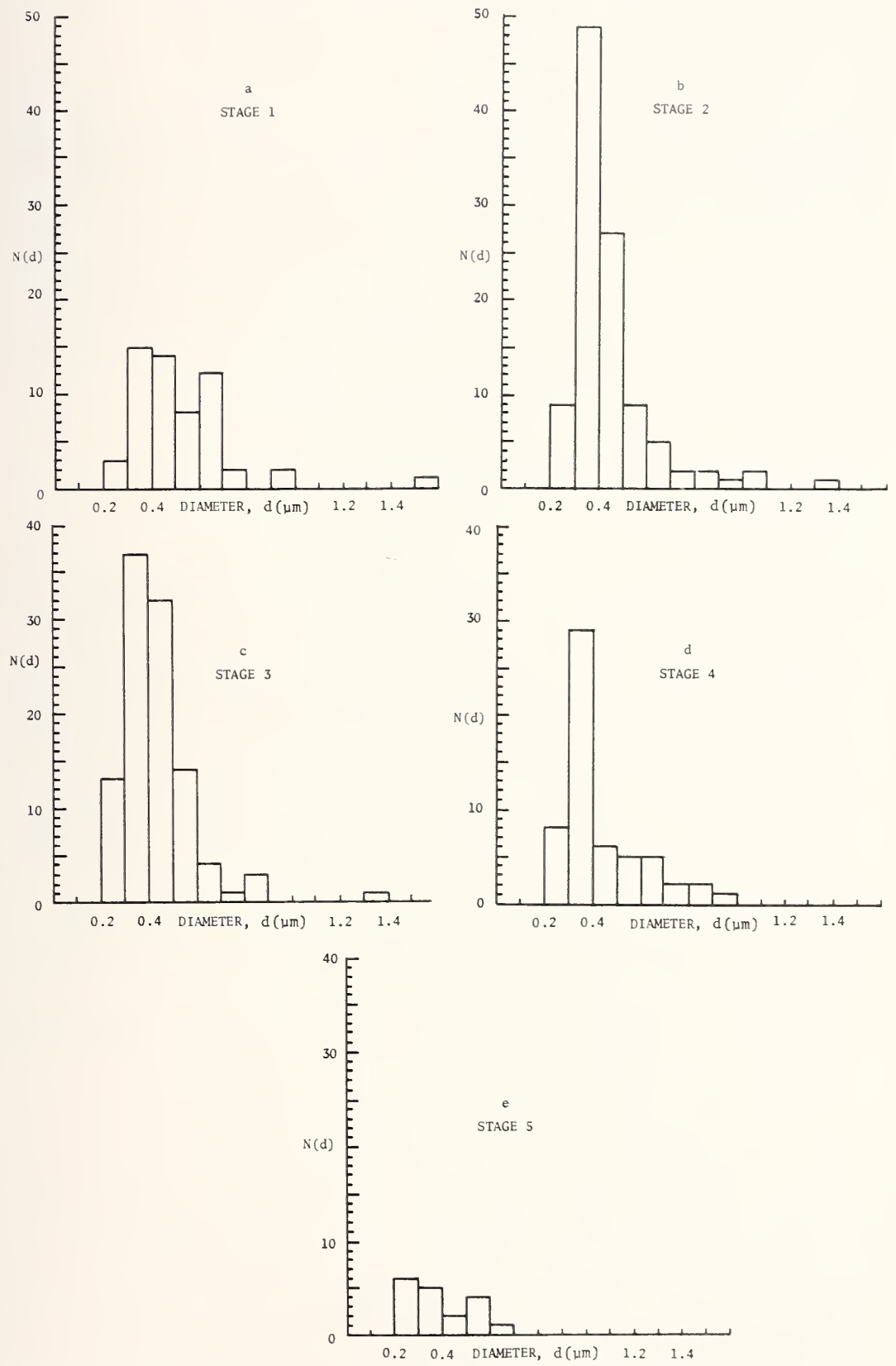

Figure 2. Diameter distributions of fissionable material collected with a cascade impactor. The diameters were calculated assuming spheres of natural $\mathrm{U}_{3} \mathrm{O}_{8}$. Figures $2 \mathrm{a}-2 \mathrm{e}$ correspond to data from stages 1 to 5 respectively. 


\section{Conclusion}

The present methods offer many advantages which can only be achieved with a combination of the more conventional methods. Methods employing chemistry, mass spectrometry, or alpha particle or gamma-ray spectrometry cannot provide data on amount and identity of isotopes present and at the same time provide data on size characteristics of the particles present. Furthermore, the present method is nondestructive allowing the samples to be subjected to subsequent analysis by alternative methods if necessary. The present method is relatively rapid, inexpensive, and provides data which under certain circumstances may be related to the inhalation risk associated with the air being sampled.

\section{References}

[1] Bair, W. J. and Thompson, R. C., Plutonium: Biomedical Research, Science 183, 715-722 (1974).

[2] See, for example Tamplin, A. R. and Cochran, R. B., Radiation Standards for Hot Particles: A Report on the Inadequacy of Existing Radiation Protection Standards Related to Internal Exposure of Man to Insoluble Particles of Plutonium and Other Alpha-Emitting Hot Particles, Natural Resources Defense Council, 1710 N Street, N.W., Washington, D.C. 20036.

[3] Price, P. B. and Walker, R. M., Chemical Etching of Charged Particle Tracks, $J$. Appl. Phys. 33, 3407-3412 (1962); Fleischer, R. L. and Price, P. B., Tracks of Charged Particles in High Polymers, Science 140, 1221-1222 (1963).

[4] Hayden, J. H., Tracking Plutonium at Rocky Flats, Bioassay, Environmental, and Analytical Chemistry 19th Annual Conference, Jackson Lake, Wyoming (1973).

[5] Lederer, C. M., Hollander, J. M., and Perlman, I., Eds., Table of Isotopes, 6th edition, Wiley and Sons, New York (1967).

[6] Gussman, R. A., Sacco, A. M., and Ladd, R. E., Design and Calibration of a High Volume Cascade Impactor, Paper No. 72-27, Annual Meeting of the Air Pollution Control Association (1972). 


\title{
ANALYTICAL DESIGN IN ACTIVATION ANALYSIS: THE ROLE OF ACCURACY AND PRECISION
}

\author{
Donald A. Becker
}

Analytical Chemistry Division

Institute for Materials Research

National Bureau of Standards

Washington, D.C. 20234 U.S.A.

In order to obtain maximum utilization of the technique of activation analysis, a logical and systematic method for the evaluation of alternate analytical procedures is required, especially for analyses requiring a high degree of accuracy and precision. The activation analysis procedure can be separated into the four basic areas: A) sampling and sample handling; B) irradiation procedures; C) radiochemical separations; and D) detection systems. Each of these areas can then be divided into a number of individual options, each option with its own characteristics, including an uncertainty value.

The resulting analytical design results when these options are fused to form a procedure which will adequately meet the requirements. This paper outlines many of the options available in each area and discusses some of their inherent limitations and uncertainties.

Keywords: Accuracy and precision; activation analysis; analytical design; irradiation procedures; radioactivity detection systems; radiochemical separations; sampling and sample handling.

\section{Introduction}

The technique of activation analysis has progressed from its comparatively recent beginning into one of the most versatile and useful analytical methods available. It is capable of analyzing almost any material for many different elements both destructively and nondestructively. However, in order to obtain maximum utilization of the technique, a logical and systematic method for the evaluation of alternate activation procedures can be very important. This is especially true for analyses requiring a high degree of accuracy and precision. 


\section{Discussion}

A diagram of the processes involved in the activation analysis procedure is presented in figure 1. The overall process is separated into the four basic areas, which are: A) sampling and sample handling; B) irradiation procedures; C) radiochemical separations; and D) detection systems. All of these areas are necessary in every analysis by activation except the third area, radiochemical separations, which is used as needed for specific analytical requirements.

\section{ACTIVATION ANALYSIS}

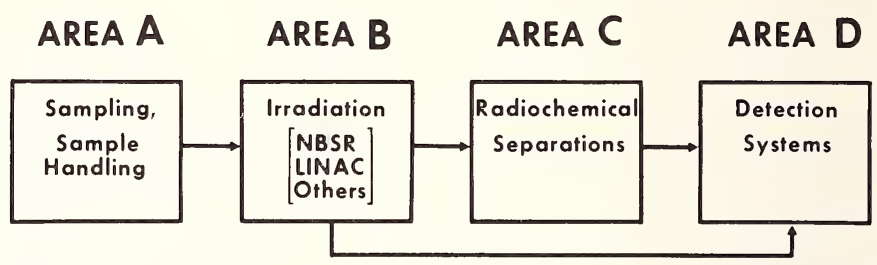

Figure 1. The four areas of interest used for analytical design in activation analysis.

All of the above basic areas can then be separated into a variety of options. Each of these options has its own individual characteristics, including an uncertainty value due to one or more random or systematic analytical biases. The inherent detection sensitivity for the activation determination of the element(s) of interest is a function of these options. Thus, the judicious choice of the best available options provides the optimum attainable analytical result for the particular analytical problem under consideration.

When a trace analytical problem presents itself, most analysts tentatively decide upon a procedure, then evaluate it. This paper describes a method to formalize this evaluation procedure. The advantages of employing this evaluation procedure are threefold: first, it forces the analyst to take the time to think critically about the "best" way to accomplish the end result; second, such a systematic evaluation makes obvious any weak or missing options which could substantially affect the overall analytical program; and third, the absence of a firm accuracy and precision assignment for any option will alert the analyst and, hopefully, will encourage a real effort to be sensitive to accuracy and precision requirements.

This proposed evaluation technique can be used by any laboratory, because it depends only on what capabilities are available to that laborato- 
ry. No laboratory will have all options available to it. It is dependent, however, upon having these capabilities critically evaluated for precision and accuracy.

The overall total uncertainty for such an analytical procedure is the aggregate of all the individual uncertainties. These individual uncertainties are composed of both random and systematic components.

The rest of this paper will be concerned with the four individual areas, selected options available to most laboratories, and examples of some of the problems affecting accuracy and precision in each of these areas.

\section{A. Sampling and Sample Handling}

To assure the validity of the analytical result, the complete sample history should be known and considered. In this first area, there are almost unlimited options available for the taking and processing of the sample for analysis. If not closely controlled and evaluated, however, many of these sampling and processing procedures can introduce significant contamination into the sample, thereby producing a positive systematic bias.

\section{Presampling Contamination}

This problem results when the system being sampled is contaminated before or during the sampling procedure. An example of this would be water sampling from a ship or boat in which contamination from that ship becomes a part of the sample. The second example is recorded by Thiers [1], who reports a significant variation of serum potassium levels due to arm compression during venipuncture. One can only surmise upon the trace element composition during such a sampling procedure.

\section{Sampling Implement Contamination}

Contamination by the sampling implement has been reported by many authors. As a result, the use of plastics has become widespread because of their generally low trace element content.

\section{Contamination or Losses During Sample Processing}

There are a multitude of processes which result in contamination or losses during processing. The effect of moisture on a sample can be sig- 
nificant; the problems involved here include attaining the "real" dry weight, trace element losses during drying procedures, and moisture pickup during weighing procedures. When a solid sample is involved, the need to obtain a representative sample introduces problems of homogeneity as well as induced contamination from grinding, sieving, etc. A liquid sample involves not only positive systematic bias due to contamination, but also negative systematic bias due to adsorption problems on container walls or particles in the solution. Liquids are also particularly susceptible to leaching of impurities from containers and desorption of previously adsorbed contaminants. These processes can occur both during sample processing, or during storage.

\section{Sample Storage Container}

The sample storage container must be chosen carefully in order to minimize the effect upon the trace element(s) of interest. Involved in this decision is the consideration of whether storage will be short term or long term. Various standard textbooks on analytical chemistry have indicated that new glass or silica is significantly worse than old containers from trace element adsorption considerations. However, much of the current analytical thought focuses on plastics for storage containers. The often seen particulate inclusions in Teflon containers should encourage the evaluation of a particular type of container for the use to which it is to be put.

\section{Storage Technique}

There are a variety of preservation techniques which can be used for the storage of various sample matrices. Included are drying (air, oven, or lyophilization) often used for biological materials. Experience in this laboratory has indicated that oven drying of biological materials can result in excessive weight loss [2]. Refrigeration or freezing is often also used for biological materials. Other storage techniques include ashing (oven or oxygen plasma), the use of preservatives (e.g., acid stabilization for water samples), or a variety of other techniques.

\section{Laboratory Atmosphere}

This aspect is very important especially for analyses at the sub $\mu \mathrm{g} / \mathrm{g}$ levels. Experience in this laboratory during the past year comparing preir- 
radiation processing in a normal laboratory atmosphere versus a class 100 clean room has indicated that reductions in blank levels from 1 to 3 orders of magnitude can be achieved $[3,4]$.

\section{Irradiation Containers}

Trace element contamination due to the irradiation container also can be a problem. The two materials primarily used for such containers are polyethylene and quartz. Polyethylene which has been cleaned before use is generally significantly lower in trace element content than is quartz. However, several types of trace elements have been known to diffuse through polyethylene irradiation containers including mercury [5], and halogens [6]. In addition, work by Brune [7] has shown that recoil effects on quartz impurities $30 \mu \mathrm{m}$ deep can contaminate the surface of a previously cleaned container.

\section{B. IRRADiation Procedures}

The options available here include the irradiation source, the particular irradiation facility within a source, irradiation time, and others. Due to lack of space only reactor neutron irradiation factors will be mentioned, although many of the same factors apply to other types of irradiation sources, such as isotopic neutron sources, LINAC, charged particle sources, etc.

The choice of the irradiation source obviously affects the sensitivity, detectability, and selection of trace elements of interest. In addition to this, the capabilities of the irradiation source and the degree to which it has been evaluated play a large part in determining the final result of the activation analysis. For example, in reactor neutron activation analysis, there are many factors which affect the accuracy and precision of the final result. These factors include:

\section{Flux Variations}

The flux variations as seen by the NBS Nuclear Reactor (NBSR) pneumatic tube facilities could introduce a significant systematic bias to the analytical results. However, these flux variations have been evaluated extensively, as illustrated by the data shown in figures 2 and 3. Figure 2 depicts the end-to-end neutron flux variation within an individual rabbit in the five pneumatic tube facilities, while figure 3 reveals the circum- 


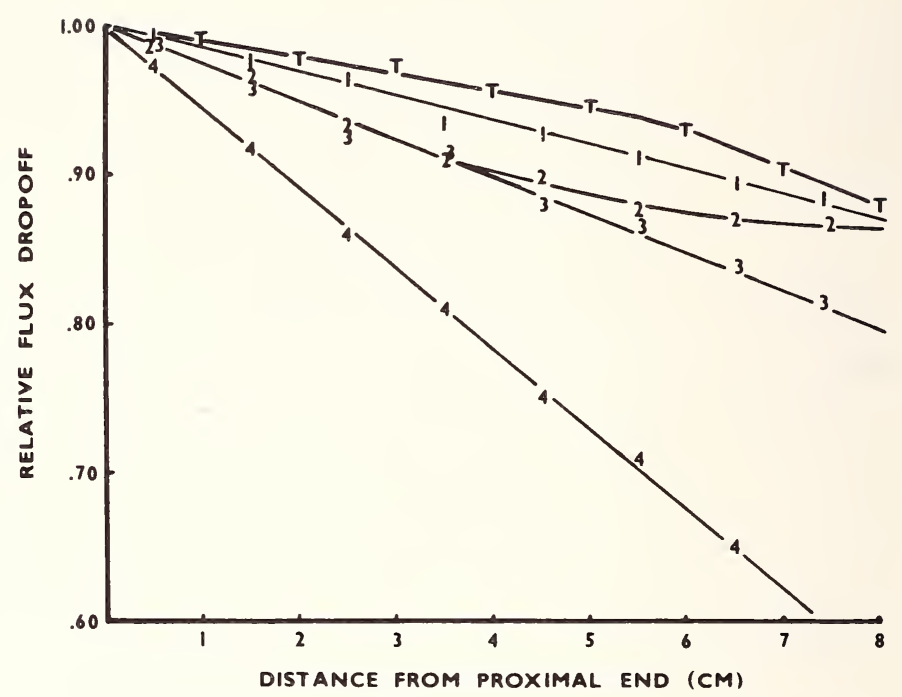

Figure 2. Axial neutron flux variation inside the NBSR pneumatic tube facilities (normalized to proximal end $=1.00$ for each tube).

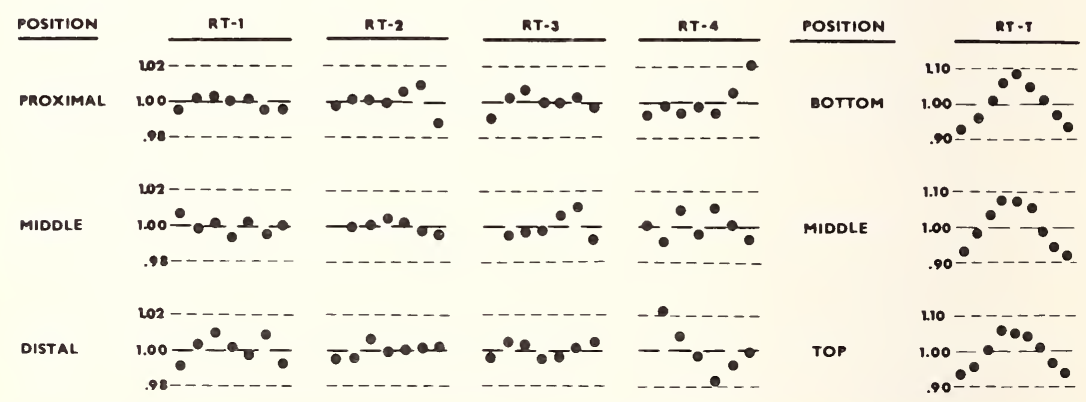

Figure 3. Circumferential neutron flux variation at three positions inside the NBSR pneumatic tube facilities (first data point at each tube position $=$ center of circle).

ferential flux variation in the same rabbit. Additional information on the procedures used in making these measurements can be found in references [8] and [9].

\section{Flux Monitoring}

Effective monitoring of the neutron flux seen by the samples and standards has been found to be necessary in the author's laboratory for high accuracy and precision analyses [8,9]. If done competently in a well- 
evaluated irradiation system, this measurement can compensate for standard to sample variations in neutron flux, variations in power levels (reactor stabilities), and such unknowns as container movement during pneumatic tube transfer. For example, a high precision analysis $[ \pm 0.5 \% \mathrm{SD}$ (1s)] revealed no detectable increase in the uncertainties obtained when the reactor power level changed during an analysis run [10]. This was attributed to very careful flux monitoring.

\section{Neutron Energy Spectrum}

Knowledge of the neutron energy spectrum is important for the evaluation of fast neutron reactions which may occur in the sample. Of greatest interest are the (n,p), (n, $\alpha)$ and $(n, 2 n)$ reactions which may occur on other elements present in the sample or standard which also form the indicator isotope.

\section{Interfering Nuclear Reactions}

This subheading includes, besides the fast neutron reactions mentioned above, the production of daughter isotopes by beta decay or electron capture, production of fission products, and burn-up effects both to produce and to remove the indicator isotope.

\section{Neutron Self Shielding}

There are a number of elements which have thermal neutron cross sections large enough to introduce neutron self-shielding effects. In general, this involves elements with elemental cross sections in excess of five barns or elements having significant resonance integrals. Methods for the estimation of these effects by calculation have been proposed [11,12]. In addition, neutron thermalization effects have been reported for aqueous solutions or for samples with a high hydrogen content [12].

\section{Radiochemical Separations}

One aspect of the rationale behind radiochemical separations is shown in figure 4. A chromium 51 gamma-ray peak is shown here both by itself. and on the top of a Compton background due to cesium 137 and cobalt 60 . The result is obvious and indicates a greatly increased detection sensitivi- 


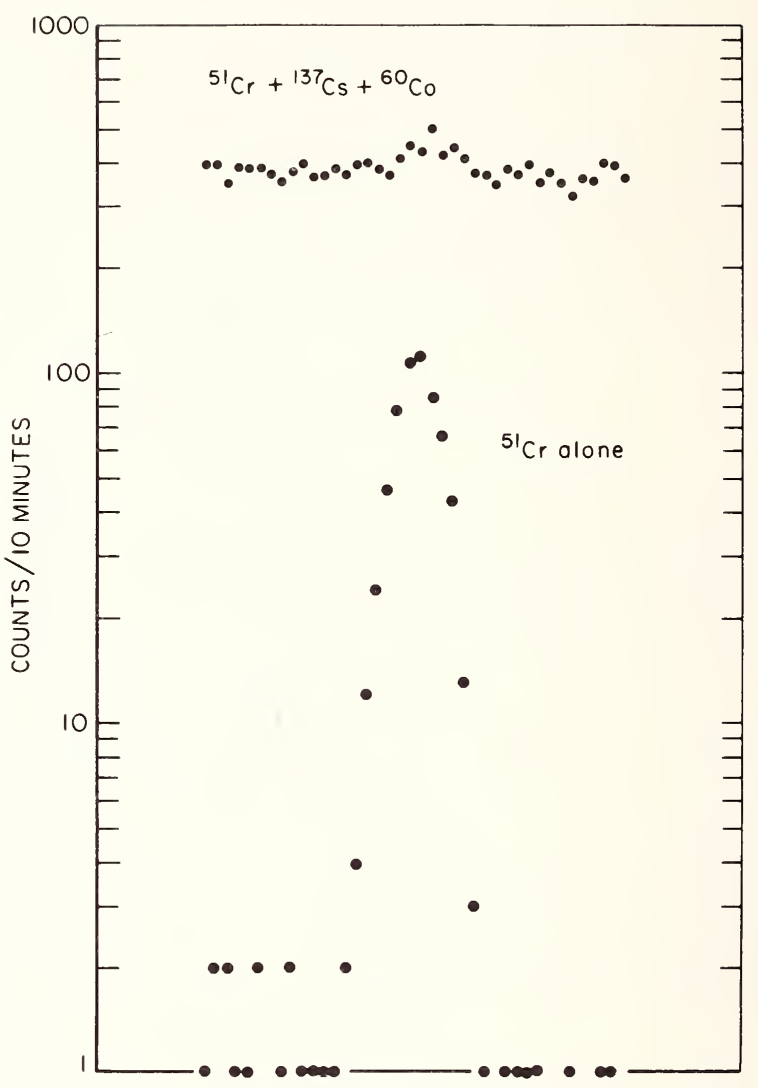

Figure 4. Effect of background on ${ }^{51} \mathrm{Cr}$ peak.

ty for the separated gamma-ray peak coupled with greatly improved analytical precision $(7.1 \%$ vs. $25.2 \%$ relative standard deviation for $\mathrm{n}=$ 10) [13]. In addition, if only one count were made of a real sample with similar characteristics, the accuracy of the determination could also suffer by as much as a factor of two due to statistical fluctuations alone.

Some of the problems affecting accuracy and precision in this area include sample dissolution difficulties (incomplete dissolution, losses during dissolution); inadequate carrier exchange with the sample trace element(s); volatilization losses (during preirradiation processing, during irradiation or when opened after irradiation, and especially during sample dissolution before carrier exchange); adsorption effects on containers (irradiation container, dissolution container, separation container); quality of the separation procedure (single element, multiple element, com- 
plicated procedures, etc.); and, finally, chemical yield measurements (reproducibility, quantitative yield highly desirable).

Each of the above aspects of radiochemical separations can introduce a random or systematic bias into the analytical procedure, and thus should be carefully evaluated and tested before being accepted as a reliable and reproducible procedure.

\section{Detection System}

This area includes radiation detection systems of all types. The choice of the best detection system for the particular trace element(s) of interest can profoundly affect both the detectability and determinability of the analyses. Basically, however, most activation analysis detection systems consist of a single gamma-ray detector ( $\mathrm{Ge}(\mathrm{Li}), \mathrm{Ge}$, or $\mathrm{NaI}(\mathrm{Tl}))$ and associated electronics, with a sample positioning device of some sort. With such a system, a variety of sample radioactivity levels and sizes can be counted rather easily. What often is not realized is that even relatively small differences between sample and standard location, or from sample to sample, in any one of many variables can often produce a significant bias in the resulting count rates.

In order to systematically evaluate the errors involved with gamma-ray counting using such a system, the counting technique was separated into eight subgroups as follows: configuration, positioning, density, homogeneity, intensity, radioisotopic purity, peak integration, and nuclear constants. These subgroups were individually examined and investigated, and have been recently discussed [14].

In particular, three important effects not often considered quantitatively will be briefly discussed here. They are configuration, photon self absorption, and homogeneity. Configuration effects are demonstrated to be extremely important as seen in figures 5 and 6 . In figure 5 , experimental results demonstrate that a 1 millimeter average height increase in sample $v s$. sample or sample $v s$. standard will produce a 3 percent bias at $5 \mathrm{~cm}$ from the detector can. Similarly, in figure 6 , a radioactive source having a diameter of $2 \mathrm{~cm}$ compared to a point source will produce a 5 percent bias when located directly on top of the detector can. Both of these measurements were made using large volume lithium drifted germanium detector systems.

Photon self-absorption effects are a function of the density of sample $v s$. standard, and are extremely important especially when the sample and standard are in different matrices. For example, a standard with a density of 1.00 compared to a sample having the density of 1.35 will introduce a 


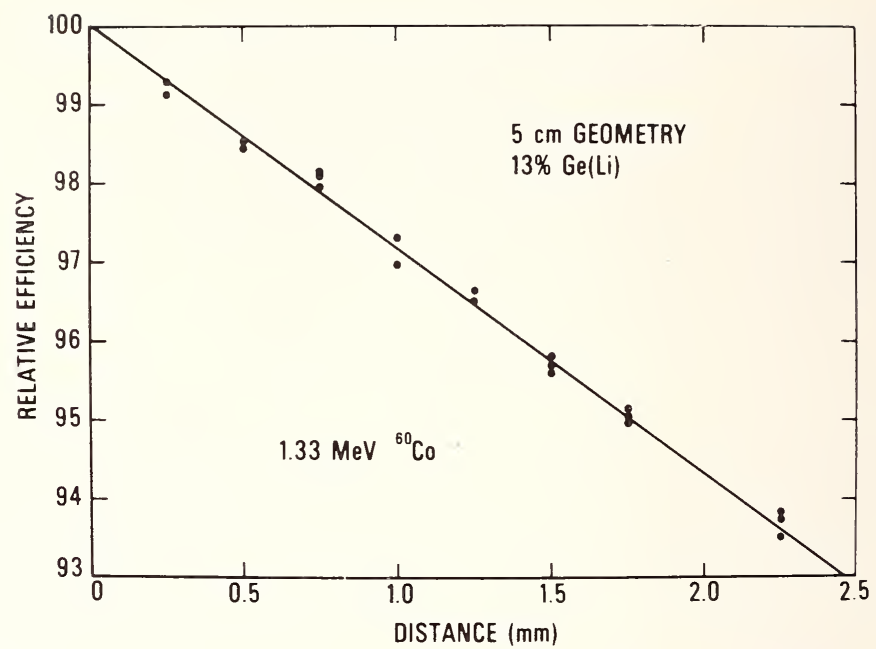

Figure 5. Experimental determination of relative photopeak efficiency vs. increased distance from the detector.

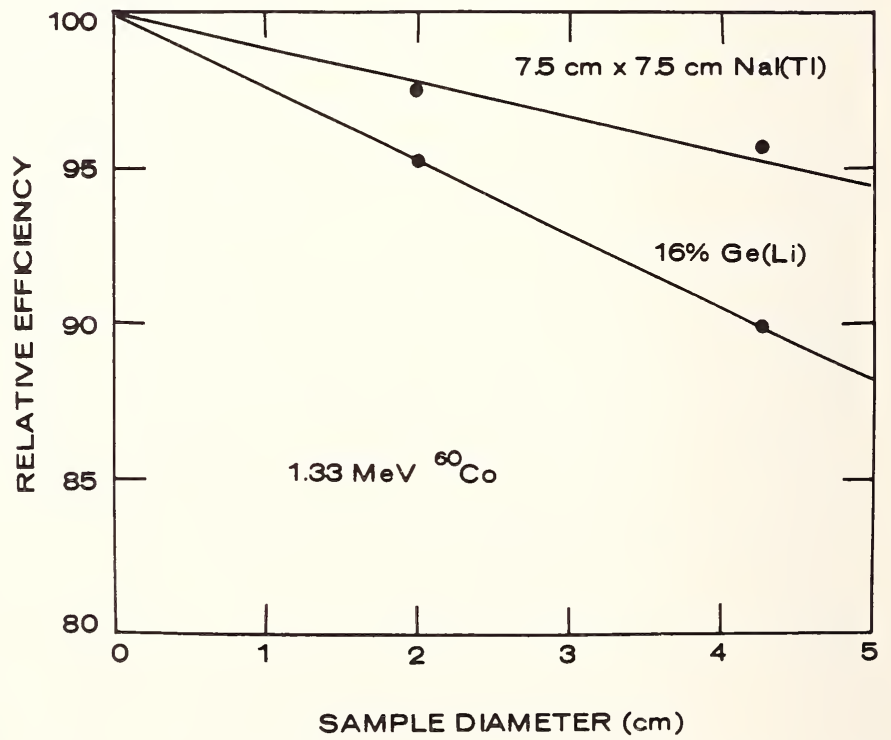

Figure 6. Effect of source diameter on the relative photopeak efficiency (thin sources, counted directly on detector can). 
1 percent bias in the analytical result due to self shielding of a $1.33 \mathrm{MeV}$ gamma ray. This effect is considerably greater for lower energy gamma rays. Thus, the use of aqueous standards for analysis of a rock sample could be expected to introduce a very significant systematic bias.

The homogeneity effect on counting may take the form of at least two variations. The first is variation of the element of interest in the sample, resulting in an effective configuration variation with effects as discussed above. This problem is very difficult to determine and is best treated by eliminating the possibility with homogenization of the sample before analysis. A second homogeneity problem may be induced in the sample matrix itself due to flux variation in the irradiation source. Thus, for a flux variation of 20 percent or more from top to bottom, apparent heterogeneity in the sample or standard could be a very serious problem.

Thus, experimental results have demonstrated that appropriate care must be shown toward the many parameters involved in the various detection systems if good analytical accuracy and precision are to be consistently obtained. In this particular instance, comparability between the sample and the standard in as many characteristics as possible assists significantly in reducing these associated uncertainties.

\section{Standards}

It has been said that for activation analysis the "ideal standard is a material closely similar, both chemically and physically, to the unknown but containing a known amount of the element to be determined...such standards are not likely to be available readily" [15]. In addition to this ideal situation, activation analysts have traditionally used two other methods of producing standards; one is the method of additions, the other is a pure element or its compound. The method of additions entails adding a known amount of the element(s) of interest to a second portion of the sample, with subsequent analysis and evaluation [16]. The use of a pure element or its compound for a standard is also widely used, but introduces the possibility of a number of sources of error. Usually this type of standard is diluted in some suitable way, either as an aqueous solution or as a solid.

In most cases activation analyses use the comparison standard technique, directly compared to the same trace element in a standard material with as closely similar a matrix as possible. The use of comparator standards eliminates the requirement for exact values for neutron flux, reaction cross sections, counting efficiencies, and half-lives to correct the 
decay back to time zero. The requirements of such a comparator standard for high accuracy and precision work include: the level of activity should not be grossly different between the sample and standard; both sample and standard should have similar dimensions in order to eliminate or reduce flux corrections and counting geometry corrections; and they should have similar neutron and gamma attenuation characteristics. Also, both the samples and standards should be carefully monitored for neutron flux using a suitable material, often a pure metal foil. Many analyses in the author's laboratory have successfully used inexpensive, analytical grade copper foil for such monitoring purposes [17].

\section{Conclusions}

Assuming all the systems discussed above are evaluated and calibrated, with contamination and other problems under control, how can this analytical design system be used? First, determine the required accuracy and precision of the analysis, by introspection (self-generated sample) or consultation (outside-generated sample). Next, select the most appropriate available option for each area. Then, obtain or produce a suitable standard for the element(s) of interest. Finally, fuse these options to generate an analytical procedure.

In the author's experience, the extra effort required before the analysis begins is found to be well worthwhile in the long run, both for the saving of time and expense, and for better analytical results.

\section{References}

[1] Thiers, R. E., Methods of Biochemical Analysis, Glick, D., Ed., Vol. 5, p. 277, Interscience Publishers, Inc., New York (1957).

[2] Becker, D. A. and LaFleur, P. D., Production and Certification of NBS Biological Standard Reference Materials, Proceedings, Fourth Annual Conference on Trace Substances in Environmental Health, p. 435, University of Missouri, Columbia, Missouri (June 1970).

[3] Rook, H. L. and Moody, J. M., Stabilization and Determination of Nanogram Quantities of Mercury in Waier, Proceedings, Second International Conference on Nuclear Methods in Environmental Research, University of Missouri, Columbia, Missouri (July 1974) (in press).

[4] Rook, H. L., Suddueth, J. E., and Becker, D. A., The Determination of ${ }^{129}$ I at Natural Levels Using Neutron Activation and Isotopic Separation, NBS Report NBSIR 74-502, National Bureau of Standards, Washington, D.C. 20234 (1974) (to be submitted to Anal. Chem.). 
[5] Bate, L. C., Loss of Mercury from Containers in Neutron Activation Analysis, Radiochem. Radioanal. Letters 6,139 (1971).

[6] Becker, D. A., unpublished work.

[7] Brune, D., Transfer of Activities from Container Material to Sample, Report AE172, Aktiebolaget Atomenergi, Stockholm (1965), also published in Radiochim. Acta 5, 14 (1966).

[8] Becker, D. A. and LaFleur, P. D., Characterization of the NBSR for Neutron Activation Analysis, in Activation Analysis Section: Summary of Activities, LaFleur, P. D. and Becker, D. A., Eds., NBS Tech. Note 548, p. 6-23, U.S. Government Printing Office, Washington, D.C. 20240 (1970).

[9] Becker, D. A. and LaFleur, P. D., Characterization of a Nuclear Reactor for Neutron Activation Analysis,J. of Radioanal. Chem. 19, 149 (1974).

[10] Becker, D. A. and Suddueth, J. E., High Accuracy and Precision Determination of Sodium and Chlorine in Serum by Neutron Activation Analysis (to be published).

[11] Høgdahl, O. T., Neutron Absorption in Pile Neutron Activation Analysis, Report MMPP-226-1, University of Michigan (1962). Also published in part in Proceedings, International Conference on Radiochemical Methods of Analysis, Saltzburg, IAEA (1964).

[12] Reynolds, S. A. and Mullins, W. T., Neutron Flux Perturbation in Activation Analysis, Inter. J. Appl. Rad. Isotopes 14, 421 (1963).

[13] Becker, D. A. and LaFleur, P. D., Neutron Activation Analysis: Application to Trace Element Analysis in Biological and Environmental Materials, Proceedings, .Fifth Annual Conference on Trace Substances in Environmental Health, University of Missouri, Columbia, Missouri (1971).

[14] Becker, D. A., Accuracy and Precision in Activation Analysis-Counting, Proceedings, Second International Conference on Nuclear Methods in Environmental Research, University of Missouri, Columbia, Missouri (July 1974) (in press).

[15] Bowens, H. J. M. and Gibbons, D., Radioactivation Analysis, p. 99, Oxford University Press, New York (1963).

[16] Thiers, R. E., Reference 1, p. 325.

[17] Becker, D. A., Production and Use of Copper Foil Flux Monitors for High Precision Neutron Activation Analysis Utilizing Short-Lived Radioisotopes, in Radiochemical Analysis: Activation Analysis, Instrumentation, Radiation Techniques, and Radioisotope Techniques, DeVoe, J. R., Ed., NBS Tech. Note 404, pp. 39-46, U.S. Government Printing Office, Washington, D.C. 20240 (1966). 



\title{
A SEARCH FOR ACCURACY IN ACTIVATION ANALYSIS OF TRACE ELEMENTS IN DIFFERENT MATRICES
}

\author{
Sandro Meloni, Maria Teresa Ganzerli-Valentini, \\ Vera Caramella-Crespi, Vera Maxia, Luigino Maggi, \\ Ugo Pisani, Renato Soma, and Pierangelo Borroni
}

\author{
Centro di Radiochimica ed Analisi \\ per Attivazione del C.N.R. \\ Istituto di Chimica Generale ed Inorganica \\ Universita di Pavia, Viale Taramelli 12 \\ 27100 Pavia, Italy
}

\begin{abstract}
Different factors may affect accuracy in activation analysis of trace elements. The evaluation of these factors often requires a number of time consuming experiments, but the statement of accuracy in activation analysis is of great value to cast some light on the overall reliability of the method itself. It can be pointed out that accuracy is often inversely proportional to the number of steps of the whole analytical procedure, from sampling to calculation of results. Several techniques of activation analysis were developed and applied to the determination of trace element content in standard reference materials and in samples chosen for intercomparison among laboratories. Emphasis was put on limiting the number of steps to improve the accuracy and on achieving the best of precision. Results are presented and discussed, together with the criteria for the choice of the most appropriate separation technique. Other sources of systematic errors, such as the reliability of the content of the reference standards and dead-time corrections when short-lived isotopes are involved, were taken into account and discussed.
\end{abstract}

Keywords: Accuracy; activation analysis; copper; gold; hafnium; palladium; platinum; precision; radiochemical separations; silver.

\section{Introduction}

The accuracy of an analytical procedure indicates how closely a series of assays agrees with the true value of the quantity being measured. The 
accuracy of analytical results is affected by systematic and random errors. The term accuracy should not be confused with precision which reflects the reproducibility of experimental results. Thus a method may yield precise but inaccurate results if systematic errors are involved with the method. Precision is a quality associated with a class of measurements and refers to the way in which repeated observations conform to themselves. The generally accepted way of indicating the precision is the standard deviation.

Precision and accuracy are factors of high importance in activation analysis as in any other analytical technique. Precision can be evaluated by repeating the determination several times and submitting the results to statistical analysis; accuracy is more difficult to evaluate as it is necessary to locate and evaluate all the sources of systematic errors. Several factors may give rise to systematic errors, such as imperfections in the measuring instruments, faulty calibrations, nonrepresentative standards, human imperfection, use of unchecked and inaccurate literature data, and shifts in the process or in the measurement method. Of course, when an activation analysis method is being set up, care must be addressed to the evaluation of accuracy. This can be carried out either by checking the method with another independent technique or better, by performing parallel analyses of well known standard reference materials.

In this paper we describe two different approaches to the evaluation of accuracy in practical activation analysis. The first case is the determination of hafnium in the NBS-SRM 360a Zircaloy-2, by instrumental activation analysis and the second one is the determination by destructive activation analysis of some noble metals and copper in different matrices, either standard reference materials or samples distributed for interlaboratory comparison.

\section{Determination of Hafnium in NBS-SRM 360a Zircaloy-2}

Hafnium assay was carried out by two independent methods of instrumental neutron activation analysis using two different nuclear reactions. Nuclear data concerning the reactions are given in table 1 . The method was set up using the reaction leading to the short-lived radioisotope; the second reaction was used to check the method as no other analytical technique for trace analysis, reliable for precision and sensitivity, was available. Usually $10 \mathrm{mg}$ samples were irradiated; 20,50, 100 and $250 \mathrm{mg}$ samples were irradiated to investigate possible matrix effects. Ap- 
TABLE 1. Nuclear data concerning the two nuclear reactions used in the assay of hafnium in Zircaloy-2

$\begin{array}{ccc}\text { Reaction } & \begin{array}{c}\text { Energy of } \\ \text { palf-life of } \\ \text { produced nuclide }\end{array} & \begin{array}{c}\text { measured gamma } \\ \text { photopeaks } \\ (\mathrm{keV})\end{array}\end{array}$

$\begin{array}{llc}{ }^{178} \mathrm{Hf}(n, \gamma){ }^{179 \mathrm{~m}} \mathrm{Hf} & 18.6 \text { second } & 217 \\ { }^{180} \mathrm{Hf}(n, \gamma)^{181} \mathrm{Hf} & 42.5 \text { day } & 133,482\end{array}$

propriate nitric solutions of hafnium, poured into the irradiation containers and dried, were used as reference standards. When measuring the ${ }^{179 m} \mathrm{Hf}$ activity, large errors may be expected if dead-time corrections different for sample and standard are applied. These were avoided by measuring the actual counting time for samples and standards (usually set for $60 \mathrm{~s}$ ), and accepting those values differing for less than 1 second. Irradiations were carried out in the LENA Reactor of the University of Pavia. When producing ${ }^{179 m} \mathrm{Hf}$, the irradiation time was 7 seconds at a flux of about $2 \times 10^{12} \mathrm{n} \cdot \mathrm{cm}^{-2} \cdot \mathrm{s}^{-1}$ in a pneumatic system; in the case of ${ }^{181} \mathrm{Hf}$ the irradiation time was 50 hours in the central thimble at a flux of about $8 \times$ $10^{12} \mathrm{n} \cdot \mathrm{cm}^{-2} \cdot \mathrm{s}^{-1}$. Activity measurements were carried out by $\mathrm{Ge}(\mathrm{Li})$ gamma spectrometry. The half life of the gamma photopeak at $217 \mathrm{keV}$, due to deexcitation of ${ }^{179 m} \mathrm{Hf}$, was measured and found to be $18.6 \pm 0.2 \mathrm{~s}$, in agreement with literature data [1]. Long activated samples were allowed to decay 2 days and then counted.

Results are given in table 2, together with the number of analyzed samples and their weight. Data obtained by the two nuclear reactions show good agreement, precision in the range 3 to 6 percent, and some kind of

TABLE 2. Hafnium content of NBS-SRM 360 a Zircaloy-2 as obtained by instrumental activation analysis through two independent nuclear reactions

Samples $\frac{\text { Reaction: hafnium content }(\mu \mathrm{g} / \mathrm{g})^{\mathrm{s}}}{{ }^{178} \mathrm{Hf}(\mathrm{n}, \gamma)^{179 m} \mathrm{Hf} \quad{ }^{180} \mathrm{Hf}(n, \gamma)^{181} \mathrm{Hf}}$

\footnotetext{
10 samples $(10 \mathrm{mg})$

2 samples $(20 \mathrm{mg})$

2 samples $(50 \mathrm{mg})$

2 samples $(100 \mathrm{mg}$ )

2 samples $(250 \mathrm{mg})$

17 samples $(10 \mathrm{mg})$
}

$\begin{array}{rc}98 \pm 3 & - \\ 102 \pm 4 & - \\ 101 \pm 5 & - \\ 91 \pm 4 & - \\ 90 \pm 3 & - \\ - & 102 \pm 7\end{array}$

s Values listed are average values and standard deviations. 
matrix effect appears for sample weights higher than $50 \mathrm{mg}$. The overall error, evaluated taking into account any possible source of errors, is in the range 5 to 7 percent. Only one literature [2] value is available: $126 \pm 9$ $\mu \mathrm{g} / \mathrm{g}$. This value has been obtained by activation analysis of large samples $(4.36 \mathrm{~g})$ with thermal neutrons from a $0.8 \mathrm{mg}{ }^{252} \mathrm{Cf}$ source and detecting gamma rays with a $\mathrm{NaI}(\mathrm{Tl})$ crystal.

\section{Determination of Some Noble Metals and Copper in Reference Materials}

The accurate determination of noble metals by neutron activation analysis always requires radiochemical separation. Reported chemical procedures are usually lengthy and tedious involving a many step chemistry, which affects sensitivity when short-lived isotopes are involved [39]. On the other hand, it is well known that the accuracy of a method is inversely proportional to the number of steps from sampling to data reduction. From this point of view a method of destructive activation analysis was developed and applied to the determination of some noble metals and copper in several reference materials. Emphasis was put on limiting the number of steps to improve the accuracy and on achieving the best precision.

The nuclear data concerning the radionuclides used in the determination are reported in table 3 .

TABLE 3. Nuclear data concerning some noble metals and copper

\begin{tabular}{llcc}
\hline Element & Radioisotope & Half-life & $\begin{array}{c}\text { Energy of } \\
\text { measured gamma } \\
\text { photopeaks } \\
(\mathrm{keV})\end{array}$ \\
\hline Palladium & ${ }^{109} \mathrm{Pd} \rightarrow{ }^{109 \mathrm{~m}} \mathrm{Ag}$ & 13.8 hour & 88 \\
Platinum & ${ }^{199} \mathrm{Pt} \rightarrow{ }^{199} \mathrm{Au}$ & 3.2 day & 159 \\
Gold & ${ }^{198} \mathrm{Au}$ & 2.69 day & 412 \\
Silver & ${ }^{110 \mathrm{~m}} \mathrm{Ag}$ & 260 day & 659,886 \\
Copper & ${ }^{64} \mathrm{Cu}$ & 12.8 hour & 511 \\
\hline
\end{tabular}

The analyzed materials were: NBS-SRM 614 and 616 Trace Elements in Glasses, air dust samples distributed for intercomparison by C.C.R. Euratom (Ispra, Italy) and copper samples doped with platinum and palladium at the $100 \mathrm{ppm}$ level, prepared and distributed for intercomparison by the Eurisotop Office of the European Communities Commission 
(Bruxelles, Belgium). Irradiations were carried out in the LENA Reactor at a flux of about $1 \times 10^{12} \mathrm{n} \cdot \mathrm{cm}^{-2} \cdot \mathrm{s}^{-1}$ and lasted 20 minutes and 2 hours respectively for $\mathrm{Pd}$ and $\mathrm{Pt}$ assay, 10 hours for $\mathrm{Au}$ and $\mathrm{Cu}$ and 50 hours for Ag. Aliquots of stock solutions containing known amounts of each element were irradiated together with samples. Activity measurements were carried out by $\mathrm{Ge}(\mathrm{Li})$ gamma spectrometry.

The dissolution step always needs particular care, as it may introduce a large error in the procedure. In fact, severe losses may be due to the presence of volatile elements and to the formation of volatile compounds. Moreover, precipitation of tiny amounts of insoluble species, such as silica, should be avoided as they may adsorb relatively large quantities of trace elements. When possible the dissolution yield should be previously checked.

In this work the following dissolution steps were applied. Eurisotop copper samples were dissolved with 2 to 4 molar nitric acid; NBS "Glasses" with a mixture of perchloric and hydrofluoric acids, and the Euratom air dust with the above mixture to which concentrated nitric acid and hydrogen peroxide were added to oxidize the organic matter. Before dissolution 0.1 to $0.2 \mathrm{mg}$ of each element was added as carrier. After dissolution, the solution was carefully evaporated to dryness and finally taken up with the proper media.

Several separation techniques are available to isolate noble metals as a group or as single elements. The choice of the proper technique plays a prominent role and often qualifies the whole procedure. As $\mathrm{Ge}(\mathrm{Li})$ gamma spectrometry is now widely used, the isolation of single isotopes is not compulsory, and therefore group separations are much more advantageous. Specific separations are sometimes required when it is necessary to eliminate the matrix or a major component interfering in the gamma spectrometry. Time requirements are relevant especially when short-lived isotopes are involved.

In this work noble metals and copper are separated by adsorption on chromatographic columns filled with inorganic materials [10]. These materials studied in our laboratory are: molybdenum dibromide (MDB), zinc ferrocyanides (ZFoC) and grained cadmium metal. Silver is adsorbed on MDB or, only when the amount of iron is not relevant, on ZFoC. Gold is separated from copper by adsorbing it on MDB; if copper is present as a macrocomponent it is necessary to hydrolize MDB before separation, to avoid precipitation of copper bromide. Copper is adsorbed on cadmium metal. Palladium is adsorbed on $\mathrm{ZFoC}$, after removal of copper on Dowex-50. All sorptions take place from nitric media (0.1-1M) except for palladium adsorbed from hydrochloric medium. Dismantled columns are submitted to gamma spectrometry. 
This separation method is rather fast $(<2 \mathrm{~h})$, the adsorption yields are $>95$ percent and the column behaviour is highly reproducible; therefore the method may be considered very reliable.

Results obtained are shown in tables 4 to 6 , where the mean values, standard deviations and the number of analyzed samples are reported. Data in table 4 show a very good agreement for palladium content between the obtained value and the Eurisotop mean value. Agreement is also good for platinum values; a better agreement is found by comparison with the reported mean value for neutron activation analysis (102 \pm 4$)$.

As far as the analysis of copper, gold and silver in NBS glasses is concerned (table 5), a good agreement is shown with the NBS certified or interim values for gold in the two glasses, for silver in SRM 614 and for copper in SRM's 614 and 616. Silver content in SRM 616 is below the detection limit for the described procedure.

Now considering the data for the Euratom air dust samples (table 6), the obtained values agree with general mean values of reported results, but they are always located at the low side of the ranges.

TABLE 4. Palladium and platinum content of " $\mathrm{Cu}-100 \mathrm{ppm} P d$ " and " $\mathrm{Cu}-100 \mathrm{ppm} \mathrm{Pt}$ " samples prepared and distributed for intercomparison by Eurisotop Office of European Communities Commission

\begin{tabular}{ccrc}
\hline Element & $\begin{array}{l}\text { No. of } \\
\text { samples }\end{array}$ & $\begin{array}{c}\text { Content } \\
\mu \mathrm{g} / \mathrm{g}\end{array}$ & $\begin{array}{c}\text { Eurisotop } \\
\text { mean value } \\
\mu \mathrm{g} / \mathrm{g}\end{array}$ \\
\hline $\mathrm{Pd}$ & 7 & $97 \pm 7$ & $96 \pm 5^{\mathrm{a}}$ \\
$\mathrm{Pt}$ & 5 & $108 \pm 2$ & $98 \pm 3^{\mathrm{b}}$
\end{tabular}

\footnotetext{
a Average of 98 individual results obtained by neutron activation, proton activation, gamma activation, x-ray fluorescence, atomic absorption, emission spectroscopy and gravimetry.

b Average of 82 individual results obtained by neutron activation, gamma activation, proton activation, x-ray fluorescence, atomic absorption, emission spectroscopy and photometry.
} 
TABLE 5. Silver, gold and copper content of NBS Trace Elements in Glasses SRM's 614 and 616

\begin{tabular}{|c|c|c|c|c|c|c|}
\hline \multirow[b]{2}{*}{ Element } & \multicolumn{3}{|c|}{$\begin{array}{c}\text { SRM } 614 \\
\text { (nominal value } 1 \text { ppm) }\end{array}$} & \multicolumn{3}{|c|}{$\begin{array}{c}\text { SRM } 616 \\
\text { (nominal value } 0.02 \mathrm{ppm} \text { ) }\end{array}$} \\
\hline & $\begin{array}{l}\text { No. of } \\
\text { ana- } \\
\text { lyzed } \\
\text { samples }\end{array}$ & $\begin{array}{c}\text { Content } \\
\mu \mathrm{g} / \mathrm{g}\end{array}$ & $\begin{array}{l}\text { Other } \\
\text { values } \\
\mu \mathrm{g} / \mathrm{g}\end{array}$ & $\begin{array}{c}\text { No. of } \\
\text { ana- } \\
\text { lyzed } \\
\text { samples }\end{array}$ & $\begin{array}{l}\text { Content } \\
\mu \mathrm{g} / \mathrm{g}\end{array}$ & $\begin{array}{l}\text { Other } \\
\text { values } \\
\mu \mathrm{g} / \mathrm{g}\end{array}$ \\
\hline $\mathrm{Cu}$ & 6 & $1.6 \pm 0.2$ & $1.34 \pm 0.07$ & 7 & $0.9 \pm 0.2$ & $0.80 \pm 0.09^{a}$ \\
\hline $\mathrm{Ag}$ & 7 & $0.6 \pm 0.1$ & $0.42 \pm 0.04^{\mathrm{a}}$ & - & $0.04^{\mathrm{b}}$ & - \\
\hline $\mathrm{Au}$ & 5 & $0.44 \pm 0.02$ & $\begin{array}{c}(0.5)^{\mathrm{a}} \\
0.55 \pm 0.05^{\mathrm{c}}\end{array}$ & 5 & $0.13 \pm 0.01$ & $(0.18 \pm 0.01)^{\mathrm{a}}$ \\
\hline
\end{tabular}

a Values reported by NBS in reference [11]. Numbers in parentheses are interim values.

b Detection limit under described experimental conditions.

- Values reported in reference [12].

TABLE 6. Silver, gold and copper content of air dust samples distributed for intercomparison by CCR Euratom (Ispra, Italy)

\begin{tabular}{cccc}
\hline Element & $\begin{array}{c}\text { No. of analyzed } \\
\text { samples }\end{array}$ & $\begin{array}{c}\text { Content } \\
\mu \mathrm{g} / \mathrm{g}\end{array}$ & $\begin{array}{c}\text { Mean values } \\
\text { and ranges } \\
\mu \mathrm{g} / \mathrm{g}\end{array}$ \\
\hline $\mathrm{Cu}$ & 8 & $614 \pm 16$ & $\begin{array}{c}651 \pm 44 \\
(614-700) \\
15 \pm 3\end{array}$ \\
$\mathrm{Ag}$ & 5 & $11 \pm 1$ & $(11.0 \pm 19.8)$ \\
$\mathrm{Au}$ & 5 & $2.25 \pm 0.07$ & $(2.6 \pm 0.2$ \\
& & & $-2.95)$
\end{tabular}

a Values obtained by neutron activation analysis and released by CCR Euratom at the working meeting held at Ispra (Italy) 8-9 July 1974. 


\section{Conclusion}

The two described different lines of action follow the general trend of investigation on accuracy, i.e., the check of the method with another independent technique and/or the comparison with certified or recommended values of Standard Reference Materials. The use of a second nuclear reaction as an independent technique, in the case of hafnium assay in Zircaloy-2, appears very useful especially when sensitivity limitations cut down the availability of other analytical methods. In destructive methods of activation analysis a good accuracy was achieved by reduction of the number of steps of the whole procedure, preliminary investigation on the yields of each step and choice of the most appropriate (for rapidity and simplicity) separation technique. Results obtained using this technique may be considered satisfactory.

\section{References}

[1] Lederer M., Hollander, J., and Perlman, I., Eds., Tables of Isotopes, John Wiley and Sons, Inc., New York (1967).

[2] Ishii, D., Jinno, K., and LaFleur, P., paper C 2508 presented at the IUPAC International Congress on Analytical Chemistry, Kyoto (Japan) (April 1972).

[3] Beamish, F. E., Chung, K. S., and Chow, A., Talanta 14, 1-32 (1967).

[4] Vincent, E. A. and Crockett, J. H., Geochim. et Cosmochim. Acta 18, 143-148 (1960).

[5] Bowen, H. J. M., Analyst 92, 118-123 (1967).

[6] Rushbrook, P. R. and Ehmann, W. D., Geochim. et Cosmochim. Acta 26, 649-657 (1962).

[7] Greenland, L. P., Geochim. et Cosmochim. Acta 31, 849-860 (1967).

[8] Crockett, J. H., Keays, R. R., and Hsich, S., J. Radioanal. Chem. 1, 487-507 (1968).

[9] Gijbels, R. and Hoste, J., Possibilities for the Application of Neutron Activation Analysis, Proc. of the Symposium on Nuclear Methods for the Preparation and Industrial use of Noble Metals, Bruxelles, Nov. 16-17, 1971, Eurisotop Report 23, III/908/73-D (1973).

[10] Caramella-Crespi, V., Pisani, U., Ganzerli-Valentini, M. T., Meloni, S., and Maxia, V., Determination of Some Noble Metals and Copper by Destructive Neutron Activation Analysis of Different Matrices, to be published in J. of Radioanal. Chem.

[11] Certificate of Analysis for Standard Reference Material, No. 614 and 616, Office of Standard Reference Materials, National Bureau of Standards, Washington, D.C. 20234.

[12] Kim, J. I. and Born, H. J., J. of Radioanal. Chem. 13, 427-442 (1973). 


\title{
SURFACE EFFECTS ON ACCURACY IN ACTIVATION ANALYSIS
}

\author{
G. Revel and M. Fedoroff \\ Centre d'Etudes de Chimie Metallurgique \\ 15 , rue Georges Urbain \\ 94400 Vitry-sur-Seine, France
}

\begin{abstract}
One of the main advantages of trace activation analysis is the possibility of eliminating surface contamination by post-irradiation removal of the superficial layers of the sample. In some cases this advantage is lost by noneffective removal, leading to inaccurate results. We discuss the main causes of ineffective removal of surface contamination. In neutron activation analysis the surface contamination takes place during sampling and irradiation. The main causes of ineffective removal of this contamination are redeposition during chemical etching and large diffusion during irradiation. For the determination of light elements $(\mathrm{O}, \mathrm{C}, \mathrm{N})$ performed by charged particle or photon irradiation, the surface contamination is increased through the diffusion of impurities by recoil and channeling. Chemical etching, used as the only method, is not convenient for a number of metallic samples - often the thickness of the dissolved layer varies along the surface due to heterogeneities in purity, crystalline structure and localized oxidation made by nonhomogeneous incident beam. Several examples investigated by tracer and metallographic methods are given. A procedure for accurate surface contamination removal is suggested.
\end{abstract}

Keywords: Accuracy; activation analysis; charged particle irradiation; diffusion; etching; metals; neutron irradiation; recoil; surface effect.

\section{Introduction}

The main advantage of activation analysis in trace determination is to achieve the theoretical sensitivity of the method. This is possible, contrary to other analytical methods, because the analysis is not sensitive to post-irradiation pollution of the sample and because surface pollution can be eliminated by post-irradiation removal of the superficial layers of a solid sample. The analysis is then performed only on the bulk of the sample. 
This advantage of activation analysis is lost in some cases by noneffective removal of the surface pollution. The purpose of this communication is to show and discuss examples of noneffective surface removal and to suggest an accurate procedure. The examples are taken from trace analysis of high-purity metals by neutron or charged particles.

It is obvious that in trace analysis, the superficial impurities greatly contribute to the total concentration in the sample. As an example the aluminium oxide film existing on the pure metal at room temperature may have a thickness of $7.5 \cdot 10^{-3} \mu \mathrm{m}$ [ 1 ]. If not removed from a cubic sample of $1 \mathrm{~cm}^{3}(2.7 \mathrm{~g})$ it gives an apparent oxygen concentration of $3 \mu \mathrm{g} / \mathrm{g}$ in aluminium. This apparent concentration can be calculated in all cases by the formula:

$$
c=S \cdot C_{s} / m
$$

where $S$ is the area of the sample, $m$ its weight and $C_{s}$ the concentration of the impurity per unit area.

\section{Surface Effects on Solid Samples}

\section{A. Contamination by Mechanical Treatment}

All mechanical treatments lead to surface contamination, which can be removed by post-irradiation etching. But some treatment and especially rolling, may introduce the contamination as inclusion into the bulk of the sample. This effect was observed in the case of iron [2]. When zonerefined iron with a carbon concentration of 6 to $7 \mu \mathrm{g} / \mathrm{g}$ is rolled, the concentration of this element reaches 75 to $100 \mu \mathrm{g} / \mathrm{g}$ even if the rollers were cleaned with a solvent. This effect decreases if the sample and the rollers are carefully polished before rolling.

\section{B. Deposition During Etching}

The primary method of removing the superficial contaminated layers of a sample is chemical etching. Some elements, especially the less electropositive metals, may deposit on the more electropositive metal during etching. This effect has been observed in the determination of copper in aluminium by thermal neutron irradiation. This deposition of copper was observed when the sample is preirradiation etched either by the bath containing 70 percent $\mathrm{H}_{3} \mathrm{PO}_{4}, 20$ percent $\mathrm{H}_{2} \mathrm{SO}_{4}, 5$ percent $\mathrm{HNO}_{3}$ or by 
$\mathrm{NaOH}$ (fig. 1). Etching of $\mathrm{Al}$ in these baths leads to apparent concentrations of about $0.1 \mu \mathrm{g} / \mathrm{g}$ of $\mathrm{Cu}$, when the actual concentration is near 0.001 $\mu \mathrm{g} / \mathrm{g}$. In this case the adequate procedure to dissolve the copper deposit is a treatment by concentrated $\mathrm{HNO}_{3}$ after etching by the 3 acids bath; however, the application of many successive 3 acids baths decreases the radioactivity of ${ }^{64} \mathrm{Cu}$ (fig. 1).

The possibility of redeposition during chemical etching must always remain in mind. As mechanical treatment of the surface is generally a more difficult procedure, chemical etching can be used but its efficiency must be controlled or a post-irradiation etching used. Generally the use of successive baths leads to accurate surface contamination removal.

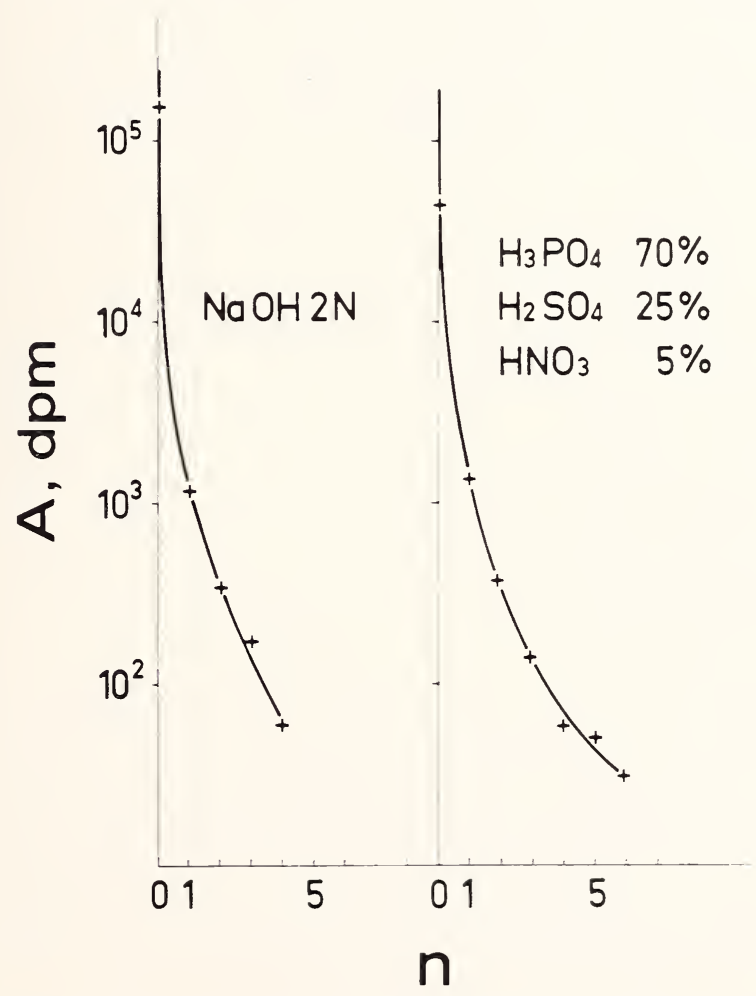

Figure 1. Variation of the radioactivity (A) of ${ }^{64} \mathrm{Cu}$ contaminating aluminium after a number (n) of successive preirradiation etchings in alkali or acid baths. 


\section{Deep Diffusion in the Sample}

In most cases the temperature during the irradiation in a reactor does not lead to deep diffusion of the surface impurities into the sample. However we have observed in some cases abnormal deep diffusion. This effect was observed during the determination of sulphur by the ${ }^{34} \mathrm{~S}(\mathrm{n}, \gamma)^{35} \mathrm{~S}$ reaction $[3,4]$. It led to quite false results for sulphur concentration, as shown by further determination by the ${ }^{32} \mathrm{~S}(\mathrm{n}, \mathrm{p})^{32} \mathrm{P}$ reaction.

This effect depends on the nature of the samples. Deep diffusion of ${ }^{35} \mathrm{~S}$ was observed in $\mathrm{Cu}, \mathrm{Zr}, \mathrm{Mo}, \mathrm{Fe}$ and $\mathrm{Ni}$. This effect has its maximum intensity in $\mathrm{Ni}$ and $\mathrm{Fe}$. In these cases ${ }^{35} \mathrm{~S}$ diffuses through more than $1 \mathrm{~mm}$ and the contamination takes place in the whole depth of the sample. On the contrary, the effect was not observed in $\mathrm{Al}$ and $\mathrm{Mg}$.

Another experimental observation is that the ${ }^{35} \mathrm{~S}$ concentration in the sample depends on the chlorine concentration of the surface (fig. 2). This dependence is explained by the fact that ${ }^{35} \mathrm{~S}$ can be produced by the ${ }^{35} \mathrm{Cl}(\mathrm{n}, \mathrm{p}){ }^{35} \mathrm{~S}$ reaction, which takes place in a thermal position of the reac-

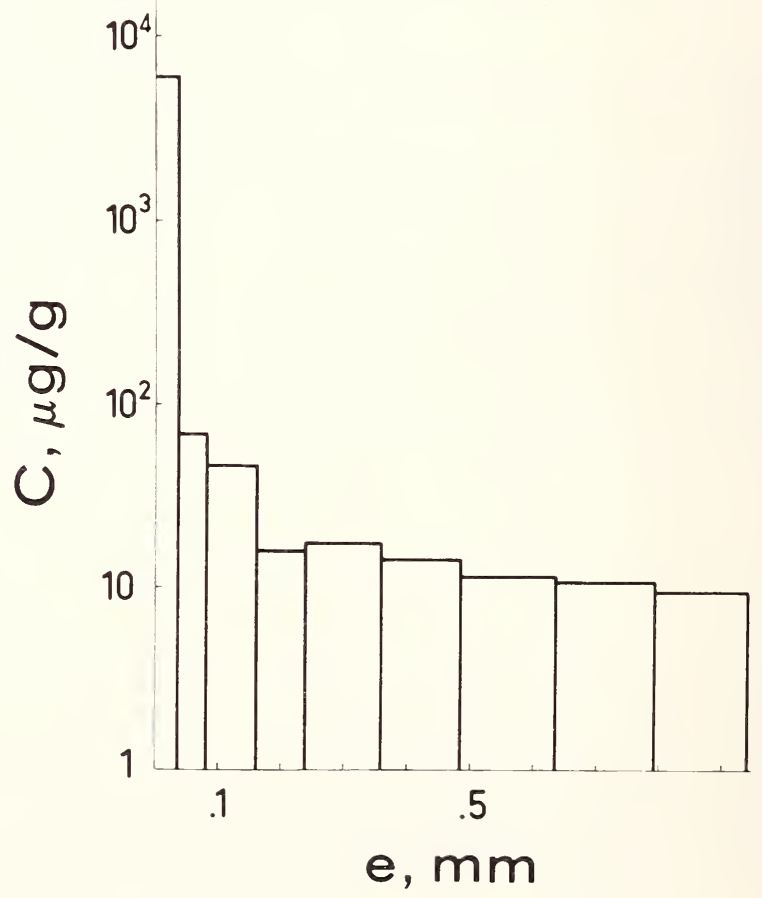

Figure 2. Apparent concentration (C) of sulphur (by ${ }^{35} \mathrm{~S}$ counting) as a function of depth (e) in nickel, whose surface was contaminated by $\mathrm{NH}_{4} \mathrm{Cl}$. 
tor. In fact. the main part of ${ }^{35} \mathrm{~S}$ present in the sample after irradiation is produced from the surface chlorine contamination.

Since the exact nature of this effect is not known up to now. we cannot avoid it. The only solution is to find another adequate nuclear reaction which is not subject to this effect. Anyhow we must always be vigilant as this example of deep diffusion is not unique [5].

\section{Surface Effects After Charged-Particle Irradiation}

Compared to thermal neutron irradiation, charged particles lead always to rather deep diffusion. The main responsible phenomenon is the recoil of the nuclides ejected by the nuclear reactions. The recoil depends on the energies of these nuclides. In most activation analysis cases it reaches several $\mu \mathrm{m}$. In fact, experiments show that diffusion of radionuclides from the surface is more intensive than predicted by recoil [6-8]. This effect may be partially explained by channelling or accelerated diffusion due to irradiation and local heating.

The main cause of inadequate removal of superficial layers is nonuniform etching. One example is the determination of oxygen in first electrolysis aluminium [9]. The concentration found by ${ }^{3} \mathrm{He}$ and proton irradiation was $20 \mu \mathrm{g} / \mathrm{g}$. These results were in agreement with those obtained by mass spectrometry and fast neutron irradiation. All these results were false due to interference for proton irradiation and inadequate etching in the other cases. As was shown by microscopic observations. intensive etching takes place at grain boundaries and by pitting. At other places the oxide pellicula is not removed. Further experiments using a more accurate etching showed that the real concentration of oxygen is 0.2 $\mu \mathrm{g} / \mathrm{g}$.

Another example is the determination of oxygen in zirconium. In this case, owing to the flux of particles and to the heating of the surface. the metal is oxidized during irradiation. This oxidation is not uniform. probably because the beam is not uniform itself. As it was shown by microscopic observation, the more oxidized points are protected against chemical etching.

These examples are not unique. In many other cases we have observed nonreproducible results which could be attributed to ineffective etching. 


\section{Conclusion}

Our experience in activation analysis showed us that in the preparation of solid samples for irradiation the procedure of chemical etching must be in each case carefully controlled. The main causes of error are redeposition and nonuniform etching. In all cases we must avoid a static etching in a single bath. It is generally better to use etching in many successive baths.

The better procedure of superficial layers removal is mechanical surface treatment coupled with chemical etching. The better mechanical treatment is cutting and not abrasion, as this last method may give inclusions.

In all cases one must avoid general or local elevation of temperature which may lead to oxidation and accelerated diffusion. The possibility of deep diffusion really exists and must be kept in mind. In the case of charged particle irradiation the elevation of temperature is always important: even with good systems of cooling and large area beams, this effect will limit the current of charged particles and consequently the sensitivity of the determination.

In conclusion, the intent of this paper is to discuss problem procedures or special cases of surface effects which lead to false results. In the majority of cases, chiefly in neutron irradiation, these effects do not affect the determinations and a quite good accuracy is achieved, which is controlled by the following procedure: preparation of an alloy with a known quantity of radioactive dopant, measurement of the observed concentration by counting, using classical neutron activation analysis after decay of the initial gross radioactivity. We can thus compare the expected concentration (calculated from weights of dopant and base metal), and the results obtained after the first counting using classical activation analysis. These experiments demonstrated that we can achieve at this time the 1 percent range in accuracy, if the counting statistics allow it.

\section{References}

[1] Chaudron, G., Monographies sur les métaux de haute pureté, p. 500, Masson, Paris (1972).

[2] Montuelle, J., Bigot, J., Durand, J. C., and Chaudron, Th., Colloque International sur les propriétés physiques, mécaniques et chimique du fer de très haute pureté, Paris, 26 Sept.-1 er Oct. 1966, Ed. du C.N.R.S., p. 17, Paris (1968).

[3] Albert, Ph., Blouri, J., Cleyrergue, Ch., Deschamps, N., and Le Hericy, J., J. Radioanal. Chem. 1, 297-311,389-396, 431-441 (1968). 
[4] Fedoroff, M., C. R. Acad.Sc. Paris 271, 399-402 (1970).

[5] Boissier-Chermette, M., Thesis, Lyon (1974).

[6] Barrandon, J. N., Debrun, J. L., and Albert, Ph., Proceedings of Second Conference on Practical Aspects of Activation Analysis with Charged Particles, p. 277, Liège (Belgium), Sept. 21-22 (1967), EUR 3896 d-f-e.

[7] Engelmann, Ch., Thesis, Orsay, France (1970).

[8] Kim, C. K. and Vasile, M. J., Anal. Chim. Acta 56, 339 (1971).

[9] Vialatte, B. and Revel, G., Anal. Chem. 44, 1077 (1972). . 



\title{
A STUDY INTO THE ACCURACY OF A REMOTE-CONTROLLED SYSTEM FOR MULTIELEMENT DETERMINATION IN FOODSTUFFS USING NAA
}

\section{Rieder Schelenz and Johannes-Friedrich Diehl}

\author{
Federal Research Center for \\ Food Preservation \\ Institute for Radiation Technology \\ D-7500 Karlsruhe \\ Federal Republic of Germany
}

\begin{abstract}
More accurate information on the trace element composition of foodstuffs is important in the field of nutrition. An appropriate approach to this problem is the application of neutron activation analysis for simultaneous multielement determination using high resolution gamma spectrometry.

A system has been developed permitting determination of at least 28 elements. utilizing remote controlled radiochemical processing of irradiated biological samples. The reliability of the method has been ascertained by participation in inter-laboratory comparisons organized by the International Atomic Energy Agency (IAEA). Results for multielement determinations are given for potatoes (peeled). milk powder. fish solubles and algae.
\end{abstract}

Keywords: Biological material: biological standards: data evaluation: distillation: food composition: gamma spectrometry: inorganic separators: isotopic exchange: multielement determination: neutron activation: radiochemical separation: trace elements.

\section{Introduction}

In the past. concerns about toxic trace elements in foodstuffs have focused on individual elements. such as mercury. lead. cadmium etc. Recently the realization has grown that the toxicological evaluation of the presence of certain amounts of an element in the diet requires information about the simultaneous presence of other elements. In the field of nutrition and food research it becomes increasingly important to obtain more 
accurate information on the trace element composition of foodstuffs and other biological materials. An appropriate approach to this problem is the application of neutron activation analysis for simultaneous multielement determination using high resolution gamma-spectrometry and electronic data evaluation with computers.

Foodstuffs and other biological materials usually contain much sodium, potassium, phosphorous and bromine. The radionuclides ${ }^{24} \mathrm{Na},{ }^{42} \mathrm{~K},{ }^{32} \mathrm{P}$ and ${ }^{82} \mathrm{Br}$ produced in such samples by neutron activation dominate the gamma spectrum to such an extent that the determination of most other elements becomes impossible, unless special procedures are applied to avoid this problem. By using inorganic separators such as hydrated antimony pentoxide (HAP) for the removal of sodium-24, titanium phosphate to remove potassium-42, and zirconium phosphate for removing phosphorous-32, as proposed by Girardi et al. [1], a multielement determination can be accomplished within reasonable time requirements.

Multielement determination in biological samples by NAA is possible either by chemical separation methods, "chemical spectrum stripping" or, by using purely instrumental techniques after a longer decay period. Both approaches are used in our laboratory [2].

\section{Method}

As indicated in the scheme (table 1) the samples are sealed in high purity quartz ampoules (available: Heraeus, 6450 Hanau, West Germany) together with multielement biological standards such as Bowen's kale powder, NBS orchard leaves or NBS bovine liver and irradiated for 24 hours. After the induced radioactivity has decreased due to the short-lived radionuclides, the biological material is decomposed according to a procedure published by Sjöstrand [3] in a siliconized wet-ashing and distillation apparatus described by Bethge [4]. After digestion of the sample a mixture of perchloric acid and glycine is added. At about $120^{\circ} \mathrm{C}$, $\mathrm{HCl}$ is formed in statu nascendi from the perchloric acid due to the reducing action of glycine. Metals present are thus converted to their chlorides. At about $250{ }^{\circ} \mathrm{C}$ the volatile chlorides, e.g., of mercury and gold, are distilled off together with bromine. The reproducibility of the distillation step was studied in 18 tracer experiments with mercury-203. Samples of freeze-dried canned tuna were spiked with radioactive mercury solutions. The yield was determined to be $93.2 \pm 3.4$ percent relative standard deviation.

From the two fractions thus obtained (table 1), distillate and residue, 
TABLE 1. Scheme for the destructive method of multielement determination by neutron activation analysis

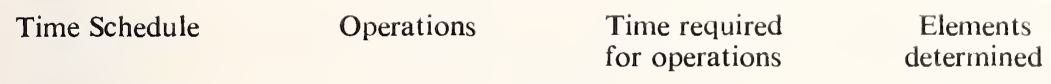

1st day

1. Irradiation

$150 \mathrm{mg}$ sample

irradiated for

24 hours at a

neutron flux of

$6 \times 10^{13} \mathrm{~cm}^{-2} \mathrm{~s}^{-1}$

2nd day

2. Cooling

3rd day

3. Digestion of sample with conc $\mathrm{HNO}_{3}$, conc $\mathrm{H}_{2} \mathrm{SO}_{4}(9: 1)$

4. Addition of $70 \%$ $\mathrm{HClO}_{4}+$ Glycine, distillation of volatiles

5. Isotopic exchange

4th day
6. Radiochemical separation of ${ }^{32} \mathrm{P}$, ${ }^{42} \mathrm{~K}$ and ${ }^{24} \mathrm{Na}$

7. Radioactive measurement with data evaluation
(a) "HAP" column
(b) Distillate
(c) Copper-foil (distillate)
(d) Copper-foil (residue)
(e) Residue

1 day

1 day

1 hour

0.5 hour

12-18 hours

0.5 hour

$50 \mathrm{~min}$

$10 \mathrm{~min}$

$\mathrm{Na}, \mathrm{K}, \mathrm{Cs}, \mathrm{Rb}$

4.5 hour

4.5 hour

12 hour
$\mathrm{Br}$

$\mathrm{Au}, \mathrm{Hg}$

$\mathrm{Ag}, \mathrm{Se}, \mathrm{Cu}$

$\mathrm{As}, \mathrm{Ba}, \mathrm{Ca}, \mathrm{Cd}, \mathrm{Ce}, \mathrm{Co}, \mathrm{Cr}$ $\mathrm{Eu}, \mathrm{Fe}, \mathrm{Ga}, \mathrm{La}, \mathrm{Mo}, \mathrm{Sb}$

Sc, $\mathrm{Sm},(\mathrm{Sn}), \mathrm{Ta}, \mathrm{Tb}$,

$\mathrm{Th}, \mathrm{W}, \mathrm{Yb}, \mathrm{Zn}$

certain elements are now removed by an isotopic exchange technique using amalgamated copper foils as previously described for the determination of mercury in foodstuffs [5]. The copper foils are placed into both fractions. The copper foil exposed to the distillate takes up mercury and gold. This achieves a fairly good separation from the bromine present in the distillate and makes it possible to detect mercury even at ppb levels.

On the copper foil of the residue are deposited copper, selenium and silver. This procedure makes possible the determination of copper using the $511 \mathrm{keV}$ photopeak without detectable interference by other radionuclides. Because selenium is retained almost quantitatively in the so called "HAP" column containing in sequence TiP, ZP and HAP. selenium is determined before passing through the "HAP" column. The 
fractions are now ready for counting; the distillate (bromine), the distillate-foil (mercury and gold) and the residue-foil (silver, selenium, copper).

The distillation residue is taken up with $9 \mathrm{~N}$ hydrochloric acid and automatically transferred to the "HAP" column, which is subsequently washed with $9 N$ hydrochloric acid, eluting the residual nonvolatile elements from the column indicated in table 1. Due to the removal of the high radioactivity caused by sodium-24, potassium-42 and phosphorous-32, the gamma-spectrometric measurement can be carried out directly after this separation step, with no dead time problems.

\section{Gamma Spectrometry and Data Evaluation}

A $35 \mathrm{~cm}^{3}$ true coaxial $\mathrm{Ge}(\mathrm{Li})$ detector is used (Ortec, resolution: 2.2 $\mathrm{keV}$ at $1.33 \mathrm{MeV}$, peak/Compton ratio 28:1), connected to a 8192-channel pulse height analyzer (Hewlett Packard 5402 B). With the aid of a 16 K computer (Hewlett Packard, Model 2100 A) and a programme written in "instrument basic," photopeaks are identified and peak areas determined by the correlation technique, essentially as described by Black [6]. Time required for evaluation of a spectrum of about 60 photopeaks is 20 minutes. Element concentration in the sample is calculated by comparing the net peak areas of "interference-free" photopeaks with those of the identically treated standard.

Especially when measuring low activities, as was found on the copperfoils from the distillate and the residue, when food samples were analyzed, the shape and height of the background may play an important role, influencing the detection limits of elements to be determined. Therefore, a circular shielding for the semiconductor detector (fig. 1) was designed and built. The shielding materials are arranged with increasing ordinal number around the detector.

The effect of this shielding on the composition of the background is shown in figure 2 . Curve I indicates a measurement without shielding and curve III with the shielding equipment shown in figure 1 . Mainly in the low energy region a considerable decrease in the pulse heights is obtained. This is very important, in particular for the determinations of mercury at the ppb level. Based on five measurements the threefold standard deviation in this energy region was found to be $0.3 \mathrm{cpm}$ for the equipment used. The number of peaks of naturally occurring gamma emitting radionuclides up to $2 \mathrm{MeV}$ was reduced from 18 to 9. 
1 Po $40 \mathrm{~mm}$ wall thickness

$2 \mathrm{Fe} 10 \mathrm{~mm}$

3Al $10 \mathrm{~mm}$

4 Plexi $10 \mathrm{~mm}$

Po thickness of lid $30 \mathrm{~mm}$

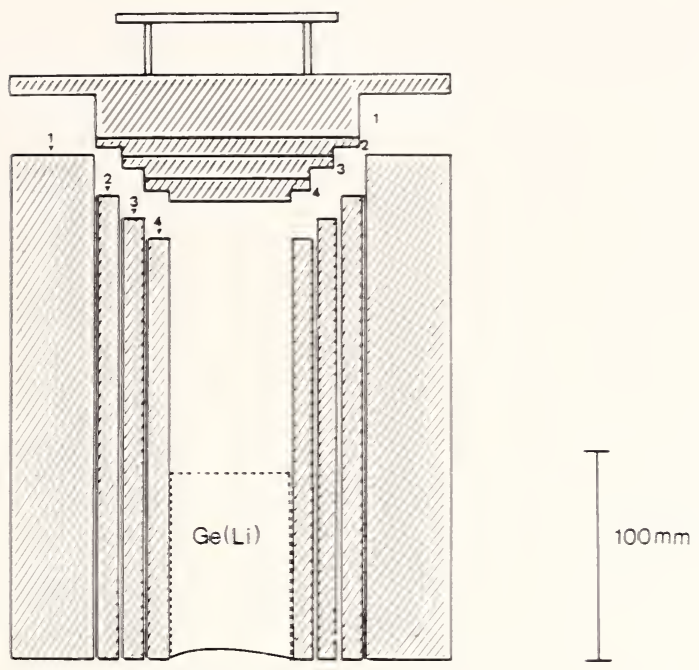

Shieiding of a $\mathrm{Ge}(\mathrm{Li})$ - semiconductor

Figure 1. Schematic of the circular shielding for the semiconductor detector.

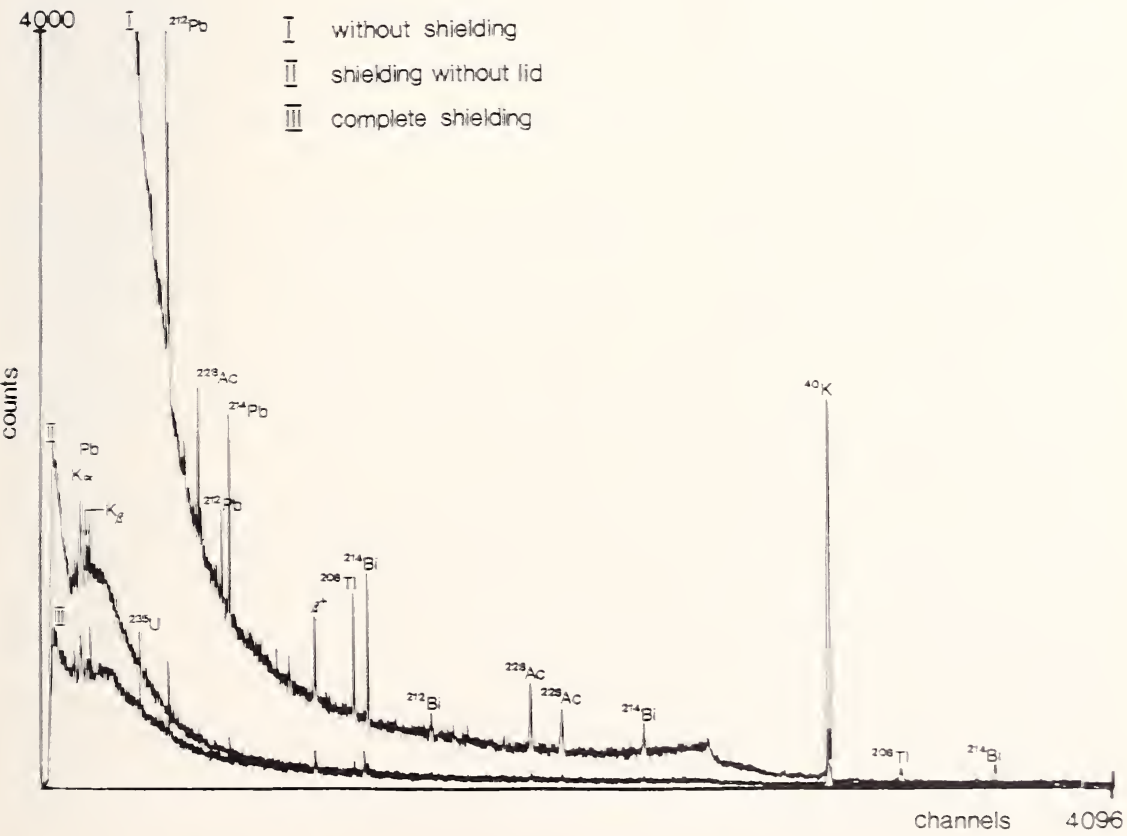

Figure 2. Composition of background curves due to the effect of shielding shown in figure 1. 
It was tested whether elements other than sodium, potassium and phosphorous are retained in the "HAP" column using the operating procedures described (table 2). Tracer studies showed that selenium and cesium are absorbed almost quantitatively by HAP and TiP. The barium activity is reduced severely by absorption on the TiP. The standard deviations obtained from at least five runs include all possible errors using the automated separation column from weighing to counting statistics. It was found that decreasing acidity does not increase the yield of the radionuclides passing through the column.

In order to study the reproducibility and accuracy of the method, Bowen's kale powder was taken as standard, and NBS bovine liver as the sample with "unknown" element concentration. The data for the standard were taken from Bowen's "best mean values" [7].

The results indicated in table 3 were obtained by repeated analyses of at least nine runs to determine 22 elements in the same material, using also short-time irradiation to determine manganese additionally. For most elements, agreement with values given by NBS and other authors [8] is quite satisfactory. Severe deviations are to be noticed for silver. We think that this is due to the values reported by Bowen which he himself considers as inconsistent [7]. The concentration found for potassium is lower than certified by NBS. Because of the 2.5 -fold higher potassium content in kale compared to bovine liver it appears possible that the very high induced radioactivity of potassium- 42 in kale is responsible for the depressed value obtained (dead time of the ADC in the measuring equipment).

Two of the most difficult elements to analyze are chromium and lanthanum. For these elements in particular there seems to be a general need to improve the precision and accuracy. In milk powder for instance, we determined chromium with about 10 percent standard deviation; analyses of the same element in potato powder indicate 60 percent standard deviation, although a fifteenfold higher concentration of chromium was present in the potato powder.

By using INAA (same irradiation conditions as for the destructive method; cooling time 5 to 10 days) the biological standards Bowen's kale powder, NBS orchard leaves and NBS bovine liver were checked mutually for the element concentrations given. The results indicate satisfactory agreement with the values certified for the determination of up to 13 elements in the different standards. For bovine liver an average chromium content of $0.03 \mathrm{ppm}$ was found, using either kale or orchard leaves as standards. This value is not in agreement with the result of 0.5 to $3.5 \mathrm{ppm}$ published by Nadkarni [9], who also used INAA. 
TABLE 2. Retention capacity of the inorganic separators titaniumphosphate (TiP) zirconiumphosphate (ZP) and hydrated antimonypentoxide (HAP) for some radionuclides in percent (medium: $9 \mathrm{~N} \mathrm{HCl}$ )

\begin{tabular}{lrrrrrr}
\hline $\begin{array}{l}\text { Radio- } \\
\text { nuclide }\end{array}$ & TiP & S.D. ${ }^{a}$ & Z.P. & S.D. & HAP & S.D. \\
\hline & & & & & & \\
\hline${ }^{65} \mathrm{Zn}$ & 10.9 & 0.6 & 10.3 & 0.6 & 10.8 & 1.0 \\
${ }^{133} \mathrm{Ba}$ & 64.8 & 1.1 & 14.2 & 2.7 & 9.5 & 2.9 \\
${ }^{60} \mathrm{Co}$ & 10.6 & 2.9 & 14.6 & 0.9 & 10.1 & 1.7 \\
${ }^{137} \mathrm{Cs}$ & 99.5 & 0.5 & 20.8 & .6 & 21.1 & 0.9 \\
${ }^{54} \mathrm{Mn}$ & 10.8 & 2.2 & 7.0 & .6 & 7.2 & 2.2 \\
${ }^{22} \mathrm{Na}$ & 22.0 & 2.3 & 17.5 & 2.9 & 99.6 & 0.5 \\
${ }^{75} \mathrm{Se}$ & 52.5 & 0.9 & 47.7 & 2.3 & 99.0 & .6 \\
\hline
\end{tabular}

a Absolute standard deviation.

TABLE 3. Element concentration found in NBS bovine liver SRM 1577 using neutron activation analysis (standard, Bowen's kale powder)

$\begin{array}{ccccc}\begin{array}{c}\text { Radionuclide } \\ \text { determined }\end{array} & \begin{array}{c}\text { Gamma-ray } \\ \text { used }[\mathrm{KeV}]\end{array} & \begin{array}{c}\text { Element con- } \\ \text { centration } \\ \text { found }[\mathrm{mg} / \mathrm{kg}]\end{array} & \begin{array}{c}\text { S.D. } \\ {[\mathrm{mg} / \mathrm{kg}]}\end{array} & \begin{array}{c}\text { NBS value } \\ {[\mathrm{mg} / \mathrm{kg}]}\end{array}\end{array}$

$\begin{array}{ccccc}{ }^{110 m} \mathrm{Ag} & 658 & 2.0 & 0.6 & (0.06) \\ { }^{76} \mathrm{As} & 1118 & 0.066 & 0.023 & (0.055) \\ { }^{196} \mathrm{Au} & 824 & 0.0049 & 0.0008 & - \\ { }^{82} \mathrm{Br} & 619 & 11.1 & 1.6 & - \\ { }^{115} \mathrm{Cd} & 528 & 0.26 & 0.03 & 0.27 \pm 0.04 \\ { }^{38} \mathrm{Cl} & 1642 & 2615 & 192 & (2600) \\ { }^{60} \mathrm{Co} & 1173 / 1333 & 0.31 & 0.06 & (0.18) \\ { }^{51} \mathrm{Cr} & 320 & 0.06 & 0.03 & - \\ { }^{64} \mathrm{Cu} & 511 & 208 & 27 & 193 \pm 10 \\ { }^{59} \mathrm{Fe} & 1099 & 310 & 28 & 300 \pm 20 \\ { }^{203} \mathrm{Hg} & 279 & 0.015 & 0.004 & 0.016 \pm 0.002 \\ { }^{12} \mathrm{~K} & 1525 & 6674 & 662 & 9700 \pm 600 \\ { }^{140} \mathrm{La} & 1595 & 0.012 & 0.009 & - \\ { }^{56} \mathrm{Mn} & 844 & 10.4 & 1.1 & 10.3 \pm 1 \\ { }^{99} \mathrm{Mo}\left({ }^{99 m} \mathrm{Tc}\right) & 141 & 3.6 & 0.7 & (3.2) \\ { }^{24} \mathrm{Na} & 1368 & 2768 & 156 & 2430 \pm 130 \\ { }^{86} \mathrm{Rb} & 1077 & 16.6 & 2.8 & 18.3 \pm 1 \\ { }^{122} \mathrm{Sb} & 564 & 0.011 & 0.009 & \pm \\ { }^{75} \mathrm{Se} & 264 & 1.3 & 0.4 & 1.1 \pm 0.1 \\ { }^{153} \mathrm{Sm} & 103 & 0.035 & 0.024 & - \\ { }^{187} \mathrm{~W} & 479 & 0.005 & 0.003 & - \\ { }^{69 m} \mathrm{Zn} & 439 & 141 & 16 & 130 \pm 10 \\ { }^{65} \mathrm{Zn} & 1115 & 159 & 8 & \end{array}$

Values in parentheses are not certified. 
It is not possible to make generalized statements about the accuracy of NAA when applied to biological samples. The choice of the standard for example depends on the material, the problem to be solved and on the information one wants about element concentration. It may be concluded from the results obtained and from the method presented that the reliability of NAA for simultaneous multielement determination is quite sufficient for most purposes in the field of food science.

\section{References}

[1] Girardi, F. and Sabbioni, E. J., J. Radioanal. Chem. 1, 169-178 (1968).

[2] Diehl, J. F. and Schelenz, R., Neutron Activation Analysis for Multielement Determination in Foodstuffs and in Other Biological Samples. Fourth Annual Symposium on Recent Advances in the Analytical Chemistry of Pollutants, Basle, 17-19 June, 1974.

[3] Sjöstrand, B., Anal. Chem. 36, 814-819 (1964).

[4] Bethge, P. O.,Anal. Chim. Acta 10,317-320 (1954).

[5] Schelenz, R. and Diehl, J. F., Z. Anal. Chem. 265,93-97 (1973).

[6] Black, W. W., Nucl. Instrum. Methods 71, 317-327 (1969).

[7] Bowen, H. J. M., International Colloquium on the Activation Analysis, Saclay (France), 2-6 October, 1972.

[8] Trace Elements in Relation to Cardiovascular Diseases (WHO/IAEA Joint Research Programme), Research Coordination Meeting, International Atomic Energy Agency, Vienna, Austria, 19-23 February, 1973, Report IAEA-157.

[9] Nadkarni, R. A. and Morrison, G. H., Anal. Chem. 45, 1957-1960 (1973). 


\title{
NEUTRON ACTIVATION ANALYSIS OF ELEMENTS EXTRACTED INTO ORGANIC MEDIA
}

\author{
Renato Stella* and Nicla Genova** \\ * Centro di Radiochimica e Analisi \\ per Attivazione del CNR \\ Istituto di Chimica Generale e Inorganica \\ Università di Pavia \\ 27100 Pavia, Italy \\ **Laboratorio Energia Nucleare Applicata \\ Università di Pavia \\ 27100 Pavia. Italy
}

It is sometimes worthwhile. especially in solvent extraction studies by radiochemical techniques, to neutron irradiate the organic phase after extraction and directly count the same. Problems arise in comparing gamma activities in the corresponding aqueous phases, eventually irradiated after extraction, and in aqueous standards. Many factors may affect, though in a different way. both the neutron activation and counting steps: the former being a function of neutron absorption in the organic medium that may be especially relevant in the case of chlorine containing solvents and of the different neutron thermalizing properties of the same.

To test these effects. tributyl phosphate in various diluents as an extractant for the chlorocomplexes of some noble metals, namely Pd. Pt. Ir. Os and Au. was used.

The effect of different thermalizing power of the media by comparing the activities of irradiated aqueous and organic samples containing same amounts of neutron absorbing elements was also evaluated.

Keywords: Chlorine effect: counting corrections: gamma intensity attenuation: neutron flux depression: organic liquid sample irradiation: palladium distribution ratios; platinum distribution ratios.

\section{Introduction}

It is sometimes worthwhile, especially in solvent extraction studies 
using radiochemical techniques, to neutron irradiate the organic phase after extraction. Problems arise in comparing gamma activities in the latter with those in the corresponding aqueous phases, eventually irradiated after extraction, or in the aqueous standards. Many factors such as differences in thermalizing power, radiation self-absorption and flux depression in the samples may affect the relative measurements [1].

The flux depression effect is especially noteworthy when a strong neutron absorber is present as a component in different concentrations in the samples to be compared. This was the case observed [2] while studying platinum and palladium chlorocomplex extraction into tributylphosphate (TBP) diluted with different hydrocarbons, of which some were chlorinated hydrocarbons. The aim of this work is to investigate the corrections that must be made when using radioanalytical techniques in solvent extraction studies to overcome radiation self-absorption and flux depression effects.

\section{Experimental}

Palladium, gold and platinum stock solutions approximately $10^{-2} M$ were prepared by dissolving in aqua regia exactly weighed amounts of very pure metals $(\mathrm{BDH})$ and finally diluting to proper volumes. Iridium (IV) stock solution was obtained by direct chlorination at $650^{\circ} \mathrm{C}$ of iridium metal mixed with pure $\mathrm{NaCl}$.

Measured aliquots of these solutions were evaporated to dryness on a steam bath, the residue was taken up in concentrated $\mathrm{HCl}$ containing a few milligrams of $\mathrm{NaCl}$ and again evaporated to dryness. This procedure was repeated twice. In the case of iridium the last evaporation step was accomplished after adding to the residue $4 \mathrm{ml}$ of $12 \mathrm{~N} \mathrm{HCl}$ and $4 \mathrm{ml}$ of 36 percent $\mathrm{H}_{2} \mathrm{O}_{2}$ to reoxidize to the quadrivalent state any iridium which had been reduced to the tervalent state. The residues were finally dissolved in a volume of water tenfold the initial one to make the final sample solution.

All other chemicals used were reagent grade.

To measured aliquots of the sample solutions, equal volumes of $12 \mathrm{~N}$ $\mathrm{HCl}$ were added, and extractions performed with a TBP-n-pentane mixture 50 percent $\mathrm{v} / \mathrm{v}$. After separating the phases $\mathrm{n}$-pentane was evaporated from the organic by gentle heating.

Carefully measured aliquots of $0.5 \mathrm{ml}$ of the TBP holding the metal complex, were mixed with $2 \mathrm{ml}$ of isooctane- $\mathrm{CCl}_{4}$ mixtures ranging from 0 to 80 percent $\mathrm{CCl}_{4}$ by volume. 
A series of six samples all containing the same amount of metal were thus prepared for each examined metal. After sealing in plastic vials 10 $\mathrm{mm}$ in diameter, they were irradiated for 4 minutes in the rabbit system of the TRIGA Mark II reactor of the University of Pavia, where a thermal flux of $4.3 \times 10^{12} \mathrm{n} \cdot \mathrm{cm}^{-2} \cdot \mathrm{s}^{-1}$ is available, and the cadmium ratio for gold is $R_{C d, A u}=3$.

After a cooling time that varied depending on the isotope of interest, the following gamma photopeaks were counted with a $\mathrm{Ge}(\mathrm{Li})$ detector linked to a multichannel analyzer: $88 \mathrm{keV}$ from ${ }^{109} \mathrm{Pd}\left(\mathrm{t}_{1 / 2}=13.47 \mathrm{~h}\right), 328 \mathrm{keV}$ from ${ }^{194} \operatorname{Ir}\left(\mathrm{t}_{1 / 2}=17.4 \mathrm{~h}\right), 411.8 \mathrm{keV}$ from ${ }^{198} \mathrm{Au}\left(\mathrm{t}_{1 / 2}=2.7 \mathrm{~d}\right)$ and $158 \mathrm{keV}$ from ${ }^{199} \mathrm{Au}\left(\mathrm{t}_{1 / 2}=3.15 \mathrm{~d}\right)$, the latter being produced by the nuclear reaction: ${ }^{198} \mathrm{Pt}(\mathrm{n}, \gamma){ }^{199} \mathrm{Pt} \stackrel{\beta}{\rightarrow}{ }^{199} \mathrm{Au}$.

\section{Results and Discussion}

Specific activities of the four isotopes are reported in figure 1 as a function of chlorine contents. Reported data were corrected for gamma absorption in the organic layer whose density is not constant. For this purpose irradiated aliquots of the metal aqueous solutions were extracted with pure TBP. Aliquots of $0.5 \mathrm{ml}$ of the latter were then diluted as in the previous experiment with $2 \mathrm{ml}$ of isooctane- $\mathrm{CCl}_{4}$ mixtures. Gamma intensity attenuations relative to the zero $\mathrm{CCl}_{4}$ sample content are reported in figure 2 as a function of the sample density. These curves were used for the already mentioned corrections.

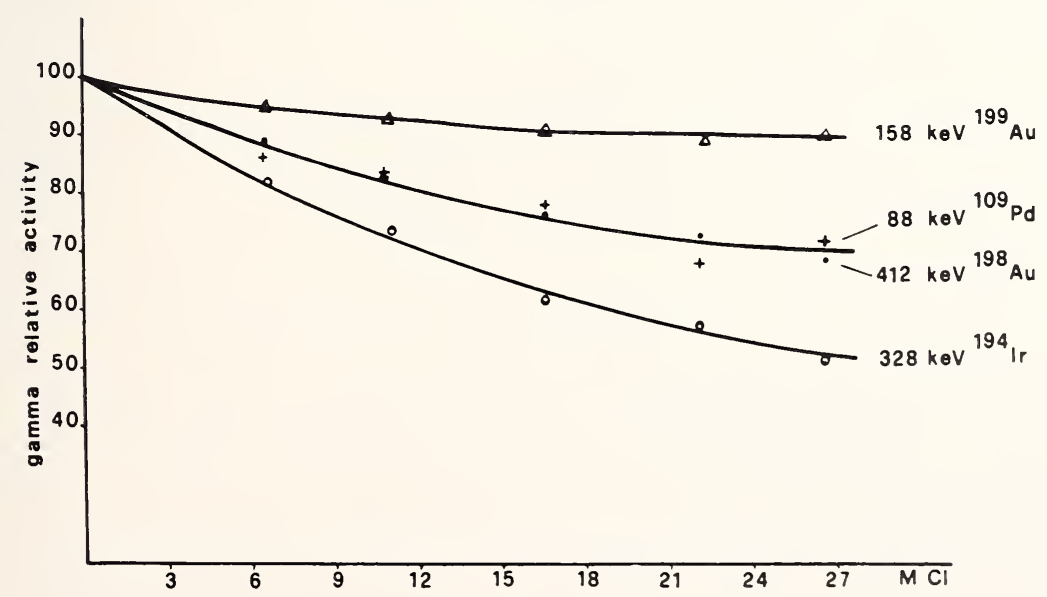

Figure 1. Relative gamma activity as a function of chlorine content in the irradiated liquid samples. 


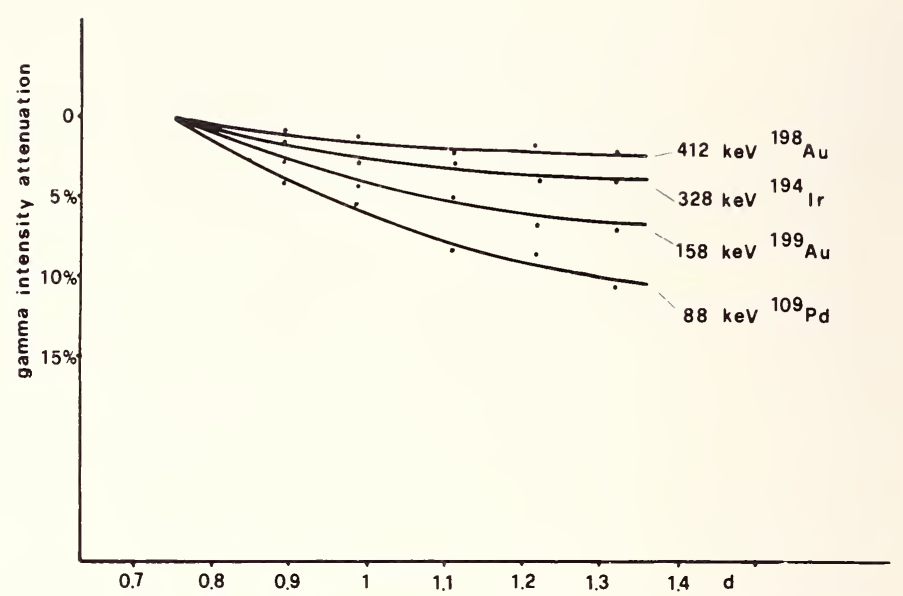

Figure 2. Gamma intensity attenuation as a function of the liquid sample density.

Platinum specific activation shows a less marked decrease as a function of chlorine content in the irradiated samples while palladium and gold behave in a fairly similar way and iridium activation seems more strongly influenced by the chlorine concentration than the other examined metals.

This effect may be at least partially explained if one looks at table 1. In accordance with the $\mathrm{I}_{o} / \sigma_{t h}$ values listed in table 1 , one should expect that the specific activities in the gold and palladium samples are affected by the same percentage decrease due to neutron flux depression, while on the other hand the iridium activity should be more negatively influenced by a neutron flux depression relatively higher for thermal than for epicadmium neutrons.

This is in fact the case, since for chlorine $\sigma=33$ [6], and $\mathrm{I}_{o}=12$ [3], the former being the thermal absorption cross-section, the latter the infinite dilution resonance integrals both referred to the natural isotopic composition.

TABLE 1. Thermal activation cross section $\sigma_{t h}$ and infinite dilution resonance integrals $\mathrm{I}_{o}$

\begin{tabular}{lrrrrr}
\hline $\begin{array}{c}\text { Produced } \\
\text { isotope }\end{array}$ & $\begin{array}{c}\text { Principal } \\
\gamma \text {-energy, keV }\end{array}$ & $\sigma_{\mathrm{th}}, 10^{-28} \mathrm{~m}^{2}$ & $I_{o}, 10^{-28} \mathrm{~m}^{2}$ & $\frac{I_{o}}{\sigma_{\text {th }}}$ & References \\
\hline${ }^{109} \mathrm{Pd}$ & 88.0 & 11.2 & 173 & 15.5 & {$[5]$} \\
${ }^{194} \mathrm{Ir}$ & 328.0 & 110.0 & 1370 & 12.5 & {$[4,3]$} \\
${ }^{198} \mathrm{Au}$ & 411.8 & 98.8 & 1551 & 15.7 & {$[5,4]$} \\
${ }^{199} \mathrm{Au}$ & 158.3 & 4.0 & - & - & {$[4]$} \\
& & & & & \\
\hline
\end{tabular}


The influence that the chemical composition and the amount of TBP may exert on the specific activity was also evaluated. For this purpose samples of 50 percent v/v TBP mixtures with 1,2-dichloro ethane (DE), chloroform and carbon tetrachloride containing constant metal amounts were irradiated as previously described. The decrease in gamma activity relative to the zero chlorine content standard is reported in table 2 and compared with the expected values deduced from figure 1 at the corresponding chlorine contents. The agreement is rather satisfactory to let us assume that, at least in the examined cases, chlorine is mostly responsible for neutron flux depression.

In a previous work [2], the distribution ratios (D values) of platinum and palladium extracted as chlorocomplexes into TBP in several diluents were measured by neutron irradiation of the organic phases. The specific activities were corrected for different neutron flux depressions from liquid samples of different chlorine contents and for gamma absorption as a function of the sample density.

TABLE 2. Gamma specific activities relative to the zero chlorine contents standard in samples of different composition

(mean of three experiments)

\begin{tabular}{|c|c|c|c|c|c|}
\hline \multirow[b]{2}{*}{ Sample $^{\mathfrak{a}}$} & \multicolumn{2}{|c|}{ Relative activity $(\gamma)$} & \multirow[b]{2}{*}{ Sample $^{a}$} & \multicolumn{2}{|c|}{ Relative activity $(\gamma)$} \\
\hline & Experin & Expected & & Experin & Expected \\
\hline${ }^{199} \mathrm{Au}$ in: & & & ${ }^{109} \mathrm{Pd}$ in: & & \\
\hline TBP-DE & 93.5 & 92.0 & TBP-DE & 70.3 & 79.5 \\
\hline $\mathrm{TBP}-\mathrm{CHCl}_{3}$ & 91.0 & 90.5 & $\mathrm{TBP}-\mathrm{CHCl}_{3}$ & 68.5 & 74.0 \\
\hline $\mathrm{TBP}-\mathrm{CCl}_{4}$ & 94.0 & 90.0 & $\mathrm{TBP}-\mathrm{CCl}_{4}$ & 65.0 & 72.0 \\
\hline${ }^{198} \mathrm{Au}$ in: & & & ${ }^{194}$ Ir in: & & \\
\hline TBP-DE & 83.0 & 79.5 & TBP-DE & 74.0 & 70.0 \\
\hline $\mathrm{TBP}-\mathrm{CHCl}_{3}$ & 75.0 & 74.0 & $\mathrm{TBP}-\mathrm{CHCl}{ }_{3}$ & 57.5 & 60.0 \\
\hline $\mathrm{TBP}-\mathrm{CCl}_{4}$ & 79.5 & 72.0 & $\mathrm{TBP}-\mathrm{CCl}_{4}$ & 54.5 & 58.0 \\
\hline
\end{tabular}

a 50 percent $\mathrm{v} / \mathrm{v}$.

In figures 3 and 4 rough D values are connected by dotted lines, corrected $\mathrm{D}$ values by solid lines. Corrections tend to increase $\mathrm{D}$ values for TBP-DE, TBP-CHCl ${ }_{3}$ and TBP-CCl ${ }_{4}$ mixtures and obviously are more remarkable for $\mathrm{Pd}$ than for $\mathrm{Pt}$. Of concern also was the $\mathrm{HCl}$ eventually extracted into TBP-n-hexane and TBP-toluene. The concentration in the equilibrated organic phase reaches the maximum value of $0.7 N$ in TBP-nhexane when is $8 N$ in the aqueous phase [7], and thus introduces only a 
little correction in the upper part of the TBP-n-hexane curve for Pt. In the corresponding Pd curve, correction due to the $88 \mathrm{keV}$ gamma attenuation still has some importance and is of the opposite sign due to the fact that TBP-n-hexane density is lower than that of the aqueous sample.

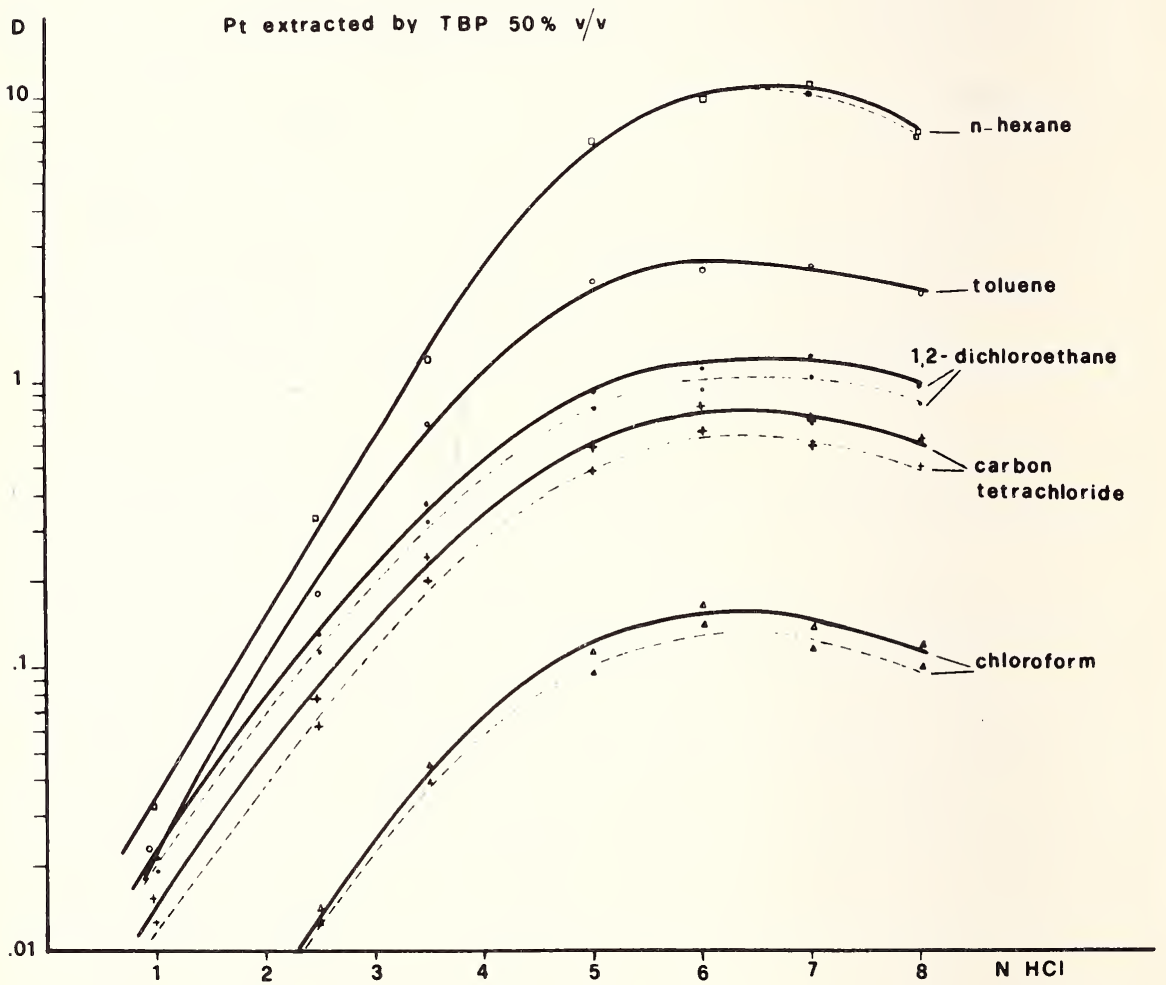

Figure 3. Platinum chlorocomplex distribution ratio as a function of TBP diluent and $\mathrm{HCl}$ concentration in the aqueous phase. (Before ( _. _.) and after (_- counting corrections.) 


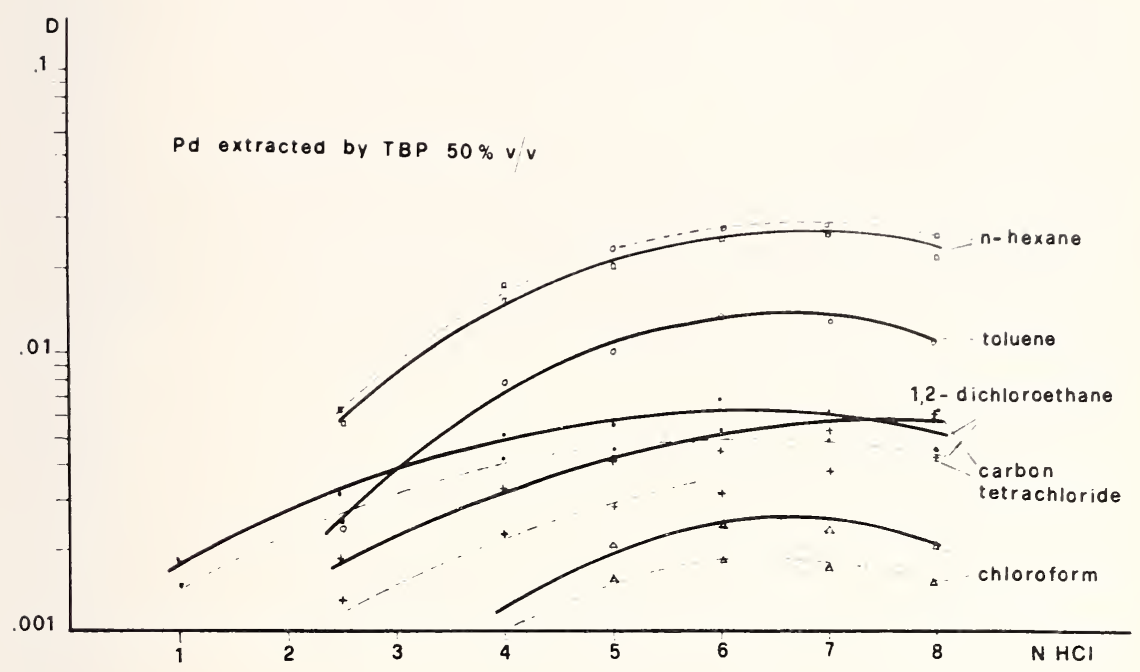

Figure 4. Palladium chlorocomplex distribution ratio as a function of $\mathrm{TBP}$ diluent and $\mathrm{HCl}$ concentration in the aqueous phase. (Before ( . . $)$ and after ( - ) counting corrections.)

\section{References}

[1] Fassò, A., Giorn. Fis. San. Radioprot. 13 (4), 313-320 (1969).

[2] Stella, R. and Genova, N., Radiochem. Radioanal. Letters 16 (6), 273-382 (1974).

[3] Drake, M. K., Nucleonics 24 (8), 108-111 (1966).

[4] Gijbels, R., Talanta 18, 587-601 (1971).

[5] Van der Linden, R., De Corte, F., Van den Winkel, P., and Hoste, J., J. Radioanal. Chem. 11, 133-141 (1972).

[6] Stelm, J. R., Goldberg, M. D., Magurno, B. A. and Wiener-Chasman, R., Neutron Cross Sections, Brookhaven National Laboratory, Associated Universities, Inc., Vol. I, BNL 325.

[7] Hanson, C. and Patel, A. N., J. Appl. Chem. 18, 209-212 (1968). 



\title{
ESTIMATION OF PRIMARY REACTION INTERFERENCES IN FAST PARTICLE ACTIVATION ANALYSIS BY CALCULATIONS USING CROSS SECTION DATA
}

\author{
Viliam Krivan \\ Max-Planck-Institut für Metallforschung \\ Institute für Werkstoffwissenschaften \\ Laboratorium für Reinststoffe \\ D-7070 Schwäbisch Gmünd \\ Federal Republic of Germany
}

Since the primary interference reactions may be a considerable source of systematic errors in fast particle activation analysis, their evaluation is of principal importance. These interferences can often be avoided or at least reduced to a negligible degree by the choice of the optimum projectile energy. In certain cases the interfering nuclear reactions cannot be avoided; then their extent must be evaluated quantitatively and corresponding corrections applied.

For the evaluation of the primary interference reactions by calculation, in addition to the appropriate projectile flux parameters, the cross section data must be known. A critical picture is provided of the present state of the cross section data required for this purpose with regard to their accuracy and completeness. Compilations from which the cross section data can be obtained are surveyed and discussed. A survey of systematics enabling the possibility to estimate unknown cross section data is given. The consequences of the uncertainty in nuclear data for the evaluation of primary reaction interferences are discussed and demonstrated by using practical examples. Handling of nuclear interferences in activation with $14 \mathrm{MeV}$ neutrons, with fast neutrons of intermediate energy and with charged particles is considered.

In most cases, the cross section data needed for these calculations are, at present, already available. Generally, the accuracy suffices to make calculations for assessing the significance of the interferences and to choose the optimum projectile energy. The application of calculations to obtain the actual correction factors is possible only in cases where the cross section data have been critically evaluated and their accuracy is well known.

Keywords: Accuracy; activation analysis; analytical chemistry; charged particle activation analysis; cross sections; excitation functions: fast neutron activation analysis; fast particle activation analy- 
sis; interferences; primary interference reactions; systematic errors; trace analysis.

\section{Introduction}

Activation analysis is based on the linear relationship between the amount of the element to be determined and the amount of the indicator radionuclide produced from this element by a nuclear reaction. However, in addition to the desired reaction, several interference reactions may take place which alter this linear relationship. They can be classified as primary, secondary, and second order reactions.

Of these reactions, the primary interference reactions are generally of greatest importance in fast particle activation analysis. Primary interference reactions are nuclear reactions induced by the principle irradiating particles in elements other than the element to be determined. They yield the same indicator radionuclide as does the desired nuclear reaction, which is the most common case, or it may proceed through a nuclear reaction followed by beta decay of the primary interference reaction product. The indicator radionuclide can be formed by primary interference reactions on elements one or some few units of $\mathrm{Z}$ above or below the element to be determined, depending on the kind of activation. In some cases, interference may occur as the result of fission of heavy elements.

The evaluation of the contribution of primary interference reactions to the production of the indicator radionuclide has been mostly performed by comparison of experimental activation curves for the desired reaction and the interfering reactions. This procedure has been applied to the estimation of primary reaction interferences, e.g., in charged particle activation analysis $[1,2]$, in photon activation analysis [3] as well as in activation analysis using cyclotron produced fast neutrons [4]. For this purpose, much additional experimental work involving irradiation is necessary. The present paper investigates to what extent the experimental evaluation may be replaced by calculations using nuclear data and what accuracy may be expected from these calculations. The activation analysis using $14 \mathrm{MeV}$ neutrons, cyclotron produced neutrons and charged particles is considered.

\section{Principle of the Estimation}

Since the bombarding particles and their parameters as well as the satu- 
ration factor are identical for both the desired and the interfering activation reaction, it is sufficient to express the reaction rates instead of activities.

In the simplest case, discrete values may be assigned to the projectile flux $\Phi$ and to the reactions cross-section $\sigma$, e.g., in activation with 14 $\mathrm{MeV}$ neutrons, the reaction rate per target nucleus $\mathrm{R}$ can be expressed as the product $\Phi \sigma$. In this case, the evaluation of the interference can be carried out by simply comparing the reaction cross sections for the desired and interfering reaction taking into consideration the atomic weight, the concentration and the isotopic abundance of the appropriate element and nuclide, respectively.

If the energy and angular distribution of the projectile particles and also the energy dependence of the reaction cross sections have to be considered, as is the case in activation with cyclotron produced neutrons, the reaction rate per target nucleus must be calculated by integrating the product of these two quantities in an energy increment over the energy range of interest by considering the angular distribution:

$$
R=\int_{0}^{E_{m}} \int_{0}^{\rho_{1}} \Phi(E, \rho) \sigma(E) 2 \pi \sin d E d \rho
$$

where $E_{m}$ is the maximum projectile energy, $\rho_{1}$ the angle given by the size of the sample and its distance from the projectile source, $\Phi(E, \rho)$ is the projectile flux for the given energy $E$ and angle $\rho$ (relative to the direction of the incident beam), $\sigma(E)$ the cross-section for the projectile energy $E$.

Figure 1 shows typical shapes of energy spectra of cyclotron produced neutrons and of charged particles. In the first case, the projectiles are already produced with such energy distribution, while for charged particles, the energy distribution shown is due to energy losses in a thick target. Thus, to calculate the reaction rate in all these cases, in addition to the knowledge of the corresponding projectile beam parameters, the cross section data for the sought reaction has to be known over the whole energy interval of the projectile spectrum. Consequently, the accuracy of estimating the primary reaction interferences depends on how accurately the projectile beam is defined and how accurately the cross sections or excitation functions are known. In general, the inaccuracy of the cross sections and excitation functions given in existing compilations mostly exceeds the inaccuracy of the beam definition several times [5]. Therefore, this will be the main subject of the following discussion of individual activation methods. Of course, time and space limitations do not permit separate dealing with interferences occurring in each individual activation reaction of importance. But still, in addition to providing a general picture of the ability to calculate primary reaction interferences, some examples of practical importance will be given. 


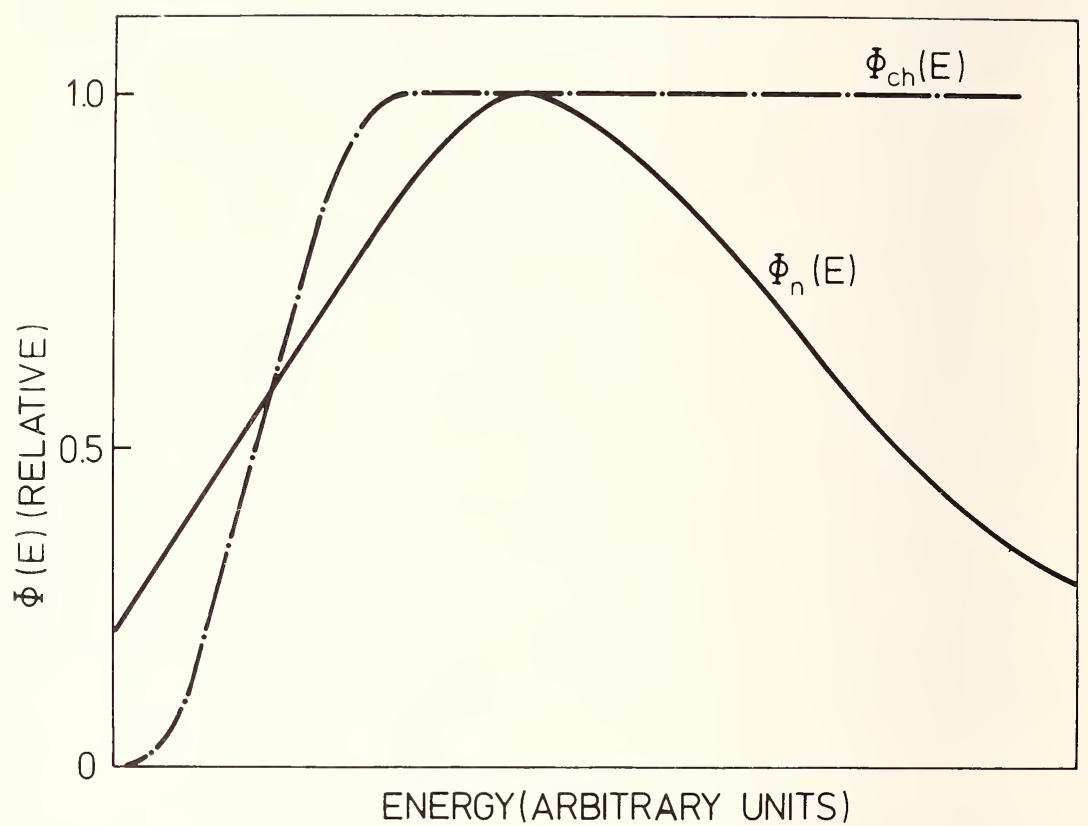

Figure 1. Typical shapes of energy spectra of neutrons produced by bombarding thick Betargets with deuterons $\left(\Phi_{n}(\mathrm{E})\right)$ and of charged particles in using thick targets $\left(\Phi_{c h}(\mathrm{E})\right)$.

\section{General Comments on Activation Cross Section Data}

From the discussion in the previous section it is evident that the existence and accuracy of the Q-values and cross section data are of principal significance for the estimation of the primary reaction interferences. Two compilations of Q-values have been published recently [6,7] covering all reaction types occurring in activation analysis, so that no problem exists any more in readily obtaining Q-values.

In most cases, the cross section data measured by different authors show large discrepancies. This may be explained by different accelerators and nonidentical experimental conditions used by different experimenters. Some compilations give recommended values in addition to the collection of measured data. But there are no entirely objective criteria for the choice of a recommended value from all existing experimental data. By means of critical analysis of the experimental conditions used in measuring the cross sections, in some cases, hints can be obtained as to which results are more reliable. As a rule, more accurate trends can be revealed 
from data measured by the same authors where the relative accuracy is higher. One of the possible ways to obtain more reliable cross sections and/or excitation functions is to carry out appropriate averaging of the available values. The difficulties in selecting the most accurate value from many existing published data are reflected also in the present state of handbooks and compilations: for the same cross sections and excitation functions, in many cases, widely differing values and energy dependences, respectively, may be found in different tabulations, or all experimental data are listed without recommendation of a certain value.

Another serious problem is that for many reactions experimental cross sections and excitation functions have not been measured. The great number of unavailable data can be explained by the limitation of the activation method by which cross sections are mostly measured and by unfavourable decay schemes. But an important factor is that measuring the cross sections is time consuming work, which, unfortunately, in spite of its usefulness does not attract enough working groups having the corresponding facilities at their disposal. The knowledge of the $14 \mathrm{MeV}$ neutron activation cross sections is the most complete of the threshold reactions. It is more critical with excitation functions where for most reactions only parts of the excitation functions are known, or the excitation functions have not been measured, at all. In cases where there is a lack of experimental cross sections, the development of special systematics providing the possibility to estimate unknown cross sections and excitation functions is of great significance. The significance of systematics can even surpass the topic of missing data. In some cases, the deviations between activities obtained using systematics and those obtained experimentally are smaller than the scatter of experimental cross sections obtained by different authors $[5,8]$. This indicates that systematics could be significant as one of the criteria in finding out the recommended values. The systematics may be based on the dependence of the cross sections, or of some characteristic magnitudes of the excitation functions, on different parameters, e.g., Z, N-Z, etc. Systematics usable in the estimation of primary reaction interferences in activation analysis based on threshold reactions will be discussed in more detail, below, for individual activation methods. Cross section data obtained by computation using nuclear theories will also be considered.

\section{Fast Neutron Activation Analysis}

In fast neutron activation analysis [9-12], the most frequently utilized 
principal reactions are that of $(n, p)$ and $(n, 2 n)$ type, but sometimes use is also made of reactions of $(n, \alpha)$ and $\left(n, n^{\prime}\right)$ type. If one of these reaction types is the principal one the others may occur as the primary interference reactions. In addition to these, in certain cases interference reactions of $(n, t),\left(n,{ }^{3} \mathrm{He}\right),(n, p x n),(n, \alpha x n),(n, 2 p)$, and $(n, 2 p x n)$ type may be energetically possible. The complex interference reactions can in practice occur only in activation with cyclotron produced neutrons. The energy spectrum of these neutrons depends on the energy of the incident particles and normally also reaches the higher energy region with its tail part. In some cases, the primary interference through $(\mathrm{n}, \gamma)$ reactions must also be considered. As examples, some of the most important principal reactions together with possible primary interference reactions are given in table 1 .

TABLE 1. Examples of primary interference reactions occurring in fast neutron activation analysis

\begin{tabular}{|c|c|c|c|c|}
\hline . & $\begin{array}{l}\text { Principal } \\
\text { reaction }\end{array}$ & $\begin{array}{c}Q \text {-value } \\
(\mathrm{MeV})\end{array}$ & $\begin{array}{l}\text { Interference } \\
\text { reactions }\end{array}$ & $\begin{array}{l}Q \text {-value } \\
(\mathrm{MeV})\end{array}$ \\
\hline & $\left.{ }^{16} \mathrm{O}(n, p)\right)^{16} \mathrm{~N}$ & -9.6 & $\begin{array}{l}{ }^{19} \mathrm{~F}(n, \alpha){ }^{16} \mathrm{~N} \\
{ }^{20} \mathrm{Ne}(n, \alpha p){ }^{16} \mathrm{~N} \\
{ }^{23} \mathrm{Na}(n, 2 \alpha)^{16} \mathrm{~N} \\
{ }^{15} \mathrm{~N}(n, \gamma){ }^{16} \mathrm{~N}\end{array}$ & $\begin{array}{r}-1.5 \\
-14.4 \\
-12.0 \\
2.5\end{array}$ \\
\hline & ${ }^{90} \mathrm{Zr}(n, 2 n)^{89 m} \mathrm{Zr}$ & -12.0 & $\begin{array}{l}\left.{ }^{92} \mathrm{Mo}(n, \alpha)\right)^{89 m} \mathrm{Zr} \\
{ }^{94} \mathrm{Mo}(n, \alpha 2 n)^{89 m} \mathrm{Zr} \\
{ }^{95} \mathrm{Mo}(n, \alpha 3 n)^{89 m} \mathrm{Zr} \\
{ }^{96} \mathrm{Ru}(n, 2 \alpha)^{89 m} \mathrm{Zr} \\
{ }^{98} \mathrm{Ru}(n, 2 \alpha 2 n)^{89 m} \mathrm{Zr}\end{array}$ & $\begin{array}{r}3.7 \\
-14.1 \\
-21.4 \\
2.0 \\
-16.3\end{array}$ \\
\hline & ${ }^{31} \mathrm{P}(n, \alpha){ }^{28} \mathrm{Al}$ & -1.9 & $\begin{array}{l}{ }^{27} \mathrm{Al}(n, \gamma)^{28} \mathrm{Al} \\
{ }^{28} \mathrm{Si}(n, p){ }^{28} \mathrm{Al} \\
{ }^{29} \mathrm{Si}(n, p n)^{28} \mathrm{Al} \\
{ }^{29} \mathrm{Si}(n, d)^{28} \mathrm{Al} \\
{ }^{30} \mathrm{Si}(n, p 2 n)^{28} \mathrm{Al} \\
{ }^{30} \mathrm{Si}(n, t)^{28} \mathrm{Al} \\
{ }^{32} \mathrm{~S}(n, \alpha p)^{28} \mathrm{Al} \\
{ }^{33} \mathrm{~S}(n, \alpha p n)^{28} \mathrm{Al} \\
{ }^{35} \mathrm{Cl}(n, 2 \alpha)^{28} \mathrm{Al}\end{array}$ & $\begin{array}{r}7.7 \\
-\quad 3.9 \\
-12.3 \\
-10.1 \\
-22.9 \\
-14.4 \\
-10.8 \\
-19.5 \\
-8.9\end{array}$ \\
\hline
\end{tabular}

Detailed data on the flux distributions of $14 \mathrm{MeV}$ neutrons are available in the literature. These data were obtained either by calculations [13$18]$ or were determined experimentally $[14,16,19-23]$. The energy of neutrons produced by generators via the $\mathrm{T}(\mathrm{d}, \mathrm{n})^{4} \mathrm{He}$ reaction depends on the emission angle. An angular variation of energy within $\sim 2 \mathrm{MeV}$ is possible, and it is especially accentuated around $90^{\circ}$ [24], as it can be seen in figure 2 . The topic of the neutron production control systems, of 


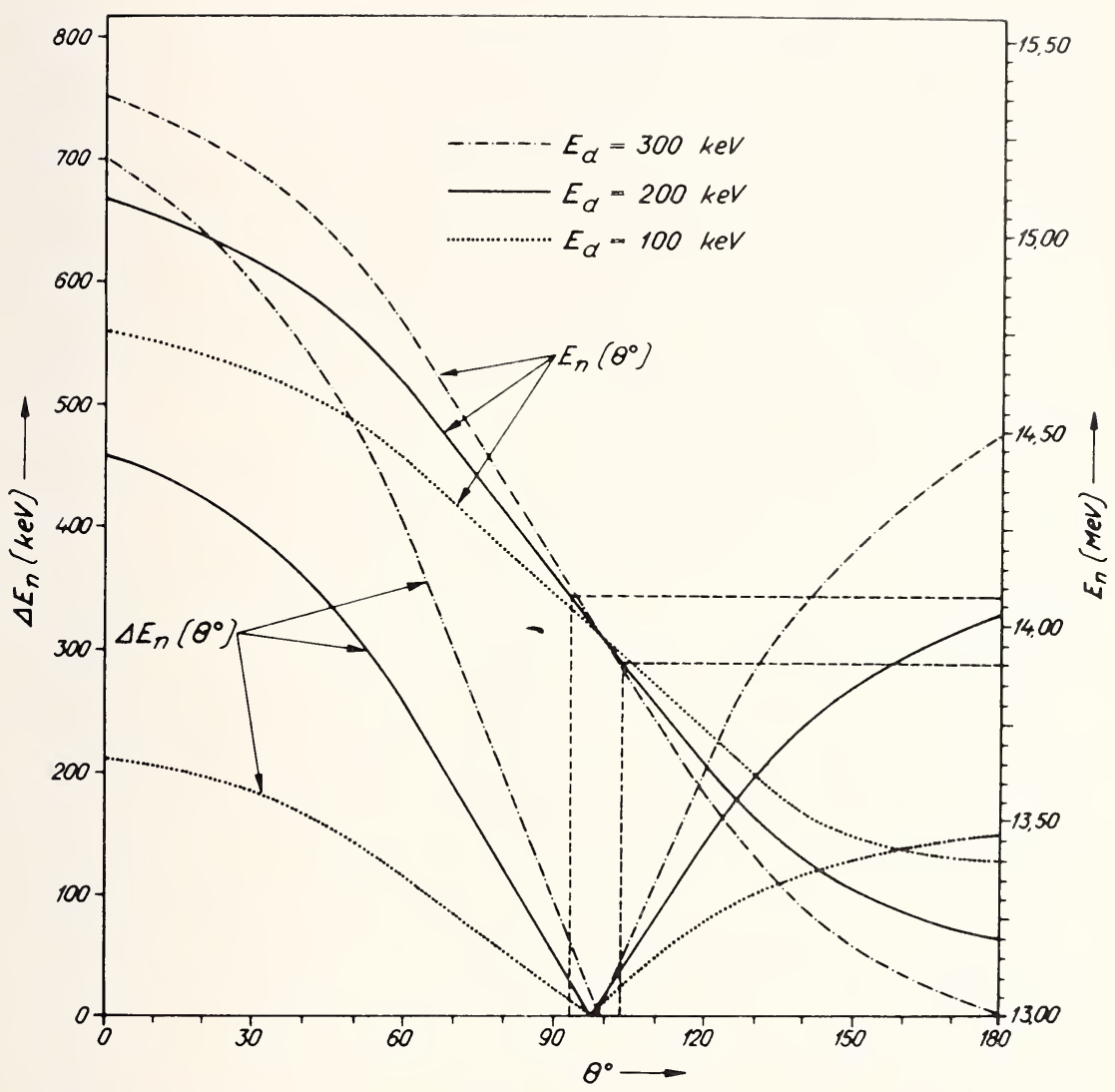

Figure 2. Dependence of energy and energy spread of neutrons on the angle of D+T reaction [24].

the neutron flux distribution, and of the deuteron beam form is comprehensively reviewed and critically discussed by Adams et al. [25] from the point of view of activation analysis.

Neutrons of intermediate energies may be obtained by using accelerated protons, deuterons, helium- 3 and $\alpha$-particles from a cyclotron to bombard a suitable target. Among all these possibilities of producing fast neutrons, bombarding a thick beryllium target with deuterons seems to be most attractive for activation analysis [26-28]. The energy and angle distribution of neutrons produced in this way can be satisfactorily described [26, 29].

Numerous data on the cross sections of nuclear reactions induced by neutrons with energies close to $14 \mathrm{MeV}$ have been published. Un- 
fortunately, these data are, in general, very inconsistent. In the $14 \mathrm{MeV}$ activation cross sections, an additional factor increasing the inconsistencies of the cross section data results from the unnormalized neutron energy. As is evident from figure 2, the neutron energy varies around the value of $14 \mathrm{MeV}$. Figure 3 shows that, in some cases, the neutron activation cross sections may be strongly energy-dependent around $14 \mathrm{MeV}$. Consequently, one should not be too free with the energy definition in measuring or comparing the $14 \mathrm{MeV}$ neutron activation cross sections. The need to reaiize the proposal by Czikai et al. [24] to normalize the neutron energy by an angular interval is very urgent.

There exist several compilations from which the activation cross sections for neutrons of energy around $14 \mathrm{MeV}$ can be obtained. Some of them survey all cross section data available without giving recommended values [30-34], others contain recommended values along with estimated accuracy based on the analysis of the existing data [8,36-38] while some contain recommended values without estimating the accuracy [24, 39-41]. For the reason discussed above, very different values may be found for the same reaction in different tabulations. Table 2 shows several examples of bad agreement between cross sections recommended in different tabulations for the same $(n, 2 n),(n, p)$, and $(n, \alpha)$ reaction. The discrepancy between the individual measured or recommended values is similar or even greater for $(n, d),(n, t),\left(n,{ }^{3} \mathrm{He}\right)$ and complex reactions. Table 2 indicates that it is very difficult to judge the accuracy of the cross section value used. From this reason it seems to be useful to provide a general picture of the expected uncertainty of the cross section data used and, at the same time, also of the calculated activities or reaction rates. This was done by the following procedure. From the cross section data published by different authors, the mean values were expressed and the deviations between these mean values and the extreme experimental values were calculated. Figure 4 shows the integrated histogram of the distribution of deviations obtained in this way for $(n, 2 n)$ reactions and a neutron energy close to $14.7 \mathrm{MeV}$. The results presented in this figure are based on the evaluation of cross sections of 114 reactions. Results obtained in the same way for $(\mathrm{n}, \mathrm{p})$ and $(\mathrm{n}, \alpha)$ reactions are shown in figure 5. They are based on the evaluation of cross sections of $162(\mathrm{p}, \mathrm{n})$ and $(\mathrm{p}, \alpha)$ reactions. The number of experimental results per reaction varied between 2 (e.g., for the ${ }^{121} \operatorname{Sb}(n, 2 n)$ ${ }^{120 m} \mathrm{Sb}$ reaction) and 20 (for the ${ }^{56} \mathrm{Fe}(\mathrm{n}, \mathrm{p})^{56} \mathrm{Mn}$ reaction). For example, the following information can be read off from the integrated histograms: the deviations are less than 30 percent in 55 percent of the cases for $(n, 2 n)$ reactions and in 44 percent of the cases for $(n, p)$ and $(n, \alpha)$ reac- 
KRIVAN

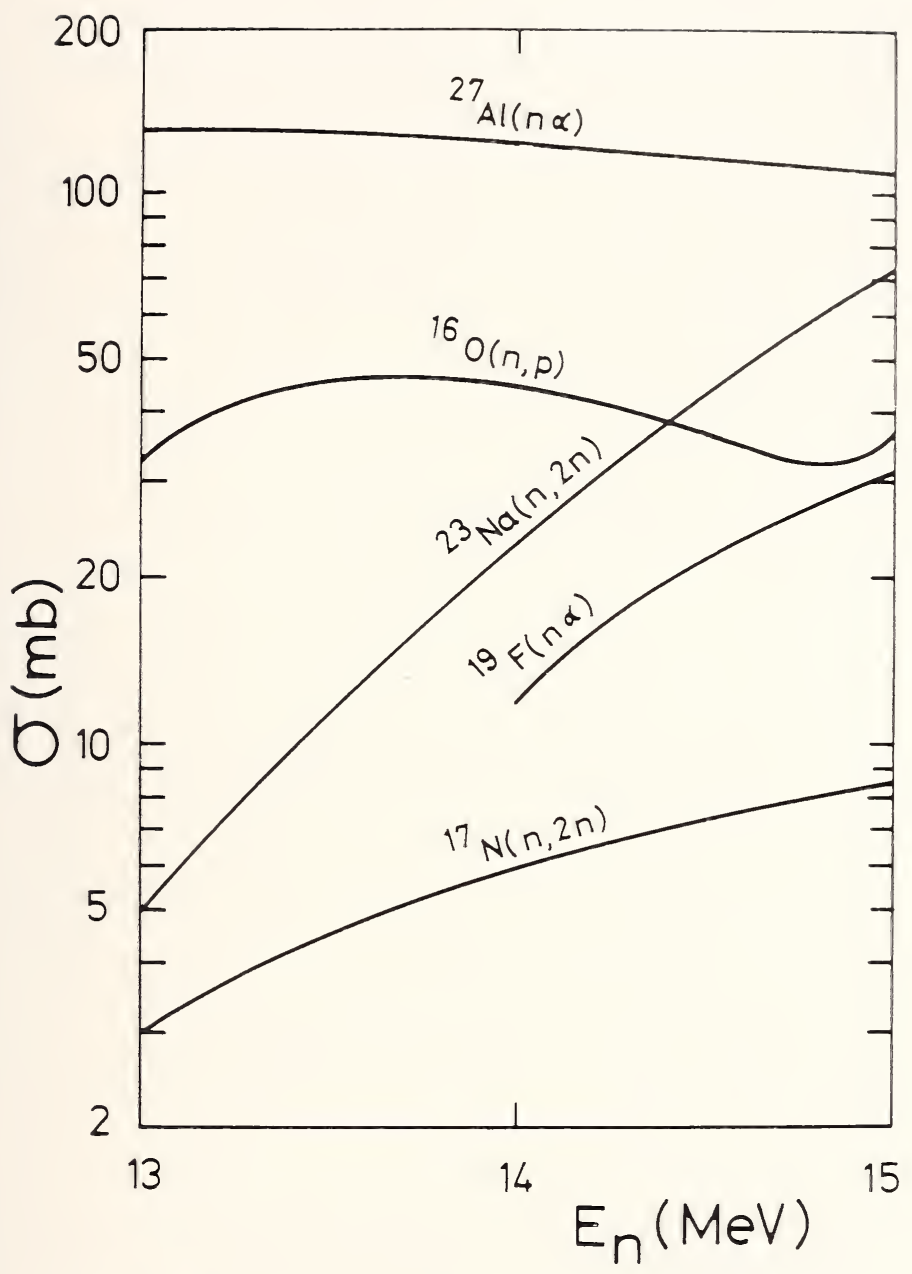

Figure 3. Examples of energy dependence of the cross sections for fast neutron induced reactions around $14 \mathrm{MeV}$. 


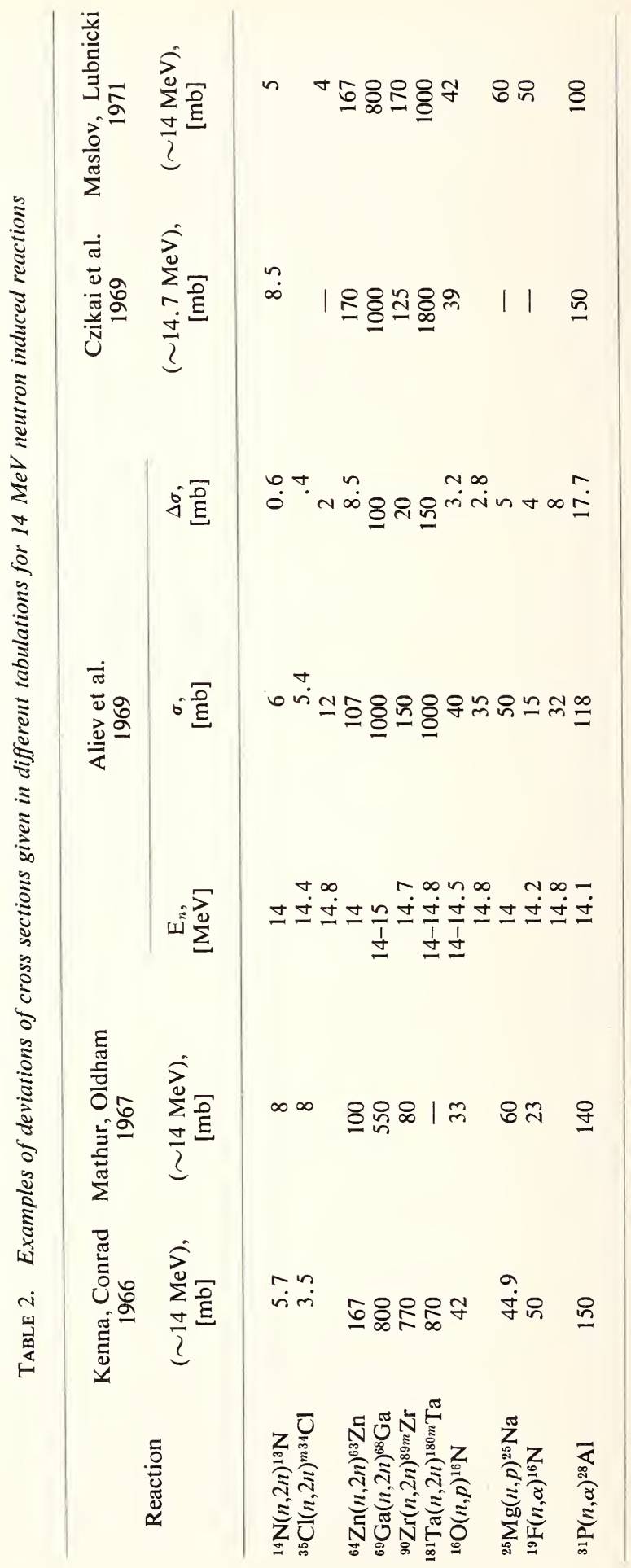




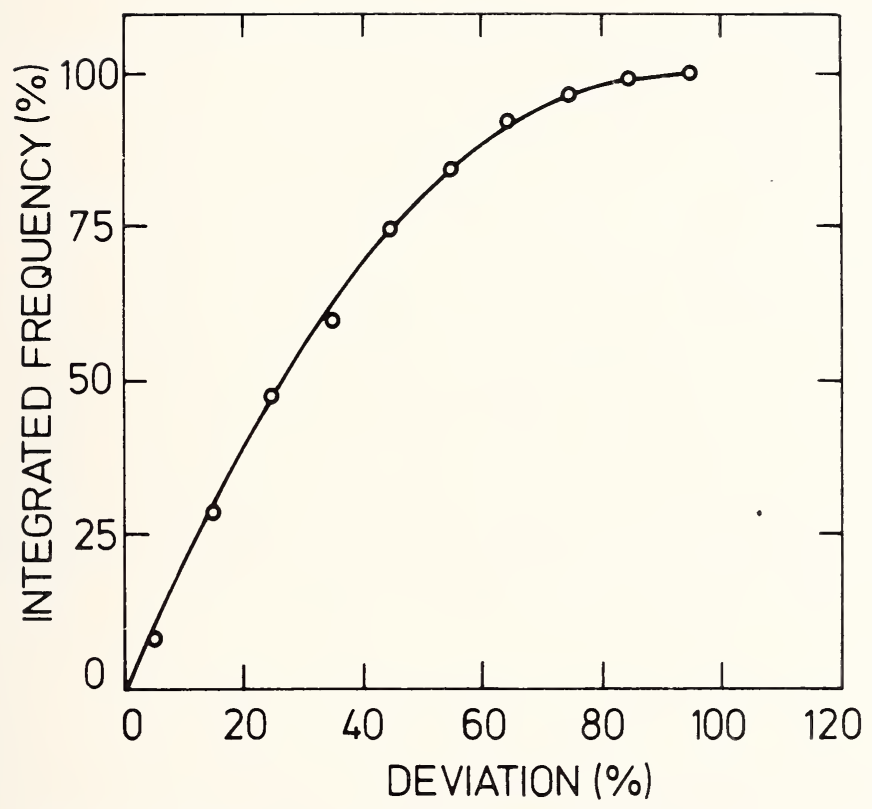

Figure 4. Integrated histogram of the frequency distribution of the deviations between the mean and extreme values of experimental neutron cross sections for $(n, 2 n)$ reactions and a neutron energy close to $14.7 \mathrm{MeV}$. 


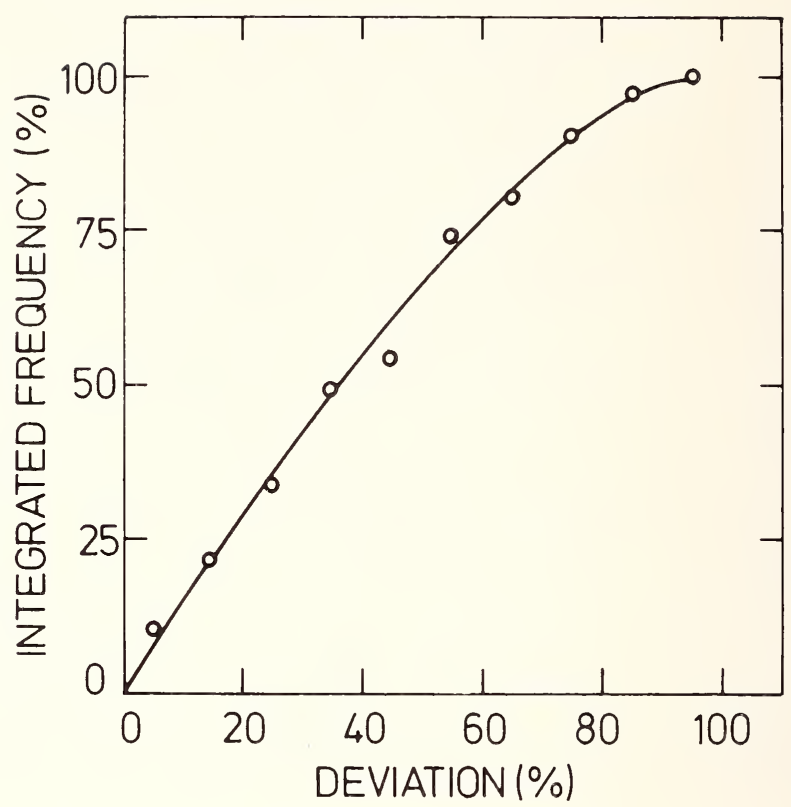

Figure 5. Integrated histogram of the frequency distribution of the deviations between the mean and extreme values of experimental neutron cross sections for $(n, p)$ and $(n, \alpha)$ reactions and a neutron energy close to $14.7 \mathrm{MeV}$.

tions. The deviations are less than 70 percent in 95 percent of the cases for $(n, 2 n)$ reactions and in 86 percent for $(n, p)$ and $(n, \alpha)$ reactions. One should note that the results obtained by these evaluations are close to the maximum possible uncertainty and that the actual uncertainty could be expected to be lower. There exist a number of reactions for which the cross sections have been evaluated and are now known with good accuracy [8], e.g., ${ }^{27} \mathrm{Al}(\mathrm{n}, \mathrm{p}),{ }^{27} \mathrm{Al}(\mathrm{n}, \alpha),{ }^{65} \mathrm{Cu}(\mathrm{n}, 2 \mathrm{n}),{ }^{63} \mathrm{Cu}(\mathrm{n}, 2 \mathrm{n}),{ }^{32} \mathrm{~S}(\mathrm{n}, \mathrm{p}),{ }^{31} \mathrm{P}(\mathrm{n}, \alpha)$, ${ }^{197} \mathrm{Au}(\mathrm{n}, 2 \mathrm{n})$ etc.

Another problem is caused by the incompleteness of the existing data sources. There exist three $14-\mathrm{MeV}$ cross section tabulations containing data for all reactions which are energetically allowed to occur, i.e., that compiled by Neuert and Pollehn [34], by Goldberg et al. [30-33] and by Aliev et al. [36]. The remaining compilations give data either for $(n, 2 n)$, $(n, p)$ and $(n, \alpha)$ reactions [24,38-41] or only for one of these reaction types $[8,35,42]$. 
Though many new and better experimental data have become available in recent years, there exist a great number of reactions for which the cross section data are still unknown. In these cases, the application of the existing systematics may be useful in estimating unknown cross section data. Systematics of cross sections have been developed for the following reaction types: $(\mathrm{n}, 2 \mathrm{n}) \quad[8,43-45], \quad(\mathrm{n}, \mathrm{p}) \quad[45,46],(\mathrm{n}, \alpha) \quad[45,46], \quad(\mathrm{n}, \mathrm{t})$ $[45,47,48],\left(n,{ }^{3} \mathrm{He}\right)[47,49]$, and $(\mathrm{n}, \gamma)[24,50,51]$. For cross sections of $(\mathrm{n}, 2 \mathrm{n})$ reactions semi-empirical and empirical formulae have been developed by Pearlstein [42], Adám and Jéki [52], and Chatterjee [53]. The comparison with the values recommended by Bödy and Czikai [8] shows that in 59 percent of all cases Pearlstein's formula gives the best approximation, whereby for mass number $\mathrm{A}<80$ the cross sections are underestimated and for $\mathrm{A}>100$ are overestimated. All three formulae give unreliable values for neutron-deficient isotopes far from the stability line. A comparison of the values obtained using Pearlstein's formula with experimental mean values shows [54] that the ratio of these two values is less than 1.25 in more than 70 percent of the cases.

The state of excitation functions for the fast neutron induced reactions is much more unsatisfactory, as compared with $14 \mathrm{MeV}$ neutron cross sections. For the most reactions only parts of the excitation functions are known, or the excitation functions have not been measured at all. To give an impression about the overall accuracy with which the excitation functions have been measured, figure 6 shows the integrated histogram of the deviations between the mean and the extreme values of maximum cross sections for $(n, 2 n),(n, p)$ and $(n, \alpha)$ reactions. The results are based on the evaluation of maximum cross sections for 49 reactions.

For the following reactions, the excitation functions have been evaluated and are known with good accuracy: ${ }^{27} \mathrm{Al}(\mathrm{n}, \mathrm{p})^{27} \mathrm{Mg},{ }^{27} \mathrm{Al}(\mathrm{n}, \alpha)^{24} \mathrm{Na}$, ${ }^{31} \mathrm{P}(\mathrm{n}, \mathrm{p})^{31} \mathrm{Si},{ }^{32} \mathrm{~S}(\mathrm{n}, \mathrm{p})^{32} \mathrm{P},{ }^{46} \mathrm{Ti}(\mathrm{n}, \mathrm{p})^{46} \mathrm{Sc},{ }^{47} \mathrm{Ti}(\mathrm{n}, \mathrm{p}){ }^{47} \mathrm{Sc},{ }^{55} \mathrm{Mn}(\mathrm{n}, 2 \mathrm{n})^{54} \mathrm{Mn}$, ${ }^{54} \mathrm{Fe}(\mathrm{n}, \mathrm{p}){ }^{54} \mathrm{Mn}, \quad{ }^{56} \mathrm{Fe}(\mathrm{n}, \mathrm{p}){ }^{56} \mathrm{Mn}, \quad{ }^{59} \mathrm{Co}(\mathrm{n}, \alpha)^{56} \mathrm{Mn}, \quad{ }^{58} \mathrm{Ni}(\mathrm{n}, \mathrm{p})^{55} \mathrm{Fe}$, ${ }^{65} \mathrm{Cu}(\mathrm{n}, 2 \mathrm{n}){ }^{64} \mathrm{Cu}, \quad{ }^{64} \mathrm{Zn}(\mathrm{n}, \mathrm{p})^{64} \mathrm{Cu}, \quad{ }^{115} \mathrm{In}\left(\mathrm{n}, \mathrm{n}^{\prime}\right){ }^{155 m} \mathrm{In}, \quad{ }^{127} \mathrm{I}(\mathrm{n}, 2 \mathrm{n}){ }^{126} \mathrm{I}$, ${ }^{197} \mathrm{Au}(\mathrm{n}, 2 \mathrm{n}){ }^{196 m} \mathrm{Au}$, and ${ }^{197} \mathrm{Au}(\mathrm{n}, 2 \mathrm{n}){ }^{196} \mathrm{Au}$. Most of these excitation functions are used in fast neutron metrology and have been recently compiled and compared by Zijp et al. [55]. The existing data on excitation functions for (n,threshold) reactions are available from several compilations [30-33,36,56-58]. The BNL 325 series [30-33], of which a new edition will be soon available is most comprehensively compiled. Since experimental data for excitation functions are available for only few ( $\mathrm{n}$,threshold) reactions, systematics of excitation functions are of substantial significance in this case. For $(n, 2 n),(n, p)$ and $(n, \alpha)$ reactions, systematics of excitation functions have been developed recently [59-60]. 


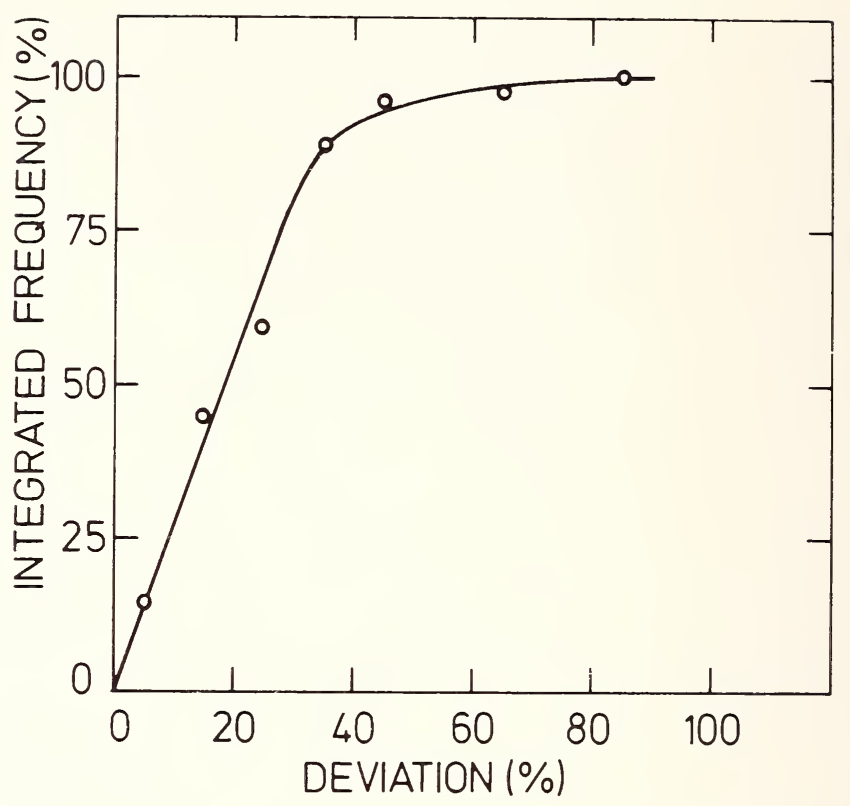

Figure 6. Integrated histogram of the frequency distribution of the deviations between the mean and extreme values of experimental maximum cross sections for $(n, 2 n),(n, p)$ and $(\mathrm{n}, \alpha)$ reactions.

The systematics are based on the dependence of the characteristic magnitudes (maximum cross section, the position of the maximum cross section, FWHM, and the asymmetry-factor at FWHM) of an excitation function on the atomic number of the target nuclide. As an example, the trends obtained for $\sigma_{\max }$ are shown in figure 7. Using these systematics, an unknown excitation function can be constructed. In this case, the systematics were checked by comparing the activities calculated using the constructed excitation functions and the experimentally determined activities for 15 reactions of $(n, 2 n),(n, p)$ and $(n, \alpha)$ types. In the case of 12 reactions, the agreement was better than a factor of 1.35 and in the case of 14 reactions better than a factor of 2 . The systematics of excitation functions for additional reaction types is in progress [14]. 


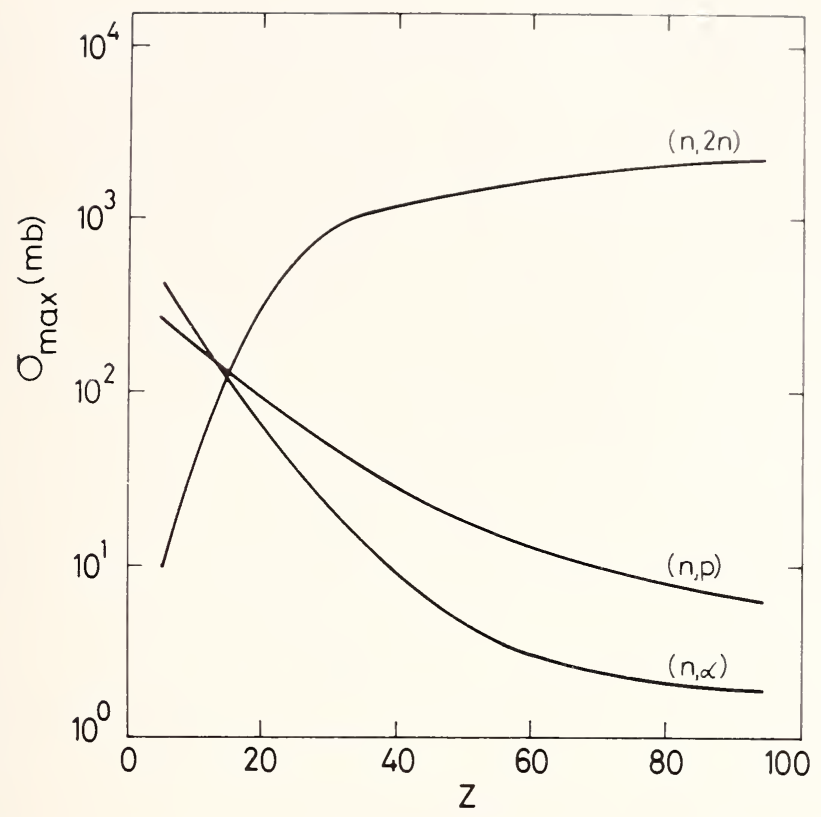

Figure 7. Maximum cross sections of excitation functions for (n,2n), (n,p), and (n, $\alpha)$ reactions as a function of $Z$ of the target nuclide [26].

\section{Charged Particle Activation Analysis}

Generally, charged particle activation analysis is more complex than neutron activation analysis. Almost exclusively light particles, i.e., protons, deuterons, ${ }^{3} \mathrm{He}$ and $\alpha$, have been applied in the charged particle activation analysis. Principal reactions of the type $(\mathrm{p}, \mathrm{n})$ and $(\mathrm{p}, \mathrm{pn})$ for protons, $(\mathrm{d}, \mathrm{p}),(\mathrm{d}, \mathrm{n})$ and $(\mathrm{d}, 2 \mathrm{n})$ for deuterons, $\left({ }^{3} \mathrm{He}, \mathrm{n}\right),\left({ }^{3} \mathrm{He}, \mathrm{p}\right)$ for ${ }^{3} \mathrm{He}$, and $(\alpha, n),(\alpha, 2 n)$ and $(\alpha, p)$ for $\alpha$-particles are mostly used. Several types of primary interference reactions may occur simultaneously. Some examples are shown in table 3.

In the charged particle activation analysis no problems occur with the angular distribution. The effect of beam divergency can be neglected. The flux of charged particles can be determined by measuring the beam intensity using a Faraday cup or using a beam monitor. As the angular distribution does not need to be considered, a correspondingly simplified version of equation (1) can be used for the calculations. A special problem of charged particle activation analysis is represented by the energy losses of the projectiles and their ranges in the sample. The knowledge of these parameters is important due to the dependence of the cross section on the 
TABLE 3. Examples of primary interference reactions in charged particle activation analysis

\begin{tabular}{|c|c|c|c|}
\hline Principal reaction & $\begin{array}{l}\text { Q-value } \\
(\mathrm{MeV})\end{array}$ & Interference reactions & $\begin{array}{l}\text { Q-value } \\
(\mathrm{MeV})\end{array}$ \\
\hline${ }^{48} \mathrm{Ti}(p, n){ }^{48} \mathrm{~V}$ & -4.8 & $\begin{array}{l}{ }^{50} \mathrm{~V}(p, p 2 n)^{48} \mathrm{~V} \\
{ }^{51} \mathrm{~V}(p, p 3 n)^{48} \mathrm{~V} \\
{ }^{50} \mathrm{Cr}\left(p,{ }^{3} \mathrm{He}\right)^{48} \mathrm{~V} \\
{ }^{50} \mathrm{Cr}(p, 2 p n){ }^{48} \mathrm{~V} \\
{ }^{52} \mathrm{Cr}(p, \alpha n)^{48} \mathrm{~V}\end{array}$ & $\begin{array}{l}-19.1 \\
-31.9 \\
-13.4 \\
-21.1 \\
-14.2\end{array}$ \\
\hline${ }^{26} \mathrm{Mg}(\alpha, p){ }^{27} \mathrm{Mg}$ & 4.2 & $\begin{array}{l}{ }^{27} \mathrm{Al}(d, 2 p)^{27} \mathrm{Mg} \\
{ }^{28} \mathrm{Si}(d, 3 p)^{27} \mathrm{Mg} \\
\left.{ }^{29} \mathrm{Si}(d, 3 p n)\right)^{27} \mathrm{Mg} \\
{ }^{30} \mathrm{Si}(d, \alpha p)^{27} \mathrm{Mg}\end{array}$ & $\begin{array}{r}-4.1 \\
-15.6 \\
-24.1 \\
-6.4\end{array}$ \\
\hline $\begin{array}{c}{ }^{16} \mathrm{O}\left({ }^{3} \mathrm{He}, p\right)^{18} \mathrm{~F} \\
+ \\
\left.{ }^{16} \mathrm{O}\left({ }^{3} \mathrm{He}, n\right)\right)^{18} \mathrm{Ne}\end{array}$ & $\begin{array}{r}2.0 \\
-3.2\end{array}$ & $\begin{array}{l}{ }^{19} \mathrm{~F}\left({ }^{3} \mathrm{He}, \alpha\right){ }^{18} \mathrm{~F} \\
{ }^{23} \mathrm{Na}\left({ }^{3} \mathrm{He}, 2 \alpha\right){ }^{18} \mathrm{~F} \\
{ }^{24} \mathrm{Mg}\left({ }^{3} \mathrm{He}, 2 \alpha p\right){ }^{18} \mathrm{~F}\end{array}$ & $\begin{array}{r}10.1 \\
-0.3 \\
-4.0\end{array}$ \\
\hline$\stackrel{\beta^{+}}{\longrightarrow}{ }^{18} \mathrm{~F}$ & & $\begin{array}{l}{ }^{25} \mathrm{Mg}\left({ }^{3} \mathrm{He}, 2 \alpha p n\right){ }^{18} \mathrm{~F} \\
{ }^{26} \mathrm{Mg}\left({ }^{3} \mathrm{He}, 2 \alpha p 2 n\right){ }^{18} \mathrm{~F} \\
{ }^{27} \mathrm{Al}\left({ }^{3} \mathrm{He}, 3 \alpha\right){ }^{18} \mathrm{~F}\end{array}$ & $\begin{array}{r}-18.9 \\
-27.0 \\
-9.5\end{array}$ \\
\hline${ }^{31} \mathrm{P}(\alpha, n){ }^{34 m} \mathrm{Cl}$ & -5.6 & $\begin{array}{l}\left.{ }^{32} \mathrm{~S}(\alpha, p n)\right)^{34 m} \mathrm{Cl} \\
{ }^{33} \mathrm{~S}(\alpha, p 2 n)^{34 m} \mathrm{Cl} \\
{ }^{34} \mathrm{~S}(\alpha, p 3 n)^{34 m} \mathrm{Cl} \\
{ }^{35} \mathrm{Cl}(\alpha, \alpha n)^{34 m} \mathrm{Cl}\end{array}$ & $\begin{array}{l}-14.5 \\
-23.2 \\
-34.6 \\
-12.6\end{array}$ \\
\hline
\end{tabular}

projectile energy. For this purpose, the tabulation of stopping powers and ranges by Williamson et al. [61] is very useful. Methodical aspects of charged particle activation analysis have been recently reviewed in detail by Ricci [62].

Concerning the discrepancies between published data, the completeness and recommended values, the state of excitation functions for charged particle induced reactions does not differ essentially from that of fast neutrons. Still, the relative number of unknown excitation functions and the disagreement between individual data is smaller for charged particles as compared with fast neutrons. Though several collections of cross section data for charged particle induced reactions have been published, each of them covered reactions with only some few nuclides, i.e., with $\mathrm{H}$ up to F [63], with $\mathrm{Ne}$ to $\mathrm{Cr}$ [64], with $\mathrm{Mn}, \mathrm{Fe}, \mathrm{Co}[65], \mathrm{Ni}, \mathrm{Cu}[66]$, $\mathrm{Li}, \mathrm{Be}, \mathrm{B}$ [67], C [68], O, H, [69], with $\mathrm{H}$ up to S [70], and with Li up to $\mathrm{Ne}[71]$. A very comprehensive compilation has been published recently by Lange et al. [72]. It covers the whole atomic number range and contains measured excitation functions for all reaction types which 
can occur in charged particle activation analysis. Recently Jäger and Münzel [54] estimated the accuracy of the evaluated activities produced in thick targets using the excitation functions for a given nuclear reaction published by different authors. In this case, the mean value was calculated and compared with the individual one. The integrated histogram is shown in figure 8. A comparison of these results with those shown in figures 4-6 indicates that the cross section data for charged particle induced reactions were more accurate as compared with cross section data for fast neutron induced reactions. However, one should note that the results for charged particles in figure 8 are based on deviations between the mean and all available experimental data while the results for fast neutrons are based on deviations between the mean and the extreme experimental values. Clearly, by using the same evaluation procedure, the difference between the distribution of deviations for charged particles and neutrons would be smaller.

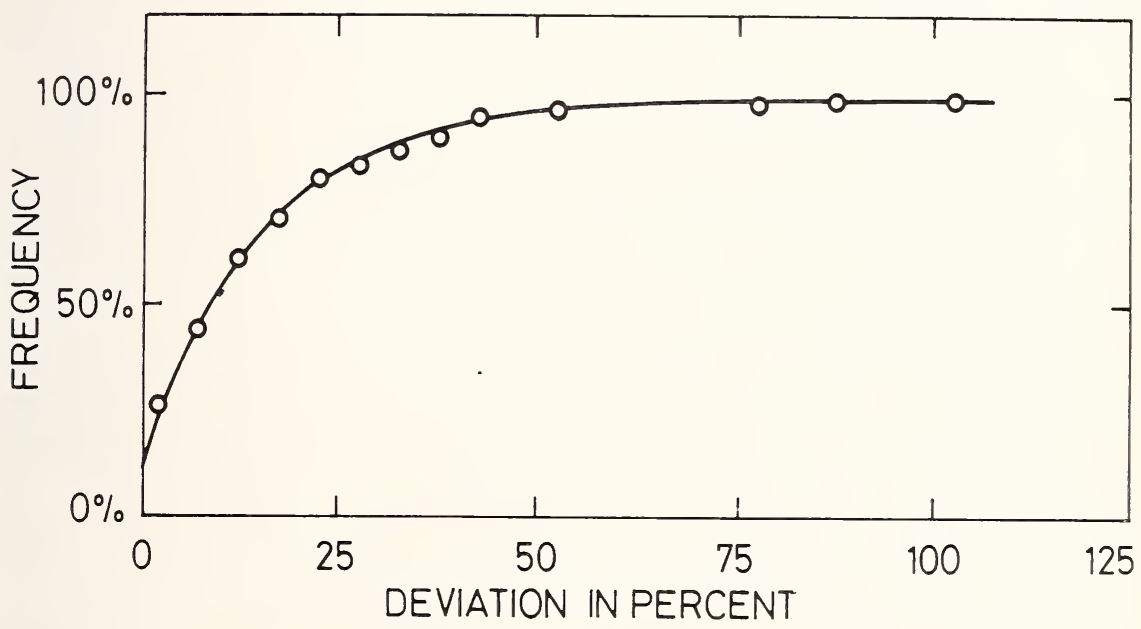

Figure 8. Integrated histogram of the frequency distribution of deviations of the thick target yields from the mean value for charged particle induced reactions [54].

If there is a demand for excitation functions not yet measured, a systematics is available for $(, \gamma),(, \mathrm{xn}),(, \mathrm{pxn}),(, 2 \mathrm{p}),(, \alpha)$ and $(, \alpha \mathrm{n})$ reactions and for $\mathrm{p}, \mathrm{d},{ }^{3} \mathrm{He}$ and $\alpha$ as projectiles [73], which is based on a semiempirical approach.

Using this systematics, unknown excitation functions and thick target yields can be estimated for the above-listed reaction types and stable target nuclides of $Z$ ranging from 10 to 83 . An examination of the uncertainty of the estimated excitation functions by calculating thick target yields and 
comparing them with the mean value of the experimental data showed [54] that in 50 percent of the cases, the deviations for the excitation functions obtained by systematics are smaller than a factor of 1.25 and in 90 percent of the cases are smaller than a factor of 2. Extreme deviations may occur in the case of double magic nuclides.

\section{Examples of Evaluation of Primary Reaction Interferences}

The estimation of primary reaction interferences by calculations using cross section data is illustrated below, using some examples of great practical importance. The calculated results are compared with those obtained experimentally.

The most important application of $14 \mathrm{MeV}$ NAA are undoubtedly the determination of oxygen via the ${ }^{16} \mathrm{O}(\mathrm{n}, \mathrm{p})^{16} \mathrm{~N}$ reaction and the determination of silicon via the ${ }^{28} \mathrm{Si}(\mathrm{n}, \mathrm{p})^{28} \mathrm{Al}$ reaction. In the oxygen determination, the indicator radionuclide can be produced also from fluorine via the ${ }^{19} \mathrm{~F}(\mathrm{n}, \alpha){ }^{16} \mathrm{~N}$ reaction, and in the silicon determination, it can be produced also from phosphorus via the ${ }^{31} \mathrm{P}(\mathrm{n}, \alpha)^{28} \mathrm{Al}$ reaction. Clearly, the evaluation of these interferences is of principal significance. Table 4 gives results of evaluation of these two interference reactions calculated using cross sections from different compilations along with data obtained experimentally. The results are given as the amount of the interfering element in milligrams producing the same activity of the indicator nuclide as $1 \mathrm{mg}$ of the element to be determined. The calculated results obtained using different compilations show a great scatter, especially for the interference of fluorine in the determination of oxygen. This is not surprising when one considers that the neutron energy is not sufficiently defined in the compilations, and that the trends of the energy dependence of the cross section for the desired ${ }^{16} \mathrm{O}(\mathrm{n}, \mathrm{p}){ }^{16} \mathrm{~N}$ and the interfering ${ }^{19} \mathrm{~F}(\mathrm{n}, \alpha){ }^{16} \mathrm{~N}$ reaction are completely different as can be seen in figure 3 . If the averages of the cross sections from the compilations are used the calculated value differs from the experimentally averaged value by -33 percent for the fluorine interference in the oxygen determination and it differs by +1.6 percent for the interference of phosphorus and by +30 percent for the interference of aluminum in the determination of silicon. In fast neutron activation analysis, higher sensitivities can be achieved if cyclotron produced neutrons are used instead of generator produced $14 \mathrm{MeV}$ neutrons [26]. The study of the interference of phosphorus in the determination of silicon is given as an example also for this variant of fast neutron activation analy- 
TABLE 4. Effects of the primary interfering reactions in the determination of oxygen and silicon by $14 \mathrm{MeV} N A A$ : comparison of calculated and experimental results

Calculated using

cross sections from $\mathrm{mg} \mathrm{F} / \mathrm{mg} \mathrm{O}^{\mathrm{a}}$

$\mathrm{mg} \mathrm{P} / \mathrm{mg} \mathrm{Sib}$

$\mathrm{mg} \mathrm{Al} / \mathrm{mg} \mathrm{Si}^{\mathrm{c}}$

$\begin{array}{llll}\text { Kenna, Conrad [40] } & 1.0 & 1.57 & 418 \\ \text { Mathur, Oldham [41] } & 1.7 & 1.43 & 336^{\mathrm{j}} \\ \text { Aliev, et al. [36] } & 3.16^{\mathrm{d}} & 2.12 & 397 \\ & 1.30^{\mathrm{e}} & & \\ \text { Maslov, Lubnickij [37] } & 1.0 & 2.50 & 420^{\mathrm{j}} \\ \text { Experimental } & 2.35^{\mathrm{f}} & 1.82^{\mathrm{h}} & 303^{\mathrm{i}} \\ & 2.5^{\mathrm{g}} & 1.92^{\mathrm{i}} & \end{array}$

a Milligrams of fluorine yielding the same activity via the ${ }^{19} \mathrm{~F}(n, \alpha){ }^{16} \mathrm{~N}$ reactions as $1 \mathrm{mg}$ oxygen via the ${ }^{16} \mathrm{O}(n, p){ }^{16} \mathrm{~N}$ reaction.

b Milligrams of phosphorus yielding the same activity via the ${ }^{31} \mathrm{P}(n, \alpha){ }^{28} \mathrm{Al}$ reaction as $1 \mathrm{mg}$ silicon via the ${ }^{28} \mathrm{Si}(n, p)^{28} \mathrm{Al}$ reaction.

${ }^{c}$ Milligrams of aluminum yielding the same activity via the ${ }^{27} \mathrm{Al}(n, \gamma){ }^{28} \mathrm{Al}$ reaction as $1 \mathrm{mg}$ of silicon via the ${ }^{28} \mathrm{Si}(n, p)^{28} \mathrm{Al}$ reaction.

${ }^{\mathrm{d}}$ Using $\sigma$ at $\mathrm{E}_{\mathrm{n}} \sim 14.2 \mathrm{MeV}$.

e Using $\sigma$ at $\mathrm{E}_{\mathrm{n}} \sim 14.8 \mathrm{MeV}$.

${ }^{f}$ Reference [74].

g Reference [75].

h Reference [76].

i Reference [77].

j For the cross section of the ${ }^{27} \mathrm{Al}(n l, \gamma)^{28} \mathrm{Al}$ reaction taken 0.53 mbarn as the average from references $[36,38,40]$

sis. Fast neutrons were produced by bombarding a thick beryllium target with deuterons. In figure 9 , the ratio of the activity of the indicator radionuclide produced by the interfering and the desired reaction is shown as a function of the deuteron energy. The experimental curve was obtained by internal irradiations at the Karlsruhe isochronous cyclotron [4]. To obtain the calculated curve, a computer program [76] based on an adapted equation (1) was used. An average curve of the published excitation functions $[30,36,58]$ was used for the calculations. The calculated curve is by 9 to 34 percent lower than the experimental one.

The determination of oxygen via the ${ }^{16} \mathrm{O}\left({ }^{3} \mathrm{He}, \mathrm{p}\right){ }^{18} \mathrm{~F}$ reaction [79] may be seen as a real breakthrough in the charged particle activation analysis. The most serious interference is represented by the ${ }^{19} \mathrm{~F}\left({ }^{3} \mathrm{He}, \alpha\right){ }^{18} \mathrm{~F}$ reaction. Figure 10 shows the ratio of the ${ }^{18} \mathrm{~F}$-activity produced via the ${ }^{19} \mathrm{~F}\left({ }^{3} \mathrm{He}, \alpha\right){ }^{18} \mathrm{~F}$ and the ${ }^{16} \mathrm{O}\left({ }^{3} \mathrm{He}, \mathrm{p}\right){ }^{18} \mathrm{~F}$ reactions as a function of the energy of ${ }^{3} \mathrm{He}$. The experimental curve was measured by Debrun et al. [1 ]. The calculations were carried out using published cross section data [80-83]. The calculated curve is by 18 to 25 percent lower than the experimental one. However, the calculated curve was not corrected for the matrix ef- 


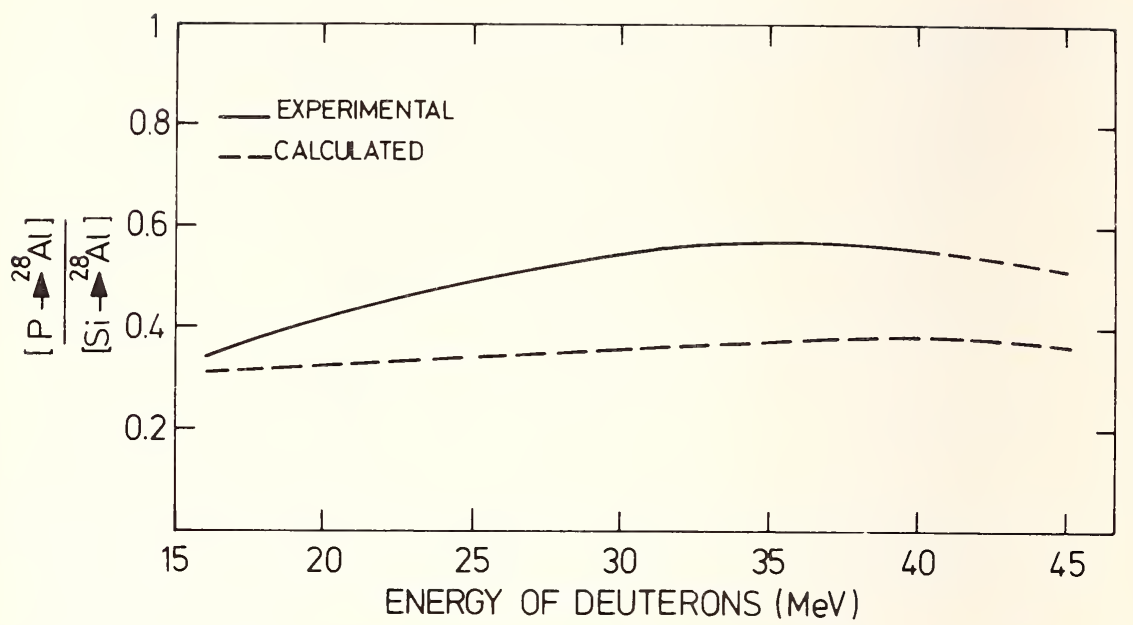

Figure 9. Energy dependence of the interference from phosphorus in the determination of silicon by activation analysis with cyclotron produced fast neutrons: Comparison of experimental [4] and calculated ratio of the ${ }^{28} \mathrm{Al}$ activity produced by the interfering reaction and the principal reaction.

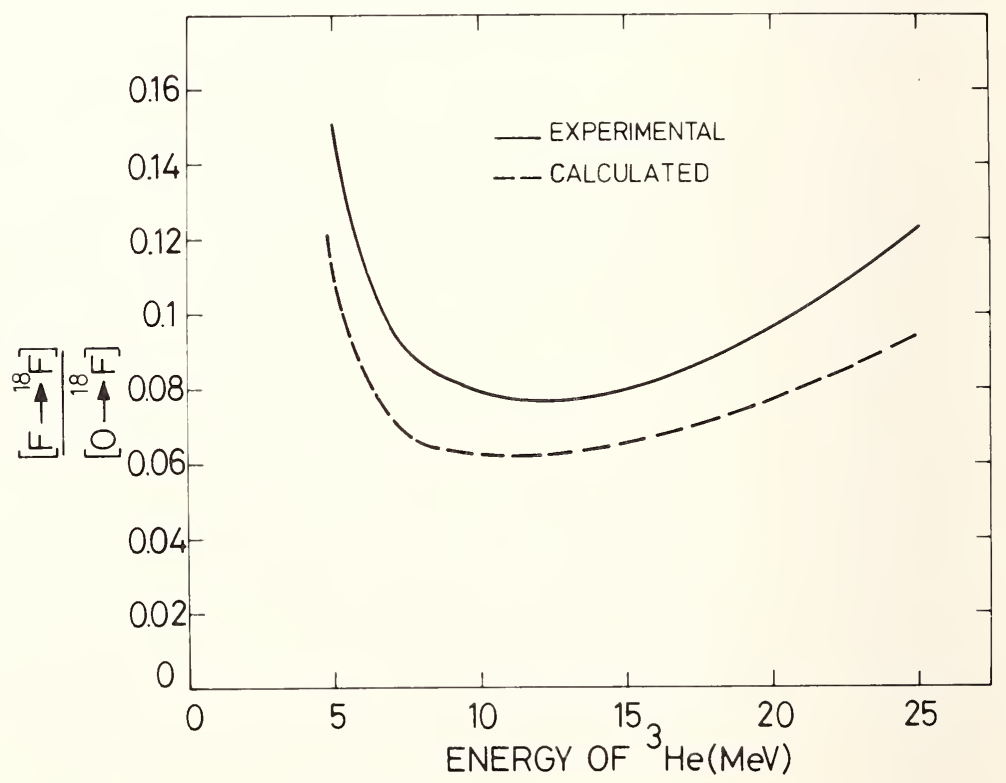

Figure 10. Energy dependence of the interference from fluorine in the determination of oxygen by activation analysis with ${ }^{3} \mathrm{He}$ : Comparison of experimental [1] and calculated ratio of the ${ }^{18} \mathrm{~F}$ activity produced by the interfering reaction and the principal reaction. 
fect as the composition of the fluorine and oxygen targets used in measuring the experimental curve was not known to the author.

A similar comparison of experimental and calculated results is given in figure 11 for the interference of beryllium via the ${ }^{9} \mathrm{Be}\left({ }^{3} \mathrm{He}, n\right)^{11} \mathrm{C}$ reaction in the determination of carbon via the ${ }^{12} \mathrm{C}\left({ }^{3} \mathrm{He}, \alpha\right){ }^{11} \mathrm{C}$ reaction. The experimental curve was measured by Debrun et al. [1], and the excitation functions used for the calculations by Markowitz and Mahony [84] and Hahn and Ricci $[82,83]$.

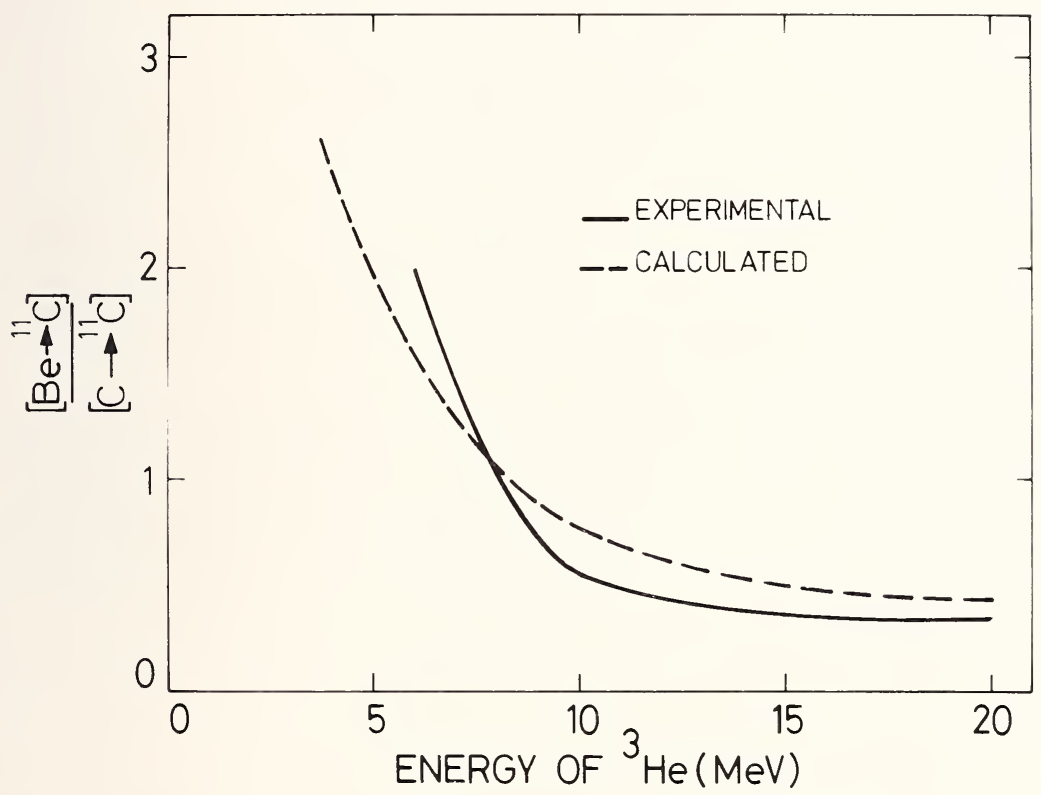

Figure 11. Energy dependence of the interference from beryllium in the determination of carbon by activation analysis with ${ }^{3} \mathrm{He}$ : Comparison of experimental [1] and calculated ratio of the ${ }^{11} \mathrm{C}$ activity produced by the interfering reaction and the principal reaction.

\section{Conclusions}

Much experimental work and costly irradiation time could be saved if primary reaction interferences were evaluated by calculations instead of measuring activation curves. To carry out such calculations, the appropriate projectile flux parameters and cross section data must be known. Absolute values for projectile flux are attainable with an accuracy between 2 to 10 percent. In general, the cross section data and their accu- 
racy constitute the main obstacle for the evaluation of the primary nuclear interferences by calculations.

On the basis of cross section data analysis for fast neutron and charged particle induced reactions the following conclusions can be made:

1 . With the exception of numerous excitation functions for fast neutron induced reactions other than that of $(n, 2 n),(n, p)$, and $(n, \alpha)$ type, cross section data needed for the purpose considered here can be obtained from existing compilations of experimental data, from semi-empirical systematics or semi-empirical formulae available.

2. At present, a critical evaluation of the available data is either completely missing or unsatisfactory for most reactions. In this situation, it seems to be useful to estimate the overall accuracy of the cross section data on the basis of all data published on the given nuclear reaction type.

3 . The overall accuracy of the cross section data obtained in the abovementioned way suffices to make calculations for assessing the significance of primary interference reactions in order to judge whether the interference effect is serious or can be neglected. The same is true for seeking the optimum energy of the incident radiation with regard to the occurrence of the interference reactions.

4. Generally, the accuracy of the cross section data presently available is not sufficient for obtaining the actual correction factors for the interference reactions. Still, there exist a number of reactions for which cross section data have been critically evaluated and the knowledge of their accuracy is acceptable for the performance of the correction for primary interference reactions.

\section{References}

[1] Debrun, J. L., Barrandon, J. H., and Albert, Ph., Modern Trends in Activation Analysis, Proceedings of the 1968 International Conference, October 7-11, 1968, Gaithersburg, Maryland, Nat. Bur. Stand. (U.S.), Spec. Publ. 312, Vol. II, pp. 77484 (1969).

[2] Rook, H. L., Schweikert, E. A., and Wainerdi, R. E., Modern Trends in Activation Analysis, Proceedings of the 1968 International Conference, October 7-11, 1968, Gaithersburg, Maryland, Nat. Bur. Stand. (U.S.), Spec. Publ. 312, Vol. II, pp. 76873 (1969).

[3] Engelmann, C., Photon Activation Analysis in Advances in Activation Analysis, Lenihan, J. M. A., Thomson, S. J., and Guinn, V. P., Eds., Vol. 2, pp. 1-56, Academic Press, New York (1972).

[4] To be published.

[5] Krivan, V., Nuclear Data in Science and Technology, Proceedings of the IAEA Symposium on Applications of Nuclear Data in Science and Technology, March 1216, 1973, Paris, Vol. II, pp. 193-225, IAEA, Vienna (1973). 
[6] Gove, N. B. and Wapstra, A. H., Nuclear Data Tables 11, 127-280 (1972).

[7] Keller, K. A., Münzel, H., Lange, J., Q-Values for Nuclear Reactions, LandoltBörnstein, Neue Serie, Gruppe I, Band 5a, Teil a, Springer, Berlin (1973).

[8] Body, Z. T. and Czikai, J., Atomic Energy Review 11, 153-65 (1973).

[9] Guinn, V. P., Proceedings of the Conference on the Use of Accelerators for Teaching and Research, April 8-10, 1968, Oak Ridge, Tenn., pp. 1-29.

[10] Nargolwalla, S. S., Proceedings of the Second Oak Ridge Conference on the Use of Small Accelerators for Teaching and Research, March 23-25, 1970, Oak Ridge, Tenn., pp. 185-204.

[11] Wood, D. E., Activation Analysis with $14 \mathrm{MeV}$ Neutron Generators in Advances in Activation Analysis, Lenihan, J. M. A., Thomson, S. J., and Guinn, V. P., Eds., Vol. 2, pp. 265-337, Academic Press, New York (1972).

[12] Van Grieken, R. and Hoste, J., Annotated Bibliography on $14 \mathrm{MeV}$ Neutrons, Office Information Booklet 65, Series: Bibliographies-8, Commission of the European Communities, Bureau Eurisotop, Brussels (1972).

[13] Op de Beeck, J. P., Radiochem. Radioanal. Letters 1, 281-8 (1969).

[14] Op de Beeck, J. P., J. Radioanal. Chem. 1, 313-23 (1968).

[15] Price, H. J., Graphical Analysis of the Theoretical Neutron Flux Produced by Fast Neutron Generators, Report, Kaman Nuclear, Kaman Nuclear Corp., Colorado Springs, Colorado (Undated).

[16] Wilkniss, P. E. and Wynne, G. J., Int. J. Appl. Rad. Isotopes 18, 77-81 (1967).

[17] Kishikawa, T. and Shinomiya, C., Bull. Chem. Society Japan 43, 1056-61 (1970).

[18] Kishikawa, T. and Shinomiya, C., Radiochem. Radioanal. Letters 7, $15-21$ (1971).

[19] Darrall, K. G. and Oldham, G., Nucl. Energy, July/August 1967, 104-6.

[20] Oldham, G. and Bibby, D. M., Nucl. Energy, November/December 1968, 167-72.

[21] Priest, H. F., Burns, F. C., and Priest, G. L., Nucl. Instr. \& Meth. 50, 141-6 (1967).

[22] Reimers, P. and Santner, E., Nucl. Instr. \& Meth. 92, 577-9 (1971).

[23] McMahon, C., The Effect of Distance and Moderator Thickness on the Neutron Energy Distribution from $14 \mathrm{MeV}$ Neutron Generators, Mineral Science Division Internal Report MS 66-93, Department of Energy, Mines and Resources, Mines Branch, Ottawa (November 1966).

[24] Czikai, J., Buszkó, M., Bödy, Z., and Demény, A., Atomic Energy Review VII. 93128 (1969).

[25] Adams, F., Op de Beeck, J., Van den Winkel, P., Gijbels, R., De Soete, D., and Hoste, J., CRC Critical Reviews in Analytical Chemistry I, 499-586 (1971).

[26] Krivan, V. and Münzel, H., J. Radioanal. Chem. 15, 575-83 (1973).

[27] Bruninx, E., Modern Trends in Activation Analysis, Proceedings of the 1968 International Conference, October 7-11, 1968, Gaithersburg, Maryland, Nat. Bur. Stand. (U.S.), Spec. Publ. 312, Vol. II, pp. 860-7 (1969).

[28] Fleischer, A. A., Modern Trends in Activation Analysis, Proceedings of the 1968 International Conference, October 7-11, 1968, Gaithersburg, Maryland, Nat. Bur. Stand. (U.S.), Spec. Publ. 312, Vol. II, pp. 868-73 (1969).

[29] Schmidt, K. and Münzel, H., Erzeugung von Neutronen durch Bestrahlung dicker Berylliumtargets mit Deuteronen im Energiebereich von 15 bis $55 \mathrm{MeV}$. KFK-Report 1288, Kernforschungszentrum Karlsruhe (September 1970).

[30] Stehn, J. R., Goldberg, M. D., Magurno, B. A., and Wiener-Chasman, R., Neutron Cross Sections, Vol. I, BNL 325, 2d edition, Suppl. 2. Brookhaven National Laboratory (May 1964). 
[31] Goldberg, M. D., Mughabghab, S. F., Magurno, B. A., and May, V. M., Neutron Cross Sections, Vol. IIA, BNL 325, 2d edition, Suppl. 2, Brookhaven National Laboratory (February 1966).

[32] Goldberg, M. D., Mughabghab, S. F., Purohit, S. N., Magurno, B. A., and May, V. M., Neutron Cross Sections, Vol. IIB, BNL 325, 2d edition, Suppl. 2, Brookhaven National Laboratory (May 1966).

[33] Goldberg, M. D., Mughabghab, S. F., Purohit, S. N., Magurno, B. A., and May, V. M., Neutron Cross Sections, Vol. IIC, BNL 325, 2d edition, Suppl. 2, Brookhaven National Laboratory (August 1966).

[34] Neuert, H. and Pollehn, H., Tables of Cross Sections of Nuclear Reactions with Neutrons in the 14 $15 \mathrm{MeV}$ Energy Range, Euratom Report EUR 122e (April 1963).

[35] Bödy, Z. T., A Compilation of ( $n, 2 n)$ Cross Sections Measured at About $14 \mathrm{MeV}$ for Nuclides $6<Z<93$, INDA(HUN)-10, IAEA, Nuclear Data Section, Vienna (Jan. 1973).

[36] Aliev, A. I., Drynkin, V. I., Leipunskaya, D. I., and Kasatkin, V. A., Handbook of Nuclear Data for Neutron Activation Analysis, Israel Program for Scientific Translations, Jerusalem (1970).

[37] Maslov, I. A. and Luknickij, V. A., Spravochnik po nejtronnomu aktivacionnomu analyzu (Handbook of Neutron Activation Analysis), Izd. Nauka, Leningrad (1971).

[38] Gillespie, A. S. and Hill, W. W., Nucleonics 19, 170-3 (1961).

[39] Koch, R. C., Activation Analysis Handbuch, Academic Press, New York (1960).

[40] Kenna, B. T. and Conrad, F. J., Tabulation of Cross Sections, Q-Values, and Sensitivities for Nuclear Reactions of Nuclides with $14 \mathrm{MeV}$ Neutrons, Report, SC-RR66-229, Sandia Laboratory, Albuquerque, New Mexico (June 1966).

[41] Mathur, S. C. and Oldham, G., Nuclear Energy, Sept.-Oct. 1967, 136-41.

[42] Pearlstein, S., Nuclear Data A3, 327-41 (1967).

[43] Czikai, J. and Petö, G., Phys. Lett. 20, 52-56 (1966).

[44] Qaim, S. M., Nucl. Phys. A185, 614-24 (1972).

[45] Qaim, S. M., Wölfle, R., and Stöcklin, G., Chemical Nuclear Data: Measurements and Application, Proceedings of the International Conference, September 20-22, 1971, University of Kent, Canterbury, The Institution of Civil Engineers, London, pp. 121-129.

[46] Qaim, S. M., Z. Naturforsch. 25, 1977-8 (1970).

[47] Qaim, S. M., Conference on Nuclear Structure-Study with Neutrons, July 31-August 5, 1972, Budapest, Proceedings, Central Institute for Physics, Budapest, 1972, pp. 50-51.

[48] Qaim, S. M. and Stocklin, G. L., J. inorg. nucl. Chem. 35, 19-30 (1973).

[49] Qaim, S. M.,J.inorg. nucl. Chem. 36, 239-44 (1974).

[50] Drake, D., Bergqvist, I., and McDaniels, D. K., Phys. Lett. 36B, 557-9 (1971).

[51] Vuletin, J., Kulisic, P., and Cindro, H., Lett. Nuovo Cimento 10, 1-3 (1974).

[52] Adam, A. and Jeki, L., Acta Phys. Hung. 26, 335-41 (1969).

[53] Chatterjee, S. and Chatterjee, A., Nucl. Phys. A125, 593-99 (1969).

[54] Jäger, V. and Münzel, H., Nuclear Data in Science and Technology, Vol. II, Proceedings of the IAEA Symposium on the Application of Nuclear Data in Science and Technology, March 12-16, 1973, Paris, IAEA, Vienna (1973), pp. 475-82.

[55] Zijp, W. L., Voorbraak, W. P., and Nolthenius, H. J., Compilation of Evaluated Cross Section Data Used in Fast Neutron Metrology, RCN-196, Reactor Centrum Netherland, Petten (Oct. 1973).

[56] Jessen, P., Bormann, M., Dreyer, F., and Neuert, H., Nucl. Data A1, 103-202 (1966). 
[57] Listien, H. and Paulsen, A., Compilation of Cross Sections for Some Neutron-Induced Threshold Reactions, Eur 119.e, Brussels, 1 (1963), 2 (1966).

[58] Alley, W. E. and Lessler, R. M., Nucl. Data Tables 11,621-826 (1973).

[59] Krivan, V. and Münzel, H., J. inorg. nucl. Chem. 34, 2093-108 (1972).

[60] Krivan, V. and Münzel, H.,J. inorg. nucl. Chem. 34, 2989-99 (1972).

[61] Williamson, C. F., Boujot, J.-P., and Picard, J., Tables of Range and Stopping Power of Chemical Elements for Charged Particles of Energy 0.05 to $500 \mathrm{MeV}$, Rapport CEA-R 3042, Saclay (July 1966).

[62] Ricci, E., Charged Particle Activation Analysis in Advances in Activation Analysis, Lenihan, J. M. A., Thomson, S. J., and Guinn, V. P., Eds., Vol. 2, pp. 221-63, Academic Press, New York (1972).

[63] Jarmie, N. and Seagrave, J. D., Charged Particle Cross Sections, Report LA-2014, Los Alamos Scientific Laboratory and University of California, Los Alamos, New Mexico (March 1957).

[64] Smith, D. B., Charged Particle Cross Sections: Ne to Cr, Report LA-2424, Los Alamos Scientific Laboratory and University of California, Los Alamos, New Mexico (1961).

[65] McGowan, F. K., Milner, W. T., and Kim, H. J., ORNL-CPX-1 (1964).

[66] McGowan, F. K., Milner, W. T., and Kim, H. J., ORNL-CPX-2 (1964).

[67] Kim, H. J., Milner, W. T., and McGowan, F. K., Nuclear Data Tables A1, 203-389 (1966).

[68] Kim, H. J., Milner, W. T., and McGowan, F. K., Nuclear Data Tables A2, 1-241 (1966).

[69] Kim, H. J., Milner, W. T., and McGowan, F. K., Nuclear Data Tables A3, 123-285 (1967).

[70] Kunz, W. and Schintlmeister, J., Nuclear Tables, Part II Nuclear Reactions, Vol. 1, Pergamon Press, Oxford (1965), Vol. 2, Pergamon Press, Oxford (1967).

[71] Lorenzen, J., and Brune, D., Excitation Functions for Charged Particle Induced Reactions in Light Elements at Low Projectile Energies, Report EA-476, Aktiebolaget Atomenergie, Studsvik, Nyköping, Sweden (1973).

[72] Lange, J., Münzel, H., Keller, K. A., and Pfennig, G., Excitation Functions for Charged-Particle Induced Reactions, Landolt-Börnstein, Neue Serie, Gruppe I, Band 5, Teil b, Springer, Berlin (1973).

[73] Keller, K. A., Lange, H., and Münzel, H., Estimation of Unknown Excitation Functions and Thick Target Yields for $p, d,{ }^{3} \mathrm{He}$ and $\alpha$ Reactions, Landolt-Börnstein, Neue Serie, Gruppe I, Band 5, Teil c, Springer, Berlin, (1974).

[74] Schmidt-Hönov, M., Interferences in the Oxygen Determination by Fast Neutron Activation Analysis and Investigation on the Cross Sections of the Involved Nuclear Reactions, Dissertation, University of Köln (1970).

[75] Hoste, J., De Soete, D., and Speecke, A., The Determination of Oxygen in Metals by $14 \mathrm{MeV}$ Neutron Activation Analysis, Euratom Report EUR 3565e, Commission of the European Commities, Brussels (September 1967).

[76] Van Grieken, R. and Dams, R., Anal. Chim. Acta 63, 369-81 (1973).

[77] Block, C. and Dams, R., Anal. Chim. Acta 71, 53-65 (1974).

[78] Münzel, H. and Schmidt, K., Berechnung der erzeugten Aktivitat bei der Bestrahlung von dunnen Targets mit energiereichen Neutronen, Ext. Bericht 10/703, Kernforschungszentrum Karlsruhe (Oktober 1970).

[79] Markowitz, S. S. and Mahony, J. O., Anal. Chem. 34, 329-35 (1962).

[80] Lamb, J. F., Lee, D. M., and Markowitz, S. S., Anal. Chem. 42, 209-12 (1970). 
[81] Lee, D. M., Lamb, J. F., and Markowitz, S. S., Anal. Chem. 43, 542-5 (1971).

[82] Hahn, R. L. and Ricci, E., Phys. Rev. 146, 650-659 (1966).

[83] Hahn, R. L. and Ricci, E., Nucl. Phys. A101, 353-68 (1967).

[84] Markowitz, S. S. and Mahony, J. D., Radiochemical Methods of Analysis, Vol. I, pp. 419-32. International Atomic Energy Agency, Vienna (1965). 


\title{
ACTIVATION ANALYSIS WITH CHARGED PARTICLES OF INTERMEDIATE ENERGY: PERFORMANCES AND APPLICATIONS
}

\author{
J. N. Barrandon and J. L. Debrun \\ Service du Cyclotron \\ Centre National de la Recherche Scientifique \\ 45045 Orleans Cedex, France
}

The subject of this communication is activation analysis with intermediate energy protons (10 to $15 \mathrm{MeV}$ ) and ${ }_{2}^{3} \mathrm{He}$ (10 to $20 \mathrm{MeV}$ ) with an emphasis on nondestructive multielemental analysis, but results obtained for single element determination after radiochemical separations are also reported.

Precision, accuracy, selectivity and sensitivity of the methods are discussed, as well as the possibilities of application in various areas including mainly metallurgy, geology, solid state physics and archeology.

In the case of nondestructive analysis with $10 \mathrm{MeV}$ protons, we will present experimental activation yields for over 130 radioisotopes corresponding to more than 50 elements. Calculated sensitivities for the determination of these elements will be compared with experimental sensitivities obtained in actual analysis of various matrixes like: $\mathrm{Ag}, \mathrm{Al}, \mathrm{Au}, \mathrm{Co}, \mathrm{Ir}, \mathrm{Nb}, \mathrm{Rh}, \mathrm{Ta}$, rocks and graphite.

For destructive analysis with higher energy protons and ${ }_{2}^{3} \mathrm{He}$, our experimental results will be compared with results obtained on identical samples by other analytical methods like colorimetry, atomic absorption and mass spectrometry.

Keywords: Activation analysis; charged particle; cyclotron; method evaluation; multielemental analysis.

\section{Introduction}

In each special case of analysis, one has to select the most appropriate technique. However, not all techniques are well known and this communication will give information on one of these not so well known methods: "Nondestructive activation analysis with protons of intermediate energy." Also, some results obtained by irradiation with higher energy protons 
or ${ }_{2}^{3} \mathrm{He}$, and chemical separations, will be discussed and compared with results obtained by other methods on the same samples.

\section{Nondestructive Analysis with $10 \mathrm{MeV}$ Protons}

Protons of $10 \mathrm{MeV}$ were chosen because with these particles and this energy, it is possible to determine about 50 elements: without nuclear interferences; with a good sensitivity; and in many matrixes in which nondestructive analysis is feasible.

There are no other charged particles that offer such possibilities. If the energy is higher than $10 \mathrm{MeV}$, nondestructive analysis becomes difficult or even impossible for most matrixes and there might be interferences; on another hand, the lower the energy, the poorer the sensitivity. The energy of $10 \mathrm{MeV}$ represents a good compromise.

Figure 1 gives the measured specific activities for 40 elements. These specific activities correspond to the following standard conditions: irradiation of 1 hour, with 1 microampere of $10 \mathrm{MeV}$ protons, activity expressed in $\gamma / \mathrm{mn}$ at the end of the bombardment and measured on the "best" radioisotope and on the "best" $\gamma$ ray of this radioisotope. All values are arbitrary normalized to the range of $10 \mathrm{MeV}$ protons in rhodium [1], for better comparison. Values corresponding to less abundant $\gamma$ rays, other radioisotopes and the rare earths are not given here because there are altogether 130 radioisotopes and about $400 \gamma$ rays, and this is too big a volume of information.

Figure 2 gives the sensitivities calculated for a Ge-Li whose specifications are: efficiency, 22 percent, and peak-to-Compton ratio, 34. The minimum detectable activity was chosen to be equal to $3 \sigma$ of the background in the region of interest and the radioisotopes are supposed to be counted (1.8) $\times \mathrm{T}$ or 60 hours maximum. On the same figure are shown the experimental sensitivities obtained for a sample of ultra-pure silicon, which is a favorable matrix. Calculated and experimental sensitivities correspond to an irradiation of 2 hours at 1.45 microamperes.

Table 1 shows the elements in which no activity is created or in which the activity decays away in a reasonable period of time. Several of these matrixes were analyzed in the course of metallurgical, archeological, geological or solid state physics studies; they include $\mathrm{Ag}, \mathrm{Al}, \mathrm{Au}, \mathrm{Co}, \mathrm{Dy}$, $\mathrm{Ho}, \mathrm{Ir}, \mathrm{Nb}, \mathrm{Pr}, \mathrm{Rh}, \mathrm{Si}, \mathrm{Ta}, \mathrm{Tb}$, and various rocks.

Tables 2 and 3 show the elements detected in $\mathrm{Rh}$ and $\mathrm{Tb}$ respectively, and the associated precision. 


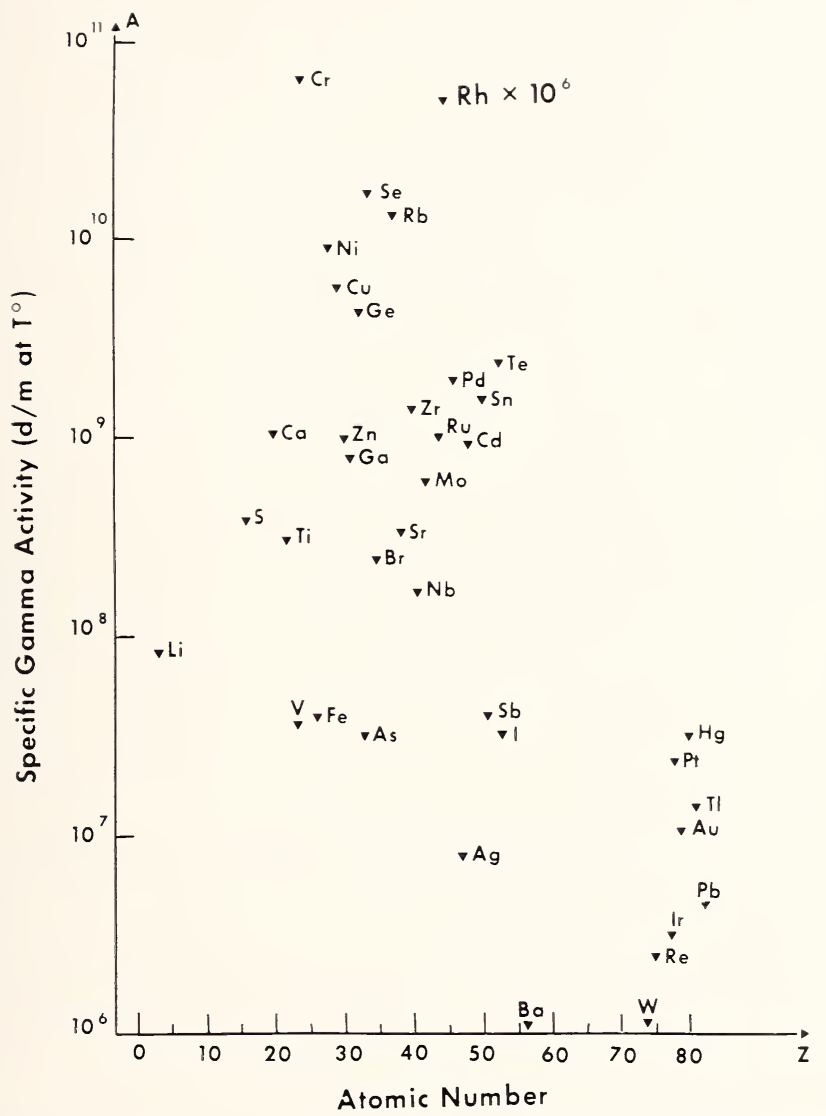

Figure 1. Specific activities of 38 elements compared to the specific activity of rhodium. 


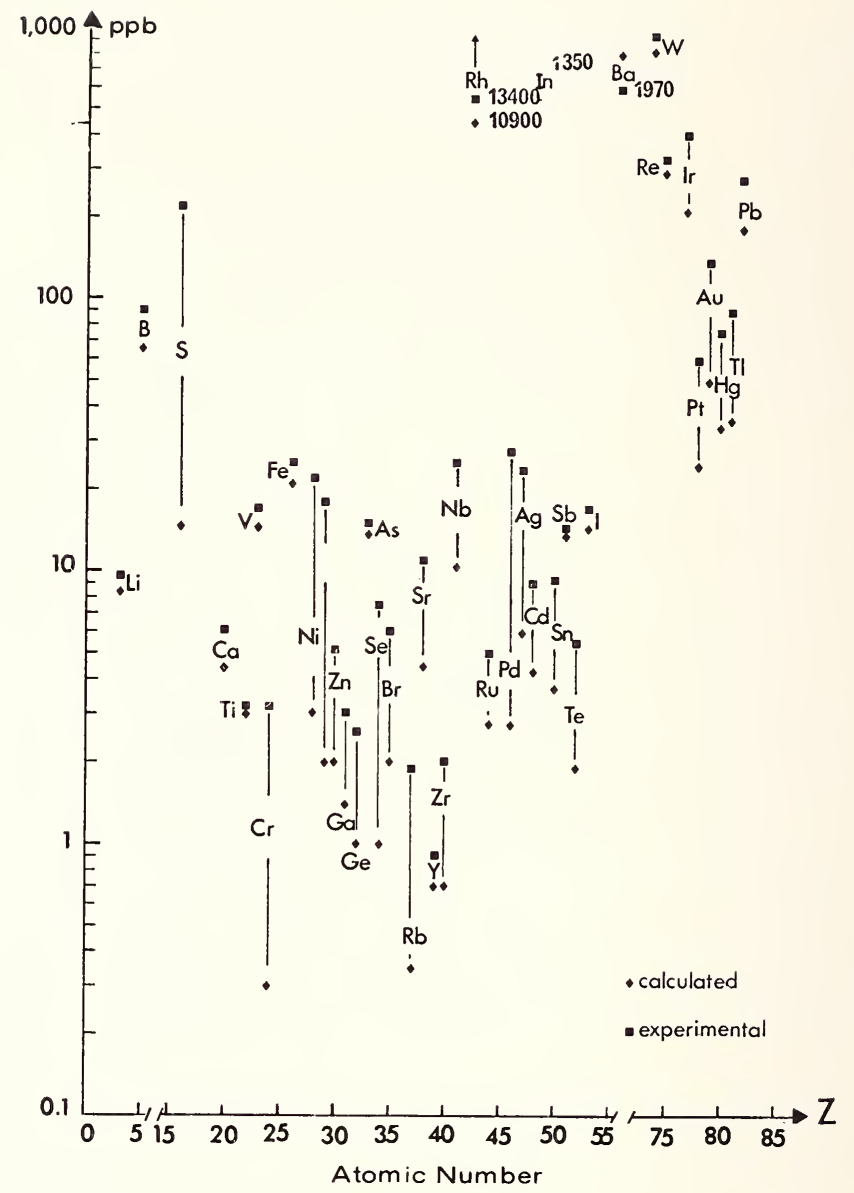

Figure 2. Calculated and experimental sensitivities.

TABLE 1. Elements which do not interfere with direct gamma spectrometry

Decay time

$\sim 0$ time

Few hours 1 to 15 days

$\mathrm{H}, \mathrm{He}, \mathrm{Be}, \mathrm{F}, \mathrm{Na}, \mathrm{Mg}$,

$\mathrm{Al}, \mathrm{Si}, \mathrm{P}, \mathrm{Cl}, \mathrm{K}, \mathrm{Mn}$,

$\mathrm{C}, \mathrm{N}, \mathrm{O}, \mathrm{S}$,

$\mathrm{Ca}, \mathrm{Sc}, \mathrm{Ni}, \mathrm{Ag}$,

$\mathrm{Co}, \mathrm{Tb}, \mathrm{Ho}, \mathrm{Ta}, \mathrm{Bi}$

$\mathrm{Ba}, \mathrm{Ce}, \mathrm{Pr}, \mathrm{Dy}$,

$\mathrm{Ir}, \mathrm{Au}, \mathrm{Tl}$ 

TABLE 3. Impurities detected in terbium samples. Activation analysis with $10 \mathrm{MeV}$ protons

$$
\text { (Type of nuclear reaction used: }{ }_{Z}^{A} \times(p, n){ }_{Z+1}^{A} Y \text { ) }
$$

\begin{tabular}{|c|c|c|c|c|}
\hline Element & $\begin{array}{c}\text { Number of } \\
\text { determinations }\end{array}$ & $\begin{array}{l}\text { Radioisotope used } \\
\text { for determination }\end{array}$ & $\begin{array}{l}\text { Mean value } \\
\text { ppm (weight) }\end{array}$ & $\begin{array}{l}\text { Standard deviation } \\
\text { of mean at } 95 \% \\
\text { confidence level }\end{array}$ \\
\hline
\end{tabular}

\begin{tabular}{ccccc}
\hline $\mathrm{Ca}$ & 6 & ${ }^{44} \mathrm{Sc}-{ }^{48} \mathrm{Sc}$ & 14.3 & 0.36 \\
$\mathrm{Ti}$ & 3 & ${ }^{48} \mathrm{~V}$ & 2.4 & .15 \\
$\mathrm{Cr}$ & 15 & ${ }^{52} \mathrm{Mn}$ & 2.8 & .24 \\
$\mathrm{Fe}$ & 17 & ${ }^{56} \mathrm{Co}$ & 98 & 3.9 \\
$\mathrm{Ni}$ & 10 & ${ }^{61} \mathrm{Cu}-{ }^{55} \mathrm{Co}$ & 18 & 2.5 \\
$\mathrm{Y}$ & 5 & ${ }^{89} \mathrm{Zr}$ & 18.7 & 0.36 \\
$\mathrm{Mo}$ & 11 & ${ }^{95} \mathrm{Tc}-{ }^{96} \mathrm{Tc}$ & 1.5 & .34 \\
$\mathrm{La}$ & 4 & ${ }^{139} \mathrm{Ce}$ & 90.4 & 1.7 \\
$\mathrm{Pr}$ & 9 & ${ }^{141} \mathrm{Nd}$ & 102.6 & 17.5 \\
$\mathrm{Gd}$ & 8 & ${ }^{156} \mathrm{~Tb}$ & 27.8 & 4.4 \\
\hline
\end{tabular}


Table 4 gives for other matrixes the elements detected and the ranges of these concentrations.

The accuracy of a method can only be known if it is applied to standard samples or if identical unknown samples are analysed by different means and different laboratories. In the particular case of nondestructive activation analysis with $10 \mathrm{MeV}$ protons and for the matrixes and the elements mentioned above, it has not been possible to really test the accuracy. For $\mathrm{Al}, \mathrm{Rh}$ and $\mathrm{Nb}$, we have obtained results by neutron activation, that were in good agreement with the results obtained by proton activation, except in a few cases where a difference reaching 20 percent was observed. In the second part of this communication, we will compare the accuracy of charged particle activation with $15 \mathrm{MeV}$ protons and $17 \mathrm{MeV}{ }_{2}^{3} \mathrm{He}$, with the accuracy of other methods. Here, we will discuss several parameters that have an influence on the accuracy. These parameters are: the quality of the beam delivered by the cyclotron, the ranges of the particles, the nuclear data, and the possible nuclear or $\gamma$ rays interferences.

The problems of the measurement of the radioactivity and of the blanks will not be discussed since the former is already well known and the latter almost nonexistent in activation analysis.

The quality of the beam is of first importance for the accuracy as well as for the precision. Usually, the analyst needs an intense beam of particles; it is therefore not possible to use a very well analysed beam, but this is not necessary as long as the mean energy and the energy dispersion remain the same throughout all the experiments. But every time the cyclotron is set up to deliver a given energy $\mathrm{E}$, there can be an uncertainty of \pm 1 on the number of revolutions accomplished by the particles. This is a source of errors because samples and standards are not always irradiated the same day, or even the same week, at least when the method of the average cross section is used [1]. A revolution of \pm 1 corresponds to $\pm 54 \mathrm{keV}$ at $10 \mathrm{MeV}$ with the cyclotron of the C.N.R.S. at ORLEANS using harmonic 2, and $\mathrm{E}_{2} \pm 66 \mathrm{keV}$ using harmonic 3. Our experiments with 10 $\mathrm{MeV}$ protons were performed with the cyclotron of the C.E.A. at ORSAY where the quality of the beam was not nearly as good as at ORLEANS, and the variation of the mean energy could be much greater than $50 \mathrm{keV}$. This could be an explanation for the differences sometimes observed between proton activation and neutron activation.

Figure 3 gives the error introduced in the determination of nickel by means of three different nuclear reactions, versus the energy of irradiation; the $(\Delta E / E)$ was taken from the curve for protons (fig. 4), using harmonic 2 which is the most favorable case. The error is different in each case because of the different shapes of the activation curves: con- 


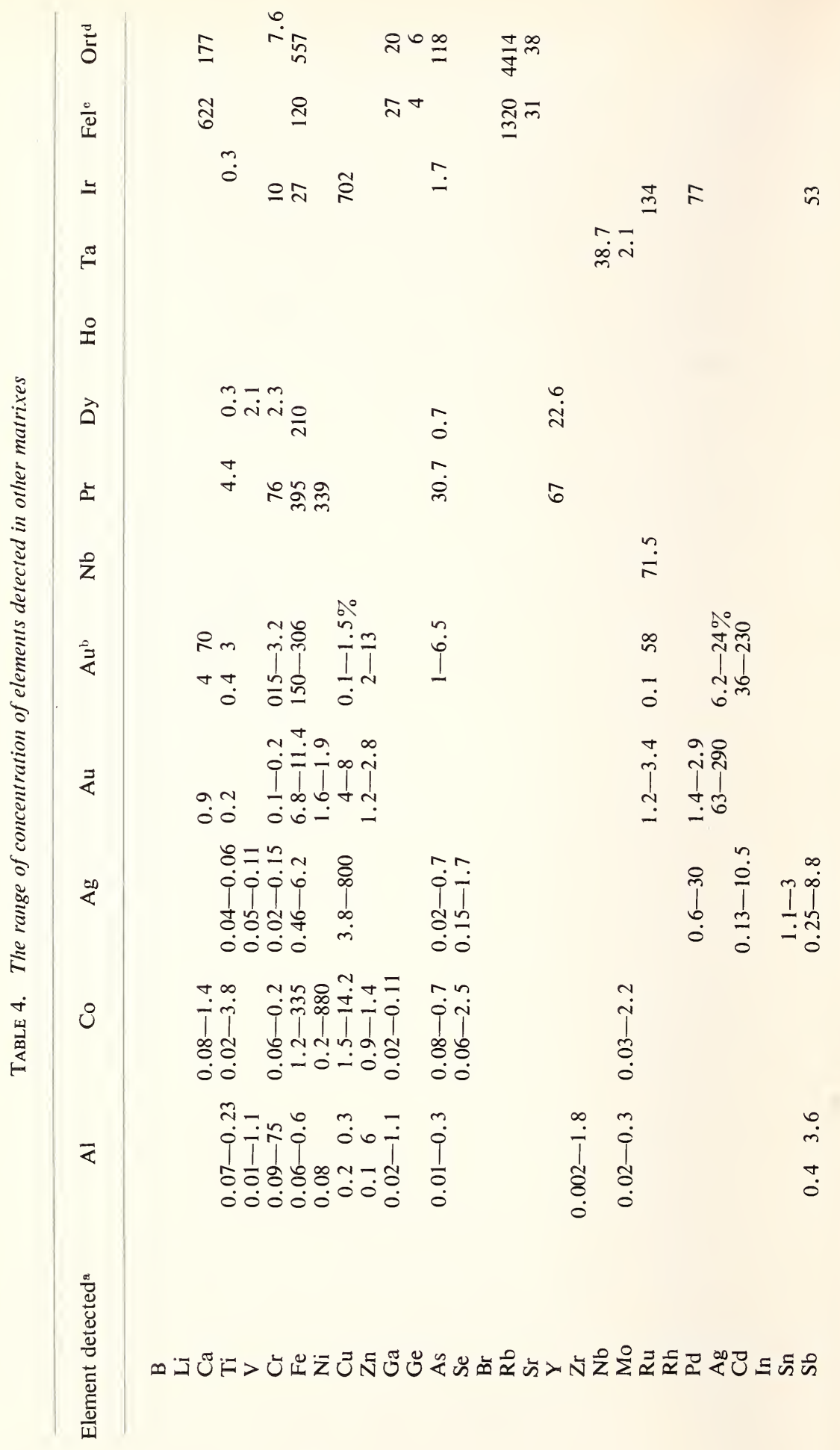




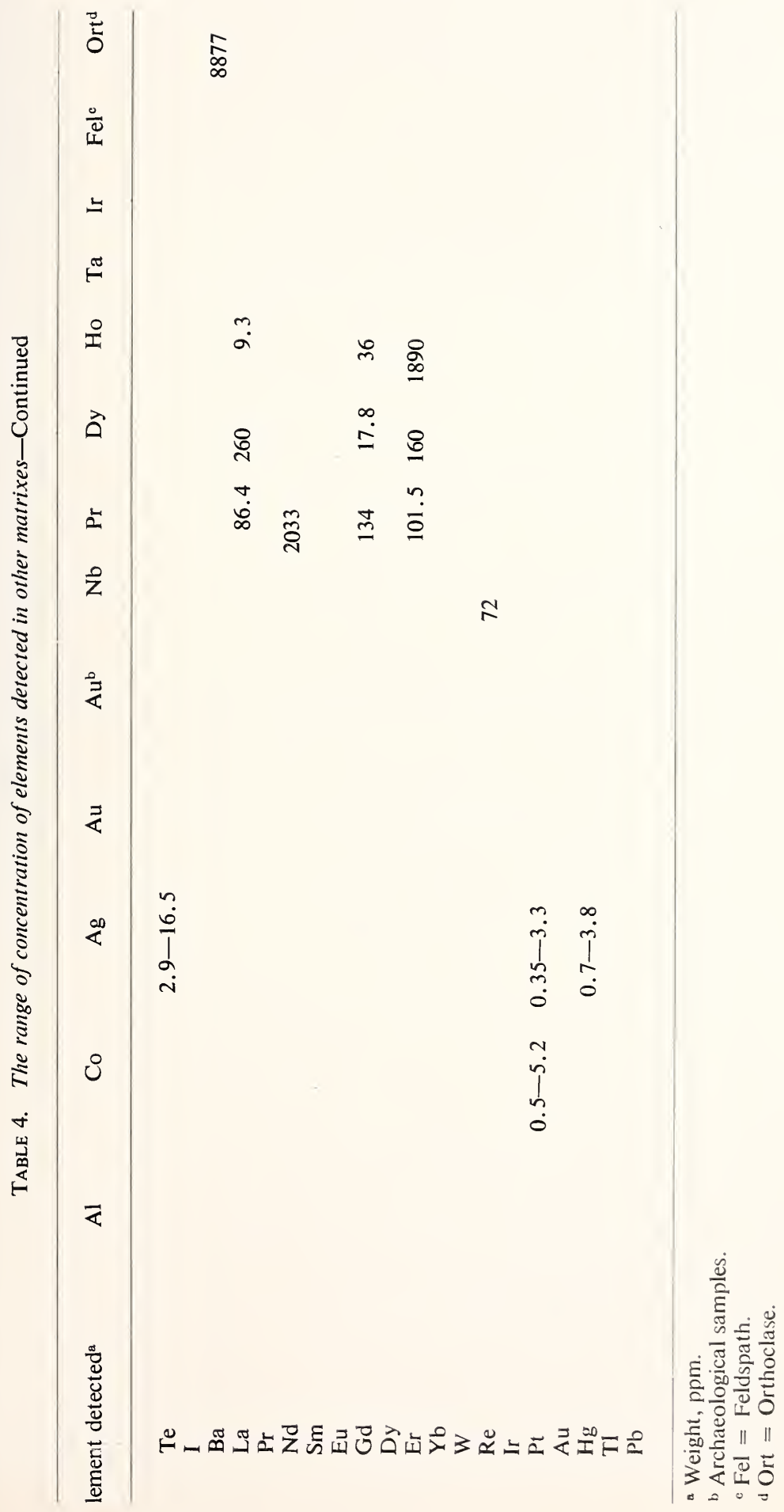




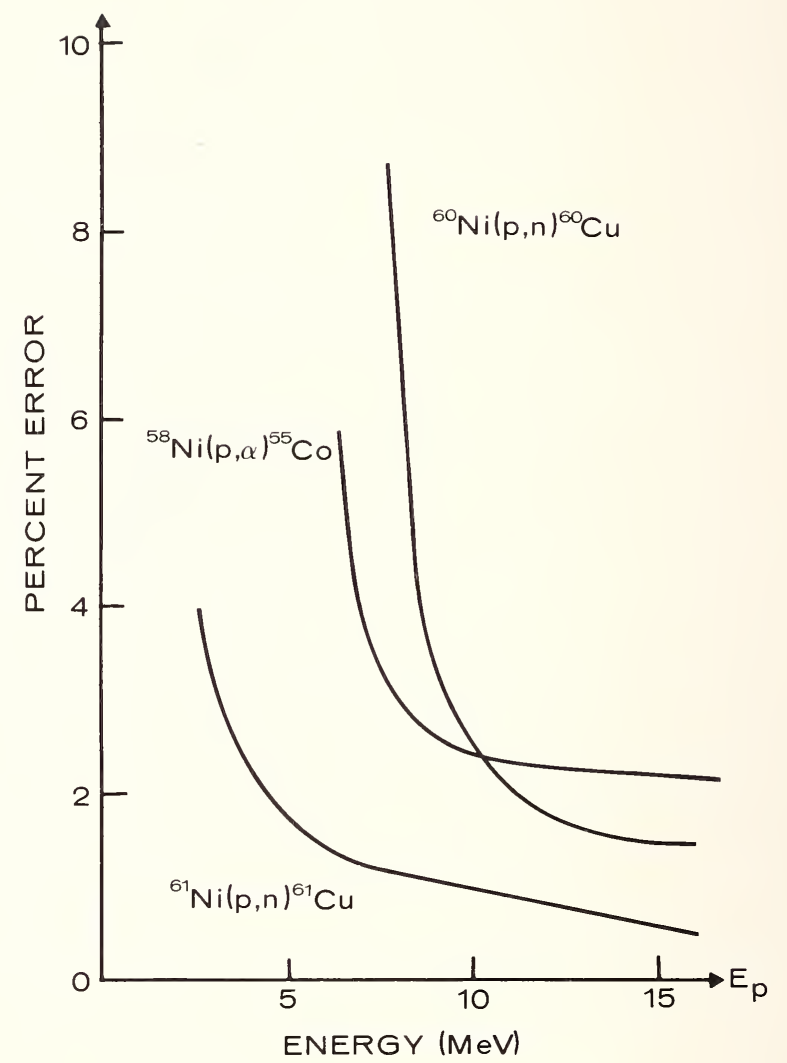

Figure 3. Error introduced in the determination of nickel. 


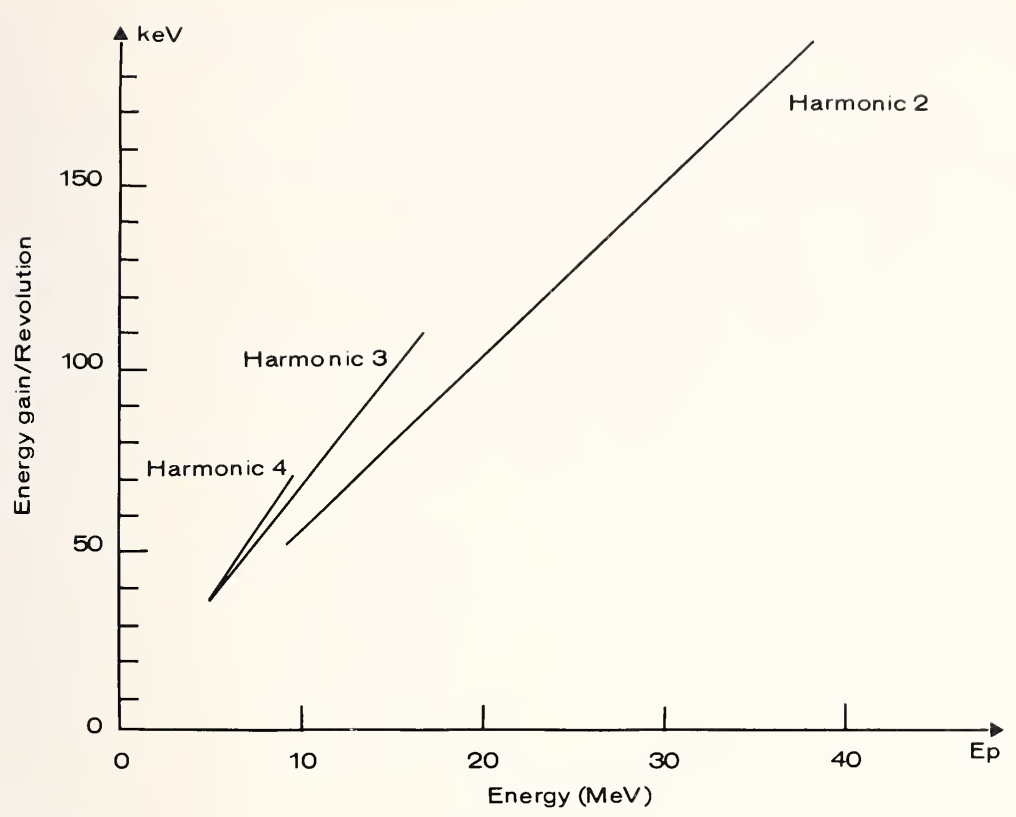

Figure 4. Energy gain per revolution for different protons incident energy.

sequently, the value of the error varies with the element to be determined and the radioisotope used for the determination, hence the usefulness and the importance of the activation curves.

These curves are not the only nuclear data of importance: the half-lives are often a source of unknown error, especially in the case of seldom used nuclides like the neutron-deficient radioisotopes. The ranges of the particles read from the published tables can lead to systematic errors, not only by themselves but also because of the possible error on the mean energy. In the case of protons it seems that the ranges are accurate, and when using the method of the average cross section only ratios of the ranges are used and the possible error is lessened; for these reasons, the systematic errors introduced by the calculated ranges are believed to be negligible if compared to the other errors.

The interferences are a problem in many methods. Here, we have to consider the nuclear and the $\gamma$ rays interferences. The former are nonexistent, or can be avoided, except in the case of $\mathrm{Li}$ and $\mathrm{B}$; the latter are quite frequent but can usually be detected, and there are so many $\gamma$ rays to be used and the half-lives are so different that it is rare to be unable to perform a given analysis because of a $\gamma$ ray interference. 


\section{Activation with $15 \mathrm{MeV}$ Protons and $17 \mathrm{MeV}$ Helium 3 Followed by Radiochemical Separations}

\section{A. Determination of Pt in Cu Samples}

In the frame of studies sponsored by the Commission of the European Communities (Bureau Eurisotop), samples of $\mathrm{Cu}$ doped with $\mathrm{Pt}$ at the 100 ppm level were prepared ${ }^{1}$ by levitation using a high frequency generator. These samples were analyzed by several laboratories and several methods; the results are shown in figure 5. Probably because of the poor quality of the beam (variation of the mean energy), the precision is not very good $(\sim 10 \%)$ but a good accuracy is obtained.

It is worth noting that the standard deviation would remain approximately the same at much lower levels than $100 \mathrm{ppm}$, because the activity was quite high for a small number of microcoulombs (intensity $\times t$ irradiation). Most other methods would be in trouble at lower levels.

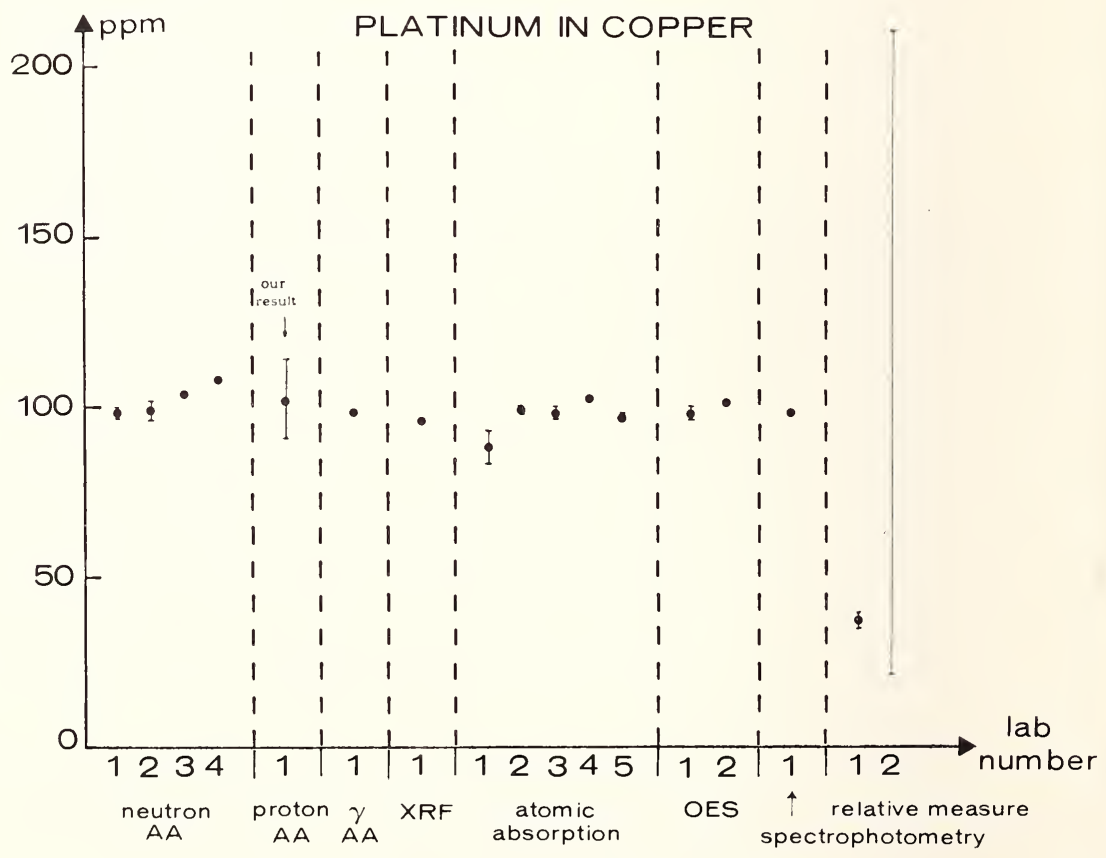

Figure 5. Results of platinum determination in copper. 


\section{B. Determination of Oxygen in Al, Cu and Ta}

The same type of work that was done for $\mathrm{Pt}$ in $\mathrm{Cu}$ was done in the case of oxygen in nonferrous and in refractoring metals. Figures 6, 7, 8 and 9 present the results obtained by the different participating laboratories.

It can be seen that the agreement among nonradioactive methods and $14 \mathrm{MeV}$ is good when the oxygen content is high ( $\sim 70 \mathrm{ppm})$. At lower levels, when all methods were used, the agreement is not as good; it is believed that not only the sensitivity of some methods is not sufficient, but also there are blank problems. Indeed, the oxygen adsorbed on the surface of the samples or the oxides formed at the surface, create a very difficult problem for all nonradioactive methods. Our own measurements $[2,3]$ of the oxygen present at the surface of different metals, indicate that there may be blanks of several ppm, the value of these blanks varying with the nature of the metal, the nature of the surface treatment, and the area to volume ratio of the samples.

In activation analysis, etching after irradiation usually solves this problem. However, etching creates another problem that has an influence on the accuracy and the precision of the oxygen determination. Indeed, the number of micrometers etched away has to be known very precisely

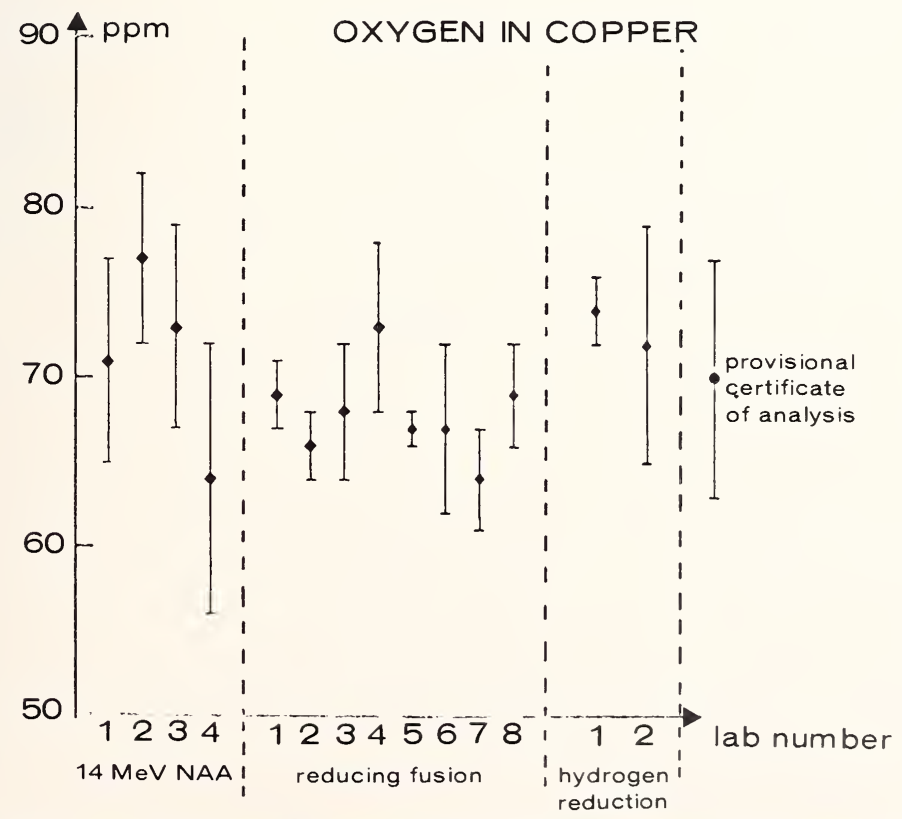

Figure 6. Results of oxygen determination in copper (phosphorus deoxidized copper). 


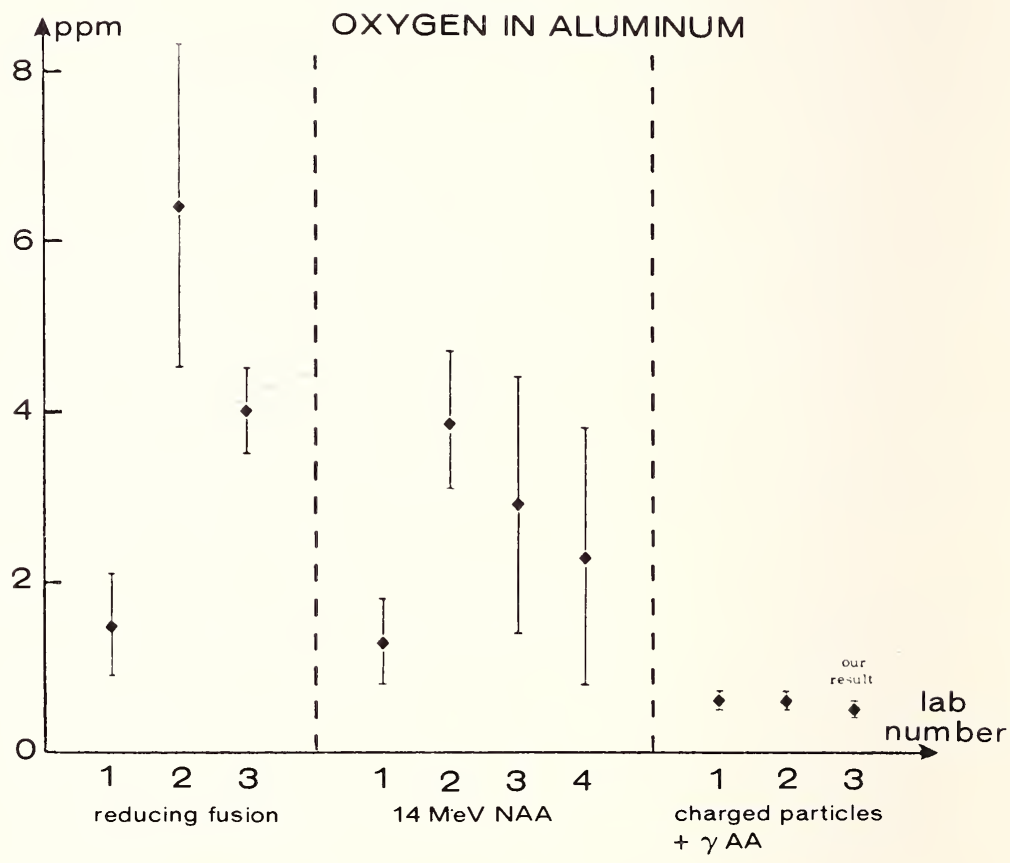

Figure 7. Results of oxygen determination in aluminum. 


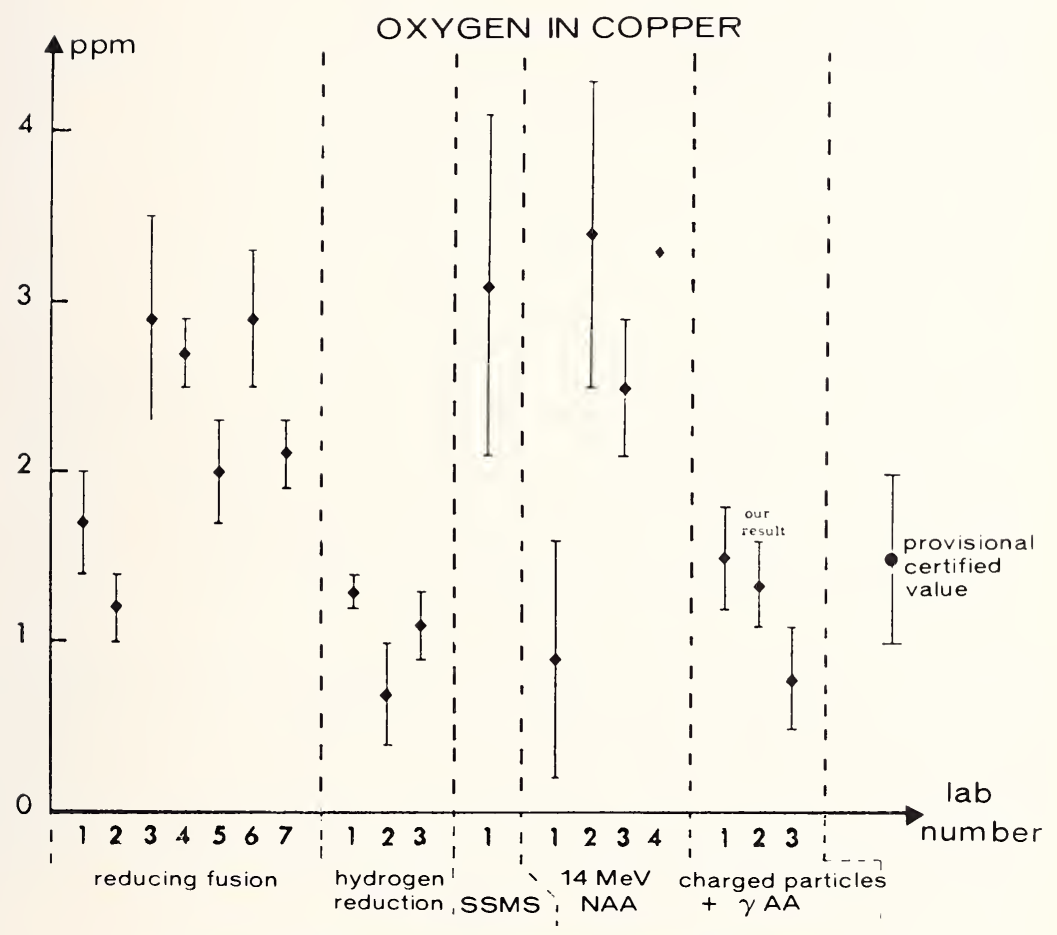

Figure 8. Results of oxygen determination in copper. 


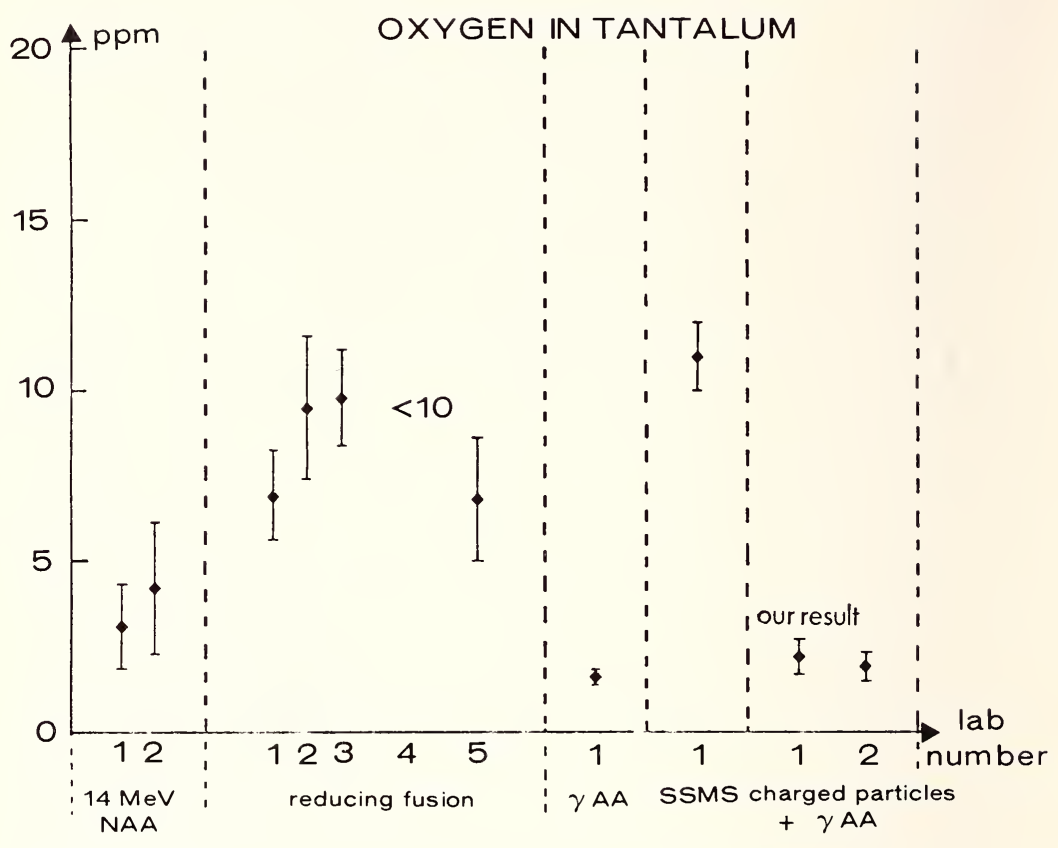

Figure 9. Results of oxygen determination in tantalum.

and this is a very difficult task. Figure 10 gives the error on oxygen determination for an error of 1 micrometer on the etching, versus the energy of irradiation; this error is calculated for $\mathrm{Al}$ and $\mathrm{Cu}$ samples and for the reactions ${ }^{16} \mathrm{O}\left({ }_{2}^{3} \mathrm{He}, \mathrm{p}\right){ }^{18} \mathrm{~F}$ and ${ }^{16}(\alpha, \mathrm{d}){ }^{18} \mathrm{~F}$. It can be seen that not only the choice between different isotopes obtained by irradiation with one particle is important, but also the choice between different particles.

\section{Conclusion}

Nondestructive multielemental trace analysis can be achieved in many matrixes if $10 \mathrm{MeV}$ protons are used. The quality of the beam is of the utmost importance from the standpoint of accuracy and precision. This is inherent to the machine which is used. Among the parameters on which the beam user can have influence, the energy of irradiation chosen and the nuclear reaction used are the most important. This is related to the existence of reaction thresholds, to the fact that activation curves are all different, and to the necessity to etch the samples. A good accuracy can be obtained with a poor associated precision if enough experiments are per- 

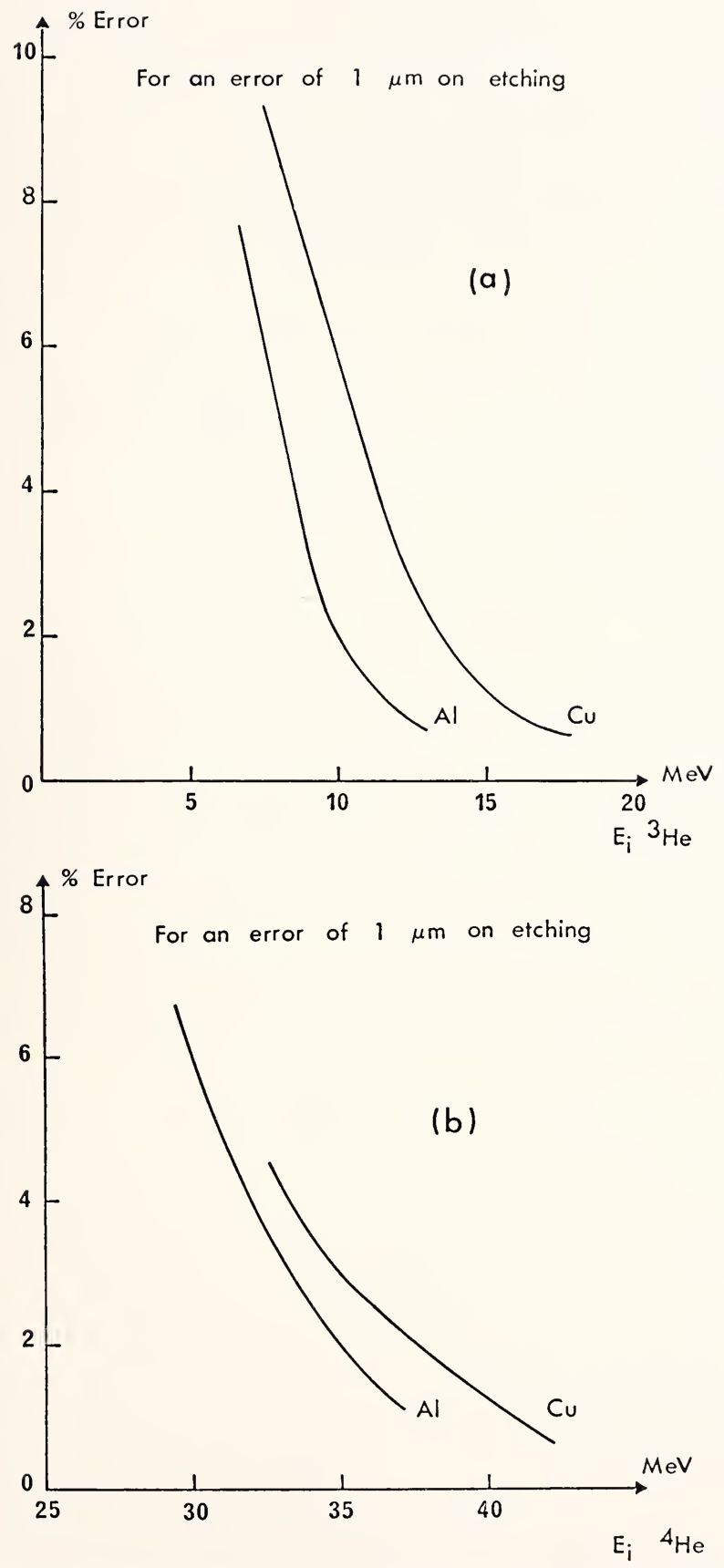

Figure 10. Error on the oxygen determination in aluminum and copper for an error of 1 micrometer on the etching versus the energy of irradiative (a) $\mathrm{E}_{i}={ }^{3} \mathrm{He}$, (b) $\mathrm{E}_{i}={ }^{4} \mathrm{He}$. 
formed with a low quality beam. With a good beam, very accurate results can be obtained with a precision that will then only be limited by the measured activity, provided that the energy and the nuclear reaction are correctly chosen.

\section{References}

[1] Ricci, E. and Hahn, R. L., Anal. Chem. 39, 794-797 (1967).

[2] Barrandon, J. N., Debrun, J. L., Cuypers, M., Quaglia, L., and Robaye, G., CR C 268, 1306 (1968).

[3] Barrandon, J. N., Debrun, J. L., Cuypers, M., Quaglia, L., and Robaye, G., Journal of Radioanalytical Chemistry 4, 115 (1970). 


\title{
ORGANOMERCURY AND TOTAL MERCURY CONTENT OF ENVIRONMENTAL MATRICES AS DETERMINED BY NEUTRON ACTIVATION ANALYSIS
}

\author{
Edoardo Orvini and Mario Gallorini
}

\author{
Centro di Radiochimica e Analisi \\ per Attivazione del C.N.R. \\ Universita di Pavia \\ Viale Taramelli 12 \\ 27100 Pavia, Italy
}

\begin{abstract}
Neutron activation analysis and the gas-chromatographic techniques are used in the determination of organomercury compounds and of total mercury in environmental matrices. In this work the benzene-cysteine separation procedure of Westöö was coupled to neutron activation analysis. In this way both the total mercury content and the organomercury fraction can be evaluated in one sample and during one radiochemical separation process. The activity of ${ }^{197} \mathrm{Hg}$ from the inorganic mercury content of the samples has been evaluated with a well-known combustion separation technique, carried out on the residual sample after benzene extraction. The activity of ${ }^{197} \mathrm{Hg}$ from organomercury compounds of the samples has been evaluated after extraction and purification with cysteine acetate from benzene fraction. The total mercury content was checked on an untreated portion of the same sample.
\end{abstract}

Keywords: Environmental matrices; extraction yields; mercury; neutron activation analysis; organomercury; solvent extraction.

\section{Introduction}

In the recent time, the interest of ecologist and analyst for mercury determination in environmental matrices has been focused on the organomercury to total mercury content ratio [1]. The determination of the total mercury content of biological and environmental matrices using neutron activation analysis procedures has been extensively investigated in these recent years, and some radiochemical separation methods are considered fully reliable for this purpose [2-4]. The methods at the 
present used in most laboratories for the determination of organomercury compounds are modifications of the procedure originally devised by Gage and developed into a gas-chromatographic method by Westöö [5-9]. When both the information on organomercury compounds content and total mercury content are required [8-10], two samples have to be processed separately. Moreover, the neutron activation analysis and the gas-chromatographic techniques are completely different, thus requiring different knowledge and equipment and involving many persons for one determination.

In this work, the benzene-cysteine separation procedure of Westöö was coupled to neutron activation analysis. In this way both the total mercury content and the organomercury fraction can be evaluated in one sample and using one analytical technique. Unfortunately, organomercury compounds, to some extent, undergo Sziland-Chalmers reaction, thus requiring that the organomercury and inorganic mercury separation be done before irradiation.

\section{Experimental}

Although the proposed method for the simultaneous determination of organo and inorganic mercury content of environmental samples follows some well tested and reliable chemical procedure, all of the steps involved in the chemical separation were tested by activation analysis assays. In particular the extraction yields of organo and inorganic mercury compounds in benzene from a strongly acidic medium and in a cysteine-acetate solution from benzene were extensively checked. The results of these tests are summarized in tables 1,2, and 3; they show that these chemical

TABLE 1. Nonextraction test for inorganic mercury with benzene from aqueous acidic medium

${ }^{197} \mathrm{Hg}$ specific activity $(\mathrm{cpm} / \mathrm{ml})$

Sample

Aqueous solution:

before extraction

after extraction

Benzene

before extraction

$\begin{array}{ccll}1 & 3.518 \times 10^{4} & 3.50 \times 10^{4} & 2.1 \times 10^{1} \\ 2 & 3.387 \times 10^{4} & 3.41 \times 10^{4} & 1.3 \times 10^{1} \\ 3 & 3.450 \times 10^{4} & 3.44 \times 10^{4} & 1.8 \times 10^{1} \\ & 3.486 \times 10^{4} & 3.450 \times 10^{4} & 1.7 \times 10^{1}\end{array}$


TABLE 2. Extraction yields of organomercury compounds with benzene from aqueous acidic medium

\begin{tabular}{|c|c|c|c|c|}
\hline \multirow{2}{*}{ Sample } & \multirow{2}{*}{ Run } & \multicolumn{2}{|c|}{${ }^{197} \mathrm{Hg}$ total activity, cpm } & \multirow{2}{*}{$\begin{array}{c}\text { Recovery yield } \\
\%\end{array}$} \\
\hline & & $\begin{array}{l}\text { Aqueous phase } \\
\text { before extraction }\end{array}$ & Benzene phase & \\
\hline a & $\begin{array}{c}1 \\
2 \\
3 \\
4 \\
\text { Total }\end{array}$ & $2.52 \times 10^{5}$ & $\begin{array}{l}2.35 \times 10^{5} \\
1.6 \times 10^{4} \\
5.00 \times 10^{2} \\
2 \times 10 \\
2.51 \times 10^{5}\end{array}$ & $\begin{array}{c}92.6 \\
6.8 \\
0.3 \\
- \\
99.7\end{array}$ \\
\hline $\mathrm{b}$ & $\begin{array}{c}1 \\
2 \\
3 \\
4 \\
\text { Total }\end{array}$ & $2.46 \times 10^{5}$ & $\begin{array}{l}2.29 \times 10^{5} \\
1.50 \times 10^{4} \\
2.46 \times 10^{2} \\
2 \times 10 \\
2.44 \times 10^{5}\end{array}$ & $\begin{array}{r}93.0 \\
6.1 \\
0.1 \\
- \\
99.2\end{array}$ \\
\hline c & $\begin{array}{c}1 \\
2 \\
3 \\
4 \\
\text { Total }\end{array}$ & $2.50 \times 10^{5}$ & $\begin{array}{l}2.30 \times 10^{5} \\
1.55 \times 10^{4} \\
1.25 \times 10^{3} \\
2.5 \times 10^{2} \\
2.47 \times 10^{5}\end{array}$ & $\begin{array}{r}92.0 \\
6.2 \\
0.5 \\
0.1 \\
98.8\end{array}$ \\
\hline Mean & & $2.49 \times 10^{5}$ & $2.41 \times 10^{5}$ & $99.2 \pm 0.6$ \\
\hline
\end{tabular}

TABLE 3. Extraction yields of organomercury compounds with cysteine from benzene

\begin{tabular}{|c|c|c|c|c|}
\hline \multirow[b]{2}{*}{ Sample } & \multirow[b]{2}{*}{ Run } & \multicolumn{2}{|c|}{${ }^{197} \mathrm{Hg}$ specific activity } & \multirow{2}{*}{$\begin{array}{c}\text { Recovery yield } \\
\%\end{array}$} \\
\hline & & Benzene phase & Residual & \\
\hline
\end{tabular}

a

$\begin{array}{ll}1 & 3.17 \times 10^{5} \\ 2 & \\ 3 & \\ 4 & \\ \text { Total } & \end{array}$

$\mathrm{b}$

$\begin{array}{ll}1 & 3.21 \times 10^{5} \\ 2 & \\ 3 & \\ 4 & \end{array}$

Total

c

1
2
3
4
Total

$2.98 \times 10^{5}$

$$
\begin{aligned}
& 1.28 \times 10^{4} \\
& 3.8 \times 10^{3} \\
& 2.8 \times 10^{3} \\
& 2.3 \times 10^{3}
\end{aligned}
$$

94.1

5.3

0.3

.1

99.8

95.0

4.3

0.3

.1

99.7

93.6

4.3

0.6

.2

98.7

Average

$3.12 \times 10^{5}$

$99.4 \pm 1.0$ 
separation steps are in fact fully reliable and reproducible and require only few extractions to be quantitative. These results were obtained by adding inactive tracer and using neutron activation analysis to assay the different phases. The second major check was done on the blank contamination of reagents used.

\section{A. Reagents}

Deionized water with a conductance of less than $0.1 \mu$ mhos was used. Hydrochloric acid and benzene were electronic grade reagents; cysteine, sodium acetate and sodium sulfate were of analytical grade (RP). All glassware was cleaned with a decontaminant solution and rinsed with demineralized water.

A blank control was done on the whole procedure, analyzing for mercury contamination in the cysteine recovered after a benzene-cysteine extraction on $20 \mathrm{ml}, 20$ percent $\mathrm{HCl}$ aqueous solution, following the same separation and activation steps of the actual analytical scheme. No mercury contamination was revealed in the reagents as used in this separation.

\section{B. Procedure for Determination of Mercury in Fish and Meat}

\section{Organomercury Content}

Five- to 10-gram samples, on a wet basis (for the canned tuna fish of NBS, 1 gram sample was run), of muscle tissue, of canned fish or meat were homogenized with $10 \mathrm{ml}$ of demineralized water, and transferred quantitatively in a $25 \mathrm{ml}$ centrifuge test tube. Two $\mathrm{ml}$ of concentrated hydrochloric acid was added and the acidic homogenate was extracted three times with $5 \mathrm{ml}$ benzene portions. The extraction was performed by sucking and injecting the homogenate and forcing it to pass through a pipette. This procedure was found to be cleaner and faster than shaking the mixture in a separatory funnel, and is always used when dealing with small volumes of extracting phase. After centrifugation, the benzene phases were recovered and transferred in a clean centrifuge test tube and centrifuged again. Ten $\mathrm{ml}$ of the clear upper benzene extract was then transferred in a centrifuge tube and extracted three times with $2 \mathrm{ml}$ portions of cysteine-acetate solution, saturated with sodium sulfate. A $2 \mathrm{ml}$ portion of the cysteine-acetate solution was then transferred into heattreated silica vial ready to be freeze-dried, sealed and irradiated. 


\section{Total Mercury Content}

Five- to 10-gram samples of canned muscle tissue were freeze-dried and a portion sealed in heat-treated silica vial for the total mercury content determination. Primary standard solution of mercury was deposited on a strip of filter paper, dried and incapsulated in a like manner. In the residual homogenate, after benzene extraction, inorganic mercury content checks were carried out, freeze drying the homogenate and sealing a portion in a silica vial for the mercury determination. For each determination of mercury content, a set of four samples was irradiated together for about 10 hours in the pneumatic facility of the LENA reactor, at a flux of approximately $4 \times 10^{12} \mathrm{n} \cdot \mathrm{cm}^{-2} \cdot \mathrm{s}^{-1}$. Namely each set of samples contains:

a. untreated portion of the sample to be analyzed for the total mercury content;

b. cysteine-acetate for organomercury determination;

c. residual homogenate for inorganic mercury determination;

d. primary standard for mercury evaluation.

The mercury content evaluation of each sample was formed via the already well known burning technique [2,3] , and evaluating the activity of the $65 \mathrm{~h}{ }^{197} \mathrm{Hg}$ measured with a $\mathrm{Ge}(\mathrm{Li})$ detector connected to a 4096 channel analyzer.

\section{Results and Discussion}

The results of some analytical work done following the previously described procedure are summarized in tables 4 and 5. The samples analyzed were an NBS tuna fish, a canned tuna fish and a canned beef meat. The organometallic to total mercury content ratio seems to be somewhat higher in the tuna sample than in the beef meat.

After these results, some consideration could be done on the proposed technique. No information on the kind of organomercury compounds present is possible via this technique. This seems not to be of relevant interest because at least 90 percent of all the organomercury compound present in nature seems to be methylmercury $[1,9]$.

Activation analysis is not used here at its best capability, because this technique gives its best results when the chemical treatments are done after irradiation. Szilard-Chalmers reaction on the organomercury compounds do not allow one to follow the easiest way. The results show that the method is reliable and gives good precision, allowing one to determine 


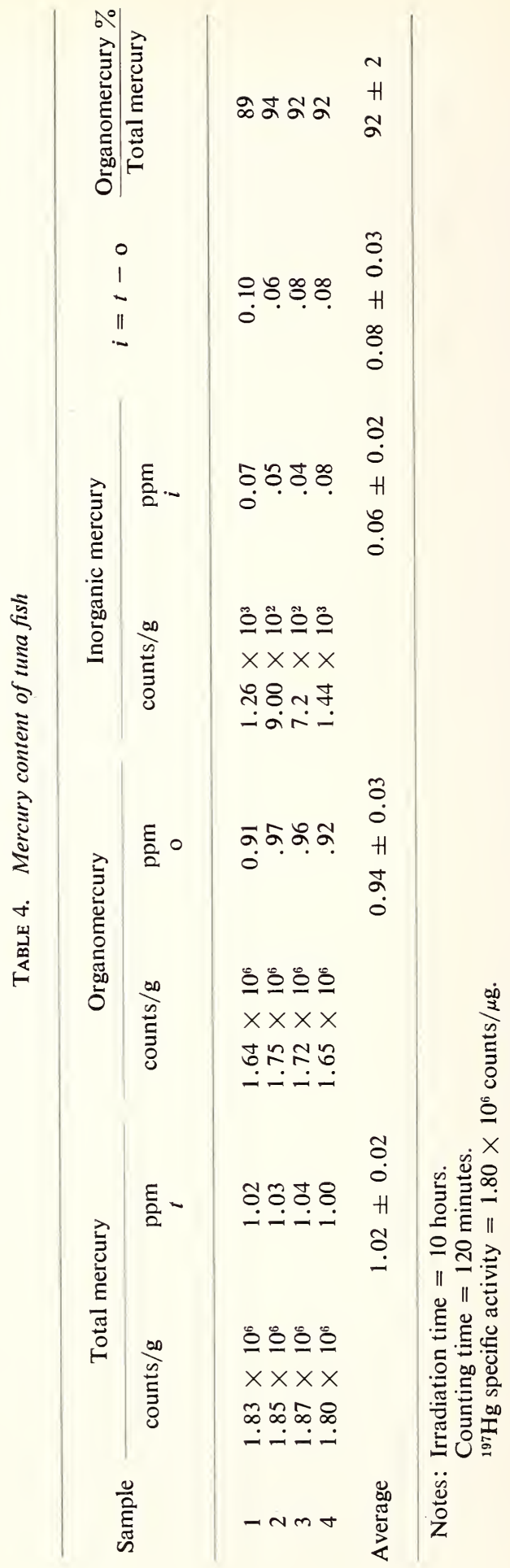




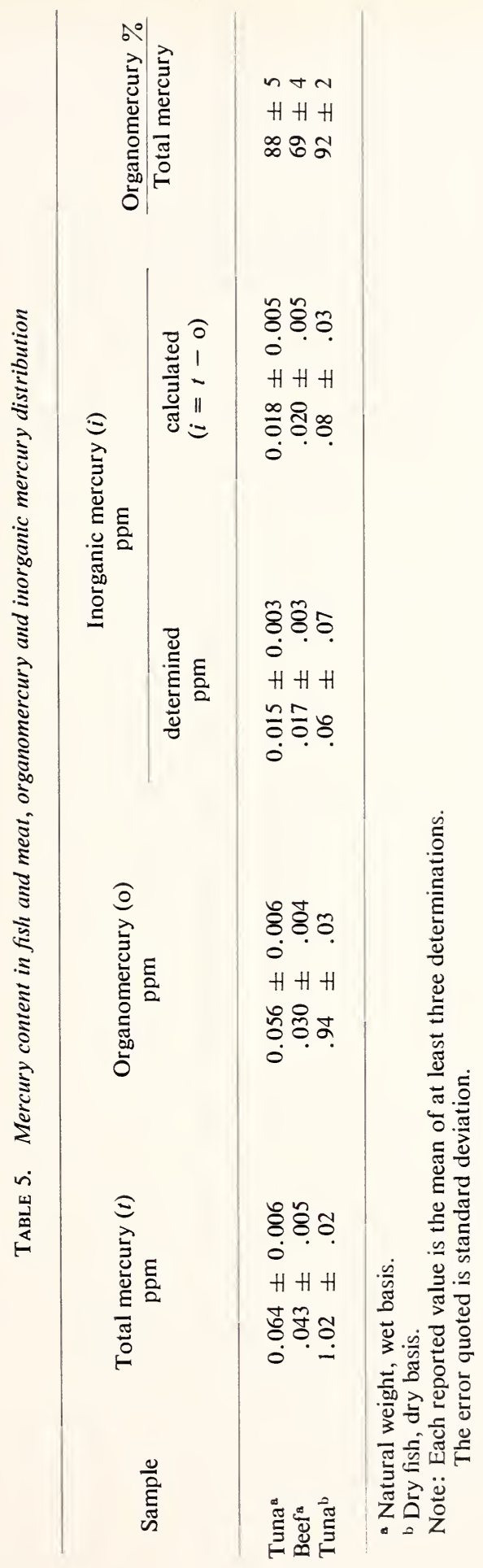


both the main species of mercury compounds relevant to environmental studies utilizing only one technique.

\section{References}

[1] Selikoff, I. J., Hazards of Mercury, Environmental Research 4, (1) (March 1971).

[2] Kosta, L. and Byrne, A. R., Activation Analysis of Mercury in Biological Sample at Nanogram Level, Talanta 16, 1297-1303 (1969).

[3] Rook, H. L., Gills, T. E., and LaFleur, P. D., A Method for Determination of Mercury, Anal. Chem. 44, 7, 1114 (1972).

[4] Orvini, E., Gills, T. E., and LaFleur, P. D., Method for Determination of Selenium, Arsenic, Zinc, Cadmium and Mercury in Environmental Matrices by Neutron Activation Analysis, Anal. Chem. 46, 9, 1294-1297 (1974).

[5] Gage, J. C., The Trace Determination of Phenyl- and Methyl-mercury Salts in Biological Material, Analyst 86, 547 (1961).

[6] Westöö,.G., Determination of Methylmercury Compounds in Foodstuffs I. Methylmercury Compounds in Fish, Identification and Determination, Acta Chem. Scand. 20,2131-2137 (1966).

[7] Westöö, G., Determination of Methylmercury Compounds in Foodstuffs II. Determination of Methylmercury in Fish, Egg, Meat and Liver, Acta Chem. Scand. 21, 1790-1800 (1967).

[8] Westöö, G., Determination of Methylmercury in Various Kinds of Biological Material, Acta. Chem. Scand. 22, 2277-2280 (1968).

[9] Westöö, G., Mercury and Methylmercury Levels in Some Animal Food Products, Var Foeda 7, 137-154 (1969).

[10] Zelenko, V. and Kosta, L., A New Method for Isolation of Methylmercury from Biological Tissues and its Determination at the Parts per Million Level by Gas Chromatography, Talanta 20, 115-123 (1973). 


\title{
EVALUATION OF ERROR IN THE INSTRUMENTAL ACTIVATION ANALYSIS OF ROCKS
}

\author{
Rolf J. Rosenberg \\ Technical Research Centre of Finland \\ Reactor Laboratory \\ SF-02150 Otaniemi - Finland
}

In this paper, the overall error in instrumental activation analysis of rocks with a TRIGA Mark II reactor and the magnitude of its components, are evaluated. The analysis of lanthanides in the USGS standard rocks G-1 and W-1 is used as an example. In view of these results the possibility to decrease the error is discussed.

The average precision of the individual results obtained in this work is 11.6 percent and the average deviation of the mean is 4.6 percent. According to a comparison of values found in the literature the average error of results obtained in different laboratories is 11.8 percent and the average deviation of the results obtained in this work from the average of all results is 7.3 percent evenly distributed on both sides of zero.

The possible components of the error have been investigated partly experimentally and partly theoretically and the following was found to give noticeable contributions when $200 \mathrm{mg}$ samples were used: The flux gradient causes an average error of 5.2 percent and thermalization in aquatic standards causes a negative error of 2.2 percent. Differences in counting geometry in measurements with the sample on the detector cause an error of 2.5 percent and differences in gamma-ray absorption cause an error that varies between 2.5 percent and below 1 percent. This error is negative when aquatic standards are used. Counting statistics causes an error that varies between 1 percent and 38 percent. The mean errors calculated from these data give a total error that varies between 9.0 and 42 percent with an average of 10.8 percent. A comparison between 10.8 percent and the average precision 12.8 percent of all results obtained in this work indicates that all errors of any importance are considered here. The mean error without counting statistics is 8.9 percent.

By rotating the samples during irradiation, counting with a few $\mathrm{cm}$ distance between sample and detector and by using rock-standards the total error can be decreased to the level of counting statistics. The same results can be obtained by decreasing the dimensions of the samples.

Keywords: Comparative analyses; error evaluation; geological materials: instrumental-neutron activation analysis. 


\section{Introduction}

The usefulness of data derived by means of analytical chemistry is proportional to their reliability. As a consequence the evaluation of error in analysis is of importance for two reasons; firstly, so that knowledge is gained of the accuracy of the analytical data, and secondly, so that the error can be diminished.

A good method for determination of the reliability of an analytical method is to be found in the comparison of the data for the same samples obtained by different laboratories. In such a comparison, the average of all the results is regarded as the true value. However, this average is no more than an approximation of the true value, and an approximation is as good as its components. The error of the mean value decreases with increase in the amount of data. This is true if all errors behave statistically.

In this work the analysis of lanthanides in USGS standard samples G-1 and $\mathrm{W}-1$ is used in such a comparison, as these make a coherent group of trace elements, from which relatively good data can be derived.

If the total error is to be diminished, the components of the error, and their magnitudes, need to be known. The potential sources of errors in activation analysis are commonly known, and some different methods for their evaluation have been published. In this work the errors are evaluated theoretically in cases where this is readily practicable, and experimentally in cases where theoretical determination is difficult. In all experimental work standard samples are utilized, and thus the results can be considered as being truly representative of the real case. The reliability of the results are tested by comparing the total error obtained in the laboratory comparison with the error calculated from the different components.

\section{The Analytical Method}

The details of instrumental activation analysis of lanthanides in rocks have been discussed elsewhere $[1,2]$. Briefly, this analysis is effected as follows: a few hours subsequent to 5 minutes irradiation in Rotary specimen track of the TRIGA Mark II reactor of the Reactor Laboratory, the samples are counted for ${ }^{165} \mathrm{Dy}$ with an Ortec $0.45 \mathrm{~cm}^{3} \mathrm{Ge}(\mathrm{Li})$ low energy detector connected through an Ortec 117 preamplifier and an Ortec 451 spectroscopy amplifier with a Nuclear Data 4400 pulse height analyzer. The system resolution is $600 \mathrm{eV} \mathrm{FWHM}$ determined with the $122 \mathrm{keV}$ line of ${ }^{57} \mathrm{Co}$. The flux is $1.2 \times 10^{12} \mathrm{n} \cdot \mathrm{cm}^{-2} \cdot \mathrm{s}^{-1}$. After an addi- 
tional $10^{13} \mathrm{n} \cdot \mathrm{cm}^{-2} \cdot \mathrm{s}^{-1}$, the samples are counted by application of the same system a few days after the irradiation for $\mathrm{La}, \mathrm{Nd}, \mathrm{Sm}, \mathrm{Ho}$ and $\mathrm{Lu}$, and more than 6 weeks after the irradiation for $\mathrm{Ce}, \mathrm{Nd}, \mathrm{Eu}, \mathrm{Gd}, \mathrm{Tb}, \mathrm{Yb}$ and Tm. In this work, data for Ho and Tm are not used. The spectra are punched upon paper tape with a FACIT 4070 punch, and processed with a UNIVAC 1108 computer, using the computer code STOAV [3]. The program calculates the elemental contents by comparison of the sample spectra with a spectrum obtained by counting a standard sample consisting of a aqueous solution of the lanthanides.

The same containers are used for the irradiations and measurements: they had been checked as containing no detectable amounts of lanthanides. For the determination of Dy, a $0.5 \mathrm{ml}$ polyethylene capsule with an external diameter of $8.5 \mathrm{~mm}$ is used. Five of these can be irradiated in the same plane in the Rotary specimen rack (Lazy Susan), and seven in the Central thimble. For the long irradiation quartz ampoules are used; these have an internal diameter of about $4 \mathrm{~mm}$, and an external diameter of 6 $\mathrm{mm}$, and 12 of them can be irradiated in a plane in the Central thimble.

\section{The Total Error}

The precision of a single determination is firstly determined by the comparison of data obtained by parallel determinations. Table 1 lists the data for G-1 and W-1 obtained in this work. The relative standard deviations of the individual results vary between 2.7 and 20 percent for different elements giving an average of 11.6 percent. The standard deviations of the means vary between 1.5 and 12 percent, giving an average of 4.6 percent. Table 2 indicates results for the same determinations, extracted from the literature. Here, the standard deviations vary between 5.8 and 20 percent, with an average of 11.8 percent, and the standard deviations of the means vary between 1.5 and 12 percent, with an average of 4.2 percent. The results given in tables 1 and 2 bear surprising resemblances. The deviations between the average results obtained in this work, and the averages derived from published results vary between 0.3 and 18 percent, distributed evenly on both sides of zero. The average of the numerical values is 7.3 percent. 


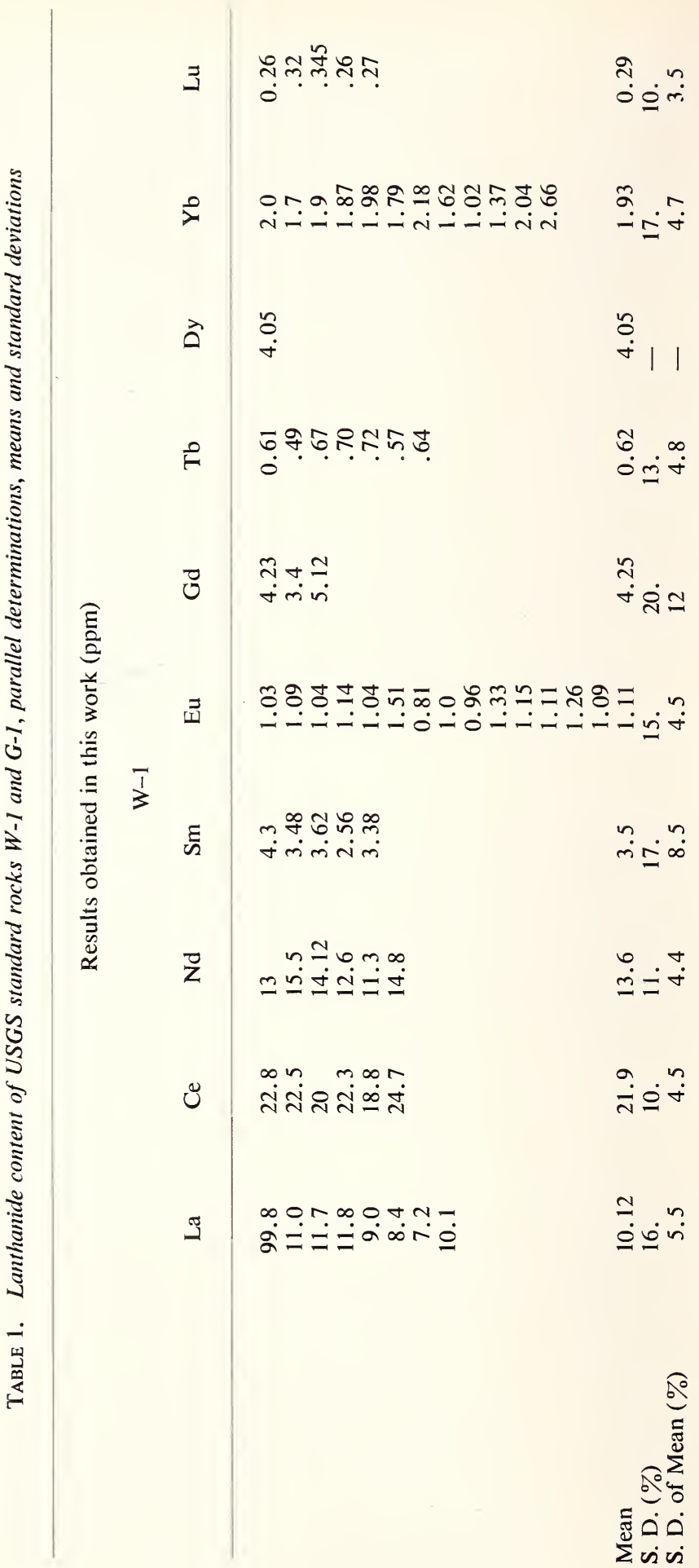




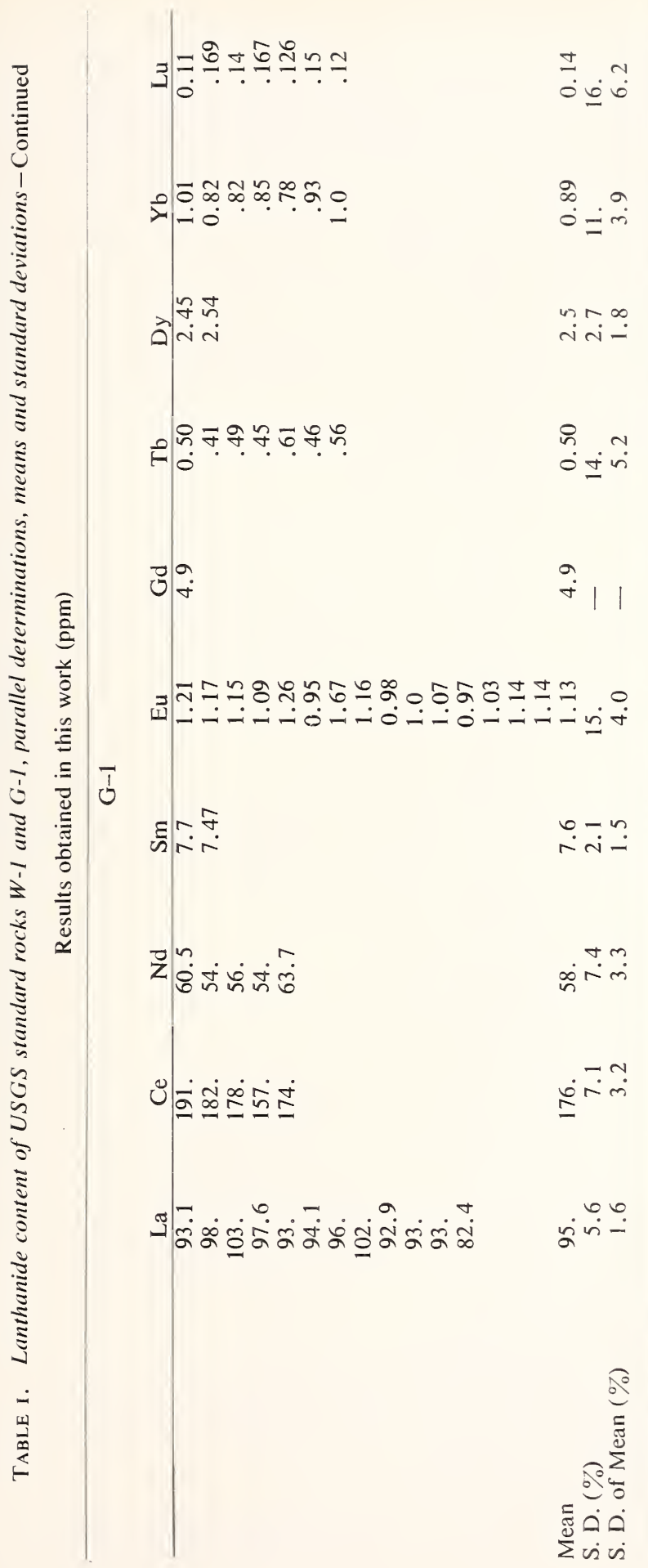




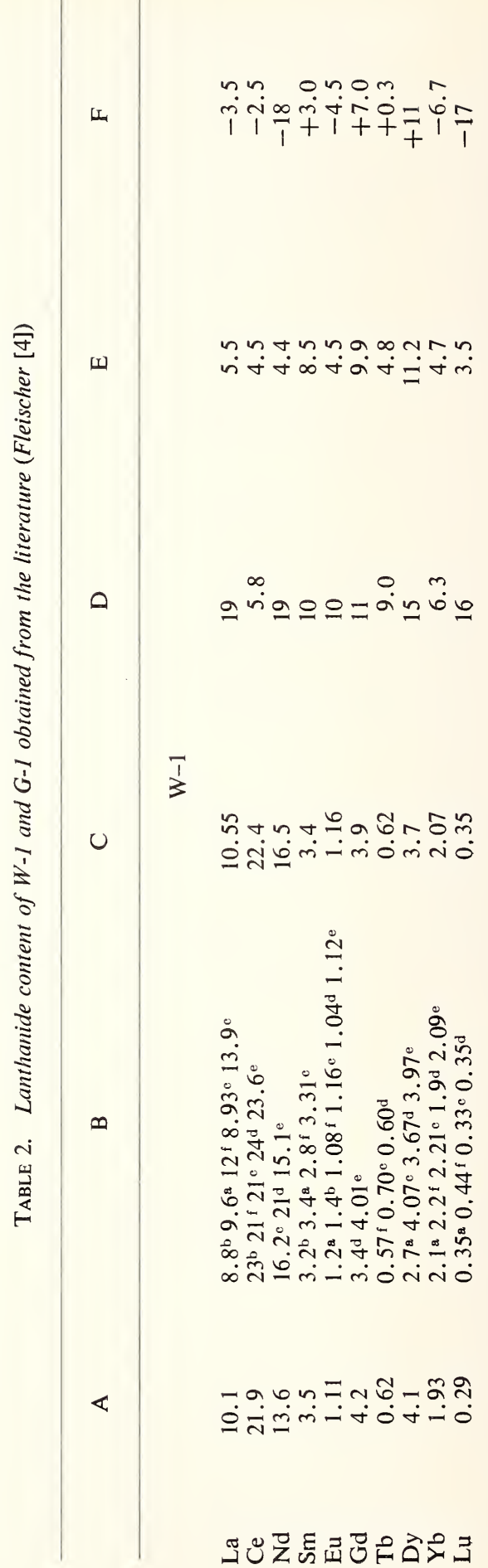




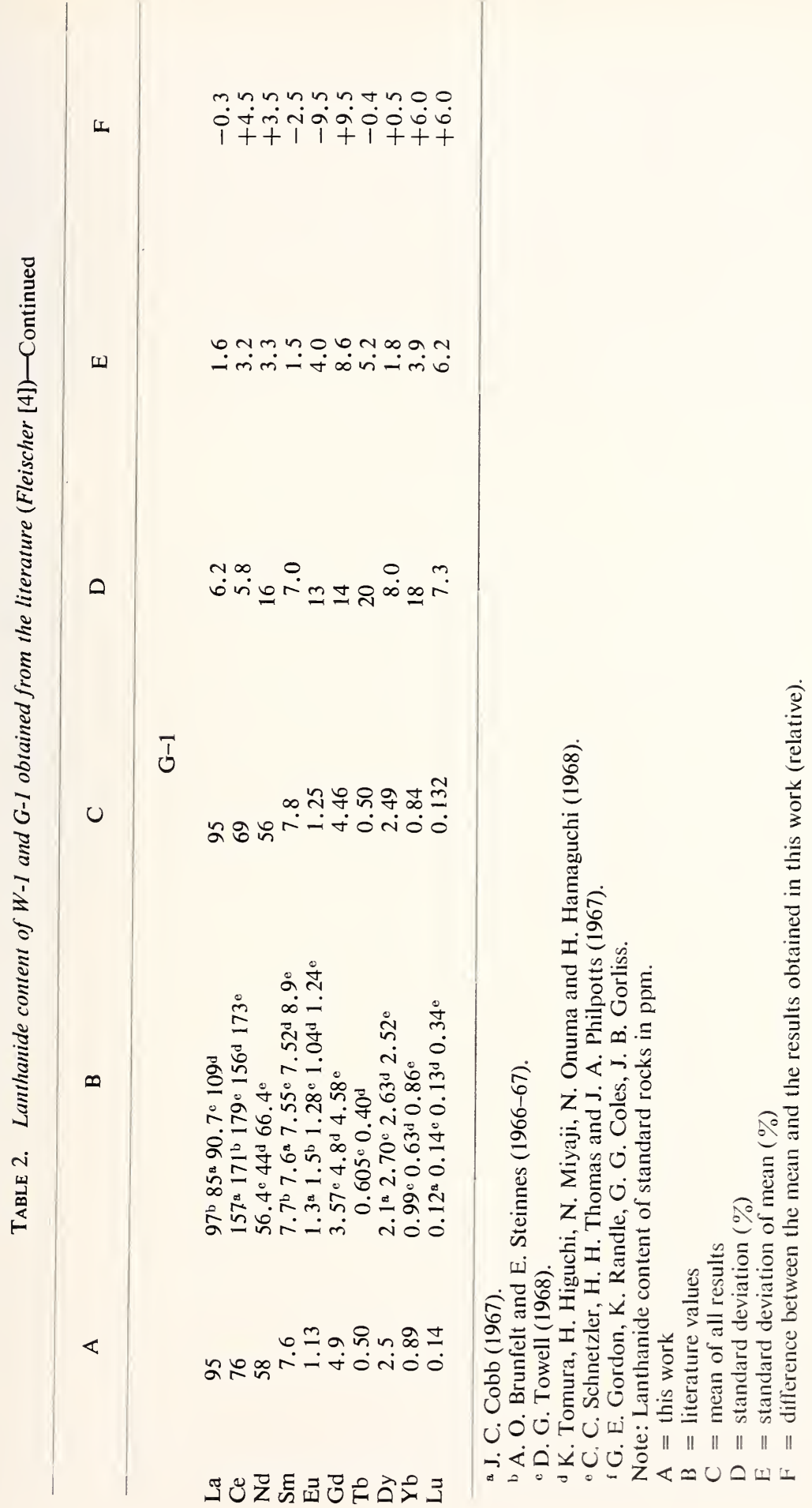




\section{Components of the Total Error}

In the following section, consideration is given to some errors that occur to a major or minor extent in every determination. Such errors as contamination and inhomogeneity of samples are not discussed here. Activation analysis essentially consists of three parts, viz, activation, counting, and data processing. Each of these has its own sources of error, which are 1) flux inhomogeneity in the reactor, and neutron perturbation in the samples, 2) counting geometry, gamma-ray attenuation, counting statistics and 3) the quantitative evaluation of gamma spectra.

\section{A. Flux deviation}

In a small reactor, such as that employed in this work, the flux deviation is considerable in all directions in the core and in the reflector where the Rotary specimen rack is located. The flux distribution in the Rotary specimen rack has been studied quite thoroughly. The radial flux depression, according to ref. [5], is 4 percent per $\mathrm{cm}$. By the irradiation of dilute Dy solutions, values between 4 and 5 percent per $\mathrm{cm}$ have been obtained in different parts of the rack. The horizontal and vertical flux variations have been studied by the irradiation of pieces of copper wire in different positions of the Rotary specimen rack. Figure 1 illustrated some of the results obtained: these indicate that the flux varies up to 1.2 percent per $\mathrm{cm}$ in the horizontal direction, and up to 3.2 percent per $\mathrm{cm}$ in the vertical direction.

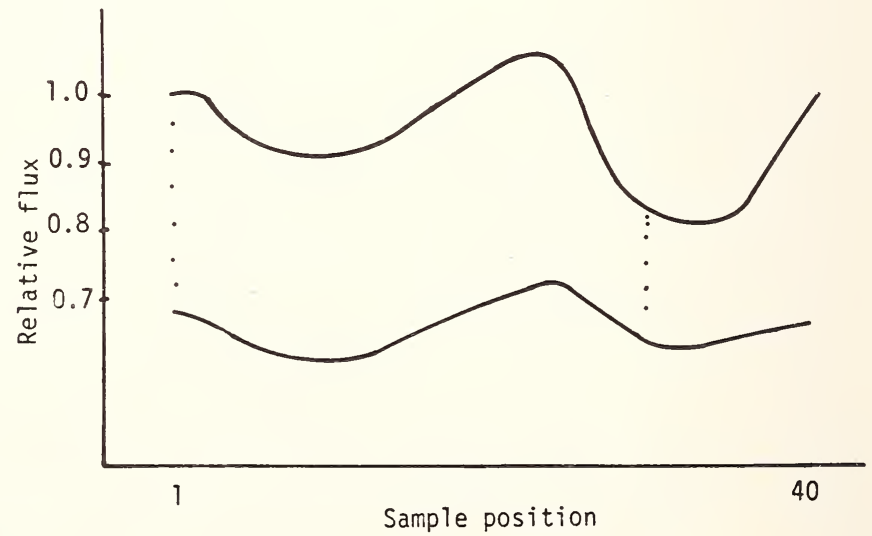

Figure 1. Relative thermal flux in the Rotary specimen rack. The distance between sample positions is $5 \mathrm{~cm}$. The two lines indicate the top and the bottom of the positions, the distance being $15.4 \mathrm{~cm}$. 
The influence of these deviations upon the induced activities of a set of samples has been investigated by the irradiation of small homogeneous rock samples, and by counting the induced ${ }^{24} \mathrm{Na}$ activity. Figure 2 indicates the sample configuration, both in the Rotary specimen rack and in the Central thimble. Table 3 shows typical relative activities produced. From these data relative standard deviations of 4.4 and 3.7 percent respectively are derived for the Rotary specimen rack and the Central thimble. Thus, when one of the samples is the standard, the mean errors induced by the flux deviation in the samples are 6.2 and 5.2 percent respectively. These values represent only order of magnitude, since the flux deviation varies in the different positions.

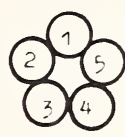

Is

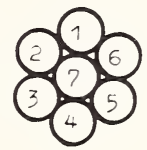

CT

Figure 2. Sample configuration in flux distribution measurement.

TABLE 3. Relative thermal flux in sample configurations shown in figure 2

\begin{tabular}{lcc}
\hline Position & $\begin{array}{c}\text { Rotary } \\
\text { specimen rack }\end{array}$ & $\begin{array}{c}\text { Central } \\
\text { thimble }\end{array}$ \\
\hline 1 & 1.00 & 1.00 \\
2 & 0.96 & 0.95 \\
3 & 1.00 & 0.92 \\
4 & 1.02 & 0.99 \\
5 & 1.08 & 1.03 \\
6 & & 0.98 \\
7 & & 1.00 \\
\hline
\end{tabular}

\section{B. Neutron Perturbation by the Sample}

Even if the incident flux is homogeneous for a set of samples, the interactions of the neutrons inside the samples differ with consequent variations in the neutron dose.

In this work, a maximum of $200 \mathrm{mg}$ of powdered rock sample. about 1 $\mathrm{g} / \mathrm{cm}^{3}$ in density, were utilized: this renders it easy to calculate. by Högdahl's method [6] that in this case the thermal neutron absorption is well below 0.1 percent. 
The thermalization of fast neutrons in the liquid standards, however, is considerable. This effect was studied experimentally. In polyethylene capsules $40 \mathrm{~mm}$ in length and $13 \mathrm{~mm}$ in diameter, copper wires were introduced diagonally over the total length. Pairs of these were irradiated in identical conditions, both filled with water and without. The specific activities in the wires irradiated in water were found to be 7.3 percent higher than those in the wires irradiated in air. By application of the method of Brune et al. [7], these results were interpolated for the sample on sizes used in the quartz ampoules and polyethylene capsules; the values obtained were 2.1 and 2.5 percent respectively.

\section{Counting Geometry}

Hertogen and Gijbels [8] have studied the influence of counting geometry with a detector similar to that employed in this work. They counted a source with a diameter of $12.7 \mathrm{~mm}$ and a thickness of $4.5 \mathrm{~mm}$, with its position being varied in both the horizontal and vertical directions. The results they obtained indicated that a transfer of the source from $10 \mathrm{~mm}$ to $11 \mathrm{~mm}$ distance from the detector induces a 10 percent diminution in the counting rate, and so does a $5 \mathrm{~mm}$ displacement in the horizontal direction from the central axis. The vertical position of a sample can be easily fixed accurately, but the normal variation in sample thickness in a set of polyethylene capsules can be approximated to 20 percent, which involves a $0.6 \mathrm{~mm}$ displacement of the center of gravity of a $100 \mathrm{mg}$ sample. From Hertogen and Gijbels' data, it can be approximated that this introduces a 6 percent error in the activity measurements. The diameter of a sample is constant, and the position can be set within $1 \mathrm{~mm}$, resulting in an approximate error of 1 percent. However, the polyethylene capsules are usually counted at such a distance from the detector surface that only slight difficulty should arise in the counting geometry.

The long-lived isotopes counted in the quartz ampoules need to be counted directly on the detector, in the position indicated in figure 3 . An attempt has been made to approximate the maximum error attributable to the normal variation in sample size and position. Five irradiated samples were counted, both with a large $\mathrm{Ge}(\mathrm{Li})$ detector at such a distance that the variation in counting geometry was negligible, and direct on the surface of the small detector. With one of the samples used as standard, the iron content of the other samples were in both cases calculated from two peaks (1098-1292 and 142-192 keV respectively). Table 4 lists the results obtained. The difference between the means of results obtained with the large detector and the small detector was found to vary between 0.9 and 


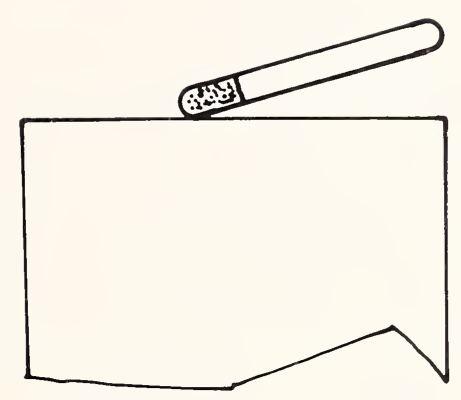

Figure 3. Sample position in counting long-lived isotopes.

TABLE 4. Comparison between iron results obtained by counting samples with a large Ge ( $\mathrm{Li}$ ) detector and a small low-energy photon detector

\begin{tabular}{|c|c|c|c|c|c|c|c|c|}
\hline Sample & A & & B & $\mathrm{C}$ & & B & $\mathrm{D}$ & $E$ \\
\hline 1. & $\begin{array}{l}2.875 \\
2.88\end{array}$ & 2.88 & \pm 0.005 & $\begin{array}{l}2.77 \\
2.85\end{array}$ & 2.81 & \pm 0.04 & 0.07 & $2.4 \%$ \\
\hline 2. & $\begin{array}{l}3.17 \\
3.19\end{array}$ & 3.18 & \pm 0.01 & $\begin{array}{l}3.15 \\
3.06\end{array}$ & 3.105 & \pm 0.045 & .075 & $2.3 \%$ \\
\hline 3. & $\begin{array}{l}3.49 \\
3.46\end{array}$ & 3.475 & \pm 0.015 & $\begin{array}{l}3.40 \\
3.51\end{array}$ & 3.455 & \pm 0.045 & .03 & $0.9 \%$ \\
\hline 4. & $\begin{array}{l}21.7 \\
20.9\end{array}$ & 21.3 & \pm 0.4 & $\begin{array}{l}20.0 \\
20.9\end{array}$ & 20.45 & \pm 0.45 & .85 & $3.9 \%$ \\
\hline
\end{tabular}

Note:

$\mathrm{A}=$ iron content obtained by counting $1099 \mathrm{keV}$ and $1292 \mathrm{keV}$ peaks respectively

$\mathrm{B}=$ mean and deviation from mean

$\mathrm{C}=$ iron content obtained by counting $143 \mathrm{keV}$ and $193 \mathrm{keV}$ peaks respectively

$\mathrm{D}=$ difference between means-absolute

$\mathrm{E}=$ difference between means-relative

3.9 percent, giving an average of 2.4 percent. This value also contains the error induced by the counting statistics, but as this is difficult to account for accurately, 2.4 percent is regarded as the maximum error induced by the difference in counting geometry. As this contains the deviation of a sample and the standard, the deviation from the mean of a set is no more than 1.5 percent.

\section{Gamma-Ray Absorption}

When low-energy gamma rays are used in analysis, it is obvious that the gamma-ray absorption in the sample can introduce a serious problem. By 
application of the formula of Smith et al. [9], and the absorption coefficients given by Wyckoff [10], calculation has been made of the absorption in water and concrete. The density of rock samples is typically 0.98 $\mathrm{g} / \mathrm{cm}^{3}$, and that of water $1 \mathrm{~g} / \mathrm{cm}^{3}$, and the sample thickness is $4 \mathrm{~mm}$. Table 5 indicates the composition of the concrete utilized. To simulate rocks of different kinds, the absorption has also been calculated for concrete containing 23.35 percent $\mathrm{Si}$, and 9.22 percent $\mathrm{Fe}$.

A rock of this composition can be regarded as the worst type of absorbing sample normally subjected to analysis. The difference in absorption between water and sample under these conditions varies between 1.3 and 2.5 percent for a $60 \mathrm{keV}$ gamma ray, and is about 0.2 percent for a 500 $\mathrm{keV}$ gamma ray.

TABLE 5. Composition of concrete used in calculations of gamma-ray absorption

1.

$\begin{array}{lc}\mathrm{H} & 0.56 \% \\ \mathrm{O} & 49.56 \\ \mathrm{Si} & 31.35 \\ \mathrm{Al} & 4.56 \\ \mathrm{Ca} & 8.26\end{array}$

2.

23.35

1.

2.

9.22

$\begin{array}{ll}\mathrm{Fe} & 1.22 \% \\ \mathrm{Mg} & 0.24 \\ \mathrm{Na} & 1.71 \\ \mathrm{~K} & 1.92 \\ \mathrm{~S} & 0.12\end{array}$

\section{E. Counting Statistics}

The counting statistics depend upon the total number of counts and the peak-to-background ratio. As a consequence, they can be influenced by the use of longer counting times, and more efficient detectors that give more favorable peak-to-background ratios. Table 6 illustrates typical errors that arise on use of the experimental conditions described in this work and ref. [2]. These are typical order-of-magnitude data, and cannot be diminished significantly except of course by increasing the content of element.

TABLE 6. Typical relative standard deviations based on counting statistics

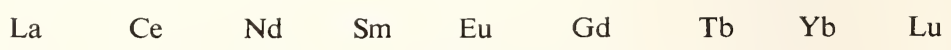

$\begin{array}{lllrllllll}\text { G-1 } & 1.04 \% & 1.31 & 6.6 & 1.0 & 1.26 & 38.0 & 4.24 & 2.96 & 7.05 \\ \text { W-1 } & 3.21 \% & 3.38 & 16.6 & 2.1 & 1.23 & 18.4 & 2.83 & 1.38 & 7.62\end{array}$




\section{F. Peak Areas}

The accuracy of analysis is highly dependent upon the representability of the peak areas calculated. Thus the net peak areas should be independent of the shape and magnitude of the Compton background, except in regard to the counting statistics. The computer code used in this work calculates the background under a straight line between the nearest minima on both sides of the peak, after a check has been made that all the channels between the background channels are over the background line. The net peak area comprises all the counts above the background line.

With a view to checking the behavior of this method, the following setup was applied. The same element was determined from several peaks in the same spectrum. Thus the differences between these results should depend only upon the counting statistics, and upon the accuracy of calculation of the net peak areas. At the same time, a variation is obtained in peak size and background size and shape. The only isotopes usable in this study were ${ }^{140} \mathrm{La}$ and ${ }^{152} \mathrm{Eu}$, and the peaks used were $328.6,815.5,1595.4$ ad $121.8,244.6,344.2$ and $244.6 .778 .6,1407.5$ respectively. Table 7 inicates the results obtained. Here are shown the statistical errors of the individual results, their mean, and, for comparative purposes, the relative standard deviation of the set of results. Obviously no statistically significant difference between these results is demonstrable; it thus seems clear that the peak area method applied here does not introduce any significant error into the analysis.

\section{Discussion}

In the previous sections, different sources of errors have been discussed briefly, and some data derived for their magnitudes. It is now important to show that all the significant sources of errors have been considered, and that their magnitudes are correct.

It was demonstrated that the individual results in this work had the same standard deviations from their means as had the laboratory results. Furthermore, the means in this work are evenly distributed around the means of all the results obtained in different laboratories. It thus seems evident that the individual results in this work are evenly distributed around a "true" value. In calculation of the composite error, the following data are used: The effects of the flux deviation in the reactor behave statistically, since the positions of the individual samples cannot be controlled. For this error an average value of 5.2 percent is applied in the 


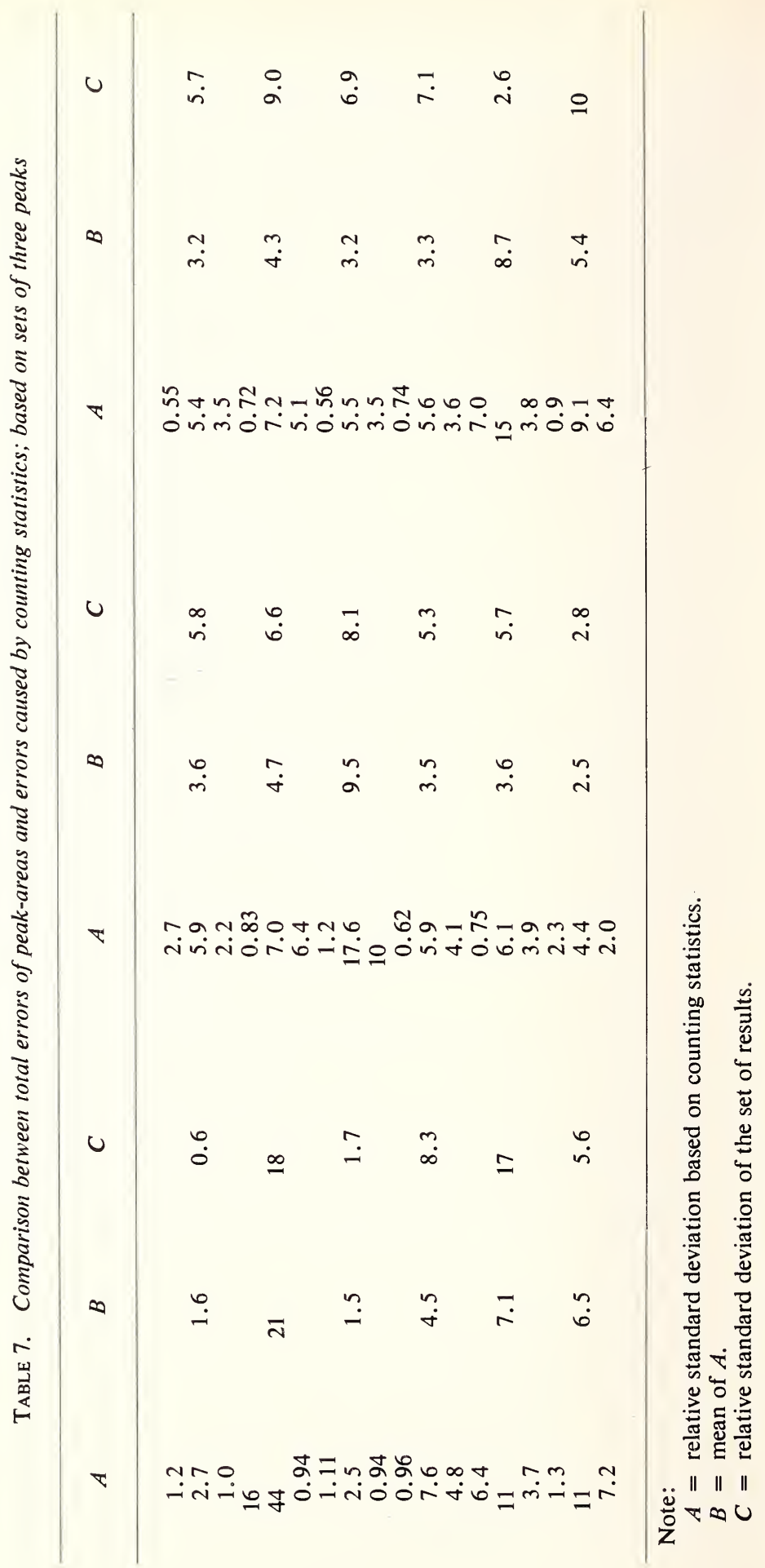




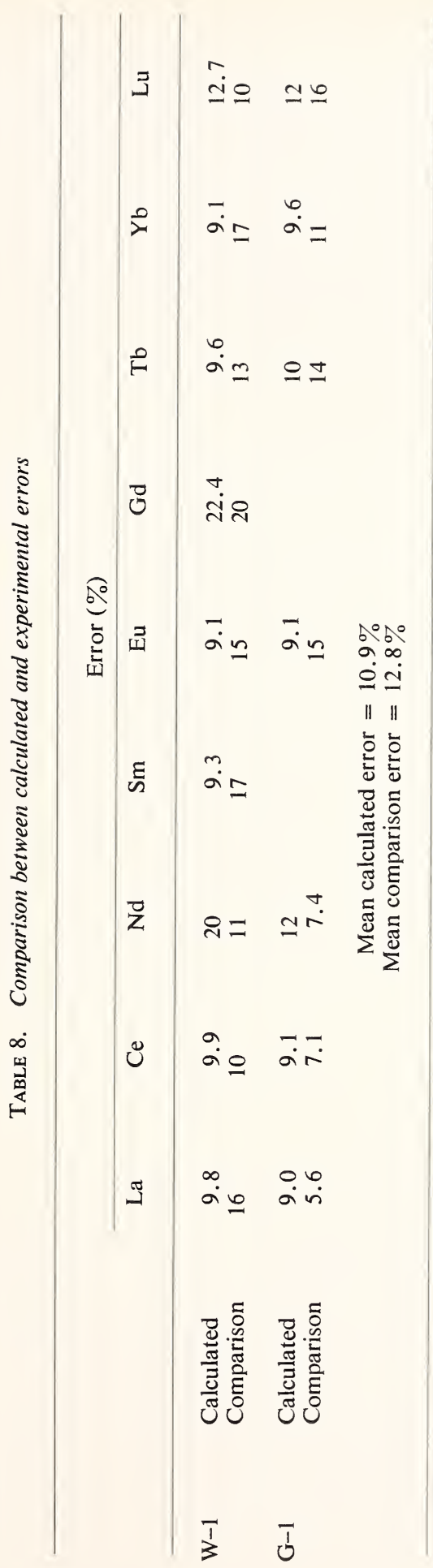


calculations. Neutron thermalization in aquatic samples induces a systematically negative 2.2 percent error, whether the maximum 2.4 percent difference in counting geometry behaves statistically. For the gamma-ray absorption, a negative error of 1 percent is used. By means of the errors induced by the counting statistics in table 6, the total error is derived as indicated in table 8. For comparative purposes the experimental errors from table 1 are also shown. The only data listed are those for which at least three values are found in table 1 . On the average, these two sets of results are in quite close agreement; it thus seems obvious that all of the significant errors have been accounted for. It was stated above that the counting statistics cannot be significantly influenced. However, the total error without counting statistics is 9 percent, and if this is eliminated, considerable advance could be made in the accuracy for most elements.

The most significant individual error arises from the flux inhomogeneity in the reactor, and in some laboratories it is common practice to eliminate this by rotating the samples during irradiation. The differences in neutron flux inside the sample and standard can be eliminated by the use of rock samples as standards; this also diminishes the effect of gamma-ray absorption. The effect of counting geometry can be reduced by pressing pellets of a mixture of graphite and sample, as was done by Hertogen and Gijbels [8]. However, with relatively little effort, rock samples can be weighed in quite equal volumes, and the quartz ampoules can be located in exactly fixed positions; this results in diminution of the error. Most of the errors mentioned can also be significantly reduced by the use of a smaller sample, although this introduces activity problems by reason of the relatively low flux of the reactor employed in this work.

\section{References}

[1] Rosenberg, R. J. and Wiik, H. B., Radiochem. Radioanal. Letters 6, 45 (1971).

[2] Rosenberg, R. J., Suomen Kemistilehti B45, 399 (1972).

[3] Rosenberg, R. J. and Seppä-Lassila, T., Reaktorilaboratorio Tiedonanto 15, Otaniemi, October 1972.

[4] Fleischer, M., Geochim. Cosmochim. Acta 33, 65 (1969).

[5] GA-2025(1961).

[6] Högdahl, O. T., Radiochemical Methods of Analysis, IAEA, Vienna 1965, Vol. I, p. 23.

[7] Brune, D. and Jirlow, K., Radiochem. Acta 8, 161 (1967).

[8] Hertogen, J. and Gijbels, R., Anal. Chim. Acta 56,61 (1971).

[9] Smith, G. W., Becker, D. A., Lutz, G. J., Currie, L. A., and DeVoe, J. R., Anal. Chim. Acta 38, 333 (1967).

[10] Wyckoff, H. O., Radiation Hygiene Handbook, Blatz, H., Ed. in Chief, New YorkToronto-London (1959). 


\title{
NEUTRON ACTIVATION ANALYSIS FOR SOME NONSTOICHIOMETRIC CONSTITUENTS IN A LARGE NUMBER OF NATURAL AND SYNTHETIC BERYL SAMPLES
}

\author{
Slobodan M. Ristic \\ Institute of Physical Chemistry \\ University Belgrade \\ 11000 Beograd, Yugoslavia
}

\begin{abstract}
About 100 natural beryls from very different locations were analyzed. An appreciable content of all five alkali metals was present, so that determination with a good precision was an important crystalochemical as well as geochemical problem. The trace amounts of $\mathrm{Cr}, \mathrm{As}, \mathrm{Sc}, \mathrm{Mn}, \mathrm{Eu}$, and Dy in some natural samples, as well as $\mathrm{Co}, \mathrm{Ni}, \mathrm{Fe}, \mathrm{Mo}, \mathrm{Sc}, \mathrm{Cs}$ and $\mathrm{Na}$ were measured in a gammaspectrometric arrangement with a 500-channel pulse height analyzer, without chemical treatment (i.e., in a nondestructive NAA procedure). The results obtained were partly controlled and compared by emission spectrography, flame spectrometry, and atomic absorption spectrometry, in order to determine the general reliability, as well as precision and accuracy in various analytical procedures and different samples.
\end{abstract}

Keywords: Cesium and scandium content; natural and synthetic beryls: neutron activation analysis.

\section{Introduction}

Mineral beryl is almost the unique source for the extraction of metallic beryllium, but is also an interesting crystalochemical individuum with potential utilization in maser and laser experimentations. The beryl crystal lattice is of a unique kind (group of cyclo-tecto-silicates) and gives rise to many peculiar geochemical problems in connection with origin and properties of natural beryls but also concerning the synthetic beryl samples. The investigations and publications on these problems are many in number, the main part of the literature being published in English. Ger- 
man, and Russian. In order to give a general approach to the problem discussed, let there be cited here only three doctoral dissertations [1-3], a few monographs, and for our purpose more closely interesting books [46] published after World War II. This paper will discuss three main aspects: 1. Beryls, natural and synthetic, as materials to be analyzed; 2 . The choice of methods for such an analysis; and 3. Trace impurities and nonstoichiometric constituents, their detection and importance.

\section{Experimental Part: Materials and Methods Employed, Experiments}

For the natural beryl samples (general notation throughout this paper: NB Nr. . .) we had at our disposal, firstly, almost the complete collection of the late Lord Rayleigh (notation: RB Nr. . ), but also some additionally acquired NB-samples, the total number being more than 100. Secondly, we also had available more than 70 synthetic beryl samples (notation: SB Nr. . .) produced by a method elaborated and described in [1], the materials for their synthesis being of the "pro analysi" grade. The specifications for apparatus and the methods employed will be mentioned briefly in the main text and discussions of the paper.

\section{A. The Beryl Crystal lattice}

The beryl crystal lattice, as is well known, possesses a special kind of "open structure" characterized by "tunnels" formed by stacking above one another the hexagonal rings constituted of six Si-O-tetrahedra. The rings have their centers on hexad axes. These tunnels afford good emplacements for some nonstoichiometric constituents and internal impurities of beryls (such as $\mathrm{He}, \mathrm{H}_{2} \mathrm{O}, \mathrm{CO}_{2}, \mathrm{Cs}^{+}$, etc.). Figures 1 and 2 show clearly the structural relations among the major constituents of the beryl lattice. This peculiar lattice construction is almost unique among the minerals, for the most closely related cordierite lattice has appreciably larger structure tunnels not so well suited for the sorption of foreign internal impurities. More recently, Baier and Pense [7] have detected by an electron microscopic technique the existence of additional squelettechannels in natural beryl crystals which may also offer good sites for some "external impurities."

Natural and synthetic beryl to be analyzed had to be treated differently for the ratio of the internal impurities (predominantly located in tunnels) to the external impurities (mainly present in squelette-channels). This 


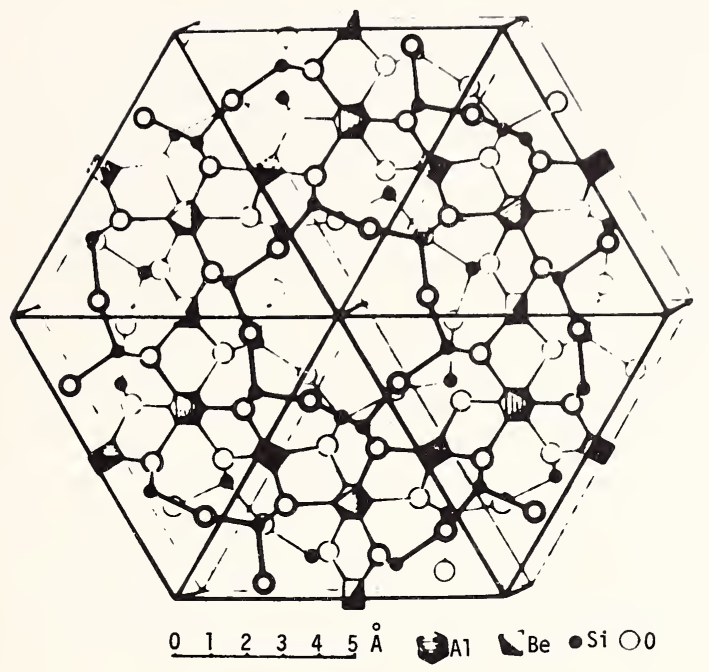

Figure 1. Mineral beryl, $\mathrm{Be}_{3} \mathrm{Al}_{2} \mathrm{Si}_{6} \mathrm{O}_{18}$, and its crystal lattice. (After Ewald U. Hermann, Structurbericht 1913 [26]).

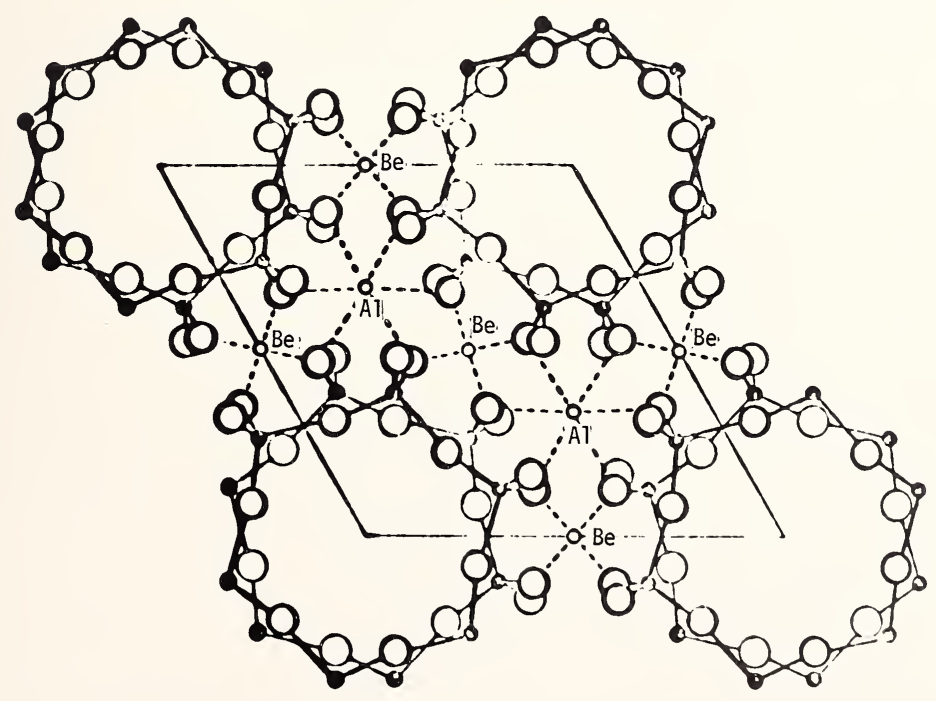

Figure 2. The structure of beryl projected on the basal plane (0001), showing the rings on the upper plane and (in fainter line) those halfway down the cell (after Bragg, 1937). 
causes a very notable effect on many important physicochemical properties but also on general chemical resistance.

The most important internal impurities in NB are alkali metals and water, having the greatest influence on their crystalochemical and other physicochemical properties. As is well established, increase in density and index of refraction accompanied by a decrease in hardness and chemical resistance, are the most pronounced effects found early by many authors $[8,9]$, and confirmed more exactly on many occasions [10,11].

As to the sites of such impurities in the beryl crystal lattice, there is still controversy among investigators. The prevailing opinion may be summarized as the so-called "cationic-block hypothesis" which suggests that the larger cations (such as $\mathrm{Cs}^{+}$) act as stoppers in the constricted parts of the beryl tunnels, the water ( $\mathrm{He}, \mathrm{Ar})$ being in their enlarged parts inside, as seen in figure 3. Some important misunderstandings persist undoubtedly, not only in connection with the sites of water molecules in tunnels $[12,13]$, and with the now well-established motion of these water molecules shown by the NMR-method [14-16] but also with the still enigmatic presence of excess $\mathrm{He}$ and $\mathrm{Ar}$ in NB-samples. The last question, posed for the first time by Lord Rayleigh [17] has been

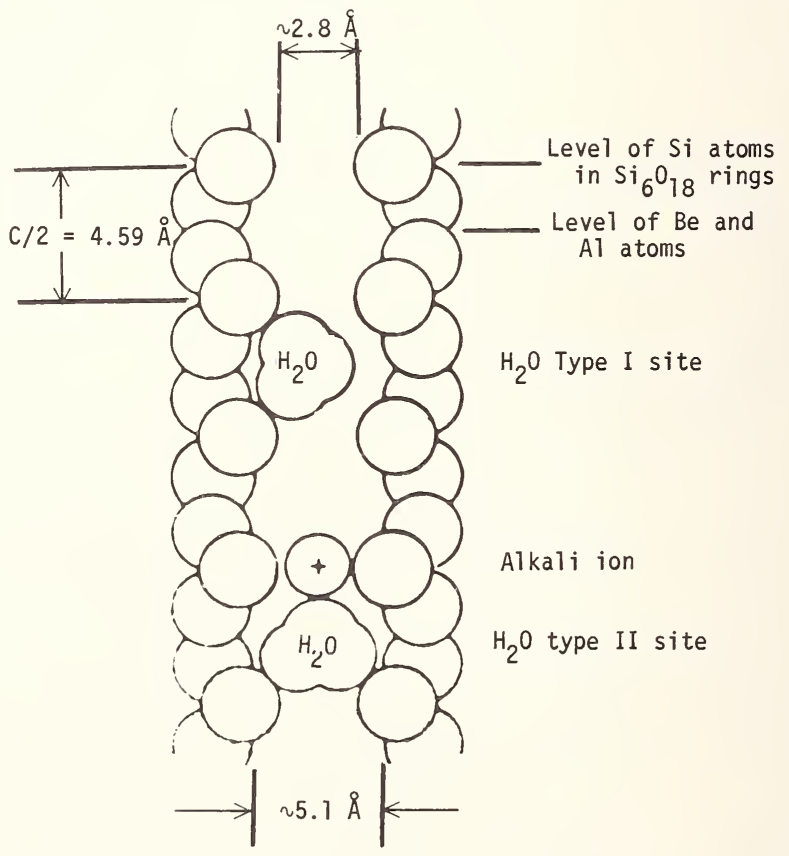

Figure 3. The positions of water molecules and larger cations in the "structure-tunnels" of the NB-crystal lattice (after Wood and Nassau, 1968). 
emphasized as an especially critical geochemical problem by the recent studies of P. E. Damon and J. L. Kulp [18] and others [19]. The final resolution of these misunderstandings and unsolved problems must be sought, in our opinion, in a much more effective utilization of the SB-samples which, surprisingly, still is to be done [18]. Synthetic beryls may be produced now in almost any size and quality (crystalline powder or larger crystals, alkali-free or alkali-rich, water-free or water-rich) so there is little doubt that one could produce even $\mathrm{He}$-rich and Ar-rich SB-specimens $[1,3]$.

To summarize, we emphasize that the alkali-rich and more "weathered" NB-samples will not pose great problems in grinding the samples for analysis, as do the much harder and chemically more resistant unweathered SB-samples.

\section{B. Choice of Methods for Beryl Analysis}

The choice of methods for beryl sample analysis must pay due attention to the aforementioned remarks, since merely grinding such samples may disturb the true constitutional relations, not only among the major and minor constituents but also, and especially among the trace constituents, which may be sometimes quite characteristic and important in many respects.

\section{The Emission Spectrographic Method}

The emission spectrographic method, applied with all due precautions, seems to be very well suited for such analysis. After some preliminary investigations on many Yugoslav NB-samples as well as SB-samples [2024 ], we found that this method gave the best results for detection and surveying the contents of all the five alkali metals, when working in the near IR-region of the spectrum $\sim 700$ to $900 \mathrm{~nm}$ ( $c$ a. 7000 to $9000 \AA)$. Such work necessitated the use of specially sensitized photoemulsions (e.g., Gevaert Scientia 52A86; Kodak IIL) and spectrographs of high resolution (Hilger E478; Steinheil GH). By using the dc arc excitation with currents up to $10 \mathrm{~A}$, and the total-burning technique of the sample in the lower cavity of a C-electrode, we have run more than 100 beryl analyses, including almost the whole collection of RB as well as many other NB and $\mathrm{SB}$. The procedure finally adopted after many trials was to crush a piece of $\mathrm{RB}$ or $\mathrm{NB}$, enveloped in filter paper, using a Plattner mortar, choosing after that a pure and representative split for pulverization in an ordinary agate mortar. This has to be done with precautions, i.e., more by a crush- 
ing than a swirling motion of the pestle to obtain the relatively small quantity needed, some 10 to $30 \mathrm{mg}$. The agate mortar was, in our experiment, not visibly damaged by such a procedure, but a "Degussit" mortar (although with a Moh's hardness of 9-synthetic corundum) showed a lesser resistance.

The spectrograms from the near IR-region for some samples of RB, NB, SB may be seen in figures 4,5 , and 6 . Three important partial conclusions are almost directly evident from them:

1: The near IR-region is the best choice for the spectrographic detection and determination of all alkali metals present, as well in NB or SBsamples.

2: All the five alkali metals are, as a rule, present in all the NB-samples (and even in SB-samples, if the purity of the materials for the synthesis was not of the spectrochemical kind).

3: The abundance of cesium in the NB-samples is generally abnormally high in comparison with other samples containing this element (e.g., plant ashes and other minerals).

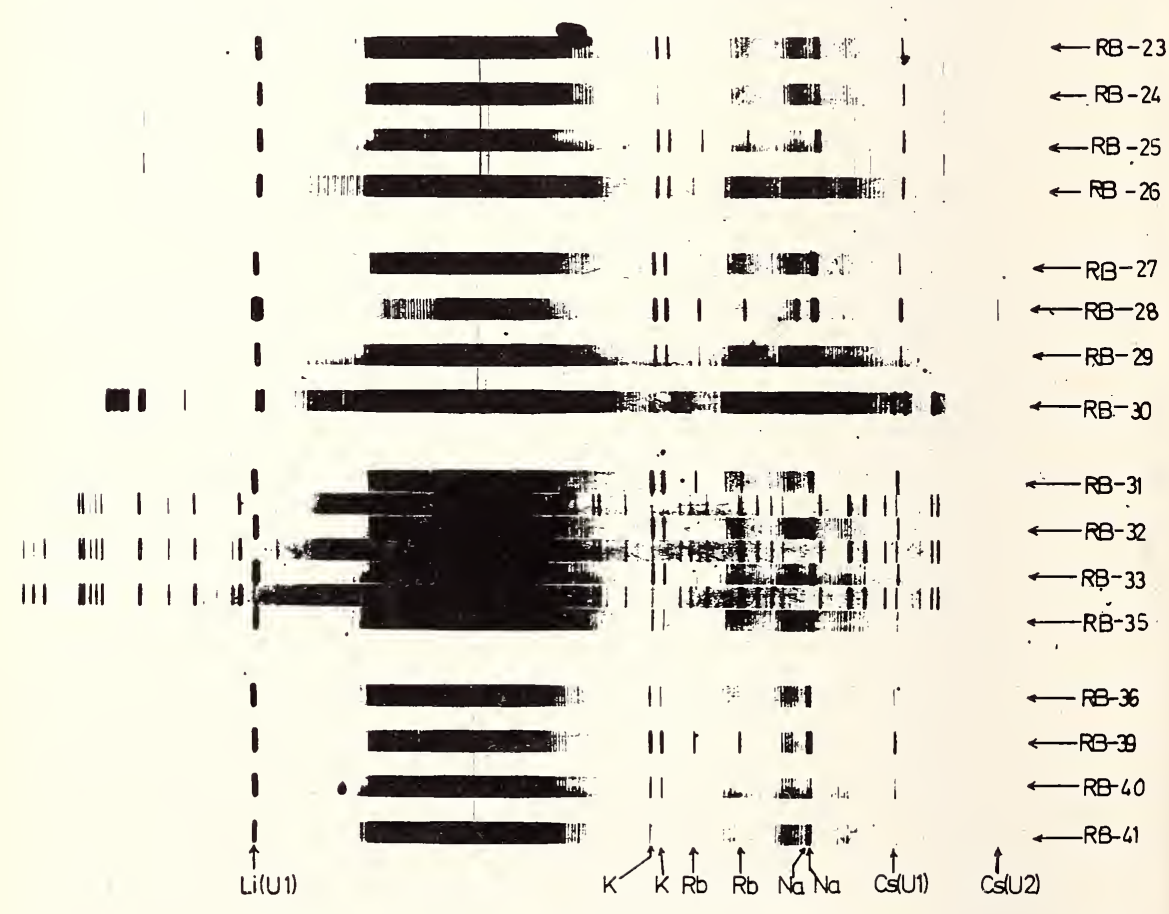

Figure 4. Near IR-emission spectrum of NB-samples: Plate XIII. Alkali metals in the RB.Nr.23-41. 
We emphasize particularly the importance of the last conclusion, for it strongly suggests some kind of selective absorption of the larger $\mathrm{Cs}^{+}$ cations in the structure tunnels of the beryl lattice. This may be taken as a strong support for the cationic-block hypothesis but also (having in view 2) some possible correlation with important although not well understood geochemical mechanisms. We will return to this question still another time, by surveying the results of the NAA.

The UV and VIS spectral region $(\sim \lambda 2350$ to $4000 \AA)$ was also taken by the same spectral instruments and procedure, but with Perutz-SilberEosin-photoemulsions. The purpose of this was to have a swift qualitative survey for the presence of transition metal ions (especially $\mathrm{Cr}^{3+}, \mathrm{Fe}^{2+}$, $\mathrm{Mn}^{2+}, \mathrm{Sc}^{3+}$ ) which are incorporated in the beryl crystal lattice by a quite different process, namely by an isomorphous substitution. Partially formulated conclusions here are:

4: There exists a characteristic constellation of the emission spectral lines and spectral bands at about $\lambda 2650 \AA$ quite specific for beryl and distinct from all other Be-minerals ( $c f .[20])$.
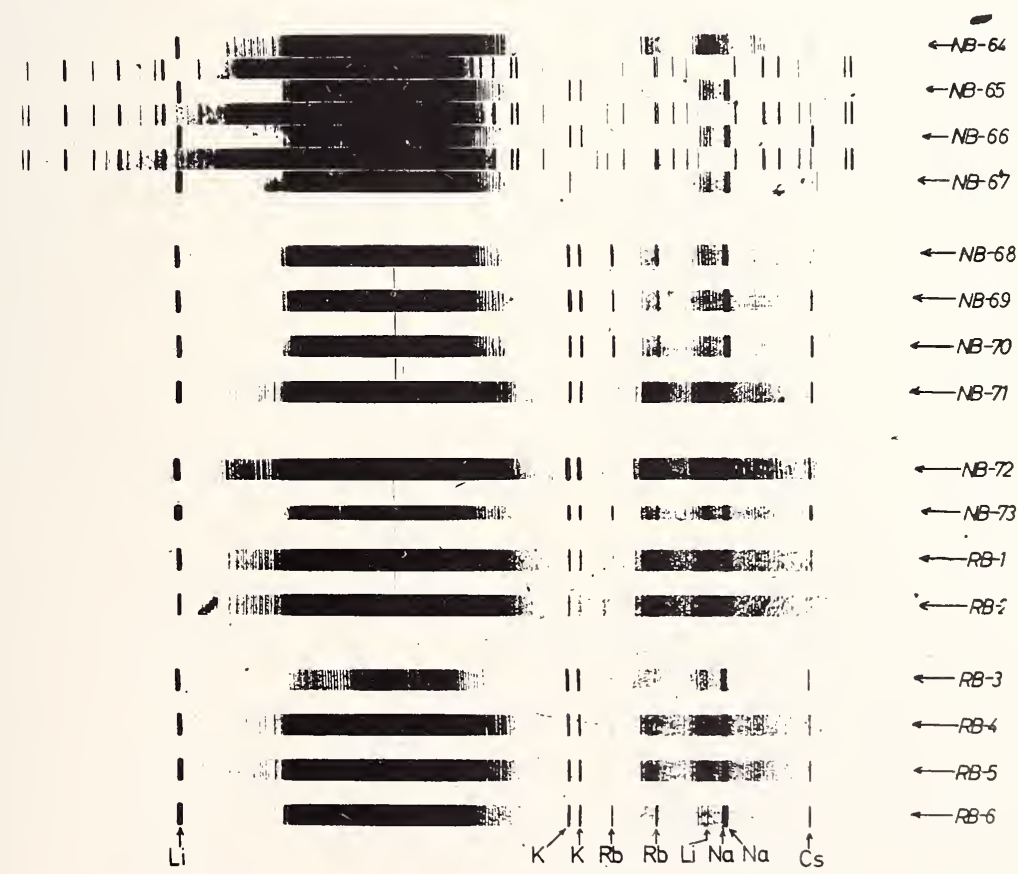

$\longleftarrow R B-5$

$\leftarrow R B-6$

Figure 5. Near IR-spectrum of NB-samples: Plate XV. Alkali metals in beryls, the first members of RB-collection, and the additionally acquired NB-samples. 
5: The presence of scandium as well as its abundance in the NB seems to be more frequent and important than it was stated by some earlier, but also by some recently made studies [2].

6: Chromophoric ions (such as $\mathrm{Cr}, \mathrm{Ni}, \mathrm{V}, \mathrm{Fe}$, and $\mathrm{Nb}$ ) are present in various amounts and in the different NB-samples, but cobalt is surely $a b$ sent from all the NB-samples investigated, which conflicts with the findings of all other investigators.

This absence of cobalt from NB-samples is unexpected from pure crystalochemical reasons, for its closest neighbors ( $\mathrm{Fe}$ and $\mathrm{Ni}$ ) in the periodic table were surely identified therein. On the other hand, in the SBsamples $\mathrm{Co}$ may be easily introduced (as well as $\mathrm{Fe}, \mathrm{Ni}$, and $\mathrm{Cr}$ ) [1] , and the rose (pink) coloration of SB, produced by this, persists without any change after an ignition over $1200^{\circ} \mathrm{C}$.

The IR-absorption spectra of $\mathrm{NB}$ and $\mathrm{SB}$ are very distinct from all other Be-minerals and also from related crystal lattices (cordierite, dioptase, milarite, ossumilite), but they could be especially useful to detect the presence of the gaseous and liquid incorporations (e.g., $\mathrm{H}_{2} \mathrm{O}$ and $\mathrm{CO}_{2}$ )as was especially emphasized recently by Wood et al. $[15,16]$. For some of our RB-samples, we not only confirmed those possibilities for a relatively easy detection of $\mathrm{H}_{2} \mathrm{O}$ and $\mathrm{CO}_{2}$ by the IR-spectra, but simplified the

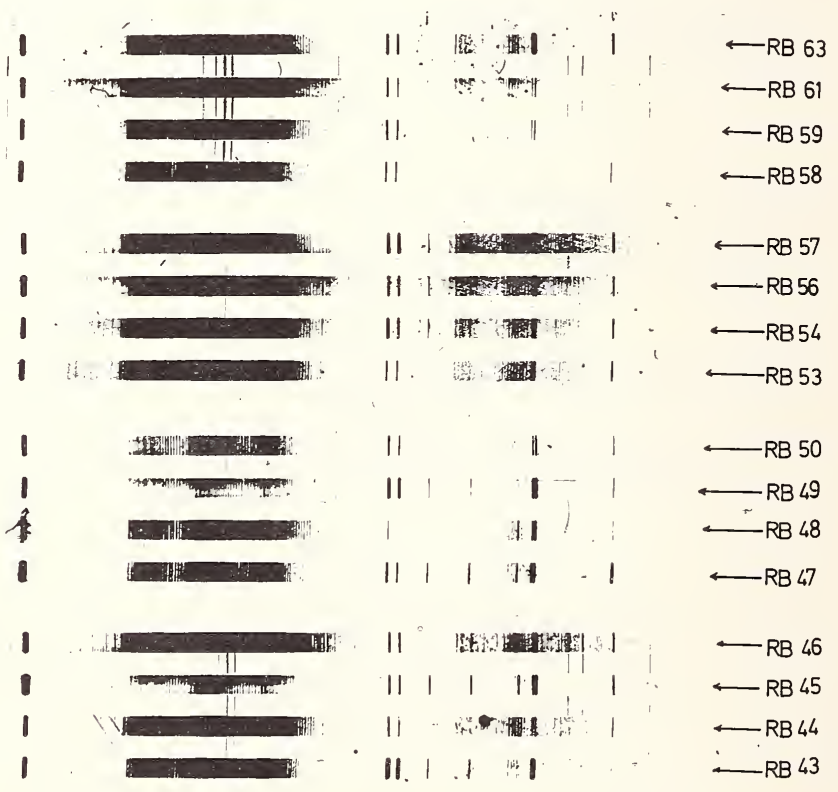

Figure 6. Near IR-emission spectrum of NB-samples: Plate XIV. Alkali metals in the RBsamples, the end of the RB-collection. 
procedure using the rock-beryl samples, instead of thin sections of quite crystalline NB. The important conclusion to be formulated here is the following:

7: The relatively simple detection of $\mathrm{H}_{2} \mathrm{O}$ and $\mathrm{CO}_{2}$-molecules in the NB-crystal lattice may be done by the absorption IR-spectra, by using the thin beryl sections (20 to $40 \mu \mathrm{m}$ ), but not the $\mathrm{KBr}$-technique or Nujolmull techniques.

\section{Instrumental Neutron Activation Analysis (NAA)}

Instrumental neutron activation analysis (NAA) is, according to our experience, the most suited method for the beryl analyses, especially for some nonstoichiometric elements present as minor or trace constituents in them (e.g., $\mathrm{Na}, \mathrm{Mn}, \mathrm{Cr}, \mathrm{Cs}, \mathrm{Sc}$, and some $\mathrm{Re}$ and $\mathrm{P}$ ), which is otherwise difficult to do by other methods. Quite surprisingly, we found very few references dealing with the applications of NAA to NB and SB-samples [22].

By a renewed occasion to work in the Radiochemical Department of the Max Planck-Institut für Chemie in Mainz, West Germany, and with kindly accorded permission to use all the laboratory facilities, especially the Nuclear Reactor-TRIGA MARK II (Director: Prof. Dr. F. Strassmann), and the Intertechnique SA40B 400-Kanal-Impulshöhenanalysator (Prof. Dr. H. Wânke of the MPI), we had the opportunity to reuse the important collection of RB as well as the other additional NB and SB-samples for an extensive study of the effectiveness of the NAA as applied to such materials. At the beginning we tried to use some slightly modified procedure which was in use in the local laboratory. The method was based on very complex chemical treatment of the substances to be analyzed. We were soon obliged to search for another procedure, less time consuming and avoiding any pretreatment which might introduce the new trace impurities in the beryl samples analyzed. After a few trials. it became clear that a purely instrumental NAA-method can be elaborated. using the sample quantities of the order of 1 gram (without any grinding and pulverizing) and reactor-irradiation times of 3 to 6 hours (the neutron flux being $7 \times 10^{-11} \mathrm{n} \cdot \mathrm{cm}^{-2} \cdot \mathrm{s}^{-1}$ ). The whole sample preparation was finally to select and weigh a representative small crystal, piece or splinter of NB or SB to be analyzed, to seal it tightly in a plastic bag. which was packed in a plastic vial with corresponding standards. All was then put into a standardized Al-container for irradiation in the TRIGA MARK IIReactor [23]. The measurements of the samples followed after intervals sufficiently long to ensure the elimination of short-lived nuclides ( $\mathrm{Na}$ and 
$\mathrm{Mn}$ ) in order to have a better precision in the measurement of the longlived nuclides here determined $(\mathrm{Cr}, \mathrm{Cs}$, and $\mathrm{Sc})$. The detection of trace amounts of Mo in all SB-samples was made through the unexpected characteristic activity of Tc. The presence of the rare-earth metals in the "pseudo-beryl" RB-30, ( $c f$. also the spectrogram of the fig. 6), was also quite unexpected. Here, through somewhat more prolonged periods of observations the half-lives were monitored, but all the measurements were still done by a purely instrumental NAA.

Some representative $\gamma$-spectra of the elements here determined in beryls by this occasion may be seen in figures 7 through 11 . We call your attention to the interference pattern between the gamma doublets of Cs and $\mathrm{Sc}(0.605,0.797 \mathrm{MeV}$ and $0.889,1.121 \mathrm{MeV}$ respectively), which was not at all too severe, since the 400 -channel analyzer used with possibilities for negative counting, facilitated very effectively the elimination of this interference.

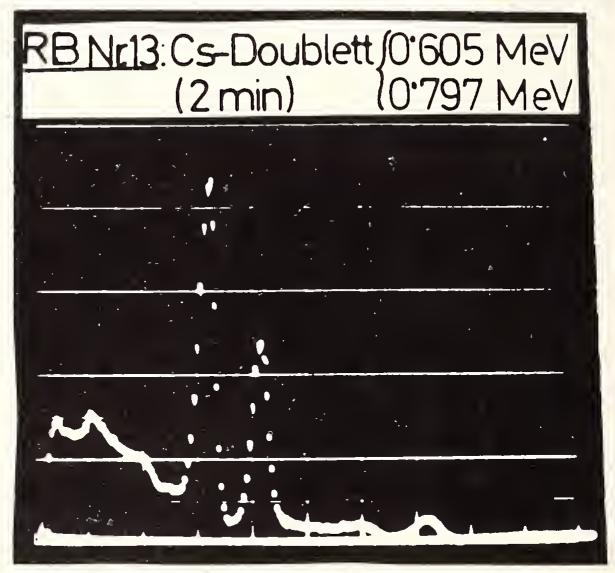

Figure 7. Cesium content in RB.Nr.13: Gamma doublet of ${ }^{134} \mathrm{Cs}$ (2 min). 


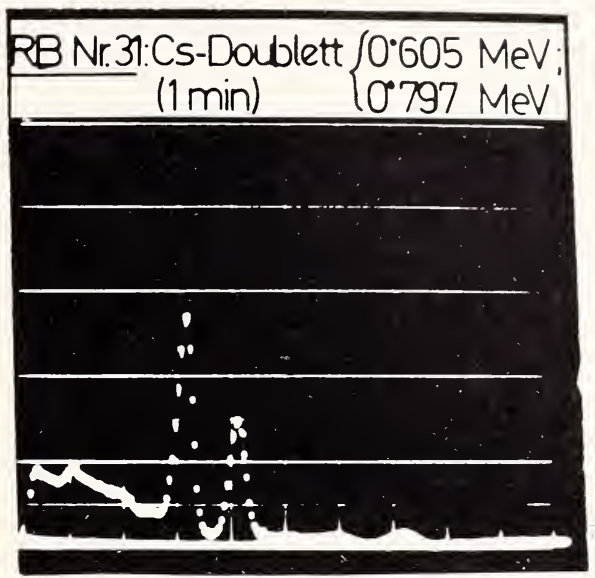

Figure 8. Cesium content in RB.Nr.31: Gamma doublet of ${ }^{134} \mathrm{Cs}$ (1 min).

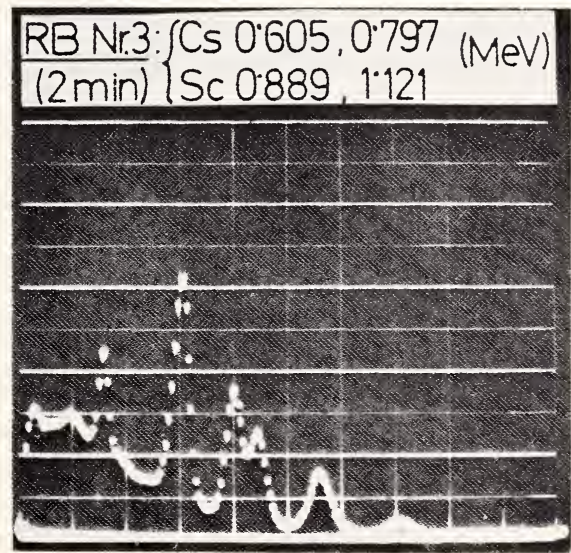

Figure 9. Cesium and scandium contents in RB.Nr.3: Interference of the low-energy component of the Cs doublet with the high-energy component of the Sc doublet (1 min). 


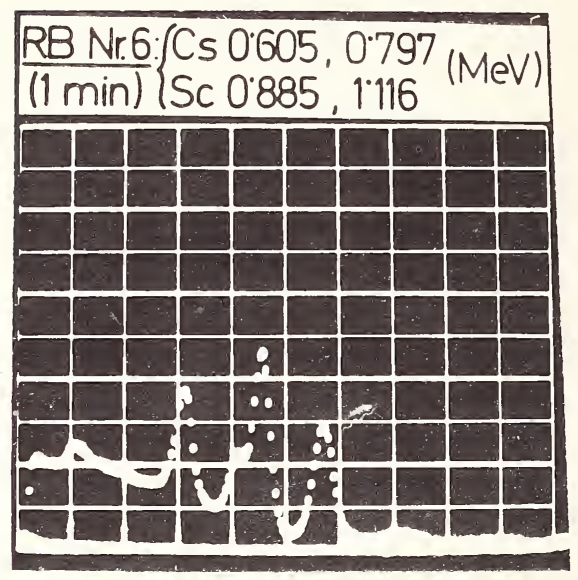

Figure 10. Scandium and cesium contents in RB.Nr.6: Interference of the low-energy component of Sc-doublet with the high-energy component of the Cs-doublet (1 $\mathrm{min}$ ).

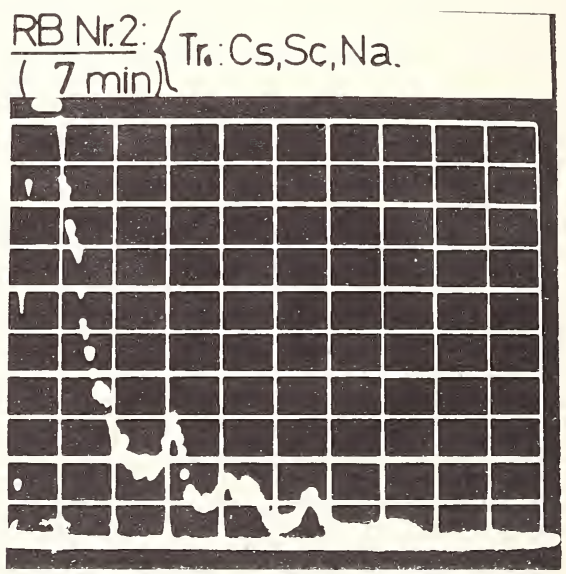

Figure 11. Traces of Cs, Sc, Na, etc. in RB.Nr.2 (7 min).

The data for the five elements readily determined by a purely instrumental NAA-method are tabulated, respecting the order of the Rayleigh's collection of beryls, but there are included also other NB and SB-samples (see table 1). In the last column of this table there is a quotient $\mathrm{Q}=$ $(\mathrm{Na} / \mathrm{Cs})$, which may be used as a criterion of the relative $\mathrm{Cs}$ and $\mathrm{Na}$ abundance, in discussing the geochemical aspects of their presence in the NB-samples. 
TABLE 1. Neutron activation analysis results of natural beryls

Sample $\begin{array}{ccc}\mathrm{Mn} & \mathrm{Na} & \mathrm{Cs} \\ (\mathrm{mg} / \mathrm{g}) & (\mathrm{mg} / \mathrm{g}) & (\mathrm{mg} / \mathrm{g})\end{array}$
Sc (mg/g) $\begin{array}{cc}\mathrm{Cr} & \mathrm{As} \\ (\mathrm{mg} / \mathrm{g}) & (\mathrm{mg} / \mathrm{g})\end{array} \quad Q=\frac{\mathrm{Na}}{\mathrm{Cs}}$

\begin{tabular}{|c|c|c|c|c|c|c|c|}
\hline 1. & 0.059 & 2.310 & 0.368 & 0.006 & $-{ }^{a}$ & - & 6.28 \\
\hline 2. & .011 & 1.186 & .013 & $<.002$ & - & - & 91.23 \\
\hline 3. & .013 & 9.370 & $0.74-117$ & $0.03-0.08$ & $12-18$ & - & $\begin{array}{r}12.00 \\
8.01\end{array}$ \\
\hline 4. & .190 & 5.603 & 0.188 & 0.005 & - & - & 29.80 \\
\hline 5. & .151 & 2.487 & .595 & .005 & - & - & 4.18 \\
\hline 6. & .798 & 10.051 & 1.142 & .577 & - & - & 8.80 \\
\hline 7. & .022 & 1.945 & 0.097 & $<.002$ & - & - & 20.05 \\
\hline 8. & .640 & 4.349 & .077 & .003 & - & - & 56.48 \\
\hline 9. & .0432 & 2.574 & .057 & .002 & - & - & 45.16 \\
\hline 10. & .0652 & 5.293 & .931 & .009 & - & - & 5.68 \\
\hline 11. & .0362 & 3.842 & .316 & .058 & - & - & 12.16 \\
\hline 12. & .034 & 4.257 & .949 & .008 & - & - & 4.49 \\
\hline 13. & .080 & 0.579 & 2.535 & .016 & - & - & 0.23 \\
\hline 14. & .014 & 1.86 & 0.116 & 0.003 & - & - & 16.03 \\
\hline 15. & .016 & 2.955 & .980 & - & - & - & 3.02 \\
\hline 16. & .0232 & 4.194 & .0935 & 0.0576 & - & - & 44.62 \\
\hline 17. & .036 & 1.63 & .185 & .004 & - & - & 8.81 \\
\hline 18. & .024 & 1.82 & .369 & .010 & - & - & 4.93 \\
\hline 19. & .044 & 1.89 & .318 & .009 & - & - & 5.94 \\
\hline 20. & .024 & 1.61 & .425 & $<.002$ & - & - & 3.79 \\
\hline 21. & .019 & 1.28 & .161 & $<.002$ & - & - & 7.95 \\
\hline 22. & .0153 & 1.983 & .029 & .006 & - & - & 68.38 \\
\hline 23. & .039 & 3.015 & .279 & .007 & - & - & 10.81 \\
\hline 24. & .026 & 1.352 & .603 & .003 & - & - & 2.24 \\
\hline 25. & .153 & 5.420 & .842 & .032 & - & - & 6.44 \\
\hline 26. & .036 & 1.785 & .386 & $<.004$ & - & - & 4.62 \\
\hline 27. & .018 & 11.727 & .078 & .167 & 0.238 & - & 150.35 \\
\hline \multirow[t]{2}{*}{28.} & .005 & 13.37 & 9.98 & .167 & - & - & 1.54 \\
\hline & & 11.21 & 8.33 & & & & 1.35 \\
\hline 29. & .035 & 1.940 & 0.266 & $<.002$ & - & - & 7.29 \\
\hline 30. & .067 & 2.801 & 3.590 & - & - & - & 0.78 \\
\hline 31. & .049 & 3.884 & 0.980 & .007 & - & - & 3.96 \\
\hline 32. & .091 & 5.474 & .502 & .003 & - & - & 10.90 \\
\hline 33. & .020 & 2.19 & .235 & .011 & - & - & 9.32 \\
\hline 34. & .016 & 3.202 & .270 & .004 & - & - & 11.86 \\
\hline 35. & .071 & 3.96 & 2.386 & 2.223 & - & - & 1.66 \\
\hline 36. & .032 & 2.14 & 0.454 & $<0.002$ & - & - & 4.71 \\
\hline 37. & .006 & 2.98 & .016 & .040 & 0.570 & - & 186.25 \\
\hline 38. & .068 & 1.868 & .141 & .002 & - & - & 13.25 \\
\hline 39. & .112 & 2.341 & .572 & .106 & - & - & 4.09 \\
\hline 40. & .048 & 10.97 & 2.523 & - & - & - & 4.35 \\
\hline 41. & .056 & 5.192 & 0.737 & .090 & - & - & 7.04 \\
\hline 42. & .011 & 6.702 & 9.201 & - & - & - & 0.73 \\
\hline 43. & .015 & 5.586 & 0.317 & .005 & - & - & 17.62 \\
\hline 44. & .052 & 11.676 & .345 & .457 & - & - & 33.84 \\
\hline 45. & .035 & 3.518 & .476 & .008 & - & - & 7.39 \\
\hline 46. & .097 & 2.110 & .781 & .097 & - & - & 2.70 \\
\hline
\end{tabular}


TABLE 1. Neutron activation analysis results of natural beryls-Continued

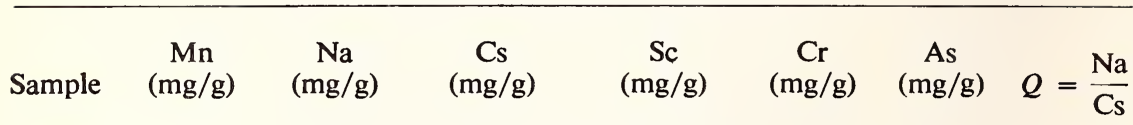

\begin{tabular}{|c|c|c|c|c|c|c|c|}
\hline 47. & 0.092 & 2.76 & 0.931 & 0.136 & 一 & - & 2.96 \\
\hline 48. & .032 & 4.448 & .685 & .004 & - & - & 6.49 \\
\hline \multirow[t]{2}{*}{49.} & .121 & 2.064 & 2.691 & .023 & - & - & 0.77 \\
\hline & & 2.392 & 2.961 & & & & 0.81 \\
\hline 50. & .008 & 0.089 & 0.005 & .006 & 一 & 一 & 17.8 \\
\hline 51. & .034 & 1.172 & .323 & .375 & - & - & 3.63 \\
\hline \multirow{2}{*}{52.} & .012 & 0.501 & .407 & .018 & $\mathrm{Tpaz}^{\mathrm{b}}$ & 2.248 & 1.23 \\
\hline & .025 & .712 & .589 & .027 & & & $(1.21)$ \\
\hline 53. & .149 & 5.230 & 1.413 & .008 & 0.097 & 一 & 3.70 \\
\hline \multirow{2}{*}{54.} & .0365 & 4.253 & 0.241 & .003 & - & - & 17.65 \\
\hline & .0324 & 4.378 & .726 & .008 & - & - & 6.03 \\
\hline 55. & .0116 & 1.924 & 194 & .002 & 一 & - & 9.92 \\
\hline 56. & .0496 & 2.849 & .214 & .002 & 一 & - & 13.31 \\
\hline 57. & .340 & 3.98 & 1.300 & .223 & 0.110 & 一 & 3.06 \\
\hline 58. & .0312 & 1.784 & 1.603 & - & - & 一 & 1.11 \\
\hline 59. & .0484 & 3.502 & 0.466 & .003 & - & - & 7.52 \\
\hline \multirow[t]{2}{*}{60.} & .0144 & 7.20 & 8.948 & - & - & - & 0.805 \\
\hline & .026 & 4.114 & 0.204 & Tpaż & Tpaż & - & 20.17 \\
\hline \multirow[t]{2}{*}{61.} & .030 & 3.816 & .240 & 0.006 & - & - & 15.9 \\
\hline & .041 & 1.665 & .161 & Tpaż & Tpaż & 一 & 10.34 \\
\hline 62. & .094 & 3.492 & 1.050 & Tpaż & 一 & 一 & 3.33 \\
\hline 63. & .0353 & 5.329 & 0.327 & 0.009 & 一 & 一 & 16.30 \\
\hline 64. & .0841 & 1.536 & .538 & Tpaż & Tpaż & - & 2.86 \\
\hline \multirow[t]{2}{*}{65.} & .211 & 6.501 & 1.366 & 0.014 & - & - & 4.76 \\
\hline & .137 & 6.835 & 1.276 & .011 & 一 & 一 & 5.36 \\
\hline 66. & .146 & 8.608 & 1.018 & .094 & 1.521 & - & 8.46 \\
\hline 67. & .0036 & 3.776 & 0.025 & .053 & 1.853 & 一 & 151.04 \\
\hline 68. & .0013 & 0.863 & .0139 & .0108 & 0.290 & 一 & 62.09 \\
\hline \multirow[t]{2}{*}{69.} & .0149 & 10.234 & .736 & .0396 & 1.060 & 一 & 13.90 \\
\hline & .0366 & 5.786 & .519 & .119 & 1.548 & - & 11.15 \\
\hline 70. & .0960 & 6.713 & .231 & - & - & - & 29.06 \\
\hline 71. & .103 & 8.572 & 2.800 & - & 一 & 一 & 3.06 \\
\hline 72. & .0287 & 7.936 & 6.315 & 一 & 一 & 一 & 1.26 \\
\hline 73. & .137 & 2.042 & 1.096 & 0.0205 & 一 & - & 1.86 \\
\hline 74. & .0822 & 2.334 & 1.755 & .128 & - & - & 1.33 \\
\hline \multirow[t]{2}{*}{75.} & .0689 & 18.821 & 0.155 & .650 & 1.648 & - & 121.43 \\
\hline & .0833 & 17.618 & .181 & .664 & 1.841 & 一 & 97.34 \\
\hline 76. & .006 & 0.172 & .180 & .005 & - & - & 0.96 \\
\hline 77. & .0106 & .820 & .050 & .009 & - & - & 16.40 \\
\hline 78. & .0082 & .401 & .308 & .038 & - & - & 1.30 \\
\hline
\end{tabular}

a $-=$ not detected.

${ }^{\mathrm{b}} \mathrm{T}$ paz $=$ traces.

For the instrumental NAA method, as applied here on beryls, let us formulate also some partial conclusions:

8: The avoidance of a contamination with external impurities is almost ideal, and in the case of such materials as beryls, this is to be emphasized as a real advantage over most other physicochemical methods of analysis. 
9: The relative speed and specificity (well recognized for all spectral methods, in general), combined with an economy in materials consumed, constitutes still another virtue of such NAA.

10: The synthetic beryl samples which allow incorporating a large number of various elements in the crystal lattice (by both aforementioned ways) give a good promise to produce quite good quality standard samples and also to develop some new technical procedures for gas-absorption and liquid-inclusions studies in beryls ( $\mathrm{He}, \mathrm{Ar}, \mathrm{CO}_{2}, \mathrm{H}_{2} \mathrm{O}$, etc.).

\section{Discussion and General Conclusion}

Beryl as an analytical material poses some special problems. Its hardness ( 7,5 Mohs scale) suggests that one should avoid any unnecessary grinding of large quantities. The presence of the open structure tunnels and megascopical to microscopical channel-pore systems in NB-samples should also not be underestimated, since all efforts for better precision and accuracy may be badly compromised by uncontrolled contaminations with the internal as well as external impurities of many kinds.

According to our experience, briefly summarized in the partial conclusions 1 to 10 , the best analytical procedure for an analysis of beryls (both NB and SB) may be achieved through an appropriate combination of spectroanalytical methods (emissions spectrographic analysis in near IR 1 to 6; IR, 7; also NMR, EPR, MS) with instrumental NAA (8 to 10). In the former methods the most serious defect is the danger of contamination. In the latter method, the danger of contamination by physical or chemical pretreatment is not only easy to avoid, but the possibilities for an improvement of this method, both in view of a higher resolution as well as in the direction of improved sensitivity (by more advanced electronics) seems quite realistic.

In ending this communication, I call attention to the graphical representation of the results given for the five metals determined in NB-samples comparatively shown with He-distribution curve for the RB-samples (as found by Rayleigh [17] (see fig. 12, the complete list of the RB- being given in the table in ref. [1]).

As is evident (fig. 12), there is no simple correlation between the $\mathrm{He}-$ contents of the RB and any of the five metals here determined (Na. Cs. $\mathrm{Mn}, \mathrm{Cr}$, and Sc). But, following a suggestion of M. H. Staatz et al. [24] to use the value $\mathrm{Q}=(\mathrm{Na} / \mathrm{Cs}$ ) ( $c f$. the last column of our table 1$)$ for the characterization of the environment wherein the NB originated. one finds that all the RB-samples may be classified into four provisional groups ac- 


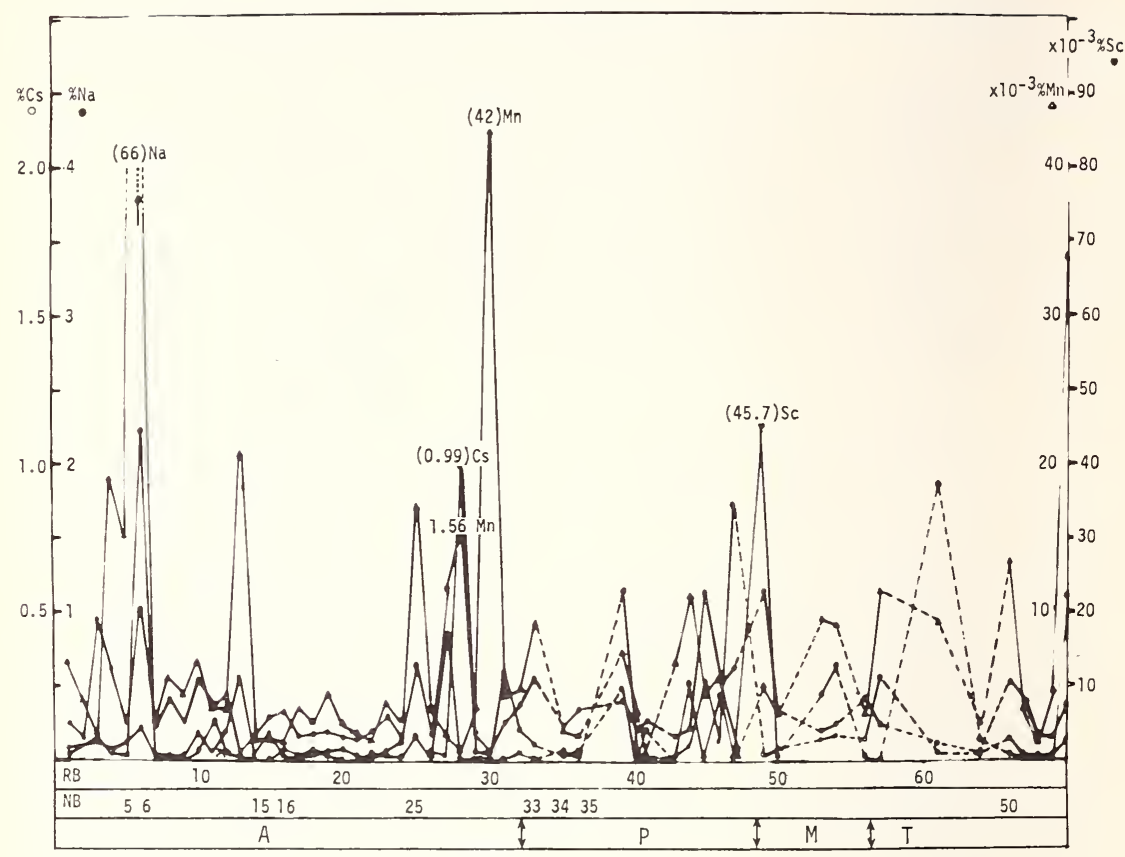

Figure 12. A view on the distribution curves of the abundances for the five elements: $\mathrm{Na}$, $\mathrm{Cs}, \mathrm{Mn}, \mathrm{Cr}$, and Sc in the Archaic (A), Paleozoic (P), Mesozoic (M), and Tertiar (T) RBsamples.

cording to their $Q$-value. The first group $(\mathrm{Q}=0$ to 1$)$ the third $(\mathrm{Q}=20$ to $50)$, the fourth $(Q=>50)$ and very sparsely represented, while the second group is abundant $(\mathrm{Q}=1$ to $20: 50$ samples from a total of 66$)$. If one takes into account the values $Q$ as given in [24] for the pegmatitic $\mathrm{NB}(\mathrm{Q}=0.33$ to 3.0$)$, for wallrock adjacent $\mathrm{NB}(\mathrm{Q}=1.33$ to 2.5$)$, for vein $\mathrm{NB}(\mathrm{Q}=0.67$ to 2.33$)$, for granitic $\mathrm{NB}(\mathrm{Q}=0.75$ to 5.0$)$ and for rhyolitic NB $(Q \leqslant 0.33)$ - one is not surprised by the aforementioned absence of any correlation: the RB-samples originate from very different geological and geochemical environments. In this sense, the recent investigations of some Russian authors to define roentgenographically the variations of the NB-crystal lattice with special influences of the nonstoichiometric constituents introduced in that lattice seems quite important (the so-called t-, o-, n-beryls of Bakakin, Rilov, and Belov) [25].

As to our final opinion, as said above and also by Damon and Kulp [18], it will be necessary to use more effectively the SB-samples, for we may now control all the "environmental conditions" governing the incorporation processes for both "internal" and "external" impurities into the beryl crystal lattice. 


\section{Acknowledgements}

The research reported here was performed partially with the support of the Institute for Chemistry, Technology and Metallurgy (Belgrade) as well as the Institute of Physical Chemistry, University of Belgrade (Yugoslavia).

Acknowledgements and special gratitude is due for hospitality and many laboratory facilities of Max-Planck-Institut für Chemie (Mainz) and Professors Drs. F. Strassmann, and H. Wânke (Johannes-Gutenberg Universität zu Mainz).

\section{References}

[1] Ristic, S., Physikalisch-chemische Untersuchungen an Beryllen im Zusammenhang mit ihrem He-Gehalt, Mainz, 1956 (Doktor-Diss.)

[2] Buchi, S., Studies on Natural History of Beryl, (Ph. D. thesis) University of Michigan (1962).

[3] Ostertag, G., Heliumafnahme in Beryll, Darmstadt, 1966 (Doktor-Diss.).

[4] Beus, A. A. and Dikov, Yu. P., Geohimiya Be v processah endogenogo mineraloobrazovanya, Moskva, 1967 (izd. "Nedra").

[5] Everest, D. A., The Chemistry of Beryllium, Elsvier, Amsterdam-London (1964).

[6] Kamenetzki, V. A., Priroda Strukturnih Primesei v Mineralah, Leningrad, 1964 (Izd. Leningr. Univerziteta).

[7] Baier, E. and Penze, J., Die Verschiedenen Kanalsysteme des Berylls, Chemie der Erde Bd. 22, 18-30 (1962).

[8] Ford, W. E., The Effect of the Presence of Alkalies in Beryl on its Optical Properties, Amer.J.Sci. 30, 128-130 (1910).

[9] Deer, W. A., Howie, R. A., and Zussman, J., Rock Forming Minerals, Vol. 1. Orthoand Ring-silicates, Longmans, Green, London (1965).

[10] Sosedko, T. A., Izmenenie Strukturi i Svoistv v Berylah s Poviseniem soderzanya Shcelocei, Zapiski Vses. Miner. Ob-va,86, 495 (1957).

[11] Feklitshev, V. T., O himitsheskom sostave mineralov grupi berila, Geohimya, 391 401 (1963).

[12] Vorma, A., Sahama, T. G., and Haapala, J., Alkali Position in the Beryl Structure, C. R. Soc. Geol. Finland 37, 119-124 (1965).

[13] Bakakin, V. V. and Bellov, N. V., Kristalohimiya berila, Geohimya, No. 5, 420-433 (1962).

[14] Wickersheim, K. A. and Buchanan, R. A., The Near IR Spectrum of Beryl. Amer. Mineralogist 44, 440-444 (1959).

[15] Wood, D. L. and Nassau, K., IR Spectra of Foreign Molecules in Beryl, J. Chem. Phys. 47,2220-2228 (1968).

[16] Wood, D. L. and Nassau, K., The Characterization of Beryl and Emerald by Visible and IR Absorption Spectroscopy, Amer. Mineralogist 53, 777-800 (1968); ib. 53, 347 (1968). 
Boutin, H., Safford, G. J., and Dinner, H. J., Low Frequency Motions of $\mathbf{H}_{2} \mathrm{O}$ Molecules in Crystals, J. Chem. Phys. 40, 2670 (1964).

[17] Lord Rayleigh (J. Strutt), The He Contained in Beryls of Different Geologic Age, Proc. Roy. Soc. (London) A142, 370-381 (1933).

Strutt, R. J., He and Radioactivity in Rare and Common Minerals, Proc. Roy. Soc. (London) A80, 572-594 (1908).

[18] Damon, P. E. and Kulp, J. L., Excess Helium and Argon in Beryl and Other Minerals, Amer. Mineralogist 43, Nos. 5-6, 433-459 (1958).

[19] Morozova, I. M., Gerling, E. K., Spinscon, V. D., Difuziya inertnih gazov iz berilov i kordieritov, etc., Geohimya, No. 10, (1969).

Ginzburg, A. I. and Panteleev, A. I., K probleme izbitocenogo argona v berilah, Geohimya, No. 10, 1118-1227 (1971).

[20] Ristic, S. et al., Alkali Metals Contents in Some Yugoslav Beryls, Proc. of the First Symposium on Geochemistry, Beograd, 1965, Geological Society of Serbia, pp. 409431.

[21] Ristic, S. et al., Influence of Foreign Incorporations on the Spectrochemical and Crystalochemical Properties of Beryl, Glasnik Shdrustva, Vol. 32, pp. 273-284 (1967).

[22] Cabell, M. J. and Smales, A. A., The Determination of Rb and Cx in Rocks, Minerals, and Meteorites by NAA, Analyst 82, 390 (1957).

Bock-Werthmann, W. and Schulze, U. W., Activation Analysis (Bibliography). AED-C-14-1; -14-02; -14-03 (1961-1964). Atomenergie-Dokumentation beim Gmelin-Institut, Frankfurt/M (West Germany).

[23] Morrison, G. H., Ed., Trace Analysis-Physical Methods, Interscience, New YorkLondon (1965).

[24] Staatz, M. H., Griffitts, W. R., and Barnett, P. R., Differences in the Minor Elements Composition of Beryl in Different Environments, American Mineralogist 50, 1783-1795 (1965).

[25] Bakakin, V. V., Rylov, G. M., and Belov, N. V., Okorelacii himitsheskogo sostava i parametrov elementarnoi yatsheiki berilov, Doklady Akad. Nauk 173, 1404-1407 (1967).

Bakakin, V. V., Rylov, G. M., and Belov, N. V., Rentgenografitsheskaya diygnostika izomorfnih raznovidnostei berila, Geohimya, No. 11, pp. 1302-1311 (1970). 


\title{
CONTROL OF SAMPLE CONFIGURATION AS AN AID TO ACCURACY IN INSTRUMENTAL NEUTRON ACTIVATION ANALYSIS
}

\author{
Robert E. Heft
}

\author{
Biomedical Division \\ Lawrence Livermore Laboratory \\ University of California \\ Livermore, California 94550 U.S.A.
}

\begin{abstract}
Errors associated with the basic calculation common to all instrumental neutron activation analyses are discussed. The sample thickness of particulate or powdered samples contained in vials or cups is likely to be nonuniform and difficult to measure. To improve the accuracy of the counting efficiency determination, a procedure was developed for potting the samples in polyethylene to form cylindrical disks of polyethylene completely containing the sample material, uniformly distributed throughout the volume of the disk. Typically, 100 milligrams of sample is intimately mixed with 1 gram of polyethylene powder by gentle grinding in an agate mortar. The mixture is placed in a 2.54 $\mathrm{cm}$ diameter polycarbonate cylindrical cup and heated to $150^{\circ} \mathrm{C}$ in a vacuum oven with a $3.81 \mathrm{~cm}$ long polycarbonate cylindrical rod of just under $2.54 \mathrm{~cm}$ diameter, in place, on top of the sample. At $150^{\circ} \mathrm{C}$ the polyethylene melts and forms a highly viscous fluid which upon cooling forms a disk of fixed diameter with parallel faces and whose thickness is measurable to 0.05 millimeter. Blank corrections for the polyethylene are found to be small, and the disks serve as secure containers for the particulates during and after irradiation: and as counting samples of precise configuration for which counting efficiency can be accurately determined.
\end{abstract}

Keywords: Gamma counting efficiency; germanium detectors; instrumental neutron activation analysis; párticulate sample geometry: trace elements in polyethylene powder.

A basic calculation common to all instrumental neutron activation analyses is the comparison of the number of photons per minute of a certain energy emitted by an activated sample with the number of photons per minute emitted by an activated reference standard. Thus, using ${ }^{60} \mathrm{Co}$ 
photons as an example and after making appropriate corrections for radioactive decay and relative neutron flux, we may write:

$\frac{\text { Micrograms cobalt }}{\text { Gram sample }}=\frac{\frac{1332 \mathrm{keV} \text { photons } / \text { minute }}{\text { Gram sample }}}{\frac{1332 \mathrm{keV} \text { photons/minute }}{\text { Microgram cobalt in Reference Standard }}}$

In order to arrive at photon emission rates, the counts per minute of the $1332 \mathrm{keV}$ photopeaks are determined by spectral analysis and the decay corrected counts per minute values are divided by appropriate counting efficiencies to produce photon emission rates.

In the simplest sense, counting efficiency is simply the ratio of photons detected to photons emitted. For $\mathrm{Ge}(\mathrm{Li})$ detectors, counting efficiencies are usually quite small and are observed to vary with photon energy, distance of the counting sample from the detector, diameter and thickness of the sample, mass of the sample and average atomic number of the sample. If all these quantities are the same for the sample and the reference standard then the counting efficiencies for the two are equal and do not affect the determination of the photons/minute ratio indicated above. However, in the general case the physical dimensions of the sample and the standard are not the same and hence, the above quantities are not the same so that the relative counting efficiencies of the standard and sample must be known to correctly calculate the stable element amounts. In the following paragraphs, the relationship of counting efficiency to sample dimensions and location is described and a method of minimizing uncertainty in counting efficiency by controlling sample configuration is given. The treatment of the counting efficiency dependence on sample/detector geometry which is given below is taken from GAMANAL, a program which provides a complete qualitative and quantitative analysis of mixtures of radioactive species by computer interpretation of high resolution gamma-ray spectra. The GAMANAL program was developed by $R$. Gunnink and J. B. Niday of Lawrence Livermore Laboratory and is completely described in a four-volume report [1].

In the GAMANAL program the total counting efficiency is treated as the product of two components - an intrinsic detector efficiency $\epsilon$ and a geometry factor $G$. For a point source and point detector, the factor $G$ is simply the inverse square of the source to detector distance $d$.

$$
\epsilon_{E}=\frac{(\mathrm{cpm})_{E}}{(\gamma \mathrm{pm})_{E}} \times d^{2}
$$

where $(\mathrm{cpnl})_{E}=$ observed counts/minute in photopeak of energy $E$ and $(\gamma \mathrm{pm})_{E}=$ emission ratio of photons of energy $E$. 
For a point source with a detector of finite size the above representation of the efficiency can still be used providing a value of $d$ is used which correctly represents the average penetration of the photons into the detector. Since the cross section for interaction of the photons with the germanium diode is energy dependent, the value of $d$ must reflect this dependence. A schematic illustration of a point source interacting with a cylindrical detector is shown in figure 1. From simple geometry:

$$
d^{2}=(x+w+p)^{2}+r^{2}
$$

where:

$x=$ distance from source to detector window

$w=$ distance from window to active detector surface

$p=$ effective penetration of gamma ray into detector

$r=$ effective radius of interaction

$d=$ distance from source to effective zone of interaction

Of the above, $w, p$, and $r$ are system parameters. $p$ is a function of detector thickness and photon energy; $r$ is a function of detector radius $\left(R_{D}\right)$, detector thickness $(T)$, and photon energy; and $w$ is fixed for a given system. Thus, the uncertainty in the geometry factor depends on the uncertainty in the source to detector window distance $x$.

The foregoing discussion applies to a point source. For a distributed source of radius $R_{S}$ and thickness $2 l$ and where $l$ is small compared to the source to detector distance, the GAMANAL model uses the following relationship to describe an equivalent point source:

$$
G=\left(\frac{d^{2}}{d^{2}+R_{S}{ }^{2} / 2}\right) e^{\mu l}\left(\frac{d^{2}-l^{2}}{d^{2}}\right) G_{p}
$$

In this equation:

$G=$ geometry factor for source of radius $R_{S}$ and thickness $2 l$

$G_{p}=d^{2}$, the geometry factor for a point source

$\mu=$ absorption coefficient for source material

The first term reduces the disc distribution to an equivalent ring of radius $R / \sqrt{2}$ the second and third terms reduce the source of thickness $2 l$ to an equivalent linear source at the midplane of the sample. The calculation of photon emission rate from counts per minute in a photopeak of energy, $E$, is given by:

$$
(\gamma \mathrm{pm})_{E}=\frac{(\mathrm{cpm})_{E}}{\epsilon_{E}}\left[\frac{d^{2}-l^{2}}{d^{2}+R_{S}{ }^{2} / 2}\right] d^{2} e^{\mu l}
$$

This relationship applies to the general case of coaxial cylindrical source and detector configuration and has been presented in order to establish the method used to determine the sensitivity of photon emission rate esti- 


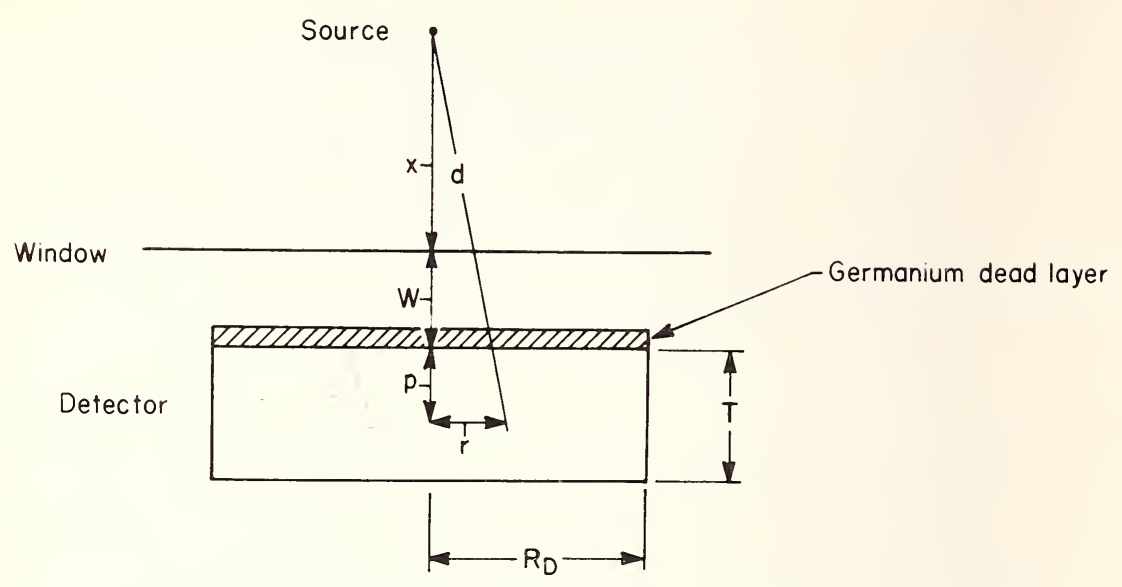

Figure 1. Schematic illustration of a point source and a cylindrical detector.

mates to variation in the geometrical parameters. A more detailed discussion of the several relationships indicated above is available in the GAMANAL report.

For the purpose of this calculation we will assume the sample to be in the form of a right circular cylinder whose radius $R_{S}$ is fixed by the wall of a cylindrical sample holder of known base thickness $b$. The sample holder is supported by a shelf whose distance from the detector window $H$ is precisely known. The half thickness of the sample is $l$ and it is the sensitivity of photon emission rate estimate to uncertainty in $l$ that we wish to determine. The dependence of $d^{2}$ on source to detector window distance $x$ is shown in equation (2); $x$ is simply the sum of $H+b+l$. The remaining quantities in equation (2) are system parameters determined by calibration experiments and are taken to be precisely known. The sensitivity as a function of variation in $l$ is summarized in table 1 . The values used for the fixed parameters are included in the table.

It is clear from the values in table 1 that the sensitivity of the photon per minute determination to sample height uncertainty is significantly reduced by moving the sample away from the detector. However, when this is done, much higher activity levels are required in order to produce the same number of photopeak counts in the spectrum. The highest counting efficiency is obtained when the sample is counted closest to the diode where the sensitivity to height uncertainty is greatest. In order to keep the photons/minute error due to height uncertainty below 1 percent at or close to the detector window, the sample height uncertainty must be kept below $0.1 \mathrm{~mm}$. 
TABLE 1. Sensitivity of derived photon per minute values to uncertainty in height of sample midplane

Sample midplane to window distance $(\mathrm{cm})$
$\%$ Error in photons/min $\mathrm{mm}$ error in distance
Relative activity level for equal cpm observed
0.16
9.0
1.0
1.41
6.9
2.6
3.41
4.7
7.1
6.91
3.0
20.6
13.93
1.9
69.8

Parameters: Detector radius $2.23 \mathrm{~cm}$.

Sample radius $1.375 \mathrm{~cm}$.

Detector surface to wind

Photon energy $662 \mathrm{keV}$.
Thickness $1.3 \mathrm{~cm}$.

Thickness $0.2 \mathrm{~cm}$.

$0.77 \mathrm{~cm}$.

Determination of sample height to within this limit is difficult mainly for particulate samples of small mass (i.e., less than 1 gram). In order to obtain precise sample geometry for such samples we have developed a technique for potting the samples in polyethylene disks. The method used is to prepare an intimate mixture of particulate sample material and polyethylene powder by grinding in an agate mortar. (The mass ratio of polyethylene to sample can be anything greater than about 1.5 to 1.) The mixture is introduced into a polycarbonate counting cup shown in figure 2. The polycarbonate cylinder is placed on top of the mixture and the as-
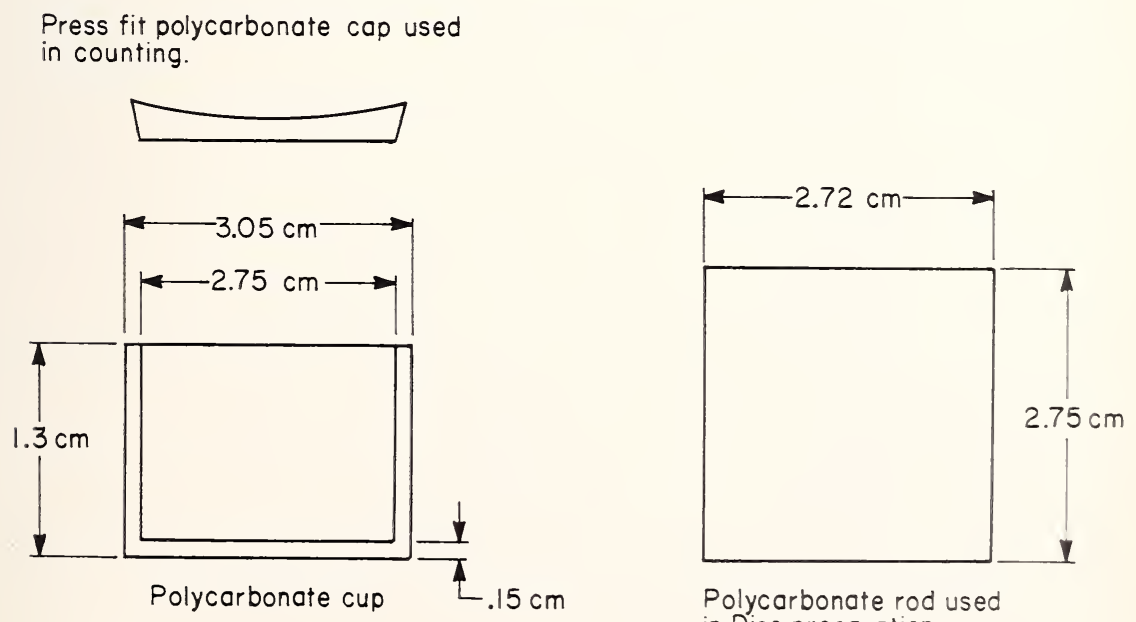

Polycarbonate rod used in Disc preparation

Figure 2. Schematic illustration of cylindrical polycarbonate cup and accessories used for making polyethylene/sample disks and for containing counting sample. 
sembly is placed in a vacuum oven which is then evacuated. This process eliminates air pockets in the final sample disk. The oven is heated to 150 ${ }^{\circ} \mathrm{C}$ and when the polyethylene has melted the assembly is removed from the oven and allowed to cool. When the cylinder is removed the disk (whose diameter is that of the cup and whose height is measurable to 0.05 $\mathrm{cm}$ ) is recovered by simply inverting the cup. Polycarbonate is one of the few materials to which liquid polyethylene has absolutely no tendency to bond. The samples thus prepared are $2.75 \mathrm{~cm}$ diameter disks with parallel faces. They can be compactly packaged for irradiation and are precisely matched to the counting cups used for the subsequent spectral analysis. The press fit lid to the cup secures the sample in place. An auxiliary benefit of this way of handling the particulate samples is that the radioactive particles are securely contained in the polyethylene and contamination hazard is substantially reduced.

The amount of trace element added to the sample by introducing the polyethylene powder is indicated in table 2. The values reported are for disks made from the polyethylene powder as received from the manufacturer. For many types of particulate samples, the trace element blank contributed by the polyethylene is sufficiently low that a blank correction can be made without seriously reducing the accuracy of the trace element analysis of the unknown. For these cases, the use of the described method of potting the sample in a polyethylene disk can be advantageously used.

TABLE 2. Trace element concentrations in polyethylene discs

Element

Sodium

Potassium

Scandium

Manganese

Chromium

Iron

Cobalt

Zinc

Bromine

Antimony

Cerium

Samarium

Europium

Tungsten

Gold
Micrograms element/gram

polyethylene
$1.32 \pm 0.06$

$(2.4 \pm .4) \times 10^{-1}$

$(8.6 \pm 3.4) \times 10^{-5}$

$(7.5 \pm 2.5) \times 10^{-3}$

$(3.8 \pm 0.4) \times 10^{-2}$

$(6.0 \pm 1.2) \times 10^{-1}$

$(1.5 \pm 0.3) \times 10^{-3}$

$(5.8 \pm .5) \times 10^{-1}$

$(2.8 \pm .5) \times 10^{-1}$

$(8.0 \pm .8) \times 10^{-4}$

$(5.2 \pm 1.0) \times 10^{-3}$

$(2.4 \pm 0.8) \times 10^{-5}$

$(5.4 \pm .5) \times 10^{-4}$

$1.38 \pm .07$

$(2.3 \pm .3) \times 10^{-2}$ 


\section{Acknowledgement}

This work was performed under the auspices of the U.S. Atomic Energy Commission. Reference to a company or product name does not imply approval or recommendation of the product by the University of California or the U.S. Atomic Energy Commission to the exclusion of others that may be suitable.

\section{Reference}

[1] Gunnink, R. and Niday, J. B., Computerized Quantitative Analysis by Gamma-Ray Spectrometry, Vol. 1. Description of the GAMANAL Program UCRL-51061 Vol. 1., Lawrence Livermore Laboratory, Livermore, California (March 1972). 



\title{
OBSERVATIONS ON THE QUANTITATIVE ELECTRON PROBE MICROANALYSIS OF PARTICLES
}

\author{
C. E. Fiori, K. F. J. Heinrich,
}

R. L. Myklebust, and M. M. Darr

\author{
Analytical Chemistry Division \\ Institute for Materials Research \\ National Bureau of Standards \\ Washington, D.C. 20234 U.S.A.
}

\begin{abstract}
Elemental analysis of high accuracy with the electron probe microanalyzer is possible for flat specimens which are thicker than the depth of penetration of the electron beam. Existing data reduction procedures convert $\mathrm{x}$-ray intensities to mass fractions of elements with typical accuracies of 1 to 2 percent (relative). These procedures will not work for specimens which are irregularly shaped and smaller than the range of the electron beam, such as dust particles. A Monte Carlo method is being developed to predict the expected $\mathrm{x}$-ray intensity from particles of various shapes, sizes and compositions. We are also developing a method to measure the mass of a particle through the emitted continucus radiation. The expected end result of this work is an algorithm for quantitative microprobe analysis of particles. Experimental parts of this work include the manufacture and analysis of glass fibers and spheres of various diameters and widely varying composition.
\end{abstract}

Keywords: Continuous radiation; electron probe microanalyzer; glass standard; lithium drifted silicon detector; Monte Carlo; particle analysis.

\section{Introduction}

The electron probe microanalyzer is an established tool for the elemental analysis of small regions localized in large solid specimens [1]. The mass of these regions is typically $10^{-11}$ grams. However, the limits of detection are typically in the range from $50 \mathrm{ppm}$ to 1 percent. The technique is thus microanalysis rather than trace analysis.

Normally, quantitative electron probe microanalysis requires a flat 
specimen thicker than the range of the electron beam. Complex data reduction procedures, including iterative approximation, have been developed and cast into computer programs [2]. For such conditions, accuracies on the order of 1 percent relative can be achieved even when standards close in composition to the specimen are not available. However, similar accuracies cannot be obtained in the analysis of particles which are smaller than the range of the electron beam, particularly if their shapes are irregular. The present publication describes several approaches tending toward the development of a practical procedure for the quantitative analysis of small particles.

\section{Monte Carlo Methods}

The quantitative procedures developed for flat, thick specimens cannot be easily adapted to the shapes of small particles. Therefore, we use Monte Carlo techniques which consist of a theoretical simulation of individual electron paths as a model of $\mathrm{x}$-ray generation. We first establish models for electron deceleration and large-angle electron scattering. Individual paths are generated by applying random numbers for the selection of individual events. A path starts when an electron enters the particle, the surface of which is defined by an equation. The path ends when it crosses this surface again or when the electron has lost its energy. The method can be adapted to different particle shapes by changing the equation for the particle surface.

The macroscopic effects of electron beam - target interactions are obtained by a summation of many individual paths. Effects which can be calculated include the production of characteristic and continuous $\mathrm{x}$-rays and that of backscattered, transmitted and secondary electrons $[3,4]$. We have adapted a method by Duncumb [5], originated for other purposes, to describe the interactions of electrons with cylindrical and spherical targets. Experimental data on x-ray production from nickel cylinders agree well with the predictions of this program (see fig. 1), which is presently being adapted to multi-element targets.

Once we know the $\mathrm{x}$-ray output from particles of defined geometrical form, those for more irregular shapes can be obtained by interpolation. Figure 2 shows that for iron particles the x-ray output from a sphere and cylinder of equal diameter are sufficiently close for such interpolations. 


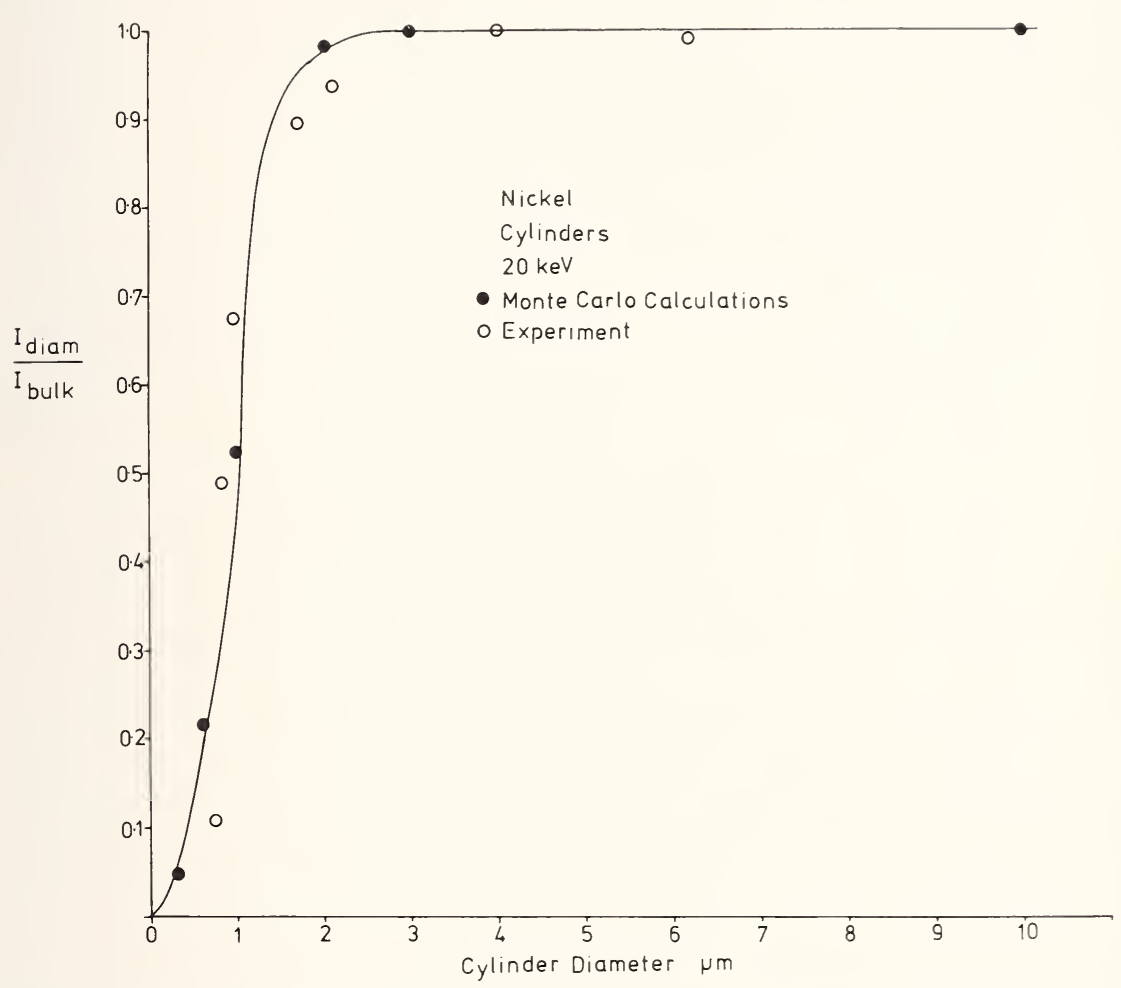

Figure 1. Measured and calculated intensity ratios versus diameter for nickel cylinders of various diameters. 


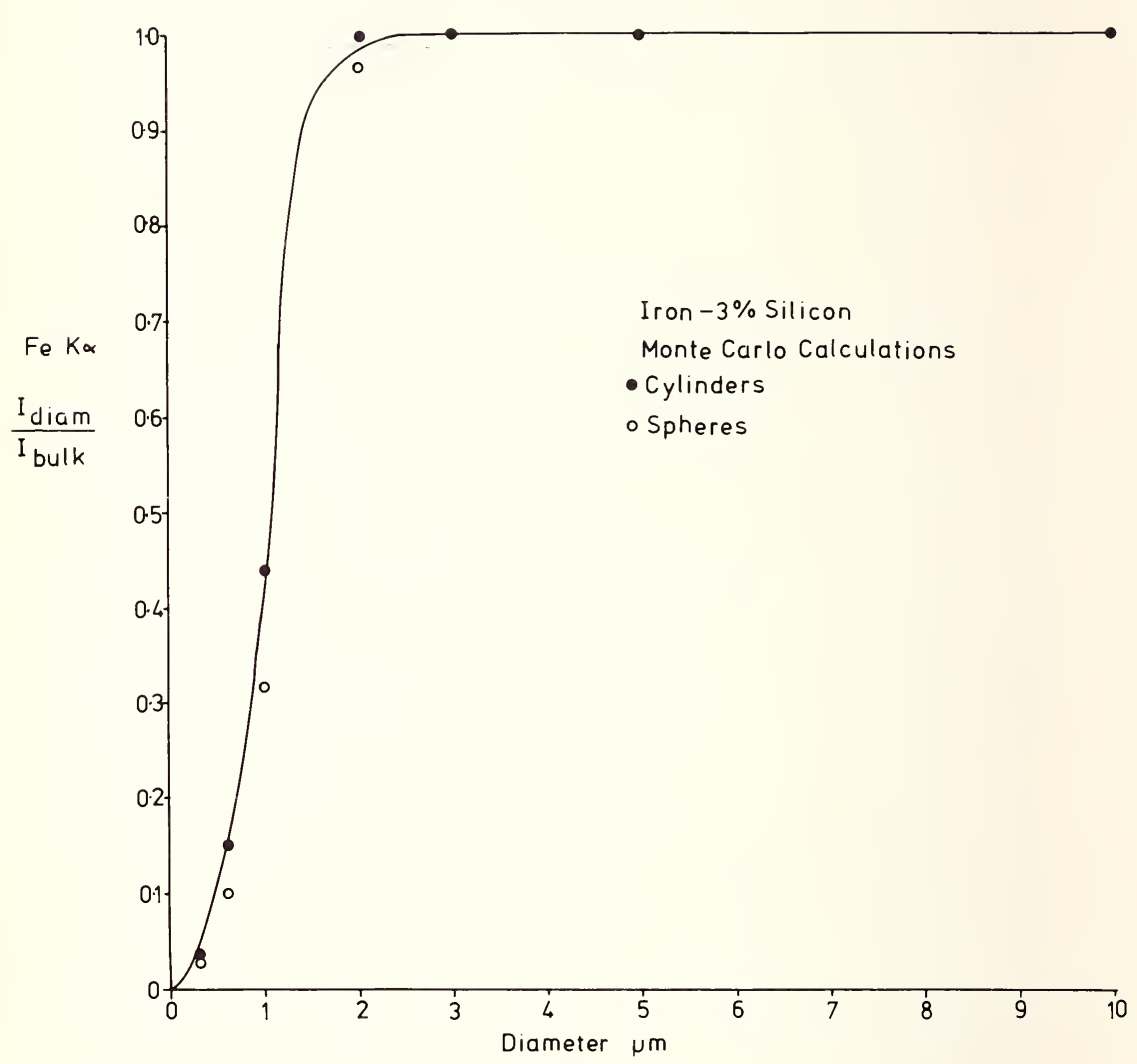

Figure 2. Calculated intensity ratios of $\mathrm{Fe}-3 \% \mathrm{Si}$ spheres and cylinders versus diameter. 


\section{The Continuous Radiation as a Measure of the Mass in a Small Particle}

The microscopic examination of a particle gives only a two-dimensional image and does not reveal the density of the particle. It is therefore advisable to obtain another measurable parameter related to the mass. We propose that the continuous radiation can be used for this purpose as advocated by Hall [6] for thin sections of biological material.

Before studying the continuous radiation from particles, we need to increase our knowledge of the continuum emitted from thick targets. The studies which we are performing in this area are of practical interest to the evaluation of the background of $\mathrm{x}$-ray spectra of thick targets, particularly with the $\mathrm{Si}(\mathrm{Li})$ detector which will also be useful in the analysis of particles due to its high efficiency $[7,8]$.

The technique used in our laboratory is an extension of the studies of Ware and Reed [9] and Lifshin [10]. The algorithm used is the following:

$$
\begin{aligned}
N_{E}=\left[\frac{a\left(E_{0}-E\right)}{E}+\frac{b\left(E_{0}-E\right)^{2}}{E}\right] & \\
& \cdot f(\chi)_{E} \cdot\left[\exp -\left((\mu / \rho)_{E}{ }^{\mathrm{Be}} z_{\mathrm{Be}}+(\mu / \rho)_{E}{ }^{\mathrm{Si}} z_{\mathrm{Si}}\right)\right]
\end{aligned}
$$

The notation used is as follows: The observed count rate within a narrow energy range of x-rays centered around the energy $E$ is $N_{E}$. The energy of the primary impinging electrons is $E_{o}$. The probability of absorption within the target of the emerging x-ray photon of energy $E$ is $\mathrm{f}(\chi)_{E}$. The mass cross-section $\left(\mathrm{g} / \mathrm{cm}^{2}\right)$ of the beryllium window and silicon dead zone of the detector are $z_{\mathrm{Be}}$ and $z_{\mathrm{Si}}$, respectively. The mass absorption coefficients, for the energy $E$, in beryllium and silicon are $(\mu / \rho)_{E}{ }^{\mathrm{Be}}$ and $(\mu / \rho)_{E}{ }^{\mathrm{Si}}$. The quantity within the first bracket of the equation is proportional to the number of photons generated within the target. The following terms describe the absorption of this radiation. The mass cross-sections $z_{\mathrm{Be}}$ and $z_{\mathrm{Si}}$ are determined once for each detector assembly. The continuous radiation under a given set of conditions can be obtained as follows. The value of $N_{E}$ is measured at two energy levels where there are no characteristic lines. Since all terms of equation (1) are known except for $a$ and $b$, we have a system of two equations with two unknowns. Therefore, $a$ and $b$ can be calculated. The value of $N_{E}$ is then defined at any energy $E$.

A minor difficulty is the determination of $\mathrm{f}(\chi)_{E}$. This term can be calculated with sufficient accuracy if it is assumed that the distribution in depth of the continuous radiation is the same as that for characteristic radiation. 
In the application of this algorithm to small particles, this term will have to be modified. A fit of the algorithm for the continuum to experimental data is shown in figure 3 . This equation has been incorporated into the computer program FRAME [11] for the analysis of thick targets with a $\mathrm{Si}(\mathrm{Li})$ detector. The next step in our research will concentrate on the effects of target composition on the intensity of the continuum.

The last phase of this work must concern itself with the experimental determination of both characteristic and continuous radiation from small targets of well-known concentration and geometrical form. For this purpose it has been necessary to prepare suitable experimental samples. For elemental targets, metal wires have been found useful. For the study of multi-element targets, the National Bureau of Standards has been preparing and testing a set of glasses of widely varying composition which are available in both fiber and bulk form. Several other laboratories have also been involved in the testing of these materials. For the production of spherical particles, a method recently proposed by M. Bayard [12] may be useful. In this method, small glass fragments are heated on a surface of vitreous carbon until they contract into spheres.

Copper

$E_{0}=20 \mathrm{keV}$

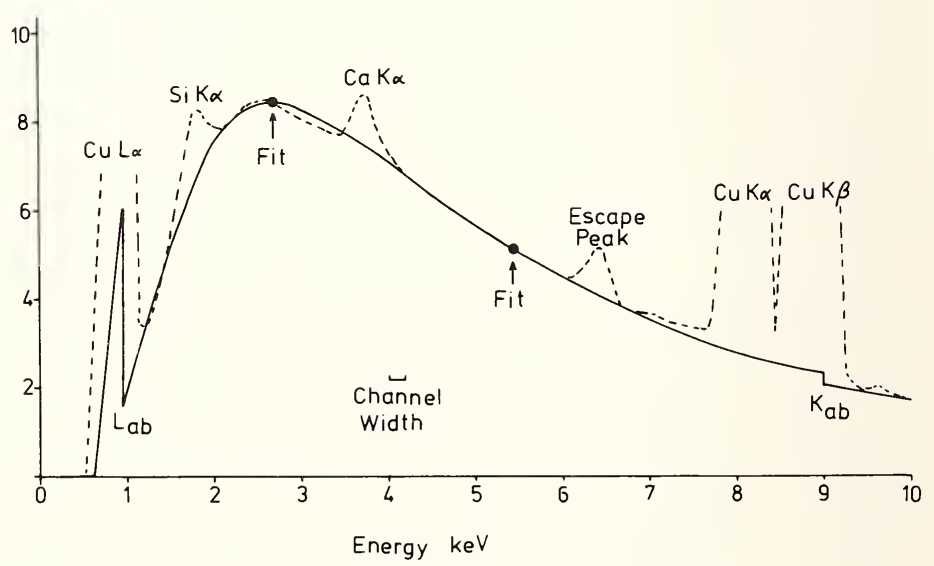

Figure 3. Fit of real data (dashed line) taken from a multi-channel analyzer and calculated background (solid curve), from the values obtained at the points labeled "Fit". $L_{a b}$ and $K_{a b}$ are the $L$ and $K$ absorption edges respectively. 


\section{The Manufacture of Glass Fibers}

Glass fibers are prepared by mixing the appropriate oxides in a platinum crucible and melting at the lowest possible temperature. The glass is stirred with a platinum rod, poured into a mold, and allowed to slowly cool. At this point, it is usually observable if a homogeneous glass has been achieved. If the material has a milky appearance, then it has crystallized and may be inhomogeneous. In the event that the glass is opaque, a corner of the block is knocked off with a hammer. If the fracture surface consists of straight line geometrical forms, then, again, crystallization has occurred. However, if the fracture surface has a conchoidal structure, then it is a glass. Uniformity of color and absence of striations (Schlieren) is also a useful indicator of homogeneity.

A piece of glass is placed in a platinum boat and heated until the melting temperature of the glass is reached. The boat has a small hole in the bottom; the optimum size of this hole is a function of the viscosity and surface tension of the glass. A small bead of glass should form at the bottom of the hole. A stringer is pulled from the bead and attached to a motordriven aluminum drum directly under the bead. When the motor is started the stringer pulls a continuous fiber from the molten bead. The diameter of the fiber is mainly a function of the drum velocity. To date we have obtained one fiber less than 2 micrometers in diameter. The remainder are four micrometers or larger in diameter. Figure 4 is a scanning electron micrograph of a number of these fibers along with a human hair as a reference.

\section{Conclusion}

Recent work in this laboratory has been directed at improving the accuracy of quantitative electron beam microanalysis of small particles. This work is based on a triple approach. (a) A Monte Carlo procedure is being adapted to the study of expected x-ray intensities from small spheres and cylinders of various materials. (b) The continuous radiation is being studied as a means to measure the mass of a small particle. (c) Glass standards, in spherical and fiber form, of widely varying composition are being manufactured to test the models being developed.

Data on x-ray emission from fibers are presently being collected and compared with the predictions given by the Monte Carlo technique. 


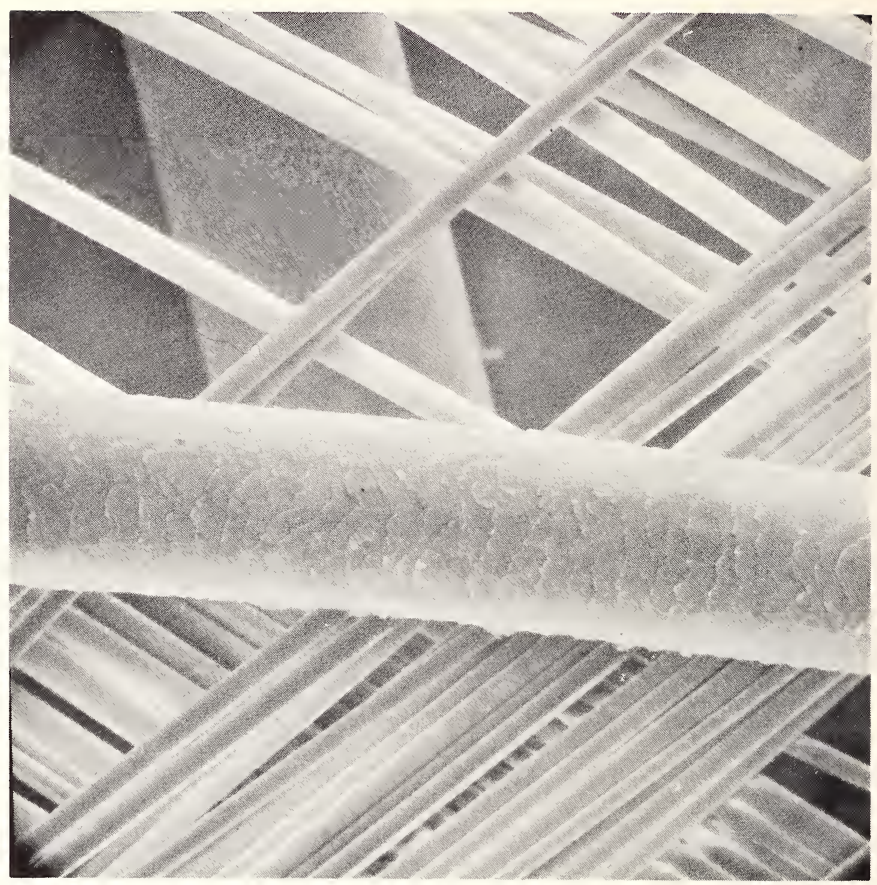

Figure 4. Scanning electron micrograph of a human hair lying over a number of glass fibers.

\section{Acknowledgements}

We are indebted to Douglas Blackburn of the Inorganic Glass Section for providing us with glass fibers. We would also like to acknowledge the cooperation of H. Yakowitz of the Metallurgy Division, and D. E. Newbury of the Analytical Chemistry Division for their work in the Monte Carlo program. Finally, we would like to thank Eric Lifshin of the General Electric Research and Development Center for valuable discussions on the nature of the continuous radiation. 


\section{References}

[1] Birks, L. S., Electron Probe Microanalysis, second edition, Wiley Interscience, New York (1971).

[2] Heinrich, K. F. J., Present State of the Classical Theory of Quantitative Electron Probe Microanalysis, Nat. Bur. Stand. (U.S.), Tech. Note 521, U.S. Government Printing Office, Washington, D.C. 20402 (1970).

[3] Oatley, C. W., The Scanning Electron Microscope, Part I, pp. 134-167, Cambridge University Press, London (1972).

[4] Berger, M. J. and Seltzer, S. M., Penetration of Electrons and Associated Bremsstrahlung Through Aluminum Targets, NASA SP-169 (1968).

[5] Curgenven, L. and Duncumb, P., Tube Investments, Ltd., Research Report No. 303, Essex, England, July 1972.

[6] Hall, T., in Quantitative Electron Probe Microanalysis, Heinrich, K., Ed., p. 269, Nat. Bur. Stand. (U.S.), Spec. Publ. 298, U.S. Government Printing Office, Washington, D.C. 20402 (1967).

[7] Fitzgerald, R., Keil, K., and Heinrich, K. F. J., Solid State Energy-Dispersion Spectrometer for Electron Microprobe Analysis, Science 159, 528-529 (1968).

[8] Heath, R. L., in Advances in X-Ray Analysis, Vol. 15, Heinrich, K. F. J., Barrett, C. S., Newkirk, J. B., and Ruud, C. O., Eds., p. 1, Plenum Press, New York (1972).

[9] Ware, N. G. and Reed, S. J. B., Background Corrections for Quantitative Electron Microprobe Analysis Using a Lithium Drifted Silicon X-Ray Detector, J. Phys. E: Scientific Instruments 1973, Vol. 6 (1973).

[10] Lifshin, E., private communication.

[11] Yakowitz, H., Myklebust, R. L., and Heinrich, K. F. J., FRA ME: An On-Line Correction Procedure for Quantitative Electron Probe Microanalysis, Nat. Bur. Stand. (U.S.), Tech. Note 769, U.S. Government Printing Office, Washington, D.C. 20402 (1973).

[12] Bayard, M., private communication. 



\title{
DETERMINATION OF ELEMENTS IN STANDARD MATERIAL (BOVINE LIVER SRM 1577)
}

\section{Y. Donev and L. M. Marichkova}

\author{
Bulgarian Academy of Sciences \\ Institute for Nuclear Research and Nuclear Energy \\ Section: Physics of Transferred Phenomena \\ in Biological Structures Bldg. Lenin No. 72 \\ Sofia, 13 Bulgaria
}

Investigations for quantitative determination of the elements: bromine, chromium, copper, iron, calcium, sodium, manganese and zinc in standard material Bovine Liver SRM 1577 have been carried out. Nondestructive neutron activation analysis was utilized. Gamma spectrometry was carried out with a $10 \mathrm{~cm}^{3} \mathrm{Ge}(\mathrm{Li})$ detector connected to a 4000 channel pulse height analyzer. In addition, some methodological procedures concerning the preparation of the standards are communicated, especially on the manipulation and origin of individual or systematic errors. Quantitative evaluations of these errors are given. Analytical results of trace element determinations, before and after cleaning, of Bulgarian polyethylene film, used as packaging material, are given.

A short description is also given of the procedure for rapid homogenization and drying of biological material at liquid nitrogen temperature for about 6 hours, where the water removed is about 85 percent. This enrichment is sufficient for investigations with the neutron activation analysis method. It is also important that the drying is carried out at $30^{\circ} \mathrm{C}$, so the danger for losses of different elements such as arsenic, selenium, silver, etc., is reduced.

Keywords: Errors of analysis; homogenization; neutron activation analysis; sample preparation; standard materials; trace element analysis.

\section{Introduction}

Neutron Activation Analysis was used in connection with the Joint Research Coordination Program WHO/IAEA for the determination of the role and the importance of some trace elements in the aetiology and 
the pathogenesis in ischaemic heart diseases. By means of this method the concentrations of the elements $\mathrm{Na}, \mathrm{K}, \mathrm{Mn}, \mathrm{Br}, \mathrm{Cu}, \mathrm{Fe}, \mathrm{Cr}$ and $\mathrm{Zn}$ were determined in the U.S. National Bureau of Standards SRM 1577, Bovine Liver. In fact, by this way, the quantitative interlaboratory control of some elements included in solution standards was carried out. In connection with these investigations a new methodology had to be developed which reduced the general error of the method.

\section{Discussion and Results}

\section{A. General Preliminary Data}

The activation of samples and standards was carried out in the vertical channels of IRT-2000 Reactor, Sofia, with a thermal neutron flux of $5 \times$ $10^{12} \mathrm{n} \cdot \mathrm{cm}^{-2} \cdot \mathrm{s}^{-1}$ and $5 \times 10^{13} \mathrm{n} \cdot \mathrm{cm}^{-2} \cdot \mathrm{s}^{-1}$, with an irradiation time corresponding to the necessary activation.

A Ge(Li) detector of $10 \mathrm{~cm}^{3}$ active volume connected to a 4000 channel Didac pulse height analyzer was used for gamma-ray spectrometry.

Irradiation of samples was carried out in sealed polyethylene capsules with an interior diameter of $2 \mathrm{~cm}$ and a height of $2.5 \mathrm{~cm}$ placed in two rows. The middle of the capsule was placed in the middle of the reactor core, where, according to Christov et al. [1] the neutron flux is homogeneous at a distance of $\pm 2 \mathrm{~cm}$. As the mass of each sample is at the maximum $2.5 \times 10^{-2} \mathrm{~g}$, the total mass in one envelope (capsule) not more than $3 \mathrm{~g}$, and the mass of each standard in the order of $5 \times 10^{-4} \mathrm{~g}$ but not more than $1 \times 10^{-3} \mathrm{~g}$ the neutron self shielding should be ignored and it is not necessary to rotate the capsule during irradiation. When the irradiation time is more than 3 hours, with the high thermal neutron flux density, the sticking of the polyethylene packaging material is avoided by laying the standards in cleaned and very thin aluminum foils, marking each to correspond to that particular standard.

The samples are sealed in polyethylene envelopes which weigh between 1.5 and $3.0 \times 10^{-2} \mathrm{~g}$, i.e., less than the weight of the standards themselves. Special attention is paid to the purity of the package as it is not removed when the gamma spectrometry is carried out. In table 1 are listed the concentrations of the following trace elements: $\mathrm{Na}, \mathrm{K}, \mathrm{Mn}, \mathrm{Cu}$, $\mathrm{Cr}, \mathrm{Zn}, \mathrm{Fe}$ and $\mathrm{Cr}$ before and after the cleaning of the surface of some polyethylene materials produced in Bulgaria, as well as a comparison with literature data [5]. It is evident from the table that after the chemical 
TABLE 1. Concentration of some contaminants found in polyethylene folio (foil) produced by different plants, before and after chemical cleaning ${ }^{3}$

\begin{tabular}{|c|c|c|c|c|c|c|c|c|}
\hline \multirow{2}{*}{ Producer } & \multicolumn{8}{|c|}{ Elements } \\
\hline & $\mathrm{K}$ & $\mathrm{Na}$ & $\mathrm{Cu}$ & $\begin{array}{c}\mathrm{Mn} \\
{\left[\times\left(10^{-2} \mathrm{~kg}\right.\right.}\end{array}$ & $\frac{\mathrm{Zn}}{\left.\left.10^{-3} \mathrm{~kg}\right)\right]}$ & $\mathrm{Br}$ & $\mathrm{Fe}$ & $\mathrm{Cr}$ \\
\hline $\begin{array}{l}\text { I Uncleaned } \\
\text { I Cleaned }\end{array}$ & $\begin{array}{l}2.1 \\
0.1\end{array}$ & $\begin{array}{l}0.4 \\
0.01\end{array}$ & & $\begin{array}{l}0.07 \\
0.03\end{array}$ & $\begin{array}{l}0.9 \\
0.8\end{array}$ & $\begin{array}{l}0.6 \\
0.5\end{array}$ & $\begin{array}{l}1.1 \\
1.0\end{array}$ & $\begin{array}{l}0.001 \\
0.001\end{array}$ \\
\hline $\begin{array}{l}\text { II Uncleaned } \\
\text { II Cleaned }\end{array}$ & $\begin{array}{l}1.8 \\
0.1\end{array}$ & $\begin{array}{l}0.3 \\
0.08\end{array}$ & & $\begin{array}{l}0.07 \\
0.04\end{array}$ & $\begin{array}{l}0.8 \\
0.7\end{array}$ & $\begin{array}{l}0.6 \\
0.5\end{array}$ & $\begin{array}{l}1.2 \\
0.7\end{array}$ & $\begin{array}{l}0.002 \\
0.001\end{array}$ \\
\hline $\begin{array}{l}\text { III Uncleaned } \\
\text { III Cleaned }\end{array}$ & $\begin{array}{l}0.00 \\
0.00\end{array}$ & $\begin{array}{l}0.4 \\
0.09\end{array}$ & $\begin{array}{l}0.01 \\
0.01\end{array}$ & $\begin{array}{l}0.0 \div \\
0.01\end{array}$ & $\begin{array}{l}0.8 \\
0.06\end{array}$ & $\begin{array}{l}0.9 \\
0.08\end{array}$ & $\begin{array}{l}1.0 \\
0.08\end{array}$ & $\begin{array}{l}0.02 \\
0.001\end{array}$ \\
\hline $\begin{array}{l}\text { Low pressure } \\
\text { polyethylene } \\
\text { [5] } \\
\text { High pressure } \\
\text { polyethylene }\end{array}$ & & 0.4 & 0.003 & 0.03 & traces & 0.6 & $\mathrm{ND}^{\circ}$ & 0.066 \\
\hline
\end{tabular}

See text for chemical cleaning procedure.

b $\mathrm{ND}=$ no data.

cleaning, only sodium and potassium were greatly decreased. while the weight of the other elements decreased several times. We should emphasize that the quantities of the elements involved are minimal. and in one envelope with a $3 \times 10^{-2} \mathrm{~g}$ weight. the trace elements are below the threshold of the method sensitivity and thus can be ignored. Table 2 gives precise analytical quantities.

For gamma spectrometry, the total absorption peaks of the elements $\mathrm{Na} . \mathrm{K} . \mathrm{Mn}, \mathrm{Cu} . \mathrm{Br}, \mathrm{Zn}$. Fe and $\mathrm{Cr}$ were measured using standard pulse height analyzer parameters, used with the peaks registered in 11 to 13

TABLE 2. Concentration of some contaminants found in $30 \mathrm{mg}$ of polyethylene packing material after cleaning

\begin{tabular}{llllll} 
Producer & Elements \\
\cline { 2 - 4 } & $\mathrm{K}$ & $\mathrm{Na} \quad \mathrm{Cu} \quad \mathrm{Mn} \quad \mathrm{Zn} \mathrm{Br}$ & $\mathrm{Fe}$ & $\mathrm{Cr}$
\end{tabular}

III Cleaned

$2.7 \quad 0.3$

0.3

$1.8 \quad 2.4$

2.4

0.03 
channels. This allows the simple calculation of their area using the following equation:

$$
I_{p}=I_{i}+\sum_{m=i+1}^{i+4} I_{m}+\sum_{n=i-1}^{i-4} I_{n}-\left(\sum_{p=i+6}^{i+9} I_{p}+\sum_{q=i-6}^{i-9} I_{q}+\frac{\sum I_{p}+\sum I_{q}}{8}\right),
$$

where

$I_{p}=$ the sum of peak impulses of total absorption without the background;

$I_{i}=$ the impulses in the channel with the maximum number of impulses;

$\sum_{m} I_{m}=$ the sum of the impulses in 4 channels after the maximum;
$\sum_{n}^{m} I_{n}=$ the sum of the impulses in 4 channels before the maximum.

The remaining part of the equation represents the sum of background impulses in 9 channels. Statistical treatment of the data is carried out at a significance level of $\mathrm{P}=0.05$.

\section{B. Preparation of a Series of Combined Standards}

It is well known that for the preparation of accurate standards there are significant difficulties. The preparation of a large series of standards as long as the solutions are fresh is recommended for many reasons. The storage of a great number of initial solutions in quartz containers is a needless expense. In practice, solutions of elements whose concentrations are approximately 1 in $1 \times 10^{-8} \mathrm{~m}^{3}$ have proved to be very convenient for our work. Also, combined standards containing elements with the above mentioned quantities, whose radioactive isotopes have almost the same half-lives, have proved to be convenient.

For the preparation of large series of combined standards Hamilton Teflon syringes (models: TF 1725, TF 1750 and TF 1001), Teflon needles with KEL-F hubs, and a repeating dispenser (model PB 600-1) were used.

The combinations of elements most used by us for the preparation of standards contain odd numbers of elements, but not more than 10. With these syringes it is easy to obtain different combinations. For the determination of weighing errors of the serial standard preparations, an identical volume was used with all three syringes. The results obtained are listed in table 3 . The weight loss is due to the evaporation of distilled 
TABLE 3. Mean weight, maximum and minimum values, as well as confidence interval of the mean weight of weighed samples from distilled water dispensed by three different repeating Teflon syringes

\begin{tabular}{|c|c|c|c|c|c|}
\hline \multirow{2}{*}{$\begin{array}{l}\text { Syringe } \\
\text { model }\end{array}$} & \multirow{2}{*}{$\begin{array}{c}\text { Total } \\
\text { volume } \\
(\mu 1)\end{array}$} & \multirow{2}{*}{$\begin{array}{l}\text { Sample } \\
\text { (n) }\end{array}$} & \multicolumn{3}{|c|}{$\begin{array}{c}\text { Weight } \\
{\left[\times\left(10^{-5} \mathrm{~kg}\right)\right]}\end{array}$} \\
\hline & & & $\begin{array}{l}\text { Syringe } \\
\text { reading }\end{array}$ & & $\begin{array}{l}\text { Amount } \\
\text { measured }\end{array}$ \\
\hline 1725 & 250 & 15 & 2.00 & $\begin{array}{l}\min \\
\max \end{array}$ & $\begin{array}{l}1.937 \pm 0.005 \\
1.90 \\
1.98\end{array}$ \\
\hline 1750 & 500 & 21 & 2.00 & & $\begin{array}{l}1.941 \pm 0.006 \\
1.88 \\
1.98\end{array}$ \\
\hline 1001 & 1000 & 19 & 2.00 & & $\begin{array}{l}1.958=0.006 \\
1.91 \\
1.99\end{array}$ \\
\hline
\end{tabular}

water, as the measurements were carried out at exactly 3 minutes after the discharge. It was established that the repeating syringe measures identical volumes, independently of whether the syringe piston position is at the top, in the middle or at the bottom of the utilized volume.

These syringes were used for the discharge of the same volume of ${ }^{51} \mathrm{Cr}$ radioactive solution, and placed in polyethylene envelopes. Due to inaccuracy of geometry calculation, a deficiency of 50 to 60 impulses was established for the preliminary counts, measured with a $\mathrm{NaI}$ detector. The results are shown in table 4. Mass evaluation is done on the basis of the mean number of counts for a weight of $0.02 \mathrm{~g}$. No significant difference between the minimum weight and the solution discharged with the three syringes was established. The mean values, together with the confidence interval, prove the convenience of these syringes for the preparation of a series of accurate standards.

The same technique was used for the verification of a series of combined standards. Using TF 1725 and TF 1750 syringes, a series containing: $1.020 \times 10^{-5} \mathrm{~g} \mathrm{Na}, 1.015 \times 10^{-4} \mathrm{~g} \mathrm{~K}$, and $1.010 \times 10^{-5} \mathrm{~g} \mathrm{Cu}$ in each standard was prepared. With the syringe TF 1001 standards of $1.004 \times 10^{-5} \mathrm{~g} \mathrm{Zn}$ and $1.001 \times 10^{-6} \mathrm{~g} \mathrm{Cr}$ were prepared. From the first series 38 standards were investigated, and from the second. 21 standards. The results obtained are given in table 5. The difference between discharged and measured quantities was carried out using SRM 1577 and Bowen's Kale, where the values of $\mathrm{Cr}$ are the ones determined by our 


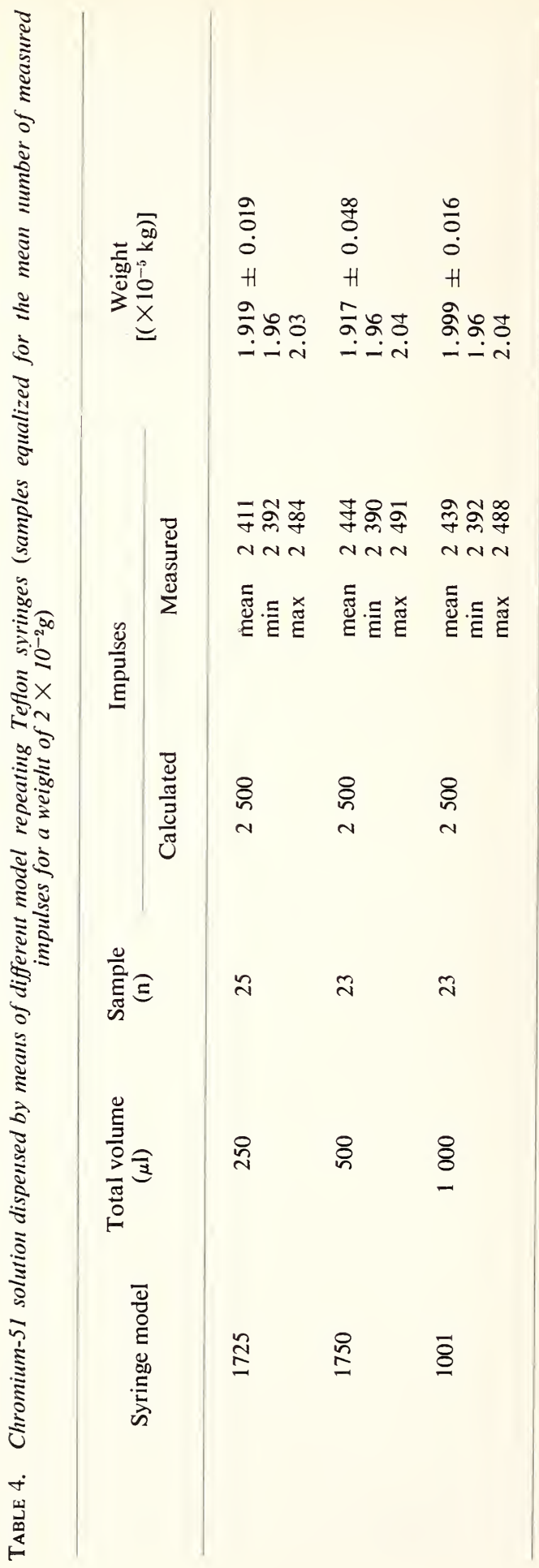




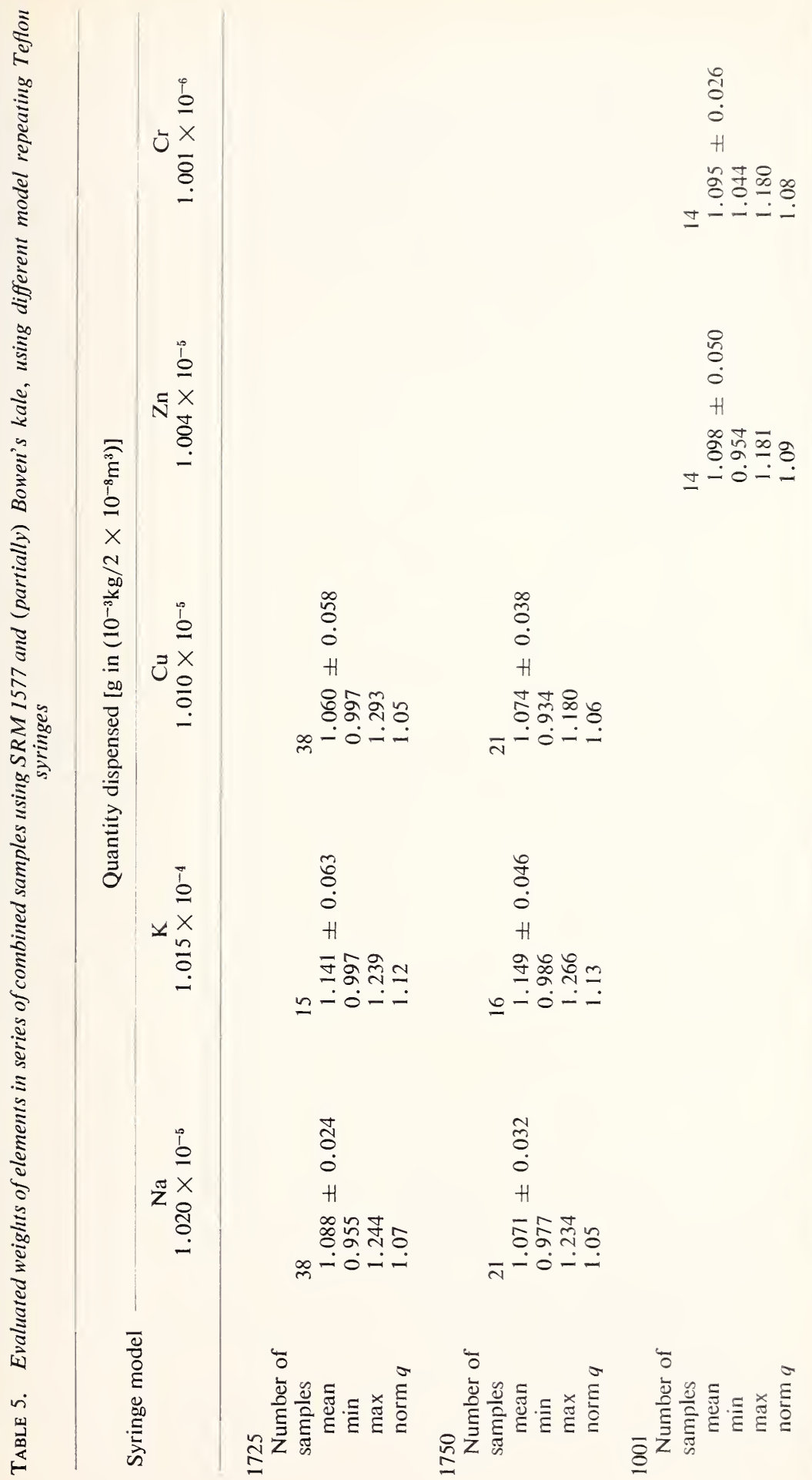


laboratory. The results obtained show relatively small confidence intervals of the mean values. The correlation coefficients close to unity prove the suitability of the chemical compounds utilized for the preparation of the standard solutions. The very close mean values of the standards prepared with different volume syringes, as well as the close confidence intervals of them, show their mutual dependence. This was confirmed also by the subsequent investigations.

In the same table, with exception of the correlation coefficients for $\mathrm{K}$, they differ from unity in the second decimal. The difference between the investigated element $\mathrm{K}$, and $\mathrm{Na}$ and $\mathrm{Cu}$ standards, is due to the more feeble activation of potassium, and consequently, the smaller number of impulses at the peak of total absorption. For reliability, only results are accepted which correspond to the formula:

$$
\mathbf{I}_{p} \geqslant \sqrt{\mathbf{I}_{b}}
$$

where:

$\mathrm{I}_{p}=$ pure impulses of the total absorption peak

$I_{b}=$ the background impulses below the total absorption peak, thus explaining the above mentioned difference.

With these already tested syringes and the already known corrective factors for each element, investigations have been carried out for the determination of the elements: $\mathrm{Na}, \mathrm{K}, \mathrm{Br}, \mathrm{Cu}, \mathrm{Mn}, \mathrm{Fe}, \mathrm{Cr}$ and $\mathrm{Zn}$ in SRM 1577. Three series of standards were prepared, namely: $\mathrm{Na}+\mathrm{K}+$ $\mathrm{Br}+\mathrm{Cu}, \mathrm{Zn}+\mathrm{Fe}+\mathrm{Cr}$ and $\mathrm{Mn}$. Together with the Bovine Liver, samples of Bowen's Kale and of 3 to 5 standards were placed. The number of the elements in the standards, their initial solutions, the purity of the chemical compounds or of the metals utilized for the preparation of the initial solutions, are given in table 6 along with the procedure for their preparation.

\section{Trace Element Concentrations in SRM 1577}

The specified and compared results, obtained by determination of elements in serial standards and Bowen's Kale, are listed in table 7. The mean values of the elements are given along with their confidence interval, at a significance of $P=0.050$. This table also shows some results obtained by other authors for the same elements [7 to 13]. It should be taken into consideration, that our investigations were carried out using neutron activation analysis only.

The quantity of bromine found varies in a very large range - from 7.49 to $12.19 \times 10^{-6} \mathrm{~g}$, a fact which we explain by the differences of the sam- 


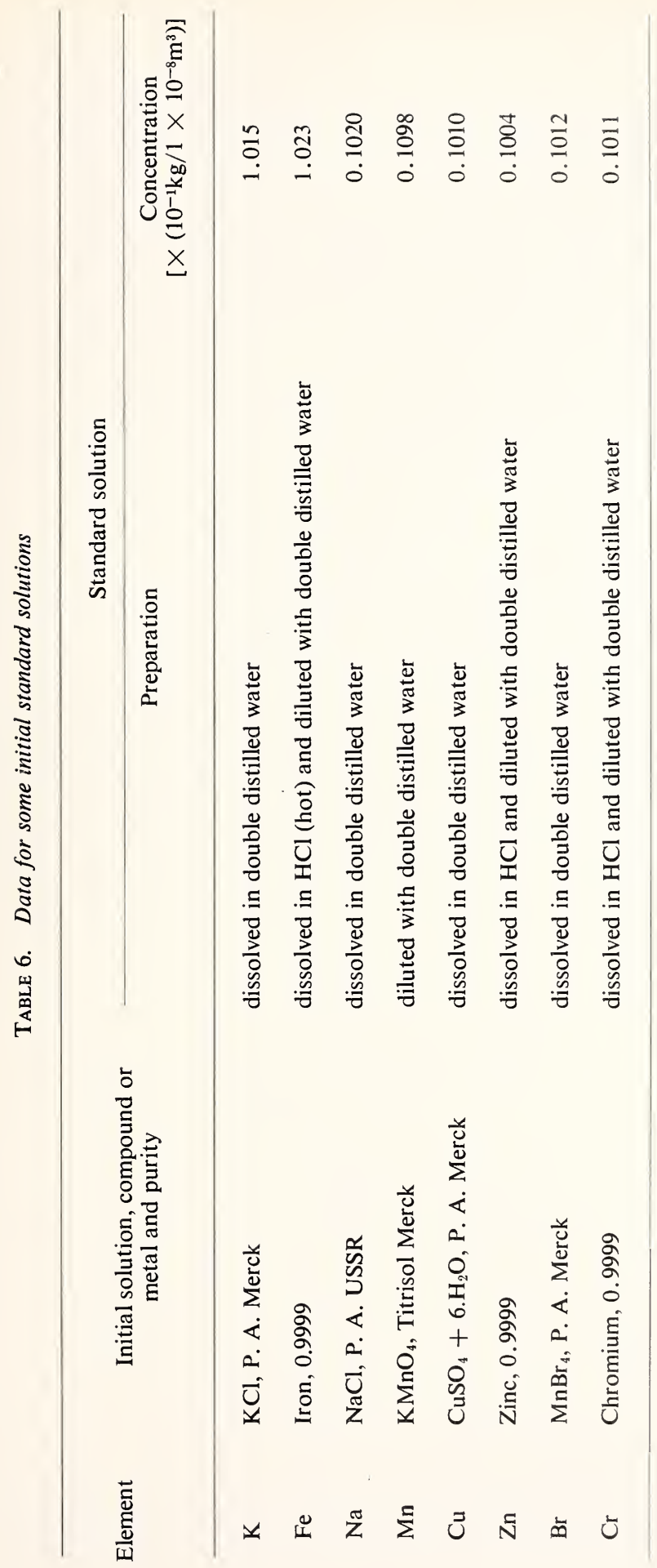




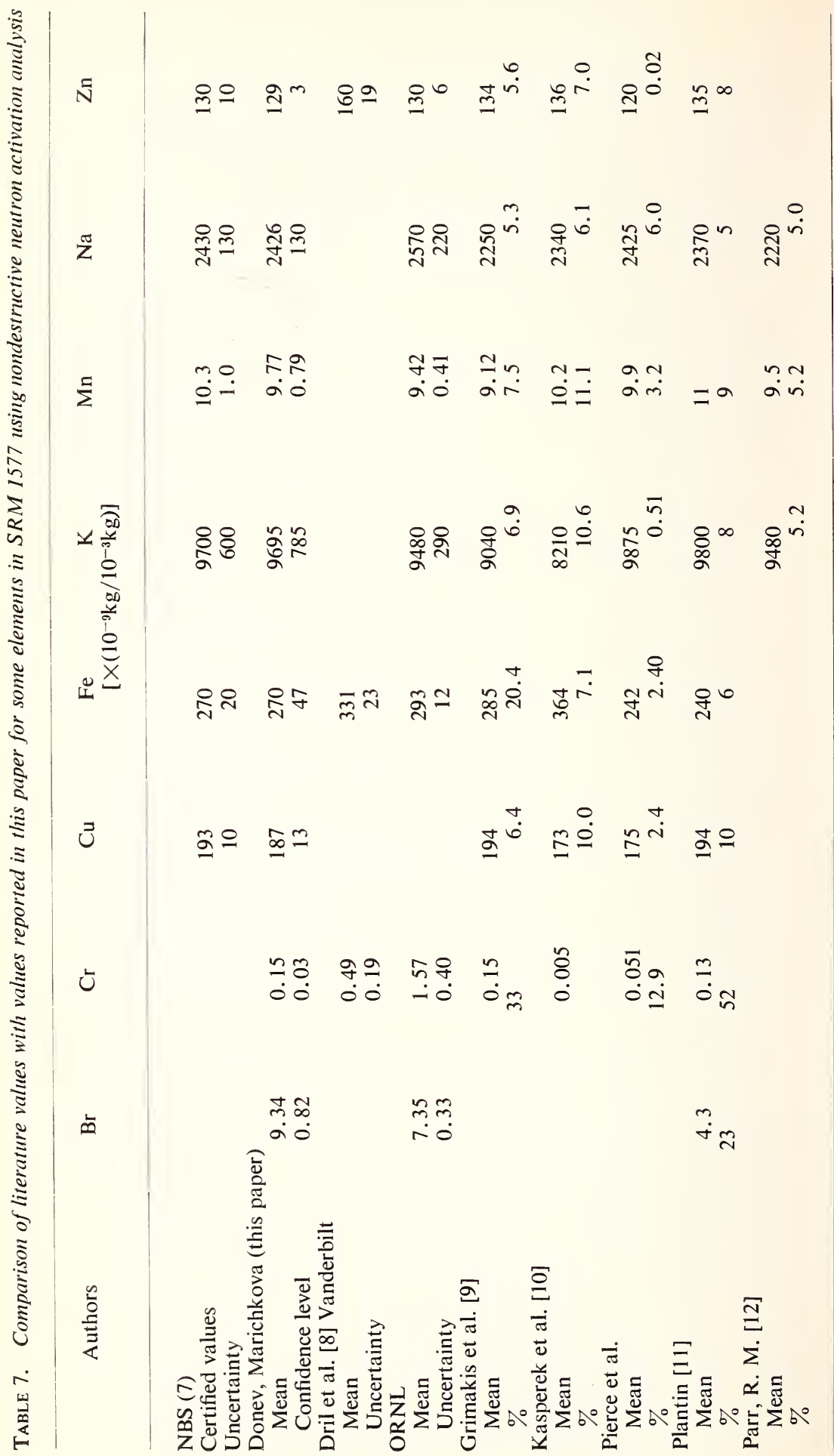


ples' mass and their activation, and consequently, the influence of the pulse pile-up effect. as well as the high value of the background.

\section{Rapid Homogenization and Drying of Biomaterials}

It is known. that the homogenization of organ sections requires much time, and the trace-element analysis is limited to special conditions for the prevention of contamination. On the other hand. the drying of the homogenates at a higher temperature than room temperature is accompanied with the risk of losing trace elements, a risk which increases with temperature. For almost 3 years we are evaluating drying, with very good results, at the temperature level of liquid nitrogen.

Homogenization and drying is carried out in one and the same homogenizer, which, by means of a conical joint, is connected to the vacuum installation. Figure 1 gives us the scheme of the combined homogenizer. It consists of a homogenizer for fine mincing, fabricated of quartz glass (1). in the tight part of which is a stroking Teflon piston with a Plexiglas handle, and in its widened part is placed a second homogenizer (3) for rough mincing. in the interior of which is working the same piston (2). After the homogenization of the biomaterials, we proceed to the laying of a thin film over the inside walls of the quartz homogenizer and to be frozen immediately to liquid nitrogen temperature. The vacuum installa-

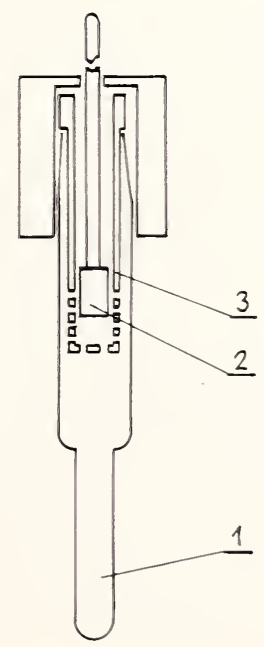

Figure 1. Scheme of the combined homogenizer. 1. Exterior homogenizer is fabricated of quartz glass and is intended for the fine and dry homogenization of biomaterials: 2. Teflon piston with Plexiglas holder; 3 . Plexiglas homogenizer for rough mincing of biomaterials. 
tion allows the simultaneous connection, by means of the ground glass joints, of 13 homogenates. The freezing is interrupted 30 minutes after initiating an ordinary vacuum pump. The temperature increases gradually, but not more than $30{ }^{\circ} \mathrm{C}$ and after 6 hours (depending on the quantity of the homogenates in the different homogenizers) the drying process is stopped. The dry homogenization is carried out in the same homogenizer with a cleaned and dried piston. The maximum volume capacity of our homogenizers is approximately $15 \times 10^{-6}\left(\sim 15 \mathrm{~cm}^{3}\right)$, and the dried residue is about 20 to 25 percent of the wet weight.

The homogenization of 13 samples including the drying and the dry mincing, as well as the operation of the vacuum installation, is managed by one laboratory assistant in 8 to 9 hours.

\section{References}

[1] Christov, V. Y., Isvestia na Phisitcheskia Institut, BAN 1-2, 210 (1963).

[2] Bowen, H. I. and Cowse, P. A., AERA -R 4309, Berkshire (1959).

[3] Plunb, R. C. and Jervis, I. E., Nucleonics, 13, 42 (1955).

[4] Morsen, P., Kernenergie 5, 839 (1962).

[5] Dubinskaya, H. A., Ljul, A. H., Pelkis, A. L., Jadernaya spectrotrosmopia i neitrono-aktivationni analis 33, (1965) Riga.

[6] Hamilton - catalog 1970.

[7] Cali J. P., NBS, Certificate of Analysis, Standard Reference Material 1577, Washington, D.C. 20234.

[8] Brill, A. B., Page, D. et al., Neutron Activation Analysis and Atomic Absorption Analysis of 18 Elements in Relation to a Study of Trace Elements in Cardiovascular Diseases, IAEA-157,(1973), pp. 5-12.

[9] Grimanis, A. P. et al., Neutron Activation Analysis Methods for the Determination of 14 Trace Elements in Tissue Samples. Results Obtained with Analytical Reference Materials IAEA-157, (1973), pp. 29-49.

[10] Kasperek, K., Schicha, H., and Weese, H., Analytical Techniques for the Determination of 15 Elements in Connection with the WHO/IAEA Joint Research Programme on Trace Elements in Cardiovascular Diseases, IAEA-157, (1973) pp. 7176.

[11] Plantin, L. O., Multielement Neutron Activation Analysis in Relation to a Study of Trace Elements in Cardiovascular Diseases, IAEA-157 (1973) pp. 91-102.

[12] Parr, R. M., Summary Report and Recommendations, IAEA-157, (1973), pp. 191204. 
NBS-114A (REV. 7-73)

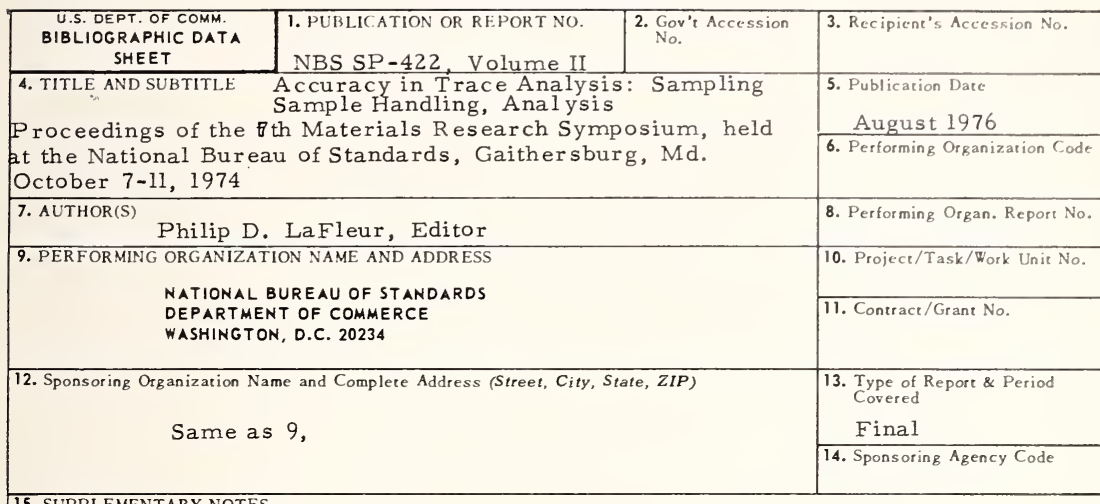

\section{SUPPLEMENTARY NOTES}

Library of Congress Catalog Card Number: 75-619250

16. ABSTRACT (A 200-word or less factual summary of most significant information. If document includes a significant bibliography or literature survey, mention it here.)

This book is the formal report of the proceedings of the 7th Materials Research Symposium: Accuracy in Trace Analysis. This volume contains the invited and contributed papers presented at the symposium, and which treat problems of sampling and sampling handling as well as the usually-discussed analytical methodology. Many important techniques and methods are described, and extensive references are presented, to give deeper insight into the problems of obtaining accurate results in trace analytical chemistry. Accordingly, this volume should provide a valuable guide for everyday analytical problems.

17. KEY WORDS (six to twelve entries; alphabetical order; capitalize only the first letter of the first key word unless a proper name; separated by semicolons)

Accuracy; analysis; analytical chemistry; sampling; sample handling; trace analysis.

18. AVAILABILITY

$$
\text { X Unlimited }
$$

— For Official Distribution. Do Not Release to NTIS

X Order From Sup. of Doc., U.S. Government Prjeting Office Washington, D.C. 20402, SD Cat. No.CI3. IO:422 vol. II

$\square$ Order From National Technical Information Service (NTIS) Springfield, Virginia 22151

\begin{tabular}{|l|c|}
\hline $\begin{array}{l}\text { 19. SECURITY CLASS } \\
\text { (THIS REPURT) }\end{array}$ & 21. NO. OF PAGES \\
UNCL ASSIFIED & 636 \\
\hline $\begin{array}{l}\text { 20. SECURITY CLASS } \\
\text { (THIS PAGE) }\end{array}$ & 22. Price \\
S20 per \\
UNCLASSIFIED & 2 -part set \\
\hline
\end{tabular}








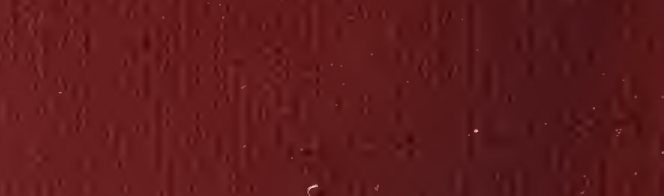

I:

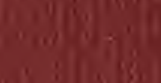

(3) $3(x)$

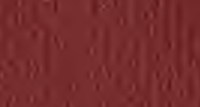

? $\left|\frac{1}{3}\right|$

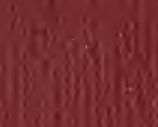

(2) 8 (1)

(4) $(3)$

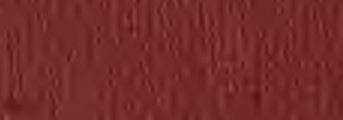

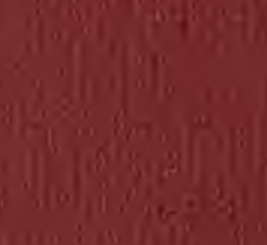

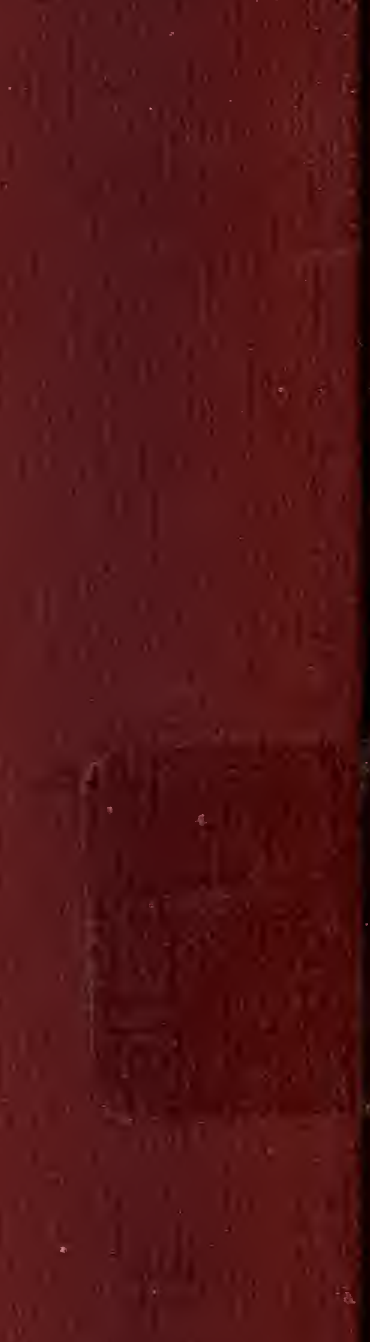

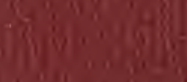

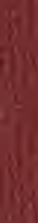

$3\left(\begin{array}{l}\text { S. } \\ \text { (3) }\end{array}\right.$

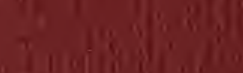

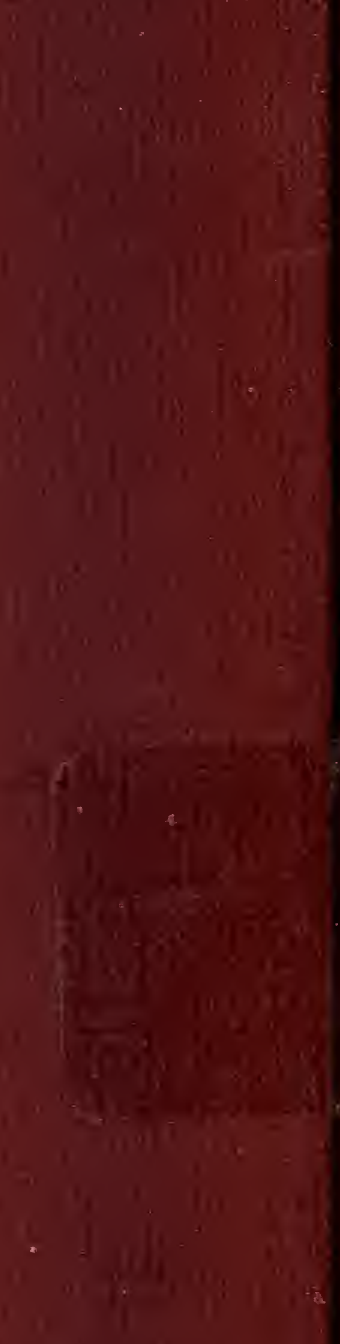

(iiv +1$)$

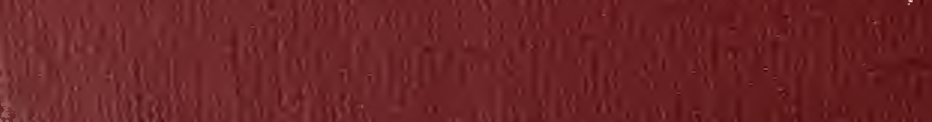

ach Som 